\title{
Code Manual for CONTAIN 2.0: A Computer Code for Nuclear Reactor Containment Analysis
}

Manuscript Completed: June 1997

Date Published: December 1997

Prepared by

K. K. Murata, D. C. Williams, J. Tills, *

R. O. Griffith, R. G. Gido, E. L. Tadios,

F. J. Davis, G. M. Martinez, K. E. Washington

Sandia National Laboratories

Albuquerque, NM 87185

Subcontractor:

*J. Tills \& Associates, Inc.

Sandia Park, NM 87047

DISTREUTTON OF THIS DOCUMENT IS UNERATED

A. Notafrancesco, NRC Project Manager

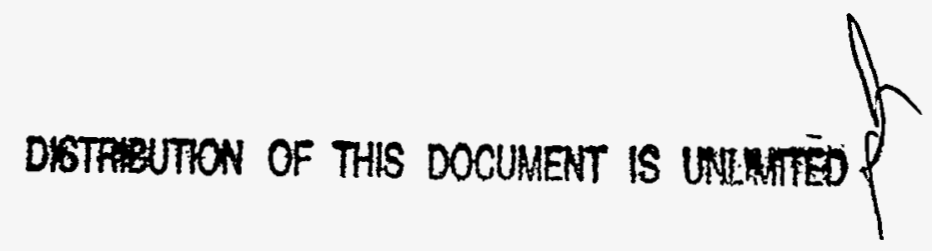

\section{Prepared for}

Division of Systems Technology

Office of Nuclear Regulatory Research

U.S. Nuclear Regulatory Commission

Washington, DC 20555-0001

NRC Job Code A1198

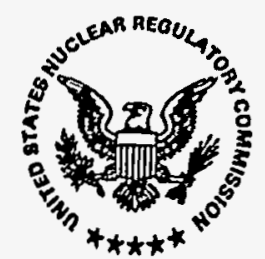




\section{AVAILABILITY NOTICE}

Avaliability of Reference Materials Cited in NRC Publications

Most documents cited in NRC publications will be available from one of the following sources:

1. The NRC Public Document Room, 2120 L Street, NW., Lower Level, Washington, DC 20555-0001

2. The Superintendent of Documents, U.S. Government Printing Office, P. O. Box 37082, Washington, DC 20402-9328

3. The National Technical Information Service, Springfield, VA 22161-0002

Although the listing that follows represents the majority of documents cited in NRC publications, it is not intended to be exhaustive.

Referenced documents available for inspection and copying for a fee from the NRC Public Document Room include NRC correspondence and internal NRC memoranda; NRC bulletins, circulars, information notices, inspection and investigation notices; licensee event reports; vendor reports and correspondence; Commission papers; and applicant and licensee documents and correspondence.

The following documents in the NUREG series are available for purchase from the Government Printing Office: formal NRC staff and contractor reports, NRC-sponsored conference proceedings, international agreement reports, grantee reports, and NRC booklets and brochures. Also available are regulatory guides, NRC regulations in the Code of Federal Regulations, and Nuclear Regulatory Commission Issuances.

Documents available from the National Technical information Service include NUREG-series reports and technical reports prepared by other Federal agencies and reports prepared by the Atomic Energy Commission, forerunner agency to the Nuclear Regulatory Commission.

Documents available from public and special technical libraries include all open literature items, such as books, journal articles, and transactions. Federal Register notices. Federal and State legislation, and congressional reports can usually be obtained from these libraries.

Documents such as theses, dissertations, foreign reports and translations, and non-NRC conference proceedings are available for purchase from the organization sponsoring the publication cited.

Single copies of NRC draft reports are available free, to the extent of supply, upon written request to the Office of Administration, Distribution and Mail Services Section, U.S. Nuclear Regulatory Commission, Washington, DC 20555-0001.

Copies of industry codes and standards used in a substantive manner in the NRC regulatory process are maintained at the NRC Library. Two White Flint North, 11545 Rockville Pike, Rockville, MD 20852-2738, for use by the public. Codes and standards are usually copyrighted and may be purchased from the originating organization or, if they are American National Standards. from the American National Standards Institute, 1430 Broadway, New York, NY 10018-3308.

\section{DISCLAIMER NOTICE}

This report was prepared as an account of work sponsored by an agency of the United States Government. Neither the United States Govemment nor any agency thereof, norany of their employees, makes any warranty, expressed or implied, or assumes any legal liability or responsibility for any third party's use, or the results of such use, of any information, apparatus, product, or process disclosed in this report, or represents that its use by such third party would not infringe privately owned rights. 


\section{DISCLAIMER}

This report was prepared as an account of work sponsored by an agency of the United States Government. Neither the United States Government nor any agency thereof, nor any of their employees, makes any warranty, express or implied, or assumes any legal liability or responsibility for the accuracy, completeness, or usefulness of any information, apparatus, product, or process disclosed, or represents that its use would not infringe privately owned rights. Reference herein to any specific commercial product, process, or service by trade name, trademark, manufacturer, or otherwise does not necessarily constitute or imply its endorsement, recommendation, or favoring by the United States Government or any agency thereof. The views and opinions of authors expressed herein do not necessarily state or reflect those of the United States Government or any agency thereof. 


\section{DISCLAIMER}

Portions of this document may be illegible electronic image products. Images are produced from the best available original document. 


\begin{abstract}
The CONTAIN 2.0 computer code is an integrated analysis tool used for predicting the physical conditions, chemical compositions, and distributions of radiological materials inside a containment building following the release of material from the primary system in a light-water reactor accident. It can also predict the source term to the environment. CONTANN 2.0 is intended to replace the earlier CONTAIN 1.12, which was released in 1991. The purpose of this Code Manual is to provide full documentation of the features and models in CONTAIN 2.0. Besides complete descriptions of the models, this Code Manual provides a complete description of the input and output from the code. CONTAIN 2.0 is a highly flexible and modular code that can run problems that are either quite simple or highly complex. An important aspect of CONTAIN is that the interactions among thermalhydraulic phenomena, aerosol behavior, and fission product behavior are taken into account. The code includes atmospheric models for steam/air thermodynamics, intercell flows, condensation/ evaporation on structures and aerosols, aerosol behavior, and gas combustion. It also includes models for reactor cavity phenomena such as core-concrete interactions and coolant pool boiling. Heat conduction in structures, fission product decay and transport, radioactive decay heating, and the thermal-hydraulic and fission product decontamination effects of engineered safety features are also modeled. To the extent possible, the best available models for severe accident phenomena have been incorporated into CONTAIN, but it is intrinsic to the nature of accident analysis that significant uncertainty exists regarding numerous phenomena. In those cases, sensitivity studies can be performed with CONTANN by means of user-specified input parameters. Thus, the code can be viewed as a tool designed to assist the knowledgeable reactor safety analyst in evaluating the consequences of specific modeling assumptions.
\end{abstract}


-

-

- 


\section{TABLE OF CONTENTS}

ABSTRACT $\ldots \ldots \ldots \ldots \ldots \ldots \ldots \ldots \ldots \ldots \ldots \ldots \ldots \ldots \ldots \ldots \ldots \ldots \ldots$

TABLE OF CONTENTS $\ldots \ldots \ldots \ldots \ldots \ldots \ldots \ldots \ldots \ldots \ldots \ldots \ldots \ldots \ldots \ldots$

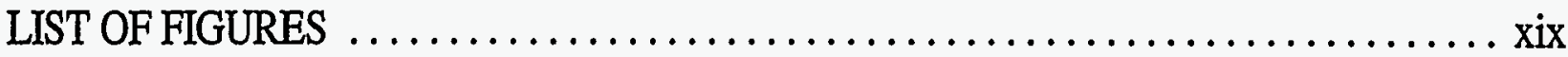

LIST OF TABLES $\ldots \ldots \ldots \ldots \ldots \ldots \ldots \ldots \ldots \ldots \ldots \ldots \ldots \ldots \ldots \ldots \ldots \ldots \ldots \ldots$

LIST OF ABBREVIATIONS $\ldots \ldots \ldots \ldots \ldots \ldots \ldots \ldots \ldots \ldots \ldots \ldots \ldots \ldots \ldots \ldots$

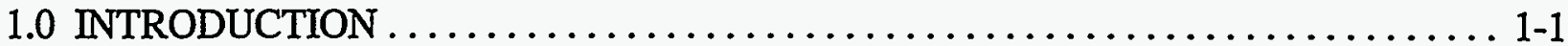

1.1 The Need for Nuclear Reactor Containment Analysis . . . . . . . . . . . 1-2

1.2 Differences Between CONTAIN 2.0 and Earlier Versions . . . . . . . . . . 1-4

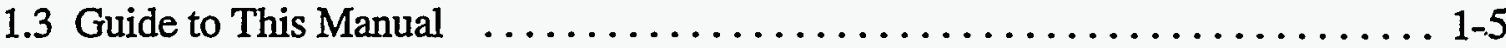

2.0 GENERAL DESCRIPTION OF THE CODE MODELS $\ldots \ldots \ldots \ldots \ldots \ldots \ldots \ldots .2-1$

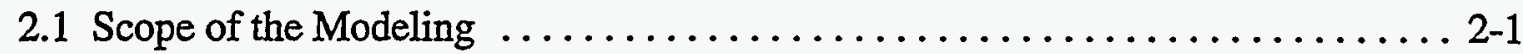

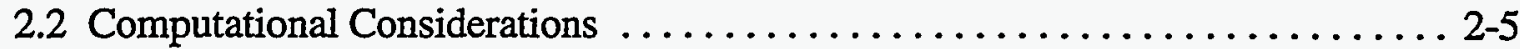

2.2.1 The Bi-Level Modeling Approach . . . . . . . . . . . . . 2-5

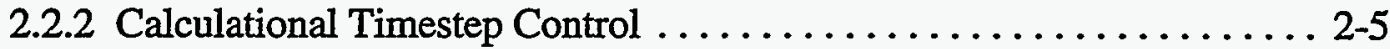

2.2.2.1 Timestep Hierarchy . . . . . . . . . . . . . . . . 2-7

2.2.2.2 Suggested Criteria for User-Specified Timesteps . . . . . . . . 2-8

2.3 Material Properties ................................ 2-10

2.4 Atmosphere/Pool Thermal-Hydraulics and Intercell Flow . . . . . . . . 2-12

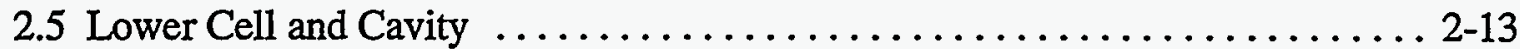

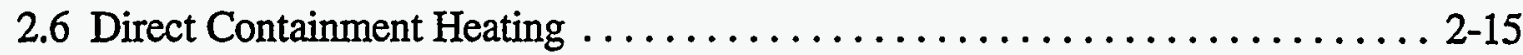

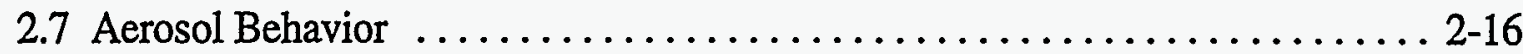

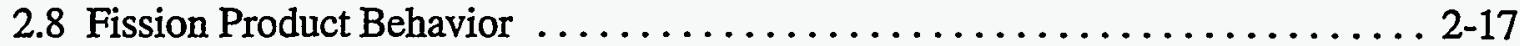

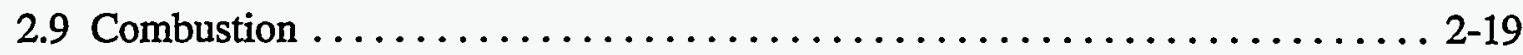

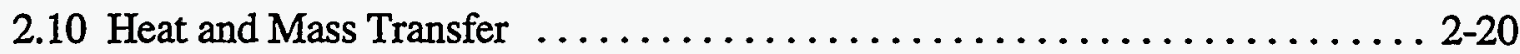

2.11 Boiling Water Reactor and Related Models . . . . . . . . . . . . . 2-22

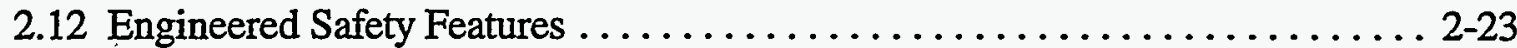

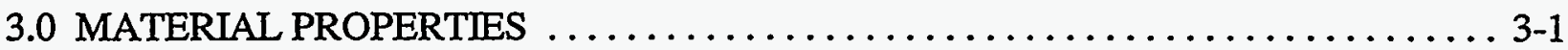

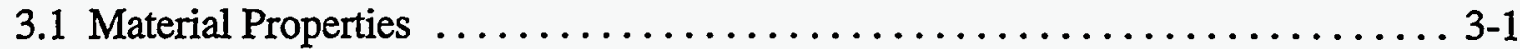

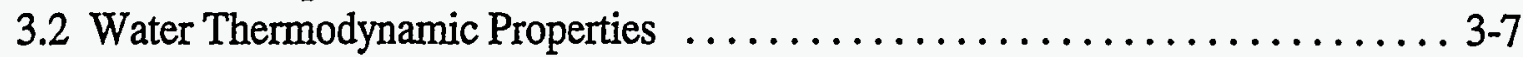

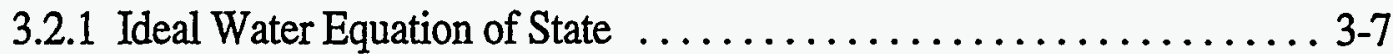

3.2 .2 Non-Ideal Water Equation of State .................... 3-8

3.2.2.1 Representation of the Water Equation of State . . . . . . . . 3-9

3.2.2.2 Extrapolation of the Water Saturation Properties . . . . . . . 3-11

3.2.2.3 Modeling Limitations . . . . . . . . . . . . . . . . 3-12

3.3 Bulk Gas Thermophysical Properties ....................... 3-14

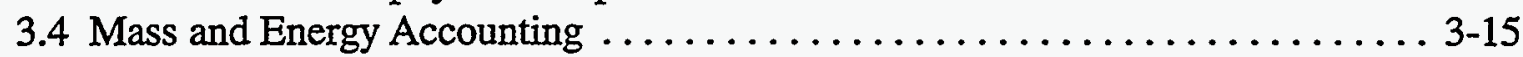




\section{TABLE OF CONTENTS (CONTINUED)}

4.0 ATMOSPHERE/POOL THERMODYNAMIC AND INTERCELL FLOW MODELS . . 4-1

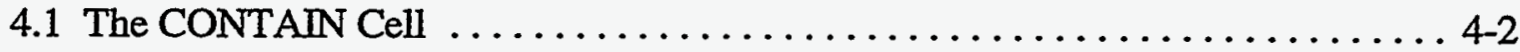

4.2 Flow Path Configurations $\ldots \ldots \ldots \ldots \ldots \ldots \ldots \ldots \ldots \ldots \ldots \ldots, 4,4$

4.3 Flow Path Modeling Options $\ldots \ldots \ldots \ldots \ldots \ldots \ldots \ldots \ldots \ldots \ldots \ldots \ldots .11$

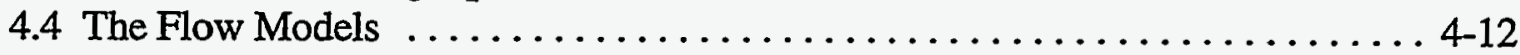

4.4.1 Inertial Flow Model .......................... 4 43

4.4.2 Quasi-Steady Flow Model ...................... 4-13

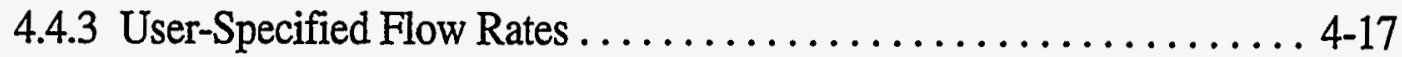

4.4.4 Critical Flow Model ........................... 4-17

4.4.5 Gravitational Head Modeling . . . . . . . . . . . .

4.4.5.1 Gas Flow Path Gravitational Heads ............... 4-19

4.4.5.2 Pool Flow Path Gravitational Heads ................ 4-25

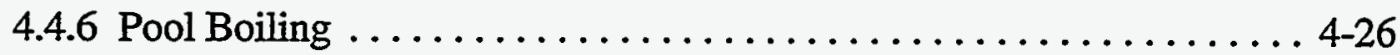

4.4.7 Gas-Pool Equilibration ....................... 4-36

4.4.8 The Gas Evolution Velocity at the Pool-Atmosphere Interface ..... . 4-39

4.4.9 The FIX-FLOW Option $\ldots \ldots \ldots \ldots \ldots \ldots \ldots \ldots \ldots \ldots \ldots \ldots \ldots \ldots \ldots \ldots \ldots$

4.4.9.1 Modeling Basis . ...................... 4-41

4.4.9.2 Guidelines for Applying the FIX-FLOW Option ....... 4-42

4.5 The Conservation and Thermodynamic State Equations $\ldots \ldots \ldots \ldots \ldots .444$

5.0 LOWER CELL AND CAVITY MODELS $\ldots \ldots \ldots \ldots \ldots \ldots \ldots \ldots \ldots \ldots \ldots \ldots, 1$

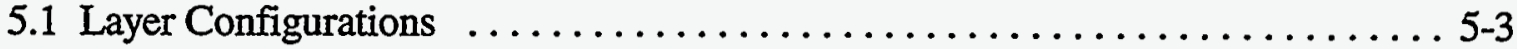

5.1.1 Layer Configuration Without CORCON $\ldots \ldots \ldots \ldots \ldots \ldots \ldots \ldots .5-3$

5.1 .2 Layer Configuration With CORCON $\ldots \ldots \ldots \ldots \ldots \ldots \ldots \ldots .6 \ldots$

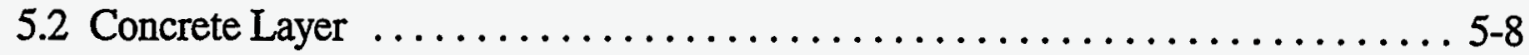

5.3 Intermediate Layers . . . . . . . . . . . . . . . . . . . . . . . .

5.3.1 Intermediate Layers Without CORCON $\ldots \ldots \ldots \ldots \ldots \ldots \ldots \ldots, 5-10$

5.3.2 Intermediate Layer With CORCON $\ldots \ldots \ldots \ldots \ldots \ldots \ldots \ldots \ldots . . \ldots \ldots$

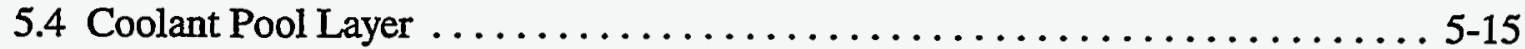

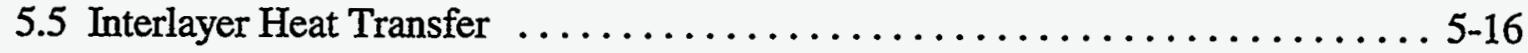

5.5.1 Interlayer Heat Transfer Coefficients ................. 5-16

5.5.2 User-Specified Heat Transfer Coefficients ............... 5-17

5.5.3 Lower Cell Conduction Modeling ..................... 5-19

5.6 Lower Cell Mass and Energy Addition ..................... 5-19

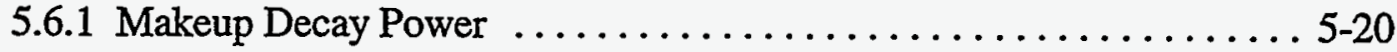

5.6.2 External Lower Cell Material Sources ................. . 5-22

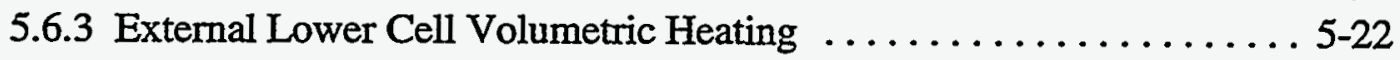

5.7 CORCON-CONTAIN Interface Considerations $\ldots \ldots \ldots \ldots \ldots \ldots \ldots \ldots, 5-22$

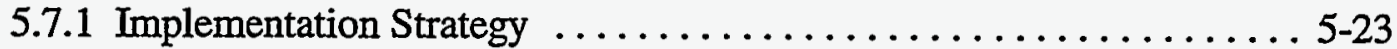

5.7.2 Obsolete Options From CORCON Mod2 ............. 5-23

5.7.3 Nonstandard Gases Released From CORCON ............ 5-23 


\section{TABLE OF CONTENTS (CONTINUED)}

5.7.4 Interfacing CONTAIN Aerosols and Fission Products with CCI Aerosol Releases ......................... 5-25

5.7.5 Restrictions in Mass and Energy Sources ............. 5-26

5.7.6 Radiative Heat Transfer to Containment Atmosphere and

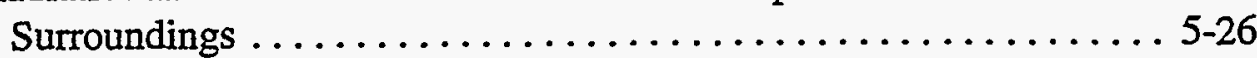

5.7.7 Fission Product Mass Additions $\ldots \ldots \ldots \ldots \ldots \ldots \ldots \ldots \ldots . . \ldots \ldots$

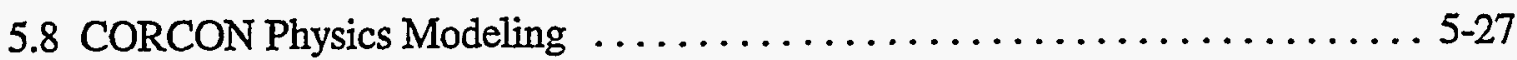

5.8.1 Broad Capabilities of CORCON Mod3 $\ldots \ldots \ldots \ldots \ldots \ldots \ldots \ldots .5-27$

5.8.2 Improvements in CORCON Mod3 $\ldots \ldots \ldots \ldots \ldots \ldots \ldots \ldots \ldots .5-28$

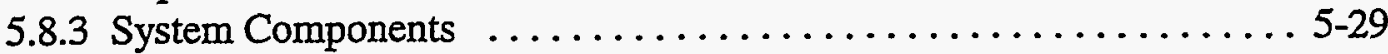

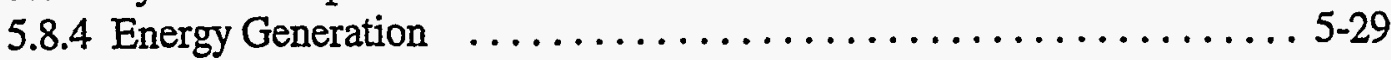

5.8.5 Melt/Concrete Heat Transfer .................... 5-30

5.8.6 Coolant Heat Transfer .......................... 5-30

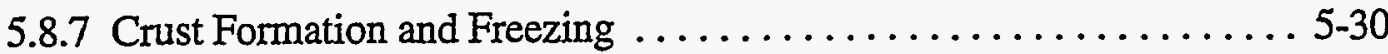

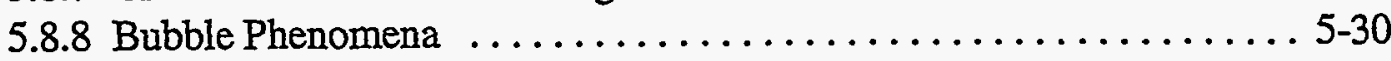

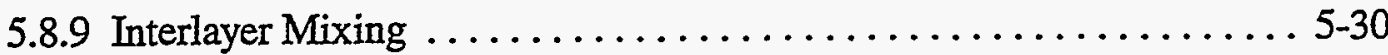

5.8.10 Pool Surface Heat Transfer $\ldots \ldots \ldots \ldots \ldots \ldots \ldots \ldots \ldots \ldots . . \ldots \ldots$

5.8.11 Concrete Decomposition and Ablation $\ldots \ldots \ldots \ldots \ldots \ldots \ldots \ldots .5,31$

5.8.12 Time-Dependent Melt Radius Option . ............... 5-31

5.8 .13 Chemical Reactions ......................... 5-31

5.8.14 Mass and Energy Transfer .................... 5-31

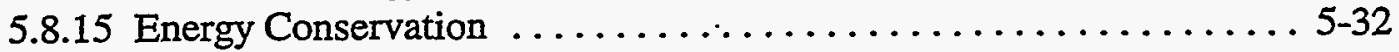

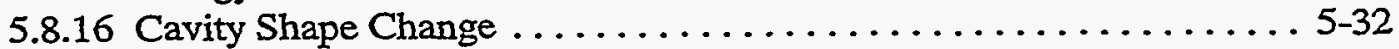

5.8.17 Aerosol Generation and Radionuclide Release $\ldots \ldots \ldots \ldots \ldots \ldots$ 5-32

5.8.18 Aerosol Removal By Overlying Water Pools ............. 5-32

5.8.19 Material Properties ........................... 5-32

5.9 Numerical Considerations and Known Limitations . . . . . . . . . . . . 5-32

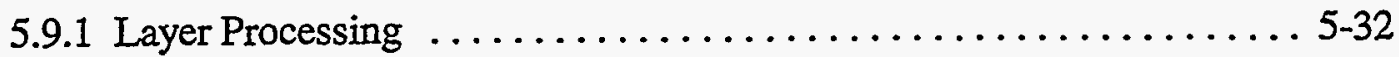

5.9.2 Assumptions and Limitations $\ldots \ldots \ldots \ldots \ldots \ldots \ldots \ldots \ldots . \ldots \ldots \ldots \ldots$

6.0 DIRECT CONTAINMENT HEATING (DCH) MODELS $\ldots \ldots \ldots \ldots \ldots \ldots \ldots, 6-1$

6.1 General Description of the CONTAIN DCH Model $\ldots \ldots \ldots \ldots \ldots \ldots \ldots, 6-2$

6.1 .1 Phenomena Modeled $. \ldots \ldots \ldots \ldots \ldots \ldots \ldots \ldots \ldots \ldots, 6.2$

6.1 .2 Multiple Debris Field Modeling Features $\ldots \ldots \ldots \ldots \ldots \ldots \ldots, 6,2$

6.2 Debris Transport and Intercell Flow $\ldots \ldots \ldots \ldots \ldots \ldots \ldots \ldots \ldots \ldots, 6,5$

6.2 .1 Gas/Debris Slip Model .......................... 6-7

6.2 .2 Gas Mass Conservation ............................ 6-9

6.2 .3 Gas Energy Conservation ...................... 6-11

6.2.4 Debris Mass Conservation ...................... 6-12

6.2.5 Debris Energy Conservation Equations ................ 6-13

6.2 .6 Choked Flow $\ldots \ldots \ldots \ldots \ldots \ldots \ldots \ldots \ldots \ldots \ldots \ldots \ldots \ldots \ldots, 15$ 


\section{TABLE OF CONTENTS (CONTINUED)}

6.2.7 Numerical Considerations of the Debris Intercell Flow Model ..... 6-16

6.2.8 Blowdown and Debris Sources ..................... 6-16

6.2.9 Reactor Pressure Vessel (RPV) Models . . . . . . . . . . . . . 6 6-18

6.2.9.1 Single-Phase Debris Discharge ................. 6-19

6.2.9.2 Two-Phase Discharge ...................... 6-19

6.2.9.2.1 Onset of Gas Blowthrough ............... 6-19

6.2.9.2.2 Gas Void Fraction and Exit Quality .......... 6-19

6.2.9.2.3 Gas Flow Area and Debris Flow Rate ........ 6-20

6.2.9.3 Vessel Hole Ablation . . . . . . . . . . . . . . . . . . . . 6-20

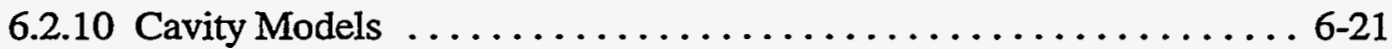

6.2.10.1 Entrainment Rate Correlations .............. 6-21

6.2.10.1.1 Whalley-Hewitt Entrainment Rate Model ..... 6-22

6.2.10.1.2 Levy Entrainment Rate Model ........... 6-23

6.2.10.1.3 Tutu Entrainment Rate Model ............ 6-23

6.2.10.2 Entrained Fraction Correlations ............... 6-25

6.2.10.2.1 Levy Entrained Fraction Correlation ......... 6-25

6.2.10.2.2 Tutu-Ginsberg Entrained Fraction Correlations . . 6-26

6.2.10.2.3 Entrainment Time ................ 6-28

6.2.10.2.4 Time-Dependent Gas Flow Rate . . . . . . . . . 6-29

6.2.10.2.5 Final RPV Hole Size ................ 6-29

6.2.10.2.6 Integration of the Entrainment Rate ........ 6-30

6.2.10.3 Debris Particle Size .................... 6-30

6.2.10.3.1 Weber Drop Size Model ............. 6-30

6.2.10.3.2 Integration of the Weber Model into the
Multifield Architecture ............... 6-31

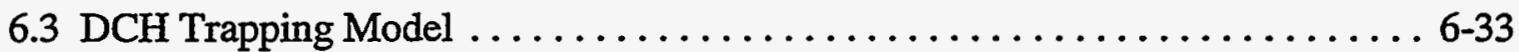

6.3.1 Rate Equations for Trapping $\ldots \ldots \ldots \ldots \ldots \ldots \ldots \ldots \ldots \ldots \ldots, 6,33$

6.3 .2 Average Velocities $\ldots \ldots \ldots \ldots \ldots \ldots \ldots \ldots \ldots \ldots \ldots \ldots, 6,36$

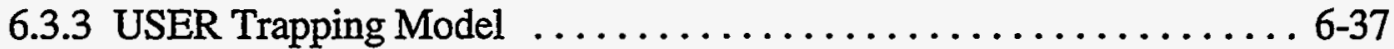

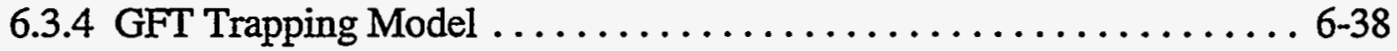

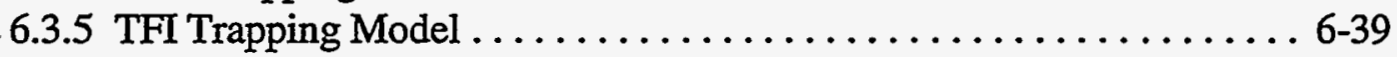

6.3.6 TOF/KU Trapping Model $\ldots \ldots \ldots \ldots \ldots \ldots \ldots \ldots \ldots \ldots .6 .41$

6.3.6.1 TOF/KU Case 1: Trapping On First Impact ......... 6-42

6.3.6.2 TOF/KU Case 2: Trapping On Structures Beyond The

First Impact ...................... 6-45

6.3.6.3 TOF/KU Case 3: No Trapping From First or Second

Kutateladze Number Criterion . . . . . . . . . . . . . 6 6-47

6.3.7 Trapping Model Sensitivity Coefficients ............... 6-48

6.4 Chemical Reactions $\ldots \ldots \ldots \ldots \ldots \ldots \ldots \ldots \ldots \ldots \ldots \ldots \ldots \ldots, 6,48$

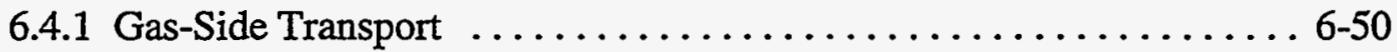

6.4.2 Drop-Side Transport $\ldots \ldots \ldots \ldots \ldots \ldots \ldots \ldots \ldots \ldots \ldots \ldots .6 .54$ 


\section{TABLE OF CONTENTS (CONTINUED)}

6.4.3 Reaction Rate Equations $. . \ldots \ldots \ldots \ldots \ldots \ldots \ldots \ldots \ldots . \ldots .6 .56$

6.4.4 DCH-Produced Hydrogen Recombination $\ldots \ldots \ldots \ldots \ldots \ldots .6 .61$

6.5 Heat Transfer ................................. 6-62

6.5 .1 Convective Heat Transfer $\ldots \ldots \ldots \ldots \ldots \ldots \ldots \ldots \ldots \ldots \ldots, 6.62$

6.5.2 Radiative Heat Transfer ......................... 6-64

6.5.3 Atmosphere-Structure Heat Transfer During DCH $\ldots \ldots \ldots \ldots \ldots 6.65$

6.6 Non-Airborne Debris Interactions . . . . . . . . . . . . . . . . . . . 6 65

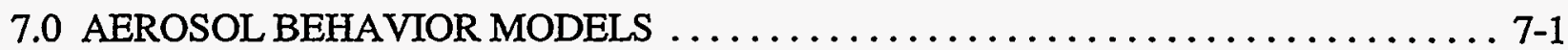

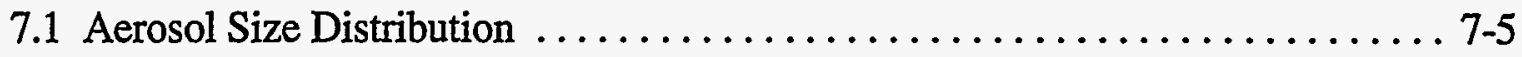

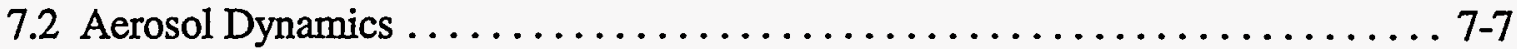

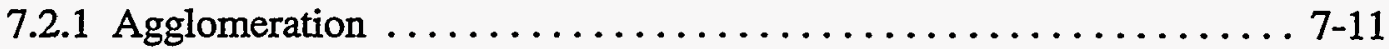

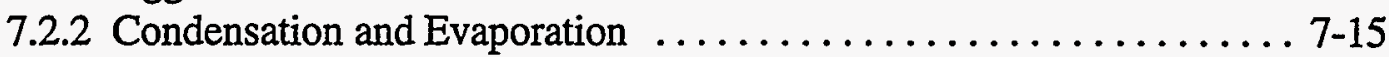

7.2.2.1 Fixed-Grid Model ........................ 7-17

7.2.2.2 Moving-Grid Model ......................... 7-19

7.2.3 Deposition ................................. 7-20

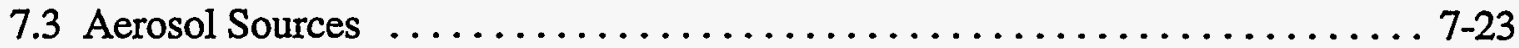

7.4 Fan Cooler Aerosol Deposition Model ........................ 7-24

7.5 Ice Condenser Aerosol Deposition Model . .................... 7-25

7.6 Containment Spray Aerosol Removal Model ................... 7-30

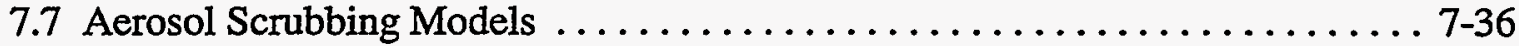

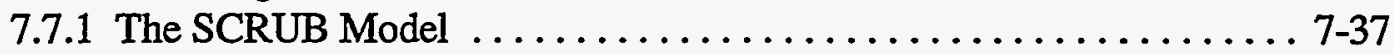

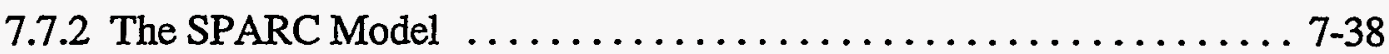

7.8 Intercell Flow of Aerosols . . . . . . . . . . . . . . . .

8.0 FISSION PRODUCT BEHAVIOR MODELS $\ldots \ldots \ldots \ldots \ldots \ldots \ldots \ldots \ldots \ldots . \ldots . \ldots . \ldots$

8.1 Introduction $\ldots \ldots \ldots \ldots \ldots \ldots \ldots \ldots \ldots \ldots \ldots \ldots \ldots \ldots \ldots \ldots, 8,1$

8.2 Fission Product Library $\ldots \ldots \ldots \ldots \ldots \ldots \ldots \ldots \ldots \ldots \ldots \ldots \ldots \ldots, 8,12$

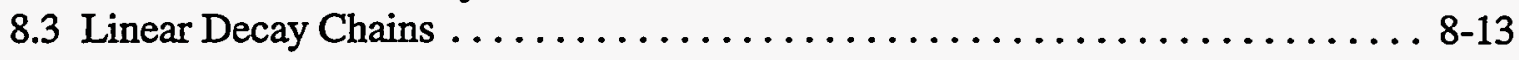

8.4 Fission Product Hosts and Targeted Release and Acceptance ........... 8-19

8.5 Fission Product Decay Heating $\ldots \ldots \ldots \ldots \ldots \ldots \ldots \ldots \ldots \ldots \ldots . .6 \ldots \ldots$

8.6 Iodine Removal Models $\ldots \ldots \ldots \ldots \ldots \ldots \ldots \ldots \ldots \ldots \ldots \ldots . \ldots \ldots .26$

8.7 User-Specified Fission Product Sources . . . . . . . . . . . . . . . . . 8-29

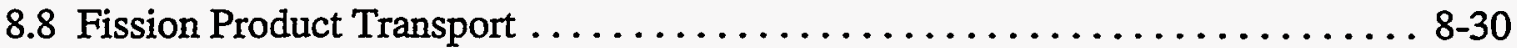

8.8.1 Gas Phase Transport of Fission Products . . . . . . . . . . .

8.8.2 Liquid Transport of Fission Products ................ 8-31

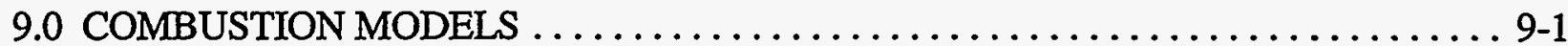

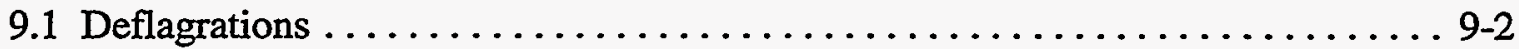

9.1.1 Deflagration Ignition and Propagation Criteria $\ldots \ldots \ldots \ldots \ldots \ldots .9 .4$

9.1.2 Flame Speed and Burn Time $\ldots \ldots \ldots \ldots \ldots \ldots \ldots \ldots \ldots . .6 .8$ 


\section{TABLE OF CONTENTS (CONTINUED)}

9.1.3 Combustion Completeness $\ldots \ldots \ldots \ldots \ldots \ldots \ldots \ldots \ldots \ldots$ 9.12

9.1.4 Burn Rate ............................ 9-13

9.2 Diffusion Flame Burning (DFB) . . . . . . . . . . . . . .

9.3 Bulk Spontaneous Recombination (BSR) . . . . . . . . . . . . . 9-20

10.0 HEAT AND MASS TRANSFER MODELS $\ldots \ldots \ldots \ldots \ldots \ldots \ldots \ldots \ldots \ldots \ldots$. $\ldots \ldots \ldots$

10.1 Convective Heat Transfer . . . . . . . . . . . . . . . . . . . .

10.1.1 Boundary Layer Properties and Nondimensional Numbers for

Heat Transfer . . . . . . . . . . . . . . . . . . . 10-3

10.1.1.1 Evaluation of the Gas Boundary Layer Composition . . . . . 10-4

10.1.1.2 Evaluation of Properties at the Boundary Layer

Temperatures ...................... 10-6

10.1.1.3 Evaluation of the Nondimensional Heat Transfer

Numbers . . . .................... 10-9

10.1.1.4 Submergence of Heat Transfer Structures . . . . . . . . 10-10

10.1.1.5 Heat Transfer Characteristic Lengths . . . . . . . . . . . 10-12

10.1.1.6 Forced Convection Velocities for Heat Transfer

Structures .................... 10-14

10.1.2 Basic Heat Transfer Correlations . . . . . . . . . . . . . . . 10-15

10.1.3 Generalized Gas-Structure Convective Heat Transfer Options . . . . 10 18

10.1.3.1 Rohsenhow Handbook Heat Transfer Correlations for

Natural Convection . . . . . . . . . . . . . . 10-18

10.1.3.2 User-Defined Natural Convection Correlations . . . . . . . 10 10-19

10.1.3.3 User-Defined Forced Convection Correlations . . . . . . . . 10 10-20

10.1.3.4 Mixed Convection . . . . . . . . . . . . . . . . . 10-20

10.1.3.5 Heat Transfer Multiplier for Gas-Structure Interfaces . . . 10 10-21

10.2 Mass Transfer Processes . . . . . . . . . . . . . . . . . . . . . . 10-22

10.2.1 Boundary Layer Properties and Nondimensional Numbers for

Mass Transfer .......................... 10-22

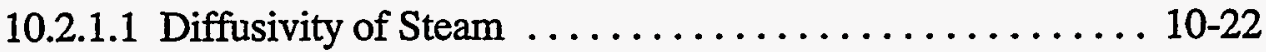

10.2.1.2 Evaluation of the Nondimensional Mass Transfer

Numbers ........................ 10-27

10.2.1.3 Mass Transfer Multipliers for Gas-Structure Interfaces . . . 10-27

10.2.2 Condensate Films on Heat Transfer Structures . . . . . . . . . . 10-28

10.2.2.1 The Film Tracking Model . . . . . . . . . . . . 10-30

10.2.2.2 Disposition of Film Runoff . . . . . . . . . . . . 10-32

10.2.3 Condensation Mass Transfer . . . . . . . . . . . . . . . 10-32

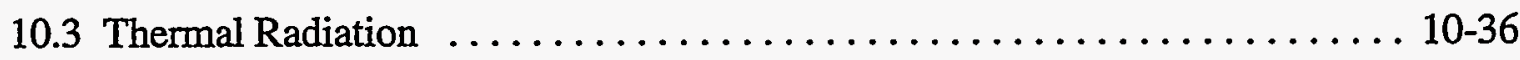

10.3.1 Net Enclosure Radiation Model . . . . . . . . . . . . . . . 10-37

10.3.2 Direct Radiative Heat Transfer Model . . . . . . . . . . . . . 10-38 


\section{TABLE OF CONTENTS (CONTINUED)}

10.3.3 Radiative Properties ........................... 10-41

10.3.3.1 Modak Radiative Property Model $\ldots \ldots \ldots \ldots \ldots \ldots . . .10-41$

10.3.3.2 Cess-Lian Radiative Property Model $\ldots \ldots \ldots \ldots \ldots \ldots$ 10-44

10.4 Boiling Heat Transfer $\ldots \ldots \ldots \ldots \ldots \ldots \ldots \ldots \ldots \ldots \ldots \ldots \ldots \ldots \ldots \ldots \ldots \ldots, 10-45$

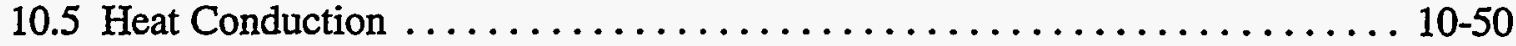

10.5.1 Containment Heat Sinks $\ldots \ldots \ldots \ldots \ldots \ldots \ldots \ldots \ldots \ldots \ldots, 10-50$

10.5.1.1 Heat Transfer Structures ...................... 10-50

10.5.1.2 Lower Cell Layers ........................ 10-54

10.5.2 Connected Structure Boundary Condition .............. 10-54

10.5.3 Heat Conduction Model ........................ 10-59

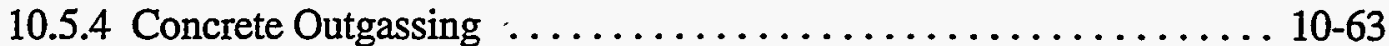

10.6 Coupled Heat and Mass Transfer . . . . . . . . . . . . . . . . . . . . . 10-69

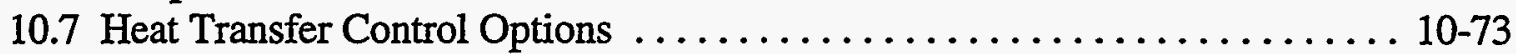

11.0 BOILING WATER REACTOR MODELS $\ldots \ldots \ldots \ldots \ldots \ldots \ldots \ldots \ldots \ldots \ldots \ldots \ldots \ldots$

11.1 Suppression Pool Vent Models . . . . . . . . . . . . . . . . . . . . 11-1

11.1.1 Multi-Node Suppression Pool Vent Flow Model ............ 11-3

11.1.2 Dedicated Suppression Pool Vent Flow Model ............. 11-8

11.1.2.1 Vent Clearing Time ....................... $11-9$

11.1.2.2 Gas Flow Model ........................ 11-17

11.2 Safety Relief Valve (SRV) Model ....................... 11-19

11.2.1 Gas-Pool Equilibration for Submerged SRV Sources ......... 11-19

11.2.2 Phase Separation Calculation for Unsubmerged SRV Sources ..... 11-23

12.0 ENGINEERED SAFETY FEATURE MODELS $\ldots \ldots \ldots \ldots \ldots \ldots \ldots \ldots \ldots \ldots \ldots \ldots \ldots, 12-1$

12.1 Fan Cooler . . . . . . . . . . . . . . . . . . . . . . . . . . . . $12-4$

12.2 Ice Condenser $\ldots \ldots \ldots \ldots \ldots \ldots \ldots \ldots \ldots \ldots \ldots \ldots \ldots \ldots \ldots \ldots \ldots \ldots \ldots \ldots \ldots, 12-10$

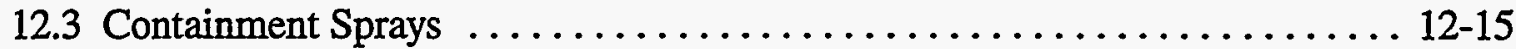

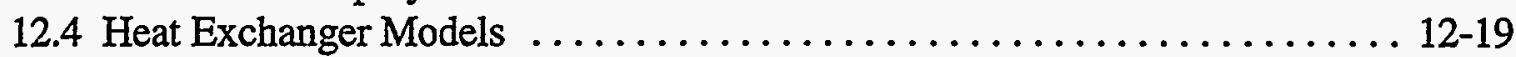

12.5 Liquid Transport System Components . . . . . . . . . . . . . . . . . .

12.5.1 Pumps ................................. 12-24

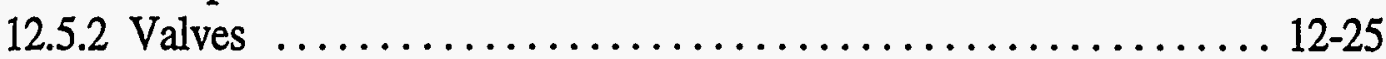

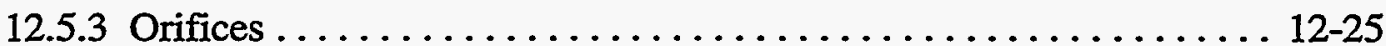

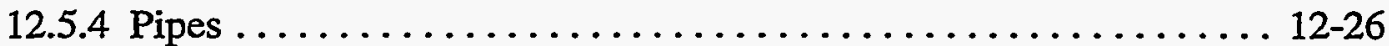

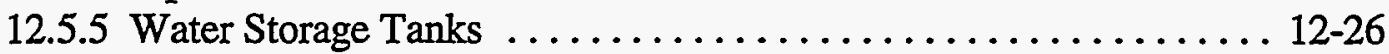

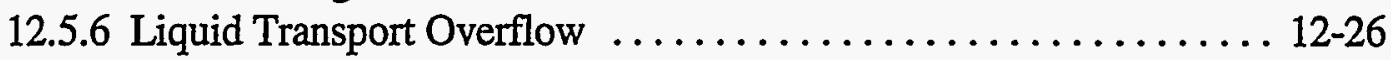




\section{TABLE OF CONTENTS (CONTINUED)}

13.0 USER GUIDANCE AND PRACTICAL AND CAUTIONARY ADVICE . . . . . . 13-1

13.1 Key Simplifying Assumptions in CONTAIN . . . . . . . . . . . . . . . 13-1

13.2 Specific Model Limitations . . . . . . . . . . . . . . . . . . . . . . . 13-2

13.2.1 Atmosphere/Pool Thermodynamics and Intercell Flow ......... 13-2

13.2.2 Lower Cell Modeling .......................... 13-3

13.2.3 DCH Modeling ............................... 13-3

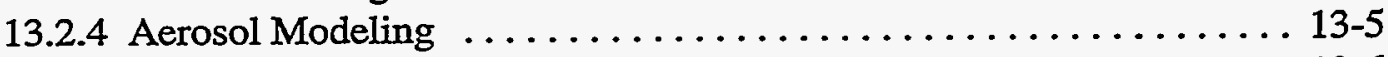

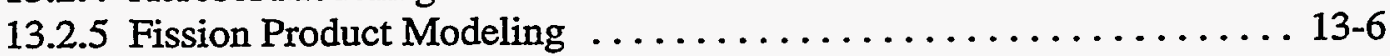

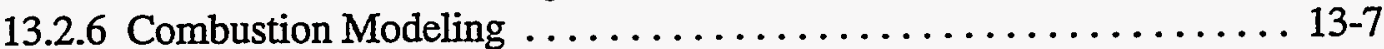

13.2.6.1 Deflagration Model ..................... 13-7

13.2.6.2. Diffusion Flame Burn (DFB) Model ............ 13-11

13.2.6.3 Bulk Spontaneous Reaction (BSR) Model .......... 13-12

13.2.7 Heat and Mass Transfer . . . . . . . . . . . . . . . . . . . 13-13

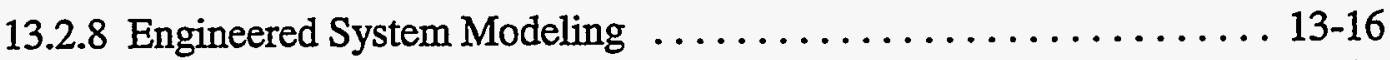

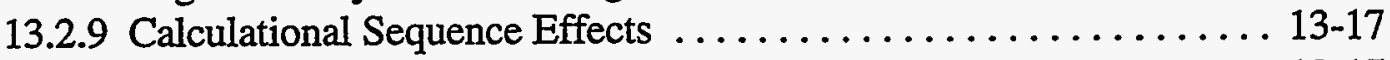

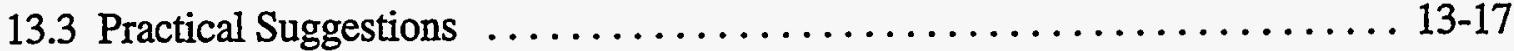

13.3.1 Atmosphere/Pool Thermodynamics and Intercell Flow ........ 13-17

13.3.1.1 Optimizing Multicell Calculations ............. 13-17

13.3.1.2 Modeling of Suspended Liquid Coolant ........... 13-17

13.3.1.3 Modeling Stratifications . .................. 13-19

13.3.1.4 Choked Flow at Less Than the Critical Pressure Difference 13-20

13.3.1.5 Modeling the Outside Environment ............ 13-22

13.3.1.6 Atmosphere and Lower Cell Source Tables .......... 13-22

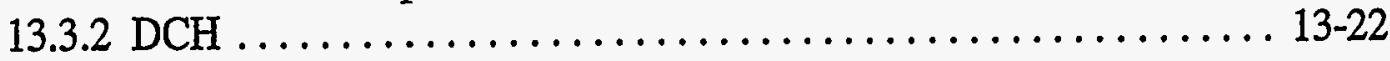

13.3.2.1 General Considerations ................... 13-22

13.3.2.2 The Standard Input Prescription for DCH Calculations ... 13-24 13.3.2.2.1 Philosophical Basis and Purpose of the Standard Prescription ................... 13-24

13.3.2.2.2 Standard Prescription Input and Model Choices . 13-28

13.3.2.3 RPV Models . ......................... 13-51

13.3.2.3.1 Hole Ablation . . . . . . . . . . . . . . 13-51

13.3.2.3.2 Gas Blowthrough ................. 13-51

13.3.2.4 Cavity Models . . . . . . . . . . . . . . . . . . . .

13.3.2.4.1 Entrainment Rate ................. 13-52

13.3.2.4.2 Entrained Fraction ................ 13-52

13.3.2.4.3 Cavity Constant $\mathrm{K}_{\mathrm{c}}$ (CCENF and CCENR) .... 13-53

13.3.2.4.4 Cavity Geometry Parameters . . . . . . . . . 13-55

13.3.2.4.5 Discharge Coefficient $\ldots \ldots \ldots \ldots \ldots \ldots \ldots .13-56$

13.3.2.4.6 Levy Standard Values ............... 13-56

13.3.2.4.7 Whalley-Hewitt Standard Value .......... 13-57

13.3.2.4.8 Weber Model . . . . . . . . . . . . . . . 13-57 


\section{TABLE OF CONTENTS (CONTINUED)}

13.3.2.5 Specific Input Parameters for RPV and Cavity Models ... 13-58

13.3.2.5.1 AHOLE1. ................... 13-58

13.3.2.5.2 CPWALL . . . . . . . . . . . . . 13-58

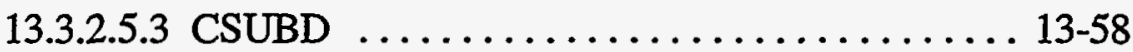

13.3.2.5.4 RHOWAL . . . . . . . . . . . . . 13-58

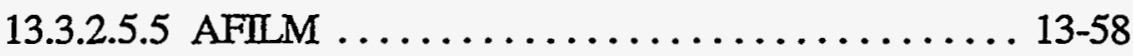

13.3.2.5.6 HYDDIA .................... 13-59

13.3.2.5.7 WETPER ................. 13-59

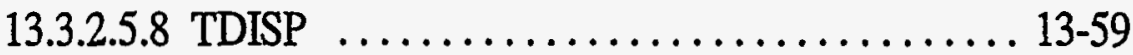

13.3.2.5.9 TSTOP . . . . . . . . . . . . . . 13-59

13.3.2.5.10 CCENF . ................... 13-59

13.3.2.5.11 ENTFR ..................... 13-60

13.3.3 Heat and Mass Transfer . . . . . . . . . . . . . . . . 13-60

13.3.3.1 Approximating the Uchida Heat Transfer Data . . . . . 13-60

13.3.3.2 Approximating Condenser Tubes . . . . . . . . . . 13-62

13.3.3.3 Approximating Boiling Heat Transfer . . . . . . . . . 13-63

13.3.3.4 Film Flow Over an Ellipsoidal Dome Shell . . . . . . . 13-63

13.3.3.5 Hints for Structure Naming and Ordering . . . . . . . . . 13-64

13.3.3.6 Simulating Quenching Phenomena and Steam Spikes ... 13-64

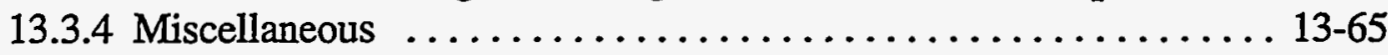

13.3.4.1 Fission Product Mass Effects . .............. 13-65

13.3.4.2 Simulation of Pool Scrubbing of Elemental Iodine . . . . 13-65

13.3.4.3 Multiple Sources for a Single Species ........... 13-66

13.3.4.4 Restarts . ........................ 13-66

13.3.4.5 Annotated Input . . . . . . . . . . . . . . . 13-66

13.3.4.6 Hints for Collapsing Multi-Cell Input Files . . . . . . . . 13-67

13.3.4.7 Role of Sensitivity Calculations in Building Input Files . . 13-68

13.3.4.8 Spray Modeling Suggestions .............. 13-68

14.0 INPUT DESCRIPTION $\ldots \ldots \ldots \ldots \ldots \ldots \ldots \ldots \ldots \ldots \ldots \ldots \ldots \ldots \ldots \ldots \ldots$

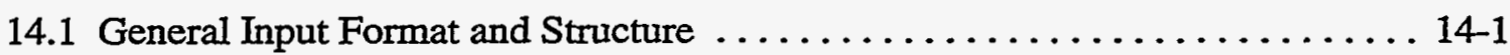

14.1.1 Ordering Requirements in Input Blocks . . . . . . . . . . . 14-6

14.2 Global Level Input $\ldots \ldots \ldots \ldots \ldots \ldots \ldots \ldots \ldots \ldots \ldots \ldots \ldots \ldots . \ldots \ldots \ldots$

14.2.1 Material, Fission Product, and Aerosol Names . . . . . . . . . . . . 14-13

14.2.1.1 User-Defined Material Definition . . . . . . . . . . . . 14-16

14.2.1.2 DCH User-Defined Material Input . . . . . . . . . . 14-19

14.2.2 Mass and Energy Accounting ................. 14-20

14.2.3 Fixed Atmosphere Conditions ................. 14-21 


\section{TABLE OF CONTENTS (CONTINUED)}

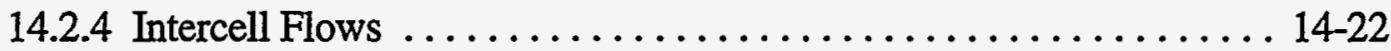

14.2.4.1 Flow Options .......................... 14-22

14.2.4.2 Engineered Vents ...................... 14-25

14.2.4.3 Suppression Pool Vent Flow Path .............. 14-31

14.2.5 Aerosol Options ............................ 14-33

14.2.6 Fission Product Options ....................... 14-40

14.2.6.1 Fission Product Decay and Heating Input ......... 14-40

14.2.6.2 Fission Product Transport Efficiency in Liquid Pathways . 14-42

14.2.7 Global DCH Input Block . . . . . . . . . . . . . . . . . 14-43

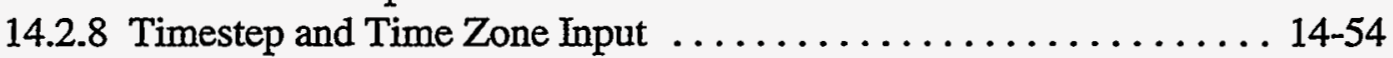

14.2.9 Output Control ............................ 14-56

14.2.9.1 Frequency of Print Output ................. 14-56

14.2.9.2 Print Output Options ....................... 14-57

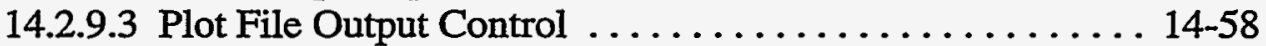

14.2.9.4 Title ............................. 14-61

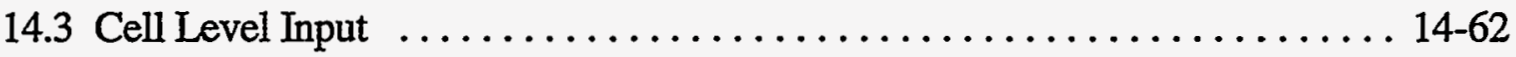

14.3.1 Upper Cell .............................. 14-67

14.3.1.1 Upper Cell Geometry .................... 14-67

14.3.1.2 Upper Cell Atmosphere Initial Conditions and Sources ... 14-69

14.3.1.3 Heat Transfer Structure Characteristics ........... 14-74

14.3.1.4 Convection and Condensation ............... 14-93

14.3.1.5 Radiation .......................... 14-94

14.3.1.6 Heat Transfer Control ...................... 14-97

14.3.1.7 Combustion Model Input . ................. 14-97

14.3.1.8 Aerosol Initial Conditions and Sources ........... 14-103

14.3.1.9 Fission Product Sources ................... 14-104

14.3.1.10 Fission Product Initial Conditions and Release Rates .. 14-106

14.3.1.11 Cell-Level DCH Input Block . . . . . . . . . . . . . 14-109

14.3.1.12 Cell Overflow ......................... 14-114

14.3.2 Lower Cell ............................. 14-115

14.3.2.1 Overall Lower Cell Input Structure . . . . . . . . . . . . . 14-115

14.3.2.2 Makeup Decay Power ..................... 14-117

14.3.2.3 Concrete Layer ..................... 14-119

14.3.2.3.1 Concrete Layer Input Without CORCON .... 14-119

14.3.2.3.2 Concrete Layer Input With CORCON . . . . . . 14-123

14.3.2.4 Intermediate Layers . . . . . . . . . . . . . . . 14-138

14.3.2.4.1 Intermediate Layer Input Without CORCON .. 14-138

14.3.2.4.2 Intermediate Layer Input With CORCON .... 14-140

14.3.2.5 Pool Layer ........................... 14-144 


\section{TABLE OF CONTENTS (CONTINUED)}

14.3.3 Engineered Safety Systems $\ldots \ldots \ldots \ldots \ldots \ldots \ldots \ldots \ldots \ldots \ldots . .14-146$

14.3.3.1 External Engineered System Source ............. 14-148

14.3.3.2 Fan Cooler .............................. 14-149

14.3.3.3 Ice Condenser .......................... 14-150

14.3.3.4 Containment Spray . . . . . . . . . . . . . . . . . 14-152

14.3.3.5 Heat Exchanger ......................... 14-153

14.3.3.6 Pump ................................ 14-154

14.3.3.7 Valve .............................. 14-154

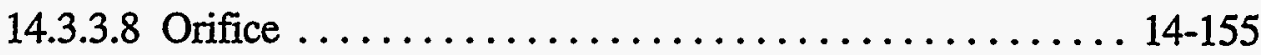

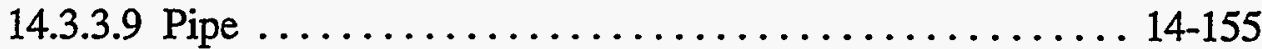

14.3.3.10 Tank ............................. 14-156

14.3.3.11 Engineered Systems Overflow .............. 14-156

14.3.4 Safety Relief Valve (SRV) Discharge . . . . . . . . . . . . 14-157

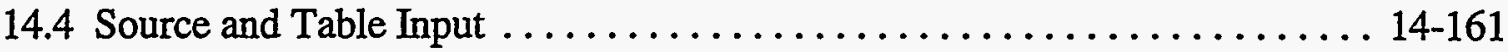

14.4.1 Source Table Input ......................... 14-161

14.4.2 Global and Cell Level Table Input .................. 14-164

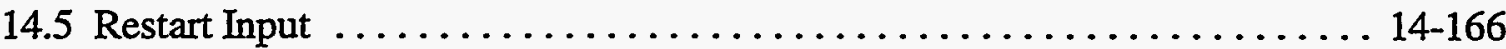

14.5.1 Global Level Input for a Restart . ................. 14-169

14.5.1.1 The TIMES Block in a Restart .............. 14-170

14.5.1.2 Print Output Options in a Restart .............. 14-170

14.5.1.3 Restart Input for DCH Calculations ............ 14-171

14.5.2 Cell Level Input for a Restart . . . . . . . . . . . . . . . . 14-171

14.5.2.1 Upper Cell Restart Block .................. 14-171

14.5.2.2 Lower Cell Restart Block ................ 14-172

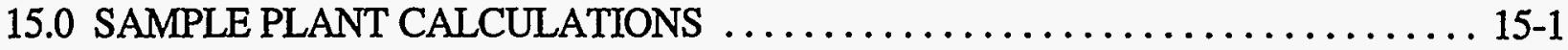

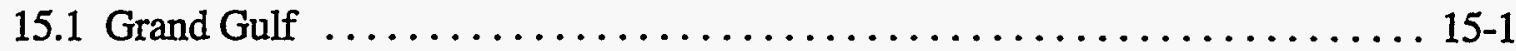

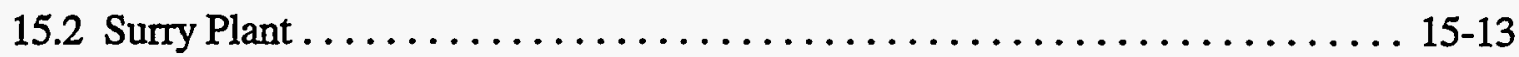

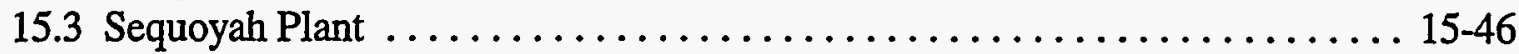

16.0 OUTPUT FILES AND POSTPROCESSING $\ldots \ldots \ldots \ldots \ldots \ldots \ldots \ldots \ldots \ldots \ldots \ldots \ldots$

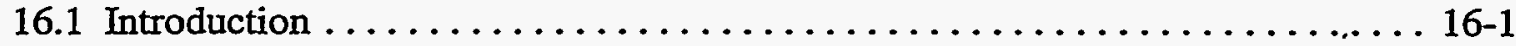

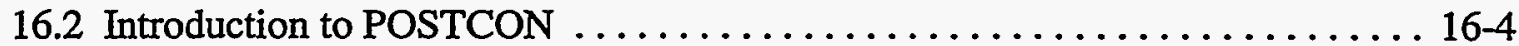

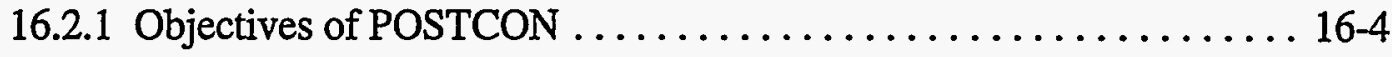

16.2.2 How to Use This Section $\ldots \ldots \ldots \ldots \ldots \ldots \ldots \ldots \ldots \ldots \ldots \ldots \ldots . . \ldots \ldots$

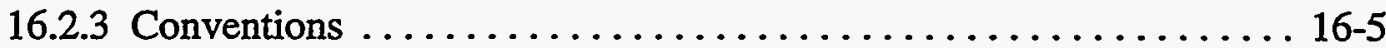

16.3 General Overview and Capabilities of POSTCON $\ldots \ldots \ldots \ldots \ldots \ldots \ldots \ldots .6 \ldots$

16.3.1 Processing the CONTAIN Binary Plot File $\ldots \ldots \ldots \ldots \ldots \ldots \ldots .6 .6$

16.3.1.1 Plot Flags and the CONTAIN Binary Plot File ......... 16-6

16.3.1.2 Extracting Data from the Binary Plot File $\ldots \ldots \ldots \ldots \ldots$ 16-6

16.3.1.3 Unit Conversions ........................ 16-7

16.3.1.4 Table Output and Vector Output ............. 16-17 


\section{TABLE OF CONTENTS (CONTINUED)}

16.3.1.5 Snapshots and Histograms $\ldots \ldots \ldots \ldots \ldots \ldots \ldots \ldots \ldots .16-18$

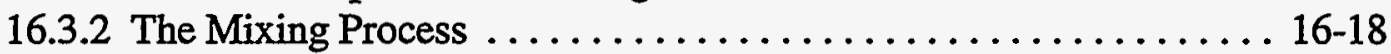

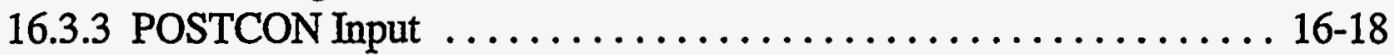

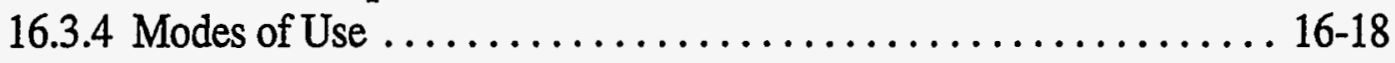

16.3.4.1 Single Pass ........................... 16-19

16.3.4.2 Multiple Passes ........................ 16-19

16.3.4.3 Processing Results from Sources Other than CONTAIN .. 16-19

16.4 Input and Output Files and the File Definition Block .............. 16-19

16.4.1 File Descriptions ............................ 16-19

16.4.1.1 Input File (PINP) . . . . . . . . . . . . . . . . . 16-19

16.4.1.2 Error File (PERR) . . . . . . . . . . . . . . . . . 16-19

16.4.1.3 Binary Plot File (PLTFL) $\ldots \ldots \ldots \ldots \ldots \ldots \ldots \ldots \ldots . .16-21$

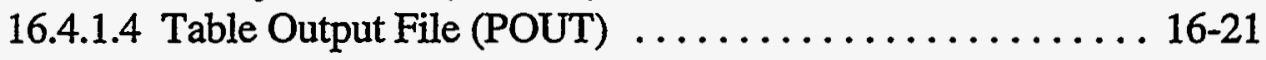

16.4.1.5 Vector File (PVEC) $\ldots \ldots \ldots \ldots \ldots \ldots \ldots \ldots \ldots \ldots \ldots \ldots \ldots \ldots \ldots, 21$

16.4.1.6 Mix File (PMIX) . ...................... 16-21

16.4.2 Renaming Input and Output Files $\ldots \ldots \ldots \ldots \ldots \ldots \ldots \ldots \ldots . \ldots \ldots$ 16-22

16.4.3 File Definition Block Input Summary ............... 16-22

16.4.4 File Definition Block Input Details . . . . . . . . . . . . . . . 16-22

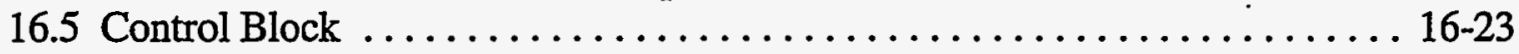

16.5.1 Control Block Input Summary ................... 16-23

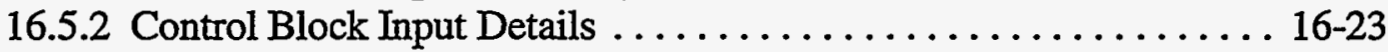

16.6 Table Definition Block . . . . . . . . . . . . . . . . . . . . . . .

16.6.1 Table Definition Block Input Summary ............... 16-27

16.6.2 Table Definition Block Input Details . . . . . . . . . . . . . 16-28

16.7 Mix Block . . . . . . . . . . . . . . . . . . . . . . . . . . . 16-31

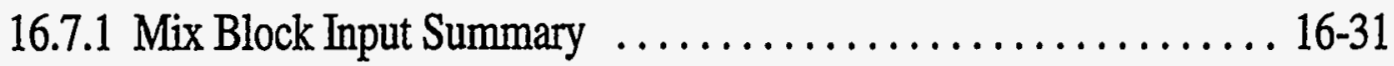

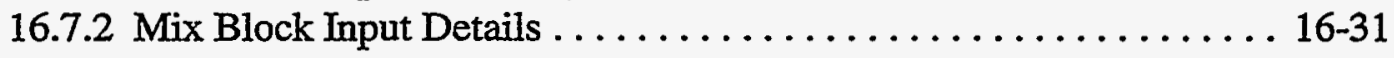

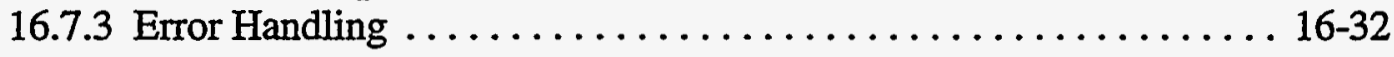

16.7.4 Adding Functions to the Mix Option ................. 16-32

16.7.4.1 Choosing a Function Name ................. 16-33

16.7.4.2 Modifying POSTCON for Unary Functions ......... 16-33

16.7.4.3 Modifying POSTCON for Binary Functions . ........ 16-33

16.7.4.4 Examples of Mix Expressions with Functions . ....... 16-33

16.7.5 Hints for Efficient Use . . . . . . . . . . . . . . . . . . . .

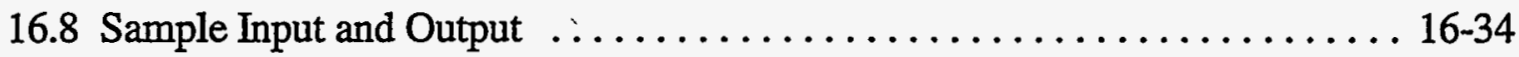

16.8.1 Example 1: Extracting Data in a Single Run ............ 16-34

16.8.2 Example 2: Unit Conversions, User-Selectable Time Zones,

and Snapshots . . . . . . . . . . . . . . . . . . . . . . . . . .

16.8.3 Example 3: Mixing . . . . . . . . . . . . . . . .

16.8.4 Example 4: More Complicated Table Definition Blocks ....... 16-35 


\section{TABLE OF CONTENTS (CONTINUED)}

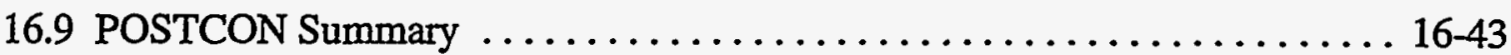

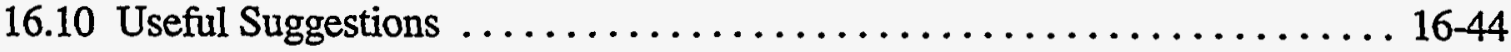

16.10.1 Upward Compatibility Issues . . . . . . . . . . . . . . . 16-44

16.10 .2 Restarts ................................... 16-44

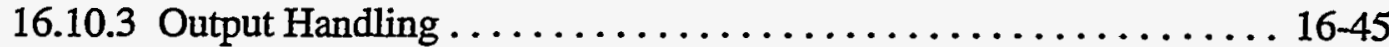

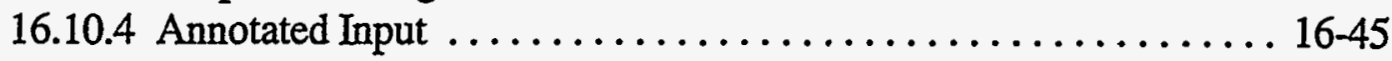

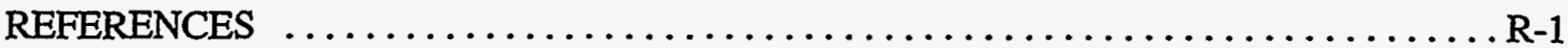

APPENDICES

APPENDIX A DETAILS OF THE MASS AND ENERGY ACCOUNTING SCHEME ... A-1

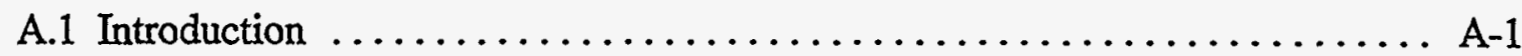

A.2 Mass and Energy Tracking Strategy $\ldots \ldots \ldots \ldots \ldots \ldots \ldots \ldots \ldots \ldots$ A-1

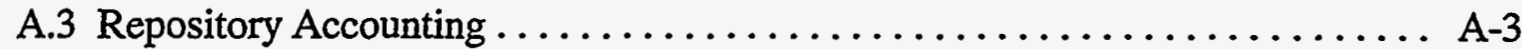

APPENDIX B ALTERNATE INPUT FORMATS AND UPWARD COMPATIBILITY . . B-1

B.1 Alternative Global Input Formats $\ldots \ldots \ldots \ldots \ldots \ldots \ldots \ldots \ldots \ldots \ldots \ldots \ldots \ldots \ldots \ldots \ldots$

B.1.1 Alternative Global CONTROL Input Block Format ........... B-1

B.1.2 Alternative Fission Product Names Keyword ............... B-2

B.1.3 Alternative FLOWS Input Block Options ............... B-2

B.1.4 Alternative Global AEROSOL Keyword Format ............. B-6

B.1.5 Alternative Global FISSION Input Block Format ............. B-7

B.2 Alternative Cell-Level Input Formats $\ldots \ldots \ldots \ldots \ldots \ldots \ldots \ldots \ldots \ldots \ldots \ldots$

B.2.1 Alternative Cell CONTROL Input Block Format ............. B-9

B.2.2 Alternative ATMOS Input Block Format ............... B-11

B.2.3 Alternative STRUC Input Block Format ................ B-12

B.2.4 Alternative STRUC Input Block Keywords ............... B-14

B.2.5 Alternative CONDENSE Input Block Keywords ............. B-14

B.2.6 Alternative Radiation Input Block Format $\ldots \ldots \ldots \ldots \ldots \ldots \ldots$ B-16

B.2.7 Alternative KMX Input Option .................... B-16

APPENDIX C VALIDATION SUMMARY $\ldots \ldots \ldots \ldots \ldots \ldots \ldots \ldots \ldots \ldots \ldots \ldots . \ldots \ldots, 1$

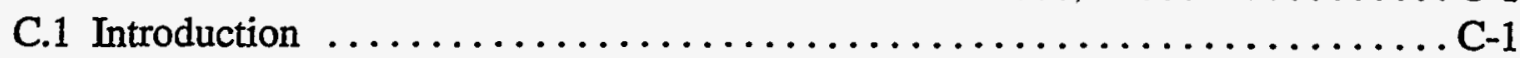

C.1.1 History of the CONTAIN Code $\ldots \ldots \ldots \ldots \ldots \ldots \ldots \ldots . . . . .1$

C.1.2 Experimental Programs ......................... 


\section{TABLE OF CONTENTS (CONCLUDED)}

C.2 Validation and Assessment of CONTAIN . . . . . . . . . . . . . . C-2

C.2.1 Objectives of Code Validation and Assessment (CV\&A) $\ldots \ldots \ldots \ldots$ C-2

C.2.2 Nature and Format of the CV\&A . . . . . . . . . . . . . C-3

C.3 Individual CV\&A Summaries ...................... -5

C.3.1 Atmosphere Thermal Hydraulics and Intercell Flow $\ldots \ldots \ldots \ldots \ldots$ C-5

C.3.2 Heat and Mass Transfer . . . . . . . . . . . . . . . . . C-5

C.3.3 Direct Containment Heating . . . . . . . . . . . . . . . C-6

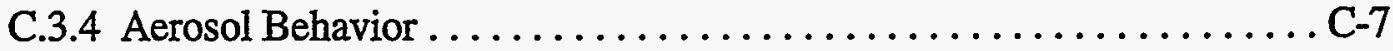

C.3.5 Hydrogen Burns . . . . . . . . . . . . . . . . . . C-7

C.3.6 Pressure Suppression Models . . . . . . . . . . . . . . C-7

C.3.7 Miscellaneous Models . . . . . . . . . . . . . . . . . . C-7

C.4 Overall CV\&A Summary . . . . . . . . . . . . . . . . . . . C-8

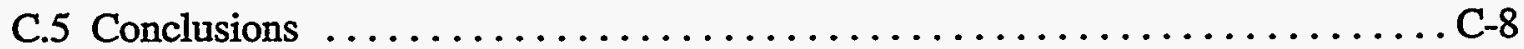

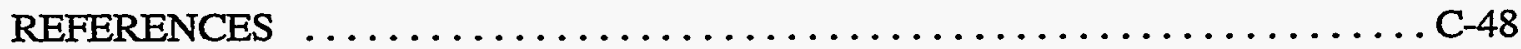

APPENDIX D QUALTTY ASSURANCE PROCEDURES . . . . . . . . . . . . . D-1

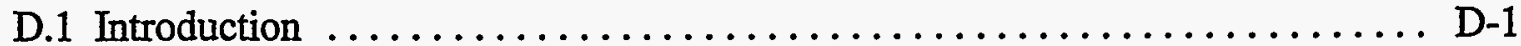

D.1.1 Quality Assurance Program Scope .................. D-1

D.1.2 CONTAIN Quality Assurance Organization .............. D-1

D.2 The CONTAIN Code Development/Maintenance System $\ldots \ldots \ldots \ldots \ldots \ldots$ D-4

D.2.1 User Support . . . . . . . . . . . . . . . . . . . D-4

D.2.1.1 Change Stimuli ..................... D-7

D.2.1.2 CONTAIN Code Manual ................. D-7

D.2.1.3 Code Releases .................... D-7

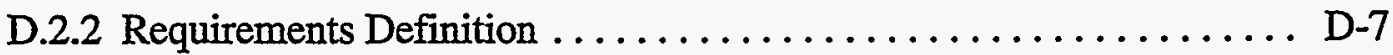

D.2.3 Development ........................... D

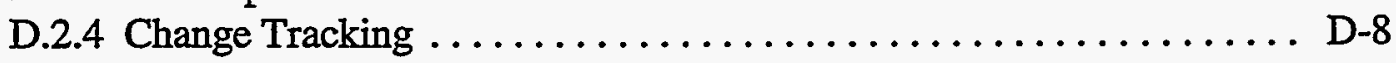

D.2.5 Configuration Control ....................... D-9

D.2.5.1 Baseline Control . . . . . . . . . . . . . . . . . D-9

D.2.5.2 The Control Mechanism ................. D-9

D.2.5.3 Control of Variant Codes . . . . . . . . . . . . D-12

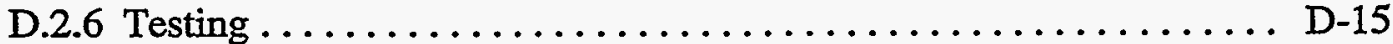

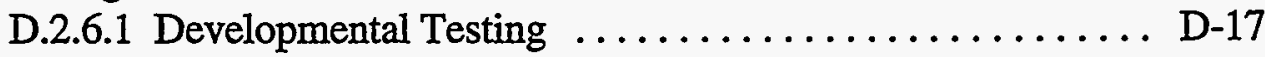

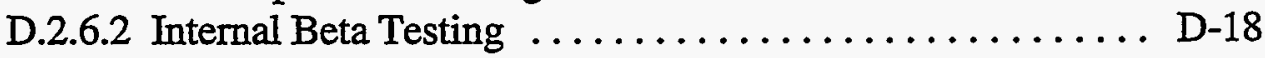

D.2.6.3 Integrated Testing . . . . . . . . . . . . . . D 18

D.2.6.4 Test Documentation .................... D-19

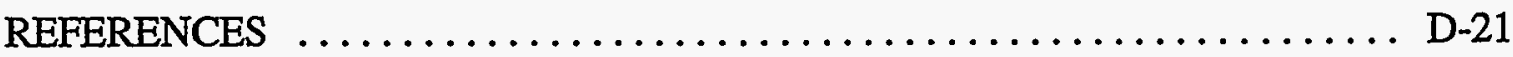




\section{LIST OF FIGURES}

Figure 2-1 Illustration of Feedback Mechanisms Treated in the CONTAIN Code. . . . . . 2-2

Figure 2-2 Reactor Containment Building Represented by Interconnected Cells ...... . 2-3

Figure 2-3 Overall Flow Diagram of CONTAIN $\ldots \ldots \ldots \ldots \ldots \ldots \ldots \ldots \ldots \ldots \ldots$

Figure 3-1 Material Properties as Modeled in CONTAIN. .................. 3-2

Figure 3-2 Example of Mass and Energy Accounting Output .............. 3-17

Figure 4-1 Atmosphere/Pool Thermodynamics and Intercell Flow as Modeled in CONTAIN . ................................ 4-3

Figure 4-2 Definition of Cell Cross-Sections and Elevations, for a Case with Three Cross-

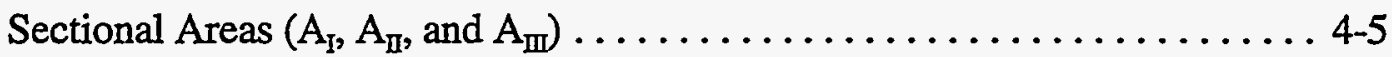

Figure 4-3 Default Attachment Points of Gas and Pool Flow Paths ............ 4-6

Figure 4-4 Definition of Flow Path and Pool Elevations .................. 4-8

Figure 4-5 Illustration of the Flow Areas Used in the Critical or Choked Flow

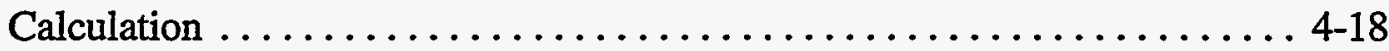

Figure 4-6 Position of the Virtual Dynamic Interfaces (VDIs) in the Flow Paths of a Four Cell Problem in Response to a Slight Increase in Density in Cell 1, Starting with a Stable Stratification Consisting of a Light Gas over a Heavy Gas ............................... 4-20

Figure 4-7 Illustration of the Flow Solver Regimes for a Pair of Cells $i$ and $j$ Connected by a Gas Flow Path, with Elevation $\mathrm{H}_{\mathrm{j}}>\mathrm{H}_{\mathrm{i}}$, for the Case in Which Neither the RESOLVHD or MSTABLE Keywords Have Been Specified $\ldots \ldots \ldots \ldots \ldots \ldots \ldots \ldots \ldots \ldots \ldots \ldots \ldots \ldots \ldots, 4-22$

Figure 5-1 . Lower Cell as Modeled in CONTAIN .................... 5-4

Figure 5-2 Layer Configuration in Lower Cell without CORCON .............. 5-5

Figure 5-3 Layer Configuration in Lower Cell with CORCON ............... 5-7

Figure 5-4 Conceptual Picture of the Implementation of CORCON Mod3 into CONTAIN and MELCOR ............................ 5-24

Figure $6-1 \quad \mathrm{DCH}$ as Modeled in CONTAIN $\ldots \ldots \ldots \ldots \ldots \ldots \ldots \ldots .6 .6 .6 .6$ Figure 6-2 Illustration of the DCH Multifield Debris Representation in CONTAIN . . . . 6-6

Figure 7-1 Aerosol Behavior as Modeled in CONTAIN . . . . . . . . . . . . 7-2 Figure 7-2 Example of Aerosol Particle Mass Concentration Distribution for a TwoComponent Aerosol ............................... 7-3

Figure 7-3 Model for Water Condensation on Aerosols ................. 7-18 Figure 7-4 Illustration of Impaction on a Horizontal Strip of a Typical Ice Condenser

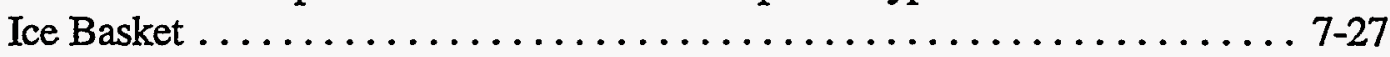

Figure 7-5 Qualitative Comparison of Aerosol Collection Efficiencies ......... 7-32 


\section{LIST OF FIGURES (CONTINUED)}

Figure 8-1 Fission Product Behavior as Modeled in CONTAIN $\ldots \ldots \ldots \ldots \ldots \ldots$ 8-2

Figure 8-2 Decay Processes in the Fission Product Library $\ldots \ldots \ldots \ldots \ldots \ldots \ldots . . .68$

Figure 8-3 Example of Linear Decay Chains Derived from a Coupled Decay Process . . 8-15

Figure 8-4 Example of Fission Product Redistribution by the Targeted Release and Acceptance Model for Fission Products Initially in the Lower Cell Fuel Layer

Figure 9-1 Combustion as Modeled in CONTAIN ..................... 9-3

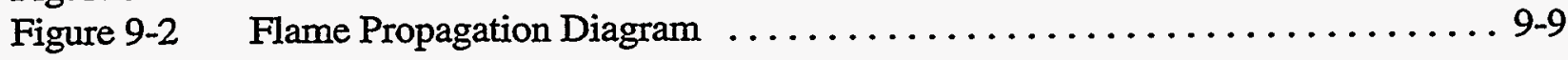

Figure 10-1 Heat and Mass Transfer as Modeled in CONTAIN . . . . . . . . . . . . 10-2

Figure 10-2 Test of the Power-Law Approximation for Thermal Conductivity at $\mathrm{T}_{\mathrm{BL}} \ldots . .10-8$

Figure 10-3 Example of a Connected Structure Configuration . . . . . . . . . . . 10-11

Figure 10-4 Steam Binary Diffusivities in Gases Calculated from the WL-HBS Model . 10-24

Figure 10-5 Comparison of WL-HBS Steam Binary Diffusivities with a Power-Law

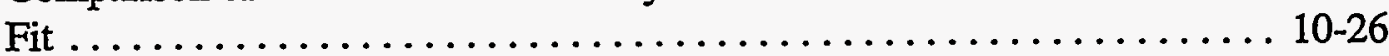

Figure 10-6 A Configuration of Structures That Could Be Used in the Film Tracking Model .................................... 10-29

Figure 10-7 The Influence of Noncondensables on the Interface Resistance . . . . . . . 10-33

Figure 10-8 Cylindrical Structure Consisting of a Steel Liner, Air Gap, and Thick

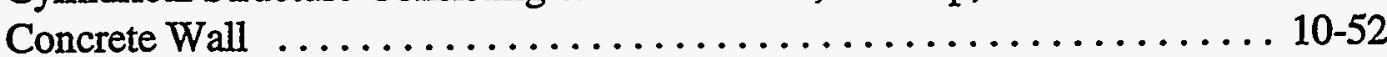

Figure 10-9 Nodes in a one-Dimensional System . . . . . . . . . . . . . . . 10-60

Figure 10-10 Illustration of the Concrete Outgassing Temperature Profile . . . . . . . . 10-67

Figure 10-11 Heat Transfer Processes at a Gas Structure Interface . ............ 10-70

Figure 11-1 Boiling Water Reactors as Modeled in CONTAIN $\ldots \ldots \ldots \ldots \ldots \ldots . . \ldots 11-2$

Figure 11-2 CONTAIN Multi-Node Vent Clearing Model for a Mark III BWR . . . . . . 11-4

Figure 11-3 Illustration of the Seven-Node Representation of Mark III Suppression

Vents Used in the Inertial Lengths Derivation . . . . . . . . . . . . . .

Figure 11-4 Suppression Pool Thermal-Hydraulics .................... 11-10

Figure 11-5 Definition of the Variables for the BWR Horizontal Vent Suppression

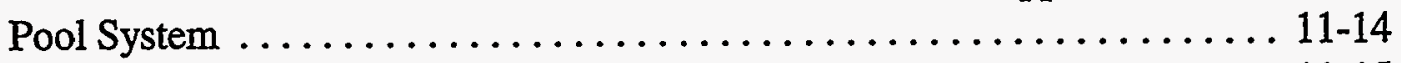

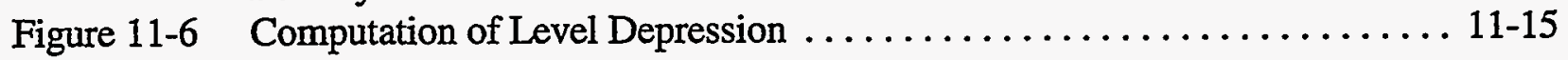

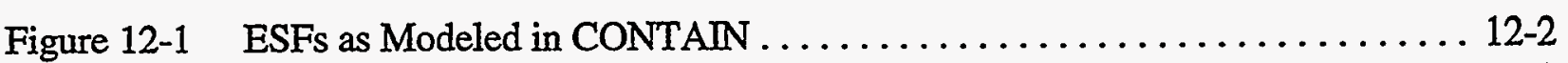

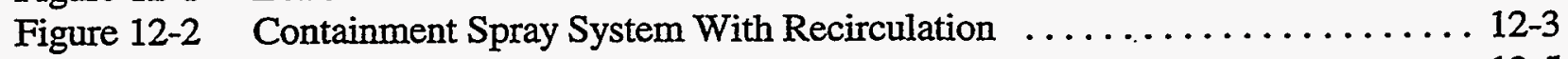

Figure 12-3 Fan Cooler System . . . . . . . . . . . . . . . . . . . . . . . 12-5

Figure 12-4 Fan Cooler Geometry Depicting One Horizontal Cooling Coil (Top View) . 12-6

Figure 12-5 Sectional View of an Ice Compartment and Containment Region

Boundaries ..................................... 12-11

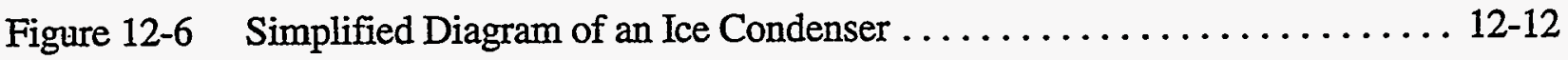




\section{LIST OF FIGURES (CONTINUED)}

Figure 12-7 CONTAIN Heat Exchanger Models $\ldots \ldots \ldots \ldots \ldots \ldots \ldots \ldots \ldots . . \ldots \ldots$

Figure 12-8 Counterflow Heat Exchanger Representation Defining the Heat Exchanger

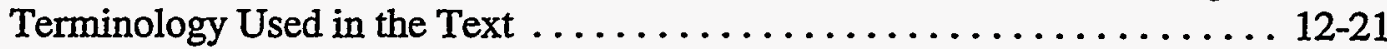

Figure 13-1 Estimated Error Resulting From Use of Bulk Noncondensable Gas Compositions in the CONTAIN Multicomponent Diffusivity Model ...... 13-15

Figure 13-2 Two Thermal Siphon Nodalizations Which Underpredict and Overpredict the Thermal Siphon Flow, Respectively, Within a Donor Approximation .. 13-21

Figure 13-3 CONTAIN Predictions Versus Experimental Results for (a) $\Delta \mathrm{P}$ and (b) Scaled $\mathrm{H}_{2}$ Production for the Standard Input Prescription (Case 1) ..... 13-27

Figure 13-4 Schematic of Approach for Defining RPV Blowdown and Airborne Debris Sources in DCH Calculations ........................ 13-34

Figure 13-5 Dispersed Debris and Liquid Water Fields Corresponding to the CONTAIN Treatment .............................. 13-42

Figure 13-6 Heat and Mass Transfer Schematics for Debris-Water Interactions . . . . . 13-42 Figure 13-7 Predicted Heat Transfer Coefficients for a Condensing Air/Stream Mixture $\left[\mathrm{T}_{\text {wall }}=322.0 \mathrm{~K}, \mathrm{~T}_{\text {init }}=300 \mathrm{~K}\right] \ldots \ldots \ldots \ldots \ldots \ldots \ldots \ldots \ldots \ldots \ldots \ldots \ldots \ldots$

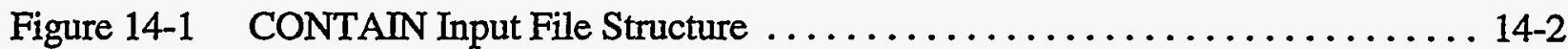

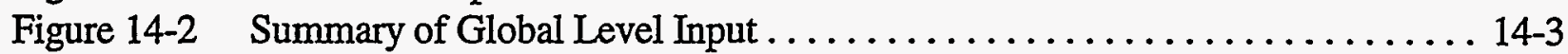

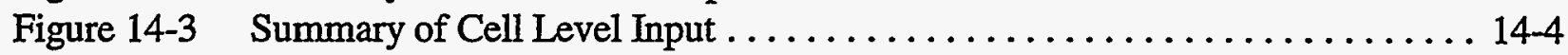

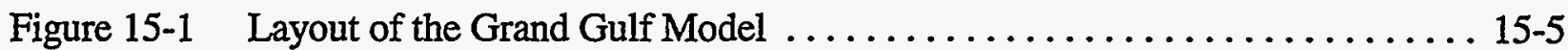

Figure 15-2 Pressure-Time Histories . . . . . . . . . . . . . . . . . . . . . . 15-7

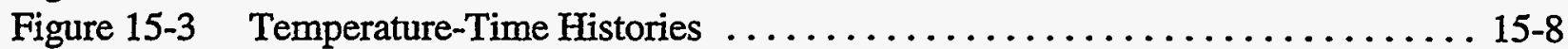

Figure 15-4 Vent Liquid Flow Rates as a Function of Time $\ldots \ldots \ldots \ldots \ldots \ldots \ldots \ldots . . \ldots 15-9$

Figure 15-5 Vent Liquid Flow Rates for First 5 Seconds $\ldots \ldots \ldots \ldots \ldots \ldots \ldots \ldots \ldots \ldots$

Figure 15-6 Vent Gas Flow Rates as a Function of Time .................. 15-11

Figure 15-7 Pool Depths for the Annulus and the Wetwell ................ 15-12

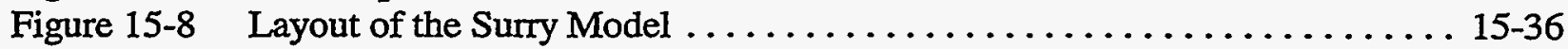

Figure 15-9 Pressure-Time Histories for the First 40,000 Seconds $\ldots \ldots \ldots \ldots \ldots \ldots 15-38$

Figure 15-10 Pressure-Time Histories for the Full Time Period . . . . . . . . . . . . . . . . . . 15-39

Figure 15-11 Temperature Histories . ............................ 15-40

Figure 15-12 Amount of Water Boiled and Evaporated from Pools ............. . 15-42

Figure 15-13 Cumulative Quantities of Gases Evolved and Amount of Zirconium

Remaining in the Melt ............................ 15-43

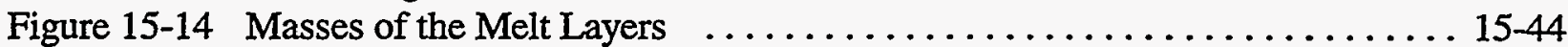

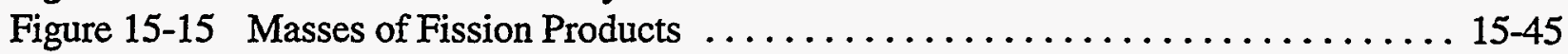

Figure 15-16 Cavity Dimensions as a Function of Time ................. 15-47

Figure 15-17 Debris Temperatures at the Lower Interfaces of the Layers $\ldots . . \ldots \ldots . .15-48$

Figure 15-18 Layout of the Sequoyah Model ....................... 15-85 


\section{LIST OF FIGURES (CONCLUDED)}

Figure 15-19 Pressure-Time Histories for the Containment Cells $\ldots . \ldots \ldots \ldots \ldots$. . . . 15-94

Figure 15-20 Flow Rates Through Paths Leaving the Lower Compartment . . . . . . . . . 15-96

Figure 15-21 Hydrogen Burned Prior to Vessel Breach ................... 15-97

Figure 15-22 Pressure-Time Histories During the DCH Event . . . . . . . . . . . . 15-98

Figure 15-23 Mass of Trapped Debris in Various Cells as a Function of Time . . . . . . . 15-99

Figure 15-24 Mass Balance Information for Hydrogen and Oxygen . . . . . . . . . . . 15-100

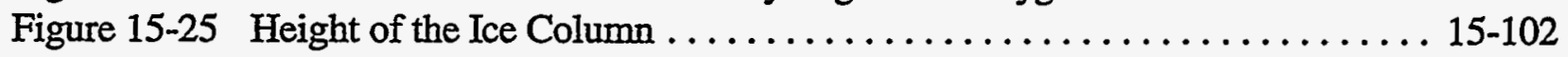

Figure 16-1 CONTAIN and POSTCON Files and Their Interrelationships $\ldots \ldots \ldots \ldots$ 16-2

Figure 16-2 The MAKEPLT Program for a Dummy Plot File $\ldots \ldots \ldots \ldots \ldots \ldots . . . \ldots 16-20$

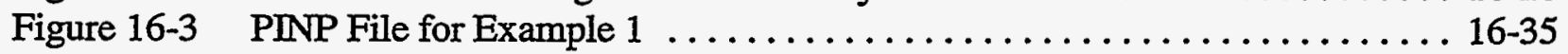

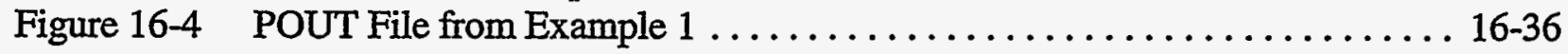

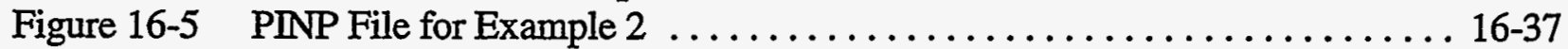

Figure 16-6 Snapshot Table from the POUT1 File for Example $2 \ldots \ldots \ldots \ldots \ldots \ldots$ 16-38

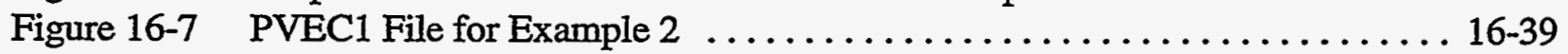

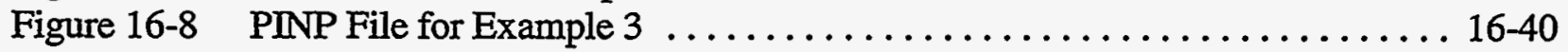

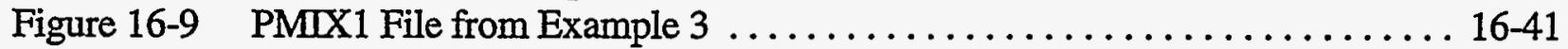

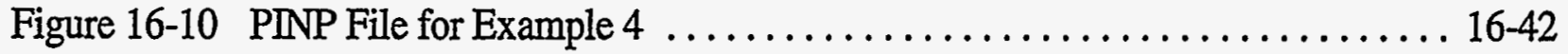

Figure C-1 Comparison of the Predicted Light Gas Concentrations in the Dome

(Cell 9) in the 15-Cell Base Case with the Experimental Measurements .... C-42

Figure C-2 Estimated Total Heat Transfer Coefficients for the LA-4 Vessel at

Location WCC2 ............................... -43

Figure C-3 CONTAIN Simulation of the University of California at Berkeley

Condenser Tube Heat Transfer Experiment for Air/Stream Mixtures . . . . . . C-44

Figure C-4 CONTAIN DCH-1 Pressure Prediction and Comparison to Experiment .... C-45

Figure C-5 Suspended Solid Aerosol Concentrations Predicted in the LA-4 Experiment,

Compared to Measurements ....................... -46

Figure C-6 Comparison of the PNL Experiment 11-6 Thermocouple Data and the CONTAIN Calculated Temperatures, Showing the Effects of

Backflow and Stratification on Temperatures in the Diffuser Inlet . . . . . . C-47

Figure D-1 CONTAIN Quality Assurance (QA) Organization $\ldots \ldots \ldots \ldots \ldots \ldots$ D-3

Figure D-2 CONTAIN Code Development/Maintenance System .............. D-5

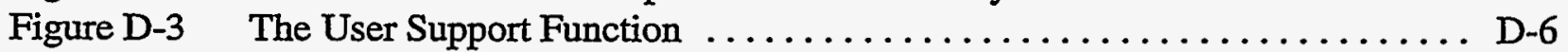

Figure D-4 Sample Change Document Cover Sheet $\ldots \ldots \ldots \ldots \ldots \ldots \ldots \ldots \ldots$ D-10

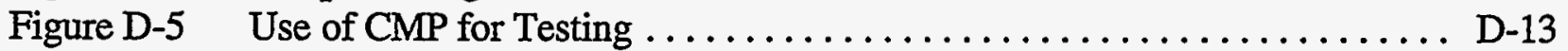

Figure D-6 Use of CMP for Operational Baseline Control $\ldots \ldots \ldots \ldots \ldots \ldots \ldots$ D-13

Figure D-7 The Configuration Control Function $\ldots \ldots \ldots \ldots \ldots \ldots \ldots \ldots \ldots$ D-14

Figure D-8 The Relationship Between the Mainstream CONTAIN Code and Its Variant

Versions ................................ D-16 


\section{LIST OF TABLES}

Table 1-1 CONTAIN Code Release History $\ldots \ldots \ldots \ldots \ldots \ldots \ldots \ldots \ldots \ldots . . \ldots \ldots$

Table 1-2 Update Sets Installed between CONTAIN 1.1 and CONTAIN 2.0,

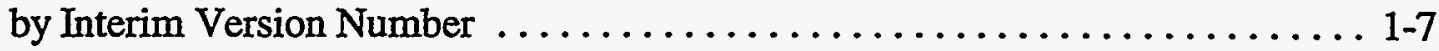

Table 1-3 Major New Models and Features of CONTAIN 2.0, by Modeling Area ....... 1-9

Table 2-1 Internal Timesteps Used Within Various Models $\ldots \ldots \ldots \ldots \ldots \ldots \ldots .2-8$

Table 2-2 Materials Modeled in CONTAIN .......................... 2-11

Table 3-1 Materials Available in CONTAN ........................... 3-3

Table 3-2 References for CONTAIN Material Properties $\ldots \ldots \ldots \ldots \ldots \ldots \ldots \ldots . . . \ldots \ldots$

Table 3-3 The Coefficients $A_{i j}$ in Equation (3-6) $\ldots \ldots \ldots \ldots \ldots \ldots \ldots \ldots \ldots \ldots \ldots \ldots \ldots$

Table 4-1 Definition of Inventory Factors for Gas and Flow Paths as a Function of the Elevation $\left(\mathrm{H}_{1}\right)$ of the Flow Path at the Donor Cell i, Cell Top Elevation $\left(\mathrm{H}_{t, i}\right)$, Cell Bottom Elevation $\left(\mathrm{H}_{\mathrm{b}, \mathrm{i}}\right)$, Pool Surface Elevation $\left(\mathrm{H}_{\mathrm{p}, \mathrm{i}}\right)$, and Gas Access

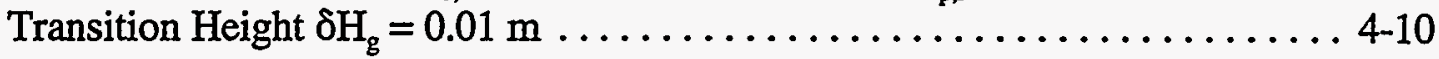

Table 4-2 Conservation of Momentum Equation for Flow Between Cell $i$ and Cell $j$. . . . 4-14

Table 4-3 Conservation of Mass Equation for the Atmosphere in Cell $i$. . . . . . . . . 4-27

Table 4-4 Conservation of Energy Equation for the Atmosphere in Cell i .......... 4-29

Table 4-5 Conservation of Mass Equation for the Coolant Pool in Cell i . . . . . . . . . 4-32

Table 4-6 Conservation of Energy Equation for the Coolant Pool in Cell i . . . . . . . . . . 4-34

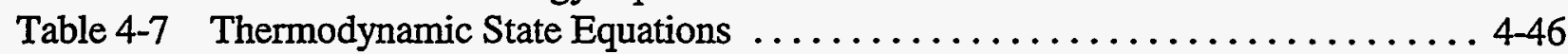

Table 5-1 Properties of CORCON Predefined Concrete Types ............... 5-9

Table 5-2 CORCON Metal and Oxide Species Names .................. 5-12

Table 5-3 CORCON Fission Product Decay Power

Element Names and Default Retention Factors ................ 5-13

Table 5-4 Melt Component Keywords and Chemical Symbols For VANESA Fission

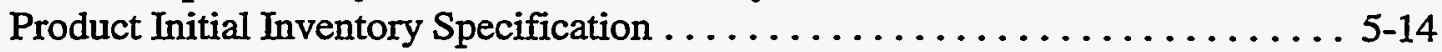

Table 5-5 VANESA Constituent Names of Released Aerosol and Fission Products ... . . 5 5-15

Table 6-1 Overview of DCH Processes Modeled in CONTAIN ................ 6-4

Table 6-2 Constants for the Tutu-Ginsberg Entrained Fraction Correlation . . . . . . . 6-28

Table 6-3 DCH Chemistry Energies of Reaction $\ldots \ldots \ldots \ldots \ldots \ldots \ldots \ldots \ldots .6 .60 .6 \%$

Table 7-1 Comparison Between Fixed- and Moving-Grid Models $\ldots \ldots \ldots \ldots \ldots \ldots$ 7-16

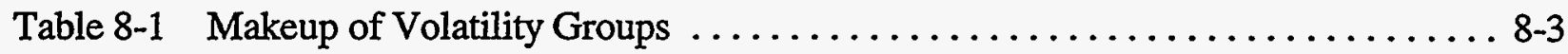

Table 8-2 Fission Product Library -- Decay Process $3 \ldots \ldots \ldots \ldots \ldots \ldots \ldots \ldots . .6 .17$

Table 8-3 Fission Product Library -- Decay Process $25 \ldots \ldots \ldots \ldots \ldots \ldots \ldots \ldots . .6 .17$

Table 8-4 Illustrative Fission Product Targeted Release Rates $\ldots \ldots \ldots \ldots \ldots \ldots \ldots$ 8-23 


\section{LIST OF TABLES (CONCLUDED)}

Table 9-1 Default Ignition and Propagation Limits $\ldots \ldots \ldots \ldots \ldots \ldots \ldots \ldots . . \ldots$ 9-5

Table 10-1 Heat and Mass Transfer Processes Considered at Various CONTAIN

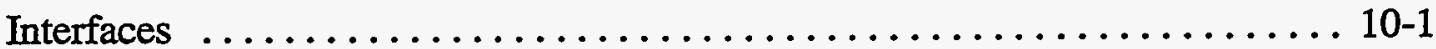

Table 10-2 Power-Law Diffusivity Constants $\ldots \ldots \ldots \ldots \ldots \ldots \ldots \ldots \ldots \ldots \ldots \ldots$ 10-25

Table 10-3 Coefficients for the Cess-Lian Correlation . . . . . . . . . . . . . . . . 10-45

Table 11-1 Example Solution for Flow Path Initial Lengths in the Three-Node

Representation of Suppression Pool Vents Shown in Figure 11-2 . . . . . . 11-8

Table 13-1 Summary of the CONTAIN Standard Input Prescription for NPP Analyses . . 13-29

Table 13-2 $\mathrm{K}_{c}$ Values and Performance Statistics for Cavity Correlations . . . . . . . . . 13-54

Table 13-3 Geometric Parameters Used in Correlations . . . . . . . . . . . . . . 13-55

Table 13-4 Standard Values Used in Assessing the Levy Correlation . . . . . . . . . . . . 13-58

Table 14-1 Types of Burns Allowed for Different Ignition Sources . . . . . . . . . . 14-98

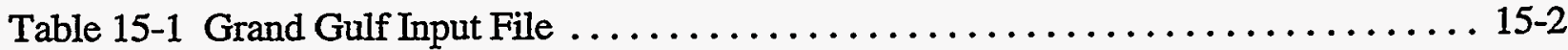

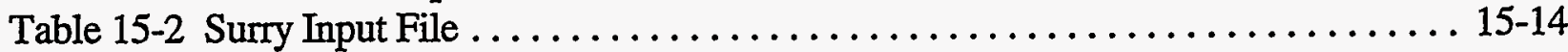

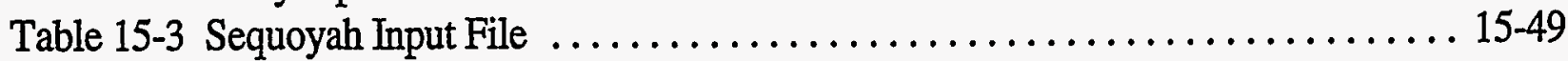

Table $15-4$ Sequoyah Restart Input File $\ldots \ldots \ldots \ldots \ldots \ldots \ldots \ldots \ldots \ldots \ldots \ldots$. $\ldots \ldots$. $\ldots \ldots$

Table $16-1$ Item Keywords $\ldots \ldots \ldots \ldots \ldots \ldots \ldots \ldots \ldots \ldots \ldots \ldots \ldots \ldots \ldots . \ldots \ldots$

Table 16-2 Default Conversion Factors and Unit Names . . . . . . . . . . . . . . 16-17

Table C-1 CONTAIN Code Release History $\ldots \ldots \ldots \ldots \ldots \ldots \ldots \ldots \ldots \ldots \ldots$. . . . . . . . .

Table C-2 Containment Test Facilities Used for CONTAIN Integral Validation . . . . . . . C-12

Table C-3 Validation Matrix for Atmosphere Thermal Hydraulics and Intercell

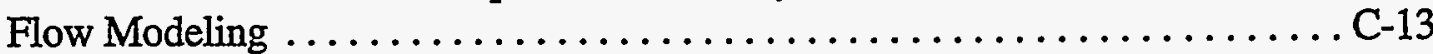

Table C-4 Validation Matrix for Heat and Mass Transfer Modeling . . . . . . . . . . . C-23

Table C-5 Validation Matrix for Heat and Mass Transfer Modeling (Non-CONTAIN

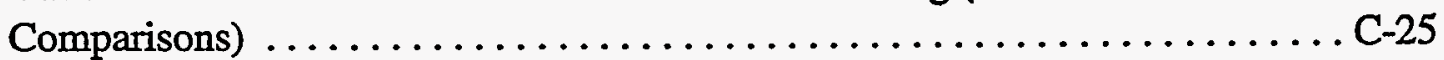

Table C-6 Validation Matrix for DCH Modeling . . . . . . . . . . . . . . . . . . C-29

Table C-7 Validation Matrix for Aerosol Modeling . . . . . . . . . . . . . . . C-36

Table C-8 Validation Matrix for Hydrogen Burn Modeling . . . . . . . . . . . . . . . C-39

Table C-9 Validation Matrix for Pressure Suppression Modeling . . . . . . . . . . . C-40

Table C-10 Validation Matrix for Miscellaneous Modeling . . . . . . . . . . . . C-41

Table D-1 Quality Assurance Functions . . . . . . . . . . . . . . . . . D-17

Table D-2 CONTAIN 2.0 Standard Tests $\ldots \ldots \ldots \ldots \ldots \ldots \ldots \ldots \ldots \ldots \ldots \ldots \ldots$ 


\section{LIST OF ABBREVIATIONS}

$\begin{array}{ll}\text { ALWR } & \text { advanced light water reactor } \\ \text { ANL } & \text { Argonne National Laboratory } \\ \text { ANSI } & \text { American National Standards Institute } \\ \text { BNL } & \text { Brookhaven National Laboratory } \\ \text { BSR } & \text { bulk spontaneous recombination } \\ \text { BWR } & \text { boiling water reactor } \\ \text { CCI } & \text { core-concrete interaction } \\ \text { CPU } & \text { central processing unit } \\ \text { DBA } & \text { design basis accident } \\ \text { DCH } & \text { direct containment heating } \\ \text { DDT } & \text { deflagration-to-detonation } \\ \text { DFB } & \text { diffusion flame burning } \\ \text { EOS } & \text { equation of state } \\ \text { ESF } & \text { engineered safety feature } \\ \text { FCI } & \text { fuel-coolant interaction } \\ \text { HEPA } & \text { High Efficiency Particulate Airborne } \\ \text { IET } & \text { Integral Effects Test } \\ \text { LFP } & \text { Limited Flight Path } \\ \text { LOCA } & \text { loss-of-coolant accident } \\ \text { LWR } & \text { light water reactor } \\ \text { MSLB } & \text { main steam line break } \\ \text { NPP } & \text { nuclear power plant } \\ \text { PCS } & \text { passive cooling system } \\ \text { PNL } & \text { Pacific Northwest Laboratory } \\ \text { PWR } & \text { pressurized water reactor } \\ \text { RCS } & \text { reactor cooling system } \\ \text { RPV } & \text { reactor pressure vessel } \\ \text { SA } & \text { severe accident } \\ \text { SNL } & \text { Sandia National Laboratories } \\ \text { SRV } & \text { safety relief valve } \\ \text { TMI } & \text { Three-Mile Island } \\ \text { USNRC } & \text { U. S. Nuclear Regulatory Commission } \\ \text { VDI } & \text { virtual dynamic interface } \\ \text { WC } & \text { Wet Cavity } \\ \text { WL-HBS } & \text { Wilke-Lee [modification of the] Hirschfelder, Bird, and Spotz [model] } \\ \text { WST } & \text { water storage tank } \\ & \end{array}$ 


\subsection{INTRODUCTION}

The CONTAIN code is an analysis tool for predicting the physical, chemical, and radiological conditions inside the containment and connected buildings of a nuclear reactor in the event of an accident. CONTAIN was developed at Sandia National Laboratories under the sponsorship of the US Nuclear Regulatory Commission (USNRC) for analyzing containment phenomena under severe accident and design basis accident conditions. It is designed to predict the thermal-hydraulic response inside containments and the release of radionuclides to the environment in the event of containment failure. The modeling capabilities of CONTAIN are also sufficiently flexible that it can be applied to the analysis of nonreactor problems, such as the migration of radioisotopes in waste repositories, and the thermal-hydraulic response of non-nuclear facilities under accident conditions. This manual focuses on the nuclear reactor containment analysis capabilities of CONTAIN, since this is its primary targeted application. Such analyses are an integral part of the USNRC's Severe Accident Research Program (SARP), where reactor safety issues are addressed through an appropriate mix of experimental and analytical research. The scope and role of the CONTAIN code as it applies to the SARP are discussed in Chapter 2.0. The role of CONTAIN and its relationship to other elements of the USNRC SARP is also discussed in the Revised Severe Accident Research Program Plan published by the USNRC. [NRC92]

The intent of this manual is to comprehensively document the models and capabilities of CONTAIN 2.0 by giving the following information. First, this manual describes the phenomenological models in CONTAIN and the underlying governing equations. The assumptions, relevant data base, and range of applicability of the models are also discussed in most instances. This information is provided for the reader who wishes to perform a "bottom-up" review or assessment of the models in the CONTAIN code. Second, this manual provides basic descriptions of the models and features in the code and the relationship among code input, models, and output. This information is provided for readers wishing to perform an engineering-level "top-down" review or assessment of the capabilities of the CONTAIN code. Third, this document provides detailed instructions and associated user guidance for preparing code input. This information is designed to assist both new and experienced code users in preparing and refining input files for the desired applications. Fourth, this document describes the postprocessing program used to process CONTAIN output into an easily usable form, including the generation of graphical results. Fifth, this document demonstrates the use of CONTAIN through the inclusion of a number of sample problems. These problems are intended to supplement the guidance provided to the code users in the basic model descriptions and input instructions. Sixth, the reader interested in an assessment of the CONTAIN models will find the CONTAIN validation data base summarized in Appendix C.

It should be noted that an extensive independent peer review of the CONTAIN code has recently been completed. [Boy95] The USNRC decided that a broad technical review of the CONTAIN code should be performed to determine its technical adequacy, and selected six technical experts to conduct an independent peer review of the code. The findings of the Peer Review Committee were extremely positive. In general, the peer review found that CONTAIN was very close to fulfilling all of its original design objectives and targeted applications, as well as the recently revised design objectives and targeted applications that were developed within the context of the NRC's severe accident code strategy. This achievement was highlighted as a "significant accomplishment" for the 
code project. In addition, the committee found that CONTAIN could be applied to both selected design basis accidents and severe accidents for both current reactors and advanced light water reactors. In the peer review committee's final report, a number of recommendations were made for improvements to the code and its documentation. [Boy95] The relatively small number of high priority recommendations have since been addressed either in the CONTAIN 2.0 code or this code manual, or resolved through separate communications with the USNRC. ${ }^{1}$

\subsection{The Need for Nuclear Reactor Containment Analysis}

Since the inception of nuclear power as a commercial energy source, safety has been recognized as a prime consideration in the design, construction, operation, maintenance, and decommissioning of nuclear power plants. Nuclear reactor systems are sufficiently complex that dismissing the possibility of an accident followed by the release of radioactivity to the environment would be imprudent. Such a release would require the failure of multiple safety systems and the breach of three physical barriers to the release of radioactivity: fuel cladding, reactor cooling system, and the containment. Probabilistic risk assessments have shown that the required multiple system failures are improbable but not negligible. The accident at Three Mile Island Unit 2 (TMI-2) demonstrated that significant core melting could occur in a commercial power reactor and that at least the first of the three physical barriers to radioactivity release can be breached. The reactivity-driven explosion at Chernobyl showed that all three barriers could be breached and the public exposed to radioactivity. Although US power reactors are not vulnerable to the type of energetic event that occurred at Chernobyl, that accident reinforced the perception that highly off-normal system and/or operator behavior, though extremely improbable, could in fact occur. Consequently, the USNRC's reactor safety research program has historically been focused on understanding system behavior and underlying phenomena associated with the low-probability, high-consequence class of accidents referred to as severe accidents. The CONTAIN code was developed as a tool to assist the analyst in understanding the complex phenomena and interactions that occur in the containment building of a nuclear reactor during such accidents.

The need for such a tool was born out of the recognition that the extreme nature of severe accident sequences presents unique difficulties for the reactor safety analyst. First, unlike many other safety engineering disciplines, very few relevant historical records of vessel failure accidents exist. Second, the reactor and its containment are extremely complex systems, and their designs vary substantially from one plant to another. Third, the fuel debris and the materials it contacts are expected to be subject to such severe conditions that the behavior of even small sub-elements of these complex systems is not easy to predict. Fourth, even though scaled experiments are vital to improving our understanding, it is often difficult to extrapolate the experimental data to the physical scale and system complexity of actual nuclear reactors. All these considerations lead to two conclusions concerning reactor safety research methodology. First, detailed, system-level, phenomenological computer models are essential tools for understanding how the reactor and its containment might

\footnotetext{
${ }^{1}$ It should be noted that the revised severe accident code strategy de-emphasizes the role of CONTAIN for source term analysis. This revised strategy was taken into account in the peer review model rankings and recommendations. Nevertheless, by direct comparison of the CONTAIN aerosol and fission product models with those of other codes, the user will find them in many cases to be state-of-the-art and suitable for many types of source term analyses.
} 
respond under accident conditions. Second, the application of such computational tools must be done intelligently with engineering judgment and with the benefit of phenomenological understanding that can only stem from experimental research. Therefore, the USNRC reactor safety research program has included both the development and application of analytical simulation tools and scaled experimental studies. Risk analysis methodologies, of course, continue to play a key integrating role in the safety assessment of nuclear power plants.

A worthwhile specific example of the importance of these research components and their interplay in the study and eventual resolution of severe accident issues occurs in the area of direct containment heating $(\mathrm{DCH})$. Here, experimental progress has resulted in major changes in our understanding of the governing processes that drive $\mathrm{DCH}$, and this change in understanding has had a significant impact on the evolution of the DCH models in CONTAIN. Since the release of CONTAIN 1.1, the USNRC has aggressively pursued the experimental investigation of DCH at several experimental scales. This effort has included thermite tests at $1 / 10$ th and 1/6th scale performed at Sandia National Laboratories, and thermite and $\mathrm{UO}_{2}$ tests at $1 / 40$ th scale performed at Argonne National Laboratory. Among other things, these tests have revealed the following: (1) containment subcompartments and structures result in a profound reduction in debris transport to the upper containment; (2) hydrogen burning can and usually does occur in a DCH event as a jet or a standing diffusion flame; (3) debris is essentially completely dispersed from the reactor cavity at modest to high driving pressures; and (4) water in containment during a DCH event can have either an aggravating or a mitigating effect depending upon the quantity involved. (Note that these lessons-learned are somewhat oversimplified). Numerous other lessons have been learned from these experiments. The point of this discussion is to emphasize that these experiments have provided invaluable insights into $\mathrm{DCH}$ that have been implemented and then refined in the CONTAIN 2.0 DCH models. The inclusion of these models and their assessment against this data base has resulted in a much more useful tool for the study of DCH in full scale plants than was available with the interim DCH models associated with CONTAIN 1.1.

Recently, the nuclear industry, with support from the US Department of Energy, has invested in the design of a new generation of advanced light water reactors (ALWRs). One objective of this initiative is to spark the revitalization of nuclear power as a viable future energy option for the United States. In light of the above-noted severe accident concerns for existing light water reactors (LWRs), great attention is being given to safety in the ALWR designs. In particular, many design features are aimed at reducing the probability of the occurrence of severe accidents, and/or mitigating the consequences. Improved safety is also being addressed in these designs through the use of more passive safety features, such as passive containment cooling. Another aspect of the strategy to spark the revitalization of the nuclear energy in this country is the development of a streamlined licensing process. At the time of this writing, many of the specifics of this revised process have not been finalized or even defined; however, it is known that some form of prelicense certification of these designs will be performed. Among other things, the certification process involves a USNRC independent assessment or confirmation of the effectiveness of the various new features in the ALWR designs. Such an assessment presents some of the same challenges as analyzing severe accidents for existing LWRs. For example, in many respects these systems are as complex and difficult to analyze as existing LWRs; therefore, extrapolation of knowledge about ALWR system performance gained from scaled experiments requires the use of detailed analysis tools. 
The safety assessment of these designs also presents some new challenges. In particular, the existing USNRC-sponsored analysis tools were not originally designed to address the passive safety features found in these designs. The USNRC reactor safety research program has therefore undertaken to adapt as necessary and apply their severe accident analysis tools to the analysis of ALWR behavior under accident conditions. The two designs that are receiving the most attention at the time of this writing are the AP600 design by Westinghouse and the simplified boiling water reactor (SBWR) design by General Electric. The certification of these designs and possibly others will require the USNRC to apply these adapted tools to the performance of the reactor and containment systems under both severe accident and design basis accident conditions. As a result, some of the new modeling capabilities in CONTAIN, such as the film tracking model discussed in Section 10.2.2.1, address the unique features of the ALWR designs.

The need for containment analysis is common to all US commercial LWR containment types. Therefore, CONTAIN 2.0 is intended to be applicable to large dry pressurized water reactor (PWR) containments, subatmospheric PWR containments, suppression pool containments of boiling water reactors (BWRs) (including horizontal and vertical vents), ice condenser containments, and passively cooled ALWR containments.

\subsection{Differences Between CONTAIN 2.0 and Earlier Versions}

The CONTAIN 2.0 code represents the third major version of CONTAIN. The first two major versions were released in 1984 and 1988 as CONTAIN 1.0 [Ber85b] and as CONTAIN 1.1, [Mur89a, Mur89b, Was91] respectively. Table 1-1 lists the code versions from 1.0 through 2.0 and summarizes the major improvements, new models, and other items contained in each version. CONTAIN 1.1 and 2.0 incorporate numerous significant improvements and advancements not included in CONTAIN 1.0. The CONTAIN 1.1 changes are summarized in References Mur89a and Was 91 . The improvements offered by CONTAIN 2.0 over CONTAIN 1.1 are equally numerous and significant.

Many of the latter improvements are embodied in interim versions. Four such versions have been released: CONTAIN 1.11, CONTAIN 1.12, CONTAIN 1.12V, and CONTAIN 1.2. Table 1-2 shows the official update sets incorporated in each of these interim versions. While CONTAIN 1.11, 1.12, $1.12 \mathrm{~V}$, and 1.2 are in use by a number of users (as approved by the USNRC on a case-by-case basis) this manual for CONTAIN 2.0 represents the first formal documentation provided since CONTAIN 1.1. Some of the models in the interim versions may have been revised or replaced; therefore, interim documentation on these versions may not agree with the descriptions provided in this code manual.

Table 1-3 indicates the code version(s) associated with the addition of a new modeling capability or code feature or the revision of an existing one and also lists the sections of this report where the reader can obtain more information about the indicated model additions or revisions. Numerous minor, but important, fixes to modeling errors or code bugs were also implemented but are not listed in this table. 


\subsection{Guide to This Manual}

This code manual includes documentation for old and new models and features of the CONTAIN code. For old models and features, the manual represents the consolidation of previously published documentation on the CONTAIN code. Information used for this consolidation includes the CONTAIN 1.0 User's Manual, [Ber85b] the CONTAIN 1.1 User's Manual, [Mur89a, Mur89b] the CONTAIN 1.1 Reference Manual, [Was91] and the POSTCON 1.0 Manual. [Was87] Other sources of information for this code manual include unpublished code change documents that describe new or updated models in the interim code revisions. Although unpublished, these change documents have been distributed to code users along with the release and distribution of CONTAIN 1.12 to USNRC approved users. Note: A code change document is generated by the developer of an update and must be approved by the revision manager before the model is finalized, according to the

Table 1-1

CONTAIN Code Release History

\begin{tabular}{|c|c|}
\hline $\begin{array}{l}\text { Code Version } \\
\text { (date) }\end{array}$ & Major Improvements and New Models \\
\hline $\begin{array}{c}1.0 \\
\text { (Aug. 1984) }\end{array}$ & First official release of code \\
\hline $\begin{array}{c}1.01 \\
\text { (December 1984) }\end{array}$ & Modifications to correct nonstandard use of character variables \\
\hline $\begin{array}{l}1.02 \\
1.03 \\
\text { (May 1985) }\end{array}$ & $\begin{array}{l}\text { Modifications to make code conform to FORTRAN- } 77 \text { standard } \\
\text { Improved lower-cell (pool and basemat) nodalization process, and engineering safety } \\
\text { systems models }\end{array}$ \\
\hline $\begin{array}{c}1.04 \\
\text { (November 1985) }\end{array}$ & $\begin{array}{l}\text { Add water aerosol deposition into structure surface film layer, time-dependent aerosol } \\
\text { size distribution input parameters, and implicit flow solver for multi-cell gas transport } \\
\text { Improved atmosphere-to-structure radiation model and hydrogen burn timestep } \\
\text { adjustment, and method to estimate liquid film boundary layer interface temperature }\end{array}$ \\
\hline $\begin{array}{c}1.05 \\
\text { (July 1986) }\end{array}$ & $\begin{array}{l}\text { Add carbon monoxide combustion, fission product targeted release and acceptance, } \\
\text { choked flow limit for intercell gas flow, and activation and deactivation keywords for } \\
\text { combustion model } \\
\text { Improved thermal properties, burn completeness correlation, and flexibility in } \\
\text { specifying combustion burn parameters }\end{array}$ \\
\hline $\begin{array}{c}1.06 \\
\text { (February 1987) }\end{array}$ & $\begin{array}{l}\text { Add integrated implementation of the CORCON-Mod2 model for core concrete } \\
\text { interactions (CCIs), VANESA model for aerosol generation and radioisotope release } \\
\text { from CCIs, water dropout model to remove water from atmosphere without using } \\
\text { aerosol dynamics model, and aerosol settling through flow paths } \\
\text { Improved models for radiant heat transfer (net enclosure model) }\end{array}$ \\
\hline
\end{tabular}


Table 1-1

CONTAIN Code Release History (Continued)

\begin{tabular}{|c|c|}
\hline $\begin{array}{l}\text { Code Version } \\
\text { (date) }\end{array}$ & Major Improvements and New Models \\
\hline $\begin{array}{c}1.10 \\
\text { (October 1987) }\end{array}$ & $\begin{array}{l}\text { Add specialized models for boiling water reactor features (safety relief valves and } \\
\text { pressure suppression pools), new flow path type (engineering vent) to provide added } \\
\text { flexibility to plant nodalization, and user-defined material properties } \\
\text { Improved method for tracking radioisotopes, the heat conduction algorithm for lower- } \\
\text { cell concrete floor model, various solution techniques including semi-implicit } \\
\text { coupling of the thermal radiation model with flow equations, and automatic recovery } \\
\text { from nonconvergence in the flow solver }\end{array}$ \\
\hline $\begin{array}{c}1.11 \\
\text { (March 1991) }\end{array}$ & $\begin{array}{l}\text { Add moving-grid calculation technique for solving the equations for aerosol growth } \\
\text { by water vapor condensation, concrete outgassing of both bound and evaporable water } \\
\text { and carbon monoxide, generalized treatment of heat structure boundary conditions, } \\
\text { flexibility for variable setting on restart, aerosol settling into pools, volumetric } \\
\text { displacement of atmosphere by pools, and an averaging scheme to approximate gas } \\
\text { flow velocities within a cell, used for calculating force convection for heat structures } \\
\text { Improved material property library; heat and mass transfer models for } \\
\text { condensation/evaporation at structure surface, and in ice condenser and fan coolers; } \\
\text { and lower cell modeling of transient pool layers } \\
\text { Improved flexibility of user control over plot files }\end{array}$ \\
\hline $\begin{array}{c}1.12 \\
\text { (March 1991) }\end{array}$ & $\begin{array}{l}\text { Add direct containment heating }(\mathrm{DCH}) \text { modeling, reactor cavity models for high } \\
\text { pressure debris dispersal and vessel blowdown, and new model for the vapor } \\
\text { saturation of noncondensable gas vented into pools } \\
\text { Improve concrete outgassing modeling to include ability to outgas from behind liners }\end{array}$ \\
\hline $\begin{array}{c}1.12 \mathrm{~V} \\
\text { (August 1993) }\end{array}$ & Workstation version of release 1.12 \\
\hline $\begin{array}{c}1.2 \\
\text { (October 1995) }\end{array}$ & $\begin{array}{l}\text { Add film flow on wall structures, energy and mass conservation tracking, hybrid flow } \\
\text { solver, pool tracking, non-ideal equation of state for water, specific reactor pressure } \\
\text { vessel and cavity models for DCH, CORCON Mod3, and fission product library } \\
\text { Improved DCH model setup and heat and mass transfer for convection and } \\
\text { condensation on structures }\end{array}$ \\
\hline $\begin{array}{c}2.0 \\
\text { (June 1997) }\end{array}$ & Improvements in the DCH and hydrogen burn models and miscellaneous bugfixes. \\
\hline
\end{tabular}

CONTAIN software quality assurance procedures. In many instances the information from the existing documentation has been rewritten for improved clarity. Therefore, when any of the previously available documents on CONTAIN appear to conflict or be inconsistent with this manual, the reader should consider this manual to be the more accurate source of information. 
The intent of this document is to provide in one publication all the information required to understand the models in CONTAIN 2.0 as well as information needed to use the code. The intended audience includes: (1) new users who are learning how to use the code, (2) experienced users who need a reference to the models, input instructions, and output, and (3) nonusers who wish to review either the broad capabilities or the specific models in CONTAIN. Given that each model is typically discussed later in this report in complete detail, the intent of this chapter and the next is to give a broader perspective, as well as to provide a road map or guide through the document to assist the reader in finding the desired information.

Table 1-2

Update Sets Installed between CONTAIN 1.1 and CONTAIN 2.0, by Interim Version Number

\begin{tabular}{||lll||}
\hline CONTAIN 1.11 & \\
\hline C110A & - & postrelease fixes for version 1.10 \\
\hline C110B & - & interface heat balance upgrade \\
\hline C110C & - & miscellaneous fixes \\
\hline C110D & - & conduction model upgrade and concrete outgassing model \\
\hline C110E & - & flow-based convective velocity model \\
\hline C110F & - & moving-grid aerosol condensation model \\
\hline C110G & - & FLINT FORTRAN corrections \\
\hline C110H & - & conduction/outgassing model revisions \\
\hline C110I & - & plot file output options \\
\hline C110J & - & miscellaneous fixes \\
\hline C110K & - & material properties upgrade \\
\hline C110L & - & miscellaneous fixes \\
\hline C110M & - & miscellaneous fixes \\
\hline C110N & - & prerelease fixes for version 1.11 \\
\hline CONTAIN 1.12 & \\
\hline C110O - & direct containment heating model \\
\hline C110P - & CORDE/GASBLOW2 models \\
\hline C110Q & - & miscellaneous fixes \\
\hline C110R & - & prerelease fixes for version 1.12 \\
\hline CONTAIN 1.2 & \\
\hline C110S - & postrelease fixes \\
\hline
\end{tabular}

${ }^{\dagger}$ Not supported in CONTAIN 2.0 
Table 1-2

Update Sets Installed between CONTAIN 1.1 and CONTAIN 2.0, by Interim Version Number (Concluded)

\begin{tabular}{|c|c|}
\hline C110T & - combustion model upgrade \\
\hline $\mathrm{C} 110 \mathrm{U}$ & - multifield DCH model \\
\hline C110V & - workstation compatibility changes \\
\hline C110w & - $\quad$ film tracking modifications \\
\hline $\mathrm{C} 110 \mathrm{X}$ & - mass and energy accounting modifications \\
\hline $\mathrm{C} 110 \mathrm{Y}$ & - $\quad$ DCH slip model \\
\hline $\mathrm{C} 110 \mathrm{Z}$ & - DCH reactor pressure vessel and cavity models \\
\hline C11AA & - nonideal water equation of state \\
\hline $\mathrm{C} 11 \mathrm{AB}$ & - $\quad$ CORCON-Mod3 upgrade \\
\hline C11AC & - fission product library \\
\hline $\mathrm{C} 11 \mathrm{AD}$ & - heat transfer flexibility improvement \\
\hline $\mathrm{C} 11 \mathrm{AE}$ & - pool tracking modifications \\
\hline $\mathrm{C} 11 \mathrm{AF}$ & - hybrid solver modifications and prerelease fixes \\
\hline \multicolumn{2}{|c|}{ CONTAIN 2.0} \\
\hline $\mathrm{C} 11 \mathrm{AG}$ & miscellaneous improvements and bugfixes \\
\hline
\end{tabular}

Note that Chapter 2 provides a broad perspective on CONTAIN and gives a general discussion of each of the major modeling areas. This chapter does not present modeling details such as governing equations; however, cross-references are provided in this chapter to the detailed modeling discussions and the model input descriptions. Detailed discussions are given in Chapters 3 through 12 with regard to each of the major modeling areas in the code. Practical advice and user guidance are provided in Chapter 13, as well as hints and tips for circumventing commonly encountered difficulties based on the experiences of CONTAIN code users and the code developers. Detailed instructions for preparing code input are provided in Chapter 14. This chapter is appropriate for guiding new users in preparing input files and is also suitable as a reference for the experienced code user. A number of sample problems and input files are provided in Chapter 15. Code output and the POSTCON postprocessing program are discussed in Chapter 16. Appendix A discusses the mass and energy accounting feature of CONTAIN 2.0. Appendix B presents a number of input formats that are considered obsolete but are available in CONTAIN 2.0 to maintain upward compatibility of input files. Appendix $\mathrm{C}$ presents a compendium of independent analyses and experimental comparisons that have served to validate CONTAIN. Finally, Appendix D discusses the quality assurance procedures that have been used in the development of CONTAIN. 
Table 1-3

Major New Models and Features of CONTAIN 2.0, by Modeling Area

\begin{tabular}{|c|c|c|}
\hline New and Upgraded Model or Feature & Code Version $^{*}$ & Code Manual Sections \\
\hline Interface Heat Balance & $\mathrm{R} 1.11, \mathrm{R} 1.2$ & $2.10,10.6$ \\
\hline Heat Conduction Model & $\mathrm{R} 1.11$ & $2.10,10.5 .3,14.3 .1 .3$ \\
\hline Concrete Outgassing & $\mathrm{N} 1.11, \mathrm{R} 1.2, \mathrm{R} 2.0$ & $2.10,10.5 .4,14.3 .1 .3$ \\
\hline Connected Structure Boundary Condition & $\mathrm{N} 1.11, \mathrm{R} 1.2$ & $2.10,10.5 .2,14.3 .1 .3$ \\
\hline Flow-based Convection Velocities & $\mathrm{N} 1.11, \mathrm{R} 1.2$ & $2.10,10.1 .1 .6$ \\
\hline $\begin{array}{l}\text { Moving Grid Aerosol Condensation } \\
\text { Model }\end{array}$ & N1.11 & $2.7,7.2 .2,14.2 .5$ \\
\hline Plot File Output Options & N1.11 & 14.2.9.3 \\
\hline Material Properties Upgrade & $\mathrm{R} 1.11$ & $2.3,3.1,3.3$ \\
\hline Direct Containment Heating & $\begin{array}{l}\mathrm{N} 1.12, \mathrm{R} 1.12 \mathrm{~V}, \mathrm{R} 1.2, \\
\mathrm{R} 2.0\end{array}$ & $\begin{array}{l}\text { 2.6, 6.0, 14.2.7 } \\
\text { 14.3.1.11, 14.5.1.3 }\end{array}$ \\
\hline Combustion Model Upgrade & $\mathrm{R} 1.12 \mathrm{~V}, \mathrm{R} 1.2, \mathrm{R} 2.0$ & $2.9,9.0,14.3 .1 .7$ \\
\hline Film Tracking Model & $\mathrm{N} 1.2$ & $2.10,10.2 .2,14.3 .1 .3$ \\
\hline Mass and Energy Accounting & $\mathrm{N} 1.2$ & $\begin{array}{l}\text { 2.3, 3.4, 14.2.2 } \\
\text { 14.2.9.2, App. A }\end{array}$ \\
\hline Nonideal Equation of State for Steam & $\mathrm{N} 1.2$ & $2.3,3.2,14.2 .4 .1$ \\
\hline CORCON-Mod3 Upgrade & $\mathrm{R} 1.2$ & $2.5,5.0,14.3 .2$ \\
\hline Heat Transfer Flexibility Upgrade & $\mathrm{R} 1.2$ & $\begin{array}{l}\text { 2.10, 10.1, 10.2, } \\
\text { 14.3.1.3 }\end{array}$ \\
\hline Pool Tracking Modifications & $\mathrm{R} 1.2$ & $\begin{array}{l}\text { 2.4, 4.0, 14.2.4, } \\
\text { 14.3.1.1 }\end{array}$ \\
\hline Hybrid Solver Modifications & $\mathrm{R} 1.2$ & $2.4,4.4 .5 .1,14.2 .4$ \\
\hline
\end{tabular}

$* \mathrm{R}=$ revision of an existing model, $\mathrm{N}=$ new model; code versions denoted by $1.11,1.12,1.12 \mathrm{~V}$, 1.2 , or 2.0 


\subsection{GENERAL DESCRIPTION OF THE CODE MODELS}

The purpose of the present chapter is to provide a general overview of each of the modeling areas in CONTAIN. This is intended to orient the reader with respect to the scope of the code and the available modeling options, without burdening the reader with the full details. Each of the modeling areas is discussed in full detail in Chapters 3-12. Additional user guidance for each modeling area is also provided in Chapter 13. Section 2.1 of the present chapter discusses the scope of the modeling, Section 2.2 discusses computational considerations, and Sections 2.3 through 2.12 are each devoted to an individual modeling area.

\subsection{Scope of the Modeling}

Where possible, best-estimate models are employed with an emphasis placed on mechanistic detail and numerical robustness. Despite use of best-estimate models, the code has reasonable computational efficiency because a control volume framework is used. The control volume approach has proven to be a useful technique for modeling a wide variety of containment configurations. It also provides a suitable framework for modeling the many different containment subsystems. To allow a wide range of applications, physical models are activated as needed for each simulation on the basis of the physical processes present.

The modeling applies primarily to processes that occur within the containment building. Therefore, the term "integral analysis" does not refer to the combined treatment of the reactor/containment/ environment system; rather, it applies to the range of phenomena analyzed for the containment system itself. Separate effects codes could be used to examine containment phenomena. Under such an approach, one code analyzes the thermal-hydraulic phenomena, another handles the fission product processes, another is used to model aerosol behavior, and so on. By contrast, CONTAIN treats all of these phenomena and others as well. The benefit of this approach is illustrated in Figure 2-1, where the ability of one process to affect another through phenomenological feedback mechanisms is shown. Today there are several other modern codes that also use this integral approach to accident analysis, although this was relatively uncommon when CONTAIN [Ber85b] was first released in 1984.

The code treats a containment system as a network of interconnected control volumes or "cells," shown schematically for a typical containment building in Figure 2-2. The cells may each represent an actual internal compartment or group of compartments in the reactor containment building, although in some cases the user may wish to partition a compartment to model phenomena such as natural convection and stratification within the compartment. The cells communicate with each other by means of mass flow of material between cells and/or heat conduction between cells through heat transfer structures. The arrows in this figure indicate possible flow directions through some of the flow paths present in the problem. Because there is considerable flexibility in specifying the properties of each cell and the connections between cells, the code is able to handle a wide variety of containment types. 


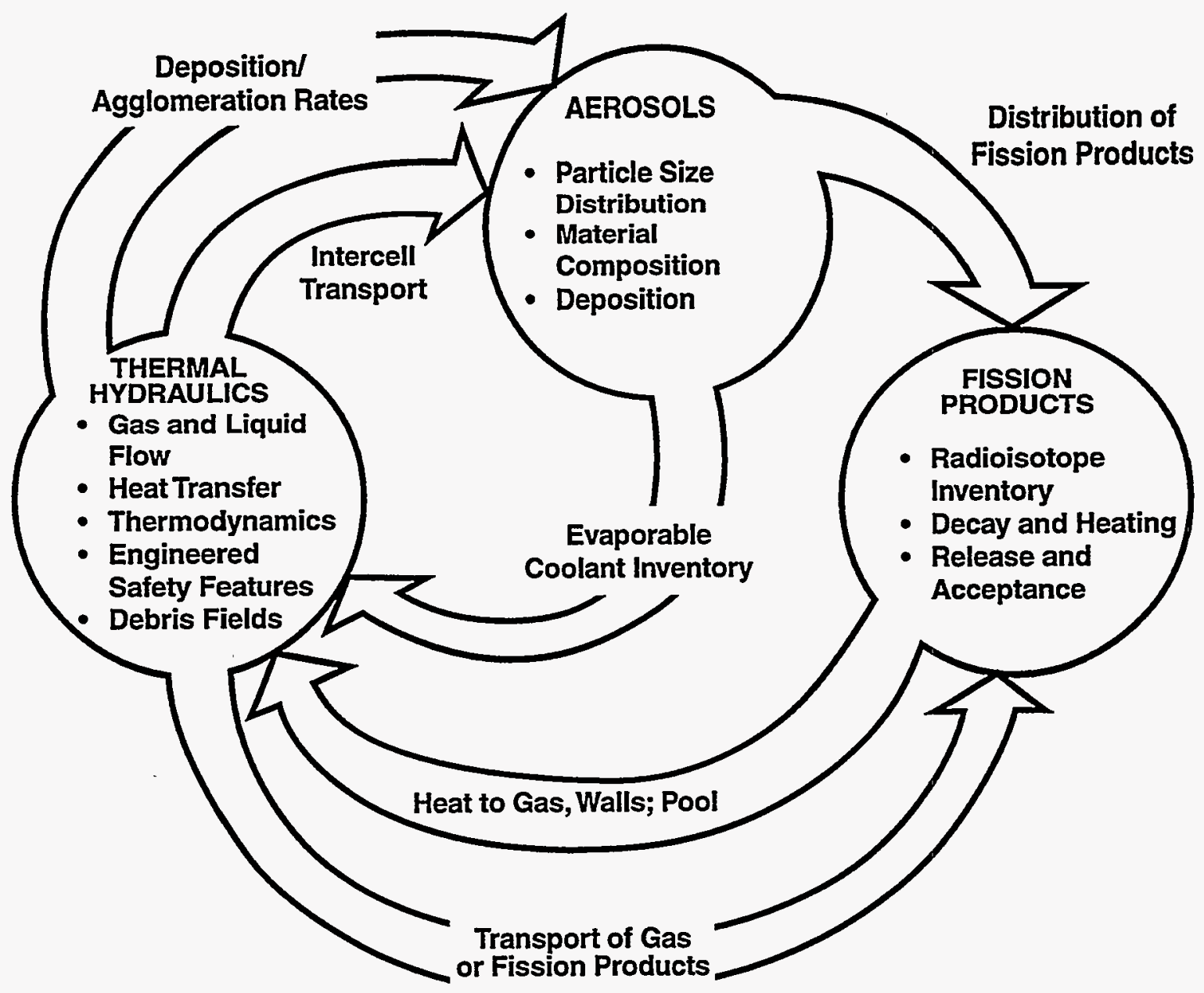

Figure 2-1. Illustration of Feedback Mechanisms Treated in the CONTAIN Code 


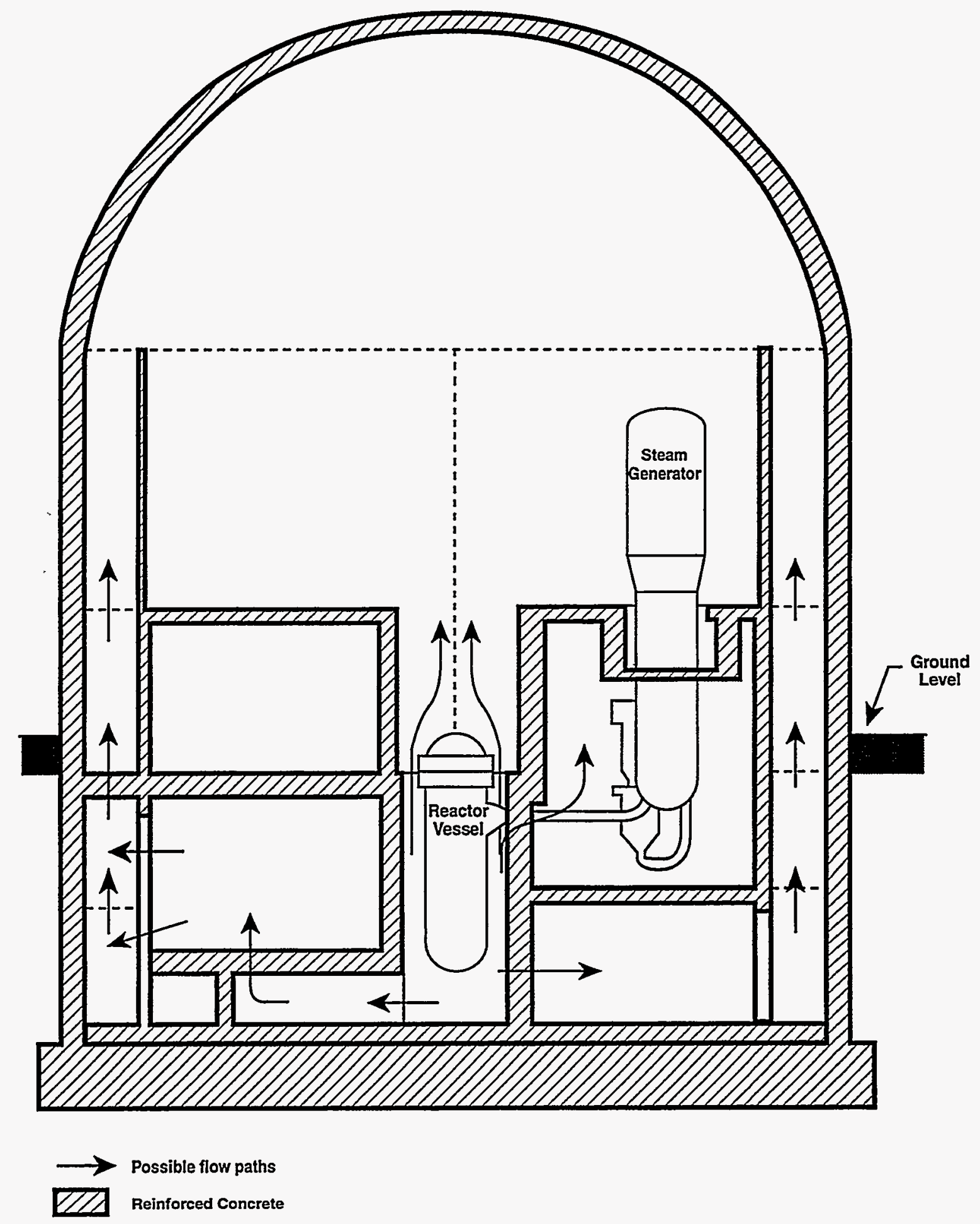

Figure 2-2. Reactor Containment Building Represented by Interconnected Cells

Rev. 0 
For completeness, the environment outside the containment building can be represented as a cell with a very large volume. This representation not only allows boundary conditions with respect to the environment to be applied to the internal cells but allows releases to the environment to be tracked in terms of the environment cell inventories.

As with many control volume codes, CONTAIN is designed to use a relatively small number of control volumes, but a relatively large number of degrees of freedom per control volume, or cell. These degrees of freedom can be localized, such as the nodes of heat transfer structure, or global fields associated with intercell flow. The global fields are associated with either the atmosphere or pool. The atmosphere fields include (1) the atmosphere bulk fluid, which includes gases, coolant vapor, and any homogeneously dispersed liquid coolant, (2) aerosols represented in terms of a number of size classes, each with a separate material composition (3) fission product distributions assigned to the gas and each aerosol component material, and (4) finely dispersed core debris, represented in terms of an arbitrary number of debris droplet fields. Such dispersed core debris can result, for example, from high pressure melt ejection from the reactor pressure vessel. The coolant pool fields include (1) the pool bulk fluid, (2) aerosols deposited into the pool, and (3) fission product distributions assigned to the pool. These fields allow complex interactions to be modeled within and between cells.

The major processes that are modeled include intercell flow, hydrogen combustion, heat and mass transfer processes (e.g., convection, condensation, condensate film flow, thermal radiation, conduction, and concrete outgassing), aerosol behavior (e.g., agglomeration, deposition, and condensation), fission product behavior (e.g., decay, heating, and transport), engineering safety features (ESFs) (e.g., containment sprays, fan coolers, and ice condensers), processes associated with but not limited to boiling water reactors (BWRs) (e.g., vent clearing, gas-pool equilibration, and aerosol scrubbing), direct containment heating (DCH) caused by high pressure ejection of finely divided core debris from the reactor vessel, and core-concrete interactions (CCIs). These modeling categories can be further divided into numerous separate phenomenological models, a few of which are noted parenthetically above. Taken collectively, these models provide the code with the capability to analyze a wide variety of $L W R$ plants and accident scenarios. Through appropriate user input, large-dry, sub-atmospheric, and ice condenser pressurized water reactor (PWR) containments can be modeled. Boiling water reactor (BWR) containments, and advanced light water reactor (ALWR) containments can also be modeled. The input flexibility also allows for the representation of experimental facilities and other nonstandard configurations. This has proven to be important for performing code validation calculations.

Since CONTAIN is not designed to treat in-vessel processes, the user in many cases must rely upon separate analyses to determine the sources of mass and energy to the containment. However, in some cases a rudimentary thermal-hydraulic model of the primary system may suffice, and the user may be able to model this directly. Also, CONTAIN does not calculate doses or model processes beyond the containment boundary. Such modeling can be performed with a separate consequence analysis code, using the results of a CONTAIN calculation. By limiting itself to the containment, the code is able to maintain reasonable computational efficiency while still incorporating detailed mechanistic models. 


\subsection{Computational Considerations}

\subsubsection{The Bi-Level Modeling Approach}

A bi-level approach to modeling is used in CONTAIN. This approach distinguishes between celllevel and global models. Cell-level models, as a rule, describe processes within a cell that are not strongly coupled to processes outside the cell. Global models, on the other hand, typically describe processes that are strongly coupled between cells.

The overall scheme under which the global and cell-level models are processed is shown in Figure 2-3. The results from the cell-level processing loop indicated in Figure 2-3 are cast in the form of sources and sinks of mass and energy for the global models. In the global loop, these sources and sinks are processed by the global level models to generate updated global conditions for use on the next pass through the cell-level loop.

The most important global process is the intercell flow of the fluids. To achieve a stable flow calculation for an arbitrary system of cells and interconnections, the flow rate through each flow path must be based on self-consistent conditions; that is, the flow must reflect changes in pressures and pool levels in the upstream and downstream cells. Thus, an implicit global analysis of intercell flows is required.

Most other phenomena treated at the global level are coupled to or affected by intercell flow. For example, the equation of state for the gas determines the pressure, which is strongly coupled to intercell flow. Therefore, for numerical stability, the thermodynamic state calculations are done simultaneously with the flow calculation. Other fields are affected by the gas flow. For example, the flow of suspended aerosols, fission products, and dispersed core debris is based on that of the gas. Since the effects of flow on these fields are taken into account at the same time gas flows are calculated, it is convenient to place certain aspects of the aerosol, fission product, and dispersed core debris modeling at the global level.

\subsubsection{Calculational Timestep Control}

This section discusses the calculational timestep hierarchy under which various models are integrated forward in time. There is a separate hierarchy that controls the various output frequencies (short edit, long edit, plot, and restart). This is discussed in Section 14.2.9.

Figure 2-3, which shows the overall scheme used for global and cell-level processing, does not show which models are processed at each of these levels. Section 2.2.2.1, which discusses the time step hierarchy, provides additional information concerning how various models are evaluated, including the timesteps used. 

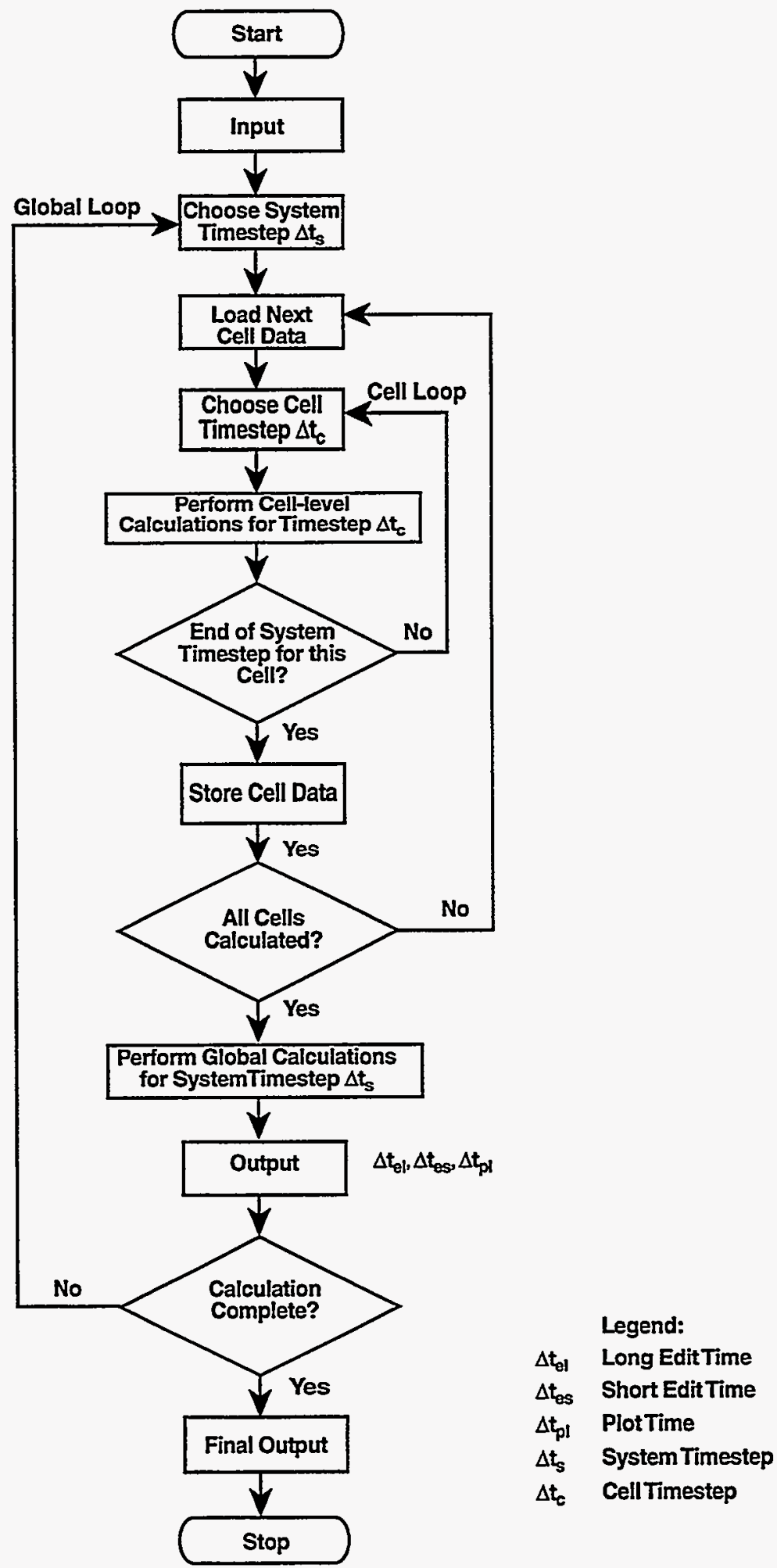

Figure 2-3. Overall Flow Diagram of CONTAIN 
Section 2.2.2.2 provides some guidance to the user in selecting reasonable maximum system and cell timesteps. These suggestions consider only some of the interactions among the explicitly coupled models. The user should be aware that other inaccuracies (not necessarily instabilities) may result from insufficiently frequent updating of information between explicitly coupled models. Depending on the level of the model, the updating frequency is controlled by either the cell or system timestep. The testing of calculated results for sensitivity to user-specified timesteps is always encouraged.

2.2.2.1 Timestep Hierarchy. Four different types of calculational timesteps are used. Two of these, the system timestep and the cell timestep ( $\Delta t_{s}$ and $\Delta t_{c}$ in Figure 2-3), are primarily under user control. The third type, the Runge-Kutta timestep, is selected automatically and used within certain models. The fourth timestep is that used by the CORCON-Mod3 module, which describes core-concrete interaction (CCIs). The maximum and minimum allowed values of this timestep are user-controlled.

The models in CONTAIN are processed in parallel. This type of processing is somewhat different from the serial, discrete-time processing normally found in single purpose codes. In parallel processing, each model, or in some cases a group of models, is integrated forward in time over the system timestep. The system timestep is defined as the maximum interval of time after which all models, except possibly CORCON-Mod3, must be in coincidence. State variables that are external to the model or model group being processed at any given time are held fixed at the last calculated value. State variables that belong to the model or model group being processed may be updated at many sub-intervals within the system timestep. These sub-intervals are determined by the model internal timestep. (In some cases, this is just the cell timestep.) After the model equations are integrated forward in time to the end of the system timestep, the state variables for that model are updated to end-of-timestep values.

The CORCON model uses its own user-specified timestep, which is chosen independently of CONTAIN timesteps. Thus, CORCON can advance beyond the end of a system timestep and generate source rates that CONTAIN will use until the CONTAIN time advances to the CORCON value. Alternately, CORCON can be run with a step smaller than the CONTAIN cell timestep. In this case, the fluxes computed by CORCON are integrated over the CONTAIN cell timestep.

In general, the values of the state variables at the sub-interval points are not available outside the model or model group. For example, the suspended aerosol concentrations are calculated every Runge-Kutta timestep. However, the values are not available outside the aerosol model except at the end of the system timestep. The exceptions occur in the models that update their state variables every cell timestep.

The maximum system and cell timesteps are specified through input. These control the frequency with which the control loops in Figure 2-3 are traversed. There is little in the way of automatic control of these timesteps; the maximum timesteps are normally the timesteps that are actually used. The exceptions are as follows: (1) Because a hydrogen deflagration is a common event whose timing cannot be predicted, the system timestep is adjusted automatically during hydrogen deflagrations. (2) Because the cell timestep cannot exceed the system timestep, the cell timestep is also adjusted during hydrogen deflagrations. 
Table 2-1 lists the internal timesteps used for various models. The internal timestep determines the frequency with which the state variables belonging to the model are updated for internal use. The convection timestep indicated for the intercell flow model is, in general, set to the smallest time required for $20 \%$ of the contents of any cell to convect out of that cell, although in some cases certain cells may be excluded from consideration in this criterion when the FIX-FLOW option discussed in Section 4.4.9 is invoked. With respect to the integration methods listed, the word "integral" implies that a closed-form solution is used. The words "explicit" and "implicit" refer to the type of numerical integration method used. (The integration method with respect to the variables at a model interface is always explicit.) The interface timestep determines the frequency with which the internal variables are updated for use outside the model in question.

Table 2-1

Internal Timesteps Used Within Various Models

\begin{tabular}{||l|c|c|c||}
\hline \multicolumn{1}{|c|}{ MODEL } & $\begin{array}{c}\text { INTERNAL } \\
\text { TIMESTEP }\end{array}$ & $\begin{array}{c}\text { INTEGRATION } \\
\text { METHOD }\end{array}$ & $\begin{array}{c}\text { INTERFACE } \\
\text { TIMESTEP }\end{array}$ \\
\hline Fission Product & System & $\begin{array}{c}\text { Integral/ } \\
\text { Matrix }\end{array}$ & System \\
\hline $\begin{array}{l}\text { Flow/Thermo- } \\
\text { dynamics }\end{array}$ & $\begin{array}{l}\text { Runge-Kutta* } \\
\text { or Convection }\end{array}$ & $\begin{array}{c}\text { Explicit* } \\
\text { or Implicit }\end{array}$ & System \\
\hline Aerosol Processes & Runge-Kutta & $\begin{array}{c}\text { Explicit/ } \\
\text { Integral }\end{array}$ & System \\
\hline Heat Transfer Structures & Cell & Implicit & Cell \\
\hline $\begin{array}{l}\text { Engineered Safety } \\
\text { Features }\end{array}$ & Cell & Explicit & Cell \\
\hline Lower Cell Model & Cell & Implicit & Cell \\
\hline
\end{tabular}

*This is the default option, but the implicit option is recommended

For example, according to Table 2-1, the temperature profile in heat transfer structures is computed every cell timestep. The method used is fully implicit with respect to the internal variables (the node temperatures). However, as a result of the explicit coupling at the interface (such as that with the atmosphere), an instability in the profile may result from too large a cell timestep. The next section will discuss the maximum stable timestep because of this explicit coupling.

2.2.2.2 Suggested Criteria for User-Specified Timesteps. This section will discuss two criteria for selecting user-specified timesteps. The first criterion specifies the system timestep required to properly incorporate the effects of global changes. The second criterion specifies the cell timestep required for stability in the presence of the explicit coupling between the atmosphere and pool and heat transfer structures. 
Flow Time Constants. The flow calculations are done with either a Runge-Kutta timestep, in the explicit flow option, or a convective timestep, in the implicit flow option. In the latter case, the flow timestep is set to the minimum of convection timestep or the system timestep. With the recommended implicit flow option, there is relatively little advantage to using a system timestep that is much larger than the flow timestep, and the system timestep should be set to a comparable value. Since the system timestep determines the frequency at which global conditions are provided to other modules, use of a system timestep much larger than the flow timestep may mean that the conditions are not updated frequently enough. In general, one should check on the sensitivity to the system timestep by reducing it by a factor of two or more in the time domain of interest.

The convection time will depend on the relative pressure differences between cells. In the blowdown of cell at high pressure, the cell contents will convect out with a time constant equal to the pressure relaxation time. A simple rule of thumb for such transient conditions is that if the system timestep is short enough to resolve a $20 \%$ change in the pressures, it should be adequate with respect to the cell convection time of the blowdown cell.

If the initial pressure difference is not large, the cell contents will not convect out significantly during the pressure relaxation to the steady state. In this case, most of the convection, if any, will occur under low, steady-state pressure differences. The timestep to use in this case is less obvious than the one to use for transients. For cases in which the gas convection time controls the flow timestep, the steady-state convection time, $t_{c}$, for cell $i$ with total volume $V_{i}$ should be obtained from

$$
t_{c}=\min \left(\frac{V_{i}}{\sum_{k} \frac{W_{k i}}{\rho_{k i}}}, \frac{V_{i}}{\sum_{j} \frac{W_{i j}}{\rho_{i j}}}\right)
$$

where the $\mathrm{k}$ sum is carried out over all flows into cell $\mathrm{i}$ and the $\mathrm{j}$ sum is carried out for all flows out of cell $\mathrm{i}, \mathrm{W}_{\mathrm{ij}}$ is the mass flow rate, and $\rho_{\mathrm{ij}}$ is the flow density in the flow path. The steady-state convection time is typically much longer than the pressure relaxation time in such cases.

Fluid-Structure Time Constant. Another important potential source of inaccuracy or instability due to explicit coupling occurs at the cell level, where a bulk fluid may transfer an excessive amount of heat into a structure, because the effect on the surface temperature is not properly taken into account. A criterion for the maximum cell timestep can be obtained if the fluid-to-structure heat transfer coefficient $\mathrm{h}$ is known. By requiring the temperature rise in the first node during a single timestep $\Delta t_{1}$ to be less than the fluid-structure temperature difference, one obtains for an insulated first node,

$$
\Delta \mathrm{t}_{1}<\rho \mathrm{c}_{\mathrm{p}} \mathrm{L} / \mathrm{h}
$$

and for a node backed by similar material,

$$
\Delta \mathrm{t}_{2}<\rho \mathrm{c}_{\mathrm{p}} \mathrm{k} / \mathrm{h}^{2}
$$


where $\rho$ is the structure density, $c_{p}$ is the specific heat of the structure, $k$ is the material thermal conductivity, and $L$ is the first node thickness. These expressions should be used as first estimates for setting cell timesteps. Stable behavior may occur even if cell timesteps are two or three times these values, but exceeding these criteria by large factors will generally result in surface temperature oscillations. Sensitivity to the cell timestep should always be checked by varying the timestep. A typical heat transfer coefficient for condensing conditions of $h=300 \mathrm{~W} / \mathrm{m}^{2}-\mathrm{K}$, an $\mathrm{L}$ of $3 \times 10^{-4} \mathrm{~m}$ and concrete properties of $\mathrm{k}=1.5 \mathrm{~W} / \mathrm{m}-\mathrm{K}, \rho=2400 \mathrm{~kg} / \mathrm{m}^{3}$ and $c_{p}=1000 \mathrm{~J} / \mathrm{kg}-\mathrm{K}$ gives values of $\Delta \mathrm{t}_{1}$ $=2.4 \mathrm{~s}$ and $\Delta \mathrm{t}_{2}=40 \mathrm{~s}$.

\subsection{Material Properties}

An important aspect of a containment code is the ability to evaluate thermodynamic and transport properties for materials typically found in LWR plant systems. Table 2-2 lists the standard materials modeled in CONTAIN and the name of each material as used in the input. Property functions are provided for the heat capacity, enthalpy, thermal conductivity, viscosity, diffusivity, and density of these materials. Appropriate mixture relationships are also included for these properties when more than one constituent is involved. Note that in addition to these materials, the user has the option of defining materials and material properties through tabular input options. It is also possible to redefine the properties of the standard materials through tabular input.

The ideal gas law is assumed for all gases in the atmosphere, except possibly steam. It should be noted that steam behaves to good approximation as an ideal gas for typical containment conditions. However, to assess non-ideal effects and address high pressure conditions, a non-ideal equation of state option for steam has been provided.

The CONTAIN heat conduction routines have limited capability to deal with phase transitions. Thus, the only CONTAIN material explicitly allowed to change phase at present is the coolant and then only the liquid-vapor phase transition is modeled. The properties of a nominally solid material above the melting point are simply an extrapolation of the properties below the melting point; the properties of a liquid-phase material are similarly extrapolated below the melting point. This limitation on phase changes fortunately does not apply to the modeling done with respect to CCIs. These are modeled through the CORCON-Mod3 module, which has its own independent set of properties functions. Note that this limitation also does not prevent modeling of ice melt in an ice condenser. The ice in this case is treated through hard-wired ice properties and represented in terms of temperature and melt-rate boundary conditions.

The material property functions were extensively revised in CONTAIN 1.11 to reflect more recent data bases and to achieve more consistency with regard to enthalpy conventions and the treatment of different phases. However, not all of the properties for each material were revised completely. Also, certain materials are no longer supported after CONTAIN 1.11. The materials presently recommended for use are specified in Table 2-2. A more complete list, with references, is provided in Tables 3-1 and 3-2. 
Table 2-2

Materials Modeled in CONTAIN

\begin{tabular}{||l|l||l|l||}
\hline \multicolumn{1}{|c|}{ Material } & $\begin{array}{c}\text { Name } \\
\text { In Code }\end{array}$ & \multicolumn{1}{c||}{ Material } & \multicolumn{1}{c||}{$\begin{array}{c}\text { Name } \\
\text { In Code }\end{array}$} \\
\hline argon & AR & oxygen & O2 \\
\hline carbon dioxide & $\mathrm{CO}$ & silicon dioxide & SIO2 \\
\hline carbon monoxide & $\mathrm{CO}$ & sodium hydroxide & NAOH \\
\hline concrete & $\mathrm{CONC}$ & solid iron & $\mathrm{FE}$ \\
\hline graphite & $\mathrm{GRAPH}$ & solid uranium & $\mathrm{U}$ \\
\hline helium & $\mathrm{HE}$ & solid uranium oxide & $\mathrm{UO} 2$ \\
\hline hydrogen & $\mathrm{H} 2$ & stainless steel & $\mathrm{SS}$ \\
\hline liquid water & $\mathrm{H} 2 \mathrm{OL}$ & water vapor & $\mathrm{H} 2 \mathrm{OV}$ \\
\hline magnesium oxide & $\mathrm{MGO}$ & zirconium & $\mathrm{ZR}$ \\
\hline nitrogen & $\mathrm{N} 2$ & zirconium oxide & ZRO2 \\
\hline \hline
\end{tabular}

The user should note that the convention for the zero of enthalpy was in some cases revised for condensed phase materials in CONTAIN 1.11. This change may be non-upward compatible if the user has specified source tables of these materials based on enthalpy. The new enthalpy convention defines the enthalpy of the stable phase of the material at $273.15 \mathrm{~K}$ to be zero; the enthalpies of materials representing other than the stable phase at $273.15 \mathrm{~K}$ are offset by either the heat of vaporization or heat of fusion, or both, from the stable phase. Note that the stable phase of water at $273.15 \mathrm{~K}$ is taken to be the liquid. The modeling of solid-liquid transitions was also revised in CONTAIN 1.11. In prior versions, the specific heat of some nominally solid materials is changed to that of the liquid above the melting point. Unfortunately, this change is not made consistently; for example, the heat of fusion is not reflected in the enthalpy, and other liquid properties, such as viscosity, are not assigned to the solid above the melt. Thus, solid-liquid transitions are no longer explicitly modeled.

It should be noted that an independent mass and energy accounting scheme is available in CONTAIN. The coolant mass and the energy of all CONTAIN and user-defined materials, except those that are used exclusively to name aerosol components and fission products, are included in the accounting scheme by default. In addition, the mass of any other CONTAIN or user-defined material may also be included as a separate line item in the accounting.

Further discussion of material properties is given in Chapter 3. The selection of materials for a given problem and the input format for user-defined materials is discussed in Section 14.2.1. As discussed in Section 14.2.1.2, DCH materials in particular must be defined by the user. Examples of DCH 
material definitions are given in the Sequoyah sample problem discussed in Section 15.3. Use of the mass and energy accounting scheme is discussed in Section 3.4 and 14.2.2.

\subsection{Atmosphere/Pool Thermal-Hydraulics and Intercell Flow}

The atmosphere/pool thermodynamics and intercell flow models deal with the thermodynamic state of the bulk fluids in a cell and the intercell flow of the atmosphere and pool fields. As discussed in Section 2.1 the bulk fluids are of two types: the atmosphere bulk fluid, consisting of the noncondensable gases, coolant vapor, and any homogeneously dispersed liquid coolant in the cell atmosphere, and the pool bulk fluid, consisting of the coolant in the pool. Besides the bulk fluids, a number of other fields are associated with the atmosphere and pool, including aerosols, fission products, and dispersed core debris in the atmosphere and deposited aerosols and fission products in the pool.

The treatment of intercell flow is typical of a control volume code. The cell fluids are assumed to be stagnant and well-mixed. Flow is assumed to occur between cells through junctions, called flow paths, that essentially determine the exchange of mass and energy between the cells. The momentum equation in this framework is actually a lumped-parameter equation for the junction flows that assumes that the flow is controlled by a fluid slug with an effective length specified by the user. The flow paths are not repositories and do not have an actual inventory associated with them. The material flowing into a flow path is placed immediately in the downstream cell without regard to the holdup of the material in the flow path.

Note that the pool tracking modifications implemented in CONTAIN 1.2, as well as the hybrid gravitational head formulation discussed below, have radically changed the treatment of intercell flows. In CONTAIN 1.2, both the atmosphere fluid and the pool fluid, if present, are treated as bulk fluids on the same footing. Within this dual fluid treatment, the atmosphere and pool are assumed to be able to occupy the same physical volume within a cell, with the pool completely displacing the atmosphere below the pool surface elevation in the case of a partially filled cell. In conjunction with this new volume-filling assumption, the effects of submergence on flow paths and heat transfer structures are now treated. In addition, the pool thermodynamic state is now calculated by the implicit flow solver, and a new type of implicit pool flow path, with features comparable to those of gas paths, is available. In order to define the CONTAIN cell geometry more precisely than in prior versions, the cell geometry has been generalized, as discussed in Section 4.1.

In contrast, in code versions prior to CONTAIN 1.2, a single bulk fluid, the atmosphere fluid, is treated implicitly with respect to intercell flow. In addition the pool is considered to occupy a volume that only partially overlaps that of the atmosphere, and while volumetric displacement effects are taken into account, the effects of submergence are not generally considered.

Flow paths modeled within the recommended implicit flow solver may now transport either the atmosphere fields or the pool fields. These fields may flow only in a path of the appropriate type, a situation requiring the definition in general of both types of CONTAIN flow paths for each physical flow path. For generalized flow paths specified through the engineered vent input, the type is defined by the user to be either GAS or POOL, respectively. The specialized flow path 
representing the dedicated suppression pool vent model for BWRs is also available, although its thermal-hydraulic modeling in some respects has been made obsolete by the generalized flow paths. Note that the use of "regular" flow paths is now considered obsolete, but if present they will be treated as gas flow paths.

The intercell flow of pool coolant may be treated both implicitly and explicitly. Pool flow paths defined in terms of engineered vents are treated implicitly, in a manner similar to that for gas flow paths. The flow of pool coolant may not be as strongly coupled to pressure heads as the gas, so that an explicit treatment may be feasible. The liquid transport system components associated with the engineered safety features and discussed in Section 12.5 may be used to treat inter-pool transfers in an explicit manner. In some cases, use of an explicit model for pools will significantly reduce the overhead of the implicit solver.

A simple containment-oriented gas-pool flow hierarchy establishes which type of flow (i.e., gas or pool or both) is allowed when flow paths are submerged. Effects related to the coverage of the inlet or outlet of a gas path by pool coolant are taken into account. These include gas-pool equilibration, blockage of gas flow path inlets by the pool, liquid head terms, and scrubbing effects. A discussion of flow path configurations and the pool-gas hierarchy for the flow paths is given in Section 4.2.

Section 4.3 gives a summary of the modeling options available for gas and pool flow paths, as well as the suppression pool vent flow path. The governing equations of the flow path models are given in Section 4.4. The latter section discusses the three basic models for intercell flow, i.e., the inertial flow model, the quasi-steady flow model, and user-specified flow rates, as well as the formulations for critical or choked flow, gravitational heads, pool boiling, gas-pool equilibration, the velocity of gas evolution from the pool, and the FLX-FLOW option for overcoming the gas Courant limit in certain situations. The reader should note that a new, "hybrid" formulation of gas gravitational heads has been implemented into CONTAIN 1.2 as the result of investigations into the over mixing associated with control volume codes. [Mur96] This formulation satisfies three important criteria regarding the treatment of stratifications. As a consequence, the strong cautionary statements made with respect to prior code versions regarding convective overmixing are no longer necessary. However, the user should consult the guidance given in Section 13.3.1.3 with respect to avoiding certain situations that may lead to excessive stability of stratifications.

Section 4.5 discusses the momentum, mass, and energy conservation equations used by the intercell flow model and summarizes all contributions to the conservation equations, except the DCH ones, which are discussed separately in Chapter 6. This section also discusses the gas and pool thermodynamic state calculations.

\subsection{Lower Cell and Cavity}

In a core-melt accident involving a breach of the primary vessel, a coolant pool and/or a bed or pool of core debris may develop in the bottom of the reactor cavity, which is typically concrete. If core debris is present, the temperature of the concrete generally increases as decay heat is transferred from the core debris to the concrete, and the concrete can undergo thermal ablation in severe cases. Ablation of the concrete presents the threat of basemat penetration while simultaneously producing 
water vapor, noncondensable gases (including combustible hydrogen and carbon monoxide), and ablation products. Further chemical reactions may take place in the core debris, generating hydrogen, carbon monoxide, and other products. These CCIs can produce aerosols, fission products, and gases that can be carried into the upper containment. The lower cell model deals with these phenomena. These processes may not be present in all cells; for example, in some cells, the lower cell system may serve simply as a sump for collecting coolant. In others, a lower cell may not be required.

The lower cell coolant pool provides one of the bulk fluids considered to be in a cell, the other being the atmosphere gas and associated fields. As a result of the pool tracking modifications introduced into CONTAIN 1.2, the pool coolant is now treated on the same footing as the gas with respect to intercell flow and thermodynamics calculations. One non-upward compatible change resulting from these modifications is that the pool coolant is assumed to fill the same cross-sectional area in a cell as the gas, and not the lower-cell layer cross-sectional area. The cell cross-sectional area is specified in the cell GEOMETRY input block, discussed in Section 14.3.1.1, and not the lower-cell GEOMETRY input block, discussed in Section 14.3.2.1. The latter may still be used, however, in determining the pool area in contact with the basemat or with other lower cell layers forming the pool substrate. The coolant pool may also be in contact with submerged heat transfer structures. The calculation of the pool thermodynamic state, including the effects of gas-pool equilibration, is carried out by the implicit flow solver, provided this recommended option is invoked. The effects of boiling are also included if the recommended BOIL keyword for the pool is invoked.

The nature of the lower cell modeling below the pool-lower-cell interface depends on whether CCIs are actively modeled. If they are actively modeled, the lower cell below the interface is controlled by the CORCON-Mod3 module in CONTAIN (see below). If CCIs are not actively modeled, the lower cell below the interface is represented by a one-dimensional system of material layers, which may include a concrete layer and multiple intermediate layers. The feature of multiple intermediate layers allows distinctly different material layers such as molten metal and oxide by-products typical of CCIs to be present. Because a high level of phenomenological uncertainty prevails concerning the configuration of core debris and other materials, the user is allowed to specify this layer configuration. When CCIs are not actively modeled, the processes modeled below the pool interface include decay heating and heat conduction.

The CORCON-MOD3 code, [Bra93] which now incorporates the VANESA code for calculating aerosol releases, [Pow86] has been integrated into CONTAIN to deal with CCIs. The CORCONMod3 module provides three types of input to CONTAIN: gas generation rates, convective or boiling heat transfer from the hot core debris upper surface, and aerosol generation rates. Both the coolant pool and atmosphere can interact with this upper surface.

Aerosols generated in the cavity are scrubbed if they pass through a coolant pool on their way to the atmosphere, as discussed in Section 7.7. The pool in this case will also scrub any fission products in aerosol form. Gases introduced into the coolant pool will essentially be equilibrated at the pool temperature before being passed to the atmosphere. The scrubbing model is discussed in Section 7.7 and also in Reference Pow86, and the gas equilibration modeling is discussed in Section 11.2.1. 
Lower cell modeling, including that in CORCON-Mod3, is discussed in Chapter 5. The input for the lower cell is discussed in Section 14.3.2. Examples of lower cell input are provided in the sample problems of Chapter 15. An example of CORCON input is provided by the Surry sample problem discussed in Section 15.2.

\subsection{Direct Containment Heating}

In severe accident scenarios in which core melting occurs, the traditional assumption has been that molten debris will slump to the bottom of the vessel. This molten debris is typically assumed to eventually fail the lower head of the vessel. One postulated scenario is that the vessel is breached while the system is still pressurized; a plausible prediction is that a control rod penetration weld will fail and then be ejected. Such a situation could lead to the ejection of molten debris as a finely dispersed collection of particles into the containment. This high-pressure melt ejection and the subsequent phenomenological processes that contribute to containment loading and other threats to containment integrity are collectively referred to as direct containment heating (DCH). This term is derived from the high efficiency at which dispersed core debris can directly exchange energy with the containment atmosphere.

The DCH modeling capability draws from standard containment models and from a suite of DCHspecific models. A high degree of integration exists between the DCH models and the standard models. This integration is an essential component of the $\mathrm{DCH}$ modeling because of the energetic nature of a DCH event. The models work together to capture the complex interactions that occur among the various physical and chemical processes. This integrated approach to DCH modeling is consistent with the spirit of other models in the code.

A few of the more important containment processes associated with DCH phenomenology are intercell flow, two-phase atmosphere and coolant pool thermodynamics, heat transfer to structures, radiative heat transfer, hydrogen transport and combustion, ice condenser behavior, core debris entrainment and transport, core-debris chemical reactions, and debris-gas energy exchange.

DCH-specific models are available with respect to the following: the transport and trapping of multiple dispersed core-debris fields, debris droplet chemistry, convection and radiation heat transfer from the debris, debris-structure interactions, and cavity dispersal processes. A treatment of vessel hole ablation, vessel blowdown, and debris entrainment is included in the cavity dispersal modeling.

A description of the $\mathrm{DCH}$-specific models used to describe the $\mathrm{DCH}$ phenomena is presented in Chapter 6. These models include the treatment of the debris fields, intercell transport, chemical interactions, heat transfer, and debris trapping. The cavity dispersal models are also described in detail. Some advice on the use and limitations of the DCH models is presented in Section 13.3.2. A complete description of the DCH input is given in Sections 14.2.7 and 14.3.1.11 for the global and cell-level input, respectively. An example of DCH input and results from a DCH calculation are provided in the Sequoyah sample plant calculation presented in Section 15.3. 


\subsection{Aerosol Behavior}

Events occurring early in an LWR accident may create fission product and core-material aerosols in the reactor primary system, which then escape to the containment. Other events occurring late in the accident may also generate aerosols through CCIs. Significant amounts of aerosols may also be produced as a result of heterogeneous condensation of water vapor in the atmosphere. The principal aerosol quantities of interest are the suspended mass, particle size distribution, chemical composition, and radiological composition of aerosol particles within containment.

The ability to model the aerosol particle size distribution and aerosol composition as a function of size is important in assessing the radiological consequences of the suspended aerosols. The aerosol models used in CONTAIN to determine these distributions are based on the MAEROS code, [Gel82] although several enhancements have been added in CONTAIN. The MAEROS approach allows the use of a number of size classes, typically 10 to 20 , to represent the particle size distribution and allows as many as eight material components to describe the chemical composition. The airborne aerosol components may each be the host for an arbitrary number of fission products, which are transported with the aerosol component in intercell flow and deposition processes. A more complete discussion of the host concept is given in Sections 8.4 and 14.3.1.10.

It should be noted that aerosol condensation modeling is one of the options available to the user for treating suspended liquid water in the atmosphere. (The other available options are discussed in the introduction to Chapter 4.) Aerosol condensation modeling allows the condensation of water vapor in the atmosphere to be treated as occurring heterogeneously on aerosols, in which case the suspended water is carried in the aerosol inventory. Because of the large masses that may be involved, such aerosol condensation may be quite important in determining the radiological consequences of the suspended aerosols. An important feature of CONTAIN is that the aerosol condensation process is tightly coupled with the atmosphere thermal-hydraulics modeling and treated in a self-consistent manner. This consistency is particularly important when hygroscopic aerosol materials are present.

The user may invoke aerosol condensation modeling by specifying $\mathrm{H} 2 \mathrm{OL}$ as the last aerosol component in the global AEROSOL input block discussed in Section 14.2.5. Two condensation models are available, the fixed-grid and moving grid models, as discussed in Section 7.2.2. The fixed-grid model is the default. The moving grid model must be invoked to treat solubility and Kelvin effects in the condensation modeling. The trade-offs between the two models are also discussed in Section 7.2.2.

Besides aerosol condensation, the aerosol model considers agglomeration and deposition processes. Three agglomeration processes are treated: Brownian, gravitational, and turbulent. Also, four deposition processes are treated with respect to unsubmerged heat transfer structure and pool surfaces: gravitational settling, diffusiophoresis, thermophoresis, and particle diffusion. Aerosol deposition specific to engineered systems operation is treated for the three engineered safety features modeled in CONTAIN: containment sprays, ice condensers, and fan coolers. Besides the above deposition mechanisms, impaction and interception mechanisms are included for sprays and ice condensers. In addition to the above processes, aerosol scrubbing is treated when gas-aerosol 
mixtures are vented into a pool from a submerged gas flow path or as the result of a submerged external source. Two different mechanistic models are available in CONTAIN to model the scrubbing process. (However, these mechanistic models are not available for ordinary gas flow paths; see the discussion in Section 7.7.) In the event that a gas flow path is not submerged, aerosols are allowed to flow between the atmospheres of the two cells connected by the flow path. In such flow, the settling of aerosols relative to the gas component of the flow is considered. All of the above processes are discussed in detail in Chapter 7.

The modeling of aerosol generation from CCIs is treated through the VANESA module of the CORCON-Mod3 code, [Bra93] which has been integrated into CONTAIN. The details of this implementation are discussed in Chapter 5.

The input required to specify global aerosol model parameters is discussed in Section 14.2.5. The geometrical specification of the deposition surfaces is generally given along with the respective thermal-hydraulic model input; e.g, the scrubbing model parameters for the dedicated suppression pool vent is given in Section 14.2.4.3. The input for aerosol initial conditions and external sources is discussed in Sections 14.3.1.8 and 14.3.4. The VANESA input is described in Section 14.3.2.3.2, and an example of CORCON/VANESA input is provided in the Surry sample problem presented in Section 15.2.

\subsection{Fission Product Behavior}

The composition of the fission product inventory in the reactor core can be determined with considerable accuracy through a knowledge of its power history and the application of any one of several well-documented "burn-up" codes. However, during a core-melt accident, the physical disposition of the radionuclides is highly uncertain. Because CONTAIN does not analyze in-vessel phenomena, the user must specify the initial conditions and/or source rates for fission products introduced to the containment. From that point on, CONTAIN models three aspects of fission product behavior: transport (which determines the locations), decay (which determines inventories of each isotope), and decay heating (which affects the thermal-hydraulic behavior). Note that mechanistic models for the effects of chemical interactions involving fission products are not available, with the exception of a model for the removal of iodine by containment sprays and the exception of the VANESA model for the aerosol generation during CCIs, discussed in Section 2.5 and Chapter 5 . However, a parametric release and acceptance model is available and may be suitable for simulating the effects of chemistry in some cases.

In CONTAIN, fission products are assigned to certain repositories, called hosts. Examples of hosts include the atmosphere gas, a suspended aerosol component material, the coolant pool, or the surface of a heat transfer structure. The transport of fission products on mobile hosts, such as the gas or aerosols, is largely based on the transport of the host. For example, transfer of half of the gas in a cell will result in transfer of half of the fission products assigned to the gas in that cell. A model for fission product transport with condensate film runoff from surfaces and flow of pool coolant is also available. 
In addition to these models, fission products can also be transferred from one host to another at userspecified rates, which can depend upon host temperatures. This flexible system, called the targeted release-and-acceptance model for fission product transport, is necessary because of the high degree of uncertainty involving the physical and chemical forms of fission products and their affinities for the various materials. The experimental and modeling data base for the radionuclide chemical effects has in general been insufficient to provide reliable mechanistic models. The targeted releaseand-acceptance formalism is provided to allow sensitivity studies to be carried out in this area.

In CONTAIN, fission products may be represented either as (1) individual parent-daughter radionuclides with a given specific power related to the decay constant, or (2) a user-specified class of fission products with a given time-dependent specific decay power. Radionuclide parent-daughter relationships are defined by transforming the radionuclide decay processes into a series of linear chains. In the individual radionuclide representation, the decay of fission products and the heating of the host materials are modeled according to the half-lives and specific power of each linear chain element. This fission product decay information may either be downloaded from an internal fission product library or be specified by the user, or both. The decay power for each host is computed from its radionuclide inventory using the linear chain data and from the fission product classes present, using the time-dependent decay power. The decay processes are assumed to cause localized heating in each host. For example, the decay power of fission products deposited on the surface of heat transfer structures is assigned entirely to the surface node of the structure.

Despite the flexibility in being able to specify individual radionuclides or fission product classes, it is usually difficult to specify in detail all of the radionuclides or classes that would contribute to decay heating, as this would require a large amount of input. Normally, only a selected subset of radionuclides is of interest for health physics or transport reasons, and it is these radionuclides that are typically specified explicitly. The remaining decay power in core debris can be handled in a more generic way, provided the decay power can be associated with a stationary distribution assigned to the lower cell. In this approach a standard ANSI decay power curve is used to calculate total decay power as a function of time since shutdown. The power associated with the explicitly specified radionuclides and fission product classes is subtracted from this total power, and the remaining power is then deposited in various lower cell layers, according to a fixed distribution specified by the user. The use of this approach is discussed in conjunction with the lower cell in Sections 5.6.1 and 14.3.2.2. In addition to this makeup decay power model, the decay heating models internal to CORCON-Mod3 may also be invoked in conjunction with the modeling of CCIs, as discussed in Sections 5.3.2 and 14.3.2.4.2.

The subjects discussed above but not already given cross-references are discussed in Chapter 8 . The specification of global fission product characteristics, including the linear decay chain information, is discussed in Sections 14.2.1 and 14.2.6. The specification of fission product initial conditions and external sources is discussed in Sections 14.3.1.9, 14.3.1.10, and 14.3.4. The specification of parameters for the targeted release and acceptance model is also discussed in Section 14.3.1.10. 


\subsection{Combustion}

Various types of combustion phenomena are modeled in CONTAIN, including deflagration of premixed combustible gas, diffusion flame burning (DFB) of combustible-gas jets entering a compartment containing oxygen, and bulk spontaneous recombination (BSR). The deflagration model is based on that in the HECTR 1.8 code (Din86 and Won88), although two additional modifications have been made. The first modification is the use of a diluent criterion that takes into account the inerting effect of excess nitrogen (i.e., beyond the ratio found in air), which becomes important if burns have previously occurred (see Equation (9-2)). The effect of nitrogen diluent is not modeled in the HECTR code. In addition, the burn completeness correlations in the HECTR 1.8 code have been revised in CONTAIN 2.0 to remove unreasonable behavior associated with those correlations.

Deflagrations can occur when the combustible gas and oxygen concentrations exceed the ignition thresholds and the atmosphere is not inerted by an excess of diluent such as steam. If default values are used, initiation of a deflagration requires an oxygen concentration in excess of 5\% and a combustible gas concentration in excess of 7\%. A deflagration can also propagate to other cells, if certain criteria are satisfied. Upward, downward, and lateral flame propagation are possible.

The purpose of the DFB model is to allow the user to explore the effects of burning hydrogen in a jet rather than in a deflagration involving premixed gases. The burning of premixed gases could be physically reasonable when igniters are first turned on, or when the igniters are on and the atmosphere subsequently deinerts. However, in cases in which hydrogen is being introduced to containment with igniters on and the atmosphere is not inerted, quasi-continuous burning such as calculated in the DFB model may be physically more reasonable.

The DFB model is a relatively simple one that is not intended to be fully mechanistic. The parametric nature of the model stems principally from the fact that the dynamics of the diffusion flame are not modeled. While the user may specify the inerting concentrations above which the diffusion flame is no longer self-sustaining, there is no modeling of the dynamics of the flame front, which determine whether the diffusion flame is stable. The diffusion flame model, given noninerted conditions and an ignition source or the presence of conditions for autoignition, simply burns the combustible gas flowing into a cell through a flowpath or from an external source, utilizing the oxygen in the cell. The burning of combustible gas entrained into the jet from the downstream cell is also modeled. The DFB (and the BSR model, below) are interfaced implicitly with the intercell flow and atmosphere thermodynamic models to prevent numerical stability problems.

The BSR model is also a simple parametric model. The model uses a recombination threshold temperature and a recombination time constant that are specified by the user. The principal motivation for this model is the need to have a physically reasonable model of spontaneous recombination for DCH parametric studies, as in Reference Gid91.

The modeling of gas combustion is discussed in detail in Chapter 9. The input for the model is discussed in Section 14.3.1.7. Ignition and propagation concentration thresholds are summarized in Table 9-1, and other ignition criteria are summarized in Table 14-1. 


\subsection{Heat and Mass Transfer}

Through heat and mass transfer processes, containment heat sinks can in some cases absorb a considerable fraction of the thermal energy introduced into the containment during a reactor accident and thus provide a mitigating effect with respect to containment loads. In CONTAIN, these sinks are of two main types: heat transfer structures, which are nominally associated with the upper cell but can be submerged, and lower cell layers, which can be specified as needed for each cell. Because of their importance, a variety of heat and mass transfer processes are modeled at a number of interfaces involving such sinks. These processes include natural and forced convection heat transfer, condensation mass and heat transfer, condensate film mass transfer, radiative heat transfer, boiling heat transfer, and heat conduction. Not all of these processes are treated at all of the interfaces. The interested reader may wish to consult Table 10-1, which indicates the types of interfaces in CONTAIN and the types of processes considered at each. Other aspects of the table are discussed in the introduction to Chapter 10 and in Section 10.6.

Gas-to-surface heat and mass transfer is treated in mostly the same manner regardless of whether the surface is that of a heat transfer structure, pool, or spray drop, or of melting ice (in an ice condenser). Thus, the discussion of heat and mass transfer in Chapter 10 is organized according to the processes mentioned above and is intended to be as general as possible. Exceptions to the general discussion are noted, and cross-references to interface-specific discussions are provided. In addition, several topics associated with heat conduction, such as heat sink boundary conditions, heat sink characteristics, and concrete outgassing, are also discussed in Chapter 10.

The discussion of convective heat transfer in Section 10.1 starts out with the treatment of boundary layer properties and the characterization of heat sinks relevant to convective heat transfer, and continues with the formulations available for the Nusselt number. It should be noted that the heat transfer modifications implemented into CONTAIN 1.2 considerably improved the treatment of the boundary layer properties that are used in the heat and mass transfer modeling. In particular, gas boundary layer composition effects, which were previously ignored, are now treated in a computationally efficient manner. In addition, a number of user options were installed for specifying alternative forms for the natural and forced convection correlations used in conjunction with heat transfer structures. The pool tracking modifications implemented in CONTAIN 1.2 also enabled heat transfer structures to be submerged in the coolant pool in a cell. Thus, the treatment of submerged heat transfer structures and of characteristic lengths for heat sinks is also discussed.

As discussed in Section 10.2, two main interfacial mass transfer processes are modeled in CONTAIN: condensation mass transfer between a gas and surface and condensate film mass transfer between two structures or between a structure and a pool, as a result of film runoff. The condensation mass transfer formulation is based on the well-known heat and mass transfer analogy, [Bir60] which relies on the Nusselt number formulation to define the Sherwood number for mass transfer. Thus, much of the previous discussion of convective heat transfer is applicable for mass transfer. The diffusivity of steam, the boundary layer property appropriate to mass transfer that is not discussed in conjunction with convective heat transfer, and the Sherwood number are discussed in detail. 
Condensate films on a structure can be created in a number of different ways: from condensation mass transfer, deposition of water aerosols, inflow of condensate runoff from other structures, or a film source table. Two models are available in CONTAIN to describe condensate film flow from a structure. The first uses a fixed film thickness parameter and allows the condensate film to flow only from a structure to a pool. The second, the film tracking model, uses film flow correlations to determine the film depth. In this model condensate films are allowed to flow from one structure to another, in a way that allows the user to simulate film flow over a general two-dimensional surface comprised of a number of structure surfaces, and from a structure to a pool.

As discussed in Section 10.3, two types of radiative heat transfer models are available: a net enclosure model and a direct radiation model. Either model can be used with modeling of the optical properties of the atmosphere that in its most general form takes into account the effects of steam, carbon monoxide, carbon dioxide, aerosols, and dispersed core debris. The direct model takes into account direct exchange between the atmosphere and the surface of a heat transfer structure and between a lower cell and a structure surface. Structure-structure direct exchange and secondary reflections are neglected in the direct model.

Boiling heat transfer correlations are available, through a model that has been adapted from the CORCON-Mod3 code. This correlation is available at the moment only with respect to CORCON or lower cell layers in contact with the pool. The user is cautioned that the modeling details depend on whether or not CORCON is active. If it is not, all of the effects on boiling heat transfer taken into account in CORCON-Mod3, such as pool subcooling and gas barbotage, are not considered. Full details are given in Section 10.4.

Section 10.5 discusses the heat sinks in CONTAIN, the method of solution of the heat conduction equation, and the model for concrete outgassing. With regard to heat transfer structures, an arbitrary number of structures within each cell can be treated. The allowed shapes are slabs, hemispheres, and partial cylinders, and each may have either a floor, wall, or roof orientation. Each structure is treated one-dimensionally and can be composed of an arbitrary number of nodes, with a different material, if necessary, for each node. The "inner" surface of the structure, as defined in Section 10.5.1 or 14.3.1.3, is considered to be exposed, or in contact with the bulk fluids, in a cell. The "outer" surface may also be exposed in the same cell or assigned an external boundary condition. It may also be connected to another structure in a different cell through a conduction boundary condition. Note that, as discussed in Section 10.5.1, the complete suite of modeling options for an atmospherestructure interface is available only for an inner surface. Fewer options are available for outer surfaces in the same cell.

The method of solving for the effects of heat conduction in heat sinks is discussed in Section 10.5.3. This method uses conventional finite difference techniques and allows the user to specify the degree of implicitness in the solution.

As discussed in Section 10.5.4, a concrete outgassing model is available for describing the release of bound and evaporable water and carbon dioxide from concrete structures. This model assumes that the outgassing is controlled by thermal conduction and thus does not explicitly model the 
diffusion of water and the released gases within the concrete. Nevertheless, it is a simple and efficient model that has been verified for certain concrete types.

The heat and mass transfer processes described above are for the most part controlled by the interface temperature, which must be determined self-consistently from the heat and mass transfer rate for each process. A discussion of the interface energy balance equation, which is solved to obtain the interface temperature, is given in Section 10.6. Finally, Section 10.7 discusses control options available to the user for activating or deactivating heat and mass transfer processes at specific interfaces.

\subsection{Boiling Water Reactor and Related Models}

CONTAIN treats a number of processes in which the partitioning of enthalpy and mass flows between pool and atmosphere is important. These are commonly associated with the design of boiling water reactors (BWRs) but not restricted to them. They include suppression pool vent clearing, gas-pool equilibration and scrubbing for gas mixtures injected under the surface of a pool, and coolant phase separation modeling for low-quality two-phase flow that is injected into the atmosphere. This modeling is discussed in the context of (1) BWR suppression pool vent models and (2) safety relief valve (SRV) discharge models.

Within the generalized treatment of pool flooding implemented in the pool tracking modifications, modeling of the above types of processes should occur automatically on the basis of a flooded geometry. For example, suppression pool vents are, in principle, no more than submerged flow paths and thus the vent clearing process should be modeled automatically in such a geometry. Within the limitations of the pool tracking modifications, this is the case. Thus, two ways of modeling suppression pool vent systems are discussed in Chapter 11 for a Mark III BWR: through a collection of ordinary gas and pool flow paths and through the dedicated suppression pool vent model originally developed to treat the BWR vent system. An approach using ordinary flow paths could presumably be developed for Mark I's and II's, but this configuration would involve water-solid cells, an aspect of the pool tracking modifications that has not been fully tested. Thus, only the dedicated model is currently recommended for the last two designs.

It should be noted that the ordinary flow path model and the dedicated model have their own advantages and disadvantages with respect to modeling gas-pool equilibration and aerosol scrubbing. The user may specify the gas-pool equilibration length for a gas flow path, but, for simplicity, all aerosols are assumed to be removed by scrubbing for a submerged gas flow path. In contrast, gaspool equilibration is always assumed to be complete for the dedicated model in forward submerged flow but is ignored in reverse submerged flow. However, two different mechanistic aerosol scrubbing models are available in conjunction with the dedicated model.

Although intended originally to describe the SRV discharge of gases, aerosols, and fission products through lines leading to the bottom of the suppression pool in a BWR, the SRV discharge model may also be used in other situations in which the discharged materials are equilibrated and scrubbed by a pool before being introduced into the atmosphere. A submerged discharge is treated in a manner similar to that from the dedicated suppression pool vent model in forward submerged flow. 
The SRV model can treat unsubmerged discharges as well as submerged discharges. In unsubmerged discharges, the partitioning between pool and atmosphere is handled through a coolant phase separation calculation. In this calculation the source materials are assumed to expand isenthalpically down to the cell pressure before mixing with the cell. Any liquid coolant present at the end of expansion is diverted to the pool, and all other materials are directed to the atmosphere. In contrast, partitioning is not considered for atmosphere sources. All materials are simply directed to the atmosphere.

The modeling of suppression pool vents is discussed in more detail in Section 11.1, and the SRV modeling is discussed in more detail in Section 11.2. The input for the ordinary flow paths is discussed in Section 14.2.4 and that for the dedicated suppression pool vent model is discussed in Section 14.2.4.3. The SRV input is discussed in Section 14.3.4.

\subsection{Engineered Safety Features}

Three major engineered safety features (ESFs) are modeled: fan coolers, ice condensers, and containment sprays. Associated with these models is a liquid transport system that provides sources and sinks for the ESFs and also allows coolant transfer between lower cell pools to be modeled. The components available for such systems include tanks, pumps, orifices, pipes, valves, and heat exchangers, as well as user-specified external sources of coolant. In addition, the overflow of coolant from one pool to another can be modeled. The status of models for the removal of suspended aerosols and fission products, as a result of ESF operation, is indicated below.

Fan Cooler. Fan coolers provide nonemergency cooling and augment the heat removal capabilities of the sprays. These coolers use large-capacity fans in conjunction with banks of finned, service-water-cooled coils to cool the containment atmosphere. Two types of fan cooler models are available. The first model is similar to that developed for the MARCH code. [Woo83] It is simple and fast, and it adequately reproduces the cooling capacity of actual plant equipment under saturated conditions. It can be used whenever the effects of superheated conditions are expected to be relatively minor.

A second, more mechanistic, fan cooler model is based on forced convective heat transfer correlations similar to those used throughout the code. This model calculates mass and heat transfer coefficients based on the atmosphere and coil conditions and can treat superheated conditions. However, it requires a more detailed knowledge of fan cooler characteristics than the simple model described above. These two fan cooler models are discussed in more detail in Section 12.1, and the input for the models is discussed in Section 14.3.3.2.

Because of the relatively cool surfaces and high condensation rates provided by fan coolers, substantial deposition of aerosols can occur. The aerosol removal resulting from diffusiophoresis is calculated if the mechanistic fan cooler model is used. This modeling is discussed in Section 7.4.

Ice Condenser. Ice condensers are used in some PWR containment systems to limit containment overpressure in the event of a loss-of-coolant accident by directing the steam released from the 
primary system through a large ice chest. In addition to reducing peak pressures and temperatures, ice condenser systems can be effective in removing aerosols.

The ice condenser model uses the intercell flow model to determine the flows into the ice compartment. Heat and mass transfer to the ice is treated by a natural/forced convection model similar to that for heat transfer structures. This heat and mass transfer modeling is discussed in more detail in Section 12.2, and the ice condenser model input is discussed in Section 14.3.3.3. Aerosol removal by the ice and supporting structures is also modeled and is discussed in Section 7.5.

Containment Spray. The containment spray system provides a high-pressure, finely divided water spray to the containment atmosphere. Heat transfer to the droplets and subsequent condensation of atmospheric steam can produce rapid reductions in temperature, pressure, and aerosol and fission product concentrations. The spray droplets, as well as much of the condensate, collect in a sump at the bottom of the containment. Generally, the initial spray water is taken from a water storage tank. When this source is exhausted, water is pumped from the sump, through a heat exchanger, and to the spray nozzles. The heat and mass transfer between the droplets and the atmosphere is calculated in a manner similar to that for heat transfer structures, but with allowance for the geometry and conditions appropriate for a spray drop. The heat and mass transfer modeling for sprays is discussed in Section 12.3, and the input for the spray model is discussed in Section 14.3.3.4.

The containment spray model allows for the removal from the containment atmosphere of aerosols, aerosolized fission products, elemental iodine, and less reactive organic iodine compounds. Aerosols and fission products removed from the atmosphere by the sprays can be diverted to the pool of any compartment. A first-order depletion rate model is used for the removal of elemental iodine $\left(I_{2}\right)$ and methyl iodide $\left(\mathrm{CH}_{3} \mathrm{I}\right)$ by sprays. The aerosol removal modeling is discussed in Section 7.6 and the iodine removal modeling is discussed in Section 8.6.

Liquid Transport System Components. The ESF modeling includes several components that might typically be used in a liquid transport and supply system. These components include a liquid storage tank, a pump, an orifice, a pipe, a valve, and a heat exchanger. These components can be combined in various ways to model coolant storage and transfer systems and auxiliary cooling systems. They can also be used to model the transfer of coolant from one pool to another. These components are discussed in Section 12.5, and the input is described in Sections 14.3.3.5 through 14.3.3.11. 


\subsection{MATERIAL PROPERTIES}

The modeling of thermodynamic and transport properties of materials is described in the following sections. An internal material property library is provided for the heat capacity, enthalpy, thermal conductivity, viscosity, and density. The emissivity properties of steam and $\mathrm{CO}_{2}$ are also provided but are discussed in Section 10.3.3 rather than in this chapter. In addition, the diffusivity of steam with respect to noncondensable gases is discussed in Section 10.2.1.1.

Note that the following discussion pertains only to the CONTAIN material properties. A separate set of material properties is maintained within the CORCON module for the purposes of calculating the effects of core-concrete interactions. The reader is directed to Reference Bra93 for a discussion of those properties.

Section 3.1 discusses the material property library and the user-defined material option available within CONTAIN. Section 3.2 discusses both the ideal-gas and non-ideal-gas equations of state for water. Section 3.3 discusses the methods by which bulk thermophysical properties of mixtures are calculated. Finally, Section 3.4 introduces the mass and energy accounting scheme available within CONTAIN.

\subsection{Material Properties}

Internal to the CONTAIN code is a library of physical properties for 50 standard materials that can be used in the modeling of containment systems. This library provides temperature-dependent specific enthalpy, specific heat, density, viscosity, and thermal conductivity for a variety of materials commonly found in nuclear reactor containments under severe accident conditions. Table 3-1 lists the standard materials available in CONTAIN and the corresponding name of each material in the library.

The material property library includes temperature-dependent functions for the following properties:

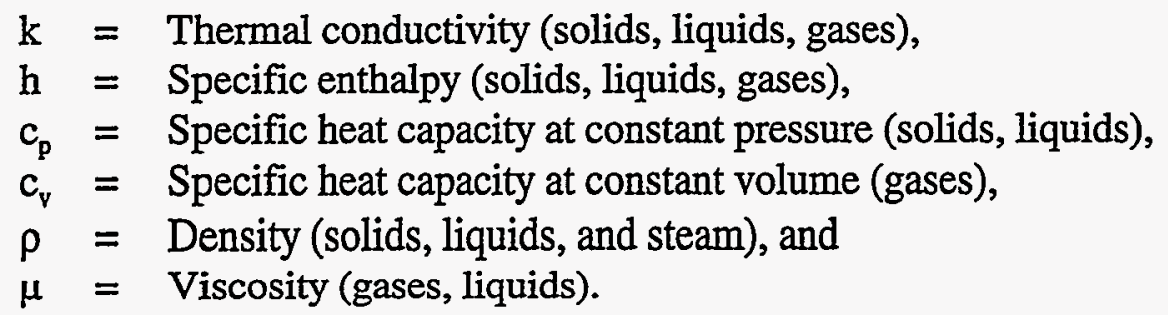

Note that viscosity functions are not needed for solids, and gas densities are determined from the equation of state. A complete listing of the property functions for each of the 50 materials in the CONTAIN 2.0 library is beyond the scope of this document. Moreover, these functions have undergone major revisions since CONTAIN 1.10 to reflect more recent data bases and to achieve more consistency with regard to enthalpy conventions and treatments of phase transitions. Refer to Reference Val88 for the correlations in CONTAIN 1.10 and those developed for CONTAIN 1.2. The user is cautioned that the changes in the enthalpy convention for materials may render obsolete source table input based on enthalpy and developed for CONTAIN 1.10 and prior versions. 


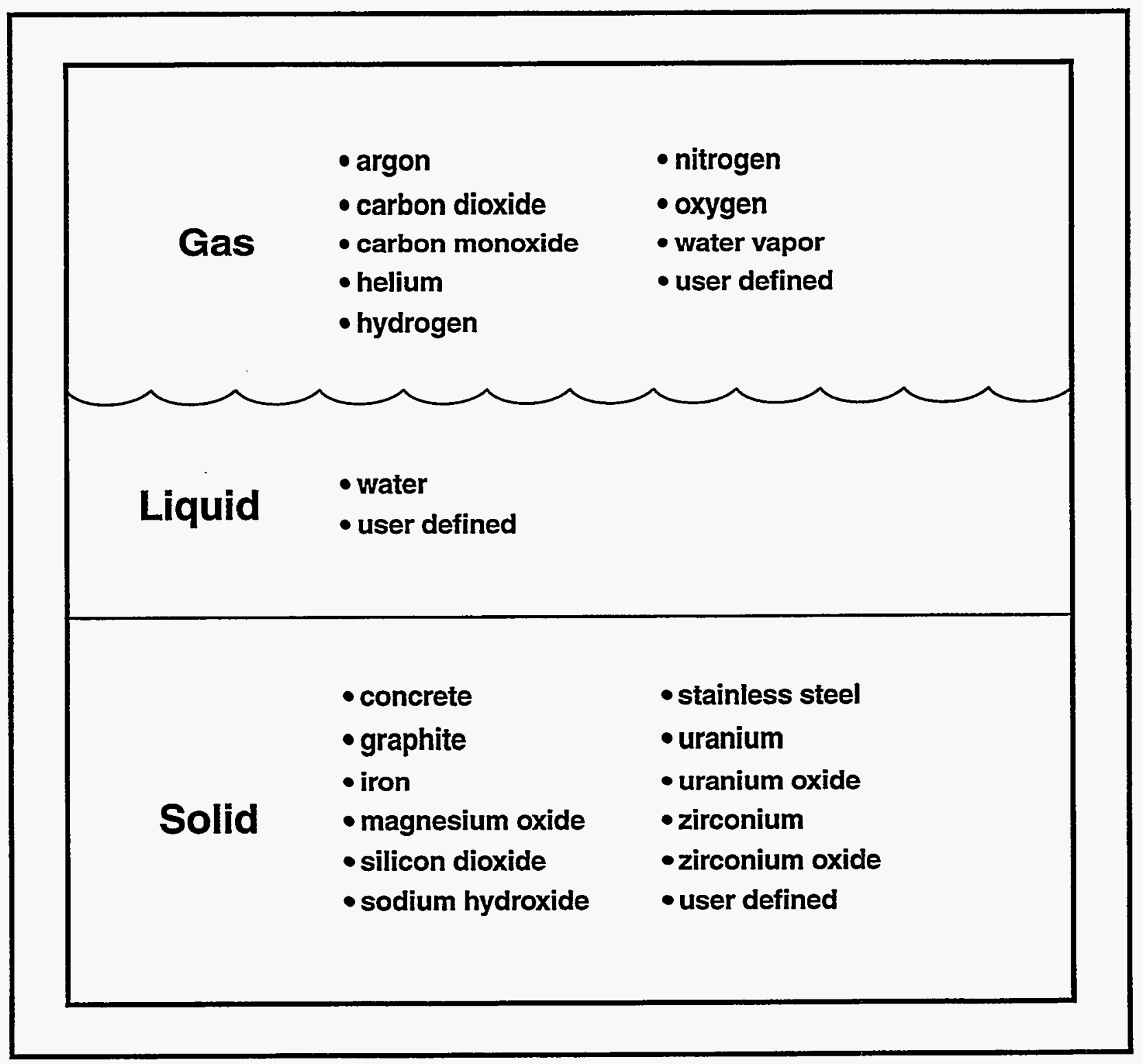

Figure 3-1. Material Properties as Modeled in CONTAIN 
Table 3-1

Materials Available in CONTAIN

\begin{tabular}{|c|c|c|c|}
\hline Material & $\begin{array}{l}\text { Name Used in } \\
\text { CONTAIN }\end{array}$ & Material & $\begin{array}{l}\text { Name Used in } \\
\text { CONTAIN }\end{array}$ \\
\hline aluminum oxide* & AL2O3 & plutonium oxide vapor & PUO2V \\
\hline argon & $\mathrm{AR}$ & plutonium vapor" & PUV \\
\hline boron carbide* & $\mathrm{B} 4 \mathrm{C}$ & potassium oxide ${ }^{*}$ & $\mathrm{~K} 2 \mathrm{O}$ \\
\hline calcium oxide ${ }^{*}$ & $\mathrm{CAO}$ & silicon dioxide & $\mathrm{SIO} 2$ \\
\hline carbon dioxide & $\mathrm{CO} 2$ & silicon trioxide & $\mathrm{SIO} 3$ \\
\hline carbon monoxide & $\mathrm{CO}$ & sodium carbonate ${ }^{\dagger}$ & $\mathrm{NA} 2 \mathrm{CO} 3$ \\
\hline chromium sesquioxide & $\mathrm{CR} 2 \mathrm{O} 3$ & sodium hydroxide & NAOH \\
\hline concrete & $\mathrm{CONC}$ & sodium monoxide ${ }^{\dagger}$ & $\mathrm{NA2O}$ \\
\hline ferrous oxide ${ }^{\dagger}$ & FEO & sodium peroxide ${ }^{\dagger}$ & $\mathrm{NA2O} 2$ \\
\hline graphite & GRAPH & sodium silicate ${ }^{\dagger}$ & NA2SIO3 \\
\hline helium & $\mathrm{HE}$ & sodium vapor ${ }^{\dagger}$ & NAV \\
\hline hydrogen & $\mathrm{H} 2$ & solid iron & FE \\
\hline ice & $\mathrm{H} 2 \mathrm{O}$ & solid plutonium ${ }^{\dagger}$ & $\mathrm{PU}$ \\
\hline iron vapor * & FEV & solid plutonium oxide ${ }^{\dagger}$ & PUO2 \\
\hline liquid iron ${ }^{\dagger}$ & FEL & solid sodium $^{\dagger}$ & $\mathrm{NA}$ \\
\hline liquid plutonium ${ }^{\dagger}$ & PUL & solid uranium & $\mathrm{U}$ \\
\hline liquid plutonium oxide & PUO2L & solid uranium oxide & $\mathrm{UO} 2$ \\
\hline liquid sodium ${ }^{\dagger}$ & NAL & stainless steel & SS \\
\hline liquid uranium ${ }^{\dagger}$ & $\mathrm{UL}$ & stainless steel oxide & SSOX \\
\hline liquid uranium oxide ${ }^{\dagger}$ & UO2L & titanium dioxide & TTO2 \\
\hline liquid water & $\mathrm{H} 2 \mathrm{OL}$ & uranium oxide vapor & $\mathrm{UO} 2 \mathrm{~V}$ \\
\hline magnesium oxide & MGO & uranium vapor* & UV \\
\hline manganese oxide ${ }^{\circ}$ & MNO & water vapor & $\mathrm{H} 2 \mathrm{OV}$ \\
\hline nitrogen & $\mathrm{N} 2$ & zirconium & $\mathrm{ZR}$ \\
\hline oxygen & $\mathrm{O} 2$ & zirconium oxide & $\mathrm{ZRO} 2$ \\
\hline
\end{tabular}

- Not supported after CONTAIN 1.10

${ }^{\dagger}$ Not recommended for use because some properties still require updating

However, note that this caution does not apply to steam or water, for which the enthalpy convention has not changed.

Not all of the properties for each material have been revised completely. These materials are marked with a dagger $(\dagger)$ in Table 3-1. Also, after CONTAIN 1.10, certain materials denoted by an asterisk (*) in Table 3-1 are no longer supported. The user is cautioned to check the property values and functions in the code before using any of these marked materials. Table 3-2 lists the references for 
Table 3-2

References for CONTAIN Material Properties

\begin{tabular}{|c|c|c|c|c|c|}
\hline \multirow[t]{2}{*}{ Name } & \multicolumn{5}{|c|}{ Reference } \\
\hline & $c_{p}$ or $c_{v}$ & $\mathbf{h}$ & $\rho$ & $\mathbf{k}$ & $\mu$ \\
\hline AL2O3 & Bar77 & $\int c d T$ & Wea85 & Tou79 & N/A \\
\hline $\mathrm{AR}$ & Van78 & $\int \mathrm{cdT}$ & ideal gas & Rei77 & Chu84 \\
\hline B4C & Bar73 & $\int \mathrm{cdT}$ & Wea85 & & N/A \\
\hline $\mathrm{CAO}$ & Bar73 & $\int c d T$ & Wea85 & Tou79 & N/A \\
\hline $\mathrm{CO} 2$ & $\operatorname{Van} 78$ & $\int c d T$ & ideal gas & Rei77 & Cha39, Rei77 \\
\hline $\mathrm{CO}$ & $\operatorname{Van} 78$ & $\int \mathrm{cdT}$ & ideal gas & Rei77 & Chu84 \\
\hline $\mathrm{CR} 2 \mathrm{O} 3$ & Bar73 & $\int c d T$ & Wea85 & & N/A \\
\hline CONC & Bak70 & $\int \mathrm{cdT}$ & Bau 78 & Bak70 & N/A \\
\hline FEO & Bar73 & $\int c d T$ & Wea85 & & N/A \\
\hline GRAPH & Bar73 & $\int c d T$ & Wea85 & Ho68 & N/A \\
\hline $\mathrm{HE}$ & $\operatorname{Van} 78$ & $\int \mathrm{cdT}$ & ideal gas & Rei77 & Cha39, Rei77 \\
\hline $\mathrm{H} 2$ & $\operatorname{Van} 78$ & $\int c d T$ & ideal gas & Rei77 & Cha39, Rei77 \\
\hline $\mathrm{H} 2 \mathrm{O}$ & Raz76 & $\int \mathrm{c} d \mathrm{~T}$, Wea85 & Raz76 & $\operatorname{Raz} 76$ & N/A \\
\hline FEL & Bar77 & $\int \mathrm{cdT}$ & Bra83 & Tou79 & Bra83 \\
\hline PUL & Bar77 & $\int \mathrm{cdT}$ & Bra83 & & Bra83 \\
\hline PUO2L & Lei71 & $\int c d T$ & & & Hag81 \\
\hline $\mathrm{UL}$ & Bar77 & $\int c d T$ & Bra83 & & Bra83 \\
\hline UO2L & Lei71 & $\int c \mathrm{dT}$ & Fin80 & & Hag81 \\
\hline $\mathrm{H} 2 \mathrm{OL}$ & $\begin{array}{c}\text { Tou79 or } \\
\text { Kee } 78\end{array}$ & See Section 3.2 & $\begin{array}{l}\text { Rey79 or } \\
\text { Kee78 } \\
\end{array}$ & Rei87 & Mak70 \\
\hline MGO & Bar73 & $\int \mathrm{cdT}$ & Wea85 & Tou79 & N/A \\
\hline MNO & Bar73 & $\int c d T$ & Wea85 & & N/A \\
\hline $\mathrm{N} 2$ & Van78 & $\int c d T$ & ideal gas & Rei77 & Cha39, Rei77 \\
\hline $\mathrm{O} 2$ & Van78 & $\int c \mathrm{dT}$ & ideal gas & Rei77 & Cha39, Rei77 \\
\hline $\mathrm{K} 2 \mathrm{O}$ & Bar73 & $\int c d T$ & Wea85 & & N/A \\
\hline $\mathrm{SIO} 2$ & Bar73 & $\int c d T$ & Wea85 & Tou79 & N/A \\
\hline $\mathrm{NA2CO} 3$ & Bar73 & $\int c d T$ & Wea85 & & N/A \\
\hline $\mathrm{NAOH}$ & Bar73 & $\int c \mathrm{dT}$ & Wea85 & Tou79 & N/A \\
\hline $\mathrm{NA} 2 \mathrm{O}$ & $\operatorname{Bar} 73$ & $\int c d T$ & Wea85 & & N/A \\
\hline
\end{tabular}


Table 3-2

References for CONTAIN Material Properties (Concluded)

\begin{tabular}{|c|c|c|c|c|c|}
\hline \multirow[t]{2}{*}{ Name } & \multicolumn{5}{|c|}{ Reference } \\
\hline & $c_{p}$ or $c_{v}$ & h & $\boldsymbol{\rho}$ & $\mathbf{k}$ & $\boldsymbol{\mu}$ \\
\hline $\mathrm{NA} 2 \mathrm{O} 2$ & Bar73 & $\int c \mathrm{dT}$ & Wea85 & & N/A \\
\hline NA2SIO3 & Bar73 & $\int \mathrm{cdT}$ & Wea85 & & N/A \\
\hline FE & Bar 77 & $\int c \mathrm{dT}$ & Wea85 & Tou79 & N/A \\
\hline $\mathrm{PU}$ & Bar77 & $\int c \mathrm{dT}$ & Tem63 & & N/A \\
\hline $\mathrm{PUO2}$ & Hag81 & $\int c d T$ & Wea85 & & N/A \\
\hline $\mathrm{U}$ & Bar 77 & $\int c d T$ & Wea85 & Tou79 & N/A \\
\hline UO2 & $\operatorname{Ker} 72$ & $\int c \mathrm{dT}$ & Wea85 & $\begin{array}{l}\text { Gol73, Bat70, } \\
\text { Wei72 }\end{array}$ & N/A \\
\hline SS & Per73 & $\int c d T$ & Per73 & Chu78 & N/A \\
\hline TIO2 & Bar73 & $\int \mathrm{cdT}$ & Wea85 & Tou79 & N/A \\
\hline $\mathrm{H} 2 \mathrm{OV}$ & $\begin{array}{c}\text { Van78 or } \\
\text { Kee78 } \\
\end{array}$ & See Section 3.2 & $\begin{array}{l}\text { equation of } \\
\text { state }\end{array}$ & Rei77 & Cha39, Rei77 \\
\hline $\mathrm{ZR}$ & Bar73 & $\int \mathrm{cdT}$ & Wea85 & Tou79 & N/A \\
\hline ZRO2 & Bar73 & $\int c \mathrm{dT}$ & Fis74 & Hag81 & N/A \\
\hline
\end{tabular}

the properties of the recommended materials, as well as the available references for the other materials.

The user should be aware of the following assumptions and conventions about the properties:

- For common gases, excluding steam, an ideal gas assumption is always made. For steam, both ideal gas and non-ideal gas properties are available (see Section 3.2)

- The only CONTAIN materials allowed to change phase are steam (H2OV) and liquid water (H2OL). ${ }^{1}$ The properties of a solid material above the melting point are in general simply extrapolated from the solid phase; the properties of a liquid material are in general extrapolated below the freezing point and above the boiling point. However, note that solidsolid transitions are taken into account by distributing the latent heat of transition over a 20$\mathrm{K}$ range. Also, note that the limitations on the modeling of phase transitions do not apply to the modeling of core-concrete interactions with the CORCON module, which uses its own set of material properties.

\footnotetext{
${ }^{1}$ The ice within an ice condenser chest is not explicitly present in an ice condenser cell. Rather, the ice condenser heat transfer model uses stored ice properties to compute the melting rate. Once the ice is melted, the melt is then introduced to the cell at the appropriate rate.
}

Rev. 0 
- A common reference temperature, $T_{\text {ref }}=273.15 \mathrm{~K}$, is used to define the arbitrary constant in the enthalpy. For all materials but steam, the constant is specified by defining the enthalpy as

$$
h_{k}(T, P \rightarrow 0)=\int_{T_{r e f}}^{T} c_{p, k}\left(T^{\prime}, P \rightarrow 0\right) d T^{\prime}
$$

where $c_{p, k}$ is the specific heat, $T$ is the temperature, and $P$ is the pressure.

For steam, the analogous definition is

$$
h_{v}\left(T, P_{v} \rightarrow 0\right)=\int_{T_{\text {ref }}}^{T} c_{p, v}\left(T^{\prime}, P_{v} \rightarrow 0\right) d T^{\prime}+\Delta H\left(T_{\text {ref }}, P_{v} \rightarrow 0\right)
$$

where $\Delta H$ is the latent heat of vaporization and $P_{v}$ is the vapor pressure.

- Volumetric displacement work by and on materials that are neither gaseous nor the liquid phase of water is ignored. Therefore, the pressure dependence of the enthalpy indicated in Equation (3-1) is ignored for such materials and the enthalpy is taken to be a function of temperature only, $h_{k}(T, P)=h_{k}(T)$. Furthermore, the specific enthalpy is assumed to be interchangeable with the specific energy for such materials.

In addition to the library of material properties, the user may define new materials and their properties through tabular input. The same method can be used to redefine the properties of one of the internally defined CONTAIN materials. The format of the user-defined property tables is discussed in 14.2.1.1. These tables are temperature-based and must be given in standard SI units. The user must ensure that the tables cover or extrapolate sensibly over the entire range of temperatures that are anticipated for a given problem. If the calculated temperature extends beyond the specified range, the enthalpy tables are linearly extrapolated beyond their end points. For all other properties, the endpoint values are assumed for such temperatures. A variable, "nwdudm," is included in the global CONTROL block (see Section 14.2) to allow the user to expand the amount of memory reserved for the user-defined tables beyond the default amount of 1000 words.

The names of materials used in a given run must be specified in the MATERIAL input block (see Section 14.2.1). The names of standard materials, which are given in Table 3-1, must follow the COMPOUND keyword. (The COMPOUND keyword must immediately follow the MATERIAL keyword.) The names of user-defined materials must follow the USERDEF keyword. These names should be alphanumeric strings of eight or fewer characters that begin with a letter and should not correspond to a keyword or the word GAS. They may be standard names from Table 3-1. The declaration of names after USERDEF implies the presence of a corresponding block of input associated with the USERDAT keyword. Note that no default properties are provided for a userdefined material, even one with a standard name.

For direct containment heating ( $\mathrm{DCH}$ ) calculations, the standard material property library cannot be used to specify the properties of the debris species. The names of debris materials must be included 
in the USERDEF list and the DCH debris material properties input according to the USERDAT input format (see Section 14.2.1.1). The one very important difference between DCH user-defined materials and other user-defined materials is that the phase type, DEBRIS, is specified after the material name in the USERDAT input block. (See Sections 6.6 and 14.2.1.2 for more information.)

To use the CORCON model, it is no longer necessary to specify the dummy melt material names used to represent CORCON materials in the CONTAIN lower cell intermediate layer representing CORCON. These are now included by default. These names are LCCHOX, LCCMET, and LCCLOX, as described in Section 5.3.

The names of user-defined fission products must also be specified in the MATERIAL block, following the keyword FP-NAMES. FP-NAMES, if used, must follow the COMPOUND block. Arbitrarily chosen names can be used for fission products, unlike the material names in the COMPOUND block, which must be chosen from Table 3-1. Nuclide names such as $\Pi 33$, or general names such as DUMI, can be used.

Names of aerosol components may be either that of a nongaseous material specified immediately after COMPOUND or a dedicated aerosol name. The latter may be specified after the keyword AERNAMES following the COMPOUND block. Such names must be unique.

\subsection{Water Thermodynamic Properties}

Water is the only material that is considered to undergo phase changes, and only the liquid-gas transition is modeled. Water phase changes are modeled in the condensation of steam onto structures and aerosols, pool boiling, and the evaporation of water films. The heat of evaporation is automatically taken into account in the enthalpy function of each phase of water. Both an ideal and a non-ideal equation of state are available as discussed in Sections 3.2.1 and 3.2.2, respectively.

\subsubsection{Ideal Water Equation of State}

The ideal water equation of state is based on an ideal gas formulation of the vapor [Van78] and on the saturation properties of the liquid. [Tou79] The specific heat of the solid is based on Reference Raz76. The specific enthalpy for the vapor is assumed given by its zero-pressure limit $h_{v}=h_{v}\left(T, P_{v} \rightarrow 0\right)$ as shown in Equation (3-2). In CONTAIN, the liquid is taken to be incompressible, with properties as a function of temperature defined along the saturation curve. The enthalpy $h_{\ell}$ of the liquid in the ideal equation of state is taken to be

$$
\mathrm{h}_{\ell}(\mathrm{T}, \mathrm{P}) \approx \mathrm{u}_{\ell}(\mathrm{T}, \mathrm{P})+\mathrm{P} / \mathrm{\rho}_{\ell}(\mathrm{T})
$$


where

$$
\mathrm{u}_{\ell}(\mathrm{T}, \mathrm{P}) \approx \mathrm{h}_{\ell}(\mathrm{T})=\int_{\mathrm{T}_{\mathrm{ref}}}^{\mathrm{T}} \mathrm{c}_{\mathrm{p} \ell}(\mathrm{T}) \mathrm{dT} \text { (ideal equation of state) }
$$

Also, $\rho_{\ell}(T)$ is the saturated liquid density, and $c_{p, l}$ is the saturated liquid specific heat at constant pressure. It should be noted that neither $h_{\ell}(T, P)$ nor $h_{\ell}(T)$ gives the standard saturated enthalpy, since $\mathrm{c}_{\mathrm{p}, \mathrm{i}}$ is defined along the saturation curve and not at a fixed pressure. In addition, the explicit pressure term in Equation (3-3) is to some extent redundant since pressure contributions are also implicitly included in the integral term. In previous code versions, the pressure dependence was ignored, and, in addition, the temperature integral was assumed to give the standard saturated enthalpy. The explicit pressure term was introduced in the present code version to allow accounting for work done on and by the liquid. Such tracking requires that a pressure dependence be explicitly present in the enthalpy. At low pressures for which the ideal equation of state is valid, the inconsistencies present in Equation (3-3) are presumably not important.

\subsubsection{Non-Ideal Water Equation of State}

This section discusses the non-ideal water equation of state. The use of a non-ideal equation of state is important at the high vapor pressures that are typical of reactor primary systems but is typically not important under reactor containment conditions. However, the non-ideal equation of state is still useful for containment problems in which high pressure primary system volumes or pressurized cavities are explicitly modeled through CONTAIN cells, for example, in the modeling of a vessel steam blowdown in a DCH problem. The non-ideal equation of state is based on properties of the vapor and saturated properties of the liquid.

The non-ideal equation of state is based primarily on the Keenan and Keyes [Kee78] analytic formulation of the steam tables. The approach is similar to that used in MELCOR, which uses an analytic representation of the single-phase regions of the equation of state and a uniform-grid tabular representation of the saturation curve properties. [Sum95] The single-phase analytic thermodynamic properties are based on Keenan and Keyes, except for those for high temperature vapor ( $T>1589$ $\mathrm{K})$, which are based on the JANAF tables. [Cha65]

The analytic representation requires a considerable amount of algebra for a full set of properties but the overhead has been reduced by selective calculation of properties inside iteration loops, and by using only saturated liquid properties. For example, CONTAIN does not require the specific heat or other second derivatives of the free energy during the flow solver pressure iteration, and thus such quantities are not calculated during the pressure iteration. The use of the non-ideal equation of state has been found to increase the computational overhead somewhat, but in test problems the increase has been less than $10 \%$. The computational overhead may be higher in applications problems with large numbers of cells. However, since the non-ideal equation of state is optional, the user can still use the old, less accurate but more efficient ideal equation of state, which is adequate for most containment conditions. 
The non-ideal equation of state is selected through the HIPRWATR keyword (for high pressure water) in the FLOWS input block. The placement of the HIIPRWATR keyword in the FLOWS (i.e., atmosphere thermodynamics and flows) input block reflects the fact that ideal gas assumptions for water have not been eliminated from all code models under this option. The limitations in the implementation of the non-ideal gas modeling are discussed below.

3.2.2.1 Representation of the Water Equation of State. The non-ideal equation of state for water was derived from the following fundamental equation from Reference Kee78, which expresses the Helmholtz free energy $\Psi$ in $\mathrm{j} / \mathrm{gm}$ in terms of the density $\rho$ in $\mathrm{g} / \mathrm{cm}^{3}$ and temperature $T$ in Kelvin:

$$
\psi=\psi_{0}(T)+R T[\ln \rho+\rho Q(\rho, \tau)],
$$

where

$$
\psi_{0}=\sum_{\mathrm{i}=1}^{6} \mathrm{C}_{\mathrm{i}} / \tau^{\mathrm{i}-1}+\mathrm{C}_{7} \ln \mathrm{T}+\mathrm{C}_{8}(\ln \mathrm{T}) / \tau
$$

and

$$
Q=\left(\tau-\tau_{c}\right) \sum_{j=1}^{7}\left(\tau-\tau_{a j}\right)^{j-2}\left[\sum_{j=1}^{8} A_{i j}\left(\rho-\rho_{a j}\right)^{i-1}+e^{-E \rho} \sum_{i=9}^{10} A_{i j} \rho^{i-9}\right]
$$

In Equations 3-4, 3-5, and 3-6, $\tau$ denotes $1000 / \mathrm{T}, \mathrm{R}=4.6151 \mathrm{bar} \mathrm{cm}^{3} / \mathrm{g}-\mathrm{K}, \tau_{\mathrm{c}} \equiv 1000 / \mathrm{T}_{\text {crit }}=1.544912$ $\mathrm{K}^{-1}, \mathrm{E}=4.8 \mathrm{~cm}^{3} / \mathrm{g}$, and

$$
\begin{aligned}
\tau_{a j} & =\tau_{c}(j=1) & \rho_{a j} & =0.634(j=1) \\
& =2.5(j>1), & & =1.0 \quad(j>1)
\end{aligned}
$$

The coefficients for $\Psi_{0}$ in joules per gram are given as follows:

$$
\begin{array}{lll}
C_{1}=1857.065 & C_{4}=36.6649 & C_{1}=46 \\
C_{2}=3229.12 & C_{5}=-20.5516 & C_{8}=-1011.249 \\
C_{3}=-419.465 & C_{6}=4.85233 &
\end{array}
$$

The coefficients $A_{i j}$ are listed in Table 3-3.

This representation in principle gives the complete water equation of state for both vapor and liquid phases. The saturation curve can be obtained by solving the analytic representation for the condition of equal chemical potentials. To avoid having to solve repeatedly for this condition, the saturation curve was previously derived from the analytic representation and the saturation properties stored in tables in the MELCOR H2O2PH subroutine. These tables have now been imported into 
Table 3-3

The Coefficients $A_{i j}$ in Equation (3-6)

\begin{tabular}{||c|c|c|c|c|c|c|c||}
\hline $\begin{array}{l}\mathrm{j} \\
\mathrm{I}\end{array}$ & \multicolumn{1}{|c|}{1} & \multicolumn{1}{c|}{2} & \multicolumn{1}{c|}{3} & \multicolumn{1}{c|}{5} & \multicolumn{1}{c|}{6} & 7 \\
\hline 1 & 29.492937 & -5.1985860 & 6.8335354 & -0.1564104 & -6.3972405 & -3.9661401 & -0.69048554 \\
\hline 2 & -132.13917 & 7.7779182 & -26.149751 & -0.72546108 & 26.409282 & 15.453061 & 2.7407416 \\
\hline 3 & 274.64632 & -33.301902 & 65.326396 & -9.2734289 & -47.740374 & -29.142470 & -5.1028070 \\
\hline 4 & -360.93828 & -16.254622 & -26.181978 & 4.3125840 & 56.323130 & 29.568796 & 3.9636085 \\
\hline 5 & 342.18431 & -177.31074 & 0 & 0 & 0 & 0 & 0 \\
\hline 6 & -244.50042 & 127.48742 & 0 & 0 & 0 & 0 & 0 \\
\hline 7 & 155.18535 & 137.46153 & 0 & 0 & 0 & 0 & 0 \\
\hline 8 & 5.9728487 & 155.97836 & 0 & 0 & 0 & 0 & 0 \\
\hline 9 & -410.30848 & 337.31180 & -137.46618 & 6.7874983 & 136.87317 & 79.847970 & 13.041253 \\
\hline 10 & -416.05860 & -209.88866 & -733.96848 & 10.401717 & 645.81880 & 399.17570 & 71.531353 \\
\hline
\end{tabular}

CONTAIN. Also imported into CONTAIN was the MELCOR treatment of the vapor at high temperatures: at temperatures greater than $1589 \mathrm{~K}$, the analytic representation of Reference Kee78 is joined onto the JANAF [Cha65] vapor phase properties using ideal gas relations.

The uniform grid used in the tabular representation of saturation properties makes looking up the table very fast. However, the analytic representation is always used for the vapor in the CONTAIN implicit flow solver, even for saturated conditions, since the single-phase representation must join continuously onto the saturation curve properties. The only way to ensure this is to use the analytic representation in conjunction with the tabular representation. In the CONTAIN implementation, the tabular properties are used to define the saturated vapor density. This density is then used in the analytic representation to define all other properties for the saturated vapor. In contrast, to avoid a similar problem for the liquid, it is treated as incompressible with an effective specific energy as a function of temperature (at zero pressure) corresponding to the saturated liquid specific energy as a function of temperature (see the next paragraph). Consequently, the tabular liquid saturation properties are almost always used for the liquid, except on the extension of the saturation curve above $640 \mathrm{~K}$, as discussed below.

While the vapor properties such as the density $P_{v}\left(T, P_{v}\right)$ and the enthalpy $h_{v}\left(T, P_{v}\right)$ follow in a straightforward fashion from the equation of state, the use of saturated liquid properties requires further clarification. The liquid specific enthalpy $h_{\ell}(T, P)$ as a function of temperature $T$ and pressure $P$ is defined in terms of the actual specific energy $u_{l}(T, P)$ for water according to

$$
\mathrm{h}_{\ell}(\mathrm{T}, \mathrm{P}) \approx \mathrm{u}_{\ell}(\mathrm{T}, \mathrm{P})+\mathrm{P} / \mathrm{p}_{\ell}(\mathrm{T})
$$


where

$$
\mathrm{u}_{\ell}(\mathrm{T}, \mathrm{P}) \approx \mathrm{u}_{\ell}\left(\mathrm{T}, \mathrm{P}_{\mathrm{s}}(\mathrm{T})\right)
$$

where $P_{s}(T)$ is the saturation pressure and $\rho_{l}(T)$ is the liquid saturation density. The specific heats are given by the standard derivatives. For example, the specific heat at constant pressure is given by

$$
c_{p, \ell}=\partial h_{\ell}(T, P) /\left.\partial T\right|_{p}
$$

and the liquid density is given by the saturated values

$$
\rho_{\ell}(T, P)=\rho_{\ell}(T)
$$

Note that $c_{p, \ell}$ is not the standard saturated liquid specific heat for the present non-ideal equation of state, whereas it is the enthalpy that is non-standard with the ideal equation of state.

It should be noted that in the CONTAIN implementation at high temperatures and pressures (above $640 \mathrm{~K}$ or $2 \times 10^{7} \mathrm{~Pa}$ on the saturation curve), the saturation curve has been modified and extended to infinity. The purpose of this extension is to provide a continuous extrapolation for the flow solver when its iterations sample conditions well outside the range of physical interest. The procedure for the extension is as follows.

3.2.2.2 Extrapolation of the Water Saturation Properties. The water saturation curve has been arbitrarily modified above $T_{0}=640 \mathrm{~K}$ for numerical reasons and redefined to extend to infinite temperatures. The extrapolation is made as follows. First, it is assumed that the saturated vapor density $\rho_{v, s}$ increases to $0.99 \rho_{c}$ and the liquid density $\rho_{l}$ decreases to $\rho_{c}$ at infinite temperature, where $\rho_{c}$ is the critical density. Furthermore, it is assumed that the density variation at infinity is exponential in temperature

$$
\begin{aligned}
& \rho_{v, s}=\left(\rho_{v, 0}-0.99 \rho_{c}\right) \exp \left(a_{v}\left(T-T_{0}\right)\right)+0.99 \rho_{c} \\
& \rho_{\ell}=\left(\rho_{\ell, 0}-\rho_{c}\right) \exp \left(a_{\ell}\left(T-T_{0}\right)\right)+\rho_{c}
\end{aligned}
$$


To ensure that the extrapolation has continuous derivatives, we use the relation

$$
\left.\frac{\mathrm{d} \rho}{\mathrm{dT}}\right|_{\mathrm{s}}=\frac{\mathrm{dP}_{\mathrm{s}} / \mathrm{dT}}{\partial \mathrm{P} /\left.\partial \rho\right|_{\mathrm{T}}}-\frac{\partial \mathrm{P} /\left.\partial \mathrm{T}\right|_{\mathrm{p}}}{\partial \mathrm{P} /\left.\partial \rho\right|_{\mathrm{T}}}
$$

It follows that

$$
\begin{aligned}
& \mathrm{a}_{\mathrm{v}}=\frac{\left[\frac{\mathrm{dP} \mathrm{s}_{\mathrm{s}} / \mathrm{dT}_{0}}{\partial \mathrm{P} /\left.\partial \rho_{\mathrm{v}}\right|_{\mathrm{T}_{0}}}-\frac{\partial \mathrm{P}_{\mathrm{v}} /\left.\partial \mathrm{T}\right|_{\rho_{0}}}{\partial \mathrm{P} /\left.\partial \rho_{\mathrm{v}}\right|_{\mathrm{T}_{0}}}\right]}{\left[\rho_{\mathrm{v}, 0}-0.99 \rho_{\mathrm{c}}\right]} \\
& \mathrm{a}_{\ell}=\frac{\left[\frac{\mathrm{dP}_{\mathrm{s}} / \mathrm{dT}_{0}}{\partial \mathrm{P} /\left.\partial \rho_{\ell}\right|_{\mathrm{T}_{0}}}-\frac{\partial \mathrm{P}_{\ell} /\left.\partial \mathrm{T}\right|_{\rho_{0}}}{\partial \mathrm{P} /\left.\partial \rho_{\ell}\right|_{\mathrm{T}_{0}}}\right]}{\left[\rho_{\ell, 0}-\rho_{\mathrm{c}}\right]}
\end{aligned}
$$

where the subscript " 0 " refers to saturated conditions at $\mathrm{T}_{0}$. These are evaluated from the tabular saturation values for the liquid and vapor calculated in subroutine "h2o2ph." Equations (3-11) and (3-13) for the vapor define the extrapolated saturated vapor density. This density defines the other extrapolated saturated thermodynamic properties of the vapor when substituted into the analytic representation given in Equations (3-4) through (3-7) with $\mathrm{T}$ and $\rho_{v, s}$ as independent variables.

The extrapolated liquid enthalpy and other properties are then obtained from the Clausius-Claperon relation

$$
\mathrm{dP}_{\mathrm{s}} / \mathrm{dT}=\frac{\mathrm{h}_{\mathrm{v}, \mathrm{s}}-\mathrm{h}_{\ell}}{\left(\mathrm{v}_{\mathrm{v}, \mathrm{s}}-\mathrm{v}_{\ell} \mathrm{T}^{\mathrm{T}}\right.}
$$

where $\mathrm{v}$ is the specific volume. To be specific, Equation (3-11) is first used with the analytic representation to define $\mathrm{v}_{\mathrm{v}, \mathrm{s}}=1 / \rho_{\mathrm{v}, s}, \mathrm{~h}_{\mathrm{v}, s}$ and $\mathrm{dP} / \mathrm{dT}=\mathrm{dP}_{\mathrm{v}, \mathrm{dT}} / \mathrm{d}$ along the saturation curve. These values are substituted into Equation (3-14) to define $h_{\ell}$, which is then used to define other liquid properties.

3.2.2.3 Modeling Limitations. As indicated previously, the non-ideal equation of state was not implemented in full generality throughout the code. A number of assumptions and modeling limitations should be noted with respect to the implementation: 
1. Definition of water vapor densities or partial pressures. In some cases, when the present code architecture does not allow convenient definition of the density or pressure to use with the non-ideal water equation of state, simplifying assumptions are made. Such cases can be enumerated as follows: (a) Source tables are presently structured so that at most one variable can be used to define the material enthalpy. If this variable is the enthalpy, it is interpreted as such; however, if this variable is the temperature, the density or pressure to use in the case of water is not specified. For either the liquid or vapor phase of water, the accompanying pressure is assumed to be the pressure just downstream of the injection point for the source, since the upstream pressure is not known. The downstream pressure is typically a cell pressure, or a cell pressure adjusted for an overlying pool head. A diagnostic is issued whenever such a pressure assumption is made in conjunction with the non-ideal equation of state. (b) The calculation of the enthalpy of water vapor evolving from the core debris during core-concrete interactions in general requires a thermodynamic state calculation when water vapor is evolved along with other gases. For simplicity, however, it is assumed that the partial pressures of the released gases are given by their molar ratios. (c) A similar situation arises with the gases evolved from concrete outgassing. In this case, however, it is assumed that the water vapor partial pressure is the cell pressure of the cell to which the outgas is directed, multiplied by the water vapor molar ratio in the outgas. This approach assumes that the outgassing surface can communicate freely with the downstream cell. (d) The definition of the enthalpy of water vapor involved with DCH chemical reactions requires specification of either a density or partial pressure. The low density (ideal gas) limiting value of the enthalpy is used in this case. None of these approximations are expected to have significant consequences for processes occurring under containment conditions. However, one should be careful to specify the correct enthalpy, as usual, in modeling a blowdown from the primary system through CONTAIN source tables.

2. Use of ideal gas relations between pressures and densities. The ideal gas relation between gas molar fractions and partial pressures is used in the derivation of the expressions for steam condensation rates, such as those for steam condensation on heat transfer structures, engineered system surfaces, and on aerosols. In addition, the ideal gas temperature dependence of the gas density at constant pressure is assumed in the definition of boundary layer gas densities. Finally, the ideal gas adiabatic relation between pressure and density is used in the derivation of choked flow rates. These aspects of gas transport modeling have not been upgraded in the non-ideal equation of state implementation. However, the bulk gas (and wall saturation) pressures used in the final transport rates have been upgraded to reflect the non-ideal equation of state, if invoked. The specific heat ratio used in the choked flow rate has also been upgraded.

3. Restrictions with respect to the explicit flow solver. The new equation of state has been implemented for use only with the implicit flow solver. This restriction was imposed on the assumption that the tabular saturation curve properties will cause timestep problems with the Runge-Kutta integrator used with the explicit solver, which expects a highly continuous function. 
4. Assumption of saturated intermediate states. Certain routines, specifically the aerosol condensation routines and the engineered systems fan cooler model, require the calculation of intermediate thermodynamic states. Such states are needed, for example, to calculate the condensation rate for the fan cooler with respect to the second and subsequent coil banks. For such calculations, it is assumed that such states are saturated or close to saturated. Therefore, calls to the saturated enthalpy and specific heat functions are used, and the complications of the pressure dependence of the vapor enthalpy avoided.

\subsection{Bulk Gas Thermophysical Properties}

This section describes the equations used in CONTAIN to obtain bulk material properties when two or more material components are present. In general a weighted averaging method is used to obtain mixture properties as described below for each property.

Bulk Gas Thermal Conductivity $\left(\mathrm{k}_{\text {mix }}\right)$. This property uses a weighting factor based on the mole fraction:

$$
\mathrm{k}_{\operatorname{mix}}=\sum_{\mathrm{i}=1}^{\mathrm{N}} \mathrm{X}_{\mathrm{i}} \mathrm{k}_{\mathrm{i}}
$$

where $\mathrm{X}_{\mathrm{i}}$ is the mole fraction and $\mathrm{k}_{\mathrm{i}}$ is the thermal conductivity of component $\mathrm{i}$. It should be noted that Equation (3-15) is an approximation for the standard method of determining mixture conductivities given in Reference Mas58 which uses a weighting factor that is also dependent on component molecular weight and viscosity. However, for steam-air mixtures, Equation (3-15) can be shown to be a good approximation for the mixture thermal conductivity.

Bulk Gas Viscosity $\left(\mu_{\text {mix }}\right)$. This property uses a weighting factor that is based on the product of the mole fraction and the square root of the molecular weight, that is,

$$
\mu_{\operatorname{mix}}=\frac{\sum_{i=1}^{N} X_{i} \mu_{i}\left(M_{i}\right)^{1 / 2}}{\sum_{i=1}^{N} X_{i}\left(M_{i}\right)^{1 / 2}}
$$

where $\mu_{\mathrm{i}}$ is the viscosity and $\mathrm{M}_{\mathrm{j}}$ is the molecular weight of component $\mathrm{i}$. [Per73]

Bulk Gas Specific Heat at Constant Volume ( $\left.c_{v, \text { mix }}\right)$ This property uses a weighting factor based on the mass fraction of each component, that is,

$$
c_{v, \operatorname{mix}}=\sum_{i=1}^{N} \Phi_{i} c_{v, i}
$$


where $\Phi_{i}$ is the mass fraction of gases, and $c_{v, i}$ is the specific heat at constant volume of gas component i.

Bulk Density $\left(\rho_{\text {mix }}\right)$ The bulk superficial gas density is defined as

$$
\rho_{\text {mix }}=\frac{m_{\text {tot }}}{V}
$$

where $\mathrm{m}_{\text {tot }}$ is the total mass of all gaseous components, and $\mathrm{V}$ is the free volume of the cell not taken up by the coolant pool (if any). Note that this bulk density is not actually used in the calculation of intercell flow. The implicit flow model uses the masses of the individual species in the upstream cell as explained in Section 4.5. The bulk density in the above equation is calculated for output purposes and for use in heat and mass transfer correlations.

Bulk Ratio of Specific Heats

$$
\gamma_{\text {mix }}=1+\frac{\mathrm{R} \mathrm{N}_{\text {tot }}}{\mathrm{m}_{\mathrm{tot}} \mathrm{c}_{\mathrm{v}, \text { mix }}}
$$

where $R$ is the universal gas constant and $N_{\text {tot }}$ is the total number of moles of all gaseous components.

\subsection{Mass and Energy Accounting}

This section discusses the CONTAIN mass and energy accounting scheme. This scheme gives an independent audit of the masses and energies in CONTAIN repositories. The audit energies are defined as a sum of the internal and potential energies, since it is the total energy that is conserved. The audit energies furthermore are obtained by using best estimate expressions, consistent with the CONTAIN thermodynamic properties functions and knowledge of thermodynamic states, for energy transfers associated with a given mass transfer rate. Such audit energies are tracked through bookkeeping arrays that are independent of the other CONTAIN interface arrays, since the energies in the latter reflect a number of approximations used in evaluating the effects of mass transfer that are not appropriate for the audit energies.

The mass and energy accounting is set up to track total energies as well as the mass of any CONTAIN thermodynamic material. In the present discussion, a thermodynamic material is defined as one either given in the CONTAIN master material list or defined explicitly by the user through user-defined material (USERDEF) input. The accounting scheme will always track energies and the combined liquid and vapor phase masses of the coolant, even if no energy accounting input is specified. The user can specify mass balances for any other thermodynamic material through use of the "nmtrac" control parameter and the TRACKMAT input discussed in Section 14.2.2. The user must specify the PRENACCT keyword to obtain energy accounting output, which is present only in the long edits. If the user initially omits the PRENACCT keyword but desires energy accounting 
output after a run is completed, this keyword may be specified on a restart. A restart at a given restart time will automatically include energy accounting information up to that time.

Aerosol materials can be either a nongaseous thermodynamic material or a dedicated aerosol material defined by the user in the AERNAMES and global AEROSOL input blocks. (If an aerosol material is declared to be the H2OV material, a usage that is now considered obsolete, the material will be treated as the liquid H2OL.) Only the aerosol components that are thermodynamic materials are included in the mass and energy accounting. Both suspended and deposited aerosol masses are considered. It should be noted that fission products, by convention, cannot be comprised of thermodynamic materials and are, therefore, excluded from the scheme.

The details of the accounting scheme are described in Appendix A. The strategy used in the mass and energy accounting is discussed in Section A.2. The repositories considered and the mass and energy contributions reported in the accounting scheme are discussed in Section A.3.

As discussed in Appendix A, the present mass and energy accounting scheme is repository-oriented. The mass and energy output is organized so that the mass and energy balance for each repository may be obtained by inspection, without having to refer to other repositories. An example of the mass and energy accounting output (enabled by the PRENACCT keyword) is given in Figure 3-2. As shown in this figure, a number of repositories and their mass and energy balances are reported. The mass reporting in this case includes only the coolant mass, given in kilograms, since "nmtrac" and TRACKMAT were not specified. In Figure 3-1, the present energy $E_{p}$ for each repository should be compared with the second column labeled " $i+e+r+i-c$," which is a combination of the first letters of the labels of the next five columns. This column contains the sum $E_{0}+E_{e}+E_{r}+E_{f}-E_{c}$, which according to Equation (A-11) should be equal to $E_{p}$, plus any work done by the repository against its geometric constraints (see Section A.3), if no energy accounting errors have occurred.

The format shown is that obtained when the implicit flow solver is used. If the explicit flow solver is used, the atmosphere mass and energy balances for each cell are replaced by a single mass and energy balance representing the sum over all cell atmospheres. The reason for reporting this sum is that the explicit solver has not been upgraded to track the change in audit energies resulting from intercell flow.

Note that because all internal interfaces are conservative with respect to audit energies, the sum of the influx energies $\mathrm{E}_{\mathrm{f}}$ over all repositories should be zero. The user can check this if desired.

It should be noted that a sizeable relative error in the energy balance, based on repository contents, does not necessarily imply that the error is significant in terms of the calculated thermodynamic state. For example, a repository may have a great deal of mass and energy cycled through it but contain only a small fraction of the total cycled mass and energy at any given time. A relatively small error in the calculation of the thermodynamic state may build up over time to a relatively large error, based on the repository contents. The error may actually be tolerable if the measure of significance is the error relative to the total mass and energy cycled through the repository. 


\begin{tabular}{|c|c|c|c|c|c|c|c|}
\hline $9 y$ and mass & vation conditio & 100.0 & & & & & \\
\hline $\begin{array}{l}\text { repository = atmos } \\
\text { energy } \\
\text { coolant }\end{array}$ & $\begin{array}{c}\text { number }=1 \\
\text { present } \\
2.60636 E+11 \\
1.19098 E+05\end{array}$ & $\begin{array}{c}\text { cell }=1 \\
1+e+r+1-c \\
2.60635 E+11 \\
1.19098 E+05\end{array}$ & $\begin{array}{c}\text { initial } \\
-4.50482 E+09 \\
2.55385 E+04\end{array}$ & $\begin{array}{c}\text { externa! } \\
0.00000 E+\infty 0 \\
0.00000 E+00\end{array}$ & $\begin{array}{c}\text { reaction } \\
0.00000 E+\infty 0 \\
0.00000 E+\infty\end{array}$ & $\begin{array}{c}\text { influx } \\
2.65140 E+11 \\
9.35592 E+04\end{array}$ & $\begin{array}{c}\text { committed } \\
0.00000 E+00 \\
0.00000 E+\infty 0\end{array}$ \\
\hline $\begin{array}{l}\text { repository = struc } \\
\text { energy } \\
\text { coolant }\end{array}$ & $\begin{array}{c}\begin{array}{c}\text { number }=1 \\
\text { present } \\
9.43296 E+10 \\
4.33135 E+02\end{array}\end{array}$ & $\begin{array}{r}c e l l=1 \\
1+3+r+1-c \\
9.43306 E+10 \\
4.33135 E+02\end{array}$ & $\begin{array}{c}\text { initial } \\
9.29716 E+10 \\
0.00000 E+\infty 0\end{array}$ & $\begin{array}{c}\text { external } \\
0.00000 E+00 \\
0.00000 E+00\end{array}$ & $\begin{array}{c}\text { reaction } \\
0.00000 E+\infty 0 \\
0.00000 E+\infty 0\end{array}$ & $\begin{array}{c}\text { influx } \\
1.35901 E+09 \\
4.33135 E+02\end{array}$ & $\begin{array}{c}\text { committed } \\
0.00000 E+00 \\
0.00000 E+00\end{array}$ \\
\hline $\begin{array}{l}\text { repository = lowcel } \\
\text { energy } \\
\text { coolant }\end{array}$ & $\begin{array}{c}\text { number }=1 \\
\text { present } \\
4.14665 E+10 \\
1.42858 E+05\end{array}$ & $\begin{array}{c}\text { cell }=1 \\
1+\mathrm{e}+\mathrm{r}+1-\mathrm{c} \\
4.14701 E+10 \\
1.42858 E+05\end{array}$ & $\begin{array}{c}\text { initial } \\
1.95154 E+11 \\
2.00000 E+05\end{array}$ & $\begin{array}{c}\text { external } \\
6.08453 E+08 \\
1.00000 E+02\end{array}$ & $\begin{array}{c}\text { reaction } \\
0.00000 E+\infty 0 \\
0.00000 E+\infty 0\end{array}$ & $\begin{array}{c}\text { influx } \\
-1.54218 E+11 \\
-5.71824 E+04\end{array}$ & $\begin{array}{c}\text { committed } \\
7.51767 E+07 \\
5.93604 E+01\end{array}$ \\
\hline $\begin{array}{l}\text { repository = waste } \\
\text { energy } \\
\text { coolant }\end{array}$ & $\begin{array}{l}\quad \begin{array}{c}\text { number } \\
\text { present }\end{array} \\
9.89510 \mathrm{E}-14 \\
0.00000 \mathrm{E}+00\end{array}$ & $\begin{array}{l}\quad c e l l=1 \\
1+e+r+1-c \\
9.89510 E-14 \\
0.00000 E+\infty 0\end{array}$ & $\begin{array}{c}\text { initial } \\
0.00000 E+\infty 0 \\
0.00000 E+\infty 0\end{array}$ & $\begin{array}{c}\text { external } \\
0.00000 E+\infty 0 \\
0.00000 E+\infty 0\end{array}$ & $\begin{array}{c}\text { reaction } \\
0.00000 E+\infty \\
0.00000 E+\infty 0\end{array}$ & $\begin{array}{l}\quad \text { influx } \\
9.89510 E-14 \\
0.00000 E+\infty\end{array}$ & $\begin{array}{c}\text { committed } \\
0.00000 E+00 \\
0.00000 E+00\end{array}$ \\
\hline $\begin{array}{l}\text { repository = atmos } \\
\text { energy } \\
\text { coolant }\end{array}$ & $\begin{array}{c}\text { number }=1 \\
\text { present } \\
4.88754 E+11 \\
2.19898 E+05\end{array}$ & $\begin{array}{c}c e l l=2 \\
1+e+r+1-c \\
4.88754 E+11 \\
2.19898 E+05\end{array}$ & $\begin{array}{c}\text { initial } \\
6.04602 E+11 \\
2.57916 E+05\end{array}$ & $\begin{array}{c}\text { external } \\
0.00000 E+\infty \\
0.00000 E+\infty\end{array}$ & $\begin{array}{c}\text { reaction } \\
0.00000 E+\infty 0 \\
0.00000 E+00\end{array}$ & $\begin{array}{c}\text { influx } \\
-1.15848 E+11 \\
-3.80182 E+04\end{array}$ & $\begin{array}{c}\text { committed } \\
0.00000 E+00 \\
0.00000 E+00\end{array}$ \\
\hline $\begin{array}{l}\text { repository = struc } \\
\text { energy } \\
\text { coolant }\end{array}$ & $\begin{array}{c}\text { number }=1 \\
\text { present } \\
9.65239 E+10 \\
1.20632 E+03\end{array}$ & $\begin{array}{c}c e l l=2 \\
1+e+r+1-c \\
9.65325 E+10 \\
1.20632 E+03\end{array}$ & $\begin{array}{c}\text { initial } \\
9.29716 E+10 \\
0.00000 E+\infty 0\end{array}$ & $\begin{array}{c}\text { external } \\
0.00000 E+\infty 0 \\
0.00000 E+\infty 0\end{array}$ & $\begin{array}{c}\text { reaction } \\
0.00000 E+\infty 0 \\
0.00000 E+\infty 0\end{array}$ & $\begin{array}{c}\text { influx } \\
3.56090 E+09 \\
1.20632 E+03\end{array}$ & $\begin{array}{c}\text { committed } \\
0.00000 E+00 \\
0.00000 E+00\end{array}$ \\
\hline $\begin{array}{l}\text { repository = lowce! } \\
\text { energy } \\
\text { coolant }\end{array}$ & $\begin{array}{c}\text { number }=1 \\
\text { present } \\
2.20642 E+05 \\
1.92021 E+00\end{array}$ & $\begin{array}{c}\text { cell }=2 \\
1+e+r+1-c \\
2.20642 E+05 \\
1.92021 E+\infty 0\end{array}$ & $\begin{array}{c}\text { initial } \\
0.00000 E+\infty 0 \\
0.00000 E+\infty 0\end{array}$ & $\begin{array}{c}\text { external } \\
-5.47505 E+06 \\
0.00000 E+\infty\end{array}$ & $\begin{array}{c}\text { reaction } \\
0.00000 E+\infty 0 \\
0.00000 E+00\end{array}$ & $\begin{array}{c}\text { influx } \\
5.69568 E+06 \\
1.92021 E+\infty 0\end{array}$ & $\begin{array}{c}\text { committed } \\
-1.48842 E+01 \\
0.00000 E+\infty 0\end{array}$ \\
\hline $\begin{array}{l}\text { repository = waste } \\
\text { energy } \\
\text { coolant }\end{array}$ & $\begin{array}{c}\quad \begin{array}{c}\text { number }=1 \\
\text { present } \\
2.21464 E-08 \\
0.00000 E+00\end{array}\end{array}$ & $\begin{array}{l}\quad \text { cell }=2 \\
1+e+r+1-c \\
2.21464 E-08 \\
0.00000 E+\infty 0\end{array}$ & $\begin{array}{c}\text { initial } \\
0.00000 E+\infty 0 \\
0.00000 E+\infty 0\end{array}$ & $\begin{array}{c}\text { external } \\
0.00000 E+\infty 0 \\
0.00000 E+\infty 0\end{array}$ & $\begin{array}{c}\text { reaction } \\
0.00000 E+\infty 0 \\
0.00000 E+\infty 0\end{array}$ & $\begin{array}{l}\quad \text { influx } \\
2.21464 E-08 \\
0.00000 E+\infty 0\end{array}$ & $\begin{array}{c}\text { committed } \\
0.00000 E+\infty 0 \\
0.00000 E+\infty\end{array}$ \\
\hline
\end{tabular}

Figure 3-2. Example of Mass and Energy Accounting Output 


\subsection{ATMOSPHERE/POOL THERMODYNAMIC AND INTERCELL FLOW MODELS}

The atmosphere/pool thermodynamics and intercell flow models deal with the thermodynamic state of the bulk fluids in a cell and with the intercell flow of the atmosphere and pool fields. As discussed in Section 2.1, the bulk fluids are of two types: the atmosphere bulk fluid, consisting of the noncondensable gases, coolant vapor, and any homogeneously dispersed liquid coolant in the cell atmosphere, and the pool bulk fluid, consisting of the coolant in the pool. The effects of the coupling of the aerosol and fission product fields to the bulk fluids are discussed elsewhere. For aerosols and fission products attached to aerosols, such effects are discussed in Section 7.8. For fission products attached to the atmosphere gas and in the pool, such effects are discussed in Section 8.8. It should be noted that the often substantial effects of dispersed core debris are also not discussed in the present section. The required modifications and additions to the equations in this section to include the latter fields are presented in Chapter 6 on the direct containment heating (DCH) models.

It should be noted that sweeping changes were made in CONTAIN 1.2 with regard to treating the pool bulk fluid on the same footing as the atmosphere bulk fluid. In CONTAIN 1.2 and later versions, both the atmosphere fluid and the pool fluid, if present, are treated as bulk fluids on the same footing. Within this dual fluid treatment, the atmosphere and pool are assumed to be able to occupy the same physical volume within a cell, with the pool completely displacing the atmosphere below the pool surface elevation in the case of a partially filled cell. Note that pool level swell from trapped gas in the pool is not modeled. In conjunction with this new volume-filling assumption, the effects of submergence on flow paths and heat transfer structures are now treated. In addition, the pool thermodynamic state is now calculated by the implicit flow solver, and a new type of implicit pool flow path, with features comparable to those of gas paths, is available. In order to define the CONTAIN cell geometry more precisely than in prior code versions, the cell geometry has been generalized, as discussed in Section 4.1.

In contrast, in code versions prior to CONTAIN 1.2, a single bulk fluid, the atmosphere fluid, is treated implicitly with respect to intercell flow. In addition the pool is considered to occupy a volume that only partially overlaps that of the atmosphere, and while volumetric displacement effects are taken into account, the effects of submergence are not generally considered.

Flow paths modeled within the recommended implicit flow solver may now transport either the atmosphere fields or the pool fields. These fields may flow only in a path of the appropriate type. For generalized flow paths specified through the engineered vent input, the type is defined by the user to be either GAS or POOL, respectively. The specialized flow path representing the dedicated suppression pool vent model for boiling water reactors (BWRs) is also available, although its thermal-hydraulic modeling in some respects has been made obsolete by the generalized flow paths. A detailed discussion of the dedicated model is given in Section 11.1. The differences between the generalized flow path modeling and the dedicated suppression pool vent modeling are also discussed as necessary below. Note that the definition of flow paths within the FLOWS input block, as opposed to the engineered vent input block, is now considered obsolete, but if present such flow paths will be treated as gas flow paths. 
The intercell flow of pool coolant may be treated either implicitly or explicitly. Pool flow paths defined in terms of engineered vents are treated implicitly, in a manner similar to that for gas flow paths. The flow of pool coolant may not be as strongly coupled to pressure heads as the gas, so that an explicit treatment may be feasible. The liquid transport system components associated with the engineered safety features and discussed in Section 12.5 may be used to treat interpool transfers in an explicit manner. In some cases, use of an explicit model for pools will significantly reduce the overhead of the implicit solver.

A simple containment-oriented gas-pool flow hierarchy establishes which type of flow (i.e., gas or pool or both) is allowed when flow paths are submerged. The flow of the atmosphere and pool fields within their respective paths is taken to be independent in the sense that interfacial shear and void fraction effects are not taken into account. However, effects related to the coverage of the inlet or outlet of a gas path by pool coolant are taken into account. These include gas-pool equilibration, blockage of gas flow path inlets by the pool, liquid head terms, and scrubbing effects. A discussion of flow path configurations and the pool-gas hierarchy for the flow paths is given in Section 4.2. Key elements of atmosphere/pool thermodynamics and intercell flow models are illustrated in Figure 4-1.

Section 4.3 gives a summary of the modeling options available for the gas and pool flow paths, as well as the suppression vent flow path. The governing equations for the flow path models are given in Section 4.4. The latter section discusses the three basic models for intercell flow, i.e., the inertial flow model, the quasi-steady flow model, and user-specified flow rates, as well as the formulations for critical or choked flow, gravitational heads, pool boiling, gas-pool equilibration, the velocity of gas evolution from the pool, and the FIX-FLOW option for overcoming the gas Courant limit in certain situations. As discussed in Section 4.4.5, the CONTAIN formulation of gas gravitational heads has been revised completely in CONTAIN 1.2 to satisfy three important criteria regarding the treatment of stratifications. As a consequence, the strong cautionary statements made with respect to previous code versions regarding convective overmixing are no longer necessary. However, the user should consult the guidance given in Section 13.3.1.3 with respect to avoiding certain situations that may lead to excessive stability of stratifications.

Section 4.5 discusses the momentum, mass, and energy conservation equations used by the intercell flow model and summarizes all contributions to the conservation equations, except the DCH ones. This section also discusses the gas and pool thermodynamic state calculations.

\subsection{The CONTAIN Cell}

The fluid control volumes, or cells, in CONTAIN are partitioned into two parts: the atmosphere and the coolant pool. A horizontal interface between the pool and atmosphere is assumed to be present at the collapsed hydrostatic level of the pool, an assumption that neglects possible level swell due to boiling and gas injected below the pool surface through submerged gas flow paths and other sources. Use of the collapsed level is consistent with the fact that although the equilibration and scrubbing of such injected gas are taken into account, a separate bubble field is not modeled in the pool. Instead, the injected gas is assumed to immediately become part of the atmosphere field of the downstream cell, without holdup in the pool. 


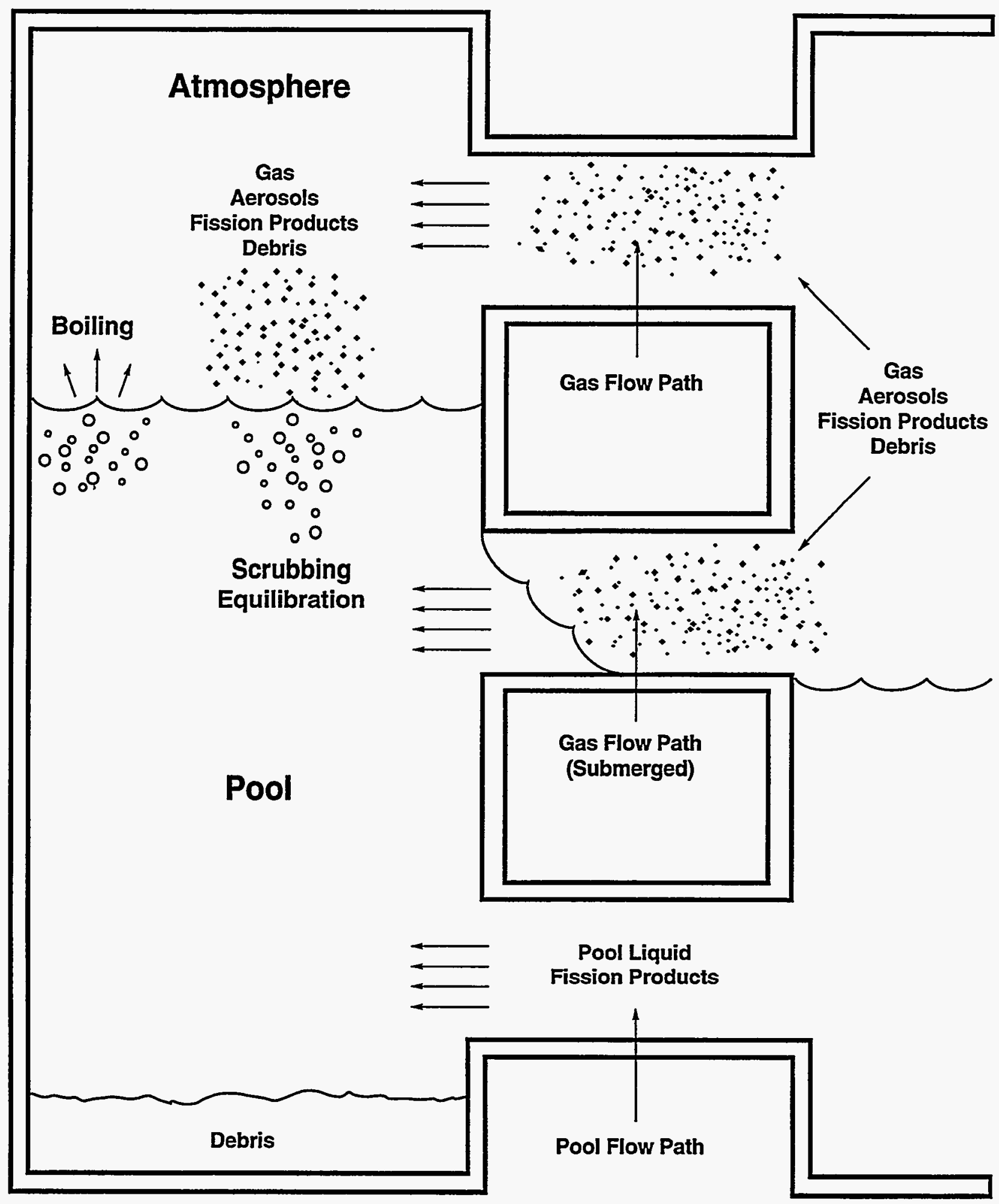

Figure 4-1. Atmosphere/Pool Thermodynamics and Intercell Flow as Modeled in CONTAIN 
The cell geometries assumed in CONTAIN 1.2 are based on a representation of the cell in which up to 10 different constant cross-sectional areas may be specified through the CELLHIST keyword as a function of height, relative to a common reference for the entire problem. The cell geometry is assumed fixed in time. The cross-sectional areas represent the area associated with the principal atmosphere and pool fluids. Heat transfer structures, the coolant film on structures, and any lower cell layers except for the coolant pool are assumed to have been excluded from the specified cell cross sections. One possible cell configuration is shown schematically in Figure 4-2 for the case of three different cross sections.

For old files lacking an explicit specification of cross-sectional areas through the new CELLHIST input, a single cross-sectional area is assumed and taken to be "volume"/"height," where the gas "volume" and "height" are parameters previously required in the cell GEOMETRY block The obsolete keyword ELEVCL in the FLOWS input is also used to define the initial gas center of elevation. The pool volume, if any, is added to the "volume" parameter to obtain the total cell volume. A diagnostic is given whenever the lower cell area is significantly different from the new cell cross-sectional area(s), as major changes in pool-atmosphere heat transfer areas and pool depths may occur. The user should also be aware that extremely large values of "height," for example, for cells representing the environment, may produce spurious effects because of the unrealistically large gravitational heads involved.

\subsection{Flow Path Configurations}

The recommended procedure with respect to flow paths is to construct them, in general, from engineered vents, which are specified through the ENGVENT input block ${ }^{1}$ discussed in Section 14.2.4.2. These flow paths have unlimited connectivity, in the sense that any number may be used to connect a given pair of cells. Beginning with CONTAIN 1.2, a given flow path modeled within this option may now transport either the atmosphere fields or the pool fields. The atmosphere and pool fields may flow only in a path of the appropriate type, defined by the user to be either GAS or POOL, respectively. Thus, it is necessary to define both a GAS and a POOL path in the ENGVENT block to represent any physical path in which both fields can flow, and it is assumed that the user will do this if necessary. The flow of the atmosphere and pool fields within their respective paths is taken to be independent, in the sense that the effects of interfacial shear and void fraction effects are neglected within the flow paths. However, gas-pool equilibration and liquid head effects related to the coverage of the inlet or outlet of a gas path by pool coolant are taken into account. In addition, a simple pool-gas hierarchy determines when gas or pool coolant can flow in a GAS or POOL path. For example, gas is not allowed to flow in a GAS path when the inlet is submerged to any appreciable degree below the surface.

CONTAIN flow paths are considered to be attached to a cell at a point, rather than a range of elevations corresponding to the physical opening height. By default, as shown in Figure 4-3, a gas path is attached at the tops of the cells connected by the path, and a pool path is attached to the bottoms of the cells. The user may specify the elevations at which each of the flow path ends are

\footnotetext{
'The option to use "regular" flow paths within the FLOWS input block is considered obsolete. However, if used, these paths are treated as type GAS. The input format for such flow paths is discussed in Appendix B.
}

Rev. 0 


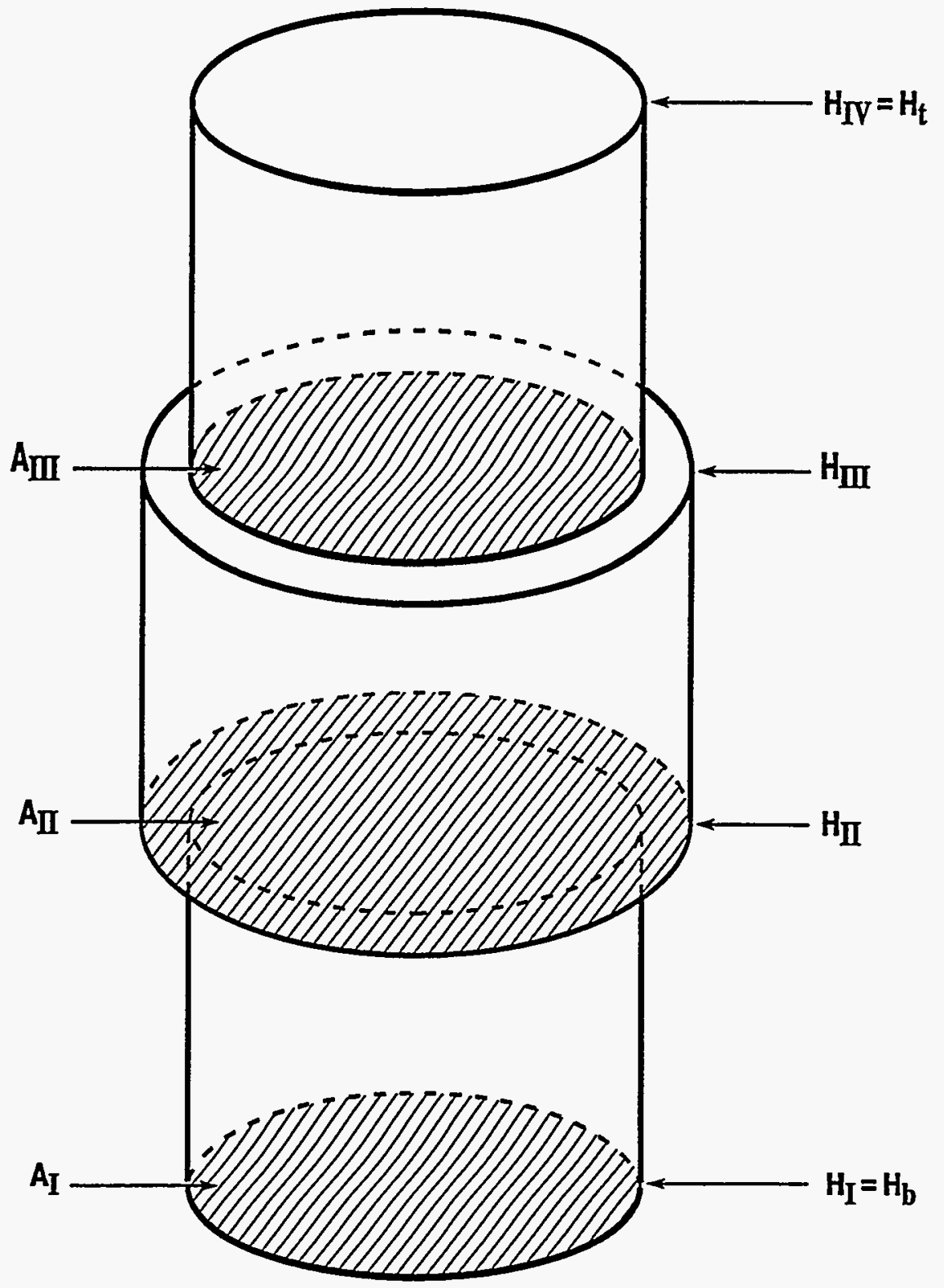

Figure 4-2. Definition of Cell Cross-Sections and Elevations, for a Case with Three CrossSectional Areas $\left(A_{\mathrm{I}}, A_{\text {II }}\right.$, and $\left.A_{\text {III }}\right)$ 


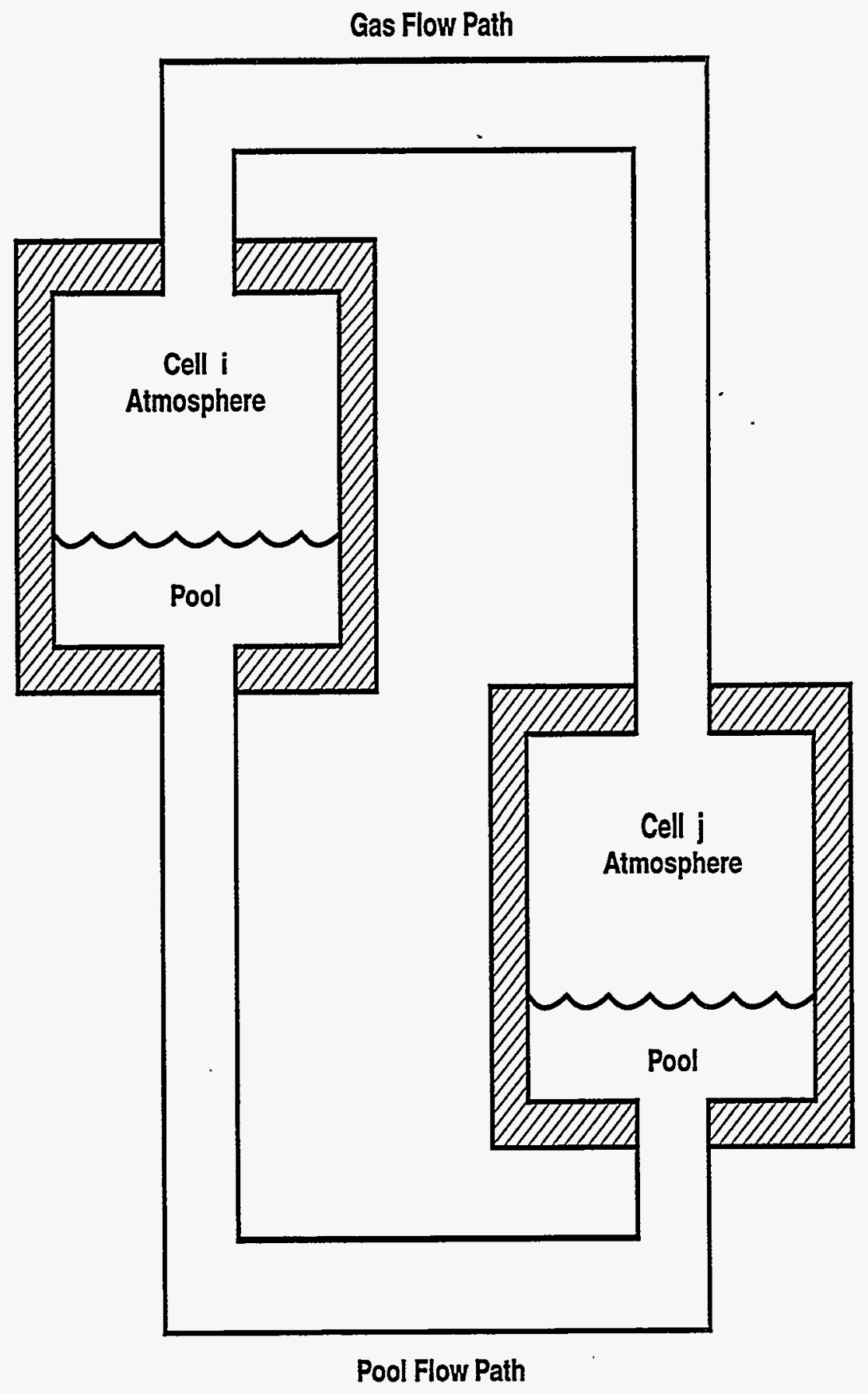

Figure 4-3. Default Attachment Points of Gas and Pool Flow Paths 
connected to the respective cells. Note that the elevation of each end must be specified separately for the gas and pool paths. Because of ambiguities related to gravitational heads, gas paths may not be connected below cell bottom, and pool paths may not be connected above the top of the cell. (The cell may be readily extended up or down to join onto the flow path, if necessary.)

It should be noted that the present defaults for the elevations of the ends of a gas flow path have changed from the pre-CONTAIN 1.2 defaults, which employed the user-specified gas centers of elevation. This change was necessary because the gas center of elevation is no longer fixed, as previously assumed, but changes with the pool height. In addition the possible submergence of the end of a gas path is now taken into account. Despite the change in default elevations, other changes in the formulation of the gas gravitational head, as discussed in Section 4.4.5, have improved the treatment of gas natural convection through the gas paths. This formulation is not dependent on user-specified gas flow path elevations, provided the gas path is not submerged and provided the new RESOLVHD or MSTABLE flow keywords are not specified. (These keywords attempt to recover the old gravitational head treatment.) However, the user is warned that if gas path elevations are specified, pool blockage and liquid head effects may produce effects on gas paths not modeled in the pre-CONTAIN 1.2 code versions.

The pool coolant level in a cell can affect not only the liquid heads for the flow paths attached to the cell but also the outflows from the cell in ways not directly reflected in the flow equations for the individual flow paths. Three types of level effects are treated: (1) if sufficiently deep, the pool may block the outflow from a gas path attached to the cell; (2) pool coolant may not flow when the pool level lies below the flow path inlet elevation; and (3) if the pool level is coincident with the elevation at which a pool path is attached to the cell, the volumetric outflow from that path may be balanced against the pool volumetric inflow for a period of time, with the pool level fixed at the pool path inlet. Treatment of the last condition in terms of a stationary level is not necessary but helps to minimize the "level hunting" associated with the point elevation assumption for flow path attachments. Such level effects are controlled by the CONTAIN pool-gas flow hierarchy.

The CONTAIN pool-gas flow hierarchy determines the degree to which gas or pool liquid, or both, are allowed to flow in multiple CONTAIN flow paths representing a given physical flow path. It should be noted that the simple CONTAIN hierarchy is intended primarily to describe separated flow processes in a containment, such as suppression vent clearing or liquid spill-over, in which a reasonably well-defined pool-gas interface exists in a cell and use of a collapsed pool level is reasonable. Such flow processes are assumed to occur with respect to "side-connected" paths, as discussed below. However, rigid adherence to a separated flow treatment would result in numerical problems, as well as a total inability to model two-phase flow phenomena such as level swell. While accurate modeling of such two-phase flow phenomena is not the intended purpose of the present set of models, some allowance for two-phase flow is desirable in the hierarchy. Therefore two-phase concurrent or countercurrent flow dependent on gravitational heads, but independent of the collapsed pool level, is assumed to occur with respect to "top-connected" paths. 


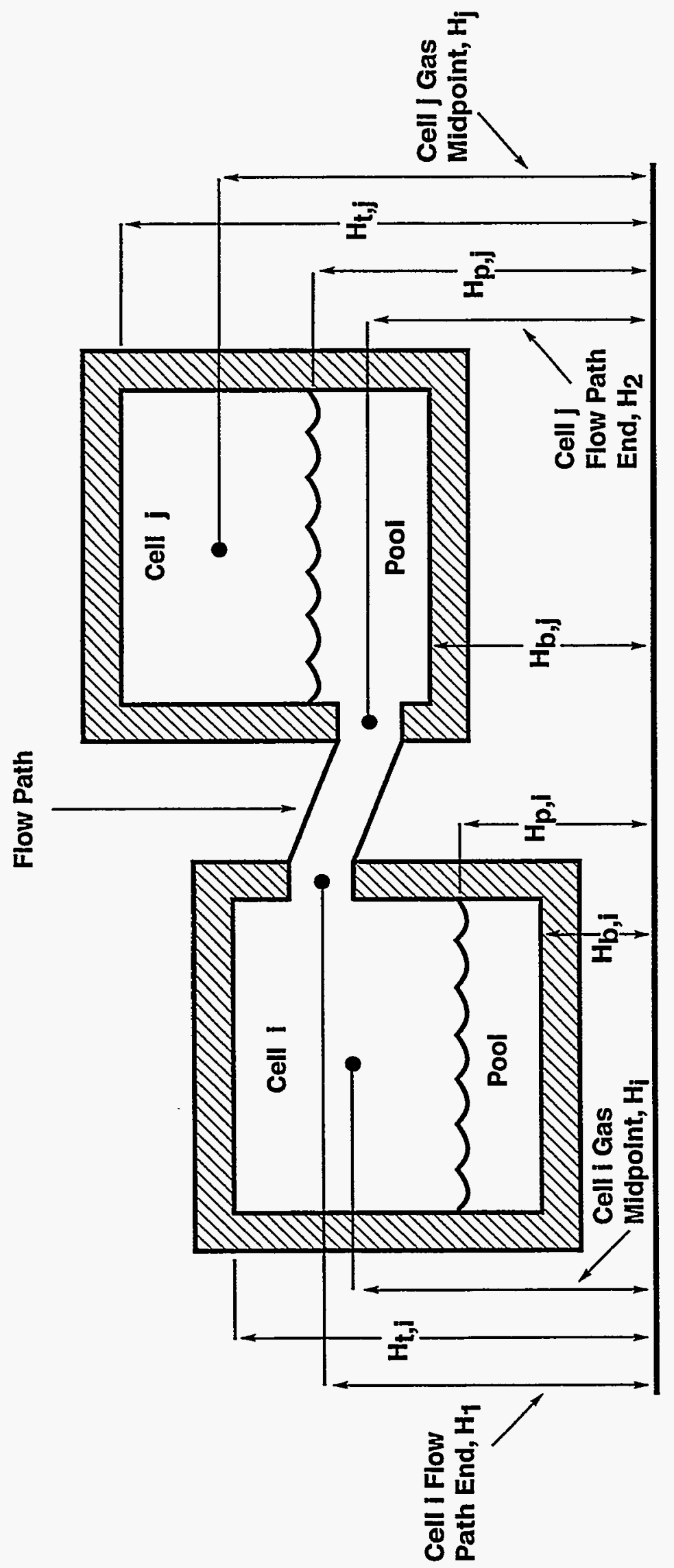

5
ต

$\stackrel{\infty}{+}$

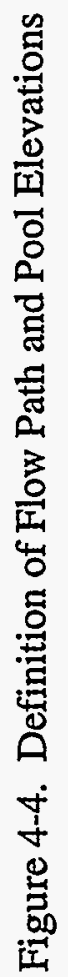

过 
A side-connected path is defined as being connected to the donor cell below the top elevation of the cell $\left(\mathrm{H}_{1}<\mathrm{H}_{\mathrm{i}, \mathrm{i}}\right.$, in terms of the elevations shown in Figure 4-4, for flow from $\mathrm{i}$ ), whereas a topconnected path is defined as connected at or above the top elevation $\left(\mathrm{H}_{1} \geq \mathrm{H}_{\mathrm{i} i}\right)$. Note that a bottomconnected path is treated in the same way as a side-connected path. Because of the differences in the treatment of these two cases, the user is warned that the point of attachment of a flow path to the cell can significantly affect the behavior of the path and therefore should be set with some care. In particular, the user should ensure that the attachment point of a side-connected path to a cell allows sufficient head space, or cell volume above the attachment point, so that the use of a collapsed-level flow hierarchy, as well as the point elevation assumption, is reasonable. It is recommended that the head space be at least $20 \%$ of the cell volume. With smaller amounts of head space, the user should anticipate an increased likelihood of stability problems related to nearly water-solid cells.

The effects of the pool-gas hierarchy may be defined very precisely in terms of quantities called inventory factors $\left(\xi_{\mathrm{ij}}\right)$. (For simplicity we will use the ij notation here and below to denote a flow path, even though more than one may connect the cells $i$ and $j$.) Such inventory factors are multipliers on the flow rates calculated from the individual flow equations for each flow path to take the hierarchy into account. For example, the pressure head for a gas path may be sufficient to cause gas to flow in the path, were it not for the fact that the inlet is completely submerged below the pool. The inventory factor zeroes out the gas flow in such a situation until the pool level approaches the inlet elevation.

Prior to discussing the inventory factors, it should be noted that for specialized applications, the user may wish to use the dedicated suppression pool vent model discussed in detail in Section 11.1. This model was implemented to model the flow of gases, aerosols, and fission products through the suppression pool vent system that connects the drywell and wetwell in a BWR, prior to the availability of the generalized flooding capabilities of CONTAIN 1.2. Its architecture is different from that of the gas and pool flow paths discussed above. First of all, only one suppression pool vent flow path may be specified per problem. Secondly, in contrast to what one would expect, coolant is not actually exchanged between pools in different cells during the vent clearing process for this model. The reason is that the dedicated model uses only one pool, that in the wetwell cell, and assumes that this pool spans both the drywell and wetwell sides of the vent. The vent clearing calculation is carried out internally in the model to determine a vent clearing time, after which the vent is assumed to be accessible to gas flow. Other ramifications of this single pool treatment are that gas-pool equilibration in reverse flow is not modeled, flooding of the vent from the drywell side cannot be modeled properly, and blockage of gas flow by the drywell pool level, if any, is not modeled.

Because of the architectural differences between the dedicated suppression pool vent model and the gas and pool flow path models, the inventory factors defined for the latter do not apply to the former. In effect the internal calculations for vent clearing in the former define the equivalent of an internal inventory factor. Thus, the external $\xi$ is simply taken to be unity for the suppression pool vent model.

Table 4-1 gives the inventory factors used for the gas, pool, and suppression pool vent flow paths. The elevations used are defined as in Figure 4-3, and it is assumed that cell $i$ is the donor cell. The 
Table 4-1

Definition of Inventory Factors for Gas and Flow Paths as a Function of the Elevation $\left(\mathrm{H}_{1}\right)$ of the Flow Path at the Donor Cell $\mathrm{i}$, Cell Top Elevation $\left(\mathrm{H}_{\mathrm{t}, \mathrm{i}}\right)$, Cell Bottom Elevation $\left(\mathrm{H}_{\mathrm{b}, \mathrm{i}}\right)$, Pool Surface Elevation $\left(\mathrm{H}_{\mathrm{p}, \mathrm{i}}\right)$, and Gas Access Transition Height $\delta \mathrm{H}_{\mathrm{g}}=0.01 \mathrm{~m}$. The other quantities are discussed in Section 4.2.

\begin{tabular}{|c|c|c|}
\hline Path Type & Condition & $\xi$ Value \\
\hline Gas & $\mathrm{H}_{1} \geq \mathrm{H}_{\mathrm{t}, \mathrm{i}}$ (top-connected) & $\xi_{0}=\min \left(1, \mathrm{~V}_{0} / \delta \mathrm{V}_{\mathrm{out}}\right)$ \\
\hline Gas & $\mathrm{H}_{\mathrm{t}, \mathrm{i}}>\mathrm{H}_{1}>\mathrm{H}_{\mathrm{p}, \mathrm{i}}$ & $\xi_{0}$ \\
\hline Gas & $\mathrm{H}_{\mathrm{i}, \mathrm{i}}>\mathrm{H}_{\mathrm{i}} ; \mathrm{H}_{1}+\delta \mathrm{H}_{\mathrm{g}}>\mathrm{H}_{\mathrm{p}, \mathrm{i}}>\mathrm{H}_{1}$ & $\left(\mathrm{H}_{\mathrm{p}, \mathrm{i}}-\mathrm{H}_{1}\right) \xi_{0} / \delta \mathrm{H}_{\mathrm{g}}$ \\
\hline Gas & $\mathrm{H}_{\mathrm{p}, \mathrm{i}}>\mathrm{H}_{1}+\delta \mathrm{H}_{\mathrm{g}}$ & 0 \\
\hline Pool & $\mathrm{H}_{1}=\mathrm{H}_{\mathrm{t}, \mathrm{i}} ; \mathrm{H}_{\mathrm{p}, \mathrm{i}}>\mathrm{H}_{\mathrm{b}, \mathrm{i}}($ top-connected $)$ & 1 \\
\hline Pool & $\mathrm{H}_{\mathrm{t}, \mathrm{i}}>\mathrm{H}_{1}>\mathrm{H}_{\mathrm{p}, \mathrm{i}}$ & 0 \\
\hline Pool & $\mathrm{H}_{\mathrm{t}, \mathrm{i}}>\mathrm{H}_{1}=\mathrm{H}_{\mathrm{p}, \mathrm{i}} \geq \mathrm{H}_{\mathrm{b}, \mathrm{i}}$ & $\delta \mathrm{V}_{\mathrm{in}} / \delta \mathrm{V}_{\text {out }}$ \\
\hline Pool & $\mathrm{H}_{\mathrm{p}, \mathrm{i}}>\mathrm{H}_{1}$ & 1 \\
\hline $\begin{array}{c}\text { Suppression Pool } \\
\text { Vent }\end{array}$ & Submerged & $\mathrm{F}_{\mathrm{vo}}{ }^{*}$ \\
\hline $\begin{array}{c}\text { Suppression Pool } \\
\text { Vent }\end{array}$ & Bypass & 1 \\
\hline \hline
\end{tabular}

"Fraction of timestep in cleared state, see Equation (11-23)

volumes $\mathrm{V}_{0}, \delta \mathrm{V}_{\text {in }}$, and $\delta \mathrm{V}_{\text {out }}$ are defined separately for the atmosphere and pool fields and denote the initial available volume, the total inflow volume, and the total outflow volume from a cell during each timestep used in the intercell transfer of the atmosphere or pool fields. The inflow volume is computed by taking the actual upstream inventory factors into account. However, the total outflow volume in this table is calculated by using a zeroeth order approximation for the inventory factors, corresponding to setting $\xi_{\mathrm{ij}}=1$ for all of the outflows.

Although Table 4-1 is for the most part self-explanatory, some discussion is helpful. A gas-access transition height, $\delta \mathrm{H}_{\mathrm{g}}$ presently hardwired to a nominal $0.01 \mathrm{~m}$, governs the cutoff of gas flow in a gas path for which the inlet end is submerged. The $\xi_{\mathrm{ij}}$ for a gas path is one at zero submergence, is zero at a submergence equal to $\delta \mathrm{H}_{\mathrm{g}}$, and is linearly dependent on the submergence in between. It should also be noted that the condition in which the collapsed pool level is fixed at the inlet elevation of a pool flow path, with pool inflow balancing pool outflow, is given by the second to last condition in the table for pool flow paths. The "suppression pool vent" entry in this table refers to the dedicated suppression pool vent model. As discussed above, because of internal calculations to the model that serve to define the equivalent of an inventory factor internally, the external $\xi$ factor for this flow path has been set to unity. 


\subsection{Flow Path Modeling Options}

The flow modeling options available for flow paths constructed of engineered vents or the suppression pool vent are described in this section. The use of engineered vents and the suppression pool vent is available only within the implicit flow solver, invoked with the IMPLICIT sub-block within the FLOWS input block. It is recommended that the IMPLICIT option always be invoked unless a compelling reason to the contrary exists.

The implicit flow solver determines the flows in flow paths of GAS, POOL, and suppression pool vent types and the thermodynamic states of the cell atmospheres and pools. By default, the size of the implicit solution space is equal to the number of cell atmospheres and number of coolant pools. The user may specify which of these are to be solved implicitly and which explicitly. When IMPLICIT is specified but some of the atmospheres and pools are to be solved explicitly, an Euler explicit method is used with respect to the atmospheres or pool pressures. In practice only environment cell atmospheres (on the order of $10^{8} \mathrm{~m}^{3}$ ) are sufficiently large to be modeled explicitly. A pool may be solved explicitly, with considerably less stringent requirements than those for an atmosphere, but that is allowed only if that pool is not connected to another pool through a pool flow path. A cell governed by the FIX-FLOW code efficiency option discussed in Section 4.4.9 also may not have a pool that is connected to another pool through a pool flow path. It should be noted that transfers between pools denoted by the user as explicit may still be effected through the liquid transport models available within the engineered systems modeling (not be confused with engineered vents) discussed in Chapter 12. If IMPLICIT is not specified, then only a very limited set of modeling options are available; for this limited set of options, an explicit Runge-Kutta method is used.

The engineered vent input options are discussed in Section 14.2.4.2. The modeling options for each engineered vent may be chosen completely independently, although the user should define two flow paths of type POOL and GAS to represent a single physical flow path in which both types of flow can occur (see Section 4.2). A number of tabular input options are available for the engineered vent. For example, the vent flow area as a function of pressure difference $\triangle P$ can be calculated reversibly or irreversibly in a RVAREA-P or IRAREA-P table, respectively. In addition, the user can specify either the mass or volumetric flow rate as a function of time through MFLOW-T and VFLOW-T tables, respectively. Each vent may be opened at a specified time, using VTOPEN, or at a given pressure differential, using VDPF or VDPB for forward or reverse opening, respectively. Each can also be closed at a specified time, using VTCLOS.

Two new keywords VEQLENB and VEQLENF for the gas-pool equilibration lengths for backward and forward flow, respectively, allow the user to control the degree of equilibration of the gas exiting a gas flow path that is submerged below the pool surface in a cell. The default for these lengths for a gas path is $0.01 \mathrm{~m}$. The details of the gas-pool equilibration modeling are given in Section 4.4.7.

The flow of aerosols, fission products, and dispersed core debris in the flow paths is discussed in Sections 7.8, 8.8, and 6.2, respectively. The treatment may be summarized as follows. For an unsubmerged gas flow path, aerosols are transported through the flow path according to the gas convective velocity and the differential settling velocity of the aerosols. The differential velocity is 
calculated from the terminal settling velocity and user input for the flow path inclination angle provided through the VCOSN keyword (see Sections 7.8 and 14.2.4.2). Fission products attached to the aerosols flow with the aerosols. In an unsubmerged gas path, fission products attached to the gas flow without slip (see Section 8.8.1). Dispersed core debris flows with the gas according to slip factors assigned by the user to each of the debris fields in the DCH input (see Sections 6.2 and 14.2.7). For submerged gas flow paths, the treatment of the scrubbing of aerosols, fission products, and dispersed core debris is presently very simple. Aerosols and fission products are simply removed from the flow and placed in the downstream pool. Core debris is removed from the flow and placed in the uppermost intermediate layer of the lower cell in the downstream cell, if such a layer has been defined. Otherwise, this debris is removed from the problem. (While such debris is no longer present in an active repository, any such removed mass and energy will be reflected in the WASTE location of the energy and mass accounting arrays.) Only fission products in the pool are allowed to flow with coolant in a pool path. The fission products flow with transport efficiency factors assigned by the user in the FPLIQUID option (see Sections 8.8.2 and 14.2.6.2).

The thermal-hydraulic details of the dedicated suppression pool vent model are discussed in Sections 11.1 and 11.2, and the input options are discussed in Section 14.2.4.3. The interested user should also read the comments about the model architecture in Section 4.2. With respect to modeling differences between a gas flow path and the dedicated model, it should be noted that although the gas-pool equilibration modeling for a submerged suppression pool vent follows the modeling described in Section 4.4.7, the gas-pool equilibration lengths, over which the gas temperature and vapor pressure are equilibrated with those of the pool, are fixed: the length is taken to be zero for forward flow from drywell to wetwell and infinite for reverse flow from wetwell to drywell (i.e., equilibration is not considered in this direction). In addition, gas or pool inertial effects are not modeled in the dedicated model.

With respect to the treatment of aerosols, fission products, and dispersed core debris, two detailed aerosol scrubbing models, the SPARC and SCRUB models, are available for the dedicated model, as discussed in Sections 7.7 and 7.8. Otherwise, the treatment is similar to that for a gas path.

If detailed modeling of aerosol scrubbing is not required, an alternative to the dedicated model is to use a system of gas and pool flow paths, which should provide more realistic modeling of a suppression pool vent system. For example, vent clearing in a MARK III can be modeled with a set of three gas and three pool paths, as discussed in more detail in Section 11.1.

\subsection{The Flow Models}

The subsections that follow describe the flow path and related models that are used in CONTAIN. There are three basic models for intercell flow: the inertial flow model, the quasi-steady flow model, and user-specified flow rates. These are discussed in Sections 4.4.1, 4.4.2, and 4.4.3, respectively. The flow in a gas path for either of the first two basic models is limited by critical or choked flow, discussed in Section 4.4.4. A revised treatment of gravitational heads, using the "hybrid" formulation, is discussed in Section 4.4.5. The next three subsections discuss quantities related to pool-gas exchange rates. Pool boiling is discussed in 4.4.6, gas-pool equilibration is discussed in Section 4.4.7, and the velocity of gas evolution from the pool is discussed in 4.4.8. Finally, the FIX- 
FLOW option for overcoming the gas Courant limit in certain situations is discussed in Section 4.4.9.

\subsubsection{Inertial Flow Model}

For gas and pool flow paths, the inertial flow model is used in all cases except in the RVAREA-P tabular option for reversible flow area versus pressure. This model takes into account the inertia of the fluid in the flow path, as well as flow losses through a user-specified loss coefficient. If a flow path is opened suddenly at a fixed pressure difference, the fluid inertia has the effect of delaying the buildup of the flow rate to the steady-state value. When two cells are close to pressure equilibrium, the inertia manifests itself in a different manner. At the point where the pressure difference becomes zero, the flow rate is generally finite because of the inertia of the flowing material. A finite flow rate will tend to reverse the direction of the pressure difference across the flow path. The reversal in the pressure difference will eventually reverse the direction of the flow. Under these conditions, damped oscillatory flow will occur.

The inertial flow model, described in detail in Table 4-2, can be viewed as a one-dimensional momentum equation for the turbulent flow regime. It is derived by integrating the equation for the time-derivative of the kinetic energy flux along streamlines for the flow path, using the assumption that all points along the streamlines are at steady state.

The inertial flow model uses an irreversible flow loss coefficient $\mathrm{C}_{\mathrm{FC}}$ that is specified by the user through the VCFC keyword (see Section 14.2.4.2). This coefficient of order unity should represent the viscous losses in the flow including entrance, exit, and Moody friction factors. Caution should be used in applying standard formulas because these may apply to dynamic pressures, whereas the CONTAIN cell pressures are stagnation pressures. One consequence is that velocity-of-approach corrections may not be necessary. Specifically, $C_{F C}=1 / 2\left(2 C_{D}^{2}\right)$ where $C_{D}$ is equal to the discharge coefficient $C$ defined in Reference Bau78 or the quantity $C_{D}$ in Reference Flo79. In the notation of Chapter 4 of Reference Ide60, the CONTAIN $C_{\mathrm{FC}}$ corresponds to the quantity $\mathrm{g} \Delta \mathrm{H} /\left(\gamma \omega_{\mathrm{o}}^{2}\right)$. In most cases discussed there, this quantity is equal to $\zeta / 2$. However, one must be careful since Reference Ide60 is not consistent in the definition of $\zeta$. In some cases, $\zeta$ is based on the upstream velocity and not the velocity $\omega_{0}$ in the flow constriction. (See Section 13.3.1.4 for other guidance concerning the specification of the flow loss coefficients in a CONTAIN calculation.)

\subsubsection{Quasi-Steady Flow Model}

The quasi-steady flow model is used in some cases in the implicit flow options; specifically, for the engineered vent with the RVAREA-P tabular option for reversible area versus pressure, as discussed in Section 14.2.4.2, and for the dedicated suppression pool vent, which is described in Sections 11.1 and 14.2.4.3.

The basic assumption of the quasi-steady flow model is that the fluid inertia is negligible. The mass flow rate is calculated by neglecting acceleration, that is, $\mathrm{dW}_{\mathrm{ij}} / \mathrm{dt}$ in Table 4-2 is set equal to zero. The resulting flow equation is 
Table 4-2

Conservation of Momentum Equation for Flow Between Cell $\mathrm{i}$ and Cell $\mathrm{j}$

For a flow path with inertia, the flow equation is a momentum equation given by [Mur96]

$$
\frac{d W_{\text {iner,ij }}}{d t}=\left(\Delta P_{i j}-C_{F C} \frac{\left|W_{\text {iner,ij }}\right| W_{\text {iner, }, \mathrm{ij}}}{\rho_{\mathrm{u}}\left(A_{i j}^{\prime}\right)^{2}}\right) \frac{A_{i j}}{L_{i j}}
$$

where for a gas path within the implicit flow option

$$
\Delta P_{i j} \quad=\quad P_{i}-P_{j}+\Delta P_{g r, i j}
$$

for a pool path within the implicit flow option

$$
\Delta P_{i j} \quad=\quad P_{i}-P_{j}+\Delta P_{p, g r, j j}
$$

and within the explicit (Runge-Kutta) flow option or for the dedicated suppression pool vent flow path in bypass (unsubmerged) state

$$
\Delta P_{i j} \quad=\quad P_{i}-P_{j}
$$

Note the other parameters are defined below. Within the implicit flow option the actual flow rate $W_{i j}$ for a gas path is limited by critical flow rate $W_{c r, j j}$ and by inventory factors $\xi_{\mathrm{ij}}$, defined below, which reflect the pool-gas hierarchy for the flow paths. For a gas flow path with inertia or the dedicated suppression pool vent flow path in bypass state:

$$
\begin{aligned}
& \mathrm{W}_{\mathrm{ij}}=\xi_{\mathrm{ij}} \min \left(\mathrm{W}_{\mathrm{iner,j \textrm {j }}} \mathrm{W}_{\mathrm{cr}, \mathrm{ij}}\right) \text { if } \mathrm{W}_{\mathrm{iner, \textrm {j } j}}>0 \text { and } \mathrm{W}_{\mathrm{cr, \textrm {j } j}}>0 \text {; } \\
& \mathrm{W}_{\mathrm{ij}}=\xi_{\mathrm{ij}} \max \left(\mathrm{W}_{\mathrm{iner,j \textrm {j }}}, \mathrm{W}_{\mathrm{cr}, \mathrm{j} j}\right) \text { if } \mathrm{W}_{\mathrm{iner,j \textrm {j }}}<0 \text { and } \mathrm{W}_{\mathrm{cr}, \mathrm{ij}}<0 \text {; } \\
& \mathrm{W}_{\mathrm{ij}}=\xi_{\mathrm{ij}} \mathrm{W}_{\mathrm{iner,j \textrm {j }}} \text { otherwise. }
\end{aligned}
$$

The flow rate $\mathrm{W}_{\mathrm{ij}}$ for a pool path is limited by the $\xi_{\mathrm{jij}}$ factors introduced through the pool-gas flow hierarchy; for a pool path with inertia

$$
\mathrm{W}_{\mathrm{ij}}=\xi_{\mathrm{ij}} \mathrm{W}_{\mathrm{iner}, \mathrm{jj}}
$$

Within the explicit (Runge-Kutta) flow option

$$
\mathrm{W}_{\mathrm{ij}}=\mathrm{W}_{\mathrm{iner,j \textrm {j }}} \text { (gas paths only) }
$$


Table 4-2

Conservation of Momentum Equation for Flow Between Cell $\mathrm{i}$ and Cell $\mathrm{j}$ (Continued)

Note that for quasi-steady flow paths without inertia, including the dedicated suppression pool vent in submerged state, the definition of $\mathrm{W}_{\mathrm{ij}}$ is discussed in Section 4.4.2. In the above

$\mathrm{W}_{\mathrm{ij}} \quad=\quad$ total mass flow rate of gases, coolant vapor, and homogeneously dispersed liquid coolant through a gas flow path or of pool coolant through a pool flow path. Note that flow paths in the following discussion, including the suppression pool vent, will be indexed by $\mathrm{ij}$, even though more than one path may be present between cells $i$ and $j$;

$\mathrm{W}_{\text {iner,ij }}=$ total mass flow rate in the inertial flow model;

$\mathrm{W}_{\mathrm{cr}, \mathrm{ij}}=$ critical flow rate for a gas path, defined in Equation (4-5);

$\xi_{\mathrm{ij}}=$ inventory factors defined in Table 4-1 that reflect the pool-gas hierarchy, as discussed in Section 4.2;

$\mathrm{t}=$ time;

$\mathrm{C}_{\mathrm{FC}}=$ user-specified irreversible flow loss coefficient for the flow path;

$\rho_{\mathrm{u}} \quad=$ the flow path density, which depends on the flow option used. For the implicit flow option, $\rho_{u}=\rho_{i}$ if $i$ is the upstream cell, and $\rho_{u}=\rho_{j}$ if $j$ is the upstream cell. For the explicit (Runge-Kutta) flow option, $\rho_{u}=\left(\rho_{i}+\right.$ $\left.\rho_{\mathrm{j}}\right) / 2$;

$\rho_{\mathrm{i}} \quad=$ gas, vapor, and homogeneously dispersed liquid density in cell i for a gas path; pool density in cell i for a pool path;

$\rho_{\mathrm{j}} \quad=$ gas, vapor, and homogeneously dispersed liquid density in cell $\mathrm{j}$ for $\mathrm{a}$ gas path; pool density in cell $j$ for a pool path;

$\mathrm{A}_{\mathrm{ij}} / \mathrm{L}_{\mathrm{ij}} \quad=$ user-specified area to length ratio of the flow path;

$\mathrm{A}_{\mathrm{ij}} \quad=$ flow path area;

$\mathrm{A}_{\mathrm{ij}}^{\prime} \quad=\quad \mathrm{A}_{\mathrm{ij}}$ for a pool flow path or gas flow path when the downstream end is not submerged below the pool in the downstream cell, and $A_{i j}^{\prime}=$ $\min \left(\mid \triangle P_{\mathrm{ij}} / 1000,1\right) \mathrm{A}_{\mathrm{ij}}$ when the downstream end is submerged; 
Table 4-2

Conservation of Momentum Equation for Flow Between Cell $i$ and Cell $j$ (Concluded)

\begin{tabular}{|c|c|}
\hline$P_{i}$ & $=$ gas pressure in cell $\mathrm{i}$ \\
\hline$P_{j}$ & $=$ gas pressure in cell $j$ \\
\hline$\Delta \mathrm{P}_{\mathrm{g}, \mathrm{j} j}$ & $\begin{array}{l}=\text { pressure head due to gravity for a gas path, as discussed in Section } \\
4.4 .5 .1 \text {; }\end{array}$ \\
\hline$\Delta P_{p, g r, i j j}$ & $\begin{array}{l}=\text { pressure head due to gravity for a pool path, as discussed in Section } \\
\text { 4.4.5.2. }\end{array}$ \\
\hline
\end{tabular}

where the quantities are as defined in Table 4-2. In the reversible-area-versus-pressure option and the dedicated suppression vent, the $A_{i j}$ is taken to be a function of the pressure difference $\Delta P_{i j}$.

Equation (4-1) can be used to solve for the mass flow rate in terms of $\Delta P_{\mathrm{ij}}$ :

$$
\mathrm{W}_{\mathrm{qs}, \mathrm{ij}}=\left(\theta_{\mathrm{ij}}-\theta_{\mathrm{ji}}\right) \sqrt{\left|\Delta \mathrm{P}_{\mathrm{ij}}\right| \rho_{\mathrm{u}} \mathrm{A}_{\mathrm{ij}}^{2} / \mathrm{C}_{\mathrm{FC}}}
$$

where $\theta_{i j}$ equals 1 and 0 for $\Delta P_{i j}>0$ and $\Delta P_{i j}<0$, respectively; $\theta_{j i}$ equals 0 and 1 for $\Delta P_{i j}>0$ and $\Delta P_{i j}$ $<0$, respectively. Except in the case of submerged gas flow through the dedicated suppression vent, the flow of gas is limited by the critical or choked flow limit; that is, the flow rate $\mathrm{W}_{\mathrm{ij}}$ in the flow path is taken to be

$$
\begin{aligned}
W_{i j} & =\xi_{i j} \min \left(W_{q s, i j}, W_{c r, i j}\right) \text { if } W_{q s, i j} \geq 0 \\
& =\xi_{i j} \max \left(W_{q s, i j}, W_{c r, i j}\right) \text { if } W_{q s, i j}<0
\end{aligned}
$$

where $W_{c, i j}$ is defined in Equation (4-5) below and the inventory factor $\xi_{\mathrm{ij}}$ is defined in Table 4-1. In the case of a submerged suppression pool vent or a pool path, critical flow is ignored and $W_{i j}$ is defined as

$$
\mathrm{W}_{\mathrm{ij}}=\xi_{\mathrm{ij}} \mathrm{W}_{\mathrm{qs}, \mathrm{ij}}
$$




\subsubsection{User-Specified Flow Rates}

The user may want simply to specify the flow rate as a function of time. The mass or volumetric flow rate can be directly specified as a function of time through tables as described in Sections 4.3 and 14.2.4.2. It should be noted that the energy equation for user-specified flow rates is taken to be the same as for pressure-driven flow at the same flow rate. This treatment neglects the work done, in some cases, by the agent required to sustain the flow. If important, such work should be calculated and added separately.

The flow area need not be specified when the flow rates are specified. However, if the area is not specified, it is assumed to be infinitesimal, and consequently the velocity in the flow path will be calculated as extremely high. Choked flow is not considered in the case of user-specified flow rates. However, the specified flow rates are modified when necessary by the inventory factors, $\xi_{\mathrm{ij}}$, in Table 4-1.

\subsubsection{Critical Flow Model}

Critical or choked flow through gas flow paths occurs when the mass flow rate reaches a fixed value independent of the pressure ratio across the flow path. The flow velocity is limited at the minimum area of the flow streamlines to the speed of sound. The critical flow rate of gas $\mathrm{W}_{\mathrm{cr}, \mathrm{j} j}$ is then given by

$$
\mathrm{W}_{\mathrm{cr}, \mathrm{ij}}=\left(\theta_{\mathrm{ij}}-\theta_{\mathrm{ji}}\right) \mathrm{A}_{\mathrm{ij}} \mathrm{v}_{\mathrm{ij}}\left[\gamma_{\mathrm{u}} \mathrm{P}_{\mathrm{u}} \rho_{\mathrm{u}} \eta_{\mathrm{u}}\right]^{1 / 2}
$$

where $\theta_{i j}$ equals 1 and 0 for $\Delta P_{i j}>0$ and $\Delta P_{i j}<0$, respectively; $\theta_{\mathrm{ji}}$ equals 0 and 1 for $\Delta P_{i j}>0$ and $\Delta P_{i j}$ $<0$, respectively; $v_{\mathrm{ij}}$ is the vena contracta factor; $\gamma_{\mathrm{u}}$ is the ratio $c_{\mathrm{p}} / c_{\mathrm{v}}$ of specific heats in the upstream cell atmosphere; and $\eta_{\mathrm{u}}$ is a dimensionless parameter given by

$$
\eta_{u}=\left[\frac{2}{1+\gamma_{u}}\right]^{\frac{\gamma_{u}+1}{\gamma_{u}-1}}
$$

The other parameters are defined in Table 4-2. Note that $v_{\mathrm{ij}}$ is generally less than unity and is defined as the ratio of the minimum area occupied by the flow streamlines to the geometric cross-sectional area $\mathrm{A}_{\mathrm{ij}}$ of the flow path [Lam45]; that is, $v_{\mathrm{ij}}=\mathrm{A}_{\text {contract }} / \mathrm{A}_{\mathrm{ijj}}$, where the relation between these areas is shown in Figure 4-5. The subscript $u$ denotes upstream conditions. 


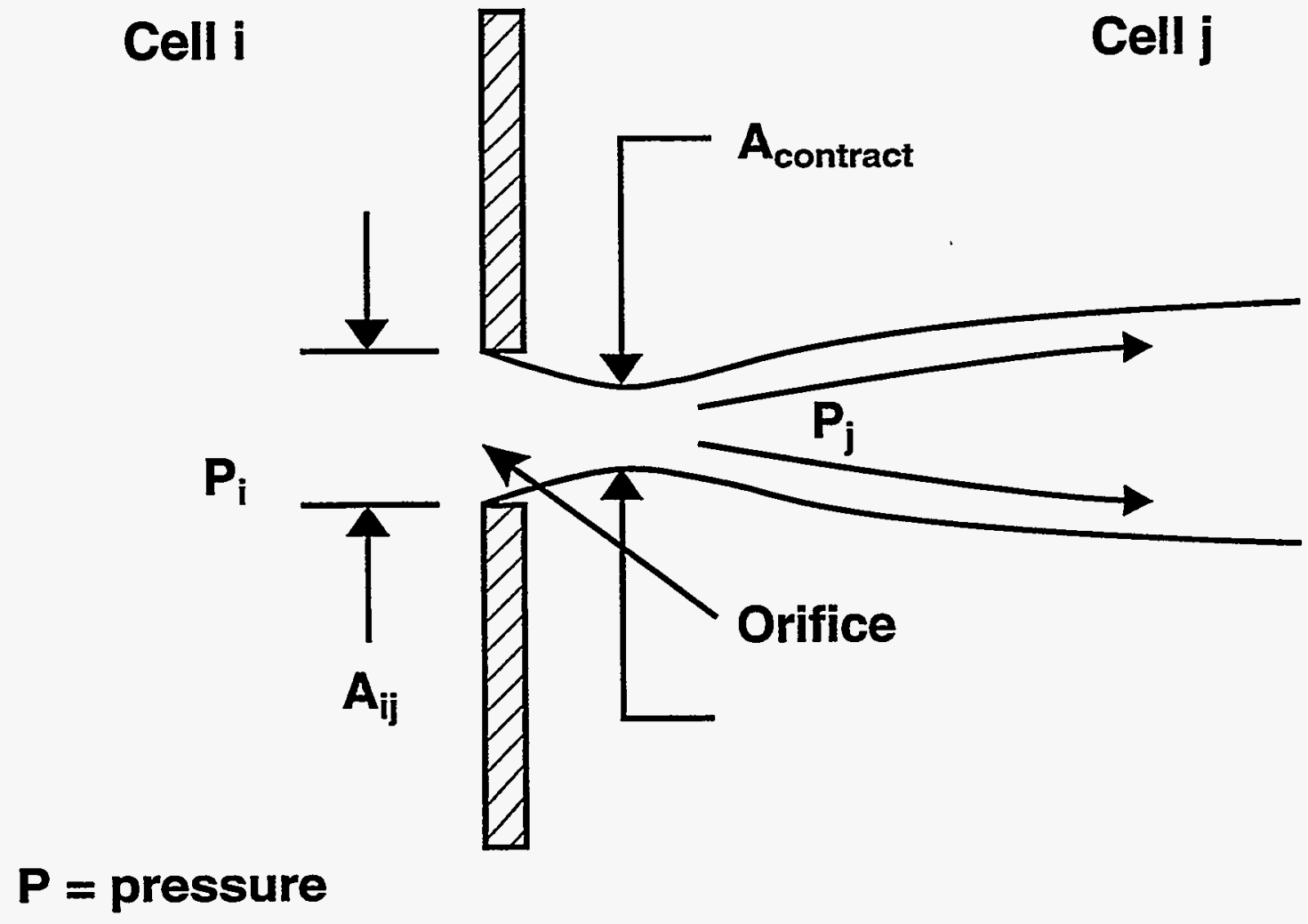

Figure 4-5. Illustration of the Flow Areas Used in the Critical or Choked Flow Calculation 


\subsubsection{Gravitational Head Modeling}

The following sections discuss the gravitational head modeling for both gas and pool flow paths that is available within the implicit flow solver. Beginning with CONTAIN 1.2, a number of important changes have been made in the modeling of gas gravitational heads as a result of investigations into the cause of the overmixing of gas stratifications typically found in control volume codes. [Mur96] These investigations have resulted in a "hybrid" flow solver formulation. The hybrid formulation refers to a treatment of gas gravitational heads that has been developed to satisfy a minimum set of requirements for modeling natural convection in the gas in a reasonable manner. These requirements are (1) consistency with the derivation of the CONTAIN momentum equation for flow paths, (2) proper treatment of stable stratifications, when present; and (3) correct limiting behavior in the well-mixed asymptotic limit. The performance of the hybrid solver has been assessed extensively and is discussed in a separate report. [Mur96] The findings from this assessment are summarized in the user guidance Section 13.3.1.3. The user should note and avoid in particular the situations discussed in that section that lead to excessive stability of stratifications. The gravitational head formulation for pool flow paths is straightforward, except for the fact that no correction is made for the gas gravitational head between the gas center of elevation, at which the pressure is defined, and the pool surface.

4.4.5.1 Gas Flow Path Gravitational Heads. In the hybrid formulation, the gas densities used to evaluate the gas contribution to the gas flow path gravitational head depend in a complicated way on the gas densities and elevations of the two cells connected by the flow path. The hybrid formulation interpolates between two methods. One method, correct in the well-mixed asymptotic limit, is based on the use of an arithmetically averaged cell gas density $\rho_{a v}=\left(\rho_{i}+\rho_{j}\right) / 2$, where $\rho_{i}$ and $\rho_{j}$ are the gas densities of the two cells $i$ and $j$ connected by a flow path. The other method, used away from the asymptotic limit, closely approximates the use of the donor density $\rho_{u}$ defined in Table 4-2, unless the user specifies that an alternative treatment be used. For numerical reasons related to the efficiency of the flow solver, the latter method is actually based on what is called a virtual dynamic interface (VDI) within a flow path. The VDI method eliminates the discontinuity in the flow that would occur at flow reversal when a strictly donor method is used. The VDI eliminates the discontinuity by defining a continuum of possible locations within the flow path at which the density changes from that of one cell to that of the other of the two cells connected by the flow path. This density crossover location is used to calculate the flow path gravitational heads. Figure 4-6 shows some possible VDI locations for the flow paths in a four-cell problem. The term "virtual" refers to the fact that this density crossover point is used only to calculate the gravitational head, since CONTAIN flow paths are not repositories and have no actual resident inventory associated with them.

The VDI has its own dynamic equation (Equation (4-11) below) based on a flow path filling considerations. However, the motion of the interface is artificially accelerated (through the parameter $\kappa$ in Equation (4-11)) so that the VDI method much of the time gives results corresponding closely to the donor treatment. Alternatives to this default flow path formulation may be specified through user input (see the discussion of the MSTABLE and RESOLVHD keywords below). Regardless of the gravitational head modeling options invoked, the gravitational head formulation will always revert back to an asymptotically correct expression, based on the averaged density and 


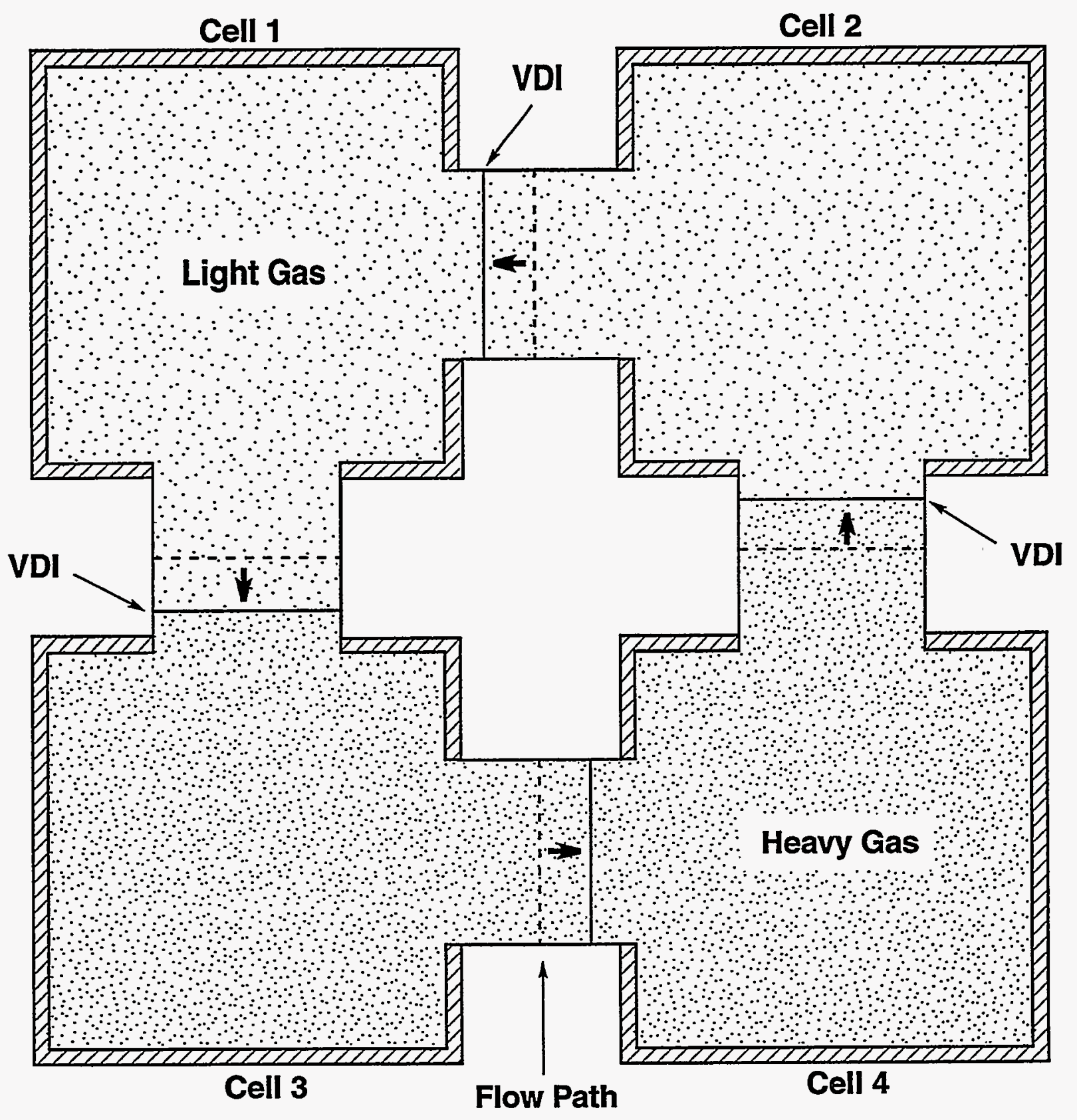

Figure 4-6. Position of the Virtual Dynamic Interfaces (VDIs) in the Flow Paths of a Four Cell Problem in Response to a Slight Increase in Density in Cell 1, Starting with a Stable Stratification Consisting of a Light Gas over a Heavy Gas 
gas center-of-volume elevations, in the well-mixed limit. Hence, the overall approach is termed a "hybrid" one.

The gas flow path gravitational head $\Delta \mathrm{P}_{\mathrm{g}, \mathrm{j} j \mathrm{j}}$ occurs in the momentum equation between two cells $\mathrm{i}$ and $\mathrm{j}$, as indicated in Table 4-2. This is taken to be a linear combination of the asymptotically correct expression for the gas head $\Delta \mathrm{P}_{g \mathrm{r}, \mathrm{j}}^{\prime}$, calculated using the averaged cell density and gas centers of elevation, the VDI expression for the gas head $\Delta \mathrm{P}_{\mathrm{gr}, \mathrm{j}}^{\prime \prime}$ used away from the asymptotic limit, and the liquid head terms present when one or both ends of the path are submerged below the pool surface in the cell to which they are attached:

$$
\Delta P_{g r, i j}=y \Delta P_{g r, i j}^{\prime}+(1-y) \Delta P_{g r, j j}^{\prime \prime}+g \Delta H_{p, i, 1} \rho_{p, i}-g \Delta H_{p, j, 2} \rho_{p, j}
$$

where $y$ is a crossover parameter, $\Delta \mathrm{H}_{\mathrm{p}, \mathrm{i}, 1}=\max \left(\mathrm{H}_{\mathrm{p}, \mathrm{i}}-\mathrm{H}_{1}, 0\right)$ is the submergence, if any, below the pool surface of the flow path end at cell $i$, and $\rho_{\mathrm{p}, \mathrm{i}}$ is the pool coolant density in $i$. The notation for the pool and flow path elevations $\mathrm{H}_{\mathrm{p}, \mathrm{i}}$ and $\mathrm{H}_{1}$, respectively, are as shown in Figure 4-4. The crossover parameter $\mathrm{y}, \Delta \mathrm{P}_{\mathrm{gr}, \mathrm{j}}^{\prime}$, and $\Delta \mathrm{P}_{\mathrm{gr}, \mathrm{j}}^{\prime \prime}$ are defined in the order given in the following discussion.

The crossover parameter $y$ determining the relative weighting of the $\Delta \mathrm{P}_{\mathrm{grij}}^{\prime}$ and $\Delta \mathrm{P}_{\mathrm{gr}, \mathrm{j} j}^{\prime \prime}$ terms depends on the value of the typically small parameter $\varepsilon$, defined as $\varepsilon=g \rho_{a v} v H_{i}-H_{j} / P_{a v}$, where $g$ is the acceleration due to gravity, and $\mathrm{H}_{i}$ and $\mathrm{H}_{j}$ are the gas centers of elevation of the two cells $i$ and $j$ connected by the flow path, as shown in Figure $4-4$, and $P_{a v}=\left(P_{i}+P_{j}\right) / 2$ is the average gas pressure. The $\varepsilon$ parameter corresponds to the absolute value of the first-order relative change in density with height that would be present if the contents of $i$ and $j$ corresponded to a well-mixed isothermal metastable stratification between $\mathrm{H}_{\mathrm{i}}$ and $\mathrm{H}_{\mathrm{j}}$. The crossover also depends on a second parameter $\delta$, which is the actual cell relative gas density difference: $\delta=\left(\rho_{\mathrm{i}}-\rho_{\mathrm{j}}\right) / \rho_{\mathrm{av}}$ if $\mathrm{H}_{\mathrm{i}}<\mathrm{H}_{\mathrm{j}} ; \delta=\left(\rho_{\mathrm{j}}-\rho_{\mathrm{i}}\right) / \rho_{\mathrm{av}}$ if $\mathrm{H}_{\mathrm{j}}<\mathrm{H}_{\mathrm{i}} ; \delta=0$ otherwise. Note that $\delta<0$ corresponds to an unstable configuration, whereas $\delta>0$ could correspond to either unstable, metastable, or stable cases.

The crossover parameter $y$ is defined according to the relation between $\rho$ and $\varepsilon$ :

$$
\begin{aligned}
& \mathrm{y}=0 \quad \text { if } \delta \geq 2 \varepsilon \text { or } \delta<0 \text { (VDI) } \\
& =2(\varepsilon-\delta / 2) / \delta \text { if } 2 \varepsilon>\delta \geq \varepsilon \quad \text { (interpolated) } \\
& =1 \quad \text { if } \varepsilon>\delta \geq 0 \quad \text { (averaged) }
\end{aligned}
$$

where the three different hybrid solver regimes (VDI, interpolated, and averaged) are also indicated. Figure 4-7 illustrates these three different regimes for $\mathrm{H}_{\mathrm{j}}>\mathrm{H}_{\mathrm{i}}$ within the one-dimensional space relating the fractional cell densities $\rho_{i} /\left(\rho_{i}+\rho_{j}\right)$ and $\rho_{j} /\left(\rho_{i}+\rho_{j}\right)$, which are subject to the obvious constraint $\rho_{\mathrm{f}} /\left(\rho_{\mathrm{i}}+\rho_{\mathrm{j}}\right)+\rho_{\mathrm{j}} /\left(\rho_{\mathrm{i}}+\rho_{\mathrm{j}}\right)=1$. This figure also gives the inherent isothermal stability regimes of the cells. Note that in a well-mixed adiabatic metastable stratification, as opposed to an isothermal one, the first-order relative density difference would be $\varepsilon / \gamma$, where $\gamma$ is the specific heat ratio, instead of $\varepsilon$. In practice, metastable stratifications are neither exactly isothermal nor adiabatic and the density difference is likely to lie somewhere in between these two values. Note that the 


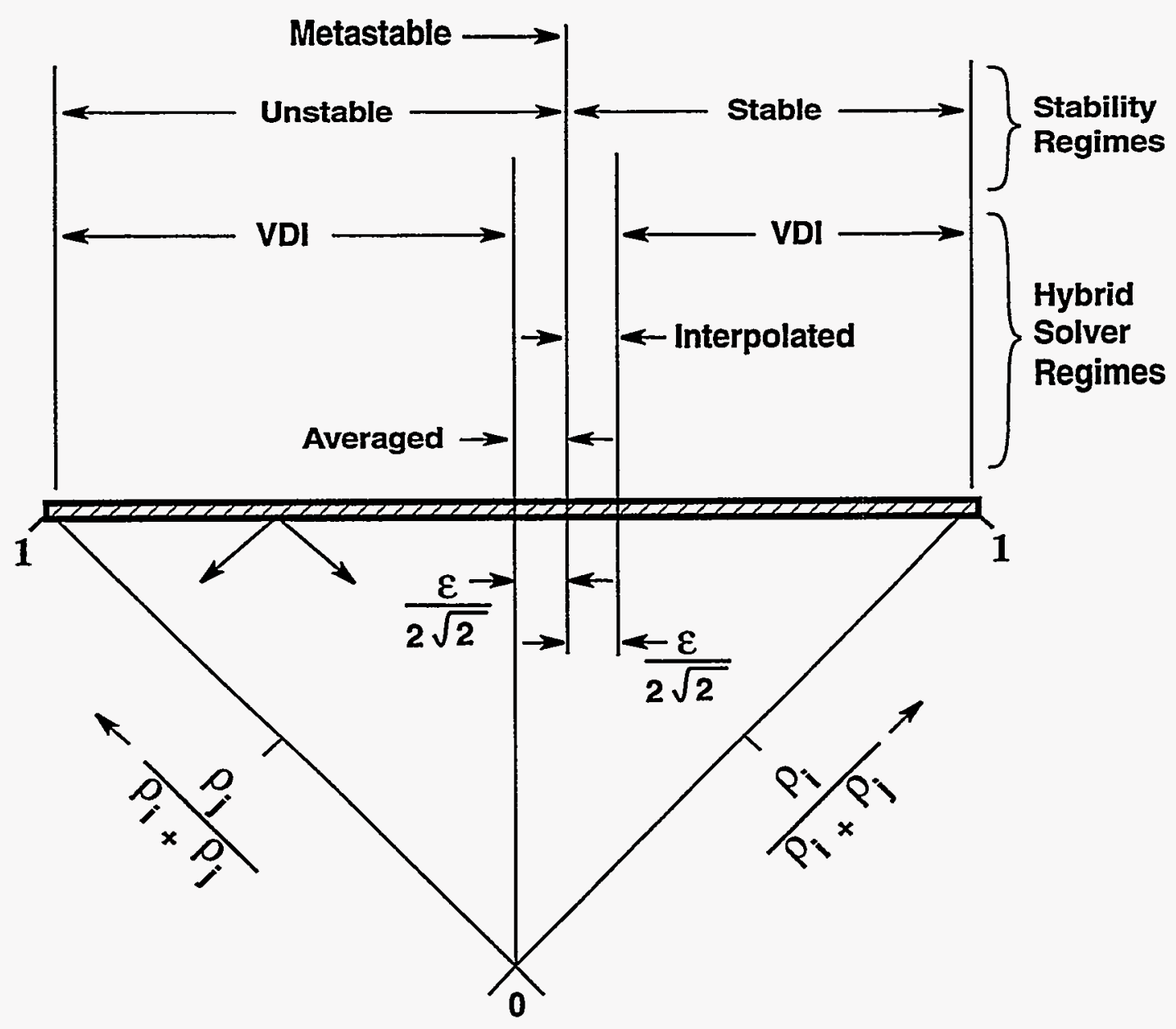

Figure 4-7. Tllustration of the Flow Solver Regimes for a Pair of Cells $i$ and $j$ Connected by a Gas Flow Path, with Elevation $\mathrm{H}_{\mathrm{j}}>\mathrm{H}_{\mathrm{i}}$, for the Case in Which Neither the RESOLVHD or MSTABLE Keywords Have Been Specified 
crossover parameter $y$ always selects the averaged expression $\Delta \mathrm{P}_{\mathrm{gr}, \mathrm{j}}^{\prime}$ (i.e., $y=1$ ) over the range of $\delta$ 's (i.e., $\varepsilon \geq \delta \geq \varepsilon / \gamma$ ) corresponding to the possible types of metastable stratifications.

The definition of the asymptotically correct head $\Delta \mathrm{P}_{\mathrm{gr}, \mathrm{ij}}^{\prime}$ is

$$
\Delta \mathrm{P}_{\mathrm{gr}, \mathrm{ij}}^{\prime}=\mathrm{g}\left(\mathrm{H}_{\mathrm{i}}^{\prime}-\mathrm{H}_{\mathrm{j}}^{\prime}\right) \rho_{\mathrm{av}}
$$

where $\mathrm{H}_{\mathrm{i}}^{\prime}=\mathrm{H}_{\mathrm{i}}-\Delta \mathrm{H}_{\mathrm{p}, \mathrm{i}, 1}$ is the gas center-of-volume elevation in $\mathrm{i}$, adjusted for submergence, if any, and $\mathrm{H}_{\mathrm{j}}^{\prime}=\mathrm{H}_{\mathrm{j}}-\Delta \mathrm{H}_{\mathrm{p}, \mathrm{j}, 2}$. This asymptotically correct head cannot be modified through user input.

The definition of the VDI head $\Delta \mathrm{P}_{\mathrm{gr}, \mathrm{j}}^{\prime \prime}$ used away from the well-mixed asymptotic limit is

$$
\Delta \mathrm{P}_{\mathrm{gri \textrm {j }}}^{\prime \prime}=\mathrm{g} \rho_{\mathrm{i}}\left(\mathrm{H}_{\mathrm{i}}^{\prime}-\mathrm{H}_{\mathrm{i}}^{\prime}\right)+\mathrm{g}\left[\mathrm{F}_{\mathrm{ij}} \rho_{\mathrm{i}}+\left(1-\mathrm{F}_{\mathrm{ij}}\right) \rho_{\mathrm{j}}\right]\left(\mathrm{H}_{1}^{\prime}-\mathrm{H}_{2}^{\prime}\right)+\mathrm{g} \rho_{\mathrm{j}}\left(\mathrm{H}_{2}^{\prime}-\mathrm{H}_{\mathrm{j}}^{\prime}\right)
$$

The definitions of the elevations $\mathrm{H}_{\mathrm{i}}^{\prime}, \mathrm{H}_{1}^{\prime}, \mathrm{H}_{\mathrm{j}}^{\prime}$, and $\mathrm{H}_{2}^{\prime}$ and the VDI parameter $\mathrm{F}_{\mathrm{ij}}$ depend on two user options, controlled by the MSTABLE and RESOLVHD keywords discussed below. The $F_{i j}$ parameter gives the location of the VDI in the flow path in terms of a fraction of the effective length of the path in the direction from $i$ to $j$. The default treatment is a dynamically calculated $F_{i j}$, as discussed below, in which the interface is assumed to span the entire elevation difference between the gas centers of volume, with adjustments for any submergence of the flow path ends. If MSTABLE is specified, then $F_{i j}$ is simply set to $1 / 2$, a value which corresponds to using the averaged density over the elevation span normally spanned by the VDI model. Specification of the RESOLVHD keyword invokes a treatment whereby the elevation difference normally spanned by the VDI is restricted to the elevation difference between the flow path ends, and the appropriate cell density is used within a cell to span the elevation change between the gas center of volume and the flow path end. In other words,

$$
\begin{aligned}
\mathrm{H}_{1}^{\prime} & =\mathrm{H}_{\mathrm{i}}^{\prime} \text { if the RESOLVHD keyword has not been specified for the path } \\
& =\mathrm{H}_{1} \text { if the RESOLVHD keyword has been specified for the path, and } \\
\mathrm{H}_{2}^{\prime} & =\mathrm{H}_{\mathrm{j}}^{\prime} \text { if the RESOLVHD keyword has not been specified for the path } \\
& =\mathrm{H}_{2} \text { if the RESOLVHD keyword has been specified for the path, }
\end{aligned}
$$

where $\mathrm{H}_{1}$ and $\mathrm{H}_{2}$ are the attachment elevations of the flow path ends to the cells $\mathrm{i}$ and $\mathrm{j}$, respectively, as shown in Figure 4-4. Note that RESOLVHD will have no effect if the flow paths are (coincidentally) attached at the cell center at each end.

The MSTABLE and RESOLVHD options have been made available to recover the old gravitational head modeling used in code versions prior to CONTAIN 1.2, and also to add flexibility to the present modeling. (Use of MSTABLE, and explicit specification of RESOLVHD and the appropriate elevations for all flow paths will allow the user to recover the "old," averaged-density gravitational head formulation recommended in prior versions, at least in cases in which the pool does not cover gas path inlets and significant displacement of gas centers of elevation by the pool does not occur.) 
In the VDI model, if MSTABLE is not specified, $F_{\mathrm{ij}}$ is determined dynamically, using an initial value $F_{i j}^{0}=1 / 2$. The motion is governed by the rate at which one considers the flow path to become filled with material from the upstream cell:

$$
\frac{d F_{i j}}{d t}=\frac{\kappa W_{i j}}{\rho_{u} A L_{i j}^{\prime}}
$$

where $\mathrm{K}$ is a dimensionless acceleration factor defined below, $\mathrm{W}_{\mathrm{ij}}$ is the mass flow rate in the path, as determined from the flow equation, $\rho_{\mathrm{u}}$ is the upstream, or donor, gas density, $\mathrm{L}_{\mathrm{ij}}^{\prime}=\max \left(\mid \mathrm{H}_{\mathrm{i}}^{\prime}-\right.$ $\mathrm{H}_{\mathrm{j}}^{\prime} \mid, \mathrm{L}_{\mathrm{ij}}$ ) is the effective filling length of the flow path, and $\mathrm{L}_{\mathrm{ij}}$ is the inertial length. Equation (4-11) is coupled to the momentum equation through Equation (4-10) above and is solved with the constraint $0 \leq F_{i j} \leq 1$. Note the value $K=1$ when $L^{\prime}=L$ would correspond to physically filling an area equal to the actual flow path area $\mathrm{A}_{\mathrm{ij}}$ over the inertial length $\mathrm{L}$, and $\kappa=\infty$ corresponds to an instantaneous flow path filling, or donor, assumption. In practice, for reasons discussed below, large values of $\kappa$ are used to accelerate the filling. Much of the time the VDI method gives essentially the same results as a straightforward donor cell approach, which corresponds to setting $F_{i j}=1$ if $W_{i j}>0$ and $F_{i j}=0$ if $W_{i j}<0$, in Equation (4-10). This occurs whenever flow in one direction has persisted for a sufficient time to cause $F_{i j}$ to be pinned at its maximum or minimum value. The time required for pinning to occur is clearly reduced as $k$ is increased.

As discussed above, if MSTABLE is specified, $F_{i j}$ is simply set equal to $1 / 2$. This value for $F_{i j}$ corresponds to treating stratifications as metastable over the vertical rise spanned by the flow path, since $F_{i j}$ cannot respond to flow to provide a restoring force for a stable stratification or a destabilizing force for an unstable stratification.

It should be noted that in the derivation of CONTAIN momentum equation in Table 4-2, the changeover in density within the flow path to that based on the donor cell is assumed to occur instantaneously. Thus, Equation (4-11) is not strictly consistent with the assumptions of the momentum equation unless $\kappa$ is effectively infinite. In practice, the value used for $\kappa$ is chosen sufficiently large $(210)$ to give results close to donor cell results but not so large that the discontinuities associated with the donor cell approach result in numerical problems. The value used is

$$
\kappa=\max \left[10, \frac{L_{i j}^{\prime}}{g\left(\Delta t_{f}\right)^{2}}\right]
$$

where $\Delta \mathrm{t}_{\mathrm{f}}$ is the flow timestep.

If $F_{\mathrm{ij}}$ were in fact solved using values of $\kappa$ close to the "physical" value, in contradiction to the momentum equation assumption, gravity wave behavior would in general be observed in the presence of stable stratifications. If one views the density difference between cells as representing a sharp stratification interface, then these gravity waves would cause unphysical mixing across the 
interface, since in CONTAIN material entering a flow path is instantaneously transported to and mixed with the downstream cell. (This unphysical mixing could be prevented through a code upgrade in which flow paths are modified to be repositories, but this upgrade is not feasible at present.)

For the above reason, the present numerical implementation of the VDI attempts to ensure that any gravity waves are artificially and strongly damped. First of all, self-consistent end-of-timestep (implicit) values of $F_{i j}$ are calculated and used in Equation (4-10) whenever the response of $F_{i j}$ is in a direction to retard the flow; beginning-of-timestep values are used otherwise. The use of such implicit values, coupled with a large $\kappa$, serve to overdamp the gravity waves. (In the limit $\kappa=\infty$, the flow must be damped to zero for stable stratifications and small perturbing forces since a "flow gap" in which no flow is possible occurs in the self-consistent donor method in such cases.)

\subsubsection{Pool Flow Path Gravitational Heads}

The gravitational head for a pool path is defined as

$$
\Delta P_{p, g r, i j}=g\left[\Delta H_{p, i, 1}^{\prime} \rho_{p, 1}+\rho_{p, a v}\left(H_{1}-H_{2}\right)-\Delta H_{p, j, 2}^{\prime} \rho_{p, 2}\right]
$$

where the pool coolant densities $\rho_{\mathrm{p}}$ are given by

$$
\begin{aligned}
\rho_{p, 1} & =\rho_{p, i} \text { and } \rho_{p, 2}=\rho_{p, j} \text { if a pool is defined in both } i \text { and } j, \\
& =\rho_{p, 2}=\rho_{p, i} \text { if a pool is defined only in } i \\
& =\rho_{p, 2}=\rho_{p, j} \text { if a pool is defined only in } j
\end{aligned}
$$

and

$$
\rho_{p, a v}=\left(\rho_{p, 1}+\rho_{p, 2}\right) / 2
$$

In contrast to a gas path, the submergence, such as $\Delta \mathrm{H}_{\mathrm{p}, \mathrm{i}, 1}^{\prime}$, used with a pool path is sometimes negative. The submergence of a side-connected $\left(\mathrm{H}_{1}<\mathrm{H}_{t, i}\right)$ pool flow path at $i$ is given as usual by

$$
\Delta \mathrm{H}_{\mathrm{p}, \mathrm{i}, 1}^{\prime}=\max \left(\mathrm{H}_{\mathrm{p}, \mathrm{i}}-\mathrm{H}_{1}, 0\right)
$$

whereas the submergence of a top-connected path $\left(\mathrm{H}_{1}=\mathrm{H}_{\mathrm{t}, \mathrm{i}}\right)$ is given by

$$
\begin{aligned}
& \Delta \mathrm{H}_{\mathrm{p}, \mathrm{i}, \mathrm{1}}^{\prime}=\mathrm{H}_{\mathrm{p}, \mathrm{i}}-\mathrm{H}_{1} \leq 0 \text { if } \mathrm{i} \text { is the donor cell } \\
& =0 \quad \text { otherwise }
\end{aligned}
$$

Similar expressions are used for $\Delta \mathrm{H}_{\mathrm{p}, 2}^{\prime}$. It should be noted that in the absence of extremely tall cells with a pool, a geometry which is not recommended, gas density contributions to pool flow path gravitational heads are negligible relative to the pool heads and are justifiably neglected. 
The reader should note that the gravitational head changes discontinuously as a function of flow path elevation at the top of the cell when the cell contains gas (so that $\mathrm{H}_{\mathrm{i}} \neq \mathrm{H}_{\mathrm{t}, \mathrm{i}}$ ). As discussed in Section 4.2 , this discontinuity reflects differences in the pool-gas flow hierarchy between the top-connected and side- or bottom-connected cases. The pool and gas flows in the former case are treated as independent of the pool level in the donor cell, except indirectly through the pressure head, whereas the flows in the latter adhere to a hierarchy controlled by the collapsed pool level.

\subsubsection{Pool Boiling}

Pool boiling is calculated either explicitly within the lower cell model or implicitly within the implicit flow solver. The former occurs when the implicit flow solver is not used or when the lower cell model determines that the remaining pool coolant will boil off in the current system timestep. The reason for doing an explicit boiling rate calculation in the lower cell model when the pool boils off is that the lower cell model can then adjust other fluxes at the atmosphere-lower cell interface to compensate for the fact that the pool is present for only part of the timestep. In this case the explicit pool mass boiling rate, $\mathrm{W}_{\mathrm{LC}, \text { boil }}$ and energy transfer rate, $\mathrm{q}_{\mathrm{Lc}, \text { boil }}$, are included in the explicit mass and heat transfer terms of the atmosphere conservation equations shown in Tables 4-3 and 4-4, respectively. Implicit boiling can occur whenever the implicit solver is used, and is based on mass and heat transfer rates to the pool known to the implicit solver at the time of processing. In the case of implicit boiling, the mass and heat transfer contributions to the pool conservation equations shown in Tables 4-5 and 4-6, respectively, are evaluated within the implicit flow solver to determine a boiling mass and heat transfer rate $\mathrm{W}_{\text {boil }}$ and $\mathrm{q}_{\text {boil }}$ to the atmosphere. Note that the explicit and implicit pool boiling rates may both be non-zero but this requires that conditions unknown to the lower cell occur in the flow step, such as flow of superheated coolant into an initially empty pool. Note that in either case, the BOIL keyword must be specified in the PHYSICS sub-block of the POOL input block for boiling to occur.

The expression for the amount of mass boiled is the same regardless of where the boiling rate is calculated. The rate calculated by the implicit solver will be discussed in detail. When the implicit solver is used, interpool flow is evaluated first, then the boiling and gas equilibration (see Section 4.4.7). The interpool flow step takes into account the flow contributions and all explicit sources except that due to boiling and to gas equilibration. The implicit pool boiling rate $\mathrm{W}_{\text {boil, }}$ follows from the standard expression for boiling rates and is defined as

$$
\begin{aligned}
& \mathrm{W}_{\text {boili, }}=\max \left[\frac{\Delta \mathrm{Q}_{s}}{\left(\mathrm{~h}_{\mathrm{v}}\left(\mathrm{T}_{\mathrm{s}, \mathrm{i}}, \mathrm{P}_{\mathrm{i}}\right)-\mathrm{h}_{\mathrm{f}}\left(\mathrm{T}_{\mathrm{s}, \mathrm{i}} \mathrm{P}_{\mathrm{i}}\right)\right) \Delta \mathrm{t}_{\mathrm{f}}}, 0\right] \\
& \mathrm{q}_{\text {boil, } \mathrm{i}}=\mathrm{W}_{\text {boili, }} \mathrm{h}_{\mathrm{v}}\left(\mathrm{T}_{\mathrm{s}, \mathrm{i}}, \mathrm{P}_{\mathrm{i}}\right)
\end{aligned}
$$


Table 4-3

Conservation of Mass Equation for the Atmosphere in Cell $\mathrm{i}$

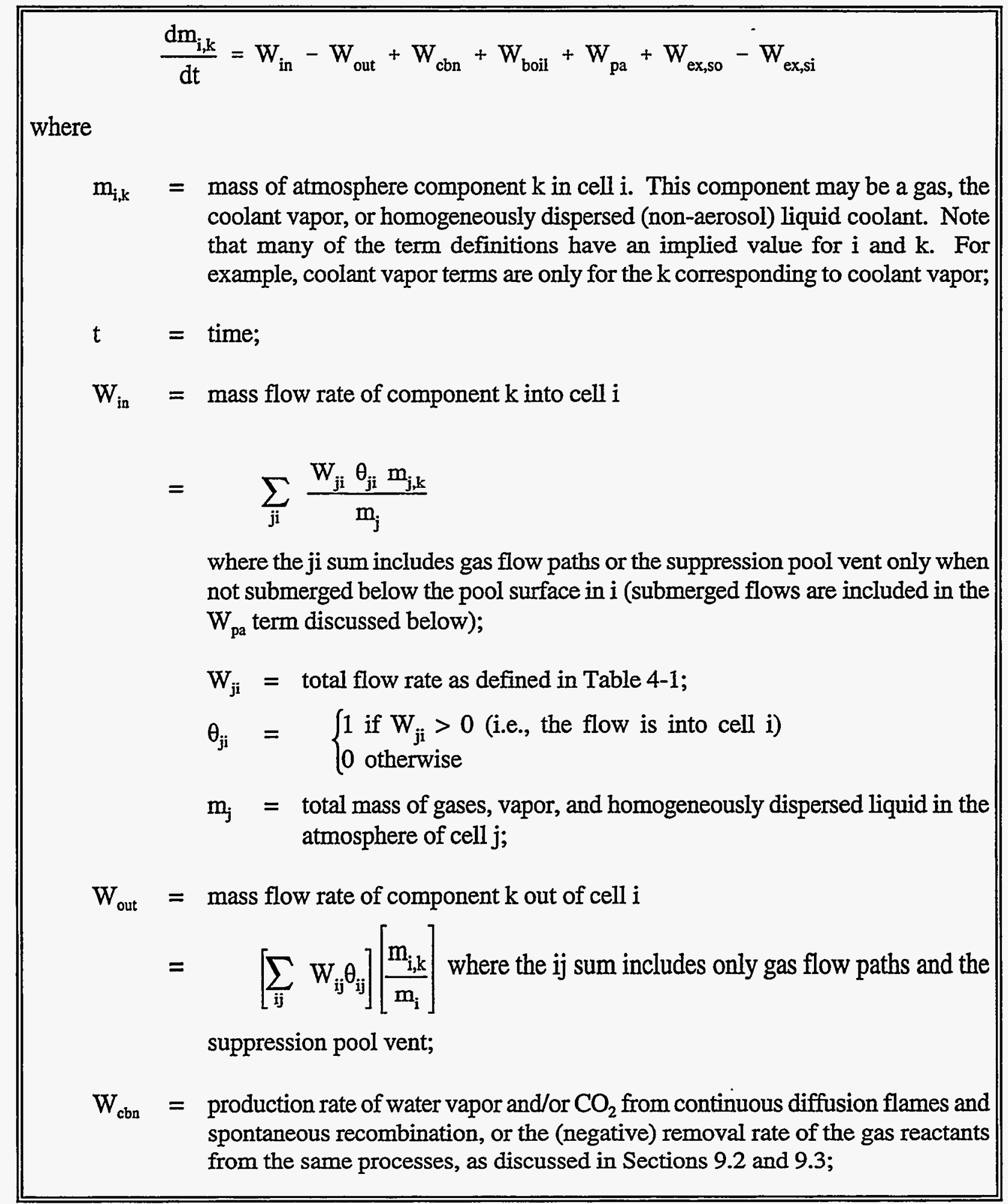


Table 4-3

Conservation of Mass Equation for the Atmosphere in Cell i (Continued)

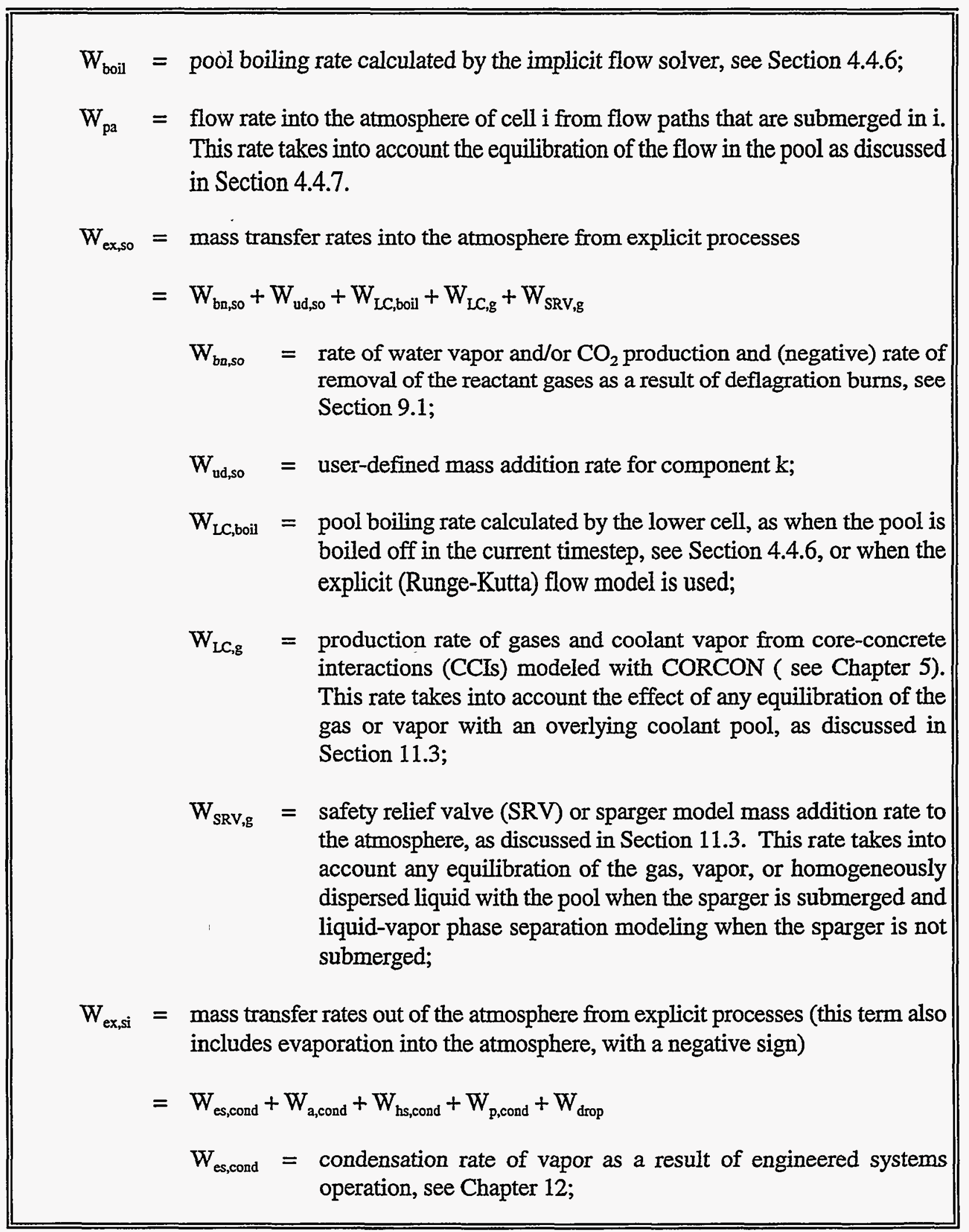


Table 4-3

Conservation of Mass Equation for the Atmosphere in Cell i (Concluded)

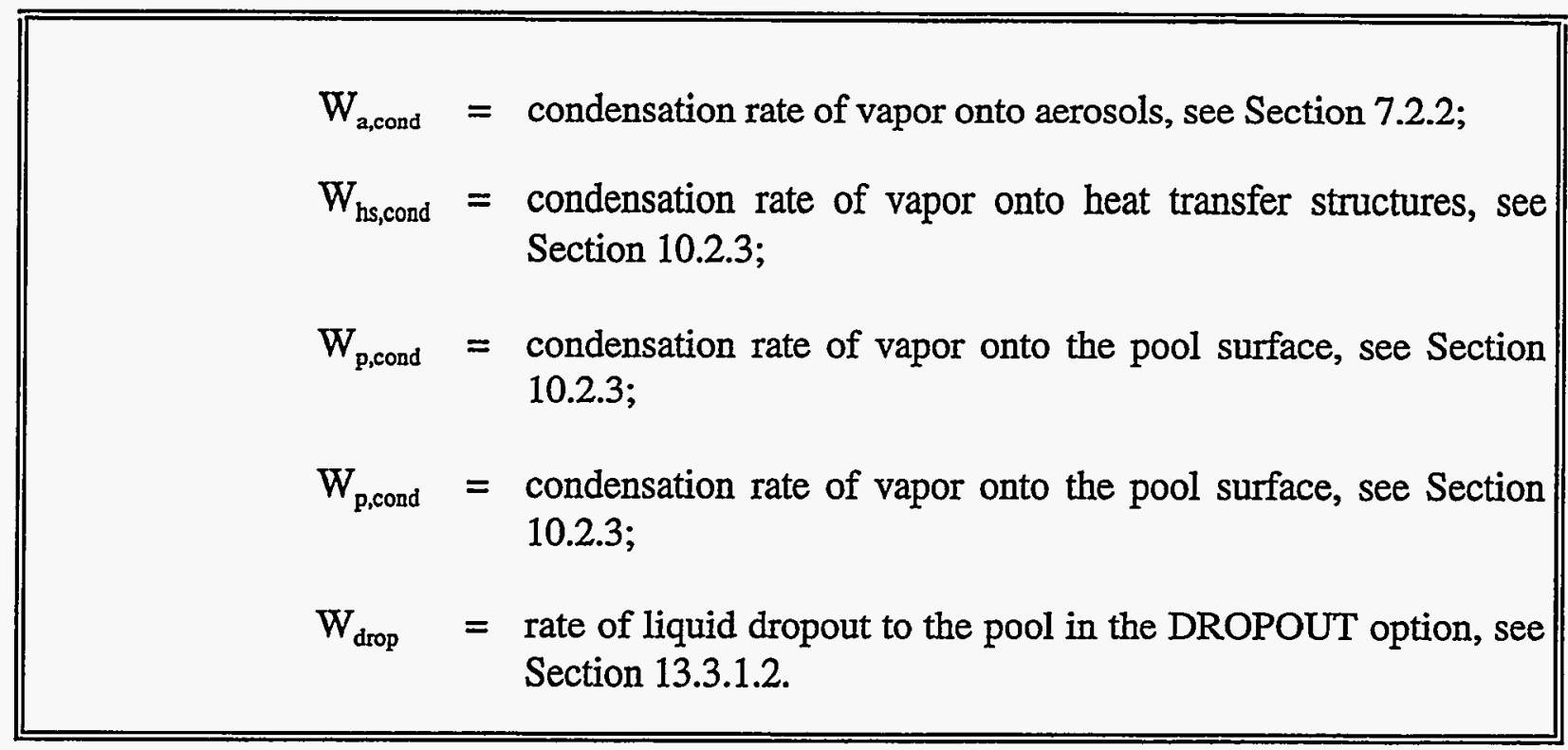

Table 4-4

Conservation of Energy Equation for the Atmosphere in Cell i

$$
\frac{d U_{i}}{d t}=q_{i n}-q_{\text {out }}+q_{c b n}+q_{\text {boil }}+q_{p a}+q_{g r}+q_{e x, s o}-q_{\text {ex }, \text { si }}+q_{g r, e x}+q_{w k}
$$

where

$$
\begin{aligned}
\frac{\mathrm{dU}_{\mathrm{i}}}{\mathrm{dt}}= & \begin{array}{l}
\text { rate of change of the total internal energy in atmosphere of cell } \mathrm{i} \text {, including gases, } \\
\text { the coolant vapor, and any homogeneously dispersed liquid coolant; }
\end{array} \\
& =\text { energy transfer rate into cell } \mathrm{i} \text { because of flow } \\
& =\sum_{\mathrm{ji}} \mathrm{W}_{\mathrm{ji}} \theta_{\mathrm{ji}} \mathrm{h}_{\mathrm{j}}
\end{aligned}
$$

where the ji sum includes gas flow paths and the suppression vent only when not submerged below the pool surface in $i$ (submerged flows are included in the $\mathrm{q}_{\mathrm{pa}}$ term discussed below);

$$
\mathrm{W}_{\mathrm{ji}} \quad=\text { total flow rate, as defined in Table 4-2; }
$$


Table 4-4

Conservation of Energy Equation for the Atmosphere in Cell i (Continued)

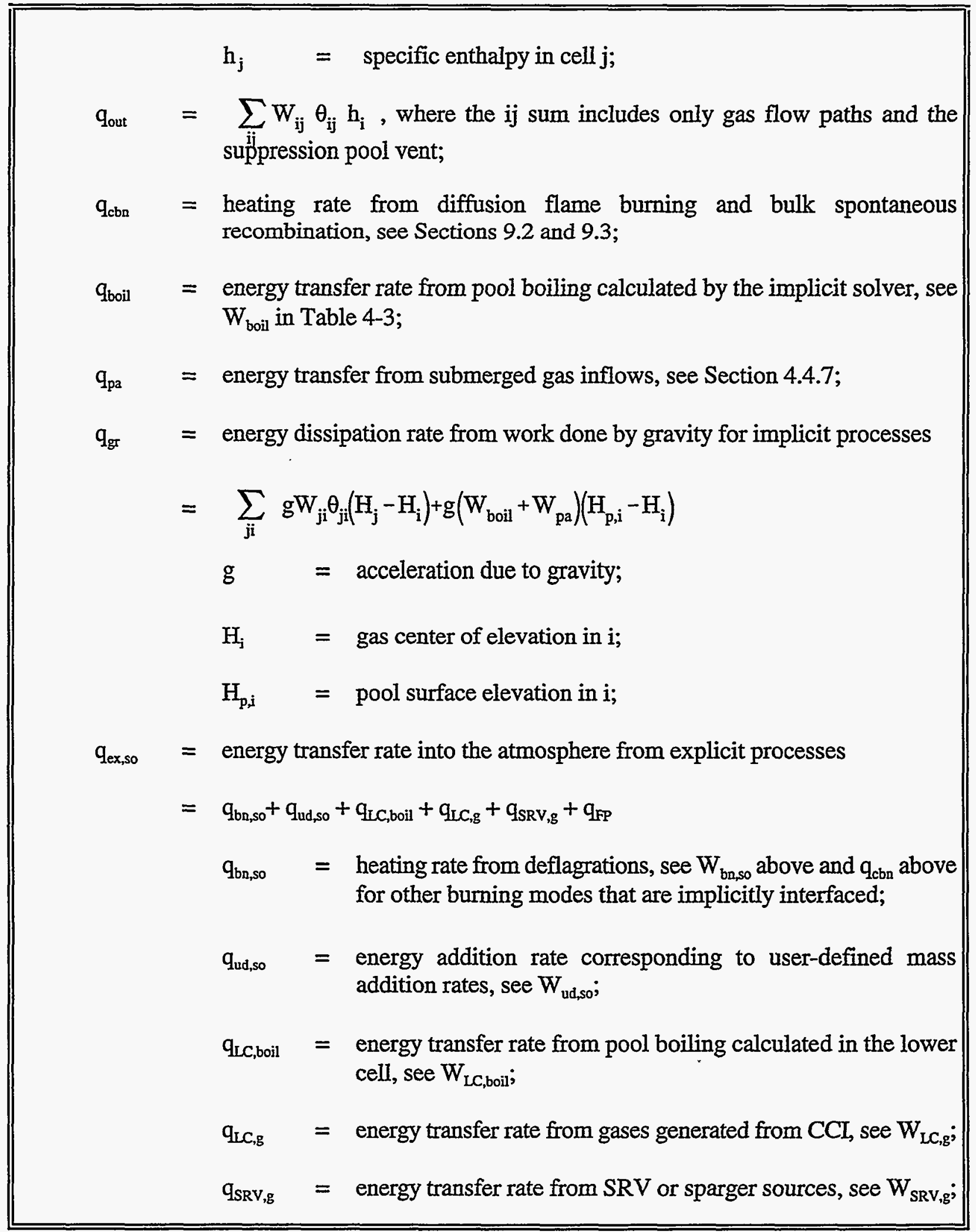


Table 4-4

Conservation of Energy Equation for the Atmosphere in Cell $i$ (Continued)

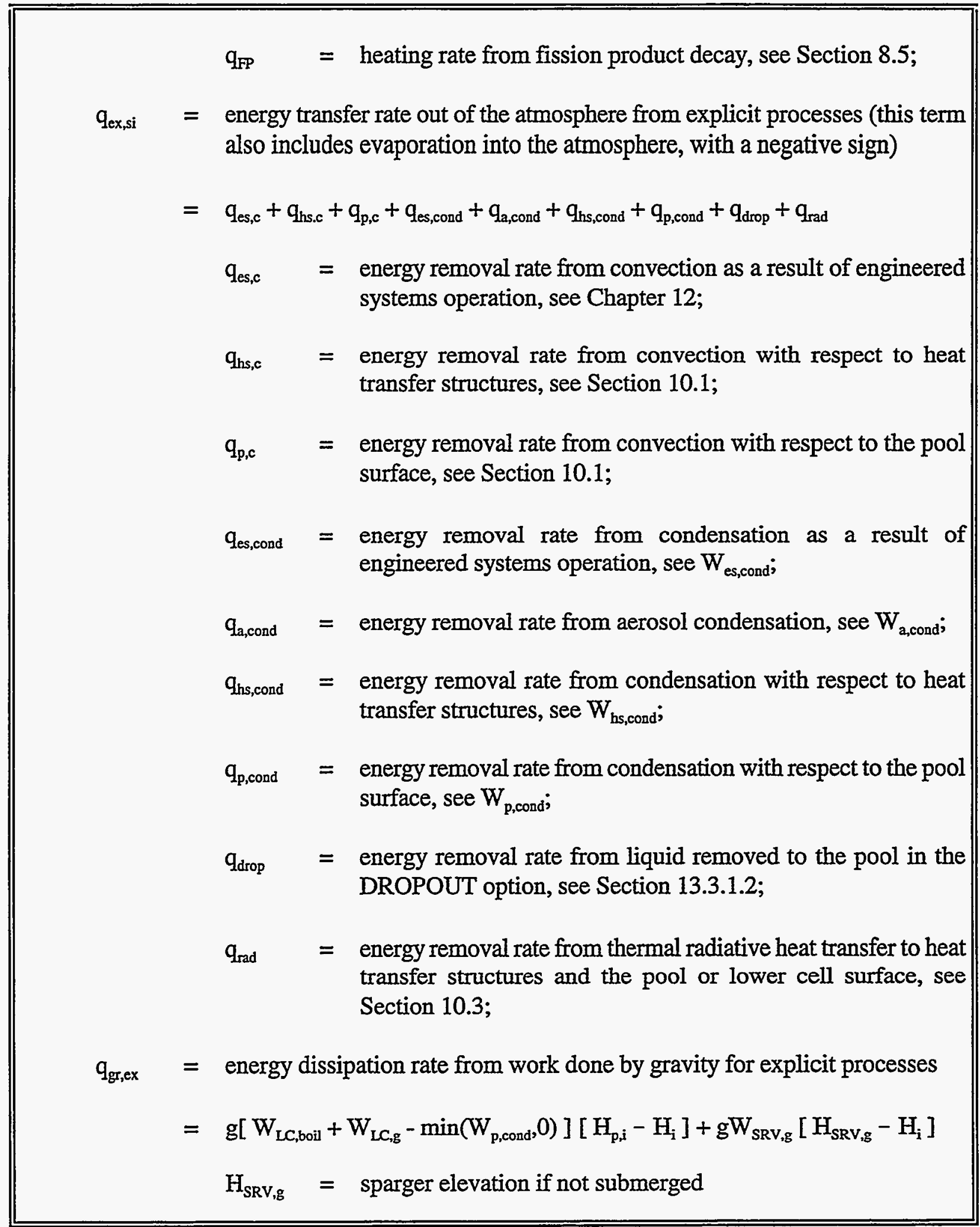


Table 4-4

Conservation of Energy Equation for the Atmosphere in Cell i (Concluded)

$$
=\mathrm{H}_{\mathrm{p}, \mathrm{i}} \text { if submerged; }
$$

$\mathrm{q}_{\mathrm{wk}} \quad=\quad$ rate of work done by the atmosphere on the pool

$=-\mathrm{P} \frac{\mathrm{dV} g}{\mathrm{dt}}$, where $\mathrm{V}_{\mathrm{g}}$ is the gas volume.

Table 4-5

Conservation of Mass Equation for the Coolant Pool in Cell i

$$
\frac{\mathrm{dm}_{\mathrm{p}, \mathrm{i}}}{\mathrm{dt}}=\mathrm{W}_{\mathrm{p}, \mathrm{in}}-\mathrm{W}_{\mathrm{p}, \text { out }}-\mathrm{W}_{\mathrm{boil}}+\mathrm{W}_{\mathrm{eq}}+\mathrm{W}_{\mathrm{p}, \mathrm{ex}, \mathrm{so}}-\mathrm{W}_{\mathrm{p}, \mathrm{ex}, \mathrm{si}}
$$

where

$$
\begin{aligned}
& \mathrm{m}_{\mathrm{p}, \mathrm{i}} \quad=\text { the pool coolant mass in cell } \mathrm{i} \text {; } \\
& \begin{aligned}
\mathrm{W}_{\mathrm{p}, \mathrm{in}}= & \text { the coolant inflow rate, defined by } \mathrm{W}_{\mathrm{p}, \mathrm{in}}=\sum_{\mathrm{ji}} \mathrm{W}_{\mathrm{ji}} \theta_{\mathrm{ji}} \text {, where the } \mathrm{ji} \text { sum } \\
& \text { includes pool flow paths only; }
\end{aligned} \\
& \mathrm{W}_{\mathrm{p}, \text { out }}=\text { the coolant outflow rate, } \mathrm{W}_{\mathrm{p}, \text { out }}=\sum_{\mathrm{ij}} \mathrm{W}_{\mathrm{ij}} \theta_{\mathrm{ij}} \text {, where the } \mathrm{ij} \text { sum includes } \\
& \text { pool flow paths only; } \\
& \mathrm{W}_{\text {boil }}=\text { pool boiling rate calculated by the implicit solver, as discussed in Table 4-3; } \\
& \mathrm{W}_{\mathrm{eq}}=\text { mass transfer rate into the pool as a result of condensation of vapor, plus the } \\
& \mathrm{W}_{\mathrm{p}, \mathrm{ex}, \mathrm{so}}=\text { mass transfer rate into the pool from explicit processes } \\
& =\mathrm{W}_{\mathrm{es}, \text { out }}+\mathrm{W}_{\mathrm{a}, \mathrm{dep}}+\mathrm{W}_{\mathrm{hs}, \text { out }}+\mathrm{W}_{\mathrm{p}, \text { cond }}+\mathrm{W}_{\mathrm{drop}}+\mathrm{W}_{\mathrm{p}, \mathrm{ud}, \mathrm{so}}+\mathrm{W}_{\mathrm{LC}, \mathrm{eq}}+\mathrm{W}_{\mathrm{SRV}, \text { eq }}+\mathrm{W}_{\mathrm{SRV}, \text { out }} \\
& \mathrm{W}_{\mathrm{es}, \text { out }}=\text { inflow rate of effluent routed to the pool as a result of } \\
& \text { engineered systems operation, or transferred to the pool } \\
& \text { through a liquid transport model, see Chapter 12; }
\end{aligned}
$$


Table 4-5

Conservation of Mass Equation for the Coolant Pool in Cell i (Concluded)

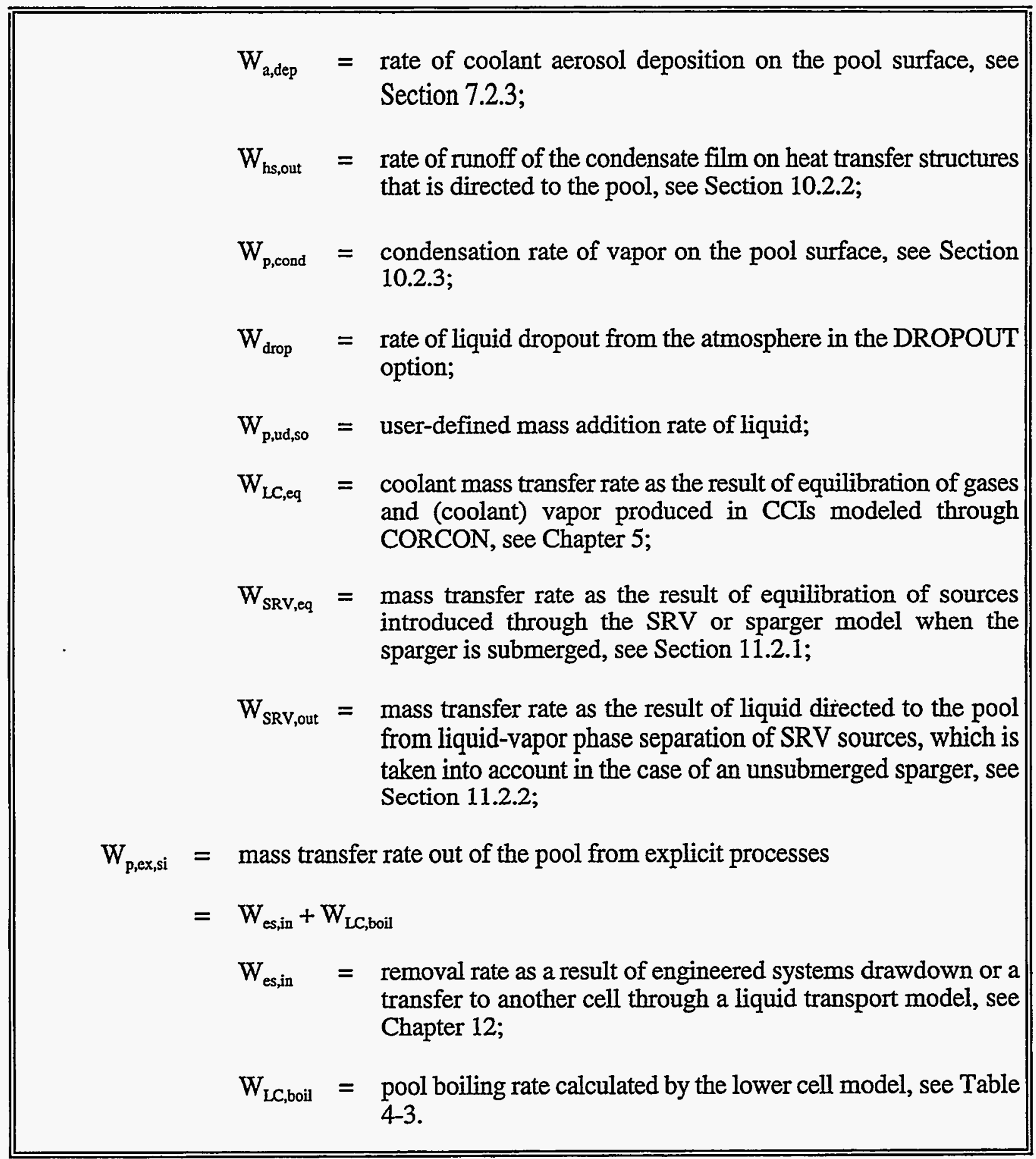


Conservation of Energy Equation for the Coolant Pool in Cell $\mathbf{i}$

$$
\frac{d U_{p, i}}{d t}=q_{p, \text { in }}-q_{p, \text { out }}-q_{b o i l}+q_{e q}+q_{p, g x}+q_{p, e x, s o}-q_{p, e x, s i}+q_{p, g r, e x}-q_{w k}
$$

where

$$
\begin{aligned}
& \mathrm{U}_{\mathrm{p}, \mathrm{i}} \quad=\text { the pool internal energy in cell } \mathrm{i} \text {; } \\
& \mathrm{q}_{\mathrm{p}, \mathrm{in}}=\begin{array}{l}
\text { the energy inflow rate, defined by } \mathrm{q}_{\mathrm{p}, \mathrm{in}}=\sum_{\mathrm{ji}} \mathrm{W}_{\mathrm{ji}} \theta_{\mathrm{ji}} \mathrm{h}_{\mathrm{p}, \mathrm{j}} \text {, where the ji sum } \\
\text { includes pool flow paths only and }
\end{array} \\
& \mathrm{h}_{\mathrm{p}, \mathrm{j}} \quad=\text { pool specific enthalpy in cell } \mathrm{j} \text {; } \\
& \begin{aligned}
\mathrm{q}_{\mathrm{p}, \text { out }}= & \begin{array}{l}
\text { the energy outflow rate, } \\
\text { pool flow paths only; }
\end{array}
\end{aligned} \\
& \mathrm{q}_{\text {boil }}=\text { energy transfer from pool boiling calculated by the implicit solver, see } \mathrm{W}_{\text {boil }} \\
& \text { in Table 4-3; } \\
& \mathrm{q}_{\mathrm{eq}}=\text { energy transfer rate into the pool as a result of the equilibration of gas flows } \\
& \text { into the pool from flow paths submerged under the pool surface, see Section } \\
& \text { 4.4.7; } \\
& \mathrm{q}_{\mathrm{p}, \mathrm{gr}}=\text { energy dissipation rate in the pool from work done by gravity for implicit } \\
& \text { processes; } \\
& =\sum_{\mathrm{ji}} \mathrm{gW}_{\mathrm{ji}} \theta_{\mathrm{ji}}\left(\mathrm{H}_{\mathrm{p}, \mathrm{j}}-\mathrm{H}_{\mathrm{p}, \mathrm{i}}\right) \text {, where the } \mathrm{ji} \text { sum extends over pool flow paths } \\
& \text { only; } \\
& \mathrm{q}_{\mathrm{p}, \mathrm{ex}, \mathrm{so}}=\text { energy transfer rate into the pool from explicit processes } \\
& \begin{aligned}
= & q_{\text {es,out }}+q_{a, \text { dep }}+q_{\text {hs,out }}+q_{p, \text { cond }}+q_{p, c}+q_{d r o p}+q_{p, h s}+q_{p, u d, s o}+q_{I C, e q}+q_{S R V, e q}+ \\
& q_{S R V, \text { out }}
\end{aligned} \\
& q_{\text {es,out }}=\text { energy transfer rate from effluent routed to the pool as a result } \\
& \text { through a liquid transport model, see Chapter 12; } \\
& \mathrm{q}_{\mathrm{a} \text {,dep }}=\text { energy transfer rate from coolant aerosols deposited on the } \\
& \text { pool surface, see Section 7.2.3; }
\end{aligned}
$$


Table 4-6

Conservation of Energy Equation for the Coolant Pool in Cell i (Continued)

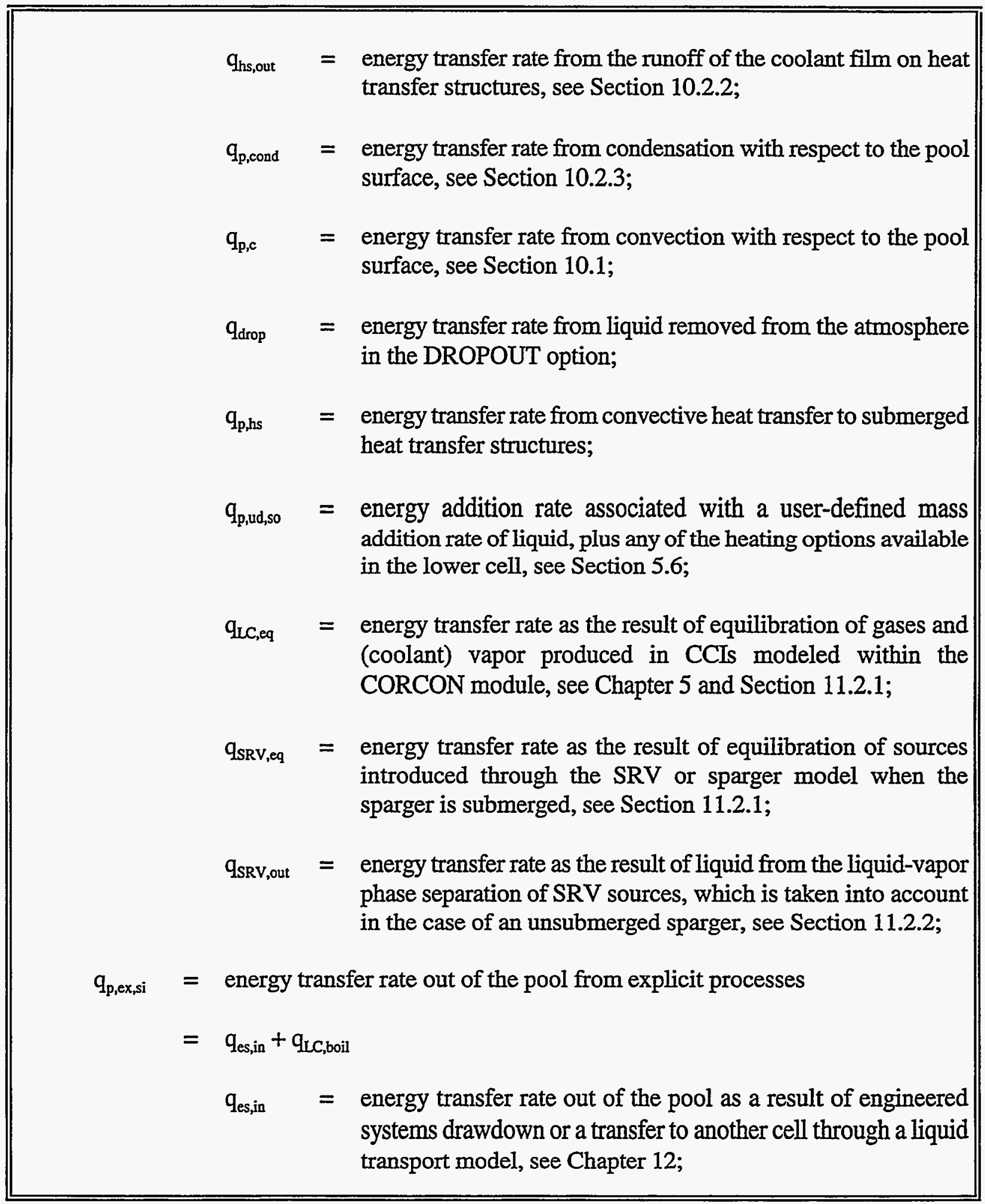


Table 4-6

Conservation of Energy Equation for the Coolant Pool in Cell i (Concluded)

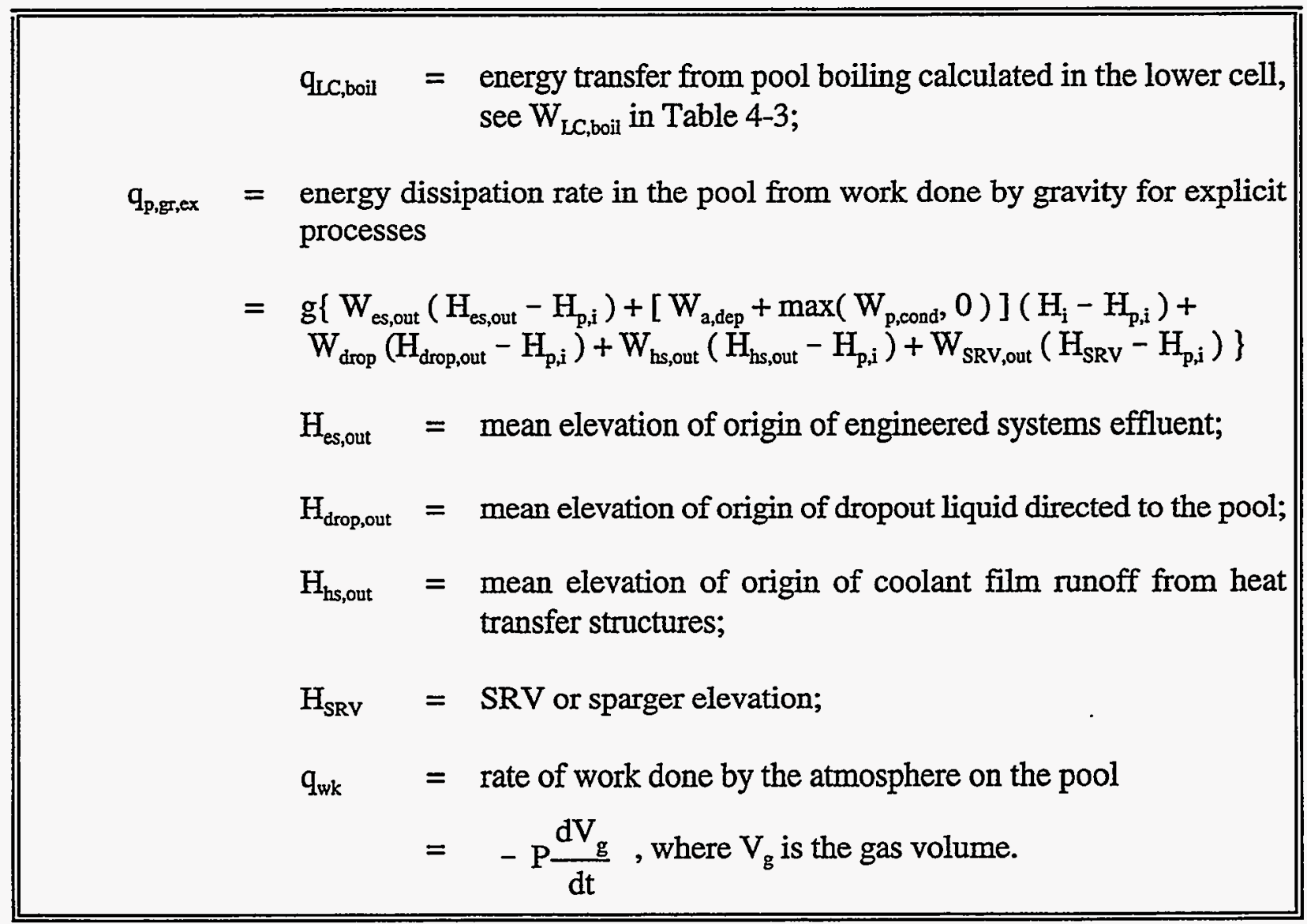

where

$$
\Delta \mathrm{Q}_{\mathrm{s}}=\mathrm{U}_{\mathrm{p}, \mathrm{i}}-\mathrm{m}_{\mathrm{p}, \mathrm{i}} \mathrm{u}_{\mathrm{f}}\left(\mathrm{T}_{\mathrm{s}, \mathrm{i}}, \mathrm{P}_{\mathrm{i}}\right)
$$

and $\Delta t_{f}$ is the flow timestep, $h_{v}$ is the coolant vapor specific enthalpy, $h_{\ell}$ is the liquid coolant specific enthalpy, $T_{s, i}$ is the pool saturation temperature, $P_{j}$ is the cell gas pressure, $m_{p, i}$ is the pool mass after flow step, but before gas equilibration, $U_{p, i}$ is the pool internal energy after the interpool flow step, but before gas equilibration, and $\mathrm{u}_{\ell}$ is the liquid coolant specific energy.

\subsubsection{Gas-Pool Equilibration}

This section discusses the modeling of gas-pool equilibration that occurs with respect to gas flow, when the flow is injected into a cell from a flow path that is submerged below the collapsed pool level in that cell. Gas flowing into a pool from a submerged gas flow path or the dedicated suppression vent in forward flow is in general equilibrated as discussed below. However, note that gas equilibration is ignored for the suppression vent in reverse flow, for reasons related to model 
architecture. A gas-pool equilibration model similar to the one presented here is used for the SRV and CORCON models, as discussed in Section 11.2.1.

With regard to gas equilibration, the gas inflow to the pool from a submerged gas flow path is assumed to equilibrate with respect to both temperature and vapor pressure over a gas equilibration length "veqlnf" or "veqlnb" for the front or back end of the path, respectively. These lengths may be specified by the user. By default, such lengths are set to $0.01 \mathrm{~m}$ for gas flow paths. For the suppression pool vent model in forward flow, the same treatment is used but the equilibration length is set to zero. Note that the behavior of the equilibrated vapor outflow from the pool depends on whether the BOIL keyword has been specified for the pool. If BOIL has not been specified, then the vapor outflow mass is taken to be zero.

The gas equilibration calculation for cell $i$ is based on the gas equilibration lengths discussed above, a self-consistent pool temperature $T_{\mathrm{p}, \mathrm{i}}^{\prime}$, and the gas inflow rates for paths submerged under the pool surface in cell $\mathrm{i}$. The self-consistent pool temperature, which is implicitly defined by the equations below, is based on the end-of-timestep conditions for the pool, and therefore includes the effects of (1) explicit sources, (2) liquid inflow and outflow through flow paths, (3) boiling (see Section 4.4.6), as well as (4) the equilibration of the gas injected into the cell below the pool surface. Gas equilibration is the last step calculated in the implicit solver, after the effects of (1) through (3) have already been taken into account. For simplicity, in the discussion below, only the effects of gas equilibration are explicitly displayed. The other effects listed above can be considered to have modified the starting conditions of the pool prior to incorporating the effects of gas equilibration.

In the case of a gas flow path or the dedicated suppression vent model, the gas inflow into cell $i$ is characterized by the gas inflow rate $\mathrm{W}_{\mathrm{ji}}=\mathrm{W}_{\mathrm{ncj \textrm {ji }}}+\mathrm{W}_{\mathrm{v}, \mathrm{ji}}$ for path $\mathrm{ji}$, where $\mathrm{W}_{\mathrm{nc}, \mathrm{ji}}$ is the noncondensable gas flow rate and $\mathrm{W}_{\mathrm{v}, \mathrm{j}}$ is taken here to be the total coolant flow rate. The latter includes the coolant vapor and homogeneously dispersed coolant liquid carried with the vapor, if present. In the discussion below, only positive inflow rates $\mathrm{W}_{\mathrm{ji}}$ corresponding to gas flow paths venting below the pool surface into cell $i$ will be considered. (As noted in prior sections, the flow is indexed by ji, even though more than one path may be present between cells $j$ and $i$.)

The energy transfer rate to the pool through a given path $\mathrm{ji}$, corresponding to the submerged gas inflow $\mathrm{W}_{\mathrm{j} i}$, is given by

$$
q_{a p, j i}=W_{j i} h_{j}
$$

where $h_{j}$ is the gas specific enthalpy in cell $j$.

The mass transfer rate from the pool surface to the atmosphere $\mathrm{W}_{\mathrm{pa}, \mathrm{i}}$ from gas outflow is broken into four parts: $W_{p a, v s,}$, the vapor contribution required to bring the pool down to saturation, if necessary, in case the inflowing gases are sufficiently hot to drive the pool above saturation; $\mathrm{W}_{\mathrm{pa}, \mathrm{ve}, \mathrm{i}}$, the vapor 
outflow associated with the equilibrated noncondensable gas; $\mathrm{W}_{\mathrm{pa}, \mathrm{vn}, \mathrm{i}}$, the vapor outflow associated with the nonequilibrated gas; and $\mathrm{W}_{\mathrm{pa}, \mathrm{nc}, \mathrm{i}}=\sum_{\mathrm{ji}} \theta_{\mathrm{ji}} \mathrm{W}_{\mathrm{nc}, \mathrm{ji}}$ the total noncondensable inflow rate.

The pool saturation flow rate $\mathrm{W}_{\mathrm{pars}, \mathrm{i}}$ follows from the standard expression for boiling rates and is defined as

$$
\begin{aligned}
\mathrm{W}_{\mathrm{pa}, v s, \mathrm{i}} & =\max \left[\frac{\Delta \mathrm{Q}_{s}}{\left(\mathrm{~h}_{\mathrm{v}}\left(\mathrm{T}_{\mathrm{s}, \mathrm{i}}, \mathrm{P}_{\mathrm{i}}\right)-\mathrm{h}_{\ell}\left(\mathrm{T}_{\mathrm{s}, \mathrm{i}}, \mathrm{P}_{\mathrm{i}}\right)\right) \Delta \mathrm{t}_{\mathrm{f}}}, 0\right] \\
=0 & \text { otherwise }
\end{aligned}
$$

where

$$
\begin{aligned}
\Delta Q_{s}= & m_{p, i}\left(h_{\ell}\left(T_{p, i}, P_{i}\right)-h_{\ell}\left(T_{s, i}, P_{i}\right)\right)-\sum_{j i} f_{j i} \theta_{j i} W_{v, j i} h_{\ell}\left(T_{s, i}, P_{i}\right) \Delta t_{f} \\
& +\sum_{j i} f_{j i} \theta_{j i}\left[\mathrm{q}_{a p, j i}+g W_{j i}\left(H_{j}-H_{p, i}\right)-W_{n c, j i}\left(T_{s, i}\right)\right] \Delta t_{f}
\end{aligned}
$$

and $\mathrm{m}_{\mathrm{p}, \mathrm{i}}$ is the initial pool mass; $\mathrm{T}_{\mathrm{p}, \mathrm{i}}$ is the initial pool temperature; $\mathrm{T}_{\mathrm{s}, \mathrm{i}}$ is the pool saturation temperature; $f_{j i}$ is the gas equilibration fraction $=1-\exp \left(-\Delta \mathrm{H}_{\mathrm{p}, \mathrm{i}, 1} \lambda_{\mathrm{j} i}\right) ; \Delta \mathrm{H}_{\mathrm{p}, \mathrm{i}, 1}$ is the submergence of the flow path end in $i ; \lambda_{j i}$ is the equilibration length associated with the flow path end at $i ; h_{l}$ is the liquid coolant specific enthalpy; $h_{v}$ is the coolant vapor specific enthalpy; and $h{ }_{n c}$ js the noncondensable gas specific enthalpy for the $\mathrm{ji}$ path; $\mathrm{g}$ is the acceleration due to gravity; $\mathrm{H}_{\mathrm{j}}$ is the gas center-of-elevation in $\mathrm{j}$; and $\mathrm{H}_{\mathrm{p}, \mathrm{i}}$ is the pool surface elevation in $\mathrm{i}$.

The contribution to the vapor flow rate associated the equilibrated outflow is defined as

$$
\begin{aligned}
W_{p a, v e, i} & =\sum_{j i} f_{j i} \theta_{j i} M_{w} W_{n c, j i} /\left[M_{n c, j i} \max \left(P_{i}-P_{s}\left(T_{p, i}^{\prime}\right), 0.01 P_{s}\left(T_{p, i}^{\prime}\right)\right)\right] & \text { if BOIL is specified } \\
& =0 & \text { otherwise }
\end{aligned}
$$

where $M_{w}$ is the molecular weight of the coolant vapor, $M_{n c, j i}$ is the molecular weight of the noncondensable gas inflow, and $P_{s}(T)$ is the pool saturation pressure at temperature $T$. The cutoff of $0.01 \mathrm{P}_{\mathrm{s}}\left(\mathrm{T}_{\mathrm{p}, \mathrm{i}}^{\prime}\right)$ in the denominator of the above equation has been implemented to prevent unrealistic amounts of vapor production for large pools.

The nonequilibrated vapor outflow contribution is defined as

$$
\mathrm{W}_{\mathrm{pa}, \mathrm{vn}, \mathrm{i}}=\sum_{\mathrm{ji}}\left(1-\mathrm{f}_{\mathrm{ji}}\right) \theta_{\mathrm{ji}} \mathrm{W}_{\mathrm{v}, \mathrm{ji}}
$$


Finally, the mass transfer rate of vapor, homogeneously dispersed liquid coolant, and noncondensables from the pool to the atmosphere, including the equilibrated and non-equilibrated parts of the flow, is given by

$$
W_{p a, i}=W_{p a v e, i}+W_{p a, v s, i}+W_{p a, v n, i}+W_{p a, n c, i}
$$

The energy addition rate to the atmosphere as a result of the mass transfer from the pool, including the equilibrated and nonequilibrated parts and referenced to the pool surface, is given by

$$
\begin{aligned}
& \mathrm{q}_{\mathrm{pa,i}}=\sum_{\mathrm{ji}}\left(1-\mathrm{f}_{\mathrm{ji}}\right) \theta_{\mathrm{ji}}\left[\mathrm{W}_{\mathrm{ji}} \mathrm{j}_{\mathrm{j}}+\mathrm{gW}_{\mathrm{ji}}\left(\mathrm{H}_{\mathrm{j}}-\mathrm{H}_{\mathrm{p}, \mathrm{i}}\right)\right]+\sum_{\mathrm{ji}} \mathrm{f}_{\mathrm{ji}} \theta_{\mathrm{ji}} \mathrm{W}_{\mathrm{nc}, \mathrm{ji}} \mathrm{h}_{\mathrm{nc}, \mathrm{ji}}\left(\mathrm{T}_{\mathrm{p}, \mathrm{i}}^{\prime}\right) \\
& +\mathrm{W}_{\mathrm{ve}, \mathrm{i}} \mathrm{h}_{\mathrm{v}}\left(\mathrm{T}_{\mathrm{p}, \mathrm{i}}^{\prime} \mathrm{P}_{\mathrm{s}}\left(\mathrm{T}_{\mathrm{p}, \mathrm{i}}^{\prime}\right)\right)+\mathrm{W}_{\mathrm{vs}, \mathrm{i}} \mathrm{h}_{\mathrm{v}}\left(\mathrm{T}_{\mathrm{s}, \mathrm{i}}, \mathrm{P}_{\mathrm{s}}\left(\mathrm{T}_{\mathrm{s}, \mathrm{i}}\right)\right)
\end{aligned}
$$

The self-consistent temperature $T_{p, i}^{\prime}$ is determined by an enthalpy balance condition, using the net energy addition rate $\mathrm{q}_{\text {eq, }}$ to the pool as a result of equilibration

$$
\begin{aligned}
q_{e q, i}=\sum_{j i} & f_{j i} \theta_{j i}\left[q_{a p, j i}+g W_{j i}\left(H_{j}-H_{p, i}\right)-W_{n c, j i} h_{n c, j i}\left(T_{p, i}^{\prime}\right)\right]-W_{p a, v e, i} h_{v}\left(T_{p, i}^{\prime}, P_{s}\left(T_{p, i}^{\prime}\right)\right) \\
& -W_{p a, v s, i} h_{v}\left(T_{s, i}, P_{i}\right)
\end{aligned}
$$

The energy balance condition is

$$
m_{p, i}^{\prime} h_{f}\left(T_{p, i}^{\prime} P_{i}\right)-m_{p, i} h_{\ell}\left(T_{p, i} P_{i}\right)=q_{e q, i} \Delta t_{f}
$$

where

$$
\mathrm{m}_{\mathrm{p}, \mathrm{i}}^{\prime}=\mathrm{m}_{\mathrm{p}, \mathrm{i}}+\mathrm{W}_{\mathrm{eq}, \mathrm{i}, \mathrm{\Delta}} \Delta \mathrm{t}_{\mathrm{f}}
$$

is the net change in the mass of the pool due to equilibration and

$$
\mathrm{W}_{\text {eq,i }}=\sum_{\mathrm{ji}} \mathrm{f}_{\mathrm{ji}} \theta_{\mathrm{ji}} \mathrm{W}_{\mathrm{v}, \mathrm{ji}}-\mathrm{W}_{\mathrm{pa}, \mathrm{ve}, \mathrm{i}}-\mathrm{W}_{\mathrm{pa}, v s, \mathrm{i}}
$$

is the net mass transfer rate to the pool due to equilibration.

\subsubsection{The Gas Evolution Velocity at the Pool-Atmosphere Interface}

The velocity of gas evolving or rising from the pool-gas interface in a cell may be non-zero for a number of reasons: (1) boiling or flashing of the pool, as discussed in Section 4.4.6; (2) injection of gas below the surface of the coolant pool from submerged gas flow paths or the dedicated suppression vent model, which can result in gases that are partially or completely equilibrated, as 
discussed in Section 4.4.7; and (3) explicitly modeled submerged sources of gas, such as the SRV discharge model discussed in Section 11.2 and the CORCON CCI model discussed in Chapter 5. A contribution not taken into account in this velocity is that due to surface condensation or evaporation of coolant at the pool surface, which is accounted for separately. The gas evolution velocity is used in a number of places. One is to offset aerosol deposition processes, as discussed in Section 7.2.3, at the pool-atmosphere interface. It is also used in the default option, as discussed in Section 10.1.1.6, to calculate the degree of forced convection present with respect to the exposed heat transfer structure surfaces. In the forced convection option, the contributions to the convective velocity from each outflow and unsubmerged inflow gas flow path connected to the cell are taken into account separately. The effect of all the submerged inflow gas paths, plus that of boiling and explicit submerged sources, are taken into account in a single gas evolution velocity $\mathrm{v}_{\mathrm{p}, \mathrm{g}, \mathrm{i}}$ (for cell i). This velocity is processed as an "inflow" velocity according to the POOLFLOW parameters in the VELCOEF input, or their defaults, to obtain the pool-gas interface contribution to the forced convection velocity. (Prior to CONTAIN 1.2, only the effect of the dedicated suppression pool vent discussed in Section 11.1.2 was taken into account, according to the SUPVENT parameters in that input. The SUPVENT keyword is considered obsolete but if specified will be treated like the POOLFLOW keyword.)

The gas evolution velocity $\mathrm{v}_{\mathrm{pg}, \mathrm{i}}$ in cell $\mathrm{i}$ is computed from the conditions calculated just above the pool surface. The total flow enthalpy for all contributions is given by

$$
W_{p g, i} h_{p g, i}=q_{p a, i}+q_{b o i l, i}+q_{L C, b o i l, i}+q_{L C, g, i}+q_{S R V, g, i}
$$

where $q_{p a, i}$ is defined in Equation (4-20) and $q_{b o i l, i}$ and $q_{L C, b o i l, i}$ represents the implicit and explicit pool boiling rates, respectively, discussed in Section 4.4.6. The other terms represent explicit submerged sources of gas as discussed in Tables 4-3 and 4-4. The total mass flow rate in this equation is given by the sum $W_{p g, i}=W_{p a, i}+W_{b o i l, i}+W_{L C, b o i l, i}+W_{L C, g, i}+W_{S R V, g, i}$. The temperature $T_{p g, i}$ and density $\rho_{p g, i}$ of the flow are obtained by solving the enthalpy equation at a total pressure equal to the cell gas pressure $\mathrm{P}_{\mathrm{i}}$, namely

$$
W_{p g, i} h_{p g, i}=\sum_{k}^{N_{g a s}} W_{p g, i, k} h_{k}\left(T_{p g, i}\right)+W_{p g, v, i} h_{v}\left(T_{p g, i}, P_{p g, v, i}\right)+W_{p g, \ell, i} h_{\ell}\left(T_{p g, i}, P_{i}\right)
$$

where $N_{\text {gas }}$ is the number of ideal gases; $W_{p g, i, k}$ is the flow rate of ideal gas $k ; h_{k}$ is the specific enthalpy function of $\mathrm{k} ; \mathrm{W}_{\mathrm{pg}, \mathrm{v}, \mathrm{i}}$ is the flow rate of coolant vapor; $h_{\mathrm{v}}$ is the coolant vapor specific enthalpy; $\mathrm{P}_{\mathrm{pg}, \mathrm{v}, \mathrm{i}}$ is the coolant vapor partial pressure; $\mathrm{W}_{\mathrm{pg}, \mathrm{l}, \mathrm{i}}$ is the flow rate of homogeneously dispersed liquid coolant and $h_{\ell}$ is the specific enthalpy of the coolant liquid.

The velocity of the flow is given by

$$
v_{p g, i}=W_{p g, i}\left(A_{p g, i} \rho_{p g, i}\right)
$$


where $A_{p g, i}$ is the area of the atmosphere-pool interface, which is the cell cross-sectional area at the collapsed pool level.

\subsubsection{The FIX-FLOW Option}

The FIX-FLOW option within the implicit flow solver is designed to improve calculational efficiency in long-term calculations in which strong gas natural convection in cells with relatively small free volumes severely limits the flow timestep because of the convective Courant condition (i.e., $\Delta t_{f} \leq 0.2 t_{c}$, where $t_{c}$ is given in Equation (2-1)). If the conditions in these cell atmospheres approach a quasi-steady state for much of the calculation, despite the strong convection, the FIXFLOW option may be useful. The option in effect removes the atmospheres in question from the implicit solver solution matrix and assumes that the gas flow rates in the flow paths connected to those atmospheres are effectively fixed boundary conditions to the rest of the problem. Note that in order for the option to apply to a cell, the cell atmosphere must be selected as being solved implicitly through the IMPLICIT option, and the coolant pool, if any, in the cell, must not be connected by pool flow paths to other cells. (The pool may be explicitly connected to other pools through liquid transport models available within the engineered systems models, discussed in Chapter 12.) The FIX-FLOW option was originally developed to treat cells modeling the passive containment cooling system air duct in the Westinghouse AP600 design.

Section 4.4.9.1 discusses the modeling basis, and Section 4.4.9.2 discusses guidelines for the applicability of the option.

4.4.9.1 Modeling Basis. The FIX-FLOW option is designed to overcome the convective Courant limit imposed by a cell on the flow timestep when conditions in the atmosphere are changing slowly, even though gas convection through the cell is occurring at a relatively high rate. By approximating these slowly changing conditions instead of completely recalculating them each timestep, the need to continually take small timesteps can be reduced. The FIX-FLOW option uses the simplest approximation to the slowly changing conditions, which is to replace selected cell atmospheres by fixed-flow-rate boundary conditions when the conditions are changing sufficiently slowly. More sophisticated approximations, such as a linearly extrapolated change, were considered but appeared to be unstable. It is shown in Section 4.4.9.2 that in order for the FLX-FLOW option to be useful, the relative rates of change of conditions must be many orders of magnitude smaller than the inverse time constants for relaxation to the quasi-steady state.

The criteria for a sufficiently slow change are based on a user-specified rate tolerance $\zeta_{\mathrm{i}}$, which is by default zero, for each cell $\mathrm{i}$. It is input as "rtol" after the FIX-FLOW keyword for each cell as described in Section 14.2.4.1. Specification of zero, the default value, indicates that the corresponding cell is excluded from consideration in the FIX-FLOW option. The rate tolerance value governs the maximum rates of change of the atmosphere temperature, mass, and volume that can be present when the fixed-flow-rate boundary conditions are invoked.

When the FIX-FLOW option is invoked, the implicit flow solver will use the first flow timestep within a system timestep to sample the rate of change $\mathrm{dT}_{\mathrm{i}} / \mathrm{dt}, \mathrm{dm} \mathrm{m}_{\mathrm{i}} / \mathrm{dt}$, and $\mathrm{dV}_{\mathrm{i}} / \mathrm{dt}$ of the temperature 
$T_{i}$, total gas mass $m_{i}$, and free volume $V_{i}$, respectively, in each cell $i$. On the first timestep, if the relative rates of change satisfy

$$
\begin{aligned}
& \left|\mathrm{T}_{\mathrm{i}}^{-1} \mathrm{dT} / \mathrm{dt}\right| \leq \zeta_{\mathrm{i}} \\
& \left|\mathrm{m}_{\mathrm{i}}^{-1} \mathrm{dm}_{\mathrm{i}} / \mathrm{dt}\right| \leq \zeta_{\mathrm{i}} \\
& \left|\mathrm{V}_{\mathrm{i}}^{-1} \mathrm{dV}_{\mathrm{i}} / \mathrm{dt}\right| \leq \zeta_{\mathrm{i}}
\end{aligned}
$$

the cell $i$ is replaced in subsequent flow timesteps within the same system timestep by fixed-flowrate boundary conditions, with the flow rates and composition taken to be those calculated in the first flow timestep. In addition, the atmospheric conditions in the cell related to the noncondensable gases and the condensable material will be held constant for those timesteps. Note that while the atmosphere thermodynamic and flow conditions will be held constant, aerosol and fission product inventories will not be held constant but will be calculated as evolving according to the assumed flow rates and thermodynamic conditions.

4.4.9.2 Guidelines for Applying the FIX-FLOW Option. In applying the FIX-FLOW option, the user should be aware of the fact that even for relatively small tolerances, mass and energy conservation problems may result and the calculated cell conditions for the cell atmospheres replaced by fixed-flow boundary conditions may deviate significantly from the correct ones. These effects in general scale with the user-specified rate tolerance $\zeta_{\mathrm{i}}$. The degree to which these effects occur depends on the detailed nature of the processes occurring within a cell. The purpose of this section is to supply guidelines to the user as to when FIX-FLOW should be invoked.

Mass and energy will in general not be conserved in a cell replaced by fixed-flow boundary conditions, since these quantities should in general be changing, albeit slowly. Under the quasisteady conditions likely to be present when the fixed-flow boundary conditions are invoked, one can estimate errors by assuming linear rates of change of mass and energy over the system timestep. Estimates for the relative error per system timestep on the atmosphere temperature and total atmospheric mass in this case can be expressed, respectively, as:

$$
\begin{aligned}
& \left|\Delta \mathrm{T}_{\mathrm{i}} / \mathrm{T}_{\mathrm{i}}\right| \leq \varsigma_{\mathrm{i}}(1-\mathrm{s}) \Delta \mathrm{t}_{\mathrm{s}} \\
& \left|\Delta \mathrm{m}_{\mathrm{i}} / \mathrm{m}_{\mathrm{i}}\right| \leq \varsigma_{\mathrm{i}}(1-\mathrm{s}) \Delta \mathrm{t}_{\mathrm{s}} \\
& \left|\Delta \mathrm{V}_{\mathrm{i}} / \mathrm{V}_{\mathrm{i}}\right| \leq \varsigma_{\mathrm{i}}(1-\mathrm{s}) \Delta \mathrm{t}_{\mathrm{s}}
\end{aligned}
$$

where $\Delta t_{s}$ is the system timestep and $\mathrm{s}$ is the ratio $\Delta t_{f} / \Delta t_{s}$ of the first flow timestep to the system timestep. The degree to which such errors accumulate and can be tolerated depends on the situation. In some cases, such as an adiabatic closed cell with a very low source rate extending over a long period of time, the cumulative errors may not be acceptable even for $\varsigma_{i}=10^{-6}$. However, as discussed below, for a cell that rapidly relaxes to a quasi-steady state and is connected to a very large reservoir (i.e., the environment), the cumulative errors may be negligible. 
The FIX-FLOW option may be useful in cases in which the atmosphere thermodynamic and flow conditions correspond to a slowly varying quasi-steady state and in which this quasi-steady state is stable if the conditions in the cell are perturbed. It is helpful to deal with a specific case. In the case of a duct cell, one would expect steady-state conditions to be established in the time $\tau$ it takes to set up natural convection flow through the duct. The natural convection flow will in general not be absolutely steady but will adjust to changes in other conditions in the duct. Such "other" conditions may, for example, include temperature changes on the surfaces of duct structures due to changes in the decay heat within the containment. In the absence of these other changes, it is assumed that the atmosphere thermodynamic and flow conditions in the duct would relax to steady-state values with a time constant $\tau$. In the presence of other slowly varying conditions, one would expect that the atmosphere thermodynamic and flow conditions will evolve slowly with time (i.e., be in a quasisteady state) and be close to their "instantaneous" steady-state values, which are defined as the values that would be eventually attained if the "other" conditions suddenly stopped changing.

The cumulative error will be analyzed here for the cell temperature, although similar analyses can be done for other quantities. The following analysis was motivated by considering the change in cell atmosphere temperature due to a change in surface temperature, for fixed-flow rates through the cell. Because of changes in the surface temperature, the "instantaneous" steady-state atmosphere temperature $\mathrm{T}_{\mathrm{qs}}$ is assumed to vary linearly in time (to lowest order)

$$
\mathrm{T}_{\mathrm{qs}}^{\mathrm{n}}=\mathrm{T}_{\mathrm{qs}}^{\mathrm{n}-1}+\left(\mathrm{dT}_{\mathrm{qs}} / \mathrm{dt}\right) \Delta \mathrm{t}_{\mathrm{s}}
$$

where $\mathrm{n}$ is the index for the system timestep $\Delta t_{s}$ and where the cell index $i$ has been suppressed for simplicity. With the standard approximation that only the first term in the expansion of the exponential describing the relaxation of the cell temperature is kept, the cell temperature $T$ varies as

$$
T^{n}=-s \Delta t_{s}\left(T^{n-1}-T_{q s}^{n-1}\right) / \tau+T^{n-1}
$$

This finite difference form is applicable either for the case in which the flow timestep is equal to the system timestep ( $=1$ ) or the case in which the flow timestep is less than the system timestep and the cell is replaced by fixed-flow boundary conditions for all but the first flow timestep in the system timestep. From Equations (4-30) and (4-31), it is easy to show that the difference $T-T_{q}$, rather than $\mathrm{T}$ itself, attains a steady-state value when $\mathrm{T}_{\mathrm{qs}}$ varies linearly in time. This steady-state value is

$$
\left(\mathrm{T}-\mathrm{T}_{\mathrm{qs}}\right)_{\mathrm{ss}}=-\left(\mathrm{dT}_{\mathrm{qs}} / \mathrm{dT}\right) \tau / \mathrm{s}
$$

If we define the cumulative error $\Delta \mathrm{T}_{\mathrm{e}}$ as the difference in cell temperature between the case in which $\mathrm{s}<1$ and the case in which the system timestep is reduced to the flow timestep, then from Equation (4-32) 


$$
\Delta \mathrm{T}_{\mathrm{e}}=-\left(\mathrm{dT}_{\mathrm{qs}} / \mathrm{dt}\right) \tau(1-\mathrm{s}) / \mathrm{s}
$$

Thus, in order to have a relatively small cumulative error for a small $s$, the time constant for change in the quasi-steady conditions must be much larger than the time constant $\tau$ for relaxation to the quasi-steady state.

In general, since we have assumed that the fixed-flow boundary conditions are being invoked, the temperature must satisfy Equation (4-29). Since the difference $T-T_{q s}$ is assumed to relax to a steady state (with a relaxation factor $1-\mathrm{s} \Delta \mathrm{t} / \tau$ ), the time derivative $\mathrm{dT}_{\mathrm{q} s} / \mathrm{dt}$ will be comparable to $\mathrm{dT} / \mathrm{dt}$. Thus, an estimate for the cumulative error is

$$
\left|\Delta \mathrm{T}_{\mathrm{e}}\right| \leq \zeta_{\mathrm{i}} \mathrm{T}_{\mathrm{qs}} \tau(1-\mathrm{s}) / \mathrm{s}
$$

This error should be evaluated to see if it is acceptable. If one assumes the conditions that $\tau=10 \mathrm{~s}$, $\Delta t_{s}=20 \mathrm{~s}, \mathrm{~s}=0.1, \mathrm{~T}_{\mathrm{qs}}=300 \mathrm{~K}$, and $\mathrm{s}_{\mathrm{i}}=10^{-5}$, then $\Delta \mathrm{T}_{\mathrm{e}} \leq 0.3 \mathrm{~K}$, which may be an acceptable temperature error. Note that for the case of the adiabatic closed cell mentioned above, $\tau$ is infinite and the error could consequently be large even if $c_{\mathrm{i}}$ is small.

The cumulative errors for conditions within cell atmospheres replaced by fixed-flow boundary conditions will produce mass and energy conservation errors, which eventually may affect other cells in the problem. It is therefore desirable that the cells to which the fixed-flow cells are connected by flow be large repositories that are not significantly affected. This condition is obviously satisfied if the former cells are environment cells. In other, less obvious cases, the effect of the mass and energy conservation errors should be explicitly checked, as discussed in Section 3.4.

The user is cautioned that $\zeta_{\mathrm{i}}$ must be carefully chosen, much like an error tolerance, to keep errors from accumulating in the calculation and to maintain calculational efficiency. Appropriate values will depend on the problem, but it is expected that values of order $10^{-5}$ to $10^{-6}$ per second will be useful for long-term containment analysis. Accuracy should be checked by reduction of $\zeta_{i}$ by a factor of 10 if there is any question about the magnitude of errors being introduced.

\subsection{The Conservation and Thermodynamic State Equations}

Tables 4-2 through 4-6 present the momentum, mass, and energy conservation equations in a form appropriate for a control volume formulation. These pertain only to the principal fluids: noncondensable gases, the coolant vapor, and liquid coolant. In particular, the modifications of these equations to include dispersed core debris fields is discussed in Section 6.2. These tables include a cross-reference for the terms in each equation.

The momentum conservation equation given in Table 4-2 is the inertial flow equation that is typically used for flow paths and has been discussed in Section 4.4.1. It relates the mass flow rate $\mathrm{W}_{\mathrm{ij}}$ between cells $\mathrm{i}$ and $\mathrm{j}$ to the total pressure difference $\Delta \mathrm{P}_{\mathrm{ij}}$ between the cells connected by the flow path. This difference includes gravitational heads for all flow paths. The irreversible flow loss coefficient $\mathrm{C}_{\mathrm{FC}}$ in the momentum equation can be specified by the user to account for entrance and 
exit losses and other frictional losses. Section 4.4.1 gives additional discussion regarding the flow loss coefficient.

The atmosphere mass conservation equation for a cell is given in Table 4-3. The equation in this table takes into account (a) the flow of principal fluids into a cell from other cells, (b) flow out of the cell to other cells, (c) pool-gas exchange terms from boiling and equilibration of gas injected into the cell below the coolant pool surface, and (d) mass sources and sinks from processes modeled explicitly outside of the flow solver.

The energy conservation equation for the atmosphere of a cell is given in Table 4-4. The equation includes volume displacement work, work associated with gravity, and the energy from sources and sinks within a cell. Note that the kinetic energy term is neglected in the energy equation, because the flow velocities must be on the order of the sound speed in order for the kinetic energy terms to become. [Ber85b] Volume displacement work terms are implicitly included in the equation through the use of the flow specific enthalpy. Similarly, volume displacement work from explicit processes is taken into account through use of the enthalpy to define the $q$ terms. The gravitational work terms are explicitly displayed. The elevations used in the work terms are dictated by the fact that the enthalpy fluxes are referenced to the gas center of elevation for transfers from an atmosphere and to the pool surface for transfers from a pool.

The coolant pool mass conservation equation is similarly given in Table 4-5, and the energy conservation equation for a pool is given in Table 4-6.

The thermodynamic state equations for the atmosphere and pool given in Table 4-7. From the component masses and the total internal energy of the atmosphere and pool, the thermodynamic state calculation determines the temperature, pressure, enthalpy, and saturation condition of the atmosphere and the temperature of the pool in the cell.

From the given internal energy and the component masses, it is necessary to invert the internal energy function to obtain the temperature. This is done iteratively for both the atmosphere and pool. An initial guess for the temperature close to the expected temperature is made. In practice, these first estimates are chosen to be close to the last known temperature. The internal energy for this temperature is then calculated and compared to the total energy that should be present. If the comparison is not acceptable, a new temperature is selected, and the iterative process is continued until acceptable convergence occurs. In the implicit method, if more than 50 iterations are required, the calculation simply will abort. In the explicit (Runge-Kutta) method, if more than 30 iterations are required, a message warning of nonconvergence is given. One should note that the inversion routines are extremely robust. Nonconvergence that is not the result of too large a timestep is usually a symptom of a calculational fault outside the solver, such as a negative mass for the condensable gas. The code does check for negative masses, but intermediate calculations are sometimes performed prior to this check. Another potential cause of nonconvergence may be the use of user-defined materials for the gas phase components of the atmosphere, particularly if data violating thermodynamic laws are specified. 


\begin{tabular}{|c|c|}
\hline \multicolumn{2}{|c|}{ Atmosphere Internal Energy: } \\
\hline$U_{\mathrm{i}}$ & $=\sum_{k=1}^{N_{g a s}} m_{k} h_{k}\left(T_{i}\right)+m_{v} h_{v}\left(T_{i}, P_{v}\right)+m_{\ell} h_{\ell}\left(T_{i}, P_{i}\right)-P_{i} V_{i}$ \\
\hline \multicolumn{2}{|c|}{ Pool Internal Energy: } \\
\hline $\mathrm{U}_{\mathrm{p}, \mathrm{i}}$ & $=\mathrm{m}_{\mathrm{p}, \mathrm{i}} \mathrm{h}_{\mathrm{l}}\left(\mathrm{T}_{\mathrm{p}, \mathrm{i}}, \mathrm{P}_{\mathrm{i}}\right)-\mathrm{P}_{\mathrm{i}} \mathrm{V}_{\mathrm{p}, \mathrm{i}}$ \\
\hline \multicolumn{2}{|l|}{ where } \\
\hline $\mathrm{U}_{\mathrm{i}}$ & $\begin{array}{l}=\text { internal energy of gases, coolant vapor, and homogeneously dispersed } \\
\text { liquid coolant in cell } \mathrm{i} \text {; }\end{array}$ \\
\hline $\mathrm{N}_{\text {gas }}$ & $=$ number of gases that are treated as ideal gases; \\
\hline $\mathrm{m}_{\mathrm{k}}$ & $=$ mass of gas component $\mathrm{k}$ in cell $\mathrm{i}$; \\
\hline $\mathrm{h}_{\mathrm{k}}\left(\mathrm{T}_{\mathrm{i}}\right)$ & $=$ specific enthalpy of ideal gas component $k$ at temperature $T_{i} ;$ \\
\hline $\mathrm{T}_{\mathbf{i}}$ & $=$ atmosphere temperature; \\
\hline $\mathrm{m}_{\mathrm{v}}$ & $=$ mass of coolant vapor; \\
\hline $\mathrm{h}_{\mathrm{v}}\left(\mathrm{T}_{\mathrm{i}}, \mathrm{P}_{\mathrm{v}}\right)$ & $\begin{aligned}= & \text { specific enthalpy of coolant vapor. See Section } 3.2 \text { for the expressions for } \\
& \mathrm{h}_{\mathrm{v}} \text { for the ideal and non-ideal equations of state; }\end{aligned}$ \\
\hline$P_{v}$ & $=$ coolant vapor partial pressure; \\
\hline $\mathbf{m}_{\mathrm{l}}$ & $=$ mass of homogeneously dispersed liquid coolant in the atmosphere; \\
\hline $\mathrm{h}_{\mathrm{f}}\left(\mathrm{T}_{\mathrm{i}}, \mathrm{P}_{\mathrm{i}}\right)$ & $\begin{aligned} &= \text { specific internal energy of liquid coolant. See Section } 3.2 \text { for the } \\
& \text { expressions for } \mathrm{h}_{\mathrm{l}} \text { for the ideal and non-ideal equations of state; }\end{aligned}$ \\
\hline$P_{i}$ & $=$ atmosphere total pressure; \\
\hline $\mathrm{V}_{\mathrm{i}}$ & $=$ free volume (cell volume - pool volume) \\
\hline$P_{p, i}$ & $=$ pool volume. \\
\hline
\end{tabular}


Table 4-7

Thermodynamic State Equations (Concluded)

\begin{tabular}{|ll||}
\hline Pressure: & \\
$\mathrm{P}_{\mathrm{i}}$ & $=\sum_{\mathrm{k}=1}^{\mathrm{N}_{\mathrm{gas}}} \frac{\mathrm{R} \mathrm{N}_{\mathrm{k}} \mathrm{T}_{\mathrm{i}}}{\mathrm{V}_{\mathrm{i}}}+\mathrm{P}_{\mathrm{v}}$ \\
$\mathrm{P}_{\mathrm{i}}$ & $=$ pressure in cell $\mathrm{i} ;$ \\
$\mathrm{P}_{\mathrm{v}}$ & $=$ partial pressure of coolant vapor; \\
$\mathrm{N}_{\mathrm{k}}$ & $=$ moles of component $k$ in the free volume.
\end{tabular}




\subsection{LOWER CELL AND CAVITY MODELS}

The lower cell system of models provide for the representation of processes in the lower regions of a cell. The principal uses of the lower cell models include the modeling of coolant pools and the underlying substrates and of core concrete interactions (CCIs). The lower cell includes models for heat conduction between the pool and substrate layers, CCIs, volumetric and radionuclide decay heating, and mass and energy sources to the pool and substrate layers. As discussed in Section 5.7.1, the CONTAIN lower cell model for radionuclide decay heating utilizes the ANSI-standard decay power curve [Ame79] and is used in conjunction with the decay heating from explicit fission products that is discussed in Section 8.5. Other models for decay heating are imbedded in the CORCON module and are discussed in Section 5.3.2.

A coolant pool, if present, is assumed to occupy the lower regions of the CONTAIN cell as depicted in Figure 4-4. The cell geometric shape (i.e., the cross-sectional area as a function of height) is defined by the cell GEOMETRY input discussed in Section 14.3.1.1. The pool is assumed to fill this cell from the bottom up, with a horizontal free surface dividing the pool and atmosphere volumes. Heat transfer structures and the pool substrate are assumed to be excluded from the user-specified cell volume.

If CORCON is not active, a conduction model solution for the heat transfer between the pool and the layers in the pool substrate is carried out. For purposes of the conduction solution, the substrate area is defined through the lower cell GEOMETRY area discussed in Section 5.1 below and in Section 14.3.2.1. (The use of cell GEOMETRY input for the pool cross-sectional area as a function of height is a non-upward compatible change from versions prior to CONTAIN 1.2, which assume a constant pool cross-sectional area given by the lower cell GEOMETRY area.) The substrate is considered to be either composed of "intermediate" and "concrete" layers, as discussed below, or represented by a basemat temperature boundary condition.

Note that a lower cell substrate is not the only way to define the region below the pool. As discussed in Chapter 10, the pool may also be in contact with the face of a submerged slab-type floor heat transfer structure, which can be at any elevation equal to or above the bottom of the pool. The bottom of the pool may also be a virtual flow boundary, characterized by a pool-type engineered vent to the cell below, in which case the pool is considered to be in contact with the pool in the cell below rather than a substrate. In the latter two cases, a lower cell GEOMETRY area for the pool substrate must still be defined but should simply be set to a negligible value.

The changes in CONTAIN 1.2 to allow the pool to flood the lower regions of a cell, including the heat transfer structures, have resulted in non-upward compatible changes with respect to aerosol deposition onto the pool surface (which is typically dominated by settling). This deposition is modeled if and only if aerosols are defined and a coolant pool is defined in the cell. The lower cell SETTLE keyword is no longer required for such deposition to occur. In addition, the aerosol deposition velocity is now offset by any gases evolving from the pool free surface. In contrast,

deposition does not occur on the part of a heat transfer structure that is submerged below the pool surface. 
CCIs are modeled through an embedded version of the CORCON Mod3 code. CORCON Mod3 includes an integrated version of VANESA for representing aerosol releases; therefore, in the discussions that follow CORCON is used to refer to both the CORCON Mod3 and VANESA models. When active, the CORCON model governs the processes occurring in the melt, which is assumed to be located at the bottom the coolant pool, if it exists. Convective and boiling heat transfer from the substrate can be modeled with either the CORCON model or the heat conduction model. In most severe accident analyses, the user will invoke CORCON to represent CCIs and related phenomena. In situations where concrete ablation is not expected to be important, the simpler heat conduction model can be used rather than CORCON. The former model has the advantages that it handles transient conduction in the concrete, is simpler to set up and use than CORCON, and runs significantly faster than the CORCON module.

The coolant pool is the repository used for liquid coolant taken from or directed to the lower cell by other modules. Such transfers can occur as the result of flow through pool-type engineered vents (see Chapter 4), operation of engineered systems (see Chapter 12), condensate runoff from heat transfer structures (see Chapter 10), coolant aerosol deposition (see Chapter 7), the flow DROPOUT model (see Chapter 4), and the steam blowdown phase separation process (see Chapter 11) that is modeled in conjunction with the safety relief valve (SRV) model. Note that if a coolant pool is not defined, any liquid coolant directed to a cell will be lost from a problem, although its mass and energy will be noted in the WASTE location of the mass and energy accounting output. To reduce the number of pools required in a problem, condensate runoff from structures in a given cell (e.g., as a result of condensation) can be directed to the pool in another cell by using the cell OVERFLOW keyword (see Chapter 10 and Section 14.3.1.12).

A number of models deal with the venting of gas/aerosol/fission-product mixtures under the pool surface. The gases are partially or completely equilibrated with the pool, depending on the model, as discussed in Section 4.4.7 and in Section 11.2.1. Also, the aerosols are scrubbed in a manner discussed in Section 7.7. For example, such mixtures are vented into the coolant pool when the downstream end of a gas-type engineered vent lies under the surface of the pool in the downstream cell. Also, when CORCON is active, any such mixtures generated by CCIs are assumed to be injected at the bottom of the coolant pool, if present. Otherwise, they are injected into the atmosphere. In addition, the user can specify such mixtures as external sources to the pool through the SRV model.

Convective, condensation, and radiative heat transfer between the pool surface and atmosphere is modeled in a manner similar to that between heat transfer structures and the atmosphere, and is therefore discussed in Chapter 10 rather than here. As with structures, the modeling of condensation heat transfer for the pool-atmosphere interface requires the specification of CONDENSE option. Convective heat transfer between the pool and the submerged portion of heat transfer structures is also discussed in Chapter 10.

Boiling heat transfer correlations are also discussed in Chapter 10. Presently, boiling heat transfer correlations are used only between the coolant pool and pool substrate. If CORCON is active, the boiling heat transfer model of CORCON Mod3 is used. This model takes into account the effects of gas barbotage and pool subcooling in the film boiling regime. If CORCON is not active and the substrate is hotter than the pool, then the somewhat simpler boiling heat transfer model from 
CORCON Mod2 is used. That model does not treat gas barbotage or subcooling effects during film boiling. It should be noted that Section 10.4 is a complete and somewhat revised version of the corresponding discussion in Reference Bra93 on boiling heat transfer, as modeled in CORCON Mod3.

A basic description of the layer configurations in the lower cell with and without CORCON active is provided in Section 5.1. Sections 5.2 through 5.4 describe specific physical models and user options associated with the concrete, intermediate, and pool layer types in the lower cell system. This includes the conduction model that is used when CORCON is not active, and special options or unique features included to link CORCON to CONTAIN. Interlayer heat transfer coefficients used in the conduction model and user-specified heat transfer coefficient option are described in Section 5.5. The use of mass and energy sources to the various layers, including decay and volumetric heating, is discussed in Section 5.6. Section 5.7 addresses any implementation specific capabilities and/or limitations of the CONTAIN implementation of CORCON not covered in the preceding sections. Section 5.8 addresses the physics modeling in CORCON. Rather than duplicate the governing equations and discussions in the CORCON Mod3 User's Manual, [Bra93] this section provides a guide for applying this existing documentation to the CONTAIN implementation of CORCON. Section 5.8 also describes any changes made to CORCON Mod3 since the publication of Reference Bra93. Section 5.9 discusses limitations and assumptions of the lower cell modeling. Key elements of the lower cell and cavity models are illustrated in Figure 5-1.

\subsection{Layer Configurations}

The first subsection below describes the layer configuration when CORCON is not active. Note that this is applicable to problems in which CORCON is invoked but CORCON is not yet active or was active and has been deactivated. Section 5.1.2 describes the layer configuration used when CORCON is invoked and active. This describes how the CONTAIN lower cell layer system represents the CORCON layer.

\subsubsection{Layer Configuration Without CORCON}

The layer configuration and the relationship of the layers to the phenomena modeled in the lower cell without CORCON is depicted in Figure 5-2. Each of these layers, with the exception of the pool layer, is assumed to have the cross-sectional area given by the lower cell GEOMETRY keyword (see Section 14.3.1.1). Layers are defined in the CONTAIN input beginning at the bottom. Thus, the first layer is the concrete layer which is shown in Figure 5-2 as being nodalized into 12 equal-size nodes. Any number of nodes greater than or equal to $5 \mathrm{can}$ be specified for the concrete layer using the "jconc" input in the cell control block. If "jconc" is specified as non-zero but smaller than 5 , the code will use 5. Only one concrete layer may be specified. Above the concrete layer are "jint" intermediate layers, each with only one node. When CORCON is not invoked, intermediate layers are allowed to have only one node. The user can specify an arbitrary number of intermediate layers by specifying the desired number as "jint" in the cell control block. Also, each layer can be given an arbitrary name when CORCON is not invoked. A pool layer is shown above the topmost intermediate layer. The pool, like an intermediate layer without CORCON, is allowed to have only a single node and is indicated by "jpool" $=1$. Only one pool layer may be specified. If specified, 


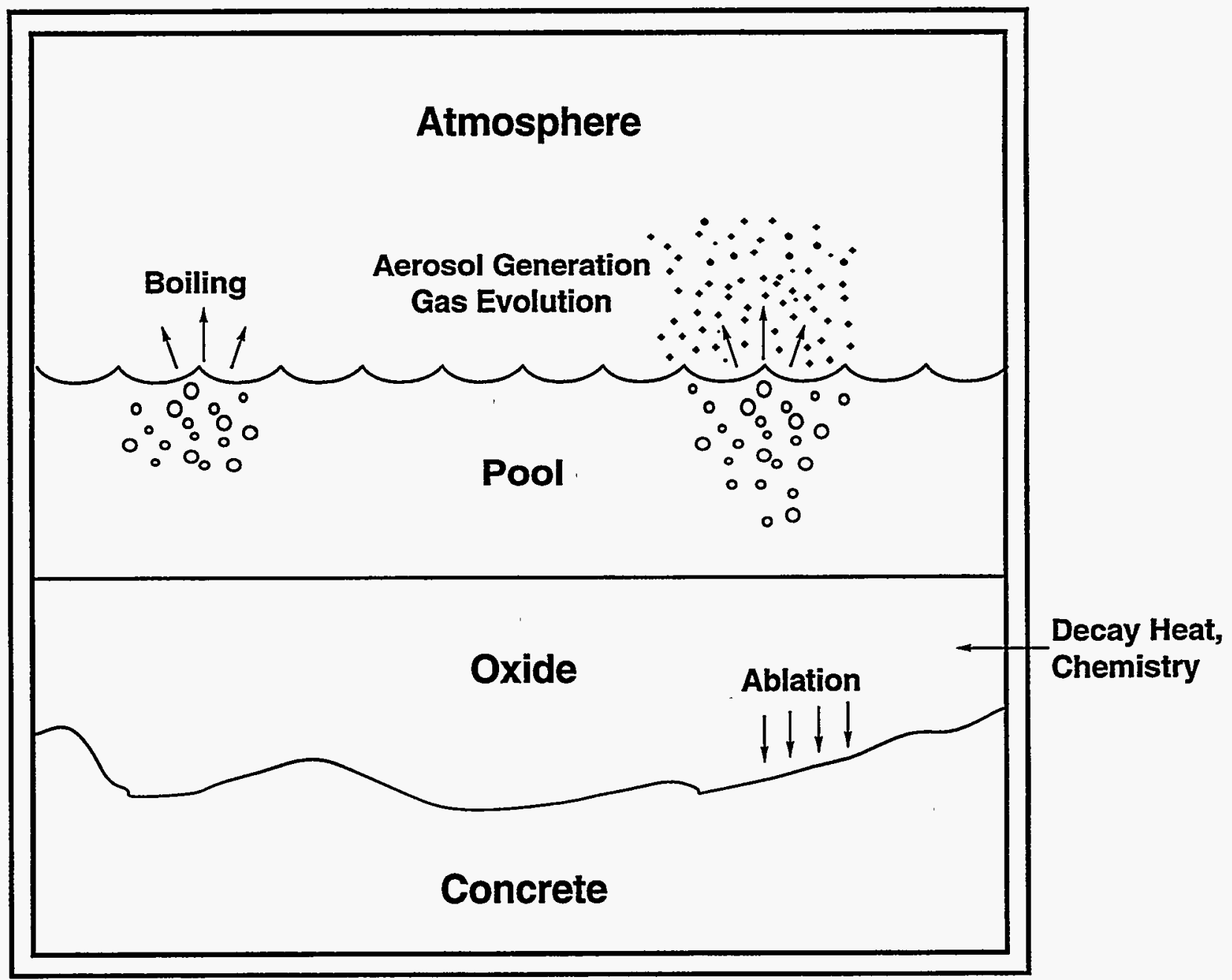

Figure 5-1. Lower Cell as Modeled in CONTAIN 


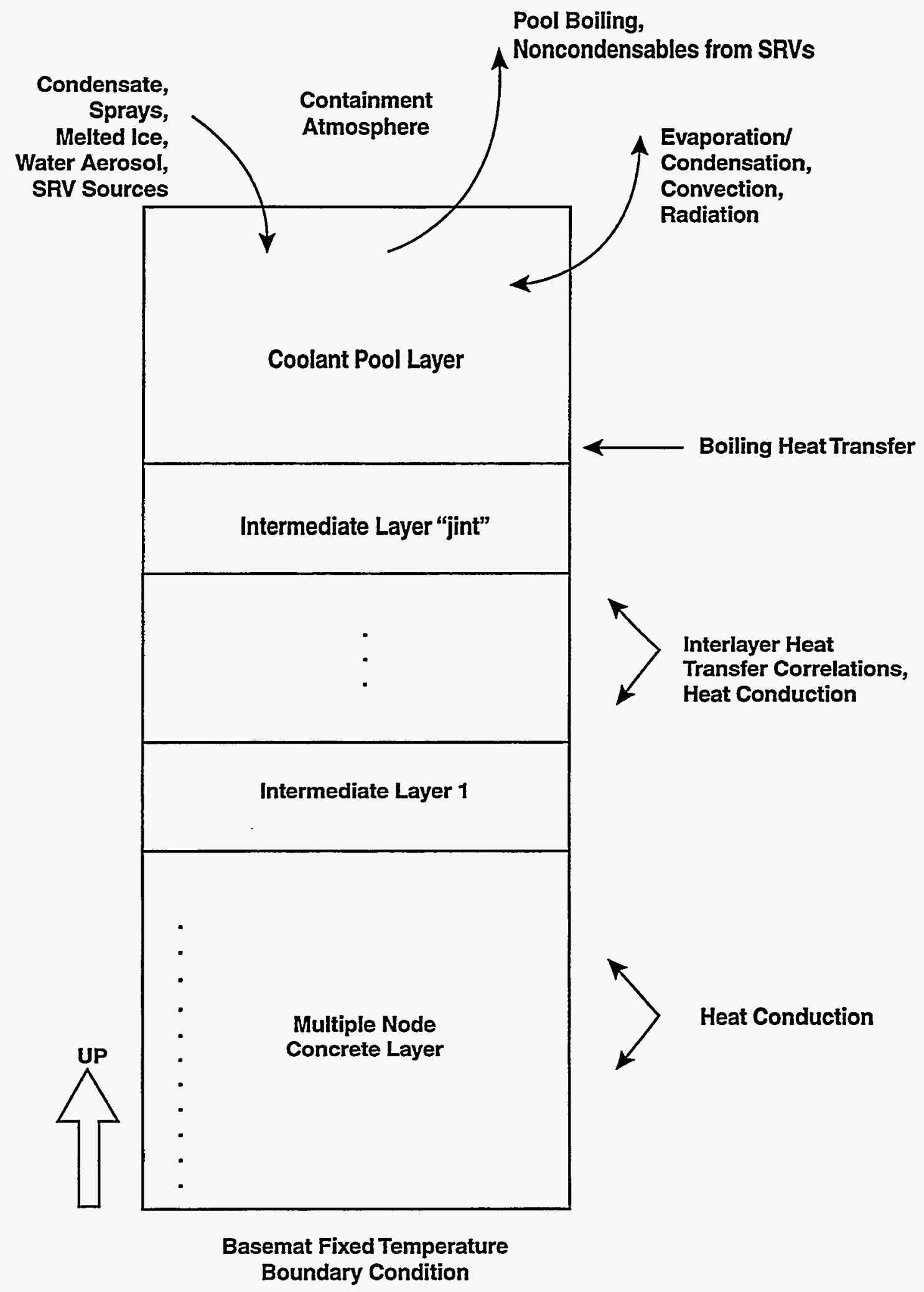

Figure 5-2. Layer Configuration in Lower Cell without CORCON

Rev. 0 
the pool layer should lie on top of all the other specified layers as shown in Figure 5-2. Each layer will be included in the one-dimensional heat conduction solution as discussed in Section 5.2. The heat transfer coefficients between these layers will be based on perfect contact if the layer is solid, otherwise the coefficient will be as described in Section 5.6.

The composition of the layers may be specified through initial conditions provided by the user and/or through material source tables. Essentially any non-gaseous material may be placed in any layer, but it is recommended that only CONC material be placed in the concrete layer, coolant in the pool layer, and core debris or concrete materials in intermediate layers. The experienced user may choose to deviate from this scheme to simulate special situations, such as simulating rebar in the concrete layer by including iron material. Alternatively, the user may also redefine the CONC material properties through the USERDEF user-defined material input.

\subsubsection{Layer Configuration With CORCON}

Figure 5-3 depicts the layer configuration in a CORCON calculation. As in the case without CORCON, layers are defined beginning at the bottom. Unlike the case without CORCON, however, the layer configuration in problems with CORCON is fixed. One must define a concrete layer and exactly one intermediate layer. A pool layer is optional, but is highly recommended, since in most applications an overlying coolant pool is at least possible.

The concrete layer is present mostly to serve as an input and output interface to the CORCON model. The CORCON concrete type is specified in the concrete layer input. Note that this concrete type is only used by the CORCON model. That is, it is ignored by the conduction model as discussed further in Section 5.2. The temperature specified in the concrete layer input is used to initialize CORCON, but the specified mass of the concrete layer is not passed to CORCON. The mass is not used by CORCON because it uses its own two-dimensional model for the concrete cavity. However, a reasonable mass should be specified in the concrete layer input of the concrete layer so that conduction can be modeled before CORCON is activated and after CORCON is deactivated. Note that the CORCON and VANESA input options are specified, in part, through the CORCON subblock of the PHYSICS block of CONCRETE layer. Note that this block includes the input specification for the melt spreading option and the interlayer mixing option.

Above the concrete layer is a single intermediate layer, which will always have the layer name of "CORCON." This required single CONTAIN intermediate layer is used to initialize and report on the five possible CORCON internal layers: HOX, HMX, MET, LMX, and LOX. The names and meanings of these five CORCON layers are discussed in Section 5.3. Each of the five CORCON internal layers is represented as a separate node within one CONTAIN intermediate layer. (As discussed in Section 5.1.1, normally only one node is available for each intermediate layer.) The intermediate layer input is used to specify the initial inventory of oxides and metals in the cavity, the degree of stratification or mixing of the CORCON layers, and the time-dependent mass and energy sources to the debris pool. Note that input for the VANESA model and its various options, the melt spreading option, and the interlayer mixing model are all part of the concrete layer input.

Figure 5-3 shows a coolant pool overlying the core debris layers. Although not shown here, CORCON can also be used without an overlying coolant pool. If a pool is included, the phenomena 


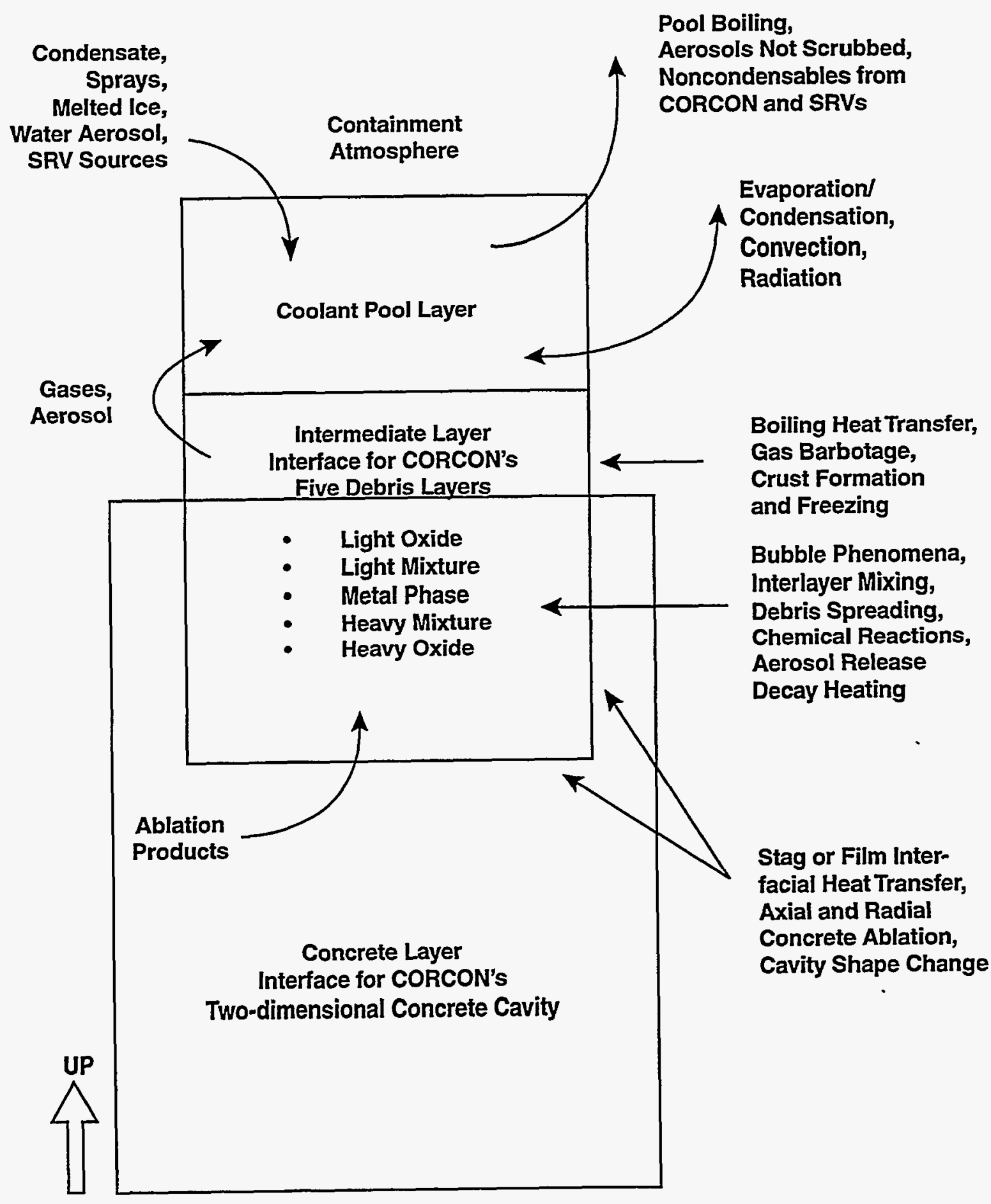

Figure 5-3. Layer Configuration in Lower Cell with CORCON 
modeled will include the scrubbing of CCI aerosols using the VANESA scrubbing model (activated with the SCRUB keyword) as discussed in Section 5.4. Equilibration of CCI gases in the coolant pool will also be modeled as discussed in Section 11.2.1. If a pool layer is defined and the pool is empty, the CORCON model will function as if a pool were not defined. However, if a pool is not defined, coolant normally directed to the pool, such as condensate runoff from structures, will be lost from the problem.

\subsection{Concrete Layer}

If CORCON is not active, the concrete layer is nodalized and heat conduction is modeled as described in Section 5.5. When used with the conduction model, the concrete layer will consist of materials specified by the user. Typically, the CONC material will be used. However, other materials besides CONC could be specified as present in the concrete layer.

If CORCON is active (i.e., the calculation is within the period specified in the CORCON TIMES block, during which CORCON is running), the concrete layer is primarily used as a vehicle for supplying CORCON initial conditions and for reporting the results of the CORCON calculation. Heat conduction in the concrete and intermediate layers is not taken into account by CONTAIN while CORCON is active. However, during the periods that CORCON is not active, heat conduction is modeled. Also, the material used with the conduction model in CORCON problems during the periods CORCON is inactive is always assumed to be the CONC material. The mass of the concrete layer will be that given by the "cmass" variable.

If CORCON is active, the shape of the concrete cavity must be specified. The concrete cavity containing the core debris is assumed to be axisymmetric. Two predefined geometries are available to describe its initial shape: a cylinder with a flat base (FLATCYL) and a cylinder with a hemispherical base (HEMICYL). Alternatively, a general but still axisymmetric initial shape may be defined by specifying the initial position of each body point (ARBISHP). The keywords shown in parentheses are specified in the GEOMETRY input of the CORCON block in the concrete input.

In a CORCON calculation the concrete type in the cavity must also be identified. Three predefined concrete types are available for analysis of CCIs in CORCON. These types are basaltic concrete (specified through the keyword BASALT), limestone/common-sand concrete (LIME), and limestone concrete (GENERIC). The concrete type is specified in the concrete layer input before the CORCON sub-block is specified. Unlike previous implementations, the concrete type specifications for CORCON and VANESA are taken to be the same, since the concrete composition for both is taken from the concrete type identified in the concrete layer. The VANESA concrete type input previously provided by the CONCCOMP keyword is obsolete, but is still allowed for upward compatibility reasons. Any CONCCOMP input specified will be ignored and the concrete type specified in the CORCON input section will be used instead. The properties and compositions of the three predefined concrete types are listed in Table 5-1. In addition the user may specify an arbitrary CORCON concrete type through the keyword OTHER, followed by the appropriate values of the mass fractions and characteristic temperatures indicated in Table 5-1. User-defined concrete input is described in detail in Section 14.3.2.3.2. 
Table 5-1

Properties of CORCON Predefined Concrete Types

\begin{tabular}{|c|c|c|c|c|}
\hline & \multicolumn{3}{|c|}{ Concrete Aggregate Type Name } \\
\hline & & $\begin{array}{c}\text { Basaltic } \\
\text { (BASALT) }\end{array}$ & $\begin{array}{l}\text { Limestone/ } \\
\text { Common Sand } \\
\text { (LIME) }\end{array}$ & $\begin{array}{l}\text { Limestone/ } \\
\text { Limestone } \\
\text { (GENERIC) }\end{array}$ \\
\hline Species & Variable & \multicolumn{3}{|c|}{ Mass Fractions } \\
\hline $\mathrm{SiO}_{2}$ & fsio2 & 0.5484 & 0.3580 & 0.0360 \\
\hline $\mathrm{TiO}_{2}$ & ftio2 & 0.0105 & 0.0018 & 0.0012 \\
\hline $\mathrm{MnO}$ & fmno & 0.0 & 0.0003 & 0.0001 \\
\hline $\mathrm{MgO}$ & fmgo & 0.0616 & 0.0048 & 0.0567 \\
\hline $\mathrm{CaO}$ & fcao & 0.0882 & 0.3130 & 0.4540 \\
\hline $\mathrm{Na}_{2} \mathrm{O}$ & fna2o & 0.0180 & 0.00082 & 0.00078 \\
\hline $\mathrm{K}_{2} \mathrm{O}$ & $\mathrm{fk} 2 \mathrm{o}$ & 0.0539 & 0.0122 & 0.0068 \\
\hline $\mathrm{Fe}_{2} \mathrm{O}_{3}$ & $\mathrm{ffe} 2 \mathrm{o} 3$ & 0.0626 & 0.0144 & 0.0120 \\
\hline $\mathrm{Al}_{2} \mathrm{O}_{3}$ & fal 203 & 0.0832 & 0.0360 & 0.0160 \\
\hline $\mathrm{Cr}_{2} \mathrm{O}_{3}$ & fer 203 & 0.0 & 0.00014 & 0.00004 \\
\hline $\mathrm{CO}_{2}$ & $\mathrm{fco} 2$ & 0.0150 & 0.21154 & 0.35698 \\
\hline $\mathrm{H}_{2} \mathrm{O}^{*}$ & fh2oe & 0.0386 & 0.0270 & 0.0394 \\
\hline $\mathrm{H}_{2} \mathrm{O} * *$ & fh20b & 0.0200 & 0.0200 & 0.0200 \\
\hline $\begin{array}{l}\text { Ablation } \\
\text { Temperature }\end{array}$ & tabl (K) & 1550.0 & 1590.0 & 1810.0 \\
\hline $\begin{array}{l}\text { Solidus } \\
\text { Temperature }\end{array}$ & tsolct $(\mathrm{K})$ & 1350.0 & 1420.0 & 1690.0 \\
\hline $\begin{array}{l}\text { Liquidus } \\
\text { Temperature }\end{array}$ & tliqct $(\mathrm{K})$ & 1650.0 & 1670.0 & 1875.0 \\
\hline
\end{tabular}

* Evaporable water

** Chemically bound water

The predefined concrete compositions do not include any steel rebar. Because rebar can be on the order of $20 \%$ of the concrete mass in reactor cavities, a provision is included for the user to specify a rebar mass fraction in the concrete. This is done by means of the REBAR keyword in the CORCON input. Note that input was previously provided in the VANESA block for specifying the rebar fraction used by the VANESA model. This input is now obsolete and will be ignored; the VANESA model will now always use the CORCON value. Rebar is by default assumed to be pure iron (material FE); however, the user can override this assumption and specify other metals for the 
rebar using the RBRCOMP keyword. Concrete, with or without reinforcing rods, is treated as a homogeneous material in the CORCON model.

The specific concrete types and the REBAR option described above apply only to the CORCON models. They do not pertain to the heat conduction model. If CORCON is invoked in a calculation but not active at a given point in time, a default concrete layer will be used in conjunction with the conduction model. The default layer will consist of the CONC material even if one of the above concrete types is specified in connection with the CORCON input and will have a total mass given by the "cmass" input variable. The properties of the CONC material are unaffected by the CORCON concrete type definitions. However, the properties of the CONC material may be specified through the CONTAIN user-defined materials option.

\subsection{Intermediate Lavers}

The first subsection discusses briefly the options available when CORCON is not invoked. These options are related primarily to heat conduction modeling. The second subsection discusses the use of intermediate layers in conjunction with CORCON. Note that the conduction model is also used in a calculation involving CORCON prior to CORCON activation and after CORCON terminates. The second subsection also inciudes a description of layer initiation and the various species names used in the input to and the output from a CORCON calculation.

\subsubsection{Intermediate Layers Without CORCON}

When CORCON is not invoked, the main physical phenomenon analyzed in the intermediate layers is conduction heat transfer. Any number of single-node intermediate layers may be present when CORCON is not invoked, and any number of CONTAIN liquid or solid materials may be present in the intermediate layers. Mass transfer between intermediate layers and phase changes within the layers are not modeled. Chemical reactions are also not modeled; therefore, the composition of these layers can be changed only through user-specified source tables as discussed in Section 5.6.2. Note, however, that trapped debris will be added to the uppermost intermediate layer in a direct containment heating calculation as described in Chapter 6. The layer mass and energy addition options (see Section 5.6) provide a great deal of flexibility in representing heat transfer problems in the cavity.

\subsubsection{Intermediate Layer With CORCON}

If CORCON is invoked, only one intermediate layer may be present. This single intermediate layer is used to initialize the CORCON debris pool layers and to report results. This layer, named CORCON, will have 5 nodes, one for each possible CORCON debris pool layer. These nodes, numbered 1 through 5 beginning at the bottom of the melt pool, correspond to the following CORCON layer names:

$\begin{array}{cll}\text { Num } & \text { Name } & \text { Description } \\ 1 & \text { HOX } & \text { Heavy oxides } \\ 2 & \text { HMX } & \text { Heterogeneous mixture of heavy oxides and metals } \\ 3 & \text { MET } & \text { Metals }\end{array}$


$4 \quad$ LMX Heterogeneous mixture of light oxides and metals

5 LOX Light oxides

The mass appearing in these nodes will be associated with special material names in the CONTAIN output and plot file. These material names are LCCHOX for heavy oxide, LCCMET for the metal layer, and LCCLOX for light oxide. LCCHOX and LCCMET can be present in the HMX layer, and LCCMET and LCCLOX can both be present in the LMX layer. The HMX layer is interpreted as a metal layer with suspended drops of heavy oxide (most likely from entrainment). The LMX layer is interpreted as a light oxide layer with suspended metal drops. These material names are also used in the plot file. The user should not specify these names in the MATERIAL list of the global COMPOUND block because they are included automatically when CORCON is invoked.

The user has the option of specifying either an initially homogeneous mixture or a stratified layer configuration via the LAYERS keyword in the INTERM block. Homogeneous mixtures are assumed located in the HMX CORCON layer. The HOX, MET, and LOX layers are used to represent an initially heterogeneous configuration. Material may move between the various layers as a result of interlayer mixing processes or density changes. Mixing, stratification, and density changes resulting from material addition (either from concrete ablation or external sources) can lead to changes in the layer configuration during a calculation. Note, however, that CORCON Mod3 does not include layer flips as did CORCON Mod2. Instead, debris movement resulting from density changes is handled more realistically by material transport between layers.

If CORCON is active, volumetric heating of the CORCON intermediate layer or concrete layer, due to Q-VOL tables (see Section 5.6.3), will not be taken into account. Fission product heating of core debris layers is calculated through either the CORCON CORESTAT decay power model or the CORCON user input tables. The user may also invoke the CONTAIN DECAY-HT makeup decay power model. When the last option is used, fission product heating from explicit fission products and DECAY-HT makeup decay power assigned to the CORCON layer will be used by CORCON (see Section 5.6.1) to normalize the overall fission product heating calculated in one of the first two options. Mass sources to the CORCON internal layers can be specified through use of CONTAIN source tables (see Section 5.6.2).

Prior to the start of a CORCON calculation, the CORCON layer is treated as a null layer. Should the CONTAIN calculation continue beyond the time that the CORCON calculations are completed, the representative $\mathrm{CORCON}$ intermediate layer will become a lower cell layer with a single node composed of UO2 and FE. The layer mass will be equal to the summed final masses of the CORCON layers with $\mathrm{UO} 2$ representing CORCON's internal oxide layers and $\mathrm{FE}$ representing the internal metal layer. After the CORCON calculations are completed, this intermediate layer is included in heat conduction modeling, along with the concrete layer and pool layer, if any. The purpose of providing an active intermediate layer is to allow a smooth transition in the heating of the containment when the CORCON calculations are completed.

Initialization of the intermediate layer for a CORCON calculation in CONTAIN requires the specification of a number of material species in various locations. The allowable species names vary depending upon whether the input pertains to the initiation of melt masses for the CORCON physics modules, or whether it pertains to the specification of fission products in the melt for the VANESA 
aerosol release model. The allowable names for each input situation are listed in Tables 5-2 through 5-5 below. Note that most of these names are taken from the CORCON and VANESA master species lists given in Table 2.1 and Table 2.2 of Reference Bra93, respectively. Because of specific changes made in the CONTAIN implementation of CORCON Mod3, the user should use the tables below rather than the tables in Reference Bra93.

The species names in Table 5-2 are used in the initialization of material masses in the oxide and metal CORCON layers of the CONTAIN intermediate layer. Any of these species may also be specified in user-specified mass sources to the intermediate layer. The metal species are also used in the RBRCOMP input for specifying the composition of the rebar. Note that condensed phase carbon is given the input name $\mathrm{C}$ in the CONTAIN implementation of CORCON, while it is named $\mathrm{C}(\mathrm{c})$ in the stand-alone implementation of CORCON. This change was necessary because parentheses are used as delimiters in the CONTAIN input parser. Internally, the name of condensed phase carbon is $\mathrm{C}(\mathrm{c})$ in both implementations.

Table 5-2

CORCON Metal and Oxide Species Names

\begin{tabular}{||c|c||c|c||}
\hline \multicolumn{2}{|c||}{ Oxides } & \multicolumn{2}{c|}{ Metals } \\
\hline SIO2 & FE2O3 & FE & U \\
\hline TIO2 & AL2O3 & CR & SI \\
\hline FEO & UO2 & NI & UAL3 \\
\hline MNO & ZRO2 & ZR & UAL2 \\
\hline MGO & CR2O3 & MN & NA \\
\hline CAO & NIO & C & CA \\
\hline SRO & FE3O4 & AL & \\
\hline BAO & MN3O4 & & \\
\hline LI2O & PUO2 & & \\
\hline NA2O & UO3 & & \\
\hline K2O & U3O8 & \\
\hline
\end{tabular}

The decay power elements in Table 5-3 below are used in the CORESTAT decay power model. In this model, the decay power is characterized by the gross fuel mass, the operating power, and the decay power element retention factors. The default retention factors and the assumed mass concentrations are shown in Table 5-3. The default retention factor shown may be replaced by specifying the appropriate "reti" value for decay power element "fpl" in the CORESTAT input block. Note that the mass concentrations may not be altered. Note that the two user input elements shown in Table 2.5 of Reference Bra93 are not available in CONTAIN. Thus, there are 25 allowable element names in the CORESTAT input block. 
Table 5-3

CORCON Fission Product Decay Power

Element Names and Default Retention Factors

\begin{tabular}{||c|c|c||c|c|c||}
\hline $\begin{array}{c}\text { Element } \\
\text { Name } \\
\text { "fpl" }\end{array}$ & $\begin{array}{c}\text { Mass } \\
\text { Concentration } \\
\text { (g-atom/MWt) }\end{array}$ & $\begin{array}{c}\text { Retention } \\
\text { Factor } \\
\text { "reti" }\end{array}$ & $\begin{array}{c}\text { Element } \\
\text { Name } \\
\text { "fpl" }\end{array}$ & $\begin{array}{c}\text { Mass } \\
\text { Concentration } \\
\text { (g-atom/MWt) }\end{array}$ & $\begin{array}{c}\text { Retention } \\
\text { Factor } \\
\text { "reti" }\end{array}$ \\
\hline $\mathrm{MO}$ & .6053 & .97 & $\mathrm{PU}$ & .7921 & .99 \\
\hline $\mathrm{TC}$ & .1545 & .97 & $\mathrm{AM}$ & .00593 & .99 \\
\hline $\mathrm{RU}$ & .3885 & .97 & $\mathrm{Y}$ & .1099 & .99 \\
\hline $\mathrm{RH}$ & .0690 & .97 & $\mathrm{LA}$ & .1662 & .99 \\
\hline $\mathrm{SB}$ & .00244 & .85 & $\mathrm{PR}$ & .1446 & .99 \\
\hline $\mathrm{TE}$ & .0627 & .85 & $\mathrm{ND}$ & .4638 & .99 \\
\hline $\mathrm{SR}$ & .2155 & .90 & $\mathrm{SM}$ & .0539 & .99 \\
\hline $\mathrm{BA}$ & .1915 & .90 & $\mathrm{EU}$ & .01705 & .99 \\
\hline $\mathrm{ZR}$ & .7352 & .99 & $\mathrm{RB}$ & .0819 & .19 \\
\hline $\mathrm{CE}$ & .3870 & .99 & $\mathrm{CS}$ & .3776 & .19 \\
\hline $\mathrm{NP}$ & .0422 & .99 & $\mathrm{BR}$ & .0053 & .10 \\
\hline $\mathrm{CM}$ & .00204 & .99 & $\mathrm{I}$ & .0320 & .10 \\
\hline $\mathrm{NB}$ & .01139 & .99 & - & - & - \\
\hline \hline
\end{tabular}

The fission product species names used to specify initial inventories for VANESA are shown in Table 5-4. (Note that this table includes three sets of columns to conserve space.) If the preferred material keyword input method is used a series of values are specified in the form "ovfp"="vfpm," where "ovfp" is the fission product name and "vfpm" is the associated mass. The names specified for "ovfp" must be taken from the keywords given in the first, third, and fifth columns of this table. The corresponding chemical symbols are shown to the right of the keywords in the second, fourth, and sixth columns. If the obsolete MELTCOMP input method is used, the values specified after the MELTCOMP keyword must be given in the order listed in the table (going down the columns first). One of these two inputs is required to specify the initial inventory of fission products in the melt. Note that the major species, shown in Table 5-4 with an asterisk, should not be specified here as they were in the previous version of CORCON Mod2 in CONTAIN. The masses of these species are now taken from the layer mass input discussed in Section 14.3.2.3.2. If the obsolete MELTCOMP method is used, zeros should be specified for the obsolete species. If the "ovfp"="vfpm" method is used, the keyword names for the obsolete species should not be specified. 
Table 5-4

Melt Component Keywords and Chemical Symbols

For VANESA Fission Product Initial Inventory Specification

\begin{tabular}{||l|l||l|l||l|l||}
\hline \hline Keyword & $\begin{array}{l}\text { Chemical } \\
\text { Symbol }\end{array}$ & Keyword & $\begin{array}{l}\text { Chemical } \\
\text { Symbol }\end{array}$ & Keyword & $\begin{array}{l}\text { Chemical } \\
\text { Symbol }\end{array}$ \\
\hline $\mathrm{CES}$ & $\mathrm{Cs}$ & $\mathrm{IOD}$ & $\mathrm{I}$ & $\mathrm{XEN}$ & $\mathrm{Xe}$ \\
\hline $\mathrm{KRY}$ & $\mathrm{Kr}$ & $\mathrm{TE}$ & $\mathrm{Te}$ & $\mathrm{BA}$ & $\mathrm{Ba}$ \\
\hline $\mathrm{SN}$ & $\mathrm{Sn}$ & $\mathrm{RU}$ & $\mathrm{Ru}$ & $\mathrm{UO} 2^{*}$ & $\mathrm{UO}_{2}$ \\
\hline $\mathrm{ZR}^{*}$ & $\mathrm{Zr}$ & $\mathrm{ZRO} 2^{*}$ & $\mathrm{ZrO}_{2}$ & $\mathrm{FE} *$ & $\mathrm{Fe}$ \\
\hline FEO* & $\mathrm{FeO}$ & $\mathrm{MO}$ & $\mathrm{Mo}$ & $\mathrm{SR}$ & $\mathrm{Sr}$ \\
\hline $\mathrm{RB}$ & $\mathrm{Rb}$ & $\mathrm{Y}$ & $\mathrm{Y}$ & $\mathrm{TC}$ & $\mathrm{Tc}$ \\
\hline $\mathrm{RH}$ & $\mathrm{Rh}$ & $\mathrm{PD}$ & $\mathrm{Pd}$ & $\mathrm{LA}$ & $\mathrm{La}$ \\
\hline $\mathrm{CE}$ & $\mathrm{Ce}$ & $\mathrm{PR}$ & $\mathrm{Pr}$ & $\mathrm{ND}$ & $\mathrm{Nd}$ \\
\hline $\mathrm{SM}$ & $\mathrm{Sm}$ & $\mathrm{PU}$ & $\mathrm{Pu}$ & $\mathrm{CR} *$ & $\mathrm{Cr}$ \\
\hline $\mathrm{MN} *$ & $\mathrm{Mn}$ & $\mathrm{NI}$ & $\mathrm{Ni}$ & $\mathrm{AG}$ & $\mathrm{Ag}$ \\
\hline $\mathrm{SB}$ & $\mathrm{Sb}$ & $\mathrm{NB}$ & $\mathrm{Nb}$ & - & - \\
\hline
\end{tabular}

"Obsolete input, since these species are taken from CORCON layer mass specification. If MELTCOMP is used, zeros should be specified for these species.

In contrast to the stand-alone implementation of CORCON Mod3, the option to define the fission product inventories in VANESA from the CORESTAT retention factors (see Table 5-3 above) and the amount of $\mathrm{UO}_{2}$ in the melt is not available. In stand-alone CORCON input terminology, this means that the "ivanfp" variable is fixed at 1 . This option was disabled in the CONTAIN implementation because the DECAY-HT option is typically used, with the default CORESTAT retention factors.

Calculated aerosol and fission product releases are mapped to CONTAIN aerosol components and fission product chain elements or groups using the AERCONST and FPTRACK input blocks. The names of the species released are given in Table 5-5. These are the names that are specified in the input when identifying the mapping between VANESA releases and CONTAIN aerosol components and fission products. If the CORESTAT decay power option is used, the inventory of the CORCON decay power elements (see Table 5-3) will be updated based on fission product releases from the melt as predicted in the VANESA module. The mapping between CONTAIN aerosol components and fission products and VANESA calculated releases is discussed in Section 5.7.4. 
Table 5-5

VANESA Constituent Names of Released Aerosol and Fission Products

\begin{tabular}{||l|l||l|l||l|l||}
\hline $\begin{array}{l}\text { VANESA } \\
\text { Constituent }\end{array}$ & $\begin{array}{l}\text { Chemical } \\
\text { Symbol }\end{array}$ & $\begin{array}{l}\text { VANESA } \\
\text { Constituent }\end{array}$ & $\begin{array}{l}\text { Chemical } \\
\text { Symbol }\end{array}$ & $\begin{array}{l}\text { VANESA } \\
\text { Constituent }\end{array}$ & $\begin{array}{l}\text { Chemical } \\
\text { Symbol }\end{array}$ \\
\hline $\mathrm{FE}$ & $\mathrm{Fe}$ & $\mathrm{AG}$ & $\mathrm{Ag}$ & $\mathrm{ZRO2}$ & $\mathrm{ZrO}_{2}$ \\
\hline $\mathrm{CR} 2 \mathrm{O} 3$ & $\mathrm{Cr}_{2} \mathrm{O}_{3}$ & $\mathrm{MN}$ & $\mathrm{Mn}$ & $\mathrm{CS} 2 \mathrm{O}$ & $\mathrm{Cs}_{2} \mathrm{O}$ \\
\hline $\mathrm{NI}$ & $\mathrm{Ni}$ & $\mathrm{CAO}$ & $\mathrm{CaO}$ & $\mathrm{BAO}$ & $\mathrm{BaO}$ \\
\hline $\mathrm{MO}$ & $\mathrm{Mo}$ & $\mathrm{AL} 2 \mathrm{O} 3$ & $\mathrm{Al}_{2} \mathrm{O}_{3}$ & SRO & $\mathrm{SrO}$ \\
\hline $\mathrm{RU}$ & $\mathrm{Ru}$ & $\mathrm{NA2O}$ & $\mathrm{Na}_{2} \mathrm{O}$ & $\mathrm{LA2O} 3$ & $\mathrm{La}_{2} \mathrm{O}_{3}$ \\
\hline $\mathrm{SN}$ & $\mathrm{Sn}$ & $\mathrm{K} 2 \mathrm{O}$ & $\mathrm{K}_{2} \mathrm{O}$ & $\mathrm{CEO} 2$ & $\mathrm{CeO}_{2}$ \\
\hline $\mathrm{SB}$ & $\mathrm{Sb}$ & $\mathrm{SIO} 2$ & $\mathrm{SiO}_{2}$ & $\mathrm{NBO}$ & $\mathrm{NbO}^{*}$ \\
\hline $\mathrm{TE}$ & $\mathrm{Te}$ & $\mathrm{UO} 2$ & $\mathrm{UO}_{2}$ & $\mathrm{CSI}$ & $\mathrm{CsI}$ \\
\hline \hline
\end{tabular}

"NB2O5 is also allowed to retain compatibility with older input files. NBO will be assumed if NB2O5 is specified.

\subsection{Coolant Pool Layer}

As discussed in the introductory Section 5.0, the coolant pool layer is a key aspect of the lower cell model because it serves as a repository for coolant for a number of models. The various modules with interfaces to the coolant pool were outlined in that introduction. The present section discusses various input options that control the physics modeling of the coolant pool. Two aspects of the physics modeling related to the venting of gas/aerosol mixtures under the pool surface are discussed elsewhere. The scrubbing models for aerosols under such conditions are discussed in Section 7.7. The modeling of the condensation/evaporation of coolant vapor within the bubbles of injected gas is discussed either in Section 4.4.7 or in Section 11.2.1, depending on the origin of the injected gas.

Note that the coolant pool layer must be unique and specified as the topmost layer in any given lower cell (this is done by defining the pool as the last layer in the lower cell input block). It can have only a single, well-mixed node. The pool may contain only coolant and deposited aerosols and fission products. Although other layers in principle can contain coolant, only the coolant in the pool layer will be accessible to other code modules. It is also worth noting that the deposited aerosol inventory in the lower cell is associated with the pool layer. Therefore, aerosol deposition onto the lower cell is modeled only if a pool layer is defined. In contrast to code versions prior to CONTAIN 1.2, such aerosol deposition is automatic and does not require the SETTLE keyword for activation. The association of aerosol inventory with only the coolant pool layer is usually not a serious limitation. If CORCON is not invoked, the simple conduction modeling done in the lower cell in the absence of a pool can to a large extent be replaced with options for a floor heat transfer structure. If CORCON is invoked, but without a coolant pool, aerosol deposition on the melt surface generally has a minor effect. 
The coolant pool layer is unique in that it is the only lower cell layer, outside of the models in CORCON, in which phase changes are allowed. As discussed in Chapter 10, surface evaporation and surface condensation of coolant vapor can occur much in the same manner as for heat transfer structures. As discussed in Section 11.2.1, pool evaporation and condensation can also occur with respect to gases vented into the pool under the pool surface. Boiling of the coolant can occur if the BOIl keyword is specified in the lower cell input block. If boiling is not activated, the pool is treated like other nodes in the conduction model, and the pool could reach unreasonable temperatures. Therefore, boiling should always be activated. The BOIL keyword is also required for proper equilibration of the coolant vapor between the pool and any gases vented under the pool surface. In the absence of the BOIL keyword, any coolant vapor in the vented gases is entirely condensed out in the pool.

If boiling is activated, any net energy flux to the coolant pool that would raise the pool above saturation is assumed to contribute instead to the pool boiling rate. When such a saturated condition is detected, the conduction model solution is carried out with the pool temperature fixed at the saturation temperature, for the purpose of calculating the conduction flux to the pool and the boiling rate.

Pool boiling is calculated fully implicitly with respect to pressure, if the implicit flow solver is invoked. Heat and mass sources to the pool are accumulated every cell timestep. They are then used to calculate a continuous boiling rate during the system timestep in which the sources are accumulated. Because of the fully implicit treatment, the pressurization due to the coolant mass and energy entering the atmosphere as a result of boiling is consistent with both the pool saturation temperature and the mass and energy flows to and from other cells. If the explicit flow solution is invoked, pool boiling is evaluated explicitly outside of the flow and atmosphere thermodynamics solution. The expressions for the pool boiling rate for both flow methods are given in Section 4.4.6.

\subsection{Interlayer Heat Transfer}

The heat transfer coefficients used in the lower cell conduction model between layers are discussed in this section. The following, with one exception, applies to the heat transfer between the uppermost lower cell layer, which could be the pool, and any additional layers below. The heat transfer across the interfaces between the atmosphere and uppermost lower cell layer and between the coolant pool layer and submerged heat transfer structures is discussed in Section 10.1.2. The one exception mentioned above is an option, discussed in Section 5.5.2, for the user to specify the total convective heat transfer coefficient for the atmosphere-pool interface.

This section consists of three parts: the first describes the overall interlayer heat transfer coefficient; the second discusses user-specified overall coefficients; and the third discusses the heat conduction model.

\subsubsection{Interlayer Heat Transfer Coefficients}

The present section discusses the overall interlayer heat transfer coefficients that are defined for use in the heat conduction model, which is used when CORCON is not active. These heat transfer correlations are applied to the interface between layers; the heat transfer between nodes of a layer 
(multiple nodes are present only in the concrete layer) are based solely on the thermal conductivity of the material in the layer, evaluated at the node temperature.

The overall heat transfer coefficient lumps together boundary heat transfer coefficients on either sides of the interface. The overall heat transfer coefficient is defined by

$$
h_{\text {eff }}=\frac{1}{\left(1 / h_{\ell}+1 / h_{u}\right)}
$$

In this formula, $h_{\ell}$ is the boundary coefficient for the layer below the interface and $h_{\mathrm{u}}$ is the boundary coefficient for the layer above the interface. At each interface, the boundary heat transfer coefficients $h_{\ell}$ and $h_{u}$ are evaluated using correlations appropriate to the layers.

The coefficient used depends on the phase (liquid or solid) of the material with the largest mass in the node adjacent to the layer interface. The boundary heat transfer coefficient for a layer with a dominant solid material is given by

$$
\mathrm{h}=\frac{2 \mathrm{k}}{\mathrm{L}}
$$

where $\mathrm{k}$ is the volume-weighted thermal conductivity of the material mixture in the layer evaluated at the surface node temperature, and $L$ is the thickness of the surface node. The node thickness, $\mathrm{L}$, is equal to the thickness of the entire layer for all layers except for the concrete layer, which is the only layer that can be nodalized in the conduction model. This coefficient is also used between the bottom-most layer and the basemat if the lowest layer is solid.

Convective heat transfer correlations are used whenever a liquid material has the largest mass in a node adjacent to a layer boundary. A number of heat transfer correlations discussed in Chapter 10 are applicable in this case. The ones used are given in Equations (10-20) through (10-22), except in the case of a coolant pool over a substrate layer above saturation. In that case, the boiling heat transfer correlations taken from the CORCON code and discussed in Section 10.4 are used. Note that the CORCON correlations are used regardless of whether CORCON is actually active or not. However, if CORCON is not active, the new modeling in CORCON Mod3 of the effects of gas barbotage and subcooling during film boiling is ignored, and modeling equivalent to CORCON Mod2 is used instead. It should be noted that for a convective layer that is not the pool, only the properties of the dominant liquid are used in the correlations. The presence of other materials is ignored with respect to the heat transfer coefficient. In addition, the properties are evaluated at the node temperature, not the conventional boundary layer temperature (i.e., the average of the node and interface temperature).

\subsubsection{User-Specified Heat Transfer Coefficients}

One of the features of the lower cell model is that it provides the user with the flexibility to explore the consequences of adopting various alternative hypotheses regarding the disposition of debris and 
water and regarding heat transfer among the different materials. There are two ways in which this flexibility is exercised: (1) The number and composition of the layers can be specified by the user; (2) through the HT-COEF option, the user can override the overall heat transfer coefficient described in Section 5.6.1 by specifying any heat transfer coefficient as either a function of layer temperature, time, or temperature difference between any two adjacent layers. In the HT-COEF option the default overall heat transfer coefficient given by Equation (5-1) is overridden by a user-specified table.

In the HT-COEF option, there are three quantities that need to be defined. The first (the independent variable) is time, layer temperature, or interlayer temperature difference; the second (the dependent variable) is the overall heat transfer coefficient. The third is the name of the layer which must be adjacent to the layer in which the HT-COEF option is invoked, for the overall coefficient to be applicable. If temperature is the independent variable, it is the temperature of the layer for which the table is specified. If a temperature difference is the independent variable, the difference refers to the bottom temperature of the layer above minus the top temperature of the layer for which the table is specified. In most cases the bottom and top temperatures are the same as the layer temperature. The only exception is the nodalized concrete layer, in which case these temperatures refer to the appropriate node temperature. Note that a given HT-COEF table is used only if the layer in which the option is specified and the declared adjacent layer have finite mass, and the declared adjacent layer is in fact physically adjacent. If different layers are physically adjacent to a layer of interest at different points in time, because of null layers, the HT-COEF option should be specified for each layer that could be physically adjacent.

The HT-COEF option can be used to specify the overall convective heat transfer coefficient between the atmosphere and the uppermost lower cell layer, by using the keyword ATMOS to identify the adjacent layer. In this case, the HT-COEF coefficient applies only to the overall convective coefficient between the atmosphere and the uppermost lower cell layer. To allow for the fact that other heat transfer processes such as radiative and condensation heat transfer are occurring, the atmosphere-side (or gas boundary layer) convective coefficient is inferred from the HT-COEF table values and a computed layer-side coefficient, using the lower-cell approximations discussed in the preceding section. Clearly, it would not make sense to specify an overall coefficient larger than the layer-side coefficient by itself. Therefore a fatal error occurs if this is detected. When coolant evaporation or condensation is allowed, the gas boundary layer convective coefficient is first computed. Then the heat and mass transfer analogy discussed in Section 10.2.1 is applied to obtain the mass transfer coefficient.

The user may also override the default heat transfer coefficient between the first non-null layer in the lower cell and the basemat. This is done by specifying the name BAS-MAT in the HT-COEF input as the declared adjacent layer.

An important use of user-specified heat transfer coefficients may be to model an enhanced heat transfer area. Because the CONTAIN calculation is based on the nominal area of the layer, the user wishing to model a significantly enhanced area, as when molten fuel is in the form of droplets suspended in the coolant layer, must increase the effective heat transfer coefficient. This can be done by multiplying the nominal heat transfer coefficient by the ratio of the true area to the nominal area. The ratio might be several thousand in the case of fragmented fuel. 
Heat transfer between layers can be set to zero through the use of the table option, but there is a simpler method. This involves the use of the keyword HT-TRAN, followed by five flags. The HT-TRAN option can be used to completely turn off all heat transfer between layers, between the basemat and the bottommost layer, and between the uppermost lower cell layer and the upper cell. Note that the heat transfer from the CORCON layer cannot be turned off if CORCON is active. The HT-TRAN option is discussed in more detail in Sections 10.7 and 14.3.1.6.

\subsubsection{Lower Cell Conduction Modeling}

If $\mathrm{CORCON}$ is not active, a simple one-dimensional heat conduction model is used to calculate heat transfer between the various layers in the lower cell, from the uppermost layer downward. The lower cell conduction algorithm is identical to that used for structures as described in Section 10.5.3 and the reader is referred to that section for details. However, only slab geometry is allowed. The crosssectional area used for the conduction solution is that given by the lower cell GEOMETRY keyword discussed in Section 14.3.2.1. In applying this model to the lower cell, the basemat below the first layer is handled as a surface temperature boundary condition. This temperature is specified following the $\mathrm{BC}$ keyword in the lower cell input block. The interlayer heat transfer coefficients used in the lower cell conduction model are described in Sections 5.5.1 and 5.5.2. The interlayer heat transfer coefficients are by default internally calculated as discussed in Section 5.5.1, but may be overridden by the user through the HT-COEF option (as discussed in Section 5.5.2).

If CORCON is specified but is not active initially, the conduction model is operative prior to the start time for the CORCON calculations and after CORCON finishes, but not while CORCON is active. Moreover, the conduction model assumes that core debris is absent prior to the CORCON start time and is present after CORCON finishes. This approach is used to allow the starting temperature of the debris in the cavity to be specified by the user (see TOXIDE and TMETAL in the intermediate layer input) without being affected by the conduction model prior to initiation of CCI phenomena modeling in CORCON.

\subsection{Lower Cell Mass and Energy Addition}

The sections below discuss various ways that mass and energy may be added to the lower cell layers. These include the decay heating by explicit fission products discussed in Section 8.5; the ANSIstandard DECAY-HT makeup decay power model discussed in Section 5.6.1 below; external material sources to the lower cell discussed in Section 5.6.2 below; and the QVOL external volumetric heating model discussed in Section 5.6 .3 below.

The application of these models is complicated by the possible presence of null layers (containing at most deposited aerosols and fission products) and the possible use of the CORCON model to describe CCIs. In the case of fission product, DECAY-HT, and QVOL heating of null layers, the heating is simply reassigned to the first node below that is not null, unless CORCON has been invoked. If CORCON has been invoked and is active, the fission product, DECAY-HT, and QVOL heating models are ignored for the intermediate and concrete layers representing CORCON, with the exception that the DECAY-HT makeup power, plus any explicit fission product heating assigned to the CORCON intermediate layer is used to scale the total decay power in the CORCON melt layers. In the case of a null coolant pool, any heating directed to the pool layer from the above three 
options is directed to the first node below that is not null, even if it represents a CORCON melt layer during the time CORCON is active. If such a node does not exist, the heating is accounted for in the WASTE repository of the mass and energy accounting scheme.

\subsubsection{Makeup Decay Power}

In many severe accident analyses, containment heating resulting from shutdown decay power needs to be represented in the calculation. However, it would be very tedious and computationally costly to specify explicitly all the fission products and their respective decay chains that contribute to the total reactor decay heat after shutdown. Therefore, a model is provided that allows the user to specify explicitly only those fission products that are of interest with respect to transport within and release from the containment, yet allows the proper amount of decay heating to be present in the problem. In particular, volatile fission products released from the fuel in the core into the containment will normally be tracked explicitly. The model bases the total shutdown decay heat on the ANSI standard ANSI/ANS-5.1-1979, which assumes light water reactor characteristics. [Ame79] The model then calculates the decay heat not represented by the explicitly specified fission products as the difference between the total decay heat and the decay heat from the specified fission products. The decay heat not carried by explicitly specified mobile fission products is referred to as "makeup power." The user specifies how the makeup power is distributed among the various lower cell layers in the problem and the stationary atmosphere interface. This model is activated by specifying the DECAY-HT input block in the lower cell input for those lower cells in which makeup power is desired.

Makeup decay power can be specified for any number of cells. In the DECAY-HT input for cell $\mathrm{i}$, a portion $\mathcal{P}_{\mathrm{i}}$ of the reactor operating power (in $\mathrm{MWt}$ ) is specified together with the parameters for the ANSI decay power model for that cell. This determines the ANSI decay power $\mathrm{P}_{\mathrm{A}, \mathrm{i}}(\mathrm{t})$ associated with the cell. The sum, $\mathcal{P}_{T}$, of the operating powers $\mathcal{P}_{i}$ should correspond to the nominal power at which the reactor operated prior to shutdown, unless not all the core debris is explicitly in the problem. In the makeup decay power modeling, the heating from all of the explicitly specified fission products in all cells is summed up to give the total explicit decay power $\mathrm{P}_{\mathrm{T}}(t)$ at each system timestep. A fraction, $\mathcal{P}_{i} / \mathcal{P}_{T}$, of this explicit decay power is compared to the value of the ANSI decay power $\mathrm{P}_{\mathrm{A}, \mathrm{i}}(\mathrm{t})$ for cell $\mathrm{i}$. For the vast majority of cases, that fraction of the explicit decay power will be smaller than the ANSI decay power. The makeup decay power $P_{i}(t)$ for cell $i$ is obtained by subtracting the former from the latter if the difference is positive; otherwise, the power is set to zero:

$$
P_{i}(t)=\max \left[P_{A, i}(t)-\mathcal{P}_{i} P_{T}(t) / \mathcal{P}_{T}, 0\right]
$$

There are two controlling times specified in the DECAY-HT input. The time "tohstr" is that at which decay heat will begin to be added to a cell with the option active. The time "tofsd" specifies the time of reactor shutdown, in terms of the problem time used in a CONTAIN run. Thus, a negative "tofsd" implies a reactor shutdown some time before the zero of time in a CONTAIN run. Decay heat will not be added to a cell unless the problem time is greater than both "tohstr" and "tofsd." Note that these parameters may have different values in different cells. The user should note, however, that the makeup power algorithm described above assumes that the makeup power comes from core debris with a common burnup and fission product release history. If the histories 
of the core debris in different cells are considerably different and significant release has occurred, then the makeup power algorithm may not be accurate.

If the makeup decay power is positive, the makeup power is allocated according to the layer distribution fractions specified by the user. All layers plus the atmosphere can accept this makeup power. Note that the condition of zero makeup power may arise through a physical inconsistency introduced by the user or in a situation in which a large fraction of the core debris is not explicitly in the problem or is modeled in a manner that does not use the lower cell or explicit fission products (e.g., a significant part of the debris may be assumed to be retained in the reactor coolant system). In the latter situation, if the user wishes to use the makeup decay power model, the decay power from the missing debris should be made part of the DECAY-HT input. (It could be placed in a lower cell that is purposely isolated from the rest of the problem.)

If CORCON is not invoked, the distribution among the layers in a given lower cell is handled as follows. Within the DECAY-HT input, the layer distribution keyword DIST-PWR is followed by a list of fractions, "dpwr," the sum of which should be less than or equal to one. These fractions define how much of the makeup power will be placed into each of the lower cell layers specified, plus the atmosphere. The first fraction in the list is associated with the first or bottommost layer, the second with the next layer up, and so forth. The last fraction refers to the stationary atmosphere interface. The user should note that the makeup power assigned to the atmosphere is associated with the lower cell interface and not with the atmosphere. In a multicell problem, the assigned makeup power thus does not flow with the atmosphere into another cell but remains fixed in the original cell.

For calculations in which the CORCON model is used along with the makeup decay power model, provision has been made to normalize the CORCON internally calculated rate of decay heating to the makeup power value. If CORCON is active, the total amount of decay heating in all CORCON layers is adjusted at each CORCON timestep to be equal to the makeup power, plus any explicit fission product heating, assigned to the representative CORCON intermediate layer. In addition, the makeup power assigned to the concrete layer is ignored during this time. Note that the DECAY-HT option should not be used with CORCON if core debris is added to CORCON through source tables since the fraction of total core power assigned to CORCON is fixed in time.

The makeup power model attempts (if possible) to place all power calculated by the makeup power model in the lower cell layers. If a layer has zero mass, exclusive of aerosols and fission products, the power for that layer will be assigned to the first node below that is not null. If all layers below are null layers, the energy will be lost to the basemat and will be accounted for in the WASTE repository of the mass and energy accounting scheme. If the "dpwr" fractions are not specified at all for a given cell or are all specified to be zero, the total makeup power for that cell will be calculated as it normally would but the power will not be distributed to the layers. If CORCON is being used in conjunction with the makeup power model, such input will also result in turning off the decay heat source to CORCON. 


\subsubsection{External Lower Cell Material Sources}

Material sources can be introduced into lower cell layers through user-specified source tables. Lower cell source table input follows the same format used elsewhere in CONTAIN. In theory material sources are allowed for concrete, intermediate, and pool layers; however, in practice there is seldom a need to specify sources to the concrete layer.

Provision has been made for the addition of materials to the CORCON layers in a time-dependent manner when CORCON is active. User-specified material source tables can be defined in the representative CORCON intermediate layer to specify mass addition rates and temperatures of any of the CORCON materials in Table 5-2 as functions of time. Only tables based on temperature, not enthalpy will be accepted. This is due to differences between CORCON's method of referencing its internal enthalpy values and CONTAIN's method. The material added will also be reflected in the VANESA inventories and also in the CORCON decay heat computation if CORESTAT is used and $\mathrm{UO}_{2}$ is added. Note that the VANESA fission product inventories will not be augmented when $\mathrm{UO}_{2}$ is added. If the layers are initially stratified or if the mixing model is turned on (regardless of the initial configuration), metal species will be added to the CORCON MET layer, and oxide species will be added to the LOX layer. Otherwise, if the layers are initially homogeneous and the mixing model is turned off, all user-specified material sources will be added to the CORCON HMX layer (see Section 5.3.2). Table 5-2 lists the species that can be specified in source tables to the CORCON intermediate layer. Note that material source tables for the concrete layer are not allowed when CORCON is invoked.

\subsubsection{External Lower Cell Volumetric Heating}

Energy sources may be introduced to the lower cell layers by means of the Q-VOL table option available for each layer. (Energy may also be introduced through material source tables or the DECAY-HT makeup power option as discussed in the previous two sections.) The values entered in the Q-VOL tables refer to rate of energy addition to the entire layer. If the layer has multiple nodes, this power is partitioned among the nodes with a mass weighting. If the layer is null, exclusive of aerosols and fission products, then the heating specified by the table will be directed to the first node below that is not null. During the periods that CORCON is active, the specified energy addition is ignored in all layers except the pool. In the case of a null pool, the pool energy addition is directed to the uppermost CORCON melt layer.

\subsection{CORCON - CONTAIN Interface Considerations}

CORCON has been integrated into CONTAIN so that it receives feedback from the upper cell atmosphere or the coolant pool, but aside from this connection, CORCON runs more or less independently from CONTAIN. When CORCON is invoked, CONTAIN's lower cell layer architecture is used to specify and initialize CORCON and to report results. The CORCON calculation acts much like a generator of external sources to the upper cell models of CONTAIN. Nonetheless, the implementation of CORCON into CONTAIN does present some new features or special limitations that the stand-alone version of CORCON is not subject to. Many of these have been described above. The purpose of this section is to describe any remaining special considerations resulting from the implementation of CORCON Mod3 into CONTAIN. This begins 
with a discussion of the implementation approach taken to ensure that the core physics modules are identical among the stand-alone, CONTAIN, and MELCOR implementations of CORCON Mod3.

\subsubsection{Implementation Strategy}

Major architecture changes were made to the CORCON Mod3 software to facilitate its implementation into CONTAIN. Most notably, an architecture was developed to allow CONTAIN and MELCOR implementations of CORCON Mod3 to share a common set of routines that embody the key physics models. Unique interface routines were then developed to link the common CORCON models to the CONTAIN code. A parallel effort was also undertaken to develop the necessary interface to link MELCOR to the common CORCON models. This strategy for linking CORCON to CONTAIN and MELCOR is illustrated in Figure 5-4. This approach has two key advantages:

- It ensures that the same physics modeling is used in the CONTAIN, MELCOR, and stand-alone implementations.

- Improvements made to the core physics models will be reflected in all three implementations with little or no code development effort.

As a result of this strategy, many of the subroutine names in CORCON Mod3 have been changed from the names referred to in Reference Bra93. All CORCON-related routines in the core physics module now begin with the two letters $\mathrm{CC}$, and all VANESA-related routines in the core physics module now begin with the two letters VA.

\subsubsection{Obsolete Options From CORCON Mod2}

A number of options were included in the CONTAIN implementation of CORCON Mod2 that have not been retained in the CORCON Mod3 implementation. Instead of specific options, CORCON Mod3 provides new user flexibility options that provide much of the required flexibility for sensitivity studies. These new user flexibility options have been fully implemented. Therefore, the decision was made to remove the previously provided user options that were not part of the new capability. The keywords that are no longer available include: CONCCOMP, EDITDELT, REBAR, FDELT, DIFCO, BUBD, PTBB, PTDIA, OXPDT, MOLEC, STABLE, MMCHEM, ISCHEM, VROVR. All of the input for these options is still accepted, but will produce an input warning message. If an existing input file containing one of these keywords is used, the user should check to see whether one of the new user flexibility options will provide a similar function.

\subsubsection{Nonstandard Gases Released From CORCON}

During CCIs, CORCON may release some gas species that are not included in the CONTAIN material library. Normally, such gases are treated by CONTAIN as nitrogen in molar-equivalent quantities. The NOUNKGAS optional keyword in the CORCON input block is provided to restrict the chemistry in CORCON to producing only gases in the CONTAIN material library: $\mathrm{CO}, \mathrm{CO}_{2}$, $\mathrm{H}_{2}$, and $\mathrm{H}_{2} \mathrm{O}$. 


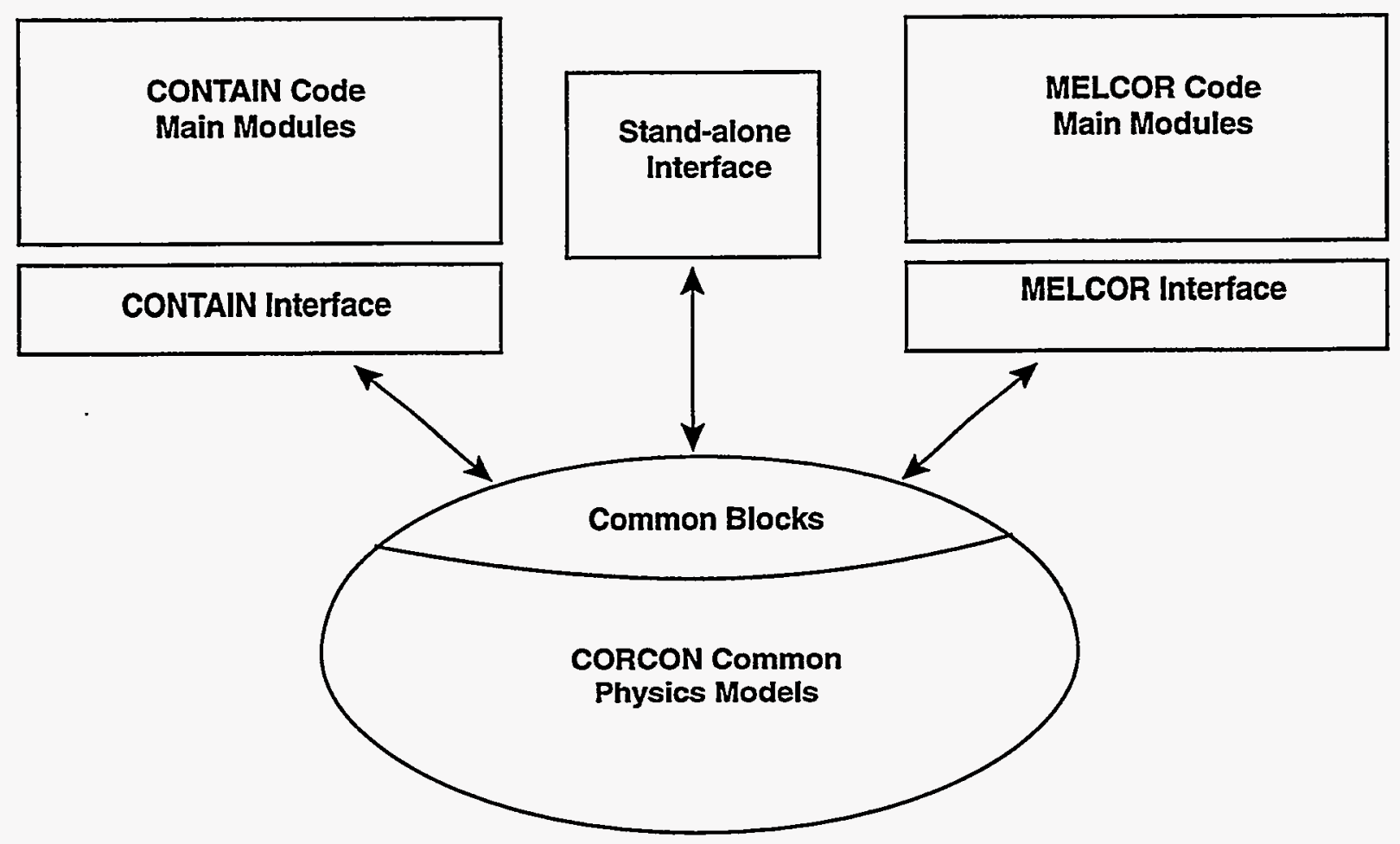

Figure 5-4. Conceptual Picture of the Implementation of CORCON Mod3 into CONTAIN and MELCOR 


\subsubsection{Interfacing CONTAIN Aerosols and Fission Products with CCI Aerosol Releases}

This section discusses the interfacing between the CONTAIN aerosol and fission product inventories and the CCI aerosol releases calculated by the VANESA submodule of CORCON. VANESA releases are expressed in terms of the constituent materials given in Table 5-5, and the released masses must in general be mapped onto the CONTAIN aerosol and fission product inventories. This mapping is assigned through the AERCONST input block for aerosols and the FPTRACK input block for fission products. Note that the CCI releases are all assumed to be in aerosol form.

Since each CONTAIN aerosol component is a possible host for fission products, the fission product mapping must specify not only the CONTAIN fission products associated with each VANESA constituent, but also the CONTAIN aerosol component to which the fission product masses are assigned. The FPTRACK input format allows the user complete flexibility in assigning the VANESA constituents to CONTAIN aerosol components and fission products. VANESA constituents may not be assigned directly to non-airborne hosts; however, this assignment may be indirectly achieved through use of the fission product targeted release formalism (see Section 8.4). Aerosols produced by the VANESA module in CORCON from CCIs may be scrubbed by an overlying coolant pool if the scrubbing model is turned on by including the SCRUB input block. This will activate the VANESA scrub model discussed in Section 7.7.1. Note that the SPARC scrubbing model that is available for scrubbing SRV aerosol sources cannot be used to scrub VANESA aerosols. If scrubbing is not modeled for CCI aerosols, the aerosols and fission products will enter the atmosphere without attenuation even in the presence of a pool.

Two schemes, SIMPLE and DETAIl, are available for mapping VANESA constituents onto the CONTAIN fission product representation. In the SIMPLE fission product tracking scheme, for each constituent in Table 5-5 that is to be tracked in the containment, a CONTAIN fission product must be defined with the exact same name. In this scheme, all releases of each fission product to be tracked will be assigned to the CONTAIN fission product with the same name. If the fission product occurs in more than one chain as a result of the linear chain decomposition (see Section 8.3), the assigned mass will be distributed among the chain elements according to the inventory factors for the fission product. The inventory factors used in the linear chain decomposition are either predefined if the fission product library is used or defined by the user for user-defined decay chains. The released mass will be associated with the aerosol component assigned in the AERCONST input block.

In the DETAIl fission product tracking scheme, the aerosol mapping is handled in the same manner as described above for the SIMPLE scheme. Unlike the SIMPLE approach, the released constituents can be mapped to any fission product defined in CONTAIN. The fission products need not be given the same name as the VANESA constituent to be tracked. This added flexibility allows the user to map different constituents to the same CONTAIN fission product. This option also allows the user to map a given VANESA constituent to more than one CONTAIN fission product, with an arbitrary distribution. 


\subsubsection{Restrictions in Mass and Energy Sources}

As discussed in Section 5.6, if CORCON has been specified, the use of material source tables and volumetric and fission product heating options for the intermediate and concrete layers is either modified or restricted. For a pool layer, these options still function as they would without CORCON. In particular, when the coolant pool layer is a null layer, the volumetric and fission product heating associated with the pool will be assigned to the first node below, even when that node is the uppermost CORCON layer. For the intermediate and concrete layers, however, these options are ignored during the time CORCON is active, with the following exceptions: (1) material source tables may still be used to add material to CORCON, but only temperatures may be specified, and (2) the DECAY-HT makeup decay power assigned to the CORCON layer, plus the decay heat of any explicit CONTAIN fission products assigned to that layer, may be used to normalize the CORCON decay power calculation.

\subsubsection{Radiative Heat Transfer to Containment Atmosphere and Surroundings}

The radiative heat transfer from the upper CORCON core-debris surface to the atmosphere may be modeled through the regular CONTAIN models. If one or more of the radiative heat transfer models discussed in Chapter 10 are invoked to model the heat transfer from the lower cell, the ones invoked will also be used to model the radiative transfer from the upper CORCON surface when it is exposed to the atmosphere. In the direct atmosphere-surface radiative transfer model discussed in Section 10.3.2 and the net enclosure model discussed in Section 10.3.1, the CORCON surface emissivity and surface area will override the CONTAIN input values for lower cell layers, when the upper CORCON surface is exposed and CORCON is active. If the net enclosure model is used with CORCON, the net enclosure input should correspond to the average geometry present when radiative transfer is important. Strictly speaking, the input is valid only for a given geometry, whereas the core-debris geometry may actually change as a function of time when CORCON is active. With time, this change in geometry will create energy conservation errors. Consequently, a warning message is given if the net enclosure radiation model is used with CORCON. In the simple lower-cell-to-structure radiative transfer model, the user-supplied value of "vufac" in the heat structure input will be used for the uppermost lower-cell layer, including the CORCON surface. However, the CONTAIN input value for the lower cell area will again be replaced by the CORCON surface area when the CORCON surface is exposed and CORCON is active. If none of the CONTAIN radiative models is specified, the internal CORCON radiative transfer model will be used as a default.

\subsubsection{Fission Product Mass Additions}

When the CORESTAT option for computing decay power is used, fission products are added to the CORCON inventories whenever UO2 is included among the materials being added to CORCON. The reason is that the fission products are assumed to be present in proportion to the mass of UO2 in the core debris and the values of the retention factors for fission products. The fission products added in association with the additional UO2 affect the decay power. However, in CONTAIN the added fission products are not used to modify the VANESA inventories. As noted in Section 5.3.2, the initial CORCON fission product inventory cannot be used to initialize the VANESA fission product inventory. VANESA fission products must be specified explicitly in the VANESA input 
block. In implementations of CORCON prior to CORCON Mod3, VANESA aerosol releases did not affect the CORCON inventories. This has been changed so that VANESA releases are now properly reflected in CORCON.

\subsection{CORCON Physics Modeling}

The physical models in CORCON Mod3 are documented in the CORCON Mod3 User's manual. [Bra93] The purpose of this section is to describe which sections of Reference Bra93 apply to the implementation of CORCON into CONTAIN. Thus, this section provides a guide to users with respect to the applicability of that reference. The first three subsections are based on sections from Reference Bra93 that summarize the modeling in CORCON Mod3 and its new features. The remaining subsections address the physical models in CORCON.

\subsubsection{Broad Capabilities of CORCON Mod3}

CORCON is a general computational model describing the interactions between molten core materials and concrete in light water reactors. The molten core debris is assumed to lie in an axisymmetric concrete cavity, with gravity acting parallel to the axis of symmetry. Several standard concretes may be used, or the user may specify a nonstandard concrete. Coolant may be present. The user also may specify addition of core material and/or coolant as a function of time.

The model includes heat transfer between core debris and concrete and between core debris and the atmosphere. If coolant is present, then the heat transfer from the core debris to the coolant is modeled. CONTAIN handles any heat and mass transfers that occur between the coolant pool and the atmosphere.

For heat transfer between the core debris and the concrete, the user may choose to model the interfacial region as a gas film or a slag film. Heat transfer across the gas film is by combined radiation and convection (the models in the code are identical to those used in CORCON Mod2). The model for heat transfer across the slag film is based on analysis of transient slag and crust growth at the interface during intermittent contact between the core debris and the concrete. Convective heat transfer between the bulk melt and the film (gas or slag) is modeled using heat transfer correlations derived for boiling and gas barbotage.

The heat transfer model for the coolant includes a representation of the full pool boiling curve. The effect of ambient pressure is included in the models for film, nucleate, and transition boiling. The effect of coolant subcooling is included in the nucleate boiling model, while the effects of subcooling and gas barbotage are included in the film boiling models. The transition boiling regime is treated using a linear interpolation with respect to the logarithm of the critical heat flux and the logarithm of the wall superheat.

Models have been added to simulate the mixing between core debris layers which occurs via droplet entrainment. Melt stratification via de-entrainment has been added as well. The user may choose to disable the mixing calculation, forcing the core debris to remain stratified into distinct oxidic and metallic layers. Here, as in CORCON Mod2, the layering configuration is determined by the relative densities of the layers. 
Both gas-phase and condensed-phase chemical reactions are modeled. The model assumes chemical equilibrium between the oxides, metals, and gases in each layer containing metals. Chemical reactions between gases and oxides in a purely oxidic layer are not treated. The user may disable the oxide-metal condensed phase reactions if desired.

CORCON Mod3 models the generation of aerosols and the release of radionuclides using the VANESA model, [Pow86] which has been fully integrated into the code. The VANESA model, which was developed originally as a stand-alone code, treats aerosol generation by vaporization and by mechanical processes (e.g., bubble bursting). Kinetic limitations to the vaporization process are considered. VANESA also models aerosol removal by an overlying water pool.

CORCON Mod3 includes a much broader range of user options than were available in CORCON Mod2. Through input the user can modify many of the more important models and parameters in the code. This capability allows the code to be applied to a broader range of accident conditions. It also allows the user greater flexibility in assessing uncertainties.

The features described above allow CORCON Mod3 to model a wide range of CCI phenomena and allow the code to be used to simulate the effects of CCIs in a wide range of severe accident scenarios. CORCON Mod3 is a state-of-the-art computer code for simulating the interaction of molten core debris with concrete in light water reactors.

\subsubsection{Improvements in CORCON Mod3}

Many improvements have been made to CORCON Mod2 during the development of CORCON Mod3. Several of the phenomenological models in CORCON Mod2 were improved, and several new models were added.

The model improvements include

- the debris-concrete heat transfer models now allow either a stable gas film or an unstable gas film with intermittent melt-concrete contact,

- the coolant heat transfer model now includes the enhancement of film boiling heat transfer by gas barbotage and coolant subcooling, and

- the models for bubble phenomena (bubble size, rise velocity, and void fraction) have been upgraded to reflect an improved understanding of bubble behavior.

The new models include

- an integrated version of the VANESA model that includes models for aerosol generation and radionuclide release from the melt, and scrubbing in overlying water pools,

- the models for condensed phase chemical reactions between oxide and metals, 
- activity coefficient models for the condensed phases (used in the aerosol generation and radionuclide release calculation),

- an aerosol scrubbing model for subcooled pools, and

- a parametric treatment of core debris spreading across the concrete floor of the reactor cavity.

In addition to these model changes, CORCON Mod3 provides the user with the capability of modifying a wide range of models and model parameters. This additional flexibility allows the code to be used in a broader range of applications.

\subsubsection{System Components (2.2 From Bra93)}

The principal components of the CORCON system are the core debris pool and the concrete cavity. An overlying coolant pool, the atmosphere, and structure surroundings above the debris are treated on the CONTAIN side of the CONTAIN-CORCON interface. Information about the concrete cavity and core debris pool components is provided in Sections 5.2 and 5.3 respectively. The coolant pool is discussed in Section 5.4. Because of differences between the CONTAIN implementation of CORCON and the stand-alone code, these sections should be consulted for information on these system components rather than Reference Bra93.

The composition of the debris pool and the concrete cavity is specified through user input in terms of a "master list" of chemical species given in Table 2.1 of Reference Bra93. As in the stand-alone code, not all of the species in the master list are available to the user for specification of initial compositions. The aluminates (species 12 to 17 ) are a holdover from the viscosity modeling of CORCON-Mod1 and are not used in CORCON-Mod3. The fission-product pseudo species (oxides 24 to 28 , metal 47 , and gases 82 to 83 ) are used in the CORCON decay power model. The initial fission product composition is determined within the code from the concentration of fission products in the fuel. The fission product composition is then updated during the calculation to account for addition of core material into the reactor cavity and release in the form of aerosols.

The VANESA model was developed using a somewhat simpler description of the melt, and considers only the major condensed phase species. VANESA focusses primarily on gas phase chemistry and it therefore includes a much more extensive species list for the gas phase. The VANESA species list is shown in Table 2.2 of Reference Bra93.

\subsubsection{Energy Generation (2.3.1 from Bra93)}

The CORESTAT decay power model included in CORCON as described in Bra93 is available in the CONTAIN implementation of CORCON. Reference Bra93 notes that the ANSI-standard decay power curve is not appropriate because of the potential for loss of important volatile radionuclides from the core debris. This problem is addressed in the CONTAIN DECAY-HT implementation of the ANSI-standard curve by allowing users to track specific radionuclides explicitly. The decay power from the explicit radionuclides in the calculation is subtracted from the ANSI-standard decay power value in the DECAY-HT model. This method is described in greater detail in Section 5.8.1. The user has the option of using the DECAY-HT model within CORCON. If this is selected, the 
decay power calculated in the DECAY-HT model for the CORCON melt layers is used to scale the decay power used in the CORCON model.

\subsubsection{Melt/Concrete Heat Transfer (2.3.2 from Bra93)}

Description of this physical process in Section 2.3.2 of Reference Bra93 is directly applicable to the CONTAIN implementation of CORCON. The default interfacial heat transfer model for both bottom and side surfaces between the debris pool and the concrete cavity is the stable gas film model. The slag film model is turned on for side surfaces with the SLAGSIDE keyword in the CORCON input block. The slag film model is turned on for bottom surfaces with the SLAGBOT keyword in the same block. The reader is referred to Section 2.3.2 of Reference Bra93 for details on the governing equations.

\subsubsection{Coolant Heat Transfer (2.3.3 from Bra93)}

Because of the importance of the boiling heat transfer correlations in both CORCON and nonCORCON calculations in CONTAIN, this section has been included in its entirety in Section 10.4. As previously noted, gas barbotage and subcooled pool effects are not included when CORCON is not invoked, since the correlations from CORCON Mod2 are used in such instances.

\subsubsection{Crust Formation and Freezing (2.3.4 from Bra93)}

The description of this physical process in Section 2.3.4 of Reference Bra93 is directly applicable to the CONTAIN implementation of CORCON.

\subsubsection{Bubble Phenomena (2.3.5 from Bra93)}

The description of this physical process in Section 2.3.5 of Reference Bra93 is directly applicable to the CONTAIN implementation of CORCON.

\subsubsection{Interlayer Mixing (2.3.6 from Bra93)}

The description of this physical process in Section 2.3.6 of Reference Bra93 is directly applicable to the CONTAIN implementation of CORCON.

\subsubsection{Pool Surface Heat Transfer (2.3.7 from Bra93)}

The description of this physical process in Section 2.3.7 of Reference Bra93 is only partially applicable to the CONTAIN implementation of CORCON. In particular, the linearization of the pool response described by Equations 112 and 113 of that reference are applicable. These equations are applied on the CORCON side of the interface to obtain a heat flux from the underlying core debris to the coolant pool. The pool boiling correlations given in Section 10.4 are used for this purpose. Heat transfer between the coolant pool and the containment atmosphere, including convection and radiation, is handled by the standard CONTAIN heat transfer models (see Chapter 10). The radiation heat flux from the atmosphere and surroundings to the pool is calculated by the radiation model and passed to the pool routine. Either the simple, the net enclosure, or the lower- 
cell-to-structure radiation model can be used with CORCON. However, as previously noted a warning is issued if the net enclosure model is used, because it assumes a fixed area for the cavity and this can lead to energy conservation problems. More detailed information about the radiation models is provided in Section 10.3.

\subsubsection{Concrete Decomposition and Ablation (2.3.8 from Bra93)}

The description of this physical process in Section 2.3.8 of Reference Bra93 is directly applicable to the CONTAIN implementation of CORCON. The model has been slightly altered to limit ablation rates to $2 \mathrm{~mm} / \mathrm{s}$ to prevent numerical problems in some of the routines. This limit is several times greater than the maximum ablation rates seen in $\mathrm{CCI}$ experiments performed to date.

\subsubsection{Time-Dependent Melt Radius Option (2.3.9 from Bra93)}

The description of this parametric model in Section 2.3.9 of Reference Bra93 is directly applicable to the CONTAIN implementation of CORCON. As noted in Bra93, as implemented this model is limited to flat-bottomed cylindrical cavities. Unlike the stand-alone implementation of CORCON Mod3, up to 100 time points may be specified. This increased number of points allows users to more realistically use the results of a mechanistic spreading calculation to drive this parametric model.

\subsubsection{Chemical Reactions (2.3.10 from Bra93)}

The description of this physical process in Section 2.3.10 of Reference Bra93 is directly applicable to the CONTAIN implementation of CORCON. Note that by default the new model for condensed phase reactions of $\mathrm{Zr}$ with silicon dioxide is by default disabled. The user must specify the CPCHEM keyword in the CORCON input block of the lower cell to enable this reaction. Another change from the stand-alone implementation is that the option to disable coking is implemented differently than as it is described in Bra93. It has been found that the approach described in Bra93, where the chemical potential of condensed carbon is set to an artificially large value, leads to slower convergence of the chemical equilibrium solver. Therefore, in the CONTAIN implementation coking is disabled by skipping the relevant reactions rather than setting the chemical potentials of condensed carbon to an artificially large value. This method was tested and shown to give equivalent results as the method described in Bra93 but with much improved computational efficiency. Note also that coking is disabled by default, whereas in previous implementations of CORCON in CONTAIN coking was enabled by default. The user must specify the COKING keyword in the CORCON input block to enable coking in the present implementation.

\subsubsection{Mass and Energy Transfer (2.3.11 from Bra93)}

The description in Section 2.3.11 of Reference Bra93 is directly applicable to the CONTAIN implementation of CORCON. 


\subsubsection{Energy Conservation (2.3.12 from Bra93)}

The description in Section 2.3.12 of Reference Bra93 is directly applicable to the CONTAIN implementation of CORCON.

\subsubsection{Cavity Shape Change (2.3.13 from Bra93)}

The description in Section 2.3.13 of Reference Bra93 is directly applicable to the CONTAIN implementation of CORCON.

\subsubsection{Aerosol Generation and Radionuclide Release (2.3.14 from Bra93)}

The description in Section 2.3.14 of Reference Bra93 is directly applicable to the CONTAIN implementation of CORCON, with the exception that the simple model for aerosol generation in CORCON Mod3 that was retained from CORCON Mod2 is not available in the CONTAIN implementation. If aerosol generation modeling is desired, the VANESA modeling must be invoked. For the interested reader, the simple model available in the stand-alone implementation of CORCON Mod3 is described near the bottom of Section 2.3.1 in Reference Bra93.

\subsubsection{Aerosol Removal By Overlying Water Pools (2.3.15 from Bra93)}

The description in Section 2.3 .15 of Reference Bra93 is directly applicable to the CONTAIN implementation of CORCON. Coolant pool behavior is also discussed in Section 5.4.

\subsubsection{Material Properties (2.4 from Bra93)}

The description in Sections 2.4.1 through 2.4.3 of Reference Bra93 is directly applicable to the CONTAIN implementation of CORCON. The equations governing the coolant pool in Section 2.4.4 are not applicable, since the coolant pool thermal hydraulic behavior is modeled on the CONTAIN side of the interface. The coolant pool equations in CONTAIN are given in Chapter 4 of the CONTAIN code manual.

\subsection{Numerical Considerations and Known Limitations}

\subsubsection{Layer Processing}

A simplified overview of the processing that occurs each timestep in the CONTAIN lower cell modules is given here. First, any radiant energy exchange between the uppermost layer and the atmosphere is taken into account. The actual radiant heat flux is computed by the upper cell radiation controller. External mass and energy sources are then added to the appropriate layers and new equilibrium conditions are found. These external sources can include sources from mechanistic upper cell models (e.g., sprays) and user-defined material source tables. The atmosphere-pool condensation model is then processed if the CONDENSE option is used (see Section 10.2.1) and a pool is present. If CORCON is not active, the interlayer heat transfer coefficients are then determined and the conduction model is called. Volumetric heating of the layers (e.g., by explicitly specified fission products, through the DECAY-HT option, and through user-specified Q-VOL 
tables) is also incorporated in the conduction solution. In either case, if a coolant pool is present, mass and energy sources to the pool are evaluated and the pool state calculated. If boiling is allowed, any energy that would raise the pool above saturation is used to boil off the pool. If CORCON is active, the conduction module is skipped because CORCON assumes a steady-state temperature profile in the concrete and models the heat transfer in the melt layers independently of CONTAIN. If the implicit flow solver is not used, boiling is evaluated in the lower cell pool routine, otherwise the boiling rate is handled in the implicit flow module.

After the completion of lower cell processing, sources accumulated in the various lower cell modules are gathered together for eventual transfer to the upper cell. If a pool is present, the gas equilibration and VANESA pool scrubbing model (SCRUB) are also invoked at this time. Any aerosol and fission product sources produced by VANESA that are not scrubbed out are transferred to the atmosphere.

\subsubsection{Assumptions and Limitations}

With the exception of the first item, the assumptions and limitations noted in Section 3.0 of Reference Bra93 also apply to the implementation of CORCON into CONTAIN. The first limitation has been relaxed by virtue of its implementation into CONTAIN. The assumptions and limitations from that reference are reproduced below for convenience. The last two items on the following list are not included in that reference.

1. The atmosphere and surroundings above the pool surface serve only to provide boundary conditions for heat and mass transfer from the pool, as the stand-alone implementation of CORCON does not include calculational procedures to update the temperature, pressure, or composition of the atmosphere or the temperature of the surroundings. The calculation of radiative heat loss from the pool surface is based on a one-dimensional model, and the convective loss is calculated using a constant heat transfer coefficient. Note: these limitations are not applicable to the implementation of CORCON in CONTAIN.

2. The calculated concrete response is based on one-dimensional steady-state ablation, with no consideration given to conduction into the concrete or to decomposition in advance of the ablation front. This assumption is probably not a source of serious error in the analysis of reactor accidents, at least for the sequences with long-term interactions between core materials and concrete. The heat fluxes involved are sufficiently large that quasi-steady ablation is approached within the first few minutes of interaction if the pool is molten; the process continues for a period of hours to days, sustained by decay heat from the fission products in the melt. The steady-state ablation assumption makes it difficult to apply the code to transient interactions that occur in the first few minutes following reactor vessel failure. The code may also be inaccurate for analysis of very long-term interactions where the debris temperature may be close to the concrete ablation temperature.

3. The solidification model is preliminary. It assumes that a crust forms on any surface whose temperature falls below the solidification temperature. The mechanical stability of the crusts is not considered. We believe that both the mechanical strength of the crust and the loads 
imposed on it by concrete decomposition gases are important in determining the true solidification behavior of the core debris.

The code also assumes that the crust has the same composition and properties as the current bulk liquid phase. This may not be true if the liquid phase composition is changing with time. Consider for example the formation of an oxide crust early in the interaction (before significant concrete ablation). This crust material will have a solidus temperature near that of the fuel oxide mixture. As concrete is incorporated into the molten phase, the molten phase solidus temperature will decrease. The code assumes that the same change in the solidus occurs for the crust. Clearly, this is not correct.

4. If the gas-film model is used, it is used for radial heat transfer even after the melt solidifies, even though the assumptions on which the model is based are no longer valid. In particular, no radial gap develops around a layer of the melt which has completely solidified. Thus, radial ablation continues with the "solid" layer continuing to conform to the changing shape of the cavity rather than behaving as a rigid penetrator. As coded, the model also assumes that the frozen material remains gas-permeable. Because the total amount of concrete eroded is largely determined by energy considerations (the available decay energy), the effect of these modeling assumptions is primarily on the calculated shape of the cavity.

5. There is no treatment of chemical reactions between the melt and the atmosphere, nor of reactions in the atmosphere. We do not consider these to be significant limitations. Meltatmosphere interactions are probably insignificant due to the limited surface area and relatively low temperature of the surface. Reactions in the atmosphere do not affect the progress of the CCIs and so can be neglected in a computer model such as CORCON. Modeling of atmosphere reactions would, however, be useful when comparing code predictions to experiment results, since the gas stream in the experiments is sampled at some distance from the melt surface.

6. The code assumes ideal chemistry when calculating bulk phase chemical reactions. Note that the code does include non-ideal chemistry modeling for vapor phase chemistry. Limitations associated with the vapor phase non-ideal modeling are discussed below in item 11.

7. The code uses flat plate pool boiling correlations to model heat transfer to an overlying coolant pool. Recent experiments show that heat transfer is greatly enhanced during the initial pour of coolant. This enhanced heat transfer may lead to rapid cooling of the melt surface, and a transition to nucleate boiling. The code predicts lower early heat fluxes than in the experiments, and long-term heat transfer by film boiling. Interestingly, the code predicts well the longer term (steady-state) coolant heat fluxes measured in the experiments.

8. The time-dependent melt radius model allows the user to mimic the spreading of a melt across a horizontal floor, but it is not a mechanistic model of spreading.

9. The code uses the same $\mathrm{Fe}-\mathrm{Cr}-\mathrm{Ni}$ phase diagram for the metal phase that was in CORCON Mod2. This treatment neglects important metallic components such as $\mathrm{Zr}$, Si, or Al that may be present in the melt at various times during a CCI. In general, these constituents will reduce 
the melting range of the metal phase relative to the range predicted by the code. Through input, the user can modify the phase diagram for the metal phase by specifying a constant solidus temperature. The liquidus temperature is then assumed to be $10 \mathrm{~K}$ greater than the specified solidus.

10. Under certain conditions, the interlayer mixing model can produce numerical instabilities, where debris layers form and disappear on alternating time steps. The source of this problem is known. Briefly, it is related to the arbitrary selection of a timestep as the duration it takes layers to mix when denser layers above less dense layers are detected. This problem is being addressed and will be corrected in future versions. In the meantime, the homogeneous layer option can be used when this is observed. Also, smaller timesteps may cause the oscillations to disappear. This limitation is not discussed in Reference Bra93.

11. The non-ideal chemistry model is presently not operational for the oxide phase. Thus the user should only use the default ideal option or the option to treat only the metal layer as non-ideal. This limitation is not discussed in Reference Bra93.

It is also worth noting that a number of sensitivity parameters are available through input to allow the user to readily explore the extent of importance of some of the approximations noted above. These sensitivity parameters are listed and briefly discussed in Section 14.3.2.3.2 under the description of the optional keywords in the USERSENS input block 


\subsection{DIRECT CONTAINMENT HEATING (DCH) MODELS}

Direct containment heating (DCH) consists of a collection of complex chemical, thermal, and physical processes resulting in the transfer of mass and energy from dispersed molten debris to the containment atmosphere and its surroundings. The CONTAIN DCH modeling capability draws from both standard containment models and from a suite of DCH-specific models. The purpose of this chapter is to describe the DCH-specific models in the CONTAIN code. Additional models important to DCH calculations (e.g., atmosphere-structure heat transfer, hydrogen combustion) are described in other sections of this manual. Guidance as to their use in DCH calculations is given in Section 13.3.2, when this use differs from normal use in non-DCH calculations.

To capture the complex interactions occurring among the various physical and chemical processes, a high degree of integration exists between the DCH and the standard models. This integrated approach to DCH modeling is consistent with the spirit of other models in CONTAIN. As is also done elsewhere in the code, detailed models are included in areas where the phenomena are well understood, and more parametric models with flexible input options are provided for phenomena that are less well understood. Parametric models are also included instead of detailed models in some cases where the phenomena are understood, but a detailed model could not be included because of inherent limitations posed by the CONTAIN control volume code architecture.

A key purpose of the DCH models in CONTAIN is to provide an analytical tool for quantifying containment loads in a DCH event. This tool is also intended to be used to gain an understanding of DCH phenomenology through the analysis and interpretation of DCH experiments. This understanding is then transferred to the use of CONTAIN in the prediction of DCH loads at full scale. Toward this end, an extensive series of CONTAIN analyses of DCH experiments has been performed, with the phenomenological insights obtained being used to develop guidance for analysis of DCH in nuclear power plant containments. [Wi195] Section 13.3.2 includes a summary of this guidance.

DCH processes can be divided into two distinct categories. The first category includes the ejection of debris under high pressure from the reactor pressure vessel (RPV), followed by the entrainment of debris from the cavity into the containment atmosphere. The second category includes the transport and trapping of debris in the containment, and the transfer of heat and chemical energy from debris to the containment atmosphere and its surroundings. Models have recently been added to CONTAIN for predicting the debris ejection and entrainment phases of the DCH process. These models are referred to collectively in this manual as the "RPV and cavity models."

In the past, the large majority of CONTAIN DCH calculations employed user-defined debris source tables in order to introduce the debris into the calculation. The new RPV and cavity models have the potential to eliminate the need for user-defined debris source tables. Because of their newness, the RPV and cavity models have not been fully validated against the available DCH database, nor have they been used to any appreciable extent in DCH full scale plant calculations. These models have been assessed against a large body of cold simulant data [Wi196]; however, they have been assessed only to a limited extent against the high temperature database. [Wil95] Use of these models is still considered exploratory. The present chapter describes the RPV models and also summarizes 
the user-defined source table capabilities. The latter are still considered the standard method of performing CONTAIN DCH calculations and guidance for defining these source tables is included in Section 13.3.1.2.2.

Section 6.1 of this chapter summarizes the DCH phenomena modeled and describes the multiple debris field modeling features. Section 6.2 describes the debris transport and intercell flow models, including the RPV and cavity models and also the options for introducing debris into the calculation via source tables when the RPV and cavity models are not used. The models for debris trapping, DCH chemistry, and DCH heat transfer are described in Sections 6.3 through 6.5. Section 6.6 extends the treatment of debris-gas heat transfer and chemical reaction to the nonairborne debris . field. Known limitations of the DCH models are summarized in Section 13.2.3 and practical guidance for the user is offered in Section 13.3.2. The description of DCH input is given in Chapter 14. Key elements of DCH models are illustrated in Figure 6-1.

\subsection{General Description of the CONTAIN DCH Model}

The DCH model is based on a multiple field representation of debris particles. With this modeling approach airborne debris can be represented by a range of particle sizes and material compositions. Any number of particle fields can be represented in the model, and each field has its own characteristic size, mass, chemical composition, and temperature. The exchange of mass and energy from the debris to the atmosphere and its surroundings are modeled in CONTAIN for each debris field. The processes that are treated are briefly discussed in the following subsection.

\subsubsection{Phenomena Modeled}

Table 6-1 lists the collection of processes contributing to the DCH phenomena that are modeled in CONTAIN. Some of these processes are modeled mechanistically; however, others are represented by parametric models that rely upon user-specified input. This input may be picked using engineering judgment or can be based upon the results of calculations performed using a stand-alone model. Table 6-1 also summarizes how the major processes that drive $\mathrm{DCH}$ are treated in the CONTAIN model. The options employing the RPV and cavity models as well as the options for user-specified debris source tables are both included in Table 6-1.

It is important to recognize that like other analyses, DCH analyses with CONTAIN require that certain information about the containment geometry be specified by the user. This includes sizes of the control volume compartments, the flow path connections, initial conditions of the containment atmosphere, structure masses and surface areas, and a variety of other information.

\subsubsection{Multiple Debris Field Modeling Features}

The CONTAIN DCH model is based on a multiple field representation of debris particles. With this modeling approach airborne debris can be represented by a range of particle sizes and material compositions. Any number of particle fields (also called "bins") can be represented in the model, and each field has its own characteristic size, mass, chemical composition, and temperature. The 


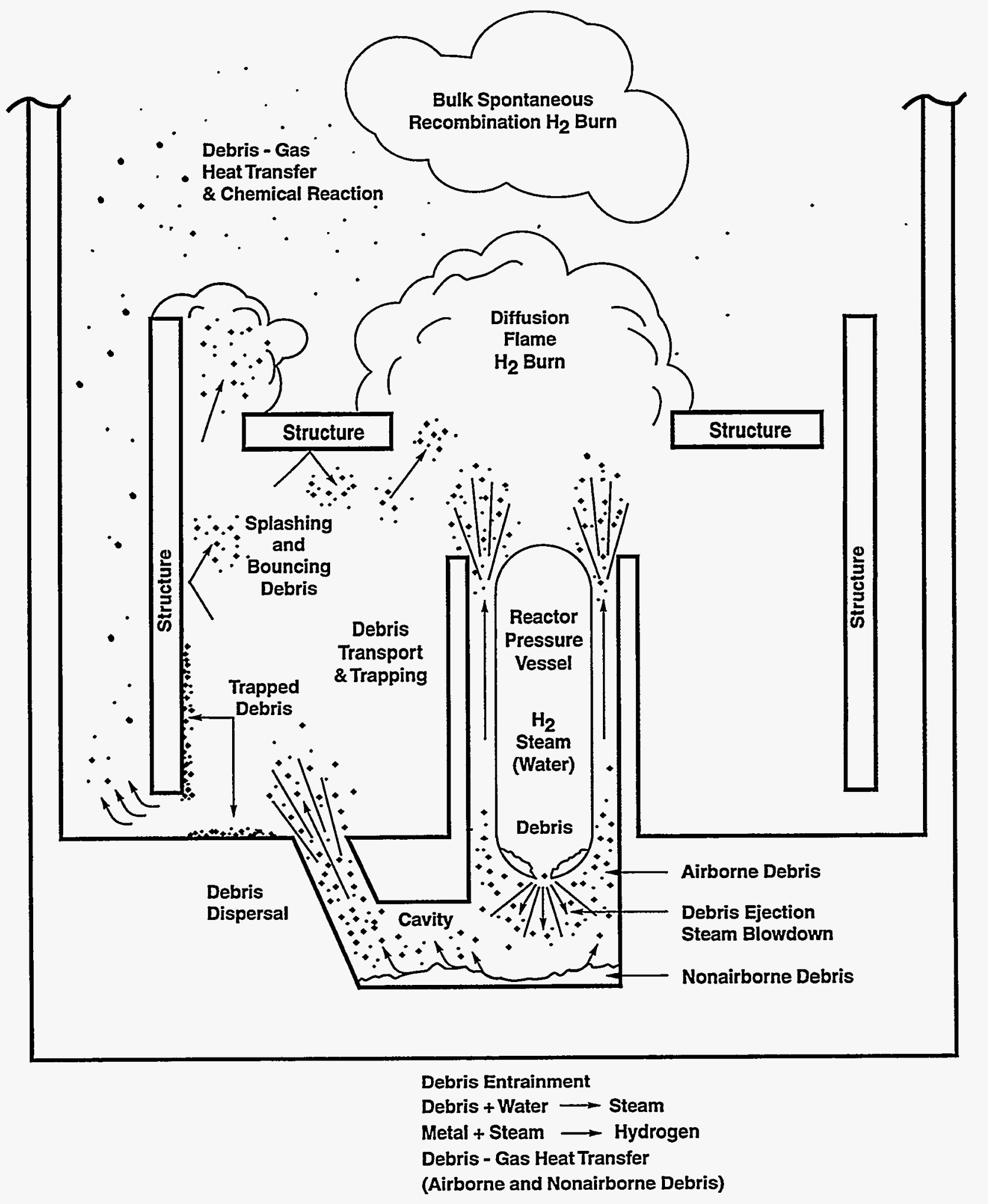

Figure 6-1. DCH as Modeled in CONTAIN 
Table 6-1

Overview of DCH Processes Modeled in CONTAIN

\begin{tabular}{|c|c|c|c|}
\hline \multirow[b]{2}{*}{ DCH Process } & \multicolumn{2}{|c|}{ Without Using the RPV and Cavity Model } & \multirow[b]{2}{*}{$\begin{array}{l}\text { With the RPV and } \\
\text { Cavity Models }\end{array}$} \\
\hline & $\begin{array}{l}\text { Representation in } \\
\text { CONTAIN }\end{array}$ & $\begin{array}{l}\text { Brief Model Description or Basis } \\
\text { for User Input }\end{array}$ & \\
\hline $\begin{array}{l}\text { Core melt ejection } \\
\text { from RPV }\end{array}$ & User input & $\begin{array}{l}\text { User-specified source tables } \\
\text { introduce debris into trapped field }\end{array}$ & Gluck correlation \\
\hline $\begin{array}{l}\text { Gas blowdown from } \\
\text { RPV }\end{array}$ & Internally calculated & $\begin{array}{l}\text { RPV modeled as a cell connected to } \\
\text { cavity with user-specified hole size } \\
\mathrm{A}(\mathrm{t})\end{array}$ & $\begin{array}{l}\text { A(t) calculated using } \\
\text { Pilch-Gluck } \\
\text { correlation [Pi192b] }\end{array}$ \\
\hline $\begin{array}{l}\text { Cavity dispersal } \\
\text { fraction }\end{array}$ & User input & Integral of cavity entrainment rate & Various correlations \\
\hline $\begin{array}{l}\text { Cavity entrainment } \\
\text { rate }\end{array}$ & User input & $\begin{array}{l}\text { Externally calculated to set the } \\
\text { mass per unit time of the source }\end{array}$ & Various correlations \\
\hline Particle size & User input & $\begin{array}{l}\text { Each field is given a mean particle } \\
\text { diameter }\end{array}$ & $\begin{array}{l}\text { Weber breakup } \\
\text { model }\end{array}$ \\
\hline Particle transport & Internally calculated & $\begin{array}{l}\text { Two-phase flow with a parametric } \\
\text { slip treatment }\end{array}$ & - \\
\hline $\begin{array}{l}\text { Trapping on } \\
\text { structures }\end{array}$ & Internally calculated & $\begin{array}{l}\text { Time of flight based on flow rate \& } \\
\text { Kutateladze criteria for first two } \\
\text { impacts; gravitational fall time } \\
\text { thereafter }\end{array}$ & - \\
\hline Chemical reactions & Internally calculated & $\begin{array}{l}\text { Gas-side \& drop-side transport } \\
\text { limitations; } \mathrm{Zr}, \mathrm{Fe}, \mathrm{Cr}, \mathrm{Al} \text { metals; } \\
\text { oxygen and steam oxidants; } \mathrm{Fe} / \mathrm{H}_{2} \mathrm{O} \\
\text { equilibrium }\end{array}$ & - \\
\hline Hydrogen burning & Internally calculated & $\begin{array}{l}\text { HECTR } 1.8 \text { correlations, } \\
\text { continuous burning, and } \mathrm{H}_{2} \text { bulk } \\
\text { spontaneous recombination at high } \\
\text { temperature and debris } \\
\text { concentrations }\end{array}$ & - \\
\hline $\begin{array}{l}\text { Convective heat } \\
\text { transfer }\end{array}$ & Internally calculated & $\begin{array}{l}\text { Forced convection: } \mathrm{N}_{\mathrm{Nu}} \text { correlation } \\
\text { for spheres }\end{array}$ & - \\
\hline $\begin{array}{l}\text { Radiation heat } \\
\text { transfer }\end{array}$ & Internally calculated & $\begin{array}{l}\text { Debris/gas and debris/wall gray } \\
\text { body law with user-specified } \\
\text { emissivity }\end{array}$ & - \\
\hline
\end{tabular}

initial values of these parameters are controlled by the user. The exchange of mass and energy from the debris to the atmosphere and its surroundings are modeled in CONTAIN for each debris field. The DCH models are evaluated multiple times, once for each field, using the composition, temperature, and other properties of that field. The composition and temperature of each field will 
evolve independently of the other debris fields in the atmosphere. Using this approach, more realism is possible than before this modeling capability was available. For example, smaller drops in the atmosphere will now react and cool off faster than larger drops if a particle size distribution is represented.

In addition to the capability to represent a range of particle sizes and compositions, there is also a capability to keep freshly dispersed debris separate, preventing it from being homogenized with previously dispersed debris. This capability is provided by modeling multiple "generations" of debris fields. When the total amount of debris introduced into all the fields defined as described above exceeds a user-specified value, the entire initial set of fields is replicated. All additional dispersed debris is introduced into the new set of fields until the total mass in it, too, reaches the user-specified maximum. Multiple generations of fields are thereby automatically provided until all the dispersed debris has been accommodated. All fields in all generations are treated in the flow, trapping, chemistry, and heat transfer models. The only distinction among the generations is that only the current generation receives newly-dispersed debris. Experience with the multigeneration capability to date suggests that time resolution often represents a relatively small effect; however, there may be cases where the time resolution feature is more important.

Figure 6-2 illustrates this concept of fields and generations. This figure also shows the trapped field that represents non-airborne debris. The non-airborne field represents trapped debris and debris in the cavity before it is entrained by the blowdown steam. Debris in the trapped field can chemically interact with and transfer heat to the blowdown steam.

The non-airborne debris field is not treated in the flow model, and is used as the repository for trapped debris in the trapping model as described in Section 6.3. The multiple generation feature does not apply to the non-airborne field.

In principle, the number of fields that can be represented is only limited by the amount of memory and CPU power available to the user. In practice, 10 or fewer fields are recommended for most calculations, not counting the additional fields generated by the multiple generation feature if it is being used.

\subsection{Debris Transport and Intercell Flow}

Modeling debris transport between control volumes requires use of the implicit flow option described in Section 14.2.4.1. Since the implicit flow model is the recommended option for almost all CONTAIN calculations, restricting the debris transport model to the implicit option should not be a serious limitation in practice.

The transport of debris in the containment is modeled as flow between control volumes using the control volume based inertial flow momentum equation shown below. In this model, debris and gas are assumed to flow together with a relative slip velocity from upstream cells to downstream cells through one or more interconnecting gas flow paths. Chapter 4 describes the intercell flow model and its solution in detail; therefore, these details will not be repeated here. The unique features of the flow model in CONTAIN when debris is present are described in this section. 


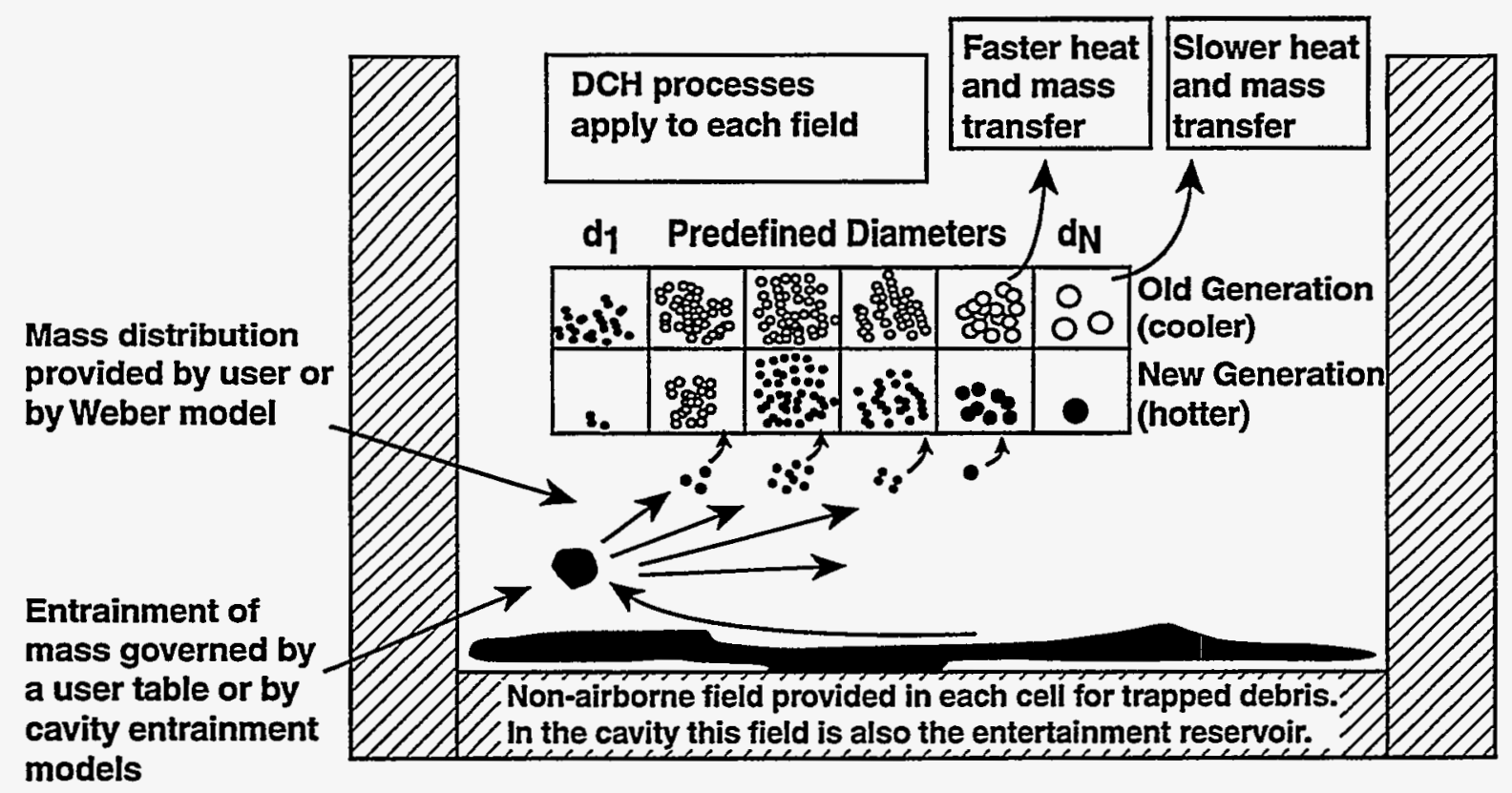

Figure 6-2. Illustration of the DCH Multifield Debris Representation in CONTAIN. In this example, 6 debris diameters and 2 generations are shown. 
The combined mass flow rate of gas and debris in a gas flow path (connecting cells $i$ and $j$ ) is governed by the following equation, within the inertial model:

$$
\begin{aligned}
& \frac{d W_{i j}^{\prime}}{d t}=\left[\Delta P_{i j}-\frac{C_{F C}\left|W_{i j}^{\prime}\right| W_{i j}^{\prime}}{\rho_{\mathrm{fl}}\left(A_{i j}^{\prime}\right)^{2}}\right] A_{i j} / L_{i j} \\
& W_{i j}=\zeta_{i j} W_{i j}^{\prime}
\end{aligned}
$$

where $W_{i j}$ is the mass flow rate of gas and debris through a flow path; $\Delta P_{i j}$ is the pressure difference between cell $i$ and $j$, including gravity head; $C_{F C}$ is the flow loss coefficient for flow path; $A_{i j}^{\prime}$ is the effective area of the flow path; $A_{i j} L_{i j}$ is the area to length ratio of the flow path; $\rho_{\mathrm{n}}$ is the effective flow density including effects of debris and gas; and $\zeta_{\mathrm{ij}}$ is the inventory factor for the flow path, as discussed in Section 4.4.

This equation is similar to the one used in the absence of DCH in CONTAIN, but with two important changes made to the variable definitions. First, the mass flow rate, $W_{i j}$, is defined here as the total flow rate of gaseous materials and debris, including all dispersed debris fields, through the flow path. The division between gas and debris is discussed in the following subsections. Second, the flow density, $\rho_{\mathrm{fl}}$, and the equivalent densities embedded in the gravitational head term of $\Delta \mathrm{P}_{\mathrm{ij}}$ are the densities of gas plus some fraction of airborne debris in each debris field in the upstream cell. The discussion in the next section on the debris/gas slip model describes how the effective flow density is obtained from the slip factor and the upstream gas and debris densities.

The detailed descriptions in Chapter 4 of the other parameters in Equation (6-1) also apply to DCH calculations; therefore, they are not repeated here. Note that the gravity head component embedded in the $\Delta \mathrm{P}_{\mathrm{ij}}$ term typically plays a small role in $\mathrm{DCH}$ calculations. DCH calculated results are also fairly insensitive to $\mathrm{C}_{\mathrm{FC}}$ values selected for flowpaths in the containment.

\subsubsection{Gas/Debris Slip Model}

The governing equations for the effective flow density, $\rho_{\mathfrak{f l}}$, which governs the debris flow rate, are based on the assumption that gas and debris flow at different velocities that can be correlated by a slip factor, s. This slip factor is defined as the ratio of the velocity of the gas phase to the velocity of the debris phase

$$
s_{n}=\frac{v_{g}}{v_{d, n}}
$$

where $s_{n}$ is the slip factor for field $n, v_{g}$ is the gas velocity, and $v_{d, n}$ is the velocity of debris in field n. The slip factor must be constant over time but can be a function of location. That is, different slip ratios can be specified for flow out of different cells. In general, the velocity of debris in fields with 
different slip factors will not be the same. The gas velocity in general will be greater than the debris velocity because the slip is constrained to be greater than or equal to one. Also, the gas velocity will always be greater than or equal to the effective flow velocity defined below, and the debris velocity will always be less than or equal to the effective flow velocity. This model for gas/debris slip was adopted from the theory of slip in two-phase fluid flow, where the ratio of the velocities of the steam and water phases are correlated with a similar slip factor. [Elw62] Note that by default slip is ignored (i.e., $\mathrm{s}_{\mathrm{n}}=1$ for all fields and all cells).

The sum of the gas and debris mass and momentum fluxes must add up to the total mass and momentum flux. Therefore, the following equations must hold true

$$
\rho_{\mathrm{fl}} \mathrm{v}_{\mathrm{fl}}=\rho_{\mathrm{g}, \mathrm{u}} \mathrm{v}_{\mathrm{g}}+\sum_{\mathrm{n}}^{\mathrm{N}_{\mathrm{fedld}}} \rho_{\mathrm{d}, \mathrm{u}, \mathrm{n}} \mathrm{v}_{\mathrm{d}, \mathrm{n}}
$$

and

$$
\rho_{\mathrm{fl}} \mathrm{v}_{\mathrm{fl}}^{2}=\rho_{\mathrm{g}, \mathrm{u}} \mathrm{v}_{\mathrm{g}}^{2}+\sum_{\mathrm{n}}^{\mathrm{N}_{\text {feclds }}} \rho_{\mathrm{d}, \mathrm{u}, \mathrm{n}} \mathrm{v}_{\mathrm{d}, \mathrm{n}}^{2}
$$

where $\rho_{\mathrm{fl}}$ is the effective flow density including effects of debris and gas; $\mathrm{v}_{\mathrm{fl}}$ is the effective velocity through flow path including effects of debris and gas; $\rho_{\mathrm{g}, \mathrm{u}}$ is the density of gas in upstream cell which is equal to the mass of gas in the upstream cell divided by the cell volume; $v_{g}$ is the velocity of gas through the flow path; $\mathbf{N}_{\text {fields }}$ is the number of airborne debris fields; $\rho_{\mathrm{d}, \mathrm{u}, \mathrm{n}}$ is the density of debris from field $\mathrm{n}$ in the upstream cell which is equal to the mass of debris in field $\mathrm{n}$ in the upstream cell divided by the cell free volume; and $v_{d, n}$ is the velocity of debris in field $n$ through the flow path.

The above equations can be solved for the effective flow density, $\rho_{\mathrm{fl}}$, and the velocity relationships, $\mathrm{v}_{\mathrm{g}} / \mathrm{v}_{\mathrm{fl}}$ and $\mathrm{v}_{\mathrm{d}, \mathrm{r}} / \mathrm{v}_{\mathrm{ff}}$,

$$
\begin{aligned}
& \rho_{\mathrm{fl}}=\zeta\left[\rho_{\mathrm{g}, \mathrm{u}}+\sum_{\mathrm{n}=1}^{N_{\mathrm{fedcls}}} \frac{\rho_{\mathrm{d}, \mathrm{u}, \mathrm{n}}}{s_{\mathrm{n}}}\right] \\
& \frac{\mathrm{v}_{\mathrm{g}}}{\mathrm{v}_{\mathrm{fl}}}=\zeta \\
& \frac{\mathrm{v}_{\mathrm{d}, \mathrm{n}}}{\mathrm{v}_{\mathrm{fl}}}=\frac{\zeta}{s_{\mathrm{n}}}
\end{aligned}
$$


where

$$
\zeta=\frac{\left|\rho_{\mathrm{g}, \mathrm{u}}+\sum_{\mathrm{n}=1}^{N_{\text {fields }}} \frac{\rho_{\mathrm{d}, \mathrm{u}, \mathrm{n}}}{s_{\mathrm{n}}}\right|}{\rho_{\mathrm{g}, \mathrm{u}}+\sum_{\mathrm{n}=1}^{N_{\text {felds }}} \frac{\rho_{\mathrm{d}, \mathrm{u}, \mathrm{n}}}{\mathrm{s}_{\mathrm{n}}^{2}} \mid}
$$

Note that when all slip ratios are one, the effective flow density, $\rho_{\mathrm{ff}}$, reduces to the sum of the gas and debris densities, which is equivalent to the heavy-gas no slip assumption. Also, as $s_{n}$ tends toward infinity, $\rho_{\mathrm{fl}}$ tends toward $\rho_{\mathrm{g}}$ as one would physically expect.

From the above equations one can write the atmosphere fluid, or gas, flow rate, $W_{g}$, and the debris field mass flow rate for each field, $W_{d}$, in terms of the total combined debris and gas flow rate, $W_{\mathrm{ij}}$,

$$
\begin{aligned}
& W_{g, i j}=\frac{\zeta \rho_{g, u}}{\rho_{f i}} W_{i j} \\
& W_{d, i j, n}=\frac{\zeta \rho_{d, u, n}}{s_{n} \rho_{\mathrm{fl}}} W_{i j}
\end{aligned}
$$

where $W_{g, i j}$ is the mass flow rate of gas, and $W_{d, i j, n}$ is the mass flow rate of debris in field $n$. Physically, we know that the sum of $W_{g, i j}$ and $W_{d, i j, n}$ over all fields must be equal to the total mass flow rate, $W_{\mathrm{ij}}$. It can be shown from the above two equations that this is true.

Gas and debris mass and energy conservation equations are shown in the following sections based on the expressions derived above. Note that the variable $\zeta$ defined in Equation (6-8) turns out to be important for evaluating the effect of flow on the distribution of gases and debris. A different value of $\zeta$ is calculated for each cell depending upon the amount of debris in each field in the cell and the slip parameter in each field in the cell.

\subsubsection{Gas Mass Conservation}

The net gas flow term in the gas mass conservation equation for cell $i$ is written in terms of the gas flow rate $W_{g, j}$ into cell $i$, and $W_{g, i j}$ out of cell $i$

$$
W_{f l o w, i, k}=\sum_{j i}\left[\frac{W_{g, j i} \theta_{j i} m_{g, j, k}}{\sum_{k}^{N_{g}} m_{g, j, k}}\right]-\left[\sum_{i j} W_{g, i j} \theta_{i j}\right]\left[\frac{m_{g, i, k}}{\sum_{k}^{N_{g}} m_{g, i, k}}\right]
$$


where the $\mathrm{ji}$ sum includes only unsubmerged gas flow paths, $\mathrm{W}_{\text {flowi,k }}$ is the net rate of change in mass of gas species $\mathrm{k}$ in cell $\mathrm{i}$ resulting from flow into and out of the cell, $\mathrm{W}_{\mathrm{g}, \mathrm{j}}$ is the flow rate of only gas (see Equation (6-9)) from cell $\mathrm{i}$ to $\mathrm{j} ; \theta_{\mathrm{ij}}$ is a conditional function ( 1 if $\mathrm{W}_{\mathrm{g}, \mathrm{j}}$ is positive, 0 otherwise); $\mathrm{m}_{\mathrm{g}, \mathrm{i}, \mathrm{k}}$ is the mass of gas species $\mathrm{k}$ in cell $\mathrm{i}$; and $\mathrm{N}_{\mathrm{g}}$ is the number of gas species in the cell.

Note that the above expression does not contain terms that include debris. Rather than using this equation directly, it is more convenient to incorporate Equation (6-9) into this equation to express $\mathrm{W}_{\text {flow, }, \mathrm{k}, \mathrm{in}}$ terms of the total effective flow rate, $\mathrm{W}_{\mathrm{fl}}$. This is done because $\mathrm{W}_{\mathrm{fl}}$ is determined directly from the flow equation (i.e., it is the flow rate actually calculated in the code), while $\mathrm{W}_{\mathrm{g}, \mathrm{j}}$ is not. For flow from cell $\mathrm{i}$ to cell $\mathrm{j}, \mathrm{W}_{\mathrm{fl}}$ is expressed as $\mathrm{W}_{\mathrm{ij}}$ in the equations below to remain consistent with the nomenclature in Chapter 4. Substituting Equations (6-5) through (6-9) into Equation (6-11), it can be shown that $\mathrm{W}_{\text {flow, }, \mathrm{k}}$ can be expressed as

$$
\begin{aligned}
& \mathrm{W}_{\text {flow }, \mathrm{i}, \mathrm{k}}=\sum_{\mathrm{ji}}\left[\frac{\mathrm{W}_{\mathrm{ji}} \theta_{\mathrm{ji}} \zeta_{\mathrm{j}} \mathrm{m}_{\mathrm{g}, \mathrm{j}, \mathrm{k}}}{\mathrm{m}_{\mathrm{F}, \mathrm{j}}}\right] \\
&-\left[\sum_{\mathrm{ij}} \mathrm{W}_{\mathrm{ij}} \theta_{\mathrm{ij}}\right]\left[\frac{\zeta_{\mathrm{i}} \mathrm{m}_{\mathrm{g}, \mathrm{i}, \mathrm{k}}}{\mathrm{m}_{\mathrm{F}, \mathrm{i}}}\right] \\
& \mathrm{m}_{\mathrm{F}, \mathrm{i}}=\zeta_{\mathrm{i}}\left[\sum_{\mathrm{k}=1}^{N_{\mathrm{g}}} \mathrm{m}_{\mathrm{g}, \mathrm{i}, \mathrm{k}}+\sum_{\mathrm{n}=1}^{N_{\text {ferds }}} \sum_{\mathrm{k}=1}^{N_{\mathrm{DCH}}} \frac{\mathrm{m}_{\mathrm{d}, \mathrm{i}, \mathrm{n}, \mathrm{k}}}{\mathrm{s}_{\mathrm{i}, \mathrm{n}}}\right]
\end{aligned}
$$

where $\theta_{\mathrm{ij}}$ is the conditional function: 1 if $\mathrm{W}_{\mathrm{ij}}$ is positive, 0 otherwise; $\zeta_{\mathrm{i}}$ is the calculated slip flow parameter for flow out of cell $i$ (see Equation (6-8)); $m_{g, i, k}$ is the mass of gas species $k$ in cell $i ; N_{g}$ is the number of gas species in a cell; $\mathrm{N}_{\text {fields }}$ is the number of debris fields; $\mathrm{N}_{\mathrm{DCH}}$ is the number of debris species in a cell; $m_{d, i, n, k}$ is the mass of debris species $k$ in field $n$ of cell $i$; and $s_{i, n}$ is the userspecified slip parameter for field $\mathrm{n}$ for flow out of cell $\mathrm{i}$.

Note that no assumptions were made in going from Equation (6-11) to Equation (6-12); thus, they are functionally identical. Equation (6-11) shows the physical basis of the model more clearly than Equation (6-12), while the latter shows how the model is actually implemented in the code. Equation (6-12) also clearly shows that these terms of the mass conservation equation reduce to the proper limits in the absence of debris, where $\zeta$ will be one and $m_{F, j}$ and $m_{F, j}$ will be the total mass of gas species only.

The above flow terms are now combined with other source and sink terms to write the mass conservation equation for gases in a DCH calculation 


$$
\begin{aligned}
\frac{d m_{g, i, k}}{d t} & =\sum_{j i}\left[\frac{\theta_{j i} W_{j i} \zeta_{j} m_{g, j, k}}{m_{F, j}}\right] \\
& -\left[\sum_{i j} \theta_{i j} W_{i j}\right]\left[\frac{\zeta_{i} m_{g, i, k}}{m_{F, i}}\right] \\
& +\left[\frac{d m_{g, i, k}}{d t}\right]_{c h e m}+\left[\frac{d m_{g, i, k}}{d t}\right]_{\text {non-DCH }}
\end{aligned}
$$

where $m_{g, i, k}$ is the mass of gas species $\mathrm{k}$ in the cell $\mathrm{i} ;[\mathrm{dm} / \mathrm{dt}]_{\text {chem }}$ represents the gas mass changes resulting from $\mathrm{DCH}$ chemical reactions (this will only be non-zero for $\mathrm{O}_{2}, \mathrm{H}_{2}$, and $\mathrm{H}_{2} \mathrm{O}$ species); and $[\mathrm{dm} / \mathrm{dt}]_{\text {non-DCH }}$ represents the gas mass sources and sinks from non-DCH processes, including the effects of gases from submerged flow paths.

The first two terms account for gas flow taking slip into consideration. The governing equations for the DCH chemical reactions are given in Section 6.4. The non-DCH processes are discussed in Chapter 4 and are therefore not described here.

The gas energy, debris mass, and debris energy conservation equations are provided in the following sections in a form similar to Equation (6-14). The above derivation is also applicable to the flow terms in the forthcoming expressions and is therefore not repeated.

\subsubsection{Gas Energy Conservation}

The energy conservation equation for the atmosphere in a DCH calculation is

$$
\begin{aligned}
\frac{d U_{i}}{d t} & =\sum_{j i}\left[\frac{\theta_{j i} W_{j i} \zeta_{j j}\left(U_{j}+P_{j} V_{j}\right)}{m_{F, j}}\right] \\
& -\left[\sum_{i j} \theta_{i j} W_{i j}\right]\left[\frac{\zeta_{i}\left(U_{i}+P_{i} V_{i j}\right)}{m_{F, i}}\right] \\
& +\left[\frac{d Q_{g, i}}{d t}\right]_{c h e m}+\left[\frac{d Q_{i}}{d t}\right]_{n o n-D C H} \\
& +\sum_{n=1}^{N_{\text {fedds }}}\left\{\left[\frac{d Q_{d, i, n}}{d t}\right]_{\text {conv }}+\left[\frac{d Q_{d, i, n}}{d t}\right]_{\text {rad, }}\right\}
\end{aligned}
$$


where $U_{i}$ is the internal energy in cell $i ; P$ is the pressure in cell $i ; V$ is the volume of cell $i$; $[\mathrm{dQ} / \mathrm{dt}]_{\mathrm{chem}}$ is the chemical energy gain rate (or loss rate, if negative); [dU/dt $]_{\text {non-DCH }}$ is the energy sources and sinks from non-DCH processes; $[\mathrm{dQ} / \mathrm{dtt}]_{\text {conv }}$ is the convective heat transfer from debris to gas; and $[\mathrm{dQ} / \mathrm{dt}]_{\mathrm{rad}, \mathrm{g}}$ is the radiative heat transfer from debris to gas.

The first two terms represent the net rate of change in atmosphere energy resulting from flow. The third term represents the energy added to the atmosphere from $\mathrm{DCH}$ chemical reactions as described in Section 6.4. This includes the local recombination energy of DCH-generated hydrogen. It does not include the debris-gas chemical reaction energy as this energy is added to the debris field. The fourth term represents energy sources and sinks from non-DCH processes. The fifth term represents convective heat transfer from debris to gas as described in Section 6.5.1 This term is equal in magnitude but opposite in sign to the counterpart term in the debris energy conservation equation given in Section 6.2.5. The last term represents radiation heat transfer from debris to gas as described in Section 6.5.2. Note that this does not include radiation from debris to structures, whereas the counterpart radiation term in the debris energy conservation equation does.

\subsubsection{Debris Mass Conservation}

The mass conservation equation for debris species $k$, debris field $n$, and cell $i$ is given by

$$
\begin{aligned}
& \frac{d m_{\mathrm{di}, \mathrm{n}, \mathrm{k}}}{\mathrm{dt}}=\sum_{\mathrm{ji}}\left[\frac{W_{\mathrm{ji}} \theta_{\mathrm{ji}} \zeta_{\mathrm{j}} \mathrm{m}_{\mathrm{d}, \mathrm{j}, \mathrm{k}, \mathrm{k}}}{\mathrm{s}_{\mathrm{j}, \mathrm{n}} \mathrm{m}_{\mathrm{F}, \mathrm{j}}}\right]
\end{aligned}
$$

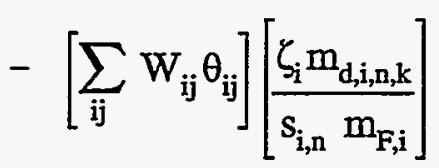

$$
\begin{aligned}
& +f_{n, k}\left[\frac{d m_{d, i, k}}{d t}\right]_{s r c}-\lambda_{i, n} m_{d, i, n, k}+\left[\frac{d m_{d, i, n, k}}{d t}\right]_{c h e m}
\end{aligned}
$$

where $m_{d, i, n, k}$ is the mass of debris species $k$ in field $n$ in the cell $i ; s_{i, n}$ is the slip factor for flow of debris in field $n$ out of cell $i ; f_{n, k}$ is the user-specified distribution factor for the source of debris species $\mathrm{k}$ into field $\mathrm{n}$; [ $\mathrm{dm} / \mathrm{dtt}]_{s c}$ is the external source of debris species $\mathrm{k}$ into cell $\mathrm{i}$, or the entrainment of debris species $\mathrm{k}$ from the non-airborne field in cell $\mathrm{i} ; \lambda_{\mathrm{i}, \mathrm{n}}$ is the debris trapping rate for field $\mathrm{n}$ in cell $\mathrm{i}$ (same for all species); and $[\mathrm{dm} / \mathrm{dt}]_{\text {chem }}$ is the debris mass change resulting from $\mathrm{DCH}$ chemical reactions. Note that $[\mathrm{dm} / \mathrm{dt}]_{\mathrm{scc}}$ is non-zero only for the current generation.

The first two terms on the right side of this equation represents the inflow and outflow of debris species $\mathrm{k}$ in field $\mathrm{n}$ in cell $\mathrm{i}$ from and to other cells, respectively. External sources of debris species $\mathrm{k}$ into field $\mathrm{n}$ of cell $\mathrm{i}$ are represented by the third term. The total source rate of debris given by the third term is the sum of any user-specified entrainment source tables, user-specified tables of debris 
entering directly into the atmosphere, and sources calculated by entrainment models. Section 6.2.8 summarizes these three methods of introducing debris into the airborne debris fields.

The $f_{n, k}$ factors are applied to the total source rate for each species to distribute the mass among the various fields. This two-dimensional parameter is specified by the user as an array in the DCH global input section following the FDISTR keyword. This is the parameter that governs how chemical species are initially distributed into fields when they enter the airborne debris fields. For example, suppose that two fields are being represented in a calculation. Dispersed metals could be separated from dispersed oxides by specifying:

$$
\begin{aligned}
& f_{1, k}=1 \text { and } f_{2, k}=0 \text { for } k=\text { metals } \\
& f_{1, k}=0 \text { and } f_{2, k}=1 \text { for } k=\text { oxides }
\end{aligned}
$$

A matrix of factors, $f_{n, k}$, is specified for the first generation of fields and all species represented in a DCH calculation to determine how sources are distributed among the fields. When the first generation fills to its capacity, a second generation of fields is generated. The first group of fields then ceases to be current and the second group becomes current. Becoming current means that $f_{n, k}$ is shifted down such that the values for the first generation of field types are all zero, and the values for the second generation of field types are set to the specified $f_{n, k}$ values. This process repeats as more generations fill and new ones are generated. The user has the flexibility to control whether or not the multiple generation feature of the DCH model will be used by specifying the number of field types and field generations. The rate at which new generations is created is governed by the userspecified maximum allowed mass in a generation of field types (see GRPLIM keyword in Section 14.2.7). By default only one generation of field types will be modeled. When metals oxidize, oxides normally stay in the same field as their parent metal. An option (see PRODSEP keyword) is provided to allow oxides to be separated from metal when one generation is used. The PRODSEP option can only be used when there is one generation; oxides are never separated from their parent metals when there is more than one generation.

The fourth term represents mass losses resulting from trapping. The governing equation for calculating the trapping rate, $\lambda$, and other details of the trapping model are described in Section 6.3. The last term in the debris mass conservation equation represents mass changes resulting from chemical reactions. The model includes reactions for $\mathrm{Zr}, \mathrm{Fe}, \mathrm{Cr}$, and $\mathrm{Al}$ metals. For reactive metals this term is negative. It is positive for the corresponding oxides of these reactive metals. The chemistry model and the governing equations are described in Section 6.4.

\subsubsection{Debris Energy Conservation Equations}

The energy conservation equation for debris in field $\mathrm{n}$ in cell $\mathrm{i}$ is given by 


$$
\begin{aligned}
\frac{d U_{d, i, n}}{d t} & =\sum_{j i}\left[\frac{\theta_{j i} W_{j i} \zeta_{j} U_{d, j, n}}{s_{j, n} m_{F, j}}\right] \\
& -\left[\sum_{i j} \theta_{i j} W_{i j}\right]\left[\frac{\zeta_{i} U_{d, i, n}}{s_{i, n} m_{F, i}}\right] \\
& +\sum_{k=1}^{N_{d c h}}\left\{f_{n, k}\left[\frac{d m_{d, i, k}}{d t}\right]_{s r c} h_{k}\left(T_{s r c}\right)-\lambda_{i, n} m_{d, i, n, k} h_{k}\left(T_{i, n}\right)\right\} \\
& +\left[\frac{d Q_{d, i, n}}{d t}\right]_{c h e m}-\left[\frac{d Q_{d, i, n}}{d t}\right]_{c o n v}-\left[\frac{d Q_{d, i, n}}{d t}\right]_{\mathrm{rad}}
\end{aligned}
$$

where $U_{d, i, n}$ is the total internal energy of debris field $n$ in cell $i ; h_{k}(T)$ is the specific enthalpy of debris material $\mathrm{k}$ at temperature $\mathrm{T} ; \mathrm{T}_{\mathrm{src}}$ is the temperature of the source debris; and $\mathrm{T}_{\mathrm{i}, \mathrm{n}}$ is the temperature of debris in field $\mathbf{n}$ in cell $i$.

The first five terms of the energy conservation equation parallel the first five terms of the debris mass conservation equation. The first two terms on the right hand side represent the inflow and outflow of energy resulting from intercell flow. The third term corresponds to the addition of energy from debris sources, such as those to represent debris ejection from the RPV or entrainment in the cavity. $\mathrm{T}_{\mathrm{scc}}$ will be the temperature of the non-airborne debris field if the source represents an entrainment rate (i.e., specified as an ENTRAIN type source table or calculated by an entrainment model). Otherwise, $T_{s c}$ will be the temperature specified in the source table. The energy associated with all of the material species that are introduced into the debris field are added together, since one energy conservation equation is used to represent all species in the field. A separate mass conservation equation is used for each species of a field. The fourth term is the energy loss associated with debris trapping, and the fifth term corresponds to the energy release from chemical reactions. The contributions from each species are added together for the trapping term. Trapping is discussed in detail in Section 6.3. The equations that govern the chemical energy term are provided in Section 6.4. Note that the chemical energy includes debris/gas chemical interactions. The energy resulting from the recombination of hydrogen produced in $\mathrm{DCH}$ with local oxygen is not included in this term, since this energy is added to the atmosphere.

The sixth term represents convective heat transfer between airborne debris and the cell atmosphere. The DCH convective heat transfer model is described in Section 6.5.1. The seventh and last term represents radiative heat transfer from airborne debris to the cell atmosphere and surrounding structures (including a coolant pool if one is present). The radiation model is described in Section 6.5.2. Convection and radiation are done on a field basis, not a species-specific basis; therefore, there is no need to have a summation over species for these two terms. There is no term for decay heat because the time scale for DCH is assumed to be sufficiently short that decay heating of 
airborne particles can be neglected. Fission products are not allowed to be hosted to debris fields for this reason.

The specific enthalpy functions, $h_{k}(T)$, are represented by user-supplied debris property tables given in the CONTANN input file as described in Section 14.2.1.2.

The total internal energy of a debris field, $U_{d, i, n}$, must not only satisfy the above equation, but must also equal the sum of the enthalpies of the debris species in the field. This relationship

$$
U_{d, i, n}=\sum_{k=1}^{N_{D C H}} m_{d, i, n, k} u_{k}\left(T_{i, n}\right)
$$

defines the temperature of each debris field. There is only one temperature that will satisfy the above equation. Obviously, this temperature must be solved for numerically.

\subsubsection{Choked Flow}

In the choked flow model an ideal gas choked flow expression is applied as an upper limit to the calculated intercell gas flow rate. This is done indirectly, since the code actually calculates first a total gas/debris flow rate and then determines the gas and debris flow rates from the specified slip factors. Given this approach, a maximum allowable total flow rate under choked flow conditions must be determined. Equation (6-9) provides the required relationship between the gas flow rate and the total flow rate, since this condition must also apply when the flow is choked. Therefore, the total flow rate limit under critical flow conditions must be given by

$$
\begin{aligned}
W_{c r, i j} & =W_{c r, g, i j}\left(\frac{\rho_{\mathrm{fl}}}{\zeta \rho_{\mathrm{g}}}\right) \\
& =\left(\theta_{\mathrm{ij}}-\theta_{\mathrm{ji}}\right) A^{\prime}{ }_{\mathrm{ij}} \mathrm{v}_{\mathrm{ij}}\left[\gamma_{\mathrm{u}} \mathrm{P}_{\mathrm{u}} \rho_{\mathrm{u}} \eta_{\mathrm{u}}\right]^{1 / 2}\left(\frac{\rho_{\mathrm{fl}}}{\zeta \rho_{\mathrm{g}}}\right)
\end{aligned}
$$

where $W_{c r, j}$ is the total critical flow rate; $W_{c r, g, j}$ is the critical gas flow rate; $A_{\mathrm{ij}}^{\prime}$ is the effective flow area; $v_{i j}$ is the vena contracta factor; $\gamma_{u}$ is the ratio of specific heat $\left(c_{p} / c_{v}\right)$ for gas in the upstream cell; $P_{u}$ is the pressure in the upstream cell; $\rho_{u}$ is the gas density in the upstream cell; and $\eta_{u}$ is a dimensionless parameter given by

$$
\eta_{u}=\left[\frac{2}{1+\gamma_{u}}\right]^{\frac{\gamma_{u}+1}{\gamma_{u}-1}}
$$


Note that only gas is considered in the heat capacity ratio. The vena contracta parameter is specified by the user and is used in place of the flow coefficient under choked conditions. This parameter is generally less than unity, and is defined as the ratio of the minimum area intersected by the flow streamlines to the geometric cross-sectional area of the flow path. [Lam45]

The last term in the critical flow rate equation is present to convert the critical gas flow rate to an effective maximum allowable total flow rate. By using this expression to calculate the total maximum flow rate, the gas flow rate will be limited to the ideal gas choked flow rate, and the debris flow rate will meet the specified gas-debris slip velocity criterion. At any time, the total flow rate $\mathrm{W}_{\mathrm{ij}}$ is given by the minimum of $\mathrm{W}_{\mathrm{ij}}$ from the conservation equations presented earlier and $\mathrm{W}_{\mathrm{c}, \mathrm{ij}}$

$$
\left|W_{i j}\right|=\min \left(\left|W_{i j}\right|,\left|W_{c r, i j}\right|\right)
$$

This choked flow model can overestimate flow rates when slip factors are small; see Section 13.3.1.4 for some additional discussion.

\subsubsection{Numerical Considerations of the Debris Intercell Flow Model}

From a numerical standpoint, it is important to understand that the code correctly solves the debris mass conservation equations, the gas mass conservation equations, the debris energy conservation equations, the gas energy conservation equations, and the inertial flow equation for the debris and gas flow rates simultaneously. This solution also includes the evaluation of cell pressures, cell temperatures, and coolant pool conditions. It is also important to understand that the debris to gas heat transfer and chemistry are explicitly coupled to these flow equations. This explicit coupling is what causes small timesteps to be required in performing DCH calculations with CONTAIN. The last two terms of the debris mass conservation equation are also calculated outside of the implicit flow solver. The explicitly coupled rates are timestep averaged values evaluated as the mass change from the process divided by the current calculational timestep.

There are $\mathrm{N}_{\mathrm{DCH}} \times \mathrm{N}_{\text {fields }}$ (one for each debris material in each debris field and generation) mass conservation equations and $\mathrm{N}_{\text {fields }}$ energy conservation equations that are solved by the code for each flow path connected to a given cell. This includes fields in the current generation and all previous generations. These same equations are then solved for all of the other cells and the flow paths that are connected to them. It should be obvious from the above discussion why DCH calculations can be computationally intensive relative to other CONTAIN calculations.

\subsubsection{Blowdown and Debris Sources}

DCH calculations necessarily must provide representations for blowdown steam and debris entering the reactor cavity from the primary system. Since CONTAIN does not include detailed primary system modeling, some means of making this information available to the code is required. There are three means of introducing the blowdown steam and gas into the calculation: 
1. Conventional atmospheric source tables representing steam and gas (e.g., hydrogen) may be defined in the cavity cell; see Sections 14.3.1.2 and 14.4.1 for the specification of atmospheric source tables.

2. A CONTAIN cell representing the primary system may be defined and filled with highpressure steam and gas at the start of the calculation, with a flow path having an appropriate time-dependent orifice area connecting the RPV cell to the cavity. The code then calculates the blowdown rate.

3. The new RPV models may be used to calculate the time-dependent flow area available for blowdown, as described in Section 6.2.9.

Likewise, there are three ways in which debris sources may be introduced into the problem:

1. Atmospheric source tables of the debris materials may be introduced in the cavity cell. The time dependence of these sources is normally defined to represent the rate at which debris is entrained by the blowdown steam, not the rate at which debris is ejected from the vessel.

2. The debris may be first introduced into the cavity trapped field using an atmosphere debris source of type TRAPBIN, and then transferred into the cavity cell atmospheric field(s) with debris source tables of type ENTRAIN; the input description is given in Section 14.3.1.2. The first set of tables may be thought of as representing melt ejection from the vessel, and the second represents melt entrainment by the blowdown steam.

3. The new RPV models (described in Section 6.2.9) and cavity entrainment models (described in Section 6.2.10) may be used to represent debris ejection from the RPV and its subsequent entrainment by the blowdown steam.

The first two methods may be used for the blowdown and debris sources in any combination with one another; the third method must be used in conjunction with the RPV and cavity models. Blowdown and debris sources may be introduced into any number of cells when the first two methods are used; when the third method is used, only one cell can be designated as an RPV cell and only one cavity cell can be designated. However, the blowdown and debris sources calculated could be supplemented by sources specified using the first two methods in either the same cell or in other cells.

If the RPV and cavity models are not being used, the user must determine the appropriate timing for both the blowdown and the debris sources. In the past, this was sometimes done with side calculations using stand-alone models. However, extensive analyses of experimental data has led to the suggestion that a simpler approach based upon experimental results for blowdown and debris entrainment rates may be just as satisfactory. Guidance for using this approach is summarized in Section 13.3.2. When this approach is being used, it is recommended that the second of the methods summarized above be used for both the blowdown sources and the debris entrainment sources. Chapter 15 gives an example of a DCH calculation for the Sequoyah plant in which this approach is used. 


\subsubsection{Reactor Pressure Vessel (RPV) Models}

The purpose of Sections 6.2.9 and 6.2.10 is to discuss RPV- and cavity-specific models in CONTAIN. The RPV models include vessel hole ablation, single-phase debris discharge, gas blowthrough, and two-phase debris/gas discharge. The cavity models include a number of correlations for the entrainment rate of debris and for the total fraction of debris dispersed from the cavity, and a Weber breakup model for determining the size of entrained debris droplets.

The user may specify one cell in a problem to represent the RPV, and one cell to represent the cavity. The code assumes that these two cells are connected by a single gas flow path. At the start of the problem, the user must place some initial amount of debris into the trapped debris bin in the RPV cell by using the TRAPBIN option for debris source tables. The initial flow area between the RPV and cavity cells must be specified by the user, and is intended to represent the initial failure of the pressure vessel by either instrument tube penetration failure or a larger scale rupture.

Prior to gas blowthrough, debris exits the RPV in single-phase flow and is transferred from the trapped debris bin in the RPV cell to the trapped debris bin in the cavity cell. Ablation of the hole in the RPV is modeled, along with the height of the debris pool in the RPV. When the debris pool depth has decreased to a critical value, gas blowthrough occurs and two-phase ejection of debris from the RPV begins. The exit quality of the two-phase discharge is modeled, and a gas flow area is passed to the CONTAIN flow solver at each timestep. As in single-phase discharge, debris exiting the RPV during two-phase discharge is removed from the trapped debris bin in the RPV cell and placed in the cavity cell trapped debris bin.

After gas blowthrough has occurred in the RPV cell, entrainment of debris from the trapped debris bin in the cavity cell is permitted. The rate of entrainment is calculated from a user-selected correlation, and is based on transient conditions in the cavity cell. Entrained debris is removed from the trapped debris bin and placed in the cell atmosphere in the appropriate multifield airborne debris bins. The size distribution of the airborne debris may be either user-specified or determined from a Weber breakup model. The airborne debris in the cavity cell may exit the cell or be trapped and returned to the trapped debris bin, where it might be re-entrained during subsequent timesteps.

The user may also specify that a specific fraction of the debris initially in the RPV cell be entrained in the cavity cell, either by selecting a specific value or by invoking a specific model to predict an entrained fraction. In this case, the code employs the user-selected entrainment rate model to estimate the entrainment rate over the duration of the RPV blowdown. The entrainment rate is then modified so that the code will produce the specified total fraction of debris dispersed from the cavity. The user must specify the conditions in the cavity cell that will be used to calculate both the entrained fraction and time-dependent entrainment rate. With this option, there is no feedback between the entrainment rate and cavity conditions as the calculation progresses; the entrainment rate is based solely on the cavity conditions provided by the user.

The original references for all of the RPV and cavity models discussed in this document have been cited in the appropriate places and are provided in the References. However, for guidance on the development and use of the various entrainment rate and entrained fraction models, it is 
recommended that the user review Reference Wil96. This reference provides guidance on the use of the cavity models, the selection of user-defined parameters, and the applicability of individual models.

6.2.9.1 Single-Phase Debris Discharge. Prior to gas blowthrough, debris exits the RPV by singlephase flow out of the hole in the vessel. The mass flow rate of debris is [Pil92b]

$$
\dot{m}_{d}=\rho_{d} A_{h} C_{d} \sqrt{\frac{2 \Delta P}{\rho_{d}}}
$$

where $\rho_{\mathrm{d}}$ is the debris density as calculated by the code from the user-supplied density tables, $A_{h}$ is the area of the hole in the RPV specified by the user in AHOLE1 or as calculated by the ablation model, $\mathrm{C}_{\mathrm{d}}$ is the discharge coefficient specified by the user in CSUBD, and $\triangle \mathrm{P}$ is the pressure difference between the RPV and cavity cells. Debris that exits the RPV cell is removed from the trapped debris bin in the RPV cell and placed in the trapped debris bin in the cavity cell.

6.2.9.2 Two-Phase Discharge. This section describes the period of two phase discharge following singie phase debris discharge from the vessel. The two phase discharge consists of gas and debris.

6.2.9.2.1 Onset of Gas Blowthrough. The onset of gas blowthrough is determined from [Pil92b]

$$
\frac{\mathrm{h}_{\mathrm{b}}}{\mathrm{d}_{\mathrm{h}}}=0.43 \frac{\mathrm{D}}{\mathrm{d}_{\mathrm{h}}} \tanh \left(\mathrm{N}_{\mathrm{Fr}}^{1 / 2} \frac{\mathrm{d}_{\mathrm{h}}}{\mathrm{D}}\right)
$$

where $h_{b}$ is the height of the debris pool at which gas blowthrough occurs; $d_{h}$ is the diameter of the RPV hole; $\mathrm{N}_{\mathrm{rr}}$ is the Froude number $\mathrm{v}_{\mathrm{d}} / \sqrt{\mathrm{gd}} \mathrm{h}_{\mathrm{h}}$, where $\mathrm{v}_{\mathrm{d}}$ is the velocity of the debris exiting the RPV; and $\mathrm{D}$ is the diameter of the RPV specified by the user. The supporting database for this correlation spans the range $3.2<\mathrm{D} / \mathrm{d}_{\mathrm{h}}<20.0$ and $0.32<\mathrm{N}_{\mathrm{Fr}}<320$. However, the database is limited to lowdensity fluids $\left(\rho \approx 1000 \mathrm{~kg} / \mathrm{m}^{3}\right)$ and small scales $(D \leq 0.0508 \mathrm{~m})$.

6.2.9.2.2 Gas Void Fraction and Exit Quality. The gas exit quality is obtained from [Pi192b]

$$
\mathrm{X}_{\mathrm{g}}=\frac{1}{1+\frac{\left(1-\alpha_{\mathrm{g}}\right)}{\alpha_{\mathrm{g}}} \frac{\mathrm{W}_{\mathrm{sl}}}{\mathrm{W}_{\mathrm{sg}}}}
$$

where the gas void fraction is 


$$
\alpha_{\mathrm{g}}=1-\left(\frac{\operatorname{arctanh}\left[\frac{\mathrm{h}}{\mathrm{h}_{\mathrm{b}}} \tanh \left(\mathrm{N}_{\mathrm{Fr}}^{1 / 2} \frac{\mathrm{d}_{\mathrm{h}}}{\mathrm{D}}\right)\right]}{\frac{\mathrm{d}_{\mathrm{h}}}{\mathrm{D}} \mathrm{N}_{\mathrm{Fr}}^{1 / 2}}\right)^{\frac{8}{3} N}
$$

and $N=0.6$. The single-phase liquid flow rate $\mathrm{W}_{\mathrm{sl}}$ is given by Equation (6-22), and the single-phase gas flow rate is determined from

$$
W_{s g}=\dot{m}_{g} \frac{A_{b}}{A_{g}}
$$

where $\dot{m}_{g}$ is the mass flow rate of gas out of the RPV and $A_{g}$ is the area for gas flow in the RPV hole. The gas void fraction is used to determine the rate at which debris exits the RPV cell during the two-phase portion of the high pressure melt ejection, and further discussion is provided in the next section. As in single-phase debris discharge, debris that exits the RPV cell is removed from the trapped debris bin in the RPV cell and placed in the trapped debris bin in the cavity cell.

6.2.9.2.3 Gas Flow Area and Debris Flow Rate. The gas flow area in the hole in the RPV during two-phase discharge can be determined from the gas exit quality using the relationship

$$
\mathrm{A}_{\mathrm{g}}=\alpha_{\mathrm{g}} \mathrm{A}_{\mathrm{h}}
$$

The mass flow rate of debris out of the RPV during two-phase discharge can be expressed as

$$
\dot{\mathrm{m}}_{\mathrm{d}}=\left(\rho_{\mathrm{d}} \mathrm{A}_{\mathrm{h}} \mathrm{C}_{\mathrm{d}} \sqrt{\frac{2 \Delta P}{\rho_{\mathrm{d}}}}\right)\left(\frac{\mathrm{A}_{\mathrm{h}}-\mathrm{A}_{\mathrm{g}}}{\mathrm{A}_{\mathrm{h}}}\right)
$$

6.2.9.3 Vessel Hole Ablation. The hole in the RPV is enlarged through ablation of the vessel wall by debris as it exits the RPV. The hole ablation rate is given by the relation [Pil94a]

$$
\frac{\mathrm{dd}_{\mathrm{h}}}{\mathrm{dt}}=\frac{2 \mathrm{~K}_{\mathrm{a}} \mathrm{h}_{\mathrm{d}, \mathrm{w}} \Delta \mathrm{T}_{\mathrm{r}}}{\rho_{\mathrm{w}}\left[\mathrm{C}_{\rho, \mathrm{w}}\left(\mathrm{T}_{\mathrm{mp}, \mathrm{w}}-\mathrm{T}_{\mathrm{w}}\right)+\mathrm{h}_{\mathrm{f}, \mathrm{w}}\right]}
$$

where $\mathrm{K}_{\mathrm{a}}$ is a constant multiplier to the ablation rate specified in ARMULT, $\Delta \mathrm{T}_{\mathrm{r}}$ is the difference between the temperature of the debris in the RPV and the melting temperature of the RPV wall as specified by TMELT, $\rho_{w}$ is the density of the RPV wall as specified by RHOWAL, $C_{p, w}$ is the specific heat of the RPV wall as specified by CPWALL, $T_{m p, w}$ is the melting temperature of the RPV wall as specified by TMELT, $T_{w}$ is the temperature of the RPV wall as specified by TWALL, and $h_{f, w}$ is the heat of fusion of the RPV wall as specified by HFWALL. 
The heat transfer coefficient between the debris and vessel wall can be written as

$$
h_{d, w}=h_{d, w}^{\prime}\left(\frac{\beta}{e^{\beta}-1}\right)\left(\frac{A_{h}-A_{g}}{A_{h}}\right)
$$

where

$$
\begin{aligned}
& \beta=\frac{\rho_{\mathrm{w}} \mathrm{C}_{\mathrm{p}, \mathrm{w}} \dot{\mathrm{D}}_{\mathrm{h}, \mathrm{r}}^{\prime}}{2 \mathrm{~h}_{\mathrm{d}, \mathrm{w}}^{\prime}} \\
& \mathrm{h}_{\mathrm{d}, \mathrm{w}}^{\prime}=0.0292 \frac{\mathrm{k}_{\mathrm{d}}}{\mathrm{L}} \mathrm{N}_{\mathrm{Re}, \mathrm{L}}{ }^{0.8} \mathrm{~N}_{\mathrm{Pr}_{\mathrm{r}}}^{0.33} \\
& \dot{\mathrm{D}}_{\mathrm{h}, \mathrm{r}}^{\prime}=\frac{2 \mathrm{~h}_{\mathrm{d}, \mathrm{w}}^{\prime} \Delta \mathrm{T}_{\mathrm{r}}}{\rho_{\mathrm{w}}\left[\mathrm{C}_{\mathrm{p}, \mathrm{w}}\left(\mathrm{T}_{\mathrm{mp}, \mathrm{w}}-\mathrm{T}_{\mathrm{w}}\right)+\mathrm{h}_{\mathrm{f}, \mathrm{w}}\right]}
\end{aligned}
$$

The thermal conductivity of the debris, $\mathrm{k}_{\mathrm{d}}$, is calculated by the code from the user-supplied conductivity tables, $L$ is the thickness of the RPV wall specified by THKWAL, and $N_{R e, L}$ and $N_{P_{r}}$ are the Reynolds number and Prandtl number, defined as $\left(\rho_{d} v_{d} L\right) / \mu_{d}$ and $\left(C_{p, d} \mu_{d}\right) / k_{d}$, respectively. The debris viscosity $\mu_{\mathrm{d}}$ is calculated by the code from the user-supplied viscosity tables. A factor of the ratio of the debris area to hole area has been included in Equation (6-30) to simulate reduced contact between the debris and vessel walls as the gas exit quality increases.

If the user has decided to specify an entrained fraction of debris or to invoke an entrained fraction model, the time-dependent RPV hole size is not determined using Equations (6-29) through (6-33) after the onset of gas blowthrough and two-phase discharge from the RPV. Instead, the hole size following gas blowthrough is determined using the method described in Section 6.2.10.2.5.

\subsubsection{Cavity Models}

6.2.10.1 Entrainment Rate Correlations. If the user specifies only an entrainment rate correlation, without specifying a correlation or a value for the entrained fractions, the amount of debris dispersed will simply equal the integral of the entrainment rate. When this option is selected, the dynamic feedback between debris entrainment and cavity conditions (e.g., gas densities and flow velocities) that control entrainment rates is included in the modeling.

Five different correlations are provided as options for calculating the entrainment rate of debris in the cavity. These include three forms of the Whalley-Hewitt model, the Levy correlation, and the Tutu correlation. Each of the five entrainment rate correlations includes a cavity constant $\mathrm{K}_{\mathrm{c}}$ as a multiplier to the entrainment rate. This factor, which is CCENR in the CONTAIN input, is specific to both the cavity being modeled and the correlation being used. It is the user's responsibility to select reasonable values for this constant, based on the guidance provided in the references cited for 
each entrainment rate model. The entrainment rates given below are actually entrainment rate fluxes; the actual entrainment rate in the cavity is determined by multiplying the entrainment rate flux by the surface area of debris in the cavity, designated as AFILM in the CONTAIN input.

6.2.10.1.1 Whalley-Hewitt Entrainment Rate Model. The entrainment rate from the Whalley-Hewitt model is determined from [Wha78, Wil96]

$$
\varepsilon=0.0025 \mathrm{~K}_{\mathrm{c}}\left(1+360 \frac{\delta}{\mathrm{D}_{\mathrm{c}}}\right)\left(\frac{\rho_{\mathrm{g}} \mathrm{v}_{\mathrm{g}}^{2} \mu_{\mathrm{d}}}{\sigma}\right)
$$

where $\varepsilon$ is the entrainment rate per unit area, $K_{c}$ is the cavity constant specified in CCENR, $D_{c}$ is the hydraulic diameter of the cavity specified in HYDDIA, $\mu_{d}$ is the debris viscosity calculated from the user-defined debris material property tables, and $\sigma$ is the surface tension of the debris specified as SURTEN in the global DHEAT block. The debris film thickness is determined from

$$
\delta=\frac{\mathrm{m}_{\mathrm{d}}}{\rho_{\mathrm{d}} \mathrm{A}_{\mathrm{ww}}}
$$

where $m_{d}$ is the mass of unentrained debris in the cavity and $A_{w w}$ is the area of the cavity walls covered by debris specified in AFILM.

An alternate form of the Whalley-Hewitt model was used in Reference Pil92b and can be written as

$$
\varepsilon=0.0395 \mathrm{~K}_{\mathrm{c}} \operatorname{Re}_{\mathrm{g}}^{-1 / 4}\left(1+360 \frac{\delta}{\mathrm{D}_{\mathrm{c}}}\right)\left(\frac{\rho_{\mathrm{g}} \mathrm{v}_{\mathrm{g}}^{2} \mu_{\mathrm{d}}}{\sigma}\right)
$$

where

$$
\operatorname{Re}_{\mathrm{g}}=\frac{\mathrm{v}_{\mathrm{g}} \rho_{\mathrm{g}} D_{\mathrm{c}}}{\mu_{\mathrm{g}}}
$$

and $\mu_{\mathrm{g}}$ is the viscosity of the gas as calculated by the code.

A third form of the Whalley-Hewitt model was proposed in Reference Wil 96 and may be written as

$$
\varepsilon=0.0025 \mathrm{~K}_{\mathrm{c}}\left(1+360 \frac{\delta}{\mathrm{D}_{\mathrm{c}}}\right)\left(\frac{\rho_{\mathrm{g}} \mathrm{v}_{\mathrm{g}}^{2}\left(1.0 \times 10^{-3}\right)}{\sigma}\right)\left(\frac{\sigma}{\sigma_{\mathrm{s}}}\right)^{0.7826}
$$

where the standard surface tension $\sigma_{s}$ is specified in SURTES. This modification was introduced to correct what was judged to be unrealistic dependencies upon viscosity and surface tension in the 
other versions of the Whalley-Hewitt model. [Wi196] For guidance on the use of these models and the selection of user-defined parameters, the user should consult Reference Wil96; see also Section 13.3.2.4.7.

6.2.10.1.2 Levy Entrainment Rate Model. The entrainment rate from the Levy model is determined from [Lev91]

$$
-\frac{\mathrm{d} \delta}{\mathrm{dt}}=\mathrm{K}_{\mathrm{c}} \mathrm{N}_{\mathrm{Eu}}^{1.5}\left(\frac{\mu_{\mathrm{g}}}{\mu_{\mathrm{d}}}\right)^{0.26} \mathrm{~F}_{\mathrm{ent}}\left(\mathrm{N}_{\mathrm{Eu}}\right)\left(\frac{2 \mathrm{P}_{\mathrm{c}}}{\sigma}\right) \sqrt{\frac{2 \mathrm{P}_{\mathrm{c}}}{\rho_{\mathrm{d}}}}(\delta) \sqrt{1+300 \frac{\delta}{\mathrm{D}_{\mathrm{c}}}} \mathrm{f}_{1} \mathrm{f}_{2}
$$

where

$$
\begin{aligned}
& F_{e n t}\left(N_{E u}\right)=N_{E u}^{0.8} \\
& f_{1}=\left[\frac{\left(\frac{d_{s, h}}{S_{s}}\right)}{\left(\frac{d_{h}}{S}\right)}\right]^{2} \sqrt{\frac{R_{s}}{\left(\frac{R}{M_{g}}\right)} \frac{T_{s}^{0}}{T^{0}}} \\
& f_{2}=\frac{\rho_{s, d}}{\rho_{d}}
\end{aligned}
$$

Here, $N_{E u}$ is the Euler number, $\left(\rho_{\mathrm{g}} \mathrm{g}_{\mathrm{g}}^{2}\right) / 2 \mathrm{P}_{\mathrm{c}}$, where $\mathrm{j}_{\mathrm{g}}$ is the superficial gas velocity in the cavity and $\mathrm{P}_{\mathrm{c}}$ is the pressure in the cavity; $\mathrm{d}_{\mathrm{s}, \mathrm{h}}$ is the standard RPV hole diameter specified by DSUBS; $\mathrm{S}_{\mathrm{s}}$ is the standard scale specified by SSCALF; $S$ is the scale specified by SCALEF; $R_{s}$ is the gas constant for the standard gas specified by RSUBS; $\mathrm{R}$ is the universal gas constant; $\mathrm{M}_{\mathrm{g}}$ is the molecular weight of the driving gas as calculated by the code; $\mathrm{T}_{\mathrm{s}}{ }^{0}$ is the initial standard gas temperature as specified by TSUBIS; $\mathrm{T}^{0}$ is the initial gas temperature; and $\rho_{\mathrm{s}, \mathrm{d}}$ is the standard density of the debris as specified by RHDEBS.

It should be emphasized that the Levy entrainment rate model requires a number of "standard" values. The user must consult the reference for the model [Lev91] in order to specify these values correctly in the input for the model. For additional guidance on the use of this model and the selection of user-defined parameters, the user should consult Reference Wi196; see also Section 13.3.2.4.

6.2.10.1.3 Tutu Entrainment Rate Model. The entrainment rate from the Tutu model [Tut91] is determined from

$$
\varepsilon=\rho_{d} A_{\min } E j_{d}
$$


The area term is determined from

$$
A_{\min }=\min \left[\text { cavity exit area, }\left(w+2 h_{c}\right) \frac{D_{c}}{4}\left(\frac{d_{h}}{D_{c}}\right)^{0.5}\right]
$$

where $\left(w+2 h_{c}\right)$ is the wetted perimeter of the cavity is specified in WETPER.

The fraction of the melt flux flowing as droplets is

$$
\mathrm{E}=\tanh \left[\mathrm{K}_{\mathrm{c}} \mathrm{N}_{\mathrm{Ku}, \mathrm{g}}^{2} \mathrm{~N}_{\mathrm{Ku}, \mathrm{d}}^{0.33}\left(\frac{\rho_{\mathrm{g}}}{\rho_{\mathrm{d}}-\rho_{\mathrm{g}}}\right)^{1.4} \mathrm{D}_{*}^{0.48}\left(1-\mathrm{e}^{-\frac{\mathrm{D}_{*}}{8}}\right)\right]
$$

where

$$
\begin{aligned}
& N_{\mathrm{Ku}, \mathrm{g}}=\left(\frac{\mathrm{j}_{\mathrm{g}} \rho_{\mathrm{g}}^{0.5}}{\left[\sigma \mathrm{g}\left(\rho_{\mathrm{d}}-\rho_{\mathrm{g}}\right]^{0.25}\right.}\right) \\
& \mathrm{N}_{\mathrm{Ku}, \mathrm{d}}=\left(\frac{\mathrm{j}_{\mathrm{d}} \rho_{\mathrm{d}}^{0.5}}{\left[\sigma \mathrm{g}\left(\rho_{\mathrm{d}}-\rho_{\mathrm{g}}\right]^{0.25}\right.}\right)
\end{aligned}
$$

The total superficial velocity of the melt can be written as

$$
j_{d}=0.5\left(j_{g}\right)\left(\frac{V_{d}}{V_{e q}}\right)
$$

where $V_{d}$ is the volume of the non-airborne debris in the cavity and

$$
\mathrm{V}_{\mathrm{eq}}=\frac{\mathrm{D}_{\mathrm{c}}}{4}\left(\frac{\mathrm{d}_{\mathrm{h}}}{\mathrm{D}_{\mathrm{c}}}\right)^{0.5} \mathrm{~A}_{\mathrm{ww}}
$$

The dimensionless pipe diameter is determined from

$$
\mathrm{D}_{*}=\mathrm{D}_{\mathrm{c}}\left(\frac{\mathrm{d}_{\mathrm{h}}}{\mathrm{D}_{\mathrm{c}}}\right)^{0.5}\left[\frac{\mathrm{g}\left(\rho_{\mathrm{d}}-\rho_{\mathrm{g}}\right)}{\sigma}\right]^{0.5}
$$

Some additional discussion of this model is given in Reference Wil96. 
6.2.10.2 Entrained Fraction Correlations. In the options described above in Section 6.2.10.1, the code determines the fraction of the debris dispersed from the cavity. Alternatively, the user has the option of specifying the dispersal fraction directly, or specifying that it is to be calculated from an integral correlation for the dispersed fraction. Six different correlations are provided as options for calculating the total fraction of debris dispersed from the cavity. These include the Levy correlation and five individual Tutu-Ginsberg correlations. Because the entrainment rate must be forced to integrate to the calculated fraction dispersed, no feedback occurs between the entrainment rate calculation and transient conditions in the system. As a result, the user must provide estimates for the cavity conditions that are required by the entrained fraction and entrainment rate models. The required conditions include the molecular weight, ratio of specific heats, density, and viscosity of gas in the cavity, as well as the cavity and containment pressures. In addition, any of the entrained fraction options requires estimates of the debris entrainment time, the time-dependent gas flow rate out of the RPV, and the final hole size in the RPV.

At the moment of gas blowthrough, the total fraction of debris that will be dispersed from the cavity is calculated with the model selected by the user. Because the entrained fraction models are all based on experiments with low temperature chemically non-reactive debris simulants and fixed RPV hole sizes, the models require fixed values for cavity conditions and a single RPV hole size. The entrained fraction of debris is calculated from the user-provided input, with either a user-specified hole size or an estimated final hole size based on the ablation rate prior to gas blowthrough.

In a side calculation removed from the main CONTAIN calculation, a time-dependent debris entrainment rate is calculated using the user-specified entrainment rate model, the cavity conditions provided by the user, and an estimated gas flow rate out of the RPV cell. The duration of the entrainment is estimated from the blowdown time of the RPV. In one option, the gas flow rate used in determining the entrainment rate is based on the fixed hole size used in the calculation of the entrained fraction. Alternatively, the user may also specify that the hole area grow linearly during the blowdown of the RPV by using the AHENF and TSTOP input parameters. Note that the use of this option has no effect on the total entrained fraction of debris, but it does affect the rate of debris entrainment in the cavity because it affects the gas flow rate out of the RPV.

At the end of the gas blowdown from the RPV cell in the side calculation, the estimated entrainment rate is modified so that the integral of the entrainment rate over the course of the gas blowdown yields the desired total entrained fraction of debris. The side calculation then terminates and the main CONTAIN calculation once again proceeds. At each timestep, the predetermined entrainment rate is used to calculate the amount of debris that is removed from the trapped debris bin and placed in the airborne debris bins in the cavity cell. Because the entrainment rate is based on the side calculation, it is completely independent of cavity conditions as the CONTAIN calculation proceeds. The models used in the entrained fraction options are described below.

6.2.10.2.1 Levy Entrained Fraction Correlation. The entrained fraction of debris predicted by the Levy correlation is determined by solving the transcendental equation [Lev91] 


$$
\begin{aligned}
& \mathrm{Y}=\log \left[\frac{\left(\sqrt{1+300 \frac{\delta^{0}}{\mathrm{D}_{\mathrm{c}}}}-1\right)\left(\sqrt{1+300 \frac{\delta}{\mathrm{D}_{\mathrm{c}}}+1}\right)}{\left(\sqrt{1+300 \frac{\delta}{\mathrm{D}_{\mathrm{c}}}-1}\right)\left(\sqrt{1+300 \frac{\delta^{0}}{\mathrm{D}_{\mathrm{c}}}}+1\right)}\right] \\
& \mathrm{Y}=\mathrm{K}_{\mathrm{c}} \mathrm{f}_{1} \mathrm{f}_{2}\left[\frac{0.36 \mathrm{~V}_{\mathrm{v}}}{\mathrm{A}_{\mathrm{h}} \sqrt{\left(\frac{\mathrm{R}}{\mathrm{M}_{\mathrm{g}}}\right) \mathrm{T}^{0}}}\right] \mathrm{Eu}_{0}^{2.3} \frac{\left(2 \mathrm{P}_{\mathrm{c}}\right)}{\sigma} \sqrt{\frac{2 \mathrm{P}_{\mathrm{c}}}{\rho_{\mathrm{d}}}}\left(\frac{\mu_{\mathrm{g}}}{\mu_{\mathrm{d}}}\right)^{0.26}
\end{aligned}
$$

where $\delta^{0}$ is the initial thickness of the debris film, the quantities $f_{1}$ and $f_{2}$ are as defined in Equations $(6-41)$ and $(6-42), M_{g}$ is the molecular weight of gas in the cavity specified by AMWCU, $V_{v}$ is the volume of the RPV calculated internally or specified by VRPVU, and $P_{c}$ is the pressure in the cavity specified by PCAVU. As with the Levy entrainment rate model, the Levy entrained fraction model requires a number of "standard" values. The user must consult the reference for the model [Lev91] in order to correctly specify these values in the input for the model. For additional guidance on the use of this model and the selection of user-defined parameters, the user should consult Reference Wil96; see also Section 13.3.2.4.

6.2.10.2.2 Tutu-Ginsberg Entrained Fraction Correlations. The entrained fraction of debris predicted by the Tutu-Ginsberg correlations is determined from the following equation [Tut90]:

$$
f=1-U F\left(x-C_{1}\right)+(F X+F Y) U F\left(x-C_{1}\right)
$$

where

$$
\begin{aligned}
& \mathrm{x}=\log _{10}\left[\mathrm{~N}_{5}\left(\mathrm{~L}_{\mathrm{c}} / \mathrm{d}_{\mathrm{h}}\right)^{\mathrm{C}_{2}}\left(\rho_{\mathrm{R}} / \rho_{\mathrm{d}}\right)^{\mathrm{C}_{3}}\left(1+\mathrm{C}_{4} \mathrm{~N}_{4} \mathrm{C}_{\mathrm{s}}\right) /\left(1+\mathrm{C}_{6} \mathrm{~N}_{1}^{\mathrm{C}_{1}}\right)\right] \\
& \mathrm{N}_{1}=\frac{\sigma \rho_{\mathrm{d}}}{\rho_{\mathrm{R}}{ }^{2} \mathrm{U}_{\mathrm{R}}{ }^{2} \mathrm{~L}_{\mathrm{c}}} \\
& \rho_{\mathrm{R}}=\frac{\mathrm{P}_{\text {con }}}{\left(\frac{\mathrm{R}}{\mathrm{M}_{\mathrm{g}}}\right) \mathrm{T}^{0}}
\end{aligned}
$$




$$
\begin{aligned}
& \mathrm{U}_{\mathrm{R}}=\mathrm{K}_{\mathrm{c}} \mathrm{f}(\gamma) \frac{\mathrm{A}_{\mathrm{h}}}{\mathrm{A}_{\mathrm{c}}}\left[\frac{\mathrm{P}_{\mathrm{v}}^{0}}{\rho_{\mathrm{R}} \sqrt{\left(\frac{\mathrm{R}}{\mathrm{M}_{\mathrm{g}}}\right) \mathrm{T}^{0}}}\right] \\
& \mathrm{f}(\gamma)=\gamma^{0.5}\left(\frac{2}{\gamma+1}\right)^{\frac{\gamma+1}{2(\gamma-1)}} \\
& \mathrm{N}_{4}=\frac{\mathrm{P}_{\mathrm{v}}^{0}\left(\frac{\mathrm{V}_{\mathrm{v}}}{\mathrm{L}_{\mathrm{c}}^{3}}\right)}{\mathrm{P}_{\text {con }}} \\
& \mathrm{N}_{5}=\rho_{\mathrm{R}} \frac{\mathrm{U}_{\mathrm{R}}{ }^{2}}{\sqrt{\rho_{\mathrm{d}} \mathrm{g \sigma}}}
\end{aligned}
$$

and

$$
\begin{aligned}
& z=\left|x-C_{1}\right| \\
& F X=C_{8} /\left\{1+C_{9} Z^{C_{10}}\right\} \\
& F Y=C_{11} e^{-\left(Z / C_{12}\right)^{C_{13}}} \\
& \mathrm{UF}(\mathrm{x}-\varphi)=0 \text { for } \mathrm{x} \leq \varphi \\
& =1 \text { for } x>\varphi
\end{aligned}
$$

The length of the cavity $\mathrm{L}_{c}$ is specified by CAVLEN, $P_{\text {con }}$ is the pressure in the containment specified by PCONU, $P_{v}^{0}$ is the initial pressure in the RPV, $A_{c}$ is the cross-sectional flow area in the cavity calculated by the code or specified by AFLOW, and $\gamma$ is the gas-specific heat ratio specified by GAMMAU.

Specific values of the constants in Equations (6-53) through (6-64) are provided for five distinct cavity configurations: Surry cavity without structures, Surry cavity with skirt only, Surry cavity with all structures, Zion cavity, and Watts-Bar cavity. Values for the constants are presented in Table 6-2. For guidance on the use of these models and the selection of user-defined parameters, the user should consult Reference Wi196 and Section 13.3.2.4. 
Table 6-2

Constants for the Tutu-Ginsberg Entrained Fraction Correlation

\begin{tabular}{||c|c|c|c|c|c||}
\hline Cavity & $\begin{array}{c}\text { Surry (no } \\
\text { structure) }\end{array}$ & $\begin{array}{c}\text { Surry } \\
\text { (skirt) }\end{array}$ & $\begin{array}{c}\text { Surry (all } \\
\text { structure) }\end{array}$ & Zion & $\begin{array}{c}\text { Watts- } \\
\text { Bar }\end{array}$ \\
\hline $\mathrm{C}_{1}$ & 0.136 & 0.481 & 0.467 & 0.839 & 0.263 \\
\hline $\mathrm{C}_{2}$ & 1.886 & 1.818 & 0.601 & 1.512 & 1.618 \\
\hline $\mathrm{C}_{3}$ & 0.748 & 0.652 & 0.133 & 0.464 & 0.440 \\
\hline $\mathrm{C}_{4}$ & 1.248 & 0.575 & 0.336 & 2.32 & 0.273 \\
\hline $\mathrm{C}_{5}$ & 1.024 & 0.680 & 0.992 & 0.324 & 1.630 \\
\hline $\mathrm{C}_{6}$ & 1.0 & 0.350 & 3.019 & 0.0 & 3.783 \\
\hline $\mathrm{C}_{7}$ & 0.630 & 0.121 & 0.558 & 0.0 & 0.522 \\
\hline $\mathrm{C}_{8}$ & 0.5 & 0.472 & 0.777 & 0.296 & 0.497 \\
\hline $\mathrm{C}_{9}$ & 0.915 & 0.688 & 0.0395 & 0.0813 & 0.858 \\
\hline $\mathrm{C}_{10}$ & 6.4 & 2.75 & 5.0 & 3.6 & 5.0 \\
\hline $\mathrm{C}_{11}$ & 0.5 & 0.528 & 0.223 & 0.704 & 0.503 \\
\hline $\mathrm{C}_{12}$ & 2.319 & 0.958 & 4.467 & 1.351 & 3.451 \\
\hline $\mathrm{C}_{13}$ & 1.8 & 8.75 & 3.0 & 6.6 & 3.0 \\
\hline \hline
\end{tabular}

6.2.10.2.3 Entrainment Time. The entrainment time is the interval over which debris is entrained in the cavity. It can be specified directly through the TDISP keyword. If TDISP is not specified, the entrainment time is estimated from an expression for the RPV blowdown time [Lev91]:

$$
t_{d}=\frac{-V_{v} \ln \left(\frac{P_{c}}{P_{v}^{0}}\right)}{(0.6065)\left(A_{h}\right) \sqrt{R^{0}}}
$$

In CONTAIN, the pressure ratio in this equation is assumed to be 0.1 , which represents the cutoff for entrainment. The blowdown time $t_{d}$ is taken to be the entrainment time, unless the user specifies through the TSTOP keyword that the RPV hole itself grows as a function of time, over the time interval given by TSTOP. In this case the entrainment time is taken to be $t_{d}+$ "tstop," to ensure that the entrainment time is adequate.

The total fraction of debris dispersed from the cavity will not be affected by the entrainment time, since the entrainment rate is forced to integrate to a specific total entrained fraction. It should be noted that while no debris entrainment can occur after the dispersal interval has passed, the debris entrainment rate might be large enough to disperse the desired fraction of debris before the dispersal interval has ended. Small entrainment times may lead to very high entrainment rates in the cavity, 
because the desired fraction of debris must be dispersed in the given time interval. However, if the entrainment rate model selected by the user already predicts high entrainment rates, increasing the entrainment time will not slow down entrainment of debris in the cavity. The entrainment rate must be controlled directly through the CCENR parameter.

6.2.10.2.4 Time-Dependent Gas Flow Rate. The use of an entrained fraction option also requires an estimate of the time-dependent gas flow rate out of the RPV. The equation is based on Tutu's formulation, [Tut91] modified to permit a time-dependent gas flow area

$$
\dot{\mathrm{m}}_{\mathrm{g}}=\mathrm{C}_{\mathrm{d}} \mathrm{A}_{\mathrm{g}}(\mathrm{t}) \sqrt{\gamma}\left(\frac{2}{\gamma+1}\right)^{\frac{\gamma+1}{2(\gamma-1)}}\left[\frac{\mathrm{P}_{\mathrm{v}}(\mathrm{t})}{\sqrt{\left(\frac{\mathrm{R}}{\mathrm{M}_{\mathrm{g}}}\right) \mathrm{T}_{\mathrm{v}}(\mathrm{t})}}\right]
$$

where $P_{v}$ is the pressure in the RPV and

$$
\begin{aligned}
& T_{v}(t)=\frac{T^{0}}{\left(\frac{M_{v}^{0}}{M_{v}(t)}\right)^{\gamma-1}} \\
& P_{v}(t)=\frac{M_{v}(t)\left(\frac{R}{M_{g}}\right) T_{v}(t)}{V_{v}}
\end{aligned}
$$

The time-dependent gas flow area $A_{g}(t)$ is controlled by specifying AHENF and TSTOP, $P_{v}(t)$ is the time-dependent pressure in the RPV calculated by the code, $T_{v}(t)$ is the time-dependent temperature in the RPV calculated by the code, $M_{v}^{0}$ is the initial mass of gas in the RPV, and $M_{y}(t)$ is the timedependent mass of gas in the RPV.

The estimate of the time-dependent gas flow rate provided by Equations (6-66) through (6-68) is used to calculate a time-dependent entrainment rate from the cavity using the model selected by the user. However, the gas flow rate has no effect on the total fraction of debris entrained from the cavity cell, since the entrainment rate is still forced to integrate to the desired total fraction dispersed.

6.2.10.2.5 Final RPV Hole Size. Because the entrained fraction models require a fixed RPV hole size, it is necessary to estimate the final size of the hole after ablation and then use this value in the entrained fraction model selected by the user. An expression for the final hole diameter can be derived by assuming that the rate of change of the hole diameter is constant during debris ejection. The final expression for the estimated RPV hole diameter has the form 


$$
\left.\mathrm{d}_{\mathrm{h}, \text { est }}=\left(\frac{12 \dot{\mathrm{d}}_{\mathrm{h}} \mathrm{M}_{\mathrm{d}}^{0}}{\mathrm{C}_{1} \pi}+\left(\mathrm{d}_{\mathrm{h}}\right)^{0}\right)^{3}\right)^{\frac{1}{3}}
$$

where

$$
C_{1}=\rho_{d} C_{d} \sqrt{\frac{2\left(P_{v}-P_{c}\right)}{\rho_{d}}}
$$

The rate of change of the hole diameter, $\dot{\mathrm{d}}_{h}$, is calculated internally by the code, $M_{d}^{0}$ is the initial mass of debris in the RPV, and $d_{h}^{0}$ is the initial RPV hole diameter at the start of the final hole size estimation in the code.

Note that the time-dependent RPV hole size used in the gas flow rate calculations described above has no effect on the entrained fraction of debris calculated by the code. The user may also select the hole diameter used in the entrained fraction model, rather than allowing the code to calculate a value, by specifying AHENF.

6.2.10.2.6 Integration of the Entrainment Rate. When an entrained fraction model is selected by the user, the entrainment rate must integrate to yield the appropriate total fraction of dispersed debris. To simplify this problem, the entrainment rate is determined from the conditions at the time of gas blowthrough; estimates of the entrainment time, gas flow rate, and final RPV hole diameter as described in the three sections above; and conditions provided by the user through input. There is no feedback with the CONTAIN calculation as a whole; the entrainment rate is estimated in a side calculation that is performed outside of the main CONTAIN calculation.

If the entrainment rate calculated by the user-specified entrainment rate model in the side calculation predicts $100 \%$ entrainment before the end of the entrainment interval estimated by Equation (6-65), the entrainment rate used in the subsequent CONTAIN calculation is multiplied by the desired entrained fraction at each timestep up to the time where $100 \%$ dispersal was reached. At this time entrainment ends, and the entrainment rate is set to zero for all future times. If the entrainment rate calculated by the user-specified entrainment rate model in the side calculation predicts less than $100 \%$ entrainment by the end of the entrainment interval estimated by Equation (6-65), the entrainment rate used in the subsequent CONTAIN calculation is multiplied by the ratio of the desired entrained fraction to the predicted entrained fraction. Entrainment continues until the entrainment interval estimated by Equation (6-65) ends, at which point the entrainment rate is set to zero for all future times.

6.2.10.3 Debris Particle Size. The following subsections discuss the Weber drop size model.

6.2.10.3.1 Weber Drop Size Model. Weber breakup occurs when liquid is broken into fragments as a result of interaction with a flowing adjacent gas. This occurs during the debris entrainment and transport process as well as in other processes not specific to $\mathrm{DCH}$, such as pneumatic atomization. The Weber number is defined as 


$$
\mathrm{N}_{\mathrm{We}}=\frac{\rho d v_{\mathrm{g}}^{2}}{\sigma}
$$

where $N_{w e}$ is the Weber number, $\rho$ is the density, $d$ is the drop diameter, $v_{g}$ is the gas velocity, and $\sigma$ is the surface tension.

Physically, the above equation defines a critical Weber number at which surface tension forces in the drop balance the forces exerted on the drop by the flowing gas. For a given flow velocity, drops with diameters above the value that will give this critical Weber number will not be stable and will break up. Therefore, the $\mathrm{N}_{\mathrm{We}}$ criterion can be applied to $\mathrm{DCH}$ problems to determine the maximum stable drop size as a function of the gas flow velocity and an assumed critical Weber number $\mathrm{N}_{\mathrm{We}, \mathrm{c}}$

$$
d_{\max } \equiv \sigma \frac{N_{W e, c}}{\rho v_{g}^{2}}
$$

The critical Weber number has been studied extensively for air/water systems and to a lesser degree for systems involving other fluids, such as molten core debris and steam. These studies are summarized in the work by Ostensen et al. ${ }^{1}$ In this work a Weber number of 6 was recommended for estimating the volumetric median particle size in DCH applications. A critical Weber number of 12 is commonly used for aerosols and has also been recommended for molten debris and other fluids. The value of 12 is used as the default critical Weber number in the CONTAIN model. Since the volumetric median diameter of the particles resulting from breakup is typically about half the critical diameter, this default is consistent with the recommendations of Ostensen et al. ${ }^{1}$ The user may specify the critical Weber number and therefore assess the impact of this parameter on containment loads.

6.2.10.3.2 Integration of the Weber Model into the Multifield Architecture. In CONTAIN, debris is grouped into a number of fields (also called bins), where the number of fields represented can be controlled by the user. The user selects the number of bins that are to be modeled, where each bin is typically a unique drop size. The user also selects the number of time generations to be modeled. A unique set of debris bins are tracked within each generation. Therefore, the total number of fields represented in the code in each cell is the product of the number of bins and the number of generations, plus one additional field for non-airborne debris. The drop size model is integrated into this treatment of debris as described below.

The maximum stable drop size predicted by the We criterion depends strongly on the velocity of the entraining fluid. In the CONTAIN default treatment, the average velocity through the cell is used as the gas velocity for the entraining fluid. Physically, the velocity used in We criterion should be the velocity across the surface of the fluid being entrained, which is the debris. In a CONTAIN calculation there is no good way to obtain this velocity. Moreover, no experimental measurements

${ }^{1}$ R. W. Ostensen, R. O. Griffith, and D. C. Williams, Sandia National Laboratories, Albuquerque, NM, letter report to the USNRC, with title "Models and Correlations for Direct Containment Heating," March 15, 1992. 
of flow velocities in the cavity are presently available so that correlation of entrainment velocity against exit velocity is not possible. The velocity of interest to the entrainment process is believed, however, to lie somewhere between the blowdown velocity and the cavity exit velocity. Physically, this is reasonable given that the blowdown gas picks up extra mass by entraining debris into the flow field and that the momentum of the blowdown gas must be conserved between the entrance and exit of the cavity. The gas is also heated, which acts to accelerate the flow. The ability to use either the average gas velocity through the cavity or the exit gas velocity from the cavity is provided through the USEVOUT keyword.

As debris is ejected from the RPV, it is transferred from the RPV non-airborne field to the cavity non-airborne field. Debris in the non-airborne field is assumed to have one particle size associated with it for purposes of interacting with the blowdown steam. As debris is entrained out of the cavity non-airborne field, the drop size models are invoked to distribute the debris among the various airborne fields. The distribution of entrained mass during a timestep into the airborne fields is done assuming that the mass is distributed log-normally. The mass median particle size, $d_{m}$, is assumed to be equal to one half the maximum stable drop size given by Equation (6-72). The mass of debris entrained into a given field during a timestep is given by

$$
\begin{aligned}
\Delta M_{i} & =\frac{\Delta M_{T} I_{i}}{\sum_{j=1}^{N_{\text {fedds }}} I_{j}} \\
I_{i} & =\int_{a_{i}}^{b_{i}} \frac{1}{x} \exp \left\{-\frac{\left[\ln (x)-\ln \left(d_{m}\right)\right]^{2}}{2 \ln ^{2}\left(\sigma_{g}\right)}\right\} d x
\end{aligned}
$$

where $\Delta M_{i}$ is the mass of debris entrained into field $i, \Delta M_{T}$ is the total mass entrained into all the fields during the timestep, $N_{\text {fields }}$ is the number of airborne debris fields, and $\sigma_{\mathrm{g}}$ is the geometric standard deviation. The integration limits $a_{i}$ and $b_{i}$ in Equation (6-73) are given by

$$
\begin{aligned}
& a_{i}=\sqrt{d_{i-1} d_{i}}, i>1 \\
& a_{i}=d_{i} / \min \left(5, \sigma_{g}^{2}\right), i=1 \\
& b_{i}=\sqrt{d_{i} d_{i+1}}, i<N_{\text {fields }} \\
& b_{i}=\min \left(5, \sigma_{g}^{2}\right) d_{i}, i=N_{\text {fields }}
\end{aligned}
$$

where $d_{i}$ is the particle diameter assigned to field $i$. The geometric standard deviation $\sigma_{g}$ is controlled by the user-specified parameter WESIG, which is the natural logarithm of $\sigma_{\mathrm{g}}$. The default value of $\sigma_{\mathrm{g}}$ is 2 ; hence, WESIG $=\ln (2)=0.693$ by default. In DCH experiments, the size distribution observed is generally broader than this, with $\sigma_{\mathrm{g}} \approx 4$ being typical. However, the value of $\mathrm{d}_{\mathrm{m}}$ calculated from Equation (6-72) will vary as the gas flow conditions in the cavity vary, which will tend to broaden the size distribution obtained for the entire calculation. 
Equations (6-73) and (6-74) may allow some particles to have sizes that are larger than $d_{\max }$ as given by Equation (6-72). Although this result is not strictly consistent with the Weber number breakup assumption as described previously, it is consistent with the size distributions observed in DCH experiments, which do not show a sharp cutoff at large particle sizes. The Weber model should be thought of as providing an estimate for $\mathrm{d}_{\mathrm{m}}$, but it does not define the entire size distribution, since this is also governed by the user-specified value of WESIG.

When the Weber number model is used, all species in the debris are distributed-proportionately among the airborne debris fields. That is, the composition of the material added to each airborne field during a timestep equals the composition in the cavity non-airborne field during that timestep, and FDISTR cannot be used to distribute the individual species separately. Section 14.2.7 provides the details as to how the debris fields are to be specified when the Weber model is used.

\subsection{DCH Trapping Model}

The process of debris removal as a result of interaction with containment structures and/or gravitational fallout is referred to as trapping. This process is still the subject of considerable uncertainty, and plant and experiment analyses have shown that trapping can have an important effect on results. Therefore, the CONTAIN model includes both a mechanistic approach to trapping as well as flexible input for performing sensitivity calculations.

In principle, the CONTAIN DCH trapping model is a simple one, where debris trapping in a given control volume is governed by a first order linear rate equation. As implemented, however, the model has several dependencies, such as the conditions in the cell atmosphere, attributes of the debris field being de-entrained, and the debris and gas inflow rates. In addition, the trapping rate is recalculated every timestep, so that debris trapping reflects changes in the particle field, atmospheric conditions, and inflow rates as the DCH event progresses. The first subsection below describes the trapping rate equation and its solution. The remaining subsections describe the models and options available in CONTAIN for calculating the trapping rates used in this model.

\subsubsection{Rate Equations for Trapping}

The debris trapping process is governed by a first order linear rate equation for the time rate of change of airborne debris mass in a field:

$$
\left[\frac{d m_{d, i, n, k}}{d t}\right]_{\text {trap }}=-\lambda_{\mathrm{i}, \mathrm{n}} m_{\mathrm{d}, \mathrm{i}, \mathrm{n}, \mathrm{k}}
$$

where $\lambda_{i, n}$ is the trapping rate for field $\mathrm{n}$ in cell $\mathrm{i}$

Notice that each particle field in each cell is governed by its own trapping rate. Therefore, these equations are solved many times throughout a calculation for each particle field, $n$, and for each cell, i. The model keeps track of the mass of trapped debris by species. 
The trapping rate for the different species in a given field and cell is assumed to be the same. The trapping rate, $\lambda_{\mathrm{i}, \mathrm{n}}$, is also assumed to be constant over a calculational timestep. Therefore, the trapping terms in the DCH mass and energy conservation equations in Section 6.2.2 are linear and can be represented by a time-averaged removal rate that depends on the initial mass of airborne debris for each material in a given field and cell. If the timestep is given by $\Delta t_{c}$, then an average trapping rate for material $\mathrm{k}$ in field $\mathrm{n}$ in cell $\mathrm{i}$ over the timestep is given by

$$
\left[\frac{\mathrm{dm}_{\mathrm{d}, \mathrm{n}, \mathrm{k}}}{\mathrm{dt}}\right]_{\text {trap }}=-\mathrm{m}_{\mathrm{d}, \mathrm{i}, \mathrm{n}, \mathrm{k}} \frac{\left[1-\exp \left(-\lambda_{\mathrm{i}, \mathrm{n}} \Delta \mathrm{t}_{\mathrm{c}}\right)\right]}{\Delta \mathrm{t}_{\mathrm{c}}}
$$

The following subsections describe the equations used to calculate the trapping rate, $\lambda_{i, n}$.

Special provisions are made in the trapping model for sending some fraction of the trapped debris material to the intermediate material layer in the lower cell. In the present model, this fraction is specified by the user. The addition of trapped debris to the lower cell is governed by:

$$
\frac{d m_{L C, i, k}}{d t}=-f_{L C, i} \sum_{n=1}^{N_{\text {ietdd }}}\left[\frac{d m_{d, i, n, k}}{d t}\right]_{\text {trap }}
$$

where $m_{L C, i, k}$ is the mass of material $k$ in the uppermost intermediate layer of the cavity in cell $i$, and $\mathrm{f}_{\mathrm{LC}, \mathrm{i}}$ is the user-supplied fraction of trapped debris to deposit into the uppermost intermediate layer of the cavity of cell $\mathrm{i}$. This is specified using the COOLFRAC keyword in the DCH-CELL input block.

The user-supplied fraction, $f_{L C, j}$, governs the fraction of the trapped debris that goes into the uppermost intermediate layer of the lower cell. The remaining fraction, $\left(1-f_{L C ;}\right)$, of the trapped debris will be placed in the non-airborne debris field. Note that this option is available in any cell, but is most useful in the cavity cell. The CONTAIN lower cell layer system is described in Chapter 5. If there is no intermediate lower cell layer, or if there is no lower cell defined at all, then all trapped debris is placed in the non-airborne debris field regardless of the $f_{\mathrm{LC}, \mathrm{i}}$ value. Trapped debris cannot be passed into the CORCON layer system if CORCON is active. Therefore, $f_{L C, i}$ will be ignored if CORCON is active and all trapped debris will be placed in the non-airborne debris field. This is not considered an important limitation because $\mathrm{DCH}$ calculations are typically performed only to predict the peak short term containment load. The $f_{L C, i}$ parameter is used if CORCON is defined but has not yet been activated; therefore, $\mathrm{DCH}$ can be used during the early phase of the accident and CORCON used in a restart or in a new calculation using the trapping results from the DCH run.

Except for the debris that is optionally sent to the lower cell, the DCH model stores trapped debris in the non-airborne debris field. This field also can be used to hold debris in the cavity that has not yet been entrained by the blowdown gas. If debris is not sent to the lower cell, the mass of debris in the non-airborne debris field is governed by the following equation 


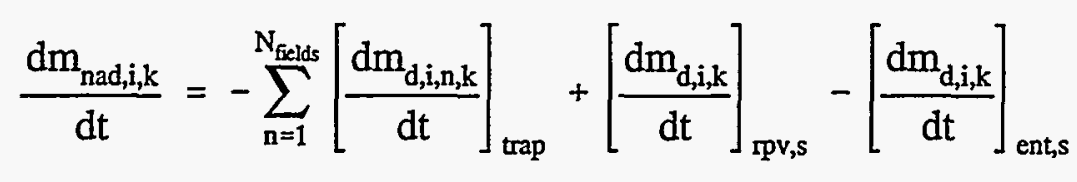

where $m_{n a d, i, k}$ is the mass of debris in cell $i$ in the non-airborne field; [dm/dt] ${ }_{\mathrm{rpv}, \mathrm{s}}$ is the discharge source rate from the RPV into the non-airborne field; and $[\mathrm{dm} / \mathrm{dt}]_{\text {ent,s }}$ is the entrainment source rate out of non-airborne field.

This equation does not include chemical interaction terms in the non-airborne debris field. These terms would be identical to those described in Section 6.4 for the airborne fields. The first term on the right side represents trapping from all airborne fields. The second term represents user sources into the non-airborne field using the TRAPBIN type source tables and/or debris transferred to the cavity trapped field by the RPV models described in Section 6.2.9. The third term represents the user-specified entrainment rate out of the non-airborne field using the ENTRAIN option for source tables or the debris entrainment rate calculated by the entrainment models described in Section 6.2.10.

The trapping rate, $\lambda$, is either provided by the user or calculated by the code based on cell conditions, particle field attributes, and inflow gas and debris velocities. Four options for determining $\lambda$ are provided. These options only differ in the way in which the trapping rate, $\lambda$, is determined. That is, all models use the first order rate Equation (6-75), and this equation is always solved in the manner described above to represent de-entrainment. The four trapping options included in the CONTAIN DCH model are:

$\begin{array}{ll}\text { USER } & \text { User-specified trapping rate } \\ \text { GFT } & \text { Gravitational fall time } \\ \text { TFI } & \text { Time to first impact and fall } \\ \text { TOF/KU } & \text { Time of flight/Kutateladze criterion }\end{array}$

The USER option is not actually a model, since it is strictly user-driven, and does not depend on field attributes, cell conditions, or inflow gas or debris conditions. The GFT model depends on cell conditions and field attributes, but is not dependent upon gas or debris inflow rates. The last two options are dependent upon cell conditions, debris field attributes, and inflow gas and debris conditions. The TOF/KU model is believed to be the most realistic of all the options and is recommended for most situations. There are some situations when use of one of the other options would be more desirable than TOF/KU. For example, one might want to use the USER option to disable trapping in a study to calculate conservative DCH loads. The equations used to calculate $\lambda$ and the trapping mechanism flags are described in the following sections. The first section below, however, discusses how velocities that are used in the trapping and heat transfer models are calculated. 


\subsubsection{Average Velocities}

The relative velocity between gas and debris, $\mathrm{v}_{\mathrm{re}}$, in the GFT and USER trapping models is calculated as the maximum of the gravitational fall velocity and the difference between the average gas velocity and the average debris velocity through the cell. The gravitational fall velocity, $\mathrm{v}_{\mathrm{gft}}$, is defined in Section 6.3.4. The average gas and debris velocities through a cell are calculated in a similar manner as the structure forced convection velocities are calculated, but with one key difference: the average velocities calculated for use in the $\mathrm{DCH}$ models do not use the structure-specific flow-path coefficients. The following equations describe the calculation of the average velocities used in the DCH trapping model and the DCH heat transfer model. Note that these velocities are used in all four (USER, GFT, TFI and TOF/KU) of the trapping models, and in the convective heat transfer model.

The average debris velocity for a given cell $i$ and field $n$, considering all flow paths, is calculated from the debris outflow rates and the debris inflow rates as follows:

$$
\begin{aligned}
& v_{\mathrm{d}, \text { in }}=\frac{1}{A_{\text {hyd }}} \sum_{\mathrm{ji}} \frac{\mathrm{W}_{\mathrm{d}, \mathrm{ji}, \mathrm{n}} \theta_{\mathrm{ji}}}{\rho_{\mathrm{d} \mathrm{j}, \mathrm{n}, \mathrm{P}}\left(P_{\mathrm{i}} / P_{\mathrm{j}}\right)} \\
& \mathrm{v}_{\mathrm{d}, \text { out }}=\frac{1}{A_{\text {hyd }}} \sum_{\mathrm{ij}} \frac{\mathrm{W}_{\mathrm{d}, \mathrm{ij}, \mathrm{n}} \theta_{\mathrm{ij}}}{\rho_{\mathrm{d}, \mathrm{i}, \mathrm{n}}} \\
& \mathrm{v}_{\mathrm{d}, \text { avg }}=\max \left[\mathrm{v}_{\mathrm{gft}}\left(\frac{\mathrm{v}_{\mathrm{d}, \mathrm{in}}+\mathrm{v}_{\mathrm{d}, \text { out }}}{2}\right)\right]
\end{aligned}
$$

where $\mathrm{W}_{\mathrm{d}, \mathrm{j}, \mathrm{n}}$ is the debris mass flow rate given by Equation (6-10), the $\mathrm{ji}$ sum corresponds to all gas and debris inflows, and the ij sum corresponds to all gas and debris outflows. The cell hydraulic area $A_{\text {hyd }}$ is equal to the cell gas volume to the two-thirds power. Note that the subscripts $i$ and the $n$ for cell $i$ and field $n$ are not included in the symbols for $v_{d, i n}, v_{d, \text { out }}$, and $v_{d, \text { avg }}$ for notational convenience; however, these values are specific to a cell and a field. It is also worth noting that the average debris velocity is not allowed to be smaller than the gravitational fall velocity.

The average gas velocity through a cell, $\mathrm{v}_{\mathrm{g}, \mathrm{avg}}$, is calculated in a similar manner, but the gas mass fluxes and gas densities are used in place of the debris values as shown below. Also, the gas velocity is calculated by assuming isothermal flow and assuming that all incoming gas flow streams mix with each other before they mix with the cell inventory. Again, this is similar to the structure forced convection velocity model discussed in Section 10.1.1.6. Note that there is only one average gas velocity through the cell which is used to calculate $\mathrm{v}_{\mathrm{re}}$ for each debris field. The controlling equations are 


$$
\begin{aligned}
& \bar{T}_{i n}=\frac{\sum_{j i} W_{g, j i} \theta_{j i} T_{g, j} c_{p, j}}{\sum_{j i} W_{g, j i} \theta_{j i} c_{p, j}} \\
& v_{g, \text { in }}=\frac{1}{A_{\text {hyd }}} \sum_{j i} \frac{W_{g, j i} \theta_{j i} R \bar{T}_{i n}}{M_{j} P_{i}} \\
& v_{g, \text { out }}=\frac{1}{A_{\text {hyd }}} \sum_{i j} \frac{W_{g, j i j} \theta_{i j}}{\rho_{g, i}} \\
& v_{g, a v g}=\frac{v_{g, \text { in }}+v_{g, \text { out }}}{2}
\end{aligned}
$$

where $T_{g, j}$ is the temperature, $c_{p j}$ is the gas heat capacity, and $M_{j}$ is the gas molecular weight in the upstream cell $j . R$ is the universal gas constant.

In the USER and GFT trapping options, if the slip s defined in Equation (6-2) is set greater than 1 for any field in any cell, then $\mathrm{v}_{\mathrm{re}}$ is calculated as follows:

$$
\mathrm{v}_{\mathrm{re}}=\max \left(\mathrm{v}_{\mathrm{gft}} \mid \mathrm{v}_{\mathrm{g}, \mathrm{avg}}-\mathrm{v}_{\mathrm{d}, \mathrm{avg}}\right)
$$

Otherwise the expression

$$
\mathrm{v}_{\mathrm{re}}=\max \left(\mathrm{v}_{\mathrm{gft}}, \mathrm{v}_{\mathrm{d}, \mathrm{avg}}\right)
$$

is used. If the former expression is desired even when the slip is essentially 1 , then the slip can be set slightly larger than 1.

The $\mathrm{v}_{\mathrm{d}, \text { avg }}$ and $\mathrm{v}_{\mathrm{g}, \text { avg }}$ averages are also used in various places in the TFI and TOF/KU trapping models as described in the following sections.

\subsubsection{USER Trapping Model}

The simplest and most parametric model is the USER model. In this model, the user simply specifies a trapping rate, $\lambda_{i}$, for each cell. The user-specified trapping rate must be the same for each particle field in a cell. That is, $\lambda_{i, n}=\lambda_{1}$ for all particle fields, $n$. This is not true for the other trapping models, since in these models, the rates depend upon the size and composition of the particles in the field. 
The user-specified trapping rate is normally a constant; however, time-varying rates can be specified through the use of user tables. To specify a constant trapping rate in a cell the value desired is given in the DCH-CELL block as explained in Section 14.3.1.11. User trapping values specified in the global DHEAT block are used as default constant trapping rates for all cells. To specify a timevarying trapping rate, the VAR-PARM keyword is used in the DCH-CELL input block. The name of the VAR-Y variable in the VAR-PARM block must be TRAPRATE to specify the trapping rate as the dependent variable in the table.

\subsubsection{GFT Trapping Model}

In this model, the trapping rate is taken to be controlled by the gravitational fall rate of a sphere in the cell atmosphere:

$$
\lambda_{\mathrm{i}, \mathrm{n}}=\frac{\mathrm{v}_{\mathrm{gft}, \mathrm{i}, \mathrm{n}}}{\mathrm{L}_{\mathrm{gft}, \mathrm{i}}}
$$

where the terminal fall velocity for debris particles in field $n$ and cell $i$ is $v_{\text {gfti, }}$. In the interest of notational convenience, this velocity is simply referred to as $v_{\text {gft }}$ hereafter, but the reader should remember that $v_{\mathrm{gft}}$ for each field and in each cell is unique. The characteristic gravitational fall height for debris particles in cell $\mathrm{i}$ is $\mathrm{L}_{\mathrm{gft}, \mathrm{i}}$. Again, the $\mathrm{i}$ subscript will be dropped for notational convenience, but the reader should remember that $\mathrm{L}_{\mathrm{gft}}$ for each cell can be unique.

The terminal fall velocity, $v_{\mathrm{gft}}$, is computed using the following drag correlation for spheres:

$$
\begin{aligned}
& v_{g f t}=\left[\frac{-C_{1}+\sqrt{C_{1}^{2}+C_{1} C_{2}}}{2}\right]^{2}\left(\frac{\mu_{g}}{\rho_{g} d}\right) \\
& C_{1}=9.06 ; C_{2}=\frac{1}{3} \sqrt{\left(\frac{g d}{2}\right)\left(\frac{\rho_{d} d}{\mu_{g}}\right)\left(\frac{\rho_{g} d}{\mu_{g}}\right)}
\end{aligned}
$$

where $g$ is the acceleration of gravity, $\mu_{\mathrm{g}}$ is the gas viscosity, $\rho_{\mathrm{g}}$ is the gas density, $\mathrm{d}$ is the particle diameter, and $\rho_{d}$ is the debris material density.

Note that $v_{\text {gft }}$ is dependent upon both cell $i$ and field $n$, through the gas density, gas viscosity, debris density, and particle diameter. The above correlation assumes that the particles are spheres, the atmosphere is stagnant, and that the particles do not physically interact with each other as they fall.

The characteristic gravitational fall height, $L_{\text {git }}$ by default, is calculated to be the cube root of the initial cell gas volume. A DCH-specific $\mathrm{L}_{\mathrm{gft}}$ value can also be specified independent of the cell volume. This is done using the LENGFT keyword in the DCH-CELL input block. 
An important aspect of the CONTAIN trapping treatment is that the GFT trapping rate is computed and used in the TOF/KU model as described in Section 6.3.6. This is based on the assumption that particles cannot de-entrain more slowly than they would fall to the floor by gravity. Details of how this is applied is provided in the following two sections. The GFT rate is not used as a bound if the USER trapping model is selected. The GFT trapping rate is also used in the TFI model to calculate the total debris flight time. The gravitational fall time, $t_{\mathrm{gft}}$ is given by

$$
t_{g f t}=\frac{L_{g f t}}{v_{g f t}}
$$

\subsubsection{TFI Trapping Model}

The TFI model is based on the assumption that debris will not stick on structures, but will strike only one structure and then rebound and fall to the floor by gravity. This model is provided primarily to facilitate performing sensitivity calculations. Production calculations and most experimental analyses should be performed with the TOF/KU model described in the following section, although use of the TFI or GFT models in the dome can be defended. The TFI and TOF/KU models both use the mass flow rates of gas and debris through the dominant flow path into a cell, and the average flow rate into a cell. By default, the code automatically determines the dominant flow path into a cell. The dominant flow path is defined as the flow path with the most debris material flowing through it. The user can optionally specify the dominant flow path as a regular flow path or an engineered vent using the FROMCELL or FROMVENT keywords. (Note that regular flow paths are now considered obsolete.) The dominant flow path may not be a dedicated suppression pool vent flow path. For any period of time when debris and gas flow is not inward through the identified flow path, the GFT model will be used. Unless there is a good reason to do otherwise, such as performing code testing, the user should let the code automatically determine the dominant flow path.

The CONTAIN flow model calculates the mass flow rate of gas and debris through the flow paths in the containment as described in Section 6.3.2. If slip between gas and debris is modeled, then the gas and debris velocities are distinct and their individual values will be used to determine flight times and $\mathrm{N}_{\mathrm{Ku}}$ numbers. However, if slip between gas and debris ignored, the average flow velocity for gas and debris is used for both. Both cases are included in the descriptions below.

The debris time of flight to the first structure, $t_{s, 1}$, is calculated by assuming the debris velocity linearly decreases from the inlet debris velocity, $\mathrm{v}_{\mathrm{d}, \mathrm{n}}$, to the debris velocity at first impact, $\mathrm{v}_{\mathrm{d}, 1}$,

$$
t_{s, 1}=\frac{L_{1}}{v_{d, n}-v_{d, 1}} \ln \left(\frac{v_{d, n}}{v_{d, 1}}\right)
$$

where $L_{1}$ is the distance to the first structure. This distance must be provided by the user. The debris velocity at first impact is assumed to be equal to the gas velocity at first impact, $\mathrm{v}_{\mathrm{g}, 1}$, if that velocity is slower than the debris inlet velocity: 


$$
\mathrm{v}_{\mathrm{d}, 1}=\min \left(\mathrm{v}_{\mathrm{d}, \mathrm{n}}, \mathrm{v}_{\mathrm{g}, 1}\right)
$$

Qualitatively, this is based on the fact that debris/gas drag and entrained ambient gas are assumed to slow down the gas until debris and gas both decrease together. The gas velocity at first impact is also used in the TOF/KU model to evaluate the Kutateladze number; therefore, its governing equations are provided in the following section.

If $v_{d, 1}$ is equal to $v_{d, n}$, then the debris flight time to the first structure is given by

$$
t_{s, 1}=\frac{L_{1}}{v_{d, n}}
$$

which is the limit of Equation (6-91) as $\mathrm{v}_{\mathrm{d}, 1}$ approaches $\mathrm{v}_{\mathrm{d}, \mathrm{n}}$. The average debris velocity to the first impact, $\mathrm{v}_{\mathrm{d} 1, \mathrm{avg}}$, is given by

$$
\mathrm{v}_{\mathrm{d} 1, \text { avg }}=\frac{\mathrm{L}_{1}}{\mathrm{t}_{\mathrm{s}, 1}}
$$

The TFI trapping rate is given by the inverse of the sum of the flight time to the first structure and the gravitational fall time:

$$
\lambda=\frac{1}{t_{s, 1}+t_{g f t}}
$$

The trapping rate given by the above equations is only calculated if flow through the identified flow path is into the cell. If this is not the case, then the trapping rate will be calculated using the GFT model.

If the slip $s$ in Equation (6-2) is greater than one for any field in any cell, then the relative velocity between gas and debris, $v_{\mathrm{re}}$, for heat and mass transfer purposes is given by

$$
\mathrm{v}_{\mathrm{re}}=\max \left(\mathrm{v}_{\mathrm{gft}},\left|\mathrm{v}_{\mathrm{g} 1, \mathrm{avg}}-\mathrm{v}_{\mathrm{d} 1, \mathrm{avg}}\right|\right)
$$

where $v_{\text {glavg }}$ is the average gas velocity to the first structure, which is defined in the following section under Case 1 of the TOF/KU model. The reader is referred to that section for a description of the governing equations for this velocity. If slip is ignored completely, then $v_{\mathrm{gl}, \mathrm{avg}}$ is dropped in the above equation. 


\subsubsection{TOF/KU Trapping Model}

Like the TFI model, this model calculates the trapping rate at each calculational cycle according to current conditions of the atmosphere, attributes of the particle field (size, composition, etc), and gas and debris inflow conditions. As the name of the model implies, the TOF/KU model uses a Kutateladze entrainment criterion to determine whether particle re-entrainment occurs after debris impacts structures. If re-entrainment is not indicated, then the debris is assumed to stick and the trapping rate is set to the inverse of the time of flight to the structure. Two de-entrainment criteria which are conceptually related to physical impacts of debris on structures are considered. The first criterion refers to debris impaction upon the first structure debris is likely to encounter as it flows into a cell. The second refers to subsequent structures that debris will impact as a result of average flow through the cell. If the Kutateladze correlation indicates debris re-entrainment for both conditions, then the trapping rate will not be based upon time of flight to structure impacts. Instead the trapping rate will be set to allow most of the debris to flow out of the cell. An option is provided to allow debris to trap at a rate characteristic of gravitational settling under this condition.

The remainder of this section describes the specific equations in the DCH model that implement this trapping strategy. First, however, it is important to understand that the flight of particles in the cell is not actually "tracked" in the TOF/KU model. Without major architectural changes, this would be impossible for a control volume code like CONTAIN to accomplish. Instead, the model relies upon estimates of particle and gas velocities and flight distances to evaluate the Kutateladze numbers for the first two phases. The magnitude of these numbers compared to Kutateladze cutoff numbers for entrainment dictate which calculated debris transport time estimate to use in the evaluation of the trapping rate at that moment in time. This trapping rate applies to all debris that is airborne at that moment in time. If the rate of change in the calculated trapping rate is slow, then this approach should be reasonable.

The Kutateladze number is a dimensionless number given by

$$
N_{\mathrm{Ku}} \equiv \frac{\rho_{\mathrm{g}} \mathrm{v}_{\mathrm{g}}^{2}}{\sqrt{\left(\rho_{\mathrm{d}, \mathrm{mat}}-\rho_{\mathrm{g}}\right) \mathrm{g} \sigma}}
$$

where $\rho_{\mathrm{d}, \text { mat }}$ is the material density of the debris, not the airborne density of debris in the cell. Physically, $N_{K u}$ represents the ratio of the kinetic force of an entraining fluid (with density $\rho_{g}$, and velocity $\mathrm{v}_{\mathrm{g}}$ ) to the geometric mean of gravitational and surface tension forces of the denser fluid being entrained (with density $\rho_{d}$ ). In the context of the TOF/KU debris trapping model, the entraining fluid is the in-flowing debris/gas jet (that tends to re-entrain any trapped debris on structures back into the jet), and the denser fluid is the molten debris in the cell (whose surface tension favors "sticking" the debris to a structure as a film). At some fluid velocity, the kinetic forces will sufficiently overcome the surface tension forces to re-entrain the debris from the surface thereby avoiding trapping on that structure. Below this velocity, debris in the cell is assumed to deentrain (or trap) at a rate that corresponds to the time of flight to the surface in question. The model uses two $\mathrm{N}_{\mathrm{Ku}}$ cutoff values, $\mathrm{N}_{\mathrm{Ku}, \mathrm{T}, 1}$ and $\mathrm{N}_{\mathrm{Ku}, \mathrm{T}, 2}$, which control the first two phases (or impacts) 
considered in the TOF/KU model. The default value for each cutoff is 10 , but the user may override this value for the first and subsequent impacts. The default is based on droplet entrainment in vertical tubes from the work in Reference Brg81.

The reader should also be aware that the equations presented below are applied to each debris field individually, even though this is not explicitly indicated by the simplified notation used below. Therefore, the trapping rate of one field will typically be different than other fields with different sizes and debris densities.

6.3.6.1 TOF/KU Case 1: Trapping On First Impact. Gases flowing into a cell are assumed to entrain gases in the cell using the Ricou-Spalding [Ric61] entrainment correlation. With this correlation, the density of the gas jet impinging the first structure used in evaluating $\mathrm{N}_{\mathrm{Ku}}$ is given by

$$
\rho_{g, 1}=\frac{\rho_{0} \rho_{a} \xi}{\left[\rho_{a}+(\xi-1) \rho_{0}\right]}
$$

where,

$$
\xi=\max \left(\frac{L_{1}}{x_{c}}, 1\right)
$$

and $L_{1}$ is the user-specified distance to the first structure as previously noted, and $x_{c}$ is a critical cutoff distance below which no entrainment occurs. This distance is given by

$$
\begin{aligned}
& \mathrm{x}_{\mathrm{c}}=\frac{\mathrm{d}_{0}}{\alpha} \\
& \mathrm{d}_{0}=\sqrt{\frac{4 \mathrm{~A}_{0}}{\pi}} \\
& \alpha=\alpha_{0} \sqrt{\frac{\rho_{\mathrm{g}, \mathrm{i}}}{\rho_{0}}} \\
& \rho_{0}=\rho_{\mathrm{g}, \mathrm{u}}\left(\frac{\mathrm{P}_{\mathrm{i}}}{\mathrm{P}_{\mathrm{u}}}\right)
\end{aligned}
$$

where $\alpha$ is the density corrected jet expansion coefficient, $d_{0}$ is the hydraulic diameter of flow path opening, $A_{0}$ is the area of the flow path opening, $\alpha_{0}$ is the user-definable jet expansion coefficient 
whose default value is $0.32, \rho_{\mathrm{g}, \mathrm{i}}$ is the density of gas in the cell, $\rho_{0}$ is the pressure corrected density of gas from the upstream cell, $P_{i}$ is the pressure in cell $i$, and $P_{u}$ is the pressure in the upstream cell.

By default the flow path area, $A_{0}$, is the area of the dominant flow path as defined in the flow input. This area can be user-specified using the ADFLOW keyword; however, if this is done, it may be desirable for the user to specify the dominant flow path explicitly using either FROMCELL or FROMVENT keywords. This is suggested because the flow path area will not change if ADFLOW is specified, while the dominant flow path may change if it is not also explicitly specified. Specifying the dominant flow path explicitly will prevent an inconsistent area from being used. There may be valid reasons for not following this suggestion; therefore, it is not enforced by the code.

The velocity of gas after it enters a cell is assumed to be constant at $v_{g, \text { in }}$ until it travels the cutoff distance $\mathrm{x}_{\mathrm{c}}$. Beyond the cutoff distance it is assumed that the velocity declines linearly as gases are entrained into the flow stream. The gas velocity at the first impact, $\mathrm{v}_{\mathrm{g}, \mathrm{i}}$, is given by the maximum of the jet velocity and a forced convective velocity

$$
\mathrm{v}_{\mathrm{g}, 1}=\max \left[\mathrm{v}_{1}, \mathrm{v}_{\mathrm{g}, \text { in }} \min \left(1, \frac{\mathrm{x}_{\mathrm{c}}}{\mathrm{L}_{\mathrm{l}}}\right)\right]
$$

where $v_{1}$ is the forced convective velocity for the inner face of the first heat transfer structure in the cell (see Section 10.1.1.6). None of the parameters in the DCH-CELL block have an effect on $v_{1}$. Because of this scheme, it is recommended that at least two structures be defined in subcompartments so that a forced convection velocity will be calculated and stored in $v_{1}$ (and $v_{2}$ used under Case 2 in the following section) for use in the TOF/KU model. If no structures are defined, then the model will use the average gas velocity through the cell, $\mathrm{v}_{\mathrm{g}, \mathrm{avg}}$ in place of $\mathrm{v}_{1}$ in the above expressions.

The Kutateladze criterion can be evaluated using one of two options. In the default RHODG = GAS option only the gas momentum is included in the numerator. If the RHODG = MIX option is invoked then debris momentum will be included in the numerator of $\mathrm{N}_{\mathrm{Ku}}$. Under the default RHODG $=$ GAS option, $N_{K u}$ for the first impact, $N_{\mathrm{Ku}, s, 1}$, is given by

$$
\mathrm{N}_{\mathrm{Ku}, \mathrm{s}, 1}=\frac{\rho_{\mathrm{g}, 1} \mathrm{v}_{\mathrm{g}, 1}^{2}}{\sqrt{\left(\rho_{\mathrm{d}, \mathrm{mat}}-\rho_{\mathrm{g}, 1}\right) \mathrm{g} \sigma}}
$$


If the RHODG = MIX option is specified, then $\mathrm{N}_{\mathrm{Ku}, \mathrm{s}, 1}$ includes the momentum of the debris:

$$
N_{\mathrm{Ku}, \mathrm{s}, 1}=\frac{\rho_{\mathrm{g}, 1} \mathrm{v}_{\mathrm{g}, 1}^{2}+\rho_{\mathrm{d}, \mathrm{u}} \mathrm{v}_{\mathrm{d}, 1}^{2}}{\sqrt{\left(\rho_{\mathrm{d}, \mathrm{mat}}-\rho_{\mathrm{g}, 1}-\rho_{\mathrm{d}, \mathrm{u}}\right) \mathrm{g} \sigma}}
$$

where the debris velocity at first impact, $\mathrm{v}_{\mathrm{d}, 1}$, is taken to be the minimum of the debris inlet velocity and the gas velocity at first impact as noted in the previous section on the TFI model. In the above equation $\rho_{\mathrm{d}, \mathrm{u}}$ is calculated as $\left(\rho_{\mathrm{fl}}-\rho_{\mathrm{g}, \mathrm{u}}\right) \mathrm{P}_{\mathrm{i}} / \mathrm{P}_{\mathrm{u}}$.

De-entrainment on the first impact is assumed to occur if $\mathrm{N}_{\mathrm{Ku}, \mathrm{s}, \mathrm{1}}$ is less than the $\mathrm{N}_{\mathrm{Ku}, \mathrm{T}, \mathrm{1}}$ cutoff value. When $\mathrm{N}_{\mathrm{Ku}, s, 1} \leq \mathrm{N}_{\mathrm{Ku}, \mathrm{T}, 1}$, the trapping rate, $\lambda$, is given by

$$
\lambda=\frac{1}{\min \left(t_{\mathrm{s}, 1}, \mathrm{t}_{\mathrm{gft}}\right)}
$$

where $t_{s, 1}$ is the debris flight time to the first structure defined in the previous section on the TFI model. The average gas velocity from the inlet to the first impact is calculated by integrating from $v_{g, i n}$ to $v_{g, 1}$ over the distance $x_{c}$ to $L_{1}$ to give

$$
v_{g 1, a v g}=\frac{v_{g, i n} L_{1}}{x_{c}+\frac{\left(L_{1}^{2}-x_{c}^{2}\right)}{2 x_{c}}} \quad \text { for } x_{c}<L_{1}
$$

If $x_{c}$ is greater than $L_{1}$ then $v_{g 1, a v g}$ will be set equal to $v_{\text {gin }}$. If the slip $s$ in Equation (6-2) is greater than one for any field in any cell, then the relative velocity between gas and debris for heat transfer and chemical reactions, $\mathrm{v}_{\mathrm{r}}$, is given by

$$
\mathrm{v}_{\mathrm{re}}=\max \left(\mathrm{v}_{\mathrm{gft}},\left|\mathrm{v}_{\mathrm{g} 1, \mathrm{avg}}-\mathrm{v}_{\mathrm{d} 1, \mathrm{avg}}\right|\right)
$$

If slip is ignored completely, then $\mathrm{v}_{\mathrm{re}}$ is given by

$$
\mathrm{v}_{\mathrm{re}}=\max \left(\mathrm{v}_{\mathrm{gft}}, \mathrm{v}_{\mathrm{d1}, \mathrm{avg}}\right)
$$


The flight time and average velocity to the first structure in Case 1 of the TOF/KU model are the same as they are for the TFI trapping model. Note, however, that the TOF/KU Case 1 trapping rate differs from the TFI rate. The TFI model takes the sum of the gravitational fall time and the first impact flight time as the time of flight, while the TOF/KU model takes the shorter of the two as the time of flight. The rates are different because if the debris reaches the first structure under Case 1 of the TOF/KU model, it is assumed to be trapped there. The main input parameter that affects this case of the TOF/KU model is the first trapping length, $\mathrm{L}_{1}$. If a direct line of travel from a primary entrance flow path to a structure can be clearly identified, then this distance should be given as $L_{1}$. Otherwise, it is recommended that $L_{1}$ be set equal to 6 times the cell gas volume divided by the surface area of all surfaces in the cell.

An alternate trapping behavior corresponding only to the time of flight to the first structure can be obtained by intentionally specifying an artificially large $\mathrm{N}_{\mathrm{Ku}, \mathrm{T}, 1}$ value. If this is done, the $\mathrm{N}_{\mathrm{Ku}}$ criterion for re-entrainment will never be met and debris will always stick on the first impact. This technique may be useful for performing sensitivity studies. In most predictive calculations, however, the default $\mathrm{N}_{\mathrm{K} \mu}$ cutoff values should be used.

If the $\mathrm{N}_{\mathrm{Ku}}$ criterion for re-entrainment on the first impact is met, sticking on the first impact is not assumed and subsequent impacts on nonhorizontal surfaces will be considered as described below.

6.3.6.2 TOF/KU Case 2: Trapping On Structures Beyond The First Impact. The velocity $v_{8,2}$ used for evaluating $\mathrm{N}_{\mathrm{Ku}}$ for impacts after the first is the second structure convective velocity, $\mathrm{v}_{2}$, if defined. This velocity is more specifically the convective velocity for the inner face of the second structure defined in the structure input block and can be controlled by changing the default hydraulic area and coefficients in that input block. Note that none of the parameters specified in the DCH-CELL input block have an effect on $v_{2}$. It is recommended that at least two structures be defined in subcompartments so that either a forced or natural convection velocity is calculated in the heat transfer module and stored in $\mathrm{v}_{2}$ for use in the TOF/KU model. If fewer than two structures are defined, then the model will use the average gas velocity through the cell, $\mathrm{v}_{\mathrm{g}, \mathrm{avg}}$, for $\mathrm{v}_{\mathrm{g}, 2} \cdot$

Under the default $\mathrm{RHODG}=\mathrm{GAS}$ option, $\mathrm{N}_{\mathrm{Ku}}$ for the second trapping criterion is given by

$$
\mathrm{N}_{\mathrm{Ku}, \mathrm{s}, 2}=\frac{\rho_{\mathrm{g}, \mathrm{i}} \mathrm{v}_{\mathrm{g}, 2}^{2}}{\sqrt{\left(\rho_{\mathrm{d}, \mathrm{mat}}-\rho_{\mathrm{g}, \mathrm{i}}\right) \mathrm{g \sigma}}}
$$

If $\mathrm{RHODG}=\mathrm{MIX}$ is specified, the second trapping $\mathrm{N}_{\mathrm{Ku}}$ number is given by

$$
N_{\mathrm{Ku}, \mathrm{s}, 2}=\frac{\rho_{\mathrm{g}, \mathrm{i}} \mathrm{v}_{\mathrm{g}, 2}^{2}+\rho_{\mathrm{d}, \mathrm{i}} \mathrm{v}_{\mathrm{d}, \mathrm{avg}}^{2}}{\sqrt{\left(\rho_{\mathrm{d}, \mathrm{mat}}-\rho_{\mathrm{g}, \mathrm{i}}-\rho_{\mathrm{d}, \mathrm{i}}\right) \mathrm{g} \sigma}}
$$


In calculating $\mathrm{v}_{\mathrm{d}, \text { avg }}$ as used here, $\mathrm{v}_{\mathrm{gft}}$ is dropped from Equation (6-81); that is, $\mathrm{v}_{\mathrm{gft}}$ does not provide a lower limit to $v_{d, a v g}$ in this context. Also notice that the density of the entraining fluid is given by $\rho_{\mathrm{g}, \mathrm{i}}$, which is the gas density in the cell and not the upstream entrained density, $\rho_{\mathrm{g}, 1}$, that was used in evaluating $\mathrm{N}_{\mathrm{Ku}, s_{1}}$. This approach is based on the assumption that the entraining fluid of interest for structure impacts, after the first one, more closely resembles the debris/gas mixture of the cell itself than the material entering from the upstream cell.

When $\mathrm{N}_{\mathrm{Ku}, \mathrm{s}, 1}>\mathrm{N}_{\mathrm{Ku}, \mathrm{T}, 1}$ and $\mathrm{N}_{\mathrm{Ku}, \mathrm{s}, 2} \leq \mathrm{N}_{\mathrm{Ku}, \mathrm{T}, 2}$, debris is assumed to re-entrain from the first structure but trap (stick) on subsequent structures. For this case the trapping rate, $\lambda$, is given by

$$
\lambda=\frac{1}{t_{s, 1}+\min \left(t_{s, 2}, t_{g t}\right)}
$$

where $t_{s, 2}$ is the average debris time of flight from the first impact to subsequent impacts before trapping. This time is not allowed to exceed the gravitational fall time, because, physically, drops normally would trap at least as fast as they would if falling under gravity to horizontal surfaces. The $t_{s, 2}$ travel time is given by

$$
t_{s, 2}=\frac{L_{2}}{v_{d, a v g}}
$$

where $\mathrm{L}_{2}$ is specified by the user. Because the second trapping criterion applies to generic second impacts, it is recommended that $L_{2}$ be set equal to a generic characteristic cell dimension equal to 6 times the cell volume divided by the sum of all exposed surface areas, unless there is a clear reason based on actual cell geometry for choosing a different value.

The average debris velocity $\mathrm{v}_{\mathrm{d} 2, \mathrm{avg}}$ from inlet to trapping on surfaces beyond the first impact is given by

$$
\mathrm{v}_{\mathrm{d} 2 \text {,avg }}=\left[\frac{\mathrm{t}_{\mathrm{s}, 1} \sqrt{\mathrm{v}_{\mathrm{d} 1, \mathrm{avg}}}+\mathrm{t}_{\mathrm{s}, 2} \sqrt{\mathrm{v}_{\mathrm{d}, \mathrm{avg}}}}{\mathrm{t}_{\mathrm{s}, 1}+\mathrm{t}_{\mathrm{s}, 2}}\right]^{2}
$$

If the slip $s$ in Equation (6-2) is greater than 1 for any field in any cell, the relative velocity between gas and debris for heat and mass transfer purposes, $\mathrm{v}_{\mathrm{re}}$, is given by

$$
\mathrm{v}_{\mathrm{re}}=\max \left(\mathrm{v}_{\mathrm{gft}},\left|\mathrm{v}_{\mathrm{g}, \mathrm{avg}}-\mathrm{v}_{\mathrm{d} 2 \text {,avg }}\right|\right)
$$


If slip is ignored completely, then $\mathrm{v}_{\mathrm{re}}$ is given by

$$
\mathrm{v}_{\mathrm{re}}=\max \left(\mathrm{v}_{\mathrm{gft}}, \mathrm{v}_{\mathrm{d} 2 \text {,avg }}\right)
$$

If $\mathrm{N}_{\mathrm{Ku}, 2,2}>\mathrm{N}_{\mathrm{Ku}, \mathrm{T}, 2}$, then debris is assumed to remain airborne until it traps by gravity or flows out of the cell. The trapping rate will be calculated as described below.

6.3.6.3 TOF/KU Case 3: No Trapping From First or Second Kutateladze Number Criterion. This section describes the case where the inflow velocity and other conditions are such that neither of the two $\mathrm{N}_{\mathrm{Ku}}$ re-entrainment criteria are met. Therefore, debris will either gravitationally settle to horizontal surfaces or flow out of the cell. The user has some control over this by specifying the third trapping length, $\mathrm{L}_{3}$, and how the debris velocity is treated with the VNOST $=\mathrm{GFT}$ and VNOST $=$ CNVEL options. Some guidance on the selection of $\mathrm{L}_{3}$ and the VNOST option is provided in the following section.

The trapping rate for this case is given by

$$
\lambda=\frac{1}{t_{s, 1}+t_{s, 2}+\min \left(t_{g f t}, t_{s, 3}\right)}
$$

where $t_{s, 3}$ is the characteristic residence time for debris that is not trapped under the first two deentrainment criteria. In the VNOST = CNVEL option (the default), the debris in the cell is assumed to travel at the average debris outlet velocity; therefore, $t_{s, 3}$ is given by

$$
t_{s, 3}=\frac{L_{3}}{v_{d, o u t}}
$$

where $L_{3}$ is a user-specified distance for this case. Four reasonable choices for this distance are (1) the GFT height, $L_{g f t}$ (2) the cell height, (3) 6 times the cell volume divided by total surface area as was also suggested for $\mathrm{L}_{2}$, and (4) a large value to approximate infinity. The fourth choice causes debris to remain airborne until it is swept out of the cell by flow. Under this approach the VNOST option has no effect. The recommended approach is to use the first choice, which is the default.

If the VNOST $=\mathrm{GFT}$ option is invoked, $t_{\mathrm{s}, 3}$ is given by

$$
t_{s, 3}=\frac{L_{3}}{v_{g f t}}
$$

Under this option the debris that is not trapped according to the two calculated $N_{K u}$ numbers is assumed to gravitationally settle to horizontal surfaces. The most logical selection of $L_{3}$ for this option is the cell height. By default $\mathrm{L}_{3}$ will be set equal to the "lengft" value, which will be set equal 
to the cell height if one is given in the GEOMETRY block. The average velocity of debris for this case, $v_{3, \text { avg }}$, will be $v_{\text {grt }}$ if VNOST $=$ GFT is specified and $v_{d, \text { out }}$ otherwise. The average velocity of debris for this case beginning with its entry into the cell is given by

$$
v_{d 3, a v g}=\left[\frac{t_{s, 1} \sqrt{v_{d 1, a v g}}+t_{s, 2} \sqrt{v_{d, a v g}}+t_{s, 3} \sqrt{v_{3, a v g}}}{t_{s, 1}+t_{s, 2}+t_{s, 3}}\right]^{2}
$$

If the slip s in Equation (6-2) is greater than one for any field in any cell, then the relative velocity is given by

$$
\mathrm{v}_{\mathrm{re}}=\max \left(\mathrm{v}_{\mathrm{gft}}\left|\mathrm{v}_{\mathrm{d} 3, \mathrm{avg}}-\mathrm{v}_{\mathrm{g}, \mathrm{avg}}\right|\right)
$$

As for the other cases, when slip is ignored, $\mathrm{v}_{\mathrm{g}, \mathrm{avg}}$ is dropped from the above equation.

\subsubsection{Trapping Model Sensitivity Coefficients}

In addition to the four trapping models and their various options and inputs, three sensitivity coefficients are provided to facilitate performing trapping sensitivity calculations. These coefficients are:

$$
\begin{array}{ll}
\lambda_{\min } & =\text { slowest allowable trapping rate (default }=0) \\
\lambda_{\max } & =\text { fastest allowable trapping rate }(\text { default }=\infty) \\
\lambda_{\operatorname{mul}} & =\text { trapping rate multiplier (default }=1 \text { ) }
\end{array}
$$

These three coefficients can be specified by the user in the input file using the TRAPMIN, TRAPMAX, and TRAPMUL keywords (see Section 6.4). Regardless of the trapping model selected, the trapping rate $\lambda^{\prime}$ actually used is obtained as follows:

$$
\lambda^{\prime}=\max \left(\min \left(\lambda_{\max }, \lambda \lambda_{\operatorname{mul}}\right), \lambda_{\min }\right)
$$

where $\lambda$ is the trapping rate defined by the above trapping rate models.

The $\lambda_{\text {mul }}$ coefficient is useful for exploring the sensitivity of a simulation to trapping rate in a relative manner. The other two are useful for limiting the calculated trapping rate to fall within known reasonable bounds.

\subsection{Chemical Reactions}

Chemical reactions are modeled to occur during a DCH event if reactive metals are present in the dispersed debris fields and if the atmosphere contains oxygen or steam. The following chemical reactions are treated in the model 


$$
\begin{gathered}
\mathrm{Zr}+\mathrm{O}_{2} \rightarrow \mathrm{ZrO}_{2} \\
\mathrm{Al}+\frac{3}{4} \mathrm{O}_{2} \rightarrow \mathrm{AlO}_{1.5} \\
\mathrm{Cr}+\frac{3}{4} \mathrm{O}_{2} \rightarrow \mathrm{CrO}_{1.5} \\
\mathrm{Fe}+\frac{1}{2} \mathrm{O}_{2} \rightarrow \mathrm{FeO} \\
\mathrm{Zr}+2 \mathrm{H}_{2} \mathrm{O} \rightarrow \mathrm{ZrO}_{2}+2 \mathrm{H}_{2} \\
\mathrm{Al}+\frac{3}{2} \mathrm{H}_{2} \mathrm{O} \rightarrow \mathrm{AlO}_{1.5}+\frac{3}{2} \mathrm{H}_{2} \\
\mathrm{Cr}+\frac{3}{2} \mathrm{H}_{2} \mathrm{O} \rightarrow \mathrm{CrO}_{1.5}+\frac{3}{2} \mathrm{H}_{2} \\
\mathrm{Fe}+\mathrm{H}_{2} \mathrm{O}
\end{gathered}
$$

As indicated, all of these reactions except for the last one for iron/steam are assumed to go to completion. These reactions are treated in a hierarchical fashion within a timestep where the order of the reactions is given by the order of the above list. Note that although a hierarchy is assumed during a timestep, normally metal is not exhausted during the short timesteps typically taken in a $\mathrm{DCH}$ calculation. As a result, the reactions of oxygen and steam are effectively calculated to proceed in parallel.

Each reaction is treated for every debris field in sequence. Therefore, the reactions are treated for drops in the first field, and then the reactions are considered for the second field, and so on. The exact same equations are applied to each debris field in a calculation; however, the reaction rate calculated for each field can and usually will be different since the models depend on the diameter, composition, and temperature of drops in the field. For example, if a debris field has no reactive metal in it, then chemical reactions will not be modeled at all for that field. Also, if two fields have different diameter drops then the reaction rate for the field with the smaller diameter will be faster than the field with the larger diameter drops. The model also considers the reaction of drops that are non-airborne if the non-airborne field is assigned a non-zero diameter (see Section 6.6).

The DCH chemistry model consists of four parts. The first part is the modeling of the transport of gases to the surface of drops. This part of the model is described in the first subsection below, where an effective reaction time for the metals in the field based on only gas-side transport limitations is derived. Note that this reaction time is also referred to as a "time constant" in the code output. Because the calculated reaction times change from timestep to timestep the term "time constant" is not used here. The second part of the model is a drop-side transport model based on the diffusion of oxidant inside the drop. This part of the model is described in the second subsection below, where an effective reaction time for metals in the field based on drop-side transport limitations is derived. The third part of the model is the combination of the gas-side and drop-side reaction rates and the hierarchy scheme used to evaluate the amount of metal that reacts in the debris fields. This part of 
the model is described in the third subsection below. The equations in the third subsection provide the mass and energy terms that go into the debris and gas mass and energy conservation equations. The final part of the model is the recombination of hydrogen produced by the chemical reactions that is described in Section 6.4.4.

\subsubsection{Gas-Side Transport}

The gas-side rate model is based on a heat/mass-transfer analogy, where the transport of oxygen and steam to the surface of drops in a field is given by a mass transfer coefficient times a density difference as follows:

$$
\begin{aligned}
\left(\frac{\mathrm{dm}}{\mathrm{dt}}\right)_{\mathrm{O}_{2}} & =\mathrm{K}_{\mathrm{O}_{2}} \mathrm{~A}_{\mathrm{d}} \rho_{\mathrm{O}_{2}}^{\prime} \\
\mathrm{K}_{\mathrm{O}_{2}} & =\frac{\mathrm{N}_{\mathrm{Sh}_{\mathrm{O}}} \boxplus_{\mathrm{O}_{2}}}{\mathrm{~d}} \\
\left(\frac{\mathrm{dm}}{\mathrm{dt}}\right)_{\mathrm{H}_{2} \mathrm{O}} & =\mathrm{K}_{\mathrm{H}_{2} \mathrm{O}} \mathrm{A}_{\mathrm{d}}\left(\rho_{\mathrm{H}_{2} \mathrm{O}}^{\prime}-\rho_{\mathrm{EQ}}\right) \text { if only Fe is present } \\
& =\mathrm{K}_{\mathrm{H}_{2} \mathrm{O}} \mathrm{A}_{\mathrm{d}} \rho_{\mathrm{H}_{2} \mathrm{O}}^{\prime} \quad \text { otherwise } \\
\mathrm{K}_{\mathrm{H}_{2} \mathrm{O}} & =\frac{\mathrm{N}_{\mathrm{Sh}_{2} \mathrm{H}_{2} \mathrm{O}} \bigoplus_{\mathrm{H}_{2} \mathrm{O}}}{\mathrm{d}}
\end{aligned}
$$

where $\mathrm{K}_{\mathrm{x}}$ is the mass transfer coefficient for oxidant $\mathrm{x}$; $\rho_{\mathrm{x}}^{\prime}$ is the density of oxidant $\mathrm{x}$ in the bulk atmosphere, corrected to $T_{B L}$, which is the boundary layer temperature between gas phase and the drop; $N_{S h x}$ is the Sherwood number for oxidant $x ; \boxplus_{x}$ is the gas diffusivity of oxidant $x$ in air; $\rho_{E Q}$ is the equilibrium density of steam at the drop surface; $A_{d}$ is the surface area for all drops in the debris field; $d$ is the diameter of debris in the field; and $x$ stands for either $\mathrm{O}_{2}$ or $\mathrm{H}_{2} \mathrm{O}$.

The Sherwood number, analogous to the Nusselt number in heat transfer, is given by the following correlation

$$
\mathrm{N}_{\mathrm{Sh}, \mathrm{x}}=2.0+0.6 \sqrt{\mathrm{N}_{\mathrm{Re}, \mathrm{g}}} \mathrm{N}_{\mathrm{Sc}, \mathrm{x}}^{1 / 3}
$$

where $\mathrm{N}_{\mathrm{Reg}, \mathrm{g}}$ is the Reynolds number for the gas density and $\mathrm{N}_{\mathrm{Sc}, \mathrm{x}}$ is the Schmidt number for oxidant $\mathrm{x}$. 
The Reynolds and Schmidt dimensionless numbers are given by

$$
\begin{aligned}
& N_{R e, g}=\frac{\rho_{g}^{\prime} V_{r e} d}{\mu_{g}\left(T_{B L}\right)} \\
& N_{S c, x}=\frac{\mu_{g}\left(T_{B L}\right)}{\rho_{g}^{\prime} \boxplus_{x}}
\end{aligned}
$$

where $\rho_{\mathrm{g}}{ }^{\prime}$ is the gas density at the gas/debris interface.

Expressions for the remaining terms in the above equations are given by

$$
\begin{aligned}
\rho_{x}^{\prime} & =\rho_{x}\left(\frac{T_{g}}{T_{B L}}\right) \\
\rho_{g}^{\prime} & =\left(\frac{m_{g}}{V_{g}}\right)\left(\frac{T_{g}}{T_{B L}}\right) \\
T_{B L} & =\frac{T_{g}+T_{d}}{2}
\end{aligned}
$$

$T_{g}$ is the gas temperature, and $T_{d}$ is the debris temperature of a specific debris field. The diameter, $\mathrm{d}$, is the diameter of drops in the field. As previously noted, the chemistry for each field is calculated separately, using the diameter, composition, and temperature specific to that field. The Reynolds number in the Sherwood correlation above is evaluated using the value of $v_{\mathrm{re}}$ calculated as described in Section 6.3.2.

Note that the density of gas, $\rho_{\mathrm{g}}^{\prime}$ in the $\mathrm{N}_{\mathrm{Re}}$ and $\mathrm{N}_{\mathrm{Sc}}$ correlations is the density of gas in the atmosphere, but modified to be at the boundary layer temperature. Likewise, $\rho_{x}^{\prime}$ is the density of oxidant $x$ in the atmosphere, modified to be at the boundary layer temperature. The viscosity $\mu_{g} / T_{\mathrm{BL}}$ is the molar average of the viscosities of the gas species in the atmosphere evaluated at the boundary layer temperature, $\mathrm{T}_{\mathrm{BL}}$.

If the debris field has iron metal in it, the equilibrium density of steam, $\rho_{\mathrm{EQ}}$, is given by 


$$
\begin{aligned}
\rho_{\mathrm{EQ}} & =\frac{\mathrm{P}_{\mathrm{EQ}} \mathrm{M}_{\mathrm{H}_{2} \mathrm{O}}}{\mathrm{RT}_{\mathrm{BL}}} \\
\mathrm{P}_{\mathrm{EQ}} & =\frac{\mathrm{P}_{\mathrm{H}_{2} \mathrm{O}, \mathrm{b}}+\mathrm{P}_{\mathrm{H}_{2}, \mathrm{~b}}}{1+\frac{\mathrm{K}}{\mathrm{X}_{\mathrm{FeO}}}}
\end{aligned}
$$

where the $P_{\mathrm{H} 20, \mathrm{~b}}$ and $\mathrm{P}_{\mathrm{H} 2, \mathrm{~b}}$ parameters are the partial pressures of steam and hydrogen in the cell atmosphere and $\mathrm{M}$ is the molecular weight. The $\mathrm{X}_{\mathrm{FeO}}$ parameter is the mole fraction of $\mathrm{FeO}$ among the other oxides in the debris field. $\mathrm{K}$ is the equilibrium constant for the iron/steam reaction and is given by

$$
K=e^{\frac{-\Delta G_{x x}}{R T_{d}}}
$$

where $\Delta G_{\mathrm{rx}}$ is the net difference in the Gibbs free energy of formation for the reactants and products of the iron/steam reaction. The correlations given in Reference Pow86 are used to evaluate the Gibbs free energies of formation.

Options also exist by which the user may either disable the equilibrium or specify that $\mathrm{X}_{\mathrm{Fe} 0}=1$ in evaluating Equation (6-127) and Equation (6-144) below; see the description of the IEQOPT input flag in Section 14.2.7 for details. The default treatment is normally recommended.

As shown by the above equations, the equilibrium density of steam at the surface of drops in the field is set to zero if there are any metals other than iron. If all the steam oxidant is consumed by non-iron metals in the field then this steam transfer rate is used unadjusted. An adjustment is made to the remaining available steam if iron is present in the field and if other metals did not consume all of the steam. This is accomplished in the code by evaluating an effective multiplier on the steam reaction rate for the $\rho_{E Q}=0$ case that would give the same amount of oxidant reaction as the non-zero case. This multiplier is then used only on the iron/steam reaction, which will only occur if iron is present and if other metals did not exhaust all steam transported to the drop. If the drop has only iron metal in it, then the steam transport rate is given by Equation (6-123). This is explained in greater detail in Section 6.4.3.

The correlations for oxygen and steam diffusivity, $\nsupseteq_{\mathrm{O} 2}$ and $\bigoplus_{\mathrm{H} 2 \mathrm{O}}$, in the cell atmosphere are derived from a binary diffusion approximation given in Reference Bir60, where the transport medium is assumed to be air:

$$
\boxplus_{\mathrm{O}_{2}}=\frac{6.40827 \times 10^{-5}\left(\mathrm{~T}_{\mathrm{BL}}\right)^{1.823}}{\mathrm{P}}
$$




$$
\mathrm{Ð}_{\mathrm{H}_{2} \mathrm{O}}=\frac{4.40146 \times 10^{-6}\left(\mathrm{~T}_{\mathrm{BL}}\right)^{2.334}}{\mathrm{P}}
$$

The molecular weights of oxidant and air and various constants specific to air are embedded within the constants in the above expressions.

The correlation for steam diffusivity is known to overestimate the diffusivity in air at elevated temperatures (above $500 \mathrm{~K}$ ). [Wil87a] On the other hand, the bulk of the metal-steam reaction in typical DCH calculations takes place in the cavity or subcompartment volumes in which the dominant noncondensable species in the debris-gas boundary layer is hydrogen, not air or nitrogen; often the hydrogen is dominant by large factors. As it happens, these errors cancel at approximately $2000 \mathrm{~K}$ and the net error does not exceed $30 \%$ over the temperature range 1200 to $3000 \mathrm{~K}$, assuming a pure steam-hydrogen mixture. [Wil87a] While it is obviously unsatisfying to rely upon this essentially fortuitous cancellation of errors, the fact is that the error is sufficiently small for the conditions that typically dominate $\mathrm{DCH}$ analysis that only a relatively sophisticated multicomponent diffusivity formulation would guarantee improvement without risking doing more harm than good. For compartmentalized geometries, the likelihood of important error in the present model is believed to be small. If large amounts of metallic debris are transported to the dome, where concentrations of noncondensables other than hydrogen may not be small, this formulation may overpredict the steam-metal reaction rate somewhat at sufficiently high temperatures.

The gas-side transport rates given by Equations (6-122) and (6-123) are used to calculate an oxygen equivalent molar gas transport rate to the surface of drops in the field

$$
\frac{\mathrm{dN}_{\mathrm{oe}}}{\mathrm{dt}}=\frac{2}{\mathrm{M}_{\mathrm{O}_{2}}}\left(\frac{\mathrm{dm}}{\mathrm{dt}}\right)_{\mathrm{O}_{2}}+\frac{1}{\mathrm{M}_{\mathrm{H}_{2} \mathrm{O}}}\left(\frac{\mathrm{dm}}{\mathrm{dt}}\right)_{\mathrm{H}_{2} \mathrm{O}}
$$

$\mathrm{N}_{o c}$ in this expression is the number of oxygen equivalents of oxidant gas at the surface of the drops in a given debris field available to react. The equation above gives the rate at which $\mathrm{N}_{o e}$ can be supplied for chemical reactions.

If only gas-side transport limits the reaction of metals, then the reaction rate of metal expressed in oxygen equivalents must equal the transport rate of gases to the drops. The metal reaction time based on gas-side transport can therefore be expressed as the ratio of metal, in oxygen equivalents, to the oxidant molar transport rate

$$
\tau_{\mathrm{g}}=\frac{\mathrm{N}_{\mathrm{met}}}{\left(\frac{\mathrm{dN} \mathrm{Ne}_{\mathrm{oe}}}{\mathrm{dt}}\right)}
$$


where $\mathrm{N}_{\text {met }}$ is the amount of metal in the debris field in oxygen equivalents and is given by

$$
N_{\text {met }}=\sum_{\substack{\mathrm{k}=\mathrm{Zr}, \mathrm{Al} \\ \mathrm{Cr}, \mathrm{Fe}}} \frac{v_{\mathrm{k}} \mathrm{m}_{\mathrm{k}}}{\mathrm{M}_{\mathrm{k}}}
$$

where $m_{k}$ is the mass of the reactive metal species " $k$ " in the debris field, $M_{k}$ is the molecular weight of the reactive metal species and $v_{k}$ are the oxygen to metal stoichiometry ratios for the metal species in the debris field. The stoichiometry ratios for $\mathrm{Zr}, \mathrm{Al}, \mathrm{Cr}$, and $\mathrm{Fe}$ are 2, 1.5, 1.5, and 1 respectively as readily seen from the reactions as written in Equation (6-121).

\subsubsection{Drop-Side Transport}

In reality, drop-side limitations may slow the reaction rate from the rate given by considering only gas-side limitations. The actual reaction rate used in the chemistry model is therefore a combination of gas-side and drop-side rates. This section describes the drop-side rate model and the next section describes how the gas-side and drop-side rate models are combined and used to represent the chemical reactions.

CONTAIN has a rate-limiting drop-side model that is based on the solution of the diffusion equation in spherical coordinates. A useful approximation to the diffusion equation solution in spherical coordinates at early times is

$$
F(y)=6 \sqrt{\frac{y}{\pi}}-3 y \quad ; y \leq 0.2
$$

where,

$$
y=\frac{4 \boxplus_{\mathrm{liq}} \mathrm{t}}{\mathrm{d}^{2}}
$$

and $\boxplus_{\mathrm{liq}}$ is the diffusivity for the drop and $t$ is time. This expression holds true until $y=0.2$ at which time over $90 \%$ of the metal has reacted. From this expression it can be seen that the reaction rate initially varies as $\mathrm{t}^{-1 / 2}$; therefore, the reaction rate is strongly time-varying early in the process. It is not practical, however, to track the history of individual debris droplets with sufficient resolution to use this parabolic reaction rate law directly in CONTAIN. Therefore, the drop-side reaction rate is set such that one obtains the correct time required to react half the metal in an initially fresh drop. In terms of a drop-side reaction time, $\tau_{d}$, this means that 


$$
1-\exp \left\{-\frac{t_{50}}{\tau_{d}}\right\}=0.5
$$

where $t_{50}$ is the time that will give $F(y)=0.5$. After a little algebra it can be shown that

$$
\mathrm{t}_{50}=\frac{0.03055 \mathrm{~d}^{2}}{4 \mathrm{Ð}_{\mathrm{liq}}}
$$

Substituting this into Equation (6-136) and solving for the effective drop-side reaction time, $\tau_{d}$, gives

$$
\tau_{\mathrm{d}}=\frac{0.011017 \mathrm{~d}^{2}}{\mathrm{\boxplus}_{\mathrm{liq}}}
$$

If the temperature of a debris field is below a user-specified cutoff temperature, $T_{\text {cutoff }}$, then the diffusivity is set to zero and the drop-side reaction time is set to infinity, thereby disabling chemical reactions for that field. The rationale for providing this option to terminate the reaction at low temperatures is to prevent reactions from continuing at unrealistically low temperatures while also preventing old, cold debris from quenching fresh debris. When a limited number of generations is used, this quenching is likely to occur if a realistic temperature dependence is specified for the drop diffusivity, $\boxplus_{\text {lig. }}$. The default value of the cutoff temperature is sufficiently low, $273.15 \mathrm{~K}$, that this feature is effectively disabled.

The drop diffusivity, $\boxplus_{\text {liq }}$, is given by

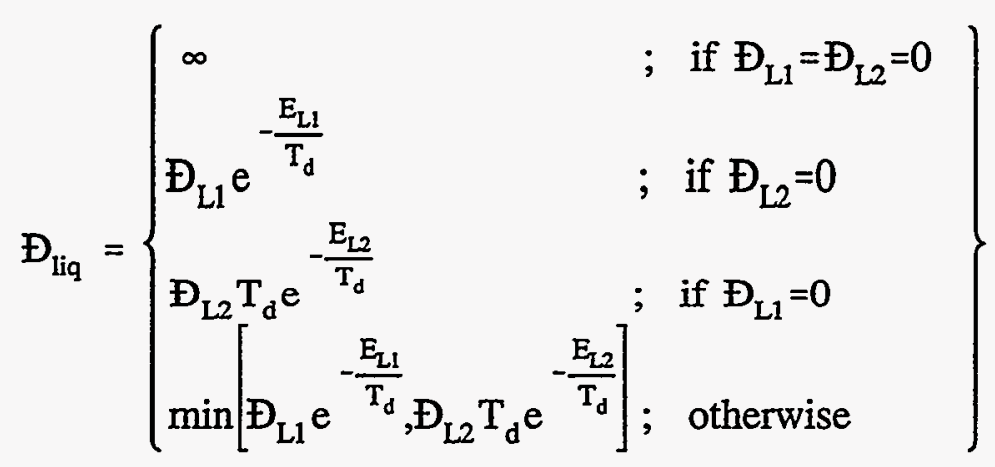

where $\bigoplus_{\mathrm{L} 1}, \mathrm{E}_{\mathrm{L} 1}, \bigoplus_{\mathrm{L} 2}$, and $\mathrm{E}_{\mathrm{L} 2}$ are specified by the user. Note that if $\bigoplus_{\mathrm{L} 1}$ and $\bigoplus_{\mathrm{L} 2}$ are set to zero by the user, then $\bigoplus_{\text {liq }}$ will be set to infinity, which corresponds to no drop-side limitations $\left(\tau_{d}=0\right)$.

A few limitations of the drop side model are now discussed. First, the drop-side reaction time given by Equation (6-138) corresponds to a fixed reaction rate that will match the time required to react half the fresh metal in a drop. Because the reaction rate is time varying approximately as $\mathrm{t}^{-1 / 2}$, the 
drop-side limited reaction rate is underestimated at early times and overestimated at late times. Second, the drop-side model is based on diffusion within a stagnant drop. Other relevant processes such as vibration or circulation within the drop may lower the drop-side resistance below the diffusion limited rate. Also, drop shattering and drop-drop physical interactions could expose fresh metal which would bypass the diffusion limited rates assumed by this model. Reliable models for these processes are not available; however, their importance can be assessed in CONTAIN by choosing large drop diffusivities or by ignoring drop-side resistance. It is now recommended that infinite diffusivity be used to bypass the drop-side model in most DCH calculations (see Section 13.3.2.2.2). This is now the default, which represents a change from code versions prior to CONTAIN 1.2 .

\subsubsection{Reaction Rate Equations}

The previous two subsections describe gas-side transport and drop-side transport limited reaction times, respectively. The reaction rate will be slower than the rate implied by either the gas-side limited or drop-side limited reaction times. The two reaction times are therefore combined to obtain an effective reaction time, $\tau_{e}$. The effective reaction time is defined as the root-sum-square of the gas-side and drop-side reaction times as follows:

$$
\tau_{e}=\sqrt{\tau_{g}^{2}+\tau_{d}^{2}}
$$

The use of a root-sum-square here has no rigorous basis; however, the intent is to account for the fact that the reaction time will be somewhere between the slower reaction time and the sum of the two reaction times.

The effective reaction time is used to calculate the reaction rate of metals in the debris field. The following discussion describes how this calculation is actually performed. First it is important to remember that each debris field is modeled separately and will therefore have its own effective reaction rate time constant. The equations below pertain to one particular field. The total mass and energy changes resulting from chemistry in a cell must include a summation over all debris fields. Another important aspect of the model is that the reaction times are not constant. That is, they are re-evaluated at the beginning of every cell timestep.

The reaction rate for metal in oxygen equivalent units for a given debris field is given by

$$
\frac{\mathrm{dN}_{\mathrm{met}}}{\mathrm{dt}}=-\frac{\mathrm{N}_{\mathrm{met}}}{\tau_{\mathrm{e}}}
$$

where $\mathrm{N}_{\text {met }}$ is the amount of metal in oxygen equivalents in a debris field given by Equation (6-133). Again, this specific rate only applies to one timestep, one cell, and one debris field. The above equation is integrated to give the amount of metal in oxygen equivalents that can react in a timestep $\Delta \mathrm{t}_{\mathrm{c}}$ : 


$$
\Delta N_{\text {met }}=N_{\text {met }}^{0}\left(1-\exp \left\{-\frac{\Delta t_{c}}{\tau_{e}}\right\}\right)
$$

The reacted amounts of all metal species in oxygen equivalents added together must not exceed $\Delta \mathrm{N}_{\text {met }}$. The reactions are also limited by the amount of oxygen and steam that can transport to the drop surface as given by Equations (6-122) and (6-123). Note that this check in the model is redundant, since $\tau_{e}$ is determined from Equations (6-122) and (6-123). The following discussion provides more details on how the calculation is actually performed.

The metal species in the debris field are reacted with the available oxygen in the following order: $\mathrm{Zr}, \mathrm{Al}, \mathrm{Cr}$, and $\mathrm{Fe}$. The reactions will be cut off if there is no oxygen remaining of that available from the gas-side transport calculation, or if the moles of metal reacted in oxygen equivalents exceeds $\Delta \mathrm{N}_{\text {met }}$. If the reactions are cut off because of oxygen limitations, the metal species are again considered in the same order listed above for reaction with steam. Again, the reaction of each metal species will proceed in the listed hierarchical order until no steam remains of that available from the gas-side transport calculation, or until the moles of metal reacted in oxygen equivalents reaches the maximum allowable amount, $\Delta \mathrm{N}_{\text {met }}$, from Equation (6-142).

The iron/steam reaction is the very last one that will be calculated to proceed. Therefore, this reaction will only proceed if other metals in the drop did not consume all the available steam from the gas-side transport Equation (6-123). The steam transport equation (with $\rho_{\mathrm{EQ}}=0$ ) is incorrect for the iron/steam reaction. Note that if iron is the only metal in the drop, the steam transport equation as originally written is correct. For the case where iron is a residual metal, any unreacted steam available for the iron reaction is reduced by a factor, $\alpha_{\mathrm{EQ}}$, to account for the fact that $\rho_{\mathrm{EQ}}>0$ for the iron/steam reaction. The parameter $\alpha_{\mathrm{EQ}}$ is defined by rewriting the steam gas-side transport Equation (6-123) as follows:

$$
\begin{aligned}
\left(\frac{\mathrm{dm}}{\mathrm{dt}}\right)_{\mathrm{H}_{2} \mathrm{O}} & =\mathrm{K}_{\mathrm{H}_{2} \mathrm{O}} \mathrm{A}_{\mathrm{d}}\left(\rho_{\mathrm{H}_{2} \mathrm{O}}^{\prime}-\rho_{\mathrm{EQ}}\right)=\mathrm{K}_{\mathrm{H}_{2} \mathrm{O}} \mathrm{A}_{\mathrm{d}} \alpha_{\mathrm{EQ}} \rho_{\mathrm{H}_{2} \mathrm{O}}^{\prime} \\
\alpha_{\mathrm{EQ}} & =\frac{\rho_{\mathrm{H}_{2} \mathrm{O}}^{\prime}-\rho_{\mathrm{EQ}}}{\rho_{\mathrm{H}_{2} \mathrm{O}}^{\prime}}
\end{aligned}
$$


It can be shown from Equation (6-127) that $\alpha_{E Q}$ is given by

$$
\alpha_{\mathrm{EQ}}=\frac{\left(\frac{\mathrm{K}}{\mathrm{X}_{\mathrm{FeO}}}-\frac{\mathrm{P}_{\mathrm{H}_{2}, \mathrm{~b}}}{\mathrm{P}_{\mathrm{H}_{2} \mathrm{O}, \mathrm{b}}}\right)}{\left(\frac{\mathrm{K}}{\mathrm{X}_{\mathrm{FeO}}}+1\right)}
$$

The $\alpha_{E Q}$ parameter is used to adjust the available steam for the iron reaction for fields that have iron and other metals present at the beginning of the timestep. The $\alpha_{\mathrm{Eq}}$ parameter is applied to account for non-zero $\rho_{\mathrm{EQ}}$ values for the iron reaction for such fields. For fields that have only iron present at the start of a timestep, the use of $\rho_{\mathrm{EQ}}$ given by Equation (6-127) in the steam transport equation is exactly equivalent to using the $\alpha_{\mathrm{EQ}}$ correction and $\rho_{\mathrm{EQ}}=0$.

It should be clear from the above discussion that simple differential equations do not describe the rate at which metals are burned. This is primarily because there is no way to know a priori which metal in the drop will use the last available mole of oxidant. In fact, there is no way of knowing $a$ priori whether the reaction will be oxidant transport limited, oxygen or steam availability limited, or metal limited. In most situations, the reaction will be oxidant transport limited. The following equations describe the amount of a given metal that reacts if metals more reactive than that metal are absent and if that metal does not run out during the timestep. The quantities in the following equations are subscripted with $\mathrm{i}$ and $\mathrm{n}$ to emphasize that the expressions pertain to a particular cell $i$ and debris field $n$. For oxygen reaction, the equations are

$$
\begin{aligned}
& \left(\Delta \mathrm{O}_{2}\right)_{\mathrm{i}, \mathrm{n}}=\left(\frac{\mathrm{dm}}{\mathrm{dt}}\right)_{\mathrm{O}_{2}} \Delta \mathrm{t}_{\mathrm{c}} \\
& \Delta \mathrm{m}_{\mathrm{d}, \mathrm{n}, \mathrm{k}, \mathrm{k}}=-2\left(\frac{\left(\Delta \mathrm{O}_{2}\right)_{i, \mathrm{n}}}{\mathrm{M}_{\mathrm{O}_{2}}}\right)\left(\frac{\mathrm{M}_{\mathrm{k}}}{v_{\mathrm{k}}}\right) ; \quad \mathrm{k}=\text { metals } \mathrm{Zr}, \mathrm{Al}, \mathrm{Cr}, \mathrm{Fe} \\
& \Delta \mathrm{m}_{\mathrm{d}, \mathrm{i}, \mathrm{k}, \mathrm{k}}=+2\left(\frac{\left(\Delta \mathrm{O}_{2}\right)_{\mathrm{i,n}}}{\mathrm{M}_{\mathrm{O}_{2}}}\right)\left(\frac{\mathrm{M}_{\mathrm{k}}}{v_{\mathrm{k}}}\right) ; \quad \mathrm{k}=\text { oxides } \mathrm{ZrO}_{2}, \mathrm{AlO}_{1.5}, \mathrm{CrO}_{1.5}, \mathrm{FeO}
\end{aligned}
$$

where $M$ is molecular weight, $v$ is the stoichiometry coefficient, and all other parameters are previously defined. Recall that $\Delta t_{c}$ is the timestep. Similar expressions apply to the reaction of metals with steam 


$$
\begin{aligned}
& \left(\Delta \mathrm{H}_{2} \mathrm{O}\right)_{\mathrm{i}, \mathrm{n}}=\left(\frac{\mathrm{dm}}{\mathrm{dt}}\right)_{\mathrm{H}_{2} \mathrm{O}} \Delta \mathrm{t}_{\mathrm{c}} \\
& \Delta \mathrm{m}_{\mathrm{d}, \mathrm{i}, \mathrm{n}, \mathrm{k}}=-\left(\frac{\left(\Delta \mathrm{H}_{2} \mathrm{O}\right)_{\mathrm{i}, \mathrm{n}}}{\mathrm{M}_{\mathrm{H}_{2} \mathrm{O}}}\right)\left(\frac{\mathrm{M}_{\mathrm{k}}}{v_{\mathrm{k}}}\right) ; \quad \mathrm{k}=\text { metals } \mathrm{Zr} \text {, Al, and } \mathrm{Cr} \\
& \Delta \mathrm{m}_{\mathrm{d}, \mathrm{i}, \mathrm{k}, \mathrm{k}}=-\left(\frac{\left(\alpha_{\mathrm{EQ}} \Delta \mathrm{H}_{2} \mathrm{O}\right)_{\mathrm{in, \textrm {n }}}}{\mathrm{M}_{\mathrm{H}_{2} \mathrm{O}}}\right)\left(\frac{\mathrm{M}_{\mathrm{k}}}{v_{\mathrm{k}}}\right) ; \quad \mathrm{k}=\text { Fe metal } \\
& \Delta \mathrm{m}_{\mathrm{d}, \mathrm{i}, \mathrm{n}, \mathrm{k}}=+\left(\frac{\left(\Delta \mathrm{H}_{2} \mathrm{O}\right)_{\mathrm{in, \textrm {n }}}}{\mathrm{M}_{\mathrm{H}_{2} \mathrm{O}}}\right)\left(\frac{\mathrm{M}_{\mathrm{k}}}{v_{\mathrm{k}}}\right) ; \quad \mathrm{k}=\text { oxides } \mathrm{ZrO}_{2}, \mathrm{AlO}_{1.5}, \mathrm{CrO}_{1.5} \\
& \Delta \mathrm{m}_{\mathrm{d}, \mathrm{n}, \mathrm{k}, \mathrm{k}}=+\left(\frac{\left(\alpha_{\mathrm{EQ}} \Delta \mathrm{H}_{2} \mathrm{O}\right)_{\mathrm{i}, \mathrm{n}}}{\mathrm{M}_{\mathrm{H}_{2} \mathrm{O}}}\right)\left(\frac{\mathrm{M}_{\mathrm{k}}}{v_{\mathrm{k}}}\right) ; \mathrm{k}=\mathrm{FeO} \text { oxide } \\
& \left(\Delta \mathrm{H}_{2}\right)_{\mathrm{i}, \mathrm{n}}=+\left(\frac{\left(\Delta \mathrm{H}_{2} \mathrm{O}\right)_{\mathrm{i}, \mathrm{n}}}{\mathrm{M}_{\mathrm{H}_{2} \mathrm{O}}}\right) \mathrm{M}_{\mathrm{H}_{2}} ; \text { (from all } \mathrm{k} \text { ) }
\end{aligned}
$$

Similar but more piecemeal expressions (using several if-then branches) are used to calculate chemical reactions under other conditions. For simplicity's sake, these more involved expressions are not shown, since the physical modeling is represented adequately by the expressions above for the simplest case.

The gas transport rate in the above expression is calculated for debris field $\mathrm{n}$ in cell $\mathrm{i}$ from Equations (6-122) and (6-123). As noted previously, the steam transport equation is evaluated with the $\rho_{\mathrm{EQ}}=$ 0 , and $\alpha_{\mathrm{EQ}}$ is used as shown above to account for non-zero values of $\rho_{\mathrm{EQ}}$. The net effect is that the correct value of $\rho_{\mathrm{EQ}}$ is used for all the metals.

The above equations are applied to each debris field sequentially. The metal masses reacted and oxide masses produced in a cell are divided by the timestep to give the chemistry terms in the debris mass conservation equation. The same procedure applies to the masses of oxygen and steam consumed and the mass of hydrogen produced, but with two important differences. First, the gas mass changes from the reaction of all debris fields must be added together. Second, hydrogen gas produced can burn if oxygen is available in the cell. This is described in the following subsection. Note that if multiple generations are included, all sizes in a generation are calculated first before moving on to the next generation.

The energy released by the chemical reactions is added to the debris field. The amount of metal burned by oxygen and steam is multiplied by the energies of reactions given in the following table. 
Table 6-3

DCH Chemistry Energies of Reaction

\begin{tabular}{||c|c|c|c||}
\hline $\begin{array}{c}\text { Metal } \\
\text { Species }\end{array}$ & $\begin{array}{c}v \\
\text { Oxygen/Metal } \\
\text { Stoichiometry } \\
\text { Ratio }\end{array}$ & $\begin{array}{c}\mathrm{Q}_{\mathrm{r} x, 02} \\
\text { Oxygen } \\
\text { Reaction } \\
\mathrm{MJ} / \mathrm{kg}\end{array}$ & $\begin{array}{c}\mathrm{Q}_{\mathrm{rx}, \mathrm{H} 20} \\
\text { Steam } \\
\text { Reaction } \\
\mathrm{MJ} / \mathrm{kg}\end{array}$ \\
\hline $\mathrm{Zr}$ & 2 & 12.023 & 5.7384 \\
\hline $\mathrm{Al}$ & 1.5 & 31.06 & 15.18 \\
\hline $\mathrm{Cr}$ & 1.5 & 10.91 & 2.442 \\
\hline $\mathrm{Fe}$ & 1 & 4.865 & -0.2679 \\
\hline
\end{tabular}

This table gives the heat of reaction per unit mass of metal-reacted referenced to $273 \mathrm{~K}$. It is important to note that the $Q_{x, H D O}$ values are for reaction with liquid phase water; therefore, the energy required to vaporize water has been subtracted from the steam reactions. If atmosphere conditions are such that water is in the vapor phase then the heat of vaporization for the water will be added back to the debris field. The energy required to heat oxygen and steam to the drop temperature is also accounted for. These energy exchanges are done in two stages. First, the model takes the difference in enthalpy between the gaseous reactants and products at the debris field temperature

$$
\begin{aligned}
H X_{d, i, n} & =\left(\Delta \mathrm{O}_{2}\right)_{i, n} h_{\mathrm{O}_{2}}\left(T_{\mathrm{d}, \mathrm{i}, \mathrm{n}}\right) \\
& +\left(\Delta \mathrm{H}_{2} \mathrm{O}\right)_{\mathrm{i}, \mathrm{n}} \mathrm{h}_{\mathrm{H}_{2}} \mathrm{O}\left(\mathrm{T}_{\mathrm{d}, \mathrm{i}, \mathrm{n}}\right) \\
& -\left(\Delta \mathrm{H}_{2}\right)_{\mathrm{i}, \mathrm{n}} \mathrm{h}_{\mathrm{H}_{2}}\left(\mathrm{~T}_{\mathrm{d}, \mathrm{i}, \mathrm{n}}\right)
\end{aligned}
$$

and adds it to the debris field and subtracts it from the gas field. This is done on a field-by-field basis. Next, because convective heat transfer results in heating of the reactants above the gas temperature as they transport toward the drop, the following correction is applied to the convection heat transfer rate as recommended by Collier [Col81]

$$
\begin{aligned}
\Delta \mathrm{Q}_{\text {cor,i,n }} & =\Delta \mathrm{Q}_{\mathrm{c}}(\xi-1) \\
\xi & =\frac{\mathrm{a}}{1-\mathrm{e}^{-\mathrm{a}}} \\
\mathrm{a} & =\frac{\mathrm{HX}_{\mathrm{d}, \mathrm{i}, \mathrm{n}}-\mathrm{HX}_{\mathrm{g}, \mathrm{in}, \mathrm{n}}}{\Delta \mathrm{Q}_{\mathrm{c}}}
\end{aligned}
$$


where

$$
\begin{aligned}
\mathrm{HX}_{\mathrm{g}, \mathrm{i}, \mathrm{n}} & =\left(\Delta \mathrm{O}_{2}\right)_{\mathrm{i}, \mathrm{n}} \mathrm{h}_{\mathrm{O}_{2}}\left(\mathrm{~T}_{\mathrm{g}, \mathrm{i}, \mathrm{n}}\right) \\
& +\left(\Delta \mathrm{H}_{2} \mathrm{O}\right)_{\mathrm{i}, \mathrm{n}} \mathrm{h}_{\mathrm{H}_{2} \mathrm{O}}\left(\mathrm{T}_{\mathrm{g}, \mathrm{i}, \mathrm{n}}\right) \\
& -\left(\Delta \mathrm{H}_{2}\right)_{\mathrm{i}, \mathrm{n}} \mathrm{h}_{\mathrm{H}_{2}}\left(\mathrm{~T}_{\mathrm{g}, \mathrm{i}, \mathrm{n}}\right)
\end{aligned}
$$

and $\Delta \mathrm{Q}_{\mathrm{c}}$ is the amount of energy exchanged by convection between the debris field and the atmosphere, and is calculated as described in Section 6.5.1.

Taking these corrections into account, the chemistry term for the debris energy equation for a particular field $\mathrm{n}$ in cell $\mathrm{i}$ is given by

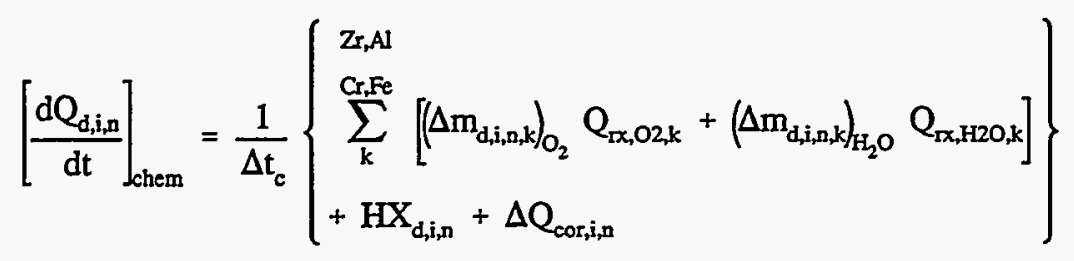

The first term in the summation represents the energy of metals burned in oxygen reactions given by Equation (6-145). The second term represents the energy of metals burned in steam reactions given by Equation (6-146). The third term is the difference in enthalpies of the gaseous reactants and products, and the last term is the Collier convection correction factor. The mass change terms are only given by Equations (6-145) and (6-146) under ideal conditions as explained previously.

The chemistry term for the gas energy equation includes the differences in enthalpies of the reactants and products, and the Collier correction for all fields:

$$
\left[\frac{\mathrm{dQ}_{\mathrm{g}, \mathrm{i}}}{\mathrm{dt}}\right]_{\mathrm{chem}}=\frac{1}{\Delta \mathrm{t}_{\mathrm{c}}}\left\{\sum_{\mathrm{n}=1}^{\mathrm{N}_{\text {sedds }}}\left[-\mathrm{HX}_{\mathrm{d}, \mathrm{i}, \mathrm{n}}-\Delta \mathrm{Q}_{\mathrm{cor}, \mathrm{i}, \mathrm{n}}\right]+2.86 \times 10^{8}\left(\Delta \mathrm{H}_{2}\right)_{\mathrm{i}, \mathrm{n}}\right\}
$$

The $\Delta \mathrm{H}_{2}$ factor in this expression represents the hydrogen (in kilogram-moles) produced in DCH chemical reactions that recombines with local oxygen in the cell atmosphere as discussed in the following subsection.

\subsubsection{DCH-Produced Hydrogen Recombination}

Consideration is given to the recombination of hydrogen produced in metal/steam reactions with unburned oxygen in the vicinity of the drop. Hydrogen produced by DCH chemical reactions is 
assumed to burn instantaneously if oxygen is available in the cell. This can only occur if oxygen is available during the same timestep when the hydrogen was produced. The rationale for this approach is that the hydrogen is assumed to be near the surface of the drop during the same timestep during which it was produced. If oxygen is not available during this timestep, then it will only burn as governed by one of the other containment combustion models (deflagration, diffusion flame buming, or bulk spontaneous recombination). The hydrogen recombination reaction can also be disabled by the user in one or more cells by specifying RCOMH2 $=$ OFF in the DHEAT or DCHCELL input blocks.

The hydrogen burning reaction is given by

$$
\mathrm{H}_{2}+0.5 \mathrm{O}_{2} \rightarrow \mathrm{H}_{2} \mathrm{O}
$$

where $2.86 \times 10^{8}$ Joules per kilogram-mole of hydrogen are released when hydrogen recombines with oxygen. This reaction is limited by the availability of oxygen in the cell and the amount of hydrogen produced by DCH during the timestep. The energy released by the recombination process is added to the atmosphere, not the drop field, as shown in Equation (6-151).

\subsection{Heat Transfer}

Models are included for convective and radiative heat transfer between the debris and the atmosphere. The $\mathrm{DCH}$ radiation model also includes provisions for direct radiation from the debris to containment structures, including the pool and ice condenser. The first subsection below describes the convection model. The second subsection describes the DCH radiation model. The models described below apply to debris in all fields, including the airborne fields and the nonairborne debris field. The models do not apply to trapped debris that is transferred to the uppermost intermediate layer in the lower cell cavity. Heat transfer for debris in the lower cell is modeled as part of the lower cell heat transfer model as described in Chapter 5 and Section 10.6.

\subsubsection{Convective Heat Transfer}

Convection heat transfer from debris to gas is assumed to be by forced convection. The heat transfer coefficient is given by the Nusselt correlation of Ranz and Marshall [Ran52, Bir60] for forced flow over a sphere

$$
\mathrm{N}_{\mathrm{Nu}, \mathrm{i}, \mathrm{n}}=2.0+0.6 \mathrm{~N}_{\mathrm{Re,g}}^{1 / 2} \mathrm{~N}_{\mathrm{Pr}, \mathrm{g}}^{1 / 3}
$$

where $\mathbf{N}_{\mathrm{Nu}, \mathrm{in}}$ is the Nusselt number for debris field $\mathrm{n}$ in cell $\mathrm{i}$. The convection heat transfer rate for each debris field is individually calculated since each field has its own temperature, $T_{d, i, n}$, and particle size, $d_{n}$.

The $\mathrm{N}_{\mathrm{Re}, \mathrm{g}}$ and $\mathrm{N}_{\mathrm{R}, \mathrm{g}}$ parameters in this expression are calculated using gas properties at the boundary layer temperature, $\mathrm{T}_{\mathrm{BL}}$, as recommended in Reference Bir60. The velocity, $\mathrm{v}_{\mathrm{re}}$, is used as the forced convection velocity in the Reynolds number. This velocity is calculated within the code from the 
calculated intercell mass flow rates and the predicted trapping behavior as described in Section 6.3. The user does have the option to override this calculated velocity with either a constant or a tabulated function of time as described in Section 14.2.7 and 14.3.1.11.

The heat transfer coefficient is obtained from the Nusselt number according to the relation

$$
h_{i, n}=\frac{N_{N u, i, n} k_{i, n}}{d_{n}}
$$

where $k_{i, n}$ is the thermal conductivity of the gas evaluated at the boundary layer temperature between the gas and the debris in field $n$ in cell $i$, and $d_{n}$ is the diameter of droplets in debris field $n$. The thermal conductivity is calculated as a mole-weighted average of the gas species as follows:

$$
k_{i, n}=\sum_{m=1}^{N_{g}} X_{m} k_{m}\left(T_{B L, n}\right)
$$

where $N_{g}$ is the number of gas species in the cell, $X_{m}$ is the mole fraction of gas species $m$ in the cell, and $T_{B L, n}$ is the boundary layer temperature between atmosphere and debris in field $n$.

The debris-to-gas convective heat transfer rate for a given field $\mathrm{n}$ in cell $\mathrm{i}$ is given by

$$
\left[\frac{d Q_{d, i, n}}{d t}\right]_{c o n v}=h_{i, n} A_{d, i, n}\left(T_{d, i, n}-T_{g, i}\right)
$$

where $A_{d, n}$ is the surface area of all drops in field $\mathrm{n}$ in cell $\mathrm{i}, \mathrm{T}_{\mathrm{d}, \mathrm{i}, \mathrm{n}}$ is the temperature of debris field $\mathrm{n}$ in cell $\mathrm{i}$, and $\mathrm{T}_{\mathrm{gi}}$ is the gas temperature in cell $\mathrm{i}$. The $[\mathrm{dQ} / \mathrm{dt}]_{\text {conv }}$ term is the convection heat transfer rate that is subtracted in the debris energy equation for field $n$ (see Equation (6-17) in Section 6.2.5), and added to the gas energy equation for all fields.

The amount of energy transferred from the debris field to the atmosphere by convection in a cell timestep, $\Delta t_{c}$, is given by

$$
\Delta Q_{c}=\left[\frac{\mathrm{dQ}_{\mathrm{d}, \mathrm{in}, \mathrm{n}}}{\mathrm{dt}}\right]_{\text {conv }} \Delta \mathrm{t}_{\mathrm{c}}
$$

The $\Delta \mathrm{Q}_{\mathrm{c}}$ term is the convection energy amount used to evaluate the Collier correction factor (see Equation (6-148)) for the effect of mass transfer on convection heat transfer as described in Section 6.4.3. 


\subsubsection{Radiative Heat Transfer}

Debris radiation to the atmosphere, all structure surfaces, the ice condenser, and the uppermost layer in the cavity are treated in the DCH radiation model. Radiation heat transfer between the debris field and the atmosphere is modeled using a simple gray body law

$$
\left[\frac{\mathrm{dQ}_{\mathrm{d}, \mathrm{i}, \mathrm{n}}}{\mathrm{dt}}\right]_{\mathrm{rad}, \mathrm{g}}=\epsilon_{\mathrm{d}-\mathrm{g}} \sigma \mathrm{A}_{\mathrm{d}, \mathrm{i}, \mathrm{n}}\left(\mathrm{T}_{\mathrm{d}, \mathrm{i}, \mathrm{n}}^{4}-\mathrm{T}_{\mathrm{g}, \mathrm{i}}^{4}\right)
$$

where $\sigma$ is the Stephan-Boltzman constant and $A_{d, i, n}$ is the surface area of all drops in debris field $n$ in cell $i$. The effective emissivity for debris-gas radiation, $\epsilon_{\mathrm{d}-\mathrm{g}}$, is specified by the user. Values of about 0.8 are typically appropriate for this parameter. The default value of $\epsilon_{\mathrm{d}-\mathrm{g}}$ is 1 .

The radiative heat transfer rate from debris field $n$ to surface $j$ is given by

$$
\begin{aligned}
{\left[\frac{d Q_{d, i, n}}{d t}\right]_{\text {rad, s,j }}=} & \epsilon_{d-s} \sigma\left(\frac{A_{d, i, n}}{\sum_{n n=1}^{N_{\text {sedds }}} A_{d, i, n n}}\right)\left(\frac{A_{i, j}}{\sum_{j j=1}^{N_{s u r f}} A_{s, j j}}\right) \times \\
& \min \left(\sum_{n n=1}^{N_{\text {sedds }}} A_{d, i, n n}, \sum_{j i=1}^{N_{\text {surf }}} A_{s, j j}\right)\left(T_{d, i, n}^{4}-T_{s, j}^{4}\right)
\end{aligned}
$$

where $\mathrm{N}_{\text {surf }}$ is the number of surfaces, $\mathrm{T}_{\mathrm{s}, \mathrm{j}}$ is the temperature of surface $\mathrm{j}$, and $\mathrm{j}$ represents all surfaces, including all exposed structure surfaces and the ice condenser area. For structure surfaces, $T_{s, j}$ is the temperature of either the first node or last, the latter being appropriate if the exposed surface is the outer surface of a structure. Note that $T_{s, j}$ is not taken to be the film interface temperature. Although using the interface temperature might be slightly more accurate than using the node temperature, doing so can cause numerical instabilities, since there is no heat capacity associated with the film temperature. In practice the two temperatures will be very close to each other for DCH calculations, since small nodes should be used for the surface nodes and water films will typically not be present. Note that water films may be present at the start of some DCH calculations and they may be surprisingly important because their evaporation represents an additional source of steam that can interact with debris. Note that the surface area for radiation is weighted in the above expression to take into account the limitations imposed by the smaller of the total debris and total structure areas.

The black body multiplier for debris-surface radiation, $\epsilon_{\mathrm{d}-s}$, is specified by the user. By default this parameter is zero and debris-to-structure direct radiation is disabled. This parameter is normally left at or close to its default value of zero in most DCH applications based on the assumption that the atmosphere is opaque from airborne debris and DCH-generated aerosols. Heat transfer from debris to heat sinks will be non-zero if a non-zero value is specified for $\epsilon_{\mathrm{d}-s^{\circ}}$. If a non-zero $\epsilon_{\mathrm{d}-\mathrm{s}}$ is given, then $\epsilon_{\mathrm{d}-\mathrm{g}}$ should also be specified to ensure that the sum of $\epsilon_{\mathrm{d}-\mathrm{g}}$ and $\epsilon_{\mathrm{d}-\mathrm{s}}$ does not exceed unity. 
The total radiative energy loss from debris field $\mathrm{n}$ used in the debris energy conservation equation (see Equation (6-17)) is given by the sum of the debris to structure radiation for all surfaces and the debris to gas radiation

$$
\left[\frac{\mathrm{dQ}_{\mathrm{d}, \mathrm{i}, \mathrm{n}}}{\mathrm{dt}}\right]_{\mathrm{rad}}=\left[\frac{\mathrm{dQ}_{\mathrm{d}, \mathrm{i}, \mathrm{n}}}{\mathrm{dt}}\right]_{\mathrm{rad}, \mathrm{g}}+\sum_{\mathrm{j}=1}^{N_{\mathrm{surf}}}\left[\frac{\mathrm{dQ}_{\mathrm{d}, \mathrm{i}, \mathrm{n}}}{\mathrm{dt}}\right]_{\mathrm{rad}, \mathrm{s}, \mathrm{j}}
$$

The first term on the right hand side of this equation is added to the gas energy conservation equation, and the last term is added to the surface node of each structure. For structures, the radiative flux is included in the surface flux used to calculate the structure interface temperature.

\subsubsection{Atmosphere-Structure Heat Transfer During DCH}

Heat transfer from the atmosphere to structures is not a DCH-specific model. The gas-to-structure heat transfer models are described in the Chapter 10 and are not repeated here. However, there is an important consideration that one must give to the gas-to-structure radiation model in DCH calculations, and DCH-specific options are provided to address this consideration. Normally, the radiative properties of the cell atmosphere are calculated by the Cess-Lian and Modak correlations described in Chapter 10. However, these correlations may not be appropriate under DCH conditions because they consider only the optically active gases in the atmosphere, while DCH events are expected to be accompanied by dense aerosol clouds that result in high atmospheric emissivities. Therefore, in CONTAIN 2.0 and higher versions, the GASSUR option permits specification of a fixed emissivity in the DCH input blocks (see Sections 14.2.7 and 14.3.1.11), and this user-specified emissivity is applied only during the DCH event. An atmospheric emissivity value of about 0.8 is thought to be appropriate for most $\mathrm{DCH}$ calculations. However, there are some potential complications that should be considered; see the discussion of DCH heat transfer in Section 13.3.2.2.2 for some additional guidance on this subject.

\subsection{Non-Airborne Debris Interactions}

Debris that is airborne and debris that is not airborne can both contribute to DCH by heat transfer and chemical reactions. Non-airborne debris includes debris that has not been entrained into the atmosphere, and debris that was once airborne that has been trapped. To enable consideration of both types of non-airborne debris in the heat transfer and chemistry models, the trapped debris field in the cavity cell must be used as an intermediate repository for debris between the RPV dispersal phase and the cavity entrainment phase. This may be accomplished by using debris sources of type TRAPBIN as summarized in Section 6.2.8 to represent debris ejection from the RPV, or by using the RPV models described in Section 6.2.9.

The governing equations for heat transfer and chemical reactions given in the previous sections also apply to non-airborne debris. There are four unique aspects to the heat transfer and chemical reactions models for non-airborne debris. First, the surface area for heat transfer and mass transfer is based on a user-specified effective diameter that applies only to debris in the non-airborne field. 
By default this diameter is not defined, thus the model is not active. A diameter must be specified by the user in a given DCH-CELL input block using the DIATRAP keyword to enable non-airborne debris interactions in that cell. The DIATRAP keyword may be used either by itself to specify a constant diameter or within the VAR-PARM block to specify a time-dependent diameter. For a given effective diameter, the debris surface area is computed according to the relation

$$
A_{\text {nad,i }}=\frac{6 \sum_{k}^{N_{\text {DCH }}} m_{\text {nad,i, },}}{\rho_{\text {nad,i }} d_{\text {nad, }, i}}
$$

where $A_{\text {nad, }}$ is the surface area for the non-airborne field in cell $i, m_{\text {nadi,k }}$ is the mass of debris species $\mathrm{k}$ in the non-airborne field in cell $\mathrm{i}, \rho_{\text {nad, }}$ is the average material density of debris in the non-airborne field in cell $i$, and $d_{\text {nadi, }}$ is the user-specified effective diameter for the non-airborne field in cell $i$.

The mass of non-airborne debris is governed by the rate of debris addition to the non-airborne debris field, the rate of entrainment from the non-airborne debris field, and the trapping rate of debris into the non-airborne debris field. Equation (6-78) in Section 6.3.1 gives the governing equation for the mass of debris in the non-airborne field that takes into account these three processes. The addition of debris to the non-airborne field and the entrainment of debris out of the non-airborne field is governed by user-specified tables or the RPV models as noted above.

The average material density of the non-airborne debris is calculated as the inverse of the mass average of specific volume of all materials in the trapped field at the temperature of the trapped field. Normally the densities are taken from the material property tables given in the USERDAT input, but they will be ignored if the DENDRP global input (or VAR-PARM table) is specified, in which case the average density of airborne and non-airborne debris will be set to the specified value.

The second unique aspect of non-airborne debris heat transfer is that heat transfer to structures or the ice surfaces in an ice condenser cell is not modeled for debris that is not airborne. Therefore, the non-airborne field is not included in the debris to surface heat transfer calculation. The third difference is that the black body multiplier for debris to gas radiation can be specified separately for non-airborne debris. This is done using the RADTRAP keyword. By default this value is equal to the value of $\epsilon_{\mathrm{d}, \mathrm{g}}$ that is used for the airborne debris fields.

The fourth and final difference for non-airborne debris is that if VELTRAP is not specified and fewer than two structures are defined in the cell, an average gas velocity through the cell is used for heat and mass transfer for non-airborne debris. This average is calculated by using the current cell gas volume to the two-thirds power for the cell hydraulic area. If VELTRAP is not specified and two or more structures are defined in the cell, then the forced convective velocity defined for the second structure is used (see Sections 10.1.1.6, 13.3.2.2.2, and 14.3.1.3). This is in contrast to airborne debris, where the trapping conditions and the gas and debris flow velocities are used to calculate an appropriate velocity for heat transfer as described in Section 6.3.2. This is not done for non-airborne debris since this field is not flowing and is not subject to trapping as are the airborne debris fields. 
The velocity for non-airborne debris heat and mass transfer can also be directly specified through the VELTRAP keyword, either by itself to specify a constant value or within the VAR-PARM table option to specify a time-dependent value. 


\subsection{AEROSOL BEHAVIOR MODELS}

The aerosol models in CONTAIN determine the mass, size distribution, and composition of suspended aerosols as well as the location and composition of deposited mass. The present chapter discusses the modeling of agglomeration, deposition, and condensation processes. It also discusses the modeling of intercell flow processes and the scrubbing processes that occur when gas-aerosol mixtures are vented into a coolant pool from a submerged flow path or external source. The aerosol models are based on the MAEROS code. [Gel82] However, several enhancements of the MAEROS models have been added for use in CONTAIN.

The CONTAIN aerosol model uses a number "nsectn" of size classes, or sections, to represent the particle size distribution for the suspended aerosols. Each section can have a different composition, in terms of the "nac" aerosol component materials present in a problem. Up to eight such aerosol components can be independently specified. (Both "nsectn" and "nac" are specified in the global CONTROL input block as described in Section 14.2.) As discussed in Section 8.4, each aerosol component is in turn a possible host for its own independently specified set of fission products. Because particle size is an important characteristic governing the release and respirability of aerosols, and because composition is important in determining consequences, the model is particularly suited to applications where health effects are important. Key elements of aerosol behavior models are illustrated in Figure 7-1.

Figure 7-2 illustrates the sectional representation of a two-component aerosol with 12 sections. The mass concentrations of component 1 in each section are given by the stair-stepped broken line above the horizontally shaded region. The mass concentrations of component 2 in each section are given by the uppermost stair-stepped solid line. Section 7.1 discusses the aerosol size distribution used in the aerosol modeling.

Section 7.2 introduces the general aerosol dynamic equation that is solved within the aerosol module. The remainder of the sections are organized according to the seven types of dynamic processes that are modeled:

- agglomeration (or coagulation), whereby two particles collide and form one larger particle, as discussed in Section 7.2.1;

- particle size and composition change as the result of condensation of water on or evaporation of water from the particle, as discussed in Section 7.2.2;

- particle deposition onto heat transfer surfaces and the lower cell coolant pool, as discussed in Section 7.2.3;

- aerosol sources, including user-specified and core-concrete interaction (CCD) source terms, as discussed in Section 7.3;

- particle removal from gases during the operation of engineered systems, as discussed in Sections 7.4 through 7.6; 


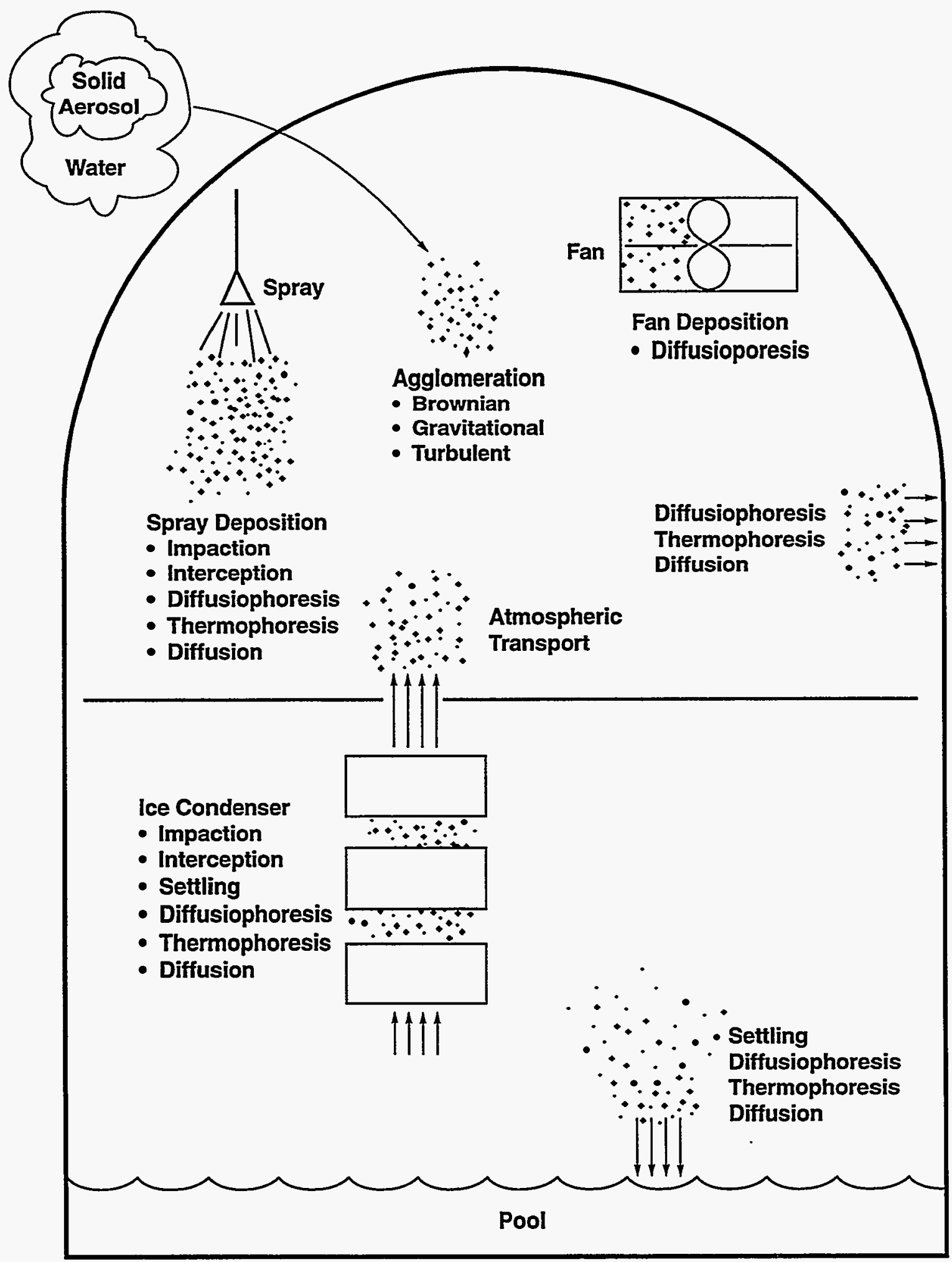

Figure 7-1. Aerosol Behavior as Modeled in CONTAIN 


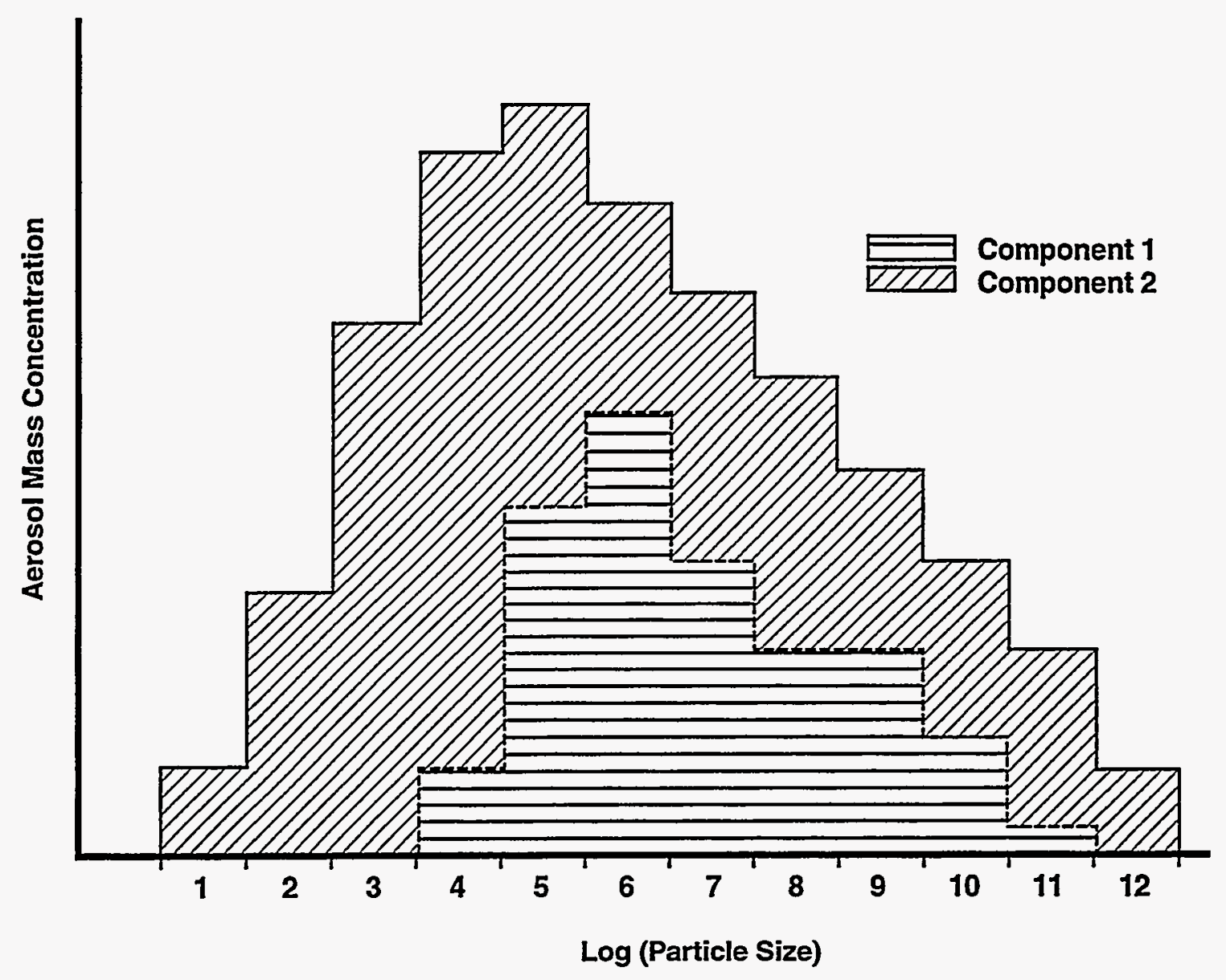

Figure 7-2. Example of Aerosol Particle Mass Concentration Distribution for a Two-Component Aerosol 
- particle scrubbing from gases vented into a coolant pool, as discussed in Section 7.7; and

- intercell flow of aerosols, with settling through flow paths and scrubbing, as discussed in Section 7.8.

Note that not all phases of aerosol transport are modeled. Aerosol processes can be classified as primary, secondary, or tertiary. By primary process, we mean a process that removes aerosols from the atmosphere or transports aerosols through it. Secondary and tertiary ones affect the aerosol after it is removed from the atmosphere. The primary processes such as deposition on surfaces are, in general, modeled. Secondary and tertiary processes following a primary process are in general not modeled. An example of a secondary process is the wash-down of deposited solid aerosols into a pool as a result of condensate draining from a surface. An example of a tertiary process is resuspension, during pool flashing, of the aerosols washed into a pool. While aerosol wash-down is not modeled, fission product wash-down into the pool may be simulated with the FPLIQUID option discussed in Section 8.8.2 and Section 14.2.6.2 or the targeted release model discussed in Sections 8.4 and 14.3.1.10.

To run an aerosol calculation, the user must specify at least the aerosol names and size distribution parameters ("amean" and "avar") for each aerosol component in the global AEROSOL block, as described in Section 14.2.5. As discussed in Section 7.1, these size distribution parameters govern the initial particle size distribution and the distribution for a source of new particles if no other options for the size distribution are specified. Initial aerosol masses and aerosol sources are specified in the cell AEROSOL block (see Section 14.3.1.8, Aerosol Initial Conditions and Sources). The aerosol component names may be selected from either the COMPOUND, USERDEF, or AERNAMES material lists (see Section 14.2.1). It should be noted that if an aerosol material name is taken from the list of CONTAIN materials, given in Table 3-1, or is a user-defined material, as discussed in Sections 3.1 and 14.2.1.1, the aerosol will be included in the mass and energy accounting scheme described in Section 3.4. (This does not mean that the aerosols are taken into account in evaluating the thermodynamic state of a repository; it means that the error of omission is evaluated.) Note that any name specified after AERNAMES must be used only to label an aerosol component. The thermodynamic properties of the associated material are unspecified and therefore are not included in the mass and energy accounting scheme.

The liquid water material $\mathrm{H} 2 \mathrm{OL}$ is treated in a special fashion when specified as an aerosol material. First of all, it must be specified as the last aerosol component if modeling of condensation on or evaporation from aerosols is desired. If $\mathrm{H} 2 \mathrm{OL}$ is specified as the last aerosol component, the amounts of any water vapor condensed on aerosols will be added to the mass of that aerosol component. Also, when aerosols with an $\mathrm{H} 2 \mathrm{OL}$ component deposit out on heat transfer structures or the coolant pool, the $\mathrm{H} 2 \mathrm{OL}$ inventory is transferred from the aerosol deposition arrays to the structure film or coolant pool inventories, respectively. The subsequent dynamics of the H2OL is thereafter controlled by the film or pool model.

Changes have occurred in aerosol deposition modeling as a result of the new coolant pool tracking modeling in CONTAIN 1.2. (In versions prior to this, the flooding of structures and flow paths is 
not taken into account.) Aerosol deposition processes are assumed to occur on the surfaces of heat transfer structures not submerged in the coolant pool in a cell. Aerosols and fission products deposited on structure surfaces are assumed to transfer to the pool in proportion to the structure surface area that is subsequently flooded. (This process should not be confused with the wash-down of deposited aerosols with condensate runoff from unsubmerged surfaces, discussed above.) As in prior versions, deposition may also occur on the surface of the coolant pool. Such deposition requires the definition of a coolant pool in the cell, as discussed in Section 5.4, but no longer requires the use of the SETTLE keyword that is required in code versions prior to CONTAIN 1.2. This deposition occurs on a surface area equal to the cell cross-sectional area at the pool height, if coolant is present; equal to the lower cell cross-sectional area, if the coolant pool is dry and CORCON is not active; or equal to the CORCON upper surface area, if the pool is dry and CORCON is active. As discussed in Section 7.2.3, pool deposition is offset by gas convection from the pool surface. Such gas convection takes into account evaporation, boiling, and any venting of noncondensable gases below the surface of the pool. It should also be noted that, as discussed in Section 7.7, aerosols and any attached fission products are completely removed from the gas vented under the surface of a coolant pool through the submerged end of a gas flow path. These aerosols and fission products are subsequently placed in the pool. This is the result of an incomplete implementation of aerosol scrubbing models for submerged gas flow paths and does not apply to the dedicated suppression pool vent flow path, for which scrubbing models are in place.

\subsection{Aerosol Size Distribution}

In CONTAIN, the aerosol particle size distribution is represented by a number "nsectn" of particle size classes called sections. The section boundaries are based on a compact spherical-equivalent diameter $d$ (the diameter of a fully dense spherical particle with the same density and mass as the particle in question) and are distributed between user-specifiable minimum and maximum diameters for the particle sizes considered in the calculation. The minimum and maximum diameters are specified by the spherical equivalent diameters "diam1" and "diam2," respectively, in the AEROSOL input block (see Section 14.2.5). The "nsectn" +1 size class boundaries are determined by partitioning the interval ["diam1,"'diam2"] geometrically; that is, the interval [ln("diam1"), $\ln \left(\right.$ "diam2")] is divided evenly. The default values of "diam1" $=10^{-7} \mathrm{~m}$ and "diam2" $=10^{-4} \mathrm{~m}$ give satisfactory results in a number of containment situations. The user is cautioned that a value smaller than $10^{-7} \mathrm{~m}$ for "diam 1 " may introduce stiffness into the calculation and increase the execution time considerably.

The CONTAIN aerosol module is designed to use a small number of sections for computational efficiency. A sensitivity study has shown that the default particle diameter range between $10^{-7}$ and $10^{-4} \mathrm{~m}$ can be handled adequately by ten sections in a typical case. [Lei84] However, it is recommended that no fewer than 20 sections be used without testing. On the other hand, one must avoid using so many sections that the geometric constraint that particle volumes must increase by a factor of two or more from section to section (see Equation (7-4) below) is violated.

Upper and lower accounting bins have been added to keep track of aerosol particles that may become larger than the maximum diameter or smaller than the minimum diameter, respectively. (Such masses are referred to as "mesh" losses in the following discussion.) Aerosol particles can become 
too small by evaporation of water. Particles can become too large by condensation of water vapor or agglomeration. Normally, the aerosols that become too large or too small are lost from the problem, and the corresponding mass and energy is added to the WASTE repository of the cell in which this happens. However, the user may specify the keywords TRAPOVFL or TRAPUNFL in the AEROSOL input block, discussed in Section 14.2.5, to redirect the aerosols. The cell OVERFLOW option (see Section 14.3.1.12) controls the disposition of mass whenever TRAPOVFL is specified. (This OVERFLOW option should not be confused with the engineered systems OVERFLOW component described in Section 12.5.6.) Specifying the TRAPOVFL keyword will result in adding the mass of particles that become too large to the aerosol deposition arrays of the coolant pool in the cell specified through the OVERFLOW option. If a coolant pool is not defined in the overflow cell, the mass is added to the WASTE location in that cell. The rationale for linking TRAPOVFL to OVERFLOW is that the oversized condition typically results from high water aerosol concentrations during blowdowns, and the OVERFLOW option directs this mass to the same location as condensate film runoff from heat transfer structures. Large amounts of mass in the upper bin typically indicate either a high aerosol condensation rate or the lack of an effective settling surface for the aerosols. Such a surface is automatically defined if a lower-cell coolant pool or floor heat transfer surfaces are defined in a cell. (Note that the SETTLE keyword is no longer required, as in versions prior to CONTAIN 1.2, to allow aerosols to settle onto the pool surface.) In addition, aerosol settling through flow paths is modeled whenever the VCOSN keyword (for flow path inclination angle) is specified in the ENGVENT input. The effect of the TRAPUNFL keyword is much simpler than that of TRAPOVFL. Specifying the TRAPUNFL keyword will prevent particles from becoming smaller than the smallest allowed size, when evaporation is occurring from particles in the smallest size class. To prevent this, the mass of particles growing smaller than the smallest allowed size is simply added back to the smallest size class.

Although the distribution of airborne particles among the "nsectn" sections is generally computed from the aerosol dynamic equations discussed in Section 7.2 and initial conditions, a specific, lognormal distribution is assumed for any initial aerosol mass or aerosol sources. A (continuum) lognormal distribution with respect to the particle diameter $d_{p}$ is given by

$$
m\left(d_{p}\right) \delta d_{p} \propto \exp \left(-\frac{1}{2}\left[\frac{\ln ^{2}\left(d_{p} / d_{m m}\right)}{\ln ^{2}\left(\sigma_{g}\right)}\right]\right) \delta d_{p} / d_{p}
$$

where $\propto$ indicates proportionality; $\mathrm{d}_{\mathrm{mm}}$ represents "amean," the user-specified spherical-equivalent mass median diameter; and $\ln \left(\sigma_{\mathrm{g}}\right)$ represents "avar," the user-specified natural logarithm of the geometric standard deviation with respect to diameter. [Yos79] Normally these two lognormal size distribution parameters are specified in the global AEROSOL input block, discussed in Section 14.2.5, and are constants that are independent of time. However, by using the AERTIM option, also described in Section 14.2.5, or by using the safety relief valve (SRV) model, discussed in Sections 11.2 and 14.3.4, the user may specify these parameters as time-dependent for aerosol sources. A different size distribution may be specified for each aerosol component. 
To obtain the initial or source distributions by section, Equation (7-1) is integrated over each section to determine the aerosol mass allocated to each section. Particle mass falling outside the size domain is redistributed within the size domain by renormalizing the section masses.

\subsection{Aerosol Dynamics}

The aerosol dynamics model calculates the aerosol suspended mass for each of the "nsectn" size classes, or sections, as a function of time for each cell. These sections are defined as discussed in Section 7.1. The composition of aerosols is described in terms of the "nac" materials specified by the user. As many as eight aerosol component materials may be used. The distribution of the component materials among the sections is governed by the initial conditions and the aerosol dynamic equations. Within each section, each particle is assumed to have the average composition, based on the component masses for that section. Particle agglomeration, deposition, intercell flow, aerosol sources, and condensation/evaporation processes are included in the model.

The modeling of the aerosol size distribution is governed by a complex integro-differential equation. In CONTAIN, this equation is converted to a sectional form using a method developed by Gelbard and Seinfeld [Gel80] and originally implemented in the MAEROS code. [Gel82] Only the discrete, sectional form of the integro-differential equation will be displayed below. To illustrate what we mean by a sectional form, the agglomeration kernel $\beta$ represents the collision rate per unit volume for two particles of two discrete sizes. The sectional form of this kernel, $\bar{\beta}_{\mathrm{ij} p}$, represents the mass accumulation rate of particles in section $\ell$, as a consequence of the agglomeration of particles of section $i$ with those of section $j$ resulting in a particle belonging to section $\ell$, based on the mass concentrations in sections $i$ and $j$. In the MAEROS approach, the integro-differential equation is reduced to sectional form by integration, assuming that the distribution of aerosol component mass within a section, with respect to the logarithm of the (spherical-equivalent) particle diameter, is constant, and that each particle within a section has the same composition. The agglomeration kernel requires a two-dimensional integration over particle sizes. The deposition and condensation rate expressions require only one-dimensional integration. When defined on a sectional basis through integration, the agglomeration kernel and the deposition and condensation rate constants are referred to collectively as the aerosol coefficients. The formulas for conversion between the discrete and sectional forms are discussed in detail in Reference Gel80 and will not be presented here.

The total mass of aerosol per unit volume of gas in section $\ell$ at time $t, Q_{\ell}(t)$, is defined in terms of the component masses for each of $\mathrm{m}=$ "nsectn" sections according to:

$$
\mathrm{Q}_{\ell}(\mathrm{t})=\sum_{\mathrm{k}=1}^{\mathrm{s}} \mathrm{Q}_{\ell, \mathrm{k}}(\mathrm{t})
$$

where $Q_{\ell, k}(t)$ is the mass of component $k$ in section $\ell$ per unit volume, and $s=$ "nac" is the total number of components. The sectional form of the integro-differential equation can be written as: 


$$
\begin{aligned}
\frac{d Q_{\ell, k}(t)}{d t} & =\frac{1}{2} \sum_{i=1}^{\ell-1} \sum_{j=1}^{\ell-1}\left[{ }^{1} \bar{\beta}_{i, j, \ell} Q_{j, k} Q_{i}+{ }^{1 b} \bar{\beta}_{i, j, \ell} Q_{i, k} Q_{j}\right] \\
& -\sum_{i=1}^{\ell-1}\left[{ }^{2 a} \bar{\beta}_{i, \ell} Q_{i} Q_{\ell, k}-{ }^{2 b} \bar{\beta}_{i, \ell} Q_{\ell} Q_{i, k}\right] \\
& -\frac{1}{2}{ }^{3} \bar{\beta}_{\ell, \ell} Q_{\ell} Q_{\ell, k}-Q_{\ell, k} \sum_{i=\ell+1}^{m}{ }^{4} \bar{\beta}_{i, \ell} Q_{i}+{ }^{1} \bar{G}_{\ell, k} Q_{\ell} \cdots \\
& -\sum_{i=1}^{N_{a}}\left[{ }^{2} \bar{G}_{\ell, i} Q_{\ell, k}-{ }^{2} \bar{G}_{\ell-1, i} Q_{\ell-1, k}\right]+{ }^{3} \bar{G}_{\ell-1, k} Q_{\ell-1}+\bar{S}_{\ell, k}-\bar{\Re}_{\ell, k} Q_{\ell, k}
\end{aligned}
$$

where $\mathrm{dQ}_{, \mathrm{k}}(\mathrm{t}) / \mathrm{dt}$ is the time rate of change of aerosol mass of component $\mathrm{k}$ per unit volume in section $\ell$ at time $t$ and the coefficients $(\bar{\beta}, \bar{G}, \bar{S}$, and $\overparen{\Re})$ are defined below. This equation describes the evolution of the aerosol size and composition distributions within one computational cell. Each cell has its own particle size and composition distributions, and the aerosols are carried from one cell to the next by intercell gas flow, as discussed in Section 7.8, but the cell dependence and flow terms are not explicitly represented in Equation (7-3). It should be noted that the $\bar{G}$ condensation terms in the above equation are written for condensing conditions within the fixed-grid option discussed in Section 7.2.2 A similar expression is used for evaporating conditions.

The coefficients in Equation (7-3) correspond to the following mechanisms:

\section{Coefficient $\quad \underline{\text { Represents }}$}

$\begin{array}{ll}\bar{\beta} & \begin{array}{l}\text { Agglomeration, } \mathrm{m}^{3} / \mathrm{s}-\mathrm{kg}, \\ \bar{G}\end{array} \\ \bar{S} & \text { Vapor condensation, } \mathrm{s}^{-1}, \\ \bar{\Re} & \text { Sources, } \mathrm{kg} / \mathrm{m}^{3}-\mathrm{s}, \\ & \text { Deposition, } \mathrm{kg} / \mathrm{m}^{3}-\mathrm{s} .\end{array}$

The six types of agglomeration coefficients in Equation (7-3) represent the following processes:

${ }^{1 a} \bar{\beta}_{\mathrm{i}, \mathrm{j}, \ell}$

${ }^{1 b} \bar{\beta}_{i, j, \ell}$

${ }^{2 a} \bar{\beta}_{i, \ell}$ addition of component $k$ in section $\ell$, through a particle in section $j$ coagulating with a particle in section $i$ to form a particle in section $\ell$. In this case, the component $k$ comes from section $j$.

addition of component $\mathrm{k}$ in section $\ell$, through a particle in section $\mathrm{i}$ coagulating with a particle in section $j$ to form a particle in section $\ell$. In this case, the component $k$ comes from section $i$.

removal of component $\mathrm{k}$ in section $\ell$, through a particle in section $\mathrm{i}$ coagulating with a particle in section $\ell$, and forming a particle larger than those in $\ell$. 

${ }^{2 b} \bar{\beta}_{i, \ell}$
addition of component $\mathrm{k}$ in section $\ell$, through a particle in section $i$ coagulating with a particle in section $\ell$, with the resulting particle remaining in section $\ell$
${ }^{3} \bar{\beta}_{\ell, \ell}$
removal of component $\mathrm{k}$ in section $\ell$, through two particles in section $\ell$ coagulating with the resulting particle promoted to a section higher than $\ell$
${ }^{4} \bar{\beta}_{i, \ell}$
removal of component $\mathrm{k}$ in section $\ell$, through a particle in section $\ell$ coagulating with a particle in section $i$, where $i>\ell$

Section 7.2.1 describes the agglomeration modeling in CONTAIN.

The three types of $\overline{\mathrm{G}}$ coefficients, as displayed in Equation (7-3), tefer to the following processes:

${ }^{1} \bar{G}_{\ell, k} \quad$ addition of component $\mathrm{k}$ to section $\ell$ through condensation of component $\mathrm{k}$ onto particles in section $\ell$. Note that condensation is limited to the water component.

${ }^{2} \overline{\mathrm{G}}_{\ell, \mathrm{i}} \quad$ removal of component $\mathrm{k}$ from section $\ell$ through condensation of water onto particles in section $\ell$, through promotion

${ }^{3} \overline{\mathrm{G}}_{\ell-1, \mathrm{k}} \quad$ addition of component $\mathrm{k}$ in section $\ell$ due to condensation vapor of water onto particles in section $\ell-1$, through promotion

Section 7.2.2 describes the condensation modeling in CONTAIN in more detail.

In Equation (7-3), particle deposition or removal is addressed by the $\bar{\Re}$ term. Deposition occurs through a number of processes, including gravitational settling, diffusion to surfaces, thermophoresis (a Brownian process causing migration of particles toward lower temperatures), and diffusiophoresis (deposition induced by condensation of water vapor onto surfaces). Section 7.2.3 describes these contributions to $\bar{\Re}$ for heat transfer structure and pool surface deposition. The engineered systems aerosol removal models described in Sections 7.4 through 7.6 also contribute to the removal rate. The ice condenser and containment spray aerosol removal models consider two deposition processes, interception and impaction, that are not considered for heat transfer structure and pool surface deposition.

External sources of aerosols may result in the addition of component mass to any section. Such sources are represented by the $\overline{\mathrm{S}}$ term in Equation (7-3).

The CONTAIN implementation uses the fact that simplifications in the coefficients and in Equation (7-3) occur if the geometric constraint

$$
\mathrm{V}_{\mathrm{i}+1} \mathrm{~V}_{\mathrm{i}}>2
$$

is satisfied, where $V_{i}$ is the particle volume at the lower boundary of section $i$. The geometric constraint ensures that the agglomeration of two particles results in a new particle that will fit into either the section that contains the larger of the two original particles or the section just above it. 
This constraint thus reduces the number of sectional agglomeration coefficients. If this geometric constraint is not satisfied by the input parameters specified by the user, the code will abort.

The calculation of the coefficients is time consuming. Therefore, the coefficients are either read in from a file or calculated on the first call to the aerosol model for use throughout the entire problem. Using a constant set of coefficients imposes some modeling constraints, however. These arise because parameters embedded in the coefficients are also effectively held fixed, despite the fact that they should vary with changing conditions during the problem. A simple multiplier in a coefficient should not be considered embedded because the coefficient can be calculated for a unit multiplier and rescaled when used. In fact, the deposition coefficients, except that for settling, are calculated for a unit forcing factor. The coefficient set employed in CONTAIN uses $8(\text { "nsectn") })^{2}+8$ ("nsectn") storage locations, not counting the condensation coefficients, which are not used when the moving grid method is employed. (For an aerosol with 20 sections this corresponds to 3360 storage locations.)

The following constraints pertain to the current coefficient set:

- The aerosol material density is assumed to be the same for all components.

- The aerosol shape, as modeled by the dynamic and agglomeration shape factors, is independent of aerosol composition.

- The medium in which the aerosol processes are assumed to occur has fixed composition and is taken to be air. In addition, gas properties appearing in expressions for deposition rates are evaluated for bulk conditions, not boundary layer conditions, unless otherwise specified.

- The degree of turbulent agglomeration is fixed throughout the problem. This is controlled by the turbulent dissipation coefficient, "turbds" specified in the global AEROSOL input block.

- Other parameters that control deposition rates do not depend on particle composition. For example, the ratio of the thermal conductivity of air to that of the aerosol material, "tkgop," also in the global AEROSOL input block, is fixed.

The pressure and temperature of the atmosphere are embedded in these coefficients and are fixed for a single set of coefficients. However, the aerosol module actually calculates four sets of coefficients at points given by combinations of two temperatures $\left(\mathrm{T}_{\min }\right.$ and $\left.\mathrm{T}_{\max }\right)$ and two pressures $\left(\mathrm{P}_{\min }\right.$ and $\left.P_{\max }\right)$. These temperatures and pressures are specified in the AEROSOL input block as "tgas1," "tgas2," "pgas1," and "pgas2," respectively. Changing thermal-hydraulic conditions during the problem are accommodated by interpolating between these sets of coefficients. The $T_{\min }, T_{\max }, P_{\min }$, and $P_{\max }$ parameters are chosen to bound the temperatures and pressures expected. At the expense of larger sets of coefficients, some of the constraints above can be removed by interpolating to accommodate other changing parameters or by separating the coefficients so that a relevant parameter is not embedded. 
For any sectional coefficient $\bar{\Im}$, the interpolated sectional coefficients are given by

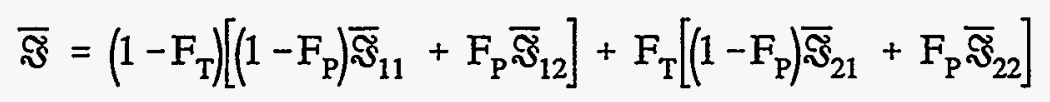

where $\bar{\Im}_{11}$ is the aerosol coefficient for some process for the lowest atmospheric temperature $\left(\mathrm{T}_{\min }\right)$ and pressure $\left(\mathrm{P}_{\min }\right), \bar{\Im}_{12}$ is the aerosol coefficient for that process for the lowest atmospheric temperature $\left(\mathrm{T}_{\min }\right)$ and highest pressure $\left(\mathrm{P}_{\max }\right),\left(\overline{\mathfrak{F}}_{21}\right)$ is the aerosol for the highest atmospheric temperature $\left(\mathrm{T}_{\max }\right)$ and lowest pressure $\left(\mathrm{P}_{\min }\right)$, and $\widetilde{\aleph}_{22}$ is the aerosol coefficient for the highest atmospheric temperature $\left(\mathrm{T}_{\max }\right)$ and pressure $\left(\mathrm{P}_{\max }\right)$.

$F_{T}$ and $F_{p}$ in Equation (7-5) are defined as

$$
F_{T}=\left(\frac{T_{g}-T_{\min }}{T_{\max }-T_{\min }}\right)
$$

and

$$
F_{P}=\left(\frac{P_{g}-P_{\text {min }}}{P_{\text {max }}-P_{\text {min }}}\right)
$$

where $T_{g}$ is the atmosphere temperature, and $P_{g}$ is the atmosphere pressure.

It should be noted that not all aerosol modeling is cast in coefficient form, because of the limitations discussed above. In particular, coefficients are not used for the aerosol deposition models for engineered systems, which are discussed in Sections 7.4 through 7.6, or for the moving grid condensation model. With regard to engineered systems, the full expressions for the deposition rates are always used and properly evaluated with respect to local boundary layer conditions. The deposition rate, however, is evaluated only for one representative particle diameter within each section, taken to be the geometric mean of the upper and lower diameters in the section. This approach is believed to be adequate. With regard to the moving grid model, the effects of condensation or evaporation are evaluated by calculating the change in particle size with time, then redistributing the time-evolved distribution into the fixed sections. The effects cannot be represented in terms of the time-independent $\overline{\mathrm{G}}$ coefficients shown in Equation (7-3).

In the following discussion, for simplicity, only the discrete forms of the agglomeration kernels and deposition rate equations will be presented. The approximations inherent in the use of the coefficient approach, if applicable, will be indicated.

\subsubsection{Agglomeration}

When two aerosol particles collide, they can combine to form a larger particle. This process is known as agglomeration or coagulation. A basic assumption about these processes is that only two 
particles can combine at a time. The agglomeration model used in MAEROS and CONTAIN treats four processes that lead to agglomeration: particle Brownian diffusion, differential gravitational settling, turbulent shear, and turbulent acceleration in eddies. The agglomeration processes depend on the physical properties of the gas and particles. Because the particles can be highly irregular, it is customary to base the agglomeration modeling on the compact spherical-equivalent particle diameter $\mathrm{d}$, the agglomeration shape factor $\gamma$, and the dynamic shape factor $\chi$. These shape factors and the three collision efficiencies $\mathrm{Col}_{\mathrm{g}}, \mathrm{Col}_{\mathrm{t} 1}$, and $\mathrm{Col}_{22}$ used in the model are discussed in more detail below, after the expressions for the agglomeration kernels are given.

The Brownian or diffusional agglomeration kernel is defined as[Gel82]

$$
\beta_{\text {diff }}=2 \pi\left(\bigoplus_{i}+\bigoplus_{j}\right)\left(\gamma_{i} d_{i}+\gamma_{j} d_{j}\right) f_{s t} / F
$$

where $f_{s t}=1$ is the sticking coefficient, $d_{i}$ and $d_{j}$ are the spherical-equivalent particle diameters of the two interacting particles, and $\gamma_{\mathrm{i}}$ is the agglomeration shape factor for the $i$ th particle, and the particle diffusivity $\bigoplus_{i}$ is given by

$$
\bigoplus_{i}=\frac{\kappa T_{g}}{3 \pi d_{i} \mu_{g} x_{i}} C_{i}
$$

In this expression, $\kappa$ is the Boltzmann constant, $\mu_{\mathrm{g}}$ is the gas viscosity (taken to be that of air), and $\chi_{i}$ is the dynamic shape factor. The $C_{1}$ factor allows for noncontinuum effects of the gas and is called the Cunningham slip correction factor

$$
\mathrm{C}_{\mathrm{i}}=1+\mathrm{N}_{\mathrm{Kn}, \mathrm{i}}\left[1.257+0.4 \exp \left(-1.1 / \mathrm{N}_{\mathrm{Kn}, \mathrm{i}}\right)\right]
$$

where $N_{K n, i}$ is the Knudsen number $2 \lambda / d_{i}$ and $\lambda$ is the mean-free path of the gas (taken to be air). The factor $F$ is defined as

$$
F=\frac{d_{i}+d_{j}}{d_{i}+d_{j}+2 g_{i, j}}+\frac{8\left(\bigoplus_{i}+\oplus_{j}\right)}{\overline{v_{i, j}}\left(d_{i}+d_{j}\right)}
$$

where

$$
g_{i, j}=\sqrt{g_{i}^{2}+g_{j}^{2}}
$$

and

$$
\overline{v_{i, j}}=\sqrt{v_{i}^{2}+v_{j}^{2}}
$$

with $g_{i}=\frac{1}{3 d_{i} l_{i}}\left[\left(d_{i}+l_{i}\right)^{3}-\left(d_{i}^{2}+l_{i}^{2}\right)^{3 / 1}\right]-d_{i}$

$$
\ell_{i}=\frac{8 \boxplus_{i}}{\pi v_{i}}
$$


and

$$
v_{i}=\sqrt{\frac{8 \kappa T_{g}}{\pi m_{i}}}
$$

The gravitational agglomeration kernel is defined as

$$
\beta_{s}=\frac{\pi}{4} \operatorname{Col}_{g}\left(\gamma_{i} d_{i}+\gamma_{j} d_{j}\right)^{2}\left|v_{s, i}-v_{s, j}\right| f_{s t}
$$

where the collision efficiency $\operatorname{Col}_{\mathrm{g}}$ is discussed below, and the settling velocity $\mathrm{v}_{\mathrm{s}, \mathrm{i}}$ is defined as

$$
v_{s, i}=\frac{\rho_{p, i} g_{i}^{2} C_{i}}{18 \mu_{g} \chi_{i}}
$$

Here, $\rho_{\mathrm{p}, \mathrm{i}}$ is the particle density and $\mathrm{g}$ is the acceleration due to gravity.

The turbulent shear and turbulent acceleration kernels are combined to yield a total kernel

$$
\beta_{\mathrm{t}}=\sqrt{\beta_{\mathrm{t} 1}^{2}+\beta_{\mathrm{t} 2}^{2}}
$$

where the turbulent shear kernel is defined as

$$
\beta_{t 1}=\sqrt{\frac{\pi^{2} \varepsilon_{t}}{120 v_{g}}} \operatorname{Col}_{t 1}\left(\gamma_{i} d_{i}+\gamma_{j} d_{j}\right)^{3} f_{s t}
$$

where $v_{g}$ is the gas kinematic viscosity (taken to be that of air). The turbulent acceleration kernel is defined as

$$
\beta_{\mathrm{t}}=\frac{0.04029 \rho_{\mathrm{g}}^{1 / 4} \varepsilon_{\mathrm{t}}^{3 / 4}}{\mu_{\mathrm{g}}^{5 / 4}} \operatorname{Col}_{\mathrm{t}}\left(\gamma_{\mathrm{i}} \mathrm{d}_{\mathrm{i}}+\gamma_{\mathrm{j}} \mathrm{d}_{\mathrm{j}}\right)^{2}\left|\frac{\rho_{\mathrm{p}, \mathrm{i}} \mathrm{C}_{\mathrm{i}} \mathrm{d}_{\mathrm{i}}^{2}}{\chi_{\mathrm{i}}}-\frac{\rho_{\mathrm{p}, \mathrm{j}} \mathrm{C}_{\mathrm{j}} \mathrm{d}_{\mathrm{j}}^{2}}{\chi_{\mathrm{j}}}\right| \mathrm{f}_{\mathrm{st}}
$$

In the above, $\varepsilon_{\mathrm{t}}$ is "turbds," the turbulent energy dissipation rate. This input parameter is specified in the global AEROSOL input block as described in Section 14.2.5. The collision efficiencies for the gravitational, turbulent shear, and turbulent acceleration processes are represented by $\mathrm{Col}_{\mathrm{g}}, \mathrm{Col}_{\mathfrak{l l}}$, and $\mathrm{Col}_{12}$, respectively, and the expressions used are discussed below. 
Except when they include significant amounts of liquid, aerosol particles are usually not spherical, and the effective aerosol densities may be significantly less than the bulk density of the materials of which the aerosols are composed. In aerosol codes, these effects may be taken into account through the use of the agglomeration shape factor $\gamma$ and the dynamic shape factor $\chi$. The shape factors $\gamma$ ("gamma") and $\chi$ ("chi") are input by the user to represent the effect of non-fully-dense shape upon aerosol collision cross sections and atmosphere drag forces, respectively. Unit values of the shape factors, which are the default, correspond to fully dense aerosols of spherical shape, while porous spherical agglomerates lead, in theory, to values somewhat greater than unity. Highly irregular aerosols and agglomerates can have shape factors substantially greater than unity, often with $\gamma$ and $\chi$ being quite different.

Given a description of the aerosol shapes and densities, shape factors could, in principle, be derived theoretically. However, this is not practical, so empirical values are obtained by fitting code calculations to the results of aerosol experiments. The values obtained may be sensitive to aerosol composition and to atmospheric conditions, especially the relative humidity. Humid conditions tend to produce more nearly spherical aerosols. Only limited information is available concerning the dependence of shape factors upon the relevant parameters (for example, particle characteristics and atmospheric conditions), and these parameters are themselves quite uncertain under accident conditions.

Agglomeration rates can be enhanced by turbulence in the containment atmosphere. In the past, very little attention has been given to estimating values of turbulent energy dissipation density $\varepsilon_{\mathrm{t}}$ appropriate for accident conditions, and uncertainty in its value may contribute to uncertainty in the aerosol agglomeration rates. The user can input the value of $\varepsilon_{\mathrm{t}}$ after keyword TURBDS or use the default value of $0.001 \mathrm{~m}^{2} / \mathrm{s}^{3}$.

Gravitational collision efficiencies $\mathrm{Col}_{\mathrm{g}}$ of unity correspond to the collision cross sections being equal to the geometric cross sections. It is well known that hydrodynamic interactions between particles can yield collision efficiencies much less than unity, especially for particles that are unequal in size. The problem of collisions between falling (spherical) aerosols has been the object of much detailed theoretical and experimental study, and may be more complex than can be represented by the simple expressions normally used in aerosol codes. The user can specify a constant value of $\mathrm{Col}_{\mathrm{g}}$ using keyword COLEFF in the global AEROSOL block; however, the default value is almost always used in practice and is given by

$$
\mathrm{Col}_{\mathrm{g}}=1.5 \mathrm{~d}_{\mathrm{j}}^{2} /\left(\mathrm{d}_{\mathrm{i}}+\mathrm{d}_{\mathrm{j}}\right)^{2}
$$

where $d_{j}$ is the smaller of the two aerosol particle diameters. Arguments have been presented [Dun84, Wil87b] that using 0.5 instead of 1.5 as the coefficient in Equation (7-11) is more accurate and that other corrections are needed when the size ratio $\mathrm{d}_{\mathrm{i}} / \mathrm{d}_{\mathrm{j}}$ is less than about 2 and/or $\mathrm{d}_{\mathrm{i}}$ is greater than about $20 \mu \mathrm{m}$. However, more recent experimental measurements of collision efficiencies by Gelbard et al. [Gel90] do not support these proposed revisions and, instead, gave collision efficiencies in reasonable agreement with Equation (7-11). These measurements involved studying the collisions of spheres at higher Reynolds numbers than those typical of aerosols and the results 
therefore may not be totally conclusive; however, arguments for modifying Equation (7-11) are not judged to be any more convincing, and hence Equation (7-11) remains the recommended option in CONTAIN.

Gravitational collision efficiencies have been a subject of considerable study in atmospheric and aerosol sciences, but much less attention has been paid to turbulent agglomeration collision efficiencies. As described in Reference Wil87b, the treatment of turbulent agglomeration is based upon that of Saffman and Turner, [Saf56] who assumed unit collision efficiencies. However, $\mathrm{Col}_{{ }_{2}}$ (the turbulent acceleration collision efficiency) is set equal to $\mathrm{Col}_{\mathrm{g}}$, although $\mathrm{Col}_{\mathrm{t}}$ is assumed to be unity. The rationale for these choices is discussed in Reference Wil87b.

Examination of the above relations for the agglomeration kernels shows that the effects of collision efficiencies, aerosol shape factors, and turbulence are coupled together in a highly nonlinear fashion. The dependence upon the various parameters differs among the different agglomeration mechanisms, and the net effects are strongly size-dependent. Hence, it is possible to give only a few generalizations of the effect to be expected.

All the agglomeration processes are enhanced by large values of the agglomeration shape factor $\gamma$, with the effect being largest for turbulent shear agglomeration and smallest for Brownian agglomeration. Large values of the dynamic shape factor $\chi$ reduce all the kernels except the turbulent shear kernel, which is unaffected. Hence, large values of the shape factors enhance the relative importance of turbulence, especially for the turbulent shear effect. Reference Wil87b includes sensitivity studies examining the implications of uncertainties in the shape factors $\gamma$ and $\chi$, the turbulent energy dissipation rate $\varepsilon_{\mathrm{t}}$, and the turbulent agglomeration collision efficiencies $\mathrm{Col}_{\mathfrak{} 1}$ and $\mathrm{Col}_{\mathrm{L}}$.

\subsubsection{Condensation and Evaporation}

The condensation of water on or evaporation of water from aerosols is calculated as part of the aerosol behavior model. The input for aerosol condensation and evaporation is part of the global aerosol block, discussed in Section 14.2.5.

A powerful aspect of the CONTAIN aerosol model is that the condensation of water on and evaporation from aerosols is coupled with the atmosphere thermal-hydraulic calculation and modeled in a self-consistent manner. This self-consistent coupling is essential to effective modeling of the dynamics of aerosol condensation, which often is controlled by a small difference between relatively large atmosphere source and sink terms. Two methods are available for modeling of aerosol condensation and evaporation. Two methods are available for computing the effects of condensation or evaporation. These methods are the fixed- and moving-grid methods discussed in Sections 7.2.2.1 and 7.2.2.2, respectively. The methods differ in their numerical algorithms and physical models as summarized in Table 7-1.

The moving-grid method has models for the solute and Kelvin effects that are not available for the fixed-grid method. The first effect is related to a reduction of vapor pressure of an aqueous solution of soluble material in the airborne particle. A particle with soluble material is hygroscopic and will 
Table 7-1

Comparison Between Fixed- and Moving-Grid Models

\begin{tabular}{|l|l|l||}
\hline \multicolumn{1}{|c|}{ Quantity } & \multicolumn{1}{c|}{ Fixed-Grid } & \multicolumn{1}{c|}{ Moving-Grid } \\
\hline Solute Effect & Not Modeled & Modeled \\
\hline Kelvin Effect & Not Modeled & Modeled \\
\hline Speed & Faster & Slower \\
\hline Numerical Diffusion & May Be Significant & Reduced Relative to Fixed-Grid \\
\hline Robustness & High & Low \\
\hline
\end{tabular}

grow faster than a particle with insoluble material. It may absorb water from the atmosphere even if the atmosphere is superheated. Particle growth will continue until the water vapor pressure above the particle surface is equal to that of the atmosphere.

The Kelvin effect considers the effect of the surface tension, which increases the water vapor pressure in the particle over that for a flat surface. The smaller the particle, the greater the surface area to volume ratio of the particle and hence the greater the effect of surface tension. For particles of pure water or with water and insoluble material, the increase in water vapor pressure because of the Kelvin effect will result in water evaporating from small particles in saturated environments. For particles with soluble material, the Kelvin effect increases the water vapor pressure in solution over that for a flat surface of solution. Thus, if a flat surface of a solution is in equilibrium with the atmosphere, the Kelvin effect would result in water vaporizing from a particle of the same composition as that of the solution.

The moving-grid method has the computational advantage of reducing numerical diffusion compared to the fixed-grid method. Numerical diffusion tends to smear discontinuous changes in the particle size distribution function. For example, because of numerical diffusion, spurious particles may remain in small particle size classes that have actually been swept clear of aerosols by condensational growth. Similarly, numerical diffusion may cause spurious particles to remain in large particle size classes that have been swept clear of aerosols by evaporation. However, reducing numerical diffusion by using the moving-grid method may require an order-of-magnitude larger amount of computer time than the fixed-grid method if the Kelvin effect is modeled.

With respect to selecting the appropriate method, if the solute effect or the Kelvin effect is to be modeled, then the moving-grid method is the only choice. However, if these effects are not to be modeled, the user may choose either method. Because the moving grid method is more accurate, it should be used whenever numerical diffusion needs to be assessed. However, if the computer time requirements are prohibitive, the fixed-grid method may be the only practical alternative.

To implement either method, the user must specify $\mathrm{H} 2 \mathrm{OL}$ as the last aerosol component in the "mapaer" input under the global AEROSOL keyword. This is discussed in Section 14.2.5, Aerosol Options. Within the fixed-grid method, one has either condensation or evaporation, but not a 
combination of the two within a given cell, since hygroscopic and Kelvin effects are not modeled. In this case, the user may employ the keywords NOCOND in the global AEROSOL input block to suppress only aerosol condensation and NOEVAP to suppress only evaporation for all cells. Within the moving grid method, the user should not employ either keyword by itself, but employing both keywords will disable both condensation and evaporation in all cells. In addition, for either method, the keyword NOCONEVA may be specified in the global AEROSOL block to disable both aerosol condensation and evaporation in all cells or only selected cells (see Section 14.2.5).

The fixed-grid and moving-grid methods also differ with regard to nucleation assumptions. The fixed-grid method attempts to simulate nucleation in a situation that would result in more rapid condensation if nucleation sites were present. The moving grid method by default does not simulate nucleation. Therefore, with the latter, in situations in which the aerosol concentration would otherwise be identically zero, the user may wish to supply a small nominal aerosol mass to allow condensation to proceed, or to select a nucleation option through the IFCOND keyword.

7.2.2.1 Fixed-Grid Model. The fixed-grid aerosol model models the condensation of water vapor onto aerosols and the evaporation of water from them. This model is the default model in CONTAIN. Considerable development work has gone into this model to ensure that CONTAIN runs efficiently for the maximum aerosol loadings that can result from condensation.

The rate equation for diffusion of water vapor to and from an aerosol is not the equation used in the MAEROS stand-alone code[Gel82] but is taken from Reference Bye65:

$$
G=2 \pi d_{p}\left(\frac{\rho_{v}}{\rho_{v, s}}-1\right)\left[\frac{M_{w} h_{l v}^{2}}{k_{g} R T_{g}^{2}}+\frac{1}{\rho_{v, s} \bigoplus_{v}}\right]^{-1}
$$

where $G$ is the vapor mass condensation rate on a particle of diameter $d_{p}, \rho_{v}$ is the steam density, $M_{w}$ is the molecular weight of water, $h_{l v}$ is the latent heat of vaporization, $k_{g}$ is the gas thermal conductivity (taken to be that of air), $R$ is the universal gas constant, $T_{g}$ is the gas temperature, $\rho_{v, s}$ is the saturated steam density, and $\bigoplus_{v}$ is the steam diffusivity (in air). This equation accounts for both the diffusivity of water vapor in air and the conduction of the heat of condensation away from the aerosol.

The rate of condensation on an aerosol particle is based on a fully dense spherical particle. Condensation is assumed to begin on existing particles, which are assumed to have a spherical core composed of either liquid or solid materials, as shown in Figure 7-3. Condensation is taken into account within the Runge-Kutta integration used for Equation (7-3).

Two solution methods are used for evaporation. For high superheat, when aerosol water evaporation is insufficient to keep the atmosphere saturated, a method of characteristics is employed. For evaporation under nearly saturated conditions, the Runge-Kutta method is used. However, use of Equation (7-12) poses problems at particle dryout, because the discontinuous change in rate at dryout causes the Runge-Kutta integrator to become inefficient. Therefore, when the Runge-Kutta method 


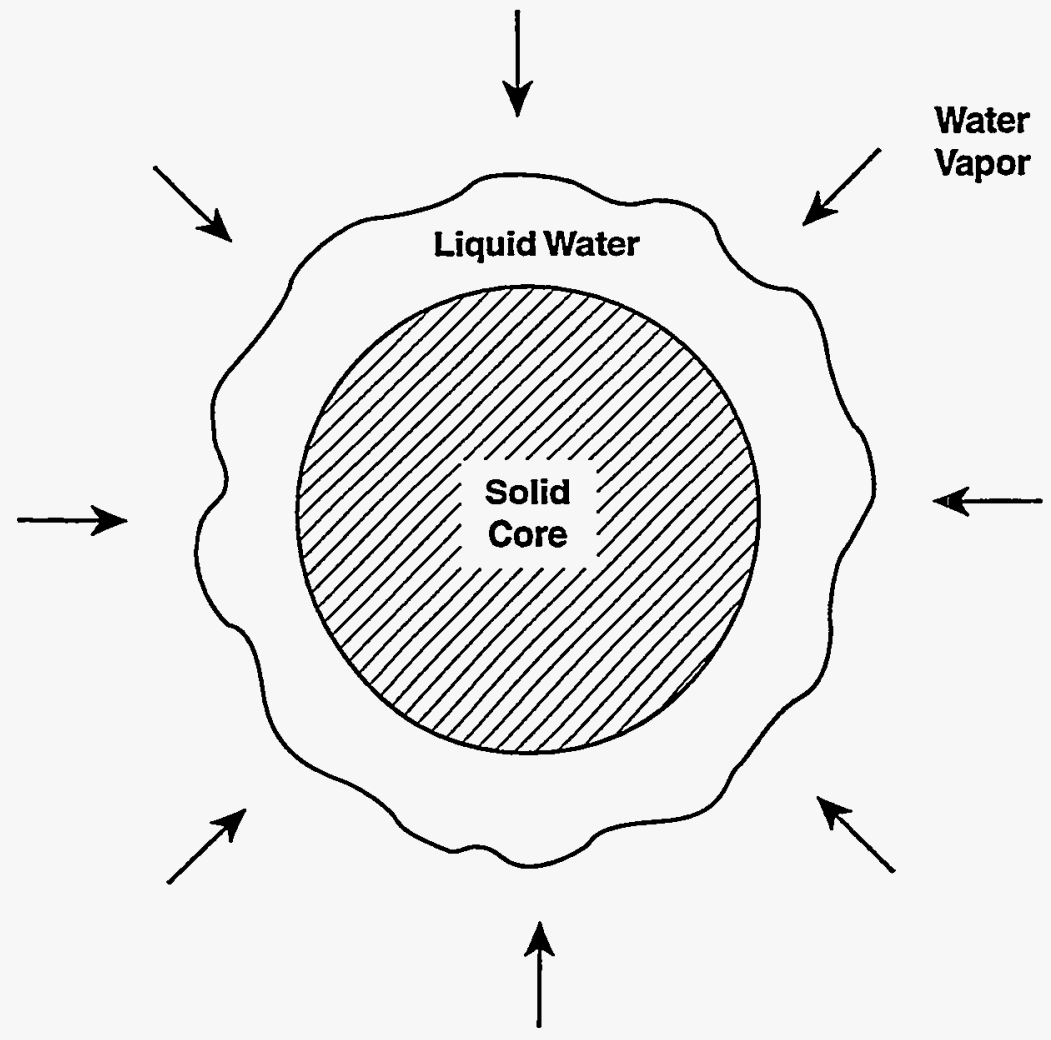

Figure 7-3. Model for Water Condensation on Aerosols 
is used during evaporation, the solid materials are assumed to inhibit evaporation when the water mass and the solid mass are comparable. The evaporation rate is under such conditions is assumed to be

$$
\mathrm{G}_{\text {evap }}=1.037 \mathrm{G} \tanh \left(2 \mathrm{~m}_{\ell} / \mathrm{m}_{\mathrm{p}}\right)
$$

where $G$ is the evaporation rate from a spherical aerosol given by Equation (7-12) and $m_{l}$ and $m_{p}$ are the liquid water mass and total mass of the aerosol particle, respectively. The amount of water present on aerosols when the rate is strongly inhibited is typically not significant.

Nucleation centers can alter the condensation rate on aerosols considerably. Even if a large number of aerosol particles are available to serve as nucleation centers, the condensation rate can decrease once the particles have grown. In the CONTAIN fixed-grid method, an attempt is made to simulate nucleation centers. A small amount of water aerosol is provided in the smallest diameter section under condensing conditions when the mass concentration in that section is zero. This is intended to promote condensation when it would not otherwise occur, or occur but at a significantly impeded rate. The mass concentration added corresponds to

$$
\mathrm{Q}_{\text {nuc }}=\max \left(10^{-20}, 0.001 \text { "abstol" } \mathrm{Q}_{\mathrm{T}}\right)
$$

where $\mathrm{Q}_{\mathrm{T}}$ is the total suspended mass concentration, and "abstol" is the scale factor for the RungeKutta absolute integration error(see Section 14.2.5). By default, "abstol" $=10^{-4}$. The added mass concentration is typically a few orders of magnitude smaller than the integration error in the mass concentration in a section.

7.2.2.2 Moving-Grid Model. This moving grid model is only used if the keyword SOLAER is included in the global AEROSOL block input. Otherwise the fixed grid model is invoked by default. The algorithm used is based on the method of characteristics. However, after the effects of condensation are calculated over a system timestep, the aerosol is remapped onto the fixed grid in order to incorporate the effects of the agglomeration and deposition calculations. The theoretical development of the moving-grid model is discussed in detail in Reference Gel90.

In the moving-grid method, particle sections are followed as they grow or shrink from water condensing on or vaporizing from the particles. Particles in a section are approximated as initially having the same chemical composition, but different sections may have different chemical compositions. There is essentially no constraint on the particle size or composition range covered by a time-evolved section, and two or more sections of particles may overlap the same particle size range.

For each timestep the growth or evaporization of a particle section is calculated based on an assumed end-of-timestep steam concentration. The water mass balance is determined from the amount of water condensed or vaporized and the assumed steam concentration. The code iterates on the end-oftimestep steam concentration until the water mass balance is satisfied. 
The condensation rate is calculated from a simplified version of the Mason equation presented in Reference Pru78:

$$
G=\frac{2 \pi d_{p} \rho_{\ell}\left(\frac{\rho_{v}}{\rho_{s, v}}-a_{w} \exp \left[\frac{4 \sigma M_{w}}{R T_{g} \rho_{\ell} d_{p}}\right]\right)}{\frac{\rho_{\ell}}{\rho_{s, v} \bigoplus_{v}}+\frac{h_{\ell v} \rho_{\ell}}{k_{g} T_{g}}\left[\frac{h_{\ell v} M_{w}}{R T_{g}}-1\right]}
$$

where $d_{p}$ is the particle diameter, $\rho_{\ell}$ is the density of water, $a_{w}$ is the activity of water, and $\sigma$ is the surface tension specified by the keyword SURTEN. The remaining terms in Equation (7-15) are previously defined in Equation (7-14). Note that Equation (7-15) includes solute and Kelvin effects, whereas Equation (7-14) does not. Simplifications have been made in Equation (7-15) with respect to the vapor diffusivity and gas conductivity: these are not corrected for finite gas mean free path effects, as they are in the original expression in Reference Pru78.

The activity can be expressed as

$$
a_{w}=\exp \left(-\sum_{s}^{v_{s} m_{s} M_{w}} \frac{m_{\ell} M_{s}}{m_{1}}\right)
$$

where $v_{s}$ is the solubility factor of the salt, given by the SOLUBLE keyword discussed in Section 14.2.5, $\mathrm{m}_{\mathrm{s}}$ is the mass of salt $\mathrm{s}$ on the particle, and $\mathrm{M}_{\mathrm{s}}$ is the molecular weight of the salt.

After the particle growth calculation is completed the aerosol is remapped from this moving grid formulation to the fixed-grid for calculation of aerosol agglomeration and deposition effects. The remapping is performed by approximating the particle mass distribution as being constant with respect to the logarithm of particle diameter.

\subsubsection{Deposition}

Containment aerosols can deposit or settle onto the surfaces of heat transfer structures and onto the coolant pool in the lower cell. Deposition on such surfaces occurs through four processes: gravitational settling, diffusion to surfaces, thermophoresis, and diffusiophoresis. Of these natural depletion processes, gravitational settling is often the dominant mechanism, although phoretic effects may be significant in some cases. In general, particle diffusion is considered to be a relatively unimportant deposition process. The velocities for each of these deposition processes are defined below. Note that deposition with respect to engineered systems is modeled somewhat differently from that for structures and pools and is discussed in Sections 7.4 through 7.6.

Gravitational Settling. The gravitational deposition removal mechanism is governed by the following particle settling velocity: 


$$
\mathrm{v}_{\mathrm{s}}=\frac{\rho_{\mathrm{p}} \mathrm{gd}_{\mathrm{p}}^{2} \mathrm{C}}{18 \mu_{\mathrm{g}} \chi}
$$

where $v_{s}$ is the downward settling terminal velocity; $\rho_{p}$ is the particle density; $g$ is the acceleration of gravity $\left(9.8 \mathrm{~m} / \mathrm{s}^{2}\right)$; $\mathrm{d}_{\mathrm{p}}$ is the particle diameter; $\mu_{\mathrm{g}}$ is bulk gas viscosity (taken to be that of dry air) at the atmosphere temperature; and $\chi$ is the dynamic shape factor. $\mathrm{C}$ is the Cunningham slip correction factor.

$$
\mathrm{C}=1+\mathrm{N}_{\mathrm{Kn}}\left[1.257+0.4 \exp \left(-1.1 / \mathrm{N}_{\mathrm{Kn}}\right)\right]
$$

where $N_{K n}$ is the Knudsen number $2 \lambda / d_{p}$ and $\lambda$ is the mean-free path of the gas.

One basic restriction of this model is that the aerosol particle Reynolds number $\mathrm{N}_{\mathrm{Re}, \mathrm{p}}$ must be much less than 1 . This is generally acceptable since $N_{R e, p}=1$ corresponds to a diameter of $70 \mu \mathrm{m}$ for a particle with the density of water, which is fairly large.

Diffusion. Another aerosol deposition mechanism results from diffusion of aerosols in a concentration gradient, that is, from a higher to a lower concentration region. The diffusional deposition velocity is given by

$$
\mathrm{v}_{\text {diff }}=\boxplus_{\mathrm{p}} / \Delta
$$

where

$$
\bigoplus_{p}=\frac{\kappa T_{g} C}{3 \pi \mu_{g} d_{p} \chi}
$$

is the particle diffusivity; $\kappa$ is the Boltzmann constant $\left(1.38 \times 10^{-23} \mathrm{~J} / \mathrm{K}\right) ; T_{\mathrm{g}}$ is the atmosphere temperature; and $\Delta$ is the user-specified diffusion boundary layer thickness, "deldif," which has a default value of $10^{-5} \mathrm{~m}$ and is specified in the global AEROSOL input block.

Thermophoresis. This aerosol deposition mechanism results from the force exerted on aerosol particles by temperature gradients in containment. The thermophoretic deposition velocity $\mathrm{v}_{\text {therm }}$ is that given in Reference Tal80

$$
v_{\text {therm }}=\frac{2.294 \mu_{g} C\left(C_{t} N_{K n}+k_{g} / k_{p}\right) q_{c}}{\chi \rho_{g} T_{g}\left(1+3 C_{m} N_{K n}\right)\left(1+2 C_{t} N_{K n}+2 k_{g} / k_{p}\right) k_{B L}}
$$

where $k_{g} / k_{p}$ is the user-specified ratio of the thermal conductivity of the gas to that for the aerosol particle; $q_{c}$ is the surface convective heat flux $\left(\mathrm{W} / \mathrm{m}^{2}\right) ; \rho_{\mathrm{g}}$ is the gas density (taken to be that of air) at the atmosphere conditions; $C_{m}=1.146$ is a slip coefficient; $C_{t}=2.20$ is a thermal accommodation coefficient [Tal80] and $\mathrm{k}_{\mathrm{BL}}$ is the actual gas boundary layer thermal conductivity. 
Diffusiophoresis. When water condenses on (evaporates from) a structure surface, composition gradients will exist in the adjacent gas which will affect aerosol deposition on the surface due to two related effects. The first is that there will be a net molar flux of gas toward (away from) the condensing (evaporating) surface, and this net flux, commonly called the Stefan flow, [Hin82] will tend to move aerosol particles with it. The second effect is that differences in the momentum transferred by molecular impacts on opposite sides of the particle will tend to drive the particle in the direction of decreasing concentration of the heavier constituent. In a strict sense, only this second component constitutes diffusiophoresis; however, in the present discussion, the term "diffusiophoresis" will be used to refer to the net result of both effects. Note that when the noncondensable gas is heavier than steam, as in air-steam mixtures, the differential molecular impact effect opposes the Stefan flow (which dominates the net result); the effects are in the same direction if the noncondensable gas is lighter than steam.

The treatment in CONTAIN is equivalent to that given in Reference Wal66 for particle sizes that are large compared with molecular mean free paths, a condition which will generally apply for containment analyses. A diffusiophoretic deposition velocity (including the Stefan flow) $v_{\text {diph }}$ for a surface is calculated from

$$
v_{\text {diph }}=\left(\frac{X_{v, B L} \sqrt{M_{w}}}{X_{v, B L} \sqrt{M_{w}}+X_{n c, B L} \sqrt{M_{n c}}}\right)\left(\frac{W_{c o n d}}{\rho_{v, B L} A_{s}}\right)
$$

where $\mathrm{X}_{\mathrm{v}, \mathrm{BL}}$ is the boundary layer mole fraction of water vapor, $\mathrm{M}_{\mathrm{w}}$ is the molecular weight of water, $\mathrm{W}_{\text {cond }}$ is the mass rate of water condensing onto a surface, $\mathrm{X}_{\mathrm{nc}, \mathrm{BL}}=1-\mathrm{X}_{\mathrm{v}, \mathrm{BL}}, \mathrm{M}_{\mathrm{nc}}$ is the molecular weight of noncondensable gases in the boundary layer, $\rho_{\mathrm{v}, \mathrm{BL}}$ is the density of water vapor in the boundary layer, and $A_{s}$ is the area of the surface. Here, the boundary layer properties are taken to be the average of the values in the bulk gas and the values at the interface between the gas and the water film on the surface. (In contrast to the other deposition velocities calculated in this section, $\mathrm{v}_{\text {diph }}$ is calculated for actual boundary layer properties because it does not rely on the aerosol coefficients.)

CONTAIN uses the four deposition velocities defined above for settling, diffusion, thermophoresis, and diffusiophoresis to calculate the aerosol removal rate term $\Re$, which is represented on a sectional basis in Equation (7-3). The contribution to $\Re$ from these processes is defined as

$$
\Re=\max \left(\sum_{j=1}^{N_{s}} \frac{A_{j}}{V_{g}}\left(v_{s} \bar{n}_{z}+v_{\text {diff }}+v_{\text {therm }}+v_{\text {diph }}-v_{p g}\right), 0\right)
$$

where $\mathrm{N}_{s}$ is the total number of heat structure surfaces and/or pool surfaces for aerosol deposition in the cell, $A_{j} / V_{g}$ is the unsubmerged surface area to cell free volume ratio, $\bar{n}_{z}$ is the mean value of the vertical component of the unit normal vector of the surface, and $v_{p g}$ is the convective velocity of gases normal to the surface. The last velocity is non-zero only for the pool and accounts for all gases evolving from the pool surface, except for the surface condensation/evaporation flux already 
included in $\mathrm{v}_{\mathrm{diph}}$. The definition of $\mathrm{v}_{\mathrm{pg}}$ is discussed in Section 4.4.8. Other contributions to $\Re$ from engineered systems operation are discussed in Sections 7.4 through 7.6.

In CONTAIN, the user should note that aerosols will not deposit onto the submerged portion of an inner surface of a heat transfer structure or on an outer surface if the outer face of the structure is not specified to be in the cell in which the structure is defined, even if aerosols are present in the cell in which the outer face resides. The outer surface of a ceiling structure is considered a floor deposition area and the outer surface of a floor structure is considered a ceiling deposition area. If surfaces with deposited aerosols and fission products are subsequently submerged, the deposited aerosols and fission products are transferred to the pool in proportion to the previously unsubmerged area that is subsequently submerged.

The user should also note that a lower cell coolant pool must be defined for deposition onto the lower cell, discussed in Chapter 5, to occur. The reason for this restriction is that the aerosol deposition arrays are associated with the lower cell coolant pool and not the lower cell system. If a pool is present, the deposition will occur on a pool area equal to the cell cross-sectional area at the pool height, as defined in Sections 4.1 and 14.3.1.1. If such a pool is logically defined but has zero mass, deposition will occur on the pool substrate. This substrate may consist either of lower cell layers or the basemat, with an area equal to the lower cell substrate area, or of the CORCON upper melt surface, if CORCON is active. The user should also note that if a pool is defined, the SETTLE keyword is no longer necessary to enable such deposition. The lower cell substrate area does not have to correspond to the entire bottom cross-sectional area of the cell, since the cell bottom may be partially or completely spanned by a floor heat transfer structure or by a flow path opening. In the latter case, aerosols may be transported through gas flow paths by settling if the VCOSN keyword is used in the ENGVENT input block to define a flow path inclination angle. (See Sections 7.8 and 14.2.4.2.)

\subsection{Aerosol Sources}

Aerosol sources, which contribute to the $\bar{S}$ term in Equation (7-3), may consist of two types, internal and external. As discussed in Chapter 5, aerosols may be produced internally through the effects of $\mathrm{CCI}$, as modeled within the CORCON Mod3 module. The size distribution of such aerosols are calculated within the VANESA routines within CORCON. Sources may also be explicitly specified by the user. Such user-supplied sources may be introduced directly into the atmosphere or in conjunction with gas sources under the surface of the coolant pool through the safety relief valve (SRV) model. When introduced through the SRV model, the aerosols will be scrubbed as described in Section 7.7 before being added to the atmosphere.

The size distribution of aerosols initially present or introduced into a cell from an external source is assumed to be lognormal as described in Section 7.1. The distribution is normally given by the constant "amean" and "avar" parameters specified in the global AEROSOL block (see Section 14.2.5). These parameters correspond to the spherical-equivalent mass median diameter and the natural logarithm of the geometric standard deviation with respect to diameter, respectively. Each aerosol component or species may have a different distribution. However, by using the AERTIM option, also described in Section 14.2.5, or the SRV model, discussed in Sections 11.2 and 14.3.4, 
the user may specify these parameters as time-dependent for aerosol sources. Aerosol dynamics will in general alter the lognormal distributions as time progresses.

At the global level, the keyword AEROSOL is used to specify the global aerosol characteristics, which are the same for all cells. The same keyword is used at the cell level to specify initial suspended masses and atmosphere sources of aerosols. For the latter, the general format for source tables is used; however, because aerosol materials are assumed to have negligible specific heat, one should not specify a temperature or enthalpy for an aerosol material. (For purposes of mass and energy accounting, aerosols are assumed to be injected at the repository temperature and pressure.) The specific format to be followed for aerosols is discussed in Section 14.3.1.8.

\subsection{Fan Cooler Aerosol Deposition Model}

Aerosol deposition driven by diffusiophoresis may be significant for a fan cooler engineered system described in Sections 12.1 and 14.3.3.2. Deposition by this process is calculated when the mechanistic fan cooler model is active as described below. Any deposited aerosols, fission products hosted by these aerosols, and the fan cooler condensate are routed to the pool in the "iclout" cell indicated by the user in the ENGINEER input block. If a pool is not defined in that cell, the condensed vapor and any removed aerosols and fission products will be accounted for in the WASTE repository of that cell.

The diffusiophoretic deposition is based upon applying Equation (7-20) to an approximate estimate of average conditions existing inside the fan cooler. The contribution to the aerosol fractional removal rate $\Re\left(\mathrm{s}^{-1}\right)$ from the fan cooler is given by

$$
\Re=\left[\frac{\mathrm{X}_{\mathrm{v}, \mathrm{av}} \sqrt{\mathrm{M}_{\mathrm{w}}}}{\mathrm{X}_{\mathrm{v}, \mathrm{av}} \sqrt{\mathrm{M}_{\mathrm{w}}}+\left(1-\mathrm{X}_{\mathrm{v}, \mathrm{av}}\right) \sqrt{\mathrm{M}_{\mathrm{nc}}}}\right] \frac{\mathrm{W}_{\mathrm{cond}}}{\rho_{\mathrm{v}, \mathrm{av}} \mathrm{V}_{\mathrm{g}}}
$$

where the subscript "av" refers to average properties as discussed below, $\mathrm{M}_{\mathrm{w}}$ is the molecular weight of water, $W_{\text {cond }}$ is the mass condensation rate within the fan cooler calculated as described in Section $12.1, \mathrm{M}_{\mathrm{nc}}$ is the actual molecular weight of the noncondensable gas present, and $\mathrm{V}_{\mathrm{g}}$ is the free volume of the cell within which the fan cooler is located. The average properties are obtained by first defining an average temperature, $\mathrm{T}_{\mathrm{av}}$, given by

$$
\mathrm{T}_{\mathrm{av}}=\left(2 \mathrm{~T}_{\mathrm{g}}+\mathrm{T}_{\mathrm{c}, \mathrm{i}}+\mathrm{T}_{\mathrm{c}, \mathrm{o}}\right) / 4
$$

where $T_{g}$ is the gas temperature and $T_{c, i}$ and $T_{c, 0}$ are, respectively, the temperature of coolant entering the fan cooler (specified by the user) and the temperature of water exiting the fan cooler (see Section 12.1). Then the vapor mole fraction $X_{v, a v}$ is assumed to be equal to $P_{s, a v} / P_{g}$, where $P_{s, a v}$ is the saturation pressure evaluated at $T_{a r}, P_{g}$ is the total gas pressure, and $\rho_{v, a v}$ is the density of water vapor evaluated at $\mathrm{P}_{\mathrm{s}, \mathrm{av}}$ and $\mathrm{T}_{\mathrm{av}}$. 


\subsection{Ice Condenser Aerosol Deposition Model}

The ice condenser provides conditions under which significant deposition of suspended aerosols from the atmosphere can occur. The aerosol deposition model for the ice condenser treats settling, impaction/interception, Brownian diffusion, diffusiophoresis, and thermophoresis. The model is based on the ICEDF model of Winegardner, Postma, and Jankowski, [Win83] with minor modifications to make it consistent with other CONTAIN models. (Also see Reference Owc85b.) In contrast to the aerosol dynamic modeling presented in Section 7.2, the ice condenser thermalhydraulic and aerosol deposition models use boundary layer values of physical quantities where appropriate and also treat noncondensable gas media other than air, using the approximations discussed in Sections 10.1 and 10.2.

The deposition modeling discussed below includes only effects attributable to the ice and ice basket structures. Note that aerosol decontamination is calculated even in the absence of ice, because the large surface areas represented by the ice baskets could be effective in removing particulates. The walls, floors, and ceiling of the ice compartment should be modeled separately as heat transfer structures. The deposition modeling for such structures is discussed in Section 7.2.3.

The aerosols and associated fission products that are removed from the atmosphere through ice and ice basket interactions are placed in the pool, if present, in the cell "iclout" specified by the user for the ice condenser. This treatment assumes that there is no holdup of aerosols and fission products in the ice compartment. One consequence is that fission product decay heating does not contribute to the melting of the ice. The "iclout" destination is also that used for the melt/condensate resulting from ice condenser operation. If a pool is not present in the designated cell, the aerosols and fission products will be placed in the waste repository of the designated cell.

The ice and ice basket contribution to the aerosol fractional removal rate $\Re\left(\mathrm{s}^{-1}\right)$ from the five mechanisms described above may be expressed as

$$
\Re=\Re_{\mathrm{s}}+\Re_{\text {diff }}+\Re_{\text {therm }}+\Re_{\text {diph }}+\Re_{\mathrm{i}}
$$

where the terms on the right represent the effects of gravitational settling, Brownian diffusion, thermophoresis, diffusiophoresis, and interception/impaction, in that order. Turbulent deposition is not modeled because it can be shown to have a negligible effect. [Win83]

Settling. The removal rate constant for gravitational settling $\Re_{s}$ is simply the product of the settling velocity and the surface area:

$$
\Re_{\mathrm{s}}=\mathrm{v}_{\mathrm{s}} \mathrm{A}_{\mathrm{s}}
$$

where $v_{s}$ is defined as in Equation (7-17), except that actual ice boundary layer values are used for gas properties, and $A_{s}$ is defined as 


$$
A_{s}=A_{s e d}+f_{s e d} A_{h t}
$$

The settling area $A_{s}$ is the sum of all upward-facing areas related to the ice and ice baskets and would normally include the ice basket wires and support structures and the ice sedimentation area. In the above equation, $A_{\text {sed }}$ is the total basket sedimentation area "areased." This may be specified by the user and is by default equal to $1535 \mathrm{~m}^{2}$. Also, $f_{\text {sed }}$ is the fraction "fracsed" of the ice heat transfer area $A_{\mathrm{ht}}$ subject to sedimentation. $f_{\text {sed }}$ may also be specified by the user and is by default equal to $1 / 2 . A_{\text {ht }}$ is obtained by scaling "arhtin," the user-specified initial ice heat transfer area by the fraction of the initial ice inventory left in the ice chest. Note that $A_{s}$ should not include the areas of heat transfer structures or the coolant pool, if any, in an ice condenser cell, since these are taken into account separately.

Diffusional Deposition. Particles exhibit a diffusivity as a result of momentum exchange with surrounding gas molecules. For the case where the gas is isothermal and has no molecular weight gradients, the particle mobility arises from Brownian diffusion. Particles experience a net flux toward surfaces because of concentration gradients; particle capture by the surface is assumed to reduce the gas phase concentration of the particles to zero on the surface.

The efficiency of deposition depends both on the diffusivity and the fluid flow pattern past a surface. The diffusional deposition processes are modeled separately for the ice baskets and ice surfaces. Note that the flow around the ice baskets is modeled in terms of the individual horizontal strips used to hold the ice, as in Figure 7-4. The diffusional deposition with respect to the ice surfaces is modeled in terms of a heat and mass transfer analogy similar to that employed to model steam condensation mass transfer, as discussed in Section 10.2.3.

The Brownian diffusion removal rate constant $\Re_{\text {diff }}$ is the sum of the contributions resulting from the flow around the ice basket wires and the flow parallel to the ice heat transfer surfaces

$$
\Re_{\text {diff }}=v_{g} A_{\text {diff }} E_{\text {diff }}+\kappa_{p} A_{\text {ht }}
$$

where $v_{g}$ is the forced convective gas velocity approaching the strip, as defined in Equation (12-7), $A_{\text {diff }}$ is the effective cylindrical area for diffusional deposition $\left(\mathrm{m}^{2}\right)$, and the capture efficiency $E_{\text {diff }}$ for diffusion is defined as

$$
E_{\text {diff }}=\frac{1}{N_{\mathrm{Pe}}}+1.727 \frac{\mathrm{N}_{\mathrm{Re}, \mathrm{c}}^{1 / 6}}{\mathrm{~N}_{\mathrm{Pe}}^{2 / 3}}
$$

where $N_{p e}$ is the Peclet number, which may be expressed as $d_{c, d} v_{g} / \bigoplus_{p} ; d_{c, d}$ is the effective cylindrical diameter for diffusion; $\boxplus_{\mathrm{p}}$ is the particle diffusivity, as defined in Equation (7-18); and $\mathrm{N}_{\mathrm{Rec}}$ is the strip Reynolds number, which may be expressed as $\rho_{\mathrm{g}} \mathrm{d}_{\mathrm{c}, \mathrm{d}} \mathrm{v}_{\mathrm{g}} / \mu_{\mathrm{g}}$. All gas quantities, for simplicity, are defined for the ice boundary layer; and it has been assumed that the strips can be treated as cylinders. 


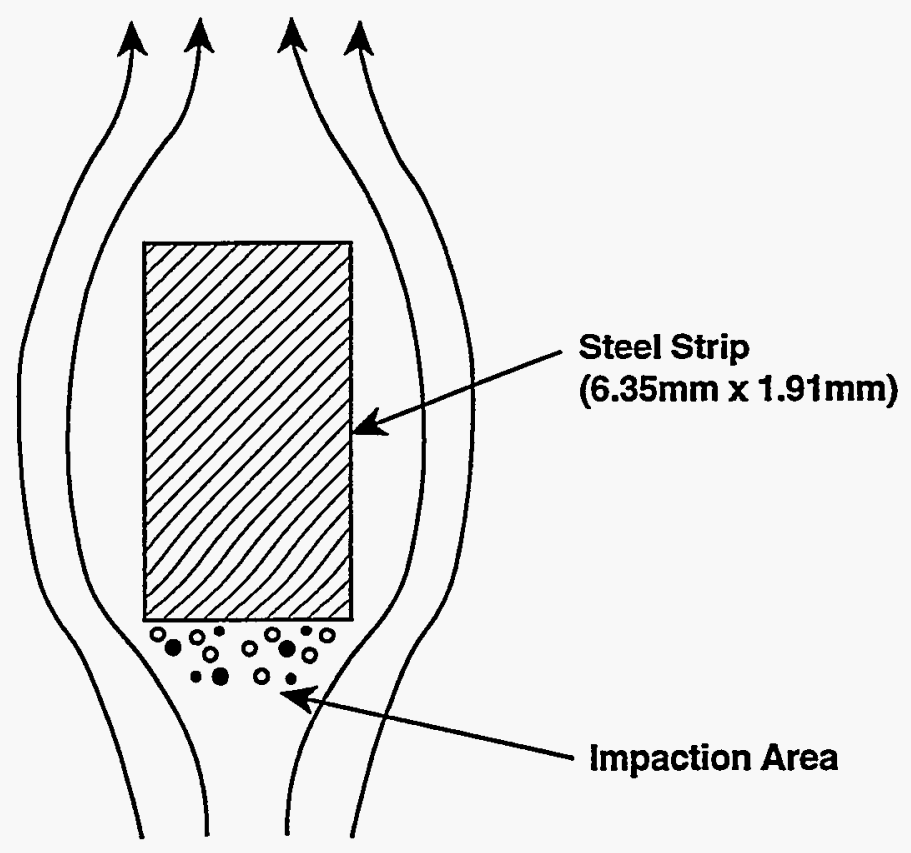

Figure 7-4. Illustration of Impaction on a Horizontal Strip of a Typical Ice Condenser Ice Basket (Based on Reference Win83) 
The effective cylindrical diameter $d_{c, d}$ for the strip for diffusion is computed by equating the cylinder perimeter to that of the rectangular strip; the equivalent diameter is $5.26 \mathrm{~mm}$. A cylinder of this diameter will exhibit the same surface area and exposure time (assuming similar velocity profiles for rectangle and cylinder) and therefore is a reasonable simulation of the strip. For this diameter, the effective cylindrical area $A_{\text {diff }}$ for the case of 1,944 baskets is predicted to be $3.43 \cdot 10^{3} \mathrm{~m}^{2}$.

The second term in Equation (7-25) accounts for diffusional deposition on the ice heat transfer surfaces from flow parallel to the ice surfaces, which have an effective heat transfer area $A_{h t}$. This is modeled in terms of the larger of a natural convective particle mass transfer coefficient $\kappa_{p, c}$ (in $\mathrm{m} / \mathrm{s}$ ) or a forced convective coefficient $\kappa_{\mathrm{p}, \mathrm{f}}$

$$
\kappa_{p}=\max \left(\kappa_{p, c}, \kappa_{p, f}\right)
$$

For unstable turbulent natural convection, the heat and mass transfer analogy defines the mass transfer coefficient as

$$
\frac{\kappa_{p, c} L}{\bigoplus_{p}}=0.14\left(N_{G r} N_{S c, p}\right)^{1 / 3}
$$

where $L$ is the length of the ice column (m), $N_{G r}$ is the Grashof number (defined below), and $N_{S c, p}$ is the particle Schmidt number, which may be expressed as $\mu_{\mathrm{g}} / \rho_{\mathrm{g}} \bigoplus_{\mathrm{p}}$.

The Grashof number used to characterize natural convection flow is

$$
N_{G r}=\frac{L^{3} g \Delta \rho_{g}}{v_{g}^{2} \rho_{g}}
$$

where $g$ is the acceleration due to gravity $\left(\mathrm{m} / \mathrm{s}^{2}\right), \Delta \rho_{\mathrm{g}}$ is the density difference between the bulk gas and the gas at the ice surface, $v_{\mathrm{g}}$ is the kinematic viscosity of gas $\left(\mathrm{m}^{2} / \mathrm{s}\right)$, and $\rho_{\mathrm{g}}$, is the bulk gas density $\left(\mathrm{kg} / \mathrm{m}^{3}\right)$. The density difference $\Delta \rho_{\mathrm{g}}$, is the total value that would result from differences in both temperature and molecular weight. Again, the gas properties are defined in the ice boundary layer.

Note that in combining Equations (7-28) and (7-29), the length scale $L$ cancels, making $\kappa_{p, c}$ independent of $L$. This lack of dependence on $L$ applies to turbulent flow; for the large distances applicable to ice compartments, turbulent flow is expected to occur over a large fraction of the surface area.

The forced convective mass transfer coefficient is defined by analogy with forced convective heat transfer to a flat plate 


$$
\frac{\kappa_{p, f} L}{\bigoplus_{p}}=0.037 N_{R e, L}^{0.8} N_{S c, p}^{1 / 3}
$$

The symbols in Equation (7-30) are as previously defined except that $N_{R e, L}$ uses the ice column height as the characteristic length.

Thermophoresis. The removal rate constant from thermophetic effects is given by

$$
\Re_{\text {therm }}=v_{\text {therm }} A_{s}
$$

where $v_{\text {therm }}$ is defined as in Equation (7-19), except that the gas properties are all defined in the ice boundary layer, and $A_{s}$ is the total heat transfer area of the ice and effluent, and $q_{c}$ in Equation (7-19) is defined as the total convective heat transfer rate $(\mathrm{J} / \mathrm{s})$ to the ice and effluent.

Diffusiophoresis. The removal rate constant from diffusiophoretic effects is given by

$$
\Re_{\text {diph }}=\mathrm{v}_{\text {diph }} \mathrm{A}_{\mathrm{s}}
$$

where $v_{\text {diph }}$ is defined as in Equation (7-20), $A_{s}$ is the total heat transfer area of the ice and effluent, and $W_{\text {cond }}$ in Equation (7-20) is defined as the total condensation rate on the ice and effluent.

Impaction/Interception Deposition. The perforated steel baskets contain a large number of horizontal strips which promote impaction and interception of particles. The individual web segments are 1.91 $\mathrm{mm}$ thick, $6.35 \mathrm{~mm}$ long (in the direction of flow), and $2.54 \mathrm{~cm}$ wide. Impaction and interception on the end of a strip are pictured schematically in Figure 7-3.

The impaction and interception efficiencies $\mathrm{E}_{\mathrm{imp}}$ and $\mathrm{E}_{\mathrm{int}}$, respectively, are defined as the fraction of approaching particles that the body captures by the two processes. The removal rate constant can be expressed as

$$
\Re_{i}=v_{g} A_{i}\left(E_{\text {imp }}+E_{\text {int }}\right)
$$

where $A_{i}$ is the effective area for impaction $\left(m^{2}\right)$. The effective area $A_{i}$ for impaction is the same as that for sedimentation on the basket wires and amounts to $1,240 \mathrm{~m}^{2}$ for a typical plant. [Win83]

The impaction efficiency is defined in terms of a correlation developed for cylinders. An empirical fit of impaction efficiency data presented in Reference Win83 can be expressed as:

$$
\mathrm{E}_{\mathrm{imp}}=\max \left(\frac{\mathrm{St}^{2}}{(\mathrm{St}+0.5)^{2}}-0.04,0\right)
$$


where St is the Stokes number, the ratio of the particle's stopping distance to the characteristic dimension of the collector surface. This is defined by

$$
S t=\frac{v_{g} \rho_{p} d_{p}^{2} C}{9 \mu_{g} d_{c, i} \chi}
$$

where $\mathrm{C}$ is the Cunningham slip correction factor for finite gas mean free path effects, given with respect to Equation (7-17), $\chi$ is the dynamic shape factor, and the collector diameter for impaction $\mathrm{d}_{\mathrm{c}, \mathrm{j}}$ should be set equal to the metal thickness, $1.91 \mathrm{~mm}$. The interception efficiency of particles by the strips is related to particle size through a formula adapted from Fuchs [Win83]:

$$
\mathrm{E}_{\mathrm{int}}=2 \frac{\gamma \mathrm{d}_{\mathrm{p}}}{\mathrm{d}_{\mathrm{c}, \mathrm{i}}}
$$

where $\gamma$ is the agglomeration shape factor.

\subsection{Containment Spray Aerosol Removal Model}

The depletion rate for airborne aerosols is defined as the product of a collection efficiency and the fraction of the cell volume swept out by spray per unit time. The collection efficiency is integrated over the falling time of a droplet, taking into account the droplet size and temperature as well as the aerosol size, and the containment conditions such as pressure. Collection mechanisms considered in deriving the efficiency are Brownian diffusion, thermophoresis, diffusiophoresis, interception, and impaction. No additional input parameters, beyond the ones specified in the global AEROSOL block, are necessary for control of aerosol washout.

It should be noted that in the following, gas properties are evaluated for boundary layer conditions between the drop and atmosphere. Since the drop is assumed to be well-mixed, the interface temperature is simply the drop temperature $T_{d}$. Thus, for example, the boundary layer temperature $T_{B L}=\left(T_{d}+T_{g}\right) / 2$, the average of the drop temperature and the gas temperature, $T_{g}$.

The aerosols and fission products removed by sprays, including the fission products attached to the aerosols, are all deposited into the pool, if present, of cell "iclout," specified in the ENGINEER input block (see Section 14.3.3). If a pool is not defined in that cell, the condensed vapor and any removed aerosols and fission products will be accounted for in WASTE repository of that cell.

The five collection mechanisms used in the modeling of the aerosol removal by spray droplets are as follows:

1. Interception, which occurs because the finite size of the particle permits its surface to contact that of the drop, even when the particle center of mass is on a trajectory that does not intersect the drop. 
2. Inertial impaction, which occurs because the particle has a finite inertia, leading the trajectory of the particle center of mass to cross the flow streamlines around the drop and thus intersect the surface of the drop.

3. Brownian diffusion, which results from molecular bombardment of the particles causing them to flow across the flow boundary layer around the drop.

4. Diffusiophoresis, which results as a response of the particle to concentration gradients and to vapor flow toward (or from) the drop surface when condensation on (or evaporation from) the drop is occurring.

5. Thermophoresis, which results from the migration of a particle down a temperature gradient because of the effect of differential molecular impacts.

Although these effects interact to some degree, they are treated as being additive. The first three effects are primarily a function of drop and particle size, while the phoretic effects are primarily a function of temperature and humidity of the atmosphere and of the drop's temperature. The latter changes rapidly at the start of the drop's fall through the atmosphere, and particle collection is therefore integrated over the drop's fall history. Under evaporating conditions, the diffusiophoretic effect becomes negative. The thermophoretic effect would also be negative in the unlikely circumstance that the drop were hotter than the atmosphere. In such cases, the total collection efficiency is still constrained to be non-negative. Under extreme conditions (such as those resulting from hydrogen burns), the drop may evaporate to aerosol size (the minimum of $100 \mu \mathrm{m}$ or the maximum aerosol diameter) during its fall. When this occurs, the drop and collected aerosols are added to the appropriate section of the aerosol distribution if water aerosols are defined. If water aerosols are not defined, then the collected aerosols are added to the appropriate section, and the water is treated as evaporated. Figure 7-5, taken from Reference Ber85a, presents collection efficiencies calculated for a typical accident scenario in which continuous spray operation brings the containment atmosphere to an approximate steady state.

The deposition rate equations below describe the five aerosol removal mechanisms. Note that the collection efficiency $E$ is defined by

$$
E=\frac{\Delta N_{p}}{\left(\pi D^{2} / 4\right) \Delta H n_{p}}
$$

where $\Delta \mathrm{N}_{\mathrm{p}}$ is the number of aerosol particles actually collected by a drop of diameter $\mathrm{D}$ as it falls a height $\Delta \mathrm{H}$ through an atmosphere containing $\mathrm{n}_{\mathrm{p}}$ particles per unit volume.

The following expressions for interception and impaction are based on Reference Fuc64. In the original expressions, there is no allowance for nonspherical aerosol particles. Therefore, an allowance for the agglomeration and dynamic shape factors ( $\gamma$ and $\chi$, respectively) has been made. However, it should be noted that the sprays keep the degree of superheat in the containment 


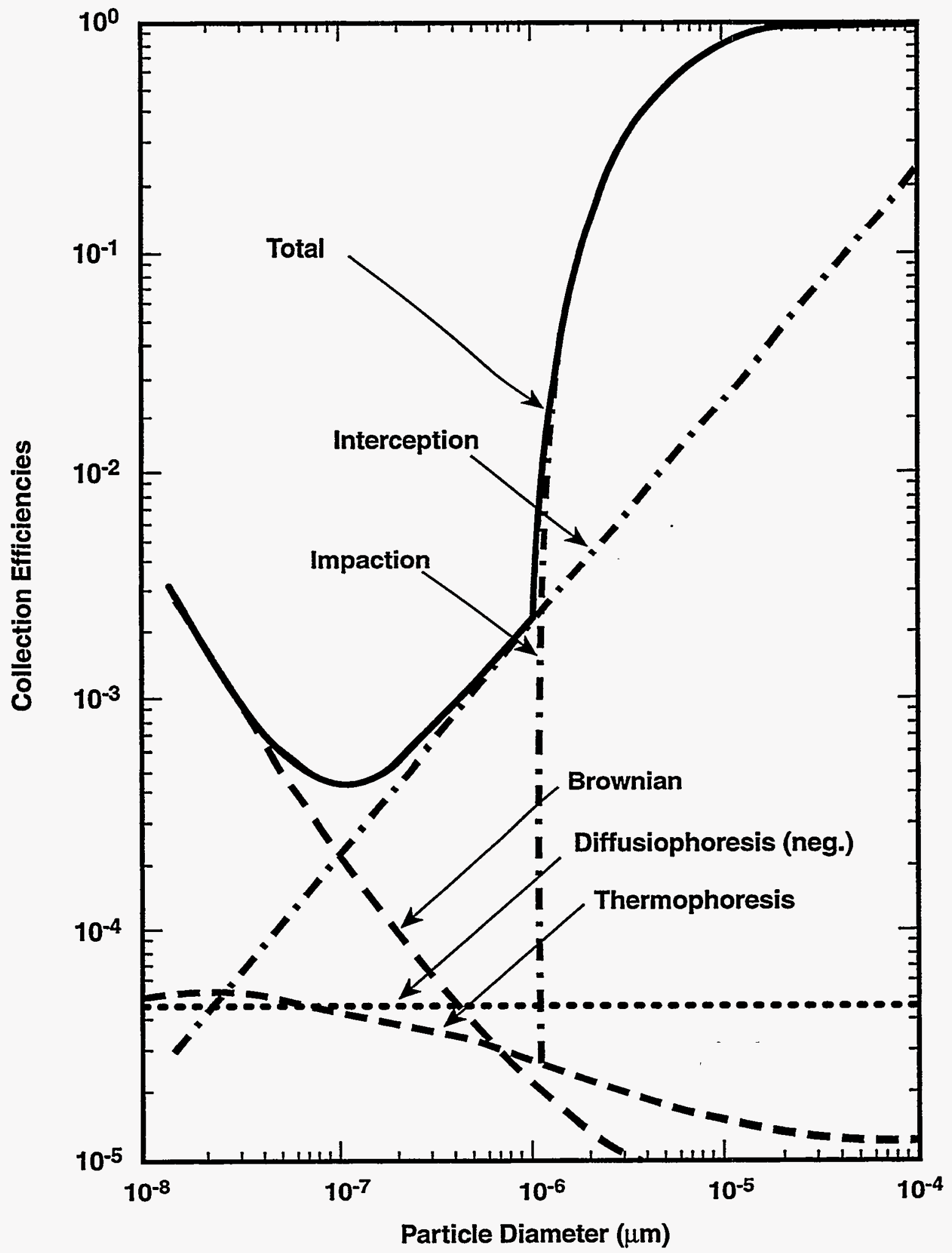

Figure 7-5. Qualitative Comparison of Aerosol Collection Efficiencies (Adapted from Reference Ber85a) 
atmosphere very small during most of the accident sequence. Under these conditions, water uptake is expected to lead to approximately spherical aerosol particles, with shape factors close to unity. Hence, the user should use the default values of unity for the shape factors in conjunction with spray modeling, except in those instances in which they are required to correct for the porosity of a particle.

\section{Interception}

Viscous flow:

$$
E_{\mathrm{I}, \mathrm{v}}=1.5\left(\gamma \mathrm{d}_{\mathrm{p}} / \mathrm{D}\right)^{2}
$$

Potential flow:

$$
E_{\mathrm{I}, \mathrm{p}}=3 \gamma \mathrm{d}_{\mathrm{p}} / \mathrm{D}
$$

where $E_{I, v}$ is the interception collection efficiency for viscous flow, $d_{p}$ is the particle diameter, $D$ is the spray drop diameter, and $E_{I p}$ is the interception collection efficiency for potential flow. Viscous flow efficiencies apply in the limit of Stokes flow around the spray drop, with a drop Reynolds number $N_{R e}<1$, while potential flow applies for $N_{R e} \gg 1$. An interpolation formula is provided for intermediate regimes as described later in this section.

\section{Inertial Impaction}

Viscous Flow:

$$
\mathrm{E}_{\mathrm{L}, \mathrm{v}}=0, \quad \text { for } \mathrm{N}_{\mathrm{st}} \leq 1.214
$$

and

$$
\mathrm{E}_{\mathrm{I}, \mathrm{V}}=\left[1+\frac{0.75 \ln \left(2 \mathrm{~N}_{\mathrm{St}}\right)}{\left(\mathrm{N}_{\mathrm{St}}-1.214\right)}\right] \text {, for } \mathrm{N}_{\mathrm{St}}>1.214
$$

where $E_{\mathbb{M}, v}$ is the inertial impaction collection efficiency for viscous flow; $N_{\mathrm{St}}$ is the Stokes number defined as

$$
N_{s t}=\frac{d_{p}^{2} \rho_{p} v_{d} C}{9 \mu_{g} D \chi}
$$

where $\rho_{\mathrm{p}}$ is the particle density, $v_{d}$ is the drop fall velocity, $C$ is the Cunningham slip correction factor defined with respect to Equation (7-17), and $\mu_{\mathrm{g}}$ is the gas viscosity, evaluated for boundary layer conditions. 
Potential Flow:

$$
E_{\mathbb{M}, \mathrm{p}}=0.0, \quad \text { for } \mathrm{N}_{\mathrm{St}} \leq 1 / 12
$$

and

$$
E_{\text {II, }}=\left(\frac{N_{S t}}{N_{S t}+0.5}\right)^{2}, \quad \text { for } N_{S t} \geq 0.2
$$

where $E_{\Pi_{\mathrm{p}}}$ is the inertial impaction collection efficiency for potential flow. Linear interpolation is used to obtain $\mathrm{E}_{\mathrm{I}, \mathrm{p}}$ for $1 / 12<\mathrm{N}_{\mathrm{St}}<0.2$.

For spray drop sizes in the range of interest, the Reynolds number based on spray drop diameter $\mathrm{N}_{\mathrm{Re}}$ ranges from about 15 to almost 1000 . Hence, $N_{R e}$ is expected always to be too large for the viscous flow relations to hold. However, it is not clear that $N_{R e}$ is large enough for the potential flow relations to provide a good approximation either, except perhaps for the larger drop sizes. In this intermediate regime, no simple expression is expected to be rigorously defendable. In early work, Reference Lan 48 suggested a simple interpolation formula as an approximation to the impaction efficiency.

$$
E_{\mathrm{II}}=\frac{E_{\mathrm{II}, \mathrm{v}}+N_{\mathrm{Re}} \mathrm{E}_{\mathrm{IM}} / 60}{1+N_{\mathrm{Re}} / 60}
$$

where $E_{\mathrm{TI}}$ is the interpolated impaction efficiency.

Equation (7-43) is used in CONTAIN. In addition, an equivalent interpolation formula is used for the interception efficiency. Little justification can be given for the latter, except to note that the interception efficiency is governed by the same flow patterns about the drop that govern the impaction efficiency; hence, the transition from the viscous flow limit to the potential flow limit might reasonably be expected to show a qualitatively similar dependence upon drop Reynolds number in both cases. Reference Wil87b includes sensitivity studies evaluating some implications of these and other uncertainties in the spray drop collection efficiencies.

Brownian Diffusion

$$
E_{\text {diff }}=\frac{4 \bigoplus_{p}\left(2+0.6 N_{R e}^{1 / 2} N_{S c, p}^{1 / 3}\right)}{v_{d} D}
$$

where $E_{\text {diff }}$ is the Brownian diffusion collection efficiency; $\oplus_{p}$ is the diffusivity of the particle as given in Equation (7-17), but evaluated for boundary layer conditions; $N_{s c, p}=\mu_{g} / \rho_{g} \boxplus_{p}$ is the particle Schmidt number; and $\mathrm{D}$ is the drop diameter. This expression is equivalent to assuming that the Sherwood number for particles is the same as that for vapor and that the mass transfer coefficient for particles can be defined analogously to the vapor mass transfer coefficient with the vapor 
diffusivity being replaced by the particle diffusivity. Details of the treatment for the vapor are given in Section 12.3.

Thermophoresis

$$
E_{\text {therm }}=\frac{4 C_{t h}\left(2+0.6 N_{R e}^{1 / 2} N_{P_{r}}^{1 / 3}\right)\left(T_{g}-T_{d}\right)}{v_{d} D}
$$

where

$$
\mathrm{C}_{\mathrm{th}}=\frac{2.294 \mu_{\mathrm{g}} \mathrm{C}\left(\mathrm{C}_{\mathrm{t}} \mathrm{N}_{\mathrm{Kn}}+\mathrm{k}_{\mathrm{g}} / \mathrm{k}_{\mathrm{p}}\right)}{\chi \rho_{\mathrm{g}} \mathrm{T}_{\mathrm{BL}}\left(1+3 \mathrm{C}_{\mathrm{m}} \mathrm{N}_{\mathrm{Kn}}\right)\left(1+2 \mathrm{C}_{\mathrm{t}} \mathrm{N}_{\mathrm{Kn}}+2 \mathrm{k}_{\mathrm{g}} / \mathrm{k}_{\mathrm{p}}\right)}
$$

Here, $\mathrm{N}_{\mathrm{pr}}$ is the Prandtl number, $\mathrm{C}$ is the Cunningham slip correction given in Equation (7-17), $N_{K n}=2 \lambda / d_{p}$ is the Knudsen number, where $\lambda$ is the molecular mean free path, $\chi$ is the dynamic shape factor, and $\mathrm{k}_{\mathrm{g}} / \mathrm{k}_{\mathrm{p}}$ is the user-specifiable ratio of the gas thermal conductivity to the particle conductivity. The constants $\mathrm{C}_{\mathrm{m}}$ and $\mathrm{C}_{\mathrm{l}}$ are related to slip and thermal accommodation, respectively (see Equation (7-19)).

\section{Diffusiophoresis}

$$
E_{\text {diph }}=4 \bigoplus_{v}\left(2+0.6 N_{R e}^{1 / 2} N_{S c}^{1 / 3}\right) \frac{M_{w}^{1 / 2} \ln \left(\frac{P_{g}-P_{v, d}}{P_{g}-P_{v}}\right)}{\left(X_{v} M_{w}^{1 / 2}+X_{n c} M_{n c}^{1 / 2}\right) V_{d} D}
$$

In Equation (7-46), $\boxplus_{v}$ is the boundary layer vapor diffusivity, $N_{S c}=\mu_{g} / \rho_{g} \bigoplus_{v}$ is the Schmidt number; $P_{g}$ is the atmosphere pressure; $P_{v, d}$ is the vapor pressure at the drop surface, $P_{v}$ is the bulk vapor pressure; $X_{v}$ is the boundary layer vapor mole fraction; $M_{w}$ is the molecular weight of water; $X_{n c}$ is the boundary layer noncondensable gas mole fraction; and $M_{n c}$ is the molecular weight of the noncondensable gas.

The expression given in Equation (7-45) for the thermophoretic collection efficiency is based upon the expression given in Equation (7-19) for thermophoretic deposition upon structures together with the temperature gradients at the drop surface implied by the heat transfer and Nusselt number relations given for the drop in Section 12.3. Likewise, the diffusiophoresis expression is given by combining the treatment of diffusiophoretic deposition on structures given in Equation (7-20) with the condensation/evaporation rate calculated for the drop as described in Section 12.3. The phoretic effects can be negative under certain conditions; for example, the diffusiophoretic effect is negative when the drop is evaporating. In such cases, the phoretic effects are still evaluated from Equations (7-45) and (7-46) and added algebraically to the other collection efficiencies. The total collection efficiency is constrained to be non-negative, however. 
The efficiency $\mathrm{E}$ of a spray drop changes during the fall. Therefore, the contribution of sprays to the aerosol removal rate coefficient $\Re$ is obtained by integrating over the drop fall height $\mathrm{H}$ under the assumption that the effect of any one spray drop over its fall is instantaneous:

$$
\Re=\frac{1}{4} \dot{\mathrm{n}}_{\mathrm{d}} \int_{0}^{\mathrm{H}} \mathrm{A}_{\mathrm{d}} \mathrm{EdH}
$$

In the above equation, $\dot{n}_{d}$ is the number density rate (per unit volume of atmosphere) at which spray drops are introduced to the atmosphere, $A_{d}$ is the drop spherical surface area, and $E$ is given by

$$
E=E_{I}+E_{\text {II }}+E_{\text {diff }}+E_{\text {therm }}+E_{\text {diph }}
$$

\subsection{Aerosol Scrubbing Models}

The process of venting gas/aerosol mixtures under the surface of a coolant pool, as in SRV operation, gives rise to scrubbing, or removal from the gas phase, of some of the aerosols in the gas bubbles rising in the pool. In CONTAIN two models are available for aerosol scrubbing; one is a model taken from the VANESA code, [Pow86] which is referred to in CONTAIN as SCRUB, and the other is an adaptation of the SPARC code. [Owc85a] These models are used for the SPVENT dedicated suppression pool vent flow model described in Section 11.1.2 and the SRVSOR SRV model described in Section 11.2. The SCRUB model is also used in conjunction with the modeling of gas/aerosol releases within the CORCON/VANESA model for CCIs, as discussed in Chapter 5, when the core debris surface is submerged below the coolant pool surface. Both the SCRUB and SPARC models have been modified slightly for integration into CONTAIN. A different approach is used for gas/aerosol mixtures exiting the downstream end of a gas flow path when it is under the pool surface. In this case the aerosols are assumed to be completely removed from gas phase. This assumption of complete removal is made only for convenience. It is expected that models similar to those for the dedicated suppression pool vent model will at some point be made available for gas flow path venting. The aerosols that are scrubbed out are deposited in the pool, with the remainder passing to the atmosphere. Fission products hosted by the aerosol materials that are scrubbed out are also deposited in the pool.

It should be noted that fission products associated with the atmosphere gas (as opposed to an aerosol component) are subject to complete removal by the coolant pool whenever the gas is vented under the pool surface. In contrast to the treatment of aerosol scrubbing, this applies both to the gas flow paths and to the dedicated suppression pool vent model. The targeted release and acceptance formalism may be useful in simulating fission product equilibrium concentrations (such as for iodine) over the pool surface in this case. (See Section 8.4.)

Both the SCRUB and SPARC models determine an overall decontamination factor $\mathrm{DF}_{\mathrm{i}}$ (i.e, the ratio of incoming mass to outgoing mass) for each of the CONTAIN aerosol size classes $i$ on the basis of gas bubble dynamics. The decontamination factors returned from these models are taken to lie in the range between 1 and $10^{5}$. The upper limit is an attempt to account for effects not modeled, such as resuspension because of bubble breaking at the pool surface. Coolant vapor evolution from the 
bubble wall during bubble rise can affect the decontamination factor. Note that these models make their own approximations for vapor equilibration in rising bubbles; in particular, the gas-pool equilibration modeling discussed in Sections 4.4.7 and 11.2.1, for the purposes of calculating thermal-hydraulic transfer rates, does not necessarily apply.

The SCRUB modeling, with default parameters, is used by default within the SPVENT and SRVSOR models, as discussed in Sections 14.2.4.3 and 14.3.4, respectively. The SPARC model is an alternative choice within these two models. In contrast, to activate the SCRUB model for use by the CORCON model, the SCRUB input block must appear explicitly in the CORCON input. If SCRUB is not specified in the CORCON input block, all of the vented aerosols will be placed directly in the atmosphere. The SPARC model is not available for use by the CORCON model.

\subsubsection{The SCRUB Model}

The SCRUB model was originally developed for the VANESA code, which has now been fully integrated in the CORCON Mod3 code. [Bra93] This integrated code has in turn been incorporated as a module of CONTAIN, as discussed in Chapter 5. The SCRUB model is a direct application of Fuchs' treatment [Fuc64] of the processes of sedimentation, impaction, and diffusion in spherical bubbles. It is assumed that the bubble is filled with an ideal gas, which expands as the bubble rises. The effects of coolant vapor evaporating from the bubble wall during the rise are not taken into account. The submergence of the vent is used as the scrubbing depth in the SPVENT and SRVSOR options, whereas the full pool depth is used as the scrubbing depth for the CORCON module. If the submergence or pool depth is zero, no scrubbing will occur. Differences in the CONTAIN implementation and the documentation of the scrubbing model for CORCON Mod3 are discussed below. For further details of the model, the reader is referred to Reference Bra93.

The SCRUB model has been modified for CONTAIN through the addition of a size-independent decontamination factor $\mathrm{DF}_{0}$ that takes into account steam condensation in bubbles at the inlet. This calculation is identical to that used in the SPARC code. $\mathrm{DF}_{0}$ has the form

$$
\mathrm{DF}_{0}=\frac{\left(\mathrm{P}_{\mathrm{H}}-\mathrm{P}_{\mathrm{s}}\right) \mathrm{M}_{\mathrm{nc}} \mathrm{W}_{\mathrm{g}}}{\mathrm{M}_{\mathrm{g}} \mathrm{P}_{\mathrm{H}} \mathrm{W}_{\mathrm{nc}}}
$$

where $P_{H}$ is the pressure at the injection elevation $H$ within the pool, $P_{s}$ is the pool saturation pressure, $M_{n c}$ is the average molecular weight of the noncondensable gas, $W_{g}$ is the total gas and vapor mass inflow rate, $M_{g}$ is the average molecular weight of the gas inflow, and $W_{n c}$ is the noncondensable gas inflow rate.

In the SCRUB input block (see Section 14.2.4.3 or 14.3.4), the user may specify the initial bubble diameter "bsizi," which is defaulted to $1 \mathrm{~cm}$, and the input parameter, "vrovr," the ratio of the gas circulation velocity to bubble rise velocity. The latter parameter controls the scrubbing efficiency arising from impaction. The default value of "vrovr" of 1 corresponds to the value for a spherical bubble. If desired, the user may specify a larger value (for example, to simulate elliptical bubbles 
with enhanced scrubbing) or a smaller value (for example, to simulate the effect of surface impurities which inhibit circulation).

\subsubsection{The SPARC Model}

The SPARC code, [Owc85a] developed at Pacific Northwest Laboratories, is a detailed scrubbing model that attempts to mechanistically treat a number of processes. As implemented, that code extends Fuchs' model explicitly to elliptic bubbles, treats deposition caused by initial steam condensation, and includes sedimentation, diffusion, and inertial deposition in rising bubbles. It mechanistically accounts for bubble growth and the deposition limiting effects of vapor evolution during bubble rise. Details of the model can be found in Reference Owc85a. Several options in the original code are not implemented as they are either not recommended by the developers or not compatible with CONTAIN models. Particle growth because of condensation, bubble interior heat transfer, and particle solubility effects are examples of models which are either incompatible or not recommended. The pool equilibrium temperature calculation is also not implemented as it conflicts with the CONTAIN pool calculation.

This model is activated by including the SPARC block in the SPVENT or SRVSOR input block. The initial bubble diameter, "bsiz," and the ratio of the major axis to minor axis of a spheroid bubble, "ratio," may be specified as described in Section 14.2.4.3 or 14.3.4.

\subsection{Intercell Flow of Aerosols}

This section describes the transport of airborne aerosols in gas flow paths and in the dedicated suppression pool vent flow path. It also implicitly describes the flow of fission products associated with aerosol component hosts, since such fission products are assumed to flow between cells in proportion to the aerosol component. The intercell flow of aerosols is in general size-dependent. For unsubmerged gas flow paths, it is often sufficient to characterize aerosol flow as occurring without slip, in proportion to the gas flow in the flow path. However, if the user has specified through the VCOSN keyword that aerosol settling be treated in an unsubmerged flow path, the aerosol flow will no longer be simply proportional to the gas flow and will depend on size.

As discussed in Section 7.7, aerosol scrubbing is modeled for submerged gas flow paths and a submerged suppression pool vent flow path. For gas flow paths an infinite decontamination factor, or DF, is used. For the suppression pool vent path, the detailed SPARC or SCRUB aerosol scrubbing models may be used to define the DF. However, such models, as well as the VCOSN option for modeling aerosol settling through flow paths, should be used judiciously with CONTAIN 1.2 and later versions because the modeling of size-dependent flow processes introduces the necessity of tracking aerosols by size class within the implicit flow solver and adds considerably to the overhead of the solver.

Within the implicit flow option, the user may specify, through the VCOSN keyword, the cosine of angle with respect to vertical of flow paths for the purpose of calculating gravitational settling effects in flow paths. If VCOSN is specified, aerosols are considered to flow at the sum of the gas velocity and the component of the aerosol gravitational settling velocity along the flow path axis. Note that 
the effects of the perpendicular component of the settling velocity, leading in some flow geometries to deposition within the path, are not modeled. In the explicit flow option, aerosol slip or decontamination effects are not considered.

The time-dependent evolution of aerosol masses is calculated in two steps. First, the effects of aerosol agglomeration, deposition, and condensation are calculated within a cell, neglecting the effects of flow. Second, the effects of flow on the airborne aerosol mass $m_{a, n, j, k}$ of component $k$ in section $i$ and cell $n$ are then calculated from

$$
\left[\frac{d m_{a, n, j, k}}{d t}\right]_{\text {flow }}=\sum_{j n}\left(v_{a, j, i} A^{\prime}{ }_{j n} \frac{m_{a, u, i, k}}{V_{u}}\right) F_{j n, i}
$$

where the sum extends over all gas flow paths connecting cell $\mathrm{j}$ to $\mathrm{n}$ and the suppression pool vent path, if present; $A_{j n}^{\prime}$ is the effective flow path area as defined in Table 4-2; $u$ denotes the upstream or donor cell; $\mathrm{V}_{\mathrm{u}}$ is the cell free volume of donor cell $\mathrm{u}$; and $\mathrm{F}_{\mathrm{i}, \mathrm{j}}$ is the attenuation factor for the $\mathrm{jn}$ path, which depends on flow direction. The aerosol velocity $v_{a j n, i}=v_{g, j n}-v_{s, j, i}$ is equal to the gas velocity $\mathrm{v}_{\mathrm{gjn}}$ minus the aerosol gravitational settling terminal velocity $\mathrm{v}_{\mathrm{s}, \mathrm{j} \mathrm{i}, \mathrm{i}}=\mathrm{v}_{\mathrm{s}, \mathrm{i}} \cos \theta_{\mathrm{j} n}$. In the latter expression, $\mathrm{v}_{\mathrm{s}, \mathrm{i}}$ is the settling velocity as defined in Equation (7-17), but logarithmically averaged over the particle diameters in size class $i$, and $\cos \theta_{\mathrm{jn}}$ is the cosine of the angle of the flow path with respect to vertical. This cosine is set through the VCOSN keyword for a gas flow path within the implicit flow option and is zero for a gas flow path by default or for the suppression pool vent path or within the explicit flow option. The attenuation factor $F_{\mathrm{ja}, \mathrm{i}}$ is set to 1 for outflows or for inflows from gas flow paths or the suppression pool vent flow path, if they are not submerged; is set to infinity for inflows through a submerged gas flow path; and is determined by the DFs calculated in the SPARC or SCRUB scrubbing model for inflows through a submerged suppression pool vent flow path $(F=1 / D F)$. The aerosols removed from the gas flow for an smaller than unity are placed, along with any associated fission products, in the coolant pool in the downstream cell.

The user should be aware that problems may arise with respect to the problem splitting that is used to handle aerosol agglomeration, deposition, and condensation on the one hand and the intercell flow of aerosols on the other. To account for the effects of aerosol agglomeration, deposition, and condensation (in the case of the fixed-grid option), a Runge-Kutta method, with automatic timestep control, is used. This calculation disregards flow. To account for the effects of flow, aerosols are redistributed with the atmosphere gases every flow timestep. During the redistribution process, the aerosol distribution is assumed not to evolve except through the flow process. The flow of the atmosphere gases is calculated with automatic timestep control. The aerosol distributions from each of these separate calculations are updated every system timestep. However, the timestep controls for each calculation individually may not ensure that the coupled problem is described adequately. The user is warned that no check is made on the adequacy of the update interval. In general, the user should check the sensitivity of the calculation with respect to the system timestep whenever both aerosol processes within a cell and intercell flow result in large effects on the aerosols within a cell during a timestep. 
As an example of difficulties that may be encountered in a calculation with too large a system timestep, the code may predict that the aerosols agglomerate and settle rapidly in a cell with a large source of aerosols before they have a chance to flow out of the cell. A more accurate calculation may predict that the aerosols may flow out of that cell before significant agglomeration occurs. Because of dilution effects in the other cells, agglomeration and settling may not be as rapid in those cells, and the total aerosol deposition may be significantly less. 


\subsection{FISSION PRODUCT BEHAVIOR MODELS}

\subsection{Introduction}

In a reactor accident, a principal concern is the risk associated with potential release of radionuclides from the containment system to the outside environment. This section describes the fission product behavior models used in CONTAIN to estimate this potential source term. The term "fission product" is used to represent all radionuclides, including actinides and other neutron activation products.

The fission product behavior modeled in CONTAIN includes radionuclide decay, decay heating, atmosphere transport processes, transport in liquid pathways, iodine scrubbing, release of fission products from hosts, and the release of fission products during core-concrete interactions. All of these processes, except for some aspects of decay heating and the last process, are discussed in the present chapter. With respect to decay heating, the heating associated with explicitly specified fission products is discussed here. However, an ANSI-standard model to describe the decay heat of fission products implicitly assumed to be present in lower cell layers is also available and is discussed in detail in Section 5.6.1. The modeling of the release of fission products (and other types of nongaseous materials) during core-concrete interactions is done within the VANESA model, which is fully integrated into the CORCON-Mod3 package for modeling such interactions. The VANESA model is therefore discussed in Chapter 5 along with the other CORCON Mod3 modeling. Key elements of fission product behavior models are illustrated in Figure 8-1.

In CONTAIN, a fission product can be either an individual radionuclide with a specific role within some decay process or a class of radionuclides. A class may be defined as an arbitrary group of radionuclides, provided each radionuclide in the class is distinct from any individually specified radionuclide and not part of any other class. Classes are defined in the input like individual radionuclides, but the effects of decay of the radionuclides in the class may be modeled only through a time-dependent specific decay power. It should be noted that a class is what was called a group in prior code versions. However, to avoid confusion the word group will be reserved for the predefined volatility groups defined in Table 8-1. A group, in contrast with a class, consists of radionuclides that are also defined individually or as part of a class. Each predefined group must consist of the radionuclides listed for each group in Table 8-1, to the extent that the listed radionuclides are included as individual radionuclides within a calculation, but may also contain any additional user-specified fission products the user explicitly assigns to the group. Such groups may be referenced in the host release model (i.e., the parametric targeted release and acceptance model discussed in Section 8.4). The user has the option to specify the release and acceptance parameters on the basis of groups, rather than individual fission products. Since the number of fission products in a calculation could be quite large, the use of groups could streamline the release and acceptance input considerably. The user also has the option to request the output of fission product inventories on the basis of groups, through the PRFPGRP keyword.

CONTAIN depends on input from the user for the initial inventory and location of fission products. If external sources of fission products are present, the fission products involved, the source rates at the point of introduction to the calculation, and the source locations must also be specified by the 


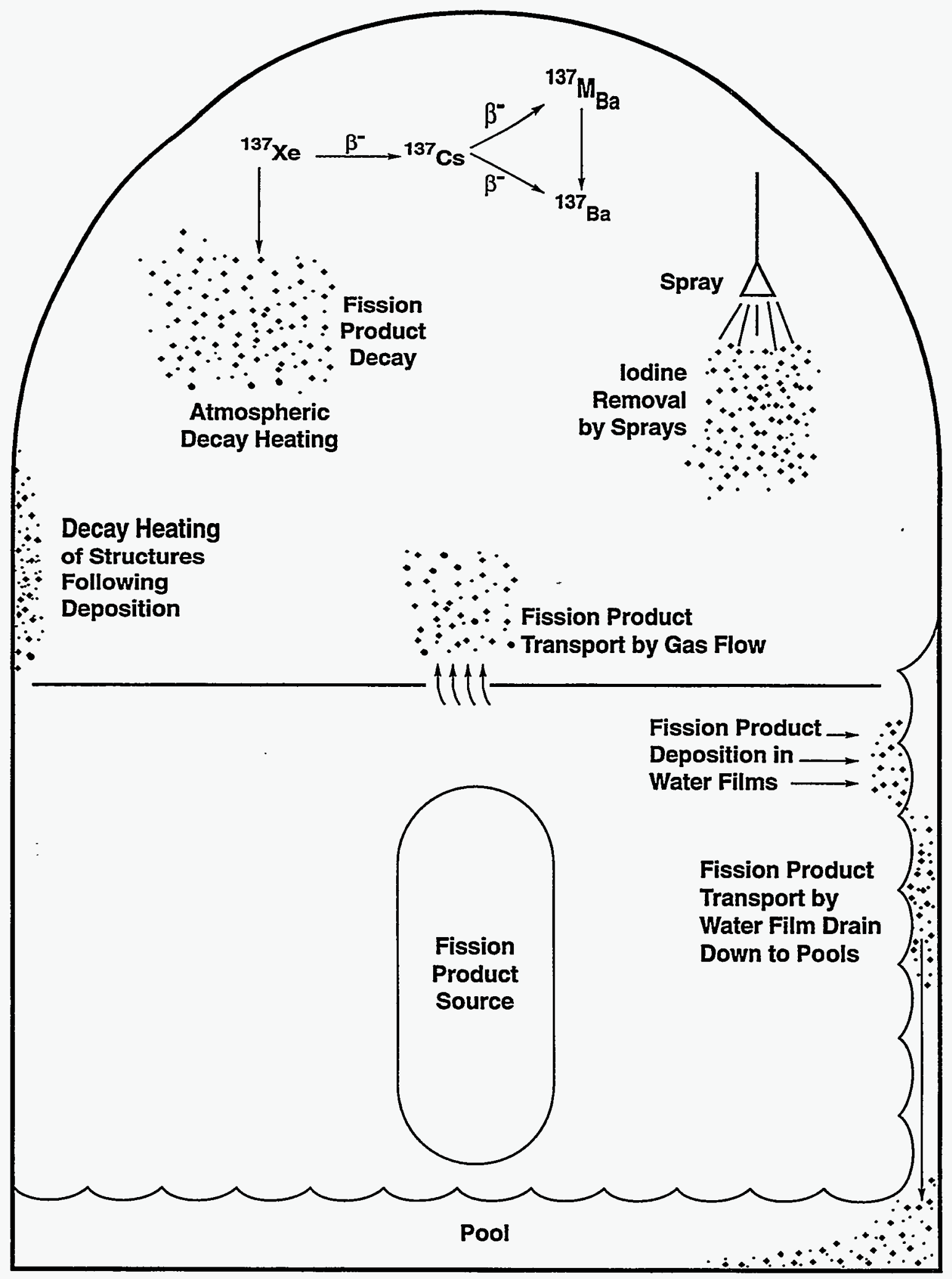

Figure 8-1. Fission Product Behavior as Modeled in CONTAIN 
Table 8-1

Makeup of Volatility Groups

\begin{tabular}{|c|c|}
\hline Group 1: Noble Gases (GROUP1) & $\begin{array}{l}\text { HE3}^{*}, \text { KR83M, KR83 } \\
\text { KR87, KR85M, KR85, } \\
\text { XE131 }{ }^{*}, \text { XE132 }{ }^{*}, \text { XE133M, XR90, XE131M, } \\
\text { XE135M, XE135, XE137, XE138, XE140 }\end{array}$ \\
\hline Group 2: Halogens (GROUP2) & $\begin{array}{l}\text { BR83, BR85, BR87, I127*, I129* I131, } \\
\text { I132M, I132, I133M, I133, I134M, I134, I135 }\end{array}$ \\
\hline Group 3: Alkali Metals (GROUP3) & 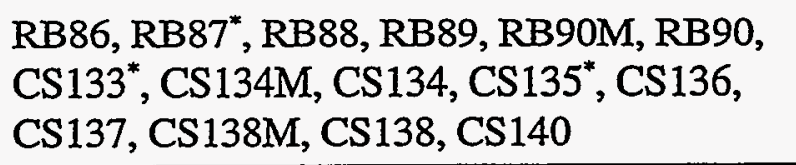 \\
\hline Group 4: Tellurium Group (GROUP4) & $\begin{array}{l}\text { SB125, SB127, SB129, SB131, TE125M, } \\
\text { TE125*, TE127M, TE127, TE129M, TE129, } \\
\text { TE131M, TE131, TE132, TE133M, TE133, } \\
\text { TE134 }\end{array}$ \\
\hline Group 5: Strontium Group (GROUP5) & SR86*, SR88*, SR89, SR90, SR91, SR92 \\
\hline Group 6: Noble Metals (GROUP6) & $\begin{array}{l}\text { TE58*, CO58, CO60M, CO60, N160*, } \\
\text { MO95*, MO97*, MO99, TC99M, TC99, }^{*} \text { RU99*, RU103, RU105, RU106, RH103M, } \\
\text { RH103*, RH105M, RH105, PD105*, RH106, } \\
\text { PD106* }\end{array}$ \\
\hline Group 7: Rare Earth Metals (GROUP7) & $\begin{array}{l}\text { Y89*, Y90, Y91M, Y91, Y92, Y93,ZR90*, } \\
\text { ZR91* }^{*} \text { ZR92*, ZR93*, ZR95, ZR97, NB95M, } \\
\text { NB95, NB97M, NB97, LA139*, LA140, } \\
\text { LA141, LA142, PR141*, PR143, PR144M, } \\
\text { PR144, PR147, ND143*, ND144*, ND147, } \\
\text { PM147, SM147* }\end{array}$ \\
\hline $\begin{array}{l}\text { Group 8: Rare Earth Metals (Actinides) } \\
\text { (GROUP8) }\end{array}$ & $\begin{array}{l}\text { CE140*, CE141, CE142, CE143, CE144, } \\
\text { PU239*, PU241, NP239, AM241* }\end{array}$ \\
\hline Group 9: Barium Group (GROUP9) & $\begin{array}{l}\text { BA134*, BA136*, BA137M, BA137*, } \\
\text { BA138*, BA139, BA140, BA141 }\end{array}$ \\
\hline Group 10: Reactor Specific (GROUP10) & $\mathrm{H3}$ \\
\hline
\end{tabular}

"End-product of decay chain. 
user. From this information, the code tracks the birth and decay rates of each individually specified radionuclide, and also accounts for the decay heating associated with the fission products. It should be noted that only beta and gamma decay processes should in general be explicitly included, because CONTAIN assumes that a decay results in no change in atomic weight. Alpha decay processes in the containment environment are sufficiently slow that they (but not the parent inventories) can be neglected during the period of a typical containment calculation. For calculations in which such processes cannot be neglected, the user may wish to include them and adjust the reported inventories for the included alpha masses.

For some studies of reactor accident scenarios, identifying and specifying the large number of decay processes and the radionuclides involved could be tedious. To alleviate this problem, a number of decay processes and radionuclides may be defined by invoking an extensive fission product data library, which provides decay information for 140 radionuclides. The decay processes considered are given in Figure 8-2, and the radionuclides involved are given in Table 8-1. The decay information available from the library is discussed in Section 8.2.

In order to model the decay process efficiently, CONTAIN uses a linear chain decomposition of the decay processes involved, as discussed in detail in Section 8.3. If the user invokes the fission product library with respect to one or more of the decay processes in Figure 8-2, the necessary information to characterize the selected decay processes is automatically loaded. For those decay processes not taken from the library, the user must input the linear chain parent-daughter relationships, inventory factors, specific powers, and half-lives of the fission products involved. It should be noted that fission product classes are treated in the input like individual radionuclides, except that the decay chains should be of length one, the half-lives set very large, and the inventory factors equal to one. The time-dependent specific decay power the user may specify for a class is typically obtained as the best curve fit through the total decay power of all radionuclides associated with the class. As discussed in Section 8.3, inventory, or distribution, factors may be required when a given radionuclide appears more than once in the linear chain decomposition. It should be noted that the use of inventory factors is new to the present code version. In prior versions, the user was required to specify directly the initial masses and source rates associated with each radionuclide occurrence within the linear chain decomposition. Each such occurrence constitutes a different "fission chain element," even though the same radionuclide is involved. For example, if all decay processes stored in the library were invoked, one would be dealing with 257 fission chain elements, taken from a set of 140 radionuclides. Consequently, the input of the chain element masses could be considerably more cumbersome than input of the radionuclide masses.

As discussed in Section 8.4, fission products in CONTAIN are associated with various "hosts," or repositories. A host can be the atmosphere gas, an aerosol component, the surface of a heat structure, or a lower cell layer or pool. Some hosts, such as the upper cell atmosphere gas or aerosols are mobile, while others, such as the wall surfaces, are fixed. In general, the location of fission products is specified by the user according to the host. Fission products are transported according to the movement of the mobile hosts. Fission product masses, as treated by CONTAIN, do not influence the dynamics of the mobile hosts, except through possible heating effects. In effect, fission products are treated as having no dynamic mass. For some hosts, such as a minor aerosol component, the fission product mass may constitute an important fraction of the mass associated 
No.1 $3_{\mathrm{H}} \stackrel{\beta^{-}}{\longrightarrow} 3_{\mathrm{He}}$

No.2 $\underbrace{58_{\mathrm{Fe}}} \underbrace{\mathrm{EC}}_{\mathrm{Co}}$

No. 3

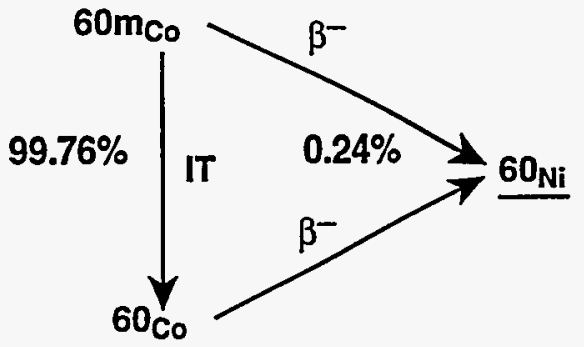

No. 4

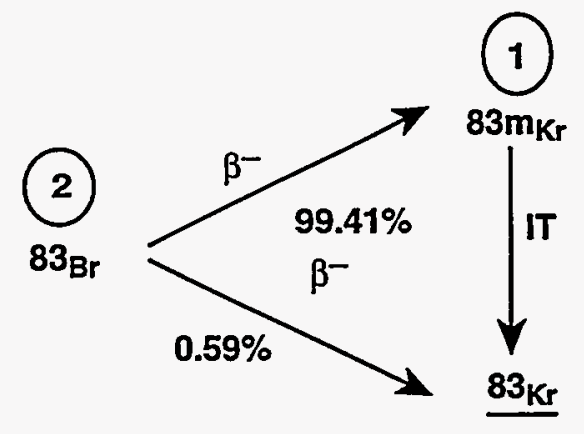

No. 5

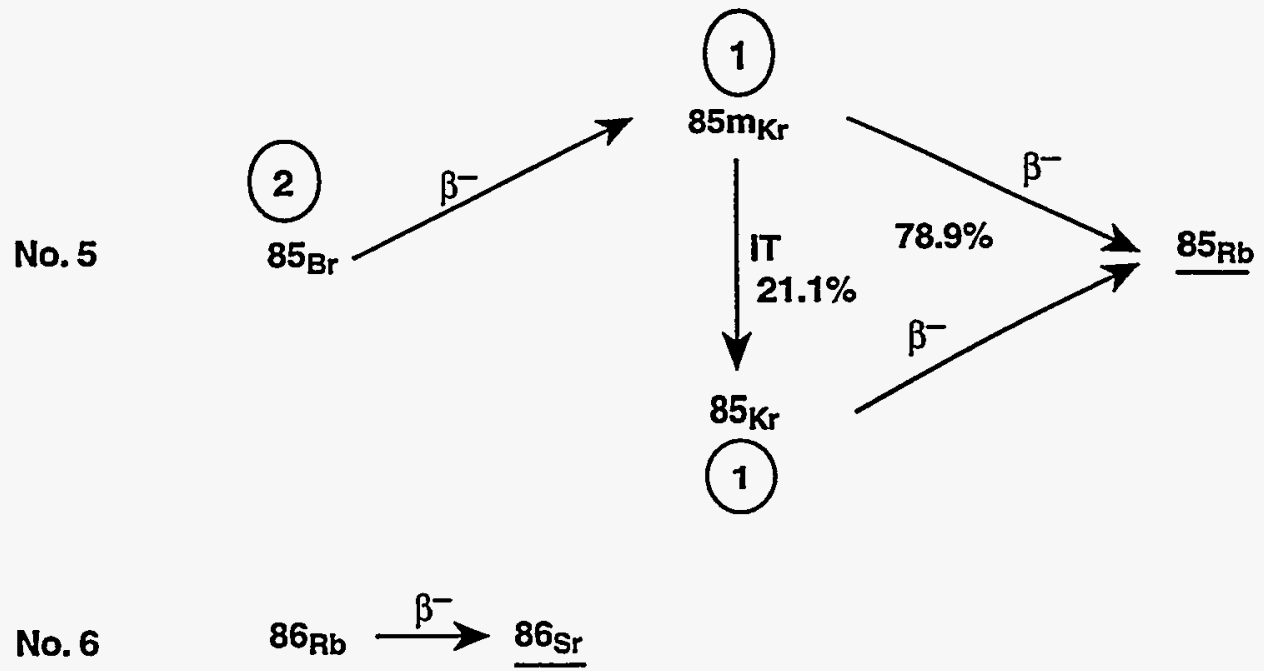

Figure 8-2. Decay Processes in the Fission Product Library. Note that the underlined radionuclides are either stable or subject to $\alpha$ decay with a relatively long half-life. Also, the circles denote the group numbers involved in a cross-group process. 
(2) (1)

No.7 $87_{\mathrm{Br}} \stackrel{\beta^{-}}{\longrightarrow} 87_{\mathrm{Kr}} \stackrel{\beta^{-}}{\longrightarrow}$ 87

(1) 3

No. $8 \quad 88_{\mathrm{Kr}} \stackrel{\beta^{-}}{\longrightarrow} 88_{\mathrm{Rb}} \stackrel{\beta^{-}}{\longrightarrow} 88_{\mathrm{Sr}}$

No. 9

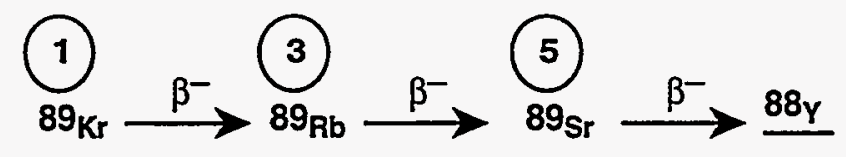

No. 10

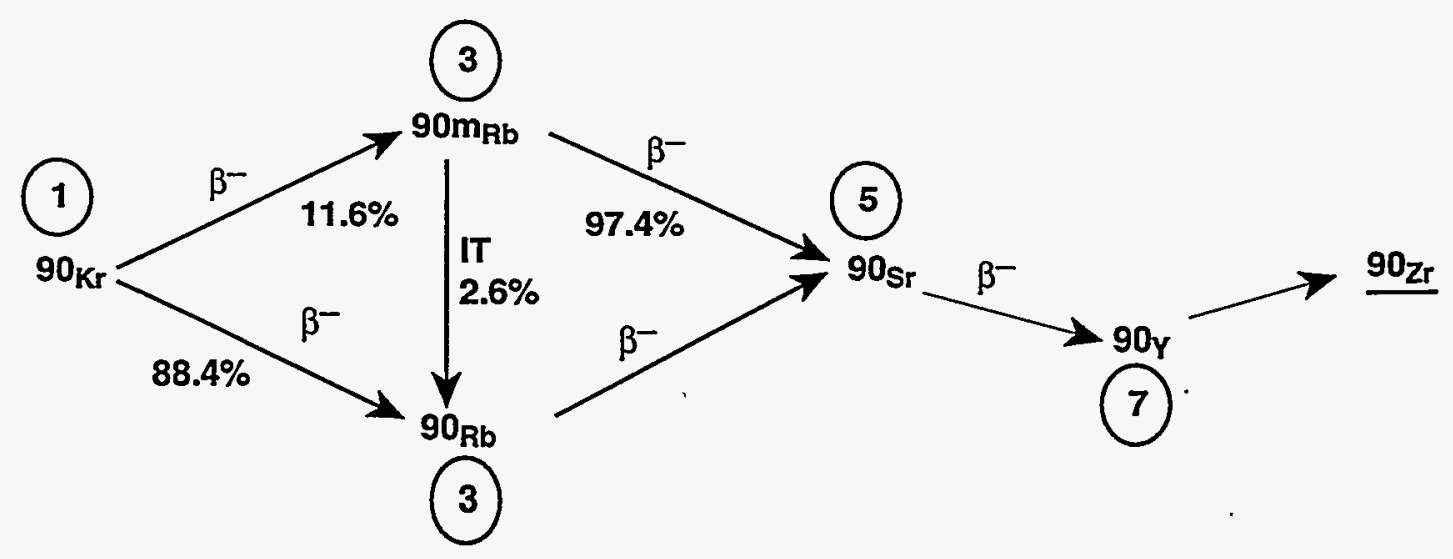

No. 11

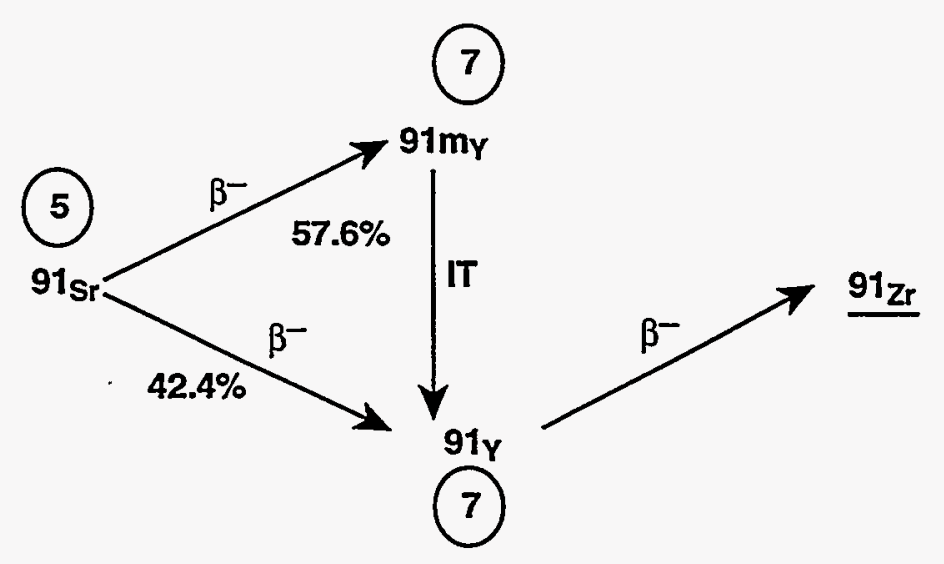

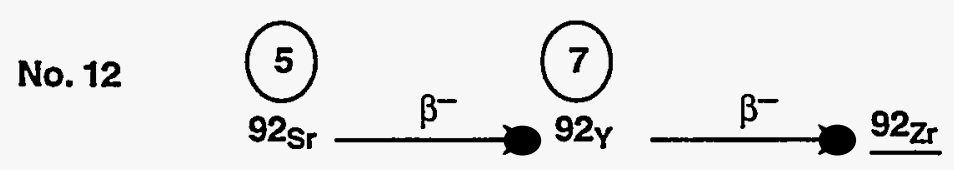

No.13 $93 \mathrm{Y} \stackrel{\beta-}{\longrightarrow} 93_{\mathrm{Zr}}$

Figure 8-2. Decay Processes in the Fission Product Library (Continued) 
No. 14

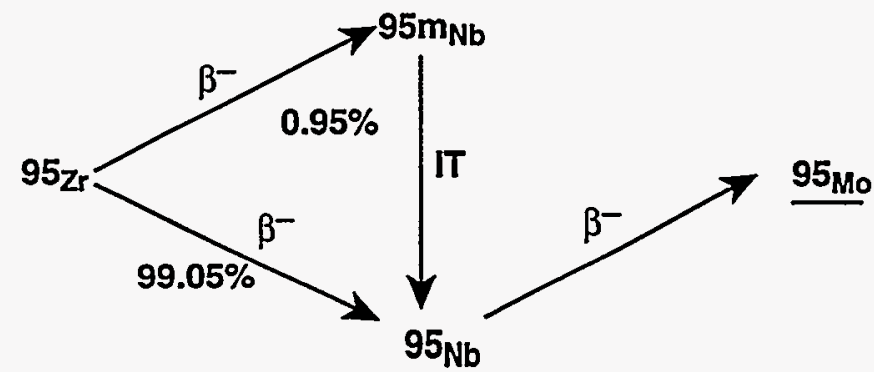

No. 15

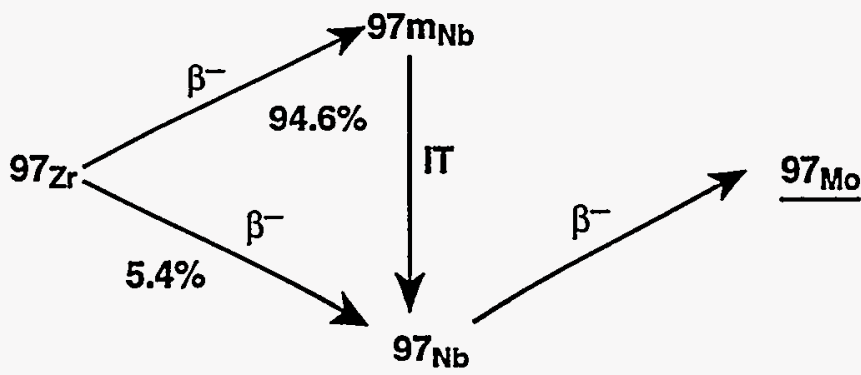

No. 16

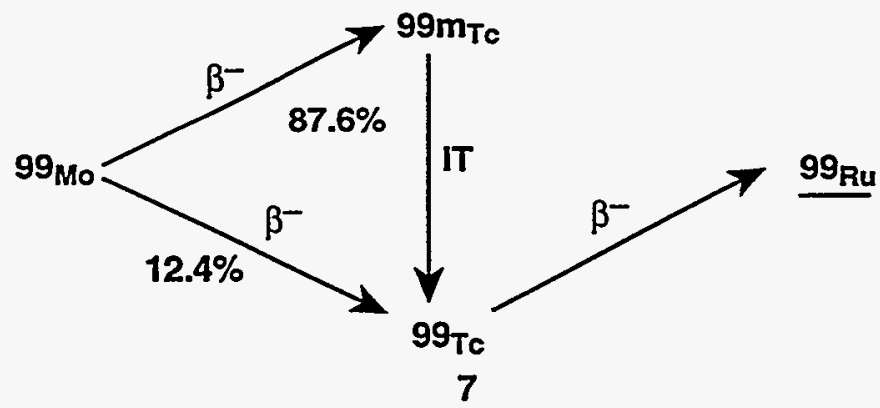

No. 17

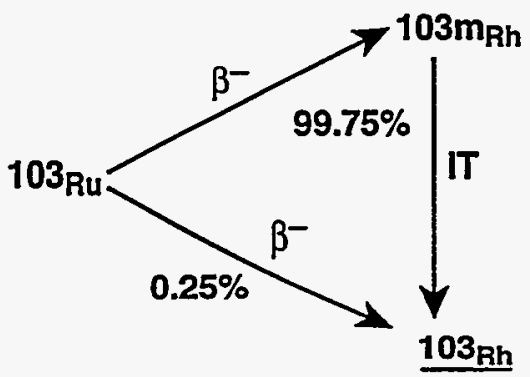

No. 18

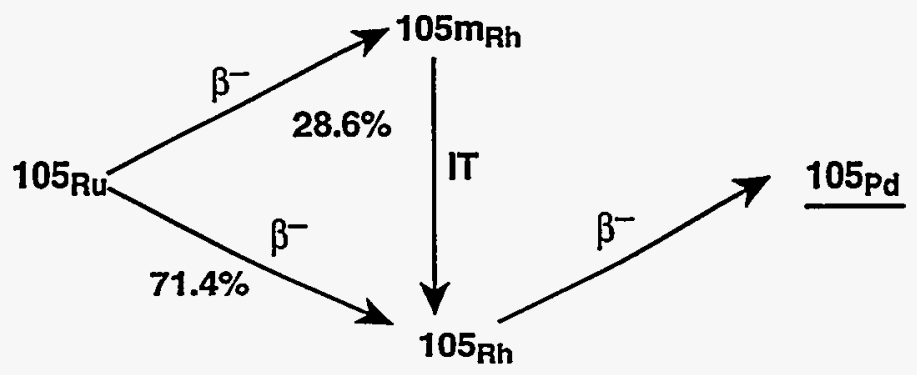

Figure 8-2. Decay Processes in the Fission Product Library (Continued)

Rev. 0 
No. $19 \quad 106_{R u} \stackrel{\beta^{-}}{\longrightarrow} 106_{R h} \stackrel{\beta^{-}}{\longrightarrow} 106_{P d}$

No. 20

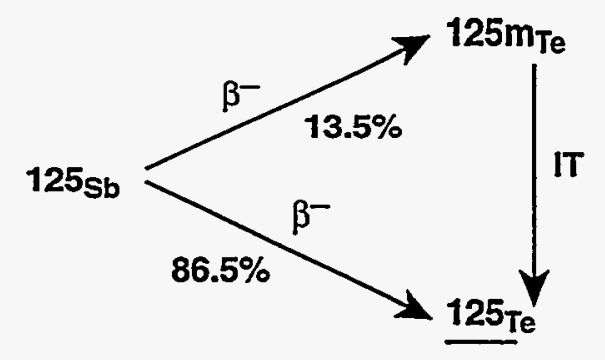

No. 21

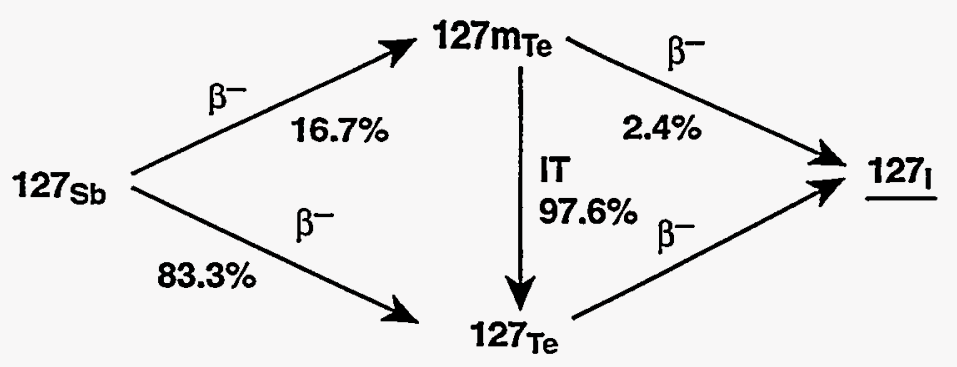

No. 22

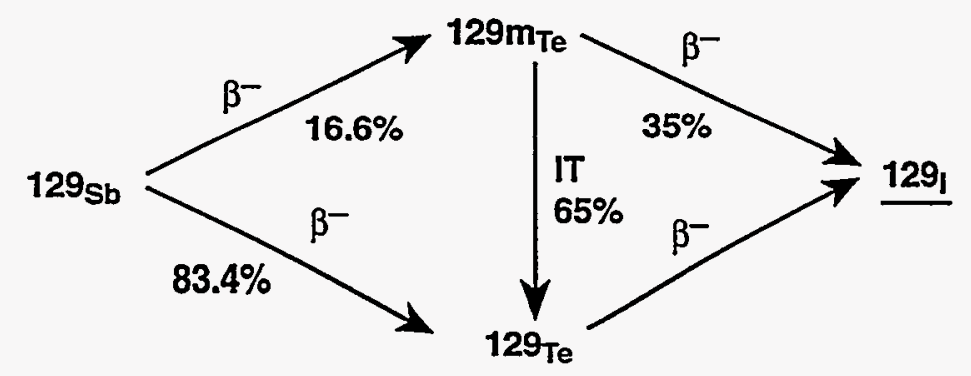

No. 23

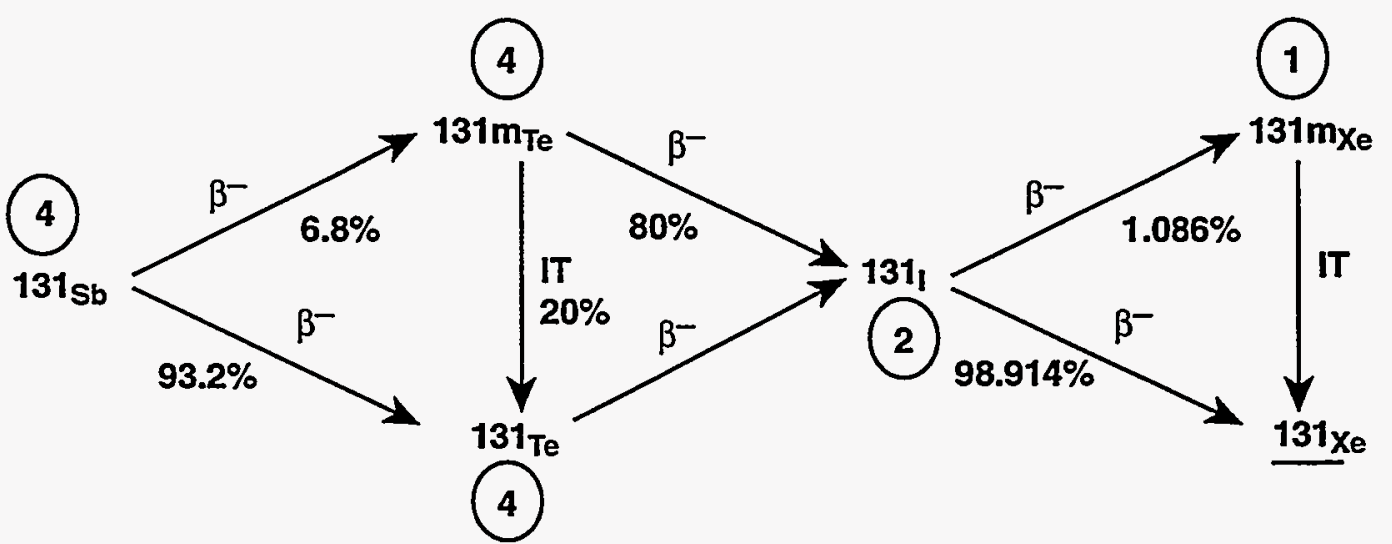

Figure 8-2. Decay Processes in the Fission Product Library (Continued) 

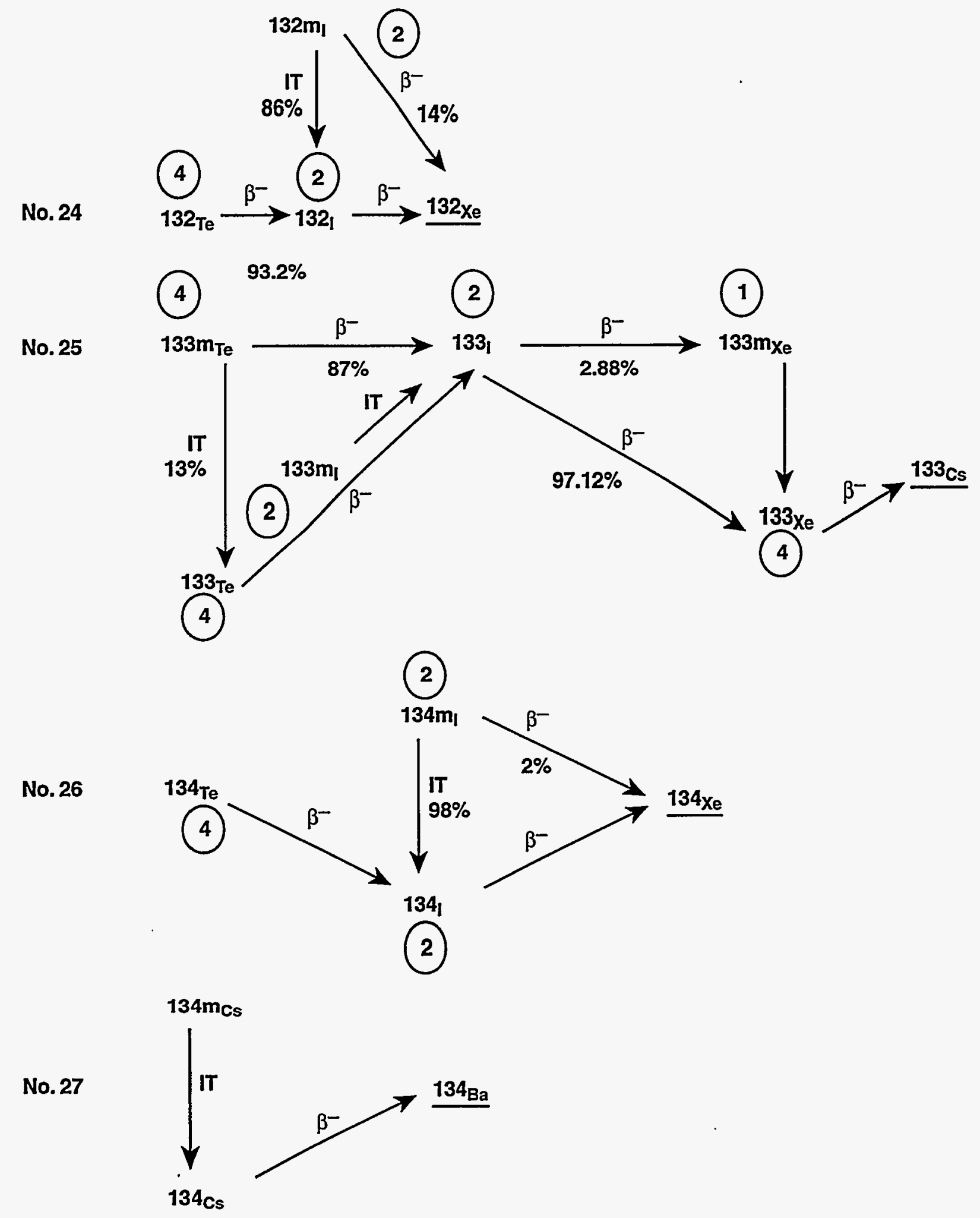

Figure 8-2. Decay Processes in the Fission Product Library (Continued) 


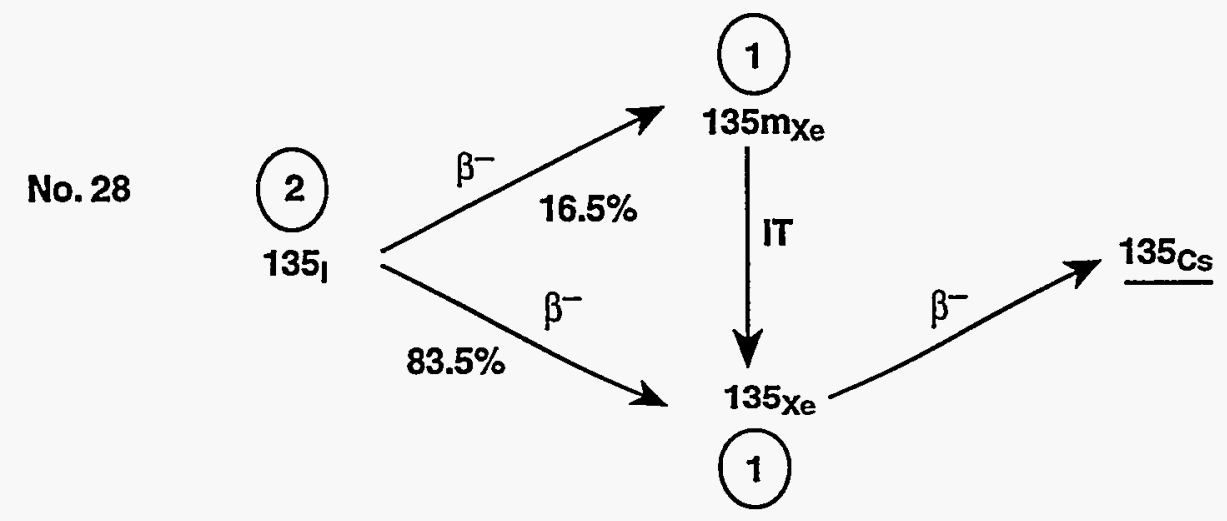

No.29 $136 \mathrm{cs} \stackrel{\beta^{-}}{\longrightarrow} 136_{\mathrm{Ba}}$

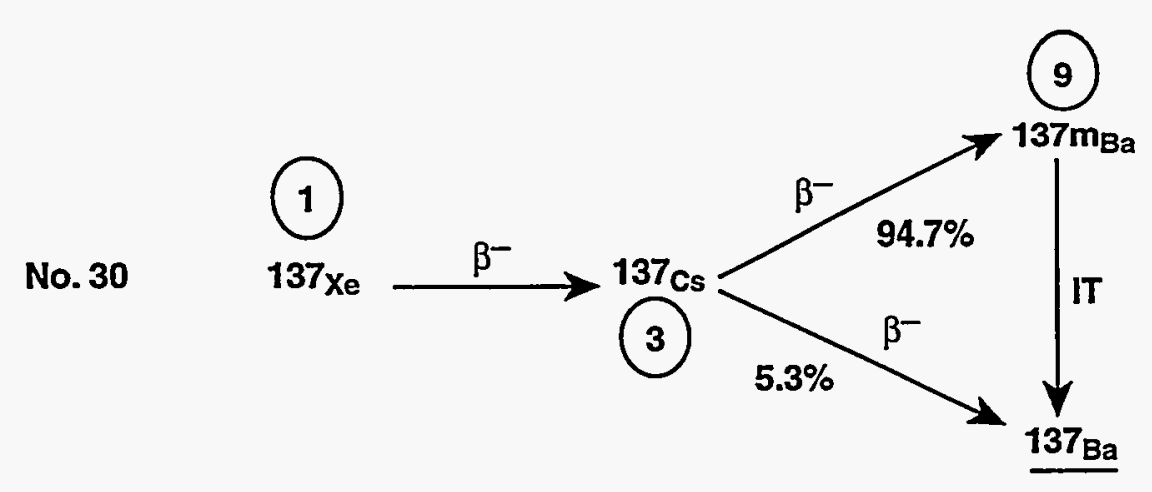

No. 31

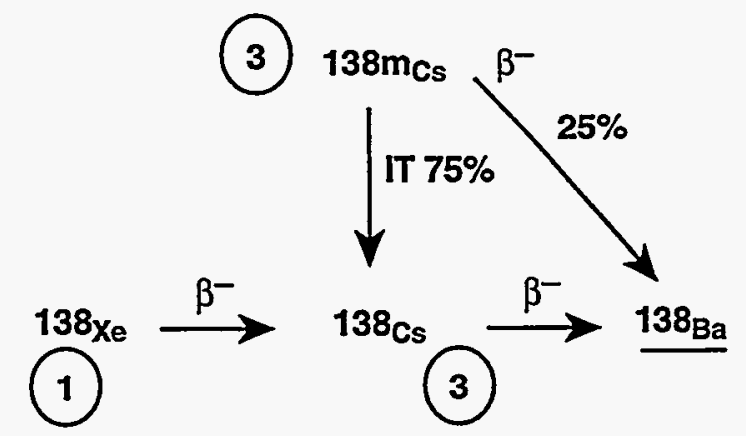

No. 32

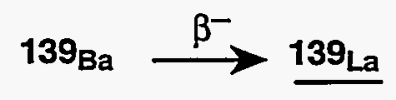

No. 33

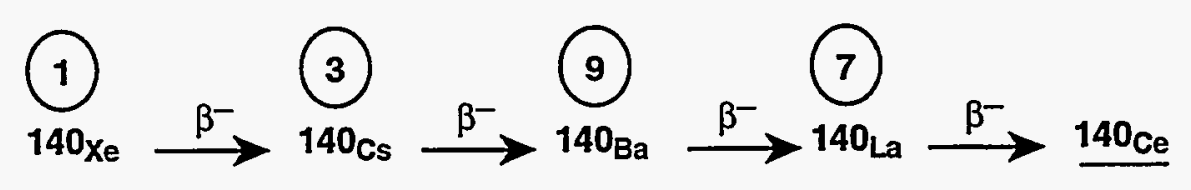

Figure 8-2. Decay Processes in the Fission Product Library (Continued) 


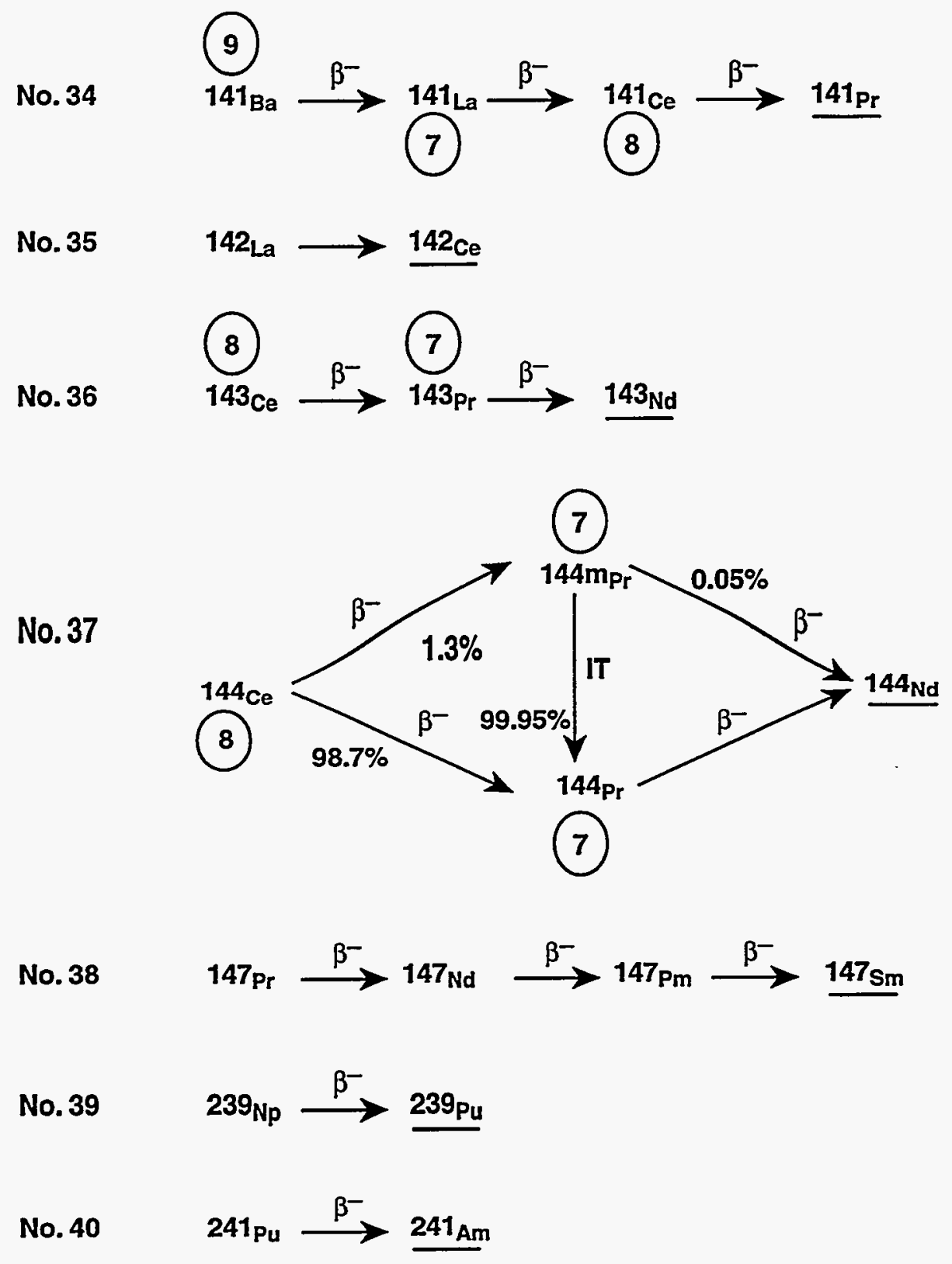

Figure 8-2. Decay Processes in the Fission Product Library (Concluded) 
with the host. In such cases, the fission product mass should be specified redundantly if possible, once in the fission product input and also as a contribution to the host material mass. Means of accounting for fission product mass effects are discussed in more detail in Section 13.3.4.1.

In addition to transport with mobile hosts, fission products should transfer from one host to another on the basis of physical or chemical compatibility. For instance, when a fission product decays, the daughter may have different chemical and physical characteristics, and therefore the subsequent host may be different from that of the parent. As an example, a solid fission product trapped in fuel material may decay to a noble gas, which then escapes. Such a process may be captured by the targeted release and acceptance model, discussed in Section 8.4. This model is also useful for other processes, such as revaporization and equilibration problems. In this model, the user-prescribed transfer rates can depend on the host temperatures; the temperature dependence is introduced through user-specified coefficients and thresholds.

The modeling of decay heating from the explicitly specified fission products is discussed in Section 8.5. Despite the increased flexibility provided by the library in defining individual radionuclides, it is usually difficult to specify in detail all of the fission products that would contribute to decay heating, as this would require a large amount of additional input. Normally, only a mobile subset of fission products is of interest for health physics or transport reasons, and it is these fission products that the user may want to specify explicitly. As mentioned above, the decay heat from fission products implicitly considered present in core materials fixed within the containment can be handled in a more generic way through the DECAY-HT option discussed in Section 5.6.1: an ANSIstandard decay power curve based on reactor operating history and fuel burnup [Ame79] is used to calculate the total decay power as a function of time since shutdown. The power associated with any fission products explicitly present in a calculation is subtracted from this total power, and the remaining power is then deposited in a number of locations, such as coolant pools or core-debris layers, as specified by the user.

Section 8.6 covers the transport of fission products within the atmosphere, Section 8.7 covers the transport of fission products within liquid pathways, and Section 8.8 presents the iodine removal models. User-specified fission product sources are covered in Section 8.9.

\subsection{Fission Product Library}

The fission product library currently includes the decay information for 140 radionuclides in the 40 coupled decay processes shown in Figure 8-2. Table 8-1 lists the radionuclides in the library, organized according to the 10 indicated volatility groups. The radionuclide name is derived by concatenating the element symbol, the mass number, and the state of the radionuclide. For example, ${ }^{129} \mathrm{Te}$ is named TE129, and ${ }^{105 \mathrm{~m}} \mathrm{Rh}$ is named RH105M. The volatility groups are used in the input for the targeted release and acceptance model and as an optional way of organizing fission product masses in the code output.

The information stored in the library consists of:

- nuclide name 
- group number for the radionuclide

- half-life

- atomic weight

- total specific decay power (W/kg)

- gamma specific decay power $(\mathrm{W} / \mathrm{kg})$

- most probable gamma energy (MeV)

- inventory factors for initializing mass distributions of a given radionuclide in the set of linear chains

The half-life, atomic weight, total specific decay power, gamma-specific decay power, and most probable gamma energy are self-explanatory. It should be noted that the latter two quantities are stored in the code but presently not used in models. The definitions of inventory factors and linear chains are discussed in Section 8.3.

Because the CONTAIN fission product decay model assumes that the atomic weight of a radionuclide remains unchanged, only radionuclides with beta and gamma decay are modeled. The available decay processes, which are shown in Figure 8-2, are selected by the user by number from the library for inclusion in a calculation. When a particular decay process is requested, CONTAIN will include all of the radionuclides in that process. The end products of the decay process are also included, and modeled with infinite half-life and zero decay power. Note that some of the end products may in reality decay over time by alpha decay, but the alpha decay is not explicitly modeled.

\subsection{Linear Decay Chains}

Fission product transmutation is modeled using the technique of linear chain resolution. [Eng68] This technique applies to the decay of explicitly specified fission products. In the technique of linear chain resolution, the differential equations for decay are decoupled by breaking a coupled decay process into a system of decoupled linear chains. The resulting chains can be treated independently so only the masses in a chain are needed to solve for the effects of decay for that chain.

To characterize decay processes not defined in the fission product library, the user must be familiar with the concepts of a fission chain element, branching ratio, and inventory factor. Linear chain decomposition replaces a coupled decay process with a set of linear chains, each representing a possible decay path, beginning with the leading radionuclide(s) of the coupled process. For example, decay process number 17 from Figure 8-2 can be broken down into two linear chains: (1) ${ }^{103} \mathrm{Ru} \rightarrow$ ${ }^{103 \mathrm{~m}} \mathrm{Rh} \rightarrow{ }^{103} \mathrm{Rh}$, and $(2){ }^{103} \mathrm{Ru} \rightarrow{ }^{103} \mathrm{Rh}$. A given radionuclide, such as ${ }^{103} \mathrm{Rh}$, may appear more than once as an element in the set of linear chains. Each occurrence of a radionuclide constitutes a "fission chain element." In the above two chains, there are a total of 5 fission chain elements and 3 radionuclides.

The branching ratio is the probability that a radionuclide will take a particular branch (i.e., decay to a particular daughter) in the decay process. Therefore, the sum of all branching ratios for a radionuclide must add to unity. The branching ratios given in Figure 8-2 indicate that ${ }^{103} \mathrm{Ru}$ will take the ${ }^{103 \mathrm{~m}} \mathrm{Rh}$ branch as in the first linear chain above with a probability of $99.75 \%$, and will take the 
${ }^{103} \mathrm{Rh}$ branch as in the second chain with a probability of $0.25 \%$. Branching ratios and decay constants are tabulated in most core inventory code libraries, and are based on data provided in ENDF/V or $/ V$ listings. [Koc81] It should be noted that the half-life to be supplied for a given radionuclide in the fission product input should be the net half-life from all decay branches, even though only one branch is taken in any given linear chain.

In the technique of linear chain resolution, the initial radionuclide inventory, and any new inventory added from an external source during the course of a calculation, must be distributed among the multiple occurrences, if present, of a given radionuclide in the linear chains. Once this initial distribution is made, any mass resulting from decay of an existing parent radionuclide will be determined from the decay rate and inventory of the parent. The initial distribution is made according to inventory factors. The inventory factor, $F_{R, i}$ is defined as the appropriate fraction of the initial mass of a given radionuclide $R$ to assign to each occurrence $i$ of the radionuclide in the set of linear chains. When merging decay paths are not present, the inventory factor for a radionuclide occurrence in a given chain is simply the probability that the radionuclide will decay to all of its daughters and granddaughters in the sequence given by the linear chain. In contrast to branching ratios, which are the probabilities of forming a particular daughter, inventory factors are essentially the probabilities of taking a particular decay path to forming the stable end product of the decay. Clearly, the sum of the inventory factors for all occurrences of a given radionuclide in the set of linear chains must be equal to one. The method for calculating inventory factors is discussed in more detail below.

In the simple example above, ${ }^{103} \mathrm{Ru}$ has two inventory factors of 0.9975 and 0.0025 , respectively, ${ }^{103 m} \mathrm{Rh}$ has an inventory factor of 1 , and ${ }^{103} \mathrm{Rh}$ has inventory factors which in CONTAIN are taken to be 1 and 0 , respectively, for the first and second chains given above. Note that a certain arbitrariness occurs in the inventory factors of radionuclides, like those of ${ }^{103} \mathrm{Rh}$, since merging paths create identical, or redundant, terminating chain segments in different linear chains. Clearly, the physical outcome of the calculation is not affected by how the mass of a radionuclide located on such a segment is distributed among the chains with the same segment.

The coupled decay process shown in Figure 8-3 will be used to illustrate how to calculate the inventory factors. In the linear chain decomposition, the decay is formulated in terms of the three chains shown at the bottom of the figure. The labels $A_{1}, A_{2}$, and so forth, denote different fission chain elements, in this case, derived from radionuclide $A$. For a decay process that is a tree, with branching but no merging paths, the inventory factor $F_{R, i}$, for radionuclide $R$ in the chain in which it appears for the $i$ th time, is determined by multiplying the branching ratio $f_{R, m}$ for the $m$ th branch taken by $R$ in that chain, by the appropriate branching ratios of all radionuclides to the right of it in the chain. For the last radionuclide of each chain from a tree-like process, the radionuclide occurrence is unique. Therefore, its inventory factor must be unity, from the sum rule, and so must its branching ratio.

The decay process in Figure 8-3 contains merging paths and therefore must first be converted to a tree structure, before applying the above prescription. The merging paths in this case create identical terminating chain segments involving $B$ and $C$ in chains 1 and 3 . The conversion to a tree structure is accomplished in this case by assuming that $\mathrm{B}_{1}$ and $\mathrm{B}_{2}$ correspond to distinct radionuclide types $\mathrm{B} 1$ 

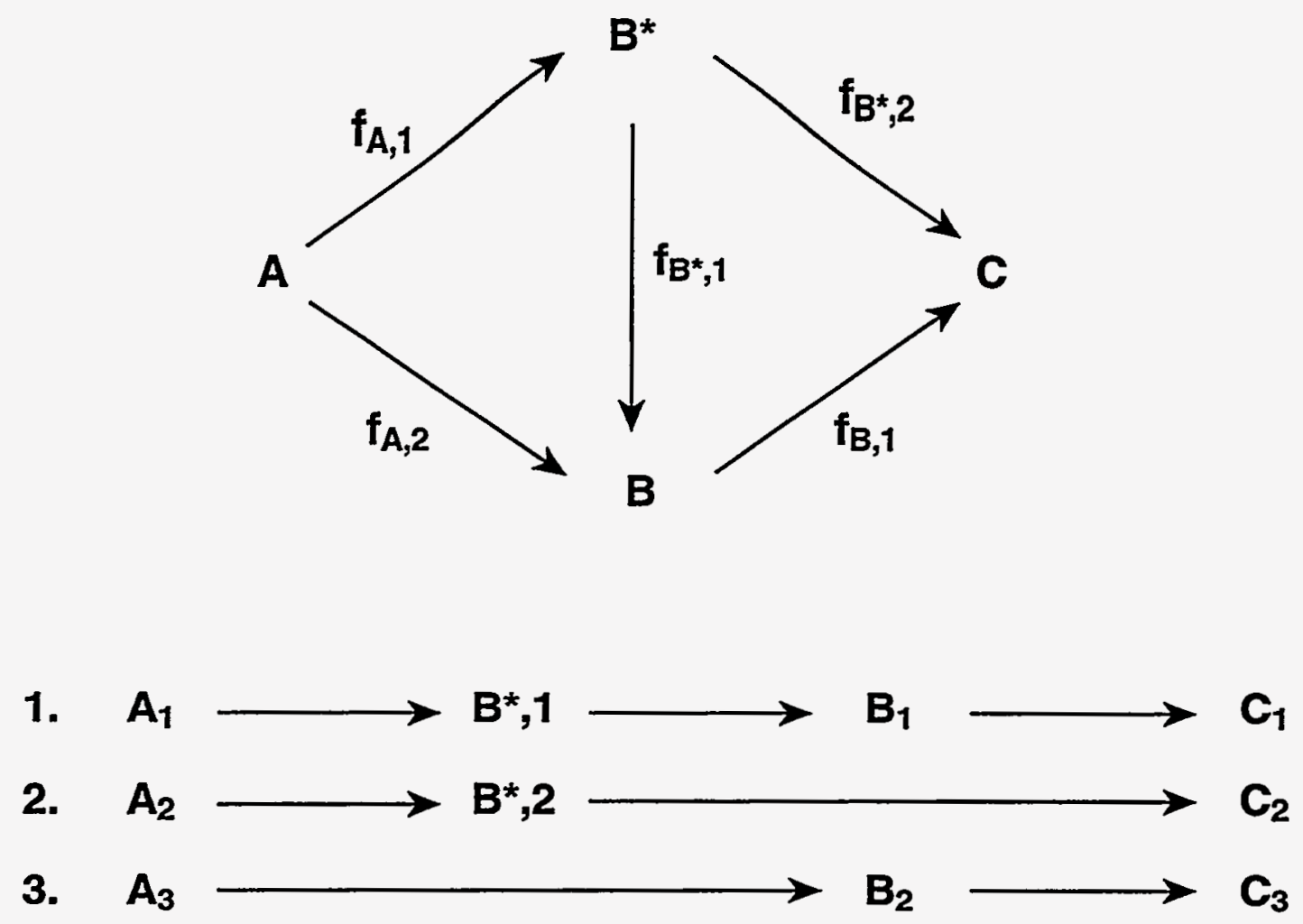

Figure 8-3. Example of Linear Decay Chains Derived from a Coupled Decay Process 
and $\mathrm{B} 2$, respectively, differing only with respect to their labeling and identical with respect to their physical properties, and by assuming that $\mathrm{C}_{1}, \mathrm{C}_{2}$, and $\mathrm{C}_{3}$ correspond to distinct types $\mathrm{C} 1, \mathrm{C} 2$, and $\mathrm{C} 3$, respectively. With this assumption, the inventory factor for $A$ in the first chain, for example, would then be $f_{A, 1}$ (for the decay of $A$ to $B^{*}$ ), multiplied by $f_{B^{*}, 1}$ (for the decay of $B^{*}$ to $B$ ), multiplied by the branch ratio from $B 1$ to $\mathrm{C} 1$. Note that the last branching ratio is taken to be unity since $\mathrm{B} 1$ can only decay to $\mathrm{C} 1$. It should be noted that only the minimum set of distinct types required to generate a tree structure should be considered.

The inventory factors for the three linear chain elements of A, according to Figure 8-2, are:

$$
\begin{aligned}
& F_{A, 1}=f_{A, 1} f_{B^{*}, 1} \\
& F_{A, 2}=f_{A, 1} f_{B^{*}, 2} \\
& F_{A, 3}=f_{A, 2}
\end{aligned}
$$

Also, one obtains

$$
\begin{aligned}
& F_{B^{*}, 1}=f_{B^{*}, 1} \\
& F_{B^{*}, 2}=f_{B^{*}, 2} .
\end{aligned}
$$

When B1 and B2 are considered to be distinct radionuclide types, each appears only once in the linear chains and therefore the sum rule requires that

$$
\begin{aligned}
& F_{B 1,1}=1 \\
& F_{B 2,1}=1 .
\end{aligned}
$$

In addition, according to the above discussion

$$
\begin{aligned}
& \mathrm{F}_{\mathrm{Cl}, 1}=1 \\
& \mathrm{~F}_{\mathrm{C} 2,1}=1 \\
& \mathrm{~F}_{\mathrm{C} 3,1}=1
\end{aligned}
$$

The procedure for obtaining the physical inventory factors $\mathrm{F}_{\mathrm{B}, \mathrm{i}}$ and $\mathrm{F}_{\mathrm{C}, \mathrm{i}}$ from the above is discussed in next paragraph.

The inventory factors defined either in the library or by the user are used to distribute initial or new mass from an external source among the possible decay chain locations. When arbitrarily distinct radionuclide types, such as B1 and B2, are involved, the manner of distribution of initial or new mass among such types is itself arbitrary, in the sense that it should not change the physical outcome of the calculation. For example, one could consider a given initial mass $m_{B}$ of $B$ to be composed of $B 1$ and $\mathrm{B} 2, \mathrm{~m}_{\mathrm{B}}=\mathrm{m}_{\mathrm{B1}}+\mathrm{m}_{\mathrm{B} 2}$, with an arbitrary distribution. In CONTAIN, the approach taken for simplicity and reasons of upward compatibility is to assume that one always considers new mass to correspond to only the first of each set of arbitrarily distinct types (e.g., to B1 but not B2) in the decay chains. This procedure is equivalent to taking the physical inventory factors to be those of the first type of each set, and setting those corresponding to the other types of each set to zero. For example, this approach is equivalent to setting $F_{B, 1}=F_{B 1,1}, F_{B, 2}=0, F_{C, 1}=F_{C 1,1}, F_{C, 2}=0$, and $F_{C, 3}=0$. 
Note that the above example is relatively simple, in the sense that each of the arbitrarily distinct types appears only once in the linear chain decomposition. In general the arbitrarily distinct types, present because of merging paths, could themselves branch in their decays. This situation would result in more than one occurrence of each type. This occurs, for example, in decay processes 23 and 25 in Figure 8-2. These cases generate inventory factors of the form $\mathrm{F}_{\mathrm{Rn}, \mathrm{i}}$ for an arbitrarily distinct radionuclide $R, n$, where both the occurrence index $i$ and the type label $n$ can have values greater than one. In such cases, the physical inventory factors for $R$ as a function of the physical occurrence $i$ are taken to be those of the first type $(n=1)$ and the corresponding $i$, and the inventory factors corresponding to other types are zeroed out. Note that in such cases it is incorrect to simply divide the inventory uniformly across all occurrences of $\mathrm{R}$ in the linear chains.

Tables 8-2 and 8-3 illustrate the results of the above procedure when applied to coupled decay processes 3 and 25 from Figure 8-2.

Table 8-2

Fission Product Library -- Decay Process 3

\begin{tabular}{||l|c|c|}
\hline Linear Decay Chain & Chain Elements & Inventory Factor \\
\hline Co60m $\rightarrow>$ Co60 --> Ni60 & Co60m & 0.9976 \\
\cline { 2 - 3 } & Co60 & 1.0000 \\
\cline { 2 - 3 } & Ni60 & 1.0000 \\
\hline Co60m $\rightarrow>$ Ni60 & Co60m & 0.0024 \\
\cline { 2 - 3 } & Ni60 & 0.0 \\
\hline
\end{tabular}

Table 8-3

Fission Product Library -- Decay Process 25

\begin{tabular}{||l|c|c||}
\hline \hline Linear Decay Chain & Chain Elements & Inventory Factor \\
\hline Te133m $\rightarrow$ I133 $\rightarrow$ Xe133m $\rightarrow>$ Xe133 $\rightarrow$ Cs133 & Te133m & 0.0251 \\
\cline { 2 - 3 } & $\mathrm{I} 133$ & 0.0288 \\
\cline { 2 - 3 } & $\mathrm{Xe133m}$ & 1.0000 \\
\cline { 2 - 3 } & $\mathrm{Xe133}$ & 1.0000 \\
\cline { 2 - 3 } & $\mathrm{Cs} 133$ & 1.0000 \\
\hline \multirow{2}{*}{ Te133m - > I133 --> Xe133 -> Cs133 } & $\mathrm{Te} 133 \mathrm{~m}$ & 0.8449 \\
\cline { 2 - 3 } & $\mathrm{I} 133$ & 0.9712 \\
\cline { 2 - 3 } & $\mathrm{Xe133}$ & 0.0 \\
\cline { 2 - 3 } & $\mathrm{Cs} 133$ & 0.0 \\
\hline
\end{tabular}


Table 8-3

Fission Product Library -- Decay Process 25 (Concluded)

\begin{tabular}{|c|c|c|}
\hline Linear Decay Chain & Chain Elements & Inventory Factor \\
\hline \multirow{6}{*}{$\begin{array}{l}\text { Te133m --> Te133 --> I133 --> Xe133m -> Xe133 } \\
-\rightarrow \text { Cs133 }\end{array}$} & $\mathrm{Te} 133 \mathrm{~m}$ & 0.0037 \\
\hline & Te133 & 0.0288 \\
\hline & $\mathrm{I133}$ & 0.0 \\
\hline & $\mathrm{Xe133m}$ & 0.0 \\
\hline & Xe133 & 0.0 \\
\hline & Cs133 & 0.0 \\
\hline \multirow{5}{*}{ Te133m --> Te133 --> I133 --> Xe133 --> Cs133 } & $\mathrm{Te} 133 \mathrm{~m}$ & 0.1263 \\
\hline & Te133 & 0.9712 \\
\hline & $\mathrm{I133}$ & 0.0 \\
\hline & Xe133 & 0.0 \\
\hline & Cs133 & 0.0 \\
\hline \multirow[t]{5}{*}{ I133m $\rightarrow$ I133 $\rightarrow>$ Xe133m $\rightarrow$ Xe133 $\rightarrow$ Cs133 } & $\mathrm{I} 133 \mathrm{~m}$ & 0.0288 \\
\hline & I133 & 0.0 \\
\hline & $\mathrm{Xe133m}$ & 0.0 \\
\hline & $\mathrm{Xe133}$ & 0.0 \\
\hline & Cs133 & 0.0 \\
\hline \multirow[t]{4}{*}{ I133m $\rightarrow$ I133 --> Xe133 --> Cs133 } & $\mathrm{I} 133 \mathrm{~m}$ & 0.9712 \\
\hline & I133 & 0.0 \\
\hline & $\mathrm{Xe133}$ & 0.0 \\
\hline & Cs133 & 0.0 \\
\hline
\end{tabular}

When the technique of linear chain resolution is used, the mathematical description of radioactive decay can be expressed as the following linear differential equations [Eng68]:

$$
\mathrm{m}_{1}{\overrightarrow{\lambda_{1}}}_{\mathrm{m}_{2}}{\overrightarrow{\lambda_{2}}}^{\mathrm{m}_{3}} \ldots \mathrm{m}_{\mathrm{j}}{\overrightarrow{\lambda_{j}}}_{\mathrm{j}} \ldots \mathrm{m}_{\mathrm{n}}
$$




$$
\begin{aligned}
\frac{\mathrm{dm}_{1}}{\mathrm{dt}} & =-\lambda_{1} \mathrm{~m}_{1}+\mathrm{S}_{1} \\
\frac{\mathrm{dm} \mathrm{m}_{2}}{\mathrm{dt}} & =\lambda_{1} \mathrm{~m}_{1}-\lambda_{2} \mathrm{~m}_{2}+\mathrm{S}_{2} \\
& \cdot \\
& \cdot \\
\frac{\mathrm{dm}}{\mathrm{dt}} & =\lambda_{\mathrm{j}-1} \mathrm{~m}_{\mathrm{j}-1}-\lambda_{\mathrm{j}} \mathrm{m}_{\mathrm{j}}+\mathrm{S}_{\mathrm{j}} \\
& \cdot \\
\frac{\mathrm{dm}}{\mathrm{n}} & =\lambda_{\mathrm{n}-1} \mathrm{~m}_{\mathrm{n}-1}+\mathrm{S}_{\mathrm{n}}
\end{aligned}
$$

Where $m_{j}$ equals $m_{j}(t)$, which is the mass of fission chain element $j$ present at time $t ; \lambda_{j}$ is the total decay constant for the radionuclide corresponding to $m_{j}$; $\mathrm{dm}_{j} / \mathrm{dt}$ is the mass rate of change; and $\mathrm{S}_{\mathrm{j}}$ is the external mass source rate of chain element $j$ (e.g., from intercell flow and/or external sources). It should be noted that $S_{j}$ is related to the mass source rate of the corresponding radionuclide through inventory factors.

This set of equations is solved analytically in CONTAIN to give

$$
m_{n}(t+\Delta t)=\sum_{k=1}^{n} m_{k}(f) \prod_{j=k}^{n} \lambda_{j} \sum_{i=k}^{n} \frac{e^{-\lambda_{1} \Delta t}}{\prod_{\substack{m=k \\ m \neq i}}^{n}\left(\lambda_{m}-\lambda_{i}\right)}
$$

where $\Delta t$ is the computational timestep. This analytic solution can be readily derived using the method of Laplace transform.

Equation (8-1) is used to calculate the mass of each fission chain element present after decay over a timestep for use by other models, such as the fission product heating model. It is important to remember that the total inventory of a radionuclide is given by the sum of the masses of all occurrences of the radionuclide among the fission chain elements.

\subsection{Fission Product Hosts and Targeted Release and Acceptance}

Fission products are typically initially assigned to a host based on the chemical affinity and physical characteristics of the fission product. These assignments may be made for the initial fission product 
inventory and for time-dependent sources of fission products. The atmosphere in each cell and each component of the suspended aerosols in each cell are always individual hosts. Each of the inner and outer surfaces of a heat transfer structure and each lower cell layer are also taken to be individual hosts. If a lower cell has been defined, then a fixed atmosphere interface host is present in addition to those for each layer. Finally, a DUMMY host and a WASTE host are provided in each cell. The former is provided as a possible release repository; the latter contains mass lost from the problem, for example, from mass transfers directed to a pool that does not exist. It should be noted that hosts may be referred to collectively. For example, a FLOOR host designation refers collectively to the FLOOR heat transfer structure surfaces present. If a distribution of fission product mass is required, it is allocated in this case according to surface area.

The transport of CONTAIN host materials may cause the fission product to change hosts; for example, aerosol deposition causes a fission product attached to the aerosols to become attached to the deposition surface (see Section 8.6). A semi-mechanistic model for the washdown of fission products from structure surfaces to a pool and transport of fission products between pools is available (see Section 8.7). In addition, host changes may be specified by the user through the targeted release and acceptance formalism described below.

The transfer of fission products from their specific locations (such as structure, aerosol, and/or lower cell layer) to other user-specified targets or hosts (such as the upper cell atmosphere gas) can be simulated in CONTAIN using user-specified transfer rates. This formalism is known as the targeted release and acceptance model.

In this formalism, the user can specify transfer rates for individual fission products between any number of host pairs. For a given host pair $\mathrm{i}, \mathrm{j}$, the fractional transfer rates per second, $\mathrm{r}_{\mathrm{i}-\mathrm{j}}$, for a particular fission product may depend on the host temperature $T_{i}$ or $T_{j}$ according to the following expressions:

$$
\begin{aligned}
& r_{i-j}=\left\{\begin{aligned}
a \exp \left(-b / T_{i}\right) & \text { for } T_{i}<T_{\text {threshold }} \\
0 & \text { for } T_{i}<T_{\text {threshold }}
\end{aligned}\right\} \text { if } a>0 \\
& r_{i-j}=\left\{\begin{aligned}
|a| \exp \left(-b / T_{j}\right) & \text { for } T_{j}>T_{\text {threshold }} \\
0 & \text { for } T_{j}<T_{\text {threshold }}
\end{aligned}\right\} \text { if } a<0
\end{aligned}
$$

where $\mathrm{a}$ and $\mathrm{b}$ are user-specified parameters and $\mathrm{T}_{\text {threshold }}$ is a user-specified threshold temperature that defines the temperature below which the release rate is set to zero, $i$ is the host specified after the FROM keyword in the TARGET input block, and $j$ is the host specified after the TO keyword (see Section 14.3.1.10). Fission products are assumed to transfer at mass rates proportional to the amount of fission product mass present. For example, the simple case of the transfer between one pair of hosts at the fractional rate, $\mathrm{r}_{\mathrm{i}-\mathrm{j}}$ per second is represented by the coupled equations: 


$$
\frac{d m_{i}}{d t}=-r_{i-j} m_{i}, \frac{d m_{j}}{d t}=r_{i-j} m_{i}
$$

where $m_{i}$ represents the fission product mass of the $i$ th host, and $m_{j}$ represents the fission product mass of the jth host.

For simple coupled equations like those given in Equation (8-3), fission product mass redistributions are calculated using an analytic solution. The trivial solution, where each host is involved in only one targeting equation, is given by

$$
\begin{aligned}
& m_{i}(t+\Delta t)=m_{i}(t) e^{-r_{i-j}-\Delta t_{s}} \\
& m_{j}(t+\Delta t)=m_{j}(t)+m_{i}(t)\left(1-e^{-r_{i-j} \Delta t_{s}}\right)
\end{aligned}
$$

where $\Delta \mathrm{t}_{\mathrm{s}}$ is the CONTAIN system timestep.

In addition to this trivial case, other cases involving fission product release from one host to more than one other host can be solved in this direct fashion. Likewise, this solution is applicable to the case where multiple hosts are releasing the same fission product to a common host. The only requirement is that no one host is releasing and accepting the same fission product. If the sets I and $\mathrm{J}$ are defined as

$$
\begin{aligned}
& I=\text { family of all releasing hosts for a fission product and } \\
& J=\text { family of all accepting hosts for a fission product, }
\end{aligned}
$$

then mathematically, $I \cap J=0$ is the requirement for the following decoupled solution to be valid:

$$
\begin{aligned}
& s_{i}=\sum_{j} r_{i-j} \quad \begin{array}{l}
\text { (sum of all release rates from host } i \\
\text { to all receiving hosts } j)
\end{array} \\
& \left.m_{i}\left(t+\Delta t_{s}\right)=m_{i}(t) e^{-s_{i} \Delta t} \quad \text { (for } i \varepsilon I\right) \\
& \left.m_{j}\left(t+\Delta t_{s}\right)=m_{j}(t)+\sum_{i} \frac{r_{i-j} m_{i}(t)\left[1-e^{-s_{i} \Delta t_{s}}\right]}{s_{i}} \text { (for } j \varepsilon J\right)
\end{aligned}
$$

where $\mathrm{j} \varepsilon \mathrm{J}$ means $\mathrm{j}$ is a member of (or is contained in) the set $\mathrm{J}$, and the same is true for i $\varepsilon \mathrm{I}$.

Certain targeting scenarios are not so simple. They lead to equations that are more highly coupled. More complex coupled equations are solved by a highly accurate exponential operator method. 
[Lee80] Note that array space for the targeted release model must be provided through the "ntgt" parameter in the global CONTROL block. The general target problem is written in matrix form as

$$
\frac{\mathrm{dm}}{\mathrm{dt}}=\mathbf{A} \overrightarrow{\mathrm{m}}
$$

where $\vec{m}$ is a vector of all fission products on hosts, and $\mathbf{A}$ is a matrix of release rates from one host to another.

The Ash exponential operator method [Lee80, Was83] is used to transmute the A matrix forward in time (analogous to scalar exponentiation) to give the solution

$$
m\left(t+\Delta t_{s}\right)=\left[e^{A \Delta t_{s}}\right] m(t)
$$

This method was chosen over implicit finite difference methods because of its ability to solve the highly coupled, large system timestep case reliably and within a reasonable computer time. [Was83] If the equations are decoupled, the direct solution method is used. Otherwise, the coupled matrix is solved using the operator method.

Targeted release rates are associated either exclusively with fission products or exclusively with the volatility groups of Table 8-1. The latter are used if the G-TARGET input option is invoked. All components of the fission product or group (i.e., the fission chain elements and/or individual fission products) will be transferred at the same specified rate. For example, when a release rate is defined for radionuclide $C$ in Figure 8-3, the masses of the fission chain elements corresponding to $C$ in chains 1,2 , and 3 will all be transferred at the same rate. To illustrate the flexibility of the targeted release formalism when coupled with fission product decay and mechanistic transport models in the code, consider how iodine and iodine decay products are affected by the release rates given in Table 8-4, which help simulate some of the processes illustrated in Figure 8-4. The decay process involved is a simplified version of decay process 30 in Figure $8-2:{ }^{137} \mathrm{I} \rightarrow{ }^{137} \mathrm{Xe} \rightarrow{ }^{137} \mathrm{Cs}$.

The hosts involved in this illustrative problem are called GAS (for the upper cell atmosphere), AEROSOL 1 (for aerosol component 1), INNER STRUC (for the inner surface of a structure), and LAYER 1 (for a lower cell layer representing core debris), and POOL for an overlying coolant pool.

Figure 8-4 illustrates how the targeted release processes fit into the overall fission product transport picture. In the example, all the initial mass is assumed to be iodine and hosted to the first lower cell layer representing core debris, and it is further assumed that a water pool exists above the core debris layer. Decay processes govern the formation of xenon and, eventually, cesium. Targeted release processes affect the mass distribution among host materials. Intercell flows transport fission products hosted to the gas and aerosols to and from other cells in multicell calculations (see Section 
Table 8-4

Illustrative Fission Product Targeted Release Rates

\begin{tabular}{|c|c|c|c|c|c|}
\hline Process & Nuclide & From & To & Rates $\left(\mathrm{s}^{-1}\right)$ & Process Represented \\
\hline 1. & ${ }^{137} \mathrm{I}$ & LAYER 1 & POOL & $10^{-1}$ & $\begin{array}{l}\text { Dissolution } \\
\text { (scrubbing) }\end{array}$ \\
\hline 2. & ${ }^{137} I$ & LAYER 1 & GAS & $10^{-3}$ & Vaporization \\
\hline 3. & ${ }^{137} \mathrm{I}$ & LAYER 1 & AEROSOL 1 & $10^{-1}$ & Aerosolization \\
\hline 4. & ${ }^{137} \mathrm{I}$ & POOL & GAS & $10^{-5}$ & Partitioning \\
\hline 5. & ${ }^{137} \mathrm{I}$ & POOL & AEROSOL 1 & $10^{-5}$ & Aerosolization \\
\hline 6. & ${ }^{137} \mathrm{Xe}$ & LAYER 1 & GAS & $10^{-3}$ & Outgassing \\
\hline 7. & ${ }^{137} \mathrm{Xe}$ & AEROSOL 1 & GAS & 5.0 & Rapid gas escape \\
\hline 8. & ${ }^{137} \mathrm{Xe}$ & WALL & GAS & 5.0 & Rapid gas escape \\
\hline 9. & ${ }^{137} \mathrm{Xe}$ & POOL & GAS & 1.0 & Rapid gas escape \\
\hline 10. & ${ }^{137} \mathrm{Cs}$ & LAYER 1 & POOL & $10^{-1}$ & $\begin{array}{l}\text { Dissolution } \\
\text { (scrubbing) }\end{array}$ \\
\hline 11. & ${ }^{137} \mathrm{Cs}$ & LAYER 1 & GAS & $10^{-5}$ & Vaporization \\
\hline 12. & ${ }^{137} \mathrm{Cs}$ & LAYER 1 & AEROSOL 1 & $10^{-1}$ & Aerosolization \\
\hline 13. & ${ }^{137} \mathrm{Cs}$ & POOL & GAS & $10^{-5}$ & Partitioning \\
\hline 14. & ${ }^{137} \mathrm{Cs}$ & POOL & AEROSOL 1 & $10^{-5}$ & Aerosolization \\
\hline 15. & ${ }^{137} \mathrm{Cs}$ & GAS & AEROSOL 1 & $10^{-1}$ & Adsorption \\
\hline
\end{tabular}

8.6). Finally, deposition mechanisms cause fission products in aerosol form to deposit on the heat structures (such as walls).

In the CONTAIN input description, the hosts involved in this illustrative example are called GAS (for the upper cell atmosphere), AEROSOL 1 (for aerosol component 1), WALL (for the collective heat structure wall area), LAYER 1 (for a lower cell layer representing core debris), and POOL (for the overlying water pool).

The processes simulated in this example are listed in Table 8-4. The gaseous iodine and xenon are assumed to transfer from the core debris to the atmosphere (processes 2 and 6) at the rate of $0.1 \%$ per second. The transfer rate of cesium vapor (process 11) is assumed to be two orders of magnitude lower at $0.001 \%$ per second. The rapid release of iodine and cesium from core debris in the form of aerosols (processes 3 and 12) is assumed to occur at the rate of $10 \%$ per second. Iodine and cesium can be trapped in the pool (processes 1 and 10) and xenon generated in the pool by decay processes can be released to the upper cell atmosphere (process 9). Any xenon hosted to the 


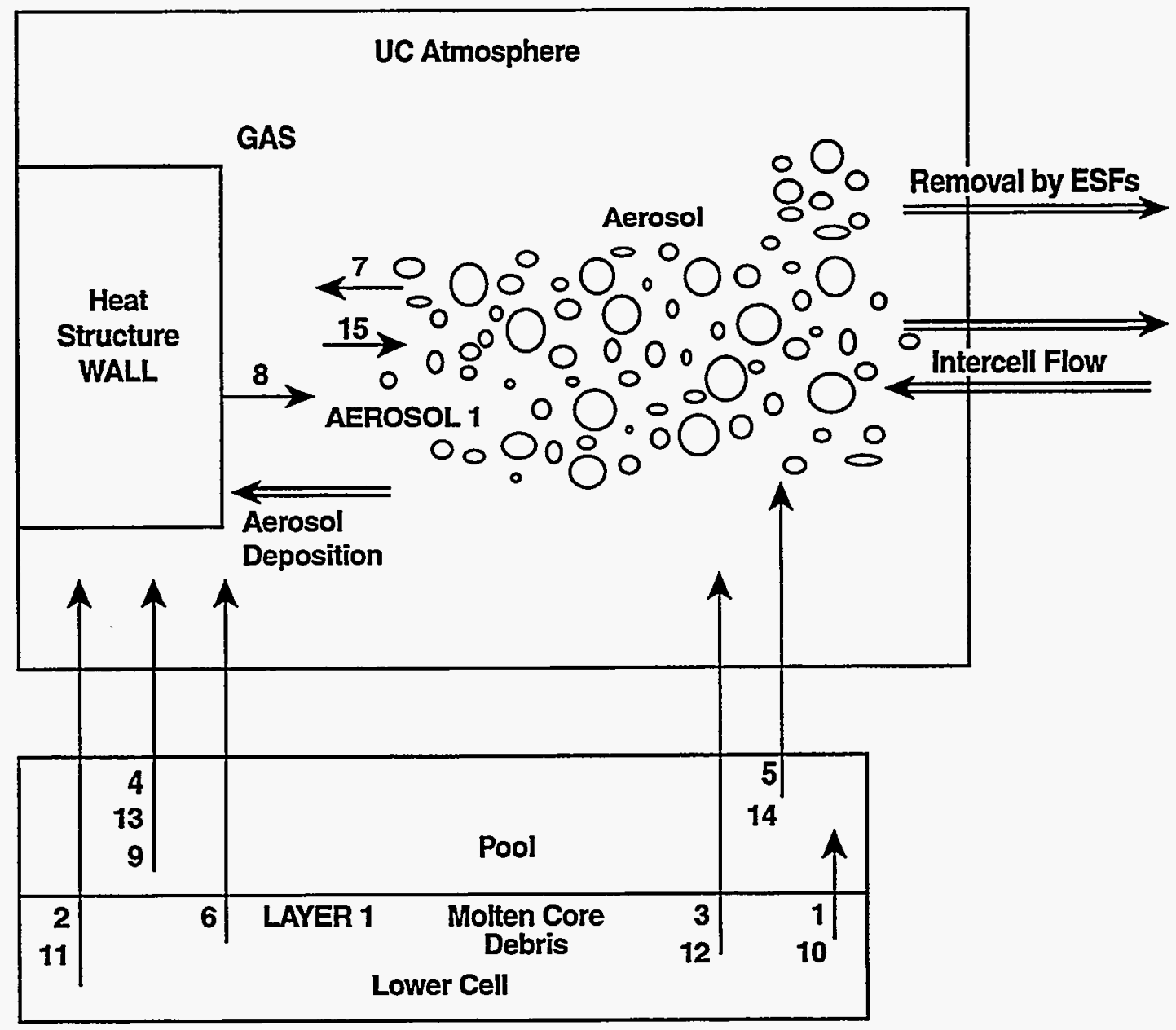

ESFs = Engineered safety features

UC = Upper Cell

$\Longrightarrow=$ Processes simulated by other source and removal models

$\longrightarrow=$ Process simulated by targeted release and acceptance model

Figure 8-4. Example of Fission Product Redistribution by the Targeted Release and Acceptance Model for Fission Products Initially in the Lower Cell Fuel Layer. The numbers correspond to the process numbers in Table 8-4. 
aerosols or to the wall of the structure (as a result of iodine decay) is assumed to be transferred to the gas at an essentially instantaneous rate of $500 \%$ per second (processes 7 and 8 ). Some iodine and cesium is assumed to be aerosolized from the pool to the upper cell atmosphere (processes 5 and 14) at a slow rate of $0.001 \%$ per second. Partitioning of iodine and cesium from the pool to the upper cell atmosphere (processes 4 and 13) is modeled at a rate of $0.001 \%$ per second. Finally, cesium hosted to the gas (as a result of release from the core debris or decay of xenon in the gas) is assumed to adsorb on the aerosols at the rate of $10 \%$ per second (process 15). Because no default values are provided, the user must specify the fission products, release rates, and hosts involved in this model.

\subsection{Fission Product Decay Heating}

Fission product heating from the explicit fission product inventories associated with various fission product hosts is discussed in this section. The types of fission product hosts considered by CONTAIN are discussed in Section 8.4. In addition to the decay heating associated with the explicit fission product inventory, an ANSI-standard model to describe the decay power of fission products implicitly assumed to be present in lower cell layers is also available and discussed in Section 5.7.1.

The fission product heating model calculates the amount of energy deposited in various hosts from the absorption of beta and gamma radiation and the recoil energy of the decaying radionuclide. A simplifying assumption is that the energy of the radioactive decay is deposited locally within the host for the decaying nucleus. Long-range heating effects, such as heating of the atmosphere by gamma rays originating at a structure surface, are not modeled. The energy associated with the decay of fission products hosted to the gas and to aerosols is assumed to be deposited in the atmosphere. The energy associated with the decay of fission products on a heat transfer structure surface is assumed to heat the first node at the structure surface. The decay energy of the explicitly specified fission products in a lower cell layer is assumed to be distributed uniformly throughout the layer, with the following exceptions: in the case of null layers (containing no thermodynamic materials), the decay heat is assumed to heat the first node below with a finite thermodynamic material mass, including the CORCON layer; when CORCON is active, the decay heat from explicit fission products assigned to the CORCON layer is ignored unless the CONTAIN ANSI-standard decay power model is invoked for that layer; and when CORCON is active, the decay heat from fission products assigned to the underlying concrete layer is lost from the calculation, since that layer is under the control of CORCON (see Section 5.7.1).

For fission products representing individual radionuclides, a constant specific decay power in watts per kilogram may be used in conjunction with the radionuclide inventory to compute the decay power for a given host. To accommodate the representation of fission product classes, a timedependent decay power option is available through the FGPPWR keyword (see Section 14.2.6.1). Up to four coefficients $\left(a_{1}\right.$ through $\left.a_{4}\right)$ may be specified for each fission product class, where the specific power $\mathrm{p}^{\prime}$ for that class is taken to be

$$
p^{\prime}=a_{1} e^{-a_{2} t}+a_{3} e^{-a_{4} t}
$$


where $t$ is the problem time in seconds and $a_{2}$ and $a_{4}$ are in units of inverse seconds. This functional form is designed so that the power will match the power of both short-lived radionuclides and longlived ones in the class. A coefficient not specified defaults to zero; for example, if only three coefficients $\left(a_{1}, a_{2}\right.$, and $\left.a_{3}\right)$ are given, $a_{4}$ will be zero. Note that $p^{\prime}$ calculated by Equation (8-5) represents the decay power as a function of the problem time, which is not necessarily the same as the time after shutdown.

\subsection{Iodine Removal Models}

This section describes the models used to determine elemental iodine $\left(\mathrm{I}_{2}\right)$ and methyl iodide $\left(\mathrm{CH}_{3} \mathrm{I}\right)$ removal by the containment sprays. The initial removal of $I_{2}$ by the containment sprays is experimentally observed to be a relatively rapid process that slows down abruptly as equilibrium is approached between competing absorption and desorption mechanisms. [Hil71] No attempt is made to model the observed late time desorption of iodine with the spray model because the experimentally observed desorption probably occurs from reservoirs (such as walls) that should be modeled outside of the spray model itself. Note that additives have been adopted in the spray systems of pressurized water reactors to enhance the rate and extent of iodine removal. The most common spray additive is sodium hydroxide.

The depletion rate for the iodine species is defined as the product of an absorption efficiency (that is, the fractional saturation attained by the drop) and the fraction of the compartment volume swept by spray per unit time. The efficiency is calculated from the diffusion rate of the iodine species through both a gas-side and a liquid-side boundary layer at the surface of a droplet. It is assumed that the drop interior is well mixed and that the drop starts its fall with zero iodine concentration. The liquid-side boundary layer is based on a stagnant film model, which provides a simple yet effective way to treat the problem. The user can input a partition coefficient $\mathrm{PC}_{\mathrm{I}}$ to characterize the solubility of the iodine species I in the spray water. This partition coefficient is used to simulate the effects of spray additives. A value of 5000 is the default for elemental iodine. A good source of recommended values for this parameter for a wide variety of spray additives is provided in Reference Gri82. Depletion rates for the organic compound methyl iodide $\left(\mathrm{CH}_{3} \mathrm{I}\right)$ have not been well established. A partition coefficient for this organic iodide "sppcmi" allows the user to model the removal rate for such relatively inert species. A value of zero is the default.

The fission products removed by sprays are deposited into the pool of the outflow cell designated by the user for the SPRAY engineered system. The waste location in that cell will be used in case a pool location is not available.

The equations used to determine the removal of $\mathrm{I}_{2}$ and $\mathrm{CH}_{3} \mathrm{I}$ have the same form. The differences are only in the diffusivities of $\mathrm{I}_{2}$ and $\mathrm{CH}_{3} \mathrm{I}$ in steam, air, and liquid water and the partition coefficients. The diffusivities are defined at the end of this section. Therefore, the equations that follow will simultaneously refer to either iodine species through use of the subscript I to stand for either $\mathrm{I}_{2}$ or $\mathrm{CH}_{3} \mathrm{I}$. To obtain the specific equation for either $\mathrm{I}_{2}$ or $\mathrm{CH}_{3} \mathrm{I}$ will only require the use of the appropriate $\mathrm{I}_{2}$ or $\left.\mathrm{CH}_{3} \mathrm{I}\right)$ parameters. 
CONTAIN models iodine removal from the atmosphere by falling spray drops as a first-order rate process expressed as

$$
\frac{\mathrm{dm}_{\mathrm{I}}}{\mathrm{dt}}=-\mathrm{m}_{\mathrm{I}} \mathrm{N}_{\mathrm{D}} \mathrm{IC}_{\text {final }}
$$

where $m_{I}$ is the mass of iodine in the atmosphere; $N_{D}$ is the number of drops per second introduced into the cell atmosphere and is equal to $\mathrm{W}_{\mathrm{sp}} / \mathrm{m}_{\mathrm{D}}$, where $\mathrm{W}_{\mathrm{sp}}$ is the spray mass flow rate, and $\mathrm{m}_{\mathrm{D}}$ is the drop mass; and $\mathrm{IC}_{\text {final }}$ is the final iodine mass in one drop divided by the total iodine mass in the atmosphere.

Note that the iodine removal could be excessive if explicitly calculated and if the cell timesteps were large. Therefore, the removal is limited as follows. Equation (8-6) is integrated over the cell timestep $\Delta t_{c}$ to produce the following expression for $F_{I}$, the ratio of iodine mass removed to the total mass of iodine in the atmosphere:

$$
\mathrm{F}_{\mathrm{I}}=\frac{\Delta \mathrm{m}_{\mathrm{I}}}{\mathrm{m}_{\mathrm{I}}}=1-\exp \left(-\mathrm{m}_{\mathrm{I}} \mathrm{N}_{\mathrm{D}} \mathrm{IC}_{\text {final }} \Delta \mathrm{t}_{\mathrm{c}}\right)
$$

The $\mathrm{IC}_{\text {final }}$ used above is the final value for the iodine mass in the drop per unit mass in the atmosphere after the drop has fallen through the atmosphere, provided the drops are assumed to initiate their fall with zero iodine mass. The final value for IC is determined by numerical integration over $N$ fall-time increments $\Delta t$. The values for $\Delta t$ are initially taken to be $1 / 10$ th of the spray fall time $H / v_{t}$, where $H$ is the fall height and $v_{t}$ is the terminal velocity.

At the end of the $i$ th increment in the cell, the iodine mass ratio in the drop to that in the atmosphere is defined to be $\mathrm{IC}_{\mathrm{i}}$, and the $\mathrm{IC}_{\mathrm{i}}$ are determined from the balance of the mass transfer from the gas phase to the drop surface and the mass transfer from the drop surface to the spray drop interior. Within an implicit method, the balance gives

$$
\mathrm{IC}_{\mathrm{i}+1}=\mathrm{IC}_{\mathrm{i}}+\mathrm{S}_{\mathrm{I}} \Delta \mathrm{t}\left(\frac{\mathrm{PC}_{\mathrm{I}}}{\mathrm{V}}-\frac{\mathrm{IC}_{\mathrm{i}+1}}{\mathrm{~V}_{\mathrm{D}}}\right)
$$

which is solved to give

$$
\mathrm{IC}_{i+1}=\frac{\mathrm{IC}_{\mathrm{i}} \mathrm{V}_{\mathrm{D}} \mathrm{V}+\mathrm{S}_{\mathrm{I}} \mathrm{PC}_{\mathrm{I}} \mathrm{V}_{\mathrm{D}} \Delta \mathrm{t}}{\mathrm{V}_{\mathrm{D}} \mathrm{V}+\mathrm{S}_{\mathrm{I}} \Delta \mathrm{t} \mathrm{V}}
$$


where $V_{D}$ is the drop volume, which can change because of condensation or evaporation of water as the drop falls; $V$ is the atmosphere volume; and $S_{I}$ is obtained from the mass balance equations and is expressed as

$$
\mathrm{S}_{\mathrm{I}}=\frac{\pi \mathrm{D}^{2} \mathrm{k}_{\ell, \mathrm{I}} \mathrm{k}_{\mathrm{g}, \mathrm{I}}}{\mathrm{PC}_{\mathrm{I}} \mathrm{k}_{\ell, \mathrm{I}}+\mathrm{k}_{\mathrm{g}, \mathrm{I}}}
$$

where $\mathrm{k}_{\mathrm{\ell}, \mathrm{I}}$ is the mass transfer coefficient in the spray drop for either $\mathrm{I}_{2}$ or $\mathrm{CH}_{3} \mathrm{I}$ as given below; and $\mathrm{k}_{\mathrm{g}, \mathrm{I}}$ is the mass transfer coefficient in the gas phase for either $\mathrm{I}_{2}$ or $\mathrm{CH}_{3} \mathrm{I}$ as given below.

The gas-phase mass transfer coefficients, based on mass density difference, are obtained from

$$
\mathrm{k}_{\mathrm{g}, \mathrm{I}}=\frac{\mathrm{D}_{\mathrm{I}, \mathrm{sa}}}{\mathrm{D}}\left(2+0.6 \mathrm{~N}_{\mathrm{Re}}^{1 / 2} \mathrm{~N}_{\mathrm{Sc}}^{1 / 3}\right)
$$

where $D_{I, s}$ is the diffusivity of the iodine species $I$ in a steam-air mixture, which is defined below; $D$ is the drop diameter; $N_{R E}=v_{t} D \rho_{1} / \mu_{a}$, where $v_{t}$ is the terminal velocity and $\mu_{a}$ is the atmosphere viscosity; and $N_{\mathrm{SC}}=\mu_{\mathrm{a}} /\left(\mathrm{\rho aD}_{\mathrm{I}, \mathrm{sa}}\right)$, here $\rho_{\mathrm{a}}$ is the atmosphere density.

The diffusivity of iodine in a steam-air mixture used in Equation (8-11) is

$$
D_{\mathrm{I}, \mathrm{sa}}=\left[\frac{\mathrm{Y}_{\mathrm{s}}}{\mathrm{D}_{\mathrm{I}, \mathrm{s}}}+\frac{\left(1-\mathrm{Y}_{\mathrm{s}}\right)}{\mathrm{D}_{\mathrm{L}, \mathrm{a}}}\right]^{-1}
$$

where $Y_{s}$ equals $P_{v} / P_{a}\left(P_{v}\right.$ is the partial pressure of the water vapor in the atmosphere, and $P_{a}$ is the atmosphere pressure); $D_{I, s}$ is the diffusivity in a steam atmosphere at temperature $T$ and pressure $P$ for either $\mathrm{I}_{2}$ or $\mathrm{CH}_{3} \mathrm{I}$; and $\mathrm{D}_{\mathrm{I}, \mathrm{a}}$ is the diffusivity in air at temperature $\mathrm{T}$ and pressure $\mathrm{P}$ for either $\mathrm{I}_{2}$ or $\mathrm{CH}_{3} \mathrm{I}$. These diffusivities (in SI units) are given by

$$
\begin{aligned}
& D_{\mathrm{I}, \mathrm{s}}=\frac{3.2801 \times 10^{-4} \mathrm{~T}^{1.5}}{\mathrm{P}\left(0.7075+\frac{454.72}{\mathrm{~T}}\right)} \\
& \mathrm{D}_{\mathrm{MI}, \mathrm{s}}=\frac{4.3306 \times 10^{-4} \mathrm{~T}^{1.5}}{\mathrm{P}\left(0.7075+\frac{475.7}{\mathrm{~T}}\right)}
\end{aligned}
$$




$$
\begin{gathered}
D_{\mathrm{I}, \mathrm{a}}=\frac{2.064 \times 10^{-4} \mathrm{~T}^{1.5}}{\mathrm{P}\left(0.7075+\frac{141.73}{\mathrm{~T}}\right)} \\
\mathrm{D}_{\mathrm{ML,a}}=\frac{2.6731 \times 10^{-4} \mathrm{~T}^{1.5}}{\mathrm{P}\left(0.7075+\frac{148.27}{\mathrm{~T}}\right)}
\end{gathered}
$$

where I2 stands for $\mathrm{I}_{2}$ and $\mathrm{MI}$ stands for $\mathrm{CH}_{3} \mathrm{I}$.

The liquid phase mass transfer coefficients, based on mass density difference, are now given. For iodine,

$$
\mathrm{k}_{\mathrm{L} 2, \ell}=\frac{6.9757 \mathrm{D}_{\mathrm{L} 2, \ell}}{\mathrm{D}}
$$

where the diffusivity of $I_{2}$ in liquid is given by

$$
\mathrm{D}_{\mathrm{1} 2 \ell}=\frac{3.906 \times 10^{-11} \mathrm{~T}_{\mathrm{D}}}{\mu_{\ell}}
$$

Here, $T_{D}$ is the drop temperature, and $\mu_{l}$ is the water viscosity at $T_{D}$. For $\mathrm{CH}_{3} \mathrm{I}$, the mass transfer coefficient is defined as

$$
\mathrm{k}_{\mathrm{ML}, \ell}=1.0801 \mathrm{k}_{\mathrm{IL}, \ell}
$$

\subsection{User-Specified Fission Product Sources}

Fission product mass sources are specified at the cell level under the FISSION keyword, which must be immediately followed by the keyword SOURCE. For sources of fission products, the general format for source tables is used. However, because fission products are assumed to have negligible specific heat, a temperature or enthalpy should not be specified for fission products. The specific format of fission product source tables is discussed in Section 14.3.1.9.

The linear chain decomposition of decay processes, as discussed in Section 8.3, may result in more than one occurrence of the same radionuclide within the set of linear chains. The distribution of source table mass for a given fission product among the occurrences is determined by the inventory factors associated with that fission product, unless a specific chain is requested. Note that the inventory factors for any radionuclide loaded from the fission product library are automatically 
supplied by the library. Such inventory factors should in general be specified, along with any userspecified linear chains, when a radionuclide appears more than once in the set of user-specified chains. For upward compatibility, the source table mass may also be directed to a specific chain. In a table with a specified chain number, all of the mass in placed in the specified chain.

Fission product mass specified through a source table is placed on the host specified through the HOST keyword. This keyword must be followed by a valid host number. Host number 1 is the atmosphere gas, and hosts number 2 to $1+$ "nac" represent the "nac" aerosol component hosts. After $1+$ "nac," there are two hosts for each cell structure (the inner surface host followed by outer surface host for each structure). These are followed by one host for each lower cell layer (beginning at the bottom), then the stationary atmosphere interface host, if a lower cell is specified. Finally, the last two hosts are the DUMMY host and the WASTE host (as discussed in Section 8.4). Since large host numbers are not uncommon in cells with multiple structures, extreme care should be taken in specifying host numbers for fission product sources on nonairborne hosts. Input files with nonairborne fission product sources written for versions prior to CONTAIN 1.1 should also be closely checked since host numbers greater than $1+$ "nac" have different meanings prior to and after CONTAIN 1.1. If a host is not specified, the host by default will be the atmosphere gas (the first host).

Note that the effects of decay, if any, on the masses specified in a source table are not modeled until the mass is introduced into the cell. It is the user's responsibility to provide source tables that reflect the radionuclide distribution at the time of introduction into the cell.

\subsection{Fission Product Transport}

This section discusses fission product transport processes that occur in conjunction with gas convection, aerosol deposition, or flow of liquid coolant. With the exceptions of the targeted release and acceptance model, discussed in Section 8.4, and the iodine depletion model for water sprays, discussed in Section 8.6, fission product transport models are based on the movement of the hosts to which the fission products are attached. Fission products attached to the gas host in a given cell are transferred with zero slip with intercell gas flow, as discussed in Section 8.8.1. Also, fission products hosted to an aerosol component are transferred according to the transport of that component in deposition or depletion processes, as discussed in Section 7.8. The transport of fission products with liquid coolant is discussed in Section 8.8.2.

\subsubsection{Gas Phase Transport of Fission Products}

The fission products attached to the gas host in a cell are transported with the gas without slip or depletion. The governing equation for the change in mass $m_{\mathrm{f}, \mathrm{i}, \mathrm{o}}$ of the $k$ th fission product species attached to the gas host in cell $i$ as a result of gas flow is therefore

$$
\left[\frac{\mathrm{dm}_{\mathrm{f}, \mathrm{i}, \mathrm{k}}}{\mathrm{dt}}\right]_{\text {flow }}=\sum_{\mathrm{ji}}\left(\mathrm{W}_{\mathrm{jij}} \frac{\mathrm{m}_{\mathrm{f}, \mathrm{u}, \mathrm{k}}}{\mathrm{m}_{\mathrm{u}}}\right)
$$


where the sum extends over all gas flow paths connected from cell $j$ to $i$ and includes the suppression pool vent path, if connected; $u$ denotes the upstream or donor cell; $\mathrm{W}_{\mathrm{ji}}$ denotes the gas flow rate; and $\mathrm{m}_{\mathrm{u}}$ denotes the gas mass in the upstream cell. Note that this equation does not allow for the presence of debris fields. As discussed in Chapter 6, when debris fields are present, the definition of $\mathrm{W}_{\mathrm{ij}}$ must be generalized.

\subsubsection{Liquid Transport of Fission Products}

The transport of fission products in the runoff of coolant films from structures or in pool-to-pool coolant flow may be modeled parametrically through transport efficiency factors, as discussed below. The materials transported in liquid pathways between repositories of coolant liquid are restricted to the coolant and to fission products carried by the coolant. Although materials such as deposited noncoolant aerosols are not transported, the fission products attached to deposited aerosols are reassigned to the repository to which the deposited aerosols are assigned. These fission products may then be transported. The transport of fission products previously associated with the deposited aerosols allows the user to assess the radiological inventories and the decay heating resulting from such transport. In situations in which the destination for such transport is not defined, such as in condensate film runoff directed to a cell (by the OVERFLOW keyword) without a pool, the fission products are assigned to the WASTE host of the destination cell. (The coolant, in addition, is tracked in the WASTE mass and energy accounting location in that cell.)

The transport of fission products in liquid pathways occurs in two instances. Fission products present on structure surfaces as the result of aerosol deposition and other mechanisms may be transported with the condensate film draining between and from the surfaces. Note that the formation of such a film, transfers between surfaces, and its runoff is controlled by the heat transfer structures input, discussed in Section 14.3.1.3, and the cell-level CONDENSE input, as discussed in Section 14.3.1.4. Fission products deposited in coolant pools may also be transported along with the coolant in the flow between pools, provided the flow is modeled through an engineered system liquid transport component, such as a PIPE, or through a pool flow path. Note that fission products on structure surfaces may also enter a pool when structures with previously deposited fission products are flooded by the pool. In such cases, the fission products allocated to the flooded surface area are transferred to the pool.

The optional FPLIQUID input block is used (see Section 14.2.6.2) to specify the "fpliq" transport efficiency factors that determine the transfer rate of fission products relative to the transfer rate of coolant. Only those fission products assigned a non-zero value of "fpliq" in the FPLIQUID input will be transferred with the film runoff and the coolant flow between pools. The transport efficiency factor for a fission product is defined as the ratio of the fraction of the fission product transferred from a repository, to the fraction of liquid transferred from that repository. Thus an efficiency factor of 1 will transport a fission product as if it is well-mixed with the coolant. The "fpliq" transport efficiency factors are assigned by fission product name and apply to all liquid pathway transfers of that fission product, except those resulting from flooding of structures, in which case all of the fission products associated with the flooded surface area are transferred. 
The governing equation for the change in mass $\mathrm{m}_{\mathrm{pf}, \mathrm{i}, \mathrm{k}}$ of the $k$ th fission product species attached to the pool host in cell $i$ as a result of pool flow is

$$
\left[\frac{\mathrm{dm}_{\mathrm{pf}, \mathrm{i}, \mathrm{k}}}{\mathrm{dt}}\right]_{\text {flow }}=\sum_{\mathrm{ji}}\left(\mathrm{W}_{\mathrm{ji}} \frac{\mathrm{m}_{\mathrm{pf}, \mathrm{u}, \mathrm{k}}}{\mathrm{m}_{\mathrm{pu}}}\right) f_{\text {liq, }, \mathrm{k}}
$$

where the sum extends over pool flow paths connected between cells $j$ and $i, u$ denotes the upstream pool, $\mathrm{m}_{\mathrm{p}, \mathrm{u}}$ denotes the upstream pool mass, and $\mathrm{f}_{\mathrm{liq}, \mathrm{k}, \mathrm{O}}, 0 \leq \mathrm{fl}_{\mathrm{iq}, \mathrm{k}} \leq 1$, denotes the value of "fpliq" assigned to the fission product in the FPLIQUID input. A similar relation holds for fission products transported with condensate film runoff. 


\subsection{COMBUSTION MODELS}

The CONTAIN code contains three combustion models: a deflagration model, a diffusion flame burning (DFB) model, and a bulk spontaneous recombination (BSR) model. Except for the BSR model, combustion models were imported from the HECTR code [Din86] and modified to accommodate the structural differences between the two codes. The deflagration model is activated by specifying the H-BURN input block; the DFB and BSR models are activated through the CONTBURN input subblock. Although default values for the parameters controlling each of the models are given for convenience, the user must ensure that these are appropriate values for the particular analysis or must supply different values. The deflagration model includes default values for ignition thresholds, inerting thresholds, flame speed, combustion completeness, and intercompartment burn propagation. The DFB model has default values for the ignition criteria for the diffusion flame. The BSR model has default values giving zero recombination rate for this form of combustion. Ignition criteria for the various types of burns are given in Table 14-1.

The assumptions that apply to the CONTAIN burn models include the following:

- Deflagration burns are assumed to occur with premixed conditions in a single confined volume like those for the tests used in Reference Won88 to obtain the correlations for flame speed and burn completeness; see also Reference Pon90. Note that the burn completeness equations given in Section 9.1.3 have been revised to avoid a problem with the expressions in Reference Won88 related to the fact that an increase in steam concentration (by replacing air with steam) results in an increase in the burn completeness, which is contrary to what would be expected.

- Deflagration burns are assumed to occur as they did for the temperature and pressure conditions that existed for the deflagration tests used in Reference Won88 to obtain the correlations for flame speed and burn completeness. Although the range of validity of the observed behavior was not specifically given in Reference Won88, the data typically were obtained at $1 \mathrm{~atm}$ pressure and between $20^{\circ} \mathrm{C}$ and $100^{\circ} \mathrm{C}$ temperature. There are no internal checks in CONTAIN on the conditions to see whether they are within the range of applicability.

- CONTAIN is limited to modeling only low-speed combustion: it does not model accelerated flames and detonation. It has been shown experimentally that mixtures that normally would burn relatively benignly in a single volume can accelerate in a multicompartment geometry. For example in the Battelle-Frankfurt Model Containment, hydrogen-air mixtures with approximately $10 \%$ hydrogen burned in the first volume with a flame velocity of 10 to $20 \mathrm{~m} / \mathrm{s}$ but accelerated to over $300 \mathrm{~m} / \mathrm{s}$ in subsequent volumes because of the turbulence generated at the junctions. These modes of combustion can generate impulsive type loads. Under these circumstances, CONTAIN's prediction of the loads, to which the containment will be subjected, may be nonconservative. 
- DFB is assumed to occur whenever ignition criteria are satisfied without the possibility of the burning flame being extinguished because of flashback or blowout. Flashback occurs when a flame is swallowed into the combustible gas source. Blowout is the opposite occurrence, when the flame front moves away so rapidly that it is extinguished. Burning is assumed to stop whenever ignition criteria are not satisfied.

- The cell gas volume for deflagration burning is assumed to be well mixed with no accounting for variation of concentrations such as may be present ahead of and behind the flame front.

- The incoming flow for DFB is assumed to come from a source that is well mixed. The single source may be the sum of several smaller sources.

- The condition for applicability of the low turbulence model given in Reference Won88 is the absence of fans or sprays. This criterion may not always be sufficient, because there could be other sources of turbulence, such as blowdown jets and natural circulation. Consideration should be given to the performance of a sensitivity study to investigate the importance of the turbulence level. Note that in CONTAIN the low turbulence model is always used except when containment sprays are active. In contrast to the recommendation in Reference Won88, the effect of fan coolers on turbulence is not taken into account.

Section 9.1 describes the deflagration model. Specifically, Section 9.1.1 describes the correlations used to determine whether a deflagration will occur by ignition or propagation from another burning region. Section 9.1.2 gives the equations used to calculate the hydrogen and carbon monoxide deflagration flame speed and corresponding total burn time, $\Delta t_{b, \text { tot }}$, given the initial molar concentrations. Section 9.1.3 gives the equations to calculate the combustion completeness of a burn. Section 9.1.4 describes the burn rate equations and the explicit cell mass changes, $m_{b n}$, resulting from the burn. Sections 9.2 and 9.3 describe the DFB model and the BSR model, respectively. Key elements of combustion models are illustrated in Figure 9-1.

\subsection{Deflagrations}

The treatment of hydrogen and carbon monoxide deflagration is derived from correlations developed by Wong [Won88] for Version 1.8 of the HECTR code. [Din86, Pon90] The deflagration model, activated with the H-BURN keyword as described in Section 14.3.1.7, initiates a deflagration when threshold conditions are satisfied and either the time is within the burn window, during which igniters or an equivalent ignition source are assumed present, or DFBs are modeled and the temperature condition for autoignition of a DFB is satisfied. The user may define the burn window by specifying "tactiv" and "tdeact" in the H-BURN input block. The burn continues for a time ("burnt") that is based on either an internally calculated or a user-specified flame speed ("flam"), and the burn can propagate to adjacent cells if conditions are favorable. 


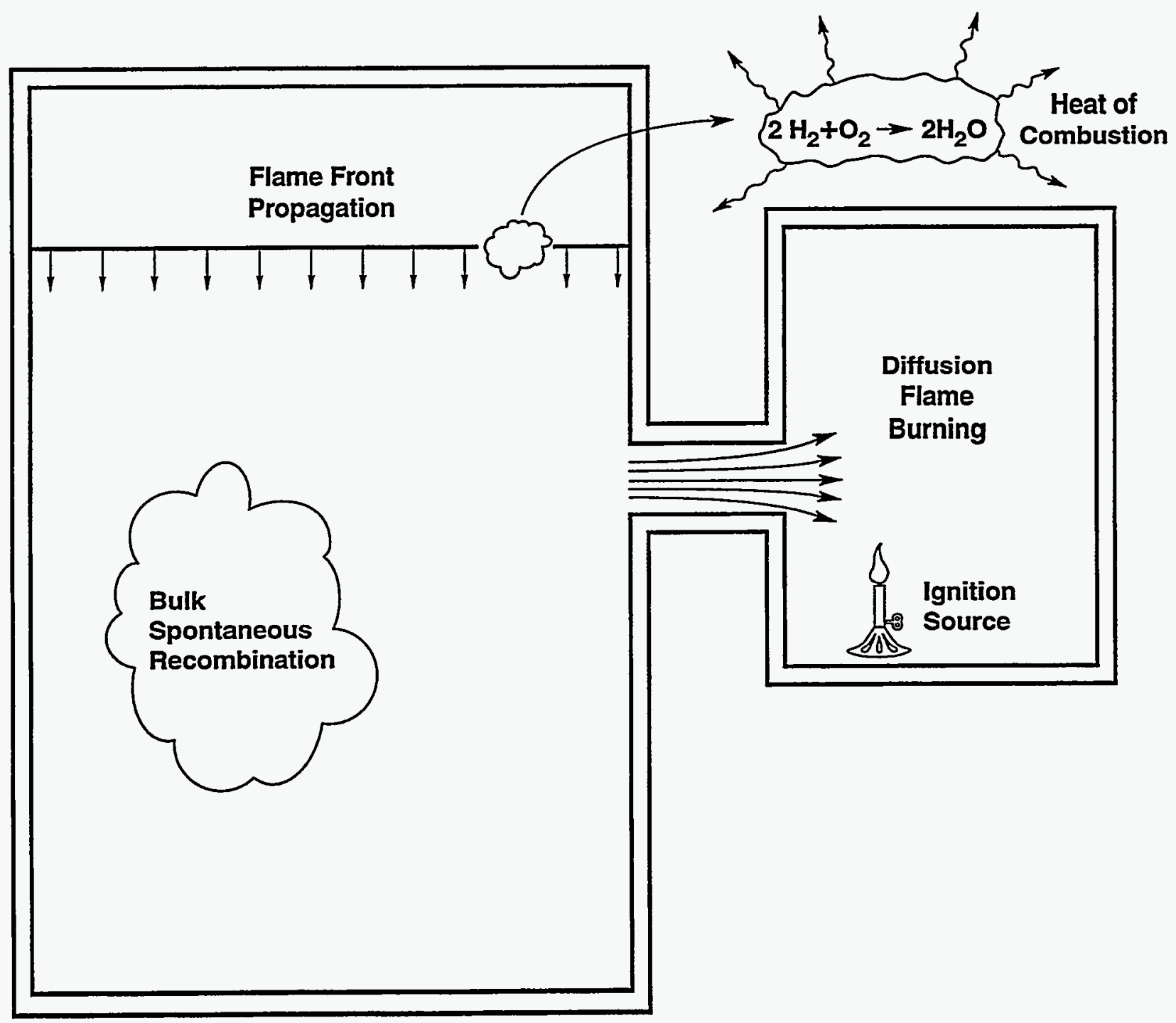

Figure 9-1. Combustion as Modeled in CONTAIN

Rev. 0 
The chemical reactions that occur during a deflagration are

$$
\begin{aligned}
& 2 \mathrm{H}_{2}+\mathrm{O}_{2} \rightarrow 2 \mathrm{H}_{2} \mathrm{O}+\mathrm{q}_{\mathrm{H} 2} \\
& 2 \mathrm{CO}+\mathrm{O}_{2} \rightarrow 2 \mathrm{CO}_{2}+\mathrm{q}_{\mathrm{CO}}
\end{aligned}
$$

\subsubsection{Deflagration Ignition and Propagation Criteria}

The ignition and propagation criteria used in CONTAIN are described in this subsection. The flame speed correlation is described in Section 9.1.2.

The molar concentrations required for igniting a mixture containing more than one combustible gas are related to the concentration required for a mixture with a single combustible gas through Le Chatelier's formula. That formula gives an effective combustible gas mole fraction $X_{c}$ defined in terms of the mole fractions of hydrogen $\left(\mathrm{X}_{\mathrm{H} 2}\right)$ and of carbon monoxide $\left(\mathrm{X}_{\mathrm{CO}}\right)$ :

$$
\mathrm{X}_{\mathrm{c}}=\mathrm{X}_{\mathrm{H} 2}+\mathrm{FX}_{\mathrm{CO}} \geq \mathrm{X}_{\mathrm{c}}^{\mathrm{crit}}
$$

where $\mathrm{F}$ is an empirical parameter that accounts for the presence of both hydrogen and carbon monoxide and $\mathrm{X}_{\mathrm{c}}^{\mathrm{crit}}$ is the threshold (or critical) mole fraction for ignition. Values for $\mathrm{F}$ in Equation (9-2) are derived from empirical values of the threshold for the two limiting cases in which only $\mathrm{H}_{2}$ or only $\mathrm{CO}$ is present. Values of $\mathrm{X}_{\mathrm{c}}^{\text {crit }}$ and the associated values of $\mathrm{F}$ for ignition, and for downward, upward, and horizontal propagation are given in Table 9-1.

The molar concentration $\mathrm{X}_{\mathrm{O} 2}$ of oxygen and effective molar concentration $\mathrm{X}_{\mathrm{d}}$ of diluents must also satisfy

$$
\begin{aligned}
& X_{\mathrm{O} 2} \geq X_{\mathrm{O} 2}^{\text {crit }} \\
& X_{d}=X_{\mathrm{H} 2 \mathrm{O}}+X_{\mathrm{CO} 2}+0.79 \Delta X_{\mathrm{N} 2} \leq X_{d}^{\text {crit }}
\end{aligned}
$$

where ${ }^{1} \Delta X_{N 2}$ is equal to $\max \left(X_{N 2}-3.774 X_{02}, 0\right)$. Table 9-1 also gives the threshold mole fraction

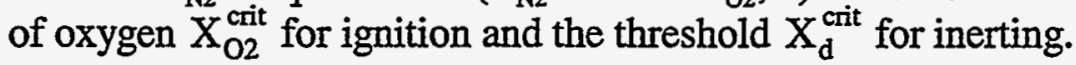

For the default values in Table 9-1, a burn is initiated if the effective combustible mole fraction is $\geq 7 \%$, the oxygen mole fraction is $z 5 \%$, and the diluent mole fraction is $\leq 55 \%$. The basis for the combustible gas concentration to be above $7 \%$ by volume is the data in Reference Low 82 . The default values for the diluent and oxygen are consistent with the data of Tamm, McFarlane, and Liu [Tam85] and Tamm, Ungurian, and Kumar. [Tam87] Ignition was observed in hydrogen-air-steam

${ }^{1}$ M. P. Sherman, Sandia National Laboratories, Albuquerque, NM, letter to R. F. Beyer, Bettis Atomic Power Laboratory with subject, "Improved Inerting Criteria for Vitiated Air," September 7, 1990. 
Table 9-1

Default Ignition and Propagation Limits

\begin{tabular}{||l|c|c|c|c||}
\hline \multicolumn{1}{|c|}{ Limits } & $\mathrm{F}$ & $\mathrm{X}_{\mathrm{c}}^{\text {crit }}$ & $\mathrm{X}_{\mathrm{O} 2}^{\text {crit }}$ & $\mathrm{X}_{\mathrm{d}}^{\text {crit }}$ \\
\hline Ignition & 0.541 & $\geq 0.07$ & $\geq 0.05$ & $\leq 0.55$ \\
\hline Upward propagation & 0.328 & 20.041 & $\geq 0.05$ & $\leq 0.55$ \\
\hline Horizontal propagation & 0.435 & $\geq 0.06$ & $\geq 0.05$ & $\leq 0.55$ \\
\hline Downward propagation & 0.600 & $\geq 0.09$ & $\geq 0.05$ & $\leq 0.55$ \\
\hline
\end{tabular}

mixtures with up to 55\% steam [Tam85] and as little as 5\% oxygen [Tam87] using hot-surface ignition devices. The propagation default values were obtained from Coward and Jones, [Cow52] who listed $4.1 \%, 6.0 \%$, and $9.0 \%$ hydrogen for the upward, horizontal, and downward propagation limits, respectively. Coward and Jones also listed the upward, horizontal, and downward propagation limits for carbon monoxide as $12.5 \%, 13.5 \%$, and $15.0 \%$, respectively. Using these values for carbon monoxide limits, values of $F$ of $0.328,0.435$, and 0.600 are obtained, respectively, when LeChatelier's formula for mixture limits is used. Dingman et al. [Din86] state that the predicted upward propagation lower flammability limit for hydrogen-carbon-monoxide-air mixtures agrees with experimental results to within $0.4 \%$. The oxygen threshold criteria for propagation is consistent with the data in References Kum85 and Her83. Kumar's data [Kum85] show that hydrogen-rich mixtures are flammable as long as the oxygen concentration is greater than $5 \%$ while Hertzberg and Cashdollar's data [Her83] indicate the value should be around 4.6\%. The inerting diluent concentration of $55 \%$ is approximately in the middle of a range of experimentally obtained values that will be discussed later.

Note that the default burn parameters are based on experiments performed with ignition sources. As a result, the user must employ judgment regarding a burn without continuous ignition sources because, for example, higher concentrations might exist before accidental ignition. For example, the 7\% threshold cannot be applied universally to all circumstances involving the ignition of hydrogen mixtures. It appears reasonable to assume that when igniters are present combustion occurs near the igniter when a concentration of approximately $5 \%$ hydrogen is reached. For instance, a value between $4.5 \%$ and $5.5 \%$ hydrogen was obtained for hydrogen-air-steam premixtures in the presence of igniters [Tam85] and in continuous injection tests in which ignition [She87] or re-ignition [Tam88] occurred when background hydrogen concentrations exceeded approximately $4.5 \%$. The same limits should also be appropriate for pilot ignition (for example, from a diffusion flame) of a premixture. Even though the igniters initiate a deflagration at approximately $5 \%$ hydrogen concentration near the igniter, this local concentration may differ considerably from the uniform concentration of combustible gas used by CONTAIN to compare to the ignition threshold. Therefore, the appropriate ignition threshold value to use may be sensitive to igniter location, the location and rate of gas release, and the time in the accident sequence. Similar points can be made when no deliberate ignition source exists. For example, in the TMI-2 event, it was estimated that the hydrogen concentration was $7.9 \%$ before ignition occurred. [Hen87] Because accidental ignition is a stochastic process, it seems reasonable that even larger concentrations of hydrogen could build 
up before ignition occurs. For example, the energy and power of the ignition source can determine what mixture compositions can be ignited. If the accidental ignition sources are weak, then higher concentrations of hydrogen may be required before ignition occurs. To provide an adequate assessment, a parametric study of the ignition criteria should be performed.

For sensitivity studies, the various concentration thresholds can be changed through input to the code, as described in Section 14.3.1.7. Note that the concentration thresholds specified for propagation apply to the adjacent cells, not the cell in which the burn originates. A range of values has been obtained for the concentrations of combustible gas and oxygen at the flammability limits although the range does not appear to be very large. A range between $4.1 \%$ [Cow52] and 5\% [Hus88] hydrogen for the upward propagation lower flammability limit and between 8\% [Her83] and 9.4\% [Sha57] hydrogen for the downward propagation direction has been reported. Carbon monoxide values range from $12.5 \%$ [Cow52] to $15 \%$ [Hus 88 ] for the downward propagation lower flammability limits. As mentioned earlier, the range is between 4.6\% [Her83] and 5\% [Kum85] for oxygen concentration thresholds. Relative to the range of combustible gas and oxygen concentrations at the flammability limit, the inerting value of the diluent can have a significant spread. For example, at approximately the same initial thermodynamic state the inerting concentration of steam in a hydrogen-air mixture varies from approximately $49 \%$ [Sha57] to 63\%. [Kum85] This compares to the 55\% value that is used in the CONTAIN default values. Kumar [Kum85] and Yeaw and Shnidman [Yea38] report that approximately 71\% excess nitrogen inerts a hydrogen-air mixture while DeSoete [DeS75] and Coward and Jones [Cow52] report approximately $72 \%$ and $74 \%$ excess nitrogen, respectively. According to Equation (9-2), if no steam or carbon dioxide is present, approximately $70 \%$ excess nitrogen is required to inert a hydrogen-air mixture. This value is obtained from the equation $\Delta \mathrm{X}_{\mathrm{N} 2}=0.55 / 0.79$ and is in reasonable agreement with the experimentally obtained values. Coward and Jones [Cow52] report an inerting concentration of carbon dioxide between $56 \%$ and $60 \%$ while the results of Benedick et al. [Ben84] indicate the inerting value to be between $52 \%$ and $54 \%$ carbon dioxide in hydrogen-air mixtures. Yeaw and Shnidman [Yea38] report a value of 56.5\% carbon dioxide. Inerting concentrations of carbon dioxide in fuel-air mixtures were obtained at ambient temperatures in contrast to steaminerting concentrations, which were generally obtained at $100^{\circ} \mathrm{C}$. Inerting concentrations of $52 \%$ [Cow52] and 41\% [Yea38] carbon dioxide in carbon monoxide-air mixtures were reported. Yeaw and Shnidman [Yea38] also reported that carbon monoxide-air mixtures were inerted with $57.5 \%$ nitrogen and $53.1 \%$ steam. The practice of using a common default value in CONTAIN for the inerting concentration of steam, carbon dioxide, and nitrogen assumes that one diluent is a good simulant for another. This practice is acceptable if the uncertainty in the inerting concentration of each diluent is not significant relative to a specific calculation.

The H-BURN input block must be specified for a cell in which hydrogen and carbon monoxide burns are to be considered (see Section 14.3.1.7). Besides the parameters specified in Table 9-1, a number of other parameters controlling the burn can be specified by the user in the H-BURN input. The cell burn time "burnt" can be specified by the user or, alternatively, is internally calculated; in the latter calculation, a cell characteristic length "chrl" is divided by the flame speed. The default for "chrl" is calculated by taking the cube root of the initial gas volume. The flame speed "flam" can be specified by the user or, alternatively, is internally calculated; that calculation uses an experimentally derived correlation that depends on initial combustible gas, oxygen, and steam concentrations. The 
fraction of initial combustible "cfrmng" left following a burn can be specified by the user or, alternatively, is internally calculated from a correlation based on the initial concentration as described in Section 9.1.3. The final combustible concentration may never be reached if the burn is oxygen-limited. By default, a burn is considered to be oxygen-limited when the oxygen mole fraction falls below a value "mormng," which by default is 0.0005 in CONTAIN 2.0 and 0.005 in prior code versions. The amount of hydrogen and carbon monoxide burned in each timestep is based on the remaining concentrations, the final combustible gas concentration desired, and the remaining burn time.

In most situations, the user should allow the code to calculate the values of "burnt," "flam," and "cfrmng." The user should have a knowledge of the correlations used and reasons for overriding them before specifying alternative values. When these values are calculated, they are calculated for each burn based on the conditions existing at the start of the burn. When specified, they are held constant throughout the run. Note that the lack of alteration to the flame speed or burn time during the course of a burn could result in errors if significant amounts of gases, including combustible gases, are introduced during a burn.

Note that by default the oxygen and diluent limits are the same for ignition and propagation from any of the directions listed. The code does allow each of these default limits to be overridden by userspecified values. A fixed burn time can also be specified by the user to override the burn time that is normally calculated from the flame speed as described in Section 9.1.2.

It is possible for a burn to propagate from one cell to an adjacent cell if (1) a connecting gas flow path is present, (2) either the gas flow is into the adjacent cell or the flame velocity exceeds the absolute value of the gas flow velocity, and (3) the combustible gas, oxygen, and water vapor concentrations in the adjacent cell allow propagation. However, propagation through a submerged boiling water reactor suppression pool vent or any submerged gas flow path is not allowed. The interconnections between the cells should be defined in the ENGVENT input block as described in Section 14.2.4.2. The criteria in Table 9-1 for the propagation of a burn to another cell depend on whether that cell is located above, alongside, or below the originating cell. The relative locations of the cells are specified through the cell elevation variable "elev," which has a default value of zero. These elevations, given in the H-BURN input block, should not be confused with other cell evaluations that govern flows. For horizontal propagation to occur between two cells, their elevations must be identical. If the elevation of cell $i$ is greater than that of cell $j$, propagation from cell $j$ to cell $i$ is upward, and propagation from cell $i$ to cell $j$ is downward. The time delay factor "kprop," which must have a value between zero and one, delays the propagation of a burn to an adjoining cell by a fraction of the total burn time in the cell from which the burn propagates. The default time delay factor is 0.5 . Because the propagation criteria have not been thoroughly verified, the performance of sensitivity studies to determine the importance of the propagation should be considered. The ability to propagate a burn into an adjacent compartment, and the total time for a burn sequence in a containment are sensitive to this parameter. Because CONTAIN is a lumped parameter code, burned and unburned gases are mixed uniformly at each timestep in the compartment where the burn takes place. These gases flow into the adjacent compartments. If the time delay factor is too large, mixtures in the adjacent compartments may be rendered nonflammable because of the nonphysical introduction of burned gases and the burn may not propagate. If the time 
delay factor is too small, the total burn time may be too short and the peak containment pressure and temperature may be over-predicted.

Figure 9-2 illustrates the burn propagation model. A flame that starts in cell 1 can propagate to cell 2 if the proper conditions exist in cell 2. The characteristic length of the cell "chrl" in the flame direction in this illustration is X. As the cells are drawn, it will take half the total burn time in cell 1 for the flame to reach the passageway to cell 2 , which corresponds to the default time delay factor "kprop" value of 0.5 . In the event of a relatively long passageway, the passage length could also be taken into account by increasing the value of "kprop." If there is more than one downstream cell, an average value of "kprop" should be used. When different cell geometries or elevations are specified, the time for propagation to adjacent cells may be greater or less than half the burn time. This fact is not accounted for in the modeling, although the default delay factor may be overridden by a user-specified value.

When conditions for a burn are satisfied, the burn does not start immediately but is delayed until the start of the next system timestep. (This could cause larger burns than warranted if the system timestep is very large.) That system timestep and those that follow are set to the minimum of all of the timesteps calculated internally for cells in which burns are in progress or have just finished. The internal timestep for a cell by default is set to one-tenth of the burn time for a period corresponding to twice the burn time. Burns within a given cell cannot be reinitiated until twice the burn time has elapsed following ignition in that cell. The user may adjust the timestep during the burn through the TSFRAC keyword and set the edit frequency through the EDMULT keyword in the TIMES input block (see Section 14.2.8). A message indicating that a burn has started is written to the event summary file TAPE21 at the time a burn starts.

\subsubsection{Flame Speed and Burn Time}

The CONTAIN default treatment of deflagration flame speeds is to use flame speed correlations derived from hydrogen-air-steam experimental data. These correlations are given in Reference Won88. The basis for these correlations comes from VGES, [Ben84] FITS, [Mar86] and NTS [Rat85, Tho88a, Tho88b] data. No equations are given for DFB or BSR because these are simple recombination equations.

All deflagrations are assumed to occur over a discrete period of time, denoted here as the burn time $\Delta t_{b, t o t}$, following ignition or propagation into a cell. The duration of each newly ignited deflagration is based by default on an experimentally derived flame speed correlation that depends on initial concentrations of combustible gas, oxygen, and inerting gases. The flame speed may also be specified by the user as "flam" in the H-BURN input block to override the calculated value. The user may also override the burn time modeling altogether by providing a user-specified discrete burn time ("burnt"). If the burn parameters are calculated by the code, they are calculated for each burn based on the conditions existing at the start of the burn. Note that the flame speed and burn time are not allowed to vary over the course of any one burn. When the burn parameters are user-specified, they are held constant throughout the calculation and apply to each deflagration that occurs. The remainder of this section describes the correlations that are used by default to calculate the flame speed and total burn time. 


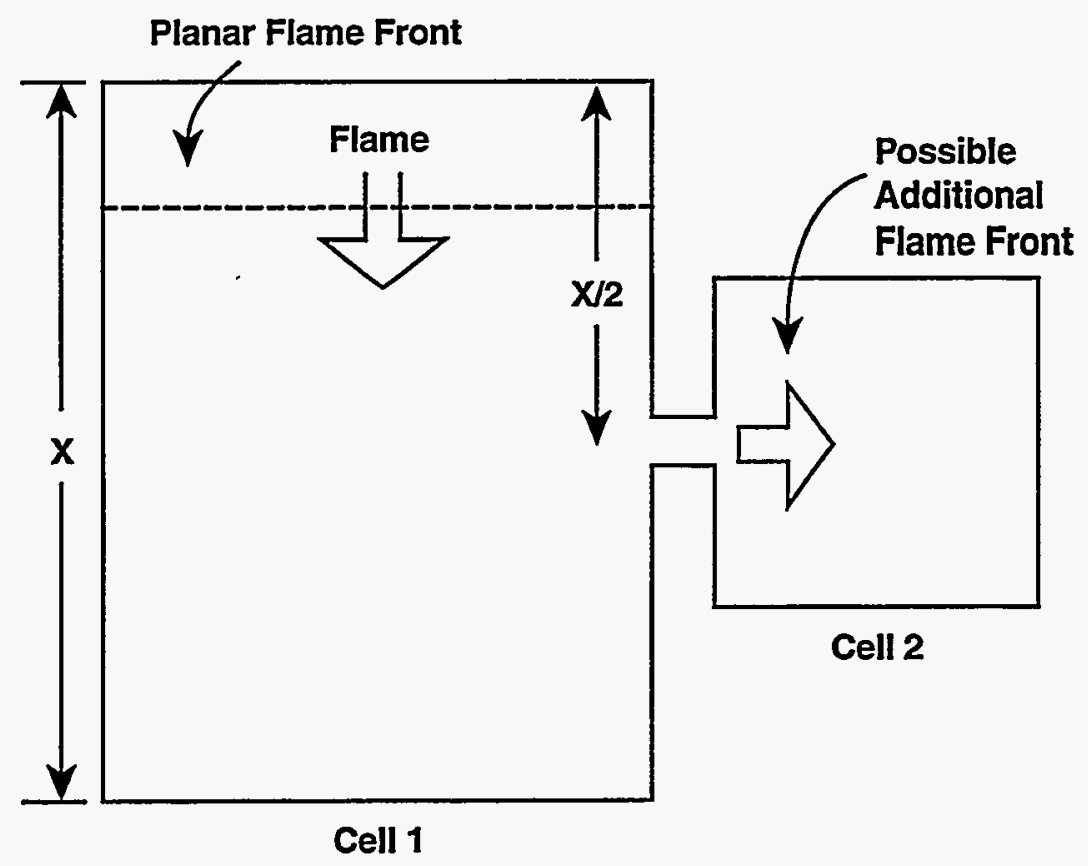

Figure 9-2. Flame Propagation Diagram 
The deflagration model assumes that the flame travels a characteristic distance in the compartment along a plane wave front at speed $v_{\mathrm{f}}$. The total burn time $\Delta \mathrm{t}_{\mathrm{b}, \text { tot }}$ for a given compartment is therefore calculated by dividing the characteristic burn length $\mathrm{L}$ by the flame speed

$$
\Delta \mathrm{t}_{\mathrm{bn}, \mathrm{tot}}=\frac{\mathrm{L}}{\mathrm{v}_{\mathrm{f}}}
$$

Normally the model assumes that the characteristic burn length is equal to the characteristic cell length, which by default is taken to be the cube root of the initial gas volume. The user is allowed to override this default value and specify a characteristic burn length ("chrl") that is more appropriate for the specific geometry of the cell. For example, if a cell is much taller than it is wide, a longer burn length than the cube root of the volume may be appropriate. Note that in the following, containment sprays are assumed to affect the turbulence level and thus the flame speed, whereas Reference Won88 assumes that fans along with sprays have this effect.

The flame speed correlations discussed in this section and the combustion completeness correlations discussed in the next section were developed from hydrogen-air-steam data. In some scenarios, carbon monoxide may also be produced. To compensate for the lack of flame speed and combustion correlations for carbon monoxide equivalent to those for hydrogen, an approximate method to account for the carbon monoxide is used. This method uses an effective combustible mole fraction $X_{c}$ as defined in Equation (9-1), where an $F$ equal to 0.541 , the ignition value, is used for the flame speed and combustion completeness correlations. Note that this value of $F$ is only based on an analogy with the ignition criteria for mixtures including carbon monoxide, not on actual experimental data for flame speed and combustion completeness for such mixtures. If large concentrations of carbon monoxide are present, user-specified values may be more appropriate.

The steam correction factor developed in Wong's NTS report [Won88] for the flame speed and combustion completeness correlations has been modified to include the dilution effects of nitrogen and carbon dioxide. The diluent mole fraction $X_{d}$ is given by Equation (9-2).

The correlations for flame speed consist of two factors. The first factor is a function of the combustible concentration in dry air, $\mathrm{X}_{c, \text { dry }}$, which is the concentration one would have if all diluent were to be removed:

$$
X_{c, d r y}=\frac{X_{c}}{1-X_{\mathrm{H}_{20}}-X_{\mathrm{CO} 2}-\Delta X_{\mathrm{N} 2}}
$$

The second factor is a diluent correction factor that takes into account the retarding effect of the diluent gases. The equations controlling flame speed are

(1) For $0 \%<X_{c, d r y} \leq 10 \%$ : 
With sprays on,

$$
v_{f}=L^{1 / 3}\left(59.65 X_{c, d r y}-1.248\right) \exp \left[X_{d}\left(a+b X_{d}\right)\right]
$$

where $\mathrm{a}=-4.877$ and $\mathrm{b}=-3.008$.

With sprays off,

$$
v_{f}=L^{1 / 3}\left(23.70 X_{c, d r y}-0.862\right) \exp \left[X_{d}\left(a+b X_{d}\right)\right]
$$

(2) For $10 \%<\mathrm{X}_{\mathrm{c}, \mathrm{dry}} \leq 18 \%$ :

With sprays on,

$$
v_{f}=L^{1 / 3}\left(2074 X_{c, d r y}^{2}-347.23 X_{c, d r y}+18.700\right) \exp \left[X_{d}\left(a+b X_{d}\right)\right]
$$

where $\mathrm{a}=-4.877$ and $\mathrm{b}=-3.008$.

With sprays off,

$$
v_{f}=L^{1 / 3}\left(1724 X_{c, d r y}^{2}-267.28 X_{c, d r y}+10.996\right) \exp \left[X_{d}\left(a+b X_{d}\right)\right]
$$

(3) For $18 \%<\mathrm{X}_{\mathrm{c}, \mathrm{dry}} \leq 25 \%$ :

Use linear interpolation between (2) and (4) with sprays on or off.

(4) For $25 \%<\mathrm{X}_{\mathrm{c}, \mathrm{dry}} \leq 35 \%$ :

With sprays on or off,

$$
v_{f}=L^{1 / 3}\left(289.73 X_{c, d r y}-33.769\right) \exp \left[X_{d}\left(a+b X_{d}\right)\right]
$$

where $a=-0.641$ and $b=-18.38$.

(5) For $35 \%<\mathrm{X}_{\mathrm{c}, \mathrm{dry}} \leq 45 \%$ :

Use linear interpolation between (4) and (6) with sprays on or off.

(6) For $45 \%<\mathrm{X}_{\mathrm{c}, \mathrm{dry}} \leq 72 \%$ :

With sprays on or off,

$$
v_{f}=L^{1 / 3}\left(145.07-199.62 X_{c, d r y}\right) \exp \left[X_{d}\left(a+b \quad X_{d}\right)\right]
$$

$$
\text { where } a=-17.279 \text { and } b=18.07 \text {. }
$$


The above correlations assume only two levels of turbulence. It is reasonable to expect, however, that the flame speed will increase with increasing turbulence up to the flame-quenching level. Turbulence can be generated not only by sprays, but also through forced convection. Gido and Koestel [Gid84] discuss how turbulence can be estimated and show how the turbulence affects the flame speed.

There is significant scatter in some of the data used to generate the correlations. In addition to the scatter, data are often sparse, especially at high steam concentrations, contributing to uncertainty in the results of the correlations. This can be particularly important in scenarios in which inert mixtures are rendered flammable as a result of steam condensation on heat sinks. Other cases occur when large quantities of steam are released into the containment prior to the release of hydrogen. Wong [Won88] recommends a parametric study be performed when using the flame speed correlations.

\subsubsection{Combustion Completeness}

The CONTAIN default values for combustion completeness were obtained from correlations derived from experimental data. The basis for these correlations comes from VGES, [Ben84] FITS, [Mar86] NTS, [Rat85, Tho88a, Tho88b] Whiteshell, [Kum84] and ACUREX [Tor83] data.

The completeness correlation used in CONTAIN 2.0 is

$$
\text { Combustion Completeness }=\max \left(\min \left(C_{1} X_{c}-C_{2}, 1\right), 0\right)
$$

where

$$
\begin{aligned}
& C_{1}=24.91, C_{2}=1.070 \text { with sprays off, and } \\
& C_{1}=25.42, C_{2}=0.925 \text { with sprays on } .
\end{aligned}
$$

The values of the constants were derived by fitting to the data given in Figures 2.3 through 2.6 of Reference Won88. This correlation gives a combustion completeness independent of steam mole fraction provided the hydrogen mole fraction is held constant, which is consistent with the data used to derive the correlation. However, the maximum steam mole fraction in these data was $40 \%$. It would be expected that the burn completeness would be reduced at still higher steam concentrations. However, the CONTAIN correlation does not capture this effect. Note also that in CONTAIN 1.2 and some previous versions a different correlation, which is given on page 19 of Reference Won88, was used. However, that correlation was found to predict that burn completeness can increase with increasing steam mole fraction under some conditions. This is considered to be unrealistic; hence that correlation has been abandoned.

Reference Won88 points out that (a) the size of the test volume had minimal effect on the deflagration burn completeness, (b) the size of the test volume had somewhat more of an effect on the deflagration flame speed, and (c) more data are needed to understand these effects. Also, Reference Pon90 notes that the correlations in this section are applicable to a complete enclosure that is modeled with one cell. The enclosure should not be divided into multiple cells without checking on the possible artifacts introduced by such a nodalization. Such artifacts include spurious dilution 
of the gas ahead of the flame front by burned gases, as a result of the well-mixed assumption used by the intercell flow model.

\subsubsection{Burn Rate}

This subsection describes how the deflagration burn time is applied to determine the burn rate and the masses consumed. The total burn time is defined in Equation (9-3).

The basic approach taken to determine the burn rate is to estimate the number of moles of combustibles and oxygen available to burn over the remainder of the burn time. The number of moles available to participate in the burn is determined from the number of moles present at a given point during the burn and by the mole fraction of combustibles or oxygen that must remain at the end of the burn. The burning rate is then set assuming a steady depletion rate of the most limiting constituent over the remaining burning time.

The actual processing of the deflagration always begins at the start of a system timestep. Thus, if one of the ignition criteria described in Section 9.1.1 is met, the cell mixture will begin to burn at the start of the next system timestep. At the start of the deflagration, the model determines the minimum mole fraction of combustibles that can remain at the end of the burn. This minimum unburned mole fraction is defined as $\mathrm{X}_{\mathrm{H} 2 \text {,inal }}$ for hydrogen and $\mathrm{X}_{\mathrm{CO}, \text { inal }}$ for carbon monoxide. They satisfy the relations:

$$
\begin{aligned}
& X_{\mathrm{H} 2 \text {, final }}=X_{\mathrm{f}} \mathrm{X}_{\mathrm{H} 2 \text {, initial }} \\
& \mathrm{X}_{\mathrm{CO}, \text { final }}=\mathrm{X}_{\mathrm{f}} \mathrm{X}_{\mathrm{CO} \text {, initial }}
\end{aligned}
$$

where

$$
\mathrm{X}_{\mathrm{f}}=1.0-\min (\max (\text { Combustion Completeness, 0), 0.995) }
$$

Note $\mathrm{X}_{\mathrm{f}}$ is the fraction of the combustible gas initially present that does not burn.

The $\mathrm{X}_{\mathrm{H} 2 \text {,initial }}$ parameter is the mole fraction of hydrogen at the start of the burn. The $\mathrm{X}_{\mathrm{Co} \text {,initial }}$ parameter is the mole fraction of carbon monoxide gas at the start of the burn. Note that these values may give a combustible concentration differing from the ignition threshold value because of the delay in initiating the burn.

The chemical reaction rate is adjusted each burn timestep to account for intercompartment flows and source injection, so that the burn finishes at the predetermined time with the predicted final mole fraction of combustible gases. Consumption or production of combustible gases and oxygen by other phenomenological processes are also accounted for in this adjustment. Note that intercompartment flow adjustments are only made at the end of the system timestep; however, the burn model automatically reduces the system timestep to force problem stability and to force flow adjustments 
to occur in the burn model at a reasonable frequency. This interaction between the intercell flow model and the burn model should not be confused with the capability to represent long continuous burns over periods of changing conditions within the containment. The CONTAIN model treats each deflagration as a discrete event, and the burn time of that event is fixed once the deflagration is calculated to occur.

The burn rate of combustible gas is calculated by estimating the number of moles of combustible gas that will remain at the end of the burn, and adjusting the burn rate such that all combustible gas in excess of this amount will be consumed at the end of the prescribed burn time. The amount of combustible gas remaining at the end of the burn is tentatively set equal to the final mole fractions of combustible gas given by Equations (9-12) and (9-13) multiplied by the total number of gas moles present at the end of the burn. The total number of gas moles at the end of the burn, $\mathrm{N}_{\text {final }}$, is estimated by taking into account the anticipated reduction in total moles resulting from combustion. This value is updated each timestep as the time progresses toward the end of the deflagration total burn time, $\Delta t_{\mathrm{bn}, \mathrm{to}}$, using the following balance equation:

$$
\mathrm{N}_{\text {final }}=\frac{\mathrm{N}-0.5 \mathrm{~N}\left(\mathrm{X}_{\mathrm{H} 2}+\mathrm{X}_{\mathrm{CO}}\right)}{\left[1-0.5\left(\mathrm{X}_{\mathrm{H} 2 \text {,final }}+\mathrm{X}_{\mathrm{CO}, \text { final }}\right)\right]}
$$

where $\mathrm{N}$ is the total moles of gas and $\mathrm{X}_{\mathrm{H} 2}$ and $\mathrm{X}_{\mathrm{CO}}$ are the molar fractions present at a given time into the burn. The unburned mole fractions of hydrogen $\mathrm{X}_{\mathrm{H} 2, \text { final }}$ and carbon monoxide $\mathrm{X}_{\mathrm{CO}, \text { final }}$ are defined in Equations (9-12) and (9-13), respectively.

An estimate of the number of moles of hydrogen remaining at the end of the burn $\mathrm{F}_{\mathrm{H} 2}$ readily follows from Equation (9-14).

$$
F_{\mathrm{H} 2}=N_{\text {final }} X_{\mathrm{H} 2 \text {, final }}
$$

The number of moles $\Delta \mathrm{N}_{\mathrm{Fz}}$ available to burn for the remainder of the burn time is tentatively given by the present number of moles of hydrogen, minus the number of moles that must remain after the burn:

$$
\Delta \mathrm{N}_{\mathrm{H} 2}=\mathrm{N}_{\mathrm{H} 2}-\mathrm{F}_{\mathrm{H} 2}
$$

An analogous approach is used to calculate the moles of carbon monoxide and oxygen tentatively available to participate in the burn for the remainder of the burn time.

$$
F_{\text {Co }}=N_{\text {final }} X_{C O \text {,final }}
$$




$$
\begin{aligned}
& \Delta N_{C O}=N_{C O}-F_{C O} \\
& F_{O 2}=N_{\text {final }} X_{O 2, \text { final }} \\
& \Delta N_{O 2}=N_{O 2}-F_{O 2}
\end{aligned}
$$

Note that $\mathrm{X}_{\mathrm{O2, \text {final }}}$ is an input parameter equal to 0.0005 by default in CONTAIN 2.0 and equal to 0.005 in previous code versions. The value can be changed by the user as described in Section 14.3.1.7.

Finally, the total moles of combustible gas tentatively available for burning over the remainder of the burn time is

$$
\Delta \mathrm{N}_{\mathrm{c}}=\Delta \mathrm{N}_{\mathrm{H} 2}+\Delta \mathrm{N}_{\mathrm{CO}}
$$

If $\Delta \mathrm{N}_{\mathrm{c}}$ is greater than $2 \Delta \mathrm{N}_{\mathrm{O} 2}$, then the burn will be oxygen-limited. In this case, the moles of hydrogen to burn will be reduced according to

$$
\Delta \mathrm{N}_{\mathrm{H} 2, \mathrm{bum}}=\Delta \mathrm{N}_{\mathrm{H} 2} \frac{2 \Delta \mathrm{N}_{\mathrm{O} 2}}{\Delta \mathrm{N}_{\mathrm{c}}}
$$

and the carbon monoxide moles to burn will also be reduced by the same factor:

$$
\Delta \mathrm{N}_{\mathrm{CO}, \mathrm{bum}}=\Delta \mathrm{N}_{\mathrm{Co}} \frac{2 \Delta \mathrm{N}_{\mathrm{O} 2}}{\Delta \mathrm{N}_{\mathrm{c}}}
$$

Otherwise, $\Delta \mathrm{N}_{\mathrm{H} 2}$ and $\Delta \mathrm{N}_{\mathrm{CO}}$ are used directly for the amounts to burn.

The molar burn rate for hydrogen is

$$
\dot{\mathrm{N}}_{\mathrm{H} 2}=\Delta \frac{\mathrm{N}_{\mathrm{H} 2, \mathrm{burm}}}{\Delta \mathrm{t}_{\mathrm{bn}}}
$$

Rev. 0 
where $\Delta \mathrm{t}_{\mathrm{bn}}$ is the remaining burn time and the molar burn rate of carbon monoxide $\dot{\mathrm{N}}_{\mathrm{CO}}$ is

$$
\dot{\mathrm{N}}_{\mathrm{CO}}=\frac{\Delta \mathrm{N}_{\mathrm{CO}, \mathrm{burm}}}{\Delta \mathrm{t}_{\mathrm{bn}}}
$$

These rates are used in Equation (9-27) below to calculate the deflagration energy release. They also are used to determine the explicit mass source or removal rates resulting from deflagrations in the mass conservation equation, shown in Table 4-3. The molar rates given in Equations (9-24) and (9-25) are converted to mass rates of hydrogen, carbon monoxide, oxygen, steam, and carbon dioxide, as follows:

$$
\begin{aligned}
& \mathrm{W}_{\mathrm{bn}, \mathrm{so}, \mathrm{H} 2}=\dot{\mathrm{N}}_{\mathrm{H} 2} \mathrm{M}_{\mathrm{H} 2} \\
& \mathrm{~W}_{\mathrm{bn}, \mathrm{so}, \mathrm{CO}}=\dot{\mathrm{N}}_{\mathrm{CO}} \mathrm{M}_{\mathrm{CO}} \\
& \mathrm{W}_{\mathrm{bn}, \mathrm{so}, \mathrm{O} 2}=\left[\frac{1}{2} \dot{\mathrm{N}}_{\mathrm{H} 2}+\frac{1}{2} \dot{\mathrm{N}}_{\mathrm{CO}}\right] \mathrm{M}_{\mathrm{O} 2} \\
& \mathrm{~W}_{\mathrm{bn}, \mathrm{so}, \mathrm{H} 2 \mathrm{O}}=\dot{\mathrm{N}}_{\mathrm{H} 2} \mathrm{M}_{\mathrm{H} 2 \mathrm{O}} \\
& \mathrm{W}_{\mathrm{bn}, \mathrm{so}, \mathrm{CO} 2}=\dot{\mathrm{N}}_{\mathrm{CO}} \mathrm{M}_{\mathrm{CO} 2}
\end{aligned}
$$

where $M$ represents the molecular weight. These reactions are exothermic, and the energy released is deposited into the atmosphere. The following expression gives the energy release rate in a burn as a function of the rate at which moles of combustibles are consumed:

$$
\mathrm{q}_{\mathrm{bn}, \mathrm{so}}=2.86 \times 10^{8} \dot{\mathrm{N}}_{\mathrm{H} 2}+2.83 \times 10^{8} \dot{\mathrm{N}}_{\mathrm{CO}}
$$

Note that $\mathrm{q}_{\mathrm{bn}, \mathrm{s}_{\mathrm{o}}}$ in Equation (9-27) corresponds to the explicit deflagration burn energy release rate defined in Table 4-4. The $\mathrm{W}_{\mathrm{bns}, \mathrm{o}}$ explicit mass source rate defined in Table 4-3 accounts for changes of the $\mathrm{H}_{2} \mathrm{O}, \mathrm{CO}_{2}, \mathrm{CO}, \mathrm{O}_{2}$, and $\mathrm{H}_{2}$ constituents.

\subsection{Diffusion Flame Burning (DFB)}

A DFB model developed for HECTR Version 1.8 [Won88] was incorporated into CONTAIN. This model allows the continuous burning of combustible gas in incoming flows providing certain conditions are met:

1. An ignition source is present. This condition is assumed satisfied if either DFB ignition criteria or the BSR existence criteria are met (see below). 
2. Sufficient oxygen is available in the receiving cell. The default value is $5 \%$ oxygen by volume. Shepherd [She87] noted that the NTS diffusion flames extinguished when oxygen concentration in the vessel dropped below $5-8 \%$ by volume. Tamanini et al. [Tam88] reported that the flame at the pool surface extinguished when the oxygen concentration dropped below $8 \%$ by volume in the HCOG $1 / 4$-scale model tests.

3. The receiving cell diluent mole fraction must be below a threshold value. The default value is $55 \%$ by volume.

4. The fraction of incoming combustible gas burned is greater than zero. The default value for the fraction of incoming gas that burns is 1 .

5. The mass inflow of combustible gas is above a threshold value (default $=0 \mathrm{~kg} / \mathrm{s}$ ).

6. The diluent to combustible mole fraction must be below a threshold value for the incoming flow. The default value is 9.0 moles diluent per mole of fuel. This value is consistent with Shepherd's calculations [She85] for the stability of a hydrogen-steam jet. This stability value, however, was calculated for a hydrogen-steam jet at $200^{\circ} \mathrm{C}$ in a $5-\mathrm{cm}$ diameter nozzle. This default value describes the stability of a diffusion flame for a specific case and cannot be applied in any general way. Diffusion flame stability depends on many factors, such as the jet diameter, velocity, temperature, and composition, and cannot be represented by a single CONTAIN default value as is currently done. The stability of a diffusion flame can determine whether a single burn or multiple burns can occur in an accident sequence and the containment loads can be quite different between the two cases. Relatively simple semiempirical relationships for the stability of a diffusion flame have been proposed, for examples see References Bro84, Kal81, and Pit89. It may be possible to estimate stability values from these relationships. While these relations have not been assessed against data for jets at elevated temperatures or jets containing large diluent concentrations, they do contain dimensionless parameters which, in principle, should allow these effects to be predicted. The conditions of the atmosphere into which the jet issues, such as the atmospheric composition and temperature, may also affect the stability of the diffusion flame. Limited data exist for these conditions, however, and include the NTS data [She87, Tho88a, Tho88b] and HCOG 1/4-scale data. [Tam88]

Two modifications have been made to the HECTR Version 1.8 DFB model. The first provides for an auto ignition temperature for the burning of incoming combustible gas. The second provides a simple bounding model for the entrainment of pre-existing combustible gases in a cell into the diffusion flame.

The DFB model is activated by specifying the keyword CONTBURN in the H-BURN input block as described in Section 14.3.1.7. In the DFB model, combustible gases flowing into a cell are assumed to burn as soon as they enter, given that the various user-specifiable concentration criteria and an ignition criterion are satisfied. 
Specifically, for DFB to occur, the incoming diluent to combustible mole fraction must be below "shratio," the receiving cell oxygen mole fraction must be above "mfocb," the receiving cell diluent mole fraction must be below "mfscb," and the combustible mass inflow rate $(\mathrm{kg} / \mathrm{s})$ must be above "h2flow." In addition, a user-specifiable fraction "cfracb" of the inflowing combustible gases is burned if these conditions are met, subject, of course, to there being sufficient oxygen. The ignition criteria are based on temperature thresholds such that the combustion will occur only if the entering gas temperature exceeds the threshold. Two such thresholds are provided. The first can be set by specifying the keyword DFTEMP, and applies during the burn window defined by the values of TACTIV and TDEACT (see Section 14.3.1.7). The default value of DFTEMP is $0 \mathrm{~K}$ and hence this threshold has no effect unless the user provides a value. Outside the burn window, the threshold is controlled by DFAUTO and the default value is effectively infinite $\left(10^{20} \mathrm{~K}\right)$. Hence the user must specify a value of DFAUTO in order for DFB combustion to occur outside the burn window, except that combustion will still occur when the receiving cell is sufficiently hot or contains sufficient hot debris that ignition is assumed to occur on the basis of BSR existence criteria, as discussed in Section 9.3. DFTEMP and DFAUTO may be used to simulate autoignition of hot incoming jets in the absence of a nearby ignition source. Note, however, that combustion in this model will terminate as soon as the jet temperature drops below the relevant threshold temperature. In reality, once the jet is ignited, combustion could continue even if the incoming gas temperature falls to lower values, provided the conditions for a stable flame are met. The CONTAIN model does not take this possibility into account.

The DFB temperature thresholds are applied independently to each unsubmerged gas flow path, and the dedicated suppression pool vent if not submerged. Therefore, hot combustible gases entering a cell through one path may burn via the DFB model, while cooler combustible gases entering the same cell through other paths may not burn. This will occur if the temperatures of some upstream cells are above the threshold, while the temperatures of other upstream cells are below the threshold.

Combustion of user-specified sources or of gas flows evolving from the surface of the coolant pool in the DFB model is modeled but is not subject to the temperature criteria defined by DFTEMP and DFAUTO. These flows always behave as if DFTEMP $=0$ were specified within the burn window and as if DFAUTO $=\infty$ were specified outside the burn window. In applying the DFB composition criteria, all user-specified sources within a given cell are combined and treated assuming that they represent a single stream of incoming mixed gas. The same is true of gas flows evolving from the pool surface.

The DFB model is based on the nominal amounts $\mathrm{N}_{\text {jet, } \mathrm{H} 2}$ and $\mathrm{N}_{\text {jet,CO}}$, of $\mathrm{H}_{2}$ and $\mathrm{CO}$, respectively, over the flow timestep $\Delta \mathrm{t}_{\mathrm{f}}$ that are burned in diffusion flames

$$
\begin{aligned}
& N_{\mathrm{jet}, \mathrm{H}_{2}}=\min \left[\sum_{\mathrm{ji}}\left(\frac{f_{\mathrm{cb}} \mathrm{W}_{\mathrm{ji}} \theta_{\mathrm{ji}} \mathrm{m}_{\mathrm{j}, \mathrm{H} 2}}{\mathrm{M}_{\mathrm{H} 2} \mathrm{~m}_{\mathrm{j}}}\right) \Delta t_{\mathrm{f}}, \mathrm{N}_{\mathrm{H} 2}^{\prime}\right] \\
& N_{\mathrm{jet}, \mathrm{CO}}=\min \left[\sum_{\mathrm{ji}}\left(\frac{\mathrm{f}_{\mathrm{cb}} \mathrm{W}_{\mathrm{ji}} \theta_{\mathrm{ji}} \mathrm{m}_{\mathrm{j}, \mathrm{CO}}}{\mathrm{M}_{\mathrm{CO}} \mathrm{m}_{\mathrm{j}}}\right) \Delta \mathrm{t}_{\mathrm{f}}, N_{\mathrm{CO}}^{\prime}\right]
\end{aligned}
$$


where the sum includes all flows entering cell $i$ that satisfy the above ignition criteria, including external sources and gas flows evolving from the surface of the pool; $f_{c b}$ is the user-specifiable fraction of inflowing combustible gas that is burned in the diffusion flame; $N_{\mathrm{H} 2}^{\prime}$ is defined as the moles of $\mathrm{H}_{2}$ initially present in the cell plus the net moles of $\mathrm{H}_{2}$ flowing into cell $\mathrm{i}$ over $\Delta \mathrm{t}_{\mathrm{f}} ; \mathrm{N}_{\mathrm{CO}}^{\prime}$ is similarly defined for $\mathrm{CO}$; and the other quantities are either defined above or in Tables 4-3 and 4-4. For sources and flows from the pool, $\mathrm{W}_{\mathrm{ji}}$ refers to the total mass rate of all sources or flows into the cell but in Equation (9-28) only positive (incoming) mass fractions $\mathrm{m}_{\mathrm{j}, \mathrm{Hz}} / \mathrm{m}_{\mathrm{j}}$ and $\mathrm{m}_{\mathrm{j}, \mathrm{co}} / \mathrm{m}_{\mathrm{j}}$ of $\mathrm{H}_{2}$ and $\mathrm{CO}$, respectively, are taken into account.

The entrainment of pre-existing combustible gases into the diffusion flames is determined assuming that the atmosphere is well-mixed in the cell, and that enough gases from the cell atmosphere are entrained into the diffusion flames to burn all of the incoming combustible gas plus the combustible gas entrained along with the oxygen. Under the well-mixed atmosphere assumption, this approach will provide a first order estimate of the amount of combustible gases entrained into jets. This model only considers entrainment, and thus does not account for burning rate limitations imposed by effects such as diffusion and mixing. The entrainment into the diffusion flames is represented by the balance equation

$$
\mathrm{N}_{\mathrm{jet}, \mathrm{cg}}+\mathrm{X}_{\mathrm{H} 2}^{\prime \prime} \mathrm{N}_{\mathrm{e}}+\mathrm{X}_{\mathrm{CO}}^{\prime \prime} \mathrm{N}_{\mathrm{e}}=2 \mathrm{X}_{\mathrm{O} 2}^{\prime \prime} \mathrm{N}_{\mathrm{e}}
$$

where $\mathrm{X}^{\prime \prime}$ is the mole fraction of the indicated gases in the downstream cell atmosphere, adjusted for flows during the timestep but excluding the moles represented by $N_{\text {jet,H2 }}$ and $N_{\text {jet, CO}}$, and $N_{e}$ is the moles of atmosphere entrained into the jet. $\mathrm{N}_{\text {jetc } c \mathrm{i}}$ is the moles of combustible gas entering the cell during the flow timestep that burn in the diffusion flame.

$$
\mathrm{N}_{\mathrm{jet,cg}}=\left[\mathrm{N}_{\mathrm{jet,H2}}+\mathrm{N}_{\mathrm{jet}, \mathrm{CO}}\right]
$$

The balance equation provided above is solved for $\mathrm{N}_{\mathrm{e}}$, and the mole fractions of hydrogen and carbon monoxide in the downstream cell are used to obtain the combustible gas moles to entrain into the jet and include in the DFB model. This solution only applies when mixtures are far from stoichiometric. If $2 \mathrm{X}_{\mathrm{O} 2}^{\prime \prime} \leq \mathrm{X}_{\mathrm{H} 2}^{\prime \prime}+\mathrm{X}_{\mathrm{CO}}^{\prime \prime}$, Equation (9-29) would imply that all of the combustible gas in the cell is assumed to be available for entrainment. Under such conditions this may produce unrealistic results; therefore, the entrained gas is limited to the amount of reactant gases in the cell and entrained amounts burned to the incoming amounts burned in the jet.

$$
\begin{aligned}
N_{\mathrm{e}} & =\min \left[\mathrm{N}_{\mathrm{total}}, \frac{\mathrm{N}_{\mathrm{jet}, \mathrm{cg}}}{\max \left(\delta, 2 \mathrm{X}_{\mathrm{O} 2}^{\prime \prime}-\mathrm{X}_{\mathrm{H} 2}^{\prime \prime}-\mathrm{X}_{\mathrm{CO}}^{\prime \prime}\right)}\right] \\
\mathrm{N}_{\mathrm{e}, \mathrm{H}_{2}} & =\min \left(\mathrm{X}_{\mathrm{H} 2}^{\prime \prime} \mathrm{N}_{\mathrm{e}}, \mathrm{N}_{\mathrm{jet}, \mathrm{H} 2}\right) \\
\mathrm{N}_{\mathrm{e}, \mathrm{CO}} & =\min \left(\mathrm{X}_{\mathrm{CO}}^{\prime \prime} \mathrm{N}_{\mathrm{e}}, \mathrm{N}_{\mathrm{jet}, \mathrm{CO}}\right)
\end{aligned}
$$


where $\mathrm{N}_{\text {total }}=\mathrm{N}_{\mathrm{H}}^{\prime \prime}+\mathrm{N}_{\mathrm{CO}}^{\prime \prime}+\mathrm{N}_{02}^{\prime \prime}$ is the total moles of reactant gas adjusted for flows but excluding the moles represented by $\mathrm{N}_{\mathrm{jet}, \mathrm{H} 2}$ and $\mathrm{N}_{\mathrm{jet}, \mathrm{CO}}$ and $\delta$ is a small number. $\mathrm{N}_{\mathrm{e}, \mathrm{Hz}}$ is the moles of hydrogen to entrain into the jet from the cell atmosphere, and $\mathrm{N}_{\mathrm{e}, \mathrm{Co}}$ is the moles of carbon monoxide to entrain into the jet from the cell atmosphere. The user can disable entrainment of pre-existing combustible gases in the DFB model by specifying the keyword NOBURNEN in the CONTBURN input block. If that keyword is not specified, any hydrogen or carbon monoxide pre-existing in cells will be subject to inclusion in the burning jet as described above.

As noted previously, the DFB threshold and ignition criteria are applied independently to each inflow from another cell. However, the sources (input tables) into a cell are combined into only one inflow, which is then evaluated relative to the DFB criteria to determine if this inflow will experience a DFB. The same is true of gas flows evolving from the surface of the coolant pool. Thus, a very high source of steam could preclude the DFB of a combustible-gas source (into the same cell). Note that the amount of combustible gas that is burned is not affected by flame speed or burn time, since the DFB model simply recombines the combustible gas with oxygen in the receiving cell, if the appropriate criteria are met.

The average molar rates of burning in diffusion flames for the entire cell and flow timestep are given by

$$
\begin{aligned}
& \dot{\mathrm{N}}_{\mathrm{H} 2}=\left(\mathrm{N}_{\mathrm{jet}, \mathrm{H} 2}+\mathrm{N}_{\mathrm{e}, \mathrm{H} 2}\right) / \Delta \mathrm{t}_{\mathrm{f}} \\
& \dot{\mathrm{N}}_{\mathrm{CO}}=\left(\mathrm{N}_{\mathrm{jet}, \mathrm{CO}}+\mathrm{N}_{\mathrm{e}, \mathrm{CO}}\right) / \Delta \mathrm{t}_{\mathrm{f}}
\end{aligned}
$$

provided the burning is not oxygen-limited. In the oxygen-limited case, these molar rates are reduced to correspond to the oxygen available for burning. The contributions to $W_{c b n}$ and $q_{c b n}$ in Tables 4-3 and 4-4 from diffusion flames are identical to Equations (9-26) and (9-27) for the case of deflagrations, and the equations will not be repeated here.

\subsection{Bulk Spontaneous Recombination (BSR)}

In a direct containment heating ( $\mathrm{DCH}$ ) event, large increases in the containment gas temperatures are predicted to occur as well as the possibility of hot debris particles being dispersed throughout the containment. It is possible that these conditions may cause the hydrogen to burn in the absence of any deliberate ignition system or accidental ignition, even if the gas composition lies outside the normal flammability limits. The BSR model was developed to treat this mode of combustion in a parametric (as opposed to mechanistic) way. Typically, the BSR model has been used in DCH calculations although, in principle, it could be applied to other accident scenarios in which hightemperature combustible mixtures exist. Thus, the BSR model allows combustible gas and oxygen

to recombine volumetrically if one of the following spontaneous recombination conditions is satisfied:

1. The bulk gas temperature exceeds a minimum spontaneous recombination temperature "srtemp." 
2. The debris temperature and mass concentration are sufficiently large.

The input for the BSR model is specified in the CONTBURN input subblock of the H-BURN block.

The default value for the minimum spontaneous recombination temperature "srtemp" in condition 1 is $773 \mathrm{~K}$. The default value of $773 \mathrm{~K}$ is in the range of reported minimum recombination temperatures [Con88, DeS75, Zab56] between $769 \mathrm{~K}$ and $805 \mathrm{~K}$ for stoichiometric hydrogen-air mixtures. The data also show that the recombination temperature decreases with increasing vessel size so that the minimum value may be even lower at containment scales. On the other hand, large departures from stoichiometric conditions and/or high steam concentrations are likely to increase the minimum temperature for recombination. Condition 2 requires that the debris temperature be greater than "'debtemp" and the mass concentration of debris that exceeds "debtemp" be greater than "debconc." The defaults for "debtemp" and "debconc" are $773 \mathrm{~K}$ and $1 \mathrm{~kg} / \mathrm{m}^{3}$, respectively.

The minimum recombination temperature has been shown experimentally to be a function of the stoichiometry of the mixture and diluent concentrations. [Con88, DeS75, Zab56] The user has the option to supply different recombination temperatures for each computational cell. However, there is no single well-defined temperature at which recombination occurs. The use of a temperature threshold is an engineering approximation. For the BSR model to physically represent the recombination that can occur in the DCH and other severe accident scenarios, the model would have to predict a recombination rate as a function of temperature, pressure, and mixture composition.

In lieu of such a model, the user must specify the fractional rate "srrate" at which the combustible gas recombines, provided one of the BSR conditions is satisfied. The default value is 0 . The SENKIN code [Lut91] (which is a driver for the CHEMKIN code [Kee92a, Kee92b]) can provide a basis for the rate of recombination for a given mixture temperature, pressure, and composition.

The molar rates of recombination are based on whether the oxygen or the combustible gas is present in excess of the stoichiometric amounts:

$$
\begin{array}{rlrl}
\dot{\mathrm{N}}_{\mathrm{H} 2} & =-\mathrm{s}_{\mathrm{r}} \mathrm{N}_{\mathrm{H} 2} & & \text { if } \mathrm{N}_{\mathrm{H} 2}+\mathrm{N}_{\mathrm{CO}}<2 \mathrm{~N}_{\mathrm{O} 2} \\
& =\frac{-2 \mathrm{~s}_{\mathrm{r}} \mathrm{N}_{\mathrm{H} 2} \mathrm{~N}_{\mathrm{O} 2}}{\mathrm{~N}_{\mathrm{H} 2}+\mathrm{N}_{\mathrm{CO}}} & & \text { otherwise } \\
\dot{\mathrm{N}}_{\mathrm{CO}} & =-\mathrm{s}_{\mathrm{r}} \mathrm{N}_{\mathrm{CO}} & & \text { if } \mathrm{N}_{\mathrm{H} 2}+\mathrm{N}_{\mathrm{CO}}<2 \mathrm{~N}_{\mathrm{O} 2} \\
& =\frac{-2 \mathrm{~s}_{\mathrm{r}} \mathrm{N}_{\mathrm{CO}} \mathrm{N}_{\mathrm{O} 2}}{\mathrm{~N}_{\mathrm{H} 2}+\mathrm{N}_{\mathrm{CO}}} & & \text { otherwise } \\
\dot{\mathrm{N}}_{\mathrm{O}_{2}} & =-1 / 2 \mathrm{~s}_{\mathrm{r}}\left(\mathrm{N}_{\mathrm{H} 2}+\mathrm{N}_{\mathrm{CO}}\right) & \text { if } \mathrm{N}_{\mathrm{H} 2}+\mathrm{N}_{\mathrm{CO}}<2 \mathrm{~N}_{\mathrm{O} 2} \\
& =-\mathrm{s}_{\mathrm{r}} \mathrm{N}_{\mathrm{O} 2} & & \text { otherwise }
\end{array}
$$


where $s_{r}$ is the user-specified spontaneous recombination rate "srrate." The contributions to $W_{b n}$ and $\mathrm{q}_{\mathrm{cbr}}$ in Tables 4-3 and 4-4 from spontaneous recombination are identical to Equations (9-26) and (9-27) for the case of deflagrations, and the equations will not be repeated here.

Note that BSR can occur during DFB, but BSR is not allowed to occur during a deflagration, to prevent a spurious transition to BSR. 


\subsection{HEAT AND MASS TRANSFER MODELS}

Heat and mass transfer processes are modeled at a number of interfaces in the CONTAIN code. The present chapter gives a general discussion of the heat and mass transfer modeling at such interfaces, in addition to a discussion of heat sink modeling and associated topics. Exceptions to this general treatment are noted in the discussion. Two types of heat sinks, heat transfer structures and lower cell layers, are discussed. The topics associated with heat sink modeling include heat sink characteristics and boundary conditions, heat conduction modeling, and the treatment of concrete outgassing. A discussion of the modeling available with respect to lower cell layers outside of these areas is given in Chapter 5.

The present chapter is organized by process type. Natural convection and forced convection heat transfer are discussed in Section 10.1, along with boundary layer properties and other quantities entering the expressions for convective heat transfer. Mass transfer processes are discussed in Section 10.2, along with quantities entering the expressions for mass transfer. Processes discussed in that section include condensate film flow on surfaces and condensation mass transfer. Radiative heat transfer is discussed in Section 10.3; boiling heat transfer is discussed in Section 10.4. The modeling of heat sinks and the associated topics mentioned above are discussed in Section 10.5. Key elements of the heat and mass transfer models are illustrated in Figure 10-1.

Table 10-1 indicates the various code interfaces addressed by the present chapter, as well as the processes considered at each interface. The pool-structure interface listed in this table was implemented in CONTAIN 1.2, in conjunction with the pool tracking modifications, which allow the pool to be treated as a bulk fluid on the same footing as the gas. One consequence of these modifications is that heat transfer structures can now be submerged in the pool, as discussed in more detail in Section 10.1.1.4. Note that the distinction made in this table with regard to whether a process is simply modeled or modeled and coupled to the interface temperature calculation refers to the role of the process in determining the interface conditions. This distinction is explained in more detail in Section 10.6, in which the coupling between the heat and mass transfer processes is discussed.

Table 10-1

Heat and Mass Transfer Processes

Considered at Various CONTAIN Interfaces *

\begin{tabular}{||l|c|c|c|c|c|c||}
\hline \multicolumn{1}{|c|}{ Interface } & $\begin{array}{c}\text { Conv. } \\
\text { HT }\end{array}$ & $\begin{array}{c}\text { Cond. } \\
\text { MT }\end{array}$ & $\begin{array}{c}\text { Rad. } \\
\text { HT }\end{array}$ & $\begin{array}{c}\text { Boiling } \\
\text { HT }\end{array}$ & $\begin{array}{c}\text { Aerosol } \\
\text { MT }\end{array}$ & $\begin{array}{c}\text { Film } \\
\text { Inflow }\end{array}$ \\
\hline Gas-Structure & C & C & C & - & C & C \\
\hline Gas-Pool & C & C & C & - & M & M \\
\hline Gas-Lower Cell & C & C & C & - & M & - \\
\hline Pool-Structure & C & - & - & - & - & - \\
\hline Pool-Lower Cell & C & - & - & M & - & - \\
\hline Gas-Engineered System & C & C & - & - & M & - \\
\hline
\end{tabular}

'HT = heat transfer; $\mathrm{MT}=$ mass transfer; $\mathrm{C}=$ modeled and coupled to interface temperature calculation; $\mathrm{M}=$ modeled but not coupled 


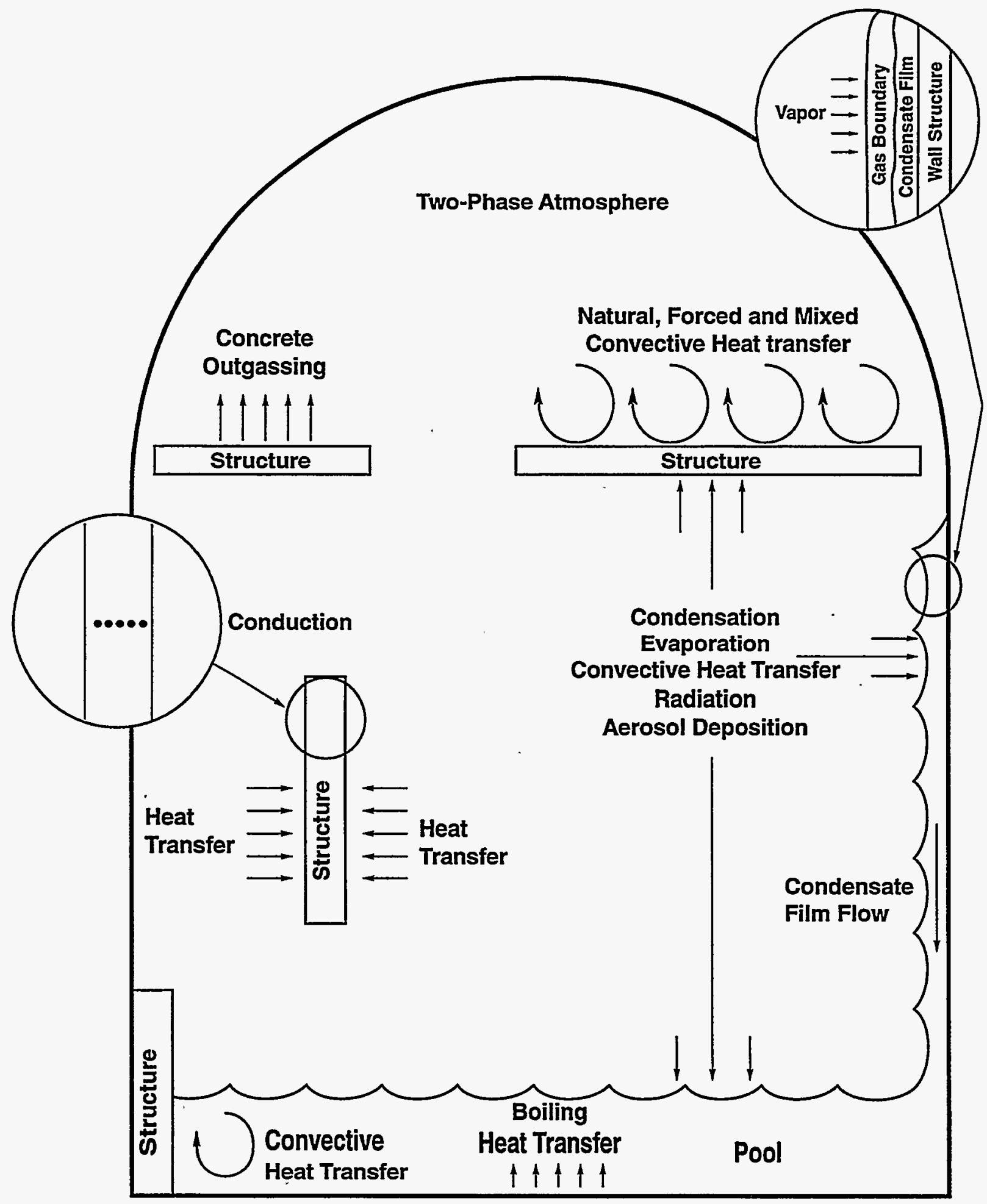

Figure 10-1. Heat and Mass Transfer as Modeled in CONTAIN 
It should be noted that steam condensation and evaporation are not modeled with regard to any surface interface in a cell unless the CONDENSE keyword has been specified for that cell. If CONDENSE has not been specified, convective heat transfer is still modeled in the same manner as if CONDENSE were specified. Note this is a change from code versions prior to CONTAIN 1.2, in which convective heat transfer for heat transfer structures is modeled by default with a fixed heat transfer coefficient of $\mathrm{h}=6.08 \mathrm{~W} / \mathrm{m}^{2}-\mathrm{K}$ when CONDENSE has not been specified.

\subsection{Convective Heat Transfer}

The convective heat transfer flux between the atmosphere or pool and a surface is in general given by

$$
q_{c}=h_{c}\left(T_{b}-T_{i f}\right)
$$

where $q_{c}$ is the convective heat transfer flux $\left(W / \mathrm{m}^{2}\right) ; T_{b}$ is the bulk fluid temperature $(\mathrm{K}) ; h_{c}$ is the convective heat transfer coefficient $\left(\mathrm{W} / \mathrm{m}^{2}-\mathrm{K}\right)$; and $\mathrm{T}_{\mathrm{if}}$ is the interface temperature at the gas-liquid interface, the gas-solid interface (for dry surfaces), or the pool-surface interface, whichever is applicable. (Note that the symbol $q$ in this chapter is reserved for heat or enthalpy flux, in $W / \mathrm{m}^{2}$.)

The convective heat transfer coefficient $h_{c}$ is related to the Nusselt number $N_{N u}$ by

$$
h_{c}=N_{N u} \frac{k_{B L}}{L}
$$

where $\mathrm{k}$ is the thermal conductivity of the bulk fluid evaluated in general in the boundary layer, and $L$ is the characteristic length for the surface.

In Section 10.1.1, the evaluation of fluid properties for the boundary layer is described, along with various dimensioned and nondimensional quantities required to evaluate the heat transfer correlations. Characteristics lengths are discussed in this subsection, as well as the effects of structure submergence. Note that there is some loss of upward compatibility with versions prior to CONTAIN 1.2, as there have been significant changes in the way these quantities are calculated and options for restoring the old treatment are not available. In Section 10.1.2, basic heat transfer correlations and tabular options for specifying forced convection Nusselt numbers for heat transfer structures are described. In Section 10.1.3, generalized heat transfer correlations for structures are described. These provide much greater flexibility in specifying heat transfer correlations than is available in prior code versions.

\subsubsection{Boundary Layer Properties and Nondimensional Numbers for Heat Transfer}

In CONTAIN, heat and mass transfer between the atmosphere or pool and various heat transfer surfaces present are normally evaluated with the aid of correlations involving various dimensionless numbers: the Grashof $\left(\mathrm{N}_{\mathrm{Gr}}\right)$, Reynolds $\left(\mathrm{N}_{\mathrm{Re}}\right)$, Sherwood $\left(\mathrm{N}_{\mathrm{Sh}}\right)$, Prandtl $\left(\mathrm{N}_{\mathrm{Pr}}\right)$, and Schmidt $\left(\mathrm{N}_{\mathrm{Sc}_{\mathrm{c}}}\right)$ numbers. These numbers, in turn, depend upon the physical properties of the fluid. In code versions 
prior to CONTAIN 1.2, there is considerable inconsistency in the way these quantities are evaluated. In some instances, properties are evaluated at the bulk fluid temperature while in other instances, properties are evaluated at an average temperature, $T_{a v}=0.5\left(T_{w}+T_{b}\right)$, where $T_{w}$ is the temperature of the first node beneath the surface and $T_{b}$ is the bulk fluid temperature. In no case in those prior versions is any difference between the composition of the boundary layer (in the case of a gas) and that of the bulk fluid taken into account.

In CONTAIN 1.2 and higher versions, all boundary layer properties are evaluated in a manner consistent with the recommendations of Reference Bir60, except those used for the engineered systems fan cooler model, which is a special case and discussed in Section 12.1. In Reference Bir60 it is recommended that properties be evaluated at a temperature equal to the mean of the surface or interface temperature and the bulk fluid temperature, and at a composition equal to the mean of the surface composition and the bulk composition (in the case of a gas). The details of the general treatment are described in the remainder of this section. The use of boundary layer properties defined in this way is a useful empirical procedure, not a rigorous physical law. Hence some simplifying approximations are used when the errors introduced by the approximations are judged to be less than the uncertainty associated with the underlying physical model.

10.1.1.1 Evaluation of the Gas Boundary Layer Composition. When condensation from a gaseous bulk fluid or evaporation into a gaseous fluid is occurring at a surface, the composition of the gas boundary layer will generally be different from the bulk composition. In the past, this difference was not taken into account in CONTAIN. It is taken into account in CONTAIN 1.2 and higher versions to the extent that the vapor mole fraction is corrected for the effect of the ongoing condensation/evaporation. The composition of the noncondensable gases in the boundary layer, however, is still assumed to be that of the bulk gas.

The vapor mole fraction for the boundary layer, $\mathrm{X}_{\mathrm{v}, \mathrm{BL}}$, is assumed to be equal to $\left(\mathrm{X}_{\mathrm{v}, \mathrm{f}}+\mathrm{X}_{\mathrm{v}, \mathrm{b}}\right) / 2$, where the subscripts $\mathrm{v}$, if, and $\mathrm{b}$ refer to coolant vapor, the gas-surface interface, and the bulk gas, respectively. The vapor mole fraction at the interface $\mathrm{X}_{\mathrm{v}, \mathrm{if}}$ is assumed to be

$$
X_{v, \text { if }}=f_{w e t}\left(X_{v, i f, w e t}-X_{v, b}\right)+X_{v, b}
$$

where

$$
X_{v, \text { if,wet }}=\frac{P_{s}\left(T_{i f}\right)}{P_{v, b}} X_{v, b}
$$

and $f_{\text {wet }}$ is the fraction of the cell timestep that the surface is considered to be wet, $P_{s}\left(T_{i f}\right)$ is the saturated vapor pressure of the coolant at the interface temperature $T_{\mathrm{if}}$, and $\mathrm{P}_{\mathrm{v}, \mathrm{b}}$ is the partial pressure of vapor in the bulk gas. 
When condensation is occurring, $f_{\text {wet }}=1$. Under other conditions, the situation can be more complicated. If the surface is solid and dry and there are no liquid water sources to wet the surface, $f_{\text {wet }}$ is zero and $X_{v, B L}$ is equal to $X_{v, b}$. It may happen, however, that a water film present at the start of a timestep dries out during that timestep. In addition, there can be sources of liquid water to the surface from water aerosol deposition, water films draining from other heat transfer structures, and/or user-specified film source tables, even though conditions are such that evaporation is occurring. If the evaporation rate exceeds the liquid source rate, it may be a poor approximation to treat the surface as being either completely dry or completely wet. Furthermore, such a treatment would run the risk of causing a frequent flipping back and forth between the dry and the wet condition, possibly resulting in an undesirable chattering effect.

Hence, when conditions are such that evaporation can occur at the structure surface, $f_{\text {wet }}$ is calculated from

$$
f_{\text {wet }}=\min \left(1, \frac{m_{\mathrm{flm}}^{0}+\mathrm{m}_{\mathrm{dep}}}{\mathrm{m}_{\mathrm{ev}}^{0}}\right)
$$

Here $\mathrm{m}_{\mathrm{flm}}^{0}$ is the mass of water film on the surface at the start of the cell timestep, $\mathrm{m}_{\mathrm{dep}}$ is the liquid water from all sources deposited or flowing onto the surface during the timestep, and $\mathrm{m}_{\mathrm{ev}}^{0}$ is the mass of water that would be evaporated from the surface during the timestep if the surface were wet throughout the timestep. For an ice surface in an ice condenser, the mass of ice melted during the timestep is also included in $\mathrm{m}_{\text {dep }}$. If both the numerator and the denominator in Equation (10-4) are equal to zero, then $f_{\text {wet }}$ is taken to be zero. In order to avoid the need for an iterative implicit treatment, the value of $f_{\text {wer }}$ from the previous cell timestep is used in Equation (10-3).

After $\mathrm{X}_{\mathrm{v}, \mathrm{f}}$ and $\mathrm{X}_{\mathrm{v}, \mathrm{BL}}$ have been calculated, a density corrected for composition is calculated for both the interface and the boundary layer conditions:

$$
\begin{aligned}
\rho\left(T_{b}, X_{v, i f}\right) & =\frac{X_{v, i f} M_{v}+X_{n c, i f} M_{n c}}{M_{b}} \rho_{b} \\
\rho\left(T_{b}, X_{v, B L}\right) & =\frac{X_{v, B L} M_{v}+X_{n c, B L} M_{n c}}{M_{b}} \rho_{b}
\end{aligned}
$$

where $M$ is the molecular weight, the subscript $n c$ refers to the noncondensable gas mixture, and $X_{n c}$ $\equiv 1-\mathrm{X}_{v}$. Note that, at this stage, the interface and boundary layer values of the density have been corrected for composition differences but not for temperature differences.

In Equations (10-3) and (10-5), the interface and boundary layer values are evaluated by applying corrections to the bulk properties, rather than by evaluating them directly from the compositions and the equation of state (EOS). The reason is that, if the non-ideal EOS for steam is being used, the bulk quantities will reflect this and hence the corrected properties will reflect the non-ideal EOS 
except for the error introduced by the fact that the correction is itself based upon the ideal gas law. For containment conditions, this error should be no more than a small fraction of the correction, even when the correction is itself small. If the properties were evaluated directly using the ideal EOS, the error in the boundary layer and interface properties could equal or exceed the correction when the latter is small. On the other hand, use of the non-ideal EOS for this purpose could be computationally expensive, since the non-ideal EOS would have to be evaluated for each structure.

The gas specific heat at constant pressure in the boundary layer, $\mathrm{c}_{\mathrm{p}, \mathrm{BL}}$, is evaluated at the temperature $T_{b}$ from

$$
c_{p, B L}=w_{v, B L} c_{p, v}+w_{n c, B L} c_{p, n c}
$$

where $w$ refers to mass fractions. Mass fractions are used because heat capacities in CONTAIN are evaluated per unit mass, not per mole. As discussed in the next section, no correction for temperature is made with respect to this specific heat.

10.1.1.2 Evaluation of Properties at the Boundary Layer Temperatures. All properties used in the heat and mass transfer calculations, with the exception of the engineered systems fan cooler model discussed in Section 12.1, are now evaluated at a boundary layer temperature $T_{B L}=\left(T_{i f}+T_{b}\right) / 2$, where $\mathrm{T}_{\mathrm{b}}$ is the bulk fluid temperature and $\mathrm{T}_{\mathrm{if}}$ is the interface temperature at the gas-liquid interface, the gas-solid interface (for dry surfaces), or the pool-surface interface, whichever is applicable. Note that $\mathrm{T}_{\mathrm{if}}$ is not the temperature of the first node beneath the surface, $\mathrm{T}_{\mathrm{w}}$, that is used in code versions prior to CONTAIN 1.2. These temperatures differ due to the temperature gradients that generally exist across the first half of the first node, the paint layer assumed to be present on the surface of heat transfer structures, and any liquid film adhering to a solid surface. The difference between $T_{\text {if }}$ and $T_{w}$ can be substantial during rapid heat and mass transfer.

For the case of heat and mass transfer from a gaseous bulk fluid, the interface temperature is calculated through an iterative process as described in Section 10.6. For the case of heat transfer from a pool to a solid surface, the interface temperature can be calculated in a considerably more straightforward fashion. Thus, the discussion below will be restricted to the more complicated case of heat transfer from a gas. The statements about fluid composition effects and the references to the Sherwood number for mass transfer should be ignored in the case of pool heat transfer.

The interface temperature calculation requires the current values of the Nusselt and Sherwood numbers and other information dependent upon the boundary layer properties. Solving for these properties in a fully implicit fashion would involve considerable computational complexity. Hence, the interface temperature calculated for the previous timestep is employed in evaluating the boundary layer properties. Developmental testing performed to date has revealed no problems resulting from this explicit treatment.

In CONTAIN, the properties of a gaseous bulk fluid at the temperature $T_{b}$ are obtained from the properties of the individual gases by using the simple mixing rules given in Section 3.3. This evaluation is only required once for each cell during a system timestep. However, each surface in 
a cell will, in general, have a different value of $\mathrm{T}_{\text {if }}$ and therefore a different value of $\mathrm{T}_{\mathrm{BL}}$ for each cell timestep. Since there can be many surfaces in a cell, a complete re-evaluation of all the gas properties at $T_{B L}$ for each surface could add considerable computational overhead.

In the procedure adopted, a gaseous bulk fluid is treated as a two-component system consisting of coolant vapor as one component and the noncondensable gas mixture present as the other. At the time the bulk atmosphere properties are evaluated, the mixing rules are also applied to calculate properties for the noncondensable gas mixture at the bulk gas temperature. When the boundary layer properties are evaluated, only the vapor viscosity and conductivity are evaluated at the temperature $\mathrm{T}_{\mathrm{BL}}$ by calling the appropriate property routines. For the noncondensable gas mixture, a simple power-law temperature dependence is used to correct the noncondensable properties calculated for the bulk temperature to the boundary layer temperature:

$$
\begin{aligned}
& \mu_{n c, B L}=\mu_{n c, b}\left(\frac{T_{B L}}{T_{b}}\right)^{0.75} \\
& k_{n c, B L}=k_{n c, b}\left(\frac{T_{B L}}{T_{b}}\right)^{0.67}
\end{aligned}
$$

where $\mu$ and $\mathrm{k}$ are, respectively, the viscosity and the thermal conductivity, and the subscript nc refers to the noncondensable gas mixture present.

In Equation (10-7), the values of the exponents were chosen to provide a reasonable fit to the CONTAIN property functions. Since the latter are not simple power laws, and since the power law giving the best fit is somewhat different for different noncondensable gases, some degree of approximation is involved. The adequacy of Equation (10-7) was tested by evaluating the viscosity and thermal conductivity functions for $\mathrm{N}_{2}, \mathrm{O}_{2}, \mathrm{CO}, \mathrm{CO}_{2}, \mathrm{Ar}, \mathrm{H}_{2}$, and $\mathrm{He}$ as a function of temperature. Values of $\mu_{\mathrm{nc}, \mathrm{BL}}$ and $\mathrm{k}_{\mathrm{nc}, \mathrm{BL}}$ were calculated from Equation (10-7), with $\mathrm{T}_{\text {if }}=400 \mathrm{~K}$ and $\mathrm{T}_{\mathrm{b}}$ varying over the range 300 to $2800 \mathrm{~K}$, and compared with the "correct" value obtained by evaluating the property functions at $\mathrm{T}_{\mathrm{BL}}$.

For the viscosity, errors were less than or equal to $1.2 \%$ for all values of $\mathrm{T}_{\mathrm{b}}$ and all gases except $\mathrm{CO}_{2}$, for which errors of up to $2 \%$ could arise. For the thermal conductivity, the fractional error could be somewhat larger; it is plotted as a function of $T_{b}$ in Figure 10-2. For design basis accident conditions, in which $\mathrm{N}_{2}$ or air is the dominant noncondensable gas and $\mathrm{T}_{\mathrm{b}}<500 \mathrm{~K}$, it is evident that any error in this approximation will be totally trivial. For severe accidents, in which a much wider range of $T_{b}$ values and bulk gas compositions could arise, errors could range up to several percent in extreme cases, but will be limited to $2 \%$ at any temperature so long as $\mathrm{N}_{2}$ or air is the dominant noncondensable gas. 


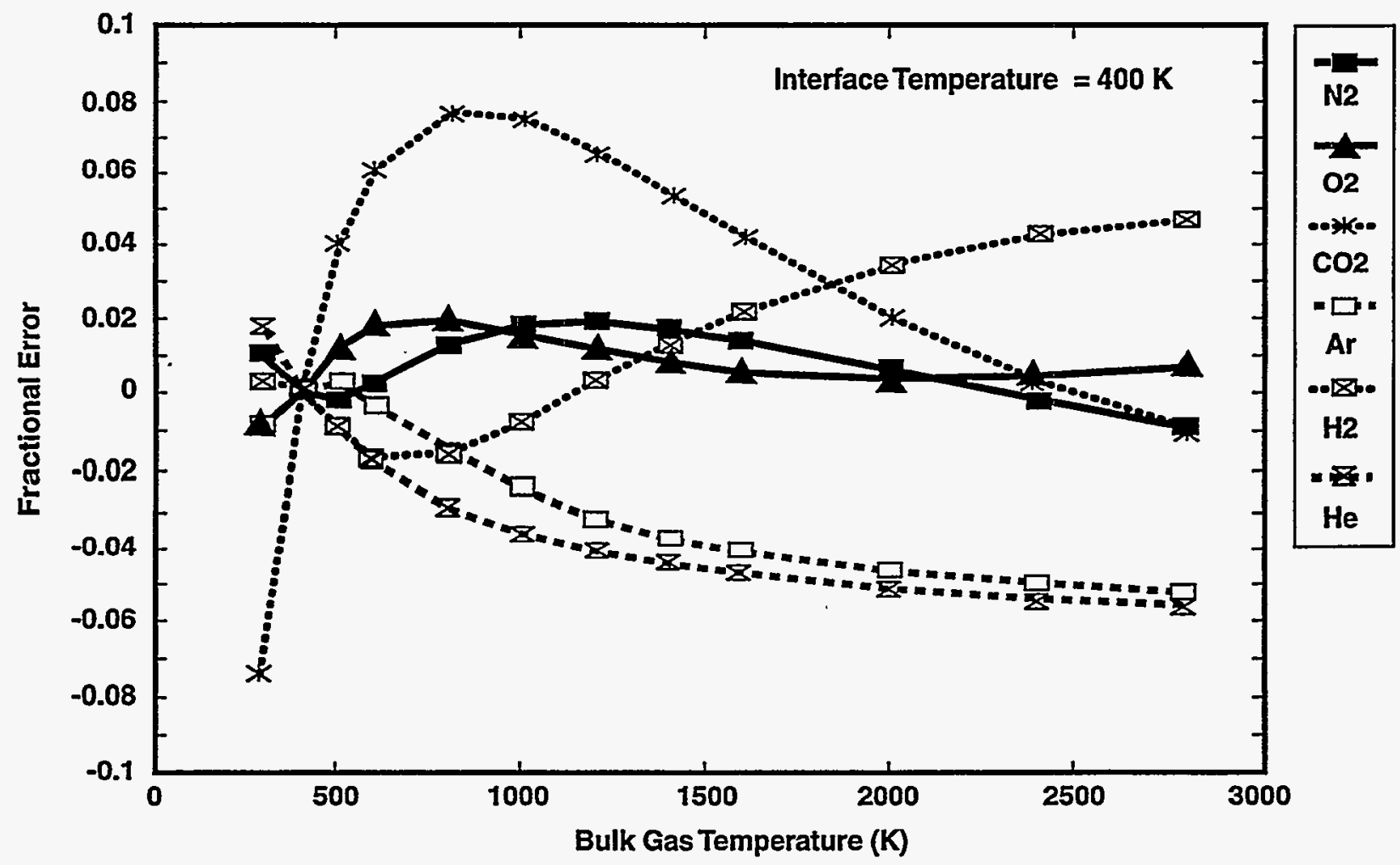

Figure 10-2. Test of the Power-Law Approximation for Thermal Conductivity at $\mathrm{T}_{\mathrm{BL}}$ 
After corrections are made for temperature as shown in Equation (10-7), the boundary layer properties are evaluated for the boundary layer mixture of vapor and noncondensable gases by using the same CONTAIN mixing rules as in Equations (3-15) and (3-16):

$$
\begin{aligned}
& \mathrm{k}_{\mathrm{BL}}=\mathrm{X}_{\mathrm{nc}, \mathrm{BL}} \mathrm{k}_{\mathrm{nc}, \mathrm{BL}}+\mathrm{X}_{\mathrm{v}, \mathrm{BL}} \mathrm{k}_{\mathrm{v}, \mathrm{BL}} \\
& \mu_{\mathrm{BL}}=\frac{\mathrm{X}_{\mathrm{nc}, \mathrm{BL}} \mu_{\mathrm{nc}, \mathrm{BL}} \sqrt{\mathrm{M}_{\mathrm{nc}}}+\mathrm{X}_{\mathrm{v}, \mathrm{BL}} \mu_{\mathrm{v}, \mathrm{BL}} \sqrt{\mathrm{M}_{\mathrm{v}}}}{\mathrm{X}_{\mathrm{nc}, \mathrm{BL}} \sqrt{\mathrm{M}_{\mathrm{nc}}}+\mathrm{X}_{\mathrm{v}, \mathrm{BL}} \sqrt{\mathrm{M}_{\mathrm{v}}}}
\end{aligned}
$$

where $\mathrm{X}$ is the mole fraction, $\mathrm{M}$ is the molecular weight, and the subscript $\mathrm{v}$ refers to the vapor; $\mathrm{X}_{\mathrm{nc}}$ $\equiv 1-\mathrm{X}_{\mathrm{v}}$. The composition-corrected densities given by Equation (10-5) are also corrected for the temperature using the ideal gas law:

$$
\begin{gathered}
\rho_{\mathrm{BL}}=\rho\left(\mathrm{T}_{\mathrm{b}}, \mathrm{X}_{\mathrm{v}, \mathrm{BL}}\right) \mathrm{T}_{\mathrm{b}} / \mathrm{T}_{\mathrm{BL}} \\
\rho_{\mathrm{if}}=\rho\left(\mathrm{T}_{\mathrm{b}}, \mathrm{X}_{\mathrm{v}, \mathrm{if}}\right) \mathrm{T}_{\mathrm{b}} / \mathrm{T}_{\mathrm{if}}
\end{gathered}
$$

No temperature correction is applied for $c_{p}$. The temperature dependence of $c_{p}$ is relatively weak compared with the temperature dependence of the gas transport properties, and simple approximations for this temperature dependence that would be guaranteed to do less harm than good under all conditions were not found. Since $c_{p}$ appears in the Nusselt number correlations only through the quantity $\operatorname{Pr}^{d}$, where $d \approx 1 / 3$ in most cases, this approximation introduces little error.

10.1.1.3 Evaluation of the Nondimensional Heat Transfer Numbers. This section discusses the evaluation of the Reynolds, Grashof, and Prandtl numbers that enter the Nusselt number correlations discussed in Sections 10.1.2 and 10.1.3. The Reynolds and Grashof numbers required in the forced and natural convection correlations, respectively, are evaluated as

$$
\begin{aligned}
& N_{\text {Re }}=\frac{\rho_{\mathrm{BL}} \mathrm{L} \mathrm{v}_{\mathrm{c}}}{\mu_{\mathrm{BL}}} \\
& \mathrm{N}_{\mathrm{Gr}}=\frac{\mathrm{L}^{3} \mathrm{~g} \max \left(|\Delta \rho|, 10^{-7}\right)\left(\rho_{\mathrm{BL}} / \mu_{\mathrm{BL}}\right)^{2}}{\rho_{\mathrm{BL}}}
\end{aligned}
$$

where $L$ is the characteristic length for the surface, as discussed in Section 10.1.1.5, $v_{c}$ is the convective gas velocity across the surface, $g$ is the acceleration of gravity, and $\Delta \rho=\rho_{\text {if }}-\rho_{b}$. Note that in contrast to code versions prior to CONTAIN 1.2, this density difference for a gaseous bulk fluid reflects both temperature and composition effects, as discussed in Sections 10.1.1.1 and 10.1.1.2 above, and not just temperature effects. The Prandtl number used to evaluate the Nusselt number correlations and the heat transfer coefficient $h$ are given by 


$$
\begin{aligned}
\mathrm{N}_{\mathrm{Pr}} & =\mu_{\mathrm{BL}} c_{\mathrm{p}, \mathrm{BL}} / \mathrm{k}_{\mathrm{BL}} \\
\mathbf{h} & =\mathrm{k}_{\mathrm{BL}} \mathrm{N}_{\mathrm{Nu}} / \mathrm{L}
\end{aligned}
$$

10.1.1.4 Submergence of Heat Transfer Structures. The pool tracking modifications made in CONTAIN 1.2 introduce a number of changes to allow the pool in a cell to be treated on the same footing as the gas. One change is related to the volumes considered to be occupied by the gas and pool. In prior code versions, the pool cross-sectional area is assumed to be contained within that of the gas. Thus, the submergence of heat transfer structures and flow paths is not considered. In CONTAIN 1.2 and higher versions, however, the pool is considered to occupy the same crosssectional area as the gas; i.e., the pool is assumed to displace the gas completely below the pool surface elevation. Thus, it is necessary to consider the submergence of heat transfer structures.

CONTAIN 1.2 and higher versions allow the modeling of the submergence of a limited subset of the structure shapes and orientations. This subset includes only SLAB structures of orientation WALL, FLOOR, and CEILING; and CLYINDER structures of orientation WALL. Note that such cylindrical wall structures are assumed to have a vertical axis. In order for a structure to be submersible, it must be a member of this subset, and in addition the user must have specified its elevation. Structures that are not submersible are assumed to be in contact only with the atmosphere.

Additional restrictions for a submersible structure include the fact that only the inner face of the structure is allowed to be in contact with the pool. Thus, a structure with an outer surface in the same cell is not allowed to be submerged. Another restriction is that the submerged surface is not allowed to undergo concrete outgassing, although this is still allowed for the unsubmerged part of a partially submerged structure, according to the average conditions and outgassing inventory remaining in the unsubmerged part.

The limited submersible subset was selected on the basis that submergence does not change the basic slab or cylindrical geometry of the submerged and unsubmerged parts of the structure. For simplicity, the heat conduction within each of the submerged and unsubmerged parts of a partially submerged structure is assumed to be governed by one-dimensional behavior. Four one-dimensional conduction regions are shown, for example, in Figure 10-3, for the case of two partially submerged structures in two different cells connected by a conduction boundary condition. (This connected structure boundary condition is discussed in more detail in Section 10.5.2.)

Although the basic geometry of the submerged and unsubmerged parts of a partially submerged structure is preserved for the limited submersible subset, it should be noted that the submergence of structures in general creates a two-dimensional heat conduction problem even in structures that would be treated accurately as one-dimensional when not submerged. The implementation of a general two-dimensional conduction solver for structures is outside the scope of the present code. A submersible structure is therefore treated in terms of two one-dimensional conduction regions, as discussed above.

Upon submergence of a structure, the structure surface areas and node masses for the unsubmerged and submerged regions are remapped vertically between the regions as the pool level and the boundary between the regions change. The masses that are remapped with pool level changes include not only the structure node masses but the liquid film, aerosols, and fission products on the 


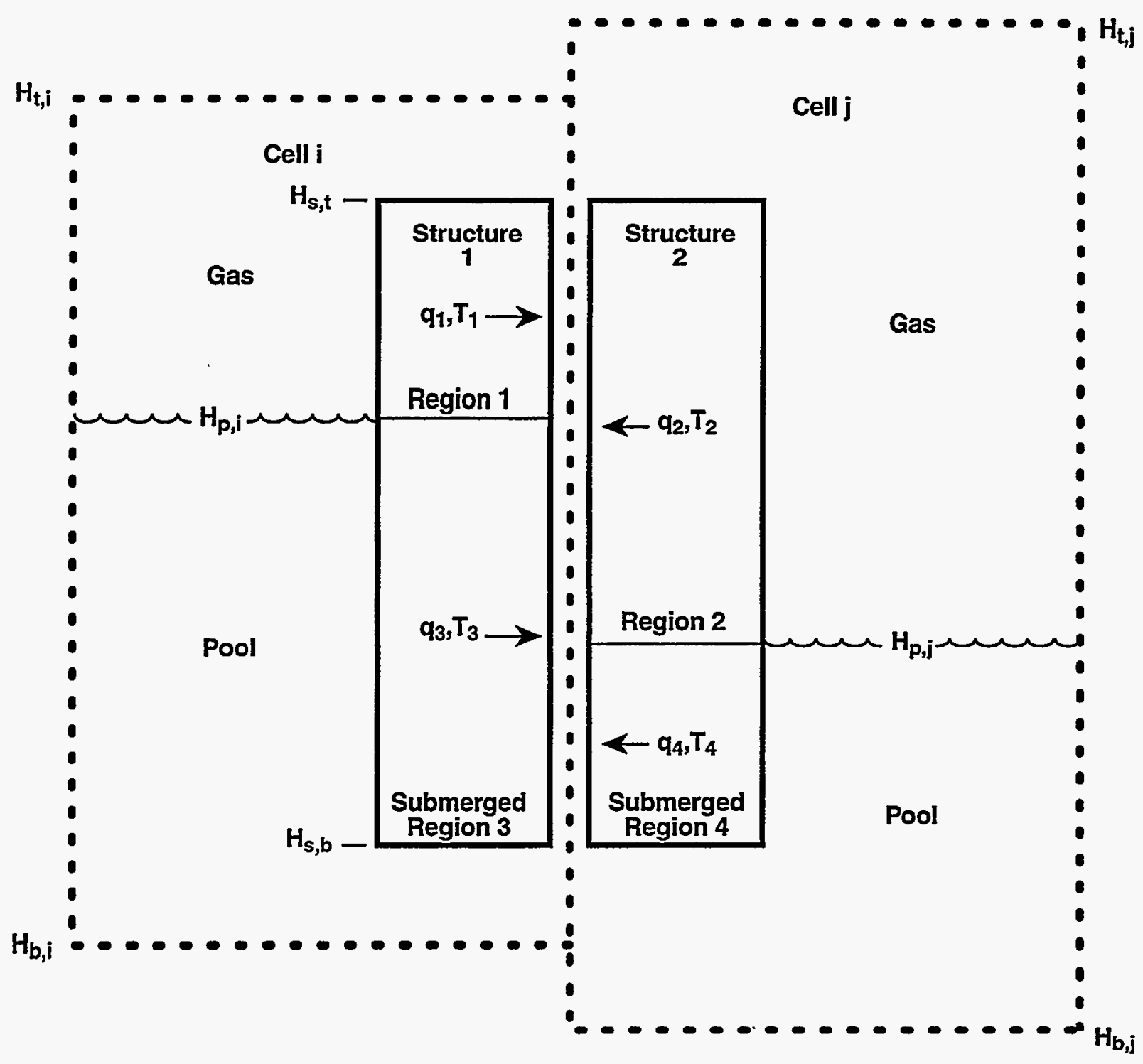

Figure 10-3. Example of a Connected Structure Configuration 
unsubmerged surface of the structure. The latter masses are considered uniformly distributed over the unsubmerged surface. With rising pool level, the masses that are present on the unsubmerged surface as it is submerged are transferred to the pool in proportion to the surface covered, without regard to any possible holdup on the structure. Also, with decreasing level, pool masses are not transferred to the unsubmerged surface from the pool but are considered entirely retained by the pool. Upon submergence, the structure node enthalpies for the unsubmerged and submerged regions are also remapped, and new structure node temperatures are calculated under the assumption that the temperature profiles within each region remain one-dimensional.

The partial submergence of a structure is considered to alter the destination of liquid film and fission products washing down from the structure. Normally the destination is controlled by the FILMFLOW input and the OVERFLOW cell designation. For partially submerged structures, this destination is reassigned to the pool of the cell containing the structure.

The degree of submergence of a SLAB or CYLINDER structure is calculated from the absolute elevation of the center of volume of the structure, which is specified through the SLELEV or CYLELEV keyword, respectively. If such a keyword is not specified, the structures will be assumed to be in contact with the atmosphere only. For connected structures, an elevation should be specified for each structure considered submersible and should refer to the center of volume of the individual structure, not the combined structure. Because of potential inconsistencies arising from structures extending below cell bottom, the code will check for such a condition and give a diagnostic if it exists.

The user should be aware of a new feature implemented specifically to accommodate submersible structures. The new feature is that the CYLINDER structure is in general no longer a half cylinder, but a partial cylinder with an azimuthal angle specified by CYLTHETA, which by default is $180^{\circ}$. This change was made to accommodate the fact that the vertical extent of cylindrical walls is given as less than full height in some input files to accommodate openings. For a submersible cylinder this practice could result in a considerable distortion in the submerged area. The CYLTHETA keyword allows more flexibility in modeling both the effective area and submergence.

10.1.1.5 Heat Transfer Characteristic Lengths. The characteristic lengths $\mathrm{L}$ used in heat transfer calculations involving both heat transfer structures and lower cell layers, including the pool, are discussed here. The characteristic lengths for engineered systems heat transfer are defined with respect to each specific system in Chapter 12.

For a heat transfer structure, the characteristic length is taken by default to the value "chrlen" specified by the user. However, if the ADJUSTCL keyword is specified, the code will use a characteristic length $L$ that includes the effects of submergence. For example, the characteristic length for an unsubmerged ceiling should change as the pool level rises toward the ceiling. In this case the characteristic length should be reduced from "chrlen" to the distance between the pool and ceiling when that distance becomes the smaller of the two. In order for this type of modeling to give continuous, or at least unambiguous, behavior in the limit that the pool level goes to zero, the distance between the ceiling and the bottom of the cell must be greater than the specified "chrlen." 
This requirement of continuous behavior gives rise to constraints on the values of "chrlen" that are allowed for each structure orientation and condition when ADJUSTCL is specified.

When ADJUSTCL is specified, the characteristic lengths used in each case have the form

$$
\mathrm{L}=\max \left(\min \left(" \operatorname{chrlen} ", \mathrm{~L}_{0}\right), 0.001\right)
$$

where the definition of $L_{0}$ and the constraint on "chrlen" in each case is given by

$$
\begin{aligned}
\mathrm{L}_{0} & =\mathrm{H}_{\mathrm{sb}}-\mathrm{H}_{\mathrm{p}, \mathrm{i}} & & \text { (unsubmerged ceiling with "chrlen" }<\mathrm{H}_{\mathrm{sb}}-\mathrm{H}_{\mathrm{b}, \mathrm{i}} \text { ) } \\
& =\mathrm{H}_{\mathrm{sb}}-\mathrm{H}_{\mathrm{b}, \mathrm{i}} & & \text { (submerged ceiling with "chrlen" }<\mathrm{H}_{\mathrm{sb}}-\mathrm{H}_{\mathrm{b}, \mathrm{i}} \text { ) } \\
& =\mathrm{H}_{\mathrm{st}}-\mathrm{H}_{\mathrm{p}, \mathrm{i}} & & \text { (unsubmerged wall with "chrlen" }<\mathrm{H}_{\mathrm{st}}-\mathrm{H}_{\mathrm{sb}} \text { ) } \\
& =\mathrm{H}_{\mathrm{p}, \mathrm{i}}-\mathrm{H}_{\mathrm{sb}} & & \text { (submerged wall with "chrlen" }<\mathrm{H}_{\mathrm{st}}-\mathrm{H}_{\mathrm{sb}} \text { ) } \\
& =\mathrm{H}_{\mathrm{t}, \mathrm{i}}-\mathrm{H}_{\mathrm{st}} & & \text { (unsubmerged floor with "chrlen" }<\mathrm{H}_{\mathrm{ti}}-\mathrm{H}_{\mathrm{st}} \text { ) } \\
& =\mathrm{H}_{\mathrm{p}, \mathrm{i}}-\mathrm{H}_{\mathrm{st}} & & \text { (submerged floor with "chrlen" }<\mathrm{H}_{\mathrm{t}, \mathrm{i}}-\mathrm{H}_{\mathrm{st}} \text { ) }
\end{aligned}
$$

where $\mathrm{H}_{\mathrm{sb}}$ is the elevation at the bottom edge of the structure, $\mathrm{H}_{\mathrm{p}, \mathrm{i}}$ is the pool surface elevation, $\mathrm{H}_{\mathrm{st}}$ is the elevation at the top of the structure, $\mathrm{H}_{\mathrm{b}, \mathrm{i}}$ is the cell bottom elevation, and $\mathrm{H}_{\mathrm{t}, \mathrm{i}}$ is the cell top elevation. Note that if "chrlen" does not satisfy the appropriate inequality in the above equations, the code will not accept the ADJUSTCL keyword until "chrlen" is changed.

The characteristic lengths used at the gas-pool and gas-lower-cell interfaces depend on whether the atmosphere or pool of a cell is considered coupled to the cell above or below, respectively. The presence or absence of coupling is determined by whether the user has invoked the CELLOVER or CELLUNDR keywords in the cell GEOMETRY block discussed in Section 14.3.1.1. If CELLOVER is used to define the overlying cell "icello" and the pool is empty or absent in "icello," then the atmosphere is considered coupled to that of "icello." Similarly, if the CELLUNDR keyword is used to define an underlying cell "icellu," then the pool is considered coupled to that of icellu." Otherwise the atmosphere and pool are considered decoupled. The characteristic length for gas-pool or gas-lower-cell interfaces, with respect to determining decoupled gas boundary layer properties, is taken to be

$$
L=\min \left(H_{t, i}-H_{p, i}, \sqrt{\frac{4 A_{\ell}}{\pi}}\right)
$$

where $A_{l}$ is the area of the pool surface or lower cell layer. The characteristic length for gas-pool interfaces, with respect to determining decoupled pool boundary layer properties, or for pool-lowercell interfaces is taken to be 


$$
L=\min \left(H_{\mathrm{p}, \mathrm{i}}-\mathrm{H}_{\mathrm{b}, \mathrm{i}}, \sqrt{\frac{4 \mathrm{~A}_{\ell}}{\pi}}\right)
$$

where $A_{l}$ is the area of the interface. For coupled atmospheres or pools, the characteristic length is simply taken to be $\sqrt{4 A_{\ell} / \pi}$ on the respective side of the gas-pool interface.

10.1.1.6 Forced Convection Velocities for Heat Transfer Structures. Forced convection heat transfer is modeled at the interface between a gaseous bulk fluid and a heat transfer structure. This modeling is discussed in detail in Sections 10.1.2 and 10.1.3.3. The purpose of the present section is to discuss the gas velocities that are used in conjunction with the forced convection Nusselt number correlations, provided the user does not invoke one of the tabular input options for specifying forced convection conditions.

In the absence of such tabular input, the gas velocity for a structure surface is calculated on the basis of the gas flows into and out of a cell. It is defined as the average of the inlet and outlet velocities for a cell:

$$
v_{c}=\frac{v_{\text {in }}+v_{\text {out }}}{2}
$$

The expression for $v_{\text {in }}$ is

$$
v_{\text {in }}=\sum_{\mathrm{ji}} \frac{C_{\text {in } \mathrm{ji}} \theta_{\mathrm{ji}}\left|W_{\mathrm{ji}}\right| R \mathrm{~T}_{\mathrm{in}}}{\mathrm{M}_{\mathrm{u}} \mathrm{A}_{\mathrm{hd}} \mathrm{P}_{\mathrm{g}}}
$$

where $C_{i n_{j i}}$ is the coefficient "valin" that may be specified by the user for each flow path and each inner and outer surface of a structure exposed within a cell, $\theta_{\mathrm{ji}}$ is the conditional function that selects flows only into $\mathrm{i}, \mathrm{W}_{\mathrm{ji}}$ is the mass flow rate of the gas and homogeneously dispersed coolant, if any, in the flow path, $R$ is the universal gas constant, $T_{\text {in }}$ is the average temperature of the inflowing gas, $M_{v}$ is the molecular weight of the gas in the upstream cell, $A_{h d}$ is the hydraulic area that may be specified for each structure, and $\mathrm{P}_{\mathrm{g}}$ is the pressure of the downstream cell. The sum in Equation (1017) and Equation (10-18) below includes gas flows into cell $\mathrm{i}$ from unsubmerged gas flow paths, from the dedicated suppression pool vent flow path, if connected and not submerged, and from the surface of the pool. In the latter case $\mathrm{W}_{\mathrm{ji}}$ is defined to be the total mass flow rate of condensable vapor and noncondensable gas evolving from the coolant pool surface, as discussed in Section 4.4.8. By default, $C_{\text {in } \mathrm{ji}}=1$ and $A_{h d}=V_{g}^{2 / 3}$, where $V_{g}$ is the cell gas volume.

$\mathrm{T}_{\mathrm{in}}$ is calculated by assuming that the flow is isothermal and that all of the incoming gas streams mix with each other before they mix with the existing cell inventory. The temperature after the streams mix in cell $i$ is given by 


$$
T_{i n}=\frac{\sum_{j i} C_{i n, j i} \theta_{j i}\left|W_{j i}\right| T_{u} c_{p, u}}{\sum_{j i} C_{i n, j i} \theta_{i j}\left|W_{j i}\right| c_{p, u}}
$$

where for unsubmerged flow paths $T_{v}$ is the upstream cell temperature and $c_{p, u}$ is the upstream cell gas specific heat at constant pressure. For gas evolving from the pool surface, these are defined as the temperature and specific heat, respectively, of the evolving gas. The expression for $\mathrm{v}_{\text {out }}$ is

$$
v_{\text {out }}=\sum_{\mathrm{ij}} \frac{C_{\text {out }, \mathrm{ij}} \theta_{\mathrm{ij}}\left|\mathrm{w}_{\mathrm{ij}}\right|}{\mathrm{A}_{\mathrm{hd}} \rho_{\mathrm{g}}}
$$

where $C_{\text {outij }}$ is the coefficient "valout," which may be specified for each flow path and each structure surface, and $\mathrm{C}_{\text {out }, \mathrm{j}}=1$ by default; and $\rho_{\mathrm{g}}$ is the density of the gas in the cell. The sum in this case includes all gas outflows through flow paths.

When the $\mathrm{C}_{\mathrm{inji}}$ and $\mathrm{C}_{\mathrm{out}, \mathrm{j}}$ parameters are left at their default values of unity, the forced flow velocity will be equal to the mean volumetric flow rate through the cell (in $\mathrm{m}^{3} / \mathrm{s}$ ) divided by the crosssectional area available for flow, $A_{\text {hd }}$. The mean volumetric flow rate, in turn, is taken to be the mean of the volumetric flow rates into and out of the cell. In practice, $C_{\mathrm{in,ji}}$ and $C_{\text {out,ij }}$ have been left equal to unity in most CONTAIN calculations. The ability to alter their values has been provided in order to permit the user to represent cases in which the geometry is such that some of the incoming or outgoing flows have more influence than others at a given structure location; e.g., flow entering via a path close to the structure may have a relatively high influence. No general prescription for defining nondefault values of these parameters is available, and it is the user's responsibility to chose appropriate values for the specific application at hand. Nondefault values of $\mathrm{A}_{\mathrm{hd}}$ are more commonly used than nondefault values of $\mathrm{C}_{\mathrm{inji}}$ and $\mathrm{C}_{\text {outij. }}$. It is appropriate to redefine $A_{\text {hd }}$ whenever the geometry is such that the cross-sectional area available for flow is expected to differ significantly from the $\mathrm{V}_{\mathrm{g}}^{2 / 3}$ default value.

\subsubsection{Basic Heat Transfer Correlations}

This section discusses a number of rather common Nusselt number, or heat transfer, correlations that are used at a number of interfaces in CONTAIN. Both natural convection and forced convection heat transfer correlations are discussed. However, boiling heat transfer is not discussed here but in Section 10.4.

This section discusses both the natural convection and forced convection heat transfer correlations that are used by default at the interface between a gaseous bulk fluid and the surface of a heat transfer structure. The natural convection correlations discussed here are also used at the interface between the gas and a lower cell layer, including the pool, and between the pool and a heat transfer structure. They are also used between the pool and a lower cell layer, provided boiling heat transfer conditions between the latter do not exist. It should be noted that no modeling of forced convection per se is presently available at interfaces involving the pool or lower cell, although it may be possible 
to simulate forced convection through tabular input of total heat transfer coefficients with respect to the pool-gas or pool-layer interfaces (see Section 5.5).

Note that the natural and forced convective correlations specific to the geometries of various engineered systems, including those for fan coolers and containment sprays, are not discussed here but in Chapter 12. In addition, although the treatment of heat transfer for an ice condenser uses the same basic correlations as those at a gas-structure interface, the implementation of user-options and the treatment of forced convection is sufficiently different to warrant a separate discussion of these matters in Section 12.2.

The correlations given below are the same as in code versions prior to CONTAIN 1.2. However, in the natural convection correlations, the driving density difference for a gaseous bulk fluid now includes composition differences as well as temperature differences, as discussed in Section 10.1.1. This change necessitates some changes in terminology when describing how natural convection correlations for horizontal surfaces are used. Formerly, it was customary to distinguish cases based upon whether the surface is hotter or cooler than the bulk gas. With the new formulation, the distinction is based upon whether the density difference corresponds to a stabilizing or a destabilizing density gradient. The density gradient is stabilizing when $\rho_{\mathrm{BL}}>\rho_{\mathrm{b}}$ for a floor or $\rho_{\mathrm{BL}}<$ $\rho_{b}$ for a ceiling, and conversely. When steam is condensing from a normal air or nitrogen atmosphere, this rule leads to the same result as the former temperature criterion, but the rule is reversed when the noncondensable gas density is less than the steam density. Thus, when condensation is occurring from a warm atmosphere rich in hydrogen (or helium) onto a cold floor, the density gradient is destabilizing, not stabilizing, and the code will select the correlation appropriate for a destabilizing density gradient.

Three standard correlations [Bau78] are available in CONTAIN for determining $\mathrm{N}_{\mathrm{Nu}}$ in Equation $(10-2)$ in various natural convection regimes. The first of the standard correlations is a laminar correlation used at the interface between the pool and a horizontal surface and between the pool and gas for the pool-side boundary layer, when a destabilizing gradient exists and the pool-side Rayleigh number $N_{R a}=N_{G r} N_{P_{r}}$ is less than $10^{7}$ :

$$
\mathrm{N}_{\mathrm{Nu}, \mathrm{c}}=0.54\left(\mathrm{~N}_{\mathrm{Pr}} \mathrm{N}_{\mathrm{Gr}}\right)^{1 / 4}
$$

Here $N_{G r}$ is the Grashof number and $N_{P r}$ is the Prandtl number as defined in Equations (10-11) and (10-12), respectively.

The following turbulent correlation is used by default at the interface between the gas and a vertical surface and between the gas and a horizontal surface for a destabilizing gradient at any Rayleigh number; it is also used between the pool and a heat transfer structure, between the gas and pool on the pool side, and between the pool and a lower cell layer, provided the surface is vertical or the pool-side Rayleigh number satisfies $N_{R a}>10^{7}$ :

$$
\mathrm{N}_{\mathrm{Nu}, \mathrm{c}}=0.14\left(\mathrm{~N}_{\mathrm{Gr}} \mathrm{N}_{\mathrm{Pr}}\right)^{0.33}
$$


As discussed in Section 10.1.3, the user may specify alternatives to this correlation in the case of an interface between the gas and a heat transfer structure. Note that alternatives to the above are not available in the case of interfaces involving the pool.

The following laminar correlation is used for either the gas or pool for a horizontal interface with a stabilizing density gradient:

$$
\mathrm{N}_{\mathrm{Nu}, \mathrm{c}}=0.27\left(\mathrm{~N}_{\mathrm{Gr}} \mathrm{N}_{\mathrm{Pr}}\right)^{1 / 4}
$$

For the natural convection correlations, only one input variable is required. This is the characteristic length, which is discussed in detail in Section 10.1.1.5. Note, however, that there is actually very little dependence of the heat transfer coefficient on this characteristic length. For laminar natural convection, the dependence is to the $-1 / 4$ power of length, while for turbulent natural convection, there is no dependence at all.

Forced convection correlations are used at the interface between the gas and a heat transfer structure, but are not considered at gas-lower-cell, pool-structure, or pool-lower-cell interfaces. The standard forced convection correlation used by default is

$$
\mathrm{N}_{\mathrm{Nu}, \mathrm{f}}=0.037 \mathrm{~N}_{\mathrm{Re}}^{0.8} \mathrm{~N}_{\mathrm{Pr}}^{0.33}
$$

The user may specify an alternative to this correlation, as discussed in Section 10.1.3.3. The gas convective velocity, $v_{c}$, required to evaluate $\mathrm{N}_{\mathrm{Re}}$ from Equation (10-10) is either calculated from the gas flow rates into and out of the cell, as discussed in Section 10.1.1.6, or specified directly or indirectly by the user through tabular input options.

The forced convection tabular input options are specified in the BCINNER or BCOUTER input block of the heat transfer structure input, as discussed in Section 14.3.1.3. Options similar to the BCINNER forced convection tabular input may also be specified in the CONDENSE block (see Appendix B), but such usage is considered obsolete.

Three forced convection tabular input options are available. When any of these options are specified, the tabular input takes precedence over the forced convection velocities calculated from gas flow rates. In the first option, the user can specify the convective velocity, $\mathrm{v}_{c}$, as a function of time. In the second the user can specify $N_{R e}$ as a function of time. In either case, either Equation (10-23) or one of the alternative forms discussed in Section 10.1.3.3 is used to evaluate $\mathrm{N}_{\mathrm{Nu}, \mathrm{f}}$. In the third option, the user can specify the value of $\mathrm{N}_{\mathrm{Nu}, \mathrm{f}}$ directly.

In the default treatment of mixed natural and forced convection, $\mathrm{N}_{\mathrm{Nu}, \mathrm{f}}$ will be used for the heat transfer calculation only if it exceeds $\mathrm{N}_{\mathrm{Nu}, \mathrm{c}}$ as calculated from the appropriate natural convection correlation; i.e., $N_{\mathrm{Nu}}=\max \left(\mathrm{N}_{\mathrm{Nu}, c}, \mathrm{~N}_{\mathrm{Nu}, f}\right)$ is the value actually used. Alternatives to this approach for treating mixed convection are discussed in Section 10.1.3.4. 


\subsubsection{Generalized Gas-Structure Convective Heat Transfer Options}

In code versions prior to CONTAIN 1.2, the user has no control over the natural convection correlations used for heat and mass transfer and has control over the forced convection correlations only through the tabular forced convection options described in Section 10.1.3. For gas-structure heat transfer, CONTAIN 1.2 now includes a substantial range of additional options for controlling convective heat transfer to structures, and these options will be described in the present section. These options are not available for interfaces between the gas and lower cell, including the pool, between the pool and a heat transfer structure, or between the pool and lower cell, or for engineered system interfaces.

The remainder of this section describes the new heat and mass transfer options that have been implemented. Input for these options is specified in the BCINNER block for the structure to which the options are to be applied; see Section 14.3.1.3 for the details. The same input specifications are available for the BCOUTER block when the outer surface of the structure is in the same cell.

10.1.3.1 Rohsenhow Handbook Heat Transfer Correlations for Natural Convection. Correlations described by Raithby and Holland in Reference Roh85 reportedly give good results for natural convection heat transfer from free planar surfaces over a wide range of $\mathrm{N}_{\mathrm{Gr}}$ values (approximately 1 through $10^{12}$ ) spanning both the laminar and turbulent regimes. There are three separate correlations: one for vertical surfaces, one for horizontal surfaces with a destabilizing density gradient across the boundary layer, and one for horizontal surfaces with stabilizing density gradients. In this correlation set, the vertical wall case and the destabilized horizontal case are not treated as being exactly the same, as they are in the default correlation set. Here, the definitions of "stabilized" and "destabilized" cases for the horizontal surfaces are the same as in Section 10.1.2.

For vertical surfaces and the destabilized horizontal case, the correlations take the form

$$
N_{N u, c}=\left(N_{N u, 1}^{m}+N_{N u, t}^{m}\right)^{1 / m}
$$

where

$$
\begin{aligned}
\mathrm{N}_{\mathrm{Nu}, \mathrm{t}} & =\mathrm{C}_{\mathrm{t}}\left(\mathrm{N}_{\mathrm{Gr}} \mathrm{N}_{\mathrm{Pr}_{\mathrm{r}}}\right)^{1 / 3} \\
\mathrm{~N}_{\mathrm{Nu}, \mathrm{l}} & =\frac{\mathrm{C}_{1}}{\ln \left(1+\mathrm{C}_{1} / \mathrm{N}_{\mathrm{Nu}, \ell}\right)} \\
\mathrm{C}_{1} & =\frac{\mathrm{C}_{0}}{\left[1+\left(0.492 / \mathrm{N}_{\mathrm{Pr}}\right)^{9 / 16}\right]^{1 / 9}} \\
\mathrm{~N}_{\mathrm{Nu}, \ell} & =\mathrm{C}_{1}\left(\mathrm{~N}_{\mathrm{Gr}} \mathrm{N}_{\mathrm{Pr}_{\mathrm{r}}}\right)^{1 / 4}
\end{aligned}
$$

In the above, for the destabilized horizontal case, $m=10, C_{t}=0.14, C_{1}=1.4$, and $C_{0}=0.580$. For vertical surfaces, $m=6, C_{1}=2.8, C_{0}=0.671$, and $C_{t}$ is given by 


$$
C_{t}=\frac{0.13 N_{P r}^{0.22}}{\left(1+0.61 N_{P r}^{0.81}\right)^{0.42}}
$$

For the stabilized horizontal case, the correlation is

$$
\mathrm{N}_{\mathrm{Nu}, \mathrm{c}}=\frac{0.527\left(\mathrm{~N}_{\mathrm{Gr}} \mathrm{N}_{\mathrm{Pr}_{\mathrm{r}}}\right)^{0.2}}{\left[1+\left(1.9 / \mathrm{N}_{\mathrm{Pr}}\right)^{0.9}\right]^{2 / 9}}
$$

If the keyword ROHAND is specified, the appropriate correlation from this set will be selected and applied, with no additional input being required.

10.1.3.2 User-Defined Natural Convection Correlations. To provide improved flexibility in heat transfer modeling, an option has been provided to permit the user to specify the constants and exponents appearing in either one or two natural convection correlations. If two correlations are specified, the user also has control over the correlation actually used. The two correlations have the same algebraic form:

$$
\begin{aligned}
& N_{\mathrm{Nu}, \mathrm{c}, 1}=\mathrm{a}_{1}+\mathrm{b}_{1} \mathrm{~N}_{\mathrm{Gr}}^{\mathrm{c}_{1}} \mathrm{~N}_{\mathrm{Pr}}^{\mathrm{d}_{1}} \\
& \mathrm{~N}_{\mathrm{Nu}, \mathrm{c}, 2}=\mathrm{a}_{2}+\mathrm{b}_{2} \mathrm{~N}_{\mathrm{Gr}}^{\mathrm{c}_{2}} \mathrm{~N}_{\mathrm{Pr}}^{d_{2}}
\end{aligned}
$$

The user specifies the quantities $a_{1}, \ldots, d_{1}, a_{2}, \ldots, d_{2}$. If one of the correlations is specified, but not both, the natural convection Nusselt number, $\mathrm{N}_{\mathrm{Nu},}$, is equated to whichever correlation is specified. If both of the correlations are specified, the following options are available:

1. $\mathrm{N}_{\mathrm{Nu}, \mathrm{c}}=\max \left(\mathrm{N}_{\mathrm{Nu}, \mathrm{c}, 1}, \mathrm{~N}_{\mathrm{Nu}, \mathrm{c}, 2}\right)$. This is the default option.

2. If the keyword STBNCOR is specified, $N_{\mathrm{Nu}, \mathrm{c}}=\mathrm{N}_{\mathrm{Nu}, \mathrm{c}, \mathrm{1}}$ when the boundary layer density gradient is stabilizing, and $N_{\mathrm{Nu}, \mathrm{c}}=\mathrm{N}_{\mathrm{Nu}, \mathrm{c}, 2}$ when the density gradient is destabilizing. This option is normally not meaningful when the structure is a wall, but its use is still permitted, in which case the stability criterion will be applied as if the structure were a floor.

3. A root-sum-power combination of the two correlations can be used:

$$
N_{N u, c}=\left(N_{N u, c, 1}^{m_{1}}+N_{N u, c, 2}^{m_{1}}\right)^{1 / m_{1}}
$$

The value of the exponent $m_{1}$ in this case is specified through the "xm1" variable. 
Note that the default correlations will not be considered at all if one or more of the user-defined correlations has been invoked. If it is desired to have the code choose between a default correlation and a user-defined correlation, then the default correlation must also be entered explicitly as one of the user-defined correlations.

10.1.3.3 User-Defined Forced Convection Correlations. Options permitting the user to specify forced convection correlations have also been provided. These options are virtually identical to the natural convection options except that $\mathrm{N}_{\mathrm{Gr}}$ is replaced by the Reynolds number $\mathrm{N}_{\mathrm{Re}}$ :

$$
\begin{aligned}
& N_{N u, f, 1}=a_{3}+b_{3} N_{R e}^{c_{3}} N_{P r}^{d_{3}} \\
& N_{N u, f, 2}=a_{4}+b_{4} N_{R e}^{c_{4}} N_{P_{r}}^{d_{4}}
\end{aligned}
$$

The user specifies the quantities $a_{3}, \ldots, d_{3}, a_{4}, \ldots, d_{4}$. If one of the correlations is specified, but not both, the forced convection Nusselt number, $\mathrm{N}_{\mathrm{Nu}, \mathrm{f}}$, is equated to whichever correlation is specified; as with the natural convection correlations, the default forced convection correlation is not considered if one or more of the user-defined forced convection correlations is invoked. If both of the correlations are invoked, the following options are available:

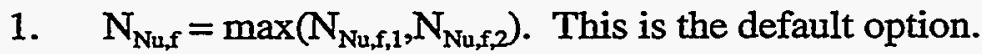

2. If the keyword STBFCOR is specified, $\mathbf{N}_{\mathrm{Nu}, \mathrm{f}}=\mathbf{N}_{\mathrm{Nu}, \mathrm{f}, \mathrm{w}}$ when the boundary layer density gradient is stabilizing, and $\mathrm{N}_{\mathrm{Nu}, \mathrm{f}}=\mathrm{N}_{\mathrm{Nu}, \mathrm{f}, 2}$ when the density gradient is destabilizing. Physically, the motivation for defining this option may be less clear than in the case of natural convection, but it may prove useful in some instances. If STBFCOR is specified for a wall, the stability criterion is applied as if it were a floor.

3. A root-sum-power combination of the two correlations can be used:

$$
N_{\mathrm{Nu}, \mathrm{f}}=\left(N_{\mathrm{Nu}, \mathrm{f}, 1}^{m_{2}}+N_{\mathrm{Nu}, \mathrm{f}, 2}^{m_{2}}\right)^{1 / m_{2}}
$$

The value of the exponent $\mathrm{m}_{2}$ is specified through the "xm2" variable.

If forced convection information has been specified through one of the tabular options and $\mathrm{N}_{\mathrm{Nu}, \mathrm{f}}$ itself has not been specified directly in the table, the resulting Reynolds numbers will be used in the above user-defined forced convection correlations. However, if $\mathrm{N}_{\mathrm{Nu}, \mathrm{f}}$ has been specified directly, any of the above user-defined forced convection correlations will be superceded by the tabular values.

10.1.3.4 Mixed Convection. The effects of mixed natural and forced convection depend in part on whether the buoyancy forces are in the same direction as the forced flow (assisted convection) or in the opposite direction (opposed convection). For assisted laminar convection, a relation that has some theoretical and experimental support is 


$$
N_{\mathrm{Nu}}=\left(N_{\mathrm{Nu}, \mathrm{c}}^{3}+N_{\mathrm{Nu}, \mathrm{t}}^{3}\right)^{1 / 3}
$$

Equation (10-28) with a minus sign between the two terms has also been used, perhaps with less justification, for the opposed laminar convection case.

For the turbulent mixed convection case, the physical situation is more complex. Even the terminology can get confusing because the effect upon $\mathrm{N}_{\mathrm{Nu}}$ is typically in the opposite direction from what it is in the case for laminar mixed convection. That is, the case of assisted convection (in the sense of aligned buoyancy and forced convection forces) typically yields $\mathrm{N}_{\mathrm{Nu}}$ values less than $\max \left(\mathbb{N}_{\mathrm{Nu},}, \mathrm{N}_{\mathrm{Nu}, \mathrm{f}}\right)$, while opposed convection has the reverse effect. (Physically, one interpretation is that the assisted convection case tends to laminarize the boundary layer, reducing heat and mass transfer, while the opposed convection case results in enhanced turbulent transport.) Quantitatively, the situation is quite complex, with the magnitude of the effects in either direction depending upon many details, including the geometrical ones.

It would be difficult if not completely impossible to provide a general mechanistic treatment of mixed convection in the CONTANN code, and no such treatment has been attempted. Instead a simple parametric representation of possible effects of mixed convection has been provided. Since the code does not attempt to make an internal choice as to whether laminar or turbulent conditions apply, the terminology "enhanced heat transfer" and. "impaired heat transfer" is used in the present discussion. "Enhanced heat transfer" cases would correspond to the assisted laminar case or the opposed turbulent convection case, and conversely for "impaired heat transfer" cases.

As in code versions prior to CONTAIN 1.2, $\mathrm{N}_{\mathrm{Nu}}$ is by default equated to $\max \left(\mathrm{N}_{\mathrm{Nu}, c}, \mathrm{~N}_{\mathrm{Nu}, f}\right)$ no matter what correlations have been used to obtain $\mathrm{N}_{\mathrm{Nu}, \mathrm{c}}$ and $\mathrm{N}_{\mathrm{Nu}, \mathrm{f}}$. However, if the MIXED keyword is specified, the following expression will be used:

$$
\mathrm{N}_{\mathrm{Nu}}=\max \left(\mathrm{N}_{\mathrm{Nu}, 1}, \mathrm{C}_{2} \max \left(\mathrm{N}_{\mathrm{Nu}, \mathrm{f}}, \mathrm{N}_{\mathrm{Nu}, \mathrm{c}}\right)\right)
$$

where

$$
N_{N u, 1}=\left[\left|N_{N u, f}^{m_{3}}+C_{1} N_{N u, c}^{m_{3}}\right|\right]^{1 / m_{3}}
$$

and the parameters $C_{2}, m_{3}$, and $C_{1}$ have default values of $0.75,3$ and -1 , respectively. The user may redefine any or all of these three parameters. $C_{1}$ might typically be chosen to be either +1 or -1 in order to represent the enhanced or the impaired heat transfer case, respectively, but there is no requirement that $\mathrm{C}_{1}$ be restricted to these values.

10.1.3.5 Heat Transfer Multiplier for Gas-Structure Interfaces. No matter how $\mathrm{N}_{\mathrm{Nu}}$ has been obtained for a heat transfer structure, the user may alter it by an overall factor that remains constant throughout the calculation. This multiplier, specified through the keyword HMXMUL, multiplies the $\mathrm{N}_{\mathrm{Nu}}$ value the code would have otherwise used. The default value for the multiplier is unity. 
Because the heat and mass transfer analogy is used to determine the condensation mass transfer coefficient, this multiplier will also affect condensation mass transfer rates (see Section 10.2.1.3).

\subsection{Mass Transfer Processes}

This section discusses a number of topics related to individual mass transfer processes at the interface between the gaseous bulk fluid and a surface. Note that a discussion of the effects of the coupling between the various heat and mass transfer processes at such an interface, through the interface temperature calculation is given in Section 10.6.

Section 10.2.1 is an extension of the discussion in Section 10.1.1 on the evaluation of the boundary layer properties and nondimensional numbers. Whereas the latter discusses quantities related to heat transfer, the former extends the discussion to quantities related to condensation mass transfer. The vapor diffusivity, the Schmidt number, and Sherwood number are discussed in this section.

Section 10.2.2 discusses the modeling of condensate films on heat transfer structures. The default film model, which uses a fixed film depth parameter, is discussed, as well as a film tracking model implemented in CONTAIN 1.2. The latter model allows films to be treated according to film flow correlations. It also allows films to be modeled on a generalized surface represented by a number of structure surfaces.

Section 10.2.3 derives the standard expression for condensation (and evaporation) mass transfer under quasi-steady conditions. For most reactor accident scenarios, the containment thermal hydraulic conditions will be such that the condensation mass transfer process will dominate much of the time. Thus, this process plays a key role in the discussion given in Section 10.6 on the coupling between various heat and mass transfer processes.

\subsubsection{Boundary Layer Properties and Nondimensional Numbers for Mass Transfer}

This section discusses a number of quantities appearing in expressions for condensation mass transfer: One quantity is the diffusivity, $\bigoplus_{v}$, of steam in the mixture of noncondensable gases present. In code versions prior to CONTAIN 1.2, the diffusivity of steam in air is used regardless of the noncondensable gas composition present. A more general model for the diffusivity has been implemented in CONTAIN 1.2 and is described in Section 10.2.1.1. The evaluation of the Schmidt and Sherwood numbers appearing in expressions for mass transfer are discussed in Section 10.2.1.2. Methods for changing the default modeling of condensation mass transfer rates are discussed in Section 10.2.1.3.

10.2.1.1 Diffusivity of Steam. In code versions prior to CONTAIN 1.2, the diffusivity assumed for steam is not a function of the gas composition. The value used is based upon a formulation given in Reference Bir60 which, when evaluated specifically for water vapor in air, reduces to

$$
\bigoplus_{\mathrm{v}, \mathrm{air}}=4.40 \times 10^{-6} \mathrm{~T}^{2.334} / \mathrm{P}
$$


where $\bigoplus_{v, \text { air }}$ is the diffusivity $\left(\mathrm{m}^{2} / \mathrm{s}\right), \mathrm{T}$ is the temperature $(\mathrm{K})$, and $\mathrm{P}$ is the pressure $(\mathrm{Pa})$. In prior code versions, Equation (10-33) is used for the diffusivity of steam throughout CONTAIN.

In general, Equation (10-33) is expected to give good results only when the dominant noncondensable gas is air (or nitrogen) and only when the temperature is relatively low, less than $500 \mathrm{~K}$. At high temperatures, Equation (10-33) can substantially overestimate the steam-air diffusivity, while at high hydrogen concentrations, Equation (10-33) can substantially underestimate the diffusivity. (For very hot steam-hydrogen mixtures, these errors cancel to a reasonable approximation, [Wil87a] a circumstance that has been used to advantage in the direct containment heating (DCH) model; see Section 6.4.)

Beginning with CONTAIN 1.2, mass transfer is calculated using diffusivities that take into account the composition of the bulk noncondensable gases. Note that there is some loss of upward compatibility with versions prior to CONTAIN 1.2, as options for restoring the old diffusivity calculation are not available. The present treatment is based upon that of Reference Bir60 for onedimensional diffusion of ideal gases. The diffusivity $\bigoplus_{i}$ of component $i$ with respect to a mixture of $\mathrm{n}$ species is given approximately by

$$
\frac{1}{c \Phi_{i}}=\frac{\sum_{j=1}^{n}\left(1 / c \bigoplus_{i, j}\right)\left(X_{j} N_{i}-X_{i} N_{j}\right)}{N_{i}-X_{i} \sum_{j=1}^{n} N_{j}}
$$

where $c$ is the molar concentration of the mixture (total moles $/ \mathrm{m}^{3}$ ), the $\bigoplus_{\mathrm{ij}}$ are the binary diffusivities for the pair $(\mathrm{i}, \mathrm{j})$; and the $\mathrm{N}_{\mathrm{i}}$ is the molar flux for each constituent.

For the case of steam condensing on (or evaporating from) a surface, the $\mathrm{N}_{\mathrm{i}}$ are equal to zero for all the noncondensable constituents once a quasi-steady boundary layer has been established, since there can be no net flux of a noncondensable species into or out of the surface. If steam is defined as the first constituent and the subscript $i$ is replaced with $v$, Equation (10-34) simplifies to

$$
\frac{1-X_{v}}{\bigoplus_{v}}=\frac{X_{n c}}{\bigoplus_{v}}=\sum_{j=2}^{n} \frac{X_{j}}{\bigoplus_{v, j}}
$$

Here $\bigoplus_{v j}$ is the binary diffusivity of steam with respect to component $j$ of the mixture, and $X_{j}$ is the mole fraction of component $j$ in the total mixture including the steam (i.e., steam is included in the total number of moles when calculating the mole fraction.)

Reference Per73 recommends the use of the Wilke-Lee modification of the Hirschfelder, Bird, and Spotz (WL-HBS) model when accurate values of binary diffusivities are desired. Binary diffusivities evaluated using this formalism, which is somewhat complex, are plotted in Figure 10-4 as a function of $\mathrm{T}(\mathrm{K}) /(300 \mathrm{~K})$ for the gases $\mathrm{H}_{2}, \mathrm{He}, \mathrm{O}_{2}, \mathrm{~N}_{2}, \mathrm{CO}_{2}$, and Ar. (The division by 300 was done to permit a single decade to span the range of abscissas of interest; this normalization has no other significance.) 


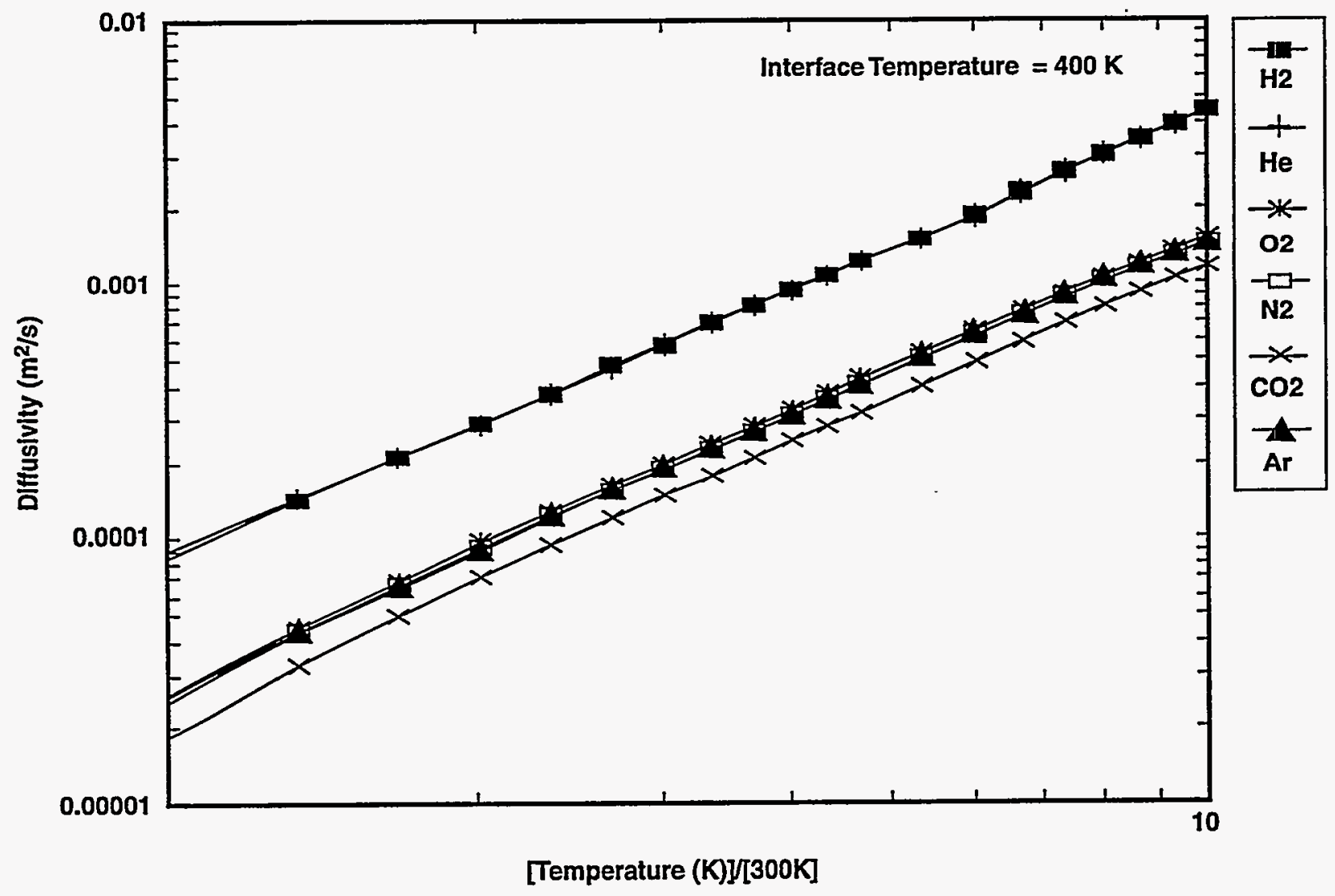

Figure 10-4. Steam Binary Diffusivities in Gases Calculated from the WL-HBS Model 
Helium is included because it frequently has been used by experimentalists as a safe simulant for hydrogen.

It is apparent from the plot that the diffusivities of steam in $\mathrm{H}_{2}$ and $\mathrm{He}$ are very similar to one another. The diffusivities of steam in $\mathrm{N}_{2}, \mathrm{O}_{2}$, and $\mathrm{Ar}$ are likewise very similar to one another, and they are considerably smaller than the $\mathrm{H}_{2}$ and $\mathrm{He}$ diffusivities. Furthermore, evaluation of the model for air and for $\mathrm{CO}$ yielded results that would be indistinguishable from $\mathrm{N}_{2}$ on the scale of the plot. For atmospheres of potential interest for reactor applications or reactor experiments, it is therefore an excellent approximation to treat the system as consisting of just four components: steam, a light noncondensable gas $\left(\mathrm{H}_{2}+\mathrm{He}\right), \mathrm{CO}_{2}$ if present, and air to represent everything else.

Implementing the WL-HBS model directly in CONTAIN would be possible; however, inspection of the plots in Figure 10-4 shows that the curves are almost straight, indicating that a power-law fit of the form

$$
\boxplus=\mathrm{AT}^{\mathrm{B}} / \mathrm{P}
$$

where A and B are constants, should be reasonably satisfactory provided one does not attempt to force a single fit to serve over too wide a temperature range. It turns out that breaking the full temperature range into two intervals, from $273 \mathrm{~K}$ to $700 \mathrm{~K}$ and from $700 \mathrm{~K}$ to $3000 \mathrm{~K}$, gives good results. The power-law fits are compared with the WL-HBS diffusivities in Figure 10-5 for $\mathrm{H}_{2}, \mathrm{~N}_{2}$, and $\mathrm{CO}_{2}$. The power law approximation is very satisfactory and has been adopted for CONTAIN 1.2. Values of $A$ and $B$ suitable for use in Equation (10-36) are tabulated in Table 10-2. For $T \leq 700 \mathrm{~K}, A_{1}$ and $B_{1}$ are to be used, and $A_{2}$ and $B_{2}$ are to be used for $T>700 \mathrm{~K}$.

Table 10-2

Power-Law Diffusivity Constants

$\mathrm{P}$ in $\mathrm{Pa}, \mathrm{T} 2=700 \mathrm{~K}$

\begin{tabular}{||c||c|c|c|c||}
\hline \hline $\mathrm{Gas}$ & $\mathrm{A} 1$ & $\mathrm{~B} 1$ & $\mathrm{~A} 2$ & $\mathrm{~B} 2$ \\
\hline $\mathrm{O} 2$ & $7.95 \mathrm{e}-05$ & 1.835 & $2.19 \mathrm{e}-04$ & 1.680 \\
\hline $\mathrm{N} 2$ & $8.84 \mathrm{e}-05$ & 1.814 & $2.20 \mathrm{e}-04$ & 1.675 \\
\hline $\mathrm{H} 2$ & $4.60 \mathrm{e}-04$ & 1.734 & $7.32 \mathrm{e}-04$ & 1.663 \\
\hline $\mathrm{He}$ & $7.41 \mathrm{e}-04$ & 1.659 & $7.18 \mathrm{e}-04$ & 1.664 \\
\hline $\mathrm{CO}$ & $7.90 \mathrm{e}-05$ & 1.832 & $2.15 \mathrm{e}-04$ & 1.679 \\
\hline $\mathrm{CO} 2$ & $4.18 \mathrm{e}-05$ & 1.888 & $1.41 \mathrm{e}-04$ & 1.702 \\
\hline $\mathrm{Air}$ & $8.54 \mathrm{e}-05$ & 1.820 & $2.20 \mathrm{e}-04$ & 1.675 \\
\hline $\mathrm{D} 2$ & $1.22 \mathrm{e}-05$ & 1.986 & $5.06 \mathrm{e}-05$ & 1.769 \\
\hline $\mathrm{Ar}$ & $7.20 \mathrm{e}-05$ & 1.843 & $2.06 \mathrm{e}-04$ & 1.683 \\
\hline
\end{tabular}

${ }^{*} \mathrm{D}=\mathrm{A}_{1} \mathrm{~T}^{\mathrm{B} 1} / \mathrm{P}$ for $\mathrm{T} \leq 700 \mathrm{~K}, \mathrm{D}=\mathrm{A}_{2} \mathrm{~T}^{\mathrm{B} 2} / \mathrm{P}$ for $\mathrm{T}>700 \mathrm{~K}$. 


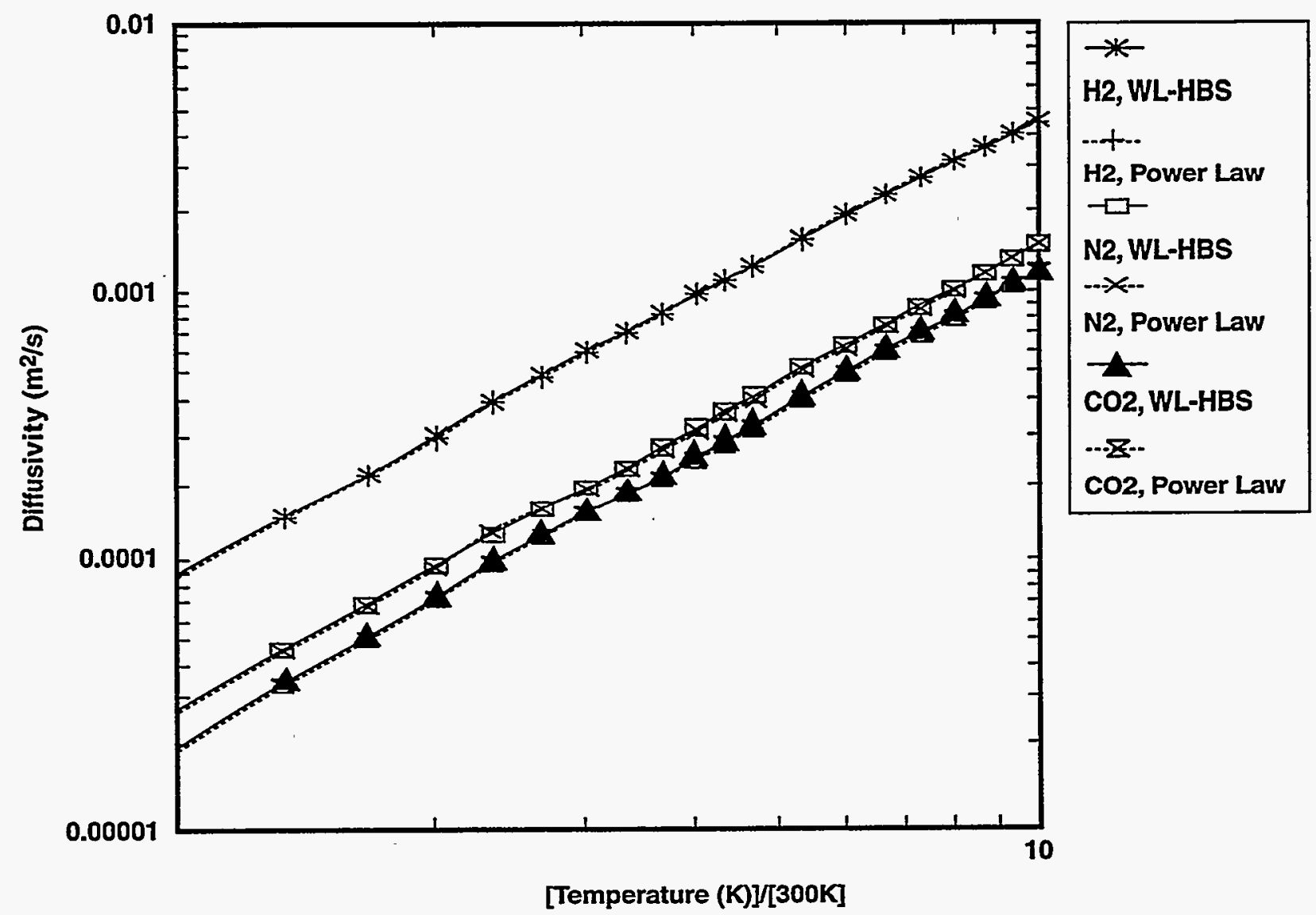

Figure 10-5. Comparison of WL-HBS Steam Binary Diffusivities with a Power-Law Fit 
In the CONTAIN implementation, the vapor diffusivity is obtained from Equation (10-34), within the four component treatment. The values of $\mathrm{A}$ and $\mathrm{B}$ used for air and $\mathrm{CO}_{2}$ are taken directly from the table. The values of A used for the light gas component are the geometric means of the values given for hydrogen and helium, and the values used for B are the arithmetic means. The resulting diffusivity corresponds to the geometric mean (i.e., the square root of the product). Since the two diffusivities never differ by more than a few percent, errors introduced by this approximation are trivial. The vapor diffusivities discussed in this chapter are evaluated at the boundary layer temperature $\mathrm{T}_{\mathrm{BL}}$ and corrected for boundary layer composition as discussed in Sections 10.1.1.1 and 10.1.1.2.

Although a more detailed treatment of the binary diffusivities would be possible, evaluation of the errors incurred by the simplifying approximations invoked here indicate that any effect of these approximations is no more than a few percent and is small compared with other uncertainties involved. The most important limitation of the present multicomponent treatment is believed to be the fact that the noncondensable gas composition, with the vapor removed, is assumed to be the same in the boundary layer as in the bulk. This composition in reality is not expected to be uniform in the boundary layer. This limitation is briefly discussed in Section 13.2.7.

10.2.1.2 Evaluation of the Nondimensional Mass Transfer Numbers. The dimensionless numbers entering specifically into the expressions for mass transfer are the Schmidt number and the Sherwood number. The Schmidt number $N_{S c}$ required by the gas-phase mass transfer correlations is given by

$$
\mathrm{N}_{\mathrm{Sc}}=\mu_{\mathrm{BL}} \mathrm{c}_{\mathrm{p}, \mathrm{BL}} / \bigoplus_{\mathrm{v}, \mathrm{BL}}
$$

where $\bigoplus_{\mathrm{v}, \mathrm{BL}}$ is the diffusivity of steam evaluated at the boundary layer temperature $T_{\mathrm{BL}}$ as discussed in Section 10.2.1.1 above.

The Sherwood number for mass transfer, $N_{S h}$, is obtained from the correlations for $N_{N u}$ described in Section 10.1 by applying the heat and mass transfer analogy. In code versions prior to CONTAIN $1.2, \mathrm{~N}_{\mathrm{Sh}}$ is obtained by multiplying $\mathrm{N}_{\mathrm{Nu}}$ by the quantity $\left(\mathrm{N}_{\mathrm{Sc}} \mathrm{N}_{\mathrm{Pr}_{\mathrm{r}}}\right)^{1 / 3}$, independently of how $\mathrm{N}_{\mathrm{Nu}}$ itself is obtained. In CONTAIN 1.2, $\mathrm{N}_{\mathrm{Sh}}$ is obtained from whatever correlation is used to obtain $\mathrm{N}_{\mathrm{Nu}}$ except that the Prandtl number $\mathrm{N}_{\mathrm{Pr}}$ is replaced by the Schmidt number $\mathrm{N}_{\mathrm{Sc}^{-}}$. This approach is used for the new heat transfer options described in Section 10.1.3 as well as for the default correlations. An exception is the case in which the user employs one of the tabular forced convection options discussed in Section 10.1.2 to specify the forced convection Nusselt number directly, in which case the forced convection Sherwood number $N_{\mathrm{Sh}, \mathrm{f}}$ is obtained by multiplying $\mathrm{N}_{\mathrm{Nu}, \mathrm{f}}$ by $\left(\mathrm{N}_{\mathrm{Sc}} / \mathrm{N}_{\mathrm{Pr}}\right)^{1 / 3}$ as before. These changes to the default treatment will normally make little or no difference to the results obtained. In general, differences in results obtained using the default correlations in CONTAIN 1.2 will be attributable to the revised interface and gas boundary layer property treatments discussed in Section 10.1.1 and/or the new diffusivity treatment to be described in Section 10.2.3, not the slight difference in how $\mathrm{N}_{\mathrm{Sh}}$ is calculated from $\mathrm{N}_{\mathrm{Nu}}$.

10.2.1.3 Mass Transfer Multipliers for Gas-Structure Interfaces. Three methods are available for changing condensation mass transfer rates at a gas-structure interface by an overall multiplier. The first is to use the generalized Nusselt number correlations discussed in Section 10.1.3 to replace the 
default correlations with ones of the same form but with larger coefficients. The second is to use the HMXMUL keyword discussed in Section 10.1.3.5 for applying a multiplier to the Nusselt number that would otherwise be used at a gas-structure interface. Since a heat and mass transfer analogy is used in CONTAIN to obtain the Sherwood number $\mathrm{N}_{\mathrm{Sh}}$ from the Nusselt number $\mathrm{N}_{\mathrm{Nu}}$, any change in $\mathrm{N}_{\mathrm{Nu}}$ will also affect $\mathrm{N}_{\mathrm{Sh}}$. In this case the ratio of heat to mass transfer would not be altered.

The third method is to use the keyword RMX2HX for specifying a multiplier by which $\mathrm{N}_{\mathrm{Sh}}$ is increased (or decreased) from the value obtained from $\mathrm{N}_{\mathrm{Nu}}$ through the heat and mass transfer analogy. This multiplier does not affect $\mathrm{N}_{\mathrm{Nu}}$. This input option is the only option that will directly alter the ratio of heat to mass transfer, besides omitting the CONDENSE keyword for a cell, which will result in no condensation at all in that cell. In all other cases, this ratio will be determined by the mass and heat transfer analogy.

\subsubsection{Condensate Films on Heát Transfer Structures}

The total mass in the water film on a heat transfer structure is required for a) the evaluation of total heat transfer to a surface, and b) to determine the reservoir of water on structures that is available for evaporation. The amount of water in the film is modeled in one of two ways. In the default modeling, a film is allowed to accumulate but not permitted to flow if the film thickness $\delta$ is less than "mndpth," the fixed minimum depth for the surface at which flow is allowed. However, the film is permitted to flow as necessary to maintain this depth. This depth may be specified by the user as "mndpth" in the heat transfer structure input block described in Section 14.3.1.3. The default for "mndpth" in this default film treatment is $0.0005 \mathrm{~m}$.

The second approach uses one-dimensional steady-state film flow correlations for either laminar or wavy laminar film flow, assuming no shear at the gas-film interface. This approach, which is applicable to the inner surfaces of structures, is referred to as the film tracking model, and is activated by the FILMFLOW input in the BCINNER input block of the structure input, as discussed in Section 14.3.1.3. Provisions have been made within the film tracking model to allow film behavior to be modeled over a generalized surface represented by a number of structure inner surfaces.

To better picture what is involved with this model, a number of structure inner surfaces are shown in Figure 10-6. This figure shows how these surfaces may be combined in a way that allows the simulation of film flow over a more general surface. Each surface is considered a "node" for the purpose of defining the flow characteristics of the surface. The mass conservation equation for the film is applied to each "half-node" of a surface, defined by a horizontal line that splits the inner surface into two equal areas. The correlations defined below are assumed to relate the average depth $\delta$ and average velocity $v_{\mathrm{f}}$ of the film along this line, and these quantities then define the mass flow rate from the upper half-node to lower half-node and the film runoff rate from the structure. The user may specify the node width $w$ along the equal-area line and the angle of inclination $\theta$ at this line. By default, these are determined by the structure type and orientation. As shown in Figure 10-6, a fraction of the film runoff from the $j$ th structure may be allocated to the $i$ th structure through the film transfer matrix $\mathscr{F}_{j i}$, which is specified by the user through the DIVERT keyword. Thus, twodimensional quasi-steady-state film flow down a series of structures can be simulated. 


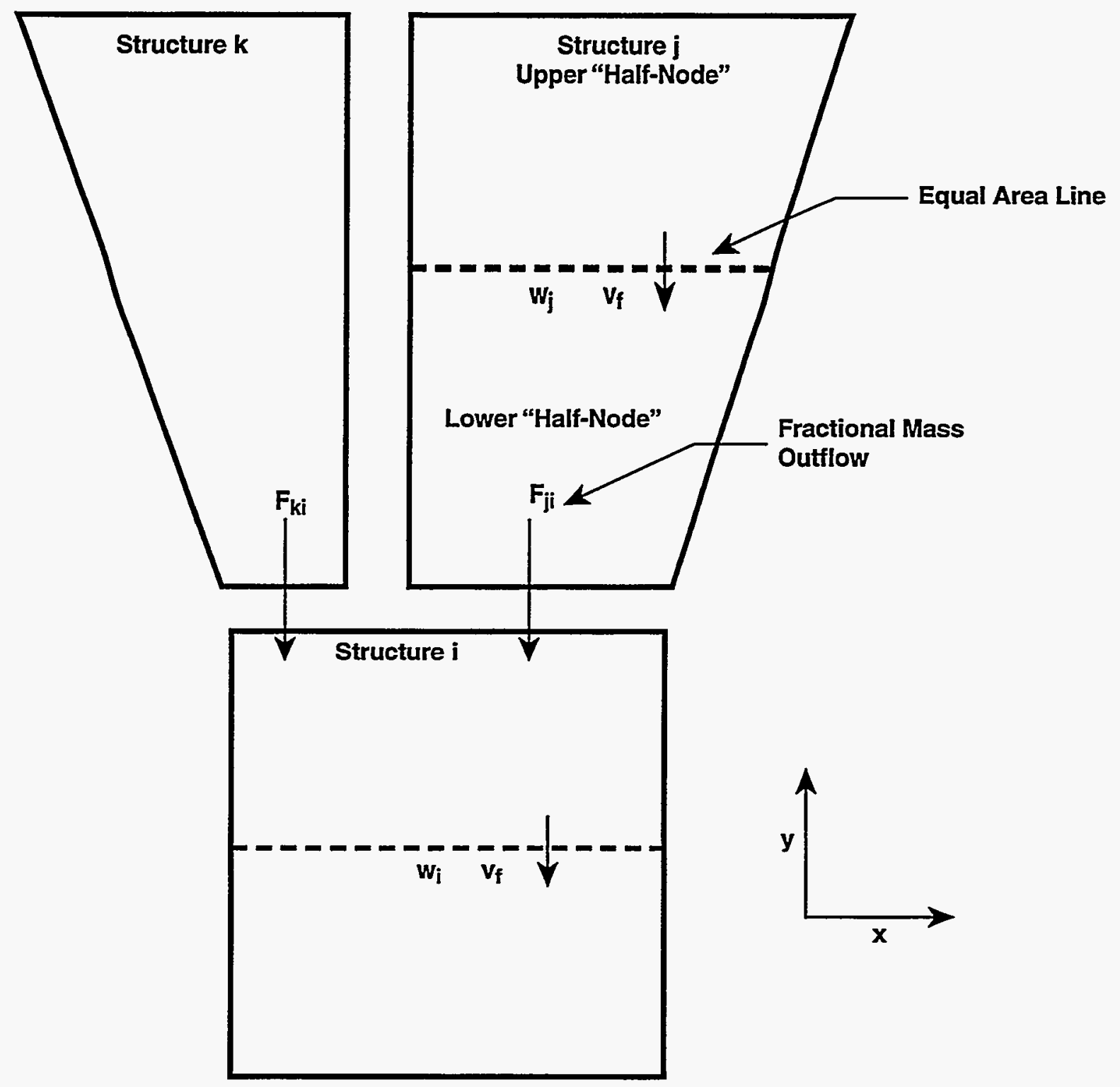

Figure 10-6. A Configuration of Structures That Could Be Used in the Film Tracking Model 
The film tracking model is discussed in detail in Section 10.2.2.1. The disposition of the film runoff is discussed in more detail in Section 10.2.2.2.

10.2.2.1 The Film Tracking Model. The film tracking model uses film flow correlations derived for films on the top side of an inclined surface $\left(0<\theta<90^{\circ}\right)$ or on a vertical surface $\left(\theta=90^{\circ}\right)$. Films on the underside of an inclined surface, such as the containment dome, are not stable, and the correlations presented here are not strictly applicable. Nevertheless, these correlations are made available for such films, for lack of more appropriate modeling, because they may be useful in parametrically representing the film behavior on such surfaces.

The expression used for the relation between film thickness and velocity depends on flow regime. This is determined by the film Reynolds number, $N_{R e, \Gamma}=4 \rho_{\ell} \delta v_{f} / \mu_{l}$, where $\rho_{l}$ is the film liquid density, $\delta$ is the average film depth, $v_{\mathrm{f}}$ the average film velocity, and $\mu_{\mathrm{l}}$ the liquid viscosity. For $\mathrm{N}_{\mathrm{Re}, \Gamma}<2328$, an analytically derived laminar flow expression is used [Wal69]

$$
\delta^{*}=0.909 \mathrm{~N}_{\mathrm{Re}, \Gamma}^{1 / 3}
$$

where the dimensionless film thickness $\delta^{*}$ is defined as

$$
\delta^{*}=\delta(g \sin \theta)^{1 / 3}\left(\rho_{\ell} / \mu_{\ell}\right)^{2 / 3}
$$

For $3 \times 10^{4}>N_{R e, \Gamma}>2328$, wavy laminar flow data have been fit to the expression [Wal69]

$$
\delta^{*}=0.115 \mathrm{~N}_{\mathrm{Re},}^{0.6}
$$

In implementing the film tracking method it is assumed that all processes but film inflow from other structures contribute to a uniformly distributed net mass of liquid $\Delta \mathrm{m}_{\mathrm{l}, \mathrm{i}}$ over the cell timestep $\Delta t_{\mathrm{c}}$

$$
\Delta \mathrm{m}_{\mathrm{n}, \mathrm{i}}=\Delta \mathrm{m}_{\mathrm{cond}}+\Delta \mathrm{m}_{\mathrm{eer}}+\Delta \mathrm{m}_{\mathrm{scc}}
$$

where $\Delta \mathrm{m}_{\text {cond }}$ is the net mass change due to condensation (or evaporation), $\Delta \mathrm{m}_{\text {eer }}$ is the mass change due to the settling of water aerosol, and $\Delta \mathrm{m}_{\text {scc }}$ is the external mass inflow, if any, that is specified through a film source table. The latter is implemented through the SOURCE input in the BCINNER input block.

With the above assumptions and the assumption that any change in the film inventory is also uniformly distributed, the runoff or outflow at the bottom of the $i$ th structure over the cell timestep $\Delta t_{c}$ is given by applying a mass balance to the lower half-node:

$$
\Delta \mathrm{m}_{\text {out }, \mathrm{i}}=\rho_{\ell} \delta\left(\mathrm{t}+\Delta \mathrm{t}_{\mathrm{c}}\right) \mathrm{v}_{\mathrm{f}}\left(\mathrm{t}+\Delta \mathrm{t}_{\mathrm{c}}\right) \mathrm{w} \Delta \mathrm{t}_{\mathrm{c}}+\Delta \mathrm{m}_{\mathrm{n}, \mathrm{i}} / 2-\left[\mathrm{m}_{\mathrm{i}}\left(\mathrm{t}+\Delta \mathrm{t}_{\mathrm{c}}\right)-\mathrm{m}_{\mathrm{i}}(\mathrm{t})\right] / 2
$$

where $t$ denotes the time at the beginning of the timestep, $w$ is the structure width, and $m_{i}$ is the structure film inventory. Also, $m_{i}\left(t+\Delta t_{c}\right)=A_{s} \delta\left(t+\Delta t_{c}\right) \rho_{l}$ where $A_{s}$ is the inner surface area of the 
structure. Note that the film depth and velocity in Equation (10-42) are defined implicitly at $t+\Delta t_{c}$ to eliminate flow surging.

The overall surface mass balance, for the upper and lower half-nodes combined, is given by

$$
\Delta \mathrm{m}_{\text {out }, \mathrm{i}}=\Delta \mathrm{m}_{\mathrm{in}, \mathrm{i}}+\Delta \mathrm{m}_{\mathrm{n}, \mathrm{i}}-\mathrm{m}_{\mathrm{i}}(\mathrm{t}+\Delta \mathrm{t})+\mathrm{m}_{\mathrm{i}}(\mathrm{t})
$$

where

$$
\Delta \mathrm{m}_{\mathrm{in}, \mathrm{i}}=\Sigma_{\mathrm{j}} \mathscr{F}_{\mathrm{ji}} \Delta \mathrm{m}_{\text {out } \mathrm{j}}
$$

represents the incoming film flow from overlying structures and where $\Delta \mathrm{m}_{\text {out,j }}$ is the film outflow from each of the overlying structures $j$, calculated as described here for the ith structure.

Equations (10-42) and (10-43) can be solved self-consistently for $\Delta \mathrm{m}_{\text {out, }}$ and $\delta\left(t+\Delta t_{\mathrm{c}}\right)$. Note that only positive $\Delta \mathrm{m}_{\text {outi }}$ are considered a valid solution of these equations. Under some conditions, a positive solution is not possible. For example, if normal processes are neglected and the initial film mass is zero, the outflow is formally negative unless the velocity at the equal-area line is such that at least one-half of the inflow $\Delta \mathrm{m}_{\mathrm{in}, \mathrm{i}}$ can be transported across the line to the lower half of the structure during the timestep. This condition is consistent with the fact that it should take any initial flow or appreciable surge a finite amount of time to flow down the structure face. If $\Delta \mathrm{m}_{\text {out, }}$ turns out negative, it is set to zero, and Equation (10-42) is solved again for $\delta$, with the left side set to zero.

To allow for film holdup because of roughness a user-specified minimum film thickness, "mndpth," for flow is used in the film tracking model as well as in the default model. If $\delta(t+\Delta t)$ as calculated above is greater than "mndpth," then the outflow will be taken to be that calculated above. If $\delta(t+\Delta t)$ $s$ "mndpth," then Equation (10-42) will be solved with $\delta(t+\Delta t)=$ "mndpth" and the resulting $\Delta \mathrm{m}_{\text {out,is }}$, if positive, will be used for the outflow. Otherwise, the outflow will be set to zero.

A finite default value of "mndpth," $0.0005 \mathrm{~m}$, is used with the default model since it is intended to model film holdup through both surface irregularities and film dynamics. However, with the film tracking model, the intent is to model film thickness through film dynamics. Thus, the default value of "mndpth" is set to zero if the film tracking model is invoked and to $0.0005 \mathrm{~m}$ otherwise.

The code architecture implemented for the film tracking model was motivated by the fact that even relatively modest film flow rates could cause numerical problems. These problems are related to the fact that in typical containment analyses, the timesteps used could be comparable to or exceed the material Courant limit for film flow, relative to the system timestep $\Delta t_{s}$ :

$$
\Delta \mathrm{m}_{\text {out },} \frac{\Delta \mathrm{t}_{\mathrm{s}}}{\Delta \mathrm{t}_{\mathrm{c}}}<0.2 \mathrm{~m}_{\mathrm{i}}(\mathrm{t})
$$

This limit corresponds to allowing changes in the film inventory on a structure by only a reasonable amount, typically $20 \%$, per system timestep. For a 3-meter-high structure and 10-second timesteps, 
this limit would correspond to a film velocity of only $0.06 \mathrm{~m} / \mathrm{s}$, which could easily be exceeded. Exceeding the Courant limit could cause accuracy and/or stability problems, depending on the film flow configuration and interfacing method.

The problem with the Courant limit has been solved in a manner consistent with the quasi-steadystate limitations of the present film modeling. In this approach, all structure outflows to other structures are simply accumulated over a system timestep and then presented as a constant inflow boundary condition to the recipient structure(s) during the following timestep. Under quasi-steadystate conditions this carryover approach to film interfacing is clearly reasonable. However, during the initial flooding of the structures from an externally specified film source, the propagation of the film will be delayed by one timestep per structure compared to what it would be without carryover; also, during the final draining, the film will persist somewhat longer. If the delay of one timestep per structure in the mass transfer is critical, then it is likely that the quasi-steady-flow correlations are themselves inadequate. Note that the carryover approach does not place obvious constraints on the nature of the film transfer matrix specified by the user. However, re-entrant or recirculating flow configurations have not been investigated and therefore should be avoided.

10.2.2.2 Disposition of Film Runoff. The disposition of film runoff is discussed in more detail in this section. For unsubmerged structures, the runoff from structures in the default model and the runoff not otherwise allocated by the film transfer matrix $\mathscr{F}_{\mathrm{ji}}$ in the film tracking model is controlled by the cell-level OVERFLOW keyword discussed in Section 14.3.1.12. Namely, it is transferred to the pool in the cell cited or, if a pool does not exist in that cell, is lost from the problem. If the OVERFLOW keyword has not been specified, the runoff is transferred to the pool in the cell in which the structure resides or, if such a pool does not exist, is lost from the problem. Partially submerged structures are treated somewhat differently. In such a case, the OVERFLOW keyword and film transfer matrix $\mathscr{F}_{\mathrm{j},}$ if specified, are ignored and the runoff is transferred entirely to the pool in which the structure is submerged.

\subsubsection{Condensation Mass Transfer}

The standard expressions for condensation mass transfer rates under quasi-steady conditions are derived in this section. Under such conditions, the condensation of vapor or evaporation of the condensed liquid on a surface is controlled by the difference in partial pressures of the condensable vapor across the gas boundary layer. Note that atmosphere-surface condensation modeling in a cell is activated through use of the CONDENSE keyword (see Section 14.3.1.4). Figure 10-7 illustrates a situation in which condensation is occurring in the presence of a boundary layer of thickness $\delta$ and a condensate film, which has formed in part or entirely from the condensation process. The partial pressure $P_{v}$ is that of the vapor and $P_{n c}$ is that of the noncondensables. The subscript $b$ denotes the bulk, and the subscript if denotes the interface. As indicated in Figure 10-7, the total pressure $P_{g}$ may be assumed constant to good approximation during this process. Although Figure 10-7 illustrates condensation, the models discussed in this section are also applicable to the evaporation process.

In order to derive the standard expressions, one must make approximations with regard to gas properties that vary over the boundary layer thickness. The CONTAIN treatment of such properties, with respect to mass transfer, is discussed in Sections 10.1.1.1, 10.1.1.2, 10.2.1.1, and 10.2.1.2. It 


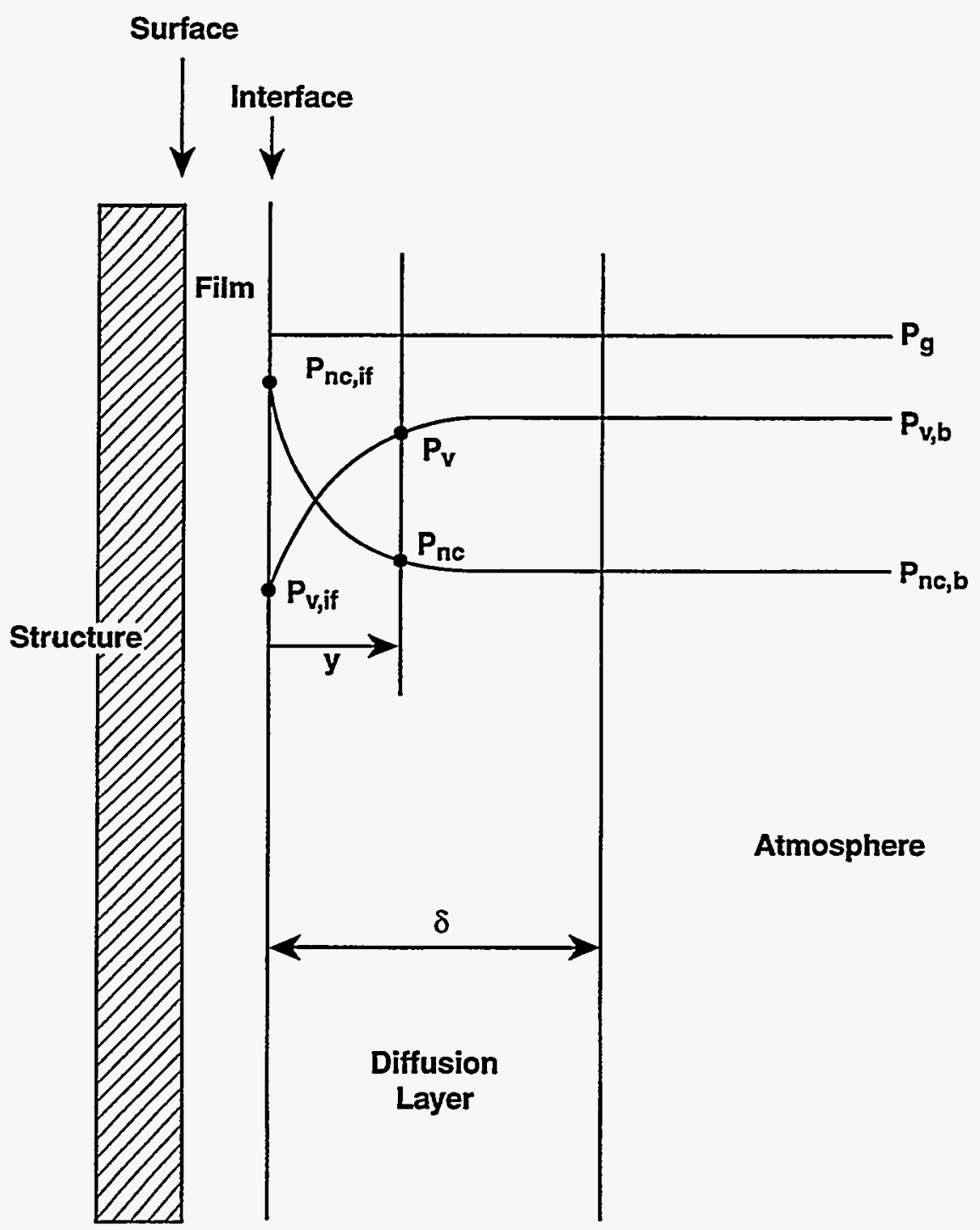

Figure 10-7. The Influence of Noncondensables on the Interface Resistance 
follows the standard recommendations [Bir60] for dealing with such gas properties. Namely, the boundary layer temperature $T_{\mathrm{BL}}$ used for such properties is taken to be fixed at the arithmetic average of the bulk temperature $T_{b}$ and the interface temperature $T_{i f}$, and the boundary layer composition is taken to be fixed at the average of the bulk and interface values. Also, as discussed in Section 10.1.1.2, the interface temperature used in the heat and mass transfer coefficients is actually taken to be the interface temperature calculated in the previous timestep for the surface in question.

For the example in Figure 10-7, both the molar fluxes ( $\left(\mathrm{kgmole} / \mathrm{m}^{2}-\mathrm{s}\right)$ of noncondensable gas $\mathrm{J}_{\mathrm{nc}}$ and condensable vapor $\mathbf{J}_{\mathrm{v}}$ passing through the interface are given by Reference Col81:

$$
\boxplus_{v} \frac{\partial C_{n c}}{\partial y}+C_{n c} v_{J}=J_{n c}
$$

and

$$
\mathrm{Ð}_{\mathrm{v}} \frac{\partial \mathrm{C}_{\mathrm{v}}}{\partial \mathrm{y}}+\mathrm{C}_{\mathrm{v}} \mathrm{v}_{\mathrm{J}}=\mathrm{J}_{\mathrm{v}}
$$

where $\bigoplus_{\mathrm{v}}$ is the boundary layer vapor diffusion coefficient $\left(\mathrm{m}^{2} / \mathrm{s}\right)$, evaluated as discussed in Section 10.2.1.1, $\mathrm{C}_{\mathrm{nc}}$ is the molar concentration of noncondensable gas $\left(\mathrm{kgmole} / \mathrm{m}^{3}\right), \mathrm{v}_{\mathrm{J}}$ is the drift-flux velocity of the bulk mixture toward the interface $(\mathrm{m} / \mathrm{s})$, and $\mathrm{C}_{\mathrm{v}}$ is the molar concentration of vapor $\left(\mathrm{kgmole} / \mathrm{m}^{3}\right)$.

Since the total pressure $P_{g}=P_{n c}+P_{v}$ is assumed constant in the diffusion process, it follows that

$$
\frac{\partial P_{n c}}{\partial y}=-\frac{\partial P_{v}}{\partial y}
$$

Furthermore, if one assumes that the diffusion takes place at a fixed boundary layer temperature $T_{B L}$ then the ideal gas law implies that

$$
\frac{\partial C_{n c}}{\partial y}=-\frac{\partial C_{v}}{\partial y}
$$

Substituting Equation (10-49) into Equation (10-47) results in

$$
J_{v}=C_{v} v_{J}-\boxplus_{v} \frac{\partial C_{n c}}{\partial y}
$$

Combining Equation (10-46) and Equation (10-50) to eliminate $\mathrm{v}_{\mathrm{J}}$ results in 


$$
J_{v}=-\bigoplus_{v} \frac{\partial C_{n c}}{\partial y}\left(\frac{C_{v}}{C_{n c}}+1\right)
$$

Substituting the ideal gas relation $\partial \mathrm{P}_{\mathrm{nc}} / \partial \mathrm{y}=\mathrm{R} \mathrm{T}_{\mathrm{BL}} \partial \mathrm{C}_{\mathrm{nc}} / \partial \mathrm{y}$, where $\mathrm{R}$ is the gas constant, into Equation $(10-51)$ results in

$$
J_{v}=-\frac{\bigoplus_{v}}{R T_{B L}} \frac{\partial P_{n c}}{\partial y}\left(\frac{C_{v}}{C_{n c}}+1\right)
$$

The above equation can be rewritten as

$$
J_{v}=-\frac{\boxplus_{v}}{R T_{B L}} \frac{\partial P_{n c}}{\partial y} \frac{P_{g}}{P_{n c}}
$$

One may integrate Equation (10-53) over the boundary layer thickness $\delta$ as follows

$$
\mathrm{J}_{\mathrm{v}} \int_{0}^{\delta} \partial \mathrm{y}=-\frac{\bigoplus_{\mathrm{v}}}{R \mathrm{~T}_{\mathrm{BL}}} \mathrm{P}_{\mathrm{g}} \int_{\mathrm{P}_{\mathrm{pccif}}}^{\mathrm{P}_{\mathrm{nc}, \mathrm{b}}} \frac{\partial \mathrm{P}_{\mathrm{nc}}}{\mathrm{P}_{\mathrm{nc}}}
$$

and obtain, after some algebra, an expression of the form

$$
j_{\text {cond }}=M_{v} J_{v}=K_{g} M_{v}\left(P_{v, b}-P_{v, i f}\right)
$$

where $j_{c o n d}$ is the condensation mass flux and $K_{g}$ is the mass transfer coefficient ( $\mathrm{kgmole} / \mathrm{m}^{2}-\mathrm{s}-\mathrm{Pa}$ ), defined as

$$
K_{g}=\frac{N_{S h} P_{g} \bigoplus_{v}}{R T_{B L} P_{n m}^{L}}
$$

In this expression $N_{S h}$ is the (uncorrected) Sherwood number defined by replacing $N_{P r}$ with $N_{S c}$ in the expression for $\mathrm{N}_{\mathrm{Nu}}$, as discussed in Section 10.2.1.2 above

$$
N_{S h}=N_{N u}\left\{N_{P r} \rightarrow N_{S c}\right\}
$$

and $\mathrm{P}_{\mathrm{nm}}$ is the logarithmic mean pressure

$$
P_{n m}=\frac{P_{v, i f}-P_{v, b}}{\ln \left(\frac{P_{g}-P_{v, b}}{P_{g}-P_{v, i f}}\right)}
$$


In Equation (10-54) the relation $\delta=\mathrm{L} / \mathrm{N}_{\mathrm{Sh}}$ has also been used, where $\mathrm{L}$ is the surface characteristic length. The modeling of such lengths is discussed in more detail in Section 10.1.1.5. Note that Equation (10-54) includes the standard high mass flux correction terms. [Bir60]

\subsection{Thermal Radiation}

When high gas temperatures occur (e.g., during a combustion event) in a compartment, thermal radiation may become a dominant heat transfer mechanism. The heat transfer is affected by the large quantities of water vapor that are typically present in containment, because of its strong emission bands and the optical depth attainable in typical containments. In addition to steam, carbon monoxide and carbon dioxide may be present as the result of core-concrete interactions and may also contribute to the emissivity of the atmosphere. Aerosols and dispersed core debris during a DCH event may also contribute.

In CONTAIN, the user can choose from two types of radiative heat transfer models. The first type is the net enclosure radiation model, which is activated through the keyword ENCLOS in the RAD-HEAT input block described in Section 14.3.1.5. This model allows for simultaneous calculations of radiative heat transfer among the cell atmosphere (which may include dispersed debris and/or aerosols), all inner surfaces of heat transfer structures, and the uppermost layer of the lower cell. With this model, radiative transfer between surfaces is modeled as well as radiation between the atmosphere and surfaces. However, because the enclosure geometry is fixed by input view factors and beam lengths, the net enclosure model should not be used if the cell geometry, including that of the coolant pool, changes appreciably during the course of a calculation. The second type of model is the direct radiative heat transfer model, activated through either of the keywords GASWAL or GEOBL (also described in Section 14.3.1.5), and the VUFAC keyword in the heat transfer structure input (described in Section 14.3.1.3). The VUFAC keyword in the heat transfer structure input invokes the modeling of direct radiative exchange between the uppermost lower cell layer and each structure inner surface. Within the direct model, the user can model radiative heat transfer from the atmosphere to both the inner and outer surfaces of a structure when both lie within a cell. As the name implies, the direct model treats the direct radiative heat transfer between the atmosphere and each structure, between the atmosphere and the uppermost layer of the lower cell, and between each structure inner surface and the uppermost layer. A limitation in this model is that only an approximate treatment of secondary reflections from other structures is possible.

For either type of model, provisions have been made to incorporate the effects of dispersed core debris on gas-surface, debris-gas, and debris-surface radiation modeling in DCH calculations. The direct model is generally recommended for simplicity in such calculations. However, the DCH modifications are viewed as special purpose and are therefore discussed in Sections 6.5.2 and 6.5.3 rather than in the present section.

Both types of radiation models take into account the effect of active gases and aerosol particles in determining the optical properties of the atmosphere. The three possible active gases considered in CONTAIN are water vapor, $\mathrm{CO}_{2}$, and $\mathrm{CO}$. If all three active gases are present, the default optical properties model, based on the method developed by Modak, is recommended. [Mod79] For those 
cases in which only water vapor is present, a Cess-Lian correlation may be specified with the CESS keyword in the RAD-HEAT input block. [Ces76] If this option is chosen, $\mathrm{CO}$ and $\mathrm{CO}_{2}$ (if present) are assumed to be transparent and do not contribute to the gas mixture emissivity.

Section 10.3.1 describes the net enclosure radiation model, and Section 10.3.2 discusses the direct radiation model. The gas mixture emissivity models used in CONTAIN are presented in Section 10.3.3.

\subsubsection{Net Enclosure Radiation Model}

The net enclosure model in CONTAIN is a treatment of radiative transfer involving a participating gas-aerosol-debris mixture and the surrounding surfaces that takes secondary reflections into account automatically. This model was adapted from a formulation for diffuse gray surfaces in Reference Sie81 (see the derivation in Reference Ber85b). A basic assumption is that the atmosphere is completely surrounded by the inner surfaces of heat transfer structures and the uppermost layer of the lower cell, if present. The surrounding surfaces can be at different temperatures with automatic accounting for secondary (surface-to-surface) reflections. Since the method requires the gas in the enclosure to be isothermal, only one cell is modeled at a time. In addition, each cell is treated as a complete enclosure. When a participating medium is not present in the cell (i.e., when water vapor, $\mathrm{CO}, \mathrm{CO}_{2}$, aerosols, and core debris are absent), the radiation model reduces to the surface radiative exchange problem with a transparent nonattenuating gas medium.

According to Kirchhoff's law, the radiation flux $q_{r a d j}$ to surface $j$ is given by

$$
q_{r a d, j}=\frac{\varepsilon_{s, j}}{1-\varepsilon_{s, j}}\left(\Psi_{j}-B_{j}\right)
$$

where $\varepsilon_{s, j}$ is the emissivity of surface $j, \psi_{j}$ is the radiosity of surface $j\left(W / m^{2}\right)$, and $B_{j}$ is the Planck blackbody radiation flux for surface $j\left(W / \mathrm{m}^{2}\right)$

$$
B_{j}=\sigma T_{w, j}^{4}
$$

where $\sigma$ is the Stefan-Boltzmann constant $\left(W / \mathrm{m}^{2}-\mathrm{K}^{4}\right)$, and $T_{\mathrm{w} j}$ is the surface node temperature for surface $\mathrm{j}$ (see Figure 10-9).

The radiosity, $\psi_{\mathbf{j}}$ in Equation (10-58), is calculated by solving a series of linear equations simultaneously.

$$
\psi_{j}-\left(1-\varepsilon_{s, j}\right) \sum_{k=1}^{N} F_{j k}\left(1-\varepsilon_{m, j k}\right) \psi_{k}=\varepsilon_{s, j} B_{j}+\left(1-\varepsilon_{s, j}\right) \sum_{k=1}^{N} F_{j k} \varepsilon_{m, j k} B_{g}
$$


where $N$ is the number of the surfaces in the enclosure, $F_{j k}$ is the user-input view factor from surface $\mathrm{j}$ to surface $\mathrm{k}$ (the fraction of radiation from surface $\mathrm{j}$ that is directed into the solid angle subtended by surface $\mathrm{k}), \varepsilon_{\mathrm{m}, \mathrm{k}}$ is the emissivity of gas mixture, $\varepsilon_{\mathrm{s}, \mathrm{j}}$ is the surface emissivity, and $\mathrm{B}_{\mathrm{g}}=\sigma\left(\mathrm{T}_{\mathrm{g}}\right)^{4}$ is the Planck blackbody radiation for the gas. The modeling of $\varepsilon_{\mathrm{m}, \mathrm{k}}$, which is a function of the userinput beam length $L_{\mathrm{jk}}$ between surfaces $\mathrm{j}$ and $\mathrm{k}$, is discussed in Section 10.3.3.

The dry surface emissivities, view factors $F_{j k}$, and characteristic beam lengths $L_{j k}$ between surfaces are provided by the user as "emsvt," "vufacn," and "beaml," respectively, in the RAD-HEAT input block described in Section 14.3.1.5. When the surface is dry, the "emsvt" value is used for $\varepsilon_{\mathrm{s}, \mathrm{j}}$. Whenever a water film is present on the surface, 0.94 , the emissivity of water, is used instead of the dry surface emissivity. Because the surface and gas temperatures of the enclosure are known, the radiosity of each surface can be obtained from Equation (10-60) through use of a standard linear equation solver. The flux $\mathrm{q}_{\mathrm{rad}, \mathrm{i}}$ is then obtained from Equation (10-58).

If the user has not input the beam length $\mathrm{L}_{\mathrm{jk}}$ between surfaces for the net enclosure model, a default value given by

$$
L=\frac{3.6 V_{g}^{0}}{\sum_{i=1}^{N} A_{s, i}}
$$

is used, where $V_{g}^{0}$ is the initial cell gas volume, and $A_{s, i}$ is the area of surface $i$.

For the view factor between surfaces, the user should only supply the view factor in one direction (say, from surface $i$ to $j$ ). The code will then calculate the view factor in the opposite direction (from surface $\mathrm{j}$ to $\mathrm{i}$ ) according to the following equation:

$$
A_{s, i} F_{i j}=A_{s, j} F_{j i}
$$

where $A_{s j}$ is the area of surface $j, F_{i j}$ is the view factor from surface $i$ to $j$, and $F_{j i}$ is the view factor from surface $j$ to $i$.

\subsubsection{Direct Radiative Heat Transfer Model}

The direct radiative heat transfer model in CONTAIN calculates the radiative heat transfer between the atmosphere and each heat transfer structure, between the atmosphere and the uppermost lower cell layer, and between the uppermost lower cell layer and each structure inner surface. The name of this model arises from the fact that secondary reflections involving three or more enclosure elements are treated in an approximate fashion, in contrast to the net enclosure radiation model. A further limitation is that direct radiation between structure surfaces is not modeled. Unlike the net enclosure radiation model, the direct model permits radiative heat transfer from the atmosphere to both the inner and outer surfaces of a structure if both lie within a cell. The direct model for gas to surface heat transfer is invoked by specifying either GASWAL or GEOBL in the RAD-HEAT input block. The modeling of direct radiative exchange between the lower cell and each heat transfer 
structure is invoked through the VUFAC keyword in the heat transfer structure input block (see Section 14.3.1.3).

Unlike the net enclosure radiation model, the direct model employs a stability-enhancing damping technique in the calculation of radiative energy exchange with the atmosphere. The total radiative heat transfer from the atmosphere $\mathrm{Q}_{\mathrm{rad}, \text { as }}$ during a system timestep $\Delta \mathrm{t}_{\mathrm{s}}$ is given by

$$
\mathrm{Q}_{\mathrm{rad}, \mathrm{as}}=\sum_{\mathrm{j}=1}^{\mathrm{N}} \mathrm{q}_{\mathrm{rad}, \mathrm{j}} \mathrm{A}_{\mathrm{s}, \mathrm{j}} \Delta \mathrm{t}_{\mathrm{s}}
$$

where $\mathrm{q}_{\mathrm{rad} j}$ is the radiative heat flux from the atmosphere to surface $\mathrm{j}$; and is given by

$$
\mathrm{q}_{\mathrm{rad}, \mathrm{j}}=\sigma \varepsilon_{\mathrm{s}, \mathrm{j}}^{\prime}\left(\varepsilon_{\mathrm{m}, \mathrm{j}} \mathrm{T}_{\mathrm{a}}^{4}-\alpha_{\mathrm{m}, \mathrm{j}} \mathrm{T}_{\mathrm{w}, \mathrm{j}}^{4}\right)
$$

where the gas mixture emissivity $\varepsilon_{\mathrm{m}, \mathrm{j}}$ and absorptivity $\alpha_{\mathrm{m}, \mathrm{j}}$ are calculated as described in Section 10.3.3 as a function of the user-specified beam length for surface $j$, and $T_{w, j}$ is the surface node temperature of surface $j . T_{a}$ in the above equation is a surrogate for the atmosphere temperature in the damping technique. Also, $\varepsilon_{\mathrm{s}, \mathrm{j}}^{\prime}$ is an effective surface emissivity.

For a gray enclosure, some of the incident radiation striking the walls is reflected back into the gas and to other parts of the enclosure. Use in Equation (10-64) of the actual surface emissivity for $\varepsilon_{s, j}$ allows for proper reduction of the primary beams (gas-to-surface or surface-to-gas), but because secondary reflections are not taken into account in the direct model, it may be more accurate to use an effective surface emissivity. [Bau78] The effective emissivity depends primarily on the surface properties, but depends also on the gas properties and beam lengths. Expressions for the effective emissivity and their limitations can be found in Reference Bau78. In some cases, it may be appropriate to use an effective emissivity equal to $\left(\varepsilon_{\mathrm{s}}+1\right) / 2$, where $\varepsilon_{\mathrm{s}}$ is the actual surface emissivity. In the direct model, the value used for $\varepsilon_{s, j}^{\prime}$ in the above equation is the dry surface emissivity input parameter "emsvt" when the surface is dry. An emissivity of $(0.94+1) / 2$, a value derived from the emissivity of water, is used when the surface is wet. Thus, an effective dry emissivity value should in general be specified for "emsvt," in the direct model.

The surrogate temperature and the radiative heat transfer $Q_{\text {rad,as }}$ for the present timestep are found from solving the following equation:

$$
\mathrm{m}_{\mathrm{g}} \mathrm{c}_{\mathrm{v}, \mathrm{g}}\left(\mathrm{T}_{\mathrm{a}}-\mathrm{T}_{\mathrm{a}}^{0}\right)=-\mathrm{Q}_{\mathrm{rad}, \mathrm{as}}+\mathrm{m}_{\mathrm{g}} \mathrm{c}_{\mathrm{v}, \mathrm{g}} \Delta \mathrm{t}_{\mathrm{s}} \chi-\mathrm{m}_{\mathrm{g}} \mathrm{c}_{\mathrm{v}, \mathrm{g}} \mathrm{d} \Delta \mathrm{t}_{\mathrm{s}} \Delta \mathrm{T}
$$

where $m_{g}$ is the atmosphere mass, $c_{v, g}$ is the specific heat of the atmosphere, $\chi$ is an auxiliary field defined below, $\mathrm{d}=0.5 / \Delta \mathrm{t}_{\mathrm{s}}$ is a damping parameter, and $\Delta \mathrm{T}=\mathrm{T}_{\mathrm{a}}^{0}-\mathrm{T}_{\mathrm{g}}$. Here, $\mathrm{T}_{\mathrm{a}}^{0}$ is the value of $\mathrm{T}_{2}$ from the last timestep, and $\mathrm{T}_{\mathrm{g}}$ is the present gas temperature. The auxiliary field $\chi$ is defined as 


$$
\chi=\chi^{0}-b \Delta t_{s} \Delta \mathrm{T}
$$

where $\chi^{0}$ is the value of $\chi$ from the previous timestep. The coefficient $b$ is a second damping parameter, set to $b=0.25 /\left(\Delta t_{\mathrm{s}}\right)^{2}$.

Equation (10-64) does not consider direct radiative exchange between the lower cell and structures. Direct exchange between the uppermost lower cell layer surface and the inner surfaces of heat transfer structures is modeled when the VUFAC keyword is specified in the structure input (see Section 14.3.1.3). The definition of the "vufac" input parameter is discussed below. The direct exchange model includes the attenuating effects of the intervening atmosphere if the GASWAL or GEOBL option has been invoked. Although this direct exchange model can be invoked even if one of the former options is not, this practice is not recommended, since the atmosphere would then be treated as nonparticipating.

The heat transfer over a system timestep as a result of direct radiative exchange between the lower cell layer surface and a given structure inner surface is calculated from the equation

$$
\mathrm{Q}_{\mathrm{rad}, \ell \mathrm{s}}=\left[1-\alpha_{\mathrm{m}}\left(\mathrm{T}_{\ell}\right)\right] \mathrm{A}_{\ell} \mathrm{C}\left(\mathrm{T}_{\ell}^{4}-\mathrm{T}_{\mathrm{w}}^{4}\right) \Delta \mathrm{t}_{\mathrm{s}}
$$

where $\alpha_{\mathrm{m}}$ is the gas absorptivity based on either GASWAL or GEOBL beam lengths for the lower cell layer; $A_{\ell}$ is the surface area of the layer; $C$ is the product of Stefan-Boltzmann constant and user-specified parameter "vufac"; $T_{l} i$ is the layer surface node temperature; and $T_{w}$ is the inner surface node temperature of the structure.

The "vufac" parameter for a given structure is a function of the emissivities of the radiating bodies and their geometrical relationships. For gray bodies, "vufac" can be defined as

$$
\frac{1}{\text { "vufac" }}=\frac{1}{\mathrm{~F}_{\ell \mathrm{s}}}+\left(\frac{1}{\varepsilon_{\ell}}-1\right)+\left(\mathrm{A}_{\ell} / \mathrm{A}_{\mathrm{s}}\right)\left(\frac{1}{\varepsilon_{\mathrm{s}}}-1\right)
$$

where $F_{l s}$ is the standard view factor (it is the fraction of the radiation from the layer surface directed into the solid angle subtended by the structure), $\varepsilon_{\ell}$ is the emissivity of the layer surface, $A_{s}$ is the area of the structure inner surface, and $\varepsilon_{s}$ is the emissivity of the structure surface.

Because of the possibility of confusion, the user should note again that direct radiative exchange between the lower cell and a structure through an intervening gas is modeled only if the "vufac" parameter is specified in the heat transfer structure input block. The attenuating effects of the gas on the direct exchange are taken into account only if one of the GASWAL or GEOBL options is also used. 


\subsubsection{Radiative Properties}

Both the net enclosure and direct radiation models, discussed in Sections 10.3.1 and 10.3.2, respectively, require the emissivity and absorptivity of the atmosphere gas mixture. These quantities are a function of the gas composition, pressure, temperature, and the average optical depth of the gas. The average optical depth is also referred to as the beam length. The present section discusses the methods available to calculate the emissivity $\varepsilon_{\mathrm{m}}$ of a gas mixture from the emissivities of the individual active gas species $\left(\mathrm{CO}, \mathrm{CO}_{2}\right.$, and $\left.\mathrm{H}_{2} \mathrm{O}\right)$ and the aerosols present in the gas.

The Modak radiative property model is used by default to calculate these properties. [Mod79] If only water vapor and/or aerosols are present to an appreciable extent in the atmosphere, the user can invoke the Cess-Lian water vapor radiative property model [Ces76] by specifying the CESS keyword in the RAD-HEAT input block. This model assumes that $\mathrm{CO}$ and $\mathrm{CO}_{2}$, if present, are transparent and do not affect radiative heat transfer.

The gas absorptivity $\alpha_{m}$ is calculated from either the Modak model or the Cess-Lian correlation by using Kirchhoff's law of radiation, which states that spectral emissivity for the emission of radiation at temperature $T$ is equal to the spectral absorptivity for radiation coming from a blackbody at the same temperature $T$. This law may be considered valid whether or not thermal equilibrium prevails. Its proper application greatly simplifies the calculation of radiative transfer.

The Modak radiative property model is described in Section 10.3.3.1. The Cess-Lian radiative property model is described in Section 10.3.3.2.

10.3.3.1 Modak Radiative Property Model. The default Modak radiative property model calculates the total gas mixture emissivity and absorptivity, taking into account the active gases $\mathrm{H}_{2} \mathrm{O}, \mathrm{CO}$, and $\mathrm{CO}_{2}$ and aerosols.

The gas mixture emissivity $\varepsilon_{\mathrm{m}}$ and absorptivity $\alpha_{\mathrm{m}}$ are given by

$$
\varepsilon_{\mathrm{m}}=\varepsilon_{\mathrm{p}}+\varepsilon_{\mathrm{g}}-\varepsilon_{\mathrm{p}} \varepsilon_{\mathrm{g}}
$$

and

$$
\alpha_{\mathrm{m}}=\alpha_{\mathrm{p}}+\alpha_{\mathrm{g}}-\alpha_{\mathrm{p}} \alpha_{\mathrm{g}}
$$

where $\varepsilon_{\mathrm{p}}$ and $\alpha_{\mathrm{p}}$ are, respectively, the aerosol emissivity and absorptivity, and $\varepsilon_{\mathrm{g}}$ and $\alpha_{\mathrm{g}}$ are, respectively, the gas emissivity and absorptivity. 
The emissivity of each of the above gases has been obtained by summing over its spectral absorption bands. [Edw73] In the approximate method adopted here, each species' spectral emissivity is approximated by curve fits, [Mod79] and an overlapping band correction is made using a temperature-dependent form of Leckner's correction $\Delta \varepsilon_{\mathrm{cw}}$. [Lec72] The emissivities $\left(\varepsilon_{\mathrm{CO} 2}, \varepsilon_{\mathrm{cO}}\right.$, and $\varepsilon_{\mathrm{H} 2 \mathrm{O}}$ ) of each species were fit to the form

$$
\ln \varepsilon=\sum_{\mathrm{i}=0}^{2} \mathrm{~T}_{\mathrm{i}}(\mathrm{x}) \sum_{\mathrm{j}=0}^{3} \mathrm{~T}_{\mathrm{j}}(\mathrm{y}) \sum_{\mathrm{k}=0}^{3} \mathrm{c}_{\mathrm{ijk}} \mathrm{T}_{\mathrm{k}}(\mathrm{z})
$$

where

$$
\begin{aligned}
& x=p_{1}+\frac{\ln (P)}{p_{2}} \\
& y=\frac{\ell_{1}+\ln (P L)}{\ell_{2}} \\
& z=\frac{T-t_{1}}{t_{2}}
\end{aligned}
$$

and $P, L$, and $T$ are, respectively, the partial pressure, beam length, and gas temperature.

The $c_{\mathrm{ijk}}$ consist of a set of 48 coefficients for $\mathrm{CO}_{2}, \mathrm{CO}$, and $\mathrm{H}_{2} \mathrm{O}$, derived from experimental data and spectral calculations; $\mathrm{p}_{1}, \mathrm{p}_{2}, \ell_{1}, \ell_{2}, \mathrm{t}_{1}$, and $\mathrm{t}_{2}$ are Gauss-Chebyshev approximation parameters for the partial pressure, the product of partial pressure and beam length, and the temperature; and $T_{n}(x)$ is a Chebyshev polynomial of order $n$.

The gas emissivity $\varepsilon_{\mathrm{g}}$ is computed from

$$
\varepsilon_{\mathrm{g}}=\varepsilon_{\mathrm{CO}}+\varepsilon_{\mathrm{CO}_{2}}+\varepsilon_{\mathrm{H}_{2} \mathrm{O}}-\Delta \varepsilon_{\mathrm{cw}}
$$

where $\varepsilon_{\mathrm{CO}}, \varepsilon_{\mathrm{CO}_{2}}$ and $\varepsilon_{\mathrm{H}_{2} \mathrm{O}}$ are the emissivities of $\mathrm{CO}, \mathrm{CO}_{2}$, and $\mathrm{H}_{2} \mathrm{O}$, respectively, and $\Delta \varepsilon_{\mathrm{cw}}$ is the overlap correction factor for $\mathrm{CO}_{2}$ and $\mathrm{H}_{2} \mathrm{O}$. The overlap correction $\Delta \varepsilon_{\mathrm{cw}}$ accounts for the $2.7-\mu \mathrm{m}$ and $15-\mu \mathrm{m}$ overlapping bands of $\mathrm{CO}_{2}$ and $\mathrm{H}_{2} \mathrm{O}$. The overlap correction originally suggested by Leckner [Lec72] has been modified to include the temperature variation of $\Delta \varepsilon_{\mathrm{cw}}$ [Edw73]:

$$
\Delta \varepsilon_{\mathrm{cw}}=\left|\frac{\xi}{(10.7+101 \delta)}-\frac{\xi^{10.4}}{111.7}\right|\left\{\log _{10}\left[101.3\left(\mathrm{P}_{\mathrm{CO}_{2}}+\mathrm{P}_{\mathrm{H}_{2} \mathrm{O}}\right) \mathrm{L}\right]\right\}^{2.76} \mathrm{~F}(\mathrm{~T})
$$

for $\left(\mathrm{P}_{\mathrm{CO}_{2}}+\mathrm{P}_{\mathrm{H}_{2} \mathrm{O}}\right) \mathrm{L} \geq 1.013 \times 10^{4} \mathrm{~Pa}-\mathrm{m}$ and $\xi \geq 0.01$; and

$$
\Delta \varepsilon_{\mathrm{cw}}=0
$$


otherwise. The parameter $\xi$ is defined by

$$
\xi=\mathrm{P}_{\mathrm{H}_{2} \mathrm{O}}\left(\mathrm{P}_{\mathrm{H}_{2} \mathrm{O}}+\mathrm{P}_{\mathrm{CO}_{2}}\right)
$$

and $\mathrm{F}(\mathrm{T})$ is given by

$$
\mathrm{F}(\mathrm{T})=-1.0204 \times 10^{-6} \mathrm{~T}^{2}+2.2449 \times 10^{-3} \mathrm{~T}-0.23469
$$

The absorptivity of a gas with respect to a radiating surface is calculated in a manner similar to that used for the emissivities using Equations (10-71) through (10-76), except that $T_{g}$ is replaced by $T_{w}$, the surface node temperature, the beam length $L$ is replaced by $L^{\prime}=L\left(T_{w} / T_{g}\right)$, and a multiplicative correction factor is applied:

$$
\alpha_{g}=\varepsilon_{g}\left\{T_{g} \rightarrow T_{w}, L \rightarrow L^{\prime}\right\}\left(\frac{T_{g}}{T_{w}}\right)^{0.65-0.2 \frac{P_{\mathrm{H}_{2} \mathrm{O}}}{\mathrm{P}_{\mathrm{H}_{2}} \mathrm{O}^{\mathrm{P}} \mathrm{CO}_{2}+\mathrm{P}_{\mathrm{co}}}}
$$

The aerosol emissivity $\varepsilon_{\mathrm{p}}$ is calculated according to the method of Felske and Tien. [Fel73] This method is applicable to absorbing particles that are small enough to produce negligible scattering. In this limit, the spectral absorption coefficient $\alpha_{\lambda}$ for the particles is proportional to $\lambda^{-1}$, the inverse wavelength. Thus,

$$
\alpha_{\lambda}=\beta f_{\sqrt{ }} \lambda
$$

where $\beta$ is a constant of proportionality and $f_{v}$ is the aerosol concentration by volume $\left(\mathrm{m}^{3}\right.$ - aerosol $/ \mathrm{m}^{3}$ - gas). In this case the emissivity due to aerosol particles $\varepsilon_{\mathrm{p}}$ is given by

$$
\varepsilon_{\mathrm{p}}=1-\frac{15}{\pi^{4}} \varphi(y)
$$

where $\varphi$ is the pentagamma function. The argument $y$ in the pentagamma function is defined as

$$
\mathrm{y}=1+\alpha_{\lambda \mathrm{m}} L \mathrm{~T}_{\mathrm{g}} \lambda_{\mathrm{m}} / \mathrm{C}_{2}
$$

where $\lambda_{m}$ is the reference wavelength $(0.94 \mu \mathrm{m})$, a value representing the infrared radiation; and $C_{2}$ is the second Planck constant, which equals $14.388 \times 10^{-3} \mathrm{~m}-\mathrm{K}$. Note that $\alpha_{\lambda \mathrm{m}} \lambda_{\mathrm{m}}$ in Equation (10-80) is independent of wavelength according to Equation (10-78). 
The term $\alpha_{\mathrm{m}}$ in Equation (10-80) is the absorption coefficient at the reference wavelength. The user can specify this value directly through the ABSORB option in the RAD-HEAT input block (see Section 14.3.1.5). Note that in this option, the aerosol emissivity will be independent of the aerosol mass concentration in the problem. The second method models the aerosol cloud emissivity as derived by Pilat and Ensor [Pil70]

$$
\alpha_{\lambda_{m}}=4000 C_{\lambda m} f_{m}
$$

where $\mathrm{C}_{\lambda \mathrm{m}}$ is "kmx", a constant of proportionality specified through the KMX option of the RAD-HEAT input block, and $f_{m}$ is the total aerosol mass concentration $\left(\mathrm{kg} / \mathrm{m}^{3}\right)$ computed by the code. $\mathrm{C}_{\mathrm{fm}}$ in this equation is provided to allow the user to account for the effects of wavelength, index of refraction, particle size distribution, and aerosol particle material density. [Pi170] For $\mathrm{C}_{\lambda m}$ $=1$, it corresponds to soot-like particles with a density of $2000 \mathrm{~kg} / \mathrm{m}^{3} . C_{\lambda \mathrm{m}}$ is related to the coefficient $\beta$ in Equation (10-78) according to $C_{\lambda m}=\beta /\left(4000 \rho_{p} \lambda_{m}\right)$, where $\rho_{p}$ is the aerosol material density. The aerosol absorptivity is calculated in a manner similar to the emissivity, except that the surface temperature is used in the expressions above instead of the gas temperature.

10.3.3.2 Cess-Lian Radiative Property Model. The user may select the Cess-Lian model for the radiative properties. If the Cess-Lian radiative property model is chosen, $\mathrm{CO}$ and $\mathrm{CO}_{2}$ are assumed to be transparent. Thus, this model should be used only when the effects of such gases are not significant. If this model is desired, the keyword CESS must be specified in the RAD-HEAT input block. Otherwise the default Modak model will be used.

The Cess-Lian model is based on an analytic correlation developed by Cess and Lian and is used to calculate steam/air emissivity and absorptivity. [Ces76] In this model, data from Hottel emittance charts [Hot67] have been fit to an equivalent single-band exponential form, which requires significantly less computation time than that for a wide-band exponential model. The gas mixture emissivity $\varepsilon_{\mathrm{m}}$ and absorptivity $\alpha_{\mathrm{m}}$ for the Cess-Lian model are defined in a similar manner to those for the Modak radiative property model, except for the fact that gas emissivity $\varepsilon_{g}$ and absorptivity $\alpha_{\mathrm{g}}$ are calculated as follows.

The value of $\varepsilon_{\mathrm{g}}$ is calculated for the steam/air mixture according to

$$
\varepsilon_{\mathrm{g}}=\mathrm{a}_{0}\left[1-\exp \left(-\mathrm{a}_{1} \sqrt{\mathrm{x}}\right)\right]
$$

where $a_{0}$ and $a_{1}$ are coefficients determined from Table 10-3, with linear interpolation between the given temperatures. 
Table 10-3

Coefficients for the Cess-Lian Correlation

\begin{tabular}{|c|c|c|}
\hline $\begin{array}{c}\mathrm{T} \\
(\mathrm{K})\end{array}$ & $\begin{array}{c}\mathrm{a}_{0} \\
\text { (dimensionless) }\end{array}$ & $\begin{array}{c}\mathrm{a}_{1} \\
\left(\mathrm{~m}^{-1 / 2} \mathrm{~atm}^{-1}\right)\end{array}$ \\
\hline 273 & 0.6838 & 1.16 \\
\hline 300 & 0.683 & 1.17 \\
\hline 600 & 0.674 & 1.32 \\
\hline 900 & 0.700 & 1.27 \\
\hline 1200 & 0.673 & 1.21 \\
\hline 1500 & 0.624 & 1.15 \\
\hline 2500 & 0.461 & 0.95 \\
\hline
\end{tabular}

The value of $x$ in Equation (10-82) is calculated by

$$
x=P_{\mathrm{H}_{2} \mathrm{O}} L\left(\frac{300}{\mathrm{~T}_{\mathrm{g}}}\right)\left\{\frac{\mathrm{P}_{\mathrm{g}}+\left[5\left(\frac{300}{\mathrm{~T}_{\mathrm{g}}}\right)^{1 / 2}-0.5\right] \mathrm{P}_{\mathrm{H}_{2} \mathrm{O}}}{(101325)^{2}}\right\}
$$

where $\mathrm{P}_{\mathrm{H}_{2} \mathrm{O}}$ is the partial pressure of water vapor, $\mathrm{L}$ is the beam length, $\mathrm{T}_{\mathrm{g}}$ is the gas temperature, and $P_{g}$ is the total gas pressure.

The gas absorptivity with respect to a radiating surface is calculated in a manner similar to Equation (10-77), by replacing $T_{g}$ in the above expressions by the surface node temperature $T_{w}$, replacing the beam length $L$ by $L^{\prime}=L\left(T_{w} / T_{g}\right)$, and applying a scaling factor

$$
\alpha_{g}=\varepsilon_{g}\left\{T_{g} \rightarrow T_{w}, L^{\prime} L^{\prime}\right\}\left(\frac{T_{g}}{T_{w}}\right)^{0.45}
$$

\subsection{Boiling Heat Transfer}

As discussed in Section 5.5, if a coolant pool is present over a lower-cell layer above the saturation temperature $T_{s}$, boiling heat transfer correlations are used. The boiling heat transfer model in the code includes the full boiling curve, based on standard pool boiling correlations as summarized by Bergles. [Brg81] If the layer is modeled within the CORCON-Mod3 module for core-concrete interactions, corrections are made for the effects of gas injection at the melt/coolant interface and 
coolant subcooling. The various correlations in Reference Brg81 are used only indirectly in CONTAIN. This is possible because the boiling heat transfer coefficient for a given fluid, water in this case, is a function of pressure and temperature only, with all of the detailed dependence of material properties on temperature and pressure contained in the one function. A series of calculations was performed outside the code, using thermal and transport properties from the Steam Tables [Kee78] to generate tables of values. These were then fit by simple analytic forms in the pressure $P$ which reproduce the tables within $3 \%$ over the pressure range of $10 \mathrm{kPa}$ to $10 \mathrm{MPa}$ (saturation temperatures from $320 \mathrm{~K}$ to $580 \mathrm{~K}$ ). The principal advantage of using these fits is that extensive libraries of water properties need not be included in the code.

Nucleate boiling is treated by using the Rohsenow [Roh52] correlation for the wall superheat $\Delta T_{C H F}$ at the point of critical heat flux and the Zuber [Zub58, Zub61] correlation (with Rohsenow's coefficient) [Roh73] for the critical heat flux $\mathrm{q}_{\mathrm{CHF}}$. These are represented as

$$
\begin{aligned}
& \Delta \mathrm{T}_{\mathrm{CHF}}=\frac{1.71 \times 10^{3} \mathrm{C}_{\mathrm{sf}}\left(10^{-5} \mathrm{P}\right)^{-0.112}}{1.0+7.02 \times 10^{-8}\left(10^{-5} \mathrm{P}\right)^{3.181}} \\
& \mathrm{q}_{\mathrm{CHF}}=\frac{1.50 \times 10^{6}\left(10^{-5} \mathrm{P}\right)^{0.415}}{1.0+5.97 \times 10^{-3}\left(10^{-5} \mathrm{P}\right)^{1.117}}
\end{aligned}
$$

respectively, where all values are in SI units (i.e., $\mathrm{q}$ is in $\mathrm{W} / \mathrm{m}^{2}, \mathrm{P}$ is in $\mathrm{Pa}$, and $\mathrm{T}$ is in $\mathrm{K}$ ) and the surface coefficient, $\mathrm{C}_{\mathrm{sf}}$, is taken as 0.01 . When subcooling is taken into account, the nucleate-boiling heat flux is represented as

$$
\mathrm{q}=\mathrm{q}_{\mathrm{CHF}, \mathrm{sub}}\left[\Delta \mathrm{T}_{\text {sat }} / \Delta \mathrm{T}_{\mathrm{CHF}}\right]^{3.03}
$$

where the exponent is that attributed to Rohsenow in Reference Brg81 and where the effect of subcooling on nucleate boiling is included through an expression recommended by Ivey [Ive62]:

$$
\mathrm{q}_{\mathrm{CHF}, \mathrm{sub}} / \mathrm{q}_{\mathrm{CHF}}=1+\mathrm{C}_{\mathrm{Tvey}} \Delta \mathrm{T}_{\text {sub }}
$$

Here $\Delta T_{\text {sub }}=T_{s}-T_{p}$, where $T_{s}$ is the saturation temperature and $T_{p}$ is the bulk pool temperature. The coefficient $\mathrm{C}_{\text {Ivey }}$ is given by

$$
C_{\text {Ivey }}=0.1\left(\frac{\rho_{\ell}}{\rho_{v}}\right)^{3 / 4} \frac{c_{p, \ell}}{h_{\ell v}}
$$

where $\mathrm{C}_{\mathrm{p}, \ell}$ is the liquid specific heat. 
This coefficient has been fit to a function of pressure according to

$$
C_{\text {Ivey }}=\frac{4.77 \times 10^{-2}\left(10^{-5} \mathrm{P}\right)^{-0.683}}{1.0-6.29 \times 10^{-3}\left(10^{-5} \mathrm{P}\right)^{0.862}}
$$

In the above $\Delta t_{\text {sat }}=T_{\text {if }}-T_{s}$ is the wall superheat, where $T_{\text {if }}$ is the surface temperature.

The film boiling regime is based on the Berenson correlations [Bra93] for the heat-transfer coefficient in film boiling and for the wall superheat at the Leidenfrost temperature (minimum film-boiling point). Respectively, these have been fit as

$$
\mathrm{q}_{\text {Leid }}=\frac{1.88 \times 10^{4}\left(10^{-5} \mathrm{P}\right)^{0.894}}{1.0+7.58 \times 10^{-3}\left(10^{-5} \mathrm{P}\right)^{0.956}}
$$

and

$$
\Delta \mathrm{T}_{\text {Leid }}=\frac{8.56 \times 10^{1}\left(10^{-5} \mathrm{P}\right)^{0.848}}{1.0+1.38 \times 10^{-1}\left(10^{-5} \mathrm{P}\right)^{0.750}}
$$

Above the Leidenfrost point, the total surface-to-coolant heat flux including radiation is represented, in accordance with Reference Brg81, as

$$
q=q_{c}\left(q_{c} / q\right)^{1 / 3}+q_{r}
$$

Here $q_{c}$ is the convective heat flux in the absence of radiation, and the factor $\left(q_{c} / q\right)^{1 / 3}$ accounts for the fact that the total heat flux contributes to the vaporization rate, which determines the thickness and thermal resistance of vapor film. The heat flux $\mathrm{q}_{\mathrm{c}}$ has an explicit variation with temperature as the 3/4 power of $\Delta \mathrm{T}_{\text {sar }}$. It is assumed that this dominates the implicit temperature dependence through material properties, so that $\mathrm{q}_{\mathrm{c}}$ may be calculated as

$$
\mathrm{q}_{\mathrm{c}}=\mathrm{q}_{\mathrm{c}, \text { Leid }}\left(\Delta \mathrm{T}_{\text {sat }} / \Delta \mathrm{T}_{\text {Leid }}\right)^{0.75}
$$

The radiative contribution $\mathrm{q}_{\mathrm{r}}$ is that derived for infinite parallel gray walls

$$
\mathrm{q}_{\mathrm{r}}=\frac{\sigma_{\mathrm{B}}\left(\mathrm{T}_{\mathrm{w}}^{4}-\mathrm{T}_{\mathrm{s}}^{4}\right)}{1 / \varepsilon_{\mathrm{w}}+1 / \varepsilon_{\mathrm{f}}-1}
$$

where $\varepsilon_{\mathrm{w}}$ is the emissivity of the wall, $\varepsilon_{\mathrm{f}}$ is that of the coolant, and $\sigma_{\mathrm{B}}$ is the Boltzmann constant. 
If boiling is occurring at the interface between the CORCON layer and the coolant pool and CORCON-Mod3 is active, the effects (discussed below) of gas barbotage (i.e., noncondensable gas injection at the interface) and coolant subcooling on film boiling heat transfer are included. If boiling is simply occurring between the coolant pool and a hot substrate not actively modeled with CORCON-Mod3, the effects of gas barbotage and subcooling on film boiling are not taken into account. Both gas barbotage and coolant subcooling can greatly increase the film boiling heat flux, while also increasing the Leidenfrost temperature at which the vapor film stabilizes.

Gas barbotage increases film boiling heat transfer by increasing agitation of the coolant, and by increasing agitation of the melt surface. In CORCON-Mod3, the enhancement to the film boiling heat flux due to gas barbotage is included as a multiplicative factor. The factor used depends on whether the surface underlying the coolant is solid or liquid.

If the surface underlying the coolant is liquid, then the enhancement is given by a correlation of experimental results advanced by Greene. ${ }^{1}$ The experimental results were for freon and water on three different molten metals, bismuth, lead, and Wood's metal. The enhancement factor proposed by Greene is

$$
\mathrm{Q}_{\ell}^{*}=\min \left(1+11.85\left(\frac{\mathrm{j}_{\mathrm{g}}^{*}}{\mathrm{Ja}^{*}}\right)^{0.91}\left(1+2 \mathrm{j}_{\mathrm{g}}^{*}\right), 5\right)
$$

where $Q_{l}^{*}$ is the ratio of the measured heat flux to the heat flux calculated using the Berenson correlation given in Equation (10-93), $\mathrm{j}_{\mathrm{g}}^{*}=\mathrm{j}_{\mathrm{g}} / \mathrm{U}_{\infty}, \mathrm{j}_{\mathrm{g}}$ is the superficial gas velocity, $\mathrm{U}_{\infty}$ is the terminal rise velocity of the noncondensable gas bubbles in the liquid metal, and $\mathrm{Ja}^{*}$ is defined by

$$
\mathrm{Ja}^{*}=\frac{\mathrm{c}_{\mathrm{p}, \mathrm{v}} \Delta \mathrm{T}_{\mathrm{sat}}}{\mathrm{h}_{\ell \mathrm{v}}+0.5 \mathrm{c}_{\mathrm{p}, \mathrm{v}} \Delta \mathrm{T}_{\mathrm{sat}}}
$$

where $c_{p, v}$ is the specific heat of the vapor at constant pressure.

If the surface underlying the coolant is solid, then the enhancement is given by a correlation advanced by Duignan. [Dui89] This correlation is based on experiments in which gas was injected through heated, drilled plates in contact with an overlying water pool. The enhancement factor used in CORCON-Mod3 is

$$
\mathrm{Q}_{\mathrm{s}}^{*}=1+0.99\left(\mathrm{j}_{\mathrm{g}}^{*} / \mathrm{Ja}^{*}\right)^{0.91}
$$

' G. A. Greene, Brookhaven National Laboratory, Upton, NY, private communication, 1990. 
When the temperature of the core debris is calculated to lie between the solidus and liquidus temperatures of the debris mixture, the two enhancement factors shown above are weighted by the surface solid fraction. The solid fraction is defined as

$$
\phi=\left(\mathrm{T}_{\text {liq }}-\mathrm{T}_{\mathrm{if}}\right) /\left(\mathrm{T}_{\text {liq }}-\mathrm{T}_{\text {sol }}\right)
$$

where $T_{\text {liq }}$ is the liquidus temperature. The gas barbotage enhancement factor is then defined as

$$
\mathrm{Q}_{\mathrm{g}}^{*}=\mathrm{Q}_{\ell}^{*}(1-\phi)+\mathrm{Q}_{s}^{*} \phi
$$

The increased agitation of the melt-coolant interface caused by gas barbotage destabilizes the vapor film, thereby increasing the temperature at which the film collapses.

The effect of gas barbotage on the minimum film boiling temperature is accounted for by the equation

$$
\Delta \mathrm{T}_{\text {Leid,g }}=\Delta \mathrm{T}_{\text {Leid }}+463.1 \mathrm{j}_{\mathrm{g}}^{0.3953}
$$

where $\Delta T_{\text {Leid,g }}$ is the minimum film boiling superheat in the presence of gas barbotage and $j_{g}$ is the superficial gas velocity.

Subcooling of the overlying coolant pool can also enhance heat transfer in the film boiling regime. When the overlying coolant pool is subcooled, energy is removed from the gas film by the overlying subcooled coolant. The net effect of this cooling is a reduction in the thickness of the vapor film. The reduced film thickness permits greater heat transfer by conduction.

The enhancement to heat transfer owing to coolant subcooling in the film boiling regime is included as a multiplicative factor. The factor is calculated using an equation of the form proposed by Siviour and Ede [Siv70] and Dhir and Purohit [Dhi78]:

$$
\mathrm{Q}_{\text {sub }}^{*}=1+\mathrm{C}_{\text {sub }}\left(\Delta \mathrm{T}_{\text {sub }}\right)^{5 / 4} /\left(\Delta \mathrm{T}_{\text {sat }}\right)^{3 / 4}
$$

where $\mathrm{Q}_{\text {sub }}^{*}$ is the coolant subcooling factor and $\mathrm{C}_{\text {sub }}$ is chosen to be 0.98 , based on comparison to experimental results in References Dhi78 and Tod82.

By reducing the thickness of the vapor film, the subcooling of the coolant reduces the stability of the film, and increases the minimum film boiling temperature. The effect of coolant subcooling on the minimum film boiling temperature is calculated using a simple linear correlation of experimental data [Dhi78, Tod82, Bra67]: 


$$
\Delta \mathrm{T}_{\text {Leid,sub }}=\Delta \mathrm{T}_{\text {Leid }}+8 \Delta \mathrm{T}_{\text {sub }}
$$

where $\Delta \mathrm{T}_{\text {Leid,sub }}$ is the minimum film boiling superheat in the presence of coolant subcooling.

In the absence of experimental data for the combined effects of coolant subcooling and gas barbotage, the following simple equations have been implemented to describe the total combined effect of these phenomena on the film boiling heat flux enhancement factor and minimum film boiling superheat:

$$
\mathrm{Q}_{\mathrm{tot}}^{*}=1+\left[\left(\mathrm{Q}_{\text {sub }}^{*}-1\right)^{2}+\left(\mathrm{Q}_{\mathrm{g}}^{*}-1\right)^{2}\right]^{1 / 2}
$$

and

$$
\Delta \mathrm{T}_{\text {Leid, ot }}=\Delta \mathrm{T}_{\text {Leid }}+\left[\left(\Delta \mathrm{T}_{\text {Leid,sub }}-\Delta \mathrm{T}_{\text {Leid }}\right)^{2}+\left(\Delta \mathrm{T}_{\text {Leid,g }}-\Delta \mathrm{T}_{\text {Leid }}\right)^{2}\right]^{[/ 2}
$$

The transition-boiling regime is represented by a simple linear interpolation in $\ln (q)$ versus $\ln \left(\Delta \mathrm{T}_{\text {sat }}\right)$ between the critical heat flux and the Leidenfrost points. The former is adjusted for subcooling and the latter for subcooling and gas barbotage, as discussed above.

\subsection{Heat Conduction}

This section discusses the modeling of heat conduction in the heat sinks used in CONTAIN and associated topics. Section 10.5.1 describes the types of heat sinks currently available in CONTAIN, the boundary conditions associated with these heat sinks, and the nodalization requirements. Section 10.5.2 discusses the special conduction boundary condition used when two heat transfer structures in different cells are connected together at their outer surfaces. Section 10.5.3 discusses the solution of the heat conduction equation. Finally, Section 10.5.4 discusses the formulation of the concrete outgassing model available for heat transfer structures.

\subsubsection{Containment Heat Sinks}

This section describes the different types of heat sinks that can be modeled with the CONTAIN code and the boundary conditions that may be applied to them. It also discusses nodalization requirements. There are essentially two types of heat sinks: heat transfer structures and lower cell layers. Heat transfer structures are discussed in Section 10.5.1.1. Lower cell layers are discussed in Section 10.5.1.2.

10.5.1.1 Heat Transfer Structures. Heat transfer structures are associated primarily with heat and mass transfer to and from the atmosphere, but a subset of the structure types is designed to be submersible in the pool, as discussed in Section 10.1.1.4. When a structure is submerged, poolstructure heat transfer is taken into account, and the heat conduction in the structure is modeled in terms of two one-dimensional regions, one above the pool and one below the pool, as indicated in 
Figure 10-3. (Figure 10-3 shows the connected structure configuration with a conduction boundary condition between the two structures, but other boundary conditions may be specified by the user, as discussed below.)

As discussed in Section 10.6, the modeling of heat transfer structures assumes that any condensate film on the surface is relatively thin and, in particular, that specific heat effects of the film are negligible. The user has two options to treat the film thickness: through a fixed user-specifiable film depth parameter or the film tracking model, as discussed in Section 10.2.2.

Each structure can consist of different materials, including gases, but only one material may be specified per node. Material names for structure layers should be taken from Table 2-2, which lists the supported materials, or from the list of user-defined materials, as discussed in Section 3.1. Gaseous materials may be used in structure interior nodes. This feature may be useful in modeling gaps between the steel liner and concrete. Such nodes are initialized at a nominal pressure of one atmosphere. Note that through the user-defined material input it is possible to redefine the properties associated with the materials in Table 2-2 or Table 3-1.

All structures are modeled as one-dimensional slabs, partial cylinders, or hemispheres with the conduction equation solved in the appropriate coordinate system for the three different geometries (see Section 10.5.3). The azimuthal angle subtended by a partial cylinder is given by the CYLTHETA keyword and is by default $180^{\circ}$. Figure 10-8 shows an example of a heat transfer structure. This is a half cylinder consisting of a steel liner, an air gap (modeled as nitrogen), and a thick concrete wall. Each structure must also have a designated orientation, either WALI, FLOOR, or ROOF. This orientation information is used in the convection and condensation models to select the proper natural convection heat transfer correlation, the default film parameters, and the degree to which gravitational settling contributes to aerosol deposition.

Each structure has two surfaces designated as the "inner" and "outer" surfaces. The precise meaning of the inner surface is that its location is given by the first number in the list of node positions supplied for the structure. (See Section 14.3.1.3, Structure Characteristics, for a complete description of all heat transfer structure input requirements and options.) For hemispherical or partial cylindrical structures, the coordinate given in the list is the radius. The center of curvature of hemispherical or cylindrical structures is determined by whether the radius is increasing or decreasing in the list. If it is increasing, the inner surface is concave; otherwise, it is convex.

The inner surface of a structure is always considered exposed to the cell in which the structure is defined. The full suite of models is available for inner surfaces. This includes heat and mass transfer models for convection, condensation/evaporation, and radiation; the models for film dynamics, pool submergence, and outgassing from the inner surface; and the modeling of aerosol deposition.

A number of options are available for the placement of the outer surface. The outer surface of a structure may be specified as exposed to the same cell as the inner surface. In this case, the outer surface of a ROOF structure is actually modeled as a FLOOR in the cell. Similarly, the outer surface of a FLOOR structure is modeled as a ROOF. The outer surface may also be connected to the outer 


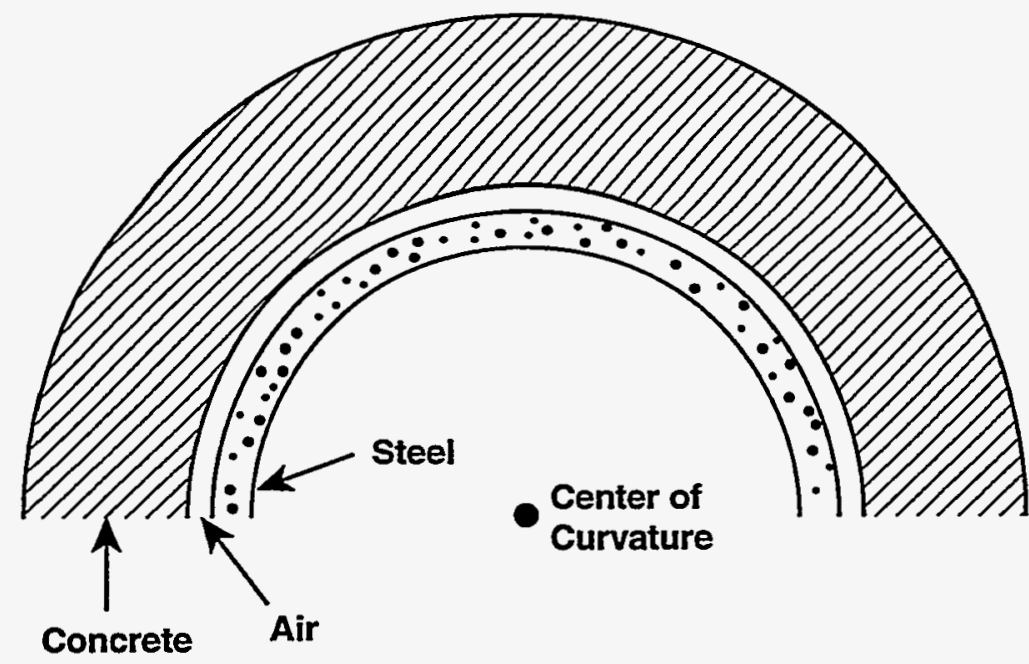

Figure 10-8. Cylindrical Structure Consisting of a Steel Liner, Air Gap, and Thick Concrete Wall 
surface of a structure in another cell, with a conduction boundary condition, as discussed in Section 10.5.2. It may be also be in a different cell or be isolated from all containment cells.

None of these choices allows the outer surface to be treated with the full suite of models available for inner surfaces. Of the allowed models for inner surfaces, only the models for convection, condensation/evaporation, direct atmosphere-to-structure radiation, specified film depth parameter, and aerosol deposition apply to those outer surfaces that are in the same cell. A structure with an outer surface in the same cell also cannot be submerged in the pool in that cell. Other modeling limitations apply to the outer surfaces that are exposed to a different cell or isolated from all cells. Such surfaces will be denoted as "external." An external surface will be present, for example, when one structure represents the physical boundary between two compartments and each compartment is modeled as a different CONTAIN cell.

A number of processes are not modeled for external surfaces: (1) surface condensation and evaporation, (2) aerosol deposition, (3) radiative heat transfer, and (4) the presence of condensate films, and (5) submergence in the pool. Finally, although convective heat transfer is modeled for external surfaces the modeling does not use the Nusselt-number correlations available for other surfaces. Instead, the heat transfer coefficient is either specified by the user or set by default to 6.08 $\mathrm{W} / \mathrm{m}^{2}-\mathrm{K}$. Note that this default is a typical wall heat transfer coefficient in the turbulent natural convection regime.

However, other options are available for external surface boundary conditions. These include (1) an adiabatic boundary condition or (2) a gas temperature boundary condition. (A gas temperature boundary condition simulates a surface exposed to a gas at the specified temperature.) Additional boundary conditions include (3) a heat flux boundary condition and (4) a surface temperature (as opposed to gas temperature) boundary condition.

If the above outer surface modeling limitations are believed to have a significant impact, such as when condensation occurs on both surfaces of a wall that separates two cells, then it may be better to divide the structure in half and put one half in each cell. Either an adiabatic boundary condition can be used for the outer surface of each half or a conduction boundary condition can be used. If the adiabatic boundary condition is used, no conduction of heat from one half to the other is allowed. However, if the conduction boundary condition is used, concrete outgassing presently may not be modeled (see Section 10.5.2). For many severe accident scenarios, the structures that divide compartments are sufficiently thick that the time scale of interest in the problem is much shorter than the time required for appreciable conduction of heat between compartments through walls; therefore, the limitations of an adiabatic boundary condition may not be significant. If the time scale of interest is relatively long, then a conduction boundary condition may be used, as long as the modeling of outgassing is not required.

Proper nodalization of each structure is the responsibility of the user. To determine how finely the structure should be nodalized, it may be useful to consider the thermal diffusion length $\delta$ defined as

$$
\delta=\left(4 \mathrm{k} \Delta \mathrm{t} / \mathrm{pc}{ }_{\mathrm{p}}\right)^{1 / 2}
$$


where $\mathrm{k}$ is the thermal conductivity, $\Delta t$ is the shortest time scale of interest, $\rho$ is the density, and $c_{p}$ is the specific heat of the material. The surface nodes that are in contact with the gas or pool should be a small fraction of this length, if accuracy in the heat transfer is desired. The user should also take care not to change the node thicknesses too abruptly from one node to the next. (Generally, any change in thickness by a factor of two or less should be acceptable.) The user should also consider stability in defining total structure thicknesses, as discussed in Section 2.2.2.2.

10.5.1.2 Lower Cell Layers. The second type of heat sink in CONTAIN arises in modeling of the lower cell, which is intended to represent processes involving deep pools, core debris layers, and concrete-lined sumps. Lower cell modeling options are discussed in detail in Chapter 5, and the details of the lower-cell interlayer heat transfer modeling are discussed in Section 5.5. In contrast to the film modeling for heat transfer structures, the pool is allowed to develop internal boundary layers at the top and bottom and is otherwise assumed to be well-mixed. When core-concrete interactions are not being modeled, a one-dimensional heat conduction model is used to calculate heat transfer between the various lower-cell layers. The conduction algorithm is identical to that used for slab-shaped heat transfer structures.

Note that a number of options available for heat transfer structures are not available for lower cell layers. Those options not available include forced-convection heat and mass transfer modeling, the modeling of concrete outgassing, and all external boundary conditions available for heat transfer structures but the specification of surface (i.e., basemat) temperature. However, models not available for structures are available for the lower cell, including the ability to treat (1) nodes with varying masses, which may be comprised of a mixture of materials in any node but that of the pool, (2) convective heat transfer for pool nodes, (3) boiling heat transfer between the pool and a substrate comprised of lower-cell layers, as discussed in Section 10.4, and (4) fission product decay heating and/or user-specified heating rates in any layer.

The nodalization requirements for the modeling of conduction in the lower-cell layers are similar to those for a heat transfer structure. The user should be forewarned, however, that the lower cell input is designed to produce relatively coarse nodes. Nodalizations for accurate solution of the interlayer conduction equation, however, can be produced through use of a large number of concrete layer nodes and multiple intermediate layers to resolve transient conduction effects.

\subsubsection{Connected Structure Boundary Condition}

The connected structure option allows the user to model heat transfer structures requiring inner surface models at each exposed face as well as the modeling of conduction heat transfer between the two faces. A typical example would be a model for a containment shell that has condensation heat transfer occurring on the interior surface, evaporative heat transfer occurring on the exterior surface, and substantial heat conduction through the shell. Such situations can be treated in terms of two structures defined in two different cells and connected at their outer faces through a conduction boundary condition. The connected-structure boundary condition in effect forces time-averaged agreement of the heat flux at the outer surfaces of the two connected structures. In the algorithm developed to implement this boundary condition, conductive outer surface boundary conditions are 
first applied to the structure in the lower numbered cell to define the heat flux, which is then applied as a flux boundary condition to the second structure.

One restriction imposed on connected structures is that concrete outgassing is not allowed. This restriction has been imposed because of the assumption in the outgassing model that a structure outgasses from its inner face. This assumption could lead to outgassing from the wrong surface, for example, when an connected structure is heated only on one surface and the outgassing region has penetrated beyond the common interface of the two structures. In addition, the robustness of the connected structure algorithm has not been checked in conjunction with an outgassing front that has penetrated to the common interface. While the effort required to generalize the connected structure algorithm to include outgassing is not expected to be a large one, this work remains to be done.

Figure 10-3 represents the most general situation regarding connected structures, namely one in which each structure is partially submerged in the pools in the respective cells. As discussed in Section 10.1.1.4, each of the submerged and unsubmerged regions of a partially submerged structure is assumed to be governed by a one-dimensional heat conduction equation. A number of different pairings of regions are possible at the interface shown in Figure 10-3, and these will be represented by pairs of digits; for example, the interface between regions 1 and 2 in the figure will be denoted by 12 .

For a given pairing of regions at the interface, say 1 and 2, the two structures are coupled through an overall heat transfer coefficient $h_{12}$ across the interface according to

$$
\frac{1}{h_{12}}=\frac{1}{h_{c}}+\frac{1}{h_{1}}+\frac{1}{h_{2}}
$$

where $h_{1}$ and $h_{2}$ represent the surface half-node conductances in regions 1 and 2, respectively. As shown in Figure 10-3, $T_{1}$ and $T_{2}$ are the surface node temperatures for that portion of the interface in which regions 1 and 2 are adjacent. The interface heat transfer coefficient $h_{c}$ is the user-specified coefficient "hgap" in the STRUC input block as described in Section 14.3.1.3.

In the one-dimensional heat conduction solution for region 1 , a single effective heat transfer coefficient and single boundary condition temperature must be defined at the region 1 interface with structure 2, regardless of whether one or two regions in structure 2 are adjacent to region 1 . The definitions of these single quantities follow from the assumption that the heat flux from region 1 is governed by a single boundary condition temperature $\left(T_{2, \text { eff }}\right)$, a single interface heat transfer coefficient $\left(\mathrm{h}_{1, \mathrm{eff}}\right)$, and a uniform surface temperature $\left(\mathrm{T}_{1}\right)$. It should be noted that the first two assumptions serve to define one-dimensional boundary conditions to be used with the conduction solver and do not affect the heat flux split among the regions. The latter is determined only by the uniform temperature assumption and the region-to-region heat transfer coefficients (such as $h_{12}$ ).

Let $\mathrm{x}_{1}$ be the fraction of unsubmerged surface area for structure 1 and $\mathrm{x}_{2}$ be the fraction of unsubmerged surface area for structure 2 . Also let $h_{12}$ be the total interface heat transfer coefficient between regions 1 and 2 , and $h_{32}$ be the total coefficient between the submerged region 3 and 2 , and so forth. For $\mathrm{x}_{1}<\mathrm{x}_{2}$, the boundary condition on region 1 is simply 


$$
\begin{aligned}
& \mathrm{T}_{2, \text { eff }}=\mathrm{T}_{2} \\
& \mathrm{~h}_{1, \text { eff }}=\mathrm{h}_{12}
\end{aligned}
$$

whereas for $x_{1} \geq x_{2}$, it is given by

$$
\begin{aligned}
& T_{2, \text { eff }}=\left(f T_{2} h_{12}+(1-f) T_{4} h_{14}\right) / h_{1, \text { eff }} \\
& h_{1, \text { eff }}=f h_{12}+(1-f) h_{14}
\end{aligned}
$$

where $\mathrm{f}=\min \left(\mathrm{x}_{1}, \mathrm{x}_{2}\right) / \max \left(\mathrm{x}_{1}, \mathrm{x}_{2}\right)$. These boundary conditions allow the conduction solution to be obtained for region 1 , in the manner discussed below. A similar set of boundary conditions allow the conduction solution to be carried out in region 3 .

In the following, structure 1 is assumed to belong to the first of the two cells to be processed by CONTAIN for the present system timestep $\Delta \mathrm{t}_{\mathrm{s}}^{\mathrm{m}}$. The flux $\mathrm{q}_{1}^{\mathrm{m}}$ leaving region 1 over all cell timesteps $\Delta t_{c}^{m}$ in the present system timestep is calculated as discussed below and used to define an averaged flux

$$
\overline{\mathrm{q}}_{1}^{\mathrm{n}}=\sum_{\mathrm{m}} \mathrm{q}_{1}^{\mathrm{m}} \Delta \mathrm{t}_{\mathrm{c}}^{\mathrm{m}} / \Delta \mathrm{t}_{\mathrm{s}}^{\mathrm{n}}
$$

where the sum is carried out over the cell timesteps in the present system timestep. When the temperature profile in region 2 is calculated (after regions 1 and 3 in structure 1 are processed for the entire system timestep), a flux-matching boundary condition is used for all cell timesteps in the system timestep for regions 2 and 4 in structure 2. The definition of the flux $q_{2}^{\mathrm{m}}$ for $\mathrm{x}_{1}<\mathrm{x}_{2}$ is given by

$$
q_{2}^{m}=-f \bar{q}_{1}^{n}-(1-f) \frac{h_{23}\left[\bar{q}_{3}^{n}+\left(1-f^{\prime}\right) h_{34}\left(\bar{T}_{4}^{m^{\prime}+\Delta m}-\bar{T}_{2}^{m^{\prime}+\Delta m}\right)\right]}{f^{\prime} h_{23}+\left(1-f^{\prime}\right) h_{34}}
$$

where $\mathrm{f}^{\prime}=\left(\mathrm{x}_{2}-\mathrm{x}_{1}\right) /\left(1-\mathrm{x}_{1}\right), \overline{\mathrm{T}}_{4}^{\mathrm{m}^{\prime}+\Delta \mathrm{m}}$ and $\overline{\mathrm{T}}_{2}^{\mathrm{m}^{\prime}+\Delta \mathrm{m}}$ are extrapolated temperatures as described below, $\mathrm{m}^{\prime}$ corresponds to the cell timestep at the end of the last system timestep, and $\Delta \mathrm{m}$ corresponds to the number of cell timesteps in the present system timestep. On the other hand, for $x_{1}>x_{2}$, the flux is given by

$$
q_{2}^{m}=-\frac{h_{12}\left[\bar{q}_{1}^{n}+(1-f) h_{14}\left(\bar{T}_{4}^{m^{\prime}+\Delta m}-\bar{T}_{2}^{m^{\prime}+\Delta m}\right)\right]}{f h_{12}+(1-f) h_{14}}
$$

It is tempting to define $\mathrm{q}_{1}{ }^{\mathrm{m}}$ explicitly in terms of $\mathrm{T}_{2, \text { eff }}$ according to 


$$
\mathrm{q}_{1}^{\mathrm{m}}=\mathrm{h}_{1, \text { eff }}\left(\mathrm{c}_{1} \mathrm{~T}_{1}^{\mathrm{m}}+\left(1-\mathrm{c}_{1}\right) \mathrm{T}_{1}^{\mathrm{m}-1}-\mathrm{T}_{2, \mathrm{eff}}^{\mathrm{m}^{\prime}}\right)
$$

where $c_{1}$ is the implicit integration weighting factor, defined as the user-specifiable "crank" parameter described in Section 14.3.1.3; m-1 corresponds to the value at the last cell timestep; and $\mathrm{m}^{\prime}$ corresponds to the value at the last system timestep. However, use of an explicit $\mathrm{T}_{2, \text { eff }}$ to define the flux within a partially implicit scheme in general gives a temperature mismatch across the interface. In contrast, within a partially implicit temperature matching scheme, there would be flux mismatch across the interface. Note that if one could solve the structures simultaneously, then one could treat all temperatures consistently, and there would not be a problem. However, as a consequence of CONTAIN architecture in which the structures in each cell are processed separately, simultaneous solution is not possible. To obtain consistency with respect to both the flux and the interface temperatures, a number of approaches for anticipating the change in the temperature $T_{2}$ in region 2 with respect to changes in the flux were investigated.

The adopted approach for calculating $\mathrm{q}_{1}^{\mathrm{m}}$ depends on the diffusivity $\alpha$ of the node in region 1 adjacent to the interface. If

$$
\alpha \Delta t_{c}{ }^{m}<y_{1}^{2} / 4
$$

where $y_{1}$ is the node thickness, then the boundary condition given in Equation (10-112) is used; that is, $q_{1}^{m}$ is calculated by iteration with the conduction solver until it satisfies Equation (10-112). Otherwise, a flux corrector is used, as discussed below.

The flux is given in terms of the flux corrector according to the relation

$$
\mathrm{q}_{1}^{\mathrm{m}}=\overline{\mathrm{q}}_{1}^{\mathrm{n}-1}+\Delta \mathrm{q}_{1}^{\mathrm{m}}
$$

where $\overline{\mathrm{q}}_{1}{ }^{\mathrm{n}-1}$ is the average flux calculated in the last system timestep and $\Delta \mathrm{q}_{1}$ is the corrector. The boundary condition used to obtain the flux corrector is

$$
q_{1}^{m}=h_{t}\left(c_{1} T_{1}^{m}+\left(1-c_{1}\right) T_{1}^{m-1}-T_{2, e f f}^{\prime m}\right)
$$

where $T_{2, \text { eff }}^{\prime}$ is a trial temperature that is used to obtain the flux corrector.

The flux corrector approach anticipates changes in $T_{2, \text { eff }}$ in two ways: (1) extrapolation of the time rate of change of $T_{2, \text { eff }}$ from the previous system timestep and (2) division of the result for the flux corrector by an extra factor of 2 . In general, extrapolating the behavior of $T_{2, \text { eff }}$ from the time derivative of $T_{2, \text { eff }}$ from the previous system timestep will cause the iteration to be less stable. However, significant improvement in temperature matching appears to be possible in some cases by extrapolating. Therefore, extrapolation is included but made conditional. The conditional approach requires two trial fluxes, $q_{1}^{\prime}$ and $q_{1}^{\prime \prime}$. $q_{1}^{\prime}$ is the flux calculated leaving structure 1 during the current cell timestep for a trial temperature boundary condition $T_{2, \text { eff }}^{\prime m}=\bar{T}_{2, \text { fff }}^{\mathrm{m}-1}$, where the latter is an 
extrapolated temperature as defined below. $q_{1}^{\prime \prime}$ is the flux for a second trial temperature $T_{2, \text { eff }}^{\prime m}=\bar{T}_{2, \text { eff }}^{\mathrm{m}-1}$ $+c_{1}\left(\mathrm{dT}_{2, \text { eff }} / \mathrm{dt}^{\mathrm{m}^{\prime}} \Delta \mathrm{t}_{\mathrm{c}}\right.$, where $\left(\mathrm{dT}_{2, \text { eff }} / \mathrm{dt}\right)^{\mathrm{m}^{\prime}}$ is the time rate of change of $\mathrm{T}_{2, \text { eff }}$ at the end of the last system timestep.

First, let $\Delta \mathrm{q}_{1}^{\prime}=\mathrm{q}_{1}^{\prime}-\overline{\mathrm{q}}_{1}^{\mathrm{n}-1}$ and $\Delta \mathrm{q}_{1}^{\prime \prime}=\mathrm{q}_{1}^{\prime \prime}-\overline{\mathrm{q}}_{1}^{\mathrm{n}-1}$. If $\mathrm{q}_{1}^{\prime}$ and $\mathrm{q}_{1}^{\prime \prime}$ are both positive, then

$$
\Delta \mathrm{q}_{1}^{\mathrm{m}}=\min \left(\Delta \mathrm{q}_{1}^{\prime}, \Delta \mathrm{q}_{1}^{\prime \prime}\right) / 2
$$

If $\mathrm{q}_{1}^{\prime}$ and $\mathrm{q}_{1}^{\prime \prime}$ are both negative, then

$$
\Delta \mathrm{q}_{1}^{\mathrm{m}}=\max \left(\Delta \mathrm{q}_{1}^{\prime}, \Delta \mathrm{q}_{1}^{\prime \prime}\right) / 2
$$

If the two trial fluxes are of opposite sign, then the average is used:

$$
\Delta \mathrm{q}_{1}^{\mathrm{m}}=\left(\Delta \mathrm{q}_{1}^{\prime}+\Delta \mathrm{q}_{1}^{\prime \prime}\right) / 4
$$

The extra factor of 2 in the denominator of the above expressions anticipates the response of structure 2 to the change in flux and is apparently necessary for stability.

The manner in which $\bar{T}_{2, \text { eff }}^{\mathrm{m}-1}$ is extrapolated for use in the next cell timestep is chosen to be consistent with the flux selected:

$$
\begin{aligned}
\overline{\mathrm{T}}_{2, \mathrm{eff}}^{\mathrm{m}} & =\overline{\mathrm{T}}_{2, \mathrm{eff}}^{\mathrm{m}-1} & & \left(\Delta \mathrm{q}_{1}^{\mathrm{m}}=\Delta \mathrm{q}_{1}^{\prime} 2\right) \\
& =\overline{\mathrm{T}}_{2, \text { eff }}^{\mathrm{m}-1}+\mathrm{c}_{1}\left(\mathrm{dT}_{2, \text { eff }} / \mathrm{dt}^{\mathrm{m}^{\prime}} \Delta \mathrm{t}_{\mathrm{c}}^{\mathrm{m}}\right. & & \left(\Delta \mathrm{q}_{1}^{\mathrm{m}}=\Delta \mathrm{q}_{1}^{\prime \prime} 2\right) \\
& =\overline{\mathrm{T}}_{2, \text { eff }}^{\mathrm{m}-1}+0.5 \mathrm{c}_{1}\left(\mathrm{dT}_{2, \text { eff }} / \mathrm{dt}\right)^{\mathrm{m}^{\prime}} \Delta \mathrm{t}_{\mathrm{c}}^{\mathrm{m}} & & \text { (otherwise) }
\end{aligned}
$$

For the first cell timestep of a system timestep, $\bar{T}_{2, \text { eff }}^{\mathrm{m}-1}$ is taken to be $\mathrm{T}_{2, \text { eff }}^{\mathrm{m}^{\prime}}$, which is value at the end of the last system timestep. Also, the end-of-system-timestep extrapolated temperatures $\bar{T}_{2}^{\mathrm{m}^{\prime}+\Delta m}$ and $\bar{T}_{4}^{m^{\prime}+\Delta \mathrm{m}}$ used above in Equations (10-110) and (10-111) are defined in a manner similar to that for $\overline{\mathrm{T}}_{2, \text { eff }}^{\mathrm{m}}$, except that the equations are applied recursively until the superscript attains a value corresponding to the last cell timestep in the present system timestep.

It is expected that the algorithm discussed here will work best on symmetrically noded halfstructures, with the same time integration factor $\mathrm{c}$ and comparable coupling to the atmosphere at the exposed faces. In case of problems with accuracy or stability with asymmetric coupling, the algorithm may work better with the stronger coupling in the lower numbered cell. 


\subsubsection{Heat Conduction Model}

This section describes the heat conduction model use for calculating the temperatures within heat transfer structures as well as within lower cell layers when core-concrete interactions are not being modeled. Note that heat transfer is modeled internally within the CORCON-Mod3 module when core-concrete interactions are actively modeled. The conduction model must deal with three different geometries: slabs, cylinders, and spheres.

The heat conduction problem is modeled by solving the standard one-dimensional heat conduction equation,

$$
\rho(\mathrm{r}) c_{p}(\mathrm{r}) \frac{\partial \mathrm{T}(\mathrm{r}, \mathrm{t})}{\partial \mathrm{t}}=\nabla \cdot \mathrm{k}(\mathrm{r}) \nabla \mathrm{T}(\mathrm{r}, \mathrm{t})+\Psi(\mathrm{r}, \mathrm{t})
$$

where $\rho(r)$ is the density, $c_{p}(r)$ is the specific heat capacity, $k(r)$ is the thermal conductivity, $T(r, t)$ is the temperature at position $r$ at time $t$, and $\Psi(r, t)$ is the volumetric energy generation rate.

Note that because the nodes in a structure or lower cell may have different materials in CONTAIN, the thermal conductivity, density, and heat capacity may all be functions of the spatial coordinate $r$, which is defined as the coordinate normal to the inner surface. For half-cylinders and hemispheres, $\mathrm{r}$ is the distance from the center of curvature of the structure. Clearly the conductance term $\nabla \cdot \mathrm{k} \nabla \mathrm{T}$ is also dependent upon the geometry of the structure.

The time evolution of the temperature profile in a heat sink is determined by solving a discretized version of the conduction equation at each cell timestep $\Delta \mathrm{t}_{\mathrm{c}}$. Therefore, the results from the preceding timestep are used as the initial conditions for the conduction equation for a given timestep. In addition the material properties are evaluated at the initial temperature at the beginning of a timestep.

The heat conduction equation is always solved using adiabatic boundary conditions. However, this does not mean that all sinks are treated as being adiabatic. The conduction model accounts for external heat transfer fluxes through the volumetric energy generation rates for the surface nodes. These energy generation rates, for example, may reflect heat transfer from convection, condensation, radiation, liquid transport, and various heating rates. Therefore, the coupling between the conduction model and the heat transfer models discussed in other sections is explicit.

The conduction equation is solved by discretizing the temperature variable in space, using the spatial nodalization specified by the user. The temperature distribution is then represented by the nodecenter temperatures, as illustrated in Figure 10-9. If $\mathrm{T}$ is the temperature vector, which is now defined for each node and governed by an equation of the form $\mathrm{dT} / \mathrm{dt}=\mathrm{F}(\mathrm{T})$, then this equation may be approximated as

$$
\frac{\mathrm{T}^{\mathrm{n}+1}-\mathrm{T}^{\mathrm{n}}}{\Delta \mathrm{t}}=\mathrm{cF}\left(\mathrm{T}^{\mathrm{n}+1}\right)+(1-\mathrm{c}) \mathrm{F}\left(\mathrm{T}^{\mathrm{n}}\right)
$$




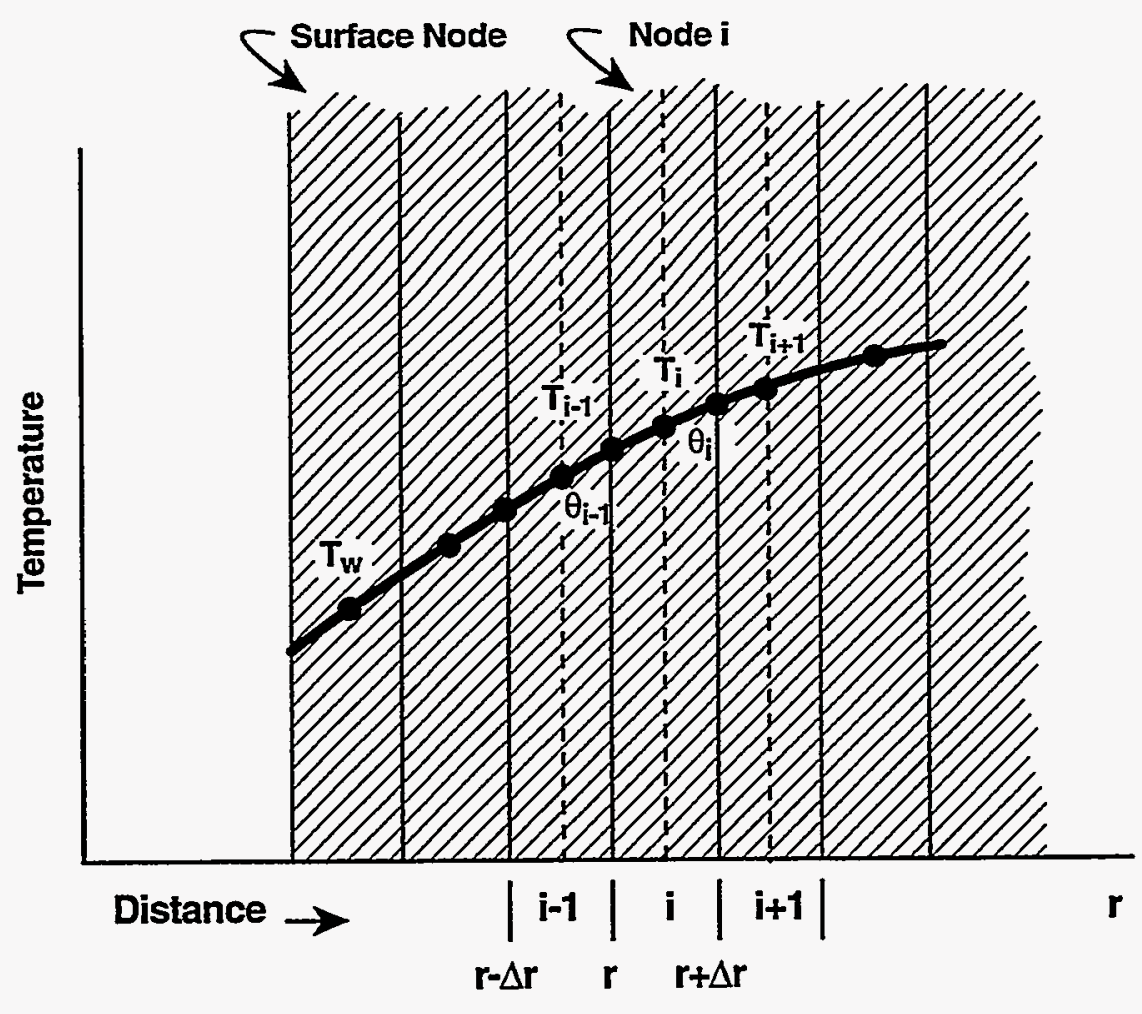

Figure 10-9. Nodes in a one-Dimensional System 
where $c$ is the user-specifiable implicit integration weighting factor "crank", and $\mathrm{T}^{\mathrm{n}}$ denotes the value of $\mathrm{T}$ at timestep $\mathrm{n}$. The default value of $\mathrm{c}=1$ represents a fully implicit treatment. For certain problems, a value of $c$ less than 1 may yield more accurate results. A value of 0.5 yields what is called Crank-Nicholson integration. Fully explicit integration is obtained when $c=0$. If values less than 0.5 are used, care should be taken in selecting the cell timestep to ensure that the Courant stability criterion is not violated (the diffusion length in Equation (10-106) during the cell timestep must not exceed the node thickness). Extremely large timesteps should also be avoided when using values between 0.5 and 1.0 ; such values will always give stable but possibly oscillatory temperatures. Note that $c=1$ will always give stable, non-oscillatory internal temperatures. However, for most problems, timesteps that give oscillatory behavior or negative temperatures when $c<1$, will also give inaccurate (albeit non-oscillatory) temperatures for $c=1$. Thus, a value of $c=1$ is not necessarily the preferred value to use. In general, if there is any doubt as to solution accuracy, a value of $c=0.5$, with appropriate timestep selection, will provide a good check on the implicit solution accuracy.

In the following, for simplicity, only the implicit integration scheme corresponding to $c=1$ will be discussed. The conduction equation for node $i$ at timestep $n$ is then given by

$$
\rho_{\mathrm{i}} \mathrm{c}_{\mathrm{p}, \mathrm{i}} \frac{\mathrm{T}_{\mathrm{i}}^{\mathrm{n}+1}-\mathrm{T}_{\mathrm{i}}^{\mathrm{n}}}{\Delta \mathrm{t}}=\nabla\left(\mathrm{k}_{\mathrm{i}} \nabla \mathrm{T}_{\mathrm{i}}^{\mathrm{n}+1}\right)+\Psi_{\mathrm{i}}
$$

where the specific heat $c_{p}$ and conductivity $k$ may depend on the node $i$. Equation (10-122) is solved by integrating over the volume $\mathrm{V}_{\mathrm{i}}$ of each node. If one then applies the divergence theorem to the conductance term, the discretized conduction equation integrated over the volume of node $i$ can be expressed as

$$
\mathrm{m}_{\mathrm{i}} \mathrm{c}_{\mathrm{p}, \mathrm{i}} \frac{\mathrm{T}_{\mathrm{i}}^{\mathrm{n}+1}-\mathrm{T}_{\mathrm{i}}^{\mathrm{n}}}{\Delta \mathrm{t}_{\mathrm{c}}}=\mathrm{k}_{\mathrm{i}}\left(\nabla \theta_{\mathrm{i}+1}^{\mathrm{n}+1} \mathrm{~A}_{\mathrm{i}+1}-\nabla \theta_{i}^{\mathrm{n}+1} A_{\mathrm{i}}\right)+\dot{Q}_{i}
$$

where $m_{i}$ is the node mass, $A_{i}$ is the area at interface $i, \nabla \theta_{i}$ is the temperature gradient at interface $i$, and $\dot{Q}_{\mathrm{I}}$ is the volume integral of the energy generation rate.

If the structure has been nodalized into $\mathrm{N}$ slabs, then there will be $\mathrm{N}$ such equations. The temperature gradient in this equation is approximated by the following first-order Taylor expansion,

$$
\nabla \theta_{\mathrm{i}} \cong \frac{\theta_{\mathrm{i}}-\mathrm{T}_{\mathbf{i}}^{\mathrm{n}+1}}{\delta \mathbf{r}}
$$

where $\theta_{i}$ is the temperature at the interface between nodes $i$ and $i+1$, and $\delta r$ is the distance between the node midpoint and the interface (see Figure 10-9). Note that this expression is the same for all three geometries. However, the location of the node midpoint, and therefore the value of $\delta \mathrm{r}$, is geometry dependent. This distance is chosen such that the volumes on both sides of the point are equal. 
The interface temperature $\theta_{\mathrm{i}}$ has still not been defined at this point. All interface temperatures can be eliminated from the equation by imposing the assumption that the heat flux on both sides of the interface between two nodes must be equal. If this is done, the temperature gradient at the interface in Equation (10-124) can be expressed in terms of differences between the node-center temperatures alone. The discretized conduction equation for a given node is then given by

$$
T_{i}^{n+1}-T_{i}^{n}=\frac{K_{i+1} A_{i+1} \Delta t_{c}}{m_{i} c_{p, i}}\left(T_{i+1}^{n+1}-T_{i}^{n+1}\right)-\frac{K_{i} A_{i} \Delta t_{c}}{m_{i} c_{p, i}}\left(T_{i}^{n+1}-T_{i-1}^{n+1}\right)+\frac{\dot{Q}_{i} \Delta t_{c}}{m_{i} c_{p, i}}
$$

The quantity $\mathrm{K}_{\mathrm{i}}$ is the effective conductance between node $i$ and $i+1$, derived by applying the flux continuity condition at the interface. This quantity depends upon the thermal conductivity of the two regions on either side of the interface, and the distances to the center of each node. This parameter is geometry-dependent because the distances to the node centers and the interface areas are both geometry-dependent. It can readily be shown that the conductances $\mathrm{K}_{i}$ and the half-node thicknesses $\delta \mathrm{r}_{\mathrm{i}}$ for the three geometries are given by

$$
\mathrm{K}_{\mathrm{i}}=\frac{1}{\left(\frac{\mathrm{k}_{\mathrm{i}}}{\delta \mathrm{r}_{\mathrm{i}}}\right)^{-1}+\left(\frac{\mathrm{k}_{\mathrm{i}+1}}{\delta \mathrm{r}_{\mathrm{i}+1}}\right)^{-1}}
$$

The $\delta r_{i}$ and $\delta r_{i+1}$ depend on the structure geometry.

For the slab geometry

$$
\delta r_{i}=\delta r_{i+1}=\frac{r_{i+1}-r_{i}}{2}
$$

For the cylindrical geometry

$$
\begin{aligned}
& \delta r_{i}=r_{i} \ln \left(\frac{\bar{r}}{r_{i}}\right) \\
& \delta r_{i+1}=r_{i+1} \ln \left(\frac{r_{i+1}}{\bar{r}}\right)
\end{aligned}
$$

where

$$
\overline{\mathrm{r}}=\left(\frac{\mathrm{r}_{\mathrm{i}+1}^{2}+\mathrm{r}_{\mathrm{i}}^{2}}{2}\right)^{1 / 2}
$$


For the spherical geometry

$$
\begin{aligned}
& \delta r_{i}=r_{i} \frac{\bar{r}-r_{i}}{\bar{r}} \\
& \delta r_{i+1}=r_{i+1} \frac{r_{i+1}-\bar{r}}{\bar{r}}
\end{aligned}
$$

where

$$
\bar{r}=\left(\frac{r_{i+1}^{3}+r_{i}^{3}}{2}\right)^{1 / 3}
$$

The $N$ linear coupled equations given by Equation (10-125) are solved by using a standard tridiagonal matrix methods.

\subsubsection{Concrete Outgassing}

The outgassing model for the release of $\mathrm{H}_{2} \mathrm{O}$ and $\mathrm{CO}_{2}$ gases from concrete heat transfer structures is described in this section. Both evaporable and bound water are considered in the modeling of steam release. The principal assumption made in the outgassing model is that the release is controlled by the rate at which thermal energy is supplied for vaporization of the available water and/or for concrete decomposition and not by gas transport effects. The concrete outgassing model has been described in greater detail in Reference Was88. The reader should consult this reference for additional information.

The heat conduction model discussed in Section 10.5.3 is tightly coupled to the concrete outgassing model in two ways. First, the energy required to decompose compounds in the concrete and/or evaporate water is accounted for in the heat conduction equation through appropriate energy sink terms. This is done in a closely coupled manner so that gas release during a timestep is consistent with the temperature at the end of the same timestep. Likewise, the end of timestep temperature is used to compute the gas release from the structure. An iterative solution technique is used to partition the heat input between that used for gas release and the heating of concrete.

Second, the thermophysical properties of the concrete are modeled as a function of temperature and the amount of gas released from the concrete. In the model, only nodes containing CONC material are allowed to outgas. Note that the properties of the CONC material as supplied by the properties routines are assumed to correspond to wet concrete. However, in the outgassing routines, the loss of evaporable water from the node is assumed to modify the specific heat of an outgassing node according to the assumption that evaporable water contributes a specific heat equal to that of liquid water. The porosity of a node, not occupied by concrete, is assumed to be equal to the mass of bound gases released, divided by the concrete density with evaporable water removed, and the mass and volume of concrete material in the node is correspondingly reduced. The conductivity is assumed 
to be reduced in proportion to this porosity. Note that the evaporable water is associated with the concrete, not the porosity volume.

Through the DESTCELL keyword in the OUTGAS input block (see Section 14.3.1.3), the user may specify the cell to which the released gas from each structure is directed. The outgassing model assumes that the released gases migrate to the innermost concrete node before being released to the destination cell at the temperature of that node and the pressure of the destination cell. (Note that migration to the outermost node is not considered even if the heating is predominantly on the outer face. The user, however, has the option of specifying which face is the inner one.) By default, unlined concrete structures will outgas into the cell associated with the structure inner face, and lined structures will not outgas. Outgassing from a structure with an inner liner of non-CONC material is permitted if the user specifies a destination cell for the outgas. Note that if more than one set of contiguous CONC nodes are present in a structure, only the block closest to the inner surface will be allowed to outgas. For reasons given in Section 10.5.2, connected structures are not allowed to outgas.

The outgassing inventories for the CONC nodes are controlled by the mass fractions for the maximum amount of evaporable water, bound water, and $\mathrm{CO}_{2}$ that may evolve from the material. These mass fractions are denoted by $\mathrm{f}_{\mathrm{e}, \mathrm{i}}^{0}, \mathrm{f}_{\mathrm{b}, \mathrm{i}}^{0}$, and $\mathrm{f}_{\mathrm{c}, \mathrm{i}}^{0}$, respectively, in the discussion below and are specified either in the OUTGAS or CONCDATA input blocks of the STRUC input (see Section 14.3.1.3).

The initial total node mass $\mathrm{M}_{\mathrm{i}}^{0}$ for the $i$ th node (assumed to be CONC) is given by

$$
M_{i}^{0}=\rho_{\text {CONC,i }}^{0} V_{i}
$$

where $\rho_{\text {CONC, } i}^{0}$ is the density of CONC material at the node initial temperature and $V_{i}$ is the volume of node $\mathrm{i}$.

The maximum masses of gases that may evolve from the node are given by

$$
\begin{aligned}
& M_{e, i}^{0}=M_{i}^{0} f_{e, i}^{0} \text { (evaporable water) } \\
& M_{b, i}^{0}=M_{i}^{0} f_{b, i}^{0} \text { (bound water) } \\
& M_{c, i}^{0}=M_{i}^{0} f_{c, i}^{0}\left(\mathrm{CO}_{2}\right)
\end{aligned}
$$

The thermal properties of all CONC nodes in the applicable structures are modified by the userdefined outgassing fractions and by the released gas inventories. If the integrated released masses from node $i$ of evaporable water, bound water, and $\mathrm{CO}_{2}$ are given by $\mathrm{G}_{\mathrm{e}, \mathrm{i}}, \mathrm{G}_{\mathrm{b}, \mathrm{i}}$, and $\mathrm{G}_{\mathrm{c}, \mathrm{i}}$, respectively, then the node mass $\mathrm{M}_{\mathrm{i}}$ is given by

$$
M_{i}=M_{i}^{0}-G_{e, i}-G_{b, i}-G_{c, i}
$$


The node effective specific heat $c_{p, i}$ is given by

$$
c_{p, i}=\left[c_{p, d r y, i}\left(M_{i}-M_{e, i}\right)+c_{p, w, i} M_{e, i}\right] M_{i}
$$

where $M_{e, i}=M_{e, i}^{0}-G_{e, i}$ is the mass of evaporable water remaining in the node, $c_{p, w, i}$ is the specific heat of water at the node temperature at the beginning of the cell timestep, and $c_{p, d r y, i}$ is the specific heat of concrete with the contribution of the evaporable water subtracted

$$
c_{p, d r y, i}=\frac{c_{p, C O N C, i} M_{i}^{0}-c_{p, w, i} M_{e, i}^{0}}{M_{i}^{0}-M_{e, i}^{0}}
$$

Here, $c_{p, c o N C, i}$ is the specific heat of the CONC material in the absence of outgassing. The effective node conductivity $\mathrm{k}_{\mathrm{i}}$ is also assumed to change according to the mass of bound water and $\mathrm{CO}_{2}$ released

$$
k_{i}=k_{C O N C, i}\left(1-f_{p, i}\right)
$$

where $\mathrm{k}_{\mathrm{CoNC}, \mathrm{i}}$ is the conductivity of the CONC material; and the time-dependent porosity $\mathrm{f}_{\mathrm{p}, \mathrm{i}}$ is given by

$$
f_{p, i}=\frac{G_{b, i}+G_{c, i}}{M_{i}^{0}-M_{e, i}^{0}}
$$

The porosity and conductivity are therefore affected only by the amount of gas released by decomposition and not by evaporation.

In the evaporable water release modeling, it is assumed that the concrete begins to dry out when it reaches a lower temperature $T_{10}$, which in the pressure-dependent formulation is the saturation temperature $T_{s}$ at the destination cell pressure $P$. The concrete evaporable water release is assumed linear in temperature between $T_{10}$ and a temperature $T_{h i}$ above that point. The upper temperature $T_{h i}$ in the pressure-dependent formulation is determined by adding a user-specified temperature range "tspan" to $\mathrm{T}_{10}$ in addition to a linear term in the cell pressure $\mathrm{P}$, according to the following expressions:

$$
\begin{aligned}
& \mathrm{T}_{10}=\mathrm{T}_{\mathrm{s}}(\mathrm{P}) \\
& \mathrm{T}_{\mathrm{hi}}=\mathrm{T}_{1 \mathrm{l}}+\text { "tspan" + "pfac" }\left(\mathrm{P}-10^{5}\right) / 10^{5}
\end{aligned}
$$

The default value of "tspan" is $10 \mathrm{~K}$ and the user-specified pressure adjustment factor, "pfac", is zero by default (see Section 14.3.1.3). Note that the user can also specify values of $T_{10}$ and $T_{h i}$ that are independent of cell pressure through a separate option using keyword TH2OE in the OUTGAS input block. This is useful for problems at constant pressure. A warning message is written to the error 
file, TAPE17, to the effect that the gas release is independent of pressure when this latter option is used.

In the evaporable water release modeling, the release is assumed to be controlled by a self-consistent temperature that takes into account heat conduction in the structure and the heats of vaporization. In the outgassing algorithm, the effects of heat conduction without outgassing are first calculated. This calculation determines the post-conduction temperature $T_{i}$ for each node $i$ and the conduction heat flux $q_{i}$ for each node interface, as shown in Figure 10-10. A self-consistent, piecewise continuous temperature profile is then constructed to determine the outgassing of evaporable water from the structure. It is assumed in the discussion below that $T_{i}$ and $q_{i}$ for the present timestep have been previously determined in the conduction algorithm.

For the purpose of determining the temperature profile, a heat flux boundary condition is imposed on each node $i$, with the conduction heat fluxes set to $q_{i}$ and $q_{i+1}$ on the left and right interfaces, respectively, of the $i$ th node. (Hereafter, the discussion will be restricted to the $i$ th node.) For simplicity, it is assumed that the temperature profile in each interior half-node corresponds to steadystate conditions without sources or sinks (i.e., it is assumed to have a linear, logarithmic, or inverse radial dependence for slabs, cylinders, and spheres, respectively). To be specific, if $T_{\mathfrak{i}}^{\prime}$ is the selfconsistent node temperature to be determined as discussed below, then the temperature profile $T(x)$ in an interior left half-node, as represented in Figure 10-10, is given by

$$
T(x)=T_{1}+\left(f(x)-f\left(x_{1}\right)\right)\left(T_{i}^{\prime}-T_{1}\right) /\left(f\left(x_{m}\right)-f\left(x_{1}\right)\right)
$$

where

$$
\begin{aligned}
f(x) & =x & & \text { (for a slab structure) } \\
& =\ln \left(x / x_{1}\right) & & \text { (cylinder) } \\
& =1 / x & & \text { (sphere) }
\end{aligned}
$$

A similar expression holds for an interior right half-node:

$$
T(x)=T_{2}+\left(f(x)-f\left(x_{2}\right)\right)\left(T_{i}^{\prime}-T_{2}\right) /\left(f\left(x_{m}\right)-f\left(x_{2}\right)\right)
$$

In Equations (10-142) and (10-143), $T_{1}=T_{i}^{\prime}+q_{i} / h_{1}$, which is the temperature at left interface in Figure 10-10; $T_{2}=T_{i}^{\prime}+q_{i+1} / h_{2}$ which is the temperature at right interface; $h_{1}$ is the left half-node effective heat transfer coefficient, based on the effective conductivity of the node material; $h_{2}$ is the right half-node effective heat transfer coefficient; $x$ is the structure coordinate (depth or radius); $x_{1}$ is the left interface coordinate value; $x_{2}$ is the right interface coordinate value; and $x_{m}$ is the coordinate corresponding to one-half of the node volume.

For the surface half-nodes, in contrast to the interior half-nodes, the temperature $T(x)$ is taken to be equal to $\mathrm{T}_{\mathrm{i}}^{\prime}$. The reason for this is that CONTAIN does not always properly distinguish between volumetric heating of surface nodes and heating by conduction. The constant temperature profile assumed for the surface half-nodes is not believed to be a serious limitation. 


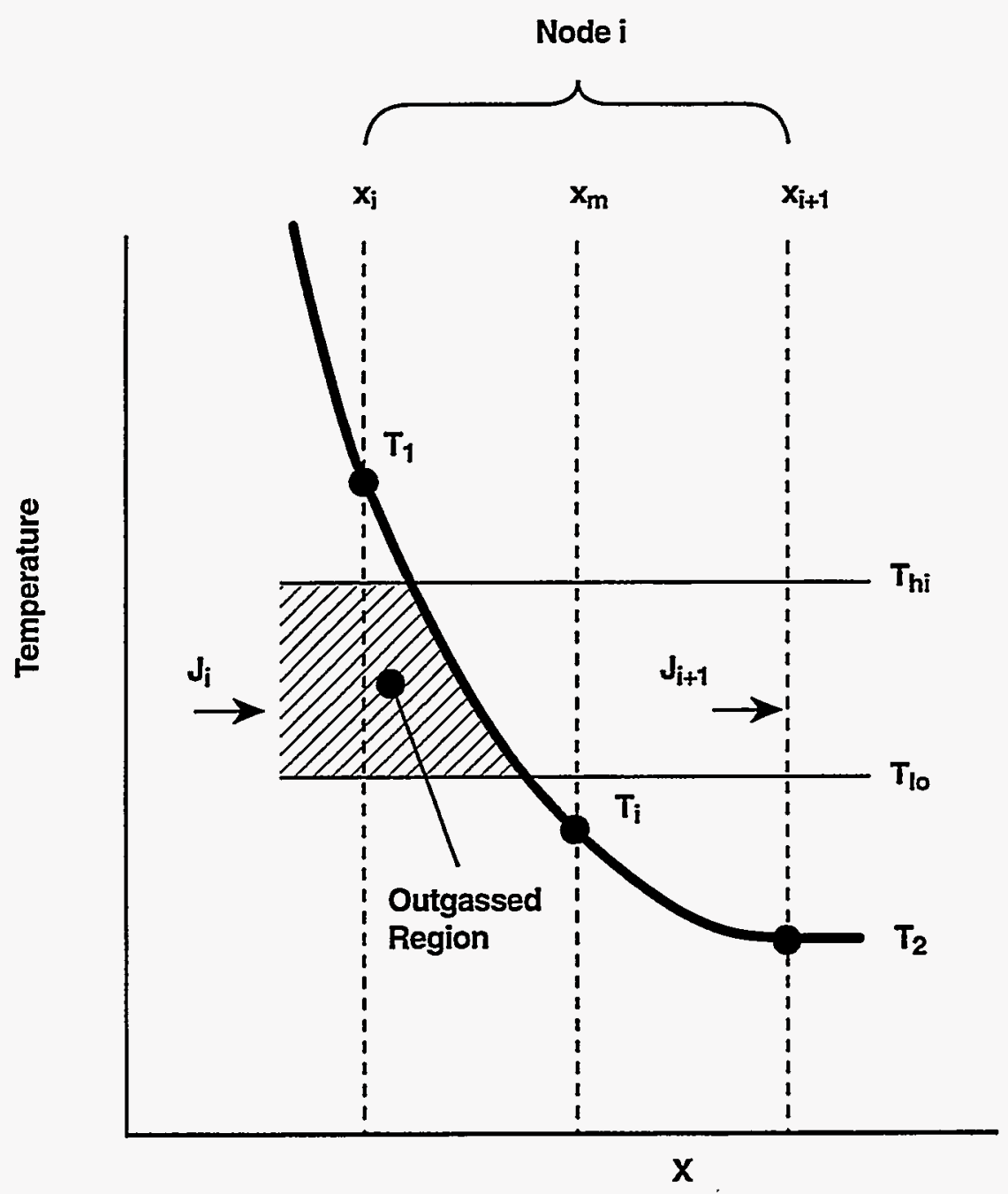

Figure 10-10. Mlustration of the Concrete Outgassing Temperature Profile 
The outgassing of evaporable water from a node is controlled by an effective temperature $T_{e, j}$. The definition of $T_{e, i}$ is

$$
\begin{gathered}
\mathrm{T}_{\mathrm{e}, \mathrm{i}}=\frac{1}{\mathrm{~V}_{\mathrm{i}}} \int \mathrm{T}(\mathrm{x}) \mathrm{dV_{ \textrm {i } }}+\mathrm{T}_{\mathrm{lo}} \\
\mathrm{T}_{\mathrm{hi}}>\mathrm{T}(\mathrm{x})>\mathrm{T}_{\mathrm{lo}}
\end{gathered}
$$

where $V_{i}$ is the node volume.

In terms of $T_{e, j}$, the evaporable water inventory released from a node up to a given point in time is given by

$$
G_{e, i}=\max \left(F_{i}, F_{i, \max }\right) M_{e, i}^{0}
$$

where the release fraction $F_{i}$ is given by

$$
F_{i}=\max \left(\left(T_{e, i}-T_{10}\right) /\left(T_{h i}-T_{10}\right), 0\right)
$$

In Equation (10-145), $F_{i, \max }$ is defined to be the largest value of $F_{i}$ attained for the node in the past, and $\mathrm{M}_{\mathrm{e}, \mathrm{i}}^{0}$ is the initial evaporable water inventory. Note that the spatial variation of the temperature $\mathrm{T}(\mathrm{x})$ within each node in the present treatment tends to smooth out the outgassing so that it will be relatively continuous even for coarse nodes.

The amount of water outgassed is easy to depict if the structure temperatures $T_{i}^{\prime}$ have been increasing with time for each node. In this case the cumulative amount of water released for the temperature profile in Figure 10-10 is proportional to the cross-hatched region marked "outgassed."

The self-consistent node temperatures $T_{i}^{\prime n}$, where $n$ denotes the present cell timestep index, is determined from energy conservation considerations. The self-consistency equation is a statement of energy conservation for the node when outgassing is taken into account:

$$
\left(q_{i}^{n} A_{i}-q_{i+1}^{n} A_{i+1}\right) \Delta t_{c}+\dot{Q}_{i} \Delta t_{c}=C_{i}\left(T_{i}^{\prime n}-T_{i}^{\prime n-1}\right)+\lambda_{f g} \Delta G_{e, i}+\lambda_{b} \Delta G_{b, i}+\lambda_{c} \Delta G_{c, i}
$$

where $A$ is the interface area; $\Delta t_{c}$ is the cell timestep; $\dot{Q}$ is the node volumetric heating rate; $C_{1}$ is the total node specific heat (equal to $c_{\mathrm{p}, \mathrm{i}} \mathrm{M}_{\mathrm{i}}$ ); $\lambda_{\mathrm{fg}}$ is the user-specifiable heat of vaporization of water, equal to $h_{v}\left(T_{f}^{\prime n-1}\right)-h_{l}\left(T_{i}^{m-1}\right)$ by default, where $h_{v}$ is the specific enthalpy of steam, $h_{l}$ is the specific enthalpy of water, $\mathrm{T}_{\mathrm{f}}^{\prime \mathrm{n}-1}$ is the temperature from the previous timestep for the innermost CONC node in the structure, and $T_{i}^{\prime n-1}$ is the self-consistent node temperature from the previous timestep for the concrete node $\mathrm{i} ; \Delta \mathrm{G}_{\mathrm{e}, \mathrm{j}}$ is the mass of evaporable water evolved during the timestep; and $\lambda_{\mathrm{b}}, \Delta \mathrm{G}_{\mathrm{b}, \mathrm{j}}, \lambda_{\mathrm{c}}$, and $\Delta \mathrm{G}_{\mathrm{c}, \mathrm{i}}$ are the analogous heats and evolved masses for bound water and $\mathrm{CO}_{2}$, respectively, as discussed below. It should be noted that the self-consistent temperatures $\mathrm{T}_{\mathrm{i}}^{\prime \mathrm{n}}$ are stored and used as the initial structure temperatures for the next timestep. 
The bound water release is calculated by default from an Arrhenius rate law:

$$
\frac{d G_{b, i}}{d t}=\left(M_{b, i}^{0}-G_{b, i}\right) \kappa_{b} \exp \left(\frac{-E_{b}}{R T^{\prime}}\right)
$$

and

$$
\Delta \mathrm{G}_{\mathrm{b}, \mathrm{i}}=\frac{\mathrm{dG}_{\mathrm{b}, \mathrm{i}}}{\mathrm{dt}} \Delta \mathrm{t}_{\mathrm{c}},
$$

where $\kappa_{\mathrm{b}}$ is the decomposition rate constant and $\mathrm{E}_{\mathrm{b}}$ is the activation energy.

A similar equation and similar parameters are used for $\mathrm{CO}_{2}$.

The Arrhenius release model may not be appropriate for complicated situations. For example, the decomposition of a mixture of $\mathrm{MgCO}_{3}$ and $\mathrm{CaCO}_{3}$, both of which produce $\mathrm{CO}_{2}$ gas, cannot be represented by a rate equation of the type given by Equation (10-148). The user does, however, have the freedom to select the rate constant $K$ and the activation energy $E$ so that average parameters for a mixture can be chosen. A simpler model, identical to the pressure-independent, temperature-range, evaporable water release model mentioned earlier, can also be used in such situations. This simpler model is activated through the TRANGE keyword in the OUTGAS input block. It is provided as an alternative that may be more appropriate than the Arrhenius model in some cases.

\subsection{Coupled Heat and Mass Transfer}

The heat and mass transfer processes discussed in this chapter generally become coupled at the heat transfer interface, through the interface energy balance equation. This equation typically is used to determine the interface temperature $T_{\text {if }}$ which drives the interface processes. This energy balance equation and the derivation of the interface temperature are discussed in this section.

To avoid separate discussions for each type of interface in CONTAIN, a general discussion of heat and mass transfer at an interface is given. This general discussion is directly applicable to the interface between the gas and a heat transfer structure. Figure 10-11 illustrates the processes that can occur at this interface. However, the reader should note that the processes considered at an interface and the manner in which they are taken into account may vary with the interface. Table 10-1 summarizes the processes that are modeled for each type of interface in CONTAIN and indicates whether or not each process is considered in the interface temperature calculation.

At an interface involving the gas, convective $\left(\mathrm{q}_{\mathrm{c}}\right)$ and condensation heat transfer $\left(\mathrm{q}_{\text {cond }}\right)$ are in general taken into account. For gas-structure interfaces the enthalpy conveyed by water aerosol deposition $\left(q_{\text {aer }}\right)$ is also taken into account and included in the interface energy conservation equation for the interface. Although water aerosol deposition and the enthalpy carried by the water aerosols are modeled at interfaces with respect to pools, lower cell layers, or engineered systems, the aerosol sensible heat is not taken into account in the interface temperature calculation. Rather, the deposited water aerosols and the associated enthalpy are simply transferred to the collecting pool. The inner 


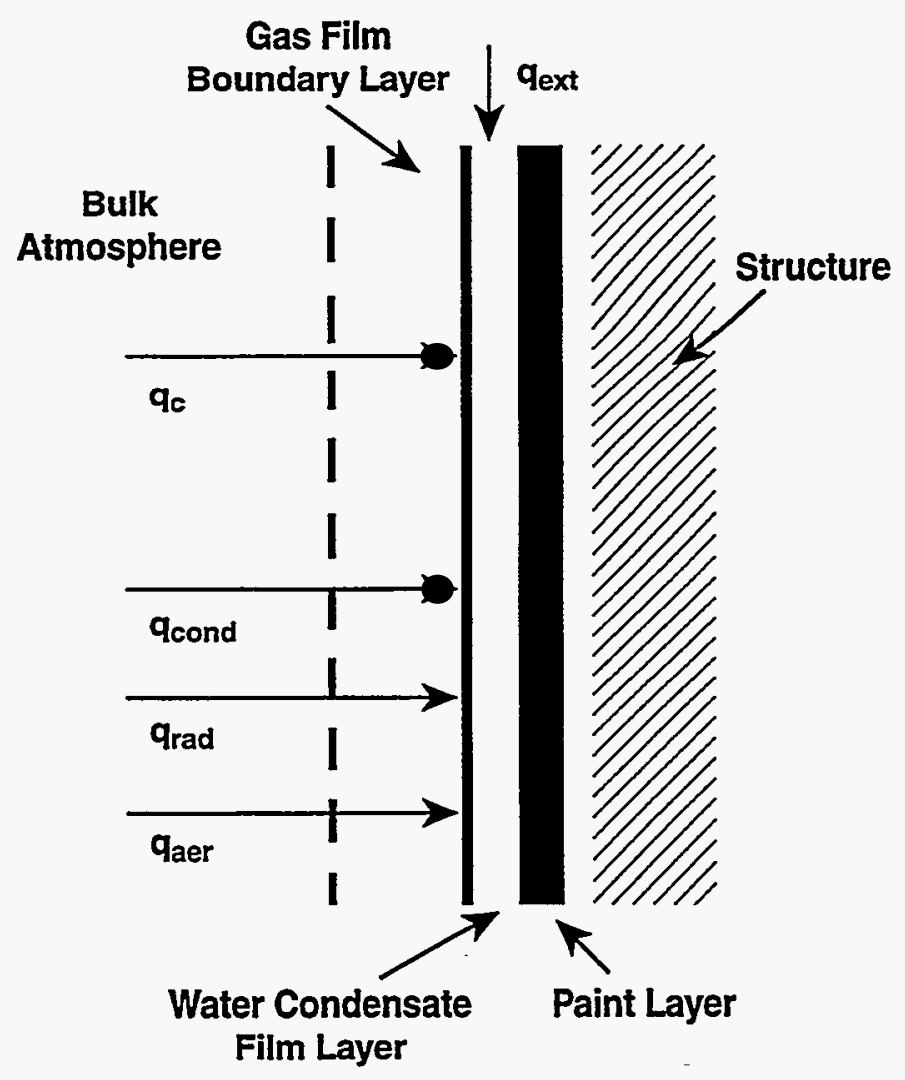

Figure 10-11. Heat Transfer Processes at a Gas Structure Interface 
surface of a heat transfer structure may also be subject to the film inflow of condensate $\left(\mathrm{q}_{\mathrm{ex}}\right)$, which may either originate on other structures or through a source table for the film. (In contrast to the discussion in Section 10.2.2, it is not necessary to separate the film source rate from the inflow rate from other structures; these are lumped together in $q_{\text {ext }}$.) For gas-structure and gas-lower-cell interfaces, thermal radiation $\left(\mathrm{q}_{\mathrm{rad}}\right)$ is modeled and is also considered in the interface energy balance equation. Note that although $\mathrm{q}_{\mathrm{rad}}$ is considered in the interface energy conservation equation, the flux itself is based on wall, or surface node temperatures, $T_{w}$ not $T_{i f}$, as discussed in Section 10.3. This is done to preserve stability when high radiative heat transfer fluxes are present.

All of these fluxes are discussed in the present chapter with the exception of the contribution $q_{\text {aer }}$ from water aerosol deposition. The deposition of suspended water aerosols on various surfaces is the result of a number of different processes. Modeling of these deposition rates for heat transfer structure and lower cell surfaces is discussed in detail in Section 7.2.3; the modeling of deposition rates for engineered systems is discussed in detail in Sections 7.4 through 7.6. For present purposes, the mass flux $\mathrm{j}_{\text {aer }}$ from water aerosol deposition may be viewed as explicitly calculated and resulting in an enthalpy flux to a surface given by

$$
q_{\text {aer }}=j_{\text {aer }} h_{\ell}
$$

where $h_{l}$ is the water enthalpy at the atmosphere temperature.

The interface energy balance equation at a gas-surface interface is derived by forming a control volume around the condensate film, or boundary layer in the case of a pool, and assuming that the film conditions instantaneously achieve steady state. This is equivalent to neglecting the specific heat effects of the film. The energy balance equation has the general form

$$
q_{c}+q_{\text {cond }}+q_{\text {rad }}+q_{\text {aer }}+q_{\text {ext }}-j_{\text {tot }} h_{\ell}\left(T_{i f}\right)=h_{\text {flm }} \Delta T+\Delta q
$$

where $j_{\text {tot }}=j_{\text {cond }}+j_{\text {aer }}+j_{\text {ext }}$ is the condensate mass flux $\left(\mathrm{kg} / \mathrm{m}^{2}-\mathrm{s}\right)$ to the interface from various processes, $h_{l}$ is the liquid coolant enthalpy, and $h_{f m}$ is the surface heat transfer coefficient, including the resistances between the interface and the location at which the surface node temperature $T_{w}$ is defined. For example, for a gas-structure interface, $h_{f l m}=\left[r_{f l m}+r_{p a i n t}+r_{n o d e}\right]$, where $r_{f m}$ is the beginning-of-timestep condensate film resistance, $r_{p a i n}$ is the surface paint resistance, and $r_{n o d e}$ is the surface half-node resistance. For a gas-structure interface, $T_{\mathrm{w}}$ is the surface node temperature, for a gas-pool interface $T_{w}$ is taken to be the bulk pool temperature, and for a gas-ice interface in an ice condenser, $T_{w}$ is taken to be the melt temperature. In the above equation, $\Delta T=T_{b}-T_{w}$, and the $\Delta q$ term, as discussed below, is used to adjust the energy balance with regard to the manner in which various mass fluxes are assumed to equilibrate with the film.

The condition $\Delta q=0$ used for heat transfer structures corresponds to the assumption that any condensate liquid introduced into the film control volume accumulates on the film from the gas side and equilibrates at the temperature $T_{\text {ir }}$. This is correct for the condensation and aerosol fluxes. For simplicity, the external film mass flux $j_{\text {ext }}$ for heat transfer structures is also assumed to equilibrate in the same manner. For consistency, condensate films are also assumed to drain off a surface at the 
interface temperature. Note that condensate film runoff is calculated explicitly after the interface conditions are calculated and therefore is not included in $\mathrm{q}_{\mathrm{ext}}$. As long as the interface temperature is used for the runoff, the energy balance is not affected by the runoff, provided a beginning-oftimestep film thickness is used to calculate the conduction through the film. More details of the film dynamics modeling are given in Section 10.2.2.

The $\Delta \mathrm{q}$ term corrects for the interface-side equilibration assumption in the case of a gas-pool interface or a gas-ice interface in the case of an ice condenser. In the case of gas-pool interface, pool liquid at temperature $T_{w}$ must enter or leave the pool-side boundary layer from the pool side as condensation or evaporation occurs, if one assumes that the boundary layer thickness is not affected by the condensation rate. This results in the contribution

$$
\Delta q=j_{\text {cond }}\left(h_{f}\left(T_{w}\right)-h_{f}\left(T_{i f}\right)\right) \quad \text { (at gas-pool interfaces) }
$$

Note that neither $j_{\text {aer }}$ or $j_{\text {ext }}$ are coupled into the film calculation at a gas-pool interface, as indicated in Table (10-1), as these fluxes are committed directly to the bulk pool.

In the case of an ice-condenser film one must take into account the fact that melt enters the film from the ice side. This results in the contribution

$$
\Delta q=j_{\text {melt }}\left(h_{\ell}\left(T_{i f}\right)-h_{f}\left(T_{w}\right)\right) \quad \text { (at gas-ice interfaces) }
$$

where $\mathrm{j}_{\text {melt }}$ is the self-consistently calculated rate of ice melt.

To solve the energy balance equation for the interface temperature, an iterative procedure is in general required. An iterative procedure is used in CONTAIN for all gas-surface interfaces, except the gas-drop interface for the containment spray model and the gas-coil interface in the fan cooler model. The iterative procedure uses a quadratic expansion of the energy balance equation in $\left(T_{\text {if }}-\right.$ $\mathrm{T}_{\mathrm{if}}^{\prime}$ ), where $\mathrm{T}_{\mathrm{if}}$ is the temperature at the gas-surface interface to be determined in the present iteration and $T_{\mathrm{if}}^{\prime}$ is the interface temperature determined from the previous iteration. Convergence is obtained when the difference between $T_{\text {if }}$ and $T_{\text {if }}^{\prime}$ becomes negligible. An iterative procedure is not required in the treatment of containment sprays because of a well-mixed drop assumption, which defines the drop temperature to be the drop bulk temperature, and in the treatment of the fan cooler because of an assumed linear dependence of the saturation vapor pressure with temperature between the coolant and gas vapor pressures and an assumed constant specific heat. The modeling of containment sprays and fan coolers is discussed in more detail in Sections 12.3 and 12.1, respectively.

In the iterative procedure, the heat and mass transfer coefficients are first evaluated at the interface temperature $T_{i f}^{0}$ last determined for the surface as a first guess. At a given iteration, selected quantities, namely, the saturation pressure and the liquid and vapor enthalpies at the interface, are 
then expanded to first order around the interface temperature $\mathrm{T}^{\prime}$ if from the last iteration. For example, the saturation vapor pressure at the interface $P_{v, \text { if }}$ is approximated as

$$
P_{v, \text { if }}=P_{s}\left(T_{i f}^{\prime}\right)+\frac{d P_{s}\left(T_{i f}^{\prime}\right)}{d T}\left(T_{i f}-T_{i f}^{\prime}\right)
$$

The energy balance equation can then be represented, after some rearrangement, in the form

$$
q^{\prime}-b \Delta T+a(\Delta T)^{2}=h_{f l m} \Delta T
$$

where $\Delta T=T_{i f}-T_{w}$. To give an example of the coefficients, for condensing conditions and $\Delta q=$ 0 , these have the form

$$
\begin{aligned}
& q^{\prime}=h_{c}\left(T_{b}-T_{w}\right)+j^{\prime}\left(h_{v, b}-\tilde{h}_{\ell}\right)+q_{r a d}+q_{a e r}+q_{e x t}-\left(j_{\text {aer }}+j_{e x t}\right) \tilde{h}_{\ell} \\
& b=h_{c}+\left(j^{\prime}+j_{\text {aer }}+j_{e x t}\right) c_{p, \ell}^{\prime}+M_{v} K_{g} \frac{d P_{s}\left(T_{i f f}^{\prime}\right)}{d T}\left(h_{v, b}-\tilde{h}_{\ell}\right) \\
& a=M_{v} K_{g} \frac{d P_{s}\left(T_{i f f}^{\prime}\right)^{\prime}}{d T} c_{p, \ell}
\end{aligned}
$$

In the above, $h_{c}$ is the convective heat transfer coefficient, $h_{v, b}$ is the vapor enthalpy in the bulk gas; $c_{p, l}^{\prime}$ is the liquid specific heat at $T_{i f}^{\prime}$. Also,

$$
\begin{aligned}
& \tilde{\mathrm{h}}_{\ell}=\mathrm{h}_{\ell}\left(\mathrm{T}_{\mathrm{if}}^{\prime}\right)-\mathrm{c}_{\mathrm{p}, \ell}^{\prime}\left(\mathrm{T}_{\mathrm{if}}^{\prime}-\mathrm{T}_{\mathrm{w}}\right) \\
& \mathrm{j}^{\prime}=\mathrm{M}_{\mathrm{v}} \mathrm{K}_{\mathrm{g}}\left[\left(\mathrm{P}_{\mathrm{v}, \mathrm{b}}-\mathrm{P}_{\mathrm{s}}\left(\mathrm{T}_{\mathrm{if}}^{\prime}\right)\right)+\frac{\mathrm{dP}_{\mathrm{s}}\left(\mathrm{T}_{\mathrm{if}}^{\prime}\right)}{\mathrm{dT}}\left(\mathrm{T}_{\mathrm{if}}^{\prime}-\mathrm{T}_{\mathrm{w}}\right)\right]
\end{aligned}
$$

Equation (10-155) is solved for $\mathrm{T}_{\mathrm{if}}$ and the assumed conditions (condensing or evaporating, and in some cases, dry surface or wet) are checked for consistency. If the assumed conditions are not consistent, an alternative is selected and $\mathrm{T}_{\text {if }}$ recalculated until consistent conditions are found. The iterations are continued until consistency and convergence in $T_{\text {if }}$ is found. In the event the surface is wet initially but dries out during the CONTAIN timestep, time-averaged heat transfer and a timeaveraged $T_{\text {if }}$ weighting the wet and dry periods are calculated.

\subsection{Heat Transfer Control Options}

The code allows the user to deactivate the heat transfer across certain interfaces on a cell-by-cell basis through the use of an optional keyword HT-TRAN followed by five user-defined flags (see Section 14.3.1.6). The heat transfer flags correspond, in the order given, to 
- heat transfer between the atmosphere and all structures, including convection, condensation, and radiative heat transfer;

- heat transfer between the first node in the lower cell model and the basemat, or between the atmosphere and basemat if all lower cell layers are empty;

- heat transfer between different layers in the lower cell, excluding the basemat layer;

- heat transfer between the uppermost solid or liquid layer in the lower cell and the atmosphere above it, or between the atmosphere and basemat if no such layer has nonzero mass. Note that the heat transfer from the upper CORCON melt layer during the modeling of core-concrete interactions is not affected by this flag. The heat transfer controlled here otherwise includes convection, condensation, and radiation;

- radiative heat transfer between the uppermost layer in the lower cell and all structures, between the atmosphere and lower cell, and between the atmosphere and all structures.

The user can control these heat transfer processes by setting specified flags in the HT-TRAN option to $\mathrm{ON}$ or OFF. The default for all flags is ON. If a flag is set to OFF, the corresponding heat transfer is set to zero.

This heat transfer control option is useful for simulating adiabatic or insulated conditions. It also provides a means by which the user can check the effectiveness of various heat transfer mechanisms. 


\subsection{BOILING WATER REACTOR MODELS}

The present section discusses miscellaneous processes in which the partitioning of flow between the atmosphere and pool is important. The processes discussed here are commonly part of the design of boiling water reactors (BWRs) but are not restricted to them. The models of such processes are of two types: (1) BWR suppression pool vent models and (2) safety relief valve (SRV) models.

The former are commonly used to model the suppression pool vent system of a BWR. This system allows the drywell to depressurize during or after a pressurization event by allowing gas to flow into a second chamber, the wetwell, after the normally submerged connecting vents have been purged of water through a vent clearing process. The flow through the suppression pool vents results in pressure suppression through gas-pool equilibration and in removal of aerosols, fission products, and dispersed core debris from the gas, through a process called scrubbing. The suppression pool vent models are discussed in Section 11.1.

The SRVs in a BWR are in discharge lines leading from the pressure vessel to spargers located in the suppression pool. In CONTAIN, the SRV model deals with an external source of gas, coolant, aerosols, and/or fission products that is considered to be introduced at a fixed elevation within a cell, which may or may not be below the pool surface. The partitioning of source materials depends on whether the source is submerged or not. If the source is submerged, the treatment is similar to that of gas mixtures vented under the pool surface in the suppression pool vent modeling. If it is not submerged, coolant phase separation is modeled, under the assumption that the source expands isenthalpically until it reaches the local pressure. The coolant liquid remaining after expansion is directed to the pool and the other materials including the coolant vapor are directed to the atmosphere. Note that this treatment is different from that of an external atmosphere source, in which all materials, except possibly core debris materials in certain cases, are directed to the atmosphere. The SRV model is discussed in Section 11.2.

The modeling used for gas-pool equilibration and for aerosol and fission product scrubbing in the BWR models is also used for non-BWR applications. The gas-pool equilibration model for suppression pool vent modeling is based on the approach discussed in Section 4.4.7 and is used for all submerged gas flow paths. The gas-pool equilibration modeling for the SRV model, discussed in Section 11.2.1, is similar to but not identical with that used by the flow path models. The SRV approach is used for all gas external sources to the pool, including those from core-concrete interactions (CCIs) with an overlying pool. CCIs are discussed in more detail in Chapter 5. The detailed SPARC and SCRUB aerosol scrubbing models, discussed in Section 7.7, are available for the dedicated suppression pool vent model and for the SRV model. The SCRUB model is also available for use with aerosol releases from CCIs. Key elements of boiling water reactor models are illustrated in Figure 11-1.

\subsection{Suppression Pool Vent Models}

The suppression pool vent models in CONTAIN are of two types. The first is a multi-node model comprised of the gas and pool flow paths discussed in Section 4.2. This type of model can be used to model the vent clearing and vent gas flow of a BWR in terms of a serial or parallel arrangement 


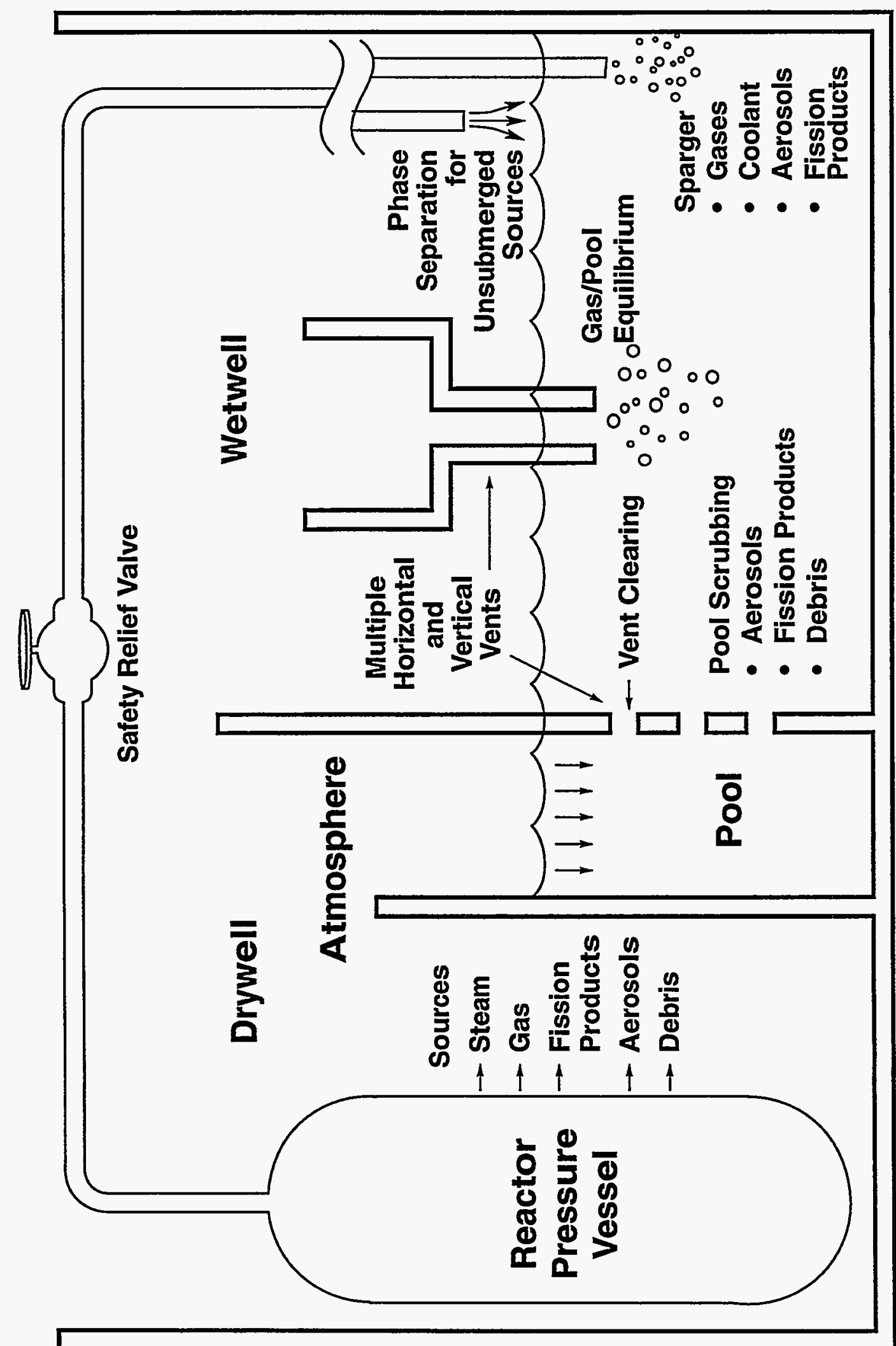

ঠิ

$\stackrel{1}{=}$

Z

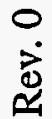


of flow paths. For example, the suppression vent system of a Mark III BWR can be represented in terms of three parallel pool and three parallel gas flow paths, as discussed in Section 11.1.1. below. A serial multi-node model presumably could also be developed for Mark I's and II's using similar techniques, although this has not been done.

As described in Sections 7.8, 8.8 and 6.2, respectively, aerosols, aerosolized fission products, and dispersed core debris are scrubbed from the gas when a gas mixture is vented under the surface of the pool in the downstream cell. Within the multi-node type of modeling the model for scrubbing is simple: these materials are assumed to be completely removed from the flow. The aerosols and associated fission products are placed in the pool, and the core debris is placed in the uppermost intermediate layer of the lower cell, if present. Otherwise, the debris is removed from the problem. Note that the debris is not equilibrated with the pool before it is removed from the flow. Rather, the equilibration occurs between the intermediate layer and the pool. Note that, in contrast, gaseous fission products are not scrubbed or removed by the pool.

The second type of model is the dedicated suppression pool vent flow path model, which models vent clearing and gas flow as occurring within a single special flow path. All three types of BWRs may be represented within the dedicated model. However, the basic assumption of this model is that the vent clearing process is controlled by quasi-steady (non-inertial) flow. Therefore, the parameters in the model may not be easy to determine for a rapid blowdown, except by calibration with a detailed inertial model. The vent clearing and gas flow modeling for the dedicated model, as well as the modeling limitations, is discussed in Section 11.1.2 below. The user should read about these limitations before using this model.

Two detailed aerosol scrubbing models, the SPARC and SCRUB models discussed in Section 7.7, are available for the dedicated model. However, the treatment of the scrubbing of gaseous fission products and dispersed core debris is the same as that in the multi-node model.

For either the multi-node or dedicated model, the gas-pool equilibration that occurs during the venting of gas mixtures below the pool surface is treated as described in Section 4.4.7. The treatment in the former case, however, is more flexible in that the user may specify a gas-pool equilibration length, as with any engineered vent gas flow path, whereas in the latter, the equilibration length is assumed to be zero. Complete equilibration is assumed for submerged venting in this case, regardless of the submergence depth.

\subsubsection{Multi-Node Suppression Pool Vent Flow Model}

The CONTAIN multi-node representation of the suppression pool vent clearing system for a Mark II BWR is shown in Figure 11-2. This shows the lower regions of the drywell annulus and wetwell in which the vent system is located. The vent system in a Mark III has three rows of round horizontal vents, with approximately 45 vents per row. In the multi-node suppression pool vent model, each row is represented by one pool and one gas flow path. The drywell annulus and wetwell are considered to be in separate cells, with separate pools, within this representation. Note that CONTAIN flow paths are interface models and do not have actual inventory associated with them. Thus, if the user wishes to include the inventory in the vents themselves in the problem, the vent 


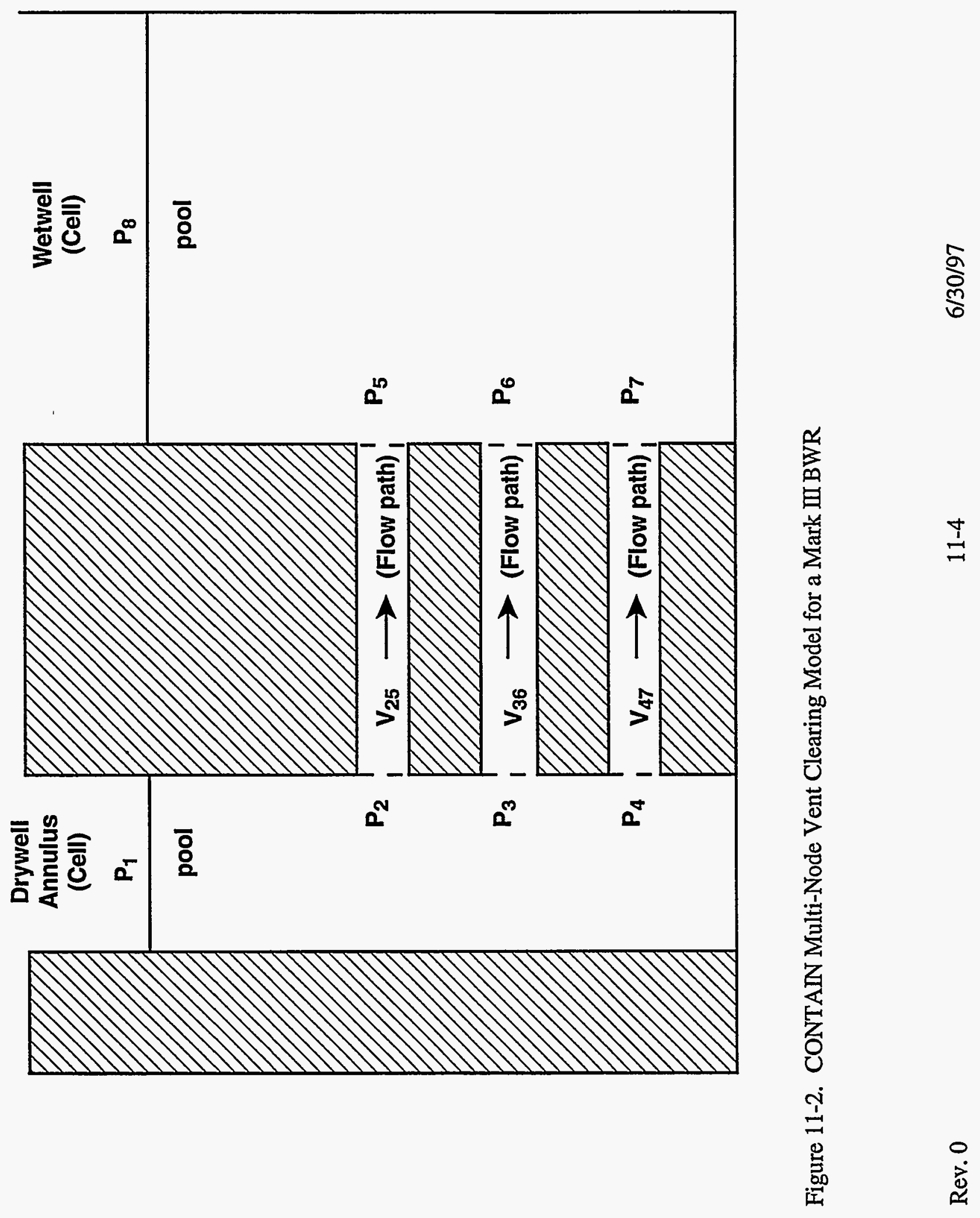


volume must be assigned to a cell. One way to do this is by slightly increasing the wetwell cell cross-sectional area below the nominal pool level to account for the volume of the vents. As expected, the thermal-hydraulic and scrubbing behavior in the multi-node model is determined by the behavior of the individual flow paths. A detailed discussion of the thermal-hydraulic behavior of the individual paths is given in Sections 4.2 and 4.3.

The usefulness of the indicated parallel flow path configuration for the modeling of vent clearing depends on the proper assignment of the inertial lengths to the pool paths. These are not immediately obvious because the annulus has a cross-section comparable to that of all the vents, and thus the inertia of the pool liquid in the annulus must be taken into account. The following discussion gives a prescription for calculating the effective inertial lengths for the pool paths, using the seven-node representation of the vent system in Figure 11-3. The same prescription could also be used for the gas paths, although the inertial lengths for these are typically not critical. Note that in the seven-node representation, the flow of liquid is assumed to be governed by standard hydraulic equations, within the approximation that slug flow is present in each node.

The effective inertial lengths of the three flow paths in Figure 11-2 may be determined by matching the initial liquid acceleration rates in that configuration with those in the configuration of Figure 11-3, for a unit step change in the drywell-wetwell pressure difference. It is assumed that the system is initially at rest, with the same pool levels and gas pressures in the drywell and wetwell. Note that effective inertial lengths based on the initial acceleration rates should result in a slightly conservative vent clearing time, since the mass of liquid in the annulus and thus the effective inertial lengths decrease with time.

The seven-node representation uses six velocities $\left(V_{12}, V_{23}, V_{34}, V_{25}, V_{36}, V_{47}\right)$, six dynamic pressures $\left(\mathrm{P}_{2}^{\prime}, \mathrm{P}_{3}^{\prime}, \mathrm{P}_{4}^{\prime}, \mathrm{P}_{5}^{\prime}, \mathrm{P}_{7}^{\prime}\right)$, and two hydrostatic pressures, $\mathrm{P}_{1}$ and $\mathrm{P}_{8}$. The vertical velocities in the wetwell are relatively small and are therefore neglected. Consequently, the dynamic pressures $\mathrm{P}_{5}^{\prime}, \mathrm{P}_{6}^{\prime}$, and $\mathrm{P}_{7}^{\prime}$ at the downstream end of the vents are related hydrostatically to $P_{8}$. The liquid in each of the six remaining nodes is assumed to move as a coherent slug, and force-balance and conservation equations can be written in the usual manner. For a constant density liquid, these equations are given by

$$
\begin{aligned}
& \Delta P_{i j}^{\prime} A_{i j}=\rho_{\ell} L_{i j}^{\prime} A_{i j} \frac{d V_{i j}}{d t} \\
& A_{12} V_{12}=A_{25} V_{25}+A_{23} V_{23} \\
& A_{23} V_{23}=A_{36} V_{36}+A_{34} V_{34} \\
& A_{34} V_{34}=A_{47} V_{47}
\end{aligned}
$$

where $\Delta \mathrm{P}_{\mathrm{ij}}^{\prime}$ is equal to $\Delta\left(\mathrm{P}_{\mathrm{i}}^{\prime}-\mathrm{P}_{\mathrm{j}}^{\prime}\right)$, the change in dynamic pressure difference between points $\mathrm{i}$ and $\mathrm{j}$, from the initial (hydrostatic) values; $A_{i j}$ is the flow area between points $i$ and $j ; \rho_{\ell}$ is the liquid density; $L_{i j}^{\prime}$ is the inertial length of liquid between points $i$ and $j$; and $d V_{i j} / d t$ is the acceleration of liquid mass from point $i$ to $j$. Note that $P_{1}^{\prime}$ is equal to $P_{1}$ and $P_{8}^{\prime}$ is equal to $P_{8}$. These equations neglect the change in liquid level within a node, as appropriate for determining the initial liquid 


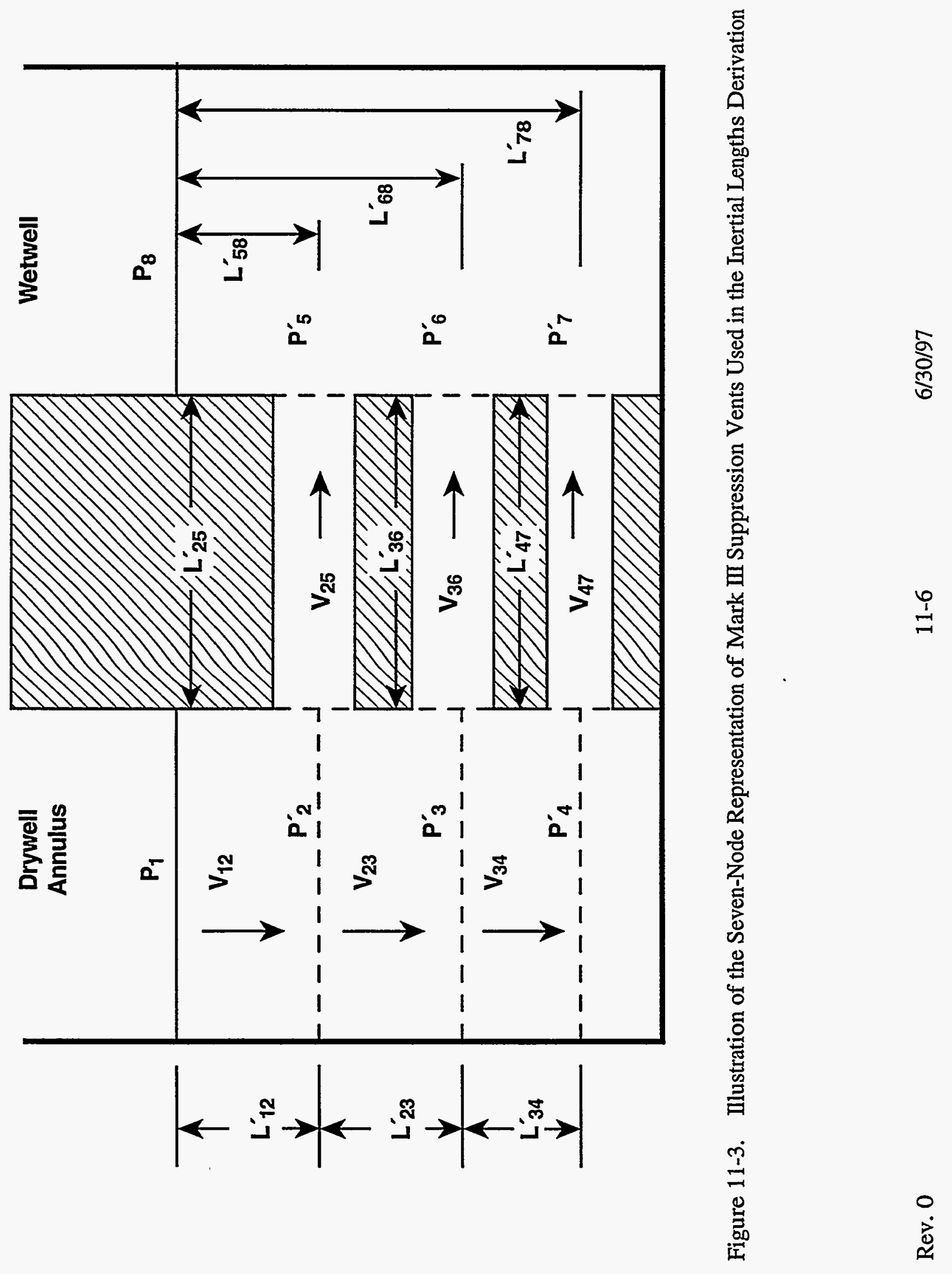


acceleration rates. Note that the time-derivatives of the velocities are the key quantities in the following discussion, and therefore the time-differentiated forms of Equations (11-2) and (11-4) are used in the following discussion.

With the CONTAIN multi-node representation, the corresponding initial acceleration equations have the form

$$
\Delta P_{i j} A_{i j}=\rho_{\ell} L_{i j} A_{i j} \frac{d V_{i j}}{d t}
$$

where $[i, j]$ is either $[2,5],[3,6]$, or $[4,7] ; \Delta P_{i j}$ is equal to $\Delta\left(P_{i}-P_{j}\right)$, the change in hydrostatic pressure difference between points $i$ and $j$, relative to the initial values; and $L_{i j}$ is the effective inertial length for liquid between points $i$ and $j$. The hydrostatic pressures $P_{i}$ are appropriate in the CONTAIN representation because momentum convection is ignored in a cell.

To compute $\mathrm{L}_{\mathrm{ij}}$, a unit step change in hydrostatic pressure, $\Delta\left(\mathrm{P}_{1}-\mathrm{P}_{8}\right)=1$, is imposed on the system and the initial time derivatives of $\mathrm{V}_{25}, \mathrm{~V}_{36}$, and $\mathrm{V}_{47}$ are assumed to be equal in Equations (11-1) and (11-5). It should be obvious that $\mathrm{L}_{\mathrm{ij}}$ is independent of the magnitude of the pressure change.

One can start by solving Equations (11-1) and the time-derivatives of Equations (11-2) through (11-4) for the unit step change in pressure. Since $P_{5}^{\prime}, P_{6}^{\prime}$, and $P_{7}^{\prime}$ are hydrostatically related to $P_{8}$ by a liquid depth that can be taken as fixed with respect to calculating initial accelerations and $P_{8}^{\prime}=P_{8}$, one can set

$$
\Delta\left(\mathrm{P}_{5}^{\prime}-\mathrm{P}_{8}^{\prime}\right)=\Delta\left(\mathrm{P}_{6}^{\prime}-\mathrm{P}_{8}^{\prime}\right)=\Delta\left(\mathrm{P}_{7}^{\prime}-\mathrm{P}_{8}^{\prime}\right)=0
$$

Substituting these into Equation (11-1) leaves six independent pressure differences and three timederivatives, $d V_{i j} / d t$, which must be determined by the nine equations represented by Equations (11-1) through (11-4). Since $L_{\mathrm{ij}}^{\prime}$ is assumed fixed at the initial value, these equations are linear and can be solved by standard methods. After the time derivatives are obtained, they are substituted into Equation (11-5), which is then solved for $\mathrm{I}_{\mathrm{ij}}$ using the hydrostatic pressure relations

$$
\Delta\left(\mathrm{P}_{1}-\mathrm{P}_{8}\right)=\Delta\left(\mathrm{P}_{2}-\mathrm{P}_{5}\right)=\Delta\left(\mathrm{P}_{3}-\mathrm{P}_{6}\right)=\Delta\left(\mathrm{P}_{4}-\mathrm{P}_{7}\right)
$$

Such relations hold for horizontal vents within a constant density liquid. The results of this solution scheme are shown in Table 11-1, for the annulus and vent areas and lengths characteristic of the Grand Gulf plant. 
Table 11-1

Example Solution for Flow Path Initial Lengths in the Three-Node

Representation of Suppression Pool Vents Shown in Figure 11-2

\begin{tabular}{||l|l||}
\hline Top Flow Path & $\mathrm{A}=17.8761$ \\
& $\mathrm{~L}=4.283$ \\
\hline Middle Flow Path & $\mathrm{A}=17.8761$ \\
& $\mathrm{~L}=6.490$ \\
\hline Bottom Flow Path & $\mathrm{A}=17.8761$ \\
& $\mathrm{~L}=8.362$ \\
\hline $\mathrm{A}=$ area $\left(\mathrm{m}^{2}\right), \mathrm{L}=$ inertial length $(\mathrm{m})$, as represented in Equation $(11-5)$, for $\mathrm{L}_{12}^{\prime}=2.10, \mathrm{~L}_{23}^{\prime}=$ \\
$\mathrm{L}_{34}^{\prime}=1.27, \mathrm{~L}_{25}^{\prime}=\mathrm{L}_{36}^{\prime}=\mathrm{L}_{47}^{\prime}=1.52$, and $\mathrm{A}_{12}=\mathrm{A}_{23}=\mathrm{A}_{34}=51.44$ in Equation $(11-1)$
\end{tabular}

\subsubsection{Dedicated Suppression Pool Vent Flow Model}

The vent clearing and gas flow rate modeling for the dedicated model is discussed in this section. The architecture for this model is different from that of the multi-node model discussed above. First of all, only one suppression pool vent flow path may be specified per problem. Secondly, in contrast to what one might expect, coolant is not actually exchanged between pools in different cells during the vent clearing process for this model. The reason is that the dedicated model uses only one pool, that in the wetwell cell, and assumes that this pool spans both the drywell and wetwell sides of the vent. Thus, the wetwell cell geometry and pool inventory should reflect the total pool volume and cross-section. Thirdly, the vent clearing calculation is carried out internally in the model to determine a vent clearing time, after which the vent is assumed to be accessible to gas flow. This vent clearing calculation keeps track of liquid levels that should be present on the drywell and wetwell sides of the vent, on the basis of a quasi-steady liquid flow model. Note, however, that the wetwell pool level reported in the output is not just that of the wetwell side, but is an average value taking into account both sides of the vent. Other limitations of the single pool treatment are that gaspool equilibration in reverse flow is not modeled and flooding of the vent from the drywell cell pool, if any, cannot be modeled properly.

The dedicated suppression pool vent flow path is activated at the global level by the keyword SPVENT as described in Section 14.2.4.3 and can be used only in conjunction with the implicit flow solver option. (The implicit flow solver option is specified by the keyword IMPLICIT in the FLOWS input block, as described in Section 14.2.4.1.) With a vent flow path, two cells are required, one for the wetwell and one for the drywell. A pool must be specified as part of the lower cell input of the wetwell cell, and this pool should include both the drywell- and wetwell-side inventory. The area of the pool specified in the lower cell input for the wetwell cell should include all of the free surface associated with these inventories. Note that the inventory of aerosols, fission products, and dispersed core debris that have been scrubbed out of the gas passing through the pool is always associated with the wetwell pool or wetwell lower cell inventory. 
Although the number of individual vents present may be specified by the user in the dedicated model, the vents are assumed to behave effectively as a single path. There is no provision for modeling the clearing of vent rows at different elevations. However, the fact that the effective area for gas flow may increase with pressure, as different rows are cleared, may be simulated through user-specifiable pressure-range parameters, corresponding to the difference between the pressure required to initiate gas flow quasi-statically and the pressure required to keep the vents fully cleared.

11.1.2.1 Vent Clearing Time. The suppression pool vent clearing time is based on the time required for the liquid level on either side of the suppression pool to drop to the level at which the vent begins to clear. The equation for the liquid level velocity is based on the Bernoulli mechanical energy balance equation for quasi-steady flow. (Figure 11-4 displays the liquid level velocities for typical BWR containments.)

The Bernoulli equation is

$$
P_{w}-P_{d}+\rho_{\ell} g\left(H_{w}-H_{d}\right)+\frac{\rho_{l}\left(v_{w}^{2}-v_{d}^{2}\right)}{2}+\Delta P_{L}=0
$$

where $P_{w}$ is the wetwell pressure; $P_{d}$ is the drywell pressure; $\rho_{l}$ is the pool density; $g$ is the acceleration of gravity $\left(9.8 \mathrm{~m} / \mathrm{s}^{2}\right) ; \mathrm{H}_{\mathrm{w}}$ is the wetwell liquid level; $\mathrm{H}_{\mathrm{d}}$ is the drywell liquid level; $\mathrm{v}_{\mathrm{w}}$ is the wetwell pool rise velocity; $v_{d}$ is the drywell-side fall velocity; and $\Delta P_{L}$ is the irreversible loss in total pressure.

Also, the amount of liquid removed from the drywell side of the vent equals the amount of liquid added to the wetwell side. Thus, $\beta v_{d}=v_{w}$, where $\beta$ is the ratio of the drywell vent area to the wetwell pool area. Substituting this definition of $\mathrm{v}_{\mathrm{w}}$ into Equation (11-8) produces

$$
P_{w}-P_{d}+\rho_{\ell} g\left(H_{w}-H_{d}\right)+\rho_{\ell}\left(\beta^{2}-1\right) \frac{v_{d}^{2}}{2}+\Delta P_{L}=0
$$

For flow from the drywell to wetwell, $\Delta \mathrm{P}_{\mathrm{L}}$ in Equation (11-9) can be rewritten [Bir60] as follows,

$$
\Delta \mathrm{P}_{\mathrm{L}}=\frac{1}{2} \rho_{\ell} \mathrm{K} \mathrm{v}_{\mathrm{w}}^{2}+\Delta \mathrm{P}_{\mathrm{L}}^{\prime}
$$

where $\mathrm{K}$ is the sudden expansion loss factor in the turbulent flow regime and $\Delta P_{\mathrm{L}}^{\prime}$ represents the additional loss terms. For sudden expansion, $K=(1 / \beta-1)^{2}$. Therefore, by substitution, Equation (11-11) becomes

$$
\Delta \mathrm{P}_{\mathrm{L}}=\frac{1}{2} \rho_{\ell}(1-\beta)^{2} \mathrm{v}_{\mathrm{d}}^{2}+\Delta \mathrm{P}_{\mathrm{L}}^{\prime}
$$

when expressed in terms of $v_{d}$, and Equation (11-9) becomes

$$
\Delta \mathrm{P}_{\mathrm{dw}}+\mathrm{g} \rho_{\ell} \Delta \mathrm{H}_{\mathrm{dw}}+\rho_{\ell} \frac{\mathrm{v}_{\mathrm{d}}^{2}}{2}\left[\beta^{2}-1+(1-\beta)^{2}\right]+\Delta \mathrm{P}_{\mathrm{L}}^{\prime}=0
$$

where $\Delta \mathrm{H}_{\mathrm{dw}}$ equals $\mathrm{H}_{\mathrm{w}}-\mathrm{H}_{\mathrm{d}}$, and $\Delta \mathrm{P}_{\mathrm{dw}}$ equals $\mathrm{P}_{\mathrm{w}}-\mathrm{P}_{\mathrm{d}}$. 


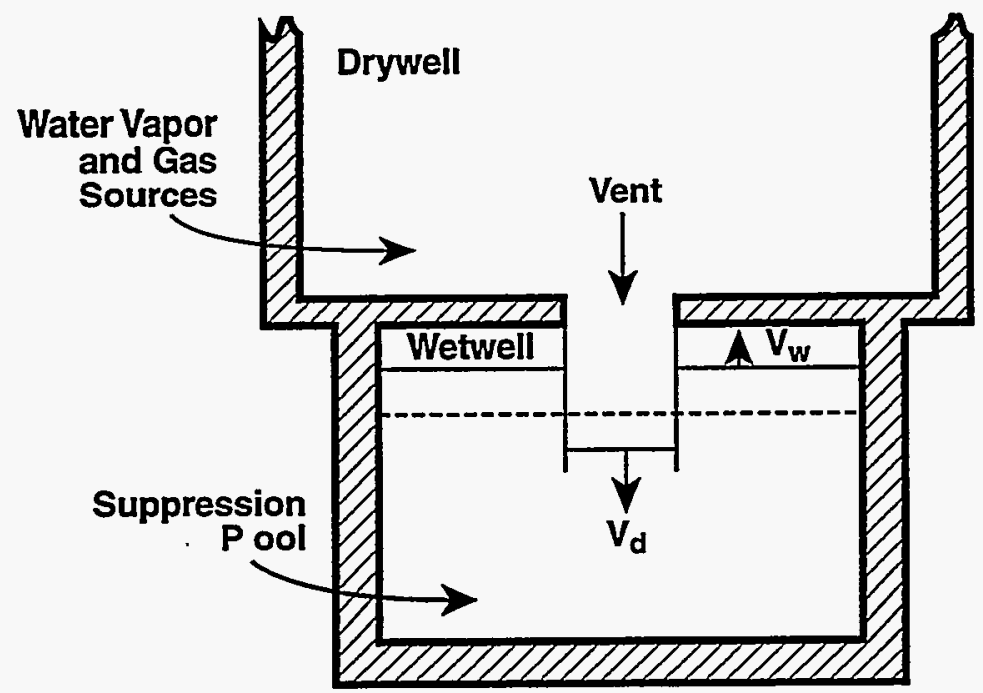

Vertical Vent System (not to scale)

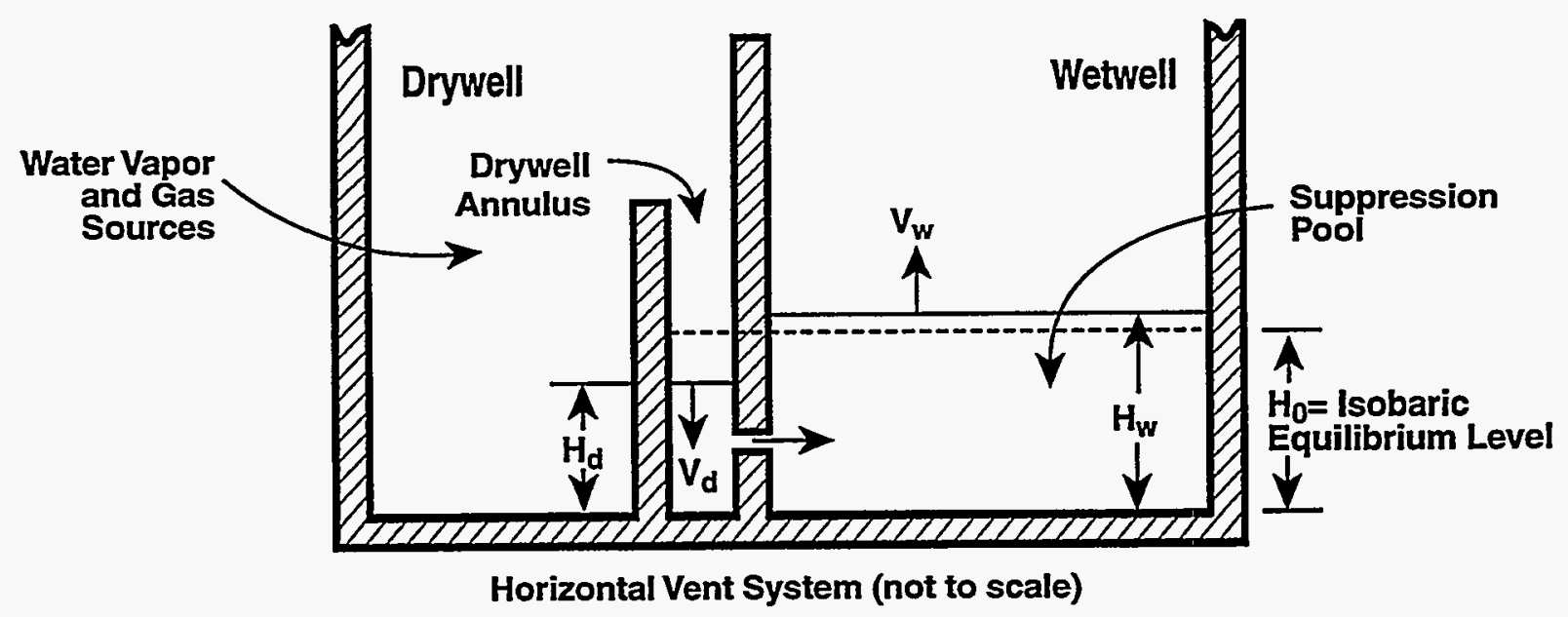

Figure 11-4. Suppression Pool Thermal-Hydraulics. Note that horizontal vents at only one elevation can be modeled in the dedicated model. Thus, horizontal vents at different elevations can only be approximated. In the figures above, the dashed lines represent the normal water levels and the solid lines represent the displaced levels. 
For additional loss terms expressed as $\Delta \mathrm{P}_{\mathrm{L}}^{\prime}=\frac{1}{2} \rho_{l} \mathrm{~K}_{\mathrm{dw}} \mathrm{v}_{\mathrm{d}}^{2}$, Equation (11-12) becomes

$$
\Delta \mathrm{P}_{\mathrm{dw}}+\mathrm{g} \rho_{\ell} \Delta \mathrm{H}_{\mathrm{dw}}+\rho_{l} \frac{\mathrm{v}_{\mathrm{d}}^{2}}{2}\left[\beta^{2}-1+(1-\beta)^{2}+\mathrm{K}_{\mathrm{dw}}\right]=0
$$

where $\mathrm{K}_{\mathrm{dw}}$ is the user-defined additive irreversible loss coefficient " $\mathrm{fdw}$ " (specified in the SPVENT input block) for flow going from the drywell to wetwell.

If the flow goes from the wetwell to drywell, then by an analogous derivation Equation (11-9) becomes

$$
-\Delta P_{d w}-g \rho_{\ell} \Delta H_{d w}+\left(1-\beta^{2}+K_{w d}\right) \rho_{\ell} \frac{v_{d}^{2}}{2}=0
$$

where $\mathrm{K}_{\mathrm{wd}}$ is the user-defined irreversible loss coefficient "fwd" for flow going from the wetwell to drywell. Note in this case, all losses are represented by $\mathrm{K}_{\mathrm{wd}}$.

The vent clearing time depends on the cell pressures and the initial liquid level in the drywell side of the suppression pool. It is also influenced by the overflow from the suppression pool, as can happen over the weir wall when the wetwell pressure is higher than the drywell pressure (see Figure 11-4). To integrate the flow in a single pass, a very complicated logic has been built into the code as discussed in the following paragraphs.

Equation (11-13) and Equation (11-14) are used to determine the levels of the suppression pool on the drywell and wetwell sides over the system timestep $\Delta t_{s}$. A displacement $\mathrm{x}$ may be used to keep track of the inventory on the drywell side:

$$
\mathrm{x}=\hat{\mathrm{H}}_{\mathrm{d}}-\mathrm{H}_{0}
$$

where the isobaric equilibrium level $\mathrm{H}_{0}$ is a constant over the timestep, and $\hat{H}_{d}$ is the drywell level that would be attained if overflow is neglected. $\mathrm{x}$ can also be expressed in terms of the wetwell height $\mathrm{H}_{w}$ as

$$
\mathrm{x}=\left(\mathrm{H}_{0}-\mathrm{H}_{\mathrm{w}}\right) / \beta
$$

By definition

$$
\frac{d x}{d t}=-v_{d}
$$

a relation that holds even when overflow is occurring.

Consider first the case of flow from drywell to wetwell. Overflow cannot occur in this direction. Therefore, by substituting Equations (11-15), (11-16), and (11-17) into Equation (11-13) 


$$
\frac{d x}{d t}=-\left(\frac{2\left[P_{d}-P_{w}+g \rho_{\ell}(1+\beta) x\right]}{F \rho_{l}}\right)^{1 / 2}
$$

where $F$ is equal to $\beta^{2}-1+(1-\beta)^{2}+\mathrm{K}_{\mathrm{dw}}$.

By defining

$$
A=\frac{2\left(P_{d}-P_{w}\right)}{F \rho_{\ell}} \text { and } B=\frac{2 g(1+\beta)}{F}
$$

the integration of Equation (11-18) is carried out in the form

$$
\int_{x_{0}}^{x_{e q}}(A+B x)^{-1 / 2} d x=-\int_{t_{0}}^{t_{0}+t_{e q}} d t
$$

where $x_{0}$ is the initial value of $x$ at the beginning of the timestep, $t_{0}$ is the time at the beginning of the timestep, $x_{e q}$ is the (non-isobaric) equilibrium position, and $t_{e q}$ is the time to attain such an equilibrium position from the initial position. Note that equilibrium may not be attainable in the present timestep.

Therefore, the time to attain equilibrium $t_{e q}$ from a non-equilibrium position $x_{0}$ is

$$
t_{e q}=\left[2 F \frac{\left[P_{d}-P_{w}+g \rho_{\ell}(1+\beta) x_{0}\right]}{\rho_{\ell} g^{2}(1+\beta)^{2}}\right]^{1 / 2}-\left[2 F \frac{\left[P_{d}-P_{w}+g \rho_{\ell}(1+\beta) x_{e q}\right.}{\rho_{\ell} g^{2}(1+\beta)^{2}}\right]^{p / 2}
$$

At equilibrium $\rho_{\ell} g H_{d}+P_{d}=\rho_{\ell} g H_{w}+P_{w}$ and therefore

$$
x_{e q}=\frac{P_{w}-P_{d}}{(1+\beta) p_{q} g}
$$

By applying Equation (11-21) to the second term of Equation (11-20), the time to attain equilibrium $t_{\text {eq }}$ becomes

$$
t_{e q}=\left(\frac{2 F\left[P_{d}-P_{w}+g \rho_{\ell}(1+\beta) x_{0}\right]}{\rho_{\ell} g^{2}(1+\beta)^{2}}\right)^{1 / 2}
$$


Figure 11-5 displays various quantities to help in understanding the logic behind the vent clearing calculation. At the beginning of the system timestep $\Delta \mathrm{t}_{\mathrm{s}}$, the code calculates the effect of pool sources added to the suppression pool. Readjustment of $\mathrm{H}_{d}$ and $\mathrm{H}_{\mathrm{w}}$ is done, if possible, so that $\mathrm{H}_{\mathrm{d}}$ is bounded within $\mathrm{H}_{v}$ and $\mathrm{H}_{\mathrm{of}}$, and so that $\mathrm{H}_{\mathrm{w}}$ lies above $\mathrm{H}_{v}$; where $\mathrm{H}_{v}$ is defined as the vent clearing height and $\mathrm{H}_{\mathrm{of}}$ is defined as the overflow height. If the isobaric equilibrium height $\mathrm{H}_{0}$ is less than $\mathrm{H}_{v}$, then a bypass situation is assumed; the vent clearing calculation is bypassed; and the information is passed to the flow model with a flag indicating the bypass state. (Such a bypass state is not necessarily a likely one for typical BWR geometries and inventories.)

The vent clearing time $t_{v c}$ for flow from drywell to wetwell is determined from Equation (11-22). The time $t_{e q}$ is the total time it takes to reach equilibrium from the beginning-of-timestep water levels. If this time is greater than $\Delta t_{s}$, that means equilibrium is not reached at the end of the timestep. However, if $\mathrm{H}_{\mathrm{v}}>\mathrm{x}_{\mathrm{eq}}+\mathrm{H}_{0}$, the vent may clear before the end of the timestep. If neither happens, and the flow is from drywell to wetwell, an equation similar to Equation (11-19) is integrated over $\Delta \mathrm{t}_{\mathrm{s}}$ to determine the displacement $\mathrm{x}$ at the end of the timestep. This $\mathrm{x}$ is used as the $\mathrm{x}_{0}$ for the next timestep.

To compute the vent clearing time $t_{v c}$, refer to Figure 11-6a. If $\hat{H}_{d}$ is above $\mathrm{H}_{v}$, and $\mathrm{H}_{0}>\mathrm{H}_{v}$, then

$$
t_{v c}=t_{e q}-t_{o p}
$$

where $t_{o p}$ is the time to achieve equilibrium starting at the vent opening height, and calculated by Equation (11-22), but with $\mathrm{x}_{0}$ equal to $\mathrm{H}_{\mathrm{v}}-\mathrm{H}_{0}$. Note that $\mathrm{t}_{\mathrm{op}}$ is really fictitious because liquid flow between the drywell and wetwell stops after the drywell level drops below $\mathrm{H}_{\mathrm{v}}$.

The fraction $F_{v o}$ of time during the system timestep $\Delta t_{s}$ that the vents are cleared is given by

$$
F_{v 0}=\frac{\min \left(\max \left(t_{0}+\Delta t_{s}-t_{v c}, 0\right), 1\right)}{\Delta t_{s}}
$$

where $t_{0}$ is the time at the beginning of the timestep.

The vent clearing time when the flow is from wetwell to drywell is determined from two sets of equations. The first set describes the vent flow up to the point of overflow and the second set describes the vent flow while overflow is occurring. The effect of overflow is, of course, to remove the gravitational head of the column of liquid on the drywell side above the overflow height. The calculations $t_{\text {eq }}, t_{\text {op }}$, and $t_{v c}$ for the case of a decreasing wetwell level are similar to those used for a decreasing drywell level. The liquid that overflows is placed in the drywell sump if modeled; otherwise it is lost from the problem. 


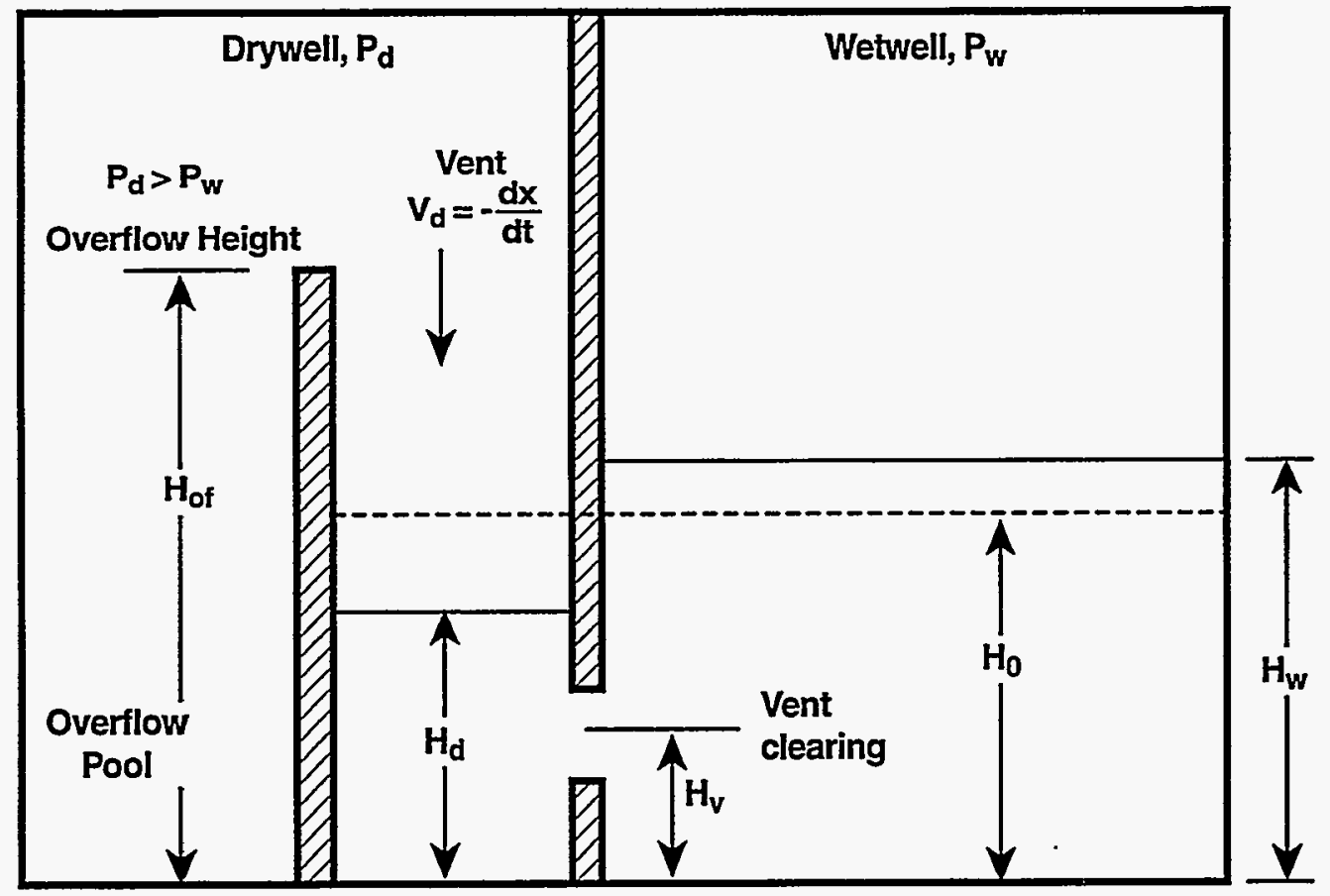

--.-- Isobaric Equilibrium Level $\mathrm{H}_{0}$ Level if Overflow is Neglected

Figure 11-5. Definition of the Variables for the BWR Horizontal Vent Suppression Pool System. 


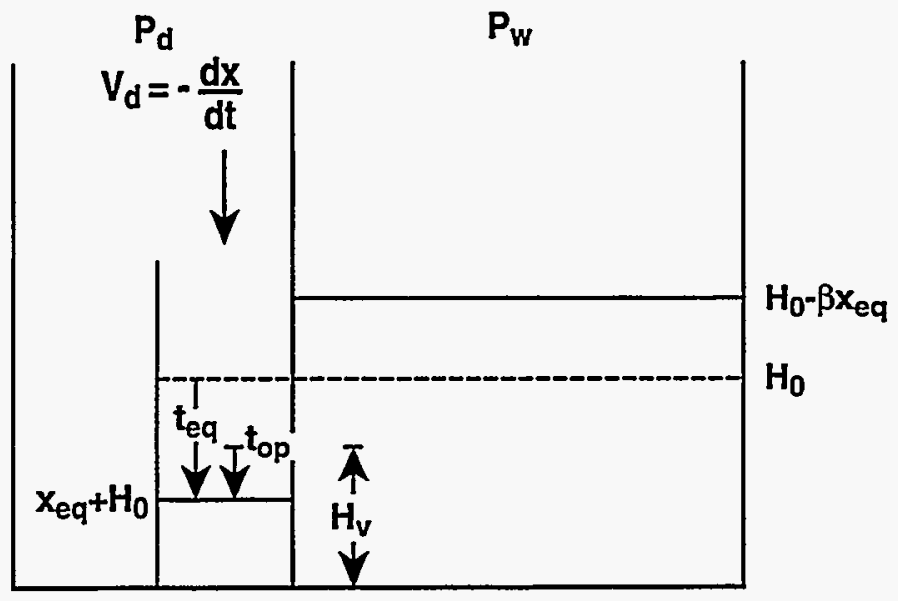

a) Drywell

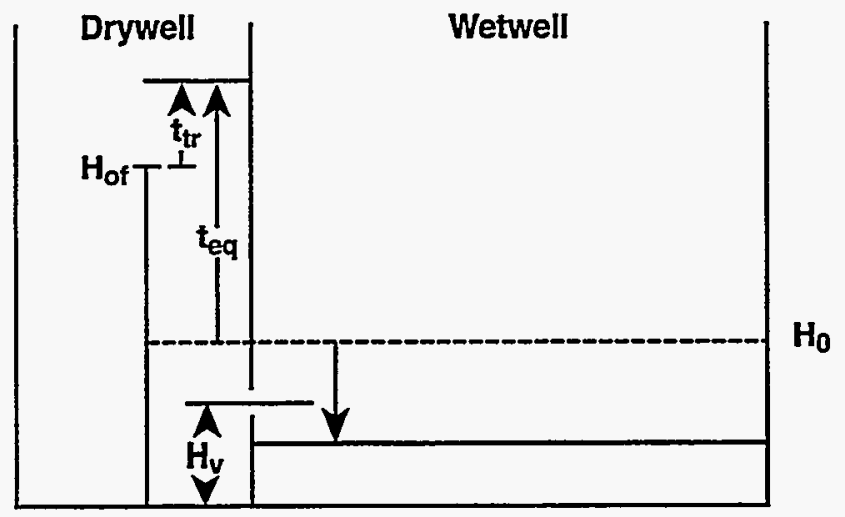

(b) Wetwell

Figure 11-6. Computation of Level Depression. 
For flow from wetwell to drywell, and assuming overflow does not occur, an equation similar to Equation (11-22) is obtained from Equations (11-14), (11-15), and (11-16),

$$
t_{e q}=\left(\frac{2 F^{\prime}\left[P_{w}-P_{d}-\rho_{\ell} g(1+\beta) x_{0}\right]}{\rho_{\ell} g^{2}(1+\beta)^{2}}\right)^{1 / 2}
$$

where $F^{\prime}$ is equal to $1-\beta^{2}+K_{d w}, x_{0}$ equals $\left(H_{0}-H_{w, 0}\right) / \beta$, and $H_{w, 0}$ is the initial value of $H_{w}$ at the beginning of the timestep.

If vent clearing occurs prior to overflow, the vent clearing time $t_{\mathrm{vc}}$ is calculated from Equation (11-24) in a manner similar to that for flow from the drywell to the wetwell. If not, it is first necessary to calculate a time to overflow $t_{\text {of }}$. As illustrated in Figure 11-6b, the time to overflow $t_{\text {of }}$ is computed as $t_{o f}=t_{e q}-t_{t r}$, where $t_{t r}$ is the (fictitious) time from overflow to equilibrium when the gravitational head of the overflow is assumed to be present. The latter is calculated by Equation (11-24) with $\mathrm{x}_{0}=\mathrm{H}_{\text {of }}-\mathrm{H}_{0}$.

The second equation set takes into account the fact that the gravitational head of the water above the overflow point is actually not present. Since $\mathrm{H}_{\mathrm{d}}=\mathrm{H}_{\mathrm{of}}$ during overflow,

$$
\mathrm{g} \rho_{\ell} \Delta H_{d w}=g \rho_{\ell}\left(H_{o f}-H_{w}\right) \quad \text { for } \hat{H}_{d}>H_{o f}
$$

By using Equation (11-16), Equation (11-25) becomes

$$
\text { g } \rho_{\ell} \Delta H_{d w}=g \rho_{\ell}\left(H_{o f}-H_{0}+\beta x\right)
$$

Note that $\mathrm{x}$ is used here as an effective level representing the total inventory on the drywell side.

By substituting Equations (11-17) and (11-26) into Equation (11-14), dx/dt becomes

$$
\frac{d x}{d t}=-\left(\frac{2\left[P_{w}-P_{d}-g \rho_{\ell}\left(H_{o f}-H_{0}+\beta x\right)\right]}{F^{\prime} \rho_{\ell}}\right)^{1 / 2}
$$

The integration of Equation (11-27) yields

$$
\bar{t}_{e q}=\left(\frac{2 F^{\prime}\left[P_{w}-P_{d}-g \rho_{l}\left(H_{o f}-H_{0}+\beta x_{0}\right)\right]}{g^{2} \beta^{2} \rho_{\ell}}\right)^{1 / 2}
$$


where $\vec{t}_{e q}$ is the time to equilibrium assuming the level $H_{d}$ is fixed at $\mathrm{H}_{\text {of }}$.

The time to equilibrium after the time overflow first occurs may be defined as

$$
\overline{\mathrm{t}}_{\mathrm{of}, \mathrm{eq}}=\left(\frac{2 \mathrm{~F}^{\prime}\left[\mathrm{P}_{\mathrm{w}}-\mathrm{P}_{\mathrm{d}}-\mathrm{g} \rho_{\ell}\left(\mathrm{H}_{\mathrm{of}}-\mathrm{H}_{0}\right)(1+\beta)\right]}{\mathrm{g}^{2} \beta^{2} \rho_{\ell}}\right)^{1 / 2}
$$

which is obtained from Equation (11-28) by substituting $\mathrm{x}_{0}=\mathrm{H}_{\mathrm{of}}-\mathrm{H}_{0}$.

There are two cases to consider when the vent clears after overflow:

1. If $\mathrm{H}_{\mathrm{d}, 0}<\mathrm{H}_{\mathrm{of}}$, where $\mathrm{H}_{\mathrm{d}, 0}$ is the initial value of $\mathrm{H}_{\mathrm{d}}$, then the vent clearing time is

$$
t_{v c}=t_{o f}+\bar{t}_{o f, e q}-\bar{t}_{o p}
$$

where $\bar{t}_{\text {op }}$ is the time from vent opening to equilibrium, given by Equation (11-28) with $x_{0}$ equal to $\left(\mathrm{H}_{0}-\mathrm{H}_{v}\right) / \beta$.

2. If $\mathrm{H}_{\mathrm{d}, 0}=\mathrm{H}_{\mathrm{of}}$, the vent clearing time is calculated as

$$
\mathrm{t}_{\mathrm{vc}}=\overline{\mathrm{t}}_{\mathrm{of}, \mathrm{eq}}-\overline{\mathrm{t}}_{\mathrm{op}}
$$

After $t_{v c}$ is obtained by one of the above methods, Equation (11-23) is used to calculate the fraction of time $F_{v o}$ that the vents are cleared for the present timestep.

11.1.2.2 Gas Flow Model. The previous section discusses the vent clearing process, for the case of submerged vents within the dedicated suppression pool vent flow model. Once the vents have cleared, the gas flow in the dedicated model is calculated according to Equations (4-2) and (4-4), within a quasi-steady flow approximation. In the latter equation, $\xi_{\mathrm{ij}}$ is taken to be $F_{v o}$, which is defined in Equation (11-23) and is the fraction of time during the timestep that the vents have cleared. The $i$ in these equations is defined as the number of the drywell cell, $j$ is defined as the number of the wetwell cell, $C_{\mathrm{FC}}$ is taken to be unity, $\Delta \mathrm{P}_{\mathrm{ij}}$ is defined as in Table 4-2 for a gas flow path, except that gravitational head corrections are not taken into account, and the area $A_{i j}=A_{\text {effo }}$, where $A_{\text {eff }}$ is discussed in the following paragraph. Note that according to Equation (4-4), for submerged vents in the dedicated model, critical flow limitations are not taken into account.

$A_{\text {eff }}$ is calculated as a user-defined function of pressure differences:

$$
\begin{array}{lll}
A_{\text {eff }}=0 & \text { for } \Delta P \leq \Delta P_{\text {min }} \\
A_{\text {eff }}=\left(\Delta P-\Delta P_{\text {min }}\right) A_{v} /\left(\Delta P_{\text {max }}-\Delta P_{\text {min }}\right) & \text { for } \Delta P_{\text {max }}>P>\Delta P_{\text {min }}
\end{array}
$$




$$
A_{\text {eff }}=A_{v} \quad \text { for } \Delta P>\Delta P_{\max }
$$

where $A_{v}$ is the total vent area, $\Delta P$ is equal to $P_{d}-P_{w}$ for flow from drywell to wetwell and is equal to $P_{w}-P_{d}$ for flow from wetwell to drywell, $\Delta P_{\min }$ is the minimum pressure difference to support the liquid head at opening, based on the current pool inventory, and $\Delta \mathrm{P}_{\max }$ is the pressure difference at maximum area, defined as:

$$
\begin{aligned}
& \Delta \mathrm{P}_{\max }=\Delta \mathrm{P}_{\min }+\Delta \mathrm{P}_{\mathrm{d}} \text { (for flow from drywell to wetwell) } \\
& \Delta \mathrm{P}_{\max }=\Delta \mathrm{P}_{\min }+\Delta \mathrm{P}_{\mathrm{w}} \text { (for flow from wetwell to drywell). }
\end{aligned}
$$

In the expressions, $\Delta P_{d}$ is the user-specified pressure range over which the effective vent gas flow area goes from zero to its maximum value for flow from the drywell to wetwell ("dpdry"), and $\Delta P_{w}$ is the range for the pressure difference analogous to $\Delta \mathrm{P}_{\mathrm{d}}$ but referring to flow from the wetwell to the drywell ("dpwet").

In the submerged case, the flow $\mathrm{W}_{\mathrm{ij}}$ is defined as the gas flow entering the vent and the downstream pool. Gas-pool equilibration processes will in general alter the composition of the flow entering the atmosphere of the downstream cell. Such processes are modeled for flow from drywell to wetwell but not flow from the wetwell to drywell, for reasons discussed below. The equilibration modeling in the former case is described in Section 4.4.7, with the equilibration length $\lambda_{i j}=0$.

Gas-pool equilibration is ignored in submerged flow from wetwell to drywell because of the limitations of the single-pool approximation used with the dedicated model. The problem is that the pool conditions on the drywell side of the vents could be different from those in the main pool but are not calculated within the single-pool approximation. Reverse flow should occur only under two circumstances: during wetwell pressurization events, in which case the wetwell loads are the principal concern, or during suction conditions in the drywell resulting from failure of the drywellwetwell vacuum breakers after a blowdown. Under the former conditions, assuming that main pool conditions exist on the drywell side of the vents could be nonconservative. Thus, it is probably better to ignore the equilibration. Under the latter conditions the drywell loads presumably have mitigated substantially, and in addition the wetwell atmosphere and pool may be reasonably well equilibrated as a result of the equilibration that previously occurred in forward flow. Thus, the modeling of equilibration effects in flow from wetwell to drywell may not be important. If gas-pool equilibration in such flow could be important, a two-pool segmented suppression pool vent model such as that discussed in Section 11.1.1 should be used to investigate the sensitivity to equilibration effects.

The flow equation in Table 4-2 is used to calculate the suppression pool vent gas flow $\mathrm{W}_{\mathrm{ij}}$ for vents in the bypass (uncovered) state. $\mathrm{C}_{\mathrm{FC}}$ in this case is taken to be unity. $\mathrm{A}_{\mathrm{ij}}$ and $\mathrm{L}_{\mathrm{ij}}$ are interpreted as the total vent area and vent inertial length, respectively, in the SPVENT input block. Finally, the pressure difference $\Delta \mathrm{P}_{\mathrm{ij}}$ used is that between the drywell and the wetwell, but gas gravitational head corrections are not taken into account. 


\subsection{Safety Relief Valve (SRV) Model}

The SRV model deals with external sources of gases, coolant, aerosols, and fission products in aerosol form that are introduced into a cell at a fixed elevation and require partitioning between the atmosphere and pool. The nature of the partitioning depends on whether the source elevation is located above or below the pool surface. If the source is submerged, gas-pool equilibration and aerosol scrubbing are modeled. In the gas-pool equilibration modeling, any coolant liquid is removed, complete temperature equilibration, and nearly (95\%) complete vapor pressure equilibration is assumed. The scrubbing of aerosols and fission products is modeled according to either the SPARC or the SCRUB model discussed in Section 7.7. If the source is not submerged, coolant phase separation is modeled under the assumption that the source volume expands isenthalpically until the source materials reach the local pressure. The coolant liquid remaining after expansion is directed to the pool, and the coolant vapor, gases, aerosols, and fission products are directed to the atmosphere. Although there is no consideration in the SRV model of the fact that some entrainment of the liquid could occur, the user has the option to calculate the entrainment of the liquid separately and then allocate the entrained liquid fraction to an atmosphere source. In contrast to SRV sources, the materials specified in an atmosphere source, with the possible exception of $\mathrm{DCH}$ materials, are directed to the atmosphere without partitioning.

The gas-pool equilibration modeling for submerged SRV sources is discussed in Section 11.2.1. The coolant phase separation modeling for an SRV source that is not submerged is discussed in Section 11.2.2.

\subsubsection{Gas-Pool Equilibration for Submerged SRV Sources}

This section discusses the gas-pool equilibration model for submerged external sources. These are assumed to equilibrate in temperature with the pool. In addition if the BOIL option has been specified for the pool, these sources are assumed to be nearly (95\%) equilibrated with respect to the pool vapor pressure. However, if the BOIL option has not been specified, coolant vapor and any homogeneously dispersed coolant liquid in the source will be completely retained in the pool.

The SRV model is activated through the SRVSOR keyword described in Section 14.3.4. Source tables are used in this model to describe the rates of introduction of the various materials. The SRV discharge is assumed to be at the elevation above pool bottom specified by the ELESRV keyword in the SRVSOR input block. Although the model is intended primarily for modeling SRVs, it could be used in any situation in which the source partitioning between atmosphere and pool is appropriate. A unique feature of SRV aerosol sources is that the lognormal size distribution parameters for the aerosol source can be specified in the source table as a function of time. If given, these values are used for the aerosol source instead of the global size distribution parameters defined for the aerosol component in the global AEROSOL block (Section 14.2.5).

The user should take care to ensure that the gas, aerosol, and fission product source table input as described in Section 14.3.4 are consistent. For example, a finite aerosol source rate would not make sense unless there is a supporting gas source rate to convey the aerosols into the pool. A finite

fission product source rate also would not make sense unless the supporting aerosol source rate for 
the component hosting the fission product is nonzero. In fact, the aerosol host source rate should exceed the fission product source rate.

However, if the supporting gas source rate for aerosols is zero and the discharge elevation is submerged below the pool surface, the aerosols will be scrubbed with the maximum decontamination factor of $10^{5}$. If the rate is zero and the discharge level is above the surface, the aerosols will be introduced directly into the atmosphere without scrubbing. The user should note that the maximum decontamination factor will also result if the gas flow consists entirely of coolant vapor, as this flow will condense completely in the pool, even for saturated pools. If a fission product is hosted by a particular aerosol component and the source mass rate for that component is zero during a given period, the fission product source mass introduced during this period will be placed in the pool if the discharge is submerged, or directly on the airborne aerosol component if the discharge is not submerged.

To account correctly for the mass and energy transferred to the pool, the equilibration calculation is broken down at each cell timestep $\Delta \mathrm{t}_{\mathrm{c}}$ into three stages of mass and energy transfer to the pool. It should be noted that the condition of the pool prior to the equilibration calculation is governed by the initial pool energy $Q_{p}$.

Stage 1 accounts for the enthalpies added to the pool because of the flow of noncondensable gas and condensation of vapor. The noncondensable gas passing through the pool is first assumed to acquire the temperature of the pool $\mathrm{T}_{\mathrm{p}}$ and the pool energy change for the timestep is calculated as

$$
\Delta Q_{p}=Q_{S R V, \text { in }}-Q_{n c}^{\prime}+g m_{t}\left(H_{S R V, \text { in }}-H_{p, i}\right)
$$

where $\Delta Q_{p}$ is the pool energy change, $Q_{\mathrm{SRV} \text {,in }}$ is the total energy associated with the gas mixture that enters the pool, $Q_{n c}^{\prime}$ is the energy associated with the noncondensable gas escaping from the suppression pool at $T_{p}, m_{t}$ is the total mass introduced by the source over the timestep, $H_{S R V, \text { in }}$ is the source elevation, and $\mathrm{H}_{\mathrm{p}, \mathrm{i}}$ is the pool surface elevation.

$\mathrm{Q}^{\prime}{ }_{\mathrm{nc}}$ in Equation (11-31) is given by

$$
\mathrm{Q}_{\mathrm{nc}}^{\prime}=\mathrm{h}_{\mathrm{nc}} \mathrm{m}_{\mathrm{nc}}
$$

where $h_{n c}$ is the specific enthalpy of the noncondensable gas evaluated at $T_{p}$, and $m_{n c}$ is the noncondensable gas mass introduced by the source over the cell timestep.

Because the noncondensable gas will not condense in the pool, the only pool mass increase comes from vapor condensation and the trapping of any homogeneously dispersed liquid coolant carried by the gas. The change of pool mass, $\Delta \mathrm{m}_{\mathrm{p}}$, is given by

$$
\Delta \mathrm{m}_{\mathrm{p}}=\mathrm{m}_{\mathrm{v}, \text { in }}
$$

where $\mathrm{m}_{\mathrm{v}, \mathrm{in}}$ is the vapor and homogeneously dispersed liquid coolant mass in the source. 
Stage 2 accounts for the flashing because of the source if the BOIL option has been specified. Otherwise, the amount flashed is set to zero. The amount of pool mass flashing $\Delta \mathrm{m}_{\mathrm{f}}$ is given by

$$
\Delta \mathrm{m}_{\mathrm{f}}=\frac{\mathrm{Q}_{\mathrm{p}}+\Delta \mathrm{Q}_{\mathrm{p}}-\left(\mathrm{m}_{\mathrm{p}}+\Delta \mathrm{m}_{\mathrm{p}}\right) \mathrm{h}_{\ell}}{\mathrm{h}_{\mathrm{v}, \mathrm{s}}-\mathrm{h}_{\ell}}
$$

if positive, and the energy carried by the flashed water is

$$
\Delta \mathrm{Q}_{\mathrm{f}}=\mathrm{h}_{\mathrm{v}, \mathrm{s}} \Delta \mathrm{m}_{\mathrm{f}}
$$

where $\mathrm{Q}_{\mathrm{p}}$ and $\mathrm{m}_{\mathrm{p}}$ are the initial pool energy and mass, respectively, and $\mathrm{h}_{2}$ and $\mathrm{h}_{v, s}$ are the saturated liquid and saturated vapor enthalpy, respectively.

Stage 3 accounts for the vapor taken up by the noncondensable gas bubbles of the vent flow. The bubble vapor pressure is assumed to be 0.95 times that of the pool vapor pressure at a self-consistent pool temperature $\mathrm{T}^{\prime}$ to avoid numerical problems. The pressure in bubbles containing the noncondensable gases at the pool surface is assumed to be the same as the atmosphere pressure $P_{i}$ in the cell $\mathrm{i}$ in question. Thus,

$$
0.95 P_{s}\left(T^{\prime}\right)+P_{n c}\left(T^{\prime}\right)=P_{i}
$$

where $\mathrm{P}_{s}\left(\mathrm{~T}^{\prime}\right)$ the saturation pressure at the new self-consistent equilibrium temperature $T^{\prime}$ of the pool, correctly adjusted for the vapor energy removed by the bubbles; and $\mathrm{P}_{\mathrm{nc}}\left(\mathrm{T}^{\prime}\right)$ is the noncondensable gas pressure in the bubble.

If the BOIL option has been specified, Equation (11-33) combined with the ideal gas assumption provides the vapor mass in the outflow $\mathrm{m}_{\mathrm{v}, \text { out }}$

$$
\mathrm{m}_{\mathrm{v}, \text { out }}=\frac{\mathrm{M}_{\mathrm{v}} 0.95 \mathrm{P}_{\mathrm{s}}\left(\mathrm{T}^{\prime}\right) \mathrm{N}_{\mathrm{nc}}}{\mathrm{P}_{\mathrm{i}}-0.95 \mathrm{P}_{\mathrm{s}}\left(\mathrm{T}^{\prime}\right)}
$$

where $M_{v}$ is the molecular weight of water vapor, and $N_{n c}$ is the moles of noncondensable gas. Otherwise $\mathrm{m}_{\mathrm{v}, \text { out }}$ is set to zero.

The linearized energy balance for the suppression pool, taking into account the fact that vaporization into the bubbles cools the pool and the gas passing through, is given by

$$
\mathrm{m}_{\mathrm{v}, \text { out }} \mathrm{h}_{\ell \mathrm{v}}\left(\mathrm{T}^{\prime}\right)=\Delta \mathrm{Q}_{\mathrm{p}}^{\prime}-\left(\mathrm{C}_{\mathrm{p}}+\mathrm{C}_{\mathrm{g}}\right) \Delta \mathrm{T}_{\mathrm{eq}}
$$

where $h_{\ell v}\left(T^{\prime}\right)$ is the latent heat of evaporation $\left(h_{v, s}-h_{\ell, s}\right)$ evaluated at $T^{\prime} ; \Delta Q_{p}^{\prime}$ equals $\Delta Q_{p}-\Delta Q_{f}-$ $\left(\Delta \mathrm{m}_{\mathrm{p}}-\Delta \mathrm{m}_{\mathrm{f}}\right) \mathrm{h}_{\mathrm{l}}\left(\mathrm{T}_{\mathrm{p}}\right)$ and represents the changes in the pool energy because of processes considered in Stage 1 and Stage 2; $C_{p}$ is the specific heat of the pool multiplied by the sum of the pool mass and any additional mass of vapor condensed in the pool minus any flashed; $C_{g}$ is the specific heat of 
noncondensable gases multiplied by the total noncondensable gas mass in the bubbles; and $\Delta T_{\text {eq }}$ is the amount of temperature change or $T^{\prime}-T_{p}$.

Equation (11-37) is approximated by doing a linear expansion in $\Delta T_{\text {vap }}$ :

$$
\mathrm{m}_{\mathrm{v}, \text { out }}=\frac{0.95 \mathrm{~B}\left(\mathrm{P}_{\mathrm{s}}\left(\mathrm{T}_{\mathrm{p}}\right)+\frac{\mathrm{dP}_{\mathrm{s}}}{\mathrm{dT}} \Delta \mathrm{T}_{\mathrm{eq}}\right)}{\Delta \mathrm{P}-0.95 \frac{d \mathrm{P}_{\mathrm{s}}}{\mathrm{dT}} \Delta \mathrm{T}_{\mathrm{eq}}}
$$

where $B$ is $M_{v} N_{n c}$, the vapor flux multiplier; $d P_{s} / d T$ is the temperature derivative of the saturation pressure at $T_{p}$; and $\Delta P$ equals $P_{i}-0.95 P_{s}\left(T_{p}\right)$.

If $\mathrm{h}_{\mathrm{ev}}$ is linearized in Equation (11-38), then Equations (11-38) and (11-39) can be solved for $\Delta \mathrm{T}_{\mathrm{eq}}$ :

$$
\Delta \mathrm{T}_{\mathrm{eq}}=\frac{1}{2 \mathrm{C}_{\mathrm{A}}}\left(\mathrm{C}_{\mathrm{B}}-\sqrt{\mathrm{C}_{\mathrm{B}}^{2}+4 \mathrm{C}_{\mathrm{A}} \mathrm{C}_{\mathrm{C}}}\right)
$$

where $C_{A}, C_{B}$, and $C_{C}$ are coefficients given by

$$
\begin{aligned}
& C_{A}=0.95\left(C_{p}+C_{g}\right) \frac{d P_{s}}{d T}-0.95 B \frac{d P_{s}}{d T} h_{\ell v}^{\prime} \\
& C_{B}=\Delta P\left(C_{p}+C_{g}\right)+0.95 \frac{d P_{s}}{d T}\left(\Delta Q_{p}^{\prime}+h_{\ell v}\left(T_{p}\right) B\right)+0.95 B P_{s} h_{\ell v}^{\prime}\left(T_{p}\right)
\end{aligned}
$$

and

$$
C_{C}=0.95 h_{e v}\left(T_{p}\right) B P_{s}-\Delta Q_{p}^{\prime} \Delta P
$$

where $\mathrm{h}_{\mathrm{lv}}^{\prime}$ equals $\mathrm{dh}_{\mathrm{lv}} / \mathrm{dT}$. After $\Delta \mathrm{T}_{\mathrm{eq}}$ is determined, $\mathrm{m}_{\mathrm{g}, \text { out }}$ is determined from Equation (11-38) with $h_{l v}\left(T^{\prime}\right) \approx h_{l v}\left(T_{p}\right)+\Delta T_{e q} h_{l v}^{\prime}\left(T_{p}\right)$.

The corresponding vaporization energy transferred to the gas $Q_{\text {vap, }}$ is given by

$$
\mathrm{Q}_{\mathrm{v}, \text { out }}=\mathrm{m}_{\mathrm{v}, \text { out }}\left(\mathrm{h}_{\mathrm{v}}\left(\mathrm{T}_{\mathrm{p}}\right)+\frac{d \mathrm{~h}_{\mathrm{v}}}{\mathrm{dT}}\left(\mathrm{T}_{\mathrm{p}}\right) \Delta \mathrm{T}_{\mathrm{eq}}\right)+\mathrm{C}_{\mathrm{P}} \Delta \mathrm{T}_{\mathrm{eq}}
$$

Thus, the mass and energy source rates to the atmosphere from submerged SRV sources, defined as $\mathrm{W}_{\mathrm{SRV}, \mathrm{g}}$ and $\mathrm{q}_{\mathrm{SRV}, \mathrm{g}}$, respectively, in Tables $4-2$ and $4-4$, are given by 


$$
\begin{aligned}
& \mathrm{W}_{\mathrm{SRV}, \mathrm{g}}=\frac{\mathrm{m}_{\mathrm{v}, \text { out }}+\Delta \mathrm{m}_{\mathrm{f}}+\mathrm{m}_{\mathrm{nc}}}{\Delta \mathrm{t}_{\mathrm{c}}} \\
& \mathrm{q}_{\mathrm{SRV}, \mathrm{g}}=\frac{\mathrm{Q}_{\mathrm{v}, \text { out }}+\Delta \mathrm{Q}_{\mathrm{f}}+\mathrm{Q}_{\mathrm{nc}}^{\prime}}{\Delta \mathrm{t}_{\mathrm{c}}}
\end{aligned}
$$

\subsubsection{Phase Separation Calculation for Unsubmerged SRV Sources}

This section discusses the coolant phase calculation done for unsubmerged SRV sources, when the SRV elevation specified by the user lies above the pool surface. In this case, the source is assumed to undergo isenthalpic expansion without mixing with the atmosphere of the cell, until the pressure of the materials introduced is equal to the cell gas pressure $\mathrm{P}_{\mathrm{i}}$. The liquid coolant remaining after expansion is directed to the pool, and the other source materials, including the noncondensable gases, coolant vapor, aerosols, and fission products, are directed to the atmosphere.

The conditions after the expansion are calculated from a constant enthalpy condition:

$$
\mathcal{H}\left(\mathrm{T}^{\prime}, \mathrm{P}_{\mathrm{i}}\right)=\mathrm{Q}_{\mathrm{SRV} \text {,in }}
$$

where $\mathcal{H}\left(\mathrm{T}^{\prime}, \mathrm{P}_{\mathrm{j}}\right)$ is the total enthalpy of the source materials introduced over a cell timestep $\Delta \mathrm{t}_{\mathrm{c}}$ and $T^{\prime}$ is a self-consistent temperature. The resulting coolant vapor and liquid coolant masses $m_{v, \text { out }}$ and $\mathrm{m}_{\ell, \text { out }}$ are obtained as part of the iterative solution for $\mathrm{T}^{\prime}$.

Then, the contribution to the atmosphere from the source can be written as

$$
\begin{aligned}
& \mathrm{W}_{\mathrm{SRV}, \mathrm{g}}=\frac{\mathrm{m}_{\mathrm{v}, \text { out }}+\mathrm{m}_{\mathrm{nc}}}{\Delta \mathrm{t}_{\mathrm{c}}} \\
& \mathrm{q}_{\mathrm{SRV}, \mathrm{g}}=\frac{\mathrm{Q}_{\mathrm{SRV}, \text { in }}-\mathrm{m}_{\ell, \text { out }} \mathrm{h}_{\ell}\left(\mathrm{T}^{\prime}\right)}{\Delta \mathrm{t}_{\mathrm{c}}}
\end{aligned}
$$

with the remainder of the coolant mass and energy directed to the pool. 


\subsection{ENGINEERED SAFETY FEATURE MODELS}

The models in CONTAIN for the three major engineered safety features (ESFs)--fan coolers, ice condensers, and containment sprays--are described in this section. Thermal-hydraulic effects and the removal of aerosols (and associated fission products) from the atmosphere are modeled, as is the removal of gaseous iodine species for spray systems (but not fan coolers or ice condensers). The heat exchangers typically used in the containment spray system and various liquid transport system components (tanks, pumps, valves, pipes, orifices, and pool overflows) associated with the ESFs are also modeled. The liquid transport system can also function independently of the basic ESF models. Restrictions on the allowed combinations of systems and components are discussed in the appropriate subsections. Boiling water reactor (BWR) suppression pools, often described as an ESF, are also modeled, but are described in Chapter 11. Key elements of the ESF models are illustrated in Figure 12-1.

The arrangements of some of the engineered system components used with an ESF are illustrated by the containment spray system of a light water reactor, shown in Figure 12-2. The spray can be used to produce steam condensation, a drop in temperature, and reduction of fission product concentrations in the atmosphere. After initiation of the spray, water from the water storage tank (WST) flows through nozzles located near the top of the containment dome. Upon reaching the floor, the spray water drains into the cavity sump (pool). When the water in the WST is exhausted, recirculated water from the sump is pumped through a cooling heat exchanger and then to the spray nozzles. The ESF framework allows a detailed description of such a system. Alternatively, the user may simply specify the mass flow rate and temperature versus time through the spray system.

The removal of aerosols from the atmosphere through the operation of ESFs is discussed in Sections 7.4 through 7.6. Except in the case of sprays, the only fission products considered in this modeling are those attached to aerosols. For sprays, however, the removal of molecular iodine and gaseous organic iodides is also modeled as described in Section 8.6. The removed aerosols and fission products are conveyed, along with the effluent from operation of the ESF, to the coolant pool, if present, in cell "iclout," which is specified in the ENGINEER input block (see Section 14.3.3). Otherwise, they are placed in the waste repository of "iclout."

The optional FPLIQUID global input block described in Section 14.2.5.1 can be specified to allow liquid transport system components or pool flow paths to carry fission products from one pool to another (see Section 8.8.2). Such fission product transport between pools occurs only in conjunction with single components, such as a pipe, that are connected between pools. Such transport does not occur, for example, when the spray system is operated in a recirculation mode between two different pools. Only those fission products with non-zero user-specified values of "fpliq" will be transported to the destination pool. The fraction of fission products transferred with the liquid is equal to the fraction of liquid transferred from a pool times "fpliq." Note that the "fpliq" values also control the washdown of fission products from structure surfaces, as discussed in Section 8.8.2.

An engineered systems source table may be used to provide a time-dependent source of coolant at a specified temperature or enthalpy to an ESF. Because of the nature of the systems modeled, the only allowed source material is water. This option otherwise is specified much like the other source 


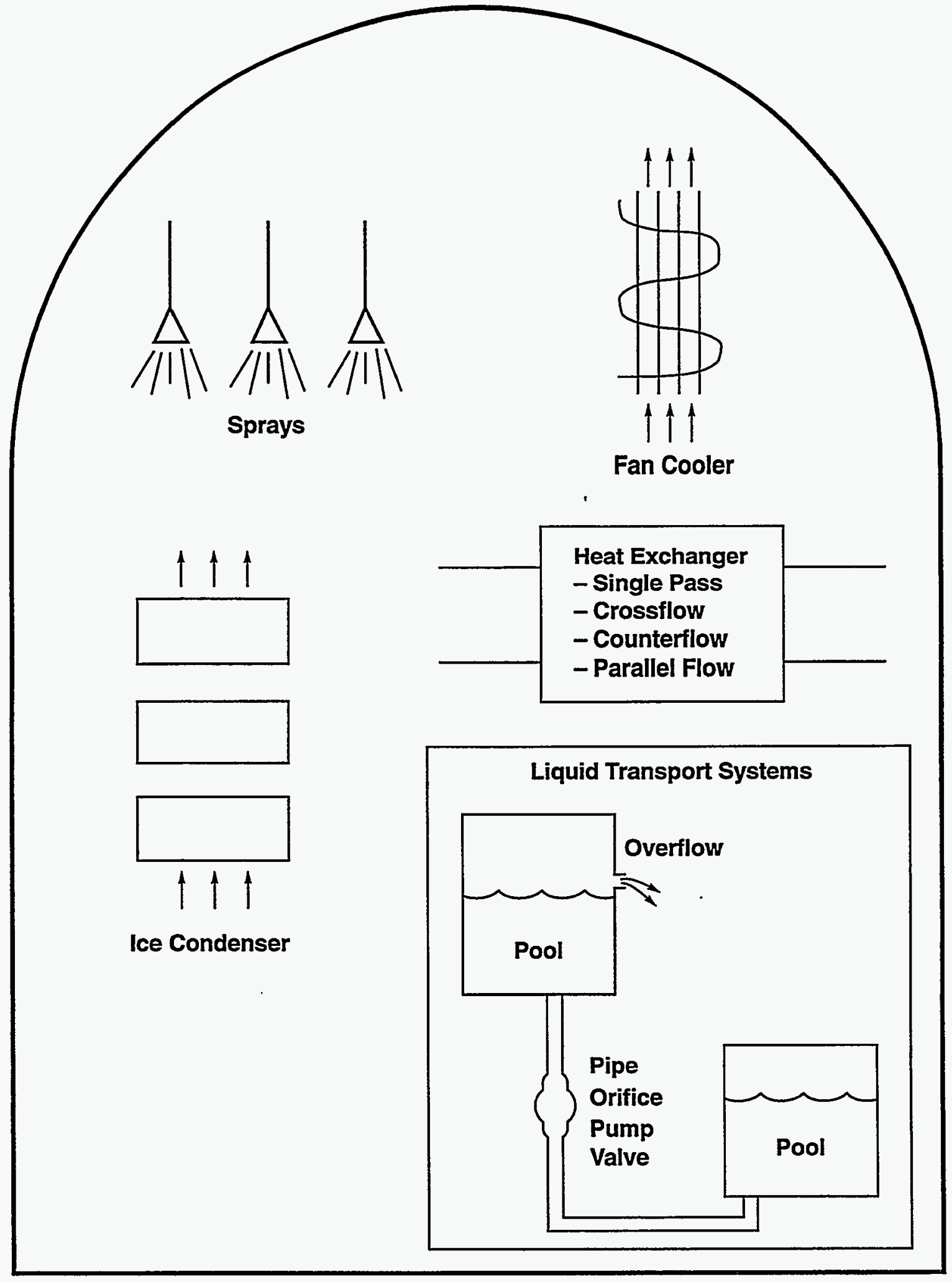

Figure 12-1. ESFs as Modeled in CONTAIN

Rev. 0 


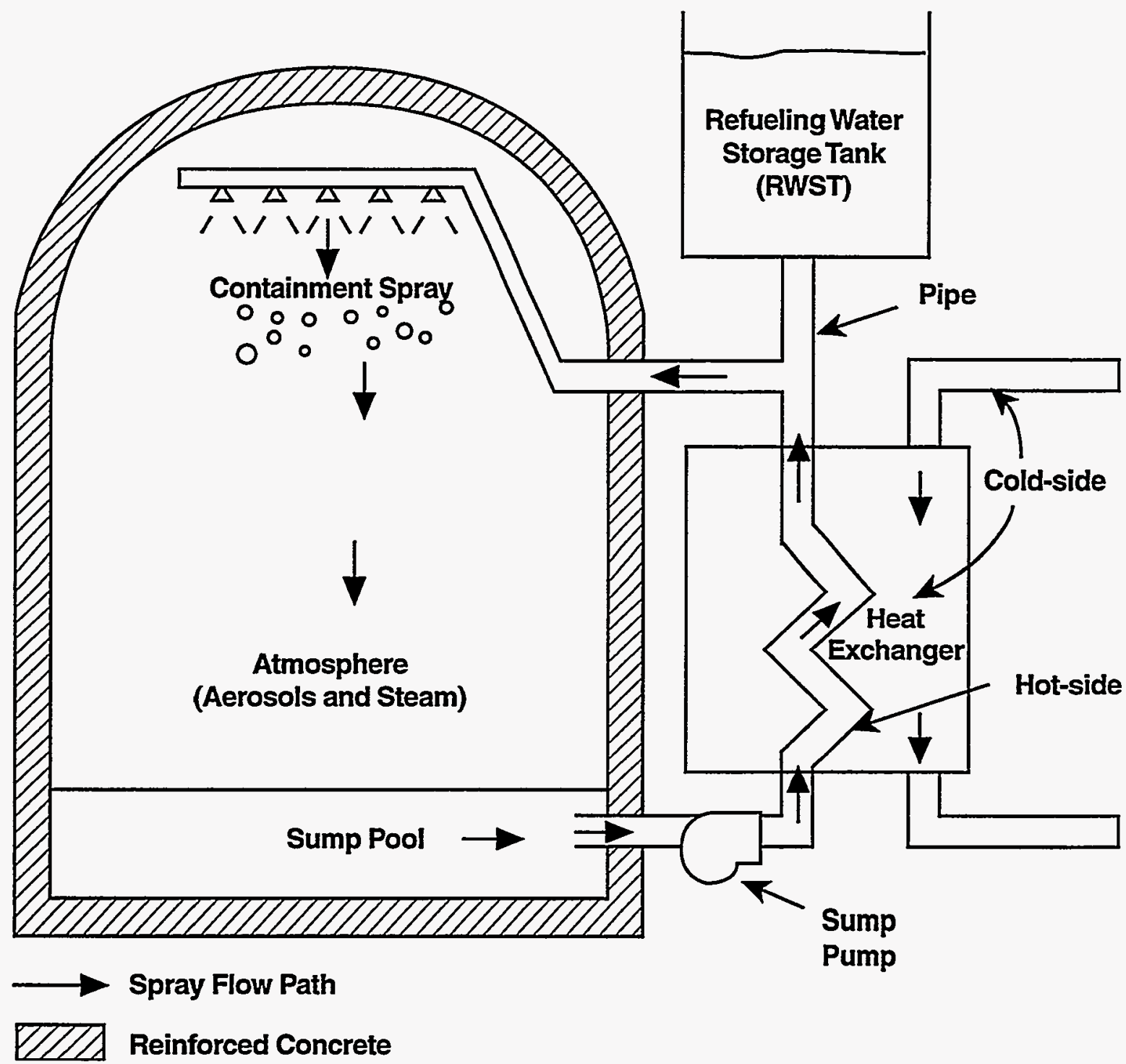

Figure 12-2. Containment Spray System With Recirculation 
tables. It is activated by the keyword SOURCE. For further details on this source table, see Sections 14.3.3.1 and 14.4.1.

\subsection{Fan Cooler}

This section discusses the thermal-hydraulic modeling of the fan cooler. The modeling of aerosol removal by the fan cooler is not discussed here but in Section 7.4.

Fan coolers are included in containment to provide non-emergency cooling and to augment the steam removal capabilities of the water sprays in the event of an accident. If operable during a severe accident, they could be important in reducing pressure and temperature and aerosol concentrations. The cooling takes place as large-capacity fans push the atmosphere gases across banks of finned, service-water-cooled coils, as illustrated in Figure 12-3. Note that the "hot side" in this figure refers to the plenum for the atmosphere gases and the "cold side" refers to plenum for the cooled water. The fan cooler models do not address the effect of fan cooler air filters, which typically use roughing and High-Efficiency Particulate Airborne (HEPA) filters.

Each cooler has several parallel coolant paths to route water back and forth across the path of the horizontally circulated atmosphere. The coils are stacked horizontally in layers with the coolant entering at the back and leaving at the front, as depicted in Figure 12-4. The geometry is therefore that of a cross-flow heat exchanger with counterflow; that is, the atmosphere inlet leg corresponds to the coolant outlet leg. For large dry pressurized water reactors, the coolers are designed to work under normal as well as accident conditions. Typical design-basis-accident temperatures are roughly 400 to $420 \mathrm{~K}$, with a saturated atmosphere, and pressures ranging between 0.3 and $0.6 \mathrm{MPa}$. The heat removal capability is in the range of 2 to $4 \times 10^{7} \mathrm{~W}$. Note that BWR and subatmospheric containments generally include fan coolers designed only for normal operating conditions, but the performance of such coolers may still be important following an accident.

CONTAIN has two fan cooler models, the March model and a more mechanistic model. The simpler of the two models is the one developed for the MARCH code. [Woo83]

March Model. This model is activated by the keyword MARCH in the FANCOOL input block (see Section 14.3.3.2). The user input for this model includes the design cooling capacity "fcqr," the associated design inlet gas temperature "fctpir," the design coolant inlet temperature "fctcli," and the design coolant mass flow rate "fcclmd." A correlation for the effective heat transfer coefficient $h_{r}$ as a function of steam vapor fraction has been derived from the capacity curves for saturated conditions as discussed in Reference Woo80. The heat transfer coefficient $h_{r}$ for design conditions is used along with the design capacity to calculate an effective heat transfer area. The cooling rate for prevailing conditions is then calculated using this area and a heat transfer coefficient corresponding to the design atmosphere vapor fraction. The default values of input parameters are those characteristic of the fan coolers in the Zion nuclear power plant. [Zio00]

The MARCH model reproduces the Zion published capacity curve with reasonable accuracy when used with the Zion fan cooler characteristics. It does not address the effect of noncondensable gases 


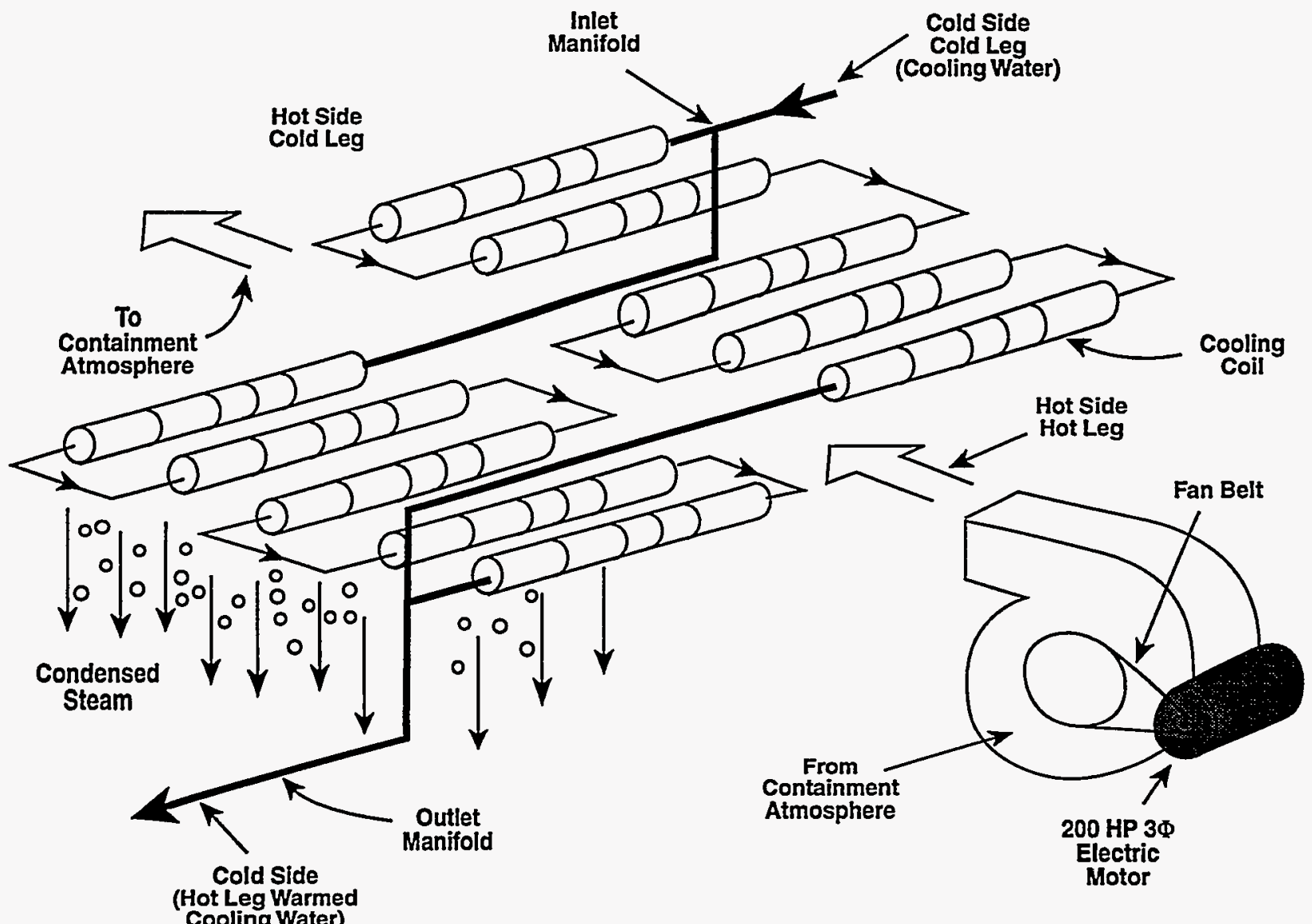

Note: arrows indicate direction of fluid flow

Figure 12-3. Fan Cooler System 


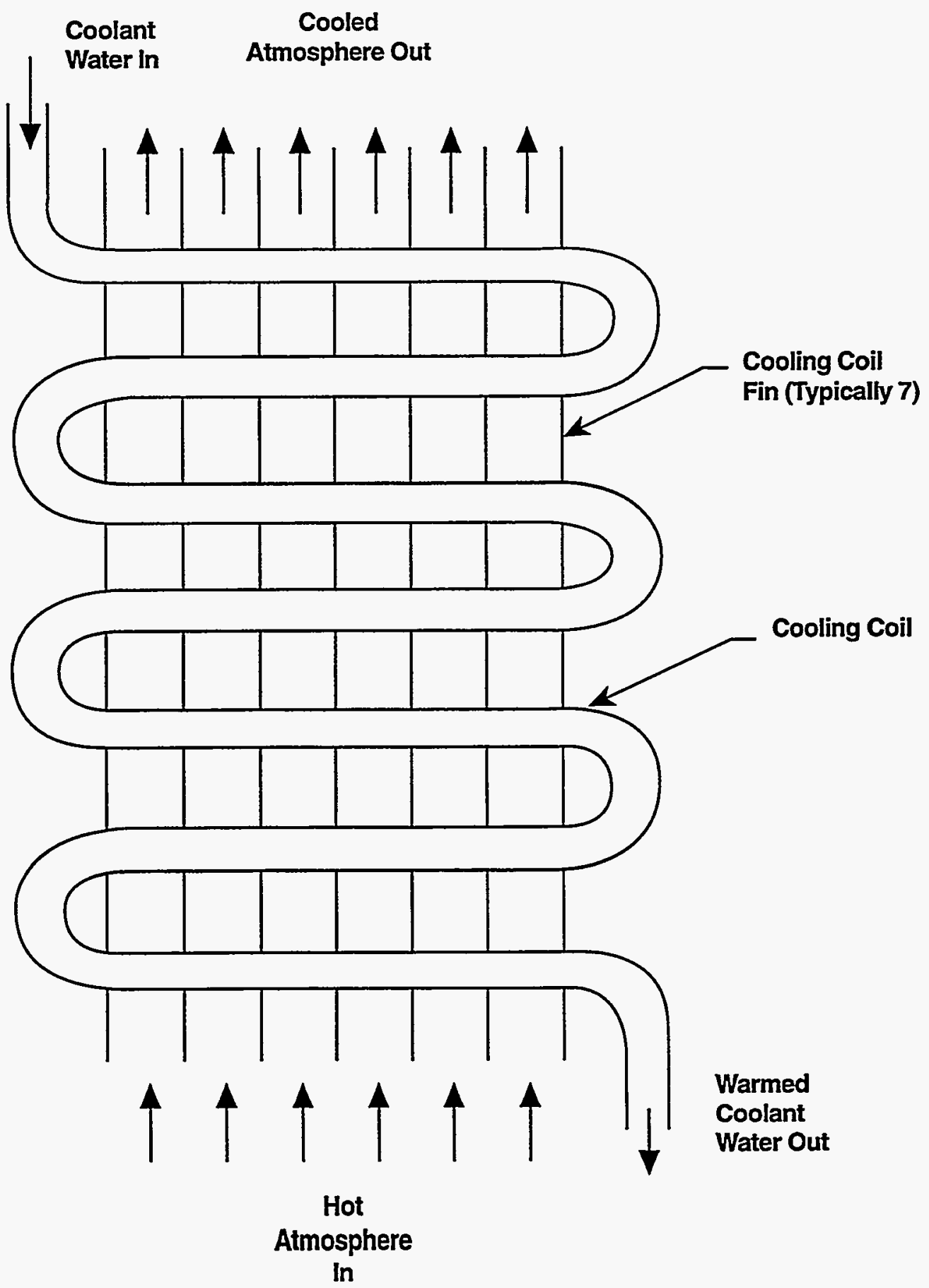

Figure 12-4. Fan Cooler Geometry Depicting One Horizontal Cooling Coil (Top View) 
other than air or superheated conditions. Also, fission product removal, condensation, and aerosol depletion are not calculated for this model.

The MARCH heat transfer coefficient [Woo83] is given by

$$
\begin{aligned}
& h_{\mathrm{r}}=1527.0+2325(X-0.26), \text { for } X \geq 0.26 \\
& h_{\mathrm{r}}=590.5+3603 \mathrm{X} \text {, for } \mathrm{X}<0.26
\end{aligned}
$$

where $h_{t}$ is the fan cooler heat transfer coefficient at design conditions, $X$ is the design steam molar fraction, which may be expressed as $P_{v, s} /\left(P_{v, s}+P_{a i r}\right)$, and $P_{v, s}$ and $P_{\text {air }}$ are the saturated water vapor and air partial pressures at the design containment atmosphere temperature. Note that $P_{\text {air }}$ is based on the assumption that the air by itself would generate a pressure of one atmosphere at $311.1 \mathrm{~K}$.

The MARCH fan cooler effective heat transfer area is then determined from

$$
A_{F C}=\frac{q_{r}}{h_{r}\left(T_{g, r}-T_{c, r}\right)-\frac{q_{r} h_{r}}{8374 W_{c, r}}}
$$

where $A_{F C}$ is the effective fan cooler area, $q_{r}$ is the design cooling capacity $(W) T_{g, r}$ is the design containment atmosphere temperature, $T_{c, r}$ is the design heat exchanger coolant temperature, the number 8374 corresponds to twice the specific heat of water in $\mathrm{J} / \mathrm{kg}-\mathrm{K}$, and $\mathrm{W}_{\mathrm{c}, \mathrm{r}}$ is the design coolant mass flow rate.

The energy removal rate from the atmosphere is then given by

$$
q=\frac{h_{r} A_{F C}\left(T_{g}-T_{c, r}\right)}{\left[1+\frac{h_{r} A_{F C}}{\left(8374 W_{c, r}\right)}\right]}
$$

Note that this model does not remove any mass from the atmosphere, not even steam.

Note that Equation (12-3) is based on the assumptions that (1) the coolant temperature is the design value, (2) the coolant mass flow rate is the design value, (3) the coolant temperature variation across each row is the same, (4) the gas temperature variation in the fan cooler is negligible, (5) the air inventory is that producing one atmosphere of pressure at $311.1 \mathrm{~K}$, and (6) the atmosphere is saturated. Studies have compared the predictions of the March model to the performance of fan coolers as described in Reference AAF72. This comparison shows that $\mathrm{q}$ from Equation (12-3) agrees with the measured values to within an accuracy of $20 \%$ for several actual fan coolers at atmosphere temperatures from 358 to $413 \mathrm{~K}$ and coolant inlet temperatures from 300 to $322 \mathrm{~K}$. 
Mechanistic Model. The mechanistic fan cooler model, activated by the keyword CONDENSE in the FANCOOL input block, is based upon condensation mass transfer and convective heat transfer coefficients that depend on the cell atmospheric conditions. The equations are given below. Note that Equations (12-4) and (12-5) for the mass and heat transfer coefficients are similar to equations presented in Sections 10.1 and 10.2 for the determination of heat and mass transfer to heat structures.

The Nusselt number used to determine the heat and mass transfer coefficients is a Reynolds-Prandtl correlation for flow over horizontal tubes. [Hol68, Kre73] This correlation is applicable to turbulent flow over tube bundles if there are 10 or more transverse rows in the direction of air flow and the pitch-to-diameter ratios are between 1.25 and 1.5. Also, it should be noted that this correlation is derived for tube banks without fins. Therefore, the mechanistic model for the fan cooler should be used with caution and the results should, when possible, be compared to published cooler performance data.

All input parameters are defined in Section 14.3.3.2 and described in the following discussion. The Reynolds number used in this correlation is based upon the coil outside diameter "fcclod" and a stream velocity based upon the air/steam flow rate and the cooler frontal area "fcflar." The effective heat transfer area per row is the input parameter "fcefar." For most applications, this parameter will not be available from cooler design data. Rough calculations based upon typical cooler fin and tube designs and prior experience indicate that a value of about ten times the cooler frontal area is appropriate. A constant heat transfer coefficient "fchntr" is used for heat transfer between the gas/ condensate-film interface and the coolant. An approximate value for this parameter found to be reasonable in parametric comparisons with published cooler capacity curves is $1000 \mathrm{~W} / \mathrm{m}^{2}-\mathrm{K}$, which is also the default value.

The two input parameters that cannot be easily characterized, the effective heat transfer area "fcefar" and the heat transfer coefficient between the gas-condensate-film interface and the coolant "fchntr," present difficulties for the user of this model. The values recommended above for these parameters will provide reasonable results for typical fan cooler designs. If accurate simulation of a cooler is important, the user should vary the values chosen so that the published cooler capacity is obtained. Note that the default values for input parameters are suitable for the Zion plant.

The calculation is carried out iteratively. The gas mixture inlet conditions and flow rate, given by the input parameter "fcwin," are known. The cooling water exit temperature $T_{c, \text { out }}$ is first estimated from the MARCH model and the input cooler capacity "fcqr." The temperature change of the cooling water from convection and condensation heat transfer in the last row of coils is calculated for the estimated cooling water exit temperature. (Recall that the last row of coils seen by the cooling water is the first row seen by the incoming air/steam mixture.) The water temperature and the gas mixture temperature, steam molar fraction, and boundary layer properties are updated prior to repeating the calculation for the next row. The process is repeated for successive rows until the water inlet temperature and exhaust gas temperature have been determined. The water inlet temperature is compared to the user-specified value "fctcli." If there is a significant difference, the calculation is repeated with a revised estimate for the coolant outlet temperature. The process is continued until there is agreement between the calculated and specified coolant inlet temperatures. 
Because the total heat transferred is relatively insensitive to changes in cooling water temperature, convergence is rapid.

For each row of the fan cooler, the heat and mass transfer to the row of coils is governed by the heat transfer coefficient $h_{c}$ and the mass transfer coefficient $K_{g}$ As discussed below, these are defined in a manner similar to the heat and mass transfer coefficients in Chapter 10, with the exception that the gas properties are evaluated at an average temperature $T_{a v}=\left(T_{g}+T\right) / 2$, where $T_{g}$ is the gas temperature for the row, and $T_{c}$ is the coolant temperature for the row. In contrast, in Chapter 10, the gas properties are evaluated at the boundary layer temperature $\left(T_{\mathrm{g}}+\mathrm{T}_{\mathrm{if}}\right) / 2$, where $\mathrm{T}_{\mathrm{if}}$ is the gassurface interface temperature of the coil. $T_{a v}$ is used to avoid the nested iterations required to solve for $T_{i f}$ simultaneously with $T_{c, o}$.

The convective heat transfer coefficient is defined as

$$
h_{c}=\frac{N_{N u} k_{a v}}{d_{c}}
$$

where $d_{c}$ is the cooling coil diameter, $k_{a v}$ is the gas conductivity at the average temperature, and the Nusselt number has the form

$$
\mathrm{N}_{\mathrm{Nu}}=0.33 \mathrm{~N}_{\mathrm{Re}}^{3 / 5} \mathrm{~N}_{\mathrm{Pr}}^{1 / 3}
$$

The gas properties in the Reynolds number $\mathrm{N}_{\mathrm{Re}}$ and the Prandt number $\mathrm{N}_{\mathrm{Pr}}$ are defined at the average temperature:

$$
N_{R e}=\frac{d_{c} v_{g} \rho_{a v}}{\mu_{a v}}
$$

where $v_{\mathrm{g}}$ is the gas velocity present at the row, $\rho_{\mathrm{av}}$ is the gas density, and $\mu_{\mathrm{av}}$ is the gas viscosity. Also,

$$
\mathrm{N}_{\mathrm{Pr}}=\frac{\mathrm{c}_{\mathrm{p}, \mathrm{av}} \mu_{\mathrm{av}}}{\mathrm{k}_{\mathrm{av}}}
$$

where $c_{p, a v}$ is the gas specific heat for the row.

The mass transfer coefficient is given by 


$$
K_{g}=\frac{N_{S h} \bigoplus_{v} \ln \left[\frac{1-X_{v, c}}{1-X_{v, g}}\right]}{R T_{a v} d_{c}\left[X_{v, g}-X_{v, c}\right]}
$$

where $\mathrm{X}_{\mathrm{v}, \mathrm{c}}$ is the vapor mole fraction at the coil surface and $\mathrm{X}_{\mathrm{v}, \mathrm{g}}$ is the vapor mole fraction in the gas. The Sherwood number is defined as

$$
\mathrm{N}_{\mathrm{Sh}}=0.33 \mathrm{~N}_{\mathrm{Re}}^{3 / 5} \mathrm{~N}_{\mathrm{Sc}}^{1 / 3}
$$

and the Schmidt number is defined as

$$
\mathrm{N}_{\mathrm{Sc}}=\frac{\mu_{\mathrm{av}}}{\rho_{\mathrm{av}} \boxplus_{\mathrm{v}}}
$$

The heat and mass transfer to the coil is calculated from the convective heat transfer $h_{c}$, the mass transfer coefficient $\mathrm{K}_{\mathrm{g}}$, and the coil-side heat transfer coefficient "fchntr," using a linearized approximation for the saturated vapor pressure as a function of temperature. Thus, $\rho_{g}, \mu_{g}, k_{g}, v_{g}, T_{g}$, and $T_{c}$ are updated for the next row.

\subsection{Ice Condenser}

This section discusses the heat and mass transfer modeling of the ice condenser. Note that the modeling of aerosol removal by the ice condenser is not discussed here but in Section 7.5.

The ice condenser containment system incorporates a large amount of subcooled ice that acts as a low temperature, passive heat sink. A typical ice condenser containment is shown in Figures 12-5 and 12-6. The only significant flow path from the lower compartment housing the reactor vessel to the upper compartment is through the ice compartment. The ice, in granulated form, is contained in perforated metal baskets approximately $0.3 \mathrm{~m}$ in diameter and stacked to a total height of about $15 \mathrm{~m}$. There are 24 annular modules, occupying approximately $300^{\circ}$ of the containment periphery. Although the ice containers are perforated, most of the flow is around the outside of the baskets, so little or no entrainment of ice or condensate occurs. Because the ice is a highly efficient heat sink, nearly all of the steam is condensed. As long as a significant amount of ice remains in the ice condenser, the peak pressure is determined largely by the accumulation of noncondensable gases in the upper compartment.

The inlet doors to the ice compartment are held closed by springs and the exit doors at the top are held closed by gravity. The doors open when there is sufficient pressure difference to overcome the forces keeping them closed. These doors may be simulated by using pressure criteria (discussed in Section 4.3) to open the flow paths representing the doors. If the user wishes to simulate the actual 


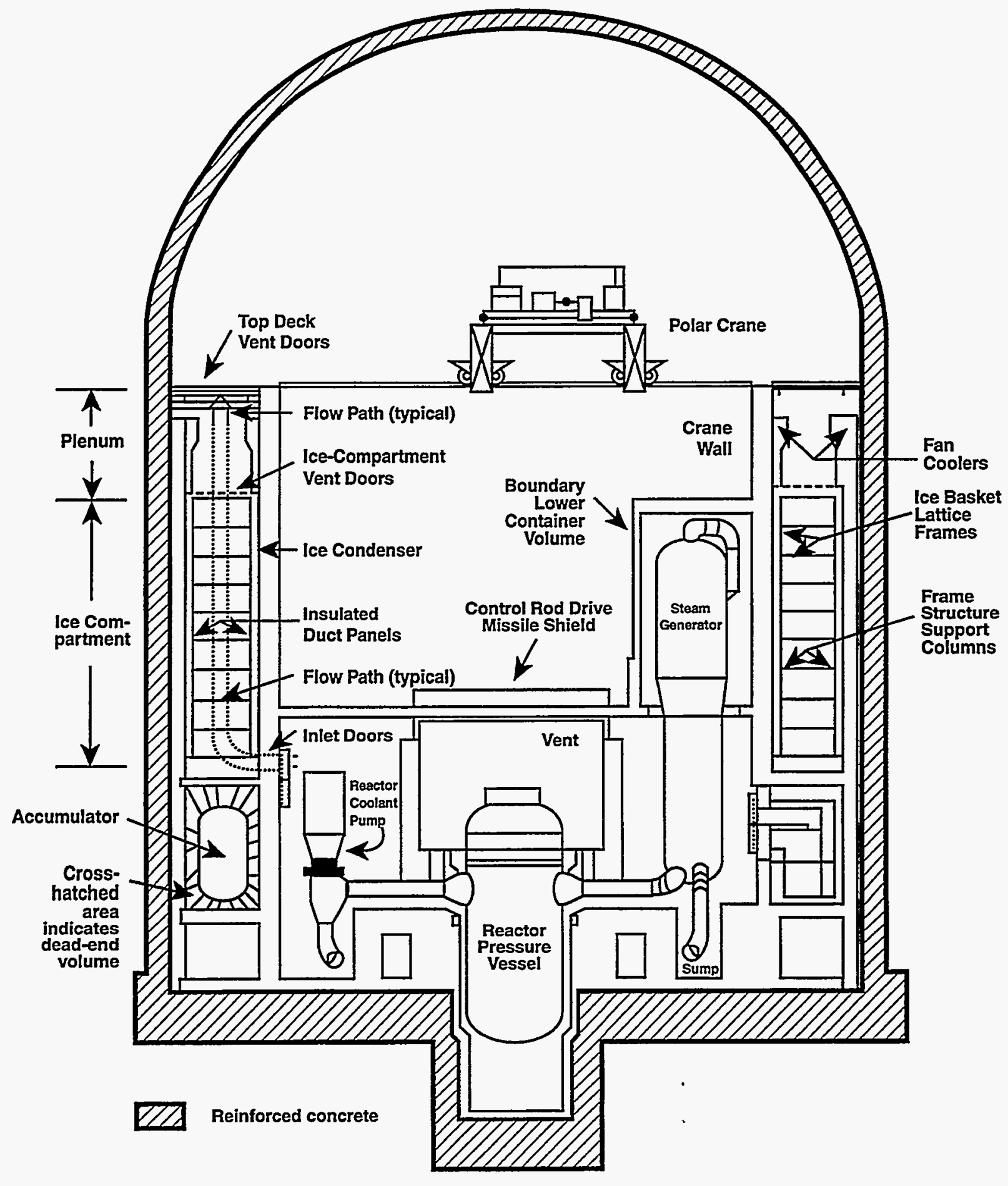

Figure 12-5. Sectional View of an Ice Compartment and Containment Region Boundaries (Adapted from Reference Win83). Note the typical flow path through the ice condenser indicated by the dotted lines at the left. 


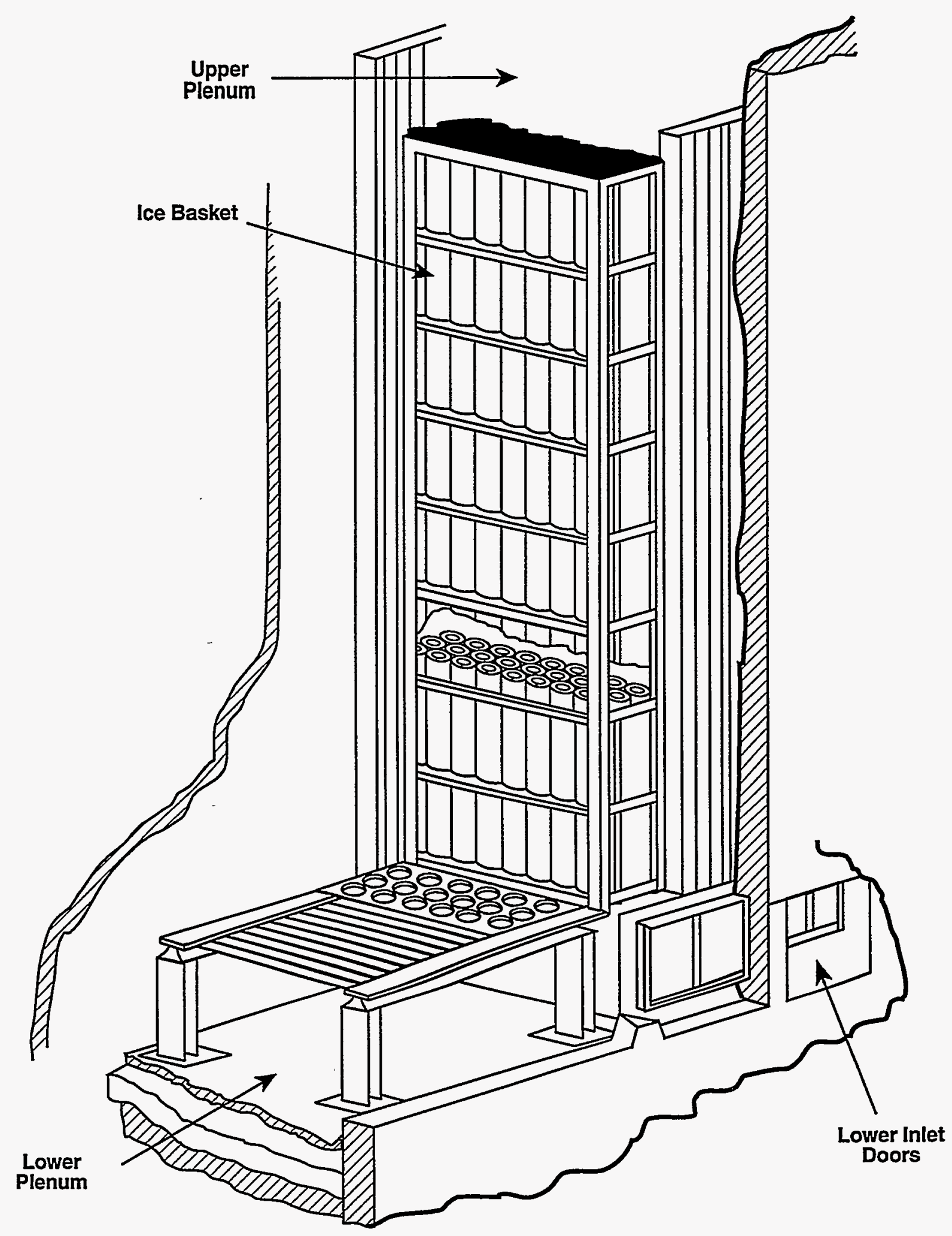

Figure 12-6. Simplified Diagram of an Ice Condenser. Note that the ice baskets are perforated cylinders supported around their circumfence by horizontal wire strips that are approximately 6-mm high and 2 -mm thick. 
spring or gravity-controlled motion of the doors, the flow area may be specified as a function of the pressure difference across it using one of the flow path tabular input options discussed in Sections 4.3 and 14.2.4.2

The ice condenser modeling was assessed against experimental results in Reference Rus $90 \mathrm{~b}$. This qualitatively demonstrated the capability of CONTAIN to predict backflow and temperature stratification. However, the CONTAIN model remains to be validated for conditions of high steam flows, such as those occurring during a large break loss-of-coolant accident, and also no database for validation exists for some severe accident scenarios, such as those involving direct containment heating.

Heat and mass transfer to the ice is treated in a manner similar to that for a wall structure (see Chapter 10). Both turbulent natural convection and forced convection are considered. Note that the expressions used for the Nusselt number are similar to equations in Section 10.1 but contain a userinput multiplier "cihtml," described in Section 14.3.3.3, that can be used to account for the roughness of the cylindrical ice columns. The default value of "cihtml" is five. Because the ice is contained in baskets, the ice height and, hence, the heat transfer area is assumed to decrease as heat is absorbed and the ice is melted. Other differences in the ice condenser treatment include a film resistance corresponding to a fixed water film thickness, which may be specified by the user and is by default $0.05 \mathrm{~mm}$. Heat and mass transfer from the gas flowing around the ice is always assumed to melt the ice as long as it remains. Thus, the ice surface is always taken to be at the melt temperature, and conditions without melting cannot be modeled. Heat and mass transfer to the ice basket structures is not considered unless they are modeled separately as heat transfer structures.

Note that radiation from the gas to the ice is not modeled. However, in direct containment heating calculations, radiation heat transfer from the core debris particles to the ice condenser is modeled as described in Section 6.5.2. In that case the ice condenser is included as one of the surfaces for calculating the debris-surface radiative heat transfer rate (see Equation (6-158)).

The condensate, melted ice, deposited aerosols, and associated fission products are diverted to the cell designated to receive the residual liquid for the engineered system in which the ice condenser is defined. This cell is the user-input parameter "iclout" specified in the ENGINEER input block.

The forced-convection velocity $\mathrm{v}_{\mathrm{g}}$ used for the ice condenser forced-convection heat transfer correlations is calculated somewhat differently from the velocity for heat transfer structures. It is based on the average velocity into the ice condenser $v_{\text {in }}$ from the cell "icllp" specified by the user and the average velocity out of the ice condenser $v_{\text {out }}$ to all cells but "icllp." The $v_{\text {in }}$ and $v_{\text {out }}$ are based on the assumption that the flow goes through an isentropic expansion. Specifically,

$$
v_{\text {in }}=\sum_{\substack{\text { flows to or } \\ \text { from "icllp" }}} \frac{W F}{A \rho_{u}}
$$

where $\mathrm{W}$ is the mass flow rate and is taken to be positive if the flow is in and negative if the flow is out; $A$ is the user-specified cross-sectional flow area "ciarfl"; and $\rho_{u}$ is the gas density of the 
upstream cell. $F$ is equal to 1 for outgoing flows, and equal to $\left(P_{u} / P_{d}\right)^{1 / \gamma_{u}}$ for incoming flows; $\gamma_{u}$ is the specific heat ratio for the upstream cell; and $P_{u}$ and $P_{d}$ are the pressures of the upstream and downstream cells, respectively. An expression similar to Equation (12-11) is used for $v_{\text {out }}$, except that the sum is carried over flows to all cells but "icllp" and the signs for W are reversed.

The forced-convection velocity $\mathrm{v}_{\mathrm{g}}$ is chosen as follows:

$$
\begin{aligned}
\mathrm{v}_{\mathrm{g}} & =\mathrm{v}_{\text {in }} \text { if } \mathrm{v}_{\text {in }}>0 \text { and } \mathrm{v}_{\text {out }}>0 \\
& =v_{\text {out }} \text { if } \mathrm{v}_{\text {in }}<0 \text { and } \mathrm{v}_{\text {out }}<0 \\
& =|| \mathrm{v}_{\text {in }}|-| \mathrm{v}_{\text {out }}|| \text { otherwise }
\end{aligned}
$$

Most of the input parameters for the ice condenser model are design data that may be taken directly from plant safety analysis reports and are input in the ICECOND input block as described in Section 14.3.3.3. These parameters include the initial height of the ice column "hitici," initial ice mass "tmsici," initial ice temperature "citice," and cross-sectional area "ciarfl" available for flow through the ice compartment.

Other input parameters require some discretion on the part of the user. Among these are the userspecified temperature "citlex" governing the temperature of the melt/condensate exiting from the ice condenser and the initial total ice condenser surface area "arhtin" available for heat transfer. The exit temperature is dependent upon the flow rate through the compartment, and tests have produced values between $335 \mathrm{~K}$ and $373 \mathrm{~K}$. In an ice condenser plant, initially over $7 \times 10^{5} \mathrm{~m}^{2}$ of ice surface area is potentially available for heat transfer. However, a reasonable assumption is that the available area is that of the outside of the baskets, or about $10^{4} \mathrm{~m}^{2}$. The user should also keep in mind the presence of the heat transfer multiplier "cihtml," by default equal to 5 , which takes into account the ice interstitial flow and roughness. The user should note, finally, that the cell "icllp" is normally the cell directly below an ice compartment cell. This may be the actual lower plenum, or the lower containment, if modeling of the actual lower plenum is not required, or the upstream cell to an ice condenser cell, in case the ice condenser is partitioned horizontally into more than one cell and the cell in question is not at the lowest level.

Note that the model for the energy transfer from the flowing gas to the ice and melted ice water includes the following accounting:

1. The increase of the ice temperature to its freezing temperature, $273.15 \mathrm{~K}$. Note that the ice is normally at approximately $264 \mathrm{~K}\left(-9^{\circ} \mathrm{C}\right)$.

2. The melting of the ice and heating of the melt/condensate to the gas-film interface temperature.

3. The heating of the melt/condensate to the exit temperature. This temperature is nominally given by the "citlex" temperature specified by the user. However, there are constraints on the temperature used. The exit temperature is constrained to be greater than or equal to the dew point of the atmosphere, greater than or equal to the melt/condensate film interface temperature, and less than or equal to the gas temperature. "citlex" is used if it satisfies these 
conditions. If "citlex" does not, then the exit temperature is set to the closest temperature satisfying these conditions, if one exists. If the exit temperature leads to heating of the melt/condensate, the heat and mass transfer processes responsible are assumed to occur in the same proportion as the original heat and mass fluxes to the ice surface that caused the ice to melt. Otherwise, the heating is ignored.

\subsection{Containment Sprays}

This section discusses the thermal-hydraulic modeling of containment sprays. The modeling of aerosol removal by the sprays is not discussed here but in Section 7.5 and the modeling of the depletion of gaseous fission product species by the sprays is discussed in Section 8.6.

The spray system provides a uniformly divided water spray to the containment atmosphere. As previously shown in Figure 12-2, heat and mass transfer to the droplets provide a rapid reduction in temperature, pressure, and fission product concentration. The sprayed water collects in a sump at the bottom of the containment. Generally, the initial spray water is from the WST. When that source is exhausted, water is pumped from the sump, through a heat exchanger, and to the spray nozzles. A model has been developed that determines the heat transfer between the droplets and atmosphere and the associated condensation of steam onto or evaporation of the droplets.

The explicit coupling between the spray model and the atmospheric thermal-hydraulics can lead to oscillations in the atmospheric temperature and pressure when the system timestep is too large. Though often small in amplitude, these oscillations can be detrimental; e.g., they can cause the saturation ratio to oscillate back and forth across unity, which can in turn cause spurious condensation on the aerosols and artificial enhancement of aerosol removal by the sprays. The best means of testing for this condition is to check the saturation ratio for oscillatory behavior. The only safe procedure is to reduce the system timestep until the oscillations go away, unless the user can establish that they cause no harmful effects in the particular calculation at hand. The maximum stable timestep decreases somewhat with increasing spray flow rate and, more noticeably, with decreasing spray drop size. The increase in computer time from reducing the system timestep may be partially recovered by reducing the number of cell timesteps per system timestep (i.e., increasing "ctmfr"; see Section 14.2.8).

The containment spray must be used in either one of two engineered system combinations with the input specified in the SPRAY input block (see Section 14.3.3.4). For the simplest system, the spray is paired with an engineered system source table, and the spray is active as long as the source is finite. A more elaborate system may be initiated using a containment pressure setpoint given by the input parameter "spstpr." In the latter system, the user must include a tank to supply an initial amount of fresh water to the spray train (see Section 12.5.5). When the tank is empty, a pump provides recirculated water, which goes through a heat exchanger and then to the spray trains. Failure of recirculation may be simulated by specifying zero flow for the pump or by drawing water from a source cell "iclin" that contains no pool. Sample input for this system is given in Section 14.3.3.4. 
As the containment spray water drops fall through the atmosphere, their diameters can increase or decrease as a result of condensation or evaporation. The heat transfer and mass transfer to the spray as the drops fall are calculated. The model for these processes is basically the same as that for heat and mass transfer used elsewhere in CONTAIN. However, the Nusselt number correlation used is that of Ranz and Marshall for forced convection around a spherical droplet. [Ran52] The rate of evaporation of, or condensation on, a spray droplet is controlled by the diffusion of water vapor through the gas boundary layer at the surface of the drop and is driven by the difference in water vapor pressure between the atmosphere and the droplet surface.

The spray calculation begins with determination of the number of droplets introduced in a cell timestep. The equations for drop height, mass, and energy are solved for the entire fall of a single droplet. The fall height is an input parameter, "sphite," that is defaulted to the cell height. The resulting transfers of mass and energy between the droplet and the atmosphere are then multiplied by the number of droplets. Note that the effects on the atmosphere resulting from a given spray drop are assumed to be instantaneous and not spread out over its fall time. This assumption is normally of concern only if the atmosphere conditions are calculated to change rapidly over the droplet fall time.

Spray droplets that reach the bottom of the cell contribute their mass and energy to the lower cell pool, if present, in the cell to which the engineered system effluent is directed. The user specifies that cell as cell "iclout" for storing residual liquid from ESFs, which by default is the cell in which the spray is defined. If no lower cell pool is specified for that cell, the water is lost from the problem. For most situations that call for the use of containment sprays, a liquid pool can be expected to form as a result of spray droplets reaching the floor. Therefore, it is recommended that the user include a lower cell model with a pool in the residual-liquid cell.

When sprays are operating, containment conditions tend to approach a quasi-steady state in which the sprays are removing about as much steam and energy from the containment as the various sources are supplying to the containment. (Important exceptions to this behavior arise during and shortly after major transient inputs of steam or energy to the containment.) Likewise, the drop itself tends to reach a quasi-steady state with respect to the containment atmosphere very early in its fall, with little change in the drop parameters (such as temperature) after the first few tenths of a meter or so of its fall.

The model assumes that (1) the gas and vapor have ideal behavior, (2) the drop is well-mixed, (3) the drops fall at all times at their terminal velocity, and (4) the effect of the ensemble of spray drops is obtained by scaling the effect of a single drop by the number of drops.

Water Drop Terminal Velocity The spray drops are assumed to fall at constant terminal velocity speed $v_{t}$ throughout their entire containment fall path. The equation for $v_{t}$ is:

$$
v_{t}=\left(\frac{\mu_{g} / \rho_{g}}{D}\right) B^{2}
$$


where $\mu_{\mathrm{g}}$ is the atmosphere gas mixture viscosity, $\rho_{\mathrm{g}}$ is the gas mixture density, and $\mathrm{D}$ is the drop diameter, and where

$$
\begin{aligned}
& B=\left(Y_{1}-9.06\right) / 2 \\
& Y_{1}=\sqrt{82.0836+9.06 \sqrt{Y_{2} / Y_{3}}} \\
& Y_{2}=64 \rho_{\ell} \rho_{g} D^{3} g \\
& Y_{3}=72 \mu_{g}^{2}
\end{aligned}
$$

In this expression $g$ is the acceleration due to gravity and $\rho_{\ell}$ is the liquid drop density.

The Reynolds number $\mathrm{N}_{\mathrm{Re}}$ is also calculated using $\mathrm{v}_{\mathrm{t}}$ from

$$
N_{R e}=\frac{\rho_{g} V_{t} D}{\mu_{g}}
$$

where all the terms have been defined previously.

Spray Droplet Heat and Mass Transfer Model The heat transfer between the atmosphere and the containment spray droplet occurs by two processes, convection and vapor condensation. A gas boundary layer in the atmosphere contributes the principal thermal resistance under condensing conditions. This is because the coolant vapor must diffuse through a region of enhanced noncondensable concentration and depleted condensable vapor concentration. The modeling for this process is taken from Reference Col81 and is very similar to the models presented in Chapter 10 for heat and mass transfer to heat structures, except the drop is assumed well-mixed and thus the presence of a drop-side boundary layer is ignored.

The total heat flux q consists of two components, one for convective heat transport across the boundary layer $q_{c}$ and one for the heat transported by the mass flux as follows

$$
\mathrm{q}=\mathrm{q}_{\mathrm{c}}+\mathrm{q}_{\text {cond }}
$$

The convective heat flux is defined as

$$
q_{c}=h_{c}\left(T_{g}-T_{d}\right)
$$


where $h_{c}$ is the convective heat transfer coefficient and is equal to $N_{N u}\left(k_{B L}(D)\right.$, where $\mathrm{Nu}_{\mathrm{Nu}}$ is the Nusselt number given below in Equation (12-19), $\mathrm{k}_{\mathrm{BL}}$ is the thermal conductivity of the boundary layer, $\mathrm{D}$ is the drop diameter; $\mathrm{T}_{\mathrm{g}}$ is the bulk gas temperature; and $\mathrm{T}_{\mathrm{d}}$ is the drop temperature.

The second component of the total heat flux, accounting for the heat transported by the mass flux, is given by

$$
q_{\text {cond }}=j_{\text {cond }} h_{v, b}
$$

where $j_{\text {cond }}$ is the vapor mass flux as determined below in Equation (12-16) and $h_{v, b}$ is the enthalpy of water vapor at the bulk gas temperature.

The mass flux $j_{\text {cond }}$ at the spray drop surface is approximated by

$$
j_{\text {cond }}=K_{g} M_{w}\left(P_{v, b}-P_{v, d}\right)
$$

where $\mathrm{K}_{\mathrm{g}}$ is the mass transfer coefficient $\left(\mathrm{kgmole} / \mathrm{s}-\mathrm{m}^{2}-\mathrm{Pa}\right)$, and

$$
=\frac{N_{S h} P_{g} \oplus_{v}}{R T_{B L} P_{n m} D}
$$

where $\mathrm{N}_{\mathrm{Sh}}$ is the Sherwood number, $\mathrm{P}_{\mathrm{g}}$ is the gas pressure $(\mathrm{Pa}), \bigoplus_{\mathrm{v}}$ is the diffusivity of water vapor $\left(\mathrm{m}^{2} / \mathrm{s}\right), \mathrm{R}$ is the universal gas constant $\left(\mathrm{Pa}-\mathrm{m}^{3} / \mathrm{kgmole}-\mathrm{K}\right), \mathrm{T}_{\mathrm{BL}}$ is the average of $\mathrm{T}_{\mathrm{g}}$ and $\mathrm{T}_{\mathrm{d}}$, and

$$
P_{n m}=\left(P_{v, b}-P_{v, d}\right) / n n\left[\left(P_{g}-P_{v, b}\right) /\left(P-P_{v, d}\right)\right]
$$

Here, $P_{v, b}$ is the partial pressure of water vapor at the gas temperature $T_{g^{\prime}}$, and $P_{v, d}$ is the saturation pressure at the drop temperature $T_{d}$.

For forced convection around a spherical droplet [Bir60] (recall that the droplets are falling through air), the Nusselt and Sherwood numbers used for calculating the convective and mass transfer coefficients are given by

$$
\mathrm{N}_{\mathrm{Nu}}=2.0+0.60\left(\mathrm{~N}_{\mathrm{Re}}\right)^{1 / 2}\left(\mathrm{~N}_{\mathrm{Pr}}\right)^{1 / 3}
$$

and

$$
\mathrm{N}_{\mathrm{Sh}}=2.0+0.60\left(\mathrm{~N}_{\mathrm{Re}}\right)^{1 / 2}\left(\mathrm{~N}_{\mathrm{Sc}}\right)^{1 / 3}
$$


In these equations, $N_{R e}$ is the drop Reynolds number, $N_{P r}$ is the Prandtl number, and $N_{S c}$ is the Schmidt number. Note that the Sherwood number is determined from Equation (12-19) by using an analogy between heat transfer and mass transfer [Kre58]:

$$
N_{S h}=N_{N u}\left\{N_{P_{r}} \rightarrow N_{S c}\right\}
$$

\subsection{Heat Exchanger Models}

The containment spray system typically uses a heat exchanger to cool the liquid water recirculated from the sump pool (Figure 12-2) to the containment spray nozzles. There is only one combination of components in which a heat exchanger can appear, and this is along with a spray, tank, and pump. An example of such a system is given in Section 14.3.3.4.

One of four heat exchanger models can be used. These correspond to (a) a single-pass shell and Utube geometry, (b) a cross-flow geometry with hot-side tubes and cold-side shell, (c) a counterflow geometry, and (d) a parallel flow geometry. These models are specified in the HEX input block (see Section 14.3.3.5) using the keywords SHELL, CROSS, COUNTER, and PARALLEL, respectively. The heat exchangers are depicted in Figure 12-7. A nonmechanistic model with a constant userspecified hot-side temperature drop is also available by specification of USER and the temperature drop across the hot side "hxdelt." Throughout this section, the terminology in Figure 12-8 will be used.

The basis for modeling the four heat exchangers illustrated in Figure 12-7 is obtained from Reference Kay64. The important quantity to model for a heat exchanger is the hot-side outlet temperature. In this formulation it is expressed as a function of an effectiveness factor, the inlet hotand cold-side temperatures, the hot- and cold-side mass flow rates, and the liquid heat capacity. The hot-side flow rate and temperature are determined from the pump capacity and pool thermal conditions, respectively. The cold-side flow rate and temperature are input parameters "hxclmd" and "hxticl." The efficiency depends upon the heat exchanger type, effective surface area, and the overall heat transfer coefficient, which correspond to the input parameters "otype," "hxarea," and "hxcoef," respectively. Because heat exchanger designs vary greatly, no default parameters are provided.

The heat exchanger modeling is based on an effectiveness factor E defined as

$$
\begin{aligned}
E=\frac{q}{q_{\max }} & =\frac{\operatorname{Cap}_{h}\left(T_{h, i}-T_{h, o}\right)}{\operatorname{Cap}_{\min }\left(T_{h, i}-T_{c, i}\right)} \\
& =\frac{\operatorname{Cap}_{c}\left(T_{c, o}-T_{c, i}\right)}{\operatorname{Cap}_{\min }\left(T_{h, i}-T_{c, i}\right)}
\end{aligned}
$$




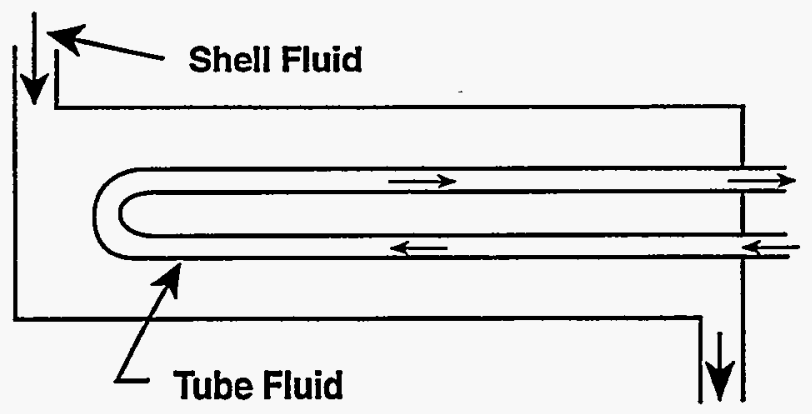

a) Single-pass Shell and U-tube Geometry

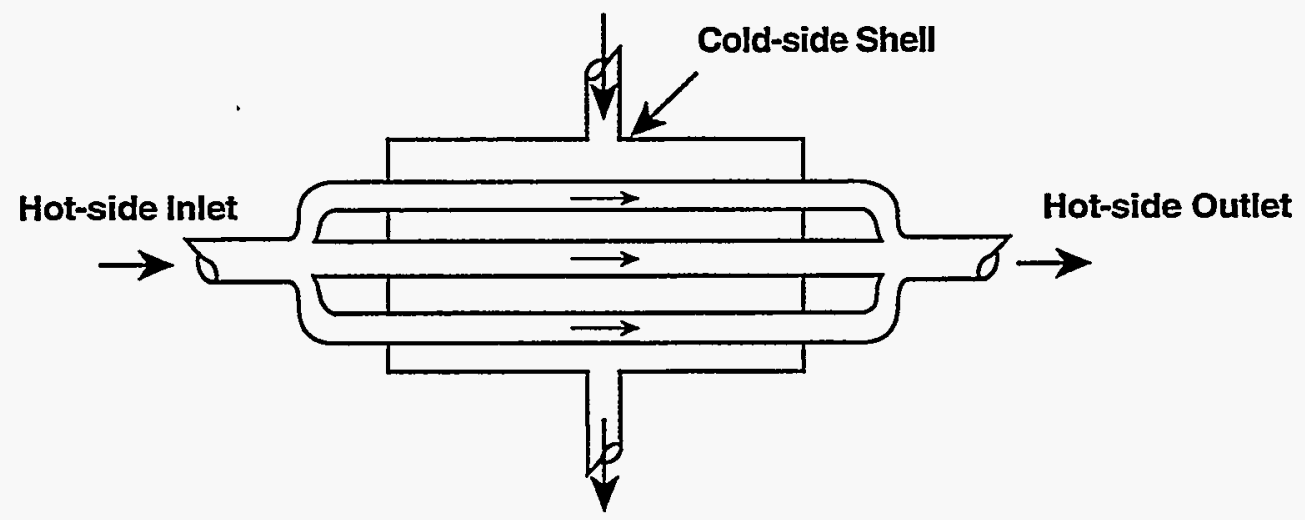

b) Cross-flow Geometry with Hot-side Tubes and Cold-side Shell

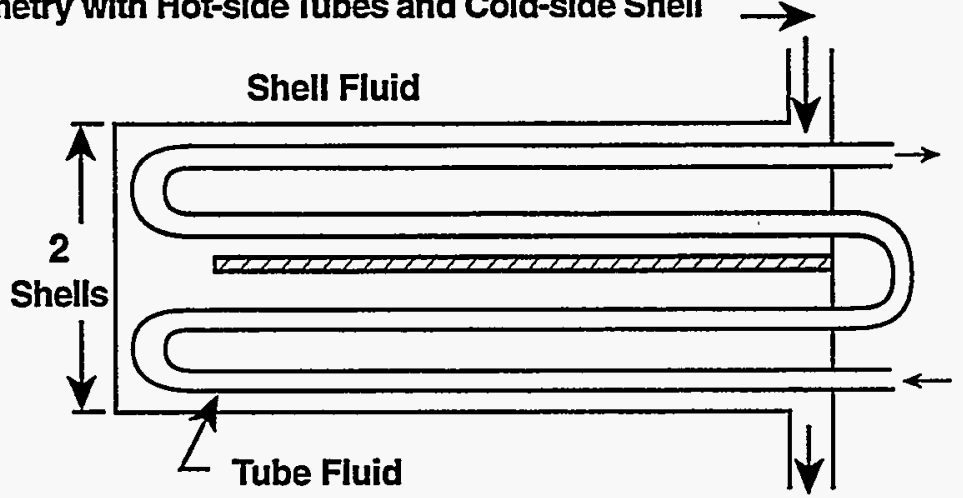

c) Counter-flow Geometry

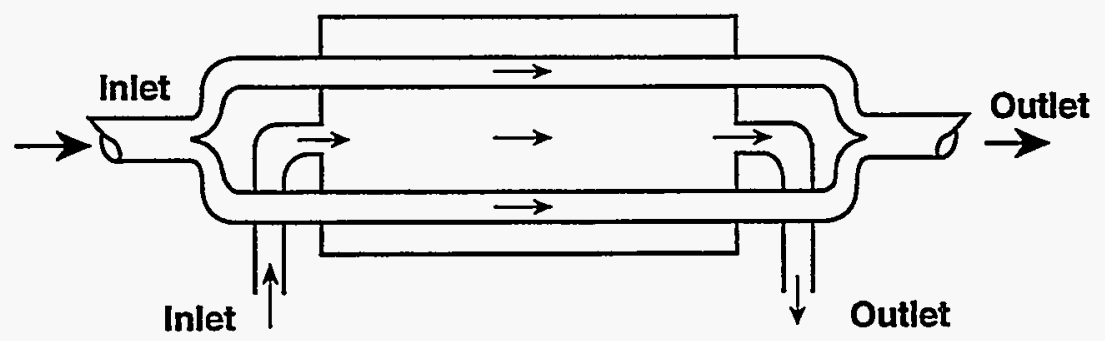

d) Parallel-flow Geometry

Note: Arrows show direction of fluid flow.

Figure 12-7. CONTAIN Heat Exchanger Models 


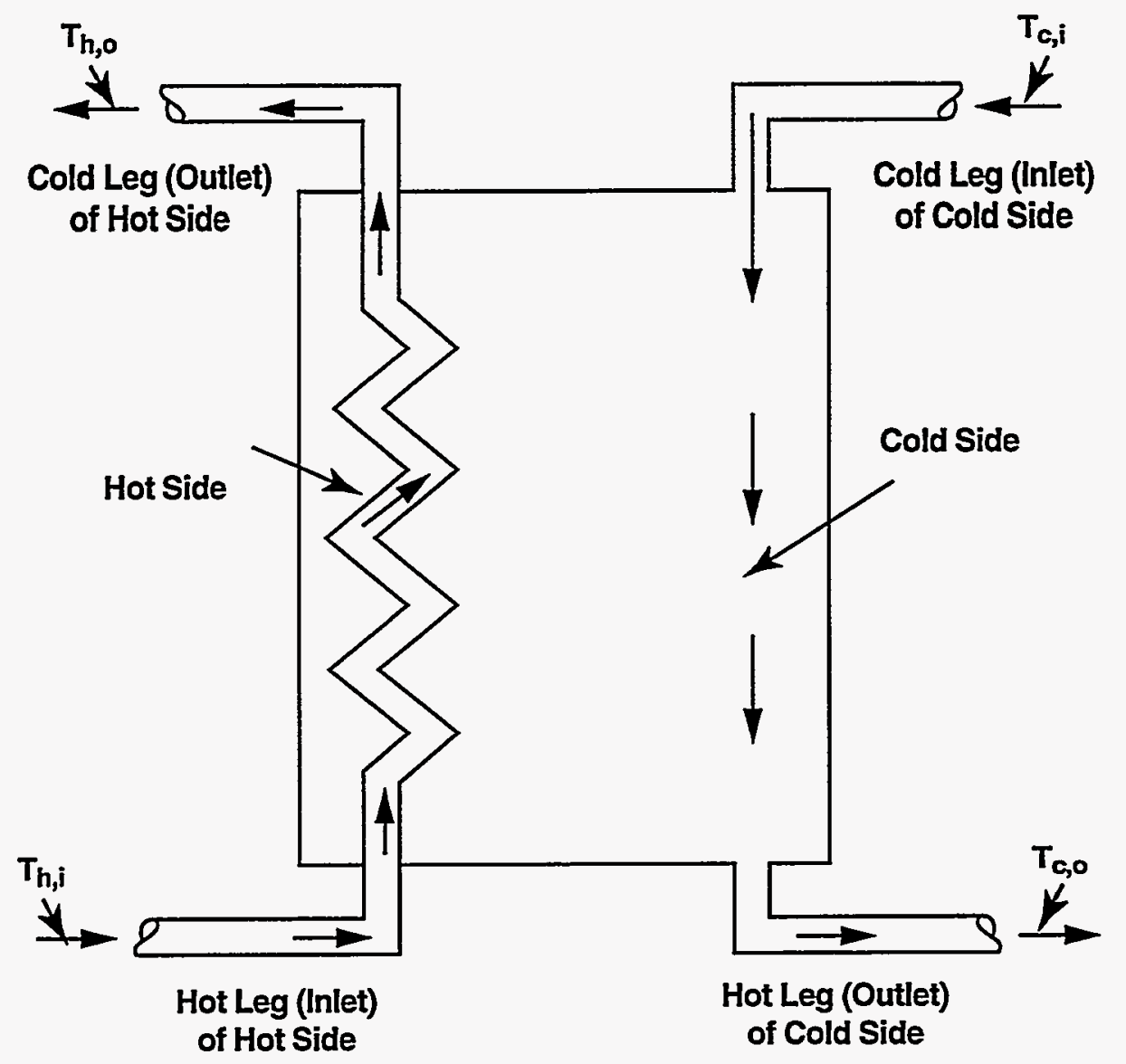

Note: Arrows indicate the direction of fluid flow.

Figure 12-8. Counterflow Heat Exchanger Representation Defining the Heat Exchanger Terminology Used in the Text 
where $q$ is the heat transfer rate, $q_{\max }$ is the maximum possible transfer rate, Cap is the hot-side fluid capacity rate $(\mathrm{J} / \mathrm{s}-\mathrm{K})$, defined as the product of the hot-side mass flow rate times the specific heat of the liquid, $T_{h, i}$ is the hot-side inlet temperature in Figure 12-8, $T_{h, o}$ is the hot-side outlet temperature, $\mathrm{Cap}_{\min }$ is the smaller of $\mathrm{Cap}_{\mathrm{h}}$ and $\mathrm{Cap}_{\mathrm{c}}, \mathrm{T}_{\mathrm{c}, \mathrm{i}}$ is the cold-side inlet temperature, $\mathrm{Cap}_{\mathrm{c}}$ is the cold-side fluid capacity rate, and $\mathrm{T}_{c, 0}$ is the cold-side outlet temperature.

It is to be noted that, given the operating conditions $T_{h, i}, T_{c, i}, C a p_{h}$, and Cap ${ }_{c}$, the efficiency E com-

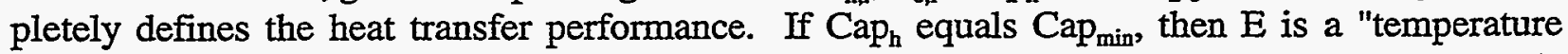
effectiveness" $\left(T_{h, i}-T_{h, o}\right) /\left(T_{h, i}-T_{c, i}\right)$ for cooling the hot fluid. But, if Cap $p_{c}$ equals Cap ${ }_{\min }$, then $E$ is the "temperature effectiveness" $\left(T_{c, 0}-T_{c, i}\right) /\left(T_{h, i}-T_{c, i}\right)$ for heating the cold fluid.

The temperature $T_{h, i}$ and hot-side mass flow rate are calculated elsewhere in CONTAIN and provided to this model. The temperature $T_{c, i}$ and the cold-side mass flow rate are specified by the user as "hxticl" and "hxclmd," respectively.

From Equation (12-22), the hot-side outlet temperature $T_{h, o}$ is given by

$$
T_{h, o}=T_{h, i}-\frac{E \operatorname{Cap}_{\min }\left(T_{h, i}-T_{c, i}\right)}{\operatorname{Cap}_{h}}
$$

The value of $T_{c, 0}$ can be similarly calculated from Equation (12-22).

For the simple nonmechanistic model, the user specifies a parameter "hxdelt" which is equal to the temperature drop $\Delta T_{h}$ across the hot side, i.e.,

$$
T_{h, o}=T_{h, i}-\Delta T_{h}
$$

Because $T_{b, j}$ is calculated by other ESF component models (for example, lower cell pool models), $T_{h, 0}$ can be easily calculated from $T_{h, i}$ and the $\Delta T_{h}$.

In general, it is possible to express the effectiveness of the heat exchanger as a function of the number of exchanger heat transfer units Ntu, the capacity-rate ratio $\mathrm{CR}$, and the flow arrangement. Ntu is defined as

$$
\mathrm{Ntu} \equiv \frac{\mathrm{A} \mathrm{h}}{\mathrm{Cap}_{\min }}
$$

where $A$ is the user-specified effective heat transfer area (the same transfer area as used in the definition of $\mathrm{h}$ ), and $\mathrm{h}$ is the user-specified overall heat transfer coefficient. 
The capacity-rate ratio $\mathrm{CR}$ is defined as

$$
\mathrm{CR}=\frac{\mathrm{Cap}_{\min }}{\mathrm{Cap}_{\max }}
$$

where Cap $\mathrm{max}_{\max }$ is the larger of $\mathrm{Cap}_{\mathrm{h}}$ and $\mathrm{Cap}_{\mathrm{c}}$.

The following sections give expressions from Reference Kay64 for the effectiveness $\mathrm{E}$ for the four types of heat exchangers shown in Figure 12-7.

Single-Pass Shell and U-Tube Effectiveness. The effectiveness factor $E$ for the heat exchanger with a single-pass shell and U-tube geometry (Figure 12-7a) is given by

$$
E=\frac{2}{\left\{[1+C R]+\sqrt{1+C^{2}}\left(1+e^{-\Gamma}\right) /\left(1-e^{-\Gamma}\right)\right\}}
$$

where

$$
\Gamma=\mathrm{Ntu} \sqrt{1+\mathrm{CR}^{2}}
$$

Equation (12-25) was derived for one shell pass and two tube passes.

Cross-Flow Heat Exchanger Effectiveness. The effectiveness $E$ for a cross-flow heat exchanger with hot-side tubes and cold-side shell (Figure 12-7b) depends on whether $\mathrm{Cap}_{\max }=\mathrm{Cap}_{\mathrm{h}}$ or cap $\mathrm{pax}_{\max }=\mathrm{Cap}_{\mathrm{c}}$. If $\mathrm{Cap}_{\max }=\mathrm{Cap}_{\mathrm{h}}$, then $\mathrm{E}$ is given by

$$
E=1-\exp (-\Gamma)
$$

where $\Gamma=C R[1-\exp (-N t u C R)]$. Otherwise, $E$ is given by

$$
E=\operatorname{CR}[1-\exp (-\Gamma)]
$$

where $\Gamma=C R[1-\exp (-N t u)]$. This model assumes that the shell fluid is mixed. 
Counterflow Heat Exchanger Effectiveness. The counterflow heat exchanger effectiveness $E$ (Figure $12-7 \mathrm{c}$ ) is given by

$$
E=\frac{1-\exp [-\mathrm{Ntu}(1-\mathrm{CR})]}{1-\mathrm{CR} \exp [-\mathrm{Ntu}(1-\mathrm{CR})]}
$$

Parallel Flow Heat Exchanger Effectiveness. The effectiveness $E$ for the parallel flow heat exchanger (Figure 12-7d) is modeled in two regimes depending on the value of CR. If $|C R-1| \leq$ 0.002 , then $E$ is given by

$$
E=\frac{N t u}{1+N t u}
$$

Otherwise, $\mathrm{E}$ is given by

$$
E=\frac{1-\exp [-\mathrm{Ntu}(1+\mathrm{CR})]}{1+\mathrm{CR}}
$$

\subsection{Liquid Transport System Components}

The models for the liquid transport system components used with ESFs or on a stand-alone basis are described in this section. Models for pumps, valves, orifices, pipes, water storage tanks, and pool overflows are included. These components provide sources and sinks of coolant for the ESF models (fan coolers, ice condensers, and containment sprays). Certain liquid transport components can also function independently from the ESFs. Note that the flow through all components except the WST and overflow model is drawn from the bottom of the pool in the inlet cell.

\subsubsection{Pumps}

Mechanical pumps are used to circulate the containment building sump pool water up to the containment spray headers. The pump can be used in one of two types of systems:

- When used by itself, the pump controls the flow of coolant liquid from one cell pool to another. The flow is directed from cell "iclin" to cell "iclout."

- When used in conjunction with a containment spray, the pump controls the flow of recirculated water from a pool to the sprays. When it is used in this mode in conjunction with a WST, the recirculation flow does not start until the WST supply is exhausted. The user must specify "pmpmdt," the pump flow rate. A sample input is shown in Section 14.3.3.4. 
Note that the user-specified pump mass flow rate "pmpmdt" is constant and is the only input required in the PUMP input block shown in Section 14.3.3.6.

\subsubsection{Valves}

The valve model input is specified in the VALVE input block described in Section 14.3.3.7. It is used only by itself in a single-component system to control transfer of coolant liquid from the pool of a donor cell "iclin" to that of a recipient cell "iclout." Two types of control are available. If the keyword PRESSURE is specified, the flow path becomes in effect a rupture disk. When the difference in hydraulic head between the donor cell and the recipient cell exceeds the input pressure difference "valopp," the flow path is opened, and the flow is characterized by the flow area "valvar" and a flow loss coefficient "valvkf," which should be equal to the sum of the Moody friction factor, any form loss factors, and entrance and exit loss factors. Alternatively, the keyword TIMES indicates that the valve is to open and close at five user-specified times given as input into the "valtim" array. The times are alternating opening and closing times with the valve assumed to be initially closed and the first time specified assumed to be an opening time. If the valve is to be initially open, the first time entry in the array should be the problem start time. No defaults are available for valve input parameters.

The mass flow rate for a given pressure difference is determined from

$$
\Delta \mathrm{P}=\mathrm{K} \frac{(\mathrm{W} / \mathrm{A})^{2}}{2 \rho}
$$

where $\Delta P$ is the pressure difference, including liquid water head, $K$ is the flow loss coefficient, $W$ is the mass flow rate, $\mathrm{A}$ is the flow area, and $\rho$ is the liquid water density.

\subsubsection{Orifices}

An orifice is modeled by specifying the keyword ORIFICE and the required input is described in Section 14.3.3.8. This is a model for a square-edged orifice component used only by itself in a single-component system to control the transfer of coolant liquid from a pool in the donor cell "iclin" to that of the recipient cell "iclout." The flow is pressure- and gravity-driven, with the driving pressure calculated from the gas pressures and the elevation difference between the bottom of the pools in the two cells. (The flow is assumed to occur between the bottoms of the pools. Thus, the flow connection cannot be uncovered and the flow interrupted while there is still water in the donor pool.) The flow is assumed to be incompressible and to occur at a steady-state rate. A flow resistance characteristic of an orifice is used. [Flo79] Input parameters that describe the geometry of the orifice are the orifice minimum diameter "orifid" and the ratio of the orifice minimum diameter to the free stream diameter "orifdr." No defaults are provided for these parameters.

The flow equation is similar to Equation (12-33) except that the loss coefficient is based on a squared-edge orifice loss coefficient that depends on (a) the ratio of the orifice diameter to the upstream pipe diameters and (b) the Reynolds number. 


\subsubsection{Pipes}

The keyword PIPE with the input parameters described in Section 14.3.3.9 invokes the pipe model. The pipe component is used like the valve model (see Equation (12-33)), except that the flow resistance is characteristic of pipe flow rather than valve flow. [Flo79] The geometry of the pipe is described by input parameters defining the pipe length "pipel," the inside diameter "pipeid," and a total irreversible loss factor "pipekf," which should be equal to the sum of the Moody friction factor, entrance and exit loss factors, and any other form loss factors.

\subsubsection{Water Storage Tanks}

The WST is used to supply water for the containment spray. It is connected with the containment spray system through valves and pumps to the containment spray nozzles. The WST provides an initial amount of water to supply the containment spray. When the tank inventory is exhausted, the containment spray system can acquire its water from the pool in cell "iclin" in the recirculation mode (see Figure 12-2).

The user should specify the initial mass of WST water "tnkmas," the water temperature "tnktem," and the constant mass flow rate "tnkflo" at which the water is delivered to the containment spray after the keyword TANK, as described in Section 14.3.3.10.

\subsubsection{Liquid Transport Overflow}

The engineered systems OVERFLOW keyword described in Section 14.3.3.11 invokes the pool overflow model. (This keyword should not be confused with the cell OVERFLOW keyword that defines the overflow cell for condensate film runoff and aerosol mesh losses.) Three required input parameters--"iclfrm," "iclto," and "flovht"--indicate the overflow cell number, the destination cell number, and the height relative to the bottom of the pool at which overflow occurs, respectively. In the pool overflow model, all coolant above the overflow height "flovht" in the cell "iclfrm" is diverted to the destination cell "iclto," provided the transfer is in the positive direction with respect to the pressure head. Note that this pressure head criterion takes into account the gas pressures in the respective cells, as well as the pool depths. Overflow may be included along with any allowable combination of components making up an engineered system. 


\subsection{USER GUIDANCE AND PRACTICAL AND CAUTIONARY ADVICE}

Much of this manual has focused on what CONTAIN is designed to do and how to implement its features. The analyst also needs to understand the types of problems for which the code is suited or not suited. One of the purposes of this section is to identify and briefly discuss some of these limitations. Another purpose of this section is to offer some practical suggestions about how to run the code. These comments are based on a substantial body of experience gained in applying CONTAIN to a wide variety of severe accident scenarios. In some cases, the suggestions are oriented towards increasing the efficiency of the process of producing useful calculations. In other cases, the idea is to caution against avoidable errors or pitfalls that have been encountered in the past. Finally, there are some suggestions about how to overcome some of the limitations of the code by simulating an effect not explicitly modeled. Such suggestions apply to manipulations of the code through input; modifications to the code itself lie beyond the scope of this document. With respect to code limitations, the CONTAIN code has also been peer reviewed by an independent committee. The report of this committee [Boy95] provides a more formal and detailed documentation of the limitations in the code.

This chapter is organized as follows. Section 13.1 addresses general limitations that are inherent in the CONTAIN modeling approach. These limitations are not expected to change with near-term improvements to the code. Section 13.2 is concerned with more detailed discussions of assumptions and approximations in individual models. Many of these deficiencies are expected to be resolved in future modifications to the code.

Section 13.3 lists suggestions that should be of use to the analyst in running the code and in getting as much useful information from it as possible. Problems and pitfalls to avoid are discussed, and there are also some ideas on how to make the code do things not obviously included in its nominal range of capabilities. Similar suggestions are made throughout this document in the context of the detailed model descriptions and the input descriptions.

\subsection{Key Simplifying Assumptions in CONTAIN}

Well-Mixed Atmosphere. The atmosphere physics modeling assumes that the atmosphere within a given cell is well-mixed. The atmosphere is taken to be uniform both in temperature and in material concentrations. This assumption may not be appropriate in many cases. During some accident scenarios, it is possible that the cell atmospheres may experience thermal stratification and/or concentration gradients. Guidelines for dealing with the presence of stratifications are discussed in Section 13.3.1.3.

Even when the above guidelines are followed, the well-mixed assumption precludes proper treatment of the entrainment of surrounding gas into nearly free jets and plumes that are much smaller in diameter than the typical cell dimension. These jets and plumes become well-mixed with the surrounding gases in the cell in which they are introduced. In contrast, the entrainment of surrounding gas into a jet or plume is expected to be more gradual, and result in a linear growth of the jet or plume diameter with distance. The well-mixed assumption may thus distort considerably the distribution of the buoyancy effects resulting from a buoyant jet or plume. 
Neglect of Momentum Convection. The neglect of momentum convection in the CONTAIN cells means that directional effects related to the convection cannot be treated. For example, the tendency of a jet to continue in same direction in the absence of a pressure gradient in that direction cannot be modeled. The neglect of momentum convection also means that the effects of buoyant jet convection on the stability of the resulting stratification cannot be modeled (see, e.g., Reference Pet94). As a consequence stratifications modeled in CONTAIN may be predicted to be too stable.

Feedback on Primary System Neglected. The code is designed to be an analytical tool for assessing accident events occurring within the containment of nuclear power plants. Because the primary system is not generally modeled, there is no feedback in terms of how events and conditions in the containment will affect conditions in the primary system. Such effects could be important if, for example, the pressure buildup in the containment building could influence the rate at which coolant, hydrogen, aerosols, or radionuclides are lost from a breach in the primary system. Although nothing prevents the user from defining additional cells to represent primary system volumes, the models were not developed with primary system conditions in mind, and it would be up to the user to assess their validity for such applications.

\subsection{Specific Model Limitations}

Specific model limitations and assumptions are discussed below. The organization of the discussions is as follows:

- Atmosphere/Pool Thermodynamics and Intercell Flow

- Lower Cell Modeling,

- Direct Containment Heating (DCH) Modeling,

- Aerosol Modeling,

- Fission Product Modeling,

- Combustion Modeling,

- Heat Transfer Modeling,

- Engineered Safety Feature (ESF) Modeling, and

- Calculational Sequence Effects.

\subsubsection{Atmosphere/Pool Thermodynamics and Intercell Flow}

No Deposition or Heat Loss in Flow Paths. Currently no provision exists for removal of mass or energy within a flow path. The aerosols and fission products cannot be deposited on the flow path walls. Sensible heat from the gas, furthermore, cannot be transferred to the flow path walls. The flow rate may be determined to some extent by viscous losses. In this case, any heating resulting from viscous effects is assumed to be retained in the flowing materials. If either the flow path inventories or heating effects are important, they must be modeled through an intervening cell.

Flow Paths as Virtual Boundaries Between Cells. Flow paths may be used to nodalize an open volume. However, the user should be aware of the modeling limitations in this case. First, not all processes important to transport between cells are modeled, and some of these, such as countercurrent flow and turbulent diffusion, may be more important for large flow areas than for 
small flow areas. Also, in the momentum equation it is assumed that the momentum and kinetic energy of the flow is dissipated completely in the downstream cell. In reality, the dissipation may occur over a number of cells; that is, momentum convection may be important. Because of this assumption, care should be used in setting the flow coefficient to obtain the proper overall flow, particularly if the nodalization is fine. (If the flow coefficient is held constant for each virtual boundary while the nodalization is refined without limit, the gas transport in the problem will go to zero!)

\subsubsection{Lower Cell Modeling}

No Conduction Heat Transfer to Walls in Lower Cell. Except for the pool, there is no heat transfer from the lower cell layers to the surrounding wall structures via conduction through these layers and the structures in contact with them. Heat transfer from the pool to structures is described in Section 10.1.2.

Fuel Coolant Interactions (FCIs). There is no mechanistic model for the interaction of core melt with water in the cavity (see 13.2.3 below). While an explosive FCI model may not be possible or even desirable, a model for the coarse mixing and heat transfer phase of FCIs could perhaps be included. The user can choose arbitrarily large surface areas between the core debris layer and the overlying water pool to simulate this effect to some degree; see Section 13.3.3.6.

\subsubsection{DCH Modeling}

The most significant known limitations of the DCH model in CONTAIN are summarized below. A more in-depth discussion of the modeling and its limitations is provided in Chapter 6, detailed guidance on the use of the DCH models is given in Section 13.3.2, and Reference Wil95 provides the validation basis for the CONTAIN DCH models and their use.

Debris Interaction With Water. There is no mechanistic model for the interaction of dispersed debris with water in the cavity. Excluding the possibility of a steam explosion, some of the effects of debris/water interactions can be represented parametrically through appropriate user input, but the results are heavily influenced by the user-controlled input assumptions. Guidance on simulating the effects of debris-water interactions is provided in Section 13.3.2.

Debris-Gas Slip. There is no mechanistic model for calculating the debris-gas slip parameter.

Choked Flow Model. The model for choked flow when debris is present is based upon applying the choking criterion to the gas flow only, and then applying the user-specified slip factors to obtain the total flow and debris transport rates as described in Section 6.2.6. This model does not capture some of the complexities involved in actual two-phase choked flow. One implication is that the model may allow unrealistically high gas and debris velocities if choking develops when slip factors are specified to be close to unity. The reason is that, under DCH conditions, high temperatures and low gas molecular weights may result in very high sound speeds in the gas phase, which in turn implies very high debris velocities under choked flow conditions if the slip factor is small. For the similar reasons, the model may underpredict the tendency for flow to become choked; i.e., the code may use 
the model for nonchoked flow when choking would actually occur. No detailed investigation of the importance of these limitations of the model has been made, but it is likely to be less important for the prediction of containment pressures than it may be for the prediction of the extent of cavity pressurization during debris dispersal.

Momentum-Driven Transport. Debris is only transported by flow with the gas. Therefore, advection-driven transport of debris, such as through a cell in which the entrance and exit flow paths are aligned, is not represented. This is believed to be an important transport mechanism in at least some containment geometries.

Trapping Limitations. A number of potentially important processes are not represented in the trapping model, such as drop splashing, surface ablation, and film interactions. Although the TOF/KU model does incorporate a number of modeling sophistications, the flight path of particles is not actually tracked in the CONTAIN model. Instead, the model relies upon estimates of flight paths based on inflow and outflow debris/gas velocities.

Non-Airborne Debris Interactions. When debris is trapped it is placed into the non-airborne debris field. Debris in this field is allowed to continue participating in DCH; however, the model does not include appropriate mechanistic models for the non-airborne field. The models used are the same ones used for the airborne fields, which are known to be incorrect because the geometry of the nonairborne debris is different than that of airborne drops. There is also no mechanistic model for estimating the velocity of the gas relative to that of the non-airborne debris field, other than the default assumption that this velocity is equal to the mean gas flow velocity through the cell. The complexities of non-airborne debris interactions are represented parametrically with an effective nonairborne debris diameter. Guidance on choosing this parameter is provided in Section 13.3.2.

Drop-Drop Interactions. The interaction of airborne debris drops with other airborne drops is not modeled. The importance of this effect is unknown. No plans are underway to address this limitation.

Concrete Ablation. There is no model for the ablation of concrete by the debris jet ejected from the reactor pressure vessel (RPV).

RPV and Cavity Models. To date, assessment of the RPV, cavity entrainment, and particle size models against experiments with high-temperature melts is limited. There appears to be a tendency for melt ejection from the RPV and gas blowthrough to occur too early in the calculation, and it was not possible to match closely both the debris fraction dispersed and the timing of debris dispersal relative to the steam blowdown in the cases considered. All the cavity entrainment rate models require the user to specify a cavity constant, $\mathrm{K}_{c}$, for which there is no mechanistic model. It has not been established whether a single value of $\mathrm{K}_{c}$ could be found that would give reasonable results for a wide range of experimental conditions using high-temperature melts. For experiments using lowtemperature melts, a considerable range of experimental conditions was successfully correlated using a single value of $\mathrm{K}_{\mathrm{c}}$ except that it could not be established whether the correlations adequately accounted for the effects of geometric scale. [Wil96] 


\subsubsection{Aerosol Modeling}

Assumption That All Aerosol Particles of a Given Size Have the Same Water Content. The aerosol module assumes that all particles within a given size class have the same composition. To do otherwise would require the code to maintain a three-dimensional mesh of composition/size information, in place of the current two-dimensional mesh, with significant increases in complexity and computational cost. However, under certain conditions involving condensation/evaporation, significant error can result in the aerosol calculation. Suppose, for example, that gas containing water aerosols flows into a cell that contains solid aerosols, and suppose further that the cell atmosphere is superheated so that the water aerosols quickly evaporate before they agglomerate significantly with the solids. In reality, the evaporation of the water would have no effect upon the solids. In CONTAIN, if the solid and water aerosol size distributions overlap, all particles in the overlapping size region will be assumed to have the average composition for that size and thus will be assumed to be part water and part solid. When the water is evaporated, the solid residue will therefore be shifted down in size. In one extreme case of this kind, an aerosol component with a mass median diameter of $10 \mu \mathrm{m}$ was found to be shifted down to the one- $\mu \mathrm{m}$ size range.

A somewhat similar problem can arise when a large amount of water first condenses upon solid aerosols, and subsequently re-evaporates. Since condensation acts to make the smaller particles grow more (in a relative sense) than the large ones, it acts to collapse the size spectrum into a smaller number of size classes. The resulting loss of size resolution is irreversible, and the initial size distribution is not fully recovered when the water evaporates. In some cases, some of the aerosol particles will be calculated to be smaller than the smallest allowed size after evaporation and will be lost from the mesh unless the TRAPUNFL keyword is specified in the global AEROSOL block (see Sections 7.1, 14.2.5, and 14.3.1.12).

No Model for Turbulent Energy Dissipation Rate. The aerosol agglomeration model includes terms for both the inertial and shear components of turbulent agglomeration. The agglomeration rates predicted depend upon the turbulent energy dissipation rate, $\varepsilon_{\mathrm{q}}$, in Equation (7-9). However, the code has no model for calculating $\varepsilon_{\mathrm{v}}$. The default value of this parameter is $0.001 \mathrm{~m}^{2} / \mathrm{s}^{3}$. The user can specify a different value through the input parameter TURBDS in the global AEROSOL block. This value will then be used in all cells and at all times during the calculation. There is not much guidance available for specifying this parameter. The default value is thought to correspond to rather quiescent conditions. Appendix E of Reference Lip85 describes a model for estimating an upper limit to $\varepsilon_{\mathrm{t}}$ assuming that only natural convection driven by temperature differences is generating turbulence. An upper limit of about $0.02 \mathrm{~m}^{2} / \mathrm{s}^{3}$ was estimated for conditions typical of station blackout accidents over long time periods. Much higher values could exist for short times during and immediately following energetic events such as blowdown of the primary system, hydrogen burns, and DCH events.

Aerosol-Atmosphere Thermal Equilibrium. The aerosols are assumed to be at the same temperature as the atmosphere. Because the mass of the suspended solid aerosols is assumed to be small compared with the total atmosphere mass, such aerosols are assumed not to contribute to the total atmosphere heat capacity in the model. This simplifying assumption is expected to be valid for most applications, but in extreme cases it could lead to energy conservation errors. 
No Aerosol Deposition in Flow Paths. Current models do not allow for aerosol deposition processes in flow paths. Deposition occurs only in cells. It is possible to model a flow path as a cell, in which case the aerosol behavior would be calculated. However, effects due to bulk movement of the gases in the path, such as impaction of aerosols on the surfaces of the flow path, would not be accounted for. Also, the efficiency of the flow solvers may be adversely affected by a relatively small cell.

Effect of Aerosols on Thermal Hydraulics. Aerosol masses are ignored in the flow density, in gravitational head terms in the flow equations, in the Grashof number for natural convection heat transfer, and in the inerting criteria for hydrogen burns. Aerosols, for example, could significantly affect natural convection near cold surfaces, as a result of fog formation.

No Insulating Effect Due to Aerosol Plateout and Buildup. As aerosols plate out on surfaces, they may well act as an insulating blanket. In dry environments, aerosol deposits have been shown to be light and fluffy, so that the aerosol layer has a high void fraction and, thus, a high gas fraction. The conductivity of the layer, therefore, could be quite low. In many light water reactor (LWR) accident scenarios, condensed water films are expected to substantially reduce this effect. Nevertheless, the insulating effects of aerosol deposits on surfaces are not modeled.

Aerosol Resuspension. There is no model for aerosol resuspension, whether from structure surfaces or from the cavity.

Electrical Charge. Electrical charge effects on aerosol dynamics are not modeled.

Aerosol Material Density. The density is the same for all aerosol components, regardless of the actual material. The user does have the ability to explore the effect of density by specifying the fixed density as an input parameter.

Particle Shape Effects. There is no mechanistic model for particle shape effects. The user can explore the sensitivity of shape effects by supplying various shape factors.

Nucleation. There is no nucleation model.

\subsubsection{Fission Product Modeling}

Chemistry. There are no mechanistic models for fission product chemistry other than the effect of sprays on iodine. A parametric workaround may be possible with the targeted release and acceptance model. See Section 13.3.4.2 for an example of the use of this model.

No Spray Washdown of Fission Products from Walls. If containment sprays are activated, fission products that are deposited on structure surfaces might reasonably be expected to wash off these surfaces. Even though a parametric model for washdown of fission products with condensate runoff is provided, such a model does not exist for spray washoff of fission products. This might, however, be simulated nonmechanistically with the targeted release formalism, i.e., by using it to transfer fission products from the wall to the pool (or to other locations). 
Gamma and Beta Heating Effects. The heat given off by many radionuclides as they decay often includes a significant fraction of gamma-ray emission energy. Much of this gamma energy is absorbed in solid objects within containment, such as walls and equipment. Relatively little would be absorbed by the gases present. Therefore, a significant fraction of the energy given off by fission products decaying in the atmosphere will not be absorbed by the atmosphere, as is assumed in the modeling. Similarly, some of the gamma rays from fission products deposited on structure surfaces would propagate away from the surface and be absorbed by other structures or be absorbed well below the structure surface, not in the surface node as is assumed in CONTAIN. Furthermore, when fission products are deposited upon a structure surface, a significant fraction of their beta energy may still be deposited in the cell atmosphere rather than in the structure. These effects are not modeled in the LWR versions of CONTAIN discussed in this document. The implications of some of these effects are explored further in Reference Wil87a. A model of these effects is present in the heavy water reactor version of CONTAIN. [Bi193]

No Scrubbing of Gaseous Fission Products in Pools. The suppression pool safety relief valve and vent models evaluate scrubbing of aerosols, and hence fission products hosted to aerosols. However, mechanistic modeling is not provided for the scrubbing of vapor phase fission products such as elemental iodine and other gaseous iodine species. To a limited degree, this effect can be simulated using targeted release and acceptance; see Section 13.3.4.2.

\subsubsection{Combustion Modeling}

The CONTAIN models for the combustion of hydrogen and carbon monoxide are intended to represent processes that are sufficiently slow that the containment loading effects may be described in terms of quasi-static pressurization only. There are no models for dynamic loading processes such as can be produced by detonations and accelerated flames. The code does not attempt to determine whether detonations or flame acceleration should be considered possible; however, the atmospheric conditions (gas composition, pressure, and temperature) reported by the code can be employed by the user in a side calculation to assess whether these phenomena could occur in a given scenario.

The discussion of the burn models given in this section applies primarily to scenarios that do not involve DCH. Guidance for the use of the combustion models in DCH calculations is included in Section 13.3.2.2.2.

All the combustion models are controlled by concentration thresholds, temperature thresholds, and combustion rates which, by default, are either given by correlations or set equal to fixed values. The user can override the defaults in all cases.

13.2.6.1 Deflagration Model. The CONTAIN model for deflagrations of gas mixtures including hydrogen (and carbon monoxide) is based upon the model in the HECTR 1.8 code [Din86, Won88, Pon90] and is believed to be a reasonably good one for many purposes. However, it does have some limitations that can contribute to uncertainties in calculations that involve hydrogen burns, and these limitations will be summarized here. 
Temperature Dependence of Burn Parameters. The default deflagration model is based upon correlations that give the ignition thresholds, combustion completeness, and flame speed (and hence burn time) as a function of the atmospheric composition. However, the initial temperature is not included in these correlations. This is a potentially important limitation because the correlations are based upon experiments performed with premixed gases [Rat85, Ben84, Kum84, Tor83, Mar86] in which the initial temperatures were less than $400 \mathrm{~K}$. Flammability thresholds are expected to decrease as the initial temperature increases, and both the completeness of combustion and the rate of combustion are expected to increase at elevated temperatures.

A review ${ }^{1}$ of the data for the lean flammability thresholds for upward and downward flame propagation at $293 \mathrm{~K}$ indicate that they are about $4.1 \%$ and $9 \%$ hydrogen, respectively but decrease to about $2 \%$ and $4.5 \%$, respectively, at $773 \mathrm{~K}$. In addition, the inerting effects of steam and other diluents may decrease as the temperature increases. This review also suggests that combustion completeness increases approximately linearly from small values at the upward flammability threshold to near $100 \%$ at concentrations somewhat less than the downward flammability threshold, and the temperature dependence cited for the flammability thresholds then implies a corresponding increase in burn completeness with increasing initial temperature. Data of Liu and McFarlane [Lui83] shown an increase in laminar flame velocities of a factor of 3 over a 300-K temperature range.

The user should be aware of these trends in applications involving hydrogen burns in which the initial gas temperatures are greater than $400 \mathrm{~K}$. The user may override the default values of the controlling parameters, and sensitivity studies utilizing this capability are warranted in any applications sensitive to the trends summarized here.

Effect of Combustible Gas Influx. Another limitation of the model is that the correlations are applied to estimate the flame speed and the burn completeness based upon the atmosphere conditions at the start of the burn. If there is a rapid influx of hydrogen or carbon monoxide during the burn, this gas will participate in the burn; however, the completeness and burn time are not readjusted to allow for the newly introduced combustible gas.

Ignition Criteria. The default ignition criteria in CONTAIN represent a compromise that is not likely to be suitable for every scenario potentially of interest, especially with respect to the minimum hydrogen concentration for ignition (7\% is the default). With strong ignition sources, such as when igniters provide a deliberate ignition source, it is likely that ignition can occur at lower concentrations, 4.5-5.5\%. [Sta92] In the analyses performed for the NUREG-1150 [NRC90] probabilistic risk assessment of the Sequoyah plant, it was assumed that combustion would initiate when hydrogen concentrations reached 5.5\% if igniters were on. [Gre90] Note, however, that the actual ignition criteria should apply to the local concentration close to the ignition source, while the CONTAIN code necessarily uses the average concentration for the cell in applying its criteria. Depending upon the relative locations of hydrogen sources and ignition sources, the average

'D. W. Stamps, Sandia National Laboratories, Albuquerque, NM, letter report to F. Eltawila, USNRC, with title "Assessment of the CONTAIN Combustion Models," December 23, 1992. 
concentration in the cell may be higher or lower than the local concentrations that actually control ignition.

If deliberate ignition sources are not present, the situation is much less clear. If AC power is available, it is customary to assume that chance sparking will still provide an ignition source somewhere within the containment; see, e.g., Reference Gre90. However, the timing of ignition is uncertain in part because of uncertainty in the timing of whatever provides the ignition source, and in part because the energy required to ignite the mixture is a strong function of the gas composition. Hence a weak spark source might fail to ignite a mixture near the lean flammability limit, but could ignite a mixture with higher combustible gas content. In the Three-Mile Island Unit 2 (TMI-2) accident, a hydrogen burn initiated with an estimated hydrogen mole fraction of about 0.08 . There were no deliberate ignition sources in this accident but $\mathrm{AC}$ power was available and steam mole fractions were low, tending to favor ignition.

If neither a deliberate ignition source nor AC power is available, initiation of combustion is especially uncertain and may not occur at all. In the NUREG-1150 analysis of station blackout accidents in the Grand Gulf plant, [Bro90] for example, it was assumed that the probability of ignition was only about 0.2 for hydrogen mole fractions of $0.04-0.08$, rising to about 0.5 for mole fractions 20.16 . Large uncertainties were assigned to these estimates, and they apply only to the specific scenarios and plant considered. For scenarios of this type, the only safe way to bound the problem would be to assume that ignition could occur at any concentration up to the maximum that corresponds to the total hydrogen input to the problem, and also include the case where ignition does not occur at all unless an identifiable ignition source is provided (e.g., power recovery, or perhaps hot core debris at the time of vessel breach).

In terms of containment loads, assuming a low ignition threshold is generally nonconservative, since a low threshold prevents development of higher hydrogen concentrations that could result in stronger burns. By this criterion, the CONTAIN default threshold of $7 \%$ can be nonconservative except for scenarios with operating igniters. If prediction of loads is an important goal of the intended application, sensitivity studies with higher ignition thresholds may be warranted. Alternatively, the TACTIV keyword may be used to suppress premature deflagrations; see Section 14.3.1.7 for details. Section 9.1.1 provides additional discussion of the ignition criteria and the supporting data base.

Flame Speed. The flame speed can have a significant effect upon the maximum containment and pressures calculated for a hydrogen burn because, when the flame speed is low, atmosphere-structure heat transfer during the burn can mitigate the pressure and temperature rises to some degree. The event summary file TAPE21 includes information about the time, location, and duration of all deflagrations occurring during a calculation; by checking the burn duration, the user can estimate whether the calculated loading is likely to be significantly reduced by a low flame speed.

The CONTAIN flame speed correlations are taken from the work of Wong, [Won88] who reports that the correlations agree with the data to within $\pm 40 \%$, with the error being smaller if some cases with low flame speeds $(<2 \mathrm{~m} / \mathrm{s})$ are not included. The general tendency was for the correlation to either predict the correct order of magnitude of the flame speed or to overpredict the flame speed. There was some tendency for the flame speed to increase with increasing scale, an effect that is not 
included in the model. The available data are sparse in some regimes of parameter space; e.g., for conditions with high hydrogen concentrations and low to moderate steam concentrations. It should also be noted that the formula used for estimating flame speeds for mixtures with substantial carbon monoxide (see Section 9.1.2) is based upon an analogy with the formula for estimating flammability limits; there is no experimental basis for its application to the flame speed.

The experiments reviewed in Reference Won88 were not performed in geometries particularly favorable to flame acceleration or deflagration-to-detonation transition (DDT). Flame acceleration and DDT are more likely to occur in channelized geometries than in open geometries, and the presence of obstacles that induce turbulence by obstructing part of the flow can substantially enhance the likelihood of flame acceleration and DDT. Flame acceleration refers to a combustion mode in which combustion propagates at a velocity much greater than the normal deflagration propagation rate; this velocity may approach, but not exceed, the speed of sound in the medium in advance of the flame front. Accelerated flames can result in important dynamic loading of containment structures. The peak dynamic pressures are generally less than for true detonations, but the integrated pressuretime history (i.e., the impulse) may be comparable to the detonation impulse.

As noted previously, dynamic loads resulting from these phenomena are not modeled in CONTAIN. However, flame acceleration implies very short combustion times with minimal mitigation by heat transfer, which may make even the quasi-static load higher than is calculated for a normal deflagration burn time. This effect can be simulated in CONTAIN by specifying very short burn times, or very high values of the flame speed. Burn times of $<1 \mathrm{~s}$ at containment scale, or flame speeds $>20 \mathrm{~m} / \mathrm{s}$, are probably adequate to capture this effect, although actual accelerated flame speeds are of the order of hundreds of meters per second. Geometries that include interconnected compartments can be conducive to flame acceleration, which has been reported to occur in such geometries with hydrogen concentrations as low as 10\%. [Kan92] Tieszen et al. [Tie93] have reviewed the available information on hydrogen behavior and concluded that, given favorable geometries, flame acceleration should not be ruled out for any gas compositions that lie within the downward flame propagation limits ( $\sim 9 \%$ hydrogen).

Default Characteristic Lengths. Note that default characteristic lengths for hydrogen burns are based on the initial gas volume specified by the user and are not allowed to change as the gas volume changes, for example, from changes in the pool volume. The characteristic length can also be specified through input:

Burn Completeness. When prediction of containment loads (pressure or thermal) is of interest, the burn completeness is of importance because the energy release is proportional to the fraction of the hydrogen that burns, for a given set of initial conditions. As in the case of the flame speed correlations, the CONTAIN correlations generally predict the completeness reasonably well or else overpredict completeness. There appears to be some tendency for completeness to increase with increasing scale, which is not reflected in the model. As was noted previously, more complete burns are expected if the temperature at burn initiation is elevated. Issues related to flame acceleration and DDT are not relevant to burn completeness, since both the experimental data and the correlations indicate that burns will be essentially complete for any gas compositions for which flame acceleration is possible. 
In comparisons with experimental results, use of these correlations in either CONTAIN or the HECTR code generally yielded favorable comparisons with experimental pressurization measurements provided the flame speed was specified to agree with the experimental value in experiments for which the flame speed prediction was inaccurate. This result suggests that the burn completeness was generally predicted adequately, even if the predicted flame speed was in error. Note, however, that these comparisons are not available for all regimes of gas composition of potential interest.

Burn Propagation. The CONTAIN model for burn propagation is described in Section 9.1.1. There are two limitations to this model that users should keep in mind. The first has to do with the fact that the flame front is not tracked within a cell, and the segregation of burned versus unburned gases by the flame front is not represented. If a burn initiates within one of several interconnected cells, the rising pressure forces gas to flow out into adjacent cells. Since the gas composition in the burning cell is assumed to be well mixed at all times, the gas entering the adjacent cells will be a mixture of burned and unburned gases. The influx of burned gases can reduce the flammability of the atmosphere in the adjacent cells, possibly suppressing propagation that should actually occur; Reference Pon 90 provides an example illustrating this behavior. In reality, the flow out of the cell in advance of the flame front would be expected to consist principally or entirely of unburned gases.

The second limitation of the model is that the flow out of the initial burn cell would be expected to generate turbulence in the adjacent cells, which could enhance burn rates in these cells and which could even result in flame acceleration and/or DDT. The CONTAIN flame speed correlation does not include this turbulence effect; see the previous discussion of flame speed.

13.2.6.2. Diffusion Flame Burn (DFB) Model. The DFB model includes a provision for specifying minimum temperatures such that the incoming gas will not burn if its temperature is less than this minimum, even if all the concentration limits are satisfied. Two minimum temperatures may be specified. The first, specified with the keyword DFTEMP, applies within the burn window defined by TACTIV and TDEACT (see Section 14.3.1.7) and the second, specified with the keyword DFAUTO, applies outside the burn window. Defaults are zero and effectively infinite $\left(10^{20}\right)$, respectively. Use of a minimum temperature may be appropriate for modeling autoignition of the incoming gas when there is no ignition source located close to the gas source. However, the model does not take into account the fact that, once ignited, the incoming jet may continue to burn even though its temperature may subsequently drop below the autoignition threshold; in the model, combustion ceases once the incoming gas temperature drops below the threshold.

The temperature thresholds are not applied to gases entering the calculation via user-defined source tables. Within the burn window, these gases will burn as they enter if the DFB concentration limits are satisfied and they will not burn outside the burn window, independently of DFTEMP and DFAUTO. Within the window, burning can be prevented by setting the DFB concentration limits to impossible values, but this will suppress all DFB in the cell.

An important limitation of the model is that there is no provision to allow the temperature thresholds to depend upon the gas composition for either the incoming flow or the receiving cell, and the composition limits for both the incoming gas and the receiving cell are independent of temperature. 
Furthermore, the composition limits for the receiving cell and the incoming gas are independent of one another. All of these simplifications are at best approximations and may be quite inaccurate in some instances. It is recommended that the user check the behavior of the DFB model for the calculation of interest, and consider whether alternative behaviors are plausible for the conditions of the calculation. If alternative behaviors are plausible, sensitivity calculations with parameters set to obtain the alternative behavior may be warranted. For example, if the default model predicts that most of the hydrogen should burn as it enters the containment, but it is not entirely clear that this is actually the correct result, then a sensitivity case might be run with the DFB parameters set to impose more stringent conditions for combustion, or with the DFB model suppressed entirely.

13.2.6.3 Bulk Spontaneous Reaction (BSR) Model. This model is intended for use when very high gas temperatures are expected to cause rapid reaction of combustible gas with oxygen, even though the normal flammability criteria for the other combustion modes are not satisfied. The BSR model is controlled by a simple user-specified threshold temperature and a user-specified fractional reaction rate (see Section 14.3.1.7). Both values are simple constants that do not depend upon any of the containment parameters. The model is only intended to simulate the consequences of this mode of hydrogen combustion; it cannot provide a mechanistic prediction as to whether it will occur in any given scenario. It is up to the user to justify the controlling parameter values chosen.

The default value of the threshold temperature, $773 \mathrm{~K}$, is close to what has been observed for autoignition of stoichiometric air-hydrogen mixtures (see Section 9.3); addition of steam and/or substantial departures from a stoichiometric composition are expected to result in higher threshold values. On the other hand, the threshold temperature may decline somewhat with increasing scale. In one approach that has been used, the threshold temperature was estimated to be that temperature at which energy release rates exceeded the energy loss rates resulting from atmosphere-structure heat transfer, with the energy release rates being calculated by the chemical kinetics code SENKIN. [Lut91] Applied to lean, steam-rich mixtures ( $4 \%$ hydrogen and $~ 50 \%$ steam), this approach predicted threshold temperatures of $\sim 800 \mathrm{~K}$; however, there may be substantial uncertainties in the kinetics data set employed by SENKIN when this model is applied to these particular conditions, implying that there may be significant uncertainty in the threshold temperatures estimated by this technique (see Appendix A of Reference Pil94b).

The user must specify a reaction rate in order to model BSR, since the default value of zero prevents BSR. Too slow a reaction rate will permit the atmosphere-structure heat transfer to cool the containment more rapidly than the reaction heats it. A chemical kinetics calculations can provide insight as to the appropriate reaction rate for a given temperature, provided a validated set of kinetics data is available. If the heating that initiates BSR in the cell of interest is very rapid (e.g., due to a sudden influx of hot gas or a large, sudden DFB), the entire cell may not be heated all at once. In this situation, the reaction rate specified may be more meaningfully defined to simulate the rate at which the superheated zone expands through the cell volume rather than to simulate reaction rates governed by chemical kinetics. This approach was used to estimate the reaction rate specified in the standard input prescription for DCH calculations (see Section 13.3.2.2.2). Whatever approach is used, it is evident that substantial uncertainties must be allowed for in estimating the reaction rate in the BSR model. 
It is normally considered inappropriate to allow the temperature rise resulting from a deflagration to initiate BSR, since the deflagration model is already intended to provide a complete description of the combustion associated with the deflagration event. Adding in a BSR event would in effect double-count burn rates and overpredict burn completeness. Hence, when a deflagration initiates in a cell, no BSR is allowed to initiate in the same cell for a period equal to twice the burn time. If a slow deflagration initiates at a time when other events (e.g., a DFB) are producing rapid heating, this suppression of BSR could be undesirable. DCH events can fall in this category, which was taken into account in defining the recommended parameter set for DCH analysis (see Section 13.3.2.2.2).

\subsubsection{Heat and Mass Transfer}

Atmosphere-Structure Radiation. The temperature difference governing atmosphere-structure radiation in CONTAIN is the temperature difference between the bulk gas $\left(T_{b}\right)$ and the bulk temperature of the first structure node $\left(T_{w}\right)$, rather than the interface temperature $\left(T_{i f}\right)$. Any modification would have to be carefully assessed because water films are not always totally opaque to thermal radiation and the degree of absorption can be a strong function of the wavelength; thus, replacing $\mathrm{T}_{\mathrm{w}}$ with $\mathrm{T}_{\mathrm{if}}$ would not a priori guarantee improvement in all instances. In addition, computational problems could result from this replacement. In any event, the user should be aware of this limitation. The net effect is that the radiant energy transfer can be overpredicted somewhat.

Aerosols Treated as Pure Absorber/Emitters. The treatment of aerosol emissivity is derived for absorbing aerosols that produce negligible scattering. The user should be cautioned that, if this condition is not satisfied, the aerosol cloud may scatter effectively and significantly reduce the radiative heat transfer or emissivity of a hot gas radiating to cold walls. This effect and others that arise in the presence of significant scattering are not taken into account in the code, and this omission may lead to calculated gas pressures and temperatures that are too low. To some extent the user can explore the importance of the effect of aerosol emissivity on the problem by varying the "kmx" or "absorb" parameters (see Sections 10.3.3 and 14.3.1.5), or by omitting aerosols from the radiation modeling. The user can also directly specify the emissivity of the gas-aerosol cloud in DCH calculations.

One-Dimensional Model. A one-dimensional linear heat conduction equation is used to represent heat conduction in structures. This is a reasonable approximation in many cases; however, in some situations two-dimensional effects may be important.

Evaporable Water Release. The model for evaporable water release from heated concrete is thermally based and does not treat the transport of gases or evaporable water liquid through the pores of the concrete. This limitation is not believed to be of major importance, particularly since the user can explore various assumptions by the model parameters. The bound water and carbon dioxide release models are mechanistically based.

Effect of Geometry on Natural Convection. The default natural convection correlations are for vertical walls, horizontal floors, and horizontal ceilings only. Walls at angles in between 0 and 90 degrees inclination must be represented as one of these three geometries. Also, walls that form 
stagnant corners are not properly represented. In these cases, the default natural convection correlations in CONTAIN, which are for free-standing surfaces, would tend to overpredict the degree of heat and mass transfer. Likewise, the default correlations for horizontal surfaces with stabilizing density gradients are based upon surfaces with free edges. In containment applications, however, surfaces of type FLOOR or ROOF may be joined to wall surfaces that extend upward or downward, respectively, at the edges of the horizontal surface, and the applicability of the default correlations is questionable under these conditions.

No Forced Convection for the Lower Cell. Forced convection models are not available for heat and mass transfer between lower cell layers, between the lower cell (including the pool) and the atmosphere or between the pool and structures. Only natural convection is modeled. The user can attempt to simulate some of the effects of forced convection by overriding the internally-calculated lower-cell heat transfer coefficients using the tabular input options described in Sections 5.5, 14.3.2.3.1, 14.3.2.4.1, and 14.3.2.5.

Mass Transfer Correction to Heat Transport. When steam condenses, sensible heat is transferred along with the latent heat between the atmosphere and structures. Mass transfer does affect sensible heat transfer; however, the CONTAIN treatment of structure heat transfer neglects a correction factor for this effect given in Collier's text on heat and mass transfer. [Col81] This correction is used in the DCH model for debris-gas heat transfer as discussed in Section 6.5.1.

Fixed View Factors. View factors in the net enclosure model are fixed. Therefore, the view factors do not change with changing conditions that affect areas or view factors, such as a filling pool or cavity erosion. A diagnostic is given when such conditions are possible.

Diffusivity of Steam. In CONTAIN 1.2 and higher versions, the steam diffusivity used in calculating condensation on structures, ice surfaces in ice condensers, and pool surfaces is based upon an approximate multicomponent treatment that assumes the noncondensable gas composition in the boundary layer is the same as that in the bulk gas. In reality, the denser components tend to be more concentrated in the boundary layer than the light components (i.e., $\mathrm{H}_{2}$ or $\mathrm{He}$ ) during periods of rapid condensation, which means that the model may overpredict the diffusivity under certain conditions. During code development, an approximate treatment for this effect was defined and used to estimate the magnitude of the resulting errors. Some results are summarized in Figure 13-1. The solid curve gives the diffusivity of steam as calculated by the CONTAIN model for a nitrogen-hydrogen mixture at $0.1 \mathrm{MPa}$ and $400 \mathrm{~K}$. The other curves show results with the approximate correction for the boundary layer composition effect for cases with $\mathrm{X}_{\mathrm{nc}, \mathrm{i}} / \mathrm{X}_{\mathrm{ncc}, \mathrm{b}}=1.5,10$, and 100. For $\mathrm{X}_{\mathrm{nc}, \mathrm{i}} / \mathrm{X}_{\mathrm{nc}, \mathrm{b}}=1.5$, the estimated error in the CONTAIN model is always $\leq 5 \%$, and it is $<20 \%$ for all cases with hydrogen $\leq 50 \%$ of the total noncondensable gas. However, the diffusivity can be overestimated by larger amounts if the noncondensable gas consists mostly (but not entirely) of hydrogen and $\mathrm{X}_{\mathrm{nc}, \mathrm{if}} / \mathrm{X}_{\mathrm{nc}, \mathrm{b}} \gg 1$. Note that meeting the latter condition requires that the atmosphere consist mostly of steam $\left(\mathrm{X}_{\mathrm{nc}, \mathrm{b}} \ll 1\right)$. Conditions required for substantial effects of this type probably arise infrequently in general containment analysis but they might arise in some special circumstances; e.g., analysis of certain advanced light water reactor passive cooling systems (PCS) under conditions for which considerable hydrogen is present. 


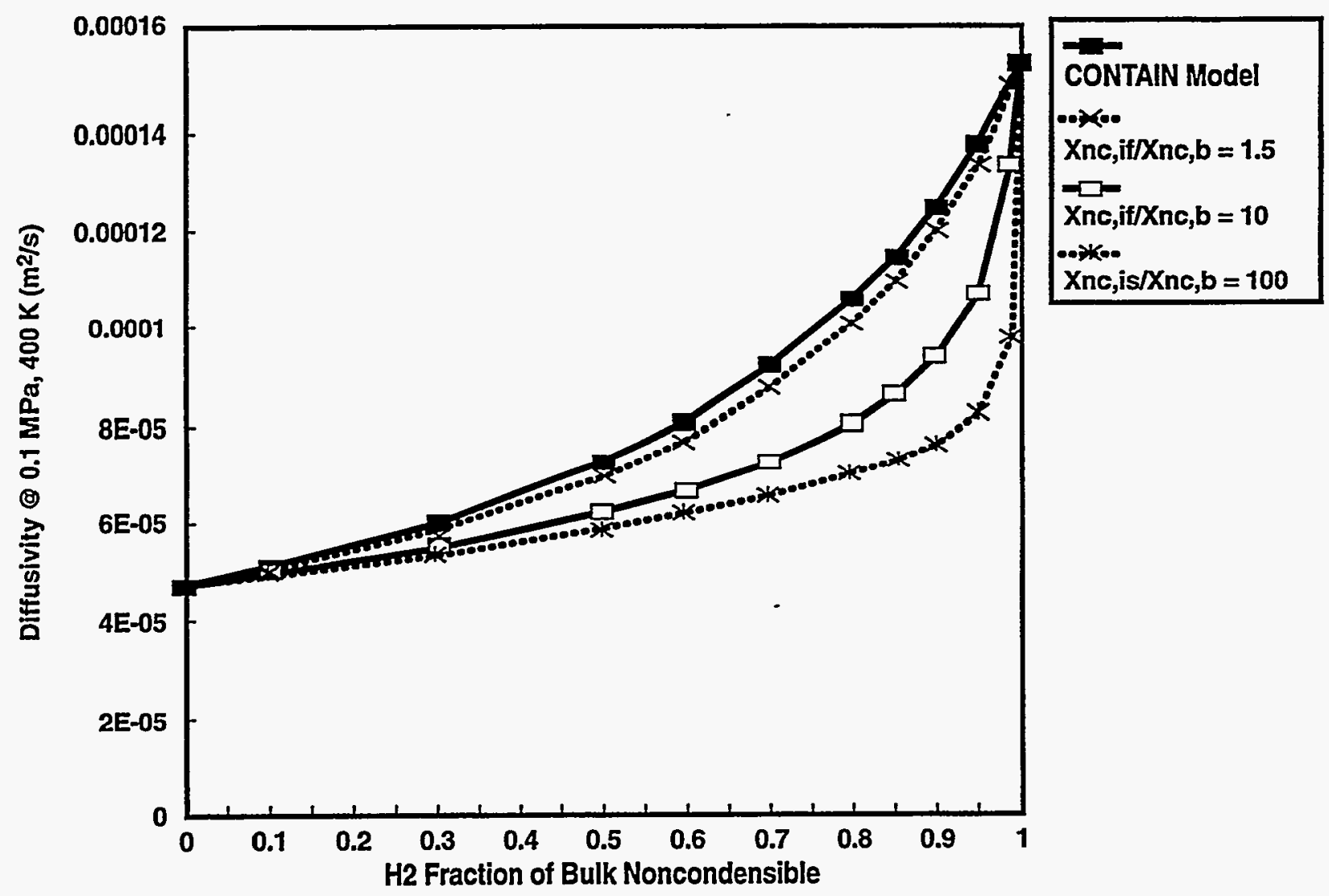

Figure 13-1. Estimated Error Resulting From Use of Bulk Noncondensable Gas Compositions in the CONTAIN Multicomponent Diffusivity Model 
Code versions earlier than CONTAIN 1.2 use Equation (10-33) for the diffusivity, which does not take into account the composition of the noncondensable gas at all and which overestimates the diffusivity of steam in air at high temperatures. The old diffusivity model is still used in several specialized applications in the code: the DCH model (see Section 6.4.1 for discussion of compensating effects that apply in this application), the SPARC model for aerosol scrubbing in suppression pools, and the models for aerosol dynamics.

\subsubsection{Engineered System Modeling}

The following list briefly summarizes the limitations for the three major ESF models: fan coolers, ice condensers, and sprays.

\section{Fan Cooler}

- a simple parametric model is provided in addition to the mechanistic condense model; if used, the simple model is plant-specific

- deposition of aerosols and fission products is not modeled in the simple model, and degradation of heat transfer surfaces by deposited aerosols is not considered in the more mechanistic model.

\section{Ice Condenser}

- temperature profile in the ice is not represented

- nonuniform melting of the ice is not modeled

- decay heating from deposited fission products is not modeled

- interaction of ice with debris in a DCH event is not modeled

\section{Spray Model}

- the atmosphere is assumed to be stagnant

- all spray droplets are assumed to be a single size within a given spray system

- interaction between spray drops are not modeled

- spray drop-to-drop interactions are ignored

- spray drops are not allowed to fall from one volume to another

- water in sprays is always pure despite its source

- $\quad$ sprays do not directly wet the containment walls

- radiation to the spray droplets is ignored

- sprays do not wash fission products off walls

The user may wish to model the upper region of the containment, where the spray nozzles are located, as a single cell, and subdivide the lower region into one or more cells. The spray model does not treat a spray falling from one cell into another cell or set of cells. If the spray carryover is perceived to be important in the lower region (e.g., with respect to aerosol scrubbing), this situation may be simulated by specifying a train of spray systems, using the pool for one to feed the next. 


\subsubsection{Calculational Sequence Effects}

The major blocks of calculations are performed in sequential order. Lower cell calculations are performed first, then upper cell calculations, and finally, ESF calculations. Because the coupling among these models is largely explicit, for some calculations time delays in the effects of one model on another may occur. For example, mass added to a lower cell pool by an engineered system (e.g., sprays) will show up in the output delayed by a timestep. This happens because the lower cell calculations are carried out before those for the engineered systems. Similarly, cell calculations are performed in sequential order according to cell number. Events occurring in higher-numbered cells that affect lower-numbered cells will not show up until the next system timestep. Users should be aware of these time-delay effects in interpreting the output. Rerunning the problem with a reduced timestep will usually reveal whether such effects are important.

\subsection{Practical Suggestions}

Many lessons have been learned through the exercise of CONTAIN for specific applications (see Appendix $C$ for relevant publications). Some of the more universally applicable suggestions are included below.

\subsubsection{Atmosphere/Pool Thermodynamics and Intercell Flow}

13.3.1.1 Optimizing Multicell Calculations. The flow option of choice is the IMPLICIT option in the FLOWS input block (Section 14.2.4.1). The implicit option allows the user to solve selected cell atmospheres and pools explicitly. Stability considerations preclude specifying explicitly solved atmospheres that are not relatively large in volume or not coupled to other cells entirely through highly restrictive flow paths. The explicit option should be used for environment cells with very large volumes (of order $10^{10} \mathrm{~m}^{3}$ ). Pools are considerably more stable than atmospheres when coupled explicitly. However, the code does not permit implicit flow paths to be coupled to an explicit pool. Explicit flow models, consisting of engineered system liquid transport system components, should be used in this case. By excluding the environment cells from the implicit solver matrix, the overhead due to multiple environment cells is reduced considerably. Robustness of the implicit solver is also improved.

It is difficult to give general guidelines on improving the convergence of the implicit solver. Shorter system timesteps often help, as will larger than default values for the "pvtmin" parameter. Cell level models that act as strong sources or sinks may cause problems if the sources or sinks cause a change in the mass of a cell by more than $20 \%$ per system timestep. User-specified sources may also cause problems. The source mass should be limited to $20 \%$ of the cell mass per system timestep. If actually a sink, the source should not be allowed to drive any of the atmosphere component masses negative.

13.3.1.2 Modeling of Suspended Liquid Coolant. This section discusses several options that deal with suspended liquid coolant in the atmosphere and control how liquid removed from the atmosphere is subsequently treated. Knowledgeable decisions in this regard require familiarity with several different modeling areas. 
The user should be aware that there are essentially three distinctly different options to treat the liquid that may arise from vapor condensation in the bulk atmosphere. The first is to carry the resulting liquid coolant along with the gas as homogeneously dispersed liquid. The second is to use the liquid DROPOUT option in the FLOWS input block to drop the liquid out into a coolant pool as soon as the liquid forms. This coolant pool may be specified through the cell OVERFLOW keyword discussed in Section 14.3.1.12. If OVERFLOW is not specified, the default is the pool in the same cell, or if that is not present, the waste location in that cell. The third is to treat the vapor condensation as occurring heterogeneously on aerosols. Once the coolant aerosols have formed, the disposition of the coolant aerosols is then controlled by the dynamics of the aerosols, as discussed in Section 7.

In the first case, the thermodynamics of the liquid coolant and its inertial mass are taken into account in the flow equations. In the second, the liquid coolant in the atmosphere is usually negligible. In the third case, the specific heat and inertial mass of the coolant aerosols are not taken into account. However, aerosol dynamics, including agglomeration, deposition, and the effects of intercell flow, are taken into account in determining the eventual disposition of the liquid. For condensation on aerosols to occur, the user should specify $\mathrm{H} 2 \mathrm{OL}$ as the last of the aerosol component materials in the global AEROSOL input block, discussed in Section 14.2.5.

In the third option, the disposition of the water aerosols is controlled by aerosol dynamics. This leads in many cases to deposition of a significant amount of water on the surfaces of heat transfer structures and pools or, in the case of aerosol scrubbing, on the walls of gas bubbles internal to the pool. Once deposited, the subsequent movement of non-coolant aerosols is ignored. However, the deposited water aerosols are transferred either to the coolant film inventory, in the case of a heat transfer structure, or to the pool inventory, in the case of pool deposition. Fission products attached to the aerosols are also transferred to the respective structure or pool hosts and are thereby subject to subsequent flow. The disposition of a coolant film is controlled by the condensate film model, discussed in Sections 10.2.2 and 14.3.1.3.

It is possible that an 'excessive amount of aerosolized water will be created in the third option. If the user specifies that all of the coolant exiting the break in a blowdown scenario be directed to and equilibrated with the atmosphere, as it would if an atmosphere source table were used, an excessive amount of liquid may be present in the atmosphere. The reason is that the entrainment and/or the equilibration of liquid before dropout is usually not complete during a blowdown, particularly if the coolant exiting the break has low quality and is not much above saturation. While CONTAIN does not have an entrainment model for blowdown coolant, an isenthalpic phase separation model is available within the safety relief valve discharge model, to treat a second limiting case, corresponding to pressure equilibration without entrainment. As discussed in Section 11.2, this model treats both gas-pool equilibration effects for submerged injection and phase separation for unsubmerged injection of an external source. In the latter case, the separated liquid goes directly to the coolant pool. These two limiting cases can be used in combination to simulate the effects of incomplete entrainment, in cases in which the partitioning of the coolant can be separately determined, or to conduct sensitivity studies. 
13.3.1.3 Modeling Stratifications. An assessment of the hybrid formulation of gravitational heads implemented in CONTAIN 1.2 has recently been completed. [Mur96] This assessment shows that the hybrid formulation offers significant improvements in the treatment of stable stratifications formed by the injection of buoyant gas at an elevated location within a plant, compared to the old formulation used in prior code versions. As discussed in that assessment, a stability criterion can be derived within the hybrid formulation for a buoyant source, and this criterion is almost always satisfied for a source injected into an open volume. On the other hand, the analogous criterion for the old formulation can never be satisfied. The old formulation consequently predicts that the region immediately below the injection point will mix significantly with the region above. Thus, one would expect the overmixing of stratifications normally associated with control volume codes to be reduced with the hybrid formulation. On the other hand, in situations with nearly well-mixed conditions, the hybrid solver should predict nearly the same results as the old formulation. Since there is almost no additional computational overhead associated with the hybrid formulation, it should be used in all cases.

The user should keep in mind the fact that momentum convection is ignored within a control volume. Thus, instability with respect to momentum convection, as discussed in References Pet94 and Hih95, is not considered in CONTAIN, and the hybrid solver should not be applied to stratifications that are unstable in that respect. With respect to stratifications that are stable against momentum convection, two classes of behavior may be expected, based on whether the stability criterion derived for the hybrid formulation is satisfied or not: the stable behavior obtained when it is satisfied and buoyancy-dominated loop-flow behavior when it is not. The latter may be viewed as unstable counterpart of stable behavior in highly confined or channeled geometries. Although stability is almost always satisfied for a source injected into an open volume, confining the source may affect the stability of the resulting stratification.

In many containment scenarios, stable behavior is expected after the initial blowdown. However, independent verification of the stability regime should be carried out. For nearly free jets or confined vertical jets in a cylindrical geometry, the user may use the guidelines of References Pet 94 and Hih95. This requires knowing the Froude number, the rise height and diameter of the jet, and the cylindrical wall diameter. For more general types of injection geometries no simple guidelines can be given. Experimental input or computational fluid dynamics calculations may be required to determine the stability regime.

The user should also keep in mind that a model for the entrainment or mixing of a buoyant jet or plume is not used: a well-mixed assumption for the injected gas is used in place of such a model. As discussed in Reference Mur96, if the stratification is stable, the details of this mixing do not appear to be important for the fully developed stratification formed after the initial transient. However, for stable transient conditions or for unstable conditions, the details of the mixing could be important in determining the flows and gas distribution. Again, experimental input or computational fluid dynamics calculations may be required to determine the mixing behavior in all but the simplest of geometries.

The user should exercise care in setting up the connections from the internal cells to the environment in thermal siphon geometries. This is related to the fact that a donor-based formulation is prone to 
introducing large "integration" errors in the gravitational head. This is especially noticeable when flows occur from short cells to tall cells with significantly different gas densities. In such cases, within a donor formulation, the density of the short cell is used to calculate the gravitational head for the first half-node of the tall cell. The use of this density may not be appropriate if the flow from the short cell mixes rapidly with the inventory of the tall cell, and in addition, the error is compounded by the large vertical span of a tall cell. Consequently, the calculated gravitational head may be in considerable error in this case. We refer to this below as the "half-node" problem within a donor formulation. It should be noted that gravitational heads will also be in error in an averagedensity treatment in this case, but typically will be only about half as large. Figure 13-2 shows two examples of a nodalization with a potential "half-node" problem, in this case representing a thermal siphon connected to an environment cell in two different ways. The hot leg of the thermal siphon heats the gas in each case so that the gas density in the hot leg is less than that in the environment. The parts of the recirculating flow loop marked "underpredicts" and "overpredicts" lead to underprediction and overprediction, respectively, of the gravitational head driving the flow, assuming that the gas from the hot leg mixes rapidly with the reservoir. To minimize this problem, one can in general use cells of approximately the same height throughout and/or invoke flow path options to refine the gravitational head definition.

The use of flow path options is appropriate for environment cells. In Figure 13-2, the "half-node" problem with respect to the tall environment cell can be minimized by explicitly specifying the elevations of the flow paths at the inlet and outlet of the thermal siphon and using the RESOLVHD keyword. This will result in the use of the environment cell gas density to calculate the gravitational head within the environment cell. However, another solution in this simple case is to set the environment cell center-of-elevation at the outlet elevation.

Guidelines for nodalizing a problem to capture a stratification are straightforward: (1) At each elevation for which a horizontal interface is present between cells, the interfaces should span the containment cross section. This should help capture the essentially one-dimensional nature of most stratifications of interest in a containment. (2) The same cell elevations should be used for all cells representing the same containment level. Slightly staggered elevations, for example, to represent minor differences in the centers of volume of the cells at a given level, should be avoided. Such slight elevation offsets may result large horizontal gas composition gradients resulting from the fact that buoyant gas will tend to accumulate in the uppermost of the cells at the same basic level. (3) Horizontal cell interfaces should be placed, if possible, at each location at which one would expect a stratified layer boundary to form (e.g., at each possible injection elevation). (4) A large variation in cell heights, except that caused by the presence of environment cells, should be avoided. As discussed above, a donor-based formulation like the hybrid formulation is particularly sensitive to gravitational head "integration" error when flows occur from a short cell to a tall cell. (5) Flow path options, as discussed above, should be used to refine gravitational head definitions for environment cells and in other situations as needed. (6) The vertical height of an environment cell should be comparable to the total height of the internal cells in the problem.

13.3.1.4 Choked Flow at Less Than the Critical Pressure Difference. To model choked flow, the flow velocity in flow paths is limited to the local sound speed, using the standard expression for the limiting flow rate of an ideal gas, assuming adiabatic flow. However, under certain conditions, the 

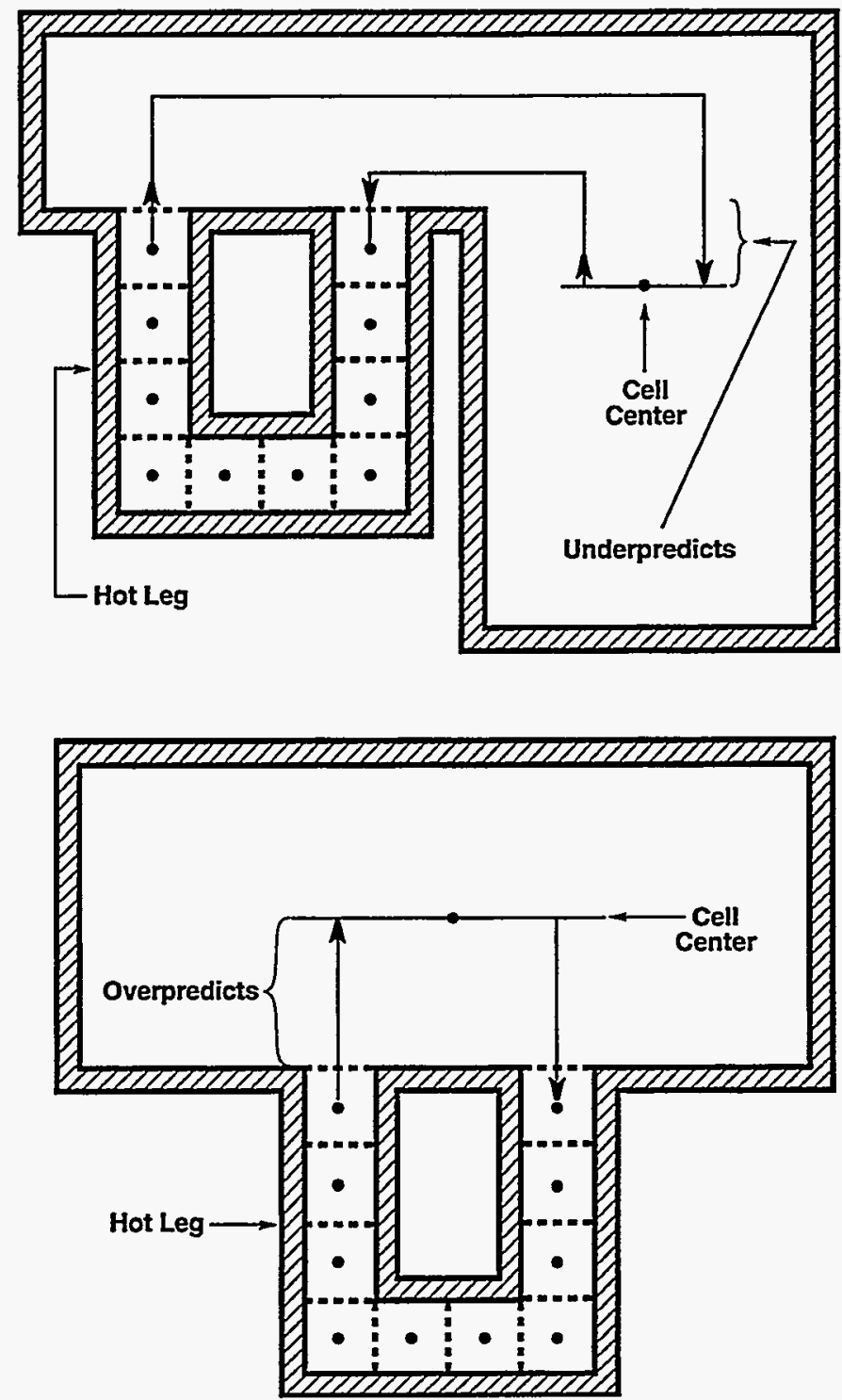

Figure 13-2. Two Thermal Siphon Nodalizations Which Underpredict and Overpredict the Thermal Siphon Flow, Respectively, Within a Donor Approximation 
user may find that choking arises at pressure differences less than the expected critical value for choked flow. The most likely cause is the specification of an unrealistically small value of the turbulent flow coefficient discussed in Sections 4.4.1 and 14.2.4.2. For an orifice, this coefficient should be of order unity. If a much smaller value is used, the flow velocities calculated for a given pressure difference will be too large, and the pressure difference at which choking begins will be too small. For realistic values of the flow coefficient, the onset of choking in the calculation will occur at approximately the correct value of the pressure difference.

13.3.1.5 Modeling the Outside Environment. The outside environment can be modeled as a very large cell (volume $>10^{10} \mathrm{~m}^{3}$ ). However, because of gravitational head effects, it is important to keep the height of the environment cell comparable to the total height of the internal cells. Also, pools of very large surface areas should not be used. A pool sump can be created through the CELLHIST input discussed in Sections 4.1 and 14.3.1.1. Also, see the discussion in 13.3.1.3 if the environment cell is involved in thermal siphon effects.

13.3.1.6 Atmosphere and Lower Cell Source Tables. Atmosphere steam sources may be associated with either the H2OV or H2OL material. Since water is a two-phase material, care must be taken in choosing the method used to define the energy state of the steam injected into the CONTAIN cell. In most cases the user should use the specific enthalpy $(\mathrm{J} / \mathrm{kg})$ option to define the steam energy state. In this manner subcooled, saturated, or superheated steam can be treated. If the temperature option is selected for $\mathrm{H} 2 \mathrm{OV}$ or $\mathrm{H} 2 \mathrm{OL}$, the source pressure will be assumed to be the repository pressure, which may not be appropriate. In general, if the steam state is given in terms of pressure and temperature, the user should externally convert to an equivalent specific enthalpy (noting that the reference state used in CONTAIN is zero enthalpy for liquid water at $273.15 \mathrm{~K}$ ), and then use the ENTH option in the atmosphere source table (Section 14.3.1.2). In the case of liquid water injected into a lower-cell pool, the material name $\mathrm{H} 2 \mathrm{OL}$ should be used.

For sources of single-phase gases such as hydrogen and for core debris material in DCH calculations, the energy state is typically specified using the gas (or debris) temperature. If the user selects to use the specific enthalpy option for the gas energy state, then the enthalpies must conform to the reference state used in the code, which is zero enthalpy for single phase gases at $273.15 \mathrm{~K}$.

\subsubsection{DCH}

13.3.2.1 General Considerations. DCH calculations are among the most complex analyses that one can perform using the CONTAIN code. The CONTAIN DCH model has undergone substantial upgrades in recent years, and an extensive series of analyses of DCH experiments has recently provided considerable insight into how the code can be used to analyze DCH events. The validation and assessment of the CONTAIN DCH models on the basis of these experiments is discussed in References Wi195 and Pi195 and their application is discussed in Reference Pil95. Because of the extensive nature of these analyses, the practical suggestions offered here for $\mathrm{DCH}$ calculations are more comprehensive than are the suggestions offered for most other types of containment analysis. Since much of this information is new, it is recommended that even experienced DCH analysts give some consideration to what is presented here. It is also recommended that users, especially new users, employ existing DCH input files as a guide to setting up new problems. The sample problem 
for the Sequoyah plant given in Chapter 15 includes DCH modeling that conforms closely to the standard input prescription described in Section 13.3.2.2. In addition, a hardcopy listing of an input file that was used in the analysis of the Zion-geometry Integral Effects Test (IET) experiments [All94] is given in Reference Was95.

Some of the recommendations made in this section are to be viewed as suggestions only, since any given problem likely will have features that cannot be taken into account here. These features may well justify substantial departures from our recommendations. In addition, some of our recommendations are based upon our judgments concerning uncertain DCH-related phenomena about which knowledgeable investigators may reasonably be expected to disagree. As always in performing complex CONTAIN calculations, results should be examined carefully to ensure that the calculated behaviors are physically reasonable, and sensitivity studies are warranted in order to determine the extent to which the results of interest are sensitive to uncertain input parameters and uncertain modeling assumptions.

There are two major parts to this presentation. The first is given in Section 13.3.2.2 and presents what is called the standardized input prescription for DCH analyses that was developed in Reference Wil95. This standardized input prescription does not include a prescription for use of the new RPV and cavity entrainment models, but it does provide guidance as to how to define debris source tables for simulating the RPV ejection and cavity entrainment processes. The second major part of this presentation describes use of the RPV and cavity models (Sections 13.3.2.3 and 13.3.2.4). Note that when these models are used, the standardized input prescription is still applicable except for those portions of the standard input that deal with setting up the debris sources. Users of the RPV and cavity entrainment models should be aware that experience with them to date has been very limited and that they can behave in complex ways; it is especially important to check the results to ensure reasonable behavior when using these models. It is recommended that users be familiar with the results of the assessment of these models that are described in Reference Wil95. Note also that this assessment concluded by recommending that these models should be used principally on a "friendly user" basis; i.e., used by analysts who are familiar with DCH phenomenology and who are willing to assume the responsibility of examining the results for possible unreasonable behavior.

Perspectives Concerning Uncertainties. The discussion of DCH analysis that follows acknowledges that important modeling uncertainties exist for some of the phenomena involved in DCH. However, this fact does not mean that the DCH loads calculated by CONTAIN will normally be heavily affected by a large number of uncertainties, because the results in any given instance will commonly be insensitive to some of the uncertain phenomena. For example, there are important uncertainties in the models controlling debris trapping and transport, yet the CONTAIN predictions of containment pressure rise $(\Delta \mathrm{P})$ and hydrogen production in the Zion- and Surry-geometry IET experiments were found to be quite insensitive to these uncertainties. [Wi195] Typically, the results of a given analysis will be sensitive to, at most, a small number of uncertain parameters or modeling assumptions; however, the identity of the more important uncertainties can be different for different DCH scenarios.

One example of this dependence upon scenario is that the dominant sensitivities may be different for compartmentalized versus open containment geometries. Containment geometries are said to 
be "compartmentalized" if the principal path for debris dispersal from the cavity communicates with a compartmentalized lower containment, the structures of which present additional barriers to debris transport to the main volumes of the containment; the geometry is said to be "open" if the principal path for debris dispersal communicates directly with the containment dome. To date, there is considerably more experience with analyzing $\mathrm{DCH}$ scenarios in compartmentalized geometries than in open geometries.

The impact of any given uncertainty on the results of DCH calculations can depend strongly upon the initial and boundary conditions of the scenario of interest. It is therefore impossible to give a quantitative estimate of the magnitudes of these uncertainties that would be applicable to all DCH analyses. In some cases, therefore, the discussion in Section 13.3.2.2 includes suggested sensitivity calculations. These recommendations are designed to provide the user with a reasonable understanding of the uncertainty for the particular case at hand.

To date, there has been only limited application of the approaches suggested here to nuclear power plant (NPP) analyses. Hence, some of the suggestions offered must be considered tentative.

13.3.2.2 The Standard Input Prescription for DCH Calculations. This section presents the standardized input prescription for DCH analysis that was reported in References Wil95 and Pil95. It discusses how to define debris source tables to simulate RPV ejection and cavity entrainment processes.

13.3.2.2.1 Philosophical Basis and Purpose of the Standard Prescription. As elsewhere in CONTAIN, the design philosophy for DCH modeling has been to provide mechanistic modeling for phenomena which are sufficiently well understood to justify a defensible mechanistic model, and to provide simpler or parametric models with flexible input options for phenomena that are not well understood, or that cannot be represented fully within the limitations imposed by the basic lumpedparameter, control-volume architecture of the CONTAIN code. When combined with the complexity of DCH phenomenology, this philosophy has led to provision of a large number of input and modeling options for DCH analysis. This flexibility in CONTAIN DCH modeling is currently viewed as being essential in order to permit the user to study uncertainties in the results of DCH calculations, as well as to permit the user to take advantage of any future refinements in DCH understanding. However, it obviously presents problems with respect to quality control and consistency, if some type of control on DCH input is not available. Hence an important goal of the CONTAIN DCH assessment effort was to develop a standardized input prescription for DCH calculations.

The standardized input prescription was defined for a number of purposes. These include providing guidance for code users and providing a means for allowing investigators working independently to obtain comparable results when consistency is desired. On the other hand, the standard prescription definitely is not offered as a "cookbook" that must be followed, or that will guarantee good results if it is followed; it is not an "official" prescription endorsed by the United States Nuclear Regulatory Commission or any other standard-setting body. It is only being offered by the CONTAIN project as a suggested starting point or guideline. The standard prescription has potentially important limitations, and sensitivity studies to explore uncertainties are likely to be a part of any study that 
uses CONTAIN. In any given analysis, there may be specific features that justify a departure from the standard prescription. One recommended use is to take the standard prescription as a starting point and to document any deviations from the standard prescription when reporting results.

Where possible, the standard inputs have been based upon separate-effects measurements obtained in the DCH experiments and/or stand-alone modeling. Separate effects measurements for phenomena such as hydrogen behavior obtained from $\mathrm{DCH}$ experiments were preferred to results of non-DCH experiments because the conditions of the latter often differ sufficiently from $\mathrm{DCH}$ conditions that applicability to DCH is doubtful in many instances. Parameters were not chosen by tuning directly to the integral results themselves (i.e., tuning to $\Delta \mathrm{P}$ or hydrogen production results), with the partial exception of the non-airborne debris parameter $d_{\text {nad }}$ discussed in Section 6.6 of this manual.

Another principle guiding the specification of the standard prescription was the desire to be mildly conservative when applying the prescription to scenarios differing from those that have been studied experimentally, especially in scenarios thought likely to be more severe than those studied experimentally. By "mildly conservative" is meant that, when available options appear to be equally defensible, the option expected to result in more conservative extrapolations is selected. The standard prescription should not be thought of as bounding, however. For example, when the more conservative choice is considered to be distinctly less plausible than a less conservative choice, the conservative choice is generally not taken to be the standard even if this choice cannot be rigorously ruled out.

The methodology applied for developing the standard prescription was as follows:

1. Using previously available information and some initial exploratory calculations, a standard input prescription for analysis of $\mathrm{DCH}$ experiments was defined.

2. The standard prescription was then "frozen" and applied to analyze a total of 22 experiments that have been performed using high-temperature melts generated by the iron-oxide aluminum thermite reaction.

3. A large number of sensitivity cases were performed, with all parameters remaining frozen at the standard values except those explicitly identified as being assigned to different values. These sensitivity studies were performed for many different reasons: to demonstrate that uncertainties in many of the input parameters actually matter little to the results, to explore the sensitivity of the results to those input parameters and modeling assumptions that do matter, to determine how important certain specific phenomena such as atmosphere-structure heat transfer are to the results, to examine model behavior and compare (as best possible) the model behavior with the behavior observed in the experiments, and to examine the degree to which alternative explanations exist for the experimental trends.

4. Using lessons learned from the results of the study, some modifications were introduced into the standard prescription for application to NPP analysis. 
The experiments that were analyzed in Steps 2 and 3 above included the Limited Flight Path (LFP) Series (six experiments), [All91] the Wet Cavity (WC) series (three experiments), [All92a,All92b] the Sandia National Laboratories Zion-geometry Integral Effects Tests (SNL/IET Zion) series (eight experiments analyzed), [Al194] the Argonne National Laboratory Zion-geometry IET (ANLIET Zion) series (three experiments analyzed), [Bin94] and SNL IET Surry-geometry (SNL/ET Surry) series (three experiments analyzed). [Bla94] The IET experiments included detailed scale models of containment structures as well as scaled reactor cavities, while the others had nonprototypic containment geometries. The SNL/IET Surry experiments were performed at 1/6-scale and $1 / 10$ scale, the ANL/IET Zion experiments were 1/40-scale, and all others were 1/10-scale. The three ANL/ET Zion cases analyzed were scaled counterparts of corresponding SNL/ET experiments and provide a test of model scalability. The Zion IET experiments analyzed all had a small amount of water in the cavity ( $3.48 \mathrm{~kg}$ at $1 / 10$-scale) except for SNLIET-8B which had a much larger amount $(62 \mathrm{~kg})$. One WC experiment (WC-2) had $11.76 \mathrm{~kg}$ of water in the cavity. Cavities were dry in all other cases.

Analysis Results for DCH Integral Experiments. Figure 13-3 compares calculated and experimental results for $\Delta P$ and hydrogen production, with the calculations being performed using the standard input prescription. Hydrogen results are plotted after scaling up to plant scale by dividing by $S^{3}$, where $S$ is the experimental linear scale factor, in order to facilitate comparison of experiments performed at different scales. Plot symbols distinguish experiments performed in open geometry, the LFP series other than LFP-8A, the SNL/ET (Zion) experiments with hydrogen combustion and without hydrogen combustion, the ANL/ET (Zion) experiments, and the SNL/IET (Surry) experiments.

In general, the CONTAIN $\triangle \mathrm{P}$ and hydrogen production results reproduce the overall trends of the experimental data reasonably well. The ability to reproduce both the $\triangle \mathrm{P}$ data and the hydrogen production data is considered important to CONTAIN validation because rates of chemical reactions producing hydrogen are calculated using a heat-mass transfer analogy. Debris-gas heat transfer and hydrogen production are closely coupled in CONTAIN, and this relationship is not sensitive to most of the acknowledged modeling uncertainties; hence, any major difference between the trends for the $\Delta \mathrm{P}$ results and the hydrogen results could be difficult to explain.

The figure shows that the CONTAIN model does a good job of accounting for the various differences between the Surry-geometry, Zion-geometry, and open-geometry experiments. It also reproduces the effects of hydrogen combustion well in the SNL/IET experiments, and comparison with the ANL/IET results reveals no major scale distortions in the model. The poorest $\Delta \mathrm{P}$ results were obtained for some of the LFP experiments, in which the code substantially overpredicted debris transport to the dome and hence overpredicted $\Delta P$. The reason may be that mean flow velocities were low in the LFP subcompartment geometries and it is likely that debris trajectories largely decoupled from the gas flow, while the CONTAIN model assumes coupled transport of debris and gas.

There are, of course, a great many issues relevant to the validation of the CONTAIN DCH model than cannot be represented in a simple summary plot like Figure 13-3. Some of these issues are noted in the discussion that follows, with additional details being given in Reference Wil95. 
(a)

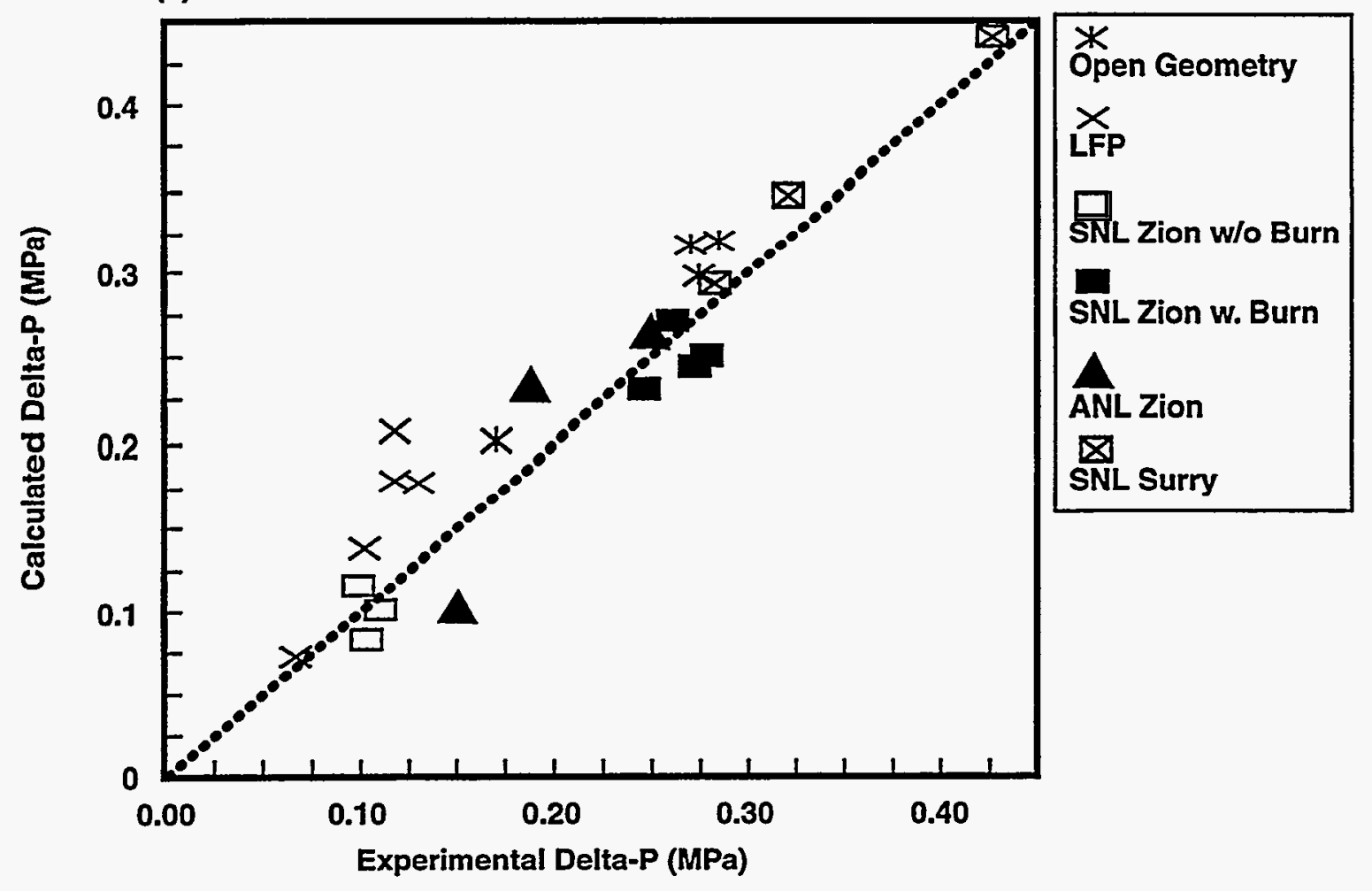

(b)

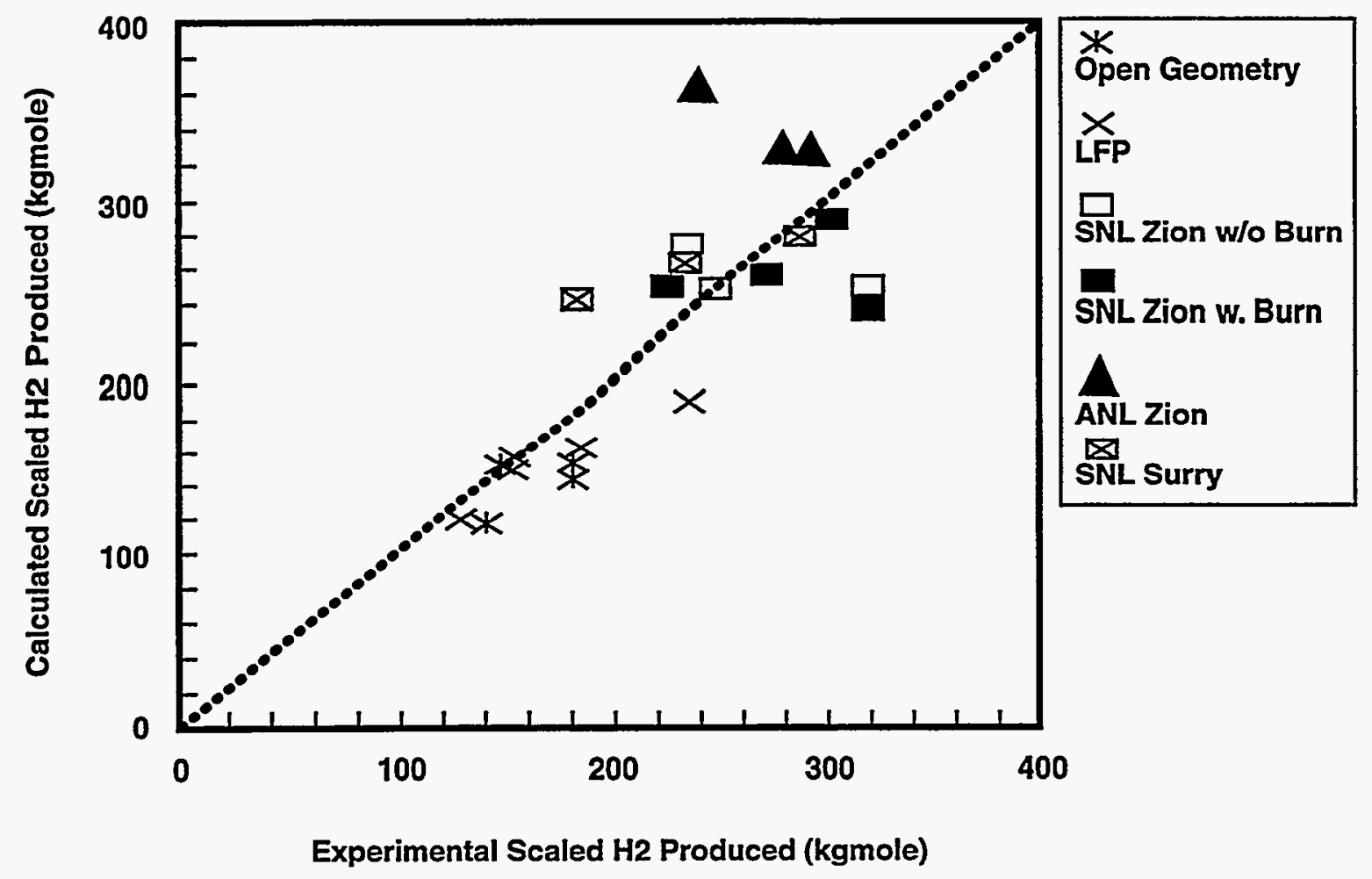

Figure 13-3. CONTAIN Predictions Versus Experimental Results for (a) $\Delta \mathrm{P}$ and (b) Scaled $\mathrm{H}_{2}$ Production for the Standard Input Prescription (Case 1) 
13.3.2.2.2 Standard Prescription Input and Model Choices. The present discussion will be limited to the final version that was recommended for NPP analysis. It is important to understand that this version is still considered applicable to experimental analysis; in fact, the modifications introduced would make very little difference in the analysis of any of the experiments considered in the CONTAIN validation effort. The experimental validation presented in Reference Wil95 and summarized in Figure 13-3 is therefore considered equally applicable to the present prescription. The principal reason for the modifications was the recognition that NPP analyses may encounter a wider range of conditions than any studied experimentally, and that the prescription used in the experimental analysis could encounter pitfalls under some conditions even though problems did not arise for the conditions of the experiments. In addition, the NPP prescription includes a few simplifications; e.g., elimination of some nondefault parameter specifications now considered to be unnecessary.

The current standard input prescription for DCH analysis is summarized in Table 13-1. All parameters and model options not given in the table are left at the CONTAIN default values.

In the analyses of the experiments, parameters describing vessel blowdown, the fraction of the debris dispersed from the cavity $\left(f_{\text {disp }}\right)$, and the time-dependence of the debris source rate $\left[S_{d}(t)\right]$ were based upon experimental results. This approach is obviously inapplicable for scenarios not studied experimentally, including NPP analysis. Hence semi-empirical means of providing this information are adopted. These will be described next.

Melt Characteristics and Vessel Failure Size. In any CONTAIN DCH analysis, definition of the melt characteristics (total mass $m_{d}$, composition, initial temperature $T_{d}^{0}$ ) must be obtained from an invessel analysis or some other information source; CONTAIN has no models for calculating these quantities. The diameter of the hole in the vessel head, $d_{h}$, likewise must be determined by the user, except that the effect of ablation on the hole size can be calculated by the RPV models if these are used.

Blowdown. In the standard prescription, the blowdown of steam from the primary system can be modeled by defining one or more cells to represent the primary system. The initial conditions for these cells should be specified to correspond to the gas composition (steam plus any hydrogen present), temperature, and pressure of the primary system immediately prior to vessel breach. If the ideal gas equation of state for steam is being used, the volume specified for the primary system should be increased so that the code will calculate the correct mass of steam for the specified conditions; if the real equation of state is used, the volume specified should correspond to the actual free volume of the primary system.

The blowdown is calculated by opening a flow path from the primary system cell(s) to the cell representing the cavity. Let $t_{b}^{0}$ represent the time the blowdown begins; it corresponds to the time of gas blowthrough in the melt ejection process, not the actual moment of vessel breach. Rather than opening the flow path area abruptly to its final size, $A_{h}=\pi \mathrm{d}_{h}^{2} / 4$, it is more realistic to specify a flow path area that increases linearly with time over an interval of duration $\tau_{\mathrm{h}}$ given by the semi-empirical relation [Wil95] 
Table 13-1

Summary of the CONTAIN Standard Input Prescription for NPP Analyses

\begin{tabular}{|c|c|}
\hline $\begin{array}{l}\text { Parameter or } \\
\text { Model Option }\end{array}$ & Values \\
\hline RPV blowdown & RPV flow path area $A_{h}(t)$ from Equations $(13-1)$ and (13-2) \\
\hline \multicolumn{2}{|l|}{ Debris sources: } \\
\hline Frac. dispersed & $f_{\text {disp }}=0.7-0.9$, from experimental results \\
\hline Time depend. & $S_{d}(t)$ based on Equations (13-3) and $(13-4)$ \\
\hline \multicolumn{2}{|l|}{ Debris particles: } \\
\hline Mass median d & $1.0 \mathrm{~mm}$ \\
\hline Size distribution & Log-normal, geom. std. dev. $\sigma_{\mathrm{g}}=4,5$ size groups \\
\hline Composition & Bulk composition all fields \\
\hline Generations & NDHGRP $=1$; however, see discussion in text \\
\hline RPV insulation & $1 / 2$ to cavity, $1 / 2$ to downstream cell \\
\hline Debris slip, s & 5 in cavity and chute, 1 elsewhere \\
\hline \multicolumn{2}{|l|}{ Trapping model: } \\
\hline Type & None in cavity and chute, TOF/KU elsewhere \\
\hline $\mathrm{L}_{1}$ & Plant geometry $\left(6 \mathrm{~V}_{\mathrm{g}} / \mathrm{S}_{\mathrm{str}}\right.$ if ambiguous) \\
\hline $\mathrm{L}_{2}$ & $6 \mathrm{~V}_{\mathrm{g}} / \mathrm{S}_{\mathrm{str}}$ \\
\hline $\mathrm{L}_{3}, \mathrm{~L}_{\mathrm{gft}}$ & $\mathrm{L}_{3}=\mathrm{L}_{\mathrm{gft}}=$ cell height \\
\hline VNOST & VNOST $=$ GFT \\
\hline RHODG & $\mathrm{RHODG}=\mathrm{MTX}$ \\
\hline \multicolumn{2}{|l|}{ Emissivity: } \\
\hline Debris-gas & RADGAS $=0.8$ \\
\hline Atm.-struc. & GASSUR $=0.8$ (during DCH) ${ }^{*}$ \\
\hline \multicolumn{2}{|l|}{ Chemistry: } \\
\hline Drop diffusivity & $\begin{array}{c}\text { LIQSIDE } 0.00 .00 .00 .0 \\
\text { (Specifies infinite diffusivity; hence no drop-side limit) }\end{array}$ \\
\hline Threshold temp. & Default; however, see discussion in text. \\
\hline
\end{tabular}


Table 13-1 (Continued)

Summary of the CONTAIN Standard Input Prescription for DCH Experimental Analyses

\begin{tabular}{|c|c|}
\hline $\begin{array}{l}\text { Parameter or } \\
\text { Model Option }\end{array}$ & Values \\
\hline \multicolumn{2}{|l|}{$\begin{array}{l}\text { Non-airborne } \\
\text { debris: }\end{array}$} \\
\hline Location & Cavity and subcompartments, not in dome \\
\hline Diameter & DIATRAP from Equation (13-5) \\
\hline Time dependence & Shut off at end of RPV blowdown time \\
\hline \multicolumn{2}{|l|}{ Cavity water: } \\
\hline Time-dependence & Scale to $S_{d}(t)$ \\
\hline Amount & Vary fraction participating in sensitivity studies \\
\hline $\begin{array}{l}\text { Co-ejected RPV } \\
\text { water }\end{array}$ & $\begin{array}{l}\text { Nominal "standard prescription" is as for cavity water; however, } \\
\text { timing of debris dispersal and water ejection should be considered. } \\
\text { See text. }\end{array}$ \\
\hline Deflagrations & $\mathrm{FLAM} \geq 10 \mathrm{~m} / \mathrm{s}$ \\
\hline \multicolumn{2}{|l|}{ DFB model: } \\
\hline MFSCB & 0.95 \\
\hline MFOCB & 0.01 \\
\hline \multicolumn{2}{|l|}{ BSR model: } \\
\hline SRRATE & $(5 \mathrm{~m} / \mathrm{s}) / \mathrm{V}_{\mathrm{g}}^{1 / 3}$ \\
\hline $\begin{array}{l}\text { Atm.-structure } \\
\text { emissivity }\end{array}$ & GASSUR $=0.8$ (during DCH) ${ }^{*}$ \\
\hline \multicolumn{2}{|l|}{ Flow velocities: } \\
\hline $\mathrm{A}_{\mathrm{h}}$, cavity & Geometric cross section \\
\hline$A_{h}$, elsewhere & Default $\left(\mathrm{V}_{\mathrm{g}}^{2 / 3}\right)$; however, see text \\
\hline Nodalization & See text and References Wil95 and Pil95 \\
\hline
\end{tabular}

${ }^{*}$ GASSUR is not available in versions earlier than CONTAIN 2.0. KMX $=-0.8$ in the RAD-HEAT block may be used; see Appendix B. 


$$
\tau_{\mathrm{h}}=(0.4)\left(\frac{\mathrm{S}}{0.1}\right)\left(\frac{0.4}{\mathrm{~d}_{\mathrm{h}} / \mathrm{S}}\right)^{2} \sqrt{\frac{6.3}{\mathrm{P}_{\mathrm{RPV}}^{0}}}
$$

Here, $\tau_{h}$ is in seconds, $d_{h}$ is the final value of the vessel failure size in meters, $\mathrm{P}_{\mathrm{RPV}}^{0}$ is the pressure in the RPV in MPa at the start of blowdown, and $S$ is the geometric linear scale factor; i.e., $S=1$ for NPP analysis, $S=0.1$ for analysis of a $1 / 10$-scale experiment, etc. In principle, the pressure $P_{R P V}^{0}$ should be corrected for the decrease in pressure corresponding to the increase in RPV free volume that results from ejection of the melt. This correction is given by

$$
\mathrm{P}_{\mathrm{RPV}}^{0}=\mathrm{P}_{\mathrm{RPV}, \mathrm{VB}}\left(1-\frac{\mathrm{V}_{\text {melt }}}{\mathrm{V}_{\mathrm{RCS}}}\right)^{\gamma}
$$

where isentropic depressurization is assumed, $\mathrm{P}_{\mathrm{RPV}, \mathrm{VB}}$ is the pressure at vessel breach prior to melt ejection, $V_{\text {melt }}$ is the volume of the melt, $V_{R C S}$ is the reactor cooling system volume, and $\gamma$ is the ratio of specific heats ( 1.33 for steam). In most cases, this correction is quite small and it is sometimes neglected in practice.

Debris Sources. Results of the CONTAIN validation effort indicate that the calculation will be insensitive to the details of the time-dependence of the debris source provided $f_{\text {disp }}$ and the degree of coherence between debris dispersal and blowdown steam are approximately correct. A simple trapezoidal time-dependence is therefore recommended for $S_{d}(t)$. In the DCH experiments that have been performed with driving pressures greater than about $4 \mathrm{MPa}, \mathrm{f}_{\text {disp }}$ values are fairly large, 0.65 0.85. Dispersal fractions may be larger at NPP scale because one factor limiting dispersal is freezing of debris on cavity surfaces and this effect would be expected to decrease with increasing scale; note, however, that comparison of the 1/40-scale ANL/IET experiments with their 1/10-scale SNL/IET counterparts did not reveal a clear scale-dependence of this type. Unless the RPV driving pressure is low, it is suggested that NPP calculations be performed assuming $f_{\text {disp }}=0.7-0.9$. CONTAIN calculations for compartmentalized containment geometries generally indicate rather limited sensitivity to $f_{\text {disp }}$ when it is varied over a range that is consistent with the experimental results, while sensitivity to $f_{\text {disp }}$ may be higher in open containment geometries.

The concept of coherence is potentially important in compartmentalized geometries because interactions of airborne debris may be limited by the amount of steam that accompanies the debris. Low coherence is a potential mitigator of DCH loads [Pil96]; however, CONTAIN calculations typically do not show high sensitivity to coherence and approximate estimates of coherence based upon experimental correlations are believed to be adequate. The standard prescription adopts, with some modifications, the representation of coherence defined by Pilch, [Pil96] who has parameterized coherence in terms of a dimensionless quantity called the coherence ratio, $R_{\tau}$. This coherence ratio may be thought of as the ratio of the characteristic time required for debris entrainment, $\tau_{e}$, to the 
characteristic time for blowdown, $\tau_{\mathrm{b}}: \mathrm{R}_{\mathrm{r}}=\tau_{\mathrm{e}} / \tau_{\mathrm{b}}$. Experimental values of $\mathrm{R}_{\tau}$ are estimated from the values of $\mathrm{P}_{\mathrm{RPV}}^{0}$ and the $R P V$ pressure at the end of the entrainment interval, $\mathrm{P}_{\mathrm{RPV}, e}$, from the relation

$$
\mathrm{R}_{\tau}=\frac{2}{\gamma-1}\left[\left(\frac{\mathrm{P}_{\mathrm{RPV}}^{0}}{\mathrm{P}_{\mathrm{RPV}, \mathrm{e}}}\right)^{\frac{(\gamma-1)}{2 \gamma}}-1\right]
$$

In the absence of an experimental estimate of the coherence, it is suggested that $R_{r}$ be estimated using a simplified version of a semi-empirical model suggested by Pilch [Pi196,Wil95]:

$$
\begin{gathered}
R_{\tau}=C_{1}\left(\frac{m_{d}^{0} V_{c}^{1 / 3}}{m_{g}^{0} V_{R C S}}\right)^{1 / 2} d_{h} \\
C_{1} \approx 4
\end{gathered}
$$

where $\mathrm{V}_{\mathrm{c}}$ is the cavity volume, $\mathrm{m}_{\mathrm{d}}^{0}$ and $\mathrm{m}_{\mathrm{g}}^{0}$ are the respective masses of debris and of steam and gas in the RPV, and $C_{1}$ is a coefficient that was estimated in Reference Wil95 by fitting to experimental values of $R_{\tau}$ calculated from Equation (13-3). Considerable variation was found in the value of $C_{1}$ obtained for different experiments; in particular, values estimated for the ANL/ET experiments $\left(\mathrm{C}_{1}\right.$ $=6.7-7.8)$ averaged at least twice as large as values obtained from their SNL/ET counterpart experiments $\left(C_{1}=1.8-3.9\right)$. If these differences are actually the result of the different experimental scales, it represents an important limitation to this approach for estimating coherence, since neither Equation (13-4) nor the complete model given in Reference Pil96 predicts any dependence of $R_{\imath}$ upon scale.

Whatever the reason for the differences between the ANL/ET and SNL/ET coherence results, these differences do suggest that substantial uncertainties should be allowed for when using Equation (134) to estimate coherence. The fact that only a limited domain of parameter space has been studied experimentally reinforces this belief. When Equation (13-4) predicts small values of $R_{t}$ (i.e., $<0.5$ ), it is suggested that sensitivity calculations be performed assuming a larger value, $R_{\tau} \approx 0.75$ to 1 , in order to determine whether the results of interest are dependent upon $R_{\tau}$ being small. Fortunately, available evidence indicates that CONTAIN calculations are usually not very sensitive to coherence, with factor-of-two variations in $\mathrm{R}_{\tau}$ resulting in changes in the calculated $\triangle \mathrm{P} \leq 10 \%$ in both NPP calculations [Wil88a] and experimental analyses. However, sensitivity might be greater in some instances; it should probably be checked for the scenario of interest unless results are available for a similar scenario.

To summarize, we offer the following procedure for defining the RPV blowdown and the debris sources in a manner consistent with the preceding discussion. For the sake of definiteness, the procedure has been made more prescriptive as to detail than is really necessary. The relationships between some of the quantities of interest is diagramed schematically in Figure 13-4. 
1. Use Equation (13-1) to define $\tau_{\mathrm{h}}$ and run a calculation for the blowdown only to obtain the RPV pressure as a function of time (solid curve, Figure 13-4). The RPV depressurization curve is drawn for a case with $\tau_{\mathrm{h}}=2 \mathrm{~s}$.

2. Using Equation (13-4), estimate the value of $R_{\tau}$ for the scenario of interest.

3. From Equation (13-3), calculate the value of $P_{R P V, e}$ corresponding to the value of $R_{\tau}$ estimated in the second step, and determine the time $t_{e}$ at which the RPV pressure falls to this value using the pressure-time history calculated in the first step. Figure 13-4 is drawn for a case with $\mathrm{P}_{\mathrm{RPV}}^{0} / \mathrm{P}_{\mathrm{RPV}, \mathrm{e}} \approx 1.6, \mathrm{R}_{\mathrm{r}} \approx 0.36$, and $\mathrm{t}_{\mathrm{e}}$ equals $3 \mathrm{~s}$.

4. Using debris sources of type TRAPBIN, introduce the total melt mass into the trapped field for the cavity cell. The timing is not very critical except that the supply of debris in the trapped field must be adequate at all times to supply the entrainment sources specified in the next step; hence it is recommended that the TRAPBIN sources should begin before the onset of blowdown at time $t_{b}^{0}$ (defined to be the zero time in Figure 13-4) and should be completed prior to the end of entrainment at time $t_{\mathrm{e}}$. Note that the standard prescription takes no credit for melt remaining in the RPV; hence all the melt is placed in the cavity. However, any melt remaining in the RPV can be taken into account when defining $f_{\text {disp. }}$. That is, $1-f_{\text {disp }}$ is equal to the fraction remaining in the cavity after dispersal plus the fraction remaining in the RPV.

5. Using debris sources of type ENTRAIN, transfer debris from the cavity trapped field to the airborne fields. Start the airborne debris source at time $t_{b}^{0}+0.25 \tau_{h}$ and terminate the source at $t_{e}$. Divide the interval between the source start time and the end time into three equal segments and use these to define a trapezoidal time dependence for the airborne debris source, normalized to integrate to the intended value of $f_{\text {disp. }}$. The suggested start time, $t_{b}^{0}+$ $0.25 \tau_{\mathrm{h}}$, is based upon the experimental observation that debris dispersal begins well before the rate of accumulator depressurization reaches its maximum. The trapezoidal timedependence is suggested as a simple approximate representation of the actual debris dispersal histories as they were inferred from the experimental cavity pressurization histories.

Debris Particle Size Distributions and Compositions. Based upon experimental measurements, the standard prescription specifies a log-normal size distribution with a geometric standard deviation of 4; with five size groups (which often appears to be adequate) the corresponding particle sizes are $0.1692,0.4834,1.0,2.069$, and $5.911 \mathrm{~mm}$. Sensitivity to particle size is typically rather small in compartmentalized geometries and larger in open geometries. Since the actual particle size is quite uncertain, sensitivity calculations with a reduced particle size may be advisable unless prior experience with similar analyses indicates sensitivity to particle size is small. In performing calculations with a reduced particle size, it is normally unnecessary to reduce the size of the smallest size group in the standard prescription because particles this small usually will quickly equilibrate with the atmosphere. Making the particles even smaller will not change the equilibrium state, and running with unnecessarily small particle sizes will force very small timesteps. Calculations are most likely to be sensitive to particle size in open containment geometries than in compartmentalized geometries. 


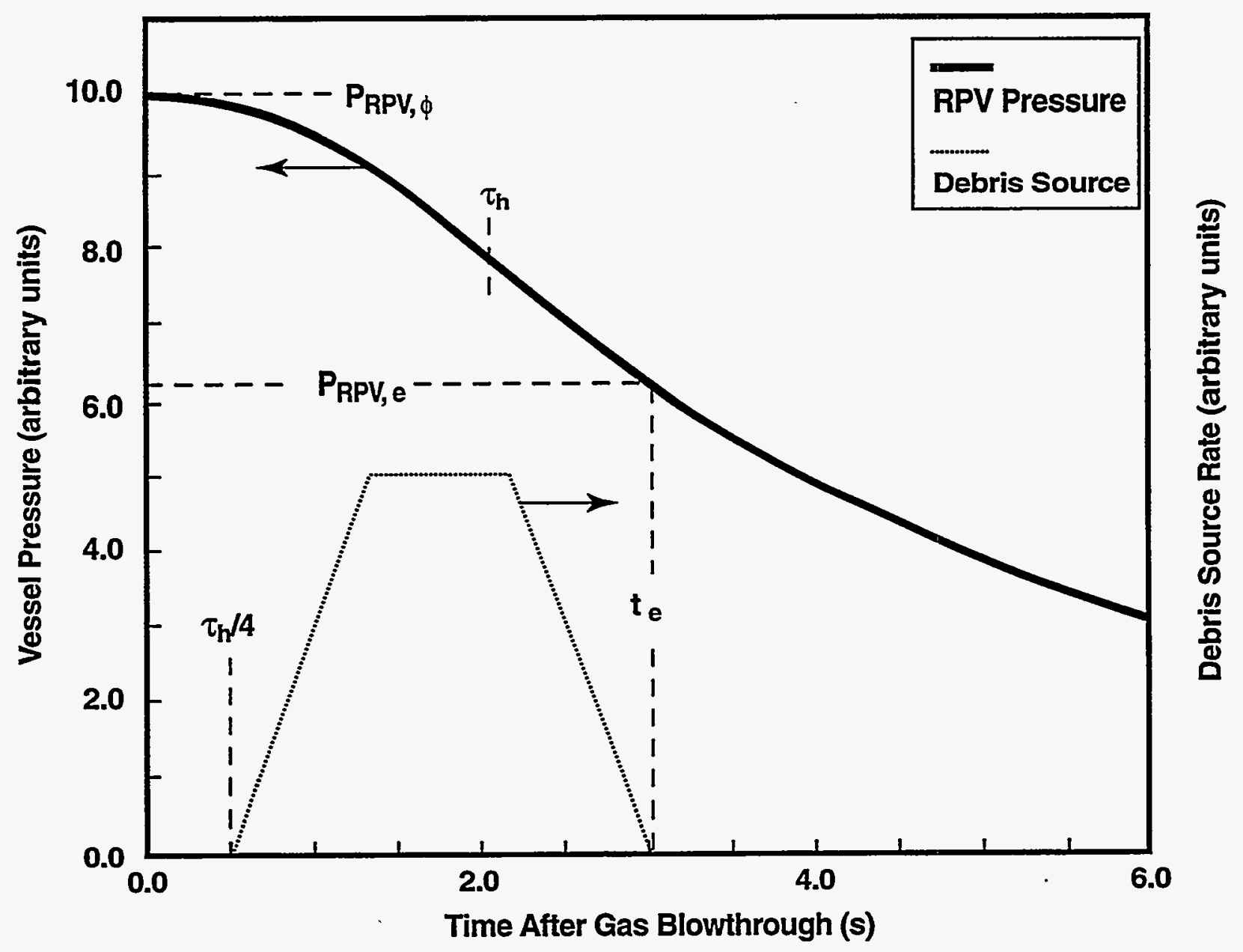

Figure 13-4. Schematic of Approach for Defining RPV Blowdown and Airborne Debris Sources in DCH Calculations 
In the standard prescription, the composition of each debris field equals the bulk composition of the debris (well-mixed assumption). Sensitivity calculations assuming separation of metal and oxide constituents may be performed, but they are believed to be unnecessary in most instances.

The CONTAIN multigeneration feature (see Sections 6.1.2 and 14.2.7) is not used in the standard prescription, which means that the code homogenizes fresh debris with aged debris. As a result, the surface area associated with aged debris (which may no longer contain reacting metal) is still credited in calculating reaction rates, which can overpredict the reaction rate. On the other hand, inclusion of aged debris may result in the debris temperature calculated being lower than is appropriate for the fresh debris. In the standard prescription, the latter effect is not important because strongly temperature-dependent reaction rate effects are not modeled in the standard prescription. It could be very important if a reaction temperature threshold, or a temperaturedependent drop-side reaction rate limit, is modeled; partly for this reason, these features are not included in the standard prescription.

Experience with the multi-generation feature is limited. Experimental analyses were generally insensitive to the debris homogenization effects in the cases that have been considered. However, the importance of these effects can be greater at full scale. A large number of generations may be required to avoid the homogenization effect in plant-scale calculations.

RPV Insulation. The RPV is typically covered with insulation consisting of thin stainless steel sheets and foils. This insulation was simulated in the SNLIET-11 experiment, and the insulation was largely stripped (apparently by melting ablation), opening up the annular gap between the RPV and the biological shield wall. The ablated insulation may have contributed to hydrogen production. [Bla94] The standard prescription includes adding the iron and chromium content of the insulation below the RPV nozzles to the airborne debris fields, with insulation above the nozzles being assumed not to participate. Half the mass is added to the cavity cell and half is added to the cell that is downstream of the annular gap. The time dependence is that of the debris sources and the temperature should correspond to the insulation temperature prior to vessel breach. Since this temperature is relatively low, the insulation does not contribute significantly to the available thermal energy, but it can contribute to the potential for hydrogen generation and combustion.

Based upon the SNL/ET-11 experimental results, it is thought that this prescription is either reasonably close to best-estimate or tending to err on the side of conservatism, but that it is unlikely to be very nonconservative. Less conservative assumptions may be used if they can be adequately defended. For example, the sample input file for the Sequoyah plant given in Chapter 15 includes only the half of the insulation directed to the cavity cell and omits the half directed to the downstream cell. The reason is that, in ice condenser plants such as Sequoyah, the gap does not communicate directly to the containment dome as is typical of many large dry containments in pressurized water reactors ( $\mathrm{PWRs}$ ); instead it communicates only to a relatively constricted volume located above the RPV and below the deck separating the upper and lower compartments of the containment. In the CONTAIN representation, the entire lower compartment was modeled as a single cell. In reality, there are only limited connections with the main volume of the lower compartment, and the volume involved is only a small fraction of the total volume of the lower 
compartment. Hence it was judged that including the insulation from the upper part of the vessel would be overly conservative.

Debris Transport and Trapping. The representation of debris-gas slip in CONTAIN is highly simplified; Section 6.2.1 may be consulted for additional information. The standard prescription consists of specifying the slip parameter $\mathrm{s}=5$ for all airborne fields in the cavity and in the chute volume connecting the cavity to the subcompartments, and specifying $s=1$ elsewhere. This prescription gave good results for the Zion- and Surry-geometry IET experiments, and $\Delta \mathrm{P}$ and hydrogen production were found to be insensitive to variations in $\mathrm{s}$ in either the cavity or the subcompartments. For the less prototypic geometries of the SNL/LFP experiments, the standard prescription overpredicted debris transport to the dome and hence overpredicted $\Delta \mathrm{P}$. Specifying a higher slip factor $(s=5)$ in the subcompartment gave more realistic results for the SNL/LFP experiments but underpredicted debris transport to the dome in the IET experiments. If cavity pressurization is of interest, the standard prescription was found to underpredict the extent of cavity pressurization relative to the dome (i.e., the difference $P_{\text {cavity }}-P_{\text {dome }}$ ) by factors of 2 to 4; considerably more realistic results were obtained assuming no slip $(s=1)$ in the cavity and chute. However, the no-slip assumption overestimates debris velocities exiting the cavity and is believed to be physically unrealistic. Compensating errors may be involved in the cavity pressurization calculation when no slip is assumed and caution is therefore warranted in using the $s=1$ specification in order to estimate the extent of cavity pressurization.

In the standard prescription, trapping in the cavity cell and chute is not modeled because the effects of any trapping in these regions is already taken into account in the value of $f_{\text {disp }}$ used to define the airborne debris sources. The time-of-flight/Kutateladze number (TOF/KU) trapping model with the options indicated in Table 13-1 is recommended as the standard prescription, although other options may be considered for the dome as noted below. In Table 13-1, the parameter $L_{1}$ refers to length of the debris flight path to the first structure impacted by debris, and $\mathrm{L}_{2}$ refers to the path length from the first to the second structure impacted, respectively. $\mathrm{L}_{3}$ is the distance from the second structure to the final collection surface of debris that does not de-entrain on either the first or second impacts, and $\mathrm{L}_{\mathrm{gft}}$ is the gravitational fall height, which is normally set equal to the cell height. The option RHODG = MIX specifies that the momentum flux of debris as well as that of the gas is credited in evaluating the Kutateladze number for the sticking criterion. The option VNOST = GFT specifies that, if debris does not stick on either the first or second structure impact, the gravitational fall velocity is used to estimate the flight time to the final collection surface. Section 6.3 .6 should be consulted for more detailed definitions of the trapping parameters listed in Table 13-1, and Section 14.3.2.11 provides the instructions for the relevant code input.

In the TOF/KU model, the first trapping length $\left(\mathrm{L}_{1}\right)$ is intended to represent the flight path length from the cell entrance point to the point of impact with the first major structure. If this distance cannot be estimated unambiguously, the generic estimate $6 \mathrm{~V}_{\mathrm{g}} / \mathrm{S}_{\mathrm{str}}$ may be used, where $\mathrm{V}_{\mathrm{g}}$ is the cell gas volume and $S_{\text {str }}$ is the total unsubmerged surface area of structures in the cell. This generic estimate is generally recommended for the second trapping length $\left(L_{2}\right)$ because the debris trajectories are expected to undergo considerable randomization following the first structure impact; different values may be used if the generic value is believed to be inappropriate for the specific cell of interest. In setting the trapping lengths, it is probably best not to take into account fine-scale structure such 
as small pipes, cabling, etc. Impact with such structures may be more likely to merely deflect the debris than de-entrain it and these structures may not survive impact by large amounts of debris.

For compartmentalized geometries, the most important debris transport issue is often the fraction of the debris transported to the dome $\left(f_{\text {dome }}\right)$. This transport fraction is governed by the relative rate at which airborne debris is transported by gas flow to downstream cells versus the rate the debris is removed from the cell atmosphere by trapping. Hence high trapping rates in the subcompartment cells tend to reduce $\mathrm{f}_{\text {dome }}$. The TOF/KU model, when used as suggested here, is thought to be either reasonably best-estimate or else tend toward conservatism in terms calculating $\mathrm{f}_{\text {dome }}$. However, it is not bounding; for example, it underestimated $f_{\text {dome }}$ by $20-40 \%$ in the Surry IET experiments. If it is thought that $f_{\text {dome }}$ is being overpredicted for the case at hand, and that this is significantly affecting the calculation, sensitivity calculations may be run with $s_{d}>1$ in the subcompartments and/or with the defaults used for RHODG and/or VNOST. In the TOF/KU model, variations in the critical Kutateladze numbers for de-entrainment $\mathrm{N}_{\mathrm{K \mu}, \mathrm{T}, 1}$ and $\mathrm{N}_{\mathrm{K \mu}, \mathrm{T}, 2}$ (see Sections 6.3.6 and Section 14.3.2.11) may be used to assess the effects of uncertainties in the de-entrainment criteria. For any trapping model, the TRAPMUL parameter may be used to vary trapping rates in either direction by an arbitrary user-specified factor. Sensitivity calculations such as these can help determine whether loads are sensitive to debris transport and trapping uncertainties, but we cannot offer defensible uncertainty ranges for these parameters that would be generally applicable to all problems.

In the dome, debris flight paths are typically long and the TOF/KU model often predicts the debris will stick on first impact, which may not be fully realistic; hence the model may be nonconservative if dome carryover is large. A sensitivity calculation with the critical Kutateladze number for sticking on the first impact $\left(\mathbb{N}_{\mathrm{Ku}, \mathrm{T}, 1}\right)$ set to a very small value can be performed to investigate the importance of this question. Alternatively, gravitational fall time trapping may be specified for the dome. These sensitivity studies may be unnecessary if $f_{\text {dome }} \leq 0.1$, since containment loads are not likely to be sensitive to trapping rates in the dome when the amount of debris reaching the dome is small.

The user is once again reminded that a major limitation of the CONTAIN models for debris transport and trapping is that debris is only transported by flow with the gas. Therefore, momentum-controlled transport of debris, such as through an intermediate cell when the entrance and exit flow paths are aligned, is not represented. A consideration of the specific containment geometry of interest is required in order to decide whether this limitation is important. Some of the effects of enhanced transport through a cell owing to this effect can be simulated in various ways; e.g., by reducing trapping rates in the intermediate cell, specifying an additional (fictitious) flow path directly connecting the upstream and the downstream cells (i.e., bypassing the intermediate cell), or by introducing an appropriate amount of debris directly into the downstream cell using source tables. None of these approaches is fully satisfactory, however: reducing trapping rates in the intermediate cell increases debris transport out all exit paths rather than just the aligned path, and unrealistically increases total airborne residence time; specifying the fictitious flow path permits gas as well as debris to bypass the intermediate cell; and introducing some of the debris directly into the downstream cell does not account for debris-gas chemical reaction and heat transfer that would actually occur in transit to the downstream cell. 
DCH Heat Transfer. In the model for heat transfer between airborne debris and the gas, the only nondefault parameter setting recommended is the black-body multiplier (RADGAS) for debris-gas radiation. Given the particle size, this debris-gas heat transfer model is considered to be adequate. Effects of any uncertainties in this model will be qualitatively similar to, and smaller in magnitude than, the effect of the uncertainties in particle size upon the heat transfer rates. If sensitivity calculations for particle size are carried out, additional sensitivity studies involving debris-gas heat transfer parameters are not needed.

The presence of dense clouds of hot aerosols during DCH events is expected to enhance atmospheric emissivities above what is calculated by the CONTAIN default atmospheric emissivity model; hence the standard input includes specifying an atmosphere emissivity (GASSUR) equal to 0.8 during the DCH event. In code versions prior to CONTAIN 2.0, this option is not available, but the user may specify $\mathrm{KMX}$ equal to -0.8 in the RAD-HEAT input block in order to obtain a similar result insofar as the DCH calculation itself is concerned. However, when this option is employed, the userspecified emissivity will be used for the entire calculation, not just during the DCH event. Hence the GASSUR option is preferred for CONTAIN 2.0. However, a sensitivity calculation for the Sequoyah plant indicated that the inappropriate use of $\mathrm{KMX}=-0.8$ prior to vessel breach had very little effect upon containment pressures $(\sim 0.001 \mathrm{MPa})$.

Although dense clouds of hot aerosols have been assumed to enhance emissivities in defining the standard prescription, concerns do exist that the emissivity could actually be reduced if the aerosol clouds are so dense that the optical mean free path is less than that of the thermal boundary layer, so that structures cannot "see" the hot gas/aerosol cloud beyond the boundary layer. In this event, the standard value could prove nonconservative. Little quantitative guidance as to the possible magnitude of this effect is currently available, however.

Sensitivity studies given in Reference Wil95 for the SNL/IET-3 and SNL/IET-11 experiments indicated that use of the default radiation treatment or using $\mathrm{KMX}=-0.4$ had at most a moderate impact on $\triangle \mathrm{P}$ ( $\leq 11 \%$ enhancement). Totally eliminating atmosphere-structure radiant heat transfer enhanced $\triangle \mathrm{P}$ by $32 \%$ in the SNLIET-11 experiment, a relatively severe event in which high atmosphere temperatures resulted in radiation being especially important. Since totally eliminating radiant transfer is clearly unrealistic, even as a bound, we believe that more realistic uncertainty estimates are provided by running sensitivity calculations with the default radiation treatment and/or with $\mathrm{KMX}=-0.4$.

DCH Chemistry. The user of code versions prior to CONTAIN 2.0 should take care to specify the drop diffusivity (LIQSIDE) as in Table 13-1, which will eliminate drop-side reaction rate limits through an infinite drop diffusivity. The default diffusivity in the previous code versions, $10^{-8} \mathrm{~m}^{2} / \mathrm{s}$, can yield strongly nonconservative results; e.g., underpredicting hydrogen production by more than a factor of two in the SNL/ET-1R and SNL/IET-3 experiments, and underpredicting $\triangle \mathrm{P}$ by $\sim 35 \%$ for SNL/ET-3, in which combustion of the DCH-produced hydrogen is a major contributor to the DCH load.

The default value of the reaction threshold temperature (THRESH $=273.15$ ) is obviously unrealistically low, but is specified for the standard prescription in order to avoid spurious quenching 
resulting from homogenizing cool aged debris with hot, fresh debris. In calculations that do not involve water, specifying a higher value $(1200 \mathrm{~K}$ or even $1800 \mathrm{~K})$ typically makes little difference because gas temperatures in locations containing substantial debris become too high to cool the debris significantly, and debris is typically either completely reacted, trapped, or both before it cools sufficiently for quenching of chemical reaction to be physically reasonable.

In scenarios involving water, calculated atmospheric temperatures can be much lower, and quenching of chemical reaction can be a physically realistic possibility. This potential mitigation effect is not captured in the standard prescription because neither the reaction threshold nor an appropriate temperature-dependent drop-side diffusivity is specified. However, specification of a reaction temperature threshold and/or a temperature-dependent drop-side diffusivity can result in very nonconservative results owing to the spurious quenching effect resulting from homogenizing fresh reacting debris with cold aged debris. Hence, if these features are to be used in calculations involving water, the multigeneration feature is required to avoid the spurious quenching effect. There is little experience in using this approach and little guidance can be given; it is up to the user to ensure that any quenching of chemical reaction calculated is realistic.

Non-Airborne Debris. DCH analysis has traditionally emphasized the interactions of airborne debris with blowdown steam and the containment atmosphere. However, analysis of the DCH experiments discussed in Section 13.3.2.2.1 shows that it is difficult to account for all the hydrogen produced in the experiments if interactions of non-airborne debris with blowdown steam are not also modeled. The CONTAIN model for these interactions is partially parametric and the standard prescription for its use is based partly upon empirical results and partly upon a stand-alone model for non-airborne debris interactions. We summarize here the standard prescription and suggested sensitivity studies with relatively little discussion.

Analysis of the Zion IET experiments shows that calculations that included only airborne debris and neglected cavity water underpredicted hydrogen production and $\Delta \mathrm{P}$ by about a factor of two. All of these experiments included at least a small amount of water in the cavity. Neither the experimental data nor the modeling were adequate to completely separate the effects of non-airborne debris and co-dispersed cavity water. In the 1/10-scale Zion IET experiments, $\Delta \mathrm{P}$ and hydrogen production were reproduced well assuming a trapped field diameter, $\mathrm{d}_{\text {nad }}$, equal to $0.01 \mathrm{~m}$ if the water is ignored, or equal to $0.02 \mathrm{~m}$ if the water is included as described below; the results given in Figure 13-4 were calculated assuming $\mathrm{d}_{\text {nad }}=0.01 \mathrm{~m}$ and included no water. The integral results provided no basis for choosing between these two prescriptions, but the calculated pressure-time histories agreed better with experimental results for the prescription including the water. On the other hand, the prescription with $\mathrm{d}_{\mathrm{nad}}=0.01 \mathrm{~m}$ gave satisfactory results for other experiments in which there was no cavity water. The calculated effects of non-airborne debris were smaller for the other experiments than for the Zion IET experiments, but the effect upon hydrogen production was still significant in almost all cases.

The non-airborne model does not capture the effects of facility scale properly. When analyzing DCH events other than $1 / 10$-scale experiments, $d_{\text {nad }}$ should be adjusted to compensate for the difference in scale. The recommended scaling law is 


$$
\begin{aligned}
& d_{\text {nad }}=0.0464 \mathrm{~S}^{2 / 3} \text { (no water) } \\
& d_{\text {nad }}=0.0928 \mathrm{~S}^{2 / 3} \text { (with water) }
\end{aligned}
$$

where $d_{\text {nad }}$ is in meters and $S$ is the linear scale factor; e.g., $S \equiv 1$ for NPP analysis, $S=0.1$ for $1 / 10$ scale experiments, etc. This scaling law was tested against the ANLMET (Zion) experiments ( $\mathrm{S}=$ $0.0255)$ and the Surry-geometry experiments in CTTF $(S=1 / 5.75)$, with reasonably good results being obtained. ${ }^{2}$

There are substantial uncertainties in the modeling of non-airborne debris interactions. It is therefore suggested that a case be run with non-airborne interactions deleted in order to determine their importance in the standard case. In addition, a conservative case may be defined for NPP analysis by specifying $\mathrm{d}_{\mathrm{nad}}=0.01 \mathrm{~m}$ (analyses without water) or $0.02 \mathrm{~m}$ (analyses with water); this in effect corresponds to taking no credit for rescaling $\mathrm{d}_{\text {nad }}$ from the values used in 1/10-scale experiments. Analyzing the 1/40-scale ANL/IET-3 experiment with these values (i.e., without scaling $d_{\text {nad }}$ ) underpredicted $\triangle \mathrm{P}$ and $\mathrm{H}_{2}$ production, with agreement being significantly poorer than that obtained using Equation (13-5). Hence, NPP analyses performed without rescaling $\mathrm{d}_{\text {nad }}$ can reasonably be expected to be conservative. Taken together, the cases suggested here should provide a reasonable bound on the uncertainties associated with non-airborne debris interactions.

DCH and Co-Dispersed Cavity Water. An acknowledged limitation of CONTAIN is that it does not have an FCI model, nor does it have a model for entrainment of water in the cavity and dispersal from the cavity. Some guidance on simulating melt-water interactions in non-DCH scenarios, such as debris quenching and steam-spike production, is summarized in Section 13.3.3.6. Here we consider ways to simulate the potential effects of cavity water and/or co-ejected RPV water in DCH scenarios. Since these effects are both potentially substantial and quite uncertain, we discuss this subject in more detail than is the case for most other DCH modeling issues considered in this section.

The starting point of the treatment is the observation that containment pressurization is primarily sensitive to certain integral quantities, not the details of the debris-water interactions. These integral quantities include energy absorbed in vaporizing water, the quantities of steam generated, energy transferred to the steam, and hydrogen generated and burned. In addition, the enhanced steam flow rates resulting from vaporization of water can affect (reduce) the degree of $\mathrm{DCH}$ mitigation provided by atmosphere-structure heat transfer and hydrogen hold-up in oxygen-starved subcompartments. The CONTAIN model can be used to simulate these effects of debris-water interactions, albeit with some important limitations acknowledged in the discussion that follows.

In calculations involving co-dispersed cavity water and/or co-ejected RPV water, the normal procedure is to specify an atmospheric source of low-enthalpy steam (i.e., steam with an enthalpy

\footnotetext{
${ }^{2}$ The analyses cited in Reference Wil95 were actually carried out with a slightly different version of the scaling law with the exponent of $S$ equal to 0.8 and with the lead constants adjusted to give the same $d_{r}$ values as Equation (13-5) at 1/10-scale. Analyses of the ANL/IET-3 experiment with both versions indicated that differences in the results were small, $\sim 1 \%$ and $\sim 7 \%$ change in $\Delta \mathrm{P}$ and hydrogen production, respectively.
} 
equivalent to that of the liquid water) in the cavity cell such that the timing of this source overlaps the time during which the debris is specified to enter the cavity. The water enters as an atmospheric component, and chemical and thermal interactions with the debris are modeled using the standard CONTAIN DCH heat and mass transfer models. No direct melt contact with liquid water is modeled, and no thermal resistance between liquid water and the heated atmosphere is modeled. Thus, the airborne water is assumed to be in thermodynamic equilibrium with the local atmosphere. On the other hand, since the standard DCH models for debris-atmosphere interaction rates are controlling, the debris is usually not in equilibrium with the atmosphere.

The treatment corresponds to the situation illustrated in Figure 13-5, in which debris and liquid water are both present as dispersed drops, with little direct liquid-liquid contact, and the water drops are small compared with the debris particles. In this case, the atmosphere does mediate the heat transfer to the water, and the controlling thermal resistance is the debris-to-gas thermal resistance, which is modeled. The controlling resistances to heat transfer $\left(R_{h x}\right)$ and mass transfer $\left(R_{m x}\right)$ are diagramed schematically in Figure 13-6. The subscripts 1,2, and 3 refer to debris-gas, gas-water, and direct debris-water interactions, respectively. Figure 13-6a corresponds to the configuration of Figure 13-5; here, $R_{h x, 1} \gg R_{h x, 2}$ and, because there is little direct debris contact with liquid water, $R_{h x, 3} \approx \infty$ and $\mathrm{R}_{\mathrm{mx}, 3} \approx \infty$ (represented as "open circuits" in the diagram). Hence the controlling processes are those that are included in the models of the code.

In an actual FCI involving direct debris-water contact, the schematic in Figure 13-6b may apply; in this case, the code may not capture all the important processes controlling the interactions. This situation may well apply when the amount of cavity water is large. It is less likely to apply when amounts of water are small because only small amounts of energy transfer, whatever the process, are required to vaporize all the water; subsequent processes are only those modeled for dry DCH scenarios.

Whether Figure 13-6a or 13-6b applies, given the amount of debris-water chemical and thermal interaction that occurs, the code does model the subsequent implications of the interaction using the same models as those used for DCH events not involving water. Effects modeled include the enthalpy required to vaporize the water (i.e., debris quenching), the effects of increased steam supply on debris-gas thermal and chemical interactions, and effects upon DCH mitigation processes involving atmosphere-structure heat transfer and hydrogen holdup in the subcompartments.

If energy transferred to the gas is insufficient to vaporize all the water, CONTAIN assumes that the remainder of the water will condense in the atmosphere as water aerosol. Except for a small amount that may deposit on structures, this water aerosol will transport with the gas flow. This water remains available as a potential atmospheric heat sink since the water aerosol can subsequently reevaporate as DCH energy is added to the containment atmosphere in the calculation. Physically, this mitigation mechanism is reasonable, although the CONTAIN model may overpredict the effect because de-entrainment of airborne water by subcompartment structures is not modeled except for the processes treated by the aerosol deposition model, which are relatively slow.

When debris and water interact, unvaporized water may be ejected ahead of the debris. The inertia of the water that must be ejected can enhance the extent of cavity pressurization, an effect sometimes 


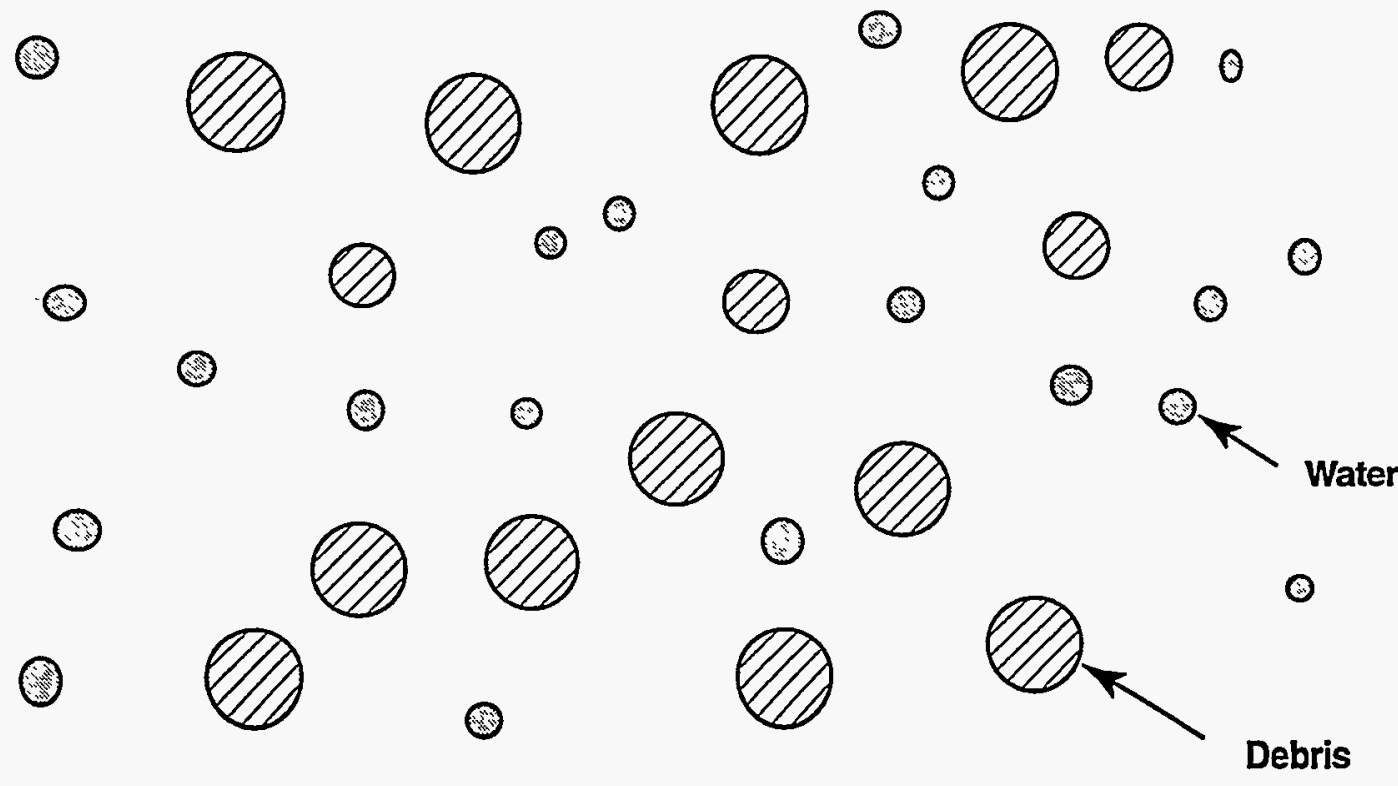

Figure 13-5. Dispersed Debris and Liquid Water Fields Corresponding to the CONTAIN Treatment

(a) Schematic Corresponding to Figure 13-5

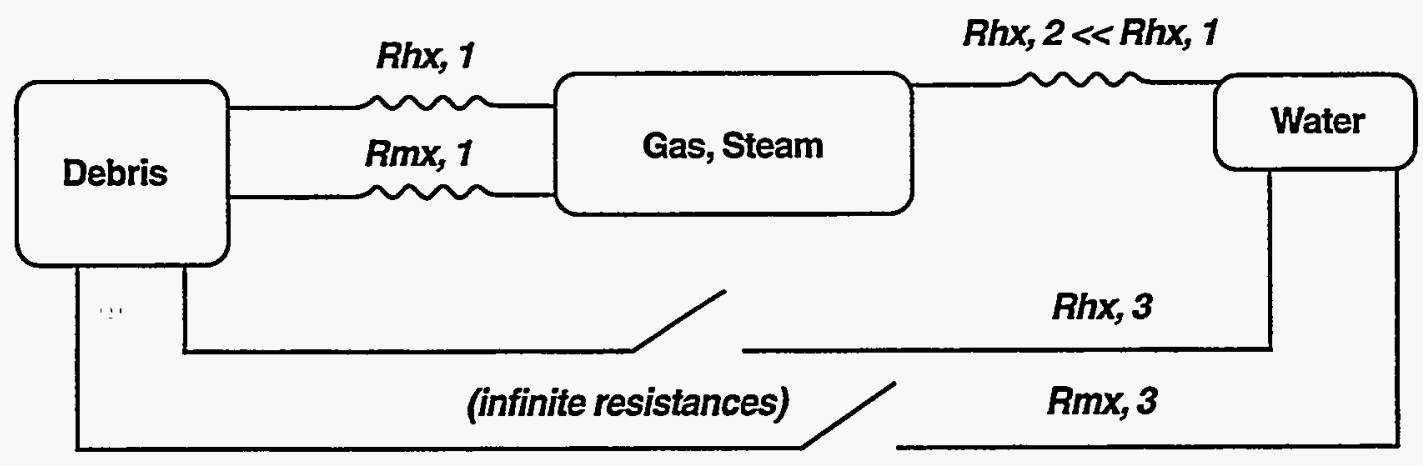

(b) Schematic for $\mathrm{FCl}$ with Direct Debris-water Contact

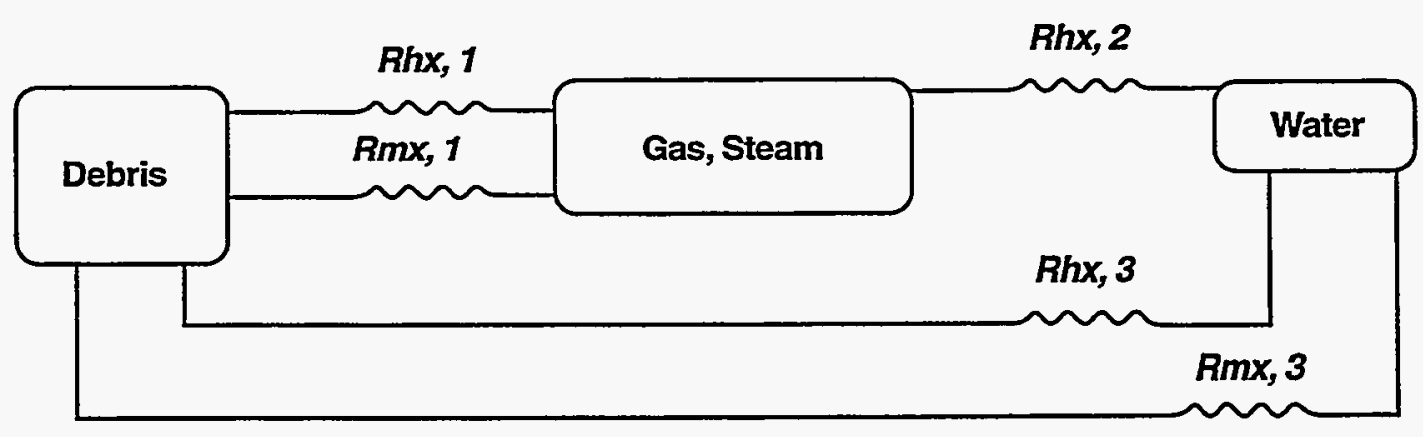

Figure 13-6. Heat and Mass Transfer Schematics for Debris-Water Interactions 
called "tamping." Since aerosols are not included in the mass treated by the flow solver, the effects of tamping by unvaporized water on cavity pressures are not modeled. This limitation can be important in predicting cavity pressurization, but it is not expected to affect the calculated containment pressurization substantially.

Note that it can be important to model water aerosols in the calculation; otherwise, when the thermal energy is insufficient to vaporize all the water, the debris oxidation and heat transfer models misinterpret the liquid portion as contributing to the density of the vapor phase. The resulting overestimate of the vapor density leads to a corresponding overprediction of chemical reaction and heat transfer rates.

Not all the water initially in the cavity necessarily interacts with the debris; some may be blown out of the cavity by the initial interaction. The water blown out of the cavity may not interact efficiently with the debris (although debris-water interactions can occur in the subcompartments as well as in the cavity). CONTAIN has no models for calculating the amount of water that is dispersed without interacting. Because the CONTAIN code cannot predict how much interaction with water will actually occur or the timing of the interaction, it is perhaps more appropriate to refer to the calculations as evaluating the potential effects of water than to refer to them as actual predictions of these effects.

Given the amount of water in the cavity, the recommended approach is to run one calculation with the total amount sourced into the cavity in parallel with the airborne debris source, and to perform sensitivity studies for smaller amounts of water in order to simulate the possible effects of some water not participating. In this way, an uncertainty range is developed for the effects of the water. The sensitivity studies may not be needed when the amount of water is small, since the usual effect of water on DCH calculations in compartmentalized geometries is to increase loads when amounts are small and decrease loads when amounts are large. (In open containment geometries, increased loads may not be predicted for any amount of water.) For small amounts, assuming all the water participates is usually the limiting case, but this assumption can be nonconservative for large amounts. Here, a "small amount" of water may be defined as an amount less than the amount the debris energy is capable of vaporizing without substantially cooling the debris, and conversely for a large amount.

It is suggested above that the water be introduced in parallel with the debris. Since CONTAIN cannot actually predict the timing of the melt-water interactions, sensitivity studies varying the timing of the water source relative to the debris source may be justified. However, it is not expected that the results obtained varying the timing will extend the uncertainty range defined by varying the amount of water as described in the preceding paragraph. Experience with varying the timing is somewhat limited, however.

Only a limited validation basis is available for this treatment. Analysis of the open-geometry WC-2 experiment with CONTAIN correctly predicted that the water has little effect upon $\triangle \mathrm{P}$, but overpredicted hydrogen production unless it was assumed that only a small fraction $(\sim 15 \%)$ of the water interacts. Analysis of the seven SNL/IET (Zion) experiments that had small amounts (3.48 $\mathrm{kg}$ ) of cavity water did not permit any conclusions to be drawn concerning the effect of the water 
insofar as the integral $\triangle \mathrm{P}$ and hydrogen production results were concerned; as noted previously, the effects of co-dispersed cavity water and non-airborne debris could not be resolved in the integral analysis. However, the analyses yielded better agreement between calculated and measured pressuretime histories when it was assumed the water did participate. Interaction with the dispersed water in the subcompartment was the suggested reason for the difference with respect to WC-2. [Wil95] The SNLIET-8B experiment is the only experiment analyzed in which relatively large amounts of water $(62 \mathrm{~kg})$ were present in the cavity. Experimental results showed that an efficient thermal interaction between debris and water did occur on DCH time scales, with interactions between dispersed water and debris in the subcompartment again being suggested as a possible reason for the apparent difference with respect to WC-2. [Bla94] About 75\% of the cavity water was vaporized, and an energy balance analysis indicated that the total net energy addition to the atmosphere (latent plus sensible heat) was considerably greater in SNL/IET-8B than in SNL/IET-7, about 146 MJ versus $80 \mathrm{MJ}$, respectively. (The two experiments were quite similar except for the amount of cavity water.) However, the oxidation efficiency for the two experiments was about equal, based upon the amounts of hydrogen produced.

Analysis of the SNLAET-8B experiment with CONTAIN, assuming all the water participated, gave reasonably good agreement for the amounts of hydrogen produced and burned and the amount of steam generated, but underpredicted $\triangle \mathrm{P}(0.146 \mathrm{MPa}$ calculated versus $0.244 \mathrm{MPa}$ observed $)$. Thus, the mitigative effect of the water was overpredicted. The inability of the code to model enhanced heat transfer rates associated with FCIs is presumably the reason. Note that the underprediction of heat transfer from debris together with the approximately correct prediction of hydrogen production implies that the heat/mass transfer ratio was not being evaluated correctly. This mismatch between the calculated heat transfer and mass transfer did not arise in any of the other experimental analyses. This result for SNLIET-8B can be understood from Figure 13-6b; interactions of debris directly with liquid water were likely important for this experiment and the CONTAIN heat/mass transfer analogy, which is based upon gas-phase transport rates, would not be expected to hold for these interactions.

It was recommended above that an uncertainty range be estimated for scenarios involving large amounts of cavity water by varying the fraction of the water assumed to participate. Application of this procedure to the SNLIET-8B experiment yielded a range of $0.146-0.385 \mathrm{MPa}$. While this range obviously does encompass the actual experimental result, it is also evident that the resulting uncertainty range can be undesirably broad.

To summarize, the limited results available suggest that debris interactions with cavity water may be quite inefficient in open containment geometries and considerably more efficient in at least some compartmentalized geometries. It also appears that the code can overestimate the mitigative effect of large amounts of water if all the water is assumed to participate. The effect of water in compartmentalized geometries may depend upon the details of the geometry, as the Surry-geometry SNL/IET-9 experiment had a small but unknown amount of condensate water in the cavity, and CONTAIN analyses of this experiment without including any water revealed no obvious discrepancies attributable to neglecting the water. The code predicts that, in compartmentalized geometries, small or moderate amounts of water can significantly enhance DCH loads under some conditions, but there are no clean experimental tests of this prediction. 
Co-Ejected Primary System Water. Water co-ejected from the RPV with the melt raises many of the same issues as co-dispersed cavity water, but there are also important differences. Co-ejected water accompanies and/or follows the melt, and therefore cannot be dispersed from the cavity as a slug in advance of the melt. Instead, much of the water may follow the dispersal of the debris. RPV water will partially flash to steam upon depressurization, and the remaining water is likely to be highly fragmented. Hence the situation may correspond more closely to the assumptions of the CONTAIN model than is the case for scenarios involving large amounts of co-dispersed cavity water.

For scenarios involving co-ejected water, a key uncertainty is the time interval over which debris is dispersed from the cavity. The only available "standard prescription" is to use Equation (13-4) to estimate the debris dispersal rate and then introduce the water source in parallel, as in the case of codispersed cavity water. However, there are several questionable features of this approach. First, we note that it may be more reasonable to estimate the rate at which water can be ejected from the RPV in a side calculation and use this rate to define the water source. In addition, Equation (13-4) is based upon experiments in which debris dispersal was driven by steam and its applicability to dispersal by flashing primary system water is doubtful. It may be noted that Equation (13-4) may be shown to be consistent with the assumption that debris dispersal rates vary as $\dot{\mathrm{m}}_{\mathrm{g}}^{1 / 2}$, where $\dot{\mathrm{m}}_{\mathrm{g}}$ is the steam blowdown rate $(\mathrm{kg} / \mathrm{s})$. If we assume that heat transfer from the debris vaporizes all the RPV water ejected during the period of active debris dispersal, the steam flow rate in the cavity will be approximately equal to the rate of water ejection from the vessel, $\dot{\mathrm{m}}_{\mathrm{w}}$. One might, therefore, estimate the dispersal time, $t_{\mathrm{w}, e}$, for the water-driven dispersal from

$$
\mathrm{t}_{\mathrm{w}, \mathrm{e}}=\mathrm{t}_{\mathrm{g}, \mathrm{e}}^{\prime}\left(\frac{\dot{\mathrm{m}}_{\mathrm{g}}^{\prime}}{\dot{\mathrm{m}}_{\mathrm{w}}}\right)^{1 / 2}
$$

Here, $t_{g, e}^{\prime}$ and $\dot{m}_{g}^{\prime}$ are respectively the dispersal time $t_{e}$ estimated from Equation (13-3) and the steam blowdown rate for a steam-driven event with the same RPV pressure as the actual scenario of interest. Many factors potentially affecting dispersal rates have been neglected here, and this approach should be used with caution and should be supplemented with sensitivity calculations.

The debris dispersal time for events involving co-ejected water should be considered quite uncertain. It is therefore recommended that users investigate sensitivity of the results to the assumed debris dispersal rate in calculations involving co-ejected RPV water.

Water on the Basement Floor. In most if not all accidents, there will be water on the basement floor. Available evidence indicates that this water does not have a large effect upon DCH. For example, the SNL/IET (Zion) experimental series included two pairs of counterpart tests with and without water on the basement floor. [All94] Comparisons of containment pressure response with the temperature response indicated that the basement water was not vaporized on DCH time scales to any large extent. Measured $\triangle \mathrm{P}$ values and hydrogen production with and without the water were the same to within $10 \%$ and $20 \%$, respectively, may be within experiment replication uncertainties. In the CONTAIN analyses of the cases with basement water, the water was not included and the analyses revealed no discrepancies that might reasonably be attributed to the neglect of the water.

Rev. 0 
Any attempt to model the effects of this water would be subject to uncertainties comparable to those involved in co-dispersed cavity water and co-ejected RPV water. In the absence of any significant evidence that basement water is important to $\mathrm{DCH}$, no attempt at defining a standard prescription for including this water has been made.

Gas Combustion. Although DCH experiments have been performed with hydrogen initially present in the atmosphere, in no case were the compositions within the flammable range. Hence the deflagration model never played an important role in the experimental analyses. However, flammable atmospheres may exist at vessel breach in some NPP scenarios. In this event, hot debris particles flying through the atmosphere are expected to provide multiple ignition sources that can substantially shorten burn times in scenarios for which the CONTAIN default flame speed correlation would normally predict a slow burn. Hence the flame speed (FLAM) is specified to be at least $10 \mathrm{~m} / \mathrm{s}$ in the standard prescription. No other nondefault deflagration parameters are specified in the standard prescription. Note, however, that hot debris might affect the combustion completeness, and sensitivity calculations may be desirable in cases in which incomplete deflagrations are predicted to occur.

Most of the combustion of DCH-produced hydrogen that occurs in a CONTAIN calculation usually occurs in the DFB model. The standard prescription sets MSFCB and MFOCB equal to 0.95 and 0.01 , respectively. These settings virtually eliminate inerting against combustion that might result from high steam concentrations or low oxygen concentrations in the receiving cell. One reason for these settings is that, with less conservative values, the composition of the receiving cell might initially permit combustion, but steam and oxygen concentrations would rise and fall, respectively, as the event proceeds, possibly terminating combustion if the default values were used. In reality, temperatures would rise rapidly in the receiving cell, which would tend to compensate for the changes in atmospheric composition, but the temperature dependence of the flammability limits is not modeled in CONTAIN. Experimentally, combustion has been observed to occur during DCH events when the default flammability limits (55\% steam and 5\% oxygen) are not satisfied. In the SNL/IET-9 experiment, for example, even the initial steam concentration was about $67 \%$ and the final steam and oxygen compositions were estimated to be $\sim 80 \%$ and $\sim 2 \%$, respectively, yet most of the DCH-produced hydrogen burned. [Bla94] A CONTAIN analysis of this experiment assuming default DFB parameters would therefore yield nonconservative results. Note also that the likelihood of jet combustion is expected to increase somewhat with increasing facility scale, other things being equal.

In both the CONTAIN model and in the experiments, DFB initiation tends to be a threshold effect, although the model appears to exaggerate the sharpness of the threshold. Given that the threshold is exceeded in the actual event, the standard prescription appears to do a good job of predicting the extent of DCH-produced hydrogen combustion and its impact upon DCH loads. However, the standard prescription clearly can be overly conservative in predicting the threshold: in the SNLIET-5 experiment, there was an initial $\mathrm{CO}_{2}$ concentration of about $76 \%$ and oxygen concentration of $4.4 \%$, and combustion of $\mathrm{DCH}$-produced hydrogen was quite limited while the standard prescription predicted efficient combustion. The initial containment atmosphere temperature in this experiment was low $(302 \mathrm{~K})$ and rose to only 400 to $450 \mathrm{~K}$ in the dome during the event; hence heating would not have had a large effect upon the flammability limits. 
If the initial atmosphere composition is not flammable, a limited amount of combustion of preexisting hydrogen will occur in a CONTAIN calculation as a result of entrainment into the diffusion flame calculated by the DFB model. However, combustion of the remaining pre-existing hydrogen usually requires exceeding the temperature threshold (SRTEMP) of the BSR model. The default temperature $(773 \mathrm{~K})$ is used in the standard input prescription. (BSR will also initiate if the debris concentration given by DEBCONC exceeds $1 \mathrm{~kg} / \mathrm{m}^{3}$ while the debris temperature given by DEBTEMP also exceeds $773 \mathrm{~K}$. Usually debris concentrations this high will result in SRTEMP also being exceeded and this second ignition criterion is not normally very important.) This value may be reasonable for some scenarios and is likely to be conservative (i.e., low) for others. It is especially likely to be conservative if substantial thermal stratification develops in the dome volume; stratification clearly did develop in the Surry-geometry IET experiments while it is less clear whether stratification developed in the Zion-geometry experiments. It is believed that the default BSR threshold temperature is unlikely to be significantly nonconservative.

Experimentally, dome temperatures in the Zion-geometry IET experiments were less than or equal to $700 \mathrm{~K}$ and some of the experiments did include pre-existing hydrogen. There is no evidence that the pre-existing hydrogen contributed significantly to DCH loads, in agreement with the CONTAIN calculations which predicted that BSR would not occur. In the Surry-geometry IET experiments, the pre-existing hydrogen was predicted to burn in the CONTAIN analyses; however, it was unclear as to whether it burned on DCH time scales in the experiments. If one views the threshold temperature as being related to the temperature at which energy generation from chemical reaction can exceed the rate of energy loss, the threshold temperature should be somewhat higher at experimental scale than at full scale because the energy loss rates decrease with increasing scale while the chemical kinetics are not scale dependent, except at sufficiently small scales that surface effects are important. The experimental analyses were generally performed with SRTEMP $\approx 850 \mathrm{~K}$. Note, however, that the argument for scale-dependence in SRTEMP may not apply if stratification effects are controlling whether BSR can occur.

The standard value of the BSR reaction rate (SRRATE) is based upon experimental measurements of temperatures in the dome for the IET experiments, [All94,Bla94] which were used to roughly estimate the rate at which the heated region of the dome expanded as the hot DCH plume mixed with the cooler dome gases. The value of SRRATE should be considered quite uncertain. Sensitivity calculations varying SRRATE may be warranted in some cases. However, experience suggests that in general the dominant issue with respect to BSR is whether the threshold is exceeded in the dome, not the exact value of SRRATE when the threshold is exceeded. Within reasonable limits, sensitivity to SRRATE is not very great in those cases that have been considered.

It should be remembered that initiation of a deflagration in a cell suppresses BSR for a period equal to the deflagration burn time plus a dead time equal to the burn time. This provides another motivation for specifying relatively rapid flame speeds in the deflagration model. Otherwise, initiation of a slow deflagration could result in prolonged suppression of BSR in a situation where BSR should actually occur. If the short deflagration burn times are considered undesirable, it is probably better to suppress the deflagration model entirely (by specifying impossible ignition criteria) and control hydrogen combustion modeling during DCH using the DFB and BSR models only, rather than allow the deflagration model to operate with default flame speeds. 
Before leaving the subject of gas combustion during $\mathrm{DCH}$, we would note that we believe it can be a mistake to place too much emphasis on identifying the "correct" values of the various threshold parameters and rate parameters controlling the hydrogen combustion models. For example, much of the combustion that occurs in the calculation often involves combustion of jets of incoming gas containing hydrogen as the gas enters cells that still contain oxygen. This combustion usually will be controlled by the DFB model. Combustion behavior of the incoming gas is actually a function of jet composition and temperature, receiving cell composition and temperatures, jet orifice size, jet flow velocities, and probably depends upon debris parameters (amounts, temperatures, compositions, particle sizes). The DFB model is not sufficiently mechanistic to capture these dependencies, and the trends in the observed DCH combustion behavior show variations that cannot be captured by the model. Hence the intent in defining the DFB standard prescription has been to set DFB parameters to ensure that most DCH-produced hydrogen does burn upon reaching an oxygen-bearing atmosphere. Since it usually did burn in the experiments and one expects large scale to favor this trend, the assumption that it will burn is considered to be the most likely outcome and it is also the conservative prescription; hence it was adopted as the standard.

It is recommended that the user monitor hydrogen combustion behavior in DCH calculations and check to ensure that the behavior being calculated seems reasonable for the conditions (gas compositions, temperatures, etc.) that exist at the time. When alternative combustion behaviors seem reasonable, they may be investigated in sensitivity calculations with the burn parameters set to obtain the alternative behaviors desired. For example, if the standard prescription predicts that BSR will occur in the dome but it is believed that stratification or other effects will actually prevent efficient combustion of the pre-existing hydrogen, the importance of this issue may be investigated by setting SRTEMP (and DEBTEMP or DEBCONC) to very high values that prevent BSR. What needs to be justified here is the belief that the pre-existing hydrogen will not burn, rather than the particular value of SRTEMP chosen. In this instance, controlling the burn behavior with SRTEMP amounts to using a chemistry parameter (i.e., the reaction threshold temperature) to investigate the importance of what is actually an uncertainty in the gas mixing dynamics. In such a case it is doubtful that one could justify any specific value of SRTEMP as being the "correct" value.

Nodalization. In setting up a CONTAIN problem, a task that always requires careful judgment on the part of the analyst is deciding how to nodalize the containment of interest. It is not possible to define a straight-forward prescription for how to nodalize an arbitrary containment. Experience with the Surry-geometry and Zion-geometry IET experiments is discussed in Reference Wil95, including calculations illustrating the sensitivity to nodalization questions. These results, plus prior experience with CONTAIN, were used to suggest some guidelines for setting up a base case. We briefly summarize these guidelines here; additional detail may be found in the reference. The following cases may be distinguished:

1. Large open volumes (e.g. the containment dome). For large open volumes, the default treatment should be to represent the volume with a single cell. Although the dome volume may not be well mixed (e.g., stratification may exist), the ability of CONTAIN to calculate gas distributions in open volumes is questionable. The new hybrid flow solver described in Chapter 4 appears to capture stratification effects under some conditions, but no attempt has been made to validate this treatment of stratification under $\mathrm{DCH}$ conditions. Note that the 
hybrid solver was not available at the time the $\mathrm{DCH}$ assessments were performed. Sensitivity calculations experimenting with different nodalizations may provide useful insights in some instances, but it will be up to the user to ensure that the results are meaningful.

2. Well-defined compartments. Each well-defined compartment should generally be assigned one computational cell. Here a "well-defined compartment" may be defined as a volume largely enclosed by structures such that flow path areas into and out of the volume are small compared with the cross section of the volume, while internal structure within the volume is sufficiently limited that, for the most part, there would be an unobstructed line-of-sight across the volume in most directions. In some cases, even well-defined compartments may be combined into a single cell if there is good reason to believe that little error can result. For example, there may exist several well-defined compartments in the same region of the containment and whose total volume is a small fraction of the containment. Lumping these volumes together may be an acceptable simplification, especially if they are far from the cavity and/or do not lie on a major flow path important to the problem (e.g., do not lie on one of the main flow paths from the cavity to the dome).

3. Subcompartments with large amounts of internal structure. These are volumes in which there is sufficient internal structure that unobstructed line-of-sight paths across the volume generally do not exist, and yet the structures do not divide the volume into subvolumes that would constitute well-defined compartments as defined above. The Zion subcompartment volume provides an example. Trapping typically prevented large amounts of debris from reaching the parts of the subcompartment farthest from the cavity, and CONTAIN's wellmixed assumption did not apply for the subcompartment volume as a whole. Ideally the portions of such volumes close to the cavity probably should be subdivided into volumes such that the dimensions of each volume are not larger than the mean unobstructed flight path as estimated from the 6V/S rule used to estimate the trapping lengths. Most of the Zion IET experimental analyses were performed using a 14-cell model, and this rule was approximately followed for parts of the subcompartment relatively close to the cavity exit. The rule was relaxed for the more distant parts of the subcompartment volume.

A simpler 5-cell model was also used in a small number of the Zion IET analyses. Calculated $\triangle \mathrm{P}$ and hydrogen production numbers agreed well with the 14-cell model values except that there was some sensitivity to ambiguity in defining the flow cross sections used to calculate flow velocities required by the trapping and non-airborne debris models. This ambiguity is described further in the discussion of flow velocities given below. The dome carryover fraction $\left(f_{\text {dome }}\right)$ calculated using the 5-cell model was higher than for the 14-cell model (which generally agreed reasonably well with experimental results), and it was more sensitive to uncertainties in the model input. It was concluded that the 14-cell model was preferable, but the more detailed model was more tedious to set up and it appeared that simpler representations might be defended if they are defined with sufficient care.

4. Non-isotropic quasi-open volumes. This term is used to refer to volumes in which the dimensions in some directions differ substantially from the dimensions in other directions. 
Examples include the annulus between the crane wall and the containment shell in the Surrygeometry IET experiments, and also the basement volume in these experiments. Although these volumes did not include a large amount of internal structure, application of the wellmixed assumption to them is doubtful because of their shape. In References Wil95 and Pi195, most of the Surry IET analyses were performed using a model in which the annulus and the basement were each represented by a single cell, but some sensitivity calculations were performed in which these volumes were divided into four and two subvolumes, respectively. This change increased the calculated $\Delta \mathrm{P}$ by $\sim 0.05 \mathrm{MPa}$. This result shows that a coarse nodalization does not always lead to a more conservative result, as is sometimes assumed. No judgment could be made as to which nodalization was more realistic.

Flow Areas for Flow Velocity Calculation. The debris trapping model and the non-airborne debris model require an estimate for the flow velocity through the cell. For the second impact in the trapping model, and for the non-airborne debris model, this is based upon the convective flow velocity calculated for the second structure in the cell. This velocity, in turn, is the volumetric gas flow rate through the cell, divided by the cross-sectional area available for flow (HYDAREA in the BCINNER subblock of the STRUC input block, Section 14.3.2.3). For the cavity, the actual flow cross section should be used. For other cells, the default $\left(\mathrm{V}_{\mathrm{g}}^{2 / 3}\right.$ where $\mathrm{V}_{\mathrm{g}}$ is the cell gas volume) is often satisfactory. However, in the case of a subcompartment with complex internal structures (Case 3 above), this value may give flow velocities that are too small when the volume is treated as a single cell. In the Zion IET analyses, the default was found to be satisfactory when the 14-cell representation was used but was less satisfactory for the 5-cell representation. The likely reason is that, in the region of the subcompartment close to the cavity where much of the debris is deentrained, the actual cross sections for flow are considerably less than $\mathrm{V}_{\mathrm{g}}^{2 / 3}$.

For the 5-cell Zion IET model, a procedure that appeared to yield more appropriate gas velocities was to sum the total flow area exiting the first subcompartment cell downstream of the cavity in the 14-cell representation. This flow area was in effect taken as representative of the flow areas controlling debris velocities in the subcompartment regions where most of the debris was being deentrained. Although generalization from a single instance is obviously questionable, it may provide the best guidance currently available for Case 3 volumes if time and resources available do not justify defining a more detailed model satisfying the rule that cell dimensions in the subcompartments should be comparable to the generic trapping length, $6 \mathrm{~V}_{\mathrm{g}} / \mathrm{S}_{\mathrm{str}}$. In order to apply this procedure to the coarse nodalization, one might determine what the first cell downstream of the cavity would include if the $6 \mathrm{~V}_{\mathrm{g}} / \mathrm{S}_{\mathrm{st}}$ rule were to be satisfied, and then use the flow exit area for this cell as being representative for the entire subcompartment cell used in the coarser nodalization. If this value of HYDAREA is considered inappropriate for evaluating the heat transfer correlations for the second structure in the cell, a "dummy" structure with a very small surface area may be defined as the second structure in the cell.

When a coarse nodalization is used in this way for the subcompartment volumes, investigation of sensitivity to HYDAREA is warranted unless it is known that the results of interest are insensitive to the trapping and non-airborne debris modeling used in the subcompartments. 
13.3.2.3 RPV Models. When the RPV and cavity models are invoked, the user should remember that both an RPV and cavity cell must be designated, and that these two CONTAIN cells must be connected by a single flow path. Furthermore, a regular flow path must be used, not an engineered vent. The characteristics of this flow path must be specified as usual in the CONTAIN input, but the user should be aware that the presence of debris in the RPV cell will start the RPV and cavity models in CONTAIN. After this point, the RPV and cavity models take control of the flow path between the RPV and cavity cells according to the input that has been provided in the RPVCAV input section.

The RPV models begin to function when debris is placed in the trapped debris bin in the RPV cell. The user may accomplish this in any manner allowed by CONTAIN; however, the user should be aware of the physical situation the RPV models were intended to model. The models are primarily designed for a situation in which a large initial mass of molten debris resides in the RPV cell, and is moved out of the RPV through single-phase and two-phase debris ejection. It is recommended that the user place all the desired debris inventory into the trapped debris bin over a period of time smaller than the current CONTAIN timestep. The user can also specify debris initially by putting mass in the ATMOS block. It is possible to add and subtract debris in the RPV cell as the calculation progresses, but the user should be aware of how the individual RPV models function.

The single-phase debris discharge model and the hole ablation model are insensitive to changes in the amount of debris in the RPV, so long as the conditions for gas blowthrough are not created in the process of adding or subtracting debris. However, the gas blowthrough model was developed with the assumption that after the height of the debris surface above the RPV hole reaches a certain critical value, the amount of debris in the RPV will continue to decrease. Adding debris to the RPV cell after gas blowthrough has occurred may produce unexpected results, because the surface of the debris pool in the RPV might be returned to a value larger than the critical pool height required for gas blowthrough. If debris is added to the RPV cell after gas blowthrough has occurred, the user should carefully scrutinize the results to ensure that the RPV models continued to function correctly.

13.3.2.3.1 Hole Ablation. The rate of hole ablation can be controlled through the use of the ARMULT parameter, which is a direct multiplier to the ablation rate; however, parts of the entrained fraction option rely on having a non-zero value for the ablation rate of the RPV hole. The safest way to create a fixed RPV hole size for a calculation is to set HFWALL to some very large value, rather than setting ARMULT to be zero.

13.3.2.3.2 Gas Blowthrough. The time at which gas blowthrough occurs is calculated internally by the code. However, it can be manipulated indirectly by changing the RPV hole size or the RPV diameter. The initial hole size can be specified through the AHOLE1 parameter. Smaller hole sizes will prolong the single-phase debris ejection period, and lengthen the time until gas blowthrough occurs. If the entrained fraction option is invoked, the hole size can then be reset through the AHENF parameter after gas blowthrough occurs. A smaller diameter, which can be specified through the DIARPV parameter, will also lengthen the time until gas blowthrough occurs. The user cannot directly select a specific gas blowthrough time. 
13.3.2.4 Cavity Models. When using the cavity models, the user should recognize that the basic physics governing debris dispersal fractions and entrainment rates is extremely complex. The new models incorporated in the CONTAIN code represent the best available models for these processes, but are still highly simplified approximations of a complex and not fully understood phenomena. The use of these models involves large uncertainties, and the models' performance and ability to predict behavior as part of an integrated systems analysis code such as CONTAIN has not yet been carefully assessed against all of the available experimental data base. As a result, the CONTAIN Project is very interested in receiving feedback from users.

The user should be extremely careful when defining all of the many user-defined parameters that are required by the entrained fraction and entrainment rate models in CONTAIN. Some guidance on parameter selection is provided below, and the user is urged to review the original references for these models when questions arise. Some of the entrainment rate and entrained fraction models are complex, and have been developed from an experimental data base in specific geometries and under low temperature conditions. The models in CONTAIN are meant to be applied to a variety of different circumstances, but the governing parameters provided in input must be carefully selected to ensure that the model performs as intended in the geometry and under the conditions of the specific CONTAIN application.

13.3.2.4.1 Entrainment Rate. An entrainment rate model must be specified through the IENRAT parameter. If no entrained fraction option is specified through the IENFRA parameter, the entrainment and ejection of debris from the cavity will be controlled completely by the selected entrainment rate model. The total fraction of debris that is dispersed from the cavity depends on the integrated entrainment rate over the course of the calculation, and can be controlled by selection of the cavity constant $\mathrm{K}_{\mathrm{c}}$ (CCENR) or the other input parameters for the selected rate model.

13.3.2.4.2 Entrained Fraction. If an entrained fraction model is invoked through the IETFRA parameter, the entrainment rate in the cavity will be modified within the code to produce the calculated or specific total fraction dispersed. At the moment gas blowthrough occurs, the gas flow area that will be used between the RPV and cavity cells for the duration of the entrainment depends on the user input. If no value of AHENF has been specified, the code will calculate the gas flow area internally. If AHENF has been specified in input, the code will use that value. If TSTOP has been specified in input without AHENF, the gas flow area will change linearly from its value at the time of gas blowthrough to the value calculated internally by the code over the time interval $t_{\text {blow }}$ to $t_{\text {blow }}$ + "tstop," where $t_{\text {blow }}$ is time of gas blowthrough. If TSTOP has been specified in conjunction with AHENF, the gas flow area will change linearly from its value at the time of gas blowthrough to the value specified in AHENF over the time interval $t_{\text {blow }}$ to $t_{\text {blow }}+$ "tstop."

When an entrained fraction model is invoked through the IENFRA parameter, it is possible to specify the entrainment interval through the input parameter TDISP. However, the user should be cautious when invoking this option. If TDISP is specified, the entrainment rate will produce the desired total fraction dispersed over the specified interval. However, the sudden end of entrainment in the cavity may produce a sharp drop in both temperature and pressure in the cavity if the entrainment rate was still high at the cutoff time, depending on the entrainment rate model and the 
other governing input parameters. It is recommended that the user allow the code to internally calculate the entrainment interval for debris dispersal from the cavity.

13.3.2.4.3 Cavity Constant $\mathrm{K}_{c}$ (CCENF and CCENR). It is hoped that future validation efforts will show that these models reproduce trends as a function of dominant parameters reasonably well, but it is not expected that they can give a valid quantitative prediction from first principles without resort to user-specified parameters. Hence, in all cases, a user-specified "cavity constant," $K_{c}$ (CCENR and CCENF in the code input), is required. CCENR is used as the value of $\mathrm{K}_{\mathrm{c}}$ when evaluating a rate correlation and CCENF is used when evaluating a dispersed fraction correlation. The Levy correlation is available both in the form of a rate correlation and an integral correlation for the dispersed fraction. If the Levy correlation is used for both the rate and the dispersed fraction, it will normally be reasonable to choose CCENR and CCENF to be equal, although this is not mandatory. The other rate correlations do not have analogous integrated dispersed fraction correlations. Likewise, there is no differential form of the Tutu-Ginsberg dispersed fraction correlations that can be used to calculate corresponding dispersal rates.

In the $\mathrm{DCH}$ assessment work, values of $\mathrm{K}_{\mathrm{c}}$ giving the best fits to the results of cavity dispersal experiments using low-temperature simulants were determined, along with standard error estimates $\left(\sigma_{e s}\right)$. These results are summarized in Table 13-2 below. Values of $K_{c}$ given correspond to either CCENR or CCENF for the Levy correlation; to CCENF for Tutu-Ginsberg; and to CCENR for the others.

Here, the standard error estimate is defined by

$$
\sigma_{\text {est }}=\sqrt{\frac{\sum_{i=1}^{N_{c}}\left(Y_{i}-X_{i}\right)^{2}}{N_{d}}}
$$

where $Y_{i}$ and $X_{i}$ are, respectively, the experimental and predicted values of the fraction dispersed $\left(F_{d}\right)$, and $N_{d}$ is the number of data points in the particular data set under consideration. As was discussed in Williams and Griffith, [Wil96] it was generally necessary to define separate values of $\mathrm{K}_{\mathrm{c}}$ in order to satisfactorily fit the Surry- and Zion-geometry data, and to fit the Brookhaven National Laboratory (BNL) 1/42-scale and the SNL 1/10-scale data.

The values tabulated here should not be used uncritically. There are many effects which are not simulated in the data base used in Williams and Griffith, [Wil96] and it would be surprising indeed if the values found suitable for the low-temperature simulants turned out to be appropriate for either full-scale DCH events or simulation of the more realistic DCH experiments performed using thermite-generated melts. A limited number of CONTAIN simulations of the latter experiments were used to further assess these models in Reference Wil96, which should be consulted for additional information. 
Table 13-2

$\mathrm{K}_{\mathrm{c}}$ Values and Performance Statistics for Cavity Correlations

\begin{tabular}{|c|c|c|c|c|c|}
\hline \multirow[b]{2}{*}{ Correlation } & & \multicolumn{2}{|c|}{ BNL (1/42 Scale) } & \multicolumn{2}{|c|}{ SNL (1/10 Scale) } \\
\hline & & Surry & Zion & Surry & Zion \\
\hline \multirow{2}{*}{ Levy } & $\mathrm{K}_{\mathrm{c}}$ & 0.57 & 0.8 & 0.048 & 0.0073 \\
\hline & $\sigma_{\text {est }}$ & 0.176 & 0.125 & 0.144 & 0.123 \\
\hline \multirow{2}{*}{ Tutu-Ginsberg } & $\mathrm{K}_{\mathrm{c}}$ & 1 & 1 & 0.6 & 0.47 \\
\hline & $\sigma_{\text {est }}$ & 0.102 & 0.088 & 0.103 & 0.153 \\
\hline \multirow{2}{*}{ Tutu } & $\mathrm{K}_{\mathrm{c}}$ & 5.3 & 16 & 1.25 & 0.4 \\
\hline & $\sigma_{\text {est }}$ & 0.115 & 0.184 & 0.191 & 0.103 \\
\hline \multirow{2}{*}{ Modified Whalley-Hewitt } & $\underline{\mathrm{K}_{\mathrm{c}}}$ & 100 & 130 & 118 & 43 \\
\hline & $\sigma_{\text {est }}$ & 0.138 & 0.137 & 0.208 & 0.143 \\
\hline
\end{tabular}

In the meantime, it is up to the user to select a value of $\mathrm{K}_{\mathrm{c}}$ that yields reasonable results. In assessing whether a result is reasonable, the two most important features to keep in mind are the fraction of the debris that is dispersed $\left(F_{d}\right)$ and the length of time during which significant amounts of debris are being dispersed. Short dispersal times imply minimal opportunity for debris to interact with blowdown steam and, hence, choosing a large value of $\mathrm{K}_{\mathrm{c}}$ resulting in everything being quickly blown out of the cavity can be nonconservative.

In judging whether the calculated debris dispersal rate is "reasonable," it may be helpful to derive an estimate of the coherence ratio $R_{\tau}$ from the calculation and compare it with the value predicted by the empirical model of Equation (13-4). In order to estimate the coherence ratio for the calculation, one may first estimate the time $t_{e}$ at which dispersal is effectively complete in the calculation; this may be taken to be the time at which the amount of debris dispersed from the cavity reaches $95 \%$ of its final value. It is best to base the estimate of $t_{e}$ upon a component of the debris which is neither produced nor consumed by chemical reactions, such as $\mathrm{UO}_{2}$. The calculated RPV pressure at time $t_{e}$ may then be equated to $P_{R P V, e}$ and used in Equation (13-3) to define the value of $\mathrm{R}_{\tau}$ corresponding to the calculation. If this value is much smaller than the value given by Equation $(13-4)$, it is likely that $\mathrm{K}_{\mathrm{c}}$ (CCENR) should be increased; and conversely. While results cited in Reference Wil95 suggest that sensitivity of DCH loads to the coherence ratio is often quite moderate, experience to date is insufficient to guarantee this will always be the case. Hence sensitivity studies in which the coherence is varied (by varying CCENR) are recommended until it is established that sensitivity is low for the class of problems being considered.

Even in the context of the experiments with cold simulants, the values of $\mathrm{K}_{\mathrm{c}}$ obtained are meaningful only for the particular cavity geometric parameters assumed in the assessments described in Williams 
and Griffith. [Wil96] In addition, the $\mathrm{K}_{\mathrm{c}}$ values apply given the values of the gas discharge coefficient assumed in the assessment, and certain "standard values" assumed in the assessment in the case of the Levy correlation. These values were generally not given in Williams and Griffith [Wil96]; for the sake of completeness, they will be given here. They also may be of some value to the user; however, there is generally redundancy between the effect of these additional parameters and the effect of $\mathrm{K}_{\mathrm{c}}$. In most cases, using different values of these additional parameters could be compensated for by changing the value of $\mathrm{K}_{\mathrm{c}}$.

In Williams and Griffith, [Wil96] an effort was made to assess the correlations in a manner consistent with what was done by the original authors. Unfortunately, the various investigators were not completely consistent in their assumptions, and this inconsistency is reflected in what follows.

13.3.2.4.4 Cavity Geometry Parameters. The cavity geometry parameters assumed are listed in Table 13-3. All are given in terms of full-scale plant values; the assessment was actually carried out after scaling down assuming linear scale factors of $1 / 42$ and $1 / 10$ for the BNL and SNL data, respectively. The name of the parameter given in the table corresponds to the CONTAIN keyword in the input stream. Blank entries imply that the indicated parameter is not required for a particular correlation. The value of AFILM given for the Levy correlation differs substantially from the others because Levy assumed debris to be spread over the floor only and Tutu assumed debris films on the walls as well as the floor; the user of these correlations should make the same assumptions. The values cited for the Whalley-Hewitt correlation correspond to the Williams-Griffith modification (see Section 6.2.10.1.1).

Table 13-3

Geometric Parameters Used in Correlations

\begin{tabular}{|c|c|c|c|c|c|}
\hline Parameter & Cavity & $\begin{array}{c}\text { Tutu- } \\
\text { Ginsberg }\end{array}$ & Tutu & $\begin{array}{c}\text { Modified } \\
\text { Whalley-Hewitt }\end{array}$ & Levy \\
\hline \multirow{2}{*}{ AFLOW } & Zion & 5.22 & -- & 7.71 & 7.71 \\
\hline & Surry & 12.6 & -- & 12.6 & 12.6 \\
\hline \multirow[t]{2}{*}{ HYDDIA } & Zion & -- & 2.72 & 2.72 & 2.72 \\
\hline & Surry & -- & 3.51 & 3.51 & 3.51 \\
\hline \multirow[t]{2}{*}{$\overline{A F I M}$} & Zion & --- & 151.9 & 151.9 & 29.8 \\
\hline & Surry & $\cdots$ & 211.3 & 211.3 & 44.7 \\
\hline \multirow[t]{2}{*}{ WETPER } & Zion & --- & 8.963 & - & -- \\
\hline & Surry & -- & 11.33 & -- & -- \\
\hline \multirow[t]{2}{*}{ CAVLEN } & Zion & 5.208 & -- & - & --- \\
\hline & Surry & 4.141 & -- & - & -- \\
\hline
\end{tabular}


13.3.2.4.5 Discharge Coefficient. The gas discharge coefficient in this discussion corresponds to the value of CONTRACT specified in the FLOWS input block for the flow path connecting the RPV and the cavity cells. The value of CONTRACT specified will affect the results of the entrainment rate models, but it will not affect the results of the integral correlations for total fraction dispersed. However, the effects of a different discharge coefficient can always be at least approximately compensated for by an appropriate change in $\mathrm{K}_{\mathrm{c}}$. Since the user of any of these cavity models must currently assume full responsibility for selecting values of $\mathrm{K}_{\mathrm{c}}$ that yield reasonable results, the user should not be excessively concerned about consistency with the values of the discharge coefficients assumed in either Williams and Griffith [Wil96] or in the original work. In particular, the value of CONTRACT in the FLOWS block should be chosen based upon what otherwise seems reasonable, and then values of $\mathrm{K}_{\mathrm{c}}$ should be identified to give acceptable results.

For the Tutu entrainment rate correlation, Williams and Griffith [Wil96] assumed that the gas discharge coefficient was equal to 0.74 in analyzing the data, as this was the value assumed by Tutu [Tut91] The same value was also assumed in assessing the modified Whalley-Hewitt correlation.

The situation is somewhat more complex for the Levy and the Tutu-Ginsberg correlations. Levy did not explicitly allow for a discharge coefficient. In effect, the influence of the discharge coefficient is incorporated into the value of $\mathrm{K}_{c}$ and the quantity identified as the "blowdown time." The latter corresponds to a gas discharge coefficient of approximately 0.89 , assuming an isothermal blowdown. The Tutu-Ginsberg correlations were fit by the original authors to the BNL data without allowing for a discharge coefficient; thus, the effects of the discharge coefficient in the BNL experiments (stated to be about 0.61 were incorporated into the fitting constants. ${ }^{3}$ Unlike the other correlations, it is not clear that the effect of different values of the discharge coefficient could be compensated for by changing just one of the fitting constants and, in any case, these constants are not available to the CONTAIN user through input. In fitting to the SNL data, Tutu and Ginsberg ${ }^{3}$ assumed that the Surry and Zion discharge coefficients were smaller than in the BNL experiments by factors of 0.6 and 0.47 , respectively. However, this interpretation was carefully considered in Williams and Griffith [Wil96] and ultimately rejected, and the factors of 0.6 and 0.47 (which are listed in Table 13-1 above) were identified as "cavity constants" in that work. These factors enter into the correlation in the same mathematical form as would a discharge coefficient; that is, the value of the "reference velocity," $U_{R}$, defined in Equation (6-57) is multiplied by $\mathrm{K}_{\mathrm{c}}$.

Although it is not presently believed that the differences between the BNL and SNL experiments noted above should be interpreted as differences in the discharge coefficient, it remains true that $\mathrm{K}_{\mathrm{c}}$ is implemented in the correlation as if it were a discharge coefficient and the user may adjust it as seems appropriate in order to take into account variations in the discharge coefficient. Using $K_{c}$ simply as a tuning parameter, as is suggested for the other correlations, seems more dubious here; certainly no justification for doing so can be found in the original work developing the correlations.

13.3.2.4.6 Levy Standard Values. Input specified for the Levy correlation requires certain standard values. The meaning and motivation of these standard values is discussed in Reference Lev91. For

\footnotetext{
${ }^{3}$ N. K. Tutu and T. Ginsberg, Brookhaven National Laboratory, Upton, NY, letter report to the USNRC with title, "A Letter Report on the Results of Melt Dispersal Experiments With the Surry and Zion Cavity Models," October 1990.
} 
several reasons, the assessment performed by Williams and Griffith [Wil96] did not use exactly the same standard values in all cases. The values used in the latter work are given in Table 13-4. The standard scale factor, SSCALF, in this table was not specified in Levy. [Lev91] However, the intent was that the ratio of scaled hole sizes be used in the correlation and hence the standard scale factor is needed if the correlation is to function as intended.

When the Levy correlation is invoked, it is recommended that the user employ the $\mathrm{K}_{\mathrm{c}}$ values taken from the fits to the BNL or the SNL data that are given in Table 13-2, along with the corresponding standard values from Table 13-4. However, the user should remember that the mathematical form of the Levy correlation is such that the standard values could actually be factored out and incorporated into the fitting constant; for any alteration in the standard values, one could find a revised value of $\mathrm{K}_{\mathrm{c}}$ which would give equivalent results. As with any of the entrainment correlations, the essential requirement is to ensure that the $\mathrm{K}_{\mathrm{c}}$ value used yields reasonable results, regardless of whatever values were specified for the other parameters.

13.3.2.4.7 Whalley-Hewitt Standard Value. The Williams-Griffith modification of the WhalleyHewitt correlation (Section 6.2.10.1.1) was developed to correct what was considered to be some unrealistic dependencies upon liquid viscosity and surface tension. [Wil96] When this version of the Whalley-Hewitt correlation is used, a value is required for the surface tension of the standard liquid used in the experimental correlation assessments. This input is introduced by the keyword SURTES and the recommended value is $0.072 \mathrm{~J} / \mathrm{m}^{2}$. This value corresponds to water, which was taken to be the standard liquid in the experimental assessments described in Reference Wil96.

13.3.2.4.8 Weber Model. To use the Weber model, the user must also invoke the RPV discharge model and one of the available cavity entrainment models. The user must also specify five or more particle size bins. Failure to use at least five bins when the Weber model is used will result in an input error. The user may want to use ten bins to obtain higher resolution on the diameters of airborne debris. Another important requirement is that the FDISTR mass distribution array may not be specified with the Weber model, since the model determines how mass is divided with respect to diameter. Each particle bin will automatically have an increasingly larger diameter when the Weber model is used, and the code assigns entrained mass into the bins based on the Weber criterion as described earlier. A side effect of this approach is that metals and oxides may not be initially separated into distinct bins when the Weber model is used. The composition of each bin will be individually tracked as always as the problem proceeds, such that smaller drops deplete their metal more quickly than larger drops. If one wants to perform sensitivity calculations where metals and oxides are initially separate, then the Weber model can be deactivated. With the Weber model deactivated, the user can then specify the FDISTR matrix.

The Weber model is highly dependent upon the average gas velocities through the cavity (or the exit velocity if the USEVOUT option is invoked). Therefore, the user is cautioned to examine this velocity to ensure that it is reasonable. This velocity is provided in the output file if the PRFLOW global keyword is specified. Note that the plot file provides the gas velocity in the cavity exit flow path(s), which may be different than the entrainment velocity used in the Weber model. It is always important to check output for reasonableness. No specific guidance can be offered at this time regarding the value of using the USEVOUT option. 
Table 13-4

Standard Values Used in Assessing the Levy Correlation

\begin{tabular}{|c|c|c|c|c|c|}
\hline Data Set & SSCALF & MW & DSUBS & RHDEBS & TSUBIS \\
\hline BNL & 0.02381 & 28.96 & 0.00953 & $10^{3}$ & 295 \\
\hline SNL & 0.1 & 28.96 & 0.038 & $10^{3}$ & 325 \\
\hline
\end{tabular}

If the entrainment velocities calculated are physically reasonable, then the default diameter ranges provided should be adequate and the DIAMIN and DIAMAX keywords need not be given. The default standard deviation of two is also recommended until further experience is obtained with the models. Note that this value will provide fairly narrow distributions for entrained debris within a given timestep; however, the net particle size distribution could be quite broad as a result of a range of entrainment velocities. The user is encouraged to utilize the user-specified diameter option to perform parameter sensitivity studies, since this option has more flexibility than the Weber model and can be more directly controlled:

\subsubsection{Specific Input Parameters for RPV and Cavity Models}

13.3.2.5.1 AHOLE1. The parameter AHOLE1 controls the initial size of the hole in the RPV at the start of single-phase debris ejection from the RPV cell. When debris is placed in the trapped debris bin in the RPV cell, the RPV and cavity models override the gas flow area between the RPV and cavity cells that was set in the FLOWS block in the input file. During single-phase debris ejection, the gas flow area between the RPV and cavity is internally set to zero. After gas blowthrough occurs, the two-phase debris ejection model calculates a time-dependent gas flow area and gives this value to the CONTAIN flow solver for gas flow between the two cells.

13.3.2.5.2 CPWALL. The parameter CPWALL is the specific heat of the RPV wall. If no value is specified in input, the code calculates a value based on Fe at the temperature TWALL. It is provided as input so that the user can simulate other materials.

13.3.2.5.3 CSUBD. The parameter CSUBD is the discharge coefficient at the RPV hole for the exiting debris. The discharge coefficient for gas flow is unaffected by the RPV and cavity models, and will be whatever the user specifies for the RPV-to-cavity flow path elsewhere in the CONTAIN input.

13.3.2.5.4 RHOWAL. The parameter RHOWAL is the density of the RPV wall. If no value is specified in the input, the code calculates a value based on $\mathrm{Fe}$ at the temperature TWALL. It is provided so that the user can simulate other materials.

13.3.2.5.5 AFICM. The parameter AFILM is the surface area in the cavity covered by debris. The product of the entrainment rate flux and the area AFILM yields the actual entrainment rate of debris in the cavity. The user can determine AFILM for a specific cavity geometry by adding up the surface areas of all structures in the cavity that are expected to be covered by debris. As a simple approximation, AFILM might include all floor and wall areas in the cavity, and exclude any ceiling 
area. It is recommended that the user select a consistent methodology for determining AFILM and use it for all analyses of a given system.

13.3.2.5.6 HYDDIA. The parameter HYDDIA is the hydraulic diameter of the cavity, defined as four times the cross-sectional flow area divided by the wetted perimeter. In this case, the wetted perimeter is defined by whether debris is expected to be present on the floor and walls only, or on the floor, walls, and ceiling of the cavity. The user should select a value that is consistent with the methodology used to obtain AFILM.

13.3.2.5.7 WETPER. The parameter WETPER is the wetted perimeter in the cavity, treating debris as a fluid. It should be defined by the user in the manner described in Sections 13.3.2.5.5 and 13.3.2.5.6 above.

13.3.2.5.8 TDISP. The parameter TDISP is the estimated debris dispersal interval. After gas blowthrough, debris entrainment in the cavity will continue for TDISP seconds. If an entrained fraction model is invoked, this parameter can be used to override the internally calculated dispersal interval. The user should be cautious when specifying a value for TDISP, since an unrealistically small value could produce a nonconservative (i.e., too small) amount of debris/steam interaction in the cavity. It is recommended that TDISP be specified only if the user fully understands the effect of doing so. Additional discussion on the use and effects of this parameter is provided in Sections 13.3.2.4.2 and 13.3.2.4.3.

13.3.2.5.9 TSTOP. The parameter TSTOP is the interval for the linear growth of the hole in the RPV from its size at the time of gas blowthrough to the size specified by AHENF or calculated internally. The TSTOP parameter may only be used when a model for the entrained fraction is invoked, and is intended to produce a more realistic blowdown of the RPV by allowing the RPV hole diameter to continue to increase after gas blowthrough has occurred. All of the models for the entrained fraction were developed assuming a single fixed hole size in the RPV, and as a result the RPV hole size used by the code after gas blowthrough occurs is normally set to a fixed value specified by AHENF or calculated internally.

However, the use of a single fixed hole size for the duration of the RPV blowdown produces a blowdown curve that does not match the available experimental data base. As a result, the TSTOP option is provided to allow the user to more accurately model the depressurization of the RPV in an accident scenario. Specifying a value of TSTOP does not affect the calculated entrained fraction of debris, but it does affect the entrainment rate by modifying the gas flow area between the RPV and cavity, and therefore changing the gas flow rate and velocities in the cavity. Users should not specify a value for the TSTOP parameter until they are familiar with the behavior of the entrainment rate and entrained fraction models, and can assess the effects of different TSTOP values.

13.3.2.5.10 CCENF. The parameter CCENF is a multiplier to the entrained fraction that is calculated when an entrained fraction model is invoked. An extensive discussion on the selection and effects of CCENF and CCENR is provided in Section 13.3.2.4.3. 
13.3.2.5.11 ENTFR. The parameter ENTFR is the entrained fraction of debris. If the user wishes to specify an entrained fraction of debris, but does not want to invoke a model to calculate a value, an entrained fraction can be specified directly by using this parameter in the input.

\subsubsection{Heat and Mass Transfer}

13.3.3.1 Approximating the Uchida Heat Transfer Data. Uchida et al. [Uch65] presented experimental data for condensation on a small vertical plate in the presence of air. The experiments were conducted in a vessel initially filled with 1 bar of air at 17 degrees $C$ (ambient air density). Steam was injected at increasing rates while the cooled plate was maintained at a constant temperature. Data for the total heat transfer coefficient, including both sensible and latent heat transfer, was correlated as a function of the air/steam mass ratio. Peterson [Pet96] has presented a diffusion layer model of the condensation process investigated experimentally by Uchida. Using this model, Peterson was able to explain the theoretical basis for the Uchida correlation. Good agreement between theory and data was shown by Peterson for conditions of natural convection in the experimental vessel. More importantly, this theory indicates that a scale dependency based on the initial air density is present but not reflected in the Uchida correlation. Since the Uchida data is used extensively in the nuclear industry to predict condensation rates on containment structures, it is useful to establish the relation between the CONTAIN method of calculating condensation rates and the data presented by Uchida.

The diffusion layer theory described by Peterson is essentially identical to the heat and mass transfer analogy method modeled in the CONTAIN code. Therefore the agreement between the theoretical estimates of Peterson and the Uchida data represent an independent verification of the heat and mass transfer modeling approach in the CONTAIN code. Figure 13-7 shows the CONTAIN-calculated heat transfer coefficients under natural convection conditions for three initial air inventories represented by initial pressures of $0.5,1$, and 2 bars. As indicated, the CONTAIN predictions for a 1-bar initial condition (ambient air density) are in good agreement with Peterson's fit of the Uchida data. The agreement confirms that the Uchida data can be reproduced with the CONTAIN heat and mass transfer model under natural convection conditions. The film resistance in the Uchida experiments is essentially zero due to the small dimensions of the condensing plate. In the CONTAIN results shown in Figure 13-7, the film resistance is zero since the results are reported at the first timestep in the calculation prior to condensate build-up. In actual containment calculations where a film does develop, agreement with Uchida can be preserved by using the MINDEPTH keyword to specify a small depth (on the order of $50 \mu \mathrm{m}$ ) for the film. This input effectively minimizes the film resistance in comparison to the gas boundary layer resistance.

Consistent with the conclusions of Peterson, the comparisons in Figure 13-7 also show that when the initial air density varies from the ambient, condensation is not well represented by the Uchida data. Thus, the data are not applicable to cases in which significant air density gradients exist in the containment, such as those resulting from steam stratification. From the above results, it is 


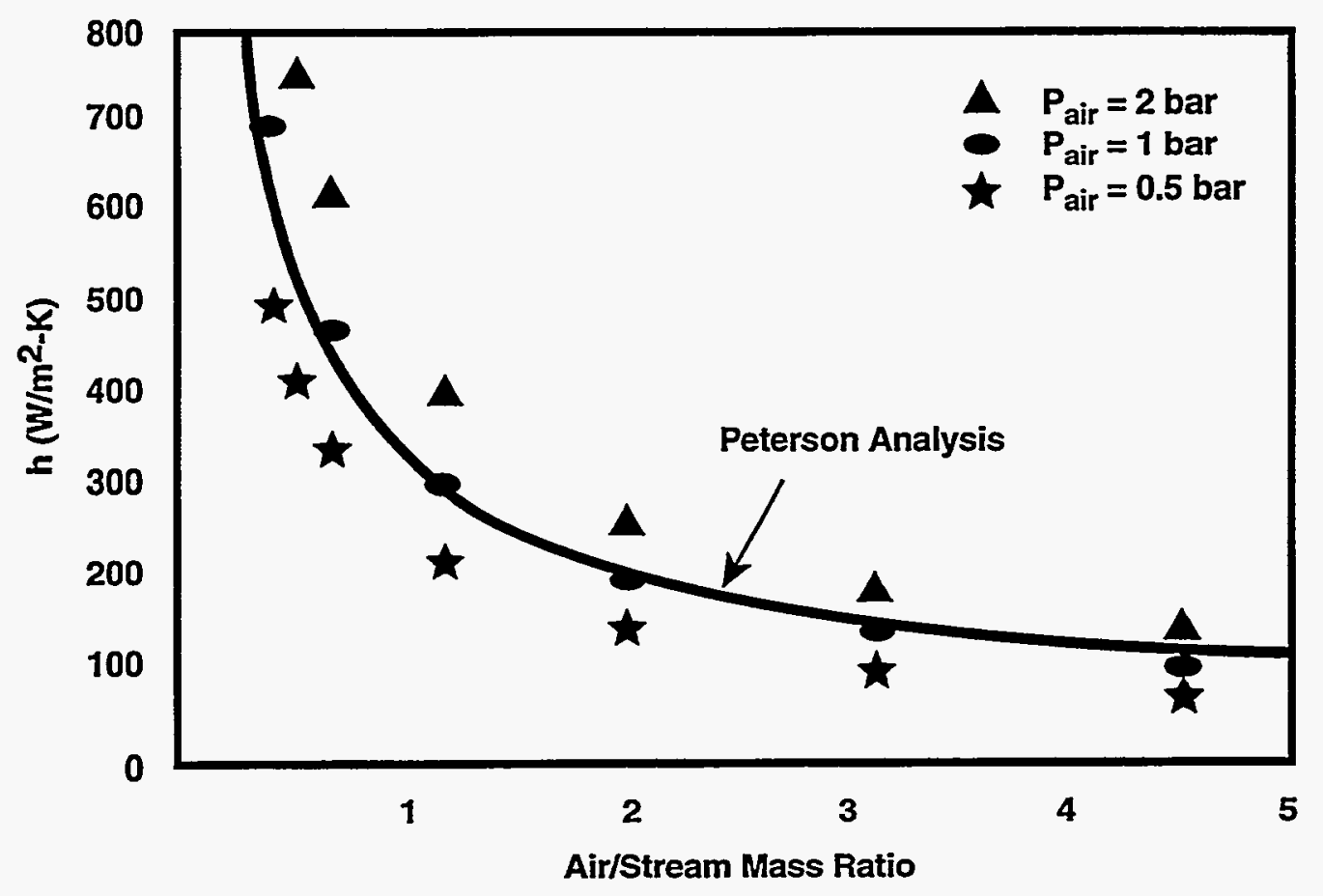

Figure 13-7. Predicted Heat Transfer Coefficients for a Condensing Air/Stream Mixture $\left[\mathrm{T}_{\text {wall }}=\right.$ $\left.322.0 \mathrm{~K}, \mathrm{~T}_{\text {init }}=300 \mathrm{~K}\right]$ 
concluded that the CONTAIN mass transfer model will reproduce the Uchida data under well mixed conditions. For air densities higher than ambient (for instance, below a steam injection elevation) CONTAIN will predict lower mass transfer rates than the Uchida correlation, and for air densities lower than ambient (generally in the dome region of the containment) CONTAIN will predict higher. While this air density dependence is taken into account in the CONTAIN model, its omission in the Uchida correlation limits the latter's usefulness for containment analysis.

13.3.3.2 Approximating Condenser Tubes. A proposed system for controlling long-term pressure in an advanced boiling water reactor containment design during a postulated design-basis loss-ofcoolant accident uses a condenser that connects between the containment drywell and wetwell. The condenser is immersed in a stagnant pool of water that is vented to the environment. Heat is passively rejected from the internal steam/air gas mixture in the containment to the environment as steam is condensed on the inside of the condenser tubes. The CONTAIN forced convective heat transfer and film tracking models can be used to simulate the internal condensation processes occurring in condenser tubes.

To model a condenser consisting of $\mathrm{N}$ tubes of diameter $\mathrm{D}$ and length $\mathrm{L}$, a representative condenser tube is divided into a number of vertical sections or segments. Due to timestep restrictions related to the Courant stability limit, the segmentation of the representative tube should be limited to only a few segments - usually as few as 6 sections for a 2-meter tube is enough to give reasonable numerical accuracy for the internal condensation rates and yet not subject the calculation to unreasonable timesteps, based on condensers sized for approximately a 10-MW capacity.

To model the cylindrical tube wall sections, a cylindrical shape is specified for the structure in the STRUC input block. Since the STRUC block "cylht" input corresponds to the height of a half cylinder, this input parameter must be doubled to represent a full tube wall. For a section length of $\ell$, "cylht" is set to $2 \mathrm{~N} \ell$ to represent the total condenser tube wall area for the section.

The heat transfer regime for convection in the tubes is forced convection. This regime can be approximated using the generalized heat and mass transfer options in the STRUC input block. A Dittus-Boelter forced convection correlation can be modeled by using a forced convection Nusselt number of the form $N_{\mathrm{Nu}}=\mathrm{a}+\mathrm{b}\left(\mathrm{N}_{\mathrm{Re}}\right)^{\mathrm{c}}\left(\mathrm{N}_{\mathrm{Pt}}\right)^{\mathrm{d}}$ where $\mathrm{N}_{\mathrm{Re}}$ is the Reynolds number, and $\mathrm{N}_{\mathrm{Pt}}$ is the Prandtl number. The parameters $a, b, c$, and $d$ that produce a Dittus-Boelter correlation are $0,0.023,0.8$, and 0.4 , respectively. The code will determine the Reynolds number based on total mass flow rate through the condenser, the cross-sectional area of the condenser $A=N \pi D^{2} / 4$, and the hydraulic diameter $\mathrm{D}$ of a representative tube, specified by setting CHRLEN $=$ "D." In the BCINNER subblock of the STRUC block the input is given as

BCINNER HYDAREA = "A" HPAINT $=1.0 \mathrm{e} 8$

FORCOR1 0.0 0.023 0.8 0.4

The HPAINT parameter is set here to simulate the bare surface for the interior of the tube.

For a vertical tube arrangement, the condensate film will flow down the tube interior wall and drain into a condensate tank. The film thickness can represent a significant part of the total resistance to 
heat flow under conditions of nearly pure steam. Therefore, a model of the film flow along the length of the tube should be included in the condenser modeling. To approximate the film flow, film tracking input is used in the BCINNER sub-block. For instance, for the third structure named V3 in cell 3 representing the third tube segment, the input is

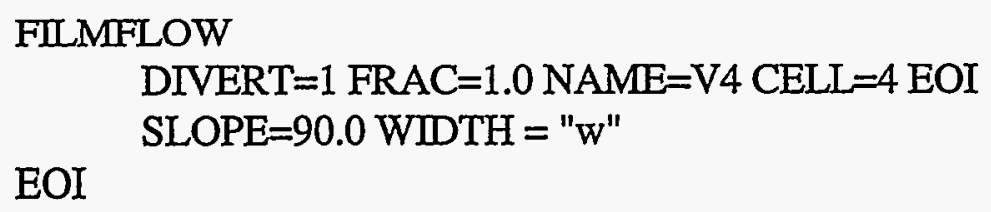

where $w$ is the total circumferential width of the film flowing down the condenser, $w=N \pi D$. The above input assumes that structure V4 lies directly below structure V3.

The CONTAIN modeling of film flow applies only for laminar flows. In general, the film Reynolds numbers for condenser flows are small enough that the condensate is believed to be well represented by laminar flow. When the gas mixture flow velocity is very high the interfacial shear of the gas flow on the slower condensate can perturb the film causing a slight decrease in the effective film thickness. However, it has been shown that for typical condenser flows with $\mathrm{N}_{\mathrm{Re}}<30000$, the shear effect is negligible and the CONTAIN laminar film flow assumption without shear effects is adequate for representing the condenser tube film resistance. To approximate a possible wavy laminar film flow, a multiplier can be used to simulate the enhancing effect that waves have on the mass transfer. It is common practice to apply a factor of 1.15 to the mass transfer coefficient calculated for waveless flow to account for waves. In the BCINNER sub-block the mass transfer coefficient can be increased by a constant factor by using the RMX2HX parameter.

13.3.3.3 Approximating Boiling Heat Transfer. Boiling heat transfer is not modeled with respect to the surface of a heat transfer structure. However, with the flexible heat transfer boundary conditions allowed in the STRUC block input, boiling heat transfer can be approximated. Under the conditions of nucleate boiling, a discrete set of data points for the heat transfer coefficient versus the surface superheat can be developed from a number of boiling correlations, such as Rohsenow's (see Section 10.4). The boiling heat transfer coefficient can be specified as a boundary condition, using the DELTA-T tabular input option in the BCOUTER sub-block. It is necessary to set TGAS equal to the saturation temperature $T_{s}$ for water at the submerged depth of the structure. During nucleate boiling, heat transfer to the water is essentially independent of the subcooling; therefore using $T_{s}$ is consistent provided the boiling correlation is defined in terms of surface superheat $T_{w}-T_{s}$, where $T_{w}$ is the structure surface node temperature. The user should be aware, however, that the table entries in the DELTA-T option correspond to the negative of the superheat; therefore, the temperature differences in the table should be input as increasing but negative values.

13.3.3.4 Film Flow Over an Ellipsoidal Dome Shell. In describing film flow over a containment dome, the dome is typically broken up into a number of heat transfer structure surfaces, or segments. The film width for liquid water flowing over spherical or ellipsoidal dome shell segments can be

\footnotetext{
${ }^{4} \mathrm{~J}$. Tills, Modeling and Analysis Department, Sandia National Laboratories, Albuquerque, NM, letter report to the USNRC with title "Letter Report on PCCS Modeling for SBWR," March 1994.
} 
determined from the definition of the width as the horizontal dividing line separating equal-area portions of the segment. The segment area is represented as a slab, and the width is given a nondefault value $w$ equal to the arc length of the dividing line. In the case in which the dome is ellipsoidal, the surface is described by the surface equation

$$
z=c\left(1-x^{2} / a^{2}-y^{2} / a^{2}\right)^{1 / 2}
$$

where $c$ and $a$ are the semi-minor and semi-major axes of the ellipsoid. The location of the equalarea line for the segment $\left(\varphi_{1}<\varphi<\varphi_{2}, \theta_{1}<\theta<\theta_{2}\right)$ is determined by numerically calculating the dome surface area $\sigma$ that lies over the circular region defined by the radius $R$ from the dome axis:

$$
\sigma=\iint\left(1+\mathrm{f}_{\mathrm{x}}+\mathrm{f}_{\mathrm{y}}\right)^{1 / 2} \mathrm{dA}
$$

where $f_{x}=\partial z / \partial x$ and $f_{y}=\partial z / \partial y$. The equation above can be used to determine the dome surface area at a given radius. From this information the equal-area lines can be determined for every dome segment.

13.3.3.5 Hints for Structure Naming and Ordering. The method for keeping track of structures in CONTAIN is to assign each structure in the STRUC input block a number corresponding to the order that the structure is listed in the input for that cell. The structure number is used to identify structures that are connected across cell boundaries. Structure input preparation can be simplified if a few guidelines are followed for naming and ordering structures. First, names can provide information that will help the user identify the structure type (wall, floor, or roof), order, and the connection to other structures in the input. For example, an unlinked wall structure that is listed as the 20th structure in cell \#1 could be given a name "w20_1." A roof structure listed as the 10th structure in cell \#1 and connected to the 3rd structure in cell \#3 could be given a name "r10_1_3." On the input line specifying the structure name one should also use comments to indicate the name of the connected structure, if any. This naming convention, if followed, will be of benefit for debugging input and in collapsing a large multi-cell input file to fewer cells if the need arises (see Section 13.3.4.6).

Since structure names are limited to eight characters, the user needs to allow for this limitation in large input files with many cells and structures. To avoid keeping track of large numbers in the structure input block for the connected structures, the user should try to order the structures such that all connected structures are listed first in the input block. This method also eliminates possible input inconsistencies if some unconnected structures are either combined later or more structures are added. Additional connected structures can be added to an existing input following the last connected structure input so as to not alter the existing structure connect input.

13.3.3.6 Simulating Quenching Phenomena and Steam Spikes. To simulate a quenching process in a pool, the user may exercise the HT-COEF option (see Section 14.3.2.3.1) to define an enhanced heat transfer coefficient between a hot intermediate layer and the coolant pool. The heat transfer coefficient should be set to a low value or to zero until the time the hot material is actually injected into the coolant, and then increased to a high value. The numerical solvers for the lower cell system and pool boiling are sufficiently robust to handle the situation in which large amounts of energy are 
dumped into a layer in a short time. The energy being added per timestep can be of the order of tens of percent of the layer's total energy. Steam spikes due to molten fuel dropping into water in the cavity have been effectively simulated in this way. The important thing to remember is that the heat transfer coefficient specified in the HT-COEF option is associated with the nominal area of the layer. To simulate an enhanced heat transfer area (due to fuel fragmentation, for example), it is necessary to multiply the heat transfer coefficient desired by the ratio of the true heat transfer area to the nominal layer area. Similarly, the steady-state behavior of a porous debris bed can be simulated with the same option.

Additional guidance for simulating debris-water interactions in DCH scenarios is given in Section 13.3.2.1.2.

\subsubsection{Miscellaneous}

13.3.4.1 Fission Product Mass Effects. Fission products are treated as being massless in terms of their effects on processes such as intercell flow and aerosol dynamics. This treatment may not be appropriate for certain types of problems. Fission product masses could, for example, significantly influence the behavior of their aerosol hosts. Thus, the user should attempt to minimize the discrepancy between the fission product masses and the aerosol masses. For example, if a fission product attached to an aerosol host is introduced via source tables, the mass of the aerosol host should be increased by the corresponding amount through source tables. If possible, the size of the aerosol particles should also reflect the presence of the fission product mass.

13.3.4.2 Simulation of Pool Scrubbing of Elemental Iodine. The CONTAIN models for boiling water reactor suppression pool vents and safety relief valves do not include scrubbing of gaseous fission products such as elemental iodine. If it is acceptable to assume that iodine will be in equilibrium between the pools and the atmosphere, a simulation of the net effect may be defined using the targeted release and acceptance formalism as described in Section 8.4. Let the partition coefficient, $K$, be defined to be the ratio of the aqueous concentration to the atmospheric concentration at equilibrium. To simulate the equilibrium behavior, the user can define both pool-togas and gas-to-pool transfers, with the (gas-to-pool)/(pool-to-gas) release rate ratio being equal to $\mathrm{KV} \mathrm{V}_{1} / \mathrm{V}_{2}$, where $\mathrm{V}_{1}$ and $\mathrm{V}_{2}$ are, respectively, the volumes of the pool and of the atmosphere. (Inclusion of $V_{1}$ and $V_{2}$ is necessary because a mass release rate is proportional to the total quantity of a fission product associated with a host, while the partition coefficient is defined in terms of concentrations.) The sum of the release rates governs the rate of approach to equilibrium. Thus, if $\mathrm{K}$ is 20 and the atmosphere volume is 10 times the pool volume, and an approach to equilibrium with a time constant of 33.3 seconds is desired, this could be achieved by the following FPM-CELL input (see Section 14.3.1.10):

FPM-CELL

TARGET

$\begin{array}{lllll}\text { MOLI2 } & 20.0 & 0.0 & \text { FROM GAS } & \text { TO LAYER POOL } \\ \text { MOLI2 } & 10.0 & 0.0 & \text { FROM LAYER POOL } & \text { TO GAS }\end{array}$

EOI

$\mathrm{EOI}$

Rev. 0

13-65

$6 / 30 / 97$ 
Temperature-dependent release rates may be specified in order to simulate the temperature dependence of the partition coefficient. Since the pool temperature would presumably govern the gas-to-pool transfer, it should be redefined as a pool-to-gas transfer with a negative rate. The reader should refer to Equation (8-2) to see how a negative rate is interpreted.

It should be noted that the assumption of rapid equilibrium is likely to be invalid for some accident scenarios. Equally important, aqueous iodine chemistry is actually very complex and will include many dependencies that cannot be simulated using the release and acceptance formalism as presently implemented. The partition coefficient, as defined above, will depend upon $\mathrm{pH}$, total iodine concentration, and possibly the amounts of the many other chemical species that might be present in the pool during a severe accident. In addition, slow iodine reactions such as hydrolysis to iodide and iodate can cause a time-dependent change in the effective value of the partition coefficient. To a limited degree, this effect could also be simulated by defining additional interhost transfers. Radiation chemistry effects can introduce additional complexities.

13.3.4.3 Multiple Sources for a Single Species. Use of CONTAIN for the analysis of LWR accidents usually requires that sources of water, steam, and hydrogen be defined as part of the input to the code. These sources may come from design-basis-accident codes or other codes that model core and reactor coolant system transport of liquids, vapors, and gases. More than one source table for the same material can be specified in the input. The fact that the effects of such tables are additive gives the user flexibility in combining sources that may have been generated by different codes.

13.3.4.4 Restarts. Long-running jobs can be efficiently handled using the restart option. Each restart file should be saved under a file name that is different from the name of any previously generated restart files. Such a backup avoids the loss of previous restart information should an error or system problem occur during the run.

The files that need to be saved to invoke a restart are the main plot file PLTFIL and the restart file TAPE10. Note that PLTFIL and TAPE10 are overwritten on a restart, beginning with the restart time. Thus subsequent restarts at the same restart time will invoke parameter values specified in the previous restart, not those defined in the original run. The output file for each CONTAIN calculation is overwritten during each run; therefore, to save the output file for subsequent restarts, the output file OUTPUT must be renamed prior to each restart. Note, if a problem terminates due to the problem time exceeding the maximum CPU time limit "cput," a restart time dump for the last edit timestep is present on the restart file. However, the restart times listed on either the error file TAPE17 or event file TAPE21 will not indicate this restart time. To restart from the last restart time dump, use a large value for "tstart" (beyond any restart time that could be on the file), for instance, "tstart" $=10^{10}$. In this manner the user can continue a calculation without having to recalculate from the last specified restart time.

13.3.4.5 Annotated Input. Users are encouraged to add comments to their input files. This is accomplished using the double ampersand sign $(\& \&)$, followed by a blank and then the appropriate comments. 
Comments at the beginning of an input file describing the nature of the file, the particular circumstances being modeled, etc., are useful for later referral. One can also use the TTTLE option. However, titles cannot be put at the very beginning of an input file, while comment lines can be. On the other hand, because titles are printed at the beginning of each long edit and become part of the plot file, they are convenient for labeling the output.

13.3.4.6 Hints for Collapsing Multi-Cell Input Files. When modeling a plant, the highest level of detail will often be determined by the format of the available data. Usually, one begins by generating CONTAIN input based on the most detailed nodalization that one believes the analysis requires and that the data will support. Later, a simpler input file with fewer cells may be required. There are a number of guidelines that will help a user when collapsing large multi-cell input files. First, in anticipation of the possible need to simplify, a detailed input file should be set up using the structure naming and ordering scheme suggested in Section 13.3.3.5. The following suggestions may also help to avoid confusion and needless debugging:

- Determine the collapsed nodalization and generate a map of cells by developing a table that shows how the "old" cells are combined into the "new" cells.

- Develop a flooding profile mapping for combining the multiple pools in the "old" input file to the "new" input file. Remember that CONTAIN is limited to only one pool per cell. Pool flow paths may require restructuring, as may the manner in which structures submerge in the pools.

- Generate a concrete structure mapping table for the "old" input file showing all of the structure connections. Use the nodalization mapping table to help develop additional columns in the mapping table that show the new cell and structure numbers.

- If the number of miscellaneous steel structures needs to be collapsed, also generate a steel structure mapping table, separating out flooded and unflooded structures. Consider combining unflooded structures into three to five thickness classes. Comment the combined structures to indicate the grouping according to the "old" structure names.

- If the "old" input file makes use of the film tracking model, generate a film flow mapping table similar to the mapping tables for connected structures.

- Generate a flow path and elevation mapping table.

- Using the mapping tables for nodalization, structure, film flow, and flow paths, begin to mark up a hardcopy of the "old" input listing. Do not modify an electronic copy of the "old" input before marking up the changes on the hardcopy. File comparison utilities are awkward to use in keeping track of changes. A hardcopy markup is generally a better approach. Try not to rename structures; instead use the comment capability to make notes on naming and ordering. 
- Modify all global input in the "old" input listing that involves cell numbering and order, like cell timestep fractions.

- After marking up the "old" hardcopy of the input, transfer the changes to an electronic copy of "old" input using copy and paste options in the editor. Save the modified electronic copy of the input.

- Begin debugging the input with the CONTAIN code.

Time spent in generating mapping tables and a good marked-up hardcopy of the "old" input file will greatly speed the collapsing process, provide a record of changes, and minimize the errors not caught by the input processor.

13.3.4.7 Role of Sensitivity Calculations in Building Input Files. Building a complicated CONTAIN input file is a time consuming effort. Sometimes the process can be simplified by eliminating some unimportant details. Determination of what is unimportant is often not clear. Therefore, sensitivity calculations may be required to justify input assumptions. Some areas that the user might consider in this regard involve nodalization, structure flooding, structure outer boundary conditions, film tracking, aerosol modeling, and liquid water inventory control.

In most cases the simplest input required to adequately capture the physical phenomena occurring within the containment is preferred. For example, the film tracking model is generally not recommended for miscellaneous steel structures or most internal concrete structures, over the default modeling option. The importance of film resistance however can be checked though sensitivity calculations. Defining structure submergence can become time consuming, and the containment data may not support this level of detail. Sensitivity calculations using bounding estimates for submergence can help the user determine whether submergence is important for the analysis. External atmospheric sources that describe RPV steaming may create problems with the water inventory in long-term calculations. Overflowing pools to the environment may solve the inventory problem. However, heat and mass transfer between the pool and the atmosphere may be affected. A sensitivity calculation that checks on the importance of such heat and mass transfer can be used to justify overflowing the pool to the environment. Connected structures often require a level of detail regarding the containment geometry that is not available. In such cases, the user may want to use an adiabatic boundary condition at the midplane separating one structural wall, floor, or roof from another. Again, sensitivity calculations can help the user verify that this simplification is justified. Alternatively, structure boundary conditions can often be analytically assessed by consideration of the time scales of the scenario being investigated.

13.3.4.8 Spray Modeling Suggestions. The CONTAIN model for water sprays discussed in Section 12.3 has a number of limitations. The model for example uses a single user-specified initial drop size. In addition the carryover of the spray droplet field from one cell to another and impingement of the sprays against structure surfaces are not modeled. Each of these limitations has a workaround. First of all, the drop size limitation applies to each spray engineered system. However, there are no limitations on the number of such systems that may be defined within a given cell. Thus, several could be defined, each with a different spray droplet diameter. Carryover of spray drops 
cannot be modeled directly. However, carryover can be simulated by defining a spray system that operates in recirculation mode in the carryover cell. The trick is to specify that the latter spray system draw its water from the pool that is used to collect the spray water reaching the bottom of the cell above. This pool should not be one that couples strongly to the thermal-hydraulics of the problem; it could, for example, be an isolated pool used only for carryover purposes. The spray system in the carryover cell should have an unrealistically high user-specified mass flow rate; in this case the actual spray mass flow rate will be determined by the spray collection rate in the pool. The carryover spray temperature in this scheme will be determined by the pool temperature. A minor disadvantage to this work-around is that the spray drop size must be a user-specified constant and thus will not depend on the size of the drops entering the pool. Impingement of spray drops against structure surfaces can be simulated by withholding part of the water actually flowing through the spray system and applying it instead to a structure surface. This is done through a structure film source table, as discussed in Section 10.2.2. The film resulting from this source will exchange heat with the structure surface. As discussed in Section 10.2.2, the user can also model the evaporation of the film, as well as its flow onto other structures. These techniques were used, for example, to model the NUPEC spray experiments, as discussed in Reference Sta95. 


\subsection{INPUT DESCRIPTION}

The input needed to run CONTAIN and to use its various options is described in this section. The overall format for the input file is discussed first, followed by the specific requirements. In general, the structure of input to CONTAIN is quite flexible. Certain restrictions on the ordering of the main input blocks are identified below, but these are quite limited. Note that all CONTAIN quantities are in SI units, unless otherwise specified.

Preparation of input for running a problem with CONTAIN need not be overly complicated. As a result of the modular use of physics routines, certain problems can be formulated rather simply. For more complex and detailed problems, the input becomes correspondingly more complicated. To simplify the task, the input has been designed with several key features, including

-

A general free-field, keyword-driven format. Physical models are activated only by the presence of associated keywords in the input stream and are otherwise inactive. Note that keywords are presented in the discussion in upper case, but depending on the system may actually be input in lower case.

- Default values for input variables. Most models allow the user to specify individual physical parameters. In many cases, however, default values will prove satisfactory.

- Separately specified global input and cell level input. Global input (for processes common to more than one cell) and each of the individual cell input sections are grouped separately to allow the user further flexibility in setting up a problem. For example, one cell may require detailed modeling, while others may simply be gas reservoirs.

The most important principle concerning input is that if a keyword is left out, the model associated with it is not activated, even if this means physically unrealistic results. Leaving CONDENSE out of the input, for example, results in no condensation mass and heat transfer in the problem.

\subsection{General Input Format and Structure}

CONTAIN has a large number of models and features, and as a consequence, a complete cataloging of input options and instructions might be somewhat overwhelming at first sight. To maintain a clear perspective, the input format will be shown as follows: first, an outline of the input file structure in this section; second, summaries of the global input and detailed instructions for each input option (Section 14.2); third, a description and instructions for the cell level input (Section 14.3); fourth, descriptions of the table input format for many of the global and cell input options (Section 14.4); and finally, the instructions necessary to restart a CONTAIN calculation (Section 14.5).

Figure 14-1 shows an outline of the input file structure. Note that there are several sections of the input. The global input, initiated by the word CONTROL, must be given next. This section has blocks of information common to all cells, including standard CONTAIN material names, the names of any user-defined materials, information on fission products and their properties, and information that defines interactions among cells. A number of cell input sections, initiated by the keyword 
CELL, must follow the global input. One such section exists for each cell in the problem. Each section has blocks of information required by the various physical models used in individual cells. Finally, the file is terminated by the keyword EOF.

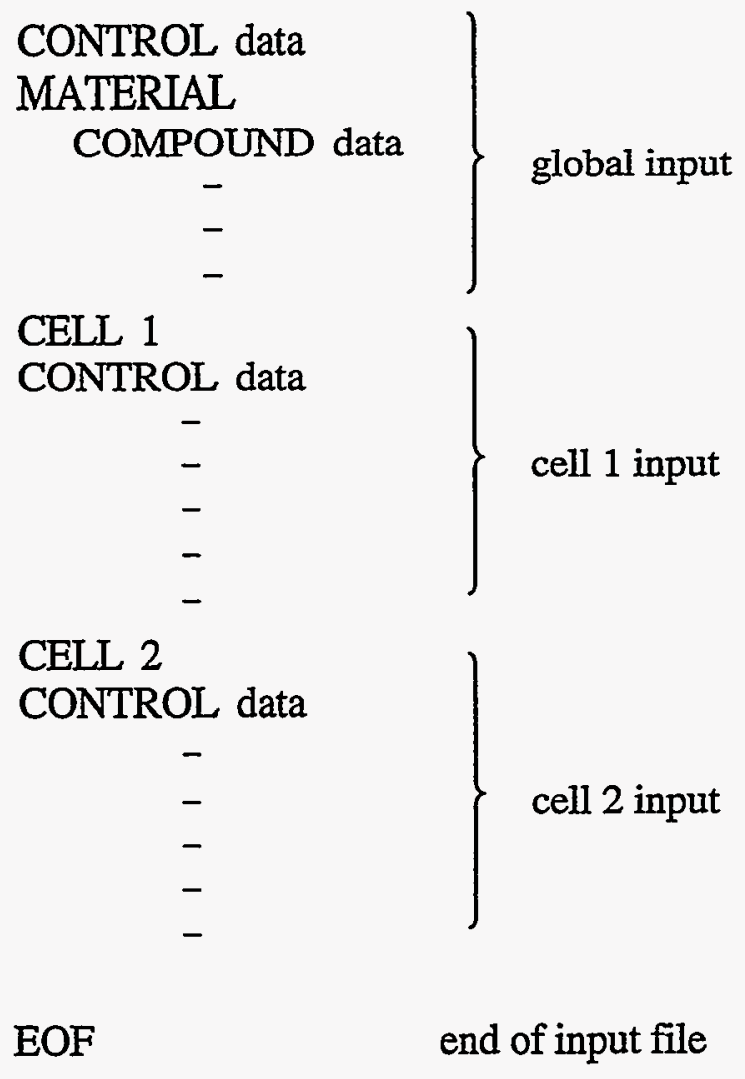

Figure 14-1. CONTAIN Input File Structure

Global and cell level input sections are shown in summary form at the next level of detail in Figures 14-2 and 14-3, respectively. Each keyword and parameter will be explained in detail below. Parentheses ( ), square brackets [ ], the symbol \&\&, and curly brackets \{\} have special meanings which will also be explained below. The purpose of the figures is to illustrate the overall pattern of the global and cell sections of input.

As shown in Figure 14-2, the first blocks of information required in the global input group specify control information, materials, and fission products. The data in these blocks enable the code to allocate the total amount of global data storage space required. Thus, the first two blocks of data in the global input must be the CONTROL and MATERIAL blocks, in that order. In the MATERIAL block, the keyword MATERIAL should be followed immediately by the COMPOUND sub-block and then by the USERDEF, FP-NAMES, or AERNAMES sub-blocks, if used.

The subsequent data consists of global blocks of data. These may occur in any order (provided they precede the cell level input). In Figure 14-2, each left-justified keyword with the exception of EOI 


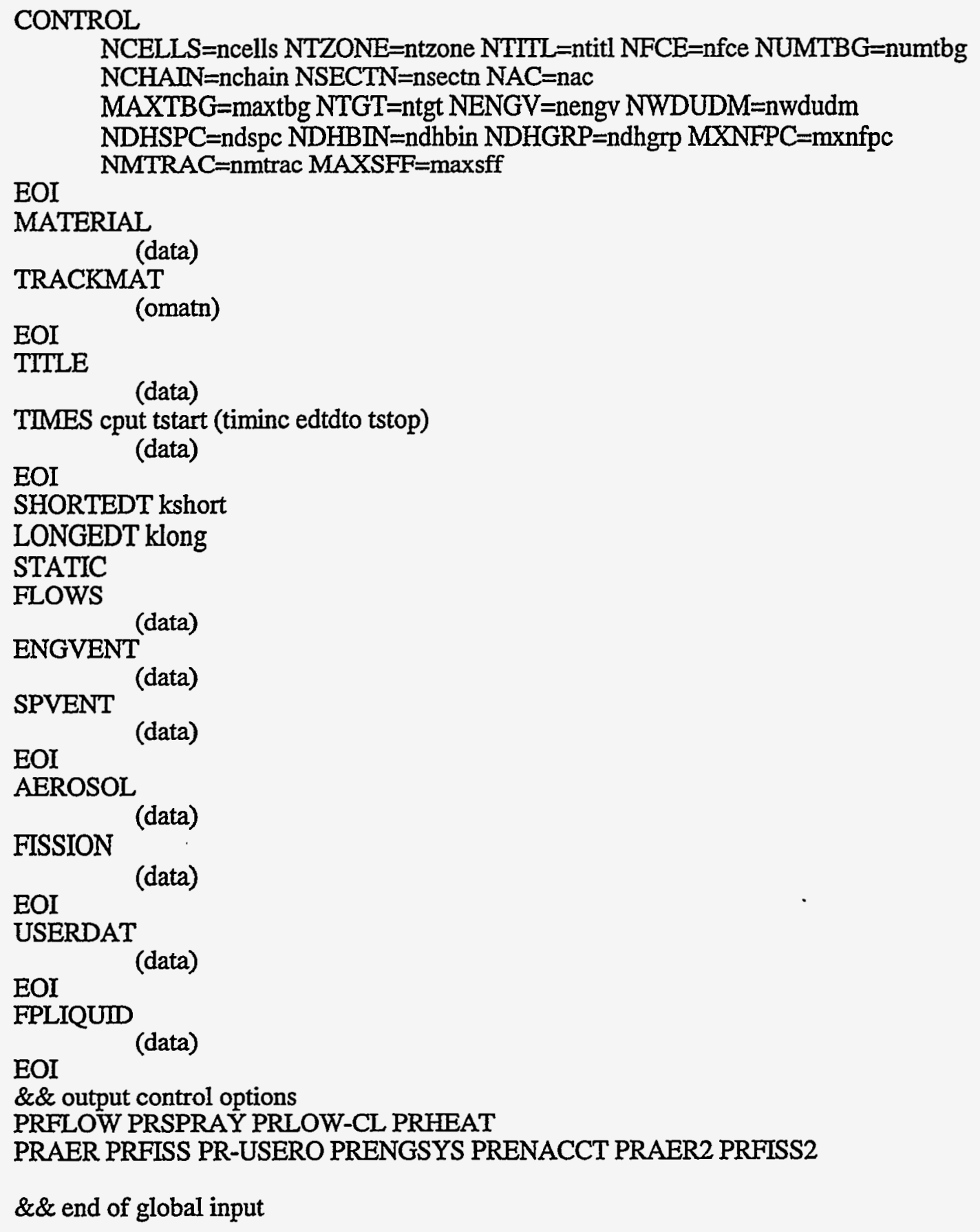

Figure 14-2. Summary of Global Level Input 


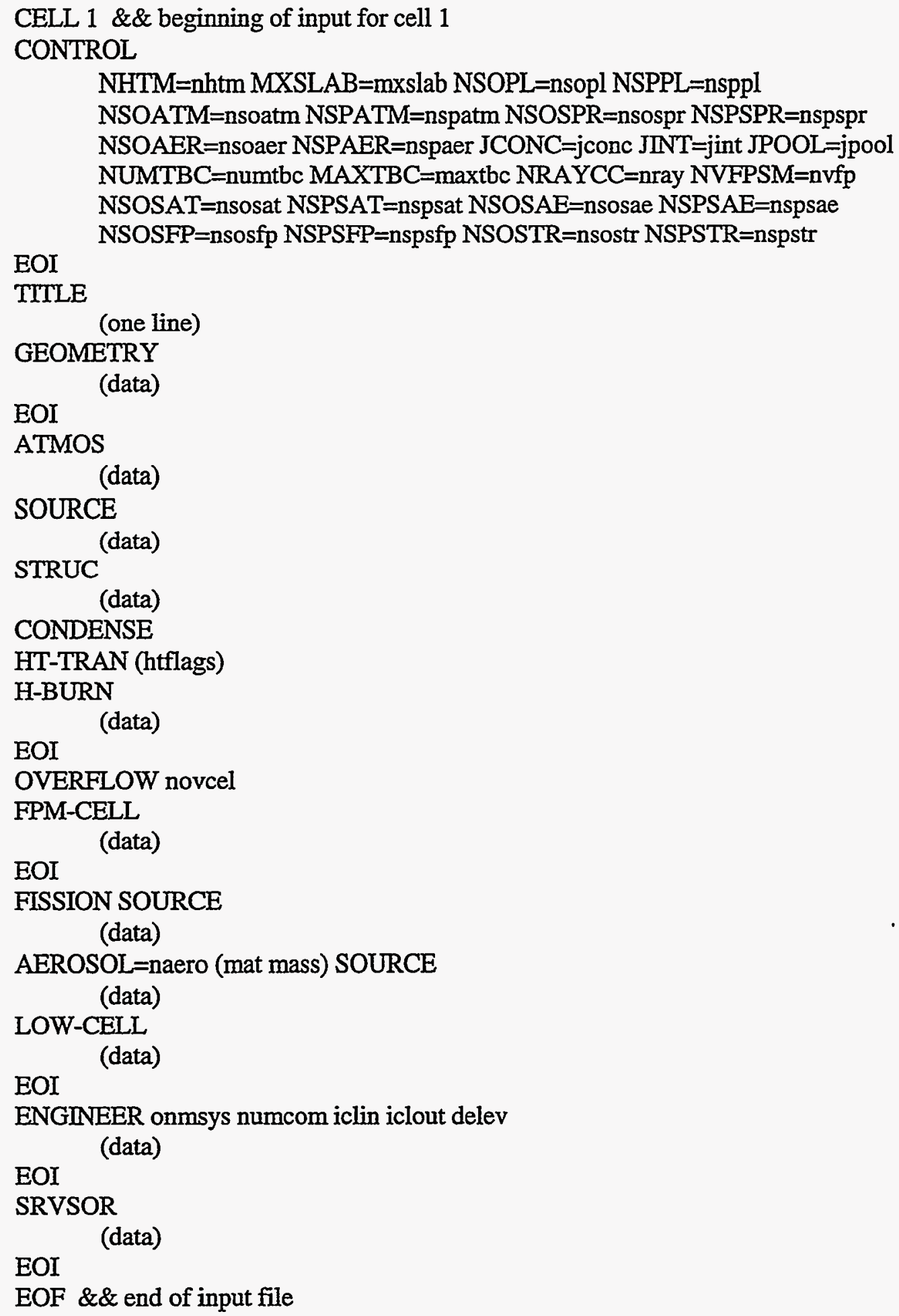

Figure 14-3. Summary of Cell Level Input 
denotes the beginning of a different main input block. The left-justified EOI (end of information) keyword simply denotes the end of the main block above it. With the exception of the reactor-type option, if a block is not specified, then the corresponding model or option is not invoked. The physical models that require global input information are the fission product model, the intercell gas flow model, and the aerosol model.

Figure 14-3 shows the structure of the cell level input for one cell. The first block of information for each cell specifies the information needed to determine the total storage required by this cell. Thus, the CONTROL block must immediately follow the CELL input line. Also, the GEOMETRY block should precede any block with physical parameters since the cell volume is often needed to calculate physical conditions.

The order of the subsequent main blocks of information is again arbitrary. Most, but not all, main blocks are shown in Figure 14-3 with the same format used in Figure 14-2. If a block for a given model is omitted in a cell, then that model is not used in that particular cell.

In the following sections, the third and final level of detail, the definition of the main input blocks, will be provided. A number of alternative input formats are available for certain blocks and are described in Appendix B. The alternative formats are obsolete but are still supported in the interest of maintaining upward compatibility with old input files. These formats will generally be of little interest since the standard formats will provide the same or more options.

In order to understand the following input instructions, the reader must be familiar with a number of conventions, both in the input data set format itself and in the instructions for writing the input. The input data consist of keywords, which are indicated in the following by uppercase letters, and values for variables, which are indicated by lowercase alphanumeric groups. (In the text, variables are enclosed in quotation marks to avoid confusion.) Appropriate values for some of the variables are character constants (i.e., names), while other values are integers or floating point numbers. In CONTAIN the convention is that all keywords and names must have 8 or fewer characters. The input data are specified in a free field format with the exception of titles, which reserve a number of entire lines of 80 or fewer characters. Acceptable and equivalent separators for keywords and input values are a blank space, a comma, an opening parenthesis, a closing parenthesis, a new line (carriage return), or an equal sign. Any number of keywords and values can be present on a line of input, up to a maximum of 80 characters per line. Each data block can be continued on as many lines as desired; however, an individual keyword or value cannot be continued from one line to the next. At any position on an input line a comment (which will not affect the input processing) can be inserted after the pair of characters \&\&. A blank space must appear after the second \&. The remainder of the input line may then contain comments of any sort. The use of $\& \&$ is very convenient for annotating input data sets with helpful comments, reminders, and brief descriptions of the problem. A few examples of the use of the comment symbol are shown in Figure 14-3. Default values are available for many of the input variables; these are listed in the appropriate subsections. Default values are not provided for input variables that depend strongly on the nodalization selected by the user. Thus, for example, the user must specify the configuration of cells to be used and all heat transfer structures in each cell. 
In the following input descriptions, rows of asterisks are used to delimit the template for an input block. The template gives all of the available keywords and variables in a special format. That format does not have to be used in the input data set itself, which is free field. However, the format defines completely the ordering requirements that must be taken into account within the input data set and indicates to a large extent the option combinations available to the user in specifying models or modeling features.

The following notation is used in the templates:

- Uppercase words are either keywords or character constants (i.e., names) that must be supplied literally in the input.

- Lowercase words are considered variables, which should be replaced by values that are either numbers or character strings. Lowercase words representing an integer value follow the FORTRAN convention of starting with a letter between $i$ and $n$, and the value should be specified as an integer. Lowercase words representing character constants (i.e., names) should be replaced by the appropriate character constant (i.e., string). (Possible character constants are also capitalized in the following discussion.) Lowercase words representing floating point values should be replaced by values in FORTRAN $F$ or $E$ format. (A decimal point must be present in numbers in the $\mathrm{E}$ format. For example, 1.E6 is acceptable but 1E6 is not.)

- Parentheses () imply that the enclosed quantity or quantities should be repeated as necessary.

- Square brackets [ ] imply that the enclosed quantity is not always required.

- Quantities within a given set of curly brackets \{ \} represent a number of alternatives, of which the user should select one. Each alternative is delimited by a curly bracket and a boldface or or by two boldface or's, as indicated by the ellipses in \{...or...or....\}.

In the following discussions of the input blocks, the templates for each block are followed by descriptor blocks that describe each keyword and variable appearing in the template.

\subsubsection{Ordering Requirements in Input Blocks}

The input specified within a given input block cannot be in an arbitrary order but must satisfy two general requirements regarding the order of variables and the order of sub-blocks. Partly because a variety of programming styles are represented in the input processing, these ordering requirements cannot be stated without lengthy definitions and considerable explanation. However, the system of input that is represented is reasonably natural and flexible in use. Thus, the user should not feel compelled to dwell too long on the ordering requirements discussed below, especially if the basic order given in the template for that input block is followed. To help the user with regard to the ordering requirements, caveats are usually given explicitly in the keyword or variable descriptor blocks when the order of specification of a sub-block or variable is restricted in a way that is not obvious. Such restrictions are also completely defined by the template format according to the rules given below. 
The ordering requirements within an input block are based on a hierarchy indicated in the template by the level of indentation used to display a group of keywords and variables. Thus, the lowest level in the hierarchy is occupied by the group placed furthest to the right.

The first type of ordering requirement is related to the order of variables: a contiguous group of variables must be specified in the input in the order given in the template. A group of variables is considered contiguous if the variables are all at the same level in the hierarchy and are not separated by a keyword at any level. (Note that a contiguous group of variables may and often does consist of only one variable, especially one following a keyword.) Furthermore, a contiguous group of variables that immediately follows a keyword at the same level in the hierarchy must be specified immediately after that keyword. Most variables follow such a keyword. However, in some cases they do not. In such cases, the leading variable of the contiguous group has the same function as a keyword in defining ordering requirements. Such leading variables are often character variables representing the name of an option or material.

As an example, a keyword PVALUE that has " $n$ " values of a variable " $p$ " associated with it might be displayed in the template in the form:

\section{PVALUE $\mathrm{n}(\mathrm{p})$}

where the parentheses imply that the enclosed item(s) should be repeated as necessary. This group of keywords and variables is considered to be at the same (the first) level in the hierarchy, since only one (the zero) level of indentation is used for the group. The variables are contiguous and follow a keyword in the template and thus must be specified in the order given immediately after the keyword. If there are four values $1.0,2.0,3.0$, and 4.0 of "p" associated with PVALUE, the corresponding input would have the form:

\section{PVALUE 41.02 .03 .04 .0}

The second type of ordering requirement involves sub-blocks of data. A sub-block at a given level in the hierarchy is basically a contiguous group of keywords and variables at the given level or lower that is not separated by keywords or variables at a higher level. The sub-block boundaries are not uniquely specified according to this definition. For present purposes additional rules are needed to make the division into sub-blocks at a given level unique. Because of the variety of ways that subblocks are recognized in the code, these rules are rather complex: (1) With the exception of an end-of-information (EOI) keyword, a keyword or leading variable at the same level as the sub-block marks the beginning of a new sub-block, as does an indentation to a lower level than the sub-block. If such a keyword or leading variable is present, it is considered included in the sub-block it begins and also is considered to terminate the sub-block at the same level immediately preceding it, if one exists. If such a keyword is present, it is called a leading keyword. An EOI keyword at a given level has a different effect: it terminates the preceding sub-block at the same level and is considered included in the sub-block it terminates. Note that a sub-block can have at most one non-EOI keyword or leading variable at the same level as the sub-block. Thus, at the lowest level in an input block, individual non-EOI keywords and leading variables each represent a different sub-block. 
A sub-block thus can begin with a leading keyword or variable and end with an EOI. It can begin with a leading keyword or variable and end with the leading keyword or variable to the next subblock; it can begin with an indentation without a leading keyword and end with an EOI. (The EOI is the only keyword at the level of the sub-block in the latter case.) From the above definitions, it follows that an EOI keyword, if present, will always be written at the same level of indentation as the sub-block that it terminates. This consistent positioning of the EOI should be quite helpful in determining which sub-block is terminated by each EOI when multiple sub-block levels are present.

With the above definitions, it is now possible to give the ordering requirements for sub-blocks: (1) A sub-block must be specified after the last keyword or leading variable that precedes it in the template and is at a higher level than the sub-block. Similarly, the sub-block must be specified before the next keyword or leading variable at a higher level. By induction, this rule constrains quantities within a sub-block to be specified in the order given, with exceptions only as allowed by the second rule. (2) However, within a contiguous group of sub-blocks at the same level, those subblocks may be specified in any order. A contiguous group of sub-blocks is defined as one that is not separated by a sub-block at a higher level. (It is not possible to have sub-blocks at a given level separated by a sub-block at a lower level because the sub-block at the lower level is by definition included in one of the higher level sub-blocks.) Since at the lowest level, individual non-EOI keywords and leading variables each represent a sub-block, the second rule implies that within a contiguous group of such items at the lowest level, those items can be specified in any order.

Two examples of the template format are given below. The first is:

*************************************************************************

KEY n (const)

[OPTION1] \{OPTION2 or OPTION3\}

EOI

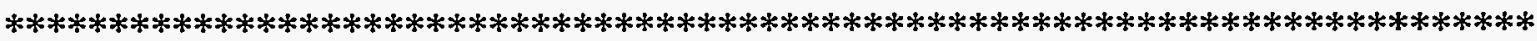

where " $n$ " is defined as the number of values of "const" to follow the keyword KEY and OPTION1, OPTION2, and OPTION3 are the three options associated with the KEY block. In this example, KEY, " $n$," "const," and EOI are considered to be at the first level in the input hierarchy, and OPTION1, OPTION2, and OPTION3 are considered to be at the second level. The "n" and "const" variables are required to immediately follow KEY in the input because of the ordering requirement for variables following keywords. OPTION1 is truly optional, as indicated by its square brackets. However, one of OPTION2 or OPTION3 must be selected, as indicated by the curly brackets. The option keywords selected are required to follow KEY, the last keyword preceding them at a higher level in the input hierarchy, and to precede EOI, the next keyword following them at a higher level. However, they are not required to follow KEY immediately as are " $n$ " and "const." Since OPTION1, OPTION2, and OPTION3 are each considered sub-blocks at the lower level, they are not restricted in the order in which they may be specified with respect to each other. (However, the curly brackets indicate that only one of OPTION2 or OPTION3 can be specified.) The EOI terminates the subblock which begins at the same level of indentation as the EOI, in this case the sub-block beginning with KEY and not with OPTION1. In the input one might thus specify 
If $\mathrm{n}$ is equal to 3 , the three required values are 1.0,2.0, and 3.0, and the indicated options have been invoked.

The second example is the template for heat transfer structures, which gives the format to be used for specifying all of the heat transfer structures in a cell. (Only the template format is discussed here. The meanings of the keywords and values are discussed in Section 14.3.1.3.)

**************EXAMPLE***********EXAMPLE**********EXAMPLE ${ }^{* * * * * * * * * * * * * * * * *}$ STRUC

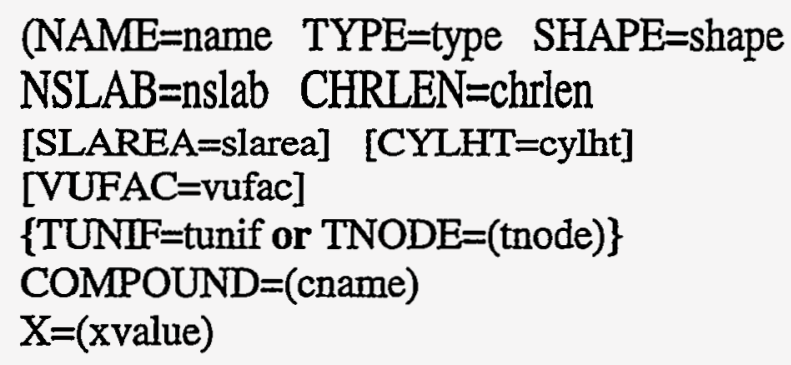

In this example three levels in the input hierarchy are represented. The keyword STRUC is at the first level, the EOI is at the second level, and all other quantities are at the third level. STRUC is the leading keyword for the sub-block at the first level, which encompasses all the quantities shown. The sub-block at the second level begins with NAME and ends with EOI. However, there is no leading keyword or variable for this sub-block, which actually. begins with an indentation. The reason for the lack of a leading quantity is that the pairs of quantities connected by the equal signs at the third level are accepted by the code in any order, and there is no quantity that should be given first. The freedom to specify these pairs in any order is indicated by their hierarchical position as sub-blocks at the third level and, therefore, according to the above rules, they may be specified in any order. (The keyword STRUC is not the leading keyword for the sub-block at the second level because it is not repeated each time a new structure is specified; only the keywords beginning with NAME and ending with EOI are repeated. Note that the EOI does not terminate the entire block but each structure. Thus it is not placed at the first level. Also, the EOI should not be placed at the third level, because that would imply that it terminates one of the pairs of quantities, such as the "X $=$ (xvalue)" input. However, according to the way the code is written, that is not its function. Thus, there is no ambiguity about where the EOI should be placed.)

\subsection{Global Level Input}

The global-level input block provides data which either is required by a global model or is common to all cells in the system being modeled. The first two blocks of data in the global input must be the CONTROL and MATERIAL blocks, in that order. In the MATERIAL block, the keyword MATERIAL should be followed immediately by the COMPOUND sub-block and then by the USERDEF, FP-NAMES, or AERNAMES sub-blocks, if used. Other main input blocks may occur in any order. 
The global CONTROL block is used to specify the storage allocation associated with the global models.

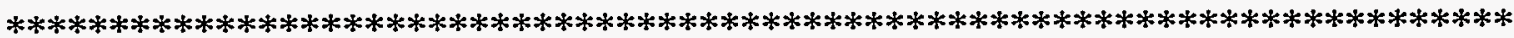

CONTROL

NCELLS=ncells NTZONE=ntzone [NTTTL=ntitl] [NFCE=nfce]

[NCHAIN=nchain] [NSECTN=nsectn] [NAC=nac] [NUMTBG=numtbg]

[MAXTBG=maxtbg] [NTGT=ntgt] [NENGV=nengv]

[NWDUDM = nwdudm] [NMTRAC=nmtrac] [MAXSFF=maxsff]

$[\mathrm{MXNFPC}=\mathrm{mxnfpc}]$

[NDHSPC $=$ ndspc]

[NDHBIN = ndhbin]

[NDHGRP $=$ ndhgrp]

EOI

$* * * * * * * * * * * * * * * * * * * * * * * * * * * * * * * * * * * * * * * * * * * * * * * * * * * * * * * * * * * * * * * * * * * * * * * * * * * * * * *$

In specifying this block, the keyword CONTROL is given first. It should be followed by the keyword and value pairs for each number the user wishes to specify. The pairs can be given in any order. Only the NCELLS and NTZONE pairs are required. The block must be terminated by an EOI.

CONTROL

NCELLS

$=$ ncells

NTZONE

$=$ ntzone

NTTTL

$=$ ntitl

NFCE

$=$ nfce

NCHAIN

$=$ nchain the keyword for starting specification of the global storage allocation.

the number of cells. This number of cell input sections must be specified following the global input section.

the number of time zones specified in the TIMES block as described in Section 14.2.7.

the number of title lines specified in the TITLE block as described in Section 14.2.8.4. Each title line can contain a maximum of 80 characters. Default $=$ 0.

the number of fission product chain elements to be defined by the user. Fission products extracted from the fission product library should not be counted in "nfce" because space for the library is allocated automatically. Default $=0$.

the number of linear chains to be defined by the user. Linear chains extracted from the fission product data library are not counted in "nchain." Default = 0 . 
NSECTN

= nsectn

NAC

$=$ nac

NUMTBG

$=$ numtbg

MAXTBG

$=$ maxtbg

NTGT

$=\mathrm{ntgt}$

the number of aerosol particle sections or sizes (20 is typical; the maximum is given by the geometric constraint $v_{i+1} / v_{i}>2$, given in Equation (7-4)). Default $=0$.

the number of aerosol components specified in the AEROSOL block as described in Section 14.2.4. Maximum $=8$. Default $=0$.

the number of global tables used. This number should be incremented by one for each global table. Examples of such tables include AERTIM, VARAREA, and engineered vent table options (e.g., AREA-T). Such tables are considered to be at the global level since these options are processed at that level. Each such table specifies one dependent variable in terms of an independent variable as explained in Section 14.4.2. Default $=0$.

the maximum number of entries used in any one global table. Default $=0$.

a number that reserves space for the targeted release and acceptance model. In theory, the exact value of "ntgt" required can be determined by following the procedure described below; however, in practice it is preferable to simply estimate "ntgt" with a sufficiently large value. For many calculations, the default of 1000 will be adequate; however, for certain large calculations, particularly when many target lines or the fission product library are used, larger values may be required.

In order to calculate the exact value of "ntgt" the total number of FROM/TO host pairs in all TARGET input blocks must be determined. A host name and a fission product name or a volatility group name are identified in each such line. Each host name corresponds to a number of actual hosts, denoted here as "nl." If the host name is a generic structure type, such as "wall," then "nl" will be the total number of wall surfaces in the cell of interest; otherwise "nl" will be 1 . The fission product or volatility group corresponds to a number of linear chain elements, denoted here as "n2." For example, if the G-TARGET option is used then " 2 " will correspond to the number of chain elements belonging to the volatility group named in the target line. If the G-TARGET option is not used, but the fission product appears in two linear chains, then " 2 " for that fission product will be 2 . The exact value of "ntgt" needed will be the sum of the product "n1" $\times$ "n2" for every FROM/TO target line in the input deck. Clearly, determining this value can be a tedious process; therefore, it is recommended that "ntgt" simply be given as an estimated large value. If the value is not large enough, the code will generate diagnostic and abort. In this event, simply increase the value of "ntgt" and try again. Default $=1000$. 
NENGV

= nengv

NWDUDM

$=$ nwdudm

NMTRAC

$=$ nmtrac

MAXSFF

$=$ maxsff

MXNFPC

$=\operatorname{mxnfpc}$ the number of engineered vents in the problem specified in the ENGVENT block as described in Section 14.2.4.2. Default $=0$.

the number of array locations reserved for the user-defined material (USERDAT) tables. The space requested must be large enough to hold the pairs of temperature and property values defined in the USERDAT block for all user-defined materials. If a direct containment heating (DCH) run is being performed, this input should be specified to increase the amount of reserved space from the default value to accommodate the $\mathrm{DCH}$ material property tables. A value of 5000 is suitable for most $\mathrm{DCH}$ calculations. Default $=$ 1000.

the maximum number of thermodynamic materials whose mass is tracked in the mass and energy accounting scheme, in addition to the coolant. Note that the combined liquid and vapor mass of the coolant is always tracked in the accounting scheme. Also, all thermodynamic materials are always tracked with respect to their energy, although only the masses of the coolant and any materials designated by the user in the TRACKMAT input are explicitly tracked in the mass accounting. In the coding for the mass accounting, it was assumed that the user will be interested in tracking only a handful of the many possible CONTAIN thermodynamic materials at any one time. Consequently, the mass tracking arrays for each repository have the same structure, and since many repositories can be present in a problem, the storage and output requirements for the mass arrays could be quite large, if not restricted to a number considerably smaller than the total number of thermodynamic materials, including default materials, in a typical problem. Thus, it is suggested that "nmtrac" be kept as small as possible $(<6)$. It can be zero if no masses but that of the coolant are to be tracked. Default $=0$.

the maximum number of structures to which the film tracking model outflow from a single structure is distributed. Default $=5$.

the maximum number of flow path connections of any type to a given cell. Default $=10$.

The following keywords in the global control input block pertain to making a DCH calculation.

NDHSPC

$=$ ndhspc

NDHBIN

$=$ ndhbin the maximum number of species that can be present in each debris field in a $D C H$ calculation. This corresponds to the $\mathrm{N}_{\mathrm{DCH}}$ parameter used in Section 6 , and sets the number of DCH species that must be given in the USERDEF and USERDAT input blocks. This is required input for a DCH calculation. Default $=0$.

the number of debris fields per generation. By default only one debris field will be modeled. Default $=1$. 
NDHGRP

$=$ ndhgrp the number of debris generations in a DCH calculation. A new generation is created with "ndhbin" fields in it when the amount of debris added to the previous generation has exceeded the allowable mass threshold, "grplim." This threshold is specified in the global DHEAT block. The maximum number of fields in the calculation is the product of "ndhbin" and "ndhgrp." This product corresponds to the number of debris fields $\mathrm{N}_{\text {fields }}$ defined in Chapter 6. Default $=1$.

EOI

the keyword required for terminating the global CONTROL block.

Unless otherwise noted, there are no preset maximum values for the control variables. However, the amount of storage space needed increases rapidly as the values of control variables increase. There is a default total working storage limit specified in the code. If the set of control parameters requires more storage than allowed by that limit, a fatal input error will occur, and a message about how to update the code to allow more storage will be printed in the error file. Note that increasing the allowable storage will require that the code be recompiled.

An additional global control keyword, NHM, is sometimes seen in older decks. This option is obsolete but still supported by the code (see Appendix B). Another format for the CONTROL block is available. This format is also discussed in Appendix B. This alternative format may appear in input files developed for earlier versions of CONTAIN. While upwardly compatible, it is considered obsolete and cannot be used to specify the newer control input parameters.

\subsubsection{Material, Fission Product, and Aerosol Names}

The material names block specifies the materials to be used in the problem. Four types of materials may be specified under the keywords COMPOUND, USERDEF, FP-NAMES, and AERNAMES, respectively.

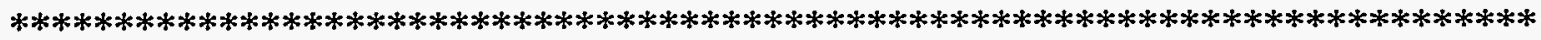

MATERIAL

COMPOUND (names)

[USERDEF (unames)]

[FP-NAMES (fname)]

[FPLIB

[EOI]

\{ALL or (nchlib)\}

[G-TARGET

EOT]

[(gname $=n f p($ fpname) $)]$

[AERNAMES (aernames)]

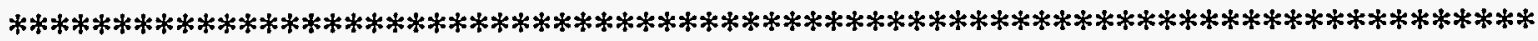

A library of material properties is provided in CONTAIN. A list of the materials included in this library is given in Table 3-1, Materials Available in CONTAIN. Any number of materials from this 
table may be specified after the COMPOUND keyword. The user may also specify tabular values for properties of materials specified after the USERDEF keyword, as discussed in Section 14.2.1.1 on the USERDAT option. The names of all materials (excluding aerosols and fission products) to be used in the calculation should be included in either the COMPOUND or USERDEF input, even though certain species are automatically added by the code in a manner that is completely transparent to the user. Fission product names used in subsequent fission product input blocks that are not invoked through the fission product library must be specified immediately after the FP-NAMES keyword. Aerosol names used in subsequent input blocks may be taken either from the materials specified after COMPOUND or from the names specified after the optional AERNAMES keyword. The COMPOUND keywords must immediately follow the MATERIAL keyword. The other keywords may be specified after COMPOUND in any order, but each should only appear once.

MATERIAL

COMPOUND

(names)

USERDEF

(unames)

FP-NAMES

(fname)

FPLIB the keyword used to initiate the material block. This keyword must be the first keyword after the global control block.

the keyword required to initiate input of material names from the library. This keyword must immediately follow the MATERIAL keyword.

the names of materials taken from Table 3-1 to be used in any of the input blocks in the input deck.

an optional keyword that initiates input of the user-defined material names.

the names of materials that will use the properties tables defined later in the USERDAT input. In most cases, the names included in this block are arbitrary, subject only to an 8-character limitation. In DCH calculations certain specific names are required for chemically reactive debris in the $\mathrm{DCH}$ debris fields. (See Section 14.2.1.2.) CONTAIN material names (see Table 3-1) may be used, in which case the internally defined properties must be replaced by user-specified properties. However, USERDEF names that are CONTAIN material names must also be specified after COMPOUND.

a keyword to initiate the input of fission product names.

the names of user-defined fission products. The "fpname" names given in the global FISSION block described in Section 14.2.6 must be included in this list. Like user-defined material names, fission product names are arbitrary. A given name may appear more than once in the linear chain decomposition of fission product decay if branching or merging decays are represented. The user should be careful not to duplicate names from the fission product library.

an optional keyword to access the fission product library. The entire library can be requested with the ALL keyword. Alternatively, specific decay processes can be requested by a list of process numbers "nchlib." 
ALL

(nchlib)

G-TARGET

gname

nfp

(fpname)

AERNAMES an optional keyword following FPLIB to request that the entire fission product data library be made available for the calculation. This choice, while convenient, will consume a lot of memory and will produce a large amount of output. To avoid these problems, the user should use the following option.

a list of coupled decay process numbers, in ascending order, from the library. The 40 coupled decay processes in the library are shown in Figure 8-2. Specifying a subset of process numbers rather than the keyword ALL after FP-NAMES is the recommended method of loading information from the library because it uses memory more efficiently and produces more concise output. CAUTION: The process numbers specified here are those for the coupled decay processes listed in Figure 8-2 and are not the linear decay chain numbers. All radionuclides involved in the specified processes and the associated decay information will be available to the calculation.

a keyword to specify that all targeted release and acceptance rate parameters will apply to the volatility groups discussed below rather than to individual fission products. The parameters themselves are specified in the TARGET sub-block in the FPM-CELL block for each cell. Use of the G-TARGET and TARGET options will in general require larger than normal "ntgt" values in the global CONTROL block, as explained under the NTGT keyword description.

All radionuclides defined in the fission product library have been assigned to the predefined volatility groups shown in Table 8-1. If the G-TARGET option is invoked, these predefined volatility groups must be used in the targeted release and acceptance model. The predefined volatility group assignment cannot be overridden by the user. The input following GTARGET is used to add user-defined fission products to the predefined volatility groups. Each of the user-defined fission products must be uniquely assigned to a predefined group if the G-TARGET option is invoked.

the name of the predefined volatility group to which the following userdefined fission products are assigned. The current valid names for "gname" are: GROUP1, GROUP2, ..., GROUP10.

the number of user-defined fission product names to follow "gname."

a list of "nfp" names of user-defined fission products to be assigned to the group "gname." Each of the "fpname" names must have been previously defined as "fname" in the FP-NAMES block.

an optional keyword that initiates input of user-defined aerosol component names. Aerosol names may also be taken from the names specified after the 
COMPOUND keyword. Such names need not be declared after AERNAMES.

(aernames)

the user-defined aerosol component names. User-defined aerosol names are also arbitrary; however, aerosol names taken from the COMPOUND list should not be duplicated. The "mapaer" names given in the global AEROSOL block described in Section 14.2.5 must be included in either this list or the compound list.

The user is cautioned that the MATERIAL block is terminated by any valid global keyword, not an EOI. As a consequence, user-defined material names, fission product names, and aerosol names should not match any of the global keywords allowed in CONTAIN input decks. This includes current and obsolete global keywords such as AEROSOL, FLOWS, TIMES, DHEAT, DUMMY, DEBUG, THERMAL, and TITLE.

14.2.1.1 User-Defined Material Definition. The user-specified properties of the materials specified in the USERDEF block of the MATERIAL input are defined in this section. Provision is made for specification of the material properties as a function of temperature. The properties that may be defined are the density (except for gases), conductivity, viscosity, specific enthalpy, and specific heat. The density may not be specified for gas phase user-defined materials because gas densities are internally evaluated using an ideal equation of state.

The property values may be entered as explicit pairs of temperature and property values. Alternatively, the temperatures at which all properties are evaluated may be given first, with the TEMPS keyword input. This is then followed by groups of property values, with the number of property values being the same as the number of temperatures specified in TEMPS, so that a one-to-one correspondence may be set up. The user may specify properties using a combination of these two methods. For example, the density and viscosity of a user-defined material may be specified with the RHO and VISC input blocks, which specify density and viscosity, respectively, along with the temperature; while the conductivity and enthalpy are specified with the TEMPS, CONDT, and ENTHT blocks. These blocks may be specified in any order, but the TEMPS block must precede any block that specifies only property values and not temperature, such as CONDT.

The user-defined material name may be a CONTAIN material name taken from the COMPOUND list in the MATERIAL block. In this case, those properties specified in the tables described in this section will override the internal properties for that material. Warning: This option can have severe effects on the accuracy and reliability of results, particularly when the coolant vapor is respecified.

Regardless of whether the user-defined material name is a CONTAIN material name or not, all properties must be defined for that material with the exception of (1) the specific heat, which will by default be defined by differentiating the enthalpy table values, (2) the density for a gas material, which is not allowed, and (3) the viscosity for a solid or debris material type.

The keyword to begin this property specification section is USERDAT and the format of the input is described as follows. 


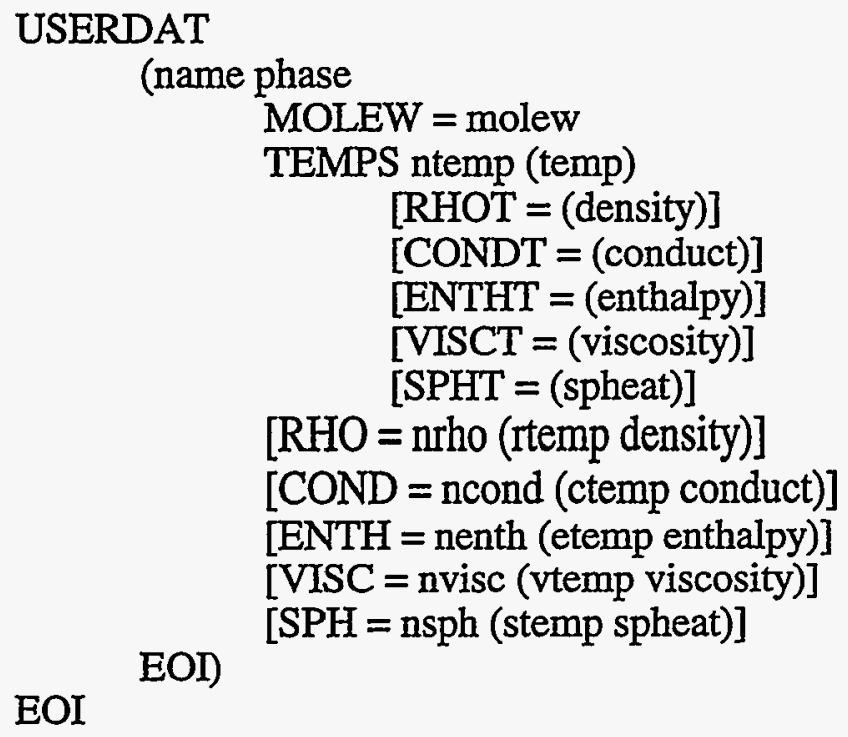

$* * * * * * * * * * * * * * * * * * * * * * * * * * * * * * * * * * * * * * * * * * * * * * * * * * * * * * * * * * * * * * * * * * * * * * * * * * * * * * * * * *$

USERDAT the keyword that initiates user definition of materials.

name

a required user-defined material name. Names given must also be specified after USERDEF in the MATERIAL block.

phase

the required phase or type of material. This must be one of the following words: GAS, LIQUID, SOLID, or DEBRIS. The DEBRIS phase is used for user-defined materials that are to be included in the DCH debris fields (see Section 14.2.1.2).

MOLEW

the required keyword for specifying the molecular weight of the material.

molew

the molecular weight $(\mathrm{kg} / \mathrm{kgmole})$.

TEMPS

the keyword for specifying a number of temperatures for the alternative format. This must precede any of the RHOT, CONDT, ENTHT, VISCT, or SPHT options.

ntemp

the number of temperature values that follow.

(temp)

temperatures paired with the values following the keywords. RHOT, ENTHT, CONDT, SPHT, and VISCT, which represent the density, enthalpy, conductivity, specific heat, and viscosity, respectively. Thus, "ntemp" property values should follow each of these keywords and "ntemp" temperature values should be given. 

RHOT
$=$ (density)
density values, paired with the "temp" values given after the TEMPS
CONDT
$=($ conduct $)$ keyword.
ENTHT
$=$ (enthalpy)
conductivity values, paired with the "temp" values given after the TEMPS keyword.
VISCT
$=($ viscosity $)$
specific enthalpy values, paired with the "temp" values given after the TEMPS keyword.
SPHT
$=($ spheat $)$
viscosity values, paired with the "temp" values given after the TEMPS keyword.
specific heat values, paired with the "temp" values given after the TEMPS keyword.

The following five keywords are used for specifying pairs of temperature-property values.

RHO the keyword for specifying density input. The density should not be specified for a gas but is required if the material is not a gas.

nrho the number of temperature-density pairs that follow.

rtemp the temperature values for which the densities are provided. $(\mathrm{K})$

density the density values. $\left(\mathrm{kg} / \mathrm{m}^{3}\right)$

COND

the keyword for specifying thermal conductivity input. The thermal conductivity is required.

ncond

the number of temperature-conductivity pairs that follow.

ctemp

the temperature values for which the conductivities are provided.

conduct

the conductivity values. (W/m-K)

ENTH

the keyword for specifying specific enthalpy input. The enthalpy is required.

nenth

the number of temperature-enthalpy pairs that follow.

etemp the temperature values for which the specific enthalpies are provided.

enthalpy the specific enthalpy values. $(\mathrm{J} / \mathrm{kg})$

VISC

the keyword for specifying viscosity input. The viscosity is required if the material phase is GAS or LIQUID. 

nvisc
the number of temperature-viscosity pairs that follow.
vtemp
the temperature values for which the viscosities are provided.
viscosity
the viscosity values. $(\mathrm{kg} / \mathrm{m}-\mathrm{s})$
SPH
the keyword for specifying specific heat input. The specific heat is by default the derivative of the enthalpy table values.
nsph
the number of temperature-specific heat pairs that follow.
stemp
the temperature values for which the specific heats are provided.
spheat
the specific heat values. $(\mathrm{J} / \mathrm{kg}-\mathrm{K})$

14.2.1.2 DCH User-Defined Material Input. DCH calculations require the user to specify the names and properties of the debris species that can be present. This is done using the user-defined material input, as described in Section 14.2.1.1. The one very important difference between DCH userdefined materials and other user-defined materials is that the phase type for DCH material is DEBRIS; i.e., the word DEBRIS is specified after the material name in the USERDAT input block. Within the code the phase number for debris will be 4 , which is distinct from the phase numbers for gas (1), liquids (2), and solids (3). The DEBRIS phase type is extremely important, since this is the only way the code knows that the DCH species given in the USERDEF list are to be treated as species in the debris field. Exactly "ndhspc" of the specified materials in the USERDEF block must be DCH materials, where "ndhspc" is given in the global control block.

The DCH model recognizes the following specific names of materials as reactive metals: $\mathrm{ZRD}$, ALD, CRD, and FED. These names and only these names may be used as reactive zirconium, aluminum, chromium, and iron, respectively. The following oxide names must also be specified if the corresponding metal is given: ZRO2D, ALOXD, CROXD, and FEOD. Again, these names and only these names will be recognized as the oxide products of the four reactive metals. Any number of additional material species with any name may be specified in addition to the special four species above. Any such species will be treated as chemically inert and will be assumed to reside in the oxide phase, even if the name suggests that it is a metal.

At least one of the DCH user-defined metals must be named in order to model chemical reactions of debris in the atmosphere. Also, if a metal is specified then its oxide must also be specified in the USERDEF block. For example, if FED is specified, then FEOD must also be specified since FED (iron) is assumed to oxidize to FEOD (iron oxide). If either of these rules is violated, an input diagnostic will be given and the calculation will not proceed. An oxide can be specified, however, without specifying its metal counterpart.

A few things are important to note about the material property data tables for $\mathrm{DCH}$. First, the oxides ALOXD and CROXD correspond to $\mathrm{AlO}_{1.5}$ and $\mathrm{CrO}_{1.5}$ respectively. These names must be used if $\mathrm{CRD}$ and ALD reactive metals are also present. Second, the molecular weight of these oxides must 
correspond to the species as they are written; for example, the molecular weight of ALOXD must be $\mathrm{M}_{\mathrm{A} 1}+1.5 \mathrm{M}_{\mathrm{O}}$. If the debris initially includes oxides of some of the reactive metals (e.g., aluminum oxide in an iron-aluminum thermite experiment), the user may assign this the standard oxide name (e.g., ALOXD). Alternatively, if it is desirable to trace the oxide produced by chemical reaction separately from that initially present, an inert material name (e.g., AL2O3D) may be assigned to the latter with identical material properties. Other results of the calculation (temperature, pressures, etc.) will not be affected by this change.

Any number of additional species may be specified as direct heating materials with phase type DEBRIS in addition to the special ones discussed above. These materials will be inert and will therefore not chemically react. However, as long as the phase type of the specified material is DEBRIS, any sources of this material to the containment will be added to the debris fields as discussed in Section 14.3.1.11. The names of user-defined DCH materials must be different than the names of materials in the CONTAIN material library. It is suggested that materials be named ending with a "d" to ensure that the $D C H$ names are distinct from the names of the materials in the CONTAIN library, listed in Table 3-1.

The CONTAIN code project has developed a standard set of material property tables for the following DCH materials: ZRD, ALD, CRD, FED, ZRO2D, ALOXD, CROXD, and UO2D. These tables, which include properties for thermal conductivity, specific enthalpy, density, and specific heat at resolutions adequate for most DCH analyses, are included in the Sequoyah sample problem discussed in Section 15.3.

The enthalpy function data includes the heat of fusion associated with debris phase transitions. The specific heat tables are the derivative of the corresponding enthalpy table, but they do not include a representation of a delta function at the phase changes. Debris specific heats are only used in the calculation of the characteristic time constant output variables, while the calculations rely solely upon the enthalpy functions. The delta function was omitted from the specific heat tables because it was discovered that including approximations to the delta function gave unrealistic values for the calculated time constants.

If the user wishes to make his or her own DCH property tables, care should be taken to include the heat of fusion in the enthalpy tables. To avoid numerical difficulties, the heat of fusion should be spread over approximately $50 \mathrm{~K}$. Also, the resolution of the tables in temperature should be approximately every $50 \mathrm{~K}$.

\subsubsection{Mass and Energy Accounting}

The mass and energy accounting scheme by default tracks the total energy and the mass of coolant. The user may wish to track the mass of other materials. The non-coolant materials to be tracked in the mass and energy accounting scheme with respect to their mass are specified through the optional "nmtrac" control parameter and the optional TRACKMAT input block. This input block can be specified anywhere in the input at the global level. Note that the energy of all such materials is always tracked in the total energy. As with other mass and energy accounting information, the mass accounting for the materials specified is obtained in the long edits by specifying the PRENACCT 
keyword. Note that the number of materials specified must be indicated through the NMTRAC keyword in the global CONTROL block.

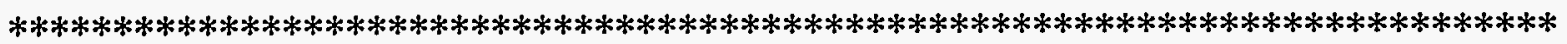

TRACKMAT

omat1 omat 2 omat 3 ... omatn

EOI

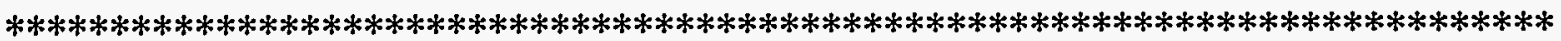

TRACKMAT keyword for specification of the materials that are to be tracked in the mass and energy accounting scheme with respect to their mass, in addition to the coolant. The combined liquid and vapor phase mass of the coolant as well as the energy of all materials with thermodynamic properties are always tracked.

omat1, omat2, names of thermodynamic materials whose mass is to be tracked in the mass ..., omatn and energy accounting scheme, excluding the coolant. At most "nmtrac" standard CONTAIN or user-defined materials may be specified. Neither the liquid or vapor phase of the coolant should be specified.

EOI input block terminator

\subsubsection{Fixed Atmosphere Conditions}

A new keyword has been added to allow the user to inhibit changes in the upper cell conditions such as atmosphere temperature. In previous versions, this inhibition is assumed if the obsolete THERMO or FLOWS keyword is not specified. However, this caused problems because these keywords could be left out accidentally. In addition, the precise behavior that should result from the omission of such keywords is ambiguous, since the atmosphere volume, among other quantities, could still change as a result of pool displacement. With the present updates, atmosphere thermodynamic calculations of the effects of other models are, by default, always taken into account. The STATIC keyword should be used in those relatively rare cases in which the time-evolution of the atmosphere state, and certain other conditions, are to be suppressed.

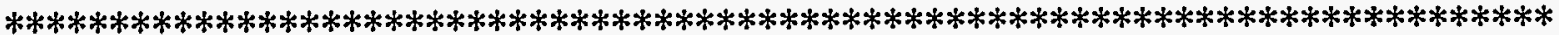

STATIC

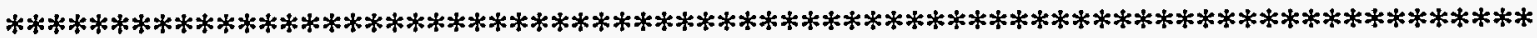

STATIC the keyword to suppress time-evolution of the atmosphere gas thermodynamic states, including changes in temperature, mass, composition, volume, and elevation. Structure submergence depths in the pools will also 
be considered fixed. Fields coupled to the atmosphere gas will still evolve, such as aerosols, core debris fields, and pools. However, of these latter fields, external sources of debris will be ignored. In general, mass and energy will not be conserved. In the case of a pool, a change in pool volume will mean that the total cell volume also will not be conserved. This keyword will be overridden if the FLOWS keyword is specified. By default, atmosphere thermodynamic state changes are taken into account and effects of source tables and heat and mass transfer processes involving the gas are incorporated.

\subsubsection{Intercell Flows}

The flow options specify intercell flow path characteristics, including those for the special BWR suppression pool vent flow path. The input is given in terms of three major input blocks: the FLOWS, ENGVENT, and SPVENT blocks. The FLOWS block describes the modeling options to be used and the solution method (implicit or explicit) to be used for the flows. The ENGVENT block describes the modeling options to be used with each of the flow paths or engineered vents. Each such path may be designated as carrying either gas or pool liquid. (The obsolete flow paths called "regular" flow paths are allowed to carry only gas.) The SPVENT block describes the liquid and gas flow characteristics and aerosol scrubbing parameters for use in the BWR suppression pool vent model.

Aerosols, fission products in the atmosphere, and other suspended materials will flow without slip with the gases through the flow paths, with three exceptions: (1) if FPCOSN or VCOSN is specified, aerosols will be allowed to settle through the flow path or vent, respectively; (2) if the flow is scrubbed in the suppression pool vent model, the scrubbed aerosols will be transferred to the pool; and (3) if flow from a gas flow path is vented under the pool surface, the aerosols will be completely removed from the flow, and placed in the pool. Fission products hosted by the aerosols will follow the aerosols in these three cases.

14.2.4.1 Flow Options. The FLOWS block determines the method to be used to integrate the mass and energy equations for the cell atmospheres. In code versions prior to CONTAIN 1.20 , it is also used to specify the characteristics of the regular flow paths. The input descriptions for such paths are discussed in more detail in Appendix B.

The flow model integration method will be the Runge-Kutta method, unless the user specifies IMPLICIT and thus selects the implicit integration method, which is the generally recommended one. A number of code features, including the ENGVENT and SPVENT options, are available only with the implicit method. While the flow model used in general with the implicit method is the inertial model, a number of special flow options use a quasi-steady flow model. (Such usage is indicated in the discussion of those options.) 


\section{FLOWS}

[MSTABLE]

[ $\{$ IMPLICIT $[=$ nimpli $]$ or

IMPLICIT

[ATMOS=nat (icell)]

EOI\}]

[POOLS=npl (jcell)]

[DROPOUT] [PERROR=perror] [PIVOTMIN=pvtmin]

[FIX-FLOW=(rtol)]

[HIPRWATR]

$* * * * * * * * * * * * * * * * * * * * * * * * * * * * * * * * * * * * * * * * * * * * * * * * * * * * * * * * * * * * * * * * * * * * * * * * * * * * * * * * * *$

FLOWS

MSTABLE

IMPLICIT

nimpli

ATMOS the keyword for initiating the specification of regular flow paths and flow solver options.

the keyword to disable the VDI formulation of the gravitational head normally used when IMPLICIT is specified, as discussed in Section 4.4.5.1. Unless RESOLVHD is also specified, in the case of an engineered vent, this keyword will cause the solver to use the asymptotically correct expression in all regimes. This asymptotically correct formulation, which uses average densities between gas centers of elevation, neglects the stability of stratifications compared to the VDI formulation in some cases and will consequently tend to overmix stable stratifications.

the keyword for invoking the implicit flow solver. Two different formats are available to specify the cell atmospheres and pools to be treated implicitly. In practice, only environment cell atmospheres are tractable when solved explicitly. Pools not coupled through flow to the rest of the problem or coupled only through explicit flow models may be solved explicitly. However, those coupled through engineered vents of type POOL must be implicit. If only IMPLICIT is specified, without further qualification, all atmospheres and pools will be treated implicitly.

the number of cells to be solved implicitly with respect to the atmospheres and pools, if present. The first "nimpli" cells according to cell number will be treated implicitly. The remaining "ncells"-"nimpli" will be treated explicitly. If "nimpli" or the ATMOS or POOLS keywords below are not given but IMPLICIT is, all cells will be solved implicitly.

a keyword to specify the atmospheres to be solved implicitly. If ATMOS or "nimpli" is not specified, but IMPLICIT is, all atmospheres will be solved implicitly. 
nat

(icell)

POOLS

npl

(jcell)

DROPOUT

PERROR

perror

PIVOTMMN

pvtmin

FIX-FLOW

(rtol) the number of cell atmospheres to be solved implicitly.

a list of "nat" cell numbers, corresponding to the cells with atmospheres to be solved implicitly.

a keyword to specify the pools to be solved implicitly. If POOLS or "nimpli" is not specified, but IMPLICIT is, all pools will be solved implicitly.

the number of pools to be solved implicitly. This may be zero.

a list of "npl" cell numbers, corresponding to the cells with pools to be solved implicitly, if "npl" is non-zero.

the keyword for removing all suspended liquid coolant from the atmosphere and depositing it in the appropriate pool, as determined by the OVERFLOW option discussed in Section 14.3.1.12.

the keyword for defining the degree to which the inventory of a cell, as measured by the pressure, is iterated to self-consistency. The error in the pressure difference between cells gives rise to an error in the flow rate. The cumulative error in the flow over the timestep leads to an inventory error. This is what is limited by "perror." (For very large flow areas, the driving pressure differences between cells have errors much less than "perror.")

the value of the self-consistency error. Default $=1 .(\mathrm{Pa})$

the keyword for defining the minimum acceptable size of a divisor in the implicit solver analogous to the pivot element in Gaussian elimination. A smaller divisor is assumed to generate an effectively singular inverse matrix and is not used.

the minimum size of the pivot element. Increasing the value somewhat should increase robustness, at the expense of efficiency. The decrease in efficiency should be acceptable for a factor of two to four increase in value. Default $=0.01$. (dimensionless).

the keyword for activating the FIX-FLOW option for implicit cell atmospheres.

the rate tolerances for the FIX-FLOW option, corresponding to $\varsigma_{i}$ in Equation (4-28) in Section 4.4.9. This tolerance will be applied to atmosphere temperature, mass, and gas volume relative rates of change to determine whether the cell atmosphere may be replaced by a fixed flow boundary condition in the implicit solver. Specify a string of tolerances, one for each cell in the problem, in order of cell number. A zero value for a tolerance 
HIPRWATR

means that the corresponding cell atmosphere is excluded from consideration in the FIX-FLOW option. Such a zero value is required for all cell atmospheres that are not solved implicitly. Default $=0$. $\left(\mathrm{s}^{-1}\right)$

keyword to specify an equation of state (EOS) for water that is accurate at high vapor pressures $\left(>5 \times 10^{5} \mathrm{~Pa}\right)$. This keyword must be used in conjunction with the IMPLICIT keyword. There are limitations regarding the implementation of the non-ideal EOS in models other than the atmosphere thermodynamic state model, as discussed in Section 3.2.2.3.

14.2.4.2 Engineered Vents. The engineered vent is an intercell gas or pool liquid flow path. The number of vents that can be specified between any two cells is not restricted. As discussed in Section 4.4.7, gas vented from a gas path under the pool surface in a cell results in equilibration of the gas with the pool over a length given by VEQLENF or VEQLENB.

The ENGVENT engineered vent input block is separate from the FLOWS input block. One must, however, select the IMPLICIT solver option in the FLOWS input block whenever engineered vents are to be specified. The keyword NENGV in the global CONTROL block must also be used to specify the total number of engineered vents in the system.

$* * * * * * * * * * * * * * * * * * * * * * * * * * * * * * * * * * * * * * * * * * * * * * * * * * * * * * * * * * * * * * * * * * * * * * * * * * *$

\section{ENGVENT}

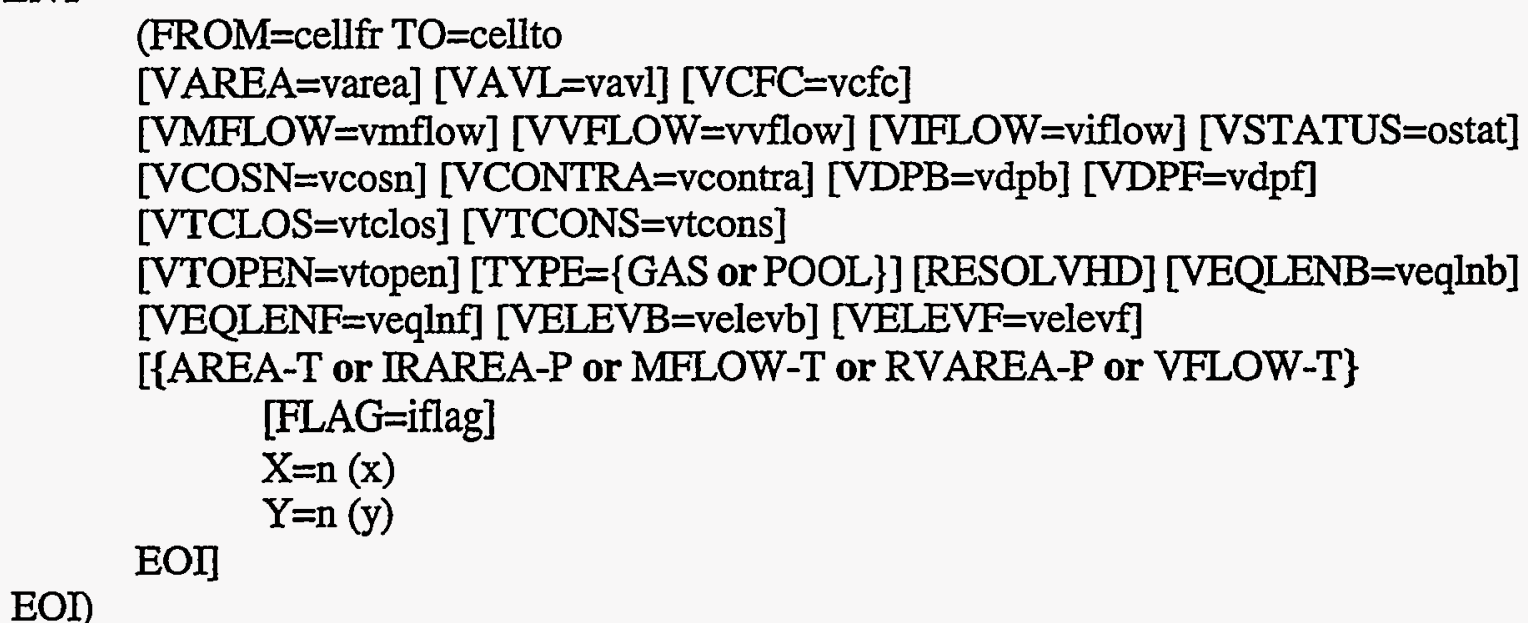

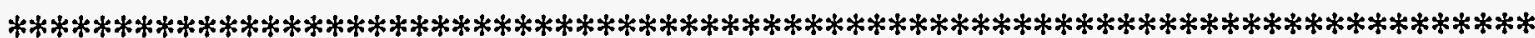

If the engineered vent modeling is to be used, the following keywords are always required:

ENGVENT keyword for specifying the engineered vent input block.

FROM the keyword for specifying the nominal donor cell. 
cellfr

the number of the cell from which flow is occurring when the flow is considered positive. (This arbitrary sign convention is also used in the code output.)

TO

the keyword for specifying the nominal acceptor cell.

cellto

the number of the cell to which flow is directed when the flow is considered positive.

The keywords from the next group should be selected as necessary to define the flow for a given vent. Note that they may be used in conjunction with a number of table options (AREA-T, IRAREA-P, MFLOW-T, RVAREA-P, and VFLOW-T) defined below. The inertial flow model is used unless otherwise specified. For this model, VAVL, VCFC, and an option to define the flow area (VAREA, AREA-T, or IRAREA-P) are required. The quasi-steady flow model is invoked when the reversible-area-versus-pressure option RVAREA-P is specified. For this model only, VCFC is required in addition to the RVAREA-P option. When any of the user- specified flow rate options are specified (VVFLOW, VMFLOW, VFLOW-T, or MFLOW-T), no other keywords from the following set are required, although VAREA should be specified to determine the gas velocity and settling rate of aerosols through the vent if VCOSN is specified.

VAREA

$=$ varea

VAVL

= vavl

VCFC

$=\mathrm{vcfc}$

VMFLOW

$=$ vmflow

VVFLOW

= vvflow

VIFLOW

= viflow the (constant) cross-sectional area of the vent when open. This should be specified unless a table option for the area is used (see below). In the user-specified flow rate options, VAREA may still be specified. Although it is not required for the gas flow calculation with those options, it may be used for calculating gas velocities and aerosol settling through the vent. $\left(\mathrm{m}^{2}\right)$

the vent area versus length used to calculate the inertial mass in the flow path. This corresponds to $A_{i j} / L_{i j}$ in Table 4-2. This input is required except in the case of the reversible pressure-dependent-area table option (RVAREA-P), which assumes quasi-steady flow, or a user-specified flow rate option. (m)

the vent turbulent flow coefficient corresponding to $\mathrm{C}_{\mathrm{FC}}$ in Table 4-2. This is required unless a user-specified flow rate option is used. (dimensionless)

the constant mass flow rate for the vent when open. A time-dependent rate can be specified through the MFLOW-T table option discussed below. $(\mathrm{kg} / \mathrm{sec})$

the constant volumetric flow rate for the vent when open. A time-dependent rate can be specified through the VFLOW-T table option discussed below. $\left(\mathrm{m}^{3} / \mathrm{sec}\right)$

the flow rate at problem start. This should be specified only if the vent is initially open and the flow model is inertial. Default $=0 .(\mathrm{kg} / \mathrm{sec})$ 
The following keywords are optional and may be specified independently of any other keyword.

VSTATUS a keyword for specifying the initial state of a flow path. This keyword may not be used on a restart.

ostat

a variable specifying the initial state of the vent. If "ostat" = OPEN, the vent will be initially open; if "ostat" = CLOSED, it will be initially closed. By default, the vent will be initially closed if VTOPEN is specified without VDPF, VDPB, or TCLOSE or if VDPF and VDPB are specified without VTOPEN or VTCLOS. Otherwise the vent is set initially open. Note that if VDPF and VDPB are specified in addition to one of the keywords VTOPEN or VTCLOS, or if VTOPEN or VTCLOS is specified in addition to VDPF and VDPB, or if VTOPEN and VTCLOS are both specified, the initial state of the flow path may not be the same as in previous revisions. A diagnostic is written to the error file in these cases.

VCOSN

$=\mathrm{v} \cos n$

the cosine of the angle between the vent axis and the vertical direction. The value is used to calculate aerosol settling through the vent from one cell to another. The angle is measured between the upward direction and the vent axis in the direction from "cellfm" to "cellto." The value should be 1 if the vent goes straight up in the direction from "cellfm" to "cellto" and - 1 if it goes straight down. Only the component of the aerosol settling velocities parallel to the vent axis is considered. Default $=0$.

VCONTRA the reduction factor for the flow area due to the vena contracta. Used only $=$ vcontra

VDPB

$=\mathrm{vdpb}$ when the flow is choked. Default $=1$. (dimensionless)

the positive-definite pressure difference to open the vent in the backward (negative flow) direction. This option sets the vent initially closed. If VDPB is specified, then VDPF, discussed below, must also be specified. VDPF and VDPB may be used with VTOPEN and VTCLOS as long as the times specified imply the vent is initially closed. $(\mathrm{Pa})$

VDPF the positive-definite pressure difference to open the vent in the forward $=\mathrm{vdpf}$

VTCLOS

$=$ vtclos (positive flow) direction. See the comments regarding usage in the discussion of VDPB above. (Pa)

the time at which the vent should close or begin to close. Default $=10^{30}$. (sec)

VTCONS the time period over which the vent should open or close after a VTOPEN, $=$ vtcons VTCLOS, VDPF, or VDPB is satisfied. The area variation on opening or closing is linear in time. Because of conflicts with the table values, this option should not be used with a table option. Default $=0$. (s) 
VTOPEN

$=$ vtopen

TYPE

$=\{$ GAS or POOL\}

RESOLVHD

VEQLENB

$=$ veqlnb

VEQLENF

$=$ veqlnf

VELEVB

$=$ velevb the time at which the vent should open or begin to open. The vent by default is initially open. Specification of this option will result in the vent being initially closed unless "vtclos" is also specified and "vtclos" < "vtopen." Default $=-10^{30}$. (s)

keyword to specify whether the path is to carry gas and its associated constituents or liquid coolant and its constituents from the pool. A given path may carry only one of these groups.

the keyword to resolve the gravitational head of an engineered vent of type GAS into three segments: the elevation change between the gas center of elevation in one cell and the elevation at which the vent is attached to the cell, the elevation change between the ends of the flow path, and the elevation change within the second cell. As discussed in Section 4.4.5.1, if RESOLVHD is specified, the appropriate cell density is always used for first and third segments. Normally, the gas gravitational head is calculated on the basis of the net elevation change between cell gas centers of elevation and either a VDI or average density. The latter is used if MSTABLE is specified in the FLOWS input block. As discussed in Section 4.4.5.1, use of RESOLVHD is not recommended unless the user has demonstrated that the resulting gravitational head is acceptable. The user invoking RESOLVHD should be aware that the default flow path elevations have changed from prior versions--gas paths are by default connected to the top of the cell to avoid flooding by the pool.

the characteristic height over which the gas exiting from a submerged back (FROM) end of a vent is equilibrated with the downstream pool as it rises. This height, designated as $\lambda_{\mathrm{ji}}$ in the discussion following Equation (4-16), is assumed to control the equilibration of both the coolant vapor in the bubbles and the gas temperatures, as discussed in that section. In contrast to the treatment in the dedicated suppression vent model, for regular flow paths and engineered vents, aerosols and fission products in the flow are removed completely from the flow and placed in the pool. As in the dedicated suppression vent model, core debris (in debris droplet fields) is completely removed and placed in the intermediate layer, if present, in the lower cell. Default $=0.01$. $(\mathrm{m})$

the characteristic height over which the gas exiting from a submerged front (TO) end of a vent, in the case of reverse flow, is equilibrated with the downstream pool as it rises. This height is defined in a manner similar to "veqlnb." (m)

the absolute elevation of the back (FROM) end of an engineered vent. The default value of "velevb" is the top of the cell to which the end is attached $\left(\mathrm{H}_{\mathrm{ti}}\right.$ as shown in Figure 4-4) for a gas path and the bottom of the cell $\left(\mathrm{H}_{\mathrm{bi}}\right)$ for 
VELEVF $=$ velevf a pool path. The maximum elevation allowed for a pool path end is the elevation of the top of the cell, and the minimum elevation allowed for a gas path end is the elevation of the bottom of the cell. The user should consider the pool-gas hierarchy discussed in Section 4.2 and the gravitational head formulation discussed in Section 4.4.5 prior to specifying this elevation and in particular should note that a flow path connected to the top of a cell is treated differently from side-connected or bottom-connected paths. In general, the robustness of the code will be improved if the gas head space associated with the onset of gas or pool flow in a side-connected path is fairly substantial (on the order of $20 \%$ of the cell volume). Side-connected paths attached very close to the top of the cell are not recommended. To avoid accidental specification of such a side connection when a top connection is intended, it is recommended that the user rely on the CELCHIST input in the cell GEOMETRY block to specify cell elevations directly, rather than rely on values that would otherwise be calculated by the code from the initial cell gas center-of-volume elevation, the cell gas "height," and the initial gas and pool volumes. (m)

the absolute elevation of the front (TO) end of an engineered vent. The default value of "velevf" is the top of the cell to which the end is attached $\left(\mathrm{H}_{\mathrm{Li}}\right.$ as shown in Figure 4-4) for a gas path and the bottom of the cell $\left(\mathrm{H}_{\mathrm{b}, \mathrm{i}}\right)$ for a pool path. The considerations involved in choosing "velevf" are similar to those for "velevb," which is discussed above. (m)

The user may introduce a number of different global table options in which the area or user-specified flow rate is specified as a function of time or pressure difference. The type of table is specified through a keyword picked from the list below and then followed by standard table keywords FLAG, $\mathrm{X}$, and $\mathrm{Y}$ as indicated in the ENGVENT input template. (These keywords are also discussed in Section 14.4.2.) If any tables described below are used in the input, the user should take them into account in setting the number of global tables "numtbg" and maximum global table size "maxtbg" in the global CONTROL block.

AREA-T

IRAREA-P

MFLOW-T the keyword for initiating the specification of a table for area versus time.

the keyword for initiating the specification of a table for area versus pressure difference. The area is treated as irreversible in this option. The table value will be used only if it is larger than the existing area. Note that the area is initialized at the value specified through the VAREA keyword, if specified; otherwise, it is initialized to zero.

the keyword for initiating the specification of a table for mass flow versus time. Note that the energy transfer associated with the flow is calculated the same way as that for pressure-driven flow. Therefore, violations of the second law of thermodynamics will occur if the flow opposes the pressure drop across the flow path. 
RVAREA-P the keyword for initiating the specification of a table for area versus pressure difference. The area is considered reversible in this option. Unlike the reversible area option for regular flow paths, the vent area is implicitly calculated as a function of pressure. The flow is calculated according to the quasi-steady flow expression, Equation (4-3), with $\mathbf{i}=$ "cellfr" and $\mathbf{j}=$ "cellto."

VFLOW-T the keyword for initiating the specification of a table for volumetric flow versus time. Note that the energy transfer associated with the flow is calculated the same way as that for pressure-driven flow. Therefore, violations of the second law of thermodynamics, will occur if the flow opposes the pressure drop across the flow path.

FLAG

iflag

$\mathrm{X}$

$\mathrm{n}$

$\mathrm{x}$

$\mathrm{Y}$

y

EOI the keyword for introducing the interpolation flag for the table.

the interpolation flag for the table. A value of 1 denotes a step-function table, whereas a value of 2 denotes a linearly interpolated table. Note that in the RVAREA-P option, a linearly interpolated table must be used.

keyword to introduce the independent variable of the table.

the number of points in the table.

the independent variable in the table. In the AREA-T, MFLOW-T, and VFLOW-T tables, the independent variable represents time (s). In the IRAREA-P and RVAREA-P tables, the independent variable represents the pressure difference $\triangle P_{i j}$ given in the conservation of momentum equation in Table 4-2 where $i=$ "cellfr" and $j=$ "cellto." Specify " $n$ " monotonically increasing values. Note that outside of the range of the independent variable of the table, the table is extrapolated. A constant value equal to the closest endpoint value is used in the extrapolation.

the keyword for introducing the dependent variable of the table.

the dependent variable of the table. In the AREA-T, IRAREA-P, and RVAREA-T tables, the dependent variable represents the flow area $\left(\mathrm{m}^{2}\right)$. In the MFLOW-T table, the dependent variable represents a mass flow rate $(\mathrm{kg} / \mathrm{s})$. In the VFLOW-T table, the dependent variable represents a volumetric flow rate $\left(\mathrm{m}^{3} / \mathrm{s}\right)$. Specify " $\mathrm{n}$ " values.

the keyword for terminating the global table options as specified and also the keyword for terminating the parameters of a given vent.

A table should not be specified if VTCONS, VMFLOW, or VVFLOW keywords are specified; these keywords represent modeling options that conflict with those of the tables. For example, VTCONS 
gives a time constant for the opening of the vent to the value given by VAREA. This method of specifying the vent characteristics obviously could conflict with the vent characteristics given by the tables, and thus the combination is not allowed. Similarly, only one table may be specified for a given vent, since each represents an independent modeling option. A table may be used in conjunction with any of the keywords which change the vent state, such as VTOPEN, VTCLOS, and VDPF. VAREA may also be used with any table that does not represent the area, but it is used in that case only to define the aerosol settling rate through the vent. Note that the table values are accessed only when the vent is open. When the vent is closed, the flow and the flow area (if specified) are zeroed out.

14.2.4.3 Suppression Pool Vent Flow Path. The suppression pool vent flow path model for a boiling water reactor is activated through the SPVENT keyword. Only one SPVENT block is allowed, and only two cells may be connected by the suppression pool vents.

$* * * * * * * * * * * * * * * * * * * * * * * * * * * * * * * * * * * * * * * * * * * * * * * * * * * * * * * * * * * * * * * * * * * * * * * * *$

\section{SPVENT}

NDRY=ndry NWET $=$ nwet

[NSVNTS=nsvnts] [AVNT=avnt] [VNTLEN=vntlen] [ELEVNT=elevnt] [DPDRY=dpdry] [DPWET=dpwet] [FDW=fdw] [FWD=fwd] [GNLEN=ginlen] [ SSCRUB

[BSIZI=bsizi] [VROVR=vrovr]

EOI or

SPARC

EOI\}]

[BSIZI=bsizi] [RATIO=ratio] [NRISE=nrise]

EOI

$* * * * * * * * * * * * * * * * * * * * * * * * * * * * * * * * * * * * * * * * * * * * * * * * * * * * * * * * * * * * * * * * * * * * * * * * * * * * * * * * *$

The following keywords specify geometrical and physical parameters of the suppression pool vent flow path system. Note that in addition to the input described here, a lower cell pool must be defined in the wetwell cell (see Section 14.3.2.5). The lower cell area must be set to the sum of the wetwell area and vent area. The pool height relative to this area may be different from the height relative to the cell cross-sections specified in the cell GEOMETRY block. Figure 11-4 illustrates the significance of the geometric parameters.

The following keywords are required:

SPVENT the keyword for beginning the specification of the suppression pool vent path.

NDRY the number of the cell on the drywell side of the suppression pool vents. $=$ ndry 
NWET

the number of the cell containing the wetwell pool.

$=$ nwet

The following keywords are optional. (The default values except for that for "elevnt" correspond to a Mark I configuration.)

NSVNTS

=nsvits

AVNT

$=$ avnt

VNTLEN

$=$ vntlen

ELEVNT

$=$ elevnt

DPDRY

$=$ dpdry

DPWET

= dpwet

FDW

$=\mathrm{fdw}$

FWD

$=$ fwd

GINLEN

$=$ ginlen the number of vent pipes of cross-sectional flow area "avnt"; used for computing the total flow area. Default $=8$.

the horizontal cross-sectional characteristic flow area for a single vent. For a Mark III, this should be the annulus flow area and "nsvnts" should be set to one. The total vent area is "nsvnts" $\times$ "avnt." Default $=6.71 .\left(\mathrm{m}^{2}\right)$

for a Mark I or II, the vertical extent of the vent pipe. For a Mark III, this is the characteristic distance from the vent to the top of the weir wall. Default $=5$. $(\mathrm{m})$

the pool height at which the vent first opens. Default $=0 .(\mathrm{m})$

the range for the pressure difference between the drywell and wetwell over which the effective vent gas flow area goes from zero to its maximum value. This range applies when the flow is from the drywell to the wetwell. The gas flow area is taken to be zero at the pressure difference required to support the liquid head present when the vents just begin to clear. The total gas flow area changes linearly from zero to "nsvnts" $x$ "avnt" over the range "dpdry." The flow solver may have difficulty converging if this range is too small. In most cases, if this range is much less than the cell pressures, the calculated results will not be sensitive to the value used. In such cases it may be adjusted to give better computational efficiency. Default $=10^{4} .(\mathrm{Pa})$

the range for the pressure difference analogous to "dpdry," but referring to flow from the wetwell to the drywell. The total gas flow area changes from zero to "nsvnts"**"avnt" over "dpwet." Default $=10^{4}$. (Pa)

the overall liquid flow loss coefficient for flow from the drywell to the wetwell, including contraction, turning, and orifice losses but not expansion losses. (See Equation (11-4).) Default $=1$. (dimensionless)

the overall liquid flow loss coefficient for flow from the wetwell to the drywell. Default $=1$. (dimensionless)

the gas inertial length to be used when the vent flow bypasses the pool (as when the level is too low to cover the vent). The bypass gas flow is calculated considering the effects of inertia in the flow. Default $=5$. (m) 
The user may choose either of two aerosol scrubbing models. The keyword SCRUB specifies the aerosol scrubbing model from the VANESA code. [Pow86] The keyword SPARC specifies the SPARC scrubbing model. [Owc85a] The SCRUB model is the model by default provided aerosols are present.

The following keywords and values may be used in conjunction with the SCRUB model from the VANESA code (see Reference Pow86):
SCRUB the keyword for specifying the VANESA aerosol scrubbing model.
BSIZI the initial bubble diameter. Default $=0.01$. (m)
$=$ bsizi
VROVR the ratio of bubble gas circulation velocity to computed rise velocity. Default $=$ vrovr $\quad=1$.

The following keywords and values may be used in conjunction with the SPARC scrubbing model (see Reference Owc85a):

SPARC the keyword for specifying the SPARC aerosol scrubbing model.

BSIZI the initial bubble diameter. This keyword replaces DIAM of the stand-alone $=$ bsizi $\quad$ version of SPARC. Default $=0.01 .(\mathrm{m})$

RATIO the ratio of the major axis to the minor axis of a symmetric oblate spheroid $=$ ratio bubble. The ratio is taken to be greater than or equal to 1 . If a value less than 1 is input, its inverse is automatically taken. Default $=1$.

NRISE the number of integration zones used for bubble rise in the scrubbing region. $=$ nrise $\quad$ Values ranging from 10 to 1000 are suggested for accuracy. Default $=10$.

\subsubsection{Aerosol Options}

The global aerosol characteristics are specified in the following input block. Note that aerosol initial conditions and sources are given on a cell-by-cell basis and are discussed in Section 14.3.1.8.

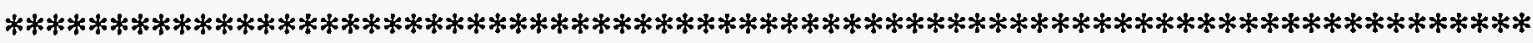

AEROSOL

[NEWCOF=newcof] [DIAM1=diam1] [DIAM2=diam2] [TGAS1=tgas1]

[TGAS2=tgas2] [PGAS1=pgas1] [PGAS2=pgas2] [TURBDS=turbds]

[COLEFF=coleff] [DENSTY=rho] [CHI=chi] [GAMMA=gamma]

[DELDIF=deldif] [TKGOP=tkgop] [NOCOND] [NOEVAP] [NOCONEVA [(ncls)]]

[RELTOL=reltol] [ABSTOL $=$ abstol]

[TRAPOVFL] [TRAPUNFL]

[AERTIM=ntb

Rev. 0 


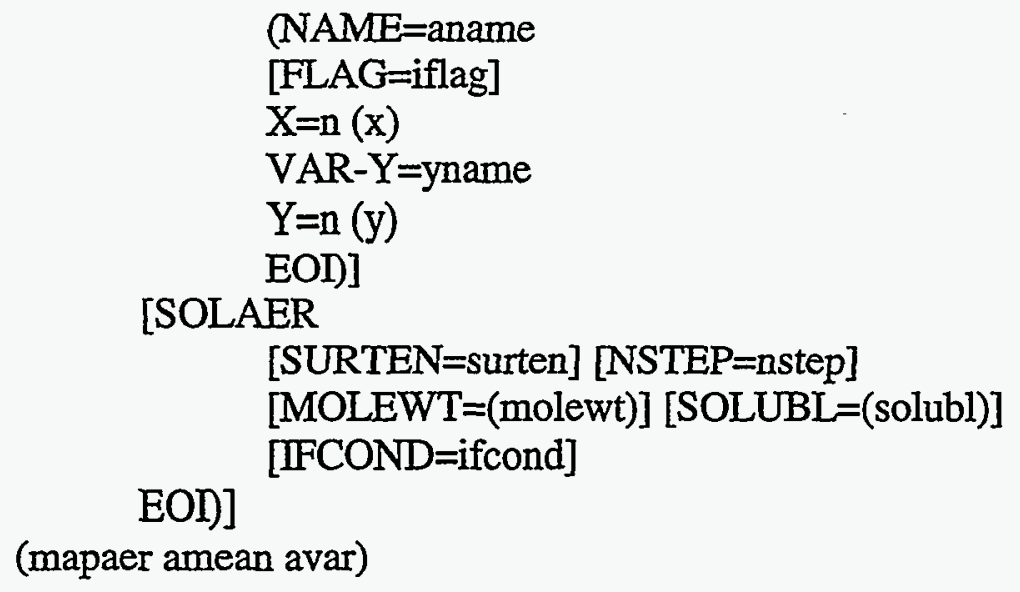

(mapaer amean avar)

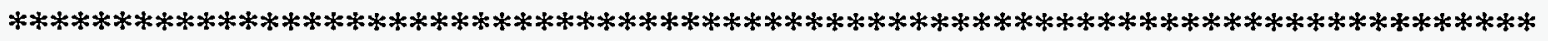

The AEROSOL keyword is required to initiate this input block. The keywords following AEROSOL are optional. The block must be terminated by values for "mapaer," "amean," and "avar" for each aerosol component defined. The number of groups of these values must equal "nac," the number of aerosol components, specified in the global CONTROL block (see Section 14.2).

AEROSOL the keyword for beginning specification of the global aerosol characteristics.
NEWCOF the flag for calculating aerosol coefficient sets. The possible values of $=$ newcof "newcof" are discussed at the end of this section. Default $=1$.
DIAM1 the minimum diameter allowed for aerosols. Default $=10^{-7} .(\mathrm{m})$
$=$ diam 1

DIAM2

$=$ diam2

TGAS1 $=$ tgas 1

TGAS2 $=$ tgas 2

PGAS1

= pgas 1

PGAS2

= pgas 2

TURBDS

$=$ turbds the maximum diameter allowed for aerosols. Default $=10^{-4} .(\mathrm{m})$

the lower temperature in the coefficient interpolation. Default $=273$. (K)

the upper temperature in the coefficient interpolation. Default $=673 .(\mathrm{K})$

the lower pressure in the coefficient interpolation. Default $=10^{5} .(\mathrm{Pa})$

the upper pressure in the coefficient interpolation. Default $=7.5 \times 10^{5} .(\mathrm{Pa})$ the turbulent energy dissipation rate $\varepsilon$ in Equation (7-11). Default $=0.001$. $\left(\mathrm{m}^{2} / \mathrm{s}^{3}\right)$ 
COLEFF
$=$ coleff

DENSTY

$=$ rho

$\mathrm{CHI}$

$=$ chi

GAMMA

= gamma

DELDIF

$=$ deldif

TKGOP

= tkgop

NOCOND

NOEVAP

NOCONEVA

[= (ncls)]

RELTOL

$=$ reltol

ABSTOL $=$ abstol

TRAPOVFL the constant collision efficiency. If a positive value is specified, it will be used. A zero value will set a flag to use an internal analytic expression (see Equation (7-11)). Default $=0$.

material density to use for all aerosol component materials. Default $=1000$. $\left(\mathrm{kg} / \mathrm{m}^{3}\right)$

the dynamic shape factor $\chi$ in Equation (7-6). Default $=1$.

the agglomeration shape factor $\gamma$ in Equation (7-6). Default $=1$.

the diffusion boundary layer thickness. Default $=10^{-5}$. (m)

the ratio of thermal conductivity of the atmosphere to that of the particle, used in Equation (7-19). Default $=0.05$.

the keyword for suppressing condensation on aerosols for all cells.

the keyword for suppressing evaporation from aerosols for all cells.

the keyword to disable aerosol condensation and evaporation for the specified cells. If the NOCONEVA keyword is given alone, aerosol condensation and evaporation in all cells will be disabled. This is equivalent to specifying both NOCOND and NOEVAP. A list of cell numbers, "ncls," can be specified following the NOCONEVA keyword to disable condensation and evaporation in just those cells.

the relative error tolerance per Runge-Kutta timestep. Default $=0.001$.

the scaling factor for the absolute error tolerance per Runge-Kutta timestep. The actual absolute error tolerance is "abstol" times the maximum total mass concentration in a size class. A "reltol" of 0.001 and an "abstol" of 0.0001 have been used successfully for problems with a large dynamic range. (For example, if one component is present initially at a relative mass concentration of one part in ten thousand compared to the total mass concentration, the relative and total mass concentrations then decay by a factor of one million.) Default $=0.0001$.

the keyword to trap aerosol particles that have become too large for the aerosol size mesh and keep them from being transferred to the waste repository of the cell in which the condition originated. Specification of this 


\section{TRAPUNFL}

keyword will result in the oversized aerosols being transferred to the lower cell pool, if defined, in the cell specified by the cell OVERFLOW keyword, as discussed in Section 7.1. If a pool is not defined in that cell, the aerosols will be placed in the waste location in that cell. In contrast to previous practice, the transferred aerosols will be assumed to have an enthalpy corresponding to the conditions of the originating cell, not the destination cell. CAUTION: This keyword represents a non-upward compatible change in the code. If the TRAPOVFL keyword is not specified, aerosols previously transferred to floors and pools will go to the waste location instead. Since these aerosols have become too large to be described in the aerosol mesh, such transfers are nonmechanistic and should be reassessed by the user.

the keyword to trap aerosol particles that have become too small for the aerosol size mesh and keep them from being transferred to the waste location of the cell in which the condition originated. Specification of this keyword will result in the undersized aerosols being retained in the smallest size class. CAUTION: This keyword represents a non-upward compatible change in the code. If the TRAPUNFL keyword is not specified, aerosols previously transferred to floors and pools or retained in the aerosol mesh will go to the waste location instead. Since these aerosols have become too small to be described in the aerosol mesh, such transfers are nonmechanistic and should be reassessed by the user.

More complete definitions of the physical parameters introduced above are given in Chapter 7.

The global aerosol input allows the user control over the disposition of the aerosol mass in particles that become either too large or too small for the aerosol mesh. This mass is reported in the aerosol waste location output and reported by default in the waste repository of the mass and energy accounting scheme for the cell in which the oversized or undersized condition arose. This is consistent with the use of the waste repository to keep track of mass and energy transfers without mechanistic basis or with an ambiguous transfer path. The TRAPOVFL and TRAPUNFL keywords alter the disposition of the oversized or undersized aerosols and enable behavior similar to that previously available. It should be noted that the enthalpy associated with the transfer of oversized aerosols corresponds to donor cell conditions, as opposed to downstream cell conditions, consistent with energy conservation. This feature may make a significant difference in pool temperature if TRAPOVFL is specified and the cell OVERFLOW option is used to direct oversized aerosols to a different cell from that in which the condition arose.

Through the AERTIM global table option, the user may specify the aerosol size distribution parameters "amean" and "avar" (discussed more fully along with the "mapaer" variable below) as a function of time. The AERTIM option uses a number of global tables to specify this time dependence, with one table specifying "amean" or "avar" for one aerosol component. The FLAG, X, and $Y$ keywords are standard table keywords defined in Section 14.4.2. Other keywords and values associated with the AERTIM option are discussed below. The user should consider the AERTIM 
tables in setting the "numtbg" and "maxtbg" parameters in the global CONTROL block (see Section 14.2).

When the time is within the range of the table, the table values of "amean" and "avar" will override the corresponding values specified after the "mapaer" variable, as discussed below. Note that the "amean" and "avar" parameters in use at a given time are global values that apply to the aerosol initial conditions and sources in any cell, with the possible exception of SRV aerosol sources. The size distribution of SRV aerosols may be specified completely independently of the global values through the SRV source tables themselves. (See Section 14.3.4.)

\begin{abstract}
AERTIM the keyword for initiating the specification of global tables for "amean" and/or "avar" as a function of time.

ntb the number of tables to follow.

NAME

$=$ aname

$\mathrm{n}$

$\mathrm{x}$

the name of the aerosol component to which the table applies. It should be among the names specified for "mapaer" below.

the number of points in the table.

the independent variable of the table, corresponding to time. Specify " $\mathrm{n}$ " values in ascending order. Note that for times prior to the first time in the table, the initial values of "amean" or "avar," specified after "mapaer" as discussed below, will be used. Within the range of the table, the table value determined according to the interpolation flag "iflag" will be used. For times greater than the last time in the table, the value of "amean" or "avar" will remain fixed at the last value determined from the table.
\end{abstract}

VAR-Y

= yname

y

EOI the name of the dependent variable in the table. The variable "yname" corresponds to either AMEAN or AVAR, depending on whether the dependent variable corresponds to "amean" or "avar," as defined below.

the dependent variable of the table, corresponding to "amean" if the AMEAN keyword has been specified after VAR-Y or to "avar" if the AVAR keyword has been specified. Specify "n" values.

the keyword required for terminating the AERTIM input block.

The following keywords are used to specify the moving-grid aerosol condensation model.

SOLAER

the keyword for beginning specification of parameters for the moving-grid model. CONTAIN will use the fixed-grid model unless this keyword is included in the input. 
SURTEN
$=$ surten

NSTEP

= nstep

MOLEWT

$=($ molewt $)$

SOLUBL

$=($ solubl $)$

IFCOND

$=$ ifcond the surface tension of a wet particle with the atmosphere that is responsible for the Kelvin effect. The default value is zero, which is used as a flag to ignore the Kelvin effect. Default $=0 .\left(\mathrm{kg} / \mathrm{s}^{2}\right)$

the number of time intervals to be used in the growth calculation for each CONTAIN system timestep. Default $=1$.

the array of "nac"-1 molecular weights for each aerosol component except the last component, which must be water. Default $=100$.

the array of "nac"-1 solubility factors for each aerosol component except the last component, which must be water. This number is equal to the number of ions formed by the dissolution of one molecule of aerosol component. For example, for $\mathrm{NaOH}$, assuming complete dissolution the solubility factor would be 2. The material is assumed to be insoluble if zero is specified. Default $=0$.

the condensation flag to add aerosol if none is present. If set to 1 , a check will be made as to whether some aerosol is present. If no aerosol exists, aerosol will be added to attain a concentration of $10^{-7} \mathrm{~kg} / \mathrm{m}^{3}$ for each component other than water. The default value of 2 results in no aerosol addition. If set to 3, water will be added to the smallest bin to attain a concentration of $10^{-10} \mathrm{~kg} / \mathrm{m}^{3}$. Default $=2$.

The last group of variables in this block specifies the aerosol components to be used in the problem and the initial values of the aerosol size distribution parameters "amean" and "avar." This group terminates the global aerosol block and thus should follow any of the keywords described above. Note that the aerosol block is not terminated with an EOI. The following group of three variables is repeated "nac" times, once for each aerosol component.

mapaer

avar an aerosol component name. This name must be one of the materials specified in the COMPOUND or AERNAMES input blocks. (See Section 14.2.1.)

a volume-equivalent mass median particle diameter to be used for initial distributions and sources of new particles. This corresponds to the $d_{m m}$ parameter in Equation (7-1). Default $=1.0 \times 10^{-6} .(\mathrm{m})$

a natural logarithm of geometric standard deviation of the particle size distribution to be used for initial distributions and sources of new particles. This corresponds to $\ln \left(\sigma_{\mathrm{g}}\right)$ in Equation (7-1). Default $=0.693$. 
These "amean" and "avar" values govern the particle size distribution for initial conditions and sources unless overridden by the AERTIM table option discussed above or by local size distribution parameters used in conjunction with SRV aerosol sources discussed in Section 14.3.4.

For aerosol condensation to be active, the last aerosol component specified for "mapaer" must be $\mathrm{H} 2 \mathrm{OL}$. If $\mathrm{H} 2 \mathrm{OL}$ is specified, the amount of liquid condensed on aerosols from the vapor phase will be added to the mass of the last component. Note that $\mathrm{H} 2 \mathrm{OV}$ may also be specified in lieu of $\mathrm{H} 2 \mathrm{OL}$; however, this use of $\mathrm{H} 2 \mathrm{OV}$ is obsolete and is not recommended.

Another format for the AEROSOL block is available. This format, discussed in Appendix B, might be present in input files developed for earlier code versions. While upwardly compatible, it is considered obsolete.

The aerosol model uses a set of coefficients that must either be calculated or read in from an aerosol database file created in a previous calculation. The user has some control over the coefficient calculation through the "newcof" parameter, as discussed below.

The coefficients depend on the number of size classes or sections, "nsectn," specified in the global CONTROL block, and all the parameters that can be set by the keywords listed above, with the exception of "newcof," "abstol," and "reltol." If present, TAPE20, the aerosol database file is first scanned for a match in all these parameters if $1 \leq$ "newcof" $\leq 4$. If an appropriate set of coefficients is found, it is read in. If a set of coefficients is found that is appropriate except for a mismatch in deposition or condensation parameters (e.g., "tkgop"), only the deposition and condensation coefficients will be calculated. The other coefficients will be read in. Coefficients not otherwise available are calculated during input processing and then used throughout the rest of the calculation. If a complete set of coefficients is calculated, the set can be appended to the end of the aerosol database file.

The aerosol routine by default is set up to interpolate between the coefficients calculated at each of the four points ("tgas1," "pgas1"; "tgas1," "pgas2"; "tgas2," "pgas1"; "tgas2," "pgas2") to account for the temperature and pressure dependence of the coefficients. For problems with no temperature or pressure variation, the user may specify that coefficients be defined at one or two points by setting "newcof" $=2,3$, or 4 as discussed below. If the user specifies such a partial set, the TAPE20 file will be scanned for a match in only the "pgas1," "pgas2," "tgas1," and "tgas2" parameters relevant to the partial set. The complete set of parameters will be read in and the relevant parameters used. If no match with partial sets on the TAPE20 file is found, the partial set will be calculated, and the coefficients not required will be set to zero.

The options selected by various values of "newcof" are as follows:

newcof $=1: \quad$ Coefficients are requested at four combinations of temperature and pressure given by "tgas1," "tgas2," "pgas1," and "pgas2." If coefficients are not available on the TAPE20 file, they will be calculated and appended to the end of that file. 
newcof $=2$ :

newcof $=3$ :

newcof $=4$ :

newcof $=99$ :
Coefficients are requested only at "tgas1" and "pgas1." This option is appropriate only for constant temperature and pressure problems.

Coefficients are requested for "pgas1" at "tgas1" and "tgas2." This option is appropriate only for constant pressure problems.

Coefficients are requested for "tgas1" at "pgas1" and "pgas2." This option is appropriate only for constant temperature problems.

Coefficients are to be recalculated regardless of availability of coefficients in the database file but are not to be appended to the end of that file. This option is useful when running CONTAIN on a machine that has difficulty processing FORTRAN "inquire" statements, or when disk space is at a premium.

A more detailed discussion of the aerosol physics modeling is found in Chapter 7.

\subsubsection{Fission Product Options}

14.2.6.1 Fission Product Decay and Heating Input. Global fission product characteristics may be specified in the following input block. These characteristics include the structure of the linear decay chains, the fission product half-lives, and the decay power coefficients. Such information for a number of radionuclides may also be loaded by simply invoking the fission product library through the FPLIB keyword discussed in Section 14.2.1. Initial fission product masses and the targeted release and acceptance parameters are defined at the cell level in the FPM-CELL input block (see Section 14.3.1.10).

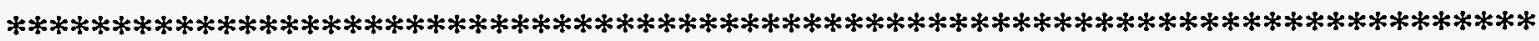

\section{FISSION}

(NFPCHN=nfpchn

FPNAME $=$ (fpname)

HFLIFE $=$ (hflife)

[ $\{$ FGPPWR=ndpcon

POWER $=(\mathrm{fpq})$ or

POWER $=(\mathrm{fpq})\}]$ )

EOI

[FINVT=finvt])

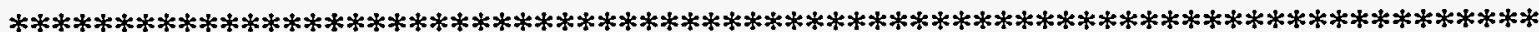

FISSION

the keyword for initiating input of the global fission product parameters. There should be only one global FISSION block in any one input file. 
NFPCHN

nfpchn

FPNAME

fpname

HFLIFE

hflife

FGPPWR

= ndpcon

POWER

fpq the keyword for specifying the number of fission product elements in a chain. It also marks the beginning of the input sub-block for that chain. All other keywords and values for that chain must be given before NFPCHN is specified again for the next chain. A total of "nchain" NFPCHN sub-blocks should be defined in the FISSION block, where "nchain" is specified in the global CONTROL block.

the number of fission chain elements in a chain. The sum of "nfpchn" over all chains must add up to the value of "nfce" given in the global CONTROL block.

the keyword for initiating the specification of the names of each element of a chain.

the fission product name for the chain element. Specify "nfpchn" names, each taken from the ones declared after FP-NAMES in the MATERIAL input block.

the keyword for initiating the input of fission product half-lives for all elements in a chain.

the half-life of a chain element (s). Exactly "nfpchn" values must be entered. The last element in a chain may be specified as stable with a zero value of "hflife." (Since there is no such thing as a zero half-life, zero is assumed to represent an infinite half-life or a zero decay constant). If a negative value is given, then its absolute value will be interpreted as the decay constant, $\lambda$, where "hflife" $=\ln (2) / \lambda .\left(s^{-1}\right)$

the number of coefficients in the decay power expression for each element in a chain. These coefficients are specified after the POWER keyword discussed below and are used to define a general time-dependent specific decay power according to Equation (8-5). Note that each chain may have its own number "ndpcon" of such coefficients. The minimum value of "ndpcon" is 1 and the maximum is 4. If FGPPWR is omitted, the value of "ndpcon" is taken to be 1 , and the decay power reduces to a constant. If specified, FGPPWR should be specified before POWER. Note that a time-dependent decay power is useful when fission product groups are modeled (see Section 8.5).

the keyword for initiating input of the power coefficients.

the value of a power coefficient. Specify "ndpcon" values for the first element in a chain, then "ndpcon" values for the second, and so on, for a total of "nfpchn" $x$ "ndpcon" values. For each element, the first value is $a_{1}$, the second, $a_{2}$, and so forth, where these coefficients are defined in Equation 
(8-5). If FGPPWR is not specified, only $\mathrm{a}_{1}$ should be specified. Default $=0$. (W/kg for odd coefficients and $\mathrm{s}^{-1}$ for even coefficients)

FINVT $=($ finvt $)$

EOI input specifying inventory factors "finvt" for each of the "nfpchn" chain elements of the current user-defined linear chain. This input is required if a user-specified branching decay process is being modeled (outside of the library), and the user wishes to rely on inventory factors to distribute initial fission product masses appropriately over the linear chains. When merging decay paths are not present, the inventory factor for a chain element represents the probability that the fission product corresponding to the chain element will decay to all of its daughters and granddaughters in the sequence given by the linear chain. (See Section 8.3.) The sum of the inventory factors for each fission product must be equal to 1 . Default $=1$ for the first occurrence of a fission product in the set of linear chains, and 0 for subsequent occurrences.

An alternative format is also available for the FISSION input block as described in Appendix B. This alternative input format includes the specification of initial fission product masses and release/acceptance functions at the global level with the FPM-CELL keyword. The FPM-CELL input block can also be given at the global level when using the input format described above; however, this is not the recommended procedure. The recommended procedure is to define the FPM-CELL input block at the cell level as described in Section 14.3.1.10.

14.2.6.2 Fission Product Transport Efficiency in Liquid Pathways. The FPLIQUID block is used to define the efficiency factors for fission product transport in liquid pathways. These factors determine the rate of fission product transport with structure condensate runoff and pool-to-pool transfers of coolant via engineered systems components, as discussed in Section 8.8.2.

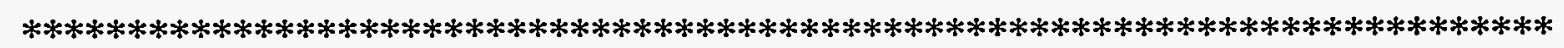

\section{FPLIQUID}

EOI

$$
\text { (fpname=fpliq) }
$$

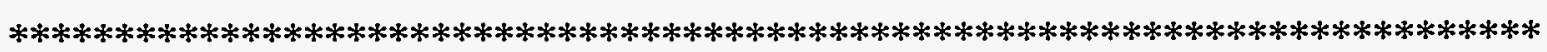

FPLIQUID the keyword for initiating the specification of transport efficiency factors for fission products in liquid pathways. This keyword should be followed by pairs of the following two variables.

fpname

a fission product name that is among the list of fission product names given in the global FISSION input block. 

be a real value between zero and one. It specifies the ratio of the relative amount of the fission product transferred to the relative amount of water transferred when condensate drains from structure surfaces to a recipient pool and when direct pool-to-pool coolant transfers occur through engineered systems components, such as a pipe. In the first case, the fission product is transferred from the structure surface to the destination pool. That pool can be designated through the cell OVERFLOW keyword discussed in Section 14.3.1.12. (This keyword should not be confused with the engineered systems OVERFLOW component discussed in Section 14.3.3.11.) In the second case, the fission product is transferred from one pool to another. Default $=0$.

the keyword required for terminating the FPLIQUID input block.

\subsubsection{Global DCH Input Block}

The DHEAT block at the global level is used to specify parameters for a CONTAIN calculation with DCH. The DHEAT block is required, in addition to the NDHBIN, NDHSPC, and possibly the NDHGRP global control parameters, to perform such a calculation. The latter parameters control the dimensionality of the airborne debris fields, or bins. In addition to the airborne fields, a trapped or nonairborne debris bin is allocated for each cell. Trapped debris may also be directed to an intermediate lower cell layer, if that exists in a cell. Additional DCH-CELL blocks at the cell level are also used to specify parameters that pertain to a particular cell. The user should note that some of the keywords in the DHEAT and DCH-CELL block are the same. The specification of such a keyword (and corresponding value, if any) in the DHEAT block sets the option or value to be used in each cell, unless overridden by the specification of the same keyword through the DCH-CELL input for a given cell. Cell-level DCH-CELL input blocks are also used to set trapping model options and to provide tabular (i.e., value versus time) input for a number of parameters. It should be noted that the tabular input for such a parameter will always override any single value specified after the corresponding keyword for the parameter. It should also be noted that the keywords ON and OFF appearing in the template below are optional, and are provided primarily for use on a restart.

The materials introduced into the debris fields must be user-defined materials of type DEBRIS. The requirements for the DCH material set are discussed in more detail in Section 14.2.1.2.

When the reactor pressure vessel (RPV) and cavity models are invoked, the user should remember that both an RPV and a cavity cell must be designated, and that these two CONTAIN cells must be connected by a single gas flow path. The characteristics of this flow path may be specified as usual in the CONTAIN input, but the user should be aware that the presence of debris in the RPV cell within the time interval defined by "strtim" and "stptim" will activate the RPV and cavity models in CONTAIN. During this time, the RPV and cavity models take control of the flow path between the RPV and cavity cells according to the input that has been provided in the RPVCAV input section. 
In the input block description of the RPV and cavity models, many of the keywords shown are optional since they are not required for the general RPV and cavity models. However, some of these keywords may be required depending on the entrainment rate (IENRAT) or entrained debris fraction (IENFRA) model specified. Additional discussions are provided in the definitions of IENRAT and IENFRA.

Note that the FDISTR or FDEVEN debris distribution options should not be used when the WEBER model is invoked. When the WEBER model is invoked, additions to the airborne debris is assumed to occur only in the cavity, and the distribution of newly entrained airborne debris is calculated in the model. Furthermore, the droplet diameter partitioning must also be logarithmic, as calculated in the DIARANGE option. Note that on a restart, the diameters of the airborne droplet fields may not be changed. Thus, if the WEBER model is to be activated on a restart, the required logarithmic size distribution should be used from the outset. Also, whenever the WEBER model is not invoked or is disabled on a restart, the FDIST or FDEVEN option must be specified in order to distribute debris from subsequent external sources in the appropriate fashion.

It is important to note that other aspects of the CONTAIN input can have an effect on the calculated DCH behavior. For example, the selection of the diffusion flame and/or spontaneous recombination options in addition to the default discrete deflagration model in the hydrogen burn input can have a dramatic effect on the calculated peak pressures in DCH. It is recommended that users of the DCH model be familiar with Section 13.3.2, which provides detailed guidance on the use of the DCH models, including the use of the combustion model in DCH calculations.

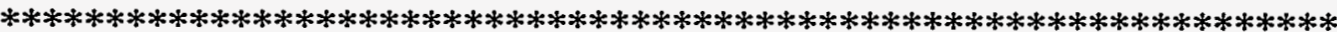

\section{DHEAT}

[STARTIME=strtim] [STOPTIME=stptim]

[\{DIADRP=diadrp or DIABIN=(diabin) or DIARANGE diamin diamax $\}$ ]

[\{FDISTR $=$ (fdistr) or FDEVEN\}]

[GRPLIM=grplim] [IEQOPT=ieqopt]

[\{SDSLIP=(sdslip) or SDEVEN=sdeven $\}]$

[DENDRP=dendrp] [SURTEN=surten] [RADGAS=radgas] [RADMUL=radmul]

[GASSUR=gassur] [DIFO2=difo2] [DIFH2O=difh2o] [HTCMUL=htcmul]

[THRESH=thresh] [LIQSIDE dl1 ell dl2 el2] [PRODSEP=[\{ON or OFF\}]]

$[\mathrm{RCOMH} 2=[\{\mathrm{ON}$ or OFF $\}]]$ [VELOCITY=velcty] [TRAPRATE $=$ trprat $]$

\section{[RPVCAV}

CAVITY=nccav
DIARPV=diarpv
[AHOLE1=ahole1]
[CSUBD=csubd]
[RHOWAL=rhowal]
AFILM=afilm
DSUBS=dsubs
SCALEF=scalef
TSUBIS=tsubis
[AFLOW=aflow

$\begin{array}{ll}\text { RPV=ncrpv [USEVOUT=[\{ON or OFF\}] } \\ \text { THKWAL=thkwal } & \text { TWALL=twall } \\ \text { [ARMULT=armult] } & \text { [CPWALL=cpwall] } \\ \text { [HFWALL=hfwall] } & \text { [IRPVGE=irpvge] } \\ \text { [TMELT=tmelt] } & \text { IENRAT =ienrat } \\ \text { CCENR=ccenr } & \text { HYDDIA=hyddia } \\ \text { RHDEBS=rhdebs } & \text { RSUBS=rsubs } \\ \text { SSCALF=sscalf } & \text { SURTES=surtes } \\ \text { WETPER =wetper } & \text { [IENFRA=ienfra] } \\ \text { [AHENF=ahenf] } & \text { [TDISP=tdisp] }\end{array}$ 


$\begin{array}{llll} & \text { [TSTOP=tstop] } & \text { [VRPVU=vrpvu] } & \text { AMWCU=amwcu } \\ & \text { CAVLEN=cavlen } & \text { CCENF=ccenf } & \text { ENTFR=entfr } \\ \text { GAMMAU=gammau } & \text { PCAVU=pcavu } & \text { PCONU=pconu } \\ & \text { RHOGU=rhogu } & \text { VISGU=visgu } & \\ & [\text { [WEBER=[\{ON or OFF }\}] & \\ & {[\text { [WECRIT=wecrit] }]} & \text { [WESIG=wesig] } & \\ & \text { EOI] } & & \\ \text { EOI EOI } & & & \end{array}$

$* * * * * * * * * * * * * * * * * * * * * * * * * * * * * * * * * * * * * * * * * * * * * * * * * * * * * * * * * * * * * * * * * * * * * * *$

DHEAT

STARTIME

$=$ strtim

STOPTIME

= stptim

DIADRP

$=\operatorname{diadrp}$

DIABIN

$=($ diabin $)$

DIARANGE

$=$ diamin diamax the keyword for initiating the input of global DCH parameters. In the following, the word "field" with respect to debris will be used to refer to the airborne debris fields, and the trapped debris bin will be referred to as such.

the start time for debris interactions with the gas field. Prior to this time, debris may be added to the debris fields or trapped bin, but it will be assumed not to interact with the gas. Caution: if debris is introduced prior to "strtim" and the WEBER model is selected, the distribution of debris will be only a nominal one. Default $=-10^{30}$. (s)

the stop time for debris interactions with the gas field. After this time, debris may be present in or added to the debris fields, but it will be assumed not to interact with the gas. Caution: if debris is introduced after "stptim" and the WEBER model is selected, the distribution of debris will be only a nominal one. Default $=10^{30}$. (s)

the drop diameter to be used for every debris field. The diameter of droplets in every airborne field will be set to the specified value. This input should not be used when the WEBER model is invoked or is going to be invoked on a subsequent restart (see DIARANGE). (m)

the drop diameter for each field specified individually. Exactly "ndhbin" values must be specified, one for each field. This is one way to specify a particle size distribution for the debris fields. This input should not be used when the WEBER model is invoked or is going to be invoked on a restart, unless the particle size distribution is logarithmic. However, that type of distribution is more easily specified in the DIARANGE option below (see DIARANGE). Note that airborne drop diameters are taken to be the same in every cell. (m)

the lower and upper drop diameters, respectively, to be represented in the airborne debris fields. The range of diameters between and including "diamin" and "diamax" will be partitioned into "ndhbin" values, spaced evenly with respect to the logarithms of the diameters. Note that "ndhbin" 
FDISTR

$=$ (fdistr)

FDEVEN

GRPLMM

$=$ grplim

IEQOPT

$=$ ieqopt

SDSLIP

$=($ sdslip $)$

SDEVEN

= sdeven must be at least 5 to use the DIARANGE option. Such a logarithmic distribution is required when the WEBER model is invoked, and in addition the ratio of "diamax" to "diamin" must be at least 10. If neither DIADRP, DIABIN, or DIARANGE is specified, the size distribution will default to the DIARANGE option with "diamin" $=10^{-4}$ and "diamax" $=0.01$. Note that the airborne drop diameters are taken to be the same in every cell. (m)

an array of dimension "ndhspc" by "ndhbin" controlling the distribution of sourced or entrained mass in all cells into the airborne debris fields. Exactly "ndhspc" $x$ "ndhbin" values must be specified. This corresponds to the matrix of $f_{n, k}$ parameters in Equation (6-16) for $1 \leq n \leq$ "ndhbin." Note that "ndhspc" values are specified in the first row, and "ndhbin" rows must be given. The sum of each column should add up to one. If they do not, the code will normalize the values to one. This input or FDEVEN must be given whenever the WEBER model is not invoked.

a keyword to specify a uniform distribution of debris sources into the airborne debris fields. This is equivalent to specifying $1 /$ "ndhbin" for each element of "fdistr."

the maximum mass of debris allowed to be dispersed into each generation. A new generation is created with "ndhbin" fields when the dispersed mass entering the previous generation has exceeded the allowable limit, "grplim." The number of generations will be limited to the maximum value specified in the global control block, "ndhgrp." Therefore, the mass dispersed into the last generation may exceed the value of "grplim." Default $=10^{20}$. (kg)

the flag to select alternate treatments for iron/steam equilibrium. A value of 0 means no equilibrium modeling (reaction can go to completion). A value of 1 means that the reaction equilibrium will be evaluated assuming unity for the mole fraction of $\mathrm{FeO}$ among the oxides. The default value of 2 means that the equilibrium will be evaluated using the calculated mole fraction of FeO. Default $=2$.

the slip ratios (i.e., the ratios of gas-to-debris velocities) for each of the airborne debris fields for debris flow out of a cell. Exactly "ndhbin" values must be specified. The slip ratios apply to flow moving out of a cell through all flow paths. These values correspond to the $s_{n}$ slip ratios in the gas and debris flow equations. This input may also be specified in the DCH-CELL block. Values greater than or equal to one must be specified. Default $=1$.

the slip ratio (i.e., the ratio of gas-to-debris velocity) for debris flow out of a cell. This input corresponds to the same parameter as the SDSLIP input, except that only one value is specified and the slip ratio for all fields is set to this single specified value. This input may also be specified in the DCH- 
CELL block. A value greater than or equal to one must be specified. Default $=1$.

DENDRP

$=$ dendrp

SURTEN

$=$ surten

RADGAS

= radgas

RADMUL

$=$ radmul

GASSUR

$=$ gassur

DIFO2

$=$ difo 2 the user-specified density for the airborne debris fields. If this is omitted, as is generally recommended, then the density of each field will be computed at each timestep from the density of the species present in the field at that time. The density of each species will be taken from the user-defined RHO or RHOT material table in the USERDAT input block. $\left(\mathrm{kg} / \mathrm{m}^{3}\right)$

surface tension of debris. SURTEN can be overridden at the cell level only if the TOF/KU model is invoked. The global value is normally used in the entrainment models, if they are invoked; see, however, the discussion of SURTEN in the DCH-CELL input described in Section 14.3.1.11. Default $=1$. $(\mathrm{N} / \mathrm{m})$

the radiation blackbody multiplier for radiative heat exchange between the debris and the atmosphere in a cell. This corresponds to debris-to-gas black body multiplier, $\varepsilon_{\mathrm{d}-\mathrm{g}}$ in Equation (6-157). The sum of "radmul" and "radgas" should be less than or equal to 1 . This input may also be specified in the DCH-CELL block. Default $=1$.

the radiation blackbody multiplier for radiative heat exchange between the debris and structure surfaces in a cell. This corresponds to the debris-tosurface black body multiplier, $\varepsilon_{\mathrm{d}-s}$, in Equation (6-158). The sum of "radmul" and "radgas" should be less than or equal to 1 . This input may also be specified in the DCH-CELL block. Default $=0$.

the value of the total gas emissivity to use with regard to gas-to-surface heat transfer, in place of the standard emissivity (see Chapter 6) during gas-debris interactions. Such surfaces include those of heat transfer structures, coolant pools, and the heat sinks of engineered systems. The value of "gassur" is used in the interval defined by the STARTIME and STOPTIME keywords to allow for deviations from the standard model, which does not take into account the presence of suspended debris. (This option supersedes the obsolete negative "kmx" option, which overrides the standard model for all time.) A zero or negative value for "gassur" will result in the use of the standard model.

the multiplier on the mass transfer coefficient for the transport of oxygen to the surface of drops as determined using the mechanistic mass transfer correlation. This parameter is not shown in Section 6.4; however, the rate given by Equation (6-122) is simply multiplied by this value. This parameter may also be specified in the DCH-CELL block. Default $=1$. 


$$
\begin{aligned}
& \text { DIFH2O } \\
& =\text { difh } 20
\end{aligned}
$$

HTCMUL

$=$ htcmul

THRESH

$=$ thresh

LIQSIDE

$=\mathrm{dll}$ ell $\mathrm{dl} 2 \mathrm{el} 2$

PRODSEP

$=[\{\mathrm{ON}$ or $\mathrm{OFF}\}]$

RCOMH2

$=[\{\mathrm{ON}$ or $\mathrm{OFF}\}]$

VELOCITY

$=$ velcty the multiplier on the mass transfer coefficient for the transport of steam to the surface of drops as determined using the mechanistic mass transfer correlation. This parameter is not shown in Section 6.4.1; however, the rate given by Equation (6-122) is simply multiplied by this value. This input may also be specified in the DCH-CELL block. Default $=1$.

the multiplier on the convective heat transfer coefficient for the convective transfer of heat between drops and the atmosphere as determined using the mechanistic heat transfer correlation. This parameter is not shown in Section 6.5.1; however, the rate given by Equation (6-155) is simply multiplied by this value. This input may also be specified in the DCH-CELL block. Default $=1$.

an optional temperature cutoff for chemical reactions for all cells. When the temperature of a debris field in a given cell falls below the "thresh" value, chemical reactions between reactive debris in that field and the atmosphere will be turned off. This input may also be specified in the DCH-CELL block. Default $=273.15$. $(\mathrm{K})$

the parameters that specify the temperature-dependent gas diffusivity used in the drop-side diffusion model. These parameters are used as shown in Equation (6-139) of Section 6.4.2. Defaults: "d11" $=10^{20}\left(\mathrm{~m}^{2} / \mathrm{s}\right)$, "el1" $=0$ $\left(\mathrm{K}^{-1}\right)$, "dl2" $=0\left(\mathrm{~m}^{2} / \mathrm{s}\right)$, "el2" $=0\left(\mathrm{~K}^{-1}\right)$. Note that an input value of "dl1" $=0$ will be interpreted as requesting the default. This input may also be specified in the DCH-CELL block.

the keywords to enable or disable the distribution of chemistry oxide products into fields according to the FDISTR array. This option is only available when there is one generation ("ndhgrp" =1). If this option is not used, then oxides stay in the field originally containing the metal that reacted. The ON or OFF keyword is optional. ON is implied if neither ON or OFF is specified. PRODSEP may also be specified in the DCH-CELL block. It may also be specified on a restart even if not specified in the initial run.

the keywords to enable or disable hydrogen recombination. With recombination specified, any hydrogen produced by debris/steam reactions is immediately combined with any available atmospheric oxygen, with the energy being released into the atmosphere. The ON or OFF keyword is optional. ON is implied if neither ON or OFF is specified. RCOMH2 may also be specified in the DCH-CELL block. It may also be specified on a restart even if not specified in the initial run.

the user-specified constant relative velocity between airborne debris and the gas, to be used for heat and mass transfer purposes. This input may also be specified in the DCH-CELL block. This input is provided for compatibility 
TRAPRATE

$$
=\text { trprat }
$$

RPVCAV

CAVITY

= nccav

RPV

$=\mathrm{ncrpv}$

USEVOUT $=[\{\mathrm{ON}$ or $\mathrm{OFF}\}]$ with older input decks and is not recommended. The preferred method is to invoke the cell-level TRAPPING model, which will calculate the debris trajectory in a cell and the relative velocity between the gas and debris. If both the VELOCITY and cell-level TRAPPING keywords are given, the VELOCITY input will take precedence.

the user-specified constant debris trapping rate. The use of such a trapping rate is only one of four modeling options. Any of the four may be selected through the TRAPPING block in the DCH-CELL input. If both a global TRAPRATE keyword and a cell-level TRAPPING block are specified, the cell-level TRAPPING input will take precedence for the cell in question. If neither are specified, the trapping rate will be set to the GFT option as described in the TRAPPING input, with a fall height set to the cube root of the initial cell gas volume. $\left(\mathrm{s}^{-1}\right)$

the keyword to begin input for the RPV and cavity DCH models.

the cell number of the cavity cell. Only one cell may be specified as the cavity cell, and both an RPV and cavity cell must be specified to activate the suite of RPV and cavity DCH models.

the cell number of the RPV cell. Only one cell may be specified as the RPV cell, and both an RPV and cavity cell must be specified in input to activate the suite of RPV and cavity DCH models.

the keyword to indicate how the gas velocity in the cavity is calculated for use in the entrainment model and Weber particle size model. If the USEVOUT keyword is specified, the gas velocity in the cavity is assumed to be the velocity of the gas leaving the cavity cell. Otherwise, the gas velocity is assumed to be the average of the cavity cell inlet and outlet gas velocities. The ON or OFF keyword is optional. If neither is specified, ON is implied. USEVOUT may be specified on a restart even if not specified in the initial run.

A number of parameters may be used to describe the RPV and control the RPV ablation models, although only the RPV diameter, wall thickness, and wall temperature are required.

$\begin{aligned} & \text { DIARPV } \\ & =\text { diarpy }\end{aligned}$
$\begin{aligned} & \text { THKWAL diameter of the RPV. (m) } \\ & =\text { thkwal }\end{aligned}$
$\begin{aligned} & \text { TWALL } \\ & =\text { twall }\end{aligned}$



AHOLE1
the initial hole size in the RPV at the start of single-phase debris ejection.
$=$ ahole 1
Default $=0.01 .\left(\mathrm{m}^{2}\right)$
ARMULT
the multiplier of the ablation rate of the hole in the RPV. Default $=1$.
$=$ armult
CPWALL
the specific heat of the RPV wall. If no value is given, the code calculates a
$=$ cpwall
value based on $\mathrm{Fe}$ at the temperature TWALL. (J/kg-K)
CSUBD
the discharge coefficient for debris at the RPV hole. Default $=0.6$.
= csubd
HFWALL
the heat of fusion of the RPV wall. Default $=2.7 \times 10^{5} .(\mathrm{J} / \mathrm{kg})$
$=$ hfwall
IRPVGE
the integer indicating the geometry of the lower head of the RPV:
$=$ irpvge
$0=$ hemispherical, $1=$ cylindrical. Default $=0$.
RHOWAL
the density of the RPV wall. If no value is given, the code calculates a value
= rhowal
based on Fe at the temperature "twall." $\left(\mathrm{kg} / \mathrm{m}^{3}\right)$
TMELT
the melting temperature of the RPV wall. Default $=1700$. $(\mathrm{K})$
$=$ tmelt

The user must select a model for the entrainment rate of debris in the cavity, and specify the area in the cavity covered by debris, the cavity coefficient for the entrainment model, and the hydraulic diameter of the cavity. A number of other parameters must be specified if the Levy entrainment rate or entrained fraction models are invoked. One of the Whalley-Hewitt options (IENRAT=3) as well as the Tutu-Ginsberg entrainment rate model (IENRAT $=5)$ requires an additional input parameter as well.

IENRAT

$=$ ienrat

AFILM

$=$ afilm

CCENR

$=$ ccenr the integer indicating which model will be used in calculating the entrainment rate in the cavity.

$$
\begin{aligned}
& 1=\text { Whalley-Hewitt (Equation (6-34)) } \\
& 2=\text { Whalley-Hewitt (Equation (6-36)) } \\
& 3=\text { Whalley-Hewitt (Equation (6-38)) } \\
& 4=\text { Levy Model } \\
& 5=\text { Tutu Model }
\end{aligned}
$$

surface area in the cavity covered by debris. $\left(\mathrm{m}^{2}\right)$

the multiplier to the entrainment rate. The constant CCENR is both cavity and model-specific. 
HYDDIA

$=$ hyddia

DSUBS

$=\mathrm{dsubs}$

RHDEBS

$=$ rhdebs

RSUBS

$=$ rsubs

SCALEF

$=$ scalef

SSCALF

= sscalf

SURTES

$=$ surtes

TSUBIS

$=$ subis

WETPER

$=$ wetper the hydraulic diameter of the cavity. (m)

the standard diameter of the hole in the RPV. This value is required when the Levy entrainment rate or entrained fraction models are invoked (IENFRA = 2 or IENRAT 4). Guidance on the selection of this value is provided in References Lev91 and Wi196b, and Section 13.3.2 of this manual. (m)

the standard debris density. This value is required when the Levy entrainment rate or entrained fraction models are invoked (IENFRA $=2$ or IENRAT = 4). Guidance on the selection of this value is provided in References Lev91 and Wi196b and Section 13.3.2.

the gas constant of the standard gas. This value is required when the Levy entrainment rate or entrained fraction models are invoked (IENFRA $=2$ or IENRAT=4). Guidance on the selection of this value is provided in References Lev91 and Wi196b, and Section 13.3.2.

the scale factor of the cavity, in relation to a full-scale plant. This value is required when the Levy entrainment rate or entrained fraction models are invoked (IENFRA $=2$ or IENRAT $=4$ ). Guidance on the selection of this value is provided in References Lev91 and Wil96b, and Section 13.3.2.

the standard scale factor of the cavity. This value is required when the Levy entrainment rate or entrained fraction models are invoked (IENFRA $=2$ or IENRAT = 4). Guidance on the selection of this value is provided in References Lev91 and Wil96b, and Section 13.3.2.

the standard surface tension of the debris simulants used in the cavity dispersal experiments. This value is required when the Williams-Griffith version of the Whalley-Hewitt entrainment rate model is invoked (IENRAT $=3$ ). Guidance on the selection of this value is provided in Reference Wil96b and in Section 13.3.2.4.7. (N/m)

the standard gas temperature in the RPV cell. This value is required when the Levy entrainment rate or entrained fraction models are invoked (IENFRA = 2 or IENRAT $=4)$. Guidance on the selection of this value is provided in References Lev91 and Wil96b, and Section 13.3.2. (K)

the wetted perimeter in the cavity. This value is required when the TutuGinsberg entrainment rate model is invoked (IENRAT $=5)$. (m)

A large number of additional parameters must be specified if any entrained fraction model is invoked. The user may also invoke a number of additional parameters to control the flow out of the cavity and the blowdown of the RPV. 
IENFRA

$=$ ienfra

AFLOW

$=$ aflow

AHENF

$=$ ahenf

TDISP

$=$ tdisp

TSTOP

= tstop

VRPVU

$=\mathrm{vrpvu}$

AMWCU

$=\mathrm{amwcu}$

CAVLEN

= cavlen the integer indicating which model will be used in calculating the total entrained fraction of debris.

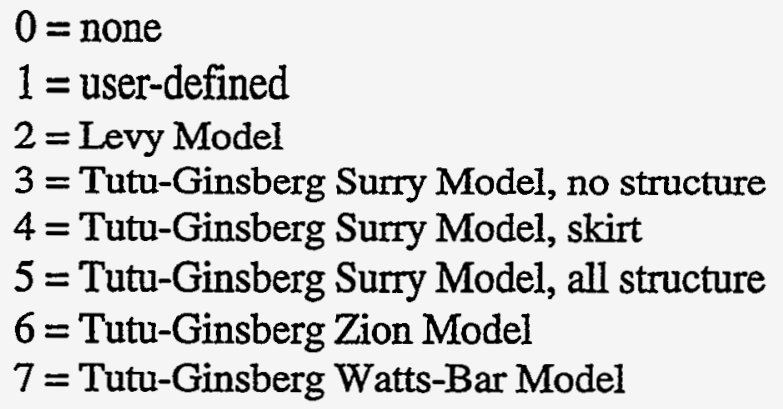

If no value for "ienfra" is specified, an entrained fraction will not be imposed on the calculation and the fraction of debris dispersed from the cavity will be based solely on the integrated dispersal determined from the selected entrainment rate model. Default $=0$.

the cross-sectional flow area in the cavity. Default $=\left(\mathrm{V}_{\mathrm{g}}\right)^{2 / 3}$, where $\mathrm{V}_{\mathrm{g}}$ is the cavity gas volume. $\left(\mathrm{m}^{2}\right)$

the area of the hole in the RPV to be used in calculating the entrained fraction of debris. If "ahenf" $=0$, calculate the final hole size in the RPV internally. Default $=0$.

the estimated debris dispersal interval. If a value is specified in input, it overrides the internally calculated estimate. (s)

the duration of the time interval for the linear growth of the hole in the RPV from its size at the time of gas blowthrough to the size specified by "ahenf" or calculated internally. (This should not be confused with "stptim.") (s)

the volume of the RPV used in estimating the dispersal interval internally. This value is only relevant if the user has invoked a model for the entrained fraction of debris. If a value is not specified in input, CONTAIN will use the volume of the specified RPV cell. Note that this will lead to underestimates of the debris dispersal time if high pressure volumes are present upstream of the RPV cell.

the molecular weight of gas in the cavity. A value must be specified if the user has invoked a model for the entrained fraction of debris.

the length of the cavity. A value must be specified if the user has invoked a Tutu-Ginsberg entrained fraction model ("ienfra" $=3$ through 7). (m) 
CCENF

$=$ ccenf

ENTFR

= entfr

GAMMAU

= gammau

PCAVU

='pcavu

PCONU

= pconu

RHOGU

$=\mathrm{rhogu}$

VISGU

= visgu the value of the cavity coefficient $\mathrm{K}_{\mathrm{c}}$ used to evaluate models for the entrained fraction. The constant "ccenf" is both cavity and model-specific, and must be specified if the user has invoked a model for the entrained fraction of debris.

the entrained fraction of debris. This value is required if and only if "ienfra" $=1$, and must be between 0 and 1 .

the ratio of gas specific heats in the cavity. A value must be specified if the user has invoked a model for the entrained fraction of debris.

the average pressure in the cavity. A value must be specified if the user has invoked a model for the entrained fraction of debris. ( $\mathrm{Pa}$ )

the average pressure in the containment. A value must be specified if the user has invoked a Tutu-Ginsberg model for the entrained fraction of debris ("ienfra" = 3 through 7). ( $\mathrm{Pa}$ )

the density of gas in the cavity. A value must be specified if the user has invoked a model for the entrained fraction of debris. $\left(\mathrm{kg} / \mathrm{m}^{3}\right)$

the viscosity of the gas in the cavity. A value must be specified if the user has invoked a model for the entrained fraction of debris. $(\mathrm{kg} / \mathrm{m}-\mathrm{s})$

The Weber model is used to calculate a particle size distribution for entrained debris, based on the conditions in the cavity cell. When the Weber model is invoked, the user may not use the FDISTR or FDEVEN options to specify the distribution of sourced mass into the airborne debris fields. Also, the user must use a logarithmic particle size distribution, as calculated in the DIARANGE option. Conversely, when the Weber model is not invoked, the FDISTR or FDEVEN option must be used to specify the distribution of any sourced mass.

WEBER

$=[\{\mathrm{ON}$ or $\mathrm{OFF}\}]$

WECRIT

$=$ wecrit enables or disables the Weber entrained drop size model. Note that userspecified distributions of sourced mass into the airborne debris fields through the FDISTR or FDEVEN options are not allowed when the Weber model is active. Also, all debris distributions will be controlled by the Weber distribution based on cavity conditions, even outside of the cavity. Note that the ON or OFF is optional. If neither is specified, then ON is implied. The keywords below will be accepted whether or not the model is active, and, if not active, the specified parameters will be stored for possible use on a subsequent restart. The WEBER input may be specified on a restart even if not specified in the initial run.

the critical Weber number. Default $=12$. 
WESIG

=wesig

EOI the natural logarithm of the geometric standard deviation of particle sizes in the assumed lognormal distribution of entrained debris during a given time stop. Default $=\ln (2)$.

the keyword required for terminating the DHEAT, RPVCAV, and WEBER blocks.

\subsubsection{Timestep and Time Zone Input}

The TIMES block establishes the maximum system timestep size, the maximum edit timestep, and the total allowable CPU calculation time. The edit timestep by definition is the interval at which information is written to the plot file. It is smaller than or equal to the interval at which long edits are written to the main output file. The TIMES block also defines the times at which restart data are written to the restart file.

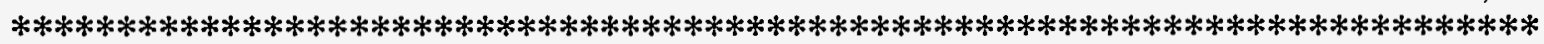

TIMES cput tstart (timinc edtdto tstop)

[ (ctmfr) or

CTFRAC $=($ ctmfr $)\}]$

[TRESTART $=\mathrm{n}($ tres)]

[TSFRAC $=$ tsfrac]

[EDMULT=edmult]

[EOI]

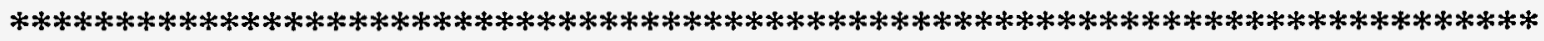

TIMES

cput

tstart a keyword initiating timestep and time zone input.

the maximum CPU time limit. (s)

the problem start time. Note that the problem start time can be positive, zero, or negative. (s)

The following set of three variables is repeated "ntzone" times, one set for each time zone:

timinc

edtdto

tstop

the end time of the time zone. (s) 
ctmfr

CTFRAC

TRESTART

$\mathbf{n}$

tres

TSFRAC

$=$ tsfrac

EDMULT

$=$ edmult

the ratio of the maximum allowed cell timestep to the system timestep. Specify a value less than or equal to one for each cell. Note that neither CTFRAC nor "ctmfr" has to be specified if the default value is acceptable. Default $=1$.

an optional keyword for defining the maximum cell timestep. It does not have to be specified if "ctmfr" values follow immediately after the time zone information.

an optional keyword to specify the times at which restart blocks are to be saved on the restart file.

the number of restart times to be defined. Maximum $=100$.

the times at which restart blocks are to be saved. Specify " $n$ " values. If no values are specified, the restart times by default are taken to be the end times of the time zones.

a timestep scaling factor for internally regulated system timesteps. These timesteps are used during certain physical processes, such as hydrogen deflagrations, and are defined by default as one tenth of the smallest characteristic time for such processes. These internal timesteps, when present, override the maximum user-specified system timesteps, whenever the former are smaller. The fraction of the characteristic time used for the internal timesteps is obtained by multiplying the default fraction of one tenth by the "tsfrac" factor. The default value of "tsfrac" is 1 for an initial run and the previous value in a restart. In support of older input decks, the keyword MULTIPLE may also be given instead of TSFRAC.

a frequency factor for edits during processes for which the system timestep is internally regulated. (See TSFRAC above.) During such processes, the edit timestep will be the actual system timestep multiplied by "edmult" if the resulting timestep is less than "edtdto." The default value of "edmult" is the ratio "edtdto"/"timinc" rounded up to the next integer for an initial run and the previous "edmult" value in a restart.

EOI

an optional terminator.

A maximum of 101 restart blocks can be present on the restart tape. The last is always the temporary restart block from the last edit time. (This block is overwritten each edit time.) Up to 100 restart blocks may also be permanently saved. These are specified through TRESTART, or if this is not used, by the "tstop" times marking the ends of the time zones. Note that on a restart, new restart blocks are added to those already present on the restart tape, and the original blocks count toward the maximum of 101 blocks allowed. 
The EDMULT keyword is useful for resolving hydrogen burns without producing massive quantities of output at other times in the run. For example, "edtdto" may be considerably larger than "timinc"; however, if "edmult" is, say, equal to 1.2 , the edit interval during a burn will be 2 (1.2 rounded up) times the actual timestep used.

\subsubsection{Output Control}

Options to control the formatted output written to the main output file and the unformatted output written to the plot file(s) are discussed in this section. Note that the latter output can be postprocessed using the POSTCON code. (See Chapter 16.)

14.2.9.1 Frequency of Print Output. Output is written to the main output file in printer carriage control format. This output consists of short summary edits and long detailed edits. The frequency at which the short edits and long edits are produced in the main output file can be controlled with the SHORTEDT and LONGEDT options.

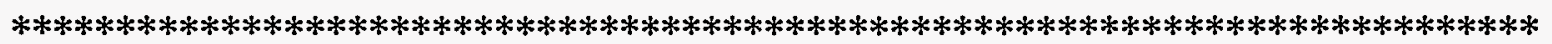

SHORTEDT=kshort

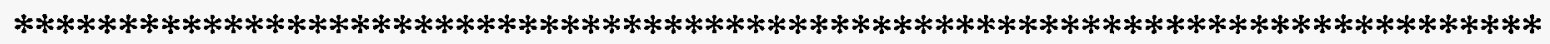

SHORTEDT the number of system timesteps between short edits. Cannot be 0; default = $=\mathrm{kshort}$ 1 .

For example, if the system timestep is $5 \mathrm{~s}$ and "kshort" is 4 , then the short edit interval will be $20 \mathrm{~s}$. The short edit interval is completely independent of other output intervals.

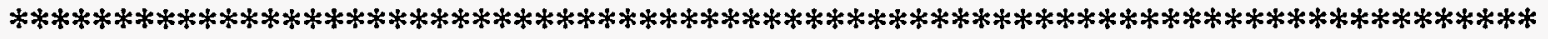

\section{LONGEDT=klong}

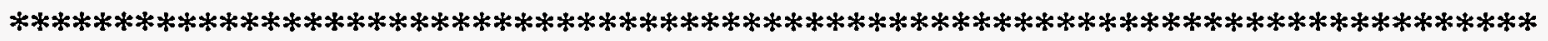

LONGEDT the number of edit timesteps between long edits. Cannot be 0 ; default $=1$. $=$ klong

The LONGEDT option controls the frequency of the long edits in the same way that the SHORTEDT option controls the short edit frequency. Note that LONGEDT and SHORTEDT have no effect on the frequency with which information is written to the plot file(s). (That frequency is governed by the basic edit timestep, which is defined by parameters such as "edtdto" discussed in Section 14.2.8 and by the plot file output control options discussed in the next section.) Thus the user can limit the quantity of long or short edits but still obtain detailed plot information on the plot file(s). A long edit will always occur at a "tstop" time, a restart time, an opening or closing time for a regular flow path, 
and a CORCON edit time regardless of the number of edit timesteps that have elapsed since the last long edit.

14.2.9.2 Print Output Options. The following single-keyword options can be used in any order to obtain the indicated block of output in the long edits.

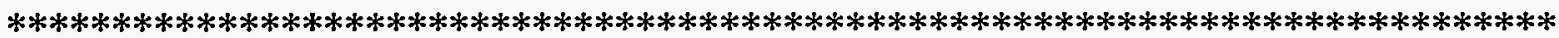

[PRFLOW]

[PRAER]

[PRAER2]

[PRLOW-CL]

[PRHEAT]

[PRFISS]

[\{PRFISS2 or PRFPGRP\}]

[PRENGSYS]

[PRBURN]

[PR-USERO]

[PRENACCT]

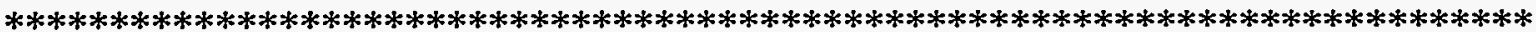

PRFLOW the intercell flow model.

PRAER a keyword which invokes detailed suspended aerosol inventories and short aerosol deposition summaries.

PRAER2 a keyword which invokes detailed suspended aerosol and aerosol deposition inventories.

PRLOW-CL a keyword which invokes the lower cell model.

PRHEAT a keyword which invokes the heat transfer structure model.

PRFISS a keyword which invokes fission product mass and decay power summaries.

PRFISS2 the keyword to specify that detailed fission product data will be output. This option will be ignored if the PRFPGRP option is specified.

PRFPGRP the keyword to specify that fission product output will be reported in terms of the volatility groups defined in Table 8-1. It applies only when the G-TARGET option is invoked. Use of PRFPGRP overrides the PRFISS2 print option.

PRENGSYS a keyword which invokes the engineered system model. 
PRBURN

PR-USERO

PRENACCT a keyword which invokes the hydrogen and carbon monoxide burn model.

a keyword which invokes user-implemented output from the USERO subroutine.

the keyword to enable output of mass and energy accounting information in the long edits.

The user may specify as many of these keywords as desired. The associated output occurs only in the long edits. (See the previous section for a discussion of the LONGEDT option.)

The PR-USERO option gives the user additional flexibility in obtaining output. It controls output from the USERO subroutine, from which essentially any variable in the code can be accessed. The user must add coding to the USERO subroutine specifying the output and the format to be used.

14.2.9.3 Plot File Output Control. Three new options are available for the specification of plot flags, plot cells, and plot write frequency control. The syntax for their use is given in this section.

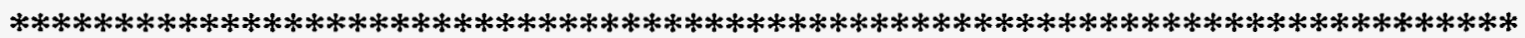

[PLFLAG (ofls) [EOI]

[PLCELL (ncls) [EOI]]

[PLAUTO

[\{ACTIVE or PASSIVE\}]

[PRESSURE (pfac)]

[TEMP (tfac)]

[GASMASS (gfac)]

[FPMASS (fpfac)]

[EOI]]

[AERMASS (aerfac)]

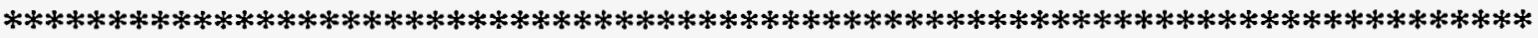

PLFLAG

(ofls)

PLCELL an optional keyword for selecting specific flags to be written to the plot file. If this keyword is not specified, all flags corresponding to active models will be written to the plot file.

a list of flag numbers, selected from Table 16-1. If PLFLAG is given, only those flags that are listed can be written to the plot file. However, the flag will not be written if the associated model is not also activated through the input. The numbers specified should correspond to a valid CONTAIN data flag. An invalid flag will be ignored by the code.

an optional keyword for selecting specific cells for writing of data to the plot file. 
ncls

PLAUTO

\{ACTIVE or PASSIVE\}

PRESSURE

(pfac)

TEMP a list of cell numbers. Only the cell arrays for the cells listed will be written to the plot file. Global arrays written as one continuous record (e.g., Flag 730) are written regardless of the cells listed.

an optional keyword for activating the automatic plot dump frequency options. The user is cautioned that use of these options, especially the ACTIVE option, can cause extensive file activity. For this reason, it should not be used on machines that have slow file handling capabilities. In particular, it is not recommended on machines that handle backspacing by rewinding the file and rereading records. Vax machines running the VMS 4.5 operating system or earlier fall into this category. Cray machines running under UNICOS and Sun Sparc machines, however, are capable of backspacing quickly.

the keywords for specifying the mode of operation of the PLAUTO feature. By default, the mode of operation is passive. In passive mode, plot writes will normally occur at the user-specified ("edtdto") plot edit intervals only if one or more of the PLAUTO criteria are met. In active mode, plot writes will always occur at the user-specified plot edit intervals, and also whenever one or more of the PLAUTO criteria are met at the end of a system timestep. Note that, upon a required change, two writes will be made in the active mode: one at the beginning and one at the end of the current system timestep. A plot write is always made at the end of a time zone and at certain other events. . Default $=$ PASSIVE.

a keyword for activating the pressure change criterion for plot writes. If this keyword is omitted, changes in cell pressure are not monitored to determine the plot dump frequency.

exactly "ncells" values specifying the amount that the pressure in each cell must change before a plot write is allowed. Three options are available. If "pfac" is zero for a given cell, then the pressure in that cell is not considered in determining whether to make a plot write. (Note that this is not the same as allowing any change at all.) If "pfac" is positive, then a relative change in pressure from the last plot write whose absolute value is greater than or equal to the value specified will allow a new plot write. If "pfac" is negative, then an absolute change greater than or equal to the absolute value of "pfac" will allow a plot write. This logic applies to all of the change criteria: "pfac," "tfac," "gfac," "fpfac," and "aerfac."

a keyword for activating a gas temperature change criterion for plot dumps. If this keyword is omitted, changes in gas temperature are not considered in determining whether to make a plot write. 
tfac

GASMASS

gfac

FPMASS

fpfac

AERMASS

aerfac exactly "ncells" values specifying the amount that the temperature in each cell must change before a plot write is allowed. The three options described under "pfac" also apply to the "tfac" input.

a keyword for activating a cell atmosphere gas mass change criterion for plot writes. If this keyword is omitted, changes in total mass of gas in the cell atmosphere are not considered in determining whether to make a plot write.

exactly "ncells" values specifying the amount that the total mass of gas in each cell can change before a plot write is allowed. The three options described under "pfac" also apply to the "gfac" input.

a keyword for activating a cell fission product mass change criterion for plot writes. If this keyword is omitted, changes in total cell fission product inventory are not considered in whether to make a plot write.

exactly "ncells" values specifying the amount that the total fission product inventory in each cell can change before a plot write is allowed. The mass monitored includes fission products on all hosts, including the pool, the DUMMY location, the WASTE location, etc. The three options described under "pfac" also apply to the "fpfac" input.

a keyword for activating a suspended aerosol mass change criterion for plot writes. If this keyword is omitted, changes in aerosol suspended mass are not monitored to determine the plot write.

exactly "ncells" values specifying the amount that the total mass of airborne aerosols in each cell can change before a plot write is allowed. Note that, unlike the FPMASS option, this criterion considers only airborne aerosol. Also, masses are monitored, not mass concentrations. The three options described under "pfac" also apply to the "aerfac" input.

EOI the input block terminator. The EOI after each of the three input blocks described above is optional.

The example involves a three-cell containment model, and detailed plots of pressure in the containment versus time are desired. In this problem a gas blowdown is implemented by allowing cell 1 to discharge into cell 2 . Cell 3 in this example is assumed to be an environment cell. A sample input block is as follows:

PLFLAG 101102105205730

PLCELL 12

PLAUTO

ACTIVE

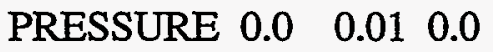


- səut! uo!̣ewrogu!

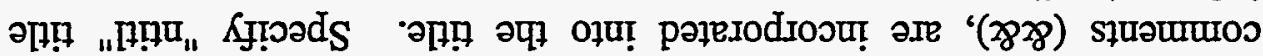

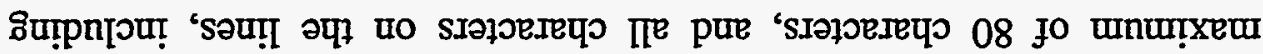

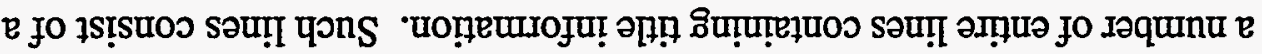

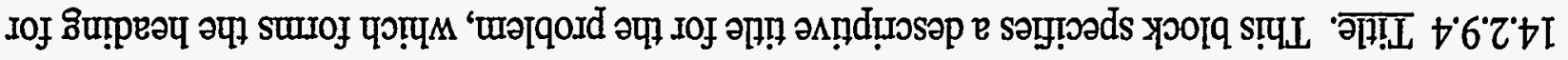

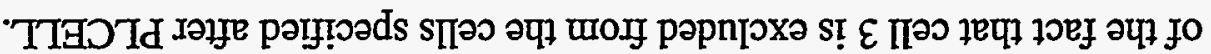

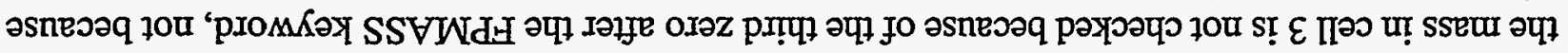

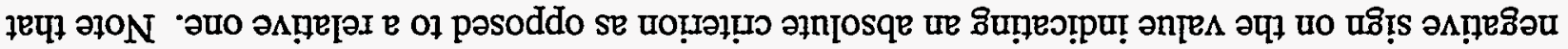

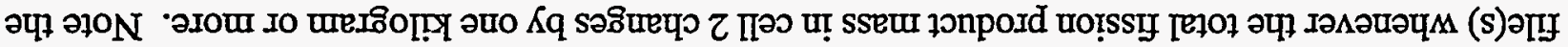

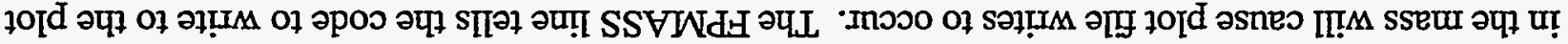

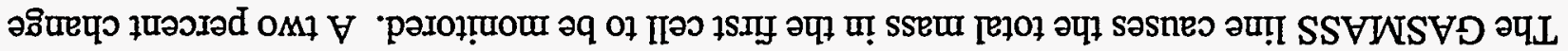

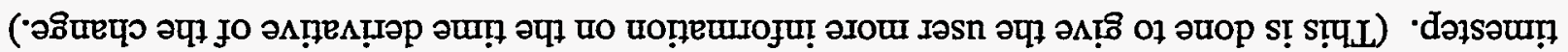

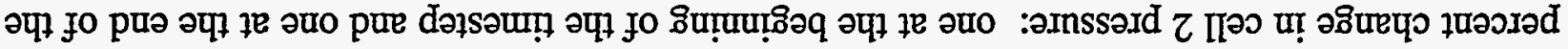

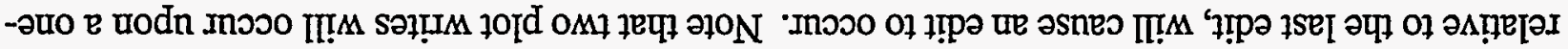

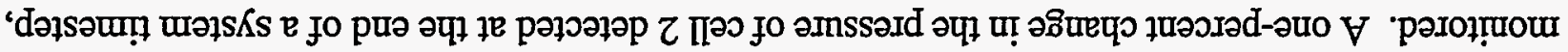

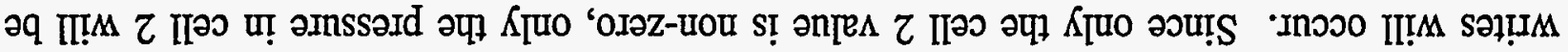

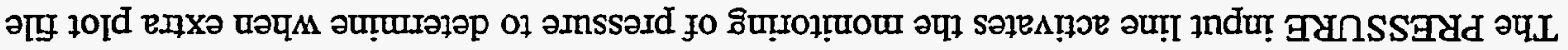

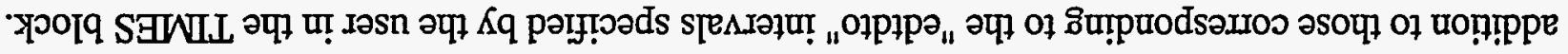

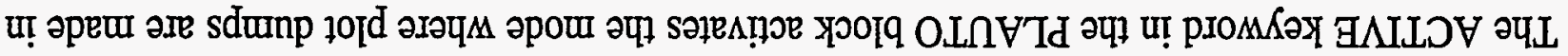

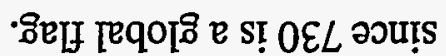

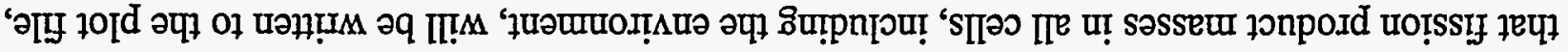

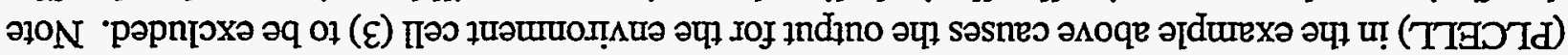

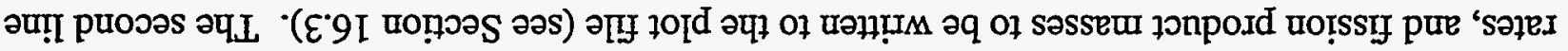

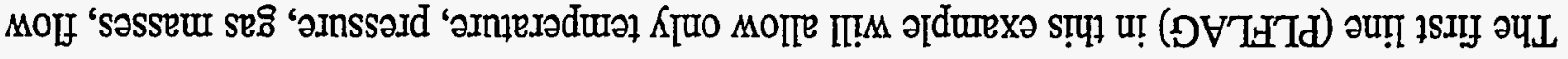




\subsection{Cell Level Input}

The cell is the basic calculational unit used in the code. A CONTAIN cell is intended to model an entire room, vault, compartment, etc. Difficulties may arise if the user tries to represent such a volume by more than one cell. In particular, the flow equations are not intended to model a continuum, and thus unrealistic flow patterns may occur within the volume.

Each cell can consist of two parts: an upper cell portion containing the cell atmosphere and a number of heat transfer structures, and a lower cell portion that may include a pool, fuel debris, concrete, and various other layers. Various physical and chemical processes can occur in both the upper and lower portions of a cell. The geometry and elevation of the cell are determined by the CELLHIST input described in Section 14.3.1.1. The cell volume is assumed to be shared by the atmosphere and lower cell pool, if any. Specific input descriptions for cell level input are found in Sections 14.3.1 through 14.3.4.

Upper Cell Models. The upper cell is the collection of models that determine the behavior of the cell. Every cell must have an upper cell, though there are many situations in which a lower cell need not be specified. Heat transfer structures are optional in the upper cell, but the atmosphere is not. There are two ways to specify a floor in a cell. It can be treated as a heat transfer structure, or it can be treated as a layer in the lower cell, where it can be in contact with the atmosphere, a coolant pool, or other layers.

Atmosphere source tables provide a way for the user to introduce external sources into the cell atmosphere. These source tables typically represent phenomena not modeled by CONTAIN, e.g., releases from the primary system, or from core-concrete interactions if the user prefers not to use the internal CORCON/VANESA module. The quantities that can be introduced through tables include mass, heat, aerosols, and fission products. Also discussed in this section are the models for heat transfer to structures. Condensation of coolant vapor onto structures may be modeled, along with normal dry convective heat transfer. Two levels of modeling are available regarding radiative heat transfer among the structures and the atmosphere. In conjunction with the radiation models, there are also two options available for modeling the emissivity of gas mixtures in the atmosphere. Finally, the hydrogen and carbon monoxide combustion models are discussed.

Upper Cell Geometry. The upper cell geometry is determined by the CELLHIST input discussed in Section 14.3.1.1. The atmosphere volume is determined by the total volume, minus the pool volume. Note that only the coolant volume in the lower cell pool is considered to affect the free volume, even though other lower cell layers may be present and other materials may be present in the pool.

Various heat transfer structures can be included to simulate roofs, walls, floors, and internal structures and equipment. The basic cell does not include any such structures automatically. They must be separately specified for each cell (see Section 14.3.1.3). If specified with an elevation, the structures' submergence below the pool surface is treated. 
Upper Cell Atmosphere Initial Conditions and Sources. The user must specify the initial conditions for the atmosphere in each cell. These initial conditions can be different from one cell to the next. Given the cell atmosphere mole fractions, pressure, temperature, and volume, the code calculates the mass of each gas present. Alternatively, the user may specify the constituent masses and temperature.

External sources of mass and energy can be provided to a cell atmosphere. Such sources can be useful for representing pheniomena such as the blowdown of coolant from the reactor coolant system into the containment. External mass and energy source rates are specified as a function of time through tables.

Atmosphere sources may consist of three types of materials: gases, coolant, dispersed debris particles (in DCH calculations). Other dispersed solids or liquids are no longer allowed except as aerosols or fission products. Materials of all three types contribute enthalpy to the atmosphere, but the ways in which their masses affect atmosphere physics are different. Gases, coolant, and debris particles are considered in both the intercell flow calculation and the atmosphere thermodynamics. In the atmosphere, the coolant is treated as a two-phase material. Therefore, sources of coolant may be either $\mathrm{H} 2 \mathrm{OV}$ or $\mathrm{H} 2 \mathrm{OL}$.

The user is cautioned not to use atmosphere source tables for aerosol materials. Aerosol sources should be specified in the cell-level aerosol AEROSOL input discussed in Section 14.3.1.8.

The input needed to activate and control models that operate at the cell level in CONTAIN is addressed in the following sections. The structure of the cell input for one cell is illustrated in Figure 14-3. The entire cell level input set is repeated "ncells" times, one set for each cell defined for the problem.

The present section discusses the CELL keyword and the cell level CONTROL block, which are given first and second, respectively, in the cell level input for each cell. In addition, the optional TITLE option for the cell is discussed.

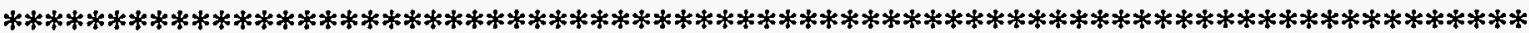

CELL ncell

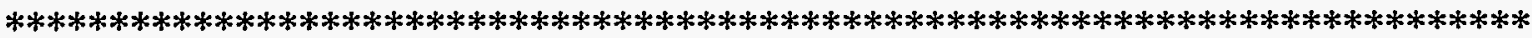

CELL the number of the cell to which the following input data blocks apply. $=$ ncell

The cell number is followed by the CONTROL keyword and the cell CONTROL information block. The cell CONTROL block is used to allocate storage space based on the models invoked in the cell. The storage allocation parameters should be set to reflect the models that will be used at any time in the calculation, not just what might be used at the initiation of the problem. For example, if a lower cell is specified for the cell, and it does not initially have a pool layer, but it is anticipated that 
a pool layer will form in the course of the calculation, then the "jpool" parameter should be given a value of 1 . This allocates the necessary storage space for the pool.

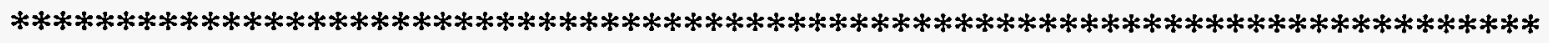

CONTROL

[NHTM=nhtm] [MXSLAB=mxslab] $[\mathrm{NSOPL}=\mathrm{nsopl}][\mathrm{NSPPL}=\mathrm{nsppl}]$

[NSOATM=nsoatm]

[NSPATM=nspatm] [NSOAER=nsoaer]

[NSPAER=nspaer] [NSOFP=nsofp] [NSPFP=nspfp] [NAENSY=naensy]

[NSOENG=nsoeng] [NSPENG=nspeng] [JCONC=jconc] [JNT=jint]

[JPOOL_-jpool]

[NUMTBC $=$ numtbc] [MAXTBC=maxtbc] [NRAYCC=nray] [NVFPSM=nvfpsm]

[NSOSAT=nsosat] [NSPSAT=nspsat] [NSOSAE=nsosae] [NSPSAE=nspsae]

EOI

[NSOSFP=nsosfp] [NSPSFP=nspsfp] [NSOSTR=nsostr] [NSPSTR=nspstr]

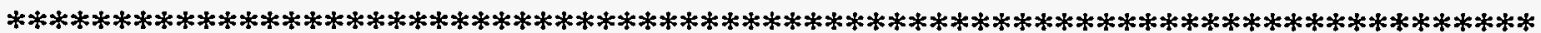

CONTROL

NHTM

$=$ nhtm

MXSLAB

$=$ mxslab

NSOPL

= nsopl

NSPPL

$=\mathrm{nsppl}$

NSOATM

= nsoatm

NSPATM

= nspatm

NSOAER

= nsoaer

NSPAER

= nspaer the keyword to begin the specification of the cell level storage allocation.

the number of heat transfer structures in the cell.

the maximum number of nodes in any heat transfer structure.

the number of external sources to the lower cell layers.

the maximum number of entries in the lower cell source tables.

the number of external sources to the upper cell atmosphere.

the maximum number of entries in the atmosphere source tables.

the number of external aerosol sources.

the maximum number of entries in the aerosol source tables. 
NSOFP

$=$ nsofp

NSPFP

$=\mathrm{nspfp}$

NAENSY

= naensy

NSOENG

= nsoeng

NSPENG

= nspeng

JCONC

$=$ jconc

JINT

$=$ jint

JPOOL

$=$ jpool

NUMTBC

$=$ numtbc

MAXTBC

$=$ maxtbc

NRAYCC

$=$ nray

NVFPSM

= nvfpsm the number of external fission product sources.

the maximum number of entries in the fission product source tables.

the number of separate engineering systems to be defined in the cell.

the number of engineered system sources.

the maximum number of entries in the engineered system source tables.

a designator indicating use of a concrete layer in the lower cell. Specify a positive integer if the concrete layer is used. If "jconc" is greater than 5 , then "jconc" is taken to be the number of nodes in the single concrete layer; if "jconc" is positive but less than 5 , the concrete layer will have 5 nodes.

a designation indicating the number of single node intermediate layers in the lower cell, if CORCON is not involved. If CORCON is involved, specify 1.

a designator indicating use of the pool layer in the lower cell. Specify 1 if the pool layer is used.

the number of cell level tables used for the cell. This number should take into account all of the tables used in the Q-VOL, HT-COEF, BCINNER, BCOUTER, and FORCED options. (Such tables are considered because these options are processed at the cell level.) Each such table defines one dependent variable in terms of an independent variable. These tables should not be confused with source tables, which have a different format and different control parameters.

the maximum number of entries in any cell level table used in the cell.

the number of CORCON rays used to model the cavity.

the total number of coefficients, representing mass fractions, used to describe the composition of the VANESA constituent materials in terms of CONTAIN fission products. See the discussion of the FPTRACK keyword in Section 14.3.2.3.2. 
NSOSAT the number of safety relief valve (SRV) gas and vapor source tables.

= nsosat

NSPSAT the maximum number of entries in the SRV gas and vapor source tables.

= nspsat

NSOSAE the number of SRV aerosol source tables.

$=$ nsosae

NSPSAE the maximum number of entries in the SRV aerosol source tables.

= nspsae

NSOSFP the number of SRV fission product source tables.

$=$ nsosfp

NSPSFP

$=\mathrm{nspsf}$

the maximum number of entries in the SRV fission product source tables.

The following keywords pertain to the film flow model. These must be used in a cell in which structure source tables simulating a surface flooding system are specified:

NSOSTR the maximum number of FILMFLOW source tables used in the current cell $=$ nsostr $\quad$ to flood structure surfaces.

NSPSTR the maximum number of time points used in any FILMFLOW source table $=$ nspstr $\quad$ specified in the current cell.

EOI the required keyword terminating the cell CONTROL block.

Unless otherwise specified, any positive integer can be assigned to each of the control variables. However, computational storage requirements can increase rapidly as the values assigned to the control variables increase.

The keyword and value pairs in the above template may be entered in any order but must be terminated by the keyword EOI. The default for each value is 0 . (Only the non-zero values need be specified.)

Another format for the CONTROL block is available. This format is discussed in Appendix B.

The CONTROL block should be followed by either the GEOMETRY block, or else the cell TITLE block and then the GEOMETRY block. The cell TITLE block is discussed below, and the GEOMETRY block in the next section. 
The cell title forms the heading for the output for the cell in the long edit:

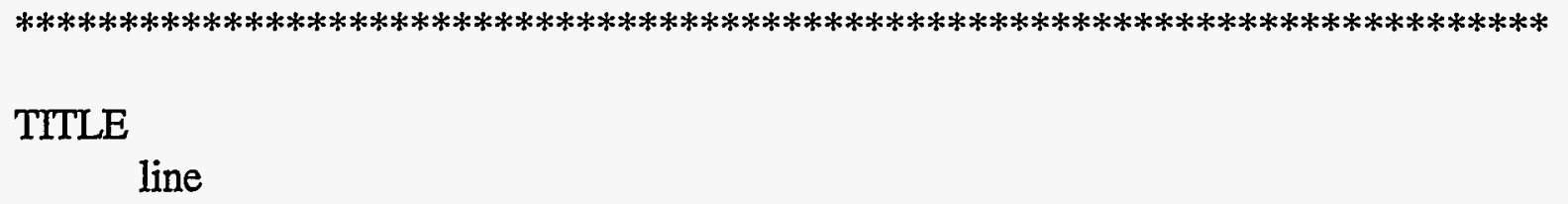

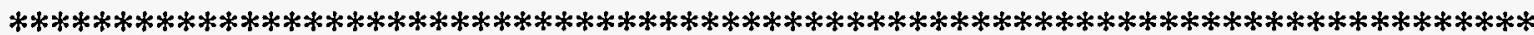

TITLE the keyword identifying the next line as a cell title.

line one line of title information describing the cell. Only one line of information is allowed for each cell.

The cell title is optional.

\subsubsection{Upper Cell}

14.3.1.1 Upper Cell Geometry. The following input block gives the initial gas volume, the cell geometry, and elevations. Note that the gas volume is adjusted for changes in the coolant volume in the lower cell pool, if any.

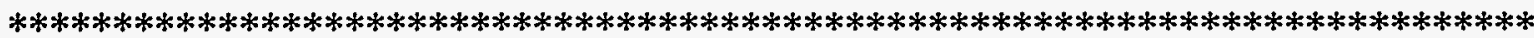

GEOMETRY

GASVOL $=$ gasvol

CELLHIST $=\mathrm{n}$ h1 area1 h2 ... hn arean hnp1

[CELLOVER = icello GASEQFR = gseqfr]

[CELLUNDR = icellu POOLEQFR = pleqfr]

EOI

$* * * * * * * * * * * * * * * * * * * * * * * * * * * * * * * * * * * * * * * * * * * * * * * * * * * * * * * * * * * * * * * * * * * * * * * * * * * * * * * *$

GEOMETRY the keyword to initiate the input of the cell geometry, initial gas volumes, and equilibration fractions. For upward compatibility, obsolete input of the form "GEOMETRY volume height" will be converted to a single cross-sectional area of "volume"/"height," which will be used instead of the CELLHIST input below. The absolute elevations of the cell top and bottom in this case will be determined from the initial gas volume, center of elevation (given in the ELEVCL input in the FLOWS block), and the coolant pool volume calculated from the lower cell input. Because the cell elevations calculated from this obsolete input may not turn out to be satisfactory, the CELLHIST input format is strongly recommended for any cell with a significant pool inventory or with pool flow paths. 
GASVOL

= gasvol

CELLHIST

n

$\mathrm{h} 1$, area1, h2, ..., hn, arean, hnpl

CELLOVER $=$ icello

GASEQFR $=$ gseqfr the initial volume of gas and any homogeneously dispersed liquid coolant in the cell. For upward compatibility, CONTAIN requires that the initial gas volume and inventory be non-zero. Because the coolant liquid in CONTAIN is treated as incompressible, a cell should never go completely water solid. The implicit flow solver will attempt to retain a gas volume at least one part in $10^{5}$ of the total cell volume in each cell.

the keyword to specify the horizontal cross-sectional area of the cell as a function of absolute elevation. If specified, the subsequent input values will override data provided by the ELEVCL input in the FLOWS input block. Also, coolant pools will be assumed to fill the cell from the cell bottom elevation according to the CELLHIST areas, not the fixed area specified by the GEOMETRY keyword in the lower cell input block. (The latter will be used only with respect to the dry pool residue and the normally dry intermediate and concrete layers.)

the number of constant horizontal cross-sectional areas used to represent the variation of the cell cross-section as a function of height. This number must be less than or equal to 10 .

the values of the cell absolute elevations (relative to the zero for the entire problem) and horizontal cross-sectional areas used to represent the cell geometry. Note that " $n "+1$ values of height " $h n$ " and " $n$ " values of area "an" should be specified in an alternating fashion, with the height " $h 1$ " at cell bottom specified first. The elevation of the top of the cell is "hnp1." The area specified between two successive values for the elevation is the crosssectional area of the cell between those two elevations. Figure 4-2 illustrates the appropriate elevations and areas for a cell with " $\mathrm{n}$ " $=3$. If $\mathrm{H}_{\mathrm{I}}=10 \mathrm{~m}, \mathrm{H}_{\text {II }}$ $=12 \mathrm{~m}, \mathrm{H}_{\text {III }}=15 \mathrm{~m}, \mathrm{H}_{\mathrm{IV}}=20 \mathrm{~m}, \mathrm{~A}_{\mathrm{I}}=100 \mathrm{~m}^{2}, \mathrm{~A}_{\mathrm{II}}=150 \mathrm{~m}^{2}$, and $A_{\text {III }}=90 \mathrm{~m}^{2}$, then the input should read "CELLHIST=3 10.0100.012.0150.015.0 90.0 20.0."

the number of the cell with which the atmosphere of the present cell is mixed and equilibrated for stability reasons, when the gas volume fraction drops below "gseqfr," and the implicit flow solver is used. The input for "gseqfr" is discussed below. This keyword may not be specified if either the intended cell "icello" or the present cell is controlled by a non-zero rate of change in the FIX-FLOW option in the FLOWS input block. Note that when gas equilibration occurs, it must occur between independent and not coupled pairs of cell gas volumes. Note this keyword also determines the characteristic length used at the atmosphere-lower-cell interface, for a shallow atmosphere, as discussed in Section 10.1.1.5.

the threshold atmosphere volume fraction of the present cell, below which the atmosphere may be mixed and equilibrated with all or part of that of cell 
"icello." The atmosphere is mixed with all or part of the atmosphere of cell "icello" so that the total volume equilibrated is, at most, equal to "gseqfr" times the present total cell volume. This mixing does not occur if cell "icello" has a finite pool mass, on the assumption that the atmospheres are not in thermal contact. The cell "icello" should be adjacent to the present cell and a nonrestrictive gas flow path should connect the gas volumes of the present cell and "icello" to minimize pressure distortions resulting from the mixing. Default $=0$.

CELLUNDR $=\mathrm{icellu}$

POOLEQFR $=$ pleqfr the number of the cell containing the pool with which the pool of present cell is mixed and equilibrated for stability reasons, when the pool volume fraction drops below "pleqfr," and the implicit flow solver is used. The input for "pleqfr" is discussed below. Note that a given "icellu" may not be used for more than one pool. Note that this keyword also determines the characteristic length used at the pool-atmosphere interface, for a shallow pool, as discussed in Section 10.1.1.5.

the threshold pool volume fraction of the present cell, below which the pool may be mixed with that of cell "icellu." The pool is mixed with all or part of the pool of cell "icellu" such that the total volume equilibrated is, at most, equal to "pleqfr" times the present total cell volume. The cell "icellu" should be adjacent to the present cell and a nonrestrictive pool flow path should connect the pools of the present cell and "icellu" to minimize pressure distortions resulting from the mixing. Default $=0$.

The keyword GEOMETRY and the geometry variables must immediately follow the cell CONTROL block or else the TITLE block if TITLE follows CONTROL.

14.3.1.2 Upper Cell Atmosphere Initial Conditions and Sources. This block specifies the initial atmosphere conditions and atmosphere sources in the upper cell. The ATMOS input format has been revised for CONTAIN 2.0. For upward compatibility, all previous formats are still accepted, as discussed in Appendix B.

Initial containment atmosphere conditions can be specified by temperature and mass or molar inventory, or by temperature and pressure and gas mass or mole fractions. Because the gas mole fraction for the coolant vapor, $\mathrm{H} 2 \mathrm{OV}$, is often given in terms of its relative humidity or quality, the coolant vapor may also be specified according to its saturation condition. Any one of the QUALTTY, SATRAT, or SATURATE keywords specifies the saturation condition. These keywords are compatible only with the use of gas mass or mole fractions. Also, when one of these keywords is given, care must be taken to not overspecify the problem (either the total pressure or coolant fraction should be omitted, depending on the initial state desired). Sample initial condition input is given at the end of this section.

It should be noted that only gas or coolant vapor material names should be specified in the atmosphere initial conditions, although DCH sources may be specified in the source block. 
Presently, DCH and other nongaseous atmospheric materials, with the exception of the coolant liquid, may not be specified in the atmosphere initial conditions. Note that the amount of coolant liquid may be specified indirectly through QUALITY or SATRAT values corresponding to a twophase initial condition. Also, if the amount of coolant vapor specified is too large to exist as vapor only under equilibrium conditions, the mass in excess of the saturated vapor mass will be placed in the liquid phase, and a diagnostic will be given.

$* * * * * * * * * * * * * * * * * * * * * * * * * * * * * * * * * * * * * * * * * * * * * * * * * * * * * * * * * * * * * * * * * * * * * * * * * * * * *$

ATMOS=nma

TGAS=tgas

[PGAS $=$ pgas]

[\{QUALITY $=x q u a l$ or SATRAT $=$ satrat or SATURATE $\}]$

[\{MOLES (ogas=xmoles) or MOLEFRAC (ogas=yfrac) or

EOI

MASSES (ogas=xmass) or MASSFRAC (ogas=xfrac) $\}$ ]

[SOURCE $=$ nso

(oname $=$ n

[DCHTYPE $=$ [ATMOSBIN or TRAPBIN or ENTRAIN\}]

[DCHBIN=isrbin] [IFLAG $=\{1$ or 2$\}]$

EOI)]

$\mathrm{T}=$ (times) $\mathrm{MASS}=$ (masses) $\{\mathrm{TEMP}=$ (temps) or $\mathrm{ENTH}=$ (enths) $\}$

$* * * * * * * * * * * * * * * * * * * * * * * * * * * * * * * * * * * * * * * * * * * * * * * * * * * * * * * * * * * * * * * * * * * * * * * * * * * * *$

ATMOS

the keyword to initiate input of atmosphere initial conditions and sources.

nma

the number of gases initially present in the atmosphere. This number should include the coolant vapor if its presence is implied through use of the QUALITY, SATRAT, or SATURATE keywords.

TGAS

the initial atmosphere temperature, which is always required. A positive

$=$ tgas

value must be specified. A temperature above the critical temperature is not allowed if coolant vapor is initially present and its amount is specified in terms of a saturation condition. (K)

PGAS

= pgas

the initial pressure of the atmosphere, which is required if (1) MASSES or MOLES is not specified and (2) the coolant saturation condition is not given through either the QUALITY, SATRAT, or SATURATE keywords, along with the coolant vapor (H2OV) mass or mole fraction. If MASSES or MOLES is specified, the initial pressure will be calculated from the constituent masses. If the saturation condition is given, along with the coolant vapor fraction, the gas pressure will be extrapolated from the vapor pressure, and the specified vapor and noncondensable fractions, if any. (Pa) 
The following three keywords, QUALITY, SATRAT, and SATURATE, all serve the same function, namely to specify the atmosphere saturation condition. The user should use only one of the three in a given ATMOS input block and only when the coolant vapor material name H2OV is not explicitly specified in that block.

QUALITY

$=\mathrm{xqual}$

SATRAT

= satrat

SATURATE

MOLES

ogas

xmoles

MOLEFRAC

yfrac

MASSES the vapor quality for initially saturated atmospheres. The quality is defined as the ratio of the vapor mass to the total coolant mass at saturation. Only values greater than zero and less than or equal to 1 may be specified. A value of one is interpreted as an atmosphere at the dew point. This option may not be used with either the MASSES or MOLES keyword. Alternatively, the amount of coolant vapor (H2OV) may be specified directly through the latter or through the MASSFRAC or MOLEFRAC keywords, in conjunction with PGAS.

the coolant saturation ratio, which is defined as the ratio of the amount of coolant relative to the amount required to saturate the atmosphere with vapor. A value greater than one will produce a two-phase condition. This option may not be used with either the MASSES or MOLES keyword. Alternatively the coolant vapor (H2OV) mass or mole fraction may be specified directly through the latter or through the MASSFRAC or MOLEFRAC keywords, in conjunction with PGAS.

a keyword equivalent to specifying SATRAT $=1$, which as discussed above places the atmosphere at the dew point. The discussion of SATRAT applies here also. Obsolete expressions in which SATURATE immediately follows a PGAS or H2OV keyword will be treated as if only SATURATE were given.

the keyword to initiate the input of moles of gas.

the name of a gas material initially present in the atmosphere.

the number of moles of species "ogas" initially present in the atmosphere. (kgmole)

the keyword to initiate the input of gas mole fractions. Note that either (1) a saturation condition keyword (i.e., QUALTTY, SATRAT, or SATURATE) and a coolant vapor (H2OV) fraction or (2) PGAS must be specified in conjunction with this keyword.

the gas mole fraction. The "yfrac" values are taken to be relative only; thus they do not have to add up to unity. The "yfrac" specified for $\mathrm{H} 2 \mathrm{OV}$ should not include the coolant liquid, if any.

the keyword to initiate the input of the masses of gases. 
the mass of species "oname" initially present in the atmosphere. If "oname" is one of the DCH materials, the mass will be placed in the trapped bin. (kg)

MASSFRAC

the keyword to initiate the input of gas mass fractions and masses of nongases. Note that either (1) a saturation condition keyword (i.e., QUALITY, SATRAT, or SATURATE) and a coolant vapor (H2OV) fraction or (2) PGAS must be specified in conjunction with this keyword.

xfrac

the mass fraction of "ogas." The values specified are interpreted as relative, thus they do not have to add up to unity. The "xfrac" for H2OV should not include the coolant liquid, if any.

EOI

the input block terminator.

The keyword SOURCE may be used here to introduce any gas or DCH material declared after the COMPOUND keyword or USERDEF keyword in the MATERIAL block to the upper cell atmosphere. Sources of other nongaseous materials are no longer accepted. For a discussion of the keywords following SOURCE, see Section 14.4.1.

SOURCE the keyword to initiate input of source information.

nso

the total number of sources to be specified after the atmosphere SOURCE keyword. This total must include atmosphere gas sources and all types of debris sources, as discussed below. It does not include source types specified in other SOURCE blocks, such as aerosol, fission product, and lower cell sources. All atmosphere gas and debris sources, and debris-entrainment related source tables should be grouped together under a single SOURCE block. The code will not operate properly if more than one atmosphere SOURCE block is provided.

oname

the name of a material to add to the atmosphere. If "oname" is one of the DCH materials, the source table can represent either (1) a user-specified external source of debris to the atmosphere, (2) a user-specified external source of debris to the trapped bin (entrainment reservoir), or (3) a userspecified entrainment rate out of the trapped bin. The DCHTYPE keyword described below is used to specify how a DCH source table is used.

DCHTYPE

the keyword to invoke either the TRAPBIN or ENTRAIN options for this source table. If DCHTYPE is not specified, the ATMOSBIN option will be used by default.

ATMOSBIN an option to specify this DCH source table as a source of debris to the atmosphere. The DCH material either is placed in a specific bin, determined by the DCHBIN input discussed below, or is divided among bins according 
to the FDISTR input (see the DHEAT input block discussed in Section 14.2.7).

TRAPBIN an option to specify the source table as a source of debris to the trapped bin. The temperature of the debris added to the trapped bin is given by the standard TEMP or ENTH source inputs.

ENTRAIN

an option to specify this DCH source table as a source of debris out of the trapped bin into the atmosphere.. The DCH material either is placed in a specific bin, determined by the DCHBIN input discussed below, or is divided among bins according to the FDISTR input (see the DHEAT input block discussed in Section 14.2.7). The temperature of the debris added to the atmosphere will be the temperature of the debris in the trapped bin. If TEMP or ENTH is given, the values will be ignored under this DCH source option.

DCHBIN

$=$ isrbin

target bin for a user-specified external DCH source or user-specified entrainment rate from the trapped bin. If DCHBIN is specified, the source will be added to the specified bin, "isrbin" in the generation that is active at the time the source is invoked. This option cannot be used for DCH source tables with DCHTYPE equal to TRAPBIN. By default, the debris will be distributed across bins according to FDISTR input (see the DHEAT input block discussed in Section 14.2.7).

The following examples demonstrate how the ATMOS input might be used (only initial conditions are illustrated).

Example 1. The pressure and temperature are known for an steam/air atmosphere, with an steam/air ratio of 0.35 .

$\operatorname{ATMOS}=3$

PGAS=1.E5 TGAS=335. MOLEFRAC N2 $=0.79$ O2 $=0.21 \mathrm{H} 2 \mathrm{OV}=.35$

EOI

In this case, the sum of the input mole fractions exceeds one, but actual mole fractions used are the properly normalized values. A two-phase condition results since the indicated vapor mole fraction is not possible (at equilibrium) at the temperature and pressure given. (The saturated vapor pressure is only $2.171 \times 10^{4}$ at $335 \mathrm{~K}$, or 0.2171 times the total pressure, but the indicated vapor mole fraction is $0.35 / 1.35=0.2592$. Consequently, a two-phase condition is assumed with the total water mole fraction set to 0.2592. A diagnostic is given in the error file in this case, and any other case, in which the specified vapor condition cannot be attained.)

Example 2. In this case the temperature, pressure, and relative humidity of $88 \%$ are known and the noncondensable gases are known to be those in air. 
$\mathrm{ATMOS}=3$

PGAS $=1.0 \mathrm{E} 5$ TGAS $=335$ SATRAT $=0.88$

MOLEFRAC N2 $=0.79 \mathrm{O} 2=0.21$

EOI

Note that the saturation ratio is given in lieu of the water vapor mole fraction. Also, note the number of gases specified after ATMOS includes the water vapor.

Example 3. The gas temperature is known, the gas pressure is not known, but the atmosphere is known to be air with condensed water (fog) at a saturation ratio of $110 \%$, and the vapor molar fraction, excluding the liquid, is known to be $20 \%$.

ATMOS $=3$

TGAS $=335$ SATRAT $=1.1$

MOLEFRAC N2 $=0.632 \mathrm{O} 2=0.168 \mathrm{H} 2 \mathrm{OV}=0.20$

EOI

In this case, the $\mathrm{H} 2 \mathrm{OV}$ mole fraction refers only to the vapor, since the specified conditions are attainable. Since the atmosphere is at or above saturation, one could specify QUALITY $=0.9091$ instead of SATRAT.

Example 4. The atmosphere is known to be pure steam at saturation at $373 \mathrm{~K}$.

ATMOS=1

TGAS=373 SATURATE

EOI

14.3.1.3 Heat Transfer Structure Characteristics. This block provides the characteristics of the heat transfer structures modeled in the cell.

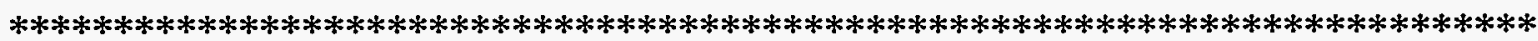

STRUC

[CRANK=crank]

[OUTGAS

[\{TRANGE or KINETIC $\}]$

[\{H2OESPAN tspan pfac or TH2OE tlohoe thihoe\}]

[ $\{\mathrm{TH} 2 \mathrm{OB}=$ tlohob thihob or $\mathrm{KH} 2 \mathrm{OB}=\mathrm{kh} 2 \mathrm{ob}$ eh2ob $\}]$

[ $\{\mathrm{TCO} 2=$ tloco2 thico2 or $\mathrm{KCO} 2=\mathrm{kco} 2 \mathrm{eco} 2\}]$

$[\mathrm{QH} 2 \mathrm{OE}=\mathrm{qh} 2 \mathrm{oe}][\mathrm{QH} 2 \mathrm{OB}=\mathrm{qh} 2 \mathrm{ob}][\mathrm{QCO} 2=\mathrm{qco} 2]$

[DESTCELL=(ndcell)]

[FH2OE=fh2oe] $[\mathrm{FH} 2 \mathrm{OB}=\mathrm{fh} 2 \mathrm{ob}][\mathrm{FCO} 2=\mathrm{fco} 2]$

EOI]

([NAME=name $]$ TYPE=type SHAPE=shape $N S L A B=$ nslab

CHRLEN=chrlen [ADJUSTCL] 
[SLAREA=slarea] [SLHITE=slhite] [SLELEV=slelev]

[CYLHITE=cylhite] [CYLELEV=cylelv] [CYLTHETA = cyltht]

[VUFAC=vufac]

$\{T U N I F=$ tunif or $T N O D E=$ (tnode) $\}$

COMPOUND $=$ (cnames)

$\mathrm{X}=$ (xvals)

[CONCDATA

[\{FH2OE=fh2oe or H2OENODE $=($ h2oenode $)\}]$

[ $\{\mathrm{FH} 2 \mathrm{OB}=$ fh $2 \mathrm{ob}$ or $\mathrm{H} 2 \mathrm{OBNODE}=(\mathrm{h} 2 \mathrm{obnode})\}]$

$[\{\mathrm{FCO} 2=\mathrm{fc0} 2$ or $\mathrm{CO} 2 \mathrm{NODE}=(\mathrm{co} 2 \mathrm{node})\}]$

EOT]

[BCINNER

$\{[R O H A N D]$ or

[NATCOR1 a1 b1 c1 d1]

[NATCOR2 a2 b2 c2 d2]

$\{[\mathrm{STBNCOR}]$ or $[\mathrm{EXPNCOM}=\mathrm{xm} 1]\}\}$

[FORCOR1 a3 b3 c3 d3]

[FORCOR2 a 4 b4 c4 d4]

[\{STBFCOR or EXPFCOM=xm2\}]

[MIXED

[CSIGN=csign]

[EXPMIX $=\mathrm{xm} 3$ ]

[FACMIN=facmin]

[HMXMUL $=\mathrm{hmxmul]}$

[RMX2HX=rmx2hx]]

VAR-PARM

[FLAG=iflag] [NAME=oname]

VAR-X=TIME

$X=n p$ (xpts)

VAR-Y $=\{$ VELOCITY or REY-NUM or NUS-FORC $\}$

EOI]

$\mathrm{Y}=\mathrm{np}$ (ypts)

[VELCOEF

[(FLOWPATH,i,j=valin valout)]

[(ENGVENT, $n=$ valin valout)]

EOI]

[POOLFLOW=valin valout]

[HYDAREA=hdarea]

[FILMFLOW

[DIVERT $=$ nstrs

(FRAC=frac $\{$ NAME=sname or NUMBER=snum $\}$

[CELL=ncell]

EOI)]

[SLOPE=theta] [WIDTH=width]

EOI]

Rev. 0 


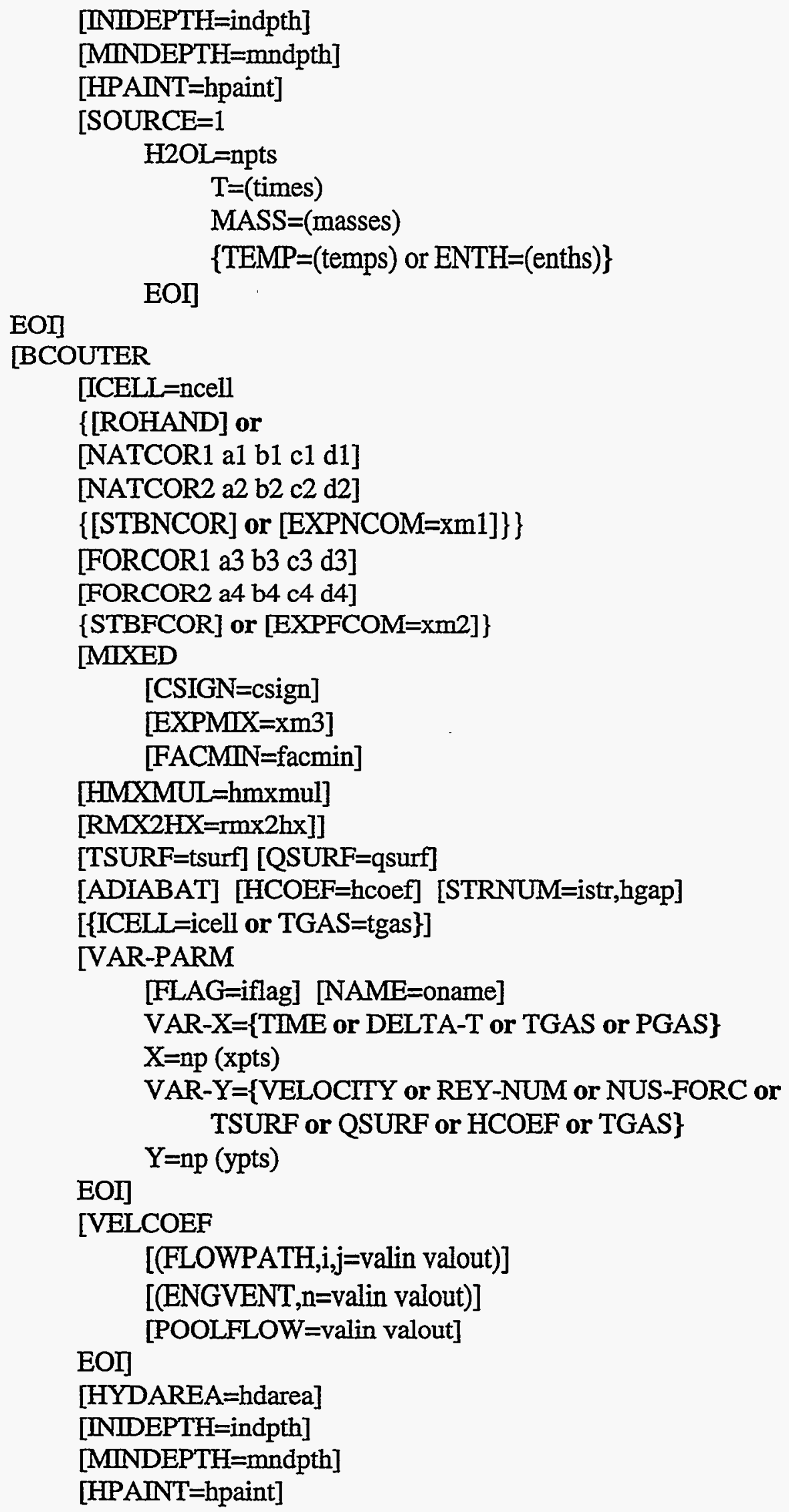




\section{EOI] \\ EOI)}

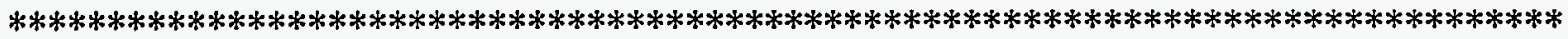

The keywords from "NAME=name" to the final EOI should be repeated for each of the "nhtm" structures. The CRANK and OUTGAS keyword blocks are given once and apply to all structures. They must be specified before the first structure is defined. The other keyword blocks may be given in any order. The TOUTER and IOUTER keywords used in versions prior to CONTAIN 1.11 do not appear in the above template since they are now obsolete (see Appendix B). The function previously served by these two keywords are now provided by TGAS and ICELL in the BCOUTER block.

The three allowed shapes for structures are SLAB, CYLINDER, and SPHERE. Cylinders and spheres are actually partial cylinders and hemispheres whose inner surfaces are defined as a ROOF, WALL, or FLOOR by the TYPE keyword. The azimuthal angle subtended by the partial cylinder is specified through the CYLTHETA keyword and is by default $180^{\circ}$. Thus, to model a whole cylinder or a complete sphere, the azimuthal angle or more than one structure should be specified. Note that the outer face of a ROOF is a floor, and the outer face of a FLOOR is a ceiling.

An inner face is by default always considered exposed in the cell in which the structure is defined. Such exposure invokes the modeling of a basic set of processes such as convective heat transfer, coolant film formation and transport, and aerosol and fission product surface deposition. While such basic processes are also modeled for the outer face if specified as exposed in the same cell as the inner face, the outer face is often best reserved for alternative boundary conditions not available for the inner face. Although the basic options are available, if the outer face is specified as exposed in the same cell, the structure may not be submerged below the pool surface, and other modeling options available for the inner face are not available for the outer face. For example, the film tracking model, concrete outgassing, and certain radiative heat transfer options are available for inner but not outer faces. In particular, the modeling of radiative heat transfer from a lower cell to structures and the net enclosure modeling option is allowed for inner but not outer faces. If the outer face is given an external boundary condition or is exposed to the gas in a different cell, the inner face may be submerged and only the heat transfer options designated as external in the BCOUTER block may be used. For such outer faces, neither submergence with respect to the outer face, surface film modeling, condensation, or aerosol deposition is modeled. If more than basic "inner" surface modeling on two sides of structure is desired and concrete outgassing is not required, the structure may be divided into two structures with a common boundary (specified through the STRNUM and ICELL keywords). Finally, the distinction between "inner" and "outer" is arbitrary. The "inner" face is the one whose node position is given first following the $\mathrm{X}$ keyword.

A certain subset of the available structure types and shapes is considered submersible in the pool of the cell, as discussed in Section 10.1.1.4. This includes slab structures of any type and partial cylindrical structures of type WALL. A submersible structure must have its elevation specified through either the SLELEV or CYLELEV keyword. If the elevation is not specified, the structure will be assumed to be in contact with the gas only. 
STRUC

CRANK

$=$ crank

OUTGAS

TRANGE

KINETIC

H2OESPAN

tspan

pfac the keyword to begin the definition of structures.

the time integration factor, defined as the quantity $c$ in Equation (10-121). Any value between 0 and 1 can be given; however, values less than 0.5 are not recommended for stability reasons. The value given here affects all structures in the cell. Default $=1$ (corresponds to fully implicit).

a keyword to activate concrete outgassing for structures in the cell. Values specified in this block are used in the outgassing model for all structures that can outgas, unless overridden by subsequent values specified for an individual structure. The OUTGAS keyword may be specified only once. In CONTAIN 1.11 a structure is allowed to outgas if and only if the CONCDATA block is given for that structure (see below) and the inner node of that structure consists of the material CONC. In CONTAIN 1.12 and later, the OUTGAS block is required for outgassing, but the CONCDATA block is not required if at least one of the values for the outgassing inventory mass fractions ("fh2oe," "fh2ob," and "fco2") is defined for all structures in the cell in the OUTGAS block, in lieu of the CONCDATA block. All structures defined after OUTGAS is specified will use these cell values if subsequent overriding CONCDATA values are not specified. Note that the outgassing will affect the thermal properties of a structure CONC node. Note also that only the innermost block of CONC nodes is allowed to outgas and then only for that part of the structure that is in contact with the atmosphere gas. The submerged part of a structure is assumed not to participate while submerged.

a keyword to select the temperature range model for bound gas release. (Evaporable water release is always modeled using the temperature range approach.) In general, this model is less mechanistic than the Arrhenius model but provides an alternative formulation. See the discussion of TH2OB and TCO2 below.

the keyword to select the Arrhenius model in Equation (10-148) for bound gas release. If neither TRANGE nor KINETIC is specified, the Arrhenius model will be used.

a keyword to allow the user to specify the parameters used in the pressure dependent evaporable water release model.

the temperature offset in Equation (10-141) describing the evaporable water release model. Default $=10$. $(\mathrm{K})$

the pressure coefficient in Equation (10-141) describing the evaporable water release model. Default $=0$. 
TH2OE

tlohoe

thihoe

TH20B

tlohob

thihob

$\mathrm{KH} 2 \mathrm{OB}$

kh2ob

eh2ob

TCO2

tloco2

thico2

$\mathrm{KCO} 2$ a keyword to allow the user to specify a fixed value of $T_{10}$ and $T_{h j}$ independent of pressure in the evaporable water release model. These parameters are discussed in conjunction with Equation (10-141).

the temperature at which outgassing of evaporable water will begin. (K)

the temperature at which all evaporable water will be released. (K)

a keyword to initiate the specification of the temperature range for the ougassing of bound water. This keyword should be used only if bound water release is modeled using the temperature range approach (see TRANGE above).

the temperature at which structure nodes will begin to release chemically bound water. Default $=473$. $(\mathrm{K})$

the temperature at which structure nodes will have released all chemically bound water. Default $=873$. $(\mathrm{K})$

a keyword to initiate the specification of the rate constant and activation energy in the Arrhenius model for bound water release. This keyword should be used only if bound water release is modeled using the Arrhenius approach (see KINETIC above).

the kinetic rate constant, $\kappa_{b}$, in Equation (10-148) for bound water release. Default $=3.29 \times 10^{10} .\left(\mathrm{s}^{-1}\right)$

the activation energy divided by the gas constant, $E_{b} / R$, in Equation (10-148) for bound water release. Default $=2560$. $(K)$

a keyword to initiate the specification of the temperature range for outgassing of $\mathrm{CO}_{2}$. This keyword should be used only if $\mathrm{CO}_{2}$ release is modeled using the temperature range approach (see TRANGE above).

the temperature at which structure nodes will begin to release $\mathrm{CO}_{2}$. Default $=873$. $(\mathrm{K})$

the temperature at which structure nodes will have released all $\mathrm{CO}_{2}$. Default $=1503$. $(\mathrm{K})$

a keyword to initiate the specification of the rate constant and activation energy in the Arrhenius rate equation for $\mathrm{CO}_{2}$ release similar to Equation (10148). This keyword should be used only if $\mathrm{CO}_{2}$ release is to be modeled using the Arrhenius approach (see KINETIC above). 
kco2

eco2

$\mathrm{QH} 2 \mathrm{OE}$

$=\mathrm{qh} 20 \mathrm{e}$

$\mathrm{QH} 2 \mathrm{OB}$

$=\mathrm{qh} 2 \mathrm{ob}$

$\mathrm{QCO} 2$

$=q c 02$

DESTCELL

$=$ (ndcell)

FH2OE

$=\mathrm{fh} 2 \mathrm{oe}$

FH2OB

$=\mathrm{fh} 2 \mathrm{ob}$

FCO2

$=\mathrm{fco} 2$ the decomposition rate constant, $\kappa_{\mathrm{c}}$ for bound $\mathrm{CO}_{2}$ release, similar to $\kappa_{\mathrm{b}}$ for bound water. Default $=3.286 \times 10^{5} .\left(\mathrm{s}^{-1}\right)$

the activation energy divided by the gas constant, $\mathrm{E} / \mathrm{R}$, for bound $\mathrm{CO}_{2}$ release similar to $E_{8} / R$ for bound water. Default $=19362$. $(K)$

the standard heat of vaporization required to release evaporable $\mathrm{H} 2 \mathrm{O}$. Default values are calculated as the heat of vaporization. $(\mathrm{J} / \mathrm{kg})$

the standard heat of decomposition required to release bound $\mathrm{H}_{2} \mathrm{O}$. Default values are calculated as the heat of vaporization. $(\mathrm{J} / \mathrm{kg})$

the standard heat of decomposition required to release $\mathrm{CO}_{2}$. Default $=4.18$ $\times 10^{6} .(\mathrm{J} / \mathrm{kg})$

the number of the cell to receive the released gas. Specify "nhtm" values, one for each structure. If the cell number is not specified or is given as zero, a structure will outgas into the cell containing the structure but only if the innermost node of the structure is composed of the material CONC. Note that only the innermost block of CONC nodes in the unsubmerged part of the structure may outgas. If a valid cell number is specified, the part of the structure not immersed in the pool will outgas into the cell specified regardless of the position of the innermost block of CONC nodes. Default $=0$.

the mass fraction of the CONC material assumed to be evaporable (as opposed to bound) water. The specific heat of a CONC node subject to nonzero values of "fh2oe" is calculated as a weighted average of the evaporable water and CONC specific heats. Otherwise the specific heat of the pure CONC material is used for the node. Use the alternative H2OENODE keyword if the mass fraction is not uniform for all CONC nodes. If a value of "fh2oe" is specified in the OUTGAS input block, it becomes the default for values of the evaporable water fraction in subsequent CONCDATA blocks. If "fh2oe" is not specified in the OUTGAS block, the default value is zero.

the mass fraction of the CONC material assumed to be bound water. With the outgassing of bound water, the porosity of a node is assumed to increase. The values and defaults for "fh $2 \mathrm{ob}$ " are set in a manner similar to those for "fh2oe."

the mass fraction of the $\mathrm{CONC}$ material assumed to be releasable $\mathrm{CO}_{2}$. With the outgassing of the releasable $\mathrm{CO}_{2}$, the porosity of a mode is assumed to increase. The values and defaults for "fco2" are set in a manner similar to those for "fh2oe." 
NAME

$=$ name

TYPE

= type

SHAPE

$=$ shape

NSLAB

$=$ nslab

CHRLEN

$=$ chrlen

ADJUSTCL

SLAREA

= slarea

SLHITE

$=$ slhite

SLELEV

= slelev the optional user-selected name for the structure. The name may have up to eight characters. Note that, although the name is optional within CONTAIN, it is used in POSTCON postprocessing, as discussed in Chapter 16.

the name of the type of structure, which may be either ROOF, WALL, or FLOOR. These names refer to the orientation of the structure inner surface.

the name of the shape of the structure, which may be either SLAB, CYLINDER, or SPHERE. See the discussion above for the actual shapes invoked by these names.

the number of nodes in the structure. The number must be less than or equal to "mxslab," defined in the cell CONTROL block.

the characteristic length of the structure to be used in heat transfer correlations. If the ADJUSTCL keyword is given with respect to a submergible structure, an interpolated value depending on the submergence is the actual length used in the heat transfer correlations, and "chrlen" becomes the asymptotic value used when the pool surface level is at the top or bottom of the cell, or both. (m)

a keyword to interpolate the characteristic length of a submergible structure according to its submergence, as discussed in Section 10.1.1.5. In this option the value of "chrlen" specified by the user becomes an asymptotic characteristic length used when the pool level is at either the top or bottom of the cell, or both. Note that continuity requires that "chrlen" be less than or equal to a maximum value, which is determined by structure and/or cell dimensions. For a FLOOR slab, for example, this maximum value is the distance between the floor top surface and the top of the cell. If "chrlen" exceeds this maximum and the ADJUSTCL option is invoked, the code will abort. The minimum value used for the interpolated characteristic length is $0.001 \mathrm{~m}$.

the area of a slab structure. $\left(\mathrm{m}^{2}\right)$

the height (vertical extent) of a slab structure of type WALL. This height is used to calculate the submergence of the structure in a cell with a pool. (m)

the absolute elevation of the center of volume of a slab structure, to be used in calculating the submergence of the structure in a cell with a pool. By default the structure is assumed to be in contact only with the gas. For connected structures, this elevation should correspond to the center of volume of the appropriate individual structure, not the combined structures. (m) 
CYLHITE

$=$ cylhite

CYLELEV
$=$ cylelv

CYLTHETA

$=$ cyltht

VUFAC

$=$ vufac

TUNIF

$=$ tunif

TNODE

tnode

COMPOUND

cname

$\mathrm{X}$

(xvals) the axial length of a cylindrical structure. The old form CYLHT = "cylht" may also be used. (m)

the absolute elevation of the center of volume of a cylindrical structure of type WALL, to be used in calculating the submergence of the structure in a cell with a pool. It should be noted that cylindrical structures only of type WALL may be submerged. By default the structure is assumed to be in contact with the gas space only. (m)

the azimuthal angle subtended by a partial cylindrical structure, in degrees. This input is provided to facilitate the modeling of submergible cylinders, since the common practice of taking penetrations into account by reducing the effective cylinder height introduces distortions in the calculated submergence. Default $=180^{\circ}$, corresponding to a half-cylinder. (degrees)

the view factor between structure and the lower cell as defined in Equation (10-36).

the initial uniform temperature of the structure. $(\mathrm{K})$

a keyword to begin the specification of the initial temperature for each of the nodes of the structure. Use either TUNIF or TNODE, but not both.

the value of the initial temperature of a node. Specify "nslab" values, going from the inner to the outer node. $(\mathrm{K})$

the keyword to begin the specification of the material constituting each node. Each material must be among the materials specified after either the COMPOUND keyword or the USERDEF keyword in the MATERIAL block.

the name of the material in a node. Specify "nslab" names, going from the inner to the outer node.

the keyword to begin the specification of the position of each of the node interfaces.

the "nslab" +1 positions of the node interfaces, starting with the position of the inner surface. For cylindrical or hemispherical structures the positions are given in terms of the radius, which must be positive definite, except possibly for the outer surface, which can have a zero radius. The values may be given in either ascending or descending order, depending on which surface is considered to be the inner or, more precisely, inward surface. 
the keyword to begin specification of concrete outgassing parameters for the present structure. Other keywords may follow to select the amount of outgassable water and $\mathrm{CO}_{2}$ in the structure. Note that the concrete composition parameters "fco2," "fh2ob," and "fh2oe" may also be specified in the OUTGAS input block for those structures that have uniform concrete composition. If the parameters specified in OUTGAS are satisfactory, CONCDATA need not be specified for a given structure.

H2OENODE

$=(\mathrm{h} 2$ oenode $)$

H2OBNODE

$=(\mathrm{h} 2$ obnode $)$

CO2NODE

$=($ co2node $)$

BCINNER

ROHAND

NATCOR1

$=\mathrm{a} 1 \mathrm{~b} 1 \mathrm{c} 1 \mathrm{~d} 1$

NATCOR2 $=\mathrm{a} 2 \mathrm{~b} 2 \mathrm{c} 2 \mathrm{~d} 2$

STBNCOR

\section{EXPNCOM} $=\mathrm{xm} 1$ the evaporable water mass fraction, defined as for "fh2oe," except that "nslab" values are specified, one for each node in the structure.

the bound water mass fraction, defined as for "fh2ob," except that the "nslab" values are specified, one for each node in the structure.

the $\mathrm{CO}_{2}$ mass fraction defined as for "fco2," except that "nslab" values are specified, one for each node in the structure.

the keyword to begin the specification of the inner surface boundary condition for a structure.

the keyword to specify that the natural convection correlations given in Equations (10-24) through (10-26) from Reference Roh85 are to be used. No other input required.

parameters to define $\mathrm{N}_{\mathrm{Nu}, \mathrm{c}, \mathrm{l}}$ as in Equation (10-27). This keyword is optional but, if specified, it must be followed immediately by the four values "a1," "b1," "c1," and "d1," in that order. These values correspond to the parameters $a_{1} \ldots d_{1}$ in Equation (10-27). Defaults: none.

parameters to define $\mathrm{N}_{\mathrm{Nuc}, 2}$ as in Equation (10-27). This keyword is optional but, if specified, it must be followed immediately by the four values "a2," "b2," "c2," and "d2," in that order. These values correspond to the parameters $a_{2} \ldots d_{2}$ in Equation (10-27). Defaults: none.

the keyword to specify that the choice between NATCOR1 and NATCOR2 is to be based upon the boundary layer stability criterion, with NATCOR1 to be used when the boundary layer density gradient is stabilizing and NATCOR2 when the gradient is destabilizing. If STBNCOR is applied to a structure of type WALL, the criterion will be applied as if it were type FLOOR.

the keyword and value to specify that the two natural convection correlations are to be combined as in Equation (10-28). The keyword must be followed immediately by "xm1," the value of the exponent $\mathrm{m}_{1}$ in Equation (10-28). "xm1" > 0 is required. Default: none. 
FORCOR 1

$=\mathrm{a} 3 \mathrm{~b} 3 \mathrm{c} 3$

d3

FORCOR2

$=\mathrm{a} 4 \mathrm{~b} 4 \mathrm{c} 4$ d4

STBFCOR

EXPFCOM $=\mathrm{xm} 2$

MTXED

CSIGN

$=$ csign

EXPMIX

$=\mathrm{xm} 3$

FACMIN

$=$ facmin

HIMXMUL

$=\mathrm{hmxmul}$

$\mathrm{RMX} 2 \mathrm{HX}$

$=\mathrm{rmx} 2 \mathrm{hx}$ parameters to define $\mathbf{N}_{\mathrm{Nu}, \mathrm{f}, 1}$ as in Equation (10-29). This keyword is optional but, if specified, it must be followed immediately by the four values "a3," "b3," "c3" and "d3," in that order. These values correspond to the parameters $a_{3} \ldots d_{3}$ in Equation (10-29). Defaults: none.

parameters to define $\mathrm{N}_{\mathrm{Nu}, \mathrm{f}, 2}$ as in Equation (10-29). This keyword is optional but, if specified, it must be followed immediately by the four values "a4," "b4," "c4," and "d4," in that order. These values correspond to the parameters $a_{4}, \ldots, d_{4}$ in Equation (10-29). Defaults: none.

the keyword to specify that the choice between FORCOR1 and FORCOR2 is to be based upon the boundary layer stability criterion, with FORCOR1 to be used when the boundary layer density gradient is stabilizing and FORCOR2 when the gradient is destabilizing. If STBFCOR is applied to a structure of type WALL, the criterion will be applied as if it were type FLOOR.

the keyword and values to specify that the two forced convection correlations are to be combined as in Equation (10-30). The keyword must be followed immediately by "xm2," the value of the exponent $\mathrm{m}_{2}$ in Equation (10-30). "xm2" > 0 is required. Default: none.

the keyword to specify that the mixed convection option described by Equation (10-32) is to be used.

the value of $C_{1}$ in Equation (10-32). Default $=-1$.

the exponent $\mathrm{m}_{3}$ in Equation (10-32). Note that "xm3" must be greater than zero. Default $=3$.

the value of $C_{2}$ in Equation (10-32). Default $=0.75$.

the multiplier applied to both $\mathrm{N}_{\mathrm{Nu}}$ and $\mathrm{N}_{\mathrm{Sh}}$. It must not be negative; it may be zero. Default $=1$.

the multiplier applied to $\mathrm{N}_{\mathrm{Sh}}$ only. It must not be negative; it may be zero. Note that if both HMXMUL and RMX2HX are specified, $\mathrm{N}_{\mathrm{Sh}}$ will be multiplied by both values while $\mathrm{N}_{\mathrm{Nu}}$ is multiplied only by the HMXMUL value. Note that the cell-level CONDENSE keyword must also be specified for condensation or evaporation to occur with respect to structure surfaces. Default $=1$. 
The user is reminded that the default natural convection correlations in Equations (10-20) through (10-22) are overridden if the user specifies even one of the three keywords ROHAND, NATCOR1, or NATCOR2. Otherwise, the default natural convection correlations remain in effect. Likewise, the default forced convection correlation in Equation (10-23) is overridden if even one of the keywords FORCOR1 or FORCOR2 is specified. Otherwise, the default forced convection correlation remains in effect. If a table option is used to define forced convection information in addition to FORCOR1 and/or FORCOR2, the resulting Reynolds numbers will be used in the userdefined forced convection correlations unless $\mathrm{N}_{\mathrm{Nu}, \mathrm{f}}$ has been specified directly in the table. In this case, FORCOR1 and/or FORCOR2 will be ignored. As before, the value of the $\mathrm{N}_{\mathrm{Nu}}$ eventually used will be based upon $\max \left(\mathrm{N}_{\mathrm{Nu}, \mathrm{c}}, \mathrm{N}_{\mathrm{Nu}, f}\right)$ unless MIXED input is specified.

The following keywords are used to specify external or forced convective boundary conditions in tabular form. The format is a standard one for global or cell level tables, as discussed in Section 14.4.2. The optional keywords FLAG and NAME are discussed in that section. Note that only one table may be specified for each surface.

VAR-PARM the keyword to begin the specification of a boundary condition table. The tabular boundary conditions can be specified as a function of time, cell temperature, gas-wall temperature difference, or cell pressure. The number of points in the table may not exceed the value of "maxtbc" specified in the cell CONTROL block.

VAR-X required keyword to specify the independent variable. The available options for the name of the independent variable to follow are:

TIME - time (s)

TGAS - gas temperature adjacent to face $(\mathrm{K})$

DELTA-T - gas temperature minus surface temperature. (K)

PGAS - pressure of cell adjacent to face $(\mathrm{Pa})$

Note that for a TIME table, the value for VAR-Y will be set to zero outside of the range of the table; for other options, end point values will be used.

When used with BCOUTER to specify the outer boundary condition, the cell temperature or pressure are those of cell "icell."

$\mathrm{X}$

the required keyword to initiate the input of the independent variable values in the table.

np the number of points in the table.

xpts specify "np" values of the independent variable in ascending order.

VAR-Y the required keyword to specify the dependent variable. For a BCINNER boundary condition or a BCOUTER boundary condition in the same cell, 
VELOCITY, REY-NUM, and NUS-FORC are the only available options for the dependent variable. The other options are available for external boundary conditions or for outer surface exposed to the gas in a cell different from that in which the structure is defined.

VELOCITY - the velocity to use in the forced convective correlation for the surface. (Available only for a TIME independent variable.) $(\mathrm{m} / \mathrm{s})$

REY-NUM - the Reynolds number to use in the forced convective correlation for the surface. (Available only for a TIME independent variable.)

NUS-FORC - the forced convective Nusselt number to use for the surface. (Available only for a TIME independent variable.)

TSURF - the surface temperature. (Not available for a DELTA-T independent variable.) $(\mathrm{K})$

QSURF - the heat flux to surface $\left(\mathrm{W} / \mathrm{m}^{2}\right)$

HCOEF - the heat transfer coefficient for convective heat transfer. The gas temperature used is the temperature dictated by either ICELL or TGAS. (W/m $\left./ \mathrm{m}^{2}-\mathrm{K}\right)$

TGAS - the gas temperature. (Not available for a DELTA-T or TGAS independent variable or when ICELL is specified.) (K)

Note that the use of tables for TSURF, QSURF, HCOEF, or TGAS is allowed whenever a constant value, specified through the TSURF, QSURF, HCOEF or TGAS keywords, is allowed. The restrictions are described above.

$\mathrm{Y}$

the required keyword to initiate the input of the dependent variable values in the table.

ypts specify "np" values of the dependent variable.

The following keywords are used to specify the parameters used to calculate the gas convective velocity at the nonsubmerged part of the structure surface in terms of the gas velocities found in the flow paths attached to the cell gas space and at the pool-gas interface. The gas velocity at the poolgas interface takes into account the effects of pool boiling, SRV venting, core-concrete interactions, and gases vented into the cell from flow paths lying below the pool surface, but not surface evaporation. If the tabular options given above for specifying the degree of forced convection are 
invoked for the structure surface, the tabular value will be used for the surface instead of the one calculated from the VELCOEF input below.

VELCOEF

FLOWPATH

$\mathrm{i}, \mathrm{j}$

valin

valout

ENGVENT

$\mathrm{n}$

POOLFLOW the keyword to begin the specification of the velocity coefficients in the convective velocity calculation.

the keyword to specify the inflow and outflow velocity coefficients for a regular flow path. It should be noted that regular flow paths are used only for gas flow, and the inflow coefficients apply only if the incoming gases are vented directly into the gas space. The effects of gases vented into the cell from below the pool surface are governed by the POOLFLOW input.

the cell indices identifying a regular flow path. One of these should be the cell in which the structure is defined.

the coefficient $C_{\mathrm{in}, \mathrm{j}}$ in Equation (10-17) used to calculate the contribution to the convective velocity at the present structure surface from the flow coming into the present cell through the flow path specified by the preceding FLOWPATH, ENGVENT, or POOLFLOW keyword. If the flow through that flow path is leaving the present cell, then "valout" is used. The applicable structure surface is determined by whether the value for "valin" is read in the BCINNER or BCOUTER input block. Default $=1$.

the coefficient $\mathrm{C}_{\text {outij }}$ in Equation (10-19) used to calculate the contribution to the convective velocity at the structure surface for flow out of the present cell. Default $=1$.

the keyword to specify the inflow and outflow velocity coefficients for an engineered vent. Only vents of type GAS are used in the gas convective velocity calculation, and the inflow coefficients apply only if the incoming gases are vented directly into the gas space. The effects of gases vented into the cell from below the pool surface are governed by the POOLFLOW input.

the number of the engineered vent, as determined from the order that engineered vents are specified in the global ENGVENT input block. This should not be confused with the cell which is connected by the vent to the present cell.

the keyword to specify the inflow and outflow velocity coefficients for the dedicated suppression vent flow path and the inflow coefficients for the total flow across the pool/atmosphere interface. (The coefficients for the two types of inflow are assumed to be identical.) Note that the flow rate entering the cell is not necessarily equal to the flow entering the pool on the upstream side. The latter may have either less or more water vapor depending on 
whether net condensation or evaporation has occurred in the equilibration process. On the downstream side, the convective velocity is based on the total flow rate at the pool surface, including contributions from submerged gas flow paths, boiling, gases from core-concrete interactions (CCIs), and SRV sources, but excluding surface condensation or evaporation losses. The obsolete keyword SUPVENT will have the same effect as POOLFLOW.

HYDAREA

the value of the hydraulic area $A_{\text {hd }}$ to use in Equations (10-17) and (10-19) to

$=$ hdarea calculate the convective velocity at the structure surface. Default $=\left(\mathrm{V}_{\mathrm{g}}\right)^{2 / 3}$ where $V_{g}$ is the cell gas volume. $\left(\mathrm{m}^{2}\right)$

A simple maximum-depth film model has been made the default model for inner surfaces and is used exclusively for outer surfaces exposed in the same cell. This model simply transfers the mass in excess of $\delta_{m}$, the minimum depth for flow, to the pool in the current cell or the overflow repository. The pool in the current cell is used for partially submerged structures; otherwise, the overflow repository is used. The overflow repository is defined as the pool, if present, in the cell specified by the cell (not engineered systems) OVERFLOW keyword; otherwise it is the waste repository in that cell. By default, the overflow cell is the cell in which the structure resides. With the default film model, a structure inner surface can be targeted to receive flow from other structures, but the outflow from that surface cannot be directed to other structures.

The alternative to the default model for inner surfaces is referred to as the film tracking model and is activated by the keyword FLMFLOW. It is described in Section 10.2.2, Film Tracking Model. The input required is described below.

The FILMFLOW input is part of the BCINNER block, which determines the boundary conditions to be used for the inner face of a structure. The BCINNER block is specified under the cell level structure input. Some of the new input options (SOURCE, MINDEPTH, INIDEPTH, and HPAINT) are available when the default model is used for the inner face. Some of these (MINDEPTH, INIDEPTH, and HPAINT) are also available in the BCOUTER block for the outer face of the structure when that face is exposed in the same cell as the structure. Note that the MINDEPTH keyword for specifying $\delta_{\mathrm{m}}$ on a specific structure face supersedes the FLMAX keyword for the common film thickness specified in the CONDENSE input block. The FLMAX keyword is not upward compatible and will cause a diagnostic to be issued.

FILMFLOW the keyword to activate the film tracking model for the current structure inner face.

DIVERT $=$ nstrs

an optional keyword to specify that some fraction of the film outflow from the current structure inner surface be diverted from the overflow repository (see discussion above for a definition of this repository) to a different structure inner surface when the former inner surface is not partially or entirely submerged. The variable "nstrs" is the number of structures to receive some fraction of the film outflow from the current structure. It is also the number of times that FRAC and the associated structure information must 
FRAC

$=$ frac

NAME

$=$ sname

NUMBER

$=$ snum

CELL

$=$ ncell

SLOPE

$=$ theta

WIDTH

= width be specified immediately after a given DIVERT keyword. Note that each FRAC grouping is terminated with an EOI. The FRAC values will be ignored and the pool in the same cell will be the target if the structure is partially submerged. If DIVERT is not specified but FILMFLOW is, then film flow correlations will be used to determine the film thickness, but all outflow will be directed to either the pool in the current cell or the overflow repository, as with the default film model.

the fraction of the film outflow to be diverted to the structure specified by the NAME or NUMBER and CELL keywords below when the current structure is not submerged. If the structure is partially submerged, the film outflow will be directed entirely to the pool in the current cell. The variable "frac" corresponds to an element of the film transfer matrix $\mathscr{F}_{\mathrm{ji}}$ discussed in Section 10.2.2, where i corresponds to the current structure and the recipient structure $\mathrm{j}$ is designated through the aforementioned keywords. The current structure may not be specified as the recipient structure. Note that while recirculating or re-entrant film flow paths may be constructed, they may adversely affect code stability and are not recommended. The sum of all "frac" values specified after a given DIVERT keyword must be less than or equal to 1 . If the sum is less than 1 and the structure is not partially submerged, a fractional amount corresponding to $\Delta \mathrm{m}_{\mathrm{i}, \mathrm{i}}$ in Equation (10-44) will be diverted to the overflow repository. Default $=0$.

the name of the structure to which "frac" applies. Either the structure name or number "snum" (see below) must be given.

the number, according to input order witbin a cell, of the structure to which "frac" applies. Either the name "sname" or number must be given.

the number of the cell containing the structure to which "frac" applies. Default $=$ current cell .

the average inclination from horizontal of the inner structure face along the horizontal equal-area line. The angle "theta" corresponds to $\theta$, discussed in Section 10.2.2.1. Note that "theta" must be positive and correspond at most to a vertical surface: $0<$ "theta" $\leq 90^{\circ}$. Unstable films on the underside of surfaces are not considered in the correlations used in the film tracking model and should be treated parametrically. Default $=90$ for walls; "cyltht" $/ 4$ for cylindrical roofs or floors; and 60 for hemispherical roofs or floors. (degrees)

the film (i.e., channel) width at the horizontal equal-area line on the structure inner face. The variable "width" corresponds to the variable $\mathrm{w}$, discussed in Section 10.2.2.1. Default = length selected according to the structure type and orientation. Defaults are "slarea"/"chrlen" for a slab in any orientation, $\pi \mathrm{R}_{1}{ }^{\text {"cyltht"}}$ /180 for a wall cylinder, and 2("cylht") for a ceiling or floor 


\section{INIDEPTH \\ = indpth \\ MINDEPTH \\ $=$ mndpth}

HPAINT

$=$ hpaint

SOURCE

$=1$

H2OL

$=$ npts

BCOUTER cylinder. Here, $R_{1}$ is the cylinder inner face radius. The default for a wall hemisphere is $\pi R_{2}$ and $3^{1 / 2} \pi R_{2}$ for a floor or ceiling hemisphere, where $R_{2}$ is the hemisphere inner radius.

the initial structure surface coolant film depth. Default $=0 .(\mathrm{m})$

the minimum film depth before flow, discussed in Section 10.2.2. In either the film tracking model or the default film model, the film depth must exceed "mndpth" before film outflow can occur. Note that the MINDEPTH keyword for specifying "mndpth" on a specific structure face supersedes the FLMAX keyword for the common film thickness that was specified in the CONDENSE input block of CONTAIN 1.1. The FLMAX keyword is not upward compatible and will cause a diagnostic to be issued. Default $=0$ for the film tracking model and 0.0005 for the default film model. (m)

the heat transfer coefficient across any additional surface layers, such as paint, that are not explicitly nodalized in the structure or represented by the coolant film. Default $=2000$. $\left(\mathrm{W} / \mathrm{m}^{2}-\mathrm{K}\right)$

a keyword to specify a FILMFLOW source table to describe the flooding of the inner surface by an external source. Only a single source table may be specified for each structure (hence, one must specify "1").

the material name and number of time points in the source table. Unlike most source tables, the material name is not arbitrary but must be the coolant (H2OL). Note that "npts" is the number of time points in the source table and is the same as the parameter " $\mathrm{n}$ " in the discussion in Section 14.4.1. (T, "times," TEMP, "temps," ENTH, and "enths" are also discussed in that section.)

the keyword to begin the specification of the outer surface boundary condition for a structure. If this keyword is omitted, the boundary condition will be adiabatic by default, unless one of the obsolete IOUTER or TOUTER keywords is used outside of this block. Note that if the outer surface is exposed to the atmosphere in the same cell as the inner surface, then the outer surface is treated in some respects like the inner surface with respect to the available options. (Differences exist with respect to the film tracking modeling, some of the radiative heat transfer options, and submergence, all of which are allowed only with respect to the inner face. A structure, furthermore, cannot be treated as submergible if the outer face is in the same cell.) If the outer surface is given an external boundary condition or is exposed to the atmosphere in a different cell from that in which the structure is defined, then only the HCOEF, TGAS, TSURF, QSURF, or ADIABAT options (including the tabular ones, if appropriate) are allowed. Conversely, 
if the outer surface is exposed to the gas in the same cell, then none of the latter options may be used. An option allowed in general is the STRNUM option to connect the outer surface to the outer surface of a structure in a different cell.

Note that more than one parameter must be specified in some cases for completeness. For example, if HCOEF is specified (or if HCOEF is specified in a VAR-PARM table), then the temperature seen by the surface must be defined by specifying either the TGAS or ICELL keyword in the BCOUTER block. If STRNUM is specified, then the ICELL keyword must be given to identify the cell for the structure "istr," to which the present structure is connected. Certain illogical combinations of keywords in the BCOUTER block (including VAR-PARM options) are not allowed. For example, the specification of TSURF and HCOEF in the same BCOUTER block is not allowed. Also, both TGAS and ICELL can never be specified in the same BCOUTER block.

TSURF

$=$ tsurf

QSURF

$=$ qsurf

ADIABAT

HCOEF

$=$ hcoef

STRNUM the constant temperature to be used as a boundary condition. This temperature represents a surface temperature; therefore, the node-center temperature reported in the CONTAIN output will be different and may actually vary. The fission product host temperatures for a surface, for example, will reflect the true constant surface temperature. (K)

the constant heat flux to the surface to be used as a boundary condition. Again, the temperature of the surface will be different from the node center temperature reported in the CONTAIN output. The true surface temperature is used, for example, in the fission product host temperature output for a surface host. $\left(\mathrm{W} / \mathrm{m}^{2}\right)$.

a keyword to specify an adiabatic boundary condition. Note that specifying QSURF $=0$ will also give an adiabatic boundary condition. This keyword is provided for convenience.

a constant heat transfer coefficient replacing the default natural convective value for an external boundary condition or an outer surface that is exposed to the gas in a different cell. When "hcoef" is used, the temperature seen by the outer surface must also be specified with either TGAS or ICELL keywords (the obsolete TOUTER and IOUTER keywords can also be used). This boundary condition should not be specified for the inner surface or for the outer surface when it is in the same cell. Default $=6.08$. $\left(\mathrm{W} / \mathrm{m}^{2}-\mathrm{K}\right)$

the keyword to specify that the outer surface is connected to another structure. In this boundary condition option, the outer surface of the structure is in thermal contact with the outer surface of another structure, which must be in a different cell. The cell number of the connecting structure must be 
istr

hgap

ICELL = icell

TGAS $=$ tgas identified by the ICELL (or IOUTER) keyword in the BCOUTER block. The STRNUM keyword is followed by "istr," the number of the structure to connect to, and "hgap," the gap conductance between the two structures. When this boundary condition is used, STRNUM should be specified in the input of each of the two structures and the specified gap conductances and connecting areas should be consistent.

the number of the structure that the outer surface is connected to.

the required gap conductance between the two structures. To model structures in perfect thermal contact, this value should be set to a large value (i.e., $10^{20}$ ). Note that the outermost half-node resistances of the connecting structures are accounted for automatically in the model. (W/ $\left./ \mathrm{m}^{2}-\mathrm{K}\right)$

the cell in which the outer surface is exposed to the gas or in which a connecting structure is specified. As discussed in the BCOUTER block above, whether "icell" is the same cell or different cell from the current cell determines the type of heat transfer option that may be selected. Note that if only "icell" is specified in the BCOUTER block and "icell" is a different cell, then the heat transfer will be controlled by the default value of "hcoef." If a structure connection (STRNUM) is specified, ICELL is required to identify the cell of the connecting structure. In order to maintain upward compatibility, the obsolete IOUTER keyword is accepted in lieu of ICELL either inside or outside of the BCOUTER block.

the gas temperature for the gas adjacent to the outer surface when a dry convective boundary condition is specified but the surface does not lie in a cell. If TGAS is specified by itself in the BCOUTER block, the default value of "hcoef" will control the heat transfer. If the surface temperature, heat flux, or structure connection boundary condition is specified (TSURF, QSURF, ADIABAT, or STRNUM), then the TGAS keyword is not allowed. In order to maintain upward compatibility, the obsolete TOUTER keyword is accepted in lieu of TGAS either inside or outside of the BCOUTER block.

An alternative input format is given in Appendix B. The format above may be used in conjunction with the alternative one, provided the alternative format is placed first in the STRUC block.

With respect to DCH calculations, the forced convection velocity is calculated for each structure either according to Equations (10-16) through (10-19) or specified (in some cases, implicitly) through VAR-PARM tables related to forced convection. (Note that the tabular input takes precedence over the calculated values.) The calculated velocity depends on coefficients for incoming and outgoing flows specified through the VELCOEF input and HYDAREA input. If no forced convection input is specified, then all structure velocities in a given cell are by default equal. 
With nondefault forced convection modeling, it should be noted that structure input order could be important because the trapping model uses the calculated forced convection velocity across the inner face of the first two structures in the TOF/KU trapping model, as described in Section 6.3. The velocities for the first two structures are the $v_{1}$ and $v_{2}$ parameters in the trapping model described in that section. If there are no structures in the cell, then the TOF/KU trapping model will be bypassed and the GFT trapping rate will be used. If only one structure is in the cell, then the trapping model will use the velocity for the first structure for both impacts considered by the TOF/KU model.

14.3.1.4 Convection and Condensation. This block activates condensation and evaporation of coolant from all surfaces (structure, lower cell, and engineered system) for the entire cell. In contrast to previous code versions, in the absence of the CONDENSE keyword for a cell, the treatment of heat transfer for surfaces within the cell of definition is not downgraded to the simplified treatments previously associated with dry heat transfer. All options associated with CONDENSE are available without CONDENSE, including the Grashof number changes, enhanced output, and user flexibility options; without CONDENSE, the Sherwood number $\mathrm{N}_{\mathrm{Sh}}$ for mass number transfer is simply set to zero, regardless of other input. Otherwise all other options previously associated with the CONDENSE option are available. The heat transfer options previously associated with dry heat transfer, such HCOEF, are presently reserved for structure surfaces that are not in the cell of definition. These include the outer surfaces of structures that are exposed to the gas in some other cell or given an external boundary condition.

The FORCED forced convection table options previously available with the CONDENSE input block have been combined with the table options previously available with the BCINNER and BCOUTER blocks of each structure. Tables for forced convection velocity, Reynold's number, and forced-convection Nusselt number (i.e., VELOCITY, REY-NUM, and NUS-NUM tables) are now available in the latter blocks. (The NUS-NUM table has been given the new name NUS-FORC for clarity, although NUS-NUM is also accepted.)

The new keyword MINDEPTH (discussed in Section 14.3.1.3) also supercedes FLMAX and allows the user to set the minimum film depth for structures in the cell; any excess condensate will either drain to a pool or be lost from the problem. The destination pool can be specified through the cell OVERFLOW option discussed in Section 14.3.1.12.

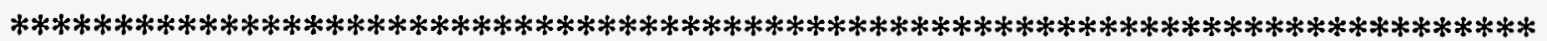

\section{CONDENSE}

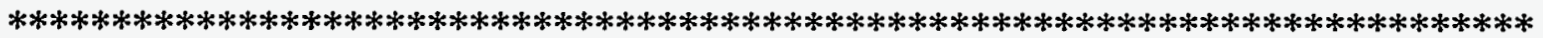

CONDENSE the keyword to enable condensation heat transfer on all surfaces but those of aerosols in the cell. In the absence of CONDENSE, the Sherwood number for mass transfer will be set to zero, but all other modeling options will be available. 
14.3.1.5 Radiation. The radiation model is activated at the cell level by the keyword RAD-HEAT. In the following descriptions, "nhtm" is the number of heat transfer structures in the cell as specified in the cell CONTROL block.

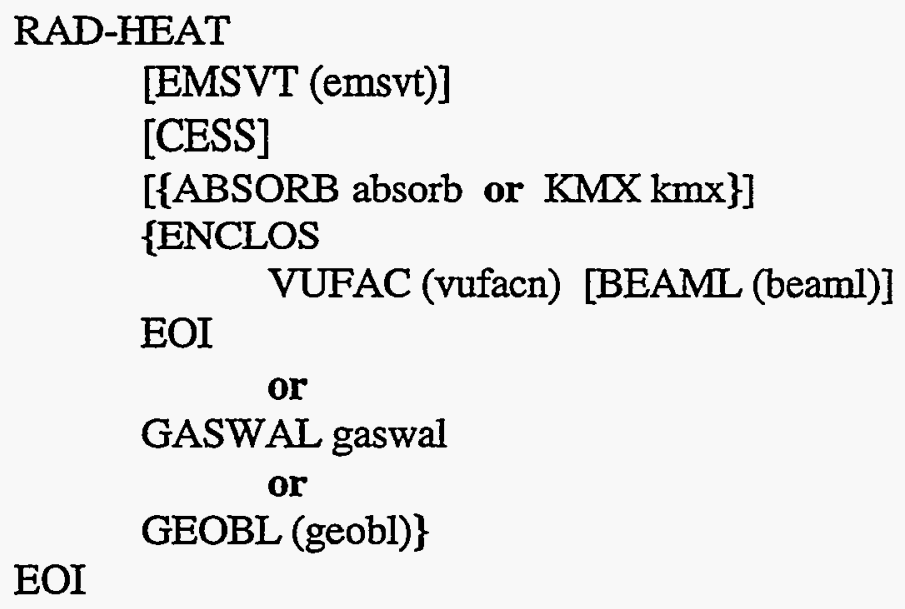

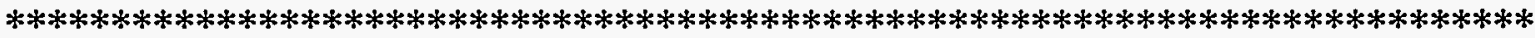

The following keywords control the gas and surface emissivities:

RAD-HEAT

EMSVT

emsvt

CESS the keyword to begin the specification of the radiation model in the cell. the keyword to begin the specification of the dry surface emissivities. the dry surface emissivity. Specify "nhtm" values if no lower cell is present. Specify "nhtm" +1 values if a lower cell model is logically present in the cell, with the last value equated to emissivity, of the uppermost lower cell layer. (The atmosphere or null layers are not taken into account in determining this layer.) A lower cell is logically present if space has been reserved for the lower cell model; i. e., if "jint" + "jpool" + "jconc" is greater than 0 . Specify an effective emissivity if the GASWAL option is chosen (see the discussion after Equation (10-64); otherwise, specify a proper dry surface emissivity. If water is present on a surface, a value of 0.94 is used for the emissivity with the net enclosure model and a value $(1+0.94) / 2$ with the GASWAL option. If "emsvt" is not specified, all dry surface emissivities default to a value of 0.8 .

the keyword specifying use of the Cess-Lian correlation for the steam emittance (see Section 10.3.3.2). If this option is activated, carbon dioxide and carbon monoxide are treated as transparent gases and therefore do not participate in the radiative heat transfer. If CESS is not specified, the default Modak model is used for the gas emittance. 
Two options are available for characterizing the contribution of the aerosols to the gas mixture radiative properties, as discussed in Section 10.3. The first option uses a constant user-specified absorption coefficient, "absorb," which is applied under all conditions. An alternative treatment of aerosol absorption uses a multiplier, "kmx," in an expression for the absorption coefficient which is proportional to the aerosol mass concentrations calculated by CONTAIN. In the model used in the latter option, small soot-like aerosols with a material density of $2000 \mathrm{~kg} / \mathrm{m}^{3}$ have been found to give a "kmx" of approximately one, as discussed in Section 10.3. If both options are specified, the ABSORB option will override the " $\mathrm{kmx}$ " option. If neither option is chosen, a value of zero will be used for the aerosol absorption coefficient. (It should be noted that the scattering effects of aerosols are presently not treated.)
ABSORB
the aerosol absorption coefficient. Default $=0 .\left(\mathrm{m}^{-1}\right)$
$=\mathrm{absorb}$
$\mathrm{KMX}$
the aerosol mass concentration multiplier. Default $=0$.
$=\mathrm{kmx}$

The user can activate either a net enclosure model through the keyword ENCLOS or the direct model through the keyword GEOBL or GASWAL. The net enclosure model treats multiple reflections correctly but does not consider the outer surfaces of structures within the cell. The direct method treats secondary reflections only approximately but allows radiative heat transfer between the atmosphere and the inner and outer structure surfaces in the cell as well as between the atmosphere and uppermost lower cell layer and between the uppermost lower cell layer and each heat transfer structure. Only one of these three keywords may be chosen.

ENCLOS

VUFAC

vufacn

BEAML the keyword to activate the net enclosure model. Note that this model should not be used if the geometry of the cell, including that of the coolant pool, changes appreciably with time.

the keyword to initiate the input of the upper triangle of the array of view factors.

the standard view factor from one surface to another surface defined in Equation (10-60). Specify the view factor from structure 1 to structure 2, and so forth, in the order used for "beaml." (The view factor from 1 to 2 corresponds to the fraction of radiation leaving 1 within the solid angle subtended by 2.) The lower triangle "vufacn" is calculated automatically using the reciprocity relation for view factors:

$$
\operatorname{area}(1) \times \operatorname{vufacn}(1 \text { to } 2)=\operatorname{area}(2) \times \operatorname{vufacn}(2 \text { to } 1)
$$

an optional keyword to initiate the input of the upper triangle of the array of beam lengths. 
the beam length between two structure inner surfaces. Specify "beaml" between structure 1 and structures 1 through "nhtm" and then between 1 and the lower cell, if logically present. Then specify "beaml" between structure 2 and structures 2 through "nhtm" and so forth. The input corresponds to rows in the upper right triangle of an "nhtm" +1 square matrix if the lower cell is present, and to the upper right triangle of an "nhtm" square matrix, otherwise. If the "beaml" input is omitted, a single value for each surface pair is calculated, using

"beaml" =

$3.6 \times$ (initial cell gas volume)/(total unsubmerged inner and lower cell surface area in cell).

The input corresponds to the upper triangle of an "nhtm" + 1 square matrix if the lower cell is logically present, and to the upper triangle of an "nhtm" square matrix, otherwise. The sum of the elements in each row of the view factor matrix (including both user-specified elements and those calculated by CONTAIN from reciprocity) must equal 1. Thus, the user will generally need to perform side calculations with the above reciprocity relation to ensure that the constraint of the row adding to 1 is satisfied.

EOI

GASWAL = gaswal

the keyword used to terminate the net enclosure input.

the geometric mean beam length for the enclosure. If specified, this single value has the effect of activating the simple atmosphere-to-structure radiation model. It is used for all structures and the uppermost lower cell layer. Note that the model for direct exchange between the lower cell and the inner surface of structures is invoked by specifying the "vufac" parameter in the structure input (see Section 14.3.1.3).

GEOBL

the keyword to activate the direct atmosphere-to-surface radiation model. Unlike the GASWAL keyword, the GEOBL keyword allows for a separate geometric beam length for each surface.

geobl

the geometric beam length for a structure or the uppermost lower cell layer. As described above for the EMSVT input, "nhtm" values for "geobl" should be specified in absence of a lower cell and "nhtm" +1 values if a lower cell is logically present.

EOI

the keyword used to terminate the RAD-HEAT radiation input block.

An obsolete radiation input format which uses the keyword RADIAT is also available. It should be noted, however, that only the direct radiation model and not the net enclosure model is available when this obsolete input format is exercised. Also, the Cess-Lian correlation is used as the default, 
as opposed to Modak, when using the RADIAT input option. The format of the RADIAT input block is given in Appendix B.

14.3.1.6 Heat Transfer Control. This block allows the user to activate or deactivate certain heat transfer mechanisms.

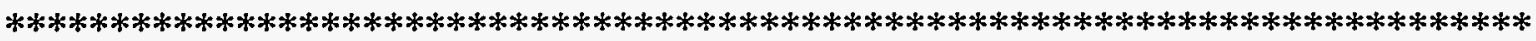

HT-TRAN htflag1 htflag2 htflag3 htflag4 htflag5

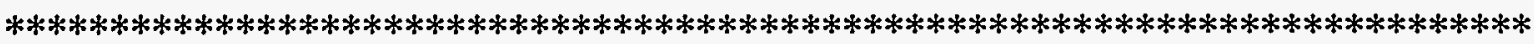

An ON or OFF value must be specified for each of the five flags following HT-TRAN. These flags control the heat transfer between the entities described below. Note that the CORCON-CONTAIN interface is not controlled by these flags.

HT-TRAN the keyword to begin specification of the heat transfer control flags

htflag1 the atmosphere to structures for all heat transfer processes.

htflag2 the first non-null layer in the lower cell to the basemat, which is assumed to be at constant temperature "txl" (see Section 14.3.2.1), or from the atmosphere to the basemat if all specified lower cell layers are null.

htflag3 between two adjacent layers in the lower cell. The heat transfer to the basemat or the atmosphere is not controlled by this flag.

htflag4 the uppermost non-null lower cell layer to the upper cell for all heat transfer processes, or from the basemat to the upper cell if all specified lower cell layers are null.

htflag5 the radiative transfer from the lower cell to the upper cell and from the atmosphere to structures. In case of conflict between "htflag5" and "htflag1" or "htflag4," the latter flags take precedence.

The processes controlled by each of the flags are discussed more fully in Section 10.7. If this optional block is not selected, then all flags are set to ON. If one or more of the heat transfer mechanisms are to be turned off, then all five flags must be included in the input.

\section{Example: HT-TRAN OFF ON OFF OFF ON}

14.3.1.7 Combustion Model Input. This optional block activates the hydrogen and carbon monoxide combustion model in CONTAIN. The three types of burns-deflagrations, diffusion flames, and bulk spontaneous recombination (BSR)--and their ignition criteria are summarized in Table 14-1. 
Table 14-1

Types of Burns Allowed for Different Ignition Sources

\begin{tabular}{||l|c|c|c|}
\hline \multirow{2}{*}{\multicolumn{1}{|c|}{ Ignition Criteria $^{*}$}} & \multicolumn{3}{|c|}{ Type of Burn } \\
\cline { 2 - 4 } & Deflagration & Diffusion Flames & BSR \\
\hline $\begin{array}{l}\text { Igniters, or equivalent } \\
\text { ignition source }\end{array}$ & $\mathrm{X}$ & $\mathrm{X}^{\mathrm{b}}$ & \\
\hline $\begin{array}{l}\text { Cell } \\
\text { Gas Temperature }\end{array}$ & & $\mathrm{X}$ & $\mathrm{X}^{\mathrm{d}}$ \\
\hline $\begin{array}{l}\text { Upstream Cell } \\
\text { Gas Temperature }\end{array}$ & $\mathrm{X}$ & $\mathrm{X}$ & \\
\hline Debris Loading $^{\mathrm{f}}$ & & $\mathrm{X}$ & $\mathrm{X}$ \\
\hline Propagation $^{\mathrm{g}}$ & $\mathrm{X}$ & & \\
\hline
\end{tabular}

${ }^{2}$ Assumed operating in the time window bounded by TACTIV and TDEACT for the burn cell DFTEMP

bAllowed for flow in unsubmerged gas flow paths only if the gas inflow temperature exceeds

'Threshold given by SRTEMP

JBSR based on gas temperature not allowed during a deflagration to prevent a spurious transition from a deflagration to BSR

Threshold given by DFAUTO outside of the time window, for unsubmerged gas inflow into the downstream cell that is not from an external source

Debris concentration > DEBCONC; debris temperature > DEBTEMP

BPropagation from a deflagrating cell through a flow path occurs after a time delay controlled by KPROP, provided the conditions in the propagation cell are suitable; namely, the flame velocity is sufficient to overcome the flow velocity from the propagation cell to the deflagrating cell, and the flow is not submerged in either cell.

The H-BURN input block is required to enable the modeling of deflagrations. In addition, the CONTBURN input block is required to model continuous burning processes like diffusion flames and BSR. Three types of inflow are modeled with respect to diffusion flames: (1) flow from each gas flow path that is not scrubbed by the pool in the inflow cell, (2) the collective gas flow from all gas paths venting under the pool surface at the inflow cell, as modified by the equilibration or partial equilibration of the gas with the pool, and (3) the (positive) mass sources to the cell, considered collectively. Each type of inflow is treated independently with respect to diffusion flame burning. For example, one diffusion flame in a cell is not allowed to ignite another diffusion flame, in the absence of all other potential ignition sources. 
The discussion below refers to a mole fraction of combustible, as well as the whole fraction of inerting gases. These are defined in terms of the constituent gas mole fractions in Equation (9-2).

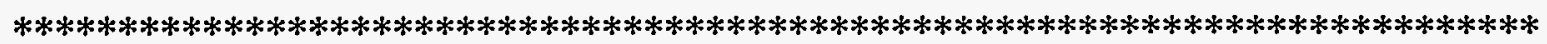

\author{
H-BURN \\ [BURNT=burnt] [CHRL=chrl] [FLAM=flam] \\ [CFRMNG=cfrmng] [MORMNG=mormng] \\ [ELEV=elev] [KPROP=kprop] [MFCIG=mfcig] [MFOIG=mfoig] \\ [MFSIG=mfsig] [MFCDN=mfcdn] [MFODN=mfodn] [MFSDN=mfsdn] \\ [MFCHZ $=\mathrm{mfchz}]$ [MFOHZ $=\mathrm{mfohz}]$ [MFSHZ=mfshz] [MFCUP=mfcup] \\ [MFOUP=mfoup] [MFSUP=mfsup] [TACTIV=tactiv] [TDEACT=tdeact] \\ [CONTBURN \\ [SHRATIO=shratio] [CFRACB $=\mathrm{cfracb}]$ \\ [SRTEMP=srtemp] [DEBCONC=debconc] [DEBTEMP=debtemp] \\ [MFSCB=mfscb] [MFOCB=mfocb] \\ [H2FLOW=h2flow] [DFTEMP=dftemp] [DFAUTO=dfauto] [SRRATE=srrate] \\ [NOBURNEN] \\ EOI] \\ [EOI]
}

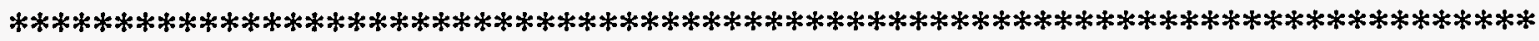

H-BURN

the keyword to activate the combustion model with respect to deflagrations. Note that the following keywords through MFSUP apply only to the deflagration model.

BURNT

the compartment burn time. Default $=$ internally calculated value. $(s)$

$=$ burnt

CHRL

$=\operatorname{chrl}$

FLAM

$=$ flam

CFRMNG

$=$ crrmng

the compartment characteristic length. Default $=$ cube root of the initial cell gas volume. (m)

the flame speed. Default $=$ internally calculated value. $(\mathrm{m} / \mathrm{s})$

the fraction of the initial amount of combustible remaining after the burn. Default $=$ internally calculated value. The user should note that the default option to have the code calculate "cfrmng," the deflagration fraction of the initial combustile remaining, may be incompatible for a mole fraction of combustible "mfoig" that is too low for ignition. For a mole fraction for ignition of less than 0.0376 , the correlation for burn completeness used in the code may return a fraction of initial combustible remaining of unity, which will result in nothing being burned. The user must therefore specify "cfrmng" through the keyword CFRMNG for a mole fraction for ignition of less than
\end{abstract}


MORMNG

$=$ mormng

\section{ELEV \\ = elev \\ KPROP \\ $=$ kprop}

MFCIG

$=\mathrm{mfcig}$

MFOIG

$=\mathrm{mfoig}$

MFSIG

$=\mathrm{mfsig}$

MFCDN

$=\operatorname{mfcdn}$

MFODN

$=\operatorname{mfodn}$

MFSDN

$=\mathrm{mfsdn}$

MFCHZ

$=\mathrm{mfchz}$
0.0376. The keyword CFRMNG serves the same function as the obsolete (but upward compatible) keyword HFNL. The keyword HFNL implies a final hydrogen concentration rather than final fraction of initial combustible and is therefore not recommended.

the mole fraction of oxygen remaining after the burn. Default $=0.0005$.

the compartment elevation, used only in the burn model to determine the relative positions of the cells for burn propagation. Default $=0$. $(\mathrm{m})$

the propagation delay factor. A deflagration will be allowed to propagate to another cell through a flow path after a time "kprop" $x$ "burnt" has elapsed after the start of the burn, if the conditions in that cell and in the flow path are suitable. The flame velocity must be sufficient to overcome the flow velocity, if positive, from the propagation cell to the deflagrating cell, and the flow path must not be submerged under the pool in either cell. A deflagration will not propagate after the time "burnt" has elapsed. For robustness, "kprop" should be rounded to the nearest "ctfrac"/10. (See the TIMES block in Section 14.2.8.) Default $=0.5$.

the mole fraction of combustible for ignition. Default $=0.07$.

the mole fraction of oxygen for ignition. Default $=0.05$.

the mole fraction of the sum of steam, carbon dioxide, and excess nitrogen for inerting against ignition sources, as given by Equation (9-2) and Table 91. Default $=0.55$.

the mole fraction of combustible for propagation downward into the cell. Default $=0.09$.

the mole fraction of oxygen for propagation downward into the cell. Default $=0.05$.

the mole fraction of the sum of steam, carbon dioxide, and excess nitrogen in the cell for inerting against propagation down into the cell as given by Equation (9-2) and Table 9-1. Default $=0.55$.

the mole fraction of combustible for propagation horizontally into the cell. Default $=0.06$. 
MFOHZ

$=\mathrm{mfohz}$

MFSHZ

$=\mathrm{mfsh} z$

MFCUP

$=$ mfcup

MFOUP

$=$ mfoup

MFSUP

$=\mathrm{mfsup}$

TACTIV

$=$ tactiv

TDEACT

$=$ tdeact

CONTBURN

SHRATIO

$=$ shratio

CFRACB

$=$ cfracb

SRTEMP

$=$ srtemp the mole fraction of oxygen for propagation horizontally into the cell. Default $=0.05$.

the mole fraction of the sum of steam, carbon dioxide, and excess nitrogen in the cell for inerting against propagation horizontally into the cell as given by Equation (9-2) and Table 9-1. Default $=0.55$.

the mole fraction of combustible for propagation up into the cell. Default = 0.041 .

the mole fraction of oxygen for propagation up into the cell. Default $=0.05$.

the mole fraction of the sum of steam, carbon dioxide, and excess nitrogen in the cell for inerting against propagation up into the cell as given by Equation (9-2) and Table 9-1. Default $=0.55$.

the time to activate igniters, or an equivalent ignition source. Note that burns are still allowed to propagate into the cell even if an ignition source is not present. Default $=-10^{30}$. (s)

the time to deactivate igniters or an equivalent ignition source. This time must be larger than "tactiv." Note that burns are still allowed to propagate into a cell even if an ignition source is not present. Default $=10^{30}$. (s)

the keyword to activate the diffusion flame and BSR models. The following describes specific modeling parameters that affect their initiation and termination. See Table 14-1.

the inflowing diluent to combustible gas molar ratio above which diffusion flames cannot occur. Default $=9.0$.

the fraction of the incoming combustible gas burned in the diffusion flame. Default $=1.0$.

the cell atmosphere temperature above which diffusion flames or BSR will occur (if there is sufficient oxygen and combustible gas). (This applies when the present cell is the downstream cell in the case of a diffusion flame.) BSR will occur according to the "srrate" parameter defined below without regard to the other parameters values in this block. Some literature values of the autoignition temperature are given in Section 9.3 and additional guidance for the use of the BSR model is given in Sections 13.2.6.3 and 13.3.2.2.2. Default $=773$. $(\mathrm{K})$ 

DEBCONC
$=$ debconc
DEBTEMP
$=$ debtemp
the concentration of debris above temperature "debtemp" above which diffusion flames or BSR may occur. (This applies when the present cell is the downstream cell in the case of diffusion flames.) Default $=1 .\left(\mathrm{kg} / \mathrm{m}^{3}\right)$
the debris temperature above which diffusion flames or BSR may occur if the corresponding debris concentration exceeds "debconc." (This applies when the present cell is the downstream cell in the case of diffusion flames.) Default $=773$. $(\mathrm{K})$

MFSCB

$=\mathrm{mfscb}$

MFOCB

$=$ mfocb

H2FLOW

$=\mathrm{h} 2$ flow

DFTEMP

$=\mathrm{dftemp}$

DFAUTO

$=$ dfauto

the maximum allowable diluent mole fraction in the receiving cell for diffusion flames to occur. Note that this could prevent diffusion flames even though the diluent to combustible ratio is less than the "shratio" above. Default $=0.55$.

the minimum downstream cell oxygen mole fraction for which diffusion flames can occur. Default $=0.05$.

the minimum combustible gas mass inflow rate for diffusion flames to occur. This has the same effect as the "shratio" parameter above except that it is an absolute check for the existence of sufficient inflow of combustible gas to sustain combustion. Default $=0 .(\mathrm{kg} / \mathrm{s})$

the minimum inflow temperature for diffusion flame burning, for gases flowing into the cell through an unsubmerged gas flow path inside the burn window. If the upstream gas temperature for a given inflow is hotter than "dftemp," the flow is not submerged in the downstream cell pool, and the time is within the burn window determined by "tactiv" and "tdeact," the gases for that inflow are allowed to burn as a diffusion flame, subject to the gas concentration requirements. It should be noted that the concentration requirements are applied separately to each such inflow. Note that "dftemp" is not applicable to submerged gas inflow or to external source inflow to a cell, as defined in the introduction to this section. The applicable ignition criteria for the latter are given in Table 14-1. Default $=0 \mathrm{~K}$ if CONTBURN is specified; = infinity if CONTBURN is not specified.

the autoignition temperature for diffusion flame burning of gases flowing into the cell outside the burn window. If the upstream gas temperature for a given inflow is hotter than "dfauto," the flow is not submerged in the downstream cell pool, and the time is outside of the burn window determined by "tactiv" and "tdeact," the gases for that inflow are allowed to burn as a diffusion flame, subject to the gas concentration requirements. If any such inflow is present, it is also assumed to function as an ignition source for deflagrations even if the inflow itself does not burn. It should be noted that the concentration requirements are applied separately to each such inflow. Note that "dfauto" is not applicable to submerged gas inflow or to external source 
SRRATE

$=$ srrate

NOBURNEN

EOI inflow to a cell, as defined in the introduction to this section. The applicable ignition criteria for the latter are given in Table 14-1. Default $=10^{20} \mathrm{~K}$.

the rate at which the combustible gas recombines with oxygen for BSR, given as a fraction per second of the maximum amount of combustible gas that should combine based on the combustible gas and oxygen present. Default $=0$. $($ fraction $/ \mathrm{s})$

a keyword to prevent the burning of hydrogen entrained into the diffusion flame from the atmosphere of the receiving cell, as discussed in Section 9.2.

the keyword used to terminate the H-BURN blocks and CONTBURN. The final EOI may be omitted if just the H-BURN keyword is specified.

14.3.1.8 Aerosol Initial Conditions and Sources. This optional block specifies initial aerosol component masses and aerosol sources in the cell.

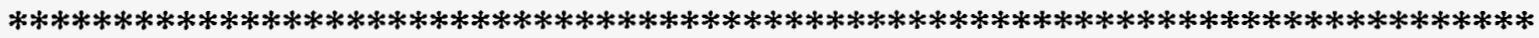

AEROSOL $=[$ naero (omat mass)]

[SOURCE=nSo

(oname=n

IFLAG=ival

$\mathrm{T}=$ (times)

MASS $=$ (masses)

EOD]

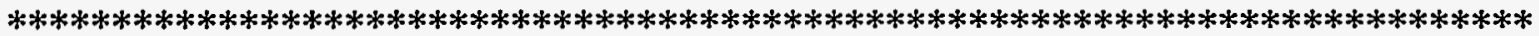

AEROSOL the keyword to begin the specification of initial masses and sources in the cell.

naero

the number of aerosol components which have non-zero initial suspended mass. Default $=0$.

The following group of two variables is repeated "naero" times:

omat a name of an aerosol component material specified in the global AEROSOL block. (See definition of "mapaer" in Section 14.2.5.)

mass

the initial total mass of the aerosol component. The initial particle size distribution will be governed by the "amean" and "avar" parameters specified in the global AEROSOL block, as discussed in Section 14.2.5. (kg)

Aerosol source tables are optional. If desired, they are initiated by the SOURCE keyword. Note that SOURCE and the keywords that follow use a standard notation for source tables discussed in Section 
14.4.1. The particle size distribution of the aerosol mass introduced through the source tables is governed by the "amean" and "avar" parameters discussed in Section 14.2.5.

SOURCE an optional keyword to initiate the input of aerosol sources.

nso the number of source tables to follow. (The input from "oname" to EOI should be repeated "nso" times.)

oname the name of the aerosol component material to be introduced by the table.

n the number of source table points.

The other keywords and values are described in Section 14.4.1. Note that neither TEMP or ENTH is used for aerosols.

14.3.1.9 Fission Product Sources. This optional block is used to specify fission product source tables.

$* * * * * * * * * * * * * * * * * * * * * * * * * * * * * * * * * * * * * * * * * * * * * * * * * * * * * * * * * * * * * * * * * * * * * * * * * * * * *$

FISSION

SOURCE=nso

(oname $=\mathrm{n}$

[IFLAG=ival] [HOST=i]

[CHAIN=j]

$\mathrm{T}=$ (times)

MASS=(masses)

EOI)

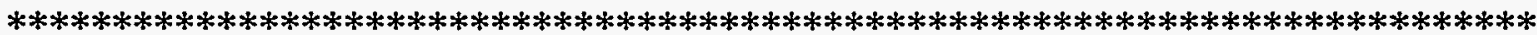

Note that many of the keywords and values used above follow a standard notation for source tables discussed in Section 14.4.1. Each fission product source table must be related to a single chain element that was given in the FISSION block.

FISSION the chain keyword to initiate the specification of fission product source tables.

SOURCE the keyword that must immediately follow FISSION.

nso the number of fission product source tables. (The parameters from "oname" to EOI should be repeated "nso" times.)

oname the name of the fission product that is to be introduced by the table. It should be a name specified in the global FISSION input block. 
an integer indicating the host to which the mass introduced by the table will be attached. Hosts are arranged in the following order: the upper cell atmosphere gas host, "nac" aerosol hosts in the order specified in the global AEROSOL input block, the inner surface host and the outer surface host for each structure, and one host for every lower cell layer. A fixed atmosphere interface host is also considered present if a lower cell is present; this interface transfers energy to the atmosphere but the fission products do not flow with the atmosphere gases. An additional DUMMY host and a WASTE host are also provided after these lower cell hosts; the DUMMY host is provided primarily for miscellaneous targeted release and acceptance purposes. The structure hosts are arranged with the inner surface of structure 1 first, the outer surface of structure 1 second, and so forth. Note that the outer surface is always considered a host, regardless of its location or boundary condition. The structure number is determined by the order in which the structures are given in the STRUC input. The lower cell hosts are arranged with the bottommost layer first, the layer above it second, and so forth, with the fixed atmosphere interface host last. Because there may be many structures and/or lower cell layers in a given cell, care should be taken when specifying host numbers for nonairborne hosts. Furthermore, old input files with sources for nonairborne fission product hosts should be examined for correctness because the fission product host numbering scheme has undergone a nonupward-compatible evolution.

CHAIN $=\mathbf{j}$

input to specify the user-specified linear chain to receive the fission product mass. This input is required only when the same user-specified fission product appears as a chain element in more than one linear decay chain (as a result of a branched decay process) and distribution of the mass according to the inventory factors for the fission product is not appropriate. The variable "j" represents the number of the user-specified linear decay chain to which the source mass of "oname" will be assigned. If CHAIN is not given, then the mass will be distributed among all the chains in which the fission product "oname" appears, according to inventory factors defined either automatically from the fission product library or by the user in the global FISSION input block.

The definitions of keywords and values not given above are discussed in Section 14.4.1. Note that the effects of decay on source material prior to the time of source will not be calculated by CONTAIN. For example, for a two-element decay chain representing F1 decaying into F2, one must generally specify a source of both F1 and F2 in the ratio appropriate to the time of the source, even if only $\mathrm{F} 1$ was present initially.

The decay and power characteristics are specified in the global input under the FISSION keyword. Initial fission product masses and release and acceptance parameters are specified in the cell input under the FPM-CELL keyword as described in the following section. 
14.3.1.10 Fission Product Initial Conditions and Release Rates. The optional FPM-CELL block is used to specify initial fission product masses on the hosts within a cell. User-specified host-to-host transfer rates for fission products within the targeted release and acceptance model are also defined in this input block. An obsolete nontargeted release and acceptance model is also available, as described in Appendix B.

The S-HOST option is an alternative to the HOST option. This option differs from the HOST option in two respects: (1) the fission product masses are specified by using the names of the fission products rather than a list of masses; and (2) they are divided up among the linear chains according to the inventory factors of the fission product.

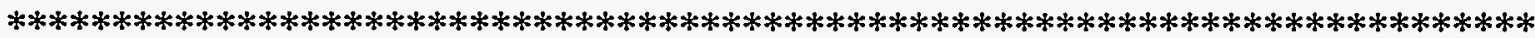

FPM-CELL

$\{($ HOST $=$ hname (masses)) or

(S-HOST=hname (fname=mass) EOI) $\}$

[TARGET

EOI

(fpname aval bval th [FROM=mame TO=aname]) EOI]

$* * * * * * * * * * * * * * * * * * * * * * * * * * * * * * * * * * * * * * * * * * * * * * * * * * * * * * * * * * * * * * * * * * * * * * * * * * * *$

FPM-CELL the keyword to initiate the specification of fission product initial masses and user-specified host-to-host transfers of fission products.

HOST

the keyword to initiate the input of a vector of fission product initial masses for a given host.

hname

a simple name or a name and qualifier that identify the host to which the fission product mass will be assigned. Valid simple names are: GAS, "aername," "strname," and DUMMY, where "aername" is a valid aerosol component name and "strname" is the name of a structure in the cell (the inner surface is the indicated host in this case). The DUMMY host is used for miscellaneous release and acceptance purposes. Names with qualifiers may be used in the following combinations: INNER "strname," OUTER "strname," AEROSOL "aername," AEROSOL "n," LAYER "n," and LAYER POOL. The INNER and OUTER qualifiers are used to specify a particular side of a given structure since each structure is a double host. Either a name ("aername") or a number ("n") may follow the AEROSOL word to specify an aerosol component. Only the pool layer may be accessed by name as shown above; all other lower cell layers must be denoted by LAYER followed by a number, "n." Layers are numbered beginning with 1 at the bottom. Three more simple names, ROOF, WALL, and FLOOR, are also accepted. These generic names refer here to all inner surfaces of structures of type ROOF, WALL, and FLOOR, respectively, and certain outer surfaces of structures of type FLOOR, WALL, and ROOF. To be considered among one of the 
masses

S-HOST

fname

mass

TARGET

fpname

aval

bval

tth generic surface types, an outer surface must be defined to be exposed in the cell in which the structure is defined. Fission products are distributed according to surface area when a generic name is specified.

the initial mass of each fission product associated with the host specified by "hname." Exactly "nfce" values must be specified (one for each chain element). (kg)

keyword to initiate keyword-driven input of fission product initial masses for a given host. This keyword may be used instead of the HOST keyword.

name of the fission product to which the following value of "mass" is assigned.

the initial mass of the fission product "fname" associated with the host "hname." If the fission product appears in more than one linear chain, the mass will be distributed among the chains in proportion to its inventory factors. NOTE: If a user-defined fission product appears in more than one user-specified chain, the user may have to specify inventory factors for the fission product in the global FISSION block.

the keyword to initiate input of targeted release and acceptance parameters for the cell. Note that the parameter "ntgt" in the global CONTROL block must be set to use this option. (See Section 14.2 and the discussion below.)

fission product or volatility group name used to specify the fission products to which the following release and acceptance parameters will apply. If the G-TARGET keyword is invoked in the global input (see Section 14.2.1), then "fpname" is assumed to be a group name. If the G-TARGET option is invoked, all fission products belonging to the group "fpname" will be assigned the specified release and acceptance parameters. The group names are limited to those of the predefined groups given in Table 8-1: GROUP1, GROUP2, GROUP3, GROUP4, GROUP5, GROUP6, GROUP7, GROUP8, GROUP9, or GROUP10. If user-specified fission products are defined, each must be assigned to one of these groups in the G-TARGET input block.

the multiplicative coefficient a in the temperature-dependent release rate given by Equation (8-2). The value of a may be negative. In that case, it is interpreted as shown in that equation. $\left(\mathrm{s}^{-1}\right)$

the exponential factor $\mathrm{b}$ in the release rate given by Equation (8-2). (K)

the host threshold temperature below which the release rate will be set to zero (see Equation (8-2)). (K) 


\begin{abstract}
FROM
$=$ rname

"mame" is nominally the releasing host in a targeted transfer; "mame" must obey the same rules as outlined above for "hname." Note that if "mame" is one of the generic surface names ROOF, WALL, or FLOOR, multiple target equations will be generated and the number "ntgt" in the global CONTROL block must be set as discussed below.

TO

$=$ aname

"aname" is nominally the receiving host in a targeted transfer; "aname" must obey the same rules as outlined above for "hname" and "mame." In order to simplify input for large problems, FROM/TO pairs may be repeated for a given fission product without repeating "fpname," "aval," "bval," and "th" as long as these parameters do not need to be changed. The TO keyword must follow the FROM keyword. Note that use of a generic surface name for "aname" will also generate multiple target equations, and in such a case, the number "ntgt" in the global CONTROL block must be set as discussed below.
\end{abstract}

EOI

the keyword used at the end of the TARGET block and also at the end of the entire FPM-CELL block to terminate the input.

Note that the keyword HOST should appear once for each host which has finite initial fission product masses. The keyword TARGET should appear only once in each FPM-CELL block, and only one FPM-CELL block should appear in the input for a given cell.

The following discussion applies only to TARGET input involving the generic fission product host names ROOF, WALL, and FLOOR. In situations where "mame" and/or "aname" are ROOF, WALL, or FLOOR, the following guidelines should be followed to determine the smallest allowable value of "ntgt" in the global control input block. Note that the generic meaning of these names will apply even if ROOF, WALL, or FLOOR is used in an attempt to reference a single structure that happens to be named either ROOF, WALL, or FLOOR. Consequently, structures named ROOF, WALL, or FLOOR cannot be explicitly referenced in the targeted release model to release or accept fission products. Thus, cell structures should not be named ROOF, WALL, or FLOOR if explicit reference to them in the targeted release input is desired.

Two variables, $n_{1}$ and $n_{2}$, are used to assist in the following description. If either ROOF, WALL, or FLOOR is specified as the FROM host, the value of $n_{1}$ is the number of structure surfaces of the corresponding orientation in the cell. The inner surface of a structure of type ROOF, WALL, or FLOOR has an orientation corresponding to the structure type. The outer surface has an orientation of the opposite type (i.e., FLOOR, WALL, and ROOF) and is included among surfaces of that orientation if the outer surface is exposed to the atmosphere in the cell in which the structure is defined. Note that while ROOF, WALL, and FLOOR refer here only to certain surfaces within the cell, the outer surfaces of structures are always hosts regardless of the cell specified or boundary condition used.) If the FROM host is not ROOF, WALL, or FLOOR, the value of $n_{1}$ is one. Identical guidelines apply to the name of the TO host in determining the value of $n_{2}$. The number of equations associated with a given FROM/TO pair is given by the product of $n_{1}$ and $n_{2}$. If multiple FROM/TO pairs are specified without repeating "fpname," this product should be formed for each pair. The sum of all $n_{1} n_{2}$ products for one "fpname" is then multiplied by the number of chain 
elements that are named "fpname." The sum of such values for each "fpname" in the targeted release input for all cells is the smallest allowed value for "ntgt."

Fortunately, "ntgt" does not have to be exactly equal to the number of targeted release equations; instead, it is only required that "ntgt" not be smaller than this number. For this reason, it is highly recommended that "ntgt" not be computed when complex targeted release and acceptance input is used. Instead, it is recommended that a large value (e.g., 1000) be specified. The fission product input routine will write an error message to the error file if "ntgt" is too small to run the problem.

14.3.1.11 Cell-Level DCH Input Block. The DCH-CELL block is used to specify DCH parameters specific to a given cell. This block need not be given if all the parameters defined in the global DHEAT block are appropriate for a given cell. However, it should be noted that additional trapping model options and tabular (i.e., value versus time) input for certain parameters (see VAR-PARM below) are available in the DCH-CELL input block but not in the DHEAT block. The list of the parameters specifiable in tabular form is given under the "oyvar" description. Note that the tabular values of a given parameter will take precedence over any single value specified after the keyword for that parameter.

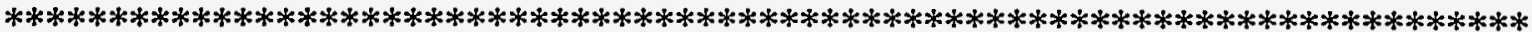

\section{DCH-CELL}

[\{SDSLIP=(sdslip) or SDEVEN=sdeven $\}]$

[DENDRP $=$ dendrp] [RADGAS $=$ radgas] $[\mathrm{RADMUL}=$ radmul $]$

[GASSUR=gassur] [DIFO2=difo2] [DIFH2O=difh2o] [HTCMUL=htcmul]

[THRESH=thresh] [LIQSDE dl1 ell dl2 el2] [PRODSEP $=[\{\mathrm{ON}$ or OFF $\}]$

$[\mathrm{RCOMH} 2=[\{\mathrm{ON}$ or OFF $\}]][$ VELOCITY $=$ velcty $]$

[DIATRAP=diatrp] [VELTRAP=veltrp] [RADTRAP=radtrp]

[\{TRAPRATE=trprat or

TRAPPING

\{GFT or TFI or TOFKU or USER=trprat\}

[\{FROMCELL=icell or FROMVENT=ivent\}] [ADFLOW=adflow]

[LEN1=xlen1] [LEN2=xlen2] [LEN3=xlen3] [LENGFT=xleng]

[KU1=xku1] [KU2=xku2] [SURTEN=surten]

[RHODG $=\{$ GAS or MEX $\}$ ] [VNOST $=\{$ GFT or CNVEL $\}]$ [TRAPMIN=trpmin] [TRAPMAX=trpmax] [TRAPMUL $=$ trpmul]

EOI\}] [COOLFRAC $=(\mathrm{cfrac})]$

[CVAR-PARM

[FLAG=iflag]

[NAME=oname]

VAR-X=oxvar $\mathrm{X}=\mathrm{nx}$ (xpts)

EOD]

VAR-Y=oyvar $Y=n y$ (ypts)

EOI

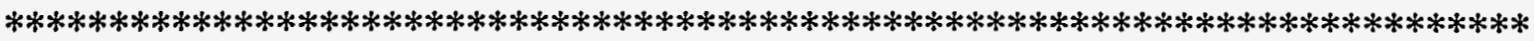


The following keywords and associated input in the DCH-CELL input block have the same meaning as they do when given in the DHEAT global input block: SDSLIP, SDEVEN, DENDRP, RADGAS, RADMUL, GASSUR, DIFO2, DIFH2O, HTCMUL, THRESH, LIQSIDE, PRODSEP, RCOMH2, VELOCITY, and TRAPRATE. Therefore, the reader should refer to the DHEAT section for the corresponding input descriptions. The primary difference between the DHEAT and DCH-CELL input for these keywords is that the values specified in the DCH-CELL block apply only to the cell being defined. On an initial run, the values defined or taken to be the default in the DHEAT block, but not redefined in a DCH-CELL block, will remain as defined in the DHEAT block. On a restart, the same logic applies, but the default values of parameters will be the values used on the initial run or previous restart, if any. If specified in the DHEAT block of a restart, the new values apply to all cells unless respecified in the DCH-CELL block of the restart also.

DCH-CELL the keyword to initiate cell-specific DCH input.

DIATRAP $=$ diatrp

VELTRAP $=$ veltrp

RADTRAP $=$ radtrp

TRAPPING

GFT

TFI

TOFKU the effective diameter for debris chemistry and heat transfer for (nonairborne) debris in the trapped bin. This diameter is used to calculate the heat and mass transfer rates as long as debris is present in the trapped bin. Unlike the airborne diameters, the trapped bin diameter may be different in each cell. If the trapped bin diameter is zero for a given cell, heat and mass transfer for the trapped bin in that cell will be ignored. Default $=0$. (m)

the user-specified relative gas-debris velocity for the trapped bin. If this input is omitted, the code will use the inner face convective velocity for the second heat transfer structure defined in the cell, or if a second structure is not present, the calculated average gas velocity through the cell, for the relative velocity. $(\mathrm{m} / \mathrm{s})$

the black body multiplier for debris-to-gas radiation heat transfer for the trapped bin. Using this parameter allows the trapped bin to radiate to the gas. By default this parameter is set to whatever is used for the current value of "radgas," the airborne debris multiplier. Note that the trapped bin does not radiate directly to structures. Default = "radgas," the value used for the airborne debris fields.

the keyword to initiate the specification of the trapping model to use to describe debris de-entrainment. If both the global TRAPRATE and cell-level TRAPPING input are given, the cell-level specification in the TRAPPING input will override the global level input for the cell in question. If a trapping model is not specified at all, then the GFT model is the default, with a fall height equal to the cube root of the initial cell gas volume.

the keyword to select the GFT trapping model discussed in Section 6.3.

the keyword to select the TFI trapping model described in Section 6.3.

the keyword to select of the TOF/KU trapping model described in Section 6.3. 
FROMCELL

$=$ icell

FROMVENT

$=$ ivent

ADFLOW

$=$ adflow

LEN1

$=$ xlen 1

LEN2

$=$ xlen 2

LEN3

$=$ xlen 3 indicates the regular flow path to use for the primary flow of debris into the cell. This path is identified by cell "icell" at the other end of the flow path. The flow rate through this flow path is used to calculate various flow parameters, including the debris velocity, in the TFI and TOF/KU trapping models as described in Section 6.3. If this input is not specified the mass flow rate through both regular flow paths and engineered vents will be monitored and the dominant flow path for gas and debris inflow will be automatically determined by the code. In contrast to earlier versions of CONTAIN, this input can now be specified with any of the trapping models, since this input affects the debris heat transfer rate as well as the trapping rate.

the engineered vent flow path to use for the primary flow for debris into the cell. The integer "ivent" is a vent number, not a cell number. The flow rate through this flow path is used to calculate various flow parameters, including the debris velocity in the TFI and TOF/KU trapping models as described in Section 6.3. If this input is not specified, the mass flow rate through both regular flow paths and engineered vents will be monitored and the dominant flow path for gas and debris inflow will be automatically determined by the code. Unlike earlier versions of CONTAIN, this input can now be specified with any of the trapping models, since this input affects the debris heat transfer rate as well as the trapping rate.

the flow area for the dominant debris transport path entering the cell. This value is used to calculate various flow and debris transport velocities as described in Section 6.3; e.g., it corresponds to the area $\mathrm{A}_{0}$ of Equation (6-100) in Section 6.3.6.1. Normally the dominant path will be determined by the code as the path which has the most debris entering the cell, but this can be overridden as discussed above in connection with FROMCELL and FROMVENT. If ADFLOW is specified, this value will be used for the flow area. The default is the flow area defined in the FLOWS or ENGVENT block for whatever flow path is the dominant flow path for entering debris. This usage applies independently of whether the dominant path is selected by the code or specified by the user.

the distance to first impact that is used in the TFI and TOF/KU trapping models to calculate flight time. Default $=$ the cube root of the initial cell gas volume. (m)

the distance to second impact that is used in the TOF/KU trapping model to calculate flight time. The default is the same as that for LEN1 above. (m)

the third length used in the TOF/KU trapping model, if the default VNOST $=$ CNVEL option is used and if conditions are such that debris does not stick on the first two impacts. The total flight time under such conditions includes the flight time to the first two structures plus the flight time to travel distance 


\section{LENGFT} $=$ xleng

KU1

$=x k u 1$

KU2

$=\mathrm{xku2}$

SURTEN

$=$ surten

RHODG $=\{$ GAS or MIX $\}$ "xlen3" at the debris exit velocity from the cell. The default is the same as that for LEN1 above. (m)

the gravitational fall height used in the GFT, TFI, and TOF/KU trapping models. The default is the same as that for LEN1 above. (m)

the Kutateladze number cutoff for the first impact used in the TOF/KU trapping model. Values of the calculated Kutateladze number greater than the value of "xku1" indicate that debris remains airborne after the first impact. Default $=10$.

the Kutateladze number cutoff for the second impact used in the TOF/KU trapping model. Values of the calculated Kutateladze number greater than the value of "xku2" indicate that debris remains airborne after the second impact. Default $=10$.

the debris surface tension used in the evaluation of the Kutateladze number for the TFI and TOF/KU trapping models. If SURTEN is specified for the cavity cell, this value will also be used in the entrainment models, if they are invoked. Default $=1$. $(\mathrm{N} / \mathrm{m})$

flag determining whether debris is to be included in the upstream and downstream densities in evaluating the $N_{\mathrm{Ku}}$ number. The default is to exclude the debris, although MIX may be a more reasonable option. The MIX option is also the more conservative of the two. Thus, the use of RHODG=MIX is recommended even though it is not the default for upward compatibility reasons. Default $=$ GAS.

VNOST $=\{\mathrm{GFT}$ or CNVEL $\}$

TRAPMIN $=$ trpmin

TRAPMAX $=$ trpmax

TRAPMUL $=$ trpmul flag determining whether or not GFT rules apply after the second impact if the $\mathrm{N}_{\mathrm{Ku}}$ re-entrainment criterion is met. Default $=$ CNVEL.

the smallest allowable value of the trapping rate. Smaller values calculated by any of the trapping model methods will be overridden by the specified value. Default $=0 .\left(\mathrm{s}^{-1}\right)$

the largest allowable value of the trapping rate. Larger values calculated by any of the trapping model methods will be overridden by the specified value. Default $=10^{20} \cdot\left(\mathrm{s}^{-1}\right)$

a multiplicative factor for the calculated trapping rate. Whether trapping is specified as a constant rate or calculated using one of the available models, the trapping rate will be multiplied by the value of "trpmul" and then limited to "trpmin" or "trpmax" range. Default $=1$. 
COOLFRAC $=$ (cfrac)

VAR-PARM

FLAG

NAME

= oname

VAR-X

= oxvar

$\mathrm{X}$

$=\mathrm{nx}$

(xpts)

VAR-Y

= oyvar the fraction of trapped debris to send to the atmosphere, structure surfaces, and the uppermost intermediate layer. Thus, exactly "nhtm" +2 values must be given, where the first value is for the atmosphere, the next "nhtm" are for the structures in the cell, and the last for the uppermost lower cell intermediate layer. At present, only the last value is used by the code, which corresponds to $\mathrm{f}_{\mathrm{LC}}$ lower cell layer fraction described in Section 6.3.1. Note that this fraction is used only if there are intermediate lower cell layers. The default for these fractions is 0 for all entries.

the keyword to initiate tabular input for any of the direct heating parameters listed under "oyvar." The tables have the standard format of the CONTAIN user table option and are used here to specify the time-dependence of the parameters. Any number of tables may be given, with each one initiated with the VAR-PARM keyword and terminated by an EOI. The total number of VAR-PARM tables plus other cell-level user tables must not exceed the value of the cell control parameter "numtbc," and the number of time points in any one table may not exceed the value of the cell control parameter "maxtbc." Note that the tabular values for a parameter override all other input for that parameter. For DCH tables, the table end-point values are used when the time is outside of the range of the table. Note that on a restart, tables should be specified in the original order, up to and including any table that has changed. The reason for this requirement is that these tables are stored according to input order, and not type.

a flag indicating the interpolation option for the parameter table. A value of 1 is for step interpolation, and 2 is for linear interpolation. Default $=1$.

the optional name for the table.

the independent variable. Note that "oxvar" must be specified as TIME.

the number of independent variable values in the table.

" $n x "$ values of the independent variable (i.e., time).

the dependent variable. Any one of the following DCH-CELL keywords may be specified for "oyvar": DENDRP, RADGAS, RADMUL, DIFO2, DIFH2O, HTCMUL, THRESH, DL1, EL1, DL2, EL2, VELOCITY, SURTEN, LEN1, LEN2, LEN3, LENGFT, KU1, KU2, DIATRAP, VELTRAP, RADTRAP, and TRAPRATE. It should be noted that the values specified for the dependent variable apply to all fields, with the exception of the RADMUL parameter, which applies only to airborne debris; the RADGAS parameter, which applies to airborne debris and also provides the default for RADTRAP; 
and the DIATRAP, VELTRAP, and RADTRAP variables, which apply to the trapped bin only.

Y

= ny

(ypts)

EOI the number of dependent variable values in the table. The value of "ny" should be equal to "nx."

"ny" values of the dependent variable.

terminator for the DCH-CELL input block. A separate terminator is required for the TRAPPING block and for each VAR-PARM table.

14.3.1.12 Cell Overflow. This input block is used to specify the cell to which condensate runoff from structures and aerosols lost from the aerosol size mesh are diverted. The condensate runoff and the fission products associated with the runoff are transferred to the pool of the overflow cell, if it is present, or lost from the problem. As discussed in Section 8.8, the amounts of fission products transferred with the runoff are determined through the "fpliq" transport efficiency factors. The "fpliq" values are assigned in the FPLIQUID input block described in Section 14.2.6.2. The treatment of aerosol mesh losses and the fission products associated with those losses are discussed in Section 7.1.

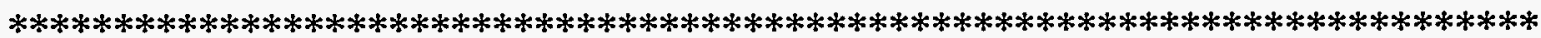

OVERFLOW=novcel

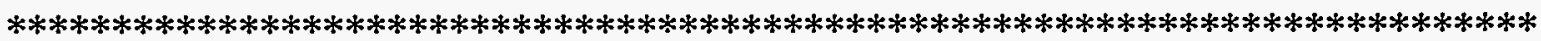

OVERFLOW

$=$ novcel specifies the number "novcel" of the destination cell for condensate runoff from structures that is not directed by FILMFLOW structure input to go to other structures. The destination cell is also used for oversized aerosols if the TRAPOVFL aerosol keyword is invoked and for coolant liquid removed from the atmosphere in the DROPOUT option in the FLOWS block. The condensate runoff that is not directed to structures and the fission products transported in that condensate will be placed in the lower cell pool, if defined, of the destination cell. If that pool is not defined and "novcel" is a valid cell number, the waste repository or location of the destination cell will be used. If "novcel" $=0$, the waste repository or location of the cell in which the runoff occurs will be used. As described in Section 7.1, aerosols that become too large for the aerosol size mesh will be treated like the condensate runoff not directed to structures, provided the TRAPOVFL keyword is specified in the global AEROSOL block; otherwise, such aerosols go to the waste location of the cell in which the oversized condition originated. CAUTION: A nonupward compatible change has been made with respect to negative values of "novcel." Such values no longer specify that aerosols which become undersized be forced to remain in the smallest size class. Instead, such retention is now specified by the global TRAPUNFL aerosol keyword. If this keyword is not specified, undersized aerosols will be treated like oversized aerosols when TRAPOVFL is not specified. 
NOTE: The cell OVERFLOW option should not be confused with the ESF OVERFLOW component (see Section 14.3.3.11) which models liquid transfers between lower cell pools.

\subsubsection{Lower Cell}

The cell level keyword LOW-CELL activates the input for the lower cell models. The lower cell consists of a series of layers in the bottom of a cell. The following layer types are considered: a concrete layer, multiple intermediate layers, a pool layer, and an atmosphere layer. For all but the atmosphere layer, a set of physics modeling options can be activated through input in the LOW-CELL block. The initial configuration of the layers and initial material masses are also specified in this input block. The atmosphere layer is used as an interface between the lower cell and the upper cell; therefore, it is automatically created, and its configuration cannot be specified by the user.

Due to the complexity of the lower cell models, the lower cell input can often be quite extensive. This is particularly true when the CORCON and VANESA models are specified. The lower cell input descriptions are therefore given at two levels of detail. The first level of detail shows the overall structure of the lower cell input. This level of detail is most useful for determining the order of the major sub-blocks in the lower cell input (see Section 14.3.2.1 below). The second level of detail gives the minor sub-blocks within each major sub-block. Complete descriptions of each keyword in the major and minor sub-blocks are given in the detailed descriptions. These descriptions are given in separate sections beginning with Section 14.3.2.2. The descriptions of the CONCRETE and INTERM input blocks are further subdivided into problems without CORCON modeling of core-concrete interactions and those with CORCON.

14.3.2.1 Overall Lower Cell Input Structure. There are two general classes of problems that can be modeled with the lower cell capabilities. The first such class consists of problems in which there are no explicitly modeled core-concrete interactions and the CORCON model is not active. Lower cell modeling is confined to heat transfer effects in such problems. The second class consists of problems in which core-concrete interactions are modeled with CORCON. In these cases the VANESA model may also be activated. The overall lower cell input structure given below applies to both general classes of lower cell modeling.

Only the major lower cell sub-blocks are shown in this section. These major sub-blocks include the sub-blocks that define the three possible types of layers (concrete, intermediate, and pool) in the lower cell. Layers in the lower cell are defined from the bottom to the top of the cavity, with the bottom layer given first in the input, the next layer up given second, and so forth. In the following, the order of layers from bottom to top is assumed to be the concrete layer, if present, then the intermediate layer(s), if present, and then the pool. This order applies to both general classes of problems.

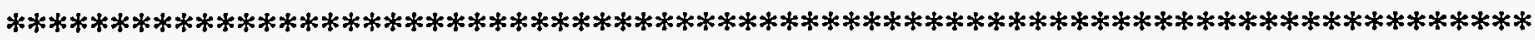

LOW-CELL

GEOMETRY=carea

$\mathrm{BC}=\mathrm{txl}$

[CRANK=crank]

[DECAY-HT

Rev. 0

$14-115$

$6 / 30 / 97$ 


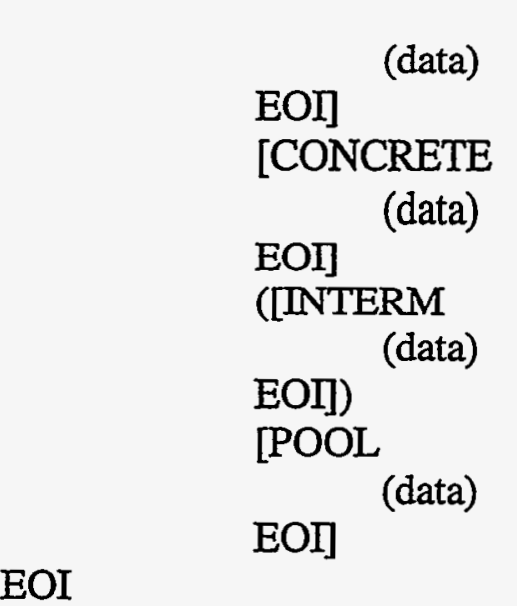

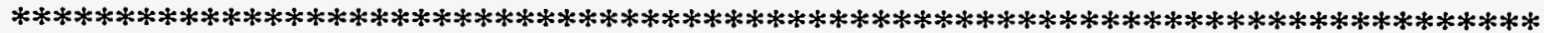

The major sub-block keywords are GEOMETRY, BC, CRANK, DECAY-HT, CONCRETE, INTERM, and POOL. A brief description of these blocks and their usage in the two general classes of problems is provided below. The required GEOMETRY and BC input blocks and the CRANK input are described in detail at the end of this section. More complete input descriptions of the optional DECAY-HT, CONCRETE, INTERM, and POOL input blocks are given in Section 14.3.2.2 through Section 14.3.2.5, respectively.

The CONCRETE layer input block is described in detail in Section 14.3.2.3. If the CORCON model is used, a concrete layer is required to specify the concrete type and other CORCON model parameters, including the starting time for the interactions. If CORCON is not specified, the concrete layer is optional but may be specified with respect to initial material masses and other parameters for the modeling of conduction heat transfer. Input for problems not involving CORCON is described in Section 14.3.2.3.1, and input for those involving CORCON is described in Section 14.3.2.3.2.

The INTERM intermediate layer input block is described in detail in Section 14.3.2.4. If the CORCON model is specified, only one intermediate layer with three nodes may be defined. This single CONTAIN intermediate layer is used to initialize the CORCON melt layers. If CORCON is not specified, multiple intermediate layers with one node each may be specified. Each such layer will be included in the heat transfer modeling. Input for problems not involving CORCON is described in Section 14.3.2.4.1 and input for those involving CORCON is described in Section 14.3.2.4.2.

The POOL layer input block is described in detail in Section 14.3.2.5. A pool layer should be specified in any problem with a lower cell. The pool layer is used as a repository for coolant in the lower cell. Even if coolant is not initially present in a problem, the pool layer should be specified if coolant is expected to accumulate in the lower cell. Aerosol settling will occur automatically onto the lower cell if a pool is defined, but not otherwise. A pool layer is also required if coolant boiling is to be modeled. Note that the pool layer must be specified to lie on top of all other specified layers; otherwise, unpredictable results may occur with respect to condensation, boiling, and pool scrubbing.

The layers specified will depend on the problem being analyzed. Only those expected to play a role in the analysis need be specified in the input. If a layer is initially empty, but could be created in the 
course of the calculations (or through user-specified material sources), then it should be specified in the lower cell input. With the exception of the intermediate layer used with CORCON, any layer may have zero initial mass. With CORCON, the user should specify the CORCON layer compositions at the CORCON start time through the intermediate layer input. Materials may thereafter be introduced into the CORCON layers to reflect time- dependent additions to the debris.

Detailed descriptions of the GEOMETRY and BC sub-blocks are given below.

GEOMETRY the area of the solid layers of the lower cell. This input should be given $=$ carea immediately after the LOW-CELL keyword. If CORCON is not invoked, "carea" is used for all solid layers and any solid coolant pool residue present when the pool is dry. If CORCON is invoked, "carea" is used for heat transfer from solid coolant pool residue to the atmosphere. For a coolant pool layer that is not completely dry, the pool depth and the pool-atmosphere heat transfer will in general be governed by the pool-atmosphere interface area determined from the cell GEOMETRY (i.e., CELLHIST) input, not the lower cell GEOMETRY input. If CORCON is invoked and the coolant pool layer is null, the CORCON melt upper surface area will be used in the heat transfer to and from the cell atmosphere. One further use of "carea" is dictated by upward compatibility: the dedicated suppression vent model uses "carea" of the wetwell cell as the total surface area of the suppression pool, including the drywell extension of the suppression pool. This total surface area determines the vent submergence, not the cell GEOMETRY input. $\left(\mathrm{m}^{2}\right)$

BC the basemat boundary condition temperature. The basemat is defined as the $=\mathrm{txl} \quad$ region below the bottommost layer in the lower cell and is assumed to be at a constant temperature "txl." This constant temperature boundary condition is used only if CORCON is not active. (For upward compatibility, an obsolete pressure boundary condition value, "pbot," may be given immediately after "txl." The "pbot" value is no longer used by the code, and any value entered will be ignored.) (K)

CRANK the time integration factor corresponding to the factor $c$ in Equation (10-121). $=$ crank Any value between 0 and 1 can be given; however, values less than 0.5 are not recommended for stability reasons. The value given here applies only to heat conduction in the lower cell. Default $=1$.

14.3.2.2 Makeup Decay Power. This optional block activates the ANSI-standard decay power model used by CONTAIN. It may be utilized in any number of cells. If DECAY-HT input has been defined in more than one cell, the total reactor power is the sum of the "rpwr" values in each cell. The general structure of the DECAY-HT input block and detailed descriptions of the DECAY-HT input options are given below.

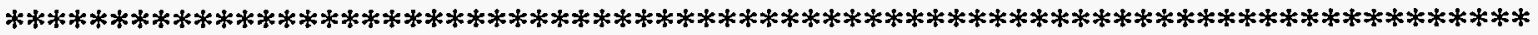

DECAY-HT rpwr

DIST-PWR (dpwr)

Rev. 0

$14-117$

$6 / 30 / 97$ 
[Q235U $=q 235 \mathrm{u}][\mathrm{Q} 238 \mathrm{U}=\mathrm{q} 238 \mathrm{u}] \quad$ [Q239PU $=\mathrm{q} 239 \mathrm{pu}]$ [P235U=p235u]

[P238U=p238u] [P239PU=p239pu] [R239U=r239u] [ROPT=ropt]

EOI

[TOFSD=tofsd] [PSCALE=pscale] [TDHSTR=tdhstr]

\begin{abstract}
$* * * * * * * * * * * * * * * * * * * * * * * * * * * * * * * * * * * * * * * * * * * * * * * * * * * * * * * * * * * * * * * * * * * * * * * * *$
\end{abstract}
DECAY-HT

the keyword to begin the specification of parameters for the decay power model.

rpwr

the reactor thermal power associated with the current cell. This is part or all of the nominal power at which the reactor operated in the time period prior to shutdown. Values of this quantity for all cells will be summed to obtain the total reactor power. (W)

DIST-PWR

the keyword to initiate input of the power distribution fraction for each layer in the lower cell, plus a stationary power distribution fraction for the atmosphere.

dpwr

the fraction of the makeup decay power to be allocated to each layer in lower cell, plus the cell atmosphere. Exactly one fraction must be specified for each layer, and for the cell atmosphere. The specified fractions apply to the concrete layer, each intermediate layer, the pool layer, and the atmosphere, in that order. If a layer has no mass, its power is allocated to the first nonnull node below, if any. Default $=0$.

Q235U

$=\mathrm{q} 235 \mathrm{u}$

the total recoverable energy per fission for U-235. Default $=199.27$. (MeV/fission)

Q238U

$=\mathrm{q} 238 \mathrm{u}$

the total recoverable energy per fission for U-238. Default $=199.59$. (MeV/fission)

Q239PU

the total recoverable energy per fission for Pu-239. Default $=210.48$.

$=\mathrm{q} 239 \mathrm{u}$ (MeV/fission)

P235U

$=\mathrm{p} 235 \mathrm{u}$

P238U

$=\mathrm{p} 238 \mathrm{u}$

the fraction of reactor power generated from U-235. Default $=0.6716$.

P239PU

$=\mathrm{p} 239 \mathrm{pu}$

the fraction of reactor power generated from U-238. Default $=0.0421$.

R239U

$=\mathrm{r} 239 \mathrm{u}$

the fraction of reactor power generated from Pu-239. Default $=0.2863$.

the ratio of U-239 atoms produced per fission at time of shutdown. Default $=0.53$. 

ROPT
the reactor operating time prior to problem start time. The reactor is assumed
$=\mathrm{ropt}$
to have been at full power during operation. Default $=5.05 \times 10^{7}$. (s)
TOFSD
the time of reactor shutdown (should be prior to problem start time). Default
$=$ tofsd
$=0$. $(s)$
PSCALE
the reactor power scale factor. Default $=0.947$.
$=$ pscale
TDHSTR
the time at which decay heat will begin to be added to the lower cell layers.
$=$ tdhstr
Default $=0$. $(s)$
EOI
the keyword used to terminate the DECAY-HT input.

If the required DIST-PWR input is not specified, the default power distribution fractions of zero will be used. The makeup power would thus be lost from the problem. Note that a portion (or all) of the makeup power may be directed to the upper cell atmosphere by setting the atmosphere layer fraction to a non-zero value.

The DECAY-HT option may be used in conjunction with the CORCON model only if time-dependent core debris sources are not used. The internally computed CORCON decay heat (see CORESTAT and DKPOWER in Section 14.3.2.4.2) will be normalized to the value computed by the makeup decay power model.

14.3.2.3 Concrete Layer. The configuration of the lower cell concrete layer is specified in the CONCRETE block. If CCIs are being modeled with CORCON, the CONCRETE input block is also used to specify the concrete type and various other input parameters for the CORCON and VANESA models. If CORCON is not used, the CONCRETE input block is used to define the initial layer masses, temperatures, user- specified sources, and heat transfer coefficients. The following two sections give the overall input structure for the CONCRETE block for both general classes of lower cell modeling.

14.3.2.3.1 Concrete Layer Input Without CORCON. The structure shown below applies to problems in which CCIs are not explicitly modeled with CORCON. Such problems involve the modeling of heat transfer in the lower cell.

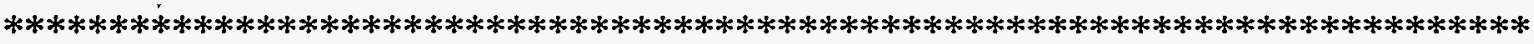

CONCRETE

[COMPOS=nma (omat cmass)]

TEMP=ctemp

[DELTA-Z=cdzin]

[PHYSICS

[SOURCE=nso

(oname $=\mathrm{n}$

IFLAG=ival

Rev. 0

14-119

$6 / 30 / 97$ 


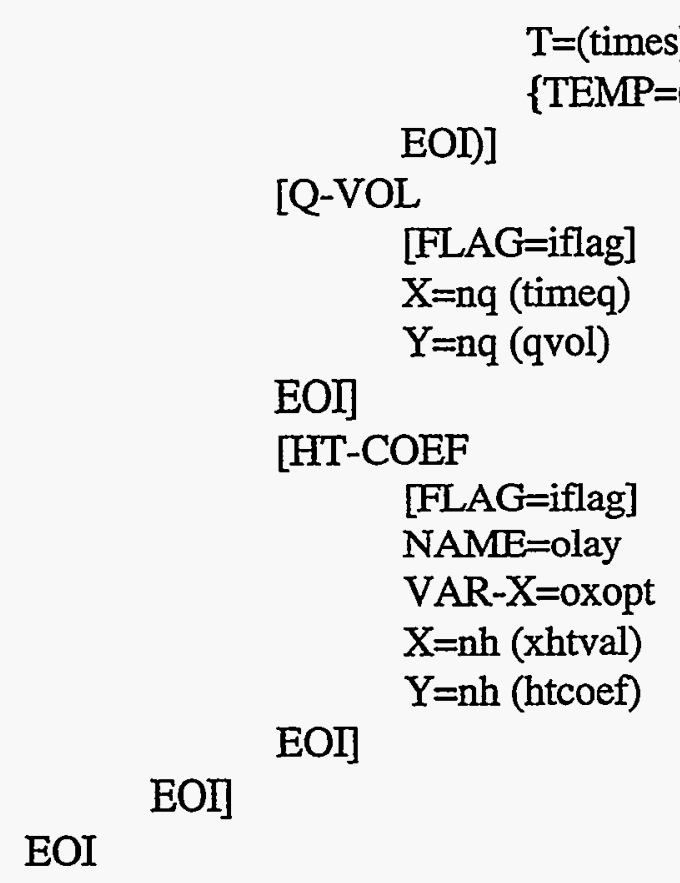

$* * * * * * * * * * * * * * * * * * * *$

CONCRETE the keyword to initiate the definition of the concrete layer.

COMPOS the keyword to initiate the specification of initial material masses in the concrete layer. If this keyword and its associated input are omitted, the concrete layer will be initially empty.

nma

the number of materials (including CONC) initially present in the concrete layer.

omat

the name of a CONTAIN material being specified as initially present in the concrete layer. This name must be among the materials specified either after the COMPOUND or USERDEF keyword in the MATERIAL input block. In most instances, at least one "omat" name will be CONC for concrete; other materials may also be included in the concrete layer to simulate steel rebar or other constituents. Note that the specific concrete types discussed in the next section under the CONCRETE keyword are only used in the CORCON and VANESA models. For upward compatibility, input for a specific concrete type may still be given here. If this is done, the code will then substitute the equivalent CONC material for the concrete type specified.

cmass

the initial mass of material "omat" in the layer. If DELTA- $Z$ is not specified, this mass will be equally divided among the nodes in the concrete layer. By default the thicknesses of the nodes and the entire concrete layer are determined by the code from the volume occupied by the materials and the area "carea" of the layer. $(\mathrm{kg})$ 
TEMP the required initial temperature of the concrete layer. $(\mathrm{K})$

= ctemp

DELTA-Z

$=$ cdzin

the optional initial concrete node thickness. If this option is used, initial material masses in the layer will be uniformly loaded into each node from the bottom to the top until either each node has been filled, or all initial mass has been exhausted. In the latter case, some of the top nodes will be empty and will therefore be excluded from the heat transfer modeling. The number of nodes corresponds to the larger of 5 or "jconc." This option is useful for fixing the initial thickness of the concrete layer as opposed to the mass. (m)

PHYSICS

the keyword to initiate input of the layer physics options. This keyword is required if any of the options described below are selected.

The SOURCE sub-block for specifying materials to be introduced into the CONCRETE layer uses a standard notation. The SOURCE keywords and values not discussed below are defined in Section 14.4.1.

SOURCE the keyword to initiate input of material source tables for the layer.

oname the name of the material to be introduced. It should be the name of a material specified after the COMPOUND keyword or USERDEF keyword in the MATERIAL input block. The material does not have to be present initially in the layer.

The Q-VOL and HT-COEF blocks use cell level tables that require the user to set appropriate values for "numtbc" and "maxtbc" in the cell CONTROL block. The standard table keywords X, Y, NAME, VAR-X, and FLAG are defined in Section 14.4.2. The variables associated with Q-VOL and HT-COEF are defined below.

Q-VOL the keyword to begin the specification of a volumetric heating table. (This option should not be confused with the DECAY-HT option.) Only one Q-VOL table may be specified in a given layer.

$\mathrm{nq}$ the number of points in the Q-VOL table.

timeq the independent variable of the Q-VOL table, which corresponds to time. Specify "nq" values in ascending order. Note that the heating is set to zero if the time is outside of the range of the table or if the layer has no mass. (s)

qvol the volumetric heating rate of the layer. Specify "nq" values. (W) 
the keyword to begin the specification of a heat transfer coefficient table. Within the range of the table, the table values will override the internally calculated overall interlayer heat transfer coefficient. Outside of the range, the internally calculated coefficient will be used. The specified heat transfer coefficient is taken to be the total, including half-node resistances, if any, between the present layer and one above it or between the present layer and atmosphere or basemat. The independent variable name "oxopt" and the name "olay," which specifies the boundary to which the heat transfer coefficient applies, must be specified. Only one HT-COEF table may be specified for a given layer. When the atmosphere is involved, the meaning of the overall interlayer heat transfer coefficient is ambiguous. The reader should consult Section 5.5.2 for a discussion of how the heat transfer coefficient is interpreted.

NAME

olay

oxopt

$\mathrm{nh}$

xhtval

htcoef the keyword to specify the boundary to which the heat transfer coefficient table applies.

either the name of a layer above the present layer or the keyword ATMOS or BAS-MAT for the atmosphere or basemat, respectively. The table values will be applied (1) when the present layer is not a null layer and (2) when the layer specified is not a null layer and is physically adjacent to the present layer or, in the case of the atmosphere or basemat, when the atmosphere or basemat is physically adjacent to the present layer. (Null layers between the present layer and the layer considered physically adjacent are ignored.) Intermediate layer names are those given after the LAY-NAM keyword (see Section 14.3.2.4). Other layer names match the type of the layer (i.e., CONCRETE or POOL).

a character flag that indicates the type of independent variable represented by "xhtval." Replace with either TIME, TEMP, or DELTA-T. These choices represent time, temperature, or temperature difference across the boundary.

the number of points in the heat transfer coefficient table.

the independent variable of the table. It represents either time (s), temperature $(K)$, or temperature difference $(K)$. The temperature difference is defined as the temperature above the boundary at which the heat transfer coefficient applies minus the temperature below the boundary. Note that if the time, temperature, or temperature difference at a given point in time lies outside of the range of the table, the internally calculated heat transfer coefficient will be used. Specify "nh" values in ascending order.

the dependent variable of the table, which represents the heat transfer coefficient across the boundary specified by "olay." ( $\left.\mathrm{W} / \mathrm{m}^{2}-\mathrm{K}\right)$ 
14.3.2.3.2 Concrete Layer Input With CORCON. The overall input structure for the CONCRETE input block when CORCON is invoked is given below. The SOURCE, Q-VOL, and HT-COEF physics options are not given in this summary, because they have no effect on the calculation when CORCON is active. These options may be specified, however, because they will affect the heat conduction model that is active before CORCON begins and after CORCON ends. The input templates for the CORCON and VANESA sub-blocks within the CONCRETE template below have been separated for clarity because of their increased length over previous versions of the code.

Invoking the CORCON model within CONTAIN requires that both a concrete and an intermediate layer be specified. The cavity geometry, concrete type, CORCON modeling options, VANESA modeling options, and fission product inventories are specified in the concrete layer input described below. The initial melt composition and any user-specified time dependent mass sources to the melt are specified in the intermediate layer input described in Section 14.3.2.4.2.

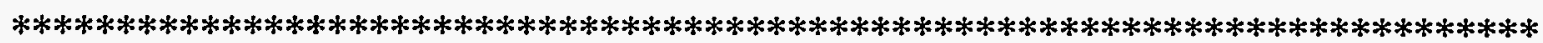

CONCRETE

COMPOS $=1$

\{CONCRETE $=\{$ BASALT or LIME or GENERIC or OTHER (fmatl) tabl efusn $\}$ [[TSOLID=tsolct] [TLIQID=tliqct] [TABLAT=tabl] [RHOCON=rhoc] [REBAR=rbr] [EMCONC=ew] [RBRCOMP EOI] (ometl=fmfrac)

$\mathrm{EO}]$

cmass

TEMP=ctemp

PHYSICS

CORCON

TIMES tstart ndelt (dtmin dtmax dedit timdt)

GEOMETRY ro zo

\{HEMICYL rs he rw hbc or

FLATCYL zt rad hit radc rw hbb nbot ncorn or

EMISIV

ARBSHP nbot rtang rw htotl (ri zi)\}

OXIDE oflag neo (torto eo)

METAL oflag nem (tortm emm)

[SURRND oflag ns (torts es)]

EOI

[CPCHEM] [COKING] [NOUNKGAS]

[SLAGBOT] [SLAGSIDE]

[SPREAD nrad htmin htmax (timi radi)]

[MIXING] [MOVIES]

Rev. 0

$14-123$

$6 / 30 / 97$ 


\section{[USERSENS}

[ $\{$ HXBOTCOR ahtb bhtb chtb or HXBOTMUL=xbm\}]

[HXSIDCOR ahts bhts chts or HXSIDMUL=xsm\}]

[\{HXINTCOR ahti bhti chti or HXINTMUL=xim\}]

[ $\{\mathrm{HBOILFLX}=\mathrm{nhb}(\mathrm{dtsat}$ bflx) or HBOILMUL=nhb (dtsat bmul) $\}]$

[MINBFTMP=tmfb] [DCRIT=dcrit]

[ENTMUL=entmul] [DNTMUL=dntmul]

[CDMUL=cdcmul] [VDCMUL=vdcmul]

[CNDMUL=cndmul] [AERSIZ=aersiz]

[\{BUBRMUL brl br2 br3 or BUBRFIX bubr\}]

[TKMMUL=ntkm (temp tkmi)] [TKOMUL=ntko (temp tkoi)]

[VSMMUL=nvsm (temp vsmi)] [VSOMUL=nvso (temp vsoi)]

[STMMUL=stmmul] [STOMUL=stomul]

[RHOMMUL=rhmmul] [RHOOMUL=rhomul]

[TSOMLT=tsomlt] [XEUT=xeut] [ZLCH=zilch]

EOI

EOI]

\section{[VANESA}

[AERCONST=numaer [(aername ncnams (ovnam))] [aername -1]

[FPTRACK

\{SIMPLE=nvanfp (ovnam) or

DETAIL=nvcons (ovnam nfp (ofpnam wfrac

$\{$ GAS or AEROSOL=aerid\}))\}]

[\{MELTCOMP (cmelt) or (ovfp=vfpm)\}]

[NONIDEAL $=\{N E T H H E R$ or METAL or OXIDE or BOTH $\}]$

[SCRUB

EOI]

[BSIZI=bsizi]

EOI

EOI]

EOI

*********************************************************************************1

The reader should note from the input block above that several of the input options provided in the previous implementation of CORCON Mod2 are no longer available. For upward compatibility purposes, input files that include these options are accepted, and a warning message is written to the error file. The ignored keywords and their associated input blocks will be identified in the discussions of the CORCON and VANESA sub-blocks which follow.

CONCRETE the keyword to initiate the definition of the concrete layer.

COMPOS

$=1$ the required composition keyword and value. The properties of the concrete layer in CORCON problems are controlled by the CONCRETE type specification. Note than when CORCON is inactive, the material CONC will 
CONCRETE

BASALT or

LIME or

GENERIC or

OTHER

fmatl

tabl

efusn

TSOLID

$=$ tsolct

TLIQID

$=$ tliqct

TABLAT

$=$ tabl

RHOCON

$=$ rhoc

REBAR

$=\mathrm{rbr}$

EMCONC

= ew be assumed to be present in the concrete layer with a mass "cmass" for the purpose of doing heat conduction calculations. The concrete type specified here through "otyp" has no effect on the properties of the CONC material.

the required keyword to initiate the concrete type specification when the CORCON model is used.

allowable values for the concrete type. The type LIME refers to limestone/ common sand concrete while GENERIC refers to limestone concrete. The keyword OTHER allows the user to specify the mass fractions present in melted concrete. Note that the OTHER keyword refers to the composition of melted concrete and is distinct from the mass fractions for solid concrete. The composition and properties of BASALT, LIME or GENERIC are given in Table 5-1.

the array of mass fractions in solid concrete. Exactly 13 mass fractions corresponding to the following species must be given in the following order: $\mathrm{SiO}_{2}, \mathrm{TiO}_{2} \mathrm{MnO}, \mathrm{MgO}, \mathrm{CaO}, \mathrm{Na}_{2} \mathrm{O}, \mathrm{K}_{2} \mathrm{O}, \mathrm{Fe}_{2} \mathrm{O}_{3}, \mathrm{Al}_{2} \mathrm{O}_{3}, \mathrm{CO}_{2}, \mathrm{H}_{2} \mathrm{O}$ (evaporable), and $\mathrm{H}_{2} \mathrm{O}$ (bound). The default values of these mass fractions for the three predefined concrete types are given in Table 5-1.

the ablation temperature of concrete. Default values are given in Table 5-1. (K)

the heat of fusion associated with concrete decomposition. For upward compatibility reasons, this value must be specified, but it is not used.

the concrete solidus temperature. Defaults are given in Table 5-1 for "otyp" = BASALT, LIME, and GENERIC. $(\mathrm{K})$

the concrete liquidus temperature. Defaults are given in Table 5-1 for "otyp" $=$ BASALT, LIME, and GENERIC. $(\mathrm{K})$

the ablation temperature of the concrete surface. Defaults are given in Table 5-1 for "otyp" = BASALT, LIME, and GENERIC. (K)

the concrete density. Defaults are given in Table 5-1 for "otyp" = BASALT, LIME, and GENERIC. $\left(\mathrm{kg} / \mathrm{m}^{3}\right)$

the mass ratio of reinforcing steel to concrete in the layer. Default $=0$.

the emissivity of the concrete surface. Used in radiation modeling between the concrete surface and the ablating material. Default $=0.8$. 
RBRCOMP

ometl

fmfrac

EOI

cmass

TEMP

= ctemp

PHYSICS

CORCON

TIMES

tstart

ndelt a keyword which initiates specification of metal composition of the rebar in the concrete. Typically rebar is pure iron, but with this input one can specify the rebar to be any combination of the 13 CORCON metal species given below.

name of one of the allowable CORCON metal species. The allowable species are listed in Table 5-2.

the mass fraction of the metal species "ometl" in the rebar. The mass of this species introduced into the melt per $\mathrm{kg}$ of concrete ablated is equal to this fraction multiplied by the "rebar" value.

the terminating keyword to be used if one or more of the concrete keywords TSOLID, TLIQID, RHOCON, REBAR, TABLAT, or EMCONC described above is given.

the required mass of concrete. Although this value is required to signal the end of the concrete composition input, the value specified is not used when CORCON is active. However, a realistic value should be specified, since it is used in the conduction model that is active prior to the start of CORCON and after the completion of CORCON.

the required initial temperature of the concrete layer. (K)

the keyword to initiate input for the layer physics options. If CORCON or VANESA are to be activated, the PHYSICS keyword is required.

the keyword to begin the specification of the CORCON core-concrete interaction model. The CORCON sub-block is a required part of the PHYSICS block of the CONCRETE layer input.

the keyword to begin the specification of time zones within the period that CORCON will be active.

the time to begin the CORCON calculation. This value may be greater than the CONTAIN starting time, in which case CONTAIN will run for a period before invoking CORCON. Note that this time should be the same as the time "timeO" input under the CORESTAT keyword in the INTERM layer input. (s)

the number of contiguous time zones during which CORCON is active. The limits on the CORCON internally computed timestep and the edit frequency may be specified in each zone. This number must be followed by "ndelt" sets of ("dtmin," "dtmax," "dedit," "timdt"). The maximum value of ndelt is 10. 
dtmin

dtmax

dedit

timdt

GEOMETRY

ro

zo

HEMICYL

IS

hc

rw

hbc

FLATCYL

zt

$\operatorname{rad}$

hit

radc the minimum allowed CORCON timestep. (s)

the maximum allowed CORCON timestep. (s)

the time interval between CORCON edits. Note that this time interval should be larger than the CONTAIN system timestep. If it is not larger, the smallest multiple of "dedit" larger than the system timestep will be used for the CORCON edit time interval. Note also that a CONTAIN long edit will occur at each CORCON edit. (s)

the end time of the time zone. (s)

the keyword to begin the selection of the geometrical model used by CORCON for the cavity shape. Schematics of the allowed cavity shapes are shown in Reference Col84.

the radial coordinate of the center of the ray system. The value of this parameter must be 0 . (m)

the axial coordinate of the center of the ray system. (m)

the keyword to select a cylindrical cavity with a hemispherical floor. The following four parameters must be specified.

the radius of the hemispherical floor as measured from the point ("ro," "zo"). (m)

the height of the cylindrical top section. (m)

the outside radius of the cylindrical top section. (m)

the height from the external base of the cavity to the base of the cylindrical top section. (m)

the keyword to select a cylindrical cavity with a flat floor. The following eight parameters must be specified.

the cylindrical $\mathrm{z}$ coordinate of cylinder top edge. (m)

the inside radius of cylinder. (m)

the height of cylinder from the floor. (m)

the radius of inner corner. (m) 
IW

hbb

nbot

ncorn

ARBSHP

nbot

rtang

Iw

htotl

ri, zi

EMISIV

OXIDE

oflag

neo

torto

eo the outside radius of the cylinder. (m)

the height from the external base of the cavity to the floor of the cavity; i.e., the thickness of the concrete at the bottom. (m)

the number of ray points equally spaced along the flat floor of the cavity.

the number of ray points equally spaced around corner (not including tangent points).

the keyword to select the arbitrarily shaped cavity option. The number of parameters which must be specified depends on the value "nrays" given in the cell CONTROL block, and the "nbot" value below. A total of "nrays" "nbot" pairs of cylindrical coordinates ("ri," "zi") must be specified to define the cavity shape.

the number of ray points equally spaced along a flat bottom.

the cylindrical radial coordinate at the point where the floor is no longer flat. (m)

the external radius of the cylindrical cavity. (m)

the height from the external base to the top of the cavity. (m)

the cylindrical radial and vertical coordinates of body points. Specify "nrays" - "nbot" pairs of values. (m)

the required keyword to begin the specification of the emissivities that will be used by CORCON. The surface emissivity will be that of either the oxide or metal depending on the material at the surface.

the keyword to begin the specification of the emissivities of the oxide layers.

a character flag indicating the type of specification being used. The use of TIME for "oflag" indicates that the emissivities are being specified as functions of time, while TEMP implies that they are being specified as functions of surface temperature.

the number of oxide emissivities in the table.

the time (s) or temperature $(\mathrm{K})$ values for the emissivity table.

the values of oxide emissivities. 
METAL

oflag

nem

tortm

emm

SURRND

oflag

ns

torts

es

CPCHEM

COKING

NOUNKGAS

SLAGBOT the keyword to begin the specification of the emissivities of the metal layers.

the same as above.

the number of metal emissivities in the table.

the time (s) or temperature $(\mathrm{K})$ values for the emissivity table.

the values of metal emissivities.

the keyword that begins specification of emissivities of the surrounding environment for the cavity. These values will be used only if a radiation model has not been specified in the CONTAIN problem definition.

the same as above.

the number of surrounding emissivities in the table.

the time (s) or temperature $(\mathrm{K})$ values for the emissivity table.

the values of surrounding emissivities.

a keyword which enables the condensed phase chemistry model. By default, condensed phase chemistry is not modeled.

a keyword which enables the coking model. By default, the coking model is not enabled. When coking is disabled, the production of condensed carbon (C(c)) during the reaction of carbon dioxide with reactive metals, such as $\mathrm{Zr}$ and $\mathrm{Al}$, is disabled. This reaction, referred to as "coking" or "carburization," is predicted by CORCON Mod2, but has not been observed to any significant extent in any previous CCI experiments. If the CORCON Mod2 modeling is desired, this keyword should be specified to enable the coking reaction.

a keyword which enables a restrictive treatment of the melt chemistry such that only $\mathrm{CO}, \mathrm{H}_{2}, \mathrm{CO}_{2}$, and $\mathrm{H}_{2} \mathrm{O}$ gases are allowed to be produced and evolve from the debris pool. In the default treatment other gases, including species not recognized by CONTAIN, are included in the chemistry. In this treatment any gases evolving from the melt pool that are not recognized by CONTAIN are assumed to be $\mathrm{N}_{2}$.

a keyword which enables the use of the slag heat transfer model for the bottom melt-concrete interface. By default the stable gas film heat transfer model is used. The latter model is that used in CORCON Mod2. 
SLAGSIDE

SPREAD

nrad

htmin

htmax

timi

radi

MIXING

MOVIES

USERSENS a keyword which enables the use of the slag heat transfer model for the side melt-concrete interface. By default the stable gas film heat transfer model is used. The latter model is that used in CORCON Mod2.

a keyword which initiates specification of the time dependent melt radius option. This option is only available when the cavity is a flat-bottomed cylinder as specified with the FLATCYL input in the CORCON GEOMETRY block above.

the number of time/radius pairs to follow. This number should be less than or equal to 100 (this is greater than the allowable number of points in the standalone CORCON Mod3 code version).

the minimum allowable melt thickness (m).

the maximum allowable melt thickness (m).

the time at which the melt radius changes to the corresponding value of "radi."

the radius specified for the melt at the corresponding "timi."

a keyword which enables the mechanistic entrainment/de-entrainment mixing models. This can be invoked regardless of the initial layer configuration. If MIXING is not specified, the layer configuration will evolve in a manner that depends on the initial layer configuration. The options for the initial layer configuration and its time evolution in the absence of MIXING are discussed in the LAYERS input in the INTERM block. Section 2.3.6 of Reference Bra93 discusses the mixing model in more detail.

As of this writing, numerical problems are known to exist with the mixing model under certain situations, where layers will entrain and de-entrain on alternate timesteps when their densities are close. When these problems arise the mixing model should not be invoked and the homogeneous layer option (LAYERS = 3) should be selected in the INTERM input. The user might also try using smaller timesteps to solve such problems. These known numerical difficulties should be corrected in future code revisions.

an optional keyword to add CORCON cavity shape information to a separate plot file.

a keyword which initiates specification of the user flexibility options in CORCON Mod3. The options and keywords available in this block closely parallel those provided in the standalone CORCON Mod3 code as described in the CORCON Mod3 User's Manual. [Bra93] 
HXBOTCOR a keyword to initiate specification of the bulk convective heat transfer coefficient for the bottom surface of the melt. This keyword defines the Nusselt number in terms of the "ahtb," "bhtb," and "chtb" parameters. The Nusselt number is defined as $\mathrm{N}_{\mathrm{Nu}}=$ "ahtb" $\mathrm{N}_{\mathrm{Re}}{ }^{\text {"bhtb" }} \mathrm{N}_{\mathrm{Pr}_{\mathrm{r}}}$ "chtb".

ahtb

a parameter in the above convective heat transfer coefficient equation.

bhtb

a parameter in the above convective heat transfer coefficient equation.

chtb

a parameter in the above convective heat transfer coefficient equation.

HXBOTMUL $=x b m$

HXSIDCOR

ahts a parameter in the above convective heat transfer coefficient equation.

bhts

a parameter in the above convective heat transfer coefficient equation.

chts

a parameter in the above convective heat transfer coefficient equation.

HXSIDMUL $=\mathrm{xsm}$

HXINTCOR

an interlayer heat transfer coefficient. This keyword defines the Nusselt number in terms of the "ahti," "bhti," and "chti" parameters. The Nusselt number is defined as $\mathrm{N}_{\mathrm{Nu}}=$ "ahti" $\mathrm{N}_{\mathrm{Re}}$ "bhti" $\mathrm{N}_{\mathrm{P}_{\mathrm{T}}}^{\text {"chti". }}$.

ahti

a parameter in the above convective heat transfer coefficient equation.

bhti

a parameter in the above convective heat transfer coefficient equation.

chti

a parameter in the above convective heat transfer coefficient equation.

HXINTMUL a multiplier on the interlayer heat transfer coefficient. If this input is given, $=$ xim 
HBOILFLX

$=\mathrm{nhb}$

dtsat

bflx

HBOILMUL $=\mathrm{nhb}$

bmul

MINBFTMP $=\mathrm{tmfb}$

DCRIT $=\mathrm{dcrit}$

ENTMUL

= entmul

DNTMUL

$=$ dntmul

CDCMUL

$=\mathrm{cdcmul}$

VDCMUL

= vdcmul

CNDMUL

$=$ cndmul

AERSIZ

= aersiz

BUBRMUL

br1 a keyword which initiates the user flexibility input table for the time dependent coolant flux as a function of $\Delta T_{\text {sal }}$, where $\Delta T_{\text {sat }}$ is the degree of subcooling. This keyword is followed by "nhb" pairs of "dtsat" and "bflx."

the temperature difference between coolant and saturation, $\Delta \mathrm{t}_{\text {sat. }}(\mathrm{K})$

the coolant heat flux table entries. $\left(\mathrm{W} / \mathrm{m}^{2}\right)$

a keyword which initiates the user flexibility input table for the time dependent multiplier on the calculated coolant flux as a function of $\Delta T_{\text {sar }}$. This keyword is followed by "nhb" pairs of "dtsat" and "bmul." The "dtsat" input is described above.

the table entry for the multiplier applied to the calculated coolant heat flux.

the minimum film boiling temperature. $(\mathrm{K})$

the critical bubble size for entrainment. (m)

the multiplier applied to the calculated entrainment rate.

the multiplier applied to the calculated droplet settling flux.

the multiplier applied to the condensed phase diffusion coefficient.

the multiplier applied to the vapor phase diffusion coefficient.

the multiplier applied to the condensation rate coefficient.

the mean aerosol particle size. Default $=1 .(\mu \mathrm{m})$

a keyword which initiates specification of the following three bubble size multipliers. The multiplier is used based on the appropriate regime.

the multiplier for the bubble size calculated with the Fritz bubble model. 
br2

br3

BUBRFIX

$=$ bubr

TKMMUL

$=$ ntkm

temp

tkmi

TKOMUL

$=$ ntko

tkoi

VSMMUL

$=\mathrm{nvsm}$

vsmi

VSOMUL

= nvso

vsoi

STMMUL

$=$ stmmul

STOMUL

= stomul

RHOMMUL

$=\mathrm{rhmmul}$ the multiplier for the bubble size calculated with the Davidson-Schuler model.

the multiplier for the bubble size calculated with the gas film bubble model.

the fixed bubble radius. If this user flexibility option is used, the bubble size will be fixed throughout the calculation at the specified "bubr" value. (m)

a keyword which initiates specification of a table of thermal conductivity multipliers versus temperature for the metal layer. "ntkm" pairs of "temp" and "tkmi" follow this keyword.

the temperature of the layer specified in the tables of thermal conductivity and viscosity multipliers. (K)

the thermal conductivity multiplier for the metal layer.

a keyword which initiates specification of a table of thermal conductivity multipliers versus temperature for the oxide layer. "ntko" pairs of "temp" and "tkoi" follow this keyword. The "temp" variable is discussed above.

the thermal conductivity multiplier for the oxide layer.

a keyword which initiates specification of a table of viscosity multipliers versus temperature for the metal layer. "nvsm" pairs of "temp" and "vsmi" follow this keyword. The "temp" variable is discussed above.

the viscosity multiplier for the metal layer.

a keyword which initiates specification of a table of viscosity multipliers versus temperature for the oxide layer. "nvso" pairs of "temp" and "vsoi" follow this keyword. The "temp" variable is discussed above.

the viscosity multiplier for the oxide layer.

the multiplier applied to the metal phase surface tension.

the multiplier applied to the oxide phase surface tension.

the multiplier applied to the metal phase density. 
RHOOMUL $=$ rhomul

TSOMLT

$=$ tsomlt

XEUT

$=$ xeut

$\mathrm{ZILCH}$

$=$ zilch

VANESA

AERCONST

numaer the multiplier applied to the oxide phase density.

the constant value for the metal phase solidus temperature. $(\mathrm{K})$

the mole fraction of concrete oxides at which the oxide phase solidus temperature has decreased to the concrete solidus temperature. This option is used to invoke the alternate phase diagram for the oxide phase as illustrated in Figure 2.16 in the CORCON Mod3 User's Manual. [Bra93]

the value of the cutoff number of moles used to determine convergence in the MLTREA routine of the chemistry model. Default $=10^{-5}$. (kg moles)

the keyword to begin the input parameters for the VANESA aerosol/ fission product model. The VANESA sub-block is an optional part of the PHYSICS block of the CONCRETE layer input.

the keyword to initiate the specification of the mapping of VANESA constituent materials onto the CONTAIN aerosol components. At least one aerosol component must be defined in the global AEROSOL block if AERCONST is invoked. If AERCONST is not specified, released VANESA constituent materials will not be reflected in the CONTAIN aerosol inventory, since the information needed to map the VANESA constituent materials onto the CONTAIN aerosol components is not provided.

the number of CONTAIN aerosol components used to represent the VANESA constituent materials. This value must not exceed the value of "nac" given in the global CONTROL block. The next group of values "aername ncnams (ovnam)" must be repeated either "numaer"- 1 or "numaer" times. If this group is specified "numaer"- 1 times, it must be followed by a final "aername" followed by -1 (negative one) for the "ncnams" parameter, but in this case the value for "ovnam" must be omitted. This input instructs the code to automatically assign the remaining VANESA constituents to the last "aername" specified. This holds also when only one aerosol component is used to track VANESA-released aerosols ("numaer" =1). In this case, "numaer" will be followed by the name of that one component followed by -1 . This tells the input processor to assign all VANESA-released constituents to this one component. If the "aername ncnams (ovnam)" group is specified "numaer" times, only the VANESA constituents explicitly identified in one of the "ovnam" arrays will be assigned to a CONTAIN aerosol component. If a VANESA constituent is not assigned to a CONTAIN aerosol component, the release of that constituent from the melt pool will not be reflected in the CONTAIN aerosol inventory. 
aername

nenams

ovnam

FPTRACK

SIMPLE

nvanfp

ovnam the name of an aerosol component, as declared in the global AEROSOL input block, or alternatively a number between 1 and "nac" corresponding to the aerosol component. All other materials not specified through "ovnum" in the AERCONST sub-block are assigned to the last "aername," if followed by -1 , or are otherwise ignored.

the number of VANESA constituents assigned to the above aerosol component.

a list of VANESA constituent names, "ncnams" in length. The 24 legal constituent names are: FE, CR2O3, NI, MO, RU, SN, SB, TE, AG, MN, CAO, AL2O3, NA2O, K2O, SIO2, UO2, ZRO2, CS2O, BAO, SRO, LA2O3, $\mathrm{CEO} 2, \mathrm{NB} 2 \mathrm{O}$, and CSI. These are also given in Table 5-5. Note that NBO in CORCON Mod3 has replaced NB2O5 from CORCON Mod2 in this list. To retain upward compatibility with older input files, the name NB2O5 will still be allowed, but will be interpreted as NBO, and a warning will be written to the error file.

the keyword to initiate the specification of the mapping of the VANESA constituent materials onto the CONTAIN fission product inventory system. This mapping allows CONTAIN to track VANESA materials that are calculated to be volatilized and/or aerosolized as a result of the core concrete interactions. If specified, this keyword and its associated input must follow the AERCONST specification. By default, VANESA constituents are not introduced into the CONTAIN fission product system. The user must specify the VANESA constituents that are to be tracked, and must supply either the SIMPLE or DETAIL tracking parameters.

the keyword to indicate that the simplified fission product tracking option will be used. With this option individual VANESA constituents may be tracked as fission products on aerosols; however, any VANESA constituent that is tracked must match the name of a fission product declared in the FISSION global input. The fission products representing VANESA constituents are assigned to aerosol component hosts in the same proportion that VANESA constituents are assigned to aerosol components in the AERCONST input.

the number of VANESA constituents to be tracked individually as CONTAIN fission products. When the SIMPLE option is used, the value of "nvanfp" must be the same as that of "nvfpsm" in the cell CONTROL block.

a list of VANESA constituent names, "nvanfp" in length. The legal names are as given above for the aerosol assignment. In the SIMPLE tracking option, these names also must have been defined previously as fission products in the global FISSION input block. 
DETAIL

nvcons

ovnam

nfp

ofpnam

wfrac

GAS

AEROSOL $=$ aerid

MELTCOMP the keyword to select the detailed fission product tracking option. With this option, the VANESA constituents may be assigned in a general manner to the airborne (gas or aerosol) hosts of the CONTAIN fission product system. (VANESA constituents may not enter the CONTAIN fission product system directly on nonairborne hosts.) With this form of fission product tracking, the parameter "nvfpsm" on the cell CONTROL line must equal the value obtained by summing the "nvcons" values of "nfp, "two parameters discussed below.

the number of VANESA constituents that will be tracked as CONTAIN fission products. The following groups of input must be repeated "nvcons" times.

the VANESA constituent name. In the DETAIl tracking option, this name need not match the name of any CONTAIN fission product; however, it must be one of the 24 allowed VANESA constituents.

the number of CONTAIN fission products associated with the VANESA constituent "ovnam." The following three keywords must be repeated "nfp" times. (This is done for each "ovnam" given.)

the name of a CONTAIN fission product specified in the global FISSION input block.

the mass fraction of VANESA constituent "ovnam" that will be assigned to the fission product "ofpnam" on host "ohost." There is no restriction on this value; therefore, the mass tracked as CONTAIN fission products will differ from the mass produced by VANESA, if the "wfrac" values do not sum to 1 .

the keyword to indicate that the fission product mass should be assigned to the atmosphere gas.

the keyword to indicate that the host is an aerosol component where "aerid" is either the name of the aerosol component or the number of the aerosol component.

the keyword to specify the composition of fission products (minor species) in the melt layer. This is one of the two possible methods for specifying the composition. The preferred method is to use the keyword-driven "ovfp" = "vfpm" format described below. The MELTCOMP format is used in the stand-alone CORCON Mod3 code and the older stand-alone VANESA code, and has been included to accommodate users who wish to use that format. If this format is used, the eight major species of the 32 required values in the "cmeit" array following the MELTCOMP keyword are now ignored: $\mathrm{UO}_{2}$, $\mathrm{Zr}, \mathrm{ZrO}_{2}, \mathrm{Fe}, \mathrm{FeO}, \mathrm{Cr}, \mathrm{Mn}$, and $\mathrm{Ni}$. These values are now taken from the 
cmelt

ovfp

$=\mathrm{vfpm}$

NONIDEAL initial oxide and metal masses given in the COMPOS block of the intermediate layer input. The expected length of the "cmelt" array is retained at 32 for upward compatibility with older CONTAIN input decks. New decks should simply specify zero for these eight entries or use the alternate format ("ovfp" = "vfpm") discussed above.

the masses of melt components following the MELTCOMP keyword. The user must provide a list of 32 numbers specifying in the following order the mass of each of the materials: $\mathrm{Cs}, \mathrm{I}, \mathrm{Xe}, \mathrm{Kr}, \mathrm{Te}, \mathrm{Ba}, \mathrm{Sn}, \mathrm{Ru}, \mathrm{UO}_{2}, \mathrm{Zr}, \mathrm{ZrO}_{2}$, $\mathrm{Fe}, \mathrm{FeO}, \mathrm{Mo}, \mathrm{Sr}, \mathrm{Rb}, \mathrm{Y}, \mathrm{Tc}, \mathrm{Rh}, \mathrm{Pd}, \mathrm{La}, \mathrm{Ce}, \mathrm{Pr}, \mathrm{Nd}, \mathrm{Sm}, \mathrm{Pu}, \mathrm{Cr}, \mathrm{Mn}, \mathrm{Ni}, \mathrm{Ag}$, $\mathrm{Sb}$, and $\mathrm{Nb}$. As explained above, the values of the major species in this list $\left(\mathrm{UO}_{2}, \mathrm{Zr}, \mathrm{ZrO}_{2}, \mathrm{Fe}, \mathrm{FeO}, \mathrm{Cr}, \mathrm{Mn}\right.$, and $\mathrm{Ni}$ ) should be set to zero, since their masses are now taken from the intermediate layer input.

an alternative input to the MELTCOMP approach for specifying initial inventory of the debris pool for the VANESA calculation. This approach may be preferred since materials not present need not be specified. The variable "ovfp" is the name of a species taken from Table 5-4 that is initially present in the melt. Only the material keywords for species present should be specified, followed by the mass "vfpm" of that species. The "ovfp" = "vfpm" input is repeated for each material initially present. Note that the mass of the major species, $\mathrm{UO}_{2}, \mathrm{Zr}, \mathrm{ZrO}_{2}, \mathrm{Fe}, \mathrm{FeO}, \mathrm{Cr}, \mathrm{Mn}$, and $\mathrm{Ni}$, should not be specified here as in the previous implementation of CORCON Mod2 in CONTAIN. Instead, the masses of these materials are initialized in VANESA from the values given in the COMPOS sub-block of the intermediate layer input. Thus, only the fission product species without an asterisk in Table 5-4 should be specified. Although the major species may be included for upward compatibility, the masses specified will be ignored. Default $=0 .(\mathrm{kg})$

a keyword which initiates the specification of the non-ideal solution phase model. This model is designed so that either the metal phase, the oxide phase, neither phase, or both phases are treated as being non-ideal. If METAL is specified, only the metal phase will be treated as non-ideal. If OXIDE is specified, only the oxide phase will be treated as non-ideal. If BOTH is specified, both phases will be treated as non-ideal. As of this writing, oxide phase non-ideal modeling is not operational. The OXIDE and BOTH input options are provided to accommodate future improvements to the VANESA modeling within CORCON. Thus, the only choices available to the user at this time are METAL and NEITHER. The default is NEITHER, which will result in both phases being treated as ideal.

It should be noted that several of the previously available options in the VANESA input block have been made inoperative. In previous versions of the code, these keywords invoked optional modeling treatments that are not compatible with the CORCON Mod3 version that is now being implemented 
into CONTAIN. The obsolete VANESA keywords from earlier CONTAIN versions include: CONCOMP, EDITDELT, REBAR, FDELT, DIFCO, BUBD, PTBB, PTDIA, OXPOT, MOLEC, STABLE, and VROVR. If any of these keywords are present in an input block, the associated input values will simply be discarded and a warning will be written to the error file notifying the user that the input is obsolete. In the case of the EDITDELT keyword, a VANESA output edit is now generated whenever a CORCON edit is also generated. This is governed by the input given in the CORCON TIMES block. In the case of the CONCOMP, the concrete type seen by the VANESA model is now taken from the CORCON input. This is also true of the REBAR keyword where the VANESA modeling now uses the appropriate rebar fraction from the CORCON input block. With regard to the obsolete FDELT keyword, the VANESA modeling now uses the same calculational timestep as CORCON. The values for the remaining obsolete keywords are set fixed at the previously defined defaults.

SCRUB the keyword to activate the VANESA aerosol scrubbing model for overlying pools. The depth of the coolant pool used by the scrubbing model is determined from the conditions of the pool layer.

BSIZI the initial bubble diameter for pool scrubbing. Default $=0.01$. (m)

$=$ bsizi

EOI the keyword used to terminate input. The CONCRETE, PHYSICS, CORCON, EMISIV, VANESA, and SCRUB blocks all must be terminated with the EOI keyword.

14.3.2.4 Intermediate Layers. This input block is used to define the characteristics of an intermediate layer in the lower cell. Like the concrete layer input, the intermediate layer input differs for problems with and without core-concrete interaction modeling with CORCON. The two sections that follow describe the intermediate layer input for problems without $\mathrm{CORCON}$ and problems with CORCON, respectively.

14.3.2.4.1 Intermediate Layer Input Without CORCON. Multiple intermediate layers may be defined in problems that do not include core-concrete interaction modeling with CORCON. The number of intermediate layers, "jint," is specified in the cell CONTROL block. The intermediate layer definition block should be repeated "jint" times. The first INTERM layer input block corresponds to the lowest intermediate layer in the lower cell system. The layers proceed upward in the order that they are defined, with the last intermediate layer corresponding to the uppermost of the intermediate layers. Each such intermediate layer will consist of one node.

Descriptions of the SOURCE, Q-VOL, and HT-COEF keywords and associated input are the same as described for the CONCRETE layer without CORCON (see Section 14.3.2.3.1). Detailed descriptions of these keywords and their associated input are therefore not repeated in this section. 
INTERM

LAY-NAM=olay

[COMPOS=nma (omat mass)]

TEMP=temp

[PHYSICS

[SOURCE=nso

[Q-VOL

(data)]

$\mathrm{EOI}$

(data)

[HT-COEF

EOI]

$$
\text { (data) }
$$

EOI]

EOI

\begin{abstract}
$* * * * * * * * * * * * * * * * * * * * * * * * * * * * * * * * * * * * * * * * * * * * * * * * * * * * * * * * * * * * * * * * * * * * * * * * * * * * * * * * * *$
INTERM the keyword to initiate the definition of an intermediate layer.

LAY-NAM the name of the intermediate layer. Intermediate layer names may be = olay anything the user desires; however, each intermediate layer name should be unique. In the HT-COEF input for specifying a heat transfer coefficient between layers, "olay" is used to identify the intermediate layer.

COMPOS the keyword to initiate the specification of materials initially in the intermediate layer. If this keyword and its associated input are omitted, this layer will be initially empty.

nma the number of materials initially present in the layer. The following two inputs must be repeated "nma" times.

omat a name of a material that is initially present in the intermediate layer. This material must be among the materials specified after either the COMPOUND or the USERDEF keywords in the MATERIAL input block.

mass the initial mass of material "omat" in the layer. $(\mathrm{kg})$

TEMP the required initial temperature of layer. (K)

$=$ temp

PHYSICS the keyword to initiate input for the layer physics options. This keyword is required if any of the options described below are to be selected.
\end{abstract}


SOURCE

nso

Q-VOL

HT-COEF the keyword to initiate the specification of material sources to the layer. The source input template for the intermediate layer is identical to that for the concrete layer shown in Section 14.3.2.3.1. Additional details are also given in Section 14.4.1, Source Table Input.

the number of source tables specified for the layer. A description of the "nso" data groups that must follow this number is given in Sections 14.3.2.3.1 and 14.4.1.

the keyword to initiate the specification of a volumetric heat source table in the layer. The input description of this option for intermediate layers is identical to that given in Section 14.3.2.3.1 for concrete layers. Only one $\mathrm{Q}-\mathrm{VOL}$ table may be specified in a given layer. Additional details on such tables are also given in Section 14.4.2, Global and Cell Level Table Input.

the keyword to initiate the specification of a heat transfer coefficient table to override the internal heat transfer coefficient either between the present layer and one above it or between it and the basemat. The input description of this option for intermediate layers is identical to that given in Section 14.3.2.3.1 for concrete layers. Only one HT-COEF table may be specified in a given layer. Additional details on such tables are also given in Section 14.4.2, Global and Cell Level Table Input.

EOI the keyword used to terminate input.

14.3.2.4.2 Intermediate Layer Input With CORCON. When the CORCON model is used, only one intermediate layer may be defined. This single CONTAIN intermediate layer is used to initialize the CORCON melt layers. This layer must be defined after the CONCRETE layer and before the POOL layer. The name for this single layer is always CORCON. The user does not have the option to change this name through the LAY-NAM keyword.

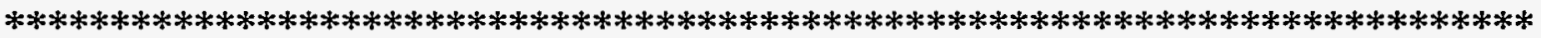

INTERM

COMPOS $=$ CORCON

[METALS=nmsi (onamen smm)]

[OXIDES=nosi (onameo smo)]

EOI

[TMETAL $=$ tmi] $[$ TOXIDE=toi] [LAYERS=ilyr]

[PHYSICS

[SOURCE $=$ nso

(oname $=\mathrm{n}$

EOD)]

IFLAG=ival T=(times) $\mathrm{MASS}=$ (masses) $\mathrm{TEMP}=$ (temps)

\{CORESTAT timeO xmtu xmwth num (fp1 reti) 


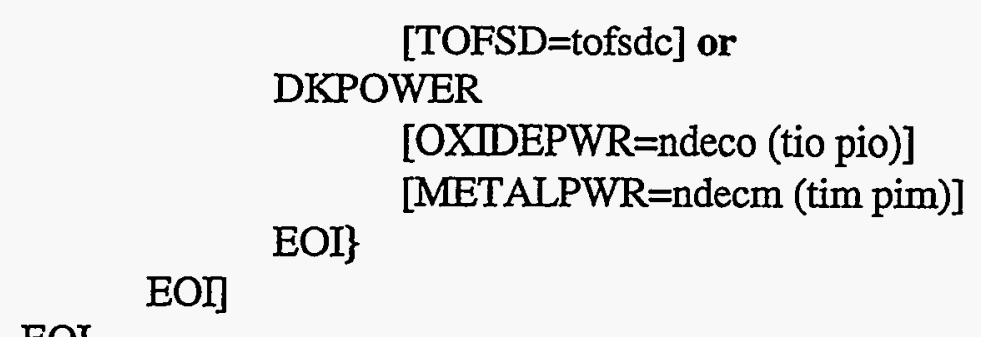

EOI

$* * * * * * * * * * * * * * * * * * * * * * * * * * * * * * * * * * * * * * * * * * * * * * * * * * * * * * * * * * * * * * * * * * * * * * * * * * * * * * *$

INTERM

the keyword to begin the specification of an intermediate layer.

COMPOS

the keyword to begin the specification of CORCON layer initial conditions. It must be followed immediately by the keyword CORCON.

CORCON

the second keyword used for specifying the CORCON layer initial conditions. Note that prior to the CORCON start time, the CORCON intermediate layer is assumed to be absent in the heat conduction calculations. However, after CORCON finishes, an intermediate layer will be constructed for use in the heat conduction calculations as described in Section 5.3.

METALS

the keyword to begin the specification of the initial CORCON metallic layer composition.

nmsi

the number of metallic species used in the CORCON metal layer.

onamem

the name of a metallic species, taken from the CORCON species list given in Table 5-2. Note that the CORCON master species list includes other materials, including FPM (a pesudo-material representing condensed phase fission product groups), and X (a pseudo-material to accommodate inert oxides in the chemical equilibrium calculation). These pseudo-materials are not allowed here. Also, condensed phase carbon, called C(c) in the master species list, is referenced here by simply specifying "C."

smm the mass of the metallic species. $(\mathrm{kg})$

OXIDES the keyword to begin the specification of the initial CORCON oxidic layer composition.

nosi

the number of oxidic species used in the CORCON oxide layer.

onameo

the name of an oxide species, taken from the CORCON species list given in Table 5-2. Note that the allowable oxide names have also been expanded from CORCON Mod2 to include UO3 and U3O8. 
smo

TMETAL

$=\mathrm{tmi}$

TOXIDE

$=$ toi

LAYERS

$=$ ilyr

PHYSICS

SOURCE the mass of the oxidic species. $(\mathrm{kg})$

the initial CORCON metallic layer temperature. (K)

the initial CORCON oxidic layer temperature. (K)

an index that specifies the initial configuration of the melt layers in CORCON. The CORCON melt layers are treated as separate nodes in the single CONTAIN intermediate layer. Altogether there are five nodes, heavy oxide (HOX), heavy mixed oxide (HMX), metal (MET), light mixed oxide (LMX), and light oxide (LOX). A value of 0 for "ilyr" implies an initially stratified layer, where metals are in the MET node, and oxides are in the HOX node. A value of 1 implies only a metallic layer, where all metals are initially in the MET node. This is only allowed if no oxides are initially present. A value of 2 implies only an oxide layer, where all oxides are initially in the HOX node. This is only allowed if no metals are initially present. A value of 3 implies a homogeneous mixture, where metals and oxides are initially mixed in the HMX node. If "ilyr" $=3$ then user-specified decay power to the well-mixed layer (the HMX node) is given in the OXIDEPWR table, otherwise the OXIDEPWR table is for the oxide layer and the METALPWR table is for the metal layer. If the mixing model is activated in the CORCON input block, the layer configuration will evolve as calculated within the model. If the mixing model is not turned on, the layer configuration will remain mixed throughout the calculation if "ilyr" $=3$ and stratified layers will develop if "ilyr" is 0,1 , or 2 . Note that the stratified layers are assumed to be gravitationally stable even if mixing is not turned on. Thus, if the HOX layer becomes less dense than the MET layer, the HOX layer materials will be combined with the LOX layers, and the HOX layers will become a null layer. Default $=0$.

the keyword to begin the specification of models for the CORCON intermediate layer.

the keyword to begin the specification of sources of material to the CORCON intermediate layer. With the exception of the allowed material names and the required temperature specification, the input description for these sources is the same as that discussed in Section 14.3.2.3.1 for the concrete layer. Time-dependent sources of the materials listed in Table 5-2 may be introduced into the CORCON layers by means of these source tables. Note: only temperature specification and not enthalpy specification of the incoming CORCON material is allowed. 
CORESTAT

timeO

xmtu

xmwth

num

fp1

reti

TOFSD

$=$ tofsdc

DKPOWER

OXIDEPWR

ndeco

tio

pio the keyword to begin the specification of one of the two CORCON models for decay heating of the CORCON layers. In this option, the gross fuel mass and operating power are specified so that the code may calculate the decay power. The user may modify the CORCON calculation by specifying new fission product retention fractions to replace those used in the code. Note that if the DECAY-HT option is also used, the decay heating calculated in the DECAY-HT option will be used to normalize the decay heating calculated in either of the CORCON options.

the value which must be equal to the CORCON start time "tstart." (s)

the core mass in metric tons of uranium. (MTU)

the core operating power. (MWt)

the number of radioactive species in the intact core inventory for which the retention factor will be modified. This value must be less than or equal to 27 . A non-zero value of "num" implies that this value is followed by "num" pairs of "fp1" and "reti."

the name of radioactive species whose retention factor is to be modified. The allowed names are given in Table 5-3. This table also shows the assumed mass concentrations at scram and the default retention factors for each species.

the user-specified retention factor for the "fp1" species.

the time of reactor shutdown. Default $=0$. (s)

the keyword to begin the specification of the second of two models for decay heating of the CORCON layers. In this method the user can specify the power to be delivered to the oxide and/or metal layers as a function of time. See also the discussion for CORESTAT.

the keyword to begin specification of an oxidic-phase power table.

the number of points in the table representing oxidic-phase power versus time. The value must be less than or equal to 30 and followed by "ndeco" pairs of values of "tio" and "pio."

the time value in oxidic-phase power table. (s)

the oxidic-phase power. (W) 
METALPWR

ndecm

tim

pim the keyword to begin specification of a metallic-phase power table.

the number of points in the table of metallic-phase power versus time. The value must be less than or equal to 30 and followed by "ndecm" pairs of values of "tim" and "pim."

the time value in the metallic-phase power table. (s)

the metallic-phase power. (W)

EOI

the keyword used to terminate the input.

14.3.2.5 Pool Layer. The POOL input block is used to specify the configuration of a coolant pool layer. The overall input format for the pool layer is the same for all problems, regardless of whether CORCON is used to model CCIs. Note that aerosol settling will occur automatically onto the lower cell if a pool layer is defined, but not otherwise. This is a non-upward compatible change from versions prior to CONTAIN 1.2. (The SETTLE keyword therefore has no purpose.)

Descriptions of the SOURCE, Q-VOL, and HT-COEF keywords and associated input are the same as described for the CONCRETE layer without CORCON (see Section 14.3.2.3.1). Detailed descriptions of these keywords and their associated input are therefore not repeated in this section.

POOL

[COMPOS=nma (omat pmass)]

TEMP=ptemp

[PHYSICS

[BOIL]

[SOURCE=nso

[Q-VOL

(data)]

(data)

EOI]

[HT-COEF

EOI]

(data)

EOI]

EOI

$* * * * * * * * * * * * * * * * * * * * * * * * * * * * * * * * * * * * * * * * * * * * * * * * * * * * * * * * * * * * * * * * * * * * * * * * * * * * *$

POOL the keyword to initiate the definition of the pool layer. 
COMPOS

nma

omat

pmass

TEMP

$=$ ptemp

PHYSICS

BOIL

SOURCE

Q-VOL

HT-COEF

EOI

the keyword to initiate the specification of the initial material in the pool layer. If this keyword and its associated input are omitted, the pool layer will be initially empty.

the number of materials initially present in the pool layer. The following two variables must be repeated "nma" times.

a name of a material initially present in the pool layer. This material must be among the materials specified after either the COMPOUND or USERDEF keywords in the MATERIAL input block. If the BOIL physics option is invoked, only coolant material (H2OL or NAL) may be present.

the initial mass of material "omat" in the pool layer. $(\mathrm{kg})$

the required initial temperature of layer. (K)

the keyword to initiate input of the layer physics options. This keyword is required if any of the options described below are to be selected.

the keyword to activate the pool boiling model. If the implicit intercell flow option has been selected, pool boiling is modeled implicitly under the direction of the implicit flow solver. Otherwise, a semi-implicit boiling algorithm is used.

the keyword to initiate the specification of material sources to this layer. With the exception of possible restrictions on the materials present when boiling is allowed, the source input description for the pool layer is identical to that given in Section 14.3.2.3.1 for the concrete layer. Additional details are also given in Section 14.4.1.

the keyword to initiate the specification of a volumetric heat source table for the pool layer. The input description of this option for a pool layer is identical to that described in Section 14.3.2.3.1 for a concrete layer. (While that section describes the concrete layer without CORCON, volumetric heating of the pool layer can also be specified with CORCON.) Only one $\mathrm{Q}-\mathrm{VOL}$ table is allowed in a given layer. Additional details on such tables are also given in Section 14.4.2.

the keyword to initiate the specification of heat transfer coefficient tables to override the internally calculated total heat transfer coefficient, with respect to the layer below. Otherwise, the input is identical to that described in Section 14.3.2.3.1 for concrete layers.

the keyword used to terminate input. 


\subsubsection{Engineered Safety Systems}

The cell level keyword ENGINEER is used to specify engineered safety features (ESFs) and/or engineered system components that form the coolant redistribution system connecting lower cell pools. The three available ESFs are the fan cooler, the ice condenser, and the containment spray, activated by the keywords FANCOOL, ICECOND, and SPRAY, respectively. Only one ESF or one redistribution system component may be defined after each ENGINEER keyword, but any number of ENGINEER keywords can be specified in a cell. Each ENGINEER keyword is considered to define an "engineered system." (The number of such systems "naensy" should be specified in the cell level CONTROL block. See Section 14.3.) For example, a given cell may include one fan cooler, two pumps, and five valves as engineered systems for that cell. Each of the pumps and each of the valves should be specified as a separate system.

In the specification of an ESF, the keyword SOURCE indicates that the inlet coolant flow to either a containment spray or the cooling coils of a fan cooler is to be specified as an external source with mass flow rate and temperature given as a function of time. A tank, activated by the keyword TANK, may also provide the source for a containment spray. If the source of the spray is recirculated water from a pool, a heat exchanger model activated by the keyword HEX must be used. Water to be recirculated may come from a pool in another cell, specified by the input variable "iclin." The residual liquids from an ESF may be diverted to the cell specified by the input variable "iclout."

The redistribution of coolant liquid from the pool of one cell to that of another may also be modeled through an engineered system component. If any one of the PIPE, ORIFICE, or VALVE components is specified, the flow rate is determined by the pressure difference between the two cells and the hydraulic head of the respective pools. Specification of a valve between the two cells allows opening and closing of the flow path. If PUMP is specified as the component, a constant flow rate is maintained from "iclin" to "iclout" as long as liquid is available in "iclin." Overflow modeling activated by the keyword OVERFLOW will allow coolant to overflow from the pool of one cell to that of another.

The complete input template for the ENGINEER input block is given below. Detailed descriptions of the three ESF models and the liquid redistribution components are given in the following sections. It should be noted that only one ESF model and certain logical combinations of components are allowed in any one engineered system as discussed in the following subsections.

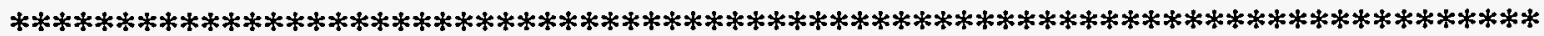

ENGINEER onmsys numcom iclin iclout delev

[SOURCE=nso

(H2OL=n

IFLAG=ival

$\mathrm{T}=$ (times)

MASS=(masses)

\{TEMP=(temps) or ENTH=(enths) $\}$

EOI)] 
[FANCOOL

\{CONDENSE

[FCQR=fcqr] [FCWIN=fcwin] [FCTCLI=fctcli] [FCCLMD=fcclmd]

[FCCLOD $=$ fcclod] $[\mathrm{NRWSFC}=$ nrwsfc] $[\mathrm{FCEFAR}=$ fcefar $]$

MARCH

[FCFLAR=fcflar] $[\mathrm{FCHNTR}=\mathrm{fchntr}]$ or

EOI]

[FCQR $=$ fcqr] $[\mathrm{FCTCLI}=\mathrm{fctcli}][\mathrm{FCCLMD}=\mathrm{fcclmd}]$

[FCTPIR=fctpir]\}

[ICECOND

[HITICI=hitici] [TMSICI=tmsici] [CIFLMX=ciflmx] [CIHTML=cihtml]

[CITICE=citice] [CITLEX=citlex] [CIARFL=ciarfl] [ARHTIN=arhtin]

[ICLLP=icllp] [AREASED=areased] [FRACSED=fracsed] [AREAIMP=areaimp]

[DIAMIMP=diamimp] [DIAMDIF=diamdif] [AREADIF=areadif]

EOI]

[SPRAY

[SPDIAM=spdiam] [SPHITE $=$ sphite] [SPPCI2=sppci2]

EOI]

[SPPCMI $=$ sppcmi] [SPSTPR $=$ spstpr] [SPSTTM $=$ spsttm]

[HEX \{otype hxticl bxclmd hxarea hxcoef or

USER hxdelt\}]

[PUMP pmpmdt]

[VALVE

\{PRESSURE valvar valvkf valopp or

TTMES valvar valkf (valtim)\}]

[ORIFICE orifid orifdr]

[PIPE pipeid pipel pipekf]

[TANK tnkmas tnktem tnkflo]

[OVERFLOW iclfrm iclto flovht]

EOI

$* * * * * * * * * * * * * * * * * * * * * * * * * * * * * * * * * * * * * * * * * * * * * * * * * * * * * * * * * * * * * * * * * * * * * * * * * * * * * * * * * *$

ENGINEER the keyword to initiate the specification of an engineered system. This system can consist of up to 10 components of which only one may be an ESF model (SPRAY, FANCOOL, or ICECOND). Multiple systems may however be specified by repeating the ENGINEER input block.

onmsys the name of the engineered system.

numcom the number of components in the engineered system. This number includes the ESF component (FANCOOL, ICECOND, or SPRAY) and the engineered system components. The SOURCE table input, if used, is also considered a component of the system. In CONTAIN versions prior to 1.1 , an accurate value for this parameter is required; however, the number of components is 
now automatically determined by the code. Any non-zero value specified is acceptable since it will be overridden at execution time.

iclin

the cell number from which the liquid to the system originates. Default $=$ current cell.

iclout

the cell number to which the system diverts residual liquid. This residual liquid may include spray droplets, condensate, melted ice, or diverted flow.

delev

the drop in elevation from the bottom of pool in "iclin" to that of "iclout," previously used in redistribution of coolant from cell to cell. It should be consistent with the cell bottom elevations calculated from GEOMETRY input; otherwise, a diagnostic will be issued. A value consistent with cell bottom elevations is now always used. (m)

14.3.3.1 External Engineered System Source. An external source table is the simplest way to specify a time-dependent mass flow rate and temperature to a spray or fan cooler engineered system. Engineered system source tables may only be used to specify liquid water for a containment spray or a fan cooler. The input format for such a source is similar to that for other sources in CONTAIN (see Section 14.4.1); however, the material name is restricted to H2OL.

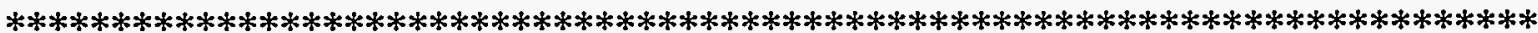

[SOURCE $=$ nSO

$(\mathrm{H} 2 \mathrm{OL}=\mathrm{n}$

[IFLAG=ival]

$\mathrm{T}=$ (times)

MASS $=$ (masses)

EOI]

$\{$ TEMP=(temps) or ENTH=(enths) $\}$

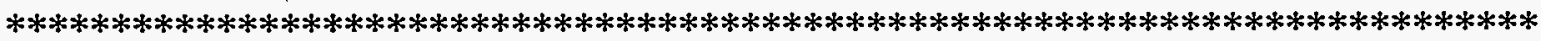

SOURCE the keyword to initiate the specification of source tables, which determine the rate at which water is supplied to the engineered system.

nso

the number of water source tables to be defined. If "nso" is greater than one, the multiple tables are additive.

H2OL

the material name required to indicate that the source consists of liquid water. Engineered system external source tables are restricted to water.

The remaining keywords and input parameters shown in the above input template are described in Section 14.4.1. 
14.3.3.2 Fan Cooler. Two fan cooler models are available as described in Section 12.1.1:a mechanistic condensation heat transfer model activated by the keyword CONDENSE and a simple heat transfer model, similar to that used in the MARCH code and activated by the keyword MARCH.

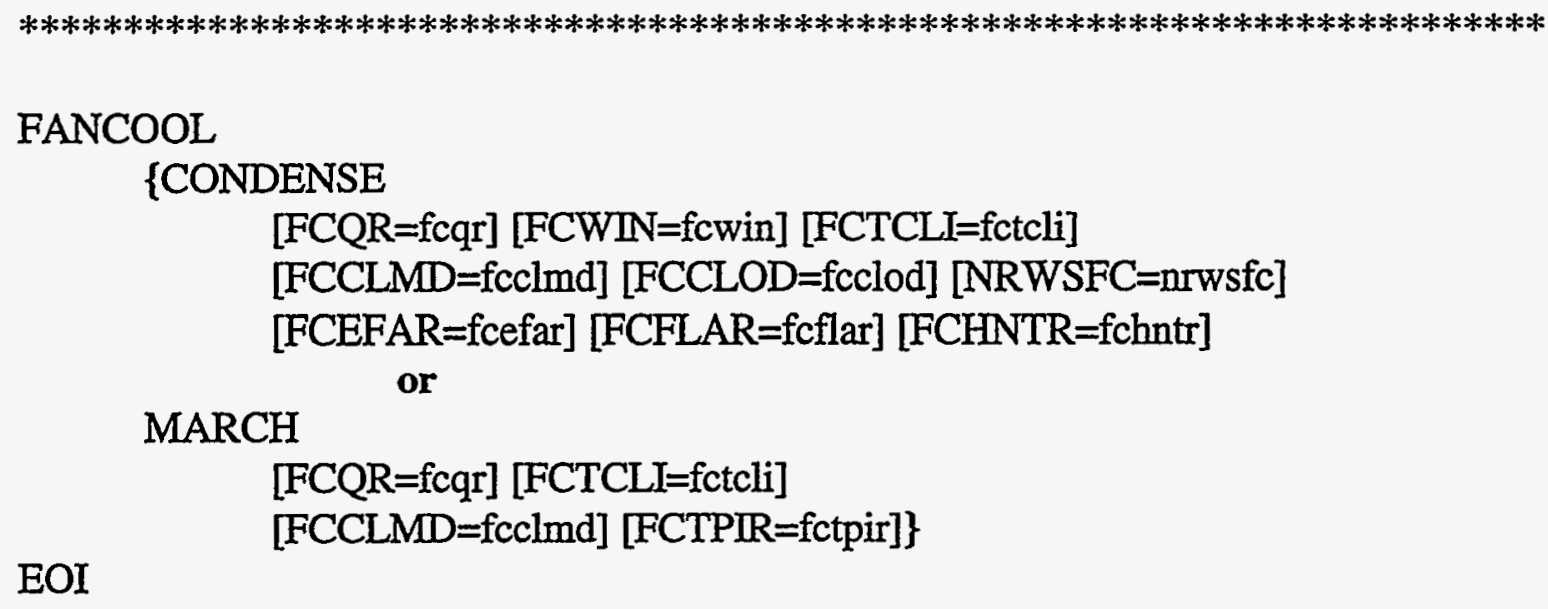

EOI

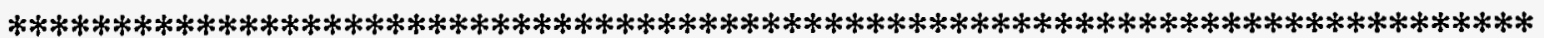

FANCOOL the keyword to begin specification of the fan cooler model.

CONDENSE the keyword to specify the mechanistic condensation heat transfer model.

FCQR

$=\mathrm{fcqr}$

FCWIN

$=$ fcwin

FCTCLI

$=$ fctcli

FCCLMD

$=$ fcclmd

FCCLOD

$=$ fcclod

NRWSFC

$=$ nrwsfc

FCEFAR

$=$ fcefar the estimated fan cooler heat removal rate. Because the CONDENSE model is iterative, this value is only used to initiate the iteration. The rated capacity should normally be used for this value. Default $=2.17 \times 10^{7}$. (W)

the volumetric flow rate of the air/steam mixture through the fan cooler. Default $=25.01 .\left(\mathrm{m}^{3} / \mathrm{s}\right)$

the cooling water inlet temperature. If an external source is specified this value is ignored. Default $=300$. $(\mathrm{K})$

the cooling water mass flow rate through cooler. If an external source is specified, this value is ignored. Default $=123.1 .(\mathrm{kg} / \mathrm{s})$

the outside diameter of the cooling tubes. Default $=0.0159$. (m)

the number of rows from front to back of cooler. Default $=12$.

the effective area for heat transfer across one row of tubes. Because tubes have closely spaced fins, this value is several times the tube area. Default = 26.5. $\left(\mathrm{m}^{2}\right)$ 

FCFLAR the frontal area of the fan cooler. Default $=3.0 .\left(\mathrm{m}^{2}\right)$
$=$ fcflar
FCHNTR the heat transfer coefficient between the tube external surface and the cooling $=$ fchntr $\quad$ water, based on "fcefar." Default $=1000 .\left(\mathrm{W} / \mathrm{m}^{2}-\mathrm{K}\right)$
MARCH the keyword to specify the simple heat transfer model.
FCQR the cooler rated capacity. Default $=2.17 \times 10^{7}$. W
$=\mathrm{fcqr}$
FCTCLI the rated coolant inlet temperature. Default $=300 .(\mathrm{K})$
$=$ fctcli
FCCLMD the rated coolant mass flow rate. Default $=123.1 .(\mathrm{kg} / \mathrm{s})$
$=$ fcclmd
FCTPIR the rated inlet air/steam temperature. Default $=405.9 .(\mathrm{K})$
$=$ fctpir
EOI the keyword used to terminate input.

If the SOURCE option is used with FANCOOL, the data provided in the SOURCE tables will override the default or user-specified values of the cooling water flow rate and temperature.

14.3.3.3 Ice Condenser. The input for the ice condenser model is described in this section. Use of the ice condenser model requires a problem with at least two cells. For realistic analysis, three or more cells are preferable. The cell in which the model is invoked is the ice chest itself. Note that the default upstream cell "icllp" is somewhat arbitrary; the user should specify the proper cell if the default is not appropriate.

\section{ICECOND}

[HITICI=hitici] [TMSICI=tmsici] [CIFLMX=ciflmx] [CIHTML=cihtml] [CITICE=citice] [CITLEX=citlex] [CIARFL=ciarfl] [ARHTIN=arhtin] [ICLLP=icllp] [AREASED=areased] [FRACSED=fracsed] [AREAIMP=areaimp] EOI [DIAMIMP=diamimp] [DIAMDIF=diamdif] [AREADIF=areadif]

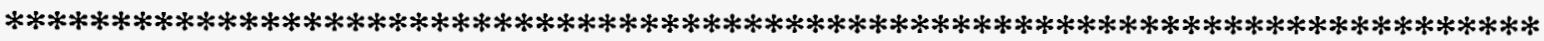

ICECOND the keyword to initiate the specification of the ice condenser model. 
HITICI

$=$ hitici

TMSICI

$=$ tmsici

CIFLMX

$=$ ciflm $\mathrm{x}$

CIHTML

$=$ cihtml

CITICE

$=$ citice

CITLEX

$=$ citlex

CIARFL

$=$ ciarfl

ARHTIN

$=$ arhtin

ICLLP

$=$ icllp

AREASED

$=$ areased

FRACSED

$=$ fracsed

AREAIMP

= areaimp

DIAMIMP

$=$ diamimp the initial height of ice in bed. Default $=14.6$. (m)

the initial mass of ice. Default $=1.1 \times 10^{6} .(\mathrm{kg})$

the fixed thickness of the water film on melting ice. Default $=5 \times 10^{-5}$. (m)

the multiplier on the Nusselt number for an ice column to account for interstitial flow and roughness. Default $=5$.

the initial ice temperature. Default $=264$. $(K)$

the temperature at which melted ice and condensate are assumed to leave the ice chest. Tests have produced values between 335 and $373 \mathrm{~K}$, depending on blowdown rate, with lower rates yielding higher temperatures. Default $=350$. (K)

the cross-sectional flow area through the ice chest. Default $=100 .\left(\mathrm{m}^{2}\right)$

the initial area of ice available for heat transfer. Default $=1 \times 10^{4} .\left(\mathrm{m}^{2}\right)$

the cell number of the upstream cell. This cell number is used only to determine the flow rate through the ice chest. The default value is normally the "iclin" value specified after the ENGINEER keyword. However, if "iclin" is the same as the current cell and if the current cell number is not 1, "icllp" will be set to the current cell number minus one.

the effective floor areas for sedimentation, including tops of baskets and support structures but not including the ice surfaces. Default $=1535 .\left(\mathrm{m}^{2}\right)$

the fraction of total ice area on which sedimentation may occur. Default $=$ 0.5 .

the total area for impaction of aerosols on the basket wires. Default $=1240$. $\left(\mathrm{m}^{2}\right)$

the effective wire cylindrical diameter for modeling impaction and interception of aerosols on the basket wires. Default $=0.00191$. (m) 
DIAMDIF

$=$ diamdif

AREADIF

$=$ areadif the effective wire cylindrical diameter for modeling diffusion of aerosols to the basket wire surfaces. Default $=0.00526$. (m)

the total area for diffusion of aerosols to the basket wires. Default $=3430$. $\left(\mathrm{m}^{2}\right)$

The ice condenser aerosol scrubbing model includes only effects attributable to the ice and basket structure. The walls, floors, and ceiling of the ice chest compartment should be modeled as structures (see Section 14.3.1.3).

14.3.3.4 Containment Spray. This input block is used to specify the containment spray model. Note that all of the spray parameters have defaults.

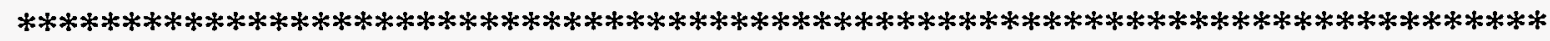

\section{SPRAY}

[SPDIAM=spdiam] [SPHITE=sphite] [SPPCI2=sppci2] [SPPCMI=sppcmi] [SPSTPR=spstpr] [SPSTTM $=$ spsttm]

EOI

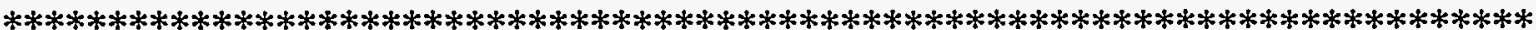

SPRAY the keyword to initiate the input for the containment spray model.

SPDIAM

the representative droplet diameter. Default $=0.001$. $(\mathrm{m})$

= spdiam

SPHITE

$=$ sphite

SPPCL2

$=$ sppci 2

SPPCMI

= sppcmi

SPSTPR

$=$ spstpr

SPSTTM

$=$ spsttm the spray fall height. Default $=$ cell height specified in the cell GEOMETRY block (see Section 14.3.1.1.) (m)

the partition coefficient used to model removal of elemental iodine. Fission products having names that begin with the letters "MOLI" are assumed to be elemental iodine. Default $=5000$.

the partition coefficient used to model removal of organic iodides. Fission products having names that begin with the letters "ORGI" are assumed to be organic iodides. Default $=0$.

the containment pressure at which the spray is initiated. If this value is 0 , the spray is initiated by a finite coolant flow rate or "spsttm." Default $=0 .(\mathrm{Pa})$

the containment temperature at which the spray is initiated. If this value is 0 , the spray is initiated by a finite coolant flow rate or "spstpr." Default $=0$. $(\mathrm{K})$ 
If the spray model is selected as the ESF in an engineered system, certain combinations of components must be specified. One combination consists of SOURCE and SPRAY, with OVERFLOW optional. The other combination consists of TANK, PUMP, HEX, and SPRAY, with OVERFLOW optional. An example of the input for the latter combination is given below.

ENGINEER SAMPLE1 4220.0

\section{SPRAY}

SPPCI2 $=6000.0$

SPSTPR $=2.0 \mathrm{e} 5$

EOI

TANK

5000.0

280.0

200.0

PUMP

200.0

HEX

\&\& engineered system has four components

$\& \&$ outlet to and recirculation from cell 2

$\& \&$ elevation difference $=0$

$\& \&$ elemental iodine partition coefficient

$\& \&$ pressure initiation at $2.0 \times 10^{5} \mathrm{~Pa}$

\&\& mass of water available in the tank

$\& \&$ source water temperature

$\& \&$ water flow rate from the tank

$\& \&$ flow rate for recirculation

USER $=50 . \quad \& \&$ user-specified temperature drop of $50 \mathrm{~K}$

EOI

14.3.3.5 Heat Exchanger. Five liquid-liquid heat exchanger options are available. The keyword SHELL invokes the appropriate heat transfer correlations for a shell and tube, single-pass heat exchanger. CROSS denotes a cross-flow heat exchanger; COUNTER, a counterflow type; PARALLEL, a parallel flow type; and USER, a user-specified constant temperature drop across the hot leg. The heat exchanger component is used only with the spray ESF.

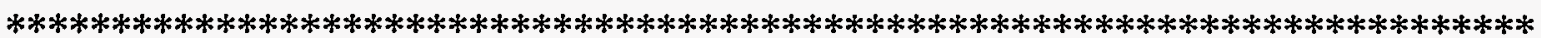

HEX \{otype hxticl hxclmd hxarea hxcoef or USER hxdelt\}

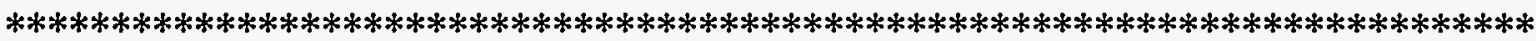

HEX

the keyword to initiate the specification of the heat exchanger component.

otype

a heat exchanger type keyword. Either SHELL, CROSS, COUNTER, or PARALLEL must be specified.

hxticl the cold side inlet temperature. $(\mathrm{K})$

hxclmd the cooling water flow rate through the cold side. $(\mathrm{kg} / \mathrm{s})$ 
hxarea the effective heat transfer area of the exchanger. $\left(\mathrm{m}^{2}\right)$

hxcoef the overall heat transfer coefficient. $\left(\mathrm{W} / \mathrm{m}^{2}-\mathrm{K}\right)$

USER the heat exchanger keyword used to specify a constant temperature drop, "hxdelt," across the hot side.

hxdelt the temperature drop across the hot side. (K)

14.3.3.6 Pump. A pump provides a constant mass rate of flow from a pool to a containment spray or to a pool in another cell.

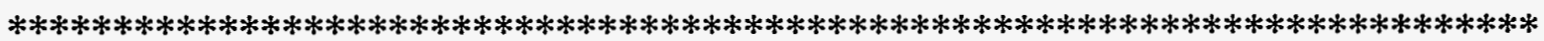

PUMP pmpmdt

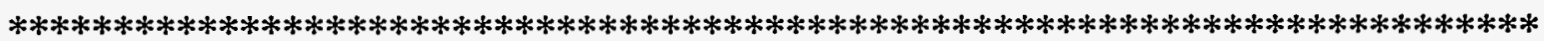

PUMP the keyword to specify a pump component.

pmpmdt the constant mass flow rate. $(\mathrm{kg} / \mathrm{s})$

When used as part of a SPRAY system that includes a TANK water supply, the pump is activated only after the TANK supply is exhausted.

14.3.3.7 Valve. The keyword VALVE may activate one of two options. Followed by the keyword PRESSURE, it simulates a pressure-activated rupture disk, i.e., once the activating pressure differential is reached, the valve opens and remains open, regardless of subsequent changes in the driving pressure. If VALVE is followed by the keyword TIMES, the user must specify five open and close times. The valve is assumed to be initially closed, and the first time specified is the open time; at the next time the valve closes; at the third time it opens again; etc. For a valve that is initially open, the first time specified would be the problem start time. If all five times are not needed, the extra times can be set to very large values which would not be encountered during the course of a run.

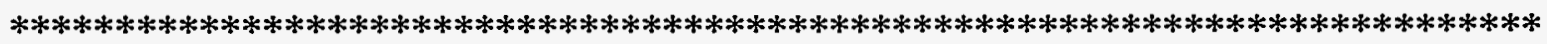

\section{VALVE}

\{PRESSURE valvar valvkf valopp or

TIMES valvar valvkf (valtim)\} 


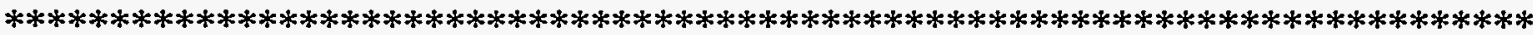

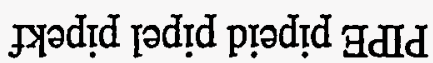

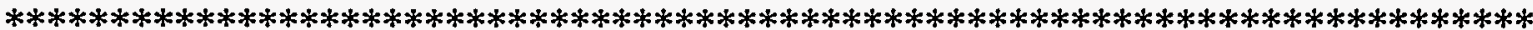

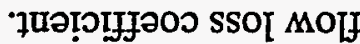

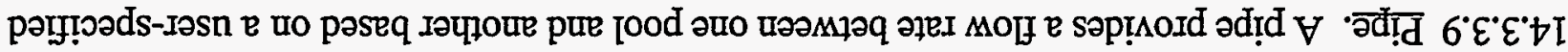

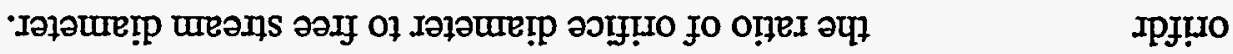

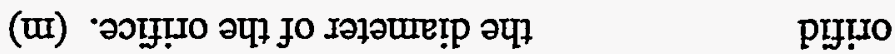

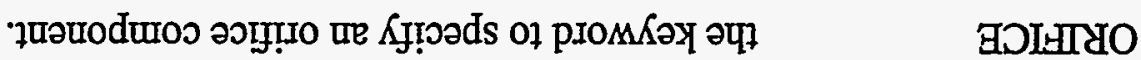

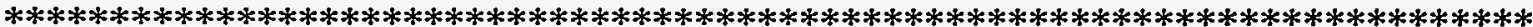

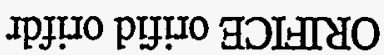

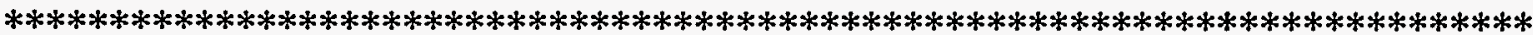

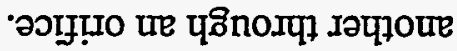

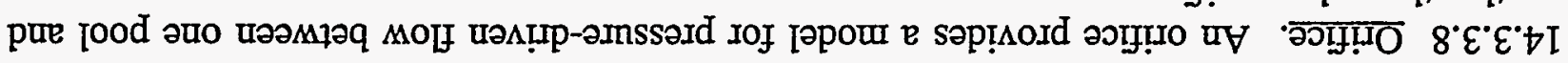

(s) · כum suturaədo

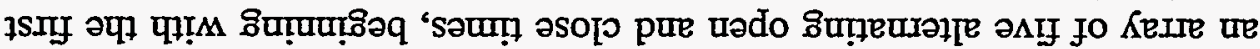

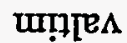

-posn st uoñdo

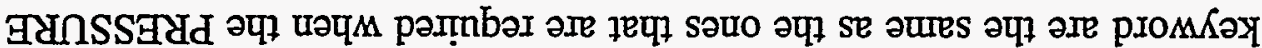

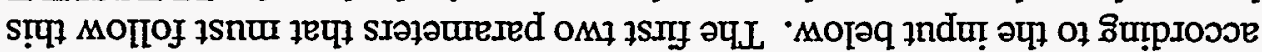

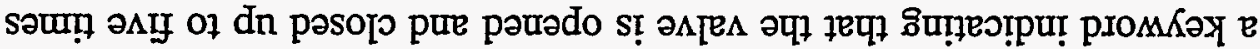

SEINIL

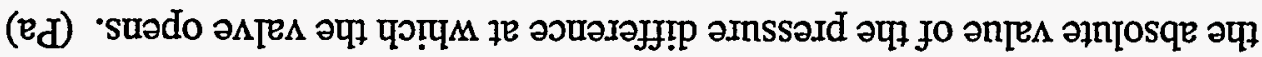

ddores

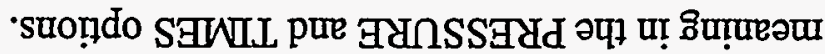

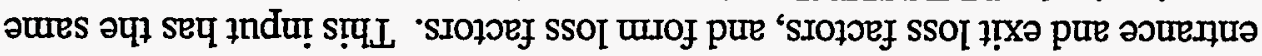

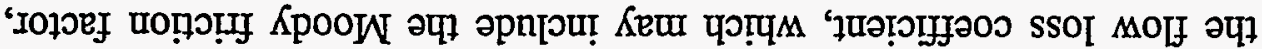

$\operatorname{PY\Lambda }[\mathrm{E} \Lambda$

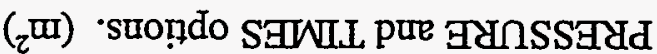

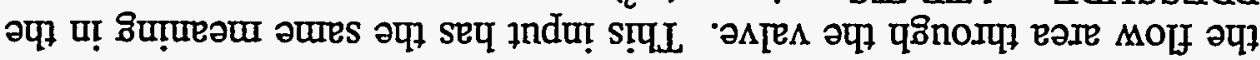
IEATE

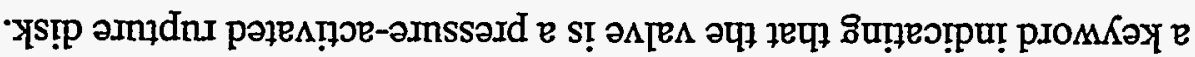

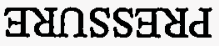

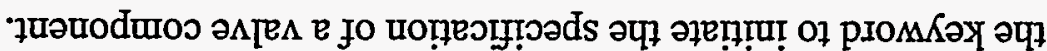


PIPE

pipeid

pipel

pipekf the keyword to specify a pipe component.

the inside diameter of the pipe. (m)

the pipe length. (m)

the flow loss coefficient, which may include the Moody friction factor, entrance and exit loss factors, and form loss factors.

14.3.3.10 Tank. A tank provides water for a containment spray. The water in the tank is used until it is exhausted, and then the spray water is drawn from the pool in cell "iclin" in a recirculation mode.

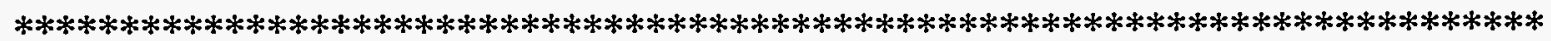

TANK tnkmas tnktem tnkflo

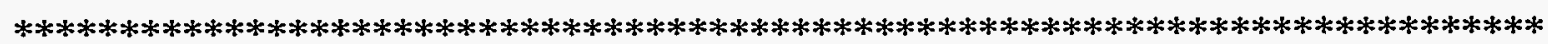

TANK the keyword to specify a tank component.

tnkmas the initial mass of liquid in the tank. $(\mathrm{kg})$

tnktem the temperature of liquid in the tank. $(\mathrm{K})$

tnkflo the flow rate from the tank. $(\mathrm{kg} / \mathrm{s})$

14.3.3.11 Engineered Systems Overflow. This input block describes the engineered systems overflow component. Overflow of coolant from the pool in one cell to that of another can be simulated through this component. It can be placed by itself in an engineered system or in a system with any other ESF model or engineered system component. Note that unphysical conditions may result if the amount of water that overflows during any given timestep is comparable to the amount in the pool.

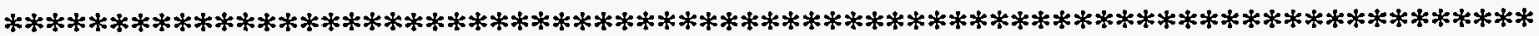

OVERFLOW iclfrm iclto flovht

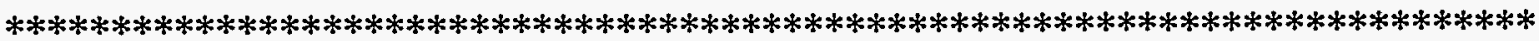

OVERFLOW the keyword to specify the overflow engineered system component.

iclfrm the cell number from which the overflow originates.

iclto the cell number to which the overflow is directed. 
flovht

the height above pool bottom in cell "iclfrm," which defines the level above which any additional coolant will be diverted to cell "iclto" if the transfer is in the direction of the positive head. Otherwise, no transfer occurs. (m)

\subsubsection{Safety Relief Valve (SRV) Discharge}

This block of input is used to activate the SRVSOR model for modeling the effects of a pool on the discharge of gases, aerosols, and fission products below the pool surface. The model may be activated in any cell with a pool specified in the LOW-CELL block. Space must be reserved for the SRV source tables in the cell level CONTROL block using the variables "nsosat," "nspsat," "nsosae," "nspsae," "nsosfp," and "nspsfp" described in Section 14.3.

The source table input in the ATMOS, AEROSOL, and FISSION blocks shown in the template below are similar to those described in Sections 14.3.1.2, Upper Cell Atmosphere Initial Conditions and Sources; 14.3.1.8, Aerosol Initial Conditions and Sources; and 14.3.1.9, Fission Product Sources, respectively. Thus, only those keywords unique to the SRVSOR block are discussed in this section.

Provided the discharge level is below the pool surface, the materials introduced through the SRVSOR sources are equilibrated in and/or scrubbed by the pool prior to being introduced into the atmosphere above the pool. The user may select either of two aerosol scrubbing models, the SCRUB model from VANESA or the SPARC model, as discussed in Section 14.2.4.3. Note that aerosol scrubbing depends strongly on the gas composition in the SRV ATMOS sources. For example, in the absence of a noncondensable gas, the aerosols will be trapped in the pool with the maximum decontamination factor $\left(10^{5}\right)$.

The only SRV fission product sources allowed are those which have an aerosol component as a host. The HOST keyword must be used and the value associated with it must lie between 2 and $1+$ "nac," a number which corresponds to an aerosol component. If the host aerosol source rate is zero for any time period during which the fission product source rate is non-zero, the fission product mass introduced will be retained entirely in the pool if the discharge level is below the pool surface and assigned directly to the airborne aerosol component if the discharge level is above the surface.

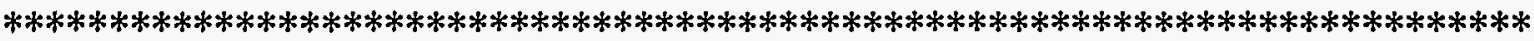

SRVSOR

[ELESRV=elesrv]

\{[SCRUB

EOI] or

[BSIZI=bsizi] [VROVR=vrovr]

[SPARC

EOI]\}

[BSIZI=bsizi] [RATIO=ratio] [NRISE=nrise]

[AEROSOL

SOURCE=nsosae 


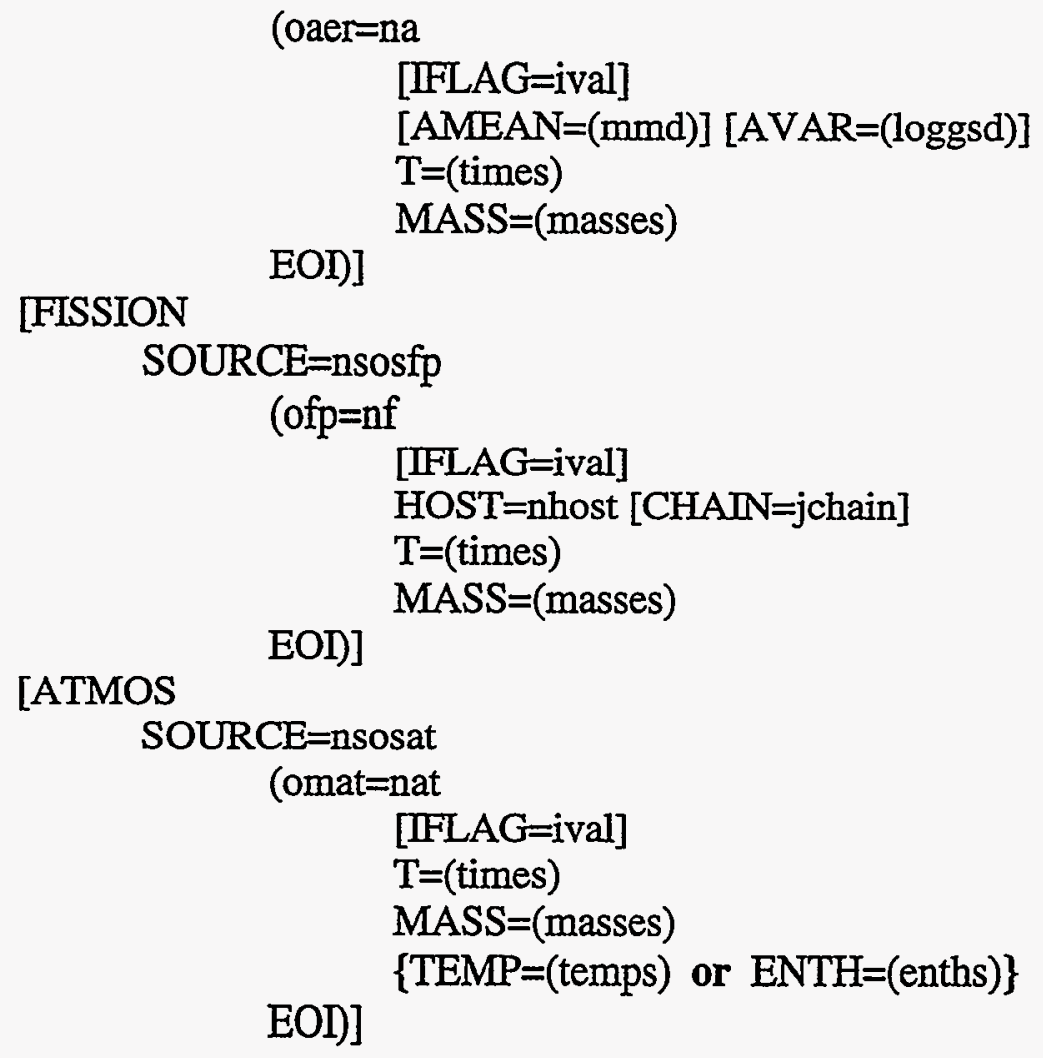

SRVSOR the keyword to activate the SRV model.

ELESRV the elevation of the SRV discharge point above pool bottom. Default $=0$. = elesrv (m)

SCRUB the keyword to specify the VANESA scrubbing model. By default this model is used if neither SCRUB nor SPARC is specified.

BSIZI

$=$ bsizi

the initial bubble diameter for pool scrubbing purposes. Default $=0.01$. (m)

VROVR

$=$ vrovr

SPARC

BSIZI

$=$ bsizi the ratio of internal gas velocity to rise velocity. This is an adjustable parameter for simulating nonspherical bubble effects and the effects of impurities on the bubble-liquid interface. Default $=1$.

the keyword to specify the SPARC scrubbing model.

the initial bubble diameter for pool scrubbing. This keyword replaces DIAM in the stand-alone version of SPARC. Default $=0.01$. (m) 
RATIO

$=$ ratio

NRISE

$=$ nrise

AEROSOL

SOURCE

nsosae

oaer

na

the ratio of major axis to minor axis in an axially symmetric oblate spheroid bubble. The ratio is taken to be greater than or equal to 1 . If a value less than 1 is specified, its inverse is automatically taken. Default $=1$.

the number of integration zones used for bubble rise in the scrubbing region. Values ranging from 10 to 1000 are suggested for accuracy. Default $=10$.

the keyword to specify SRV aerosol sources. Note that the keywords and values associated with these sources but not discussed below are discussed in Sections 14.3.1.8, Aerosol Initial Conditions and Sources, and 14.4.1, Source Table Input.

the keyword to specify that source table input follows.

the number of aerosol source tables to be specified. This number should agree with the value specified in the cell CONTROL block.

the name of the aerosol component declared in the global AEROSOL block.

the number of time points in the aerosol table. The value of "nspsae" in the cell CONTROL block must be greater than or equal to "na."

One unique feature available for SRV aerosol sources, but not for other types of aerosol sources, is that aerosol size distribution parameters for the aerosol source may be specified as a function of time through the source table using the AMEAN and AVAR keywords described below.

AMEAN

$=(\mathrm{mmd})$

AVAR $=(\operatorname{loggs} \mathrm{d})$

FISSION a keyword to specify the values of the mass median diameter "mmd." Specify "na" values of "mmd." The definition of "mmd" is identical to that of "amean," defined in the global AEROSOL input block discussed in Section

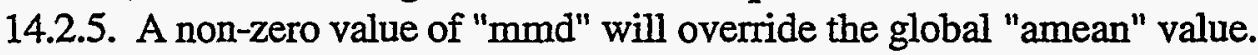
A zero value for "mmd" will result in the global "amean" value being used instead. Default = global value of "amean." (m)

a keyword to specify the logarithm of the geometric standard deviation "loggsd." Specify "na" values of "loggsd." The definition of "loggsd" is identical to that of "avar," defined in the global AEROSOL input block discussed in Section 14.2.5. A non-zero value of "loggsd" will override the global "avar" value. A zero value of "loggsd" will result in the global value "avar" being used instead. Default = global value of "avar."

a keyword to specify SRV fission product sources. The keywords and values associated with these sources but not specified below are discussed in Sections 14.3.1.9, Fission Product Sources and 14.4.1, Source Table Input. 
SOURCE

nsosfp

ofp

nf

HOST

$=$ nhost

CHAIN

$=$ jchain

ATMOS

SOURCE

nsosat

omat

nat a keyword to specify that source table input follows.

the number of fission product source tables to be specified. This number should agree with the value specified in the cell CONTROL block.

the name of the fission product declared in the global FISSION block.

the number of points in the fission product source table. The value of "nspsfp" in the cell CONTROL block must be greater than or equal to "nf."

required keyword to specify the host number associated with the fission product in the table. Specify a number between 2 and $1+$ "nac" which corresponds to the aerosol host.

the number of the fission product chain that is to receive the "ofp" mass. This should be given if "ofp" appears more than once in the linear chain decomposition.

a keyword to specify SRV gas and condensable vapor sources. The keywords and values associated with these sources but not discussed below are discussed in Sections 14.3.1.2, Upper Cell Atmosphere Initial Conditions and Sources, and 14.4.1, Source Table Input. (The former section describes atmosphere sources that enter the atmosphere directly without equilibrating with the pool.)

a keyword to specify that source table input follows.

the number of gas source tables to be specified. This number should agree with the value specified in the cell CONTROL block.

the name of a gas or the condensable vapor. This material must be among the materials specified after either the COMPOUND or USERDEF keywords in the global MATERIAL input block.

the number of points in the gas source table. The value of "nspsat" defined in the cell CONTROL block must be greater than or equal to "nat."

An example of the SRVSOR input is given below. The elevation of the discharge point is one meter above pool bottom. The aerosol sources, in addition to the usual time and mass rate specifications, use the optional keywords for specifying size distribution parameters, AMEAN and AVAR. The SCRUB aerosol scrubbing option, by default, is in effect, with default parameters. The UO2 aerosol is intended to be the first aerosol component in this example, and the fission product LA2O3 is associated with this component through the HOST $=2$ specification. The control parameters that need to be specified in the cell level CONTROL block to accommodate the tables are "nsosat"=2, "nspsat"=4, "nsosae"=1, "nspsae"=4, "nsosfp"=1, and "nspsfp"=4. 
SRVSOR input example:

SRVSOR

ELESRV $=1.0$

ATMOS SOURCE $=2$

$\mathrm{H} 2 \mathrm{OV}=4 \mathrm{IFLAG}=1$

$\mathrm{T}=28200.30000 .31800 .33600$.

MASS $=14.9610 .166 .637 .43$

$\mathrm{ENTH}=2.85 \mathrm{e} 62.92 \mathrm{e} 63.07 \mathrm{e} 63.24 \mathrm{e} 6$

EOI

$\mathrm{H} 2=4 \mathrm{IFLAG}=1$

$\mathrm{T}=28200.30000 .31800 .33600$.

MASS $=0.0 .0 .0020 .033$

TEMP $=569.3594 .1645 .0705 .9$

EOI

\section{AEROSOL SOURCE $=1$}

$\mathrm{UO} 2=4$ IFLAG $=1$

$\mathrm{T}=0.37300 .40506 .42648$.

MASS $=0.9 .576 \mathrm{e}-5$ 2.971e-3 0 .

$\mathrm{AVAR}=0.70 .720 .720 .7$

AMEAN= 1.e-6 1.1e-6 1.2e-6 1.e-6

EOI

FISSION SOURCE $=1$

$\mathrm{LA} 2 \mathrm{O} 3=4 \mathrm{IFLAG}=1$

$\mathrm{HOST}=2$

$\mathrm{T}=0.37300 .40506 .42648$.

MASS $=0.4 .683 \mathrm{e}-51.453 \mathrm{e}-30$.

EOI

EOI
\&\& safety relief valve input

$\& \&$ elevation of discharge

$\& \&$ gas sources

$\& \&$ steam; step interpolation

$\& \&$ time points

$\& \&$ mass rates

$\& \&$ specific enthalpies

$\& \&$ end of first source table

\&\& hydrogen; step interpolation

$\& \&$ time points

$\& \&$ mass rates

$\& \&$ temperatures

$\& \&$ end of second table

$\& \&$ aerosol source

$\& \&$ uo2 aerosol

$\& \&$ time points

$\& \&$ mass rates

$\& \& \log$ of standard deviation

$\& \&$ mass median diameter

$\& \&$ end of table

$\& \&$ fission product source

$\& \& 1 \mathrm{a} 2 \mathrm{o} 3$ fission product

$\& \&$ host is first aerosol

$\& \&$ component

$\& \&$ time points

$\& \&$ mass rates

$\& \&$ end of table

\&\& end of SRVSOR input

\subsection{Source and Table Input}

\subsubsection{Source Table Input}

Provisions are made for specifying external sources of mass and energy. The input description for these sources uses a standard format to the extent possible for both regular materials and for fission products and aerosols. This section lists the definitions of the standard keywords and values used in the source tables. The reader should note that although the basic definitions of most of the keywords and values used in the source tables are the same, the source tables for a particular model may require special keywords and values or impose special requirements on the values associated 
with standard keywords. Thus the present section cannot entirely replace the discussion of source tables in conjunction with the particular models that use them. If a definition of a common keyword or value appears in conjunction with the discussion of a particular model, that definition supersedes the definition given below.

Upper cell atmosphere material sources are discussed in Section 14.3.1.2; upper cell aerosol sources are discussed in Section 14.3.1.8; fission product sources are discussed in Section 14.3.1.9; lower cell material sources are discussed in Sections 14.3.2.3 to 14.3.2.5; engineered system sources are discussed in Section 14.3.3.1; and SRV sources are discussed in Section 14.3.4.

Source tables should not be confused with the global and cell level tables discussed in Section 14.4.2 and elsewhere. Unlike source tables, the latter types of tables establish a functional relationship between one independent and one dependent variable.

The common keywords and values used in source tables appear in the following template:

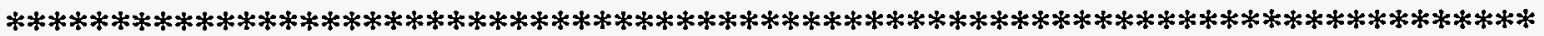

SOURCE $=$ nSo

(oname $=\mathrm{n}$

[IFLAG=ival]

$\mathrm{T}=$ (times)

MASS $=$ (masses)

EOI)

[\{TEMP=(temps) or ENTH=(enths) $\}]$

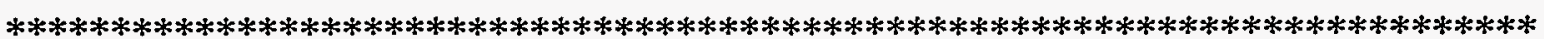

SOURCE a keyword to indicate that source table input follows.

nso

the number of source tables to follow. In the template above, the items between "oname" and EOI should be repeated "nso" times.

oname

the name of the source material. Depending on the particular type of source table, this name should be the name of a compound, aerosol, or fission product. The names used should be defined in the global input or be available as a default.

n

the number of source table points used for material "oname." Each entry ("times," "masses," "temps," and "enths") in the source table for this material must consist of this number of points. The minimum is two points. Space for the source table must generally be provided in the cell CONTROL block for the cell in which the source table is used. The name of the control parameter depends on the model invoking the source table.

IFLAG a keyword to introduce the interpolation flag for the table. 
ival

if "ival" = 1 , the mass rate and temperature or enthalpy, if appropriate, are treated as step-functions. If "ival" $=2$, linear interpolation is used between table values. With the step-function option ("ival" $=1$ ), the value returned from the table remains constant in the interval between adjacent source times. When the code calculational time reaches a particular source time the mass rate changes to the tabular value for that time. With the "ival" $=2$ option, the mass rate and other quantities are assumed to vary linearly between the specified tabular values. The mass rate is taken to be zero outside of the time range of the table.

$\mathrm{T}$

times

MASS

masses

TEMP

temps

ENTH

enths

EOI a keyword to introduce time values.

the source times. Specify " $n$ " monotonically increasing values. (s)

a keyword to introduce mass values.

the source mass rates. Specify " $n$ " values. Note that the source rate is taken to be zero outside of the time range of the table. $(\mathrm{kg} / \mathrm{s})$

a keyword to introduce source temperature values. The temperature is converted internally to an equivalent source specific enthalpy. For compounds that are not CORCON materials, either TEMP or ENTH should be specified, but not both. For CORCON materials only TEMP should be specified. For aerosol or fission product source tables, neither TEMP nor ENTH should be specified since only the mass source rate is pertinent.

the source temperatures. Specify " $n$ " values. (K)

a keyword to introduce specific enthalpy values. See the discussion above for TEMP on when to use TEMP or ENTH.

the source specific enthalpies. Specify " $n$ " values. (J/kg)

the keyword used to terminate source table input. Note that each source table is terminated by an EOI; however, the SOURCE block itself is not terminated by an EOI.

For all but engineered system source tables, both positive and negative mass rate values are allowed. Positive values signify mass additions, negative values signify mass removal from the cell. However, note that net negative masses are not allowed in any cell. If negative mass sources are specified, care should be taken to avoid conditions wherein the net mass of the material in question could go negative in any cell. For most models, the code checks for negative masses and sets the mass to zero if this condition is detected. In some cases, however, intermediate calculations are performed prior to the negative mass check, and thus some error could be introduced into the calculation. In some models, a diagnostic is given in the error file if a negative mass condition is

Rev. 0

$14-163$

$6 / 30 / 97$ 
encountered. Aerosol suspended masses are checked for negative masses and reset but a diagnostic is not given. Fission product masses are not checked by the code to detect a possible negative mass condition.

The following example indicates how the injection of steam into a cell atmosphere could be modeled. That steam may be generated, for example, from a blowdown of the primary system. Only one source is needed, and thus "nso" $=1$. The source material is injected into the cell as steam, so the "oname" is H2OV. Linear interpolation is desired, so "ival" $=2$. There are fourteen times, mass flow rates, and enthalpies defined in the tables, and " $n "=14$. Initially, the flow rate is small but rapidly builds and then slowly decreases as the pressure driving the steam flow drops, and the steam remaining in the tank decreases. At $23.2 \mathrm{~s}$, the blowdown is complete, with the mass flow rate dropping to 0 .

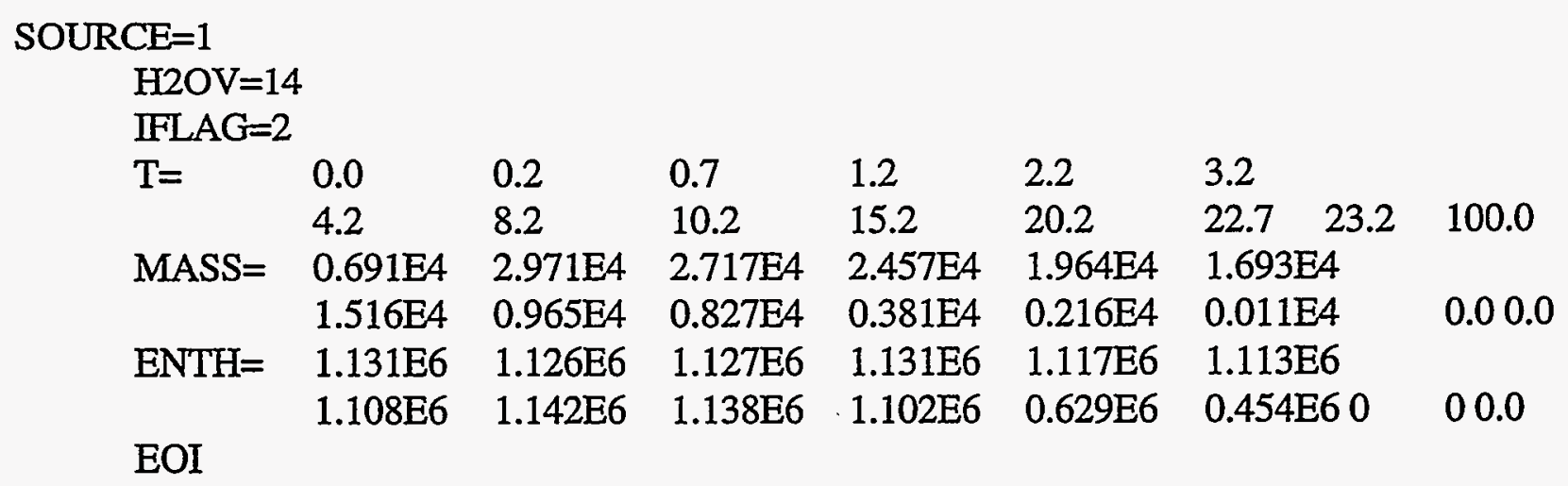

\subsubsection{Global and Cell Level Table Input}

This section gives the generic definitions of the standard keywords and values used in global and cell level tables. Such tables provide a standard format to specify the relationship between one dependent and one independent variable, such as a heat transfer coefficient versus time, and should not be confused with the source tables discussed in the preceding section.

The distinction between global and cell level tables is made on the basis of the level of the model using the table format. The tables used in global models, such as the flow model, are considered global, whereas tables used in cell level models, such as the lower cell model, are considered at the cell level. Global tables require that the user specify "numtbg" and "maxtbg" in the global CONTROL block; whereas cell level tables require specification of "numtbc" and "maxtbc" in the appropriate cell level CONTROL block.

The reader should note that although the keywords used in all global and cell level tables are the same, the definition and utilization of the values associated with the keywords depends on the application. Also a particular application may impose special requirements on the values that may be selected. Thus the present section cannot replace the discussion of such values in conjunction with the particular models that use them. If a definition of a table keyword or value appears in conjunction with the discussion of a particular model, that definition supersedes the generic definition given below. 
In the input for global and cell level tables, a keyword initiating the input of one or more tables is given. That keyword may or may not be followed by application-specific parameters that are then followed by the body of the table. Because the application-specific parameters are nonstandard, the template below shows only the body of one table.

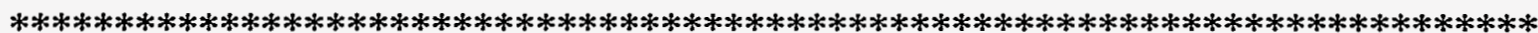

otabky

[FLAG=iflag]

NAME=oname

VAR-X $=x$ name $X=n(x)$

VAR-Y=yname $Y=n(y)$

EOI

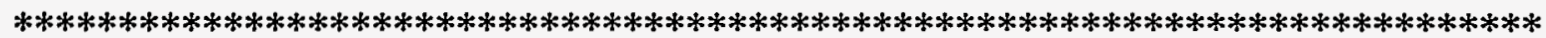
otabky
a module-specific keyword initiating the specification of a particular type of table, for example, "otabky" = VAR-PARM.
FLAG
$=$ iflag
NAME
= oname
VAR-X
$=$ Xname
$\mathrm{X}$
n
$\mathrm{x}$
VAR-Y
$=$ yname
$\mathbf{Y}$
y
the table interpolation parameter. The value "iflag" $=1$ specifies step function interpolation between table points, whereas "iflag" $=2$ specifies linear interpolation between table points. Default $=1$.
the name of the table. The table name in some cases is required to identify the table type. In tables where the name is optional, it may still be given for identification purposes.
the name of the independent variable. In some tables only one type of independent variable is considered, in which case this input is optional.
the keyword to specify the values of the independent variable.
the number of independent variable entries in the table. It is also the number of dependent variable entries.
the independent variable values. Exactly " $n$ " monotonically increasing values should be specified.
the name of the dependent variable. In some tables only one type of dependent variable is considered, in which case this input is optional.
the keyword to specify the values of the dependent variable.
the dependent variable values. Exactly " $n$ " values should be specified. 
Note that the value of " $y$ " returned from the table for values of " $x$ " outside of the range of the table depends on the application. In some applications, the table is ignored in this case, and the value of " $y$ " is calculated in some other fashion. In other applications the " $y$ " value is determined by using the appropriate endpoint value. The method used is discussed with each specific application.

\subsection{Restart Input}

A calculation can be restarted at the times for which restart blocks are present in the restart file from the preceding run. Such restart block times are controlled by the TIMES input block. (See Sections 14.2.7, Timestep and Time Zone Input, and 14.5.1.1, The TIMES Block in a Restart.) One may use a restart to continue a given problem or alter the course of the calculation. In general, model parameters may be changed for certain supported models, but new models may not be invoked and initially invoked models may not be deactivated. The exceptions to this rule are indicated by the presence of an optional ON or OFF keyword following the model keyword.

The calculation in a restart is controlled by an input file similar to that used to initiate the calculation. In the simplest case (e.g., when a CPU time limit was encountered in the prior run), the only input required is the RESTART keyword, a TIMES block, and an EOF.

The keyword RESTART indicates to CONTAIN that the restart file and plot file(s) generated in the previous run should be used. As discussed in Section 14.5.1.1, restart calculations begin at the time "tstart" specified in the new TIMES block, if found to match the time of a restart block in the restart file; or that failing, at the time of the block closest to and following "tstart," if such a block is found; or if neither is found, at the time of the last restart block. In a restart run, plot information is added to the existing plot file, and new restart blocks are also added to the existing restart file. New data will be added to the plot file beginning at the restart time and any subsequent data initially present will be overwritten; however, note that the old plot data at the restart time will be preserved so that any discontinuous changes will appear as such in the plots. In contrast, new restart blocks will be added to the restart file and old blocks will be replaced, starting at the time of the restart block used to restart. Any changes in model parameters on restart will therefore become embedded in the restart file at the restart time. Prudence dictates that the user backup the restart and plot files before any attempt at restart. Note that the main output file, the error file, and the event summary file will always be completely overwritten in a restart. Therefore, these files from the initial run should be renamed before a restart is performed if they are to be retained.

On restart the user may change physical or output parameters but is not allowed to change the number of nodes, fields, or entities used for various models, or directly change mass and energy inventories or inventory distributions, if the repository is an internal one. (A discussion of what constitutes an internal repository is given in Section A.2 on the details of the mass and energy accounting system) The major modeling options that one may respecify on restart are indicated by the explicit presence of the corresponding keywords, such as H-BURN, in the RESTART input template below. If specified on a restart, such an option, or any sub-option within that option, in general should also have been invoked in the initial run. However, in some cases, sub-options not 
in the initial run can be invoked on a restart. Such options typically have ON or OFF keywords associated with them.

If specified on a restart, an previously invoked model or modeling option should not be changed in a manner that would increase storage allocation requirements beyond that initially allocated for each entity specified. Such allocation is controlled by the original set of CONTROL block parameters and by the array storage allocation schemes used in the code. Note that the restrictions discussed above on nodalization changes prohibits changes in the size of the associated arrays. Other arrays, such as those for user tables, are also present. Unless the user is aware of the storage allocation schemes used for such arrays, prudence dictates that those array sizes also be kept the same as in the initial run.

The template for the restart input has the same basic structure as that for an initial run: the global input is given first, and the cell input. For parameters not specified in a restart, a rule different from that in an initial run generally governs default values: parameters remain the same unless specifically changed. The only exception lies in the selection of restart times for the restart run, as discussed below with respect to the restart TIMES block. Thus, in general a parameter need not be specified if its value is not to be changed. Note, however, that if multiple entities are defined within a given input block in the initial run--as is possible for engineered systems, engineered vents, source tables, and user-defined tables--a sufficient number of parameters should be specified for the code to determine which entity is being referred to. For example, if one wishes to change the area of the third of five engineered vents, the following engineered vent input block could be used in a restart:

\section{ENGVENT}

$$
\begin{aligned}
& \text { FROM }=3 \text { TO }=5 \text { EOI } \\
& \text { FROM }=4 \text { TO }=5 \text { EOI } \\
& \text { FROM }=1 \text { TO }=4 \text { VAREA }=1.0 \quad \text { EOI }
\end{aligned}
$$

Note that the cell numbers following FROM and TO in the above example should be those used in the initial run. These keywords are simply place markers for the first and second vents. For the cell input, it follows that only those cells for which changes are made need to be specified.

The input template for a restart has the following form:

********************************************************************************

RESTART

TIMES cput tstart (timinc edtdto tstop) [\{(ctfrac) or CTFRAC $=(\mathrm{ctmfr})\}]$ [TRESTART $=\mathrm{n}$ (tres)] [TSFRAC $=$ tsfrac]

[EOI] [EDMULT=edmult]

[LONGEDT=klong]

[SHORTEDT=kshort] 


\section{[PRFLOW}

[\{ON or OFF\}]]

[PRAER

[\{ON or OFF $\}]]$

[PRAER2

[\{ON or OFF\}]]

[PRLOW-CL

[\{ON or OFF\}]]

[PRHEAT

[\{ON or OFF $\}]]$

[PRFISS

[\{ON or OFF\}]]

[\{PRFISS2

[\{ON or OFF\}] or

PRFPGRP

[\{ON or OFF\}\}]

[PRBURN

[\{ON or OFF $\}]]$

[PRENACCT

[\{ON or OFF\}]]

[PRENGSYS

[\{ON or OFF\}]]

[FLOWS

(data)]

[ENGVENT

((data)

EOI)]

[SUPVENT

(data)

EOI]

[DHEAT

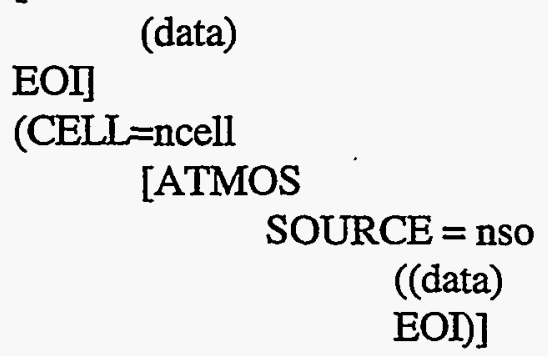

[FISSION

SOURCE $=$ nso

((data)

EOI)]

[AEROSOL

SOURCE $=$ nso

((data) 
[H-BURN

EOI)]

[EOI]]

\author{
(data)
}

[DCH-CELL

(data)

EOI]

[(ENGINEER onmsys numcom iclin iclout delev

EOI)] (data)

[HT-TRAN (htflags)]

[LOW-CELL

[CORCON

TIMES tstart ndelt (dtmin dtmax dedit timdt)]

[SOURCE $=$ nso

((data)

EOI)

EOI)]

EOF

$* * * * * * * * * * * * * * * * * * * * * * * * * * * * * * * * * * * * * * * * * * * * * * * * * * * * * * * * * * * * * * * * * * * * * * * * * * * * * * * * * * * * *$

In the above template, the notation "(data)" indicates that the keywords and options available in a restart are those normally available at that position within the input block. The user should refer to the appropriate section for those keywords and options.

In the following sections, keywords or input parameters that are unique to a restart run or that have different meanings or structures in a restart run are described. The input template shown above includes all of the input that is allowed in a restart run; therefore, portions of this template are not repeated in the following sections.

\title{
14.5.1 Global Level Input for a Restart
}

The global level restart input is described in detail in this and the following two sections. Restart input decks should begin with the single word RESTART. For upward compatibility, a machine name can precede RESTART in support of old input decks, but any name given will be ignored. At the global level only the TIMES block, the print flag options, and global DCH input following RESTART require special discussion.

Note that control parameter and file reassignments are not allowed on restart. The plot and restart files produced in the initial run must be available to CONTAIN in a restart, and these files must have the same names that they had in the initial run.

RESTART a keyword used to denote a restart. 
14.5.1.1 The TIMES Block in a Restart. The TIMES block for a restart has exactly the same format as the TIMES block for an initial run. That format is discussed in Section 14.2.8, Timestep and Time Zone Input. The number of time zones specified in a restart run must be the same as that originally specified; however, the problem restart time can be any time within the new time zones. Values specified in a restart will override values specified in the previous run.

Only one parameter in the TIMES block, "tstart," has a meaning somewhat different from that in an initial run:

tstart

the problem restart time. If a restart from a specific restart block is desired, use a time slightly less than or equal to the time of the block. Such restart blocks, with one exception, are listed in the error file or the event summary file from the previous run. That exception is the temporary restart block from the last edit time encountered in the previous run. The time for that block is not given in the error or event summary files. However, the temporary block will be the one used if the restart time is set greater than the times of the listed restart blocks.

As indicated in Section 14.2.8, the user can put as many as 101 restart blocks on the restart tape. One of these restart blocks will always be a temporary block corresponding to the last edit time encountered (a plot file write and a restart dump are always made at an edit time). In general such blocks are saved permanently in the restart file only at times specified through the TRESTART option or supplied as a default to that option. The exception is a temporary restart block which is used to restart a run. That block becomes a permanent part of the restart file.

Note that if TRESTART is not specified in a restart, the times at which restart blocks will be permanently saved in the restart run will be the end times of the time zones present on restart, not the restart times defined in the previous run. This selection of default restart block times on a restart constitutes a change from the practice prior to CONTAIN 1.1. This change, strictly speaking, violates the convention that in a restart the default values are those present in the previous run. However, it appears that many users expect that changing the end times of the time zones in a restart will also change the times that new restart blocks are saved on the restart file.

14.5.1.2 Print Output Options in a Restart. The output options for the long edits written to the main output file are the same for a restart as those for an initial run (see Section 14.2.9, Output Control). However, in order to have the flexibility to turn off an option used in the previous run, each output option keyword may be followed by either ON or OFF. The word ON will enable the option, and the word OFF will disable it. A print flag not set in the initial run can be set on a restart. Note that the keywords PRFISS2 and PRFPGRP pertain to mutually exclusive options and therefore specifying one as ON will toggle the other off. If an output option keyword is omitted entirely then the status of that option will be the same as in the previous run. 
Example:

PRFLOW OFF

PRLOW-CL ON

In the above example, flow output is suppressed, and lower cell output is activated.

14.5.1.3 Restart Input for DCH Calculations. There is a potential pitfall in defining input for restarts of DCH problems that users must keep in mind. There are a number of DCH input parameters that can be specified for all cells in the global DHEAT input block and subsequently reset for specific cell(s) in the DCH-CELL block(s). If one of these variables appears in the global DHEAT block for a restart, this value will by default be applied to all cells, even if cell-specific values were previously set in the parent run. Hence, if cell-specific values are desired in the restart, they must be specified in the DCH-CELL blocks for the cells of interest in the restart also. It is necessary to do this only if the variable appears in the restart global DHEAT block; otherwise all values remain at the values set in the parent run unless changed in the restart.

\subsubsection{Cell Level Input for a Restart}

Certain model parameters within a cell as described below may be changed in a restart run. If no changes are desired in a given cell, that cell can be omitted from the restart input.

14.5.2.1 Upper Cell Restart Block. The upper cell models and parameters that may be modified on restart are shown in the restart input template between the keywords CELL and LOW-CELL. Only those portions of the upper cell restart input that differ from the initial input are described. Note that the H-BURN and SOURCE input, which is followed by ON or OFF in versions prior to CONTAIN 1.2 , no longer uses ON or OFF but the same format as that in an initial run.

ATMOS SOURCE $=$ nso

FISSION SOURCE $=$ nso a keyword sequence to initiate the respecification of all atmospheric sources in the cell. Note that the first number required after SOURCE is "nso," the number of tables to follow. The "((data) ... EOI)" sequence in the input template is explained in detail in Sections 14.3.1.2, Upper Cell Atmosphere Initial Conditions and Sources, and 14.4.1, Source Table Input. The number of tables and number of data points in the tables must not be greater than those initially specified. If the number of tables is less than the original, only the first "nso" tables will be replaced, and the rest will remain unchanged.

a keyword sequence to initiate the respecification of all fission product sources in the cell. Note that the first number required after SOURCE is "nso," the number of tables to follow. The "((data) ... EOI)" sequence in the input template is explained in detail in Sections 14.3.1.9, Fission Product Sources and 14.4.1, Source Table Input. The number of tables and the number of data points in the tables must not be greater than those initially specified. If the number of tables is less than the original, only the first "nso" tables will be replaced, and the rest will remain unchanged. 
AEROSOL SOURCE = nso

HT-TRAN a keyword sequence to specify a new set of aerosol sources. These replace the current sources in the cell. Note that the first number required after SOURCE is "nso," the number of tables to follow. The number of tables and the number of data points in the tables must not be greater than those initially specified. If the number of tables is less than the original, only the first "nso" tables will be replaced, and the rest will remain unchanged. If the number of tables is less than the original, only the first "nso" tables will be replaced, and the rest will remain unchanged.

a keyword to turn specific aspects of heat transfer in the problem on or off. This option can be invoked on a restart even if not used in the initial run. This keyword is followed by five ON or OFF keywords which specify the status of the various heat transfer options. These status keywords are discussed in Section 14.3.1.6.

14.5.2.2 Lower Cell Restart Block. The lower cell models and parameters that may be modified on restart are explicitly shown in the restart input template after the LOW-CELL keyword.

LOW-CELL the keyword to invoke changes to the lower cell modeling.

CORCON

TIMES

tstart

ndelt

dtmin

dtmax

dedit

timdt

SOURCE

= nso a keyword sequence to initiate changes in the timestep and edit frequency values specified in the previous run for the CORCON calculation. The TIMES keyword initiates the specification of the range of times during which CORCON will be active. Note that CORCON may be active for only one time interval during a given calculation.

the time to begin the CORCON calculation. This value will be ignored if the CORCON model is already active at the time of the restart. (s)

the number of time zones to be used for the CORCON calculation. In each time zone the bounds on the CORCON timestep and the edit frequency may be specified. This value must be followed by "ndelt" sets of ("dtmin," "dtmax," "dedit," "timdt"). The maximum value of "ndelt" is 10 and is not limited by the initial value of "ndelt."

the minimum allowed CORCON timestep. (s)

the maximum allowed CORCON timestep. (s)

the time interval between CORCON edits. (s)

the end time of the time zone. (s)

a keyword sequence to initiate the respecification of all lower cell sources in the cell. Such sources normally are specified independently for each layer. 
Here they must all be specified together, in the order presented in the lower cell input in the initial run. Also note that the first number required after SOURCE is "nso," the total number of tables to follow. Note that the total number of tables may not exceed the number in the initial run and the number of tables for each layer must be remain the same as in the initial run. If the total number of tables is less than the original, only the first "nso" tables will be replaced, and the rest will remain unchanged. The input represented by the sequence "((data) ... EOI)" in the input template is shown, for example, in Section 14.3.2.3.1, Concrete Layer Input Without CORCON, and described in Section 14.4.1, Source Table Input. 


\subsection{SAMPLE PLANT CALCULATIONS}

In this chapter, sample input files and calculations are presented for three different accident scenarios, involving three different nuclear reactor containment types. Section 15.1 describes a accident scenario involving a recirculation line break in the Grand Gulf plant, which is a boiling water reactor (BWR) plant with a Mark III containment. Section 15.2 describes the long-term response to a station blackout scenario in the Surry plant, which is a pressurized water reactor (PWR) with a sub-atmospheric dry containment. Section 15.3 describes an accident scenario that includes the effects of direct containment heating (DCH) in a PWR with an ice condenser containment. The last scenario is assumed to occur in the Sequoyah plant.

For each of these three sample problems, a problem description is given, along with a discussion of the major code options used. Each problem description is followed by a brief discussion of results. Plots of the results and complete listings of the input files are given in each case.

\subsection{Grand Gulf}

In the first sample problem, a Mark III BWR containment is modeled, using the Grand Gulf plant geometry. In the CONTAIN model for Grand Gulf, the containment is divided into three computational cells: the drywell (Cell 1), annulus (Cell 2), and wetwell (Cell 3). The accident scenario that is modeled is a recirculation line break transient. The following paragraphs discuss how each of the cells is modeled and what models are invoked.

The input file is listed in Table 15-1, and the model layout is shown in Figure 15-1. The drywell in the containment model presented here is taken to be a cell with a height of $31.09 \mathrm{~m}$ and a constant cross-sectional area of $243.01 \mathrm{~m}^{2}$. A pool is modeled in the lower cell but it initially starts out dry, so that the total cell volume is specified with the GASVOL keyword. The annulus has a cross-sectional area of $51.441 \mathrm{~m}^{2}$ and its volume is bounded vertically by the height of the weir wall at $7.4104 \mathrm{~m}$. The annulus pool is initialized with $2.8574 \times 10^{5} \mathrm{~kg}$ of water, leaving a gas volume of $93.749 \mathrm{~m}^{3}$. The wetwell is $69.6 \mathrm{~m}$ high with a cross-sectional area of $619.38 \mathrm{~m}^{2}$. The suppression pool in the wetwell has an initial inventory of $3.4399 \times 10^{6} \mathrm{~kg}$ of water, and the wetwell atmosphere has an initial volume of $39650 \mathrm{~m}^{3}$. Cell elevations are specified through the CELLHIST input. Note that heat transfer structures are not present in this containment model.

Intercell flow is modeled using a number of engineered vents, which are specified in the ENGVENT input block. The flow path between the drywell and annulus is simply the annulus area, with flow losses associated with the contraction flow from the drywell to the annulus. The annulus is connected to the wetwell by three rows of 45 horizontal vents. Each row of vents is modeled in CONTAIN as a single liquid and a single gas flow path. The liquid and gas flow path parameters take all 45 vents in each row into account. The flow losses for these paths take contraction, elbow, and expansion effects into account. 
Table 15-1

Grand Gulf Input File

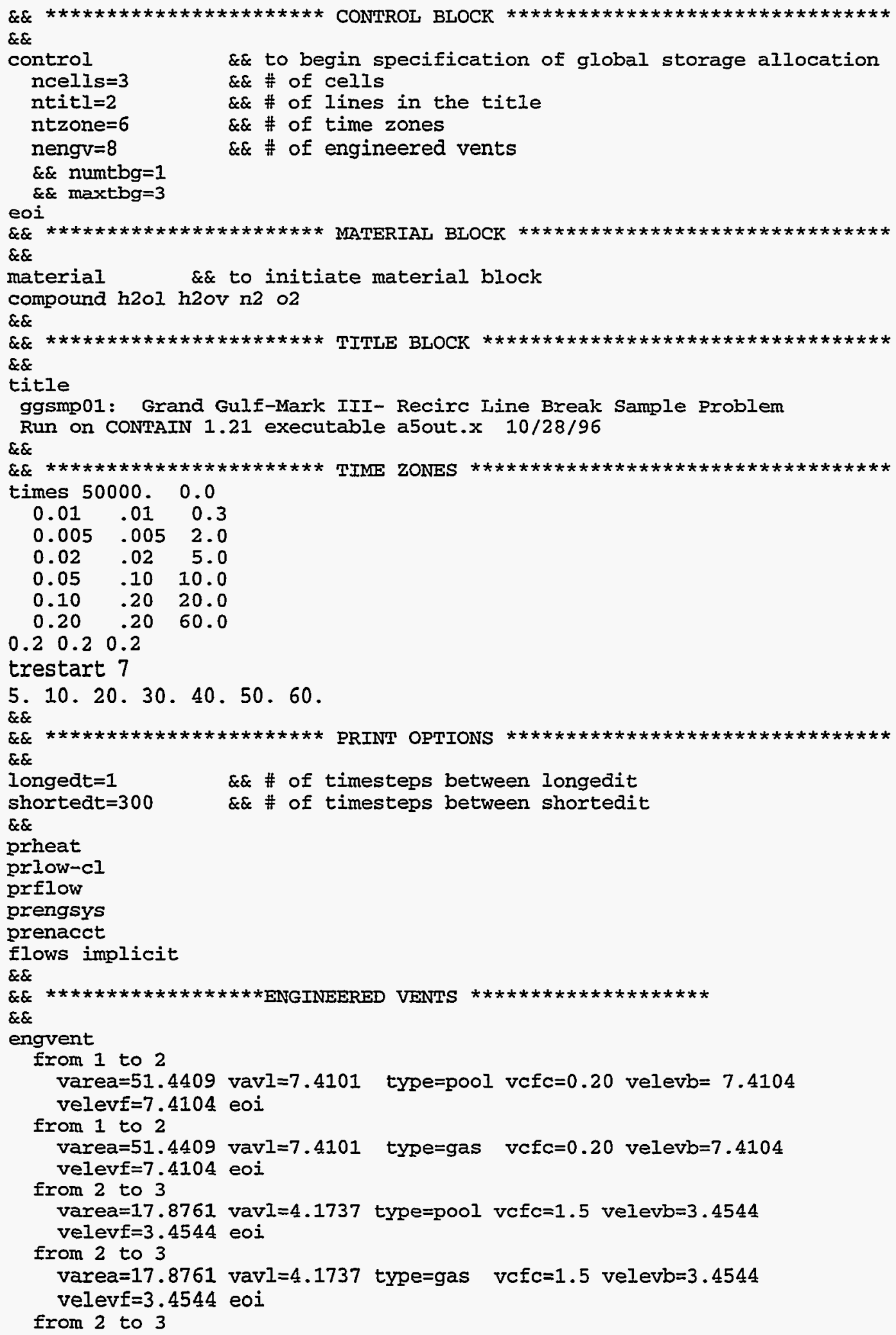


Table 15-1

Grand Gulf Input File (Continued)

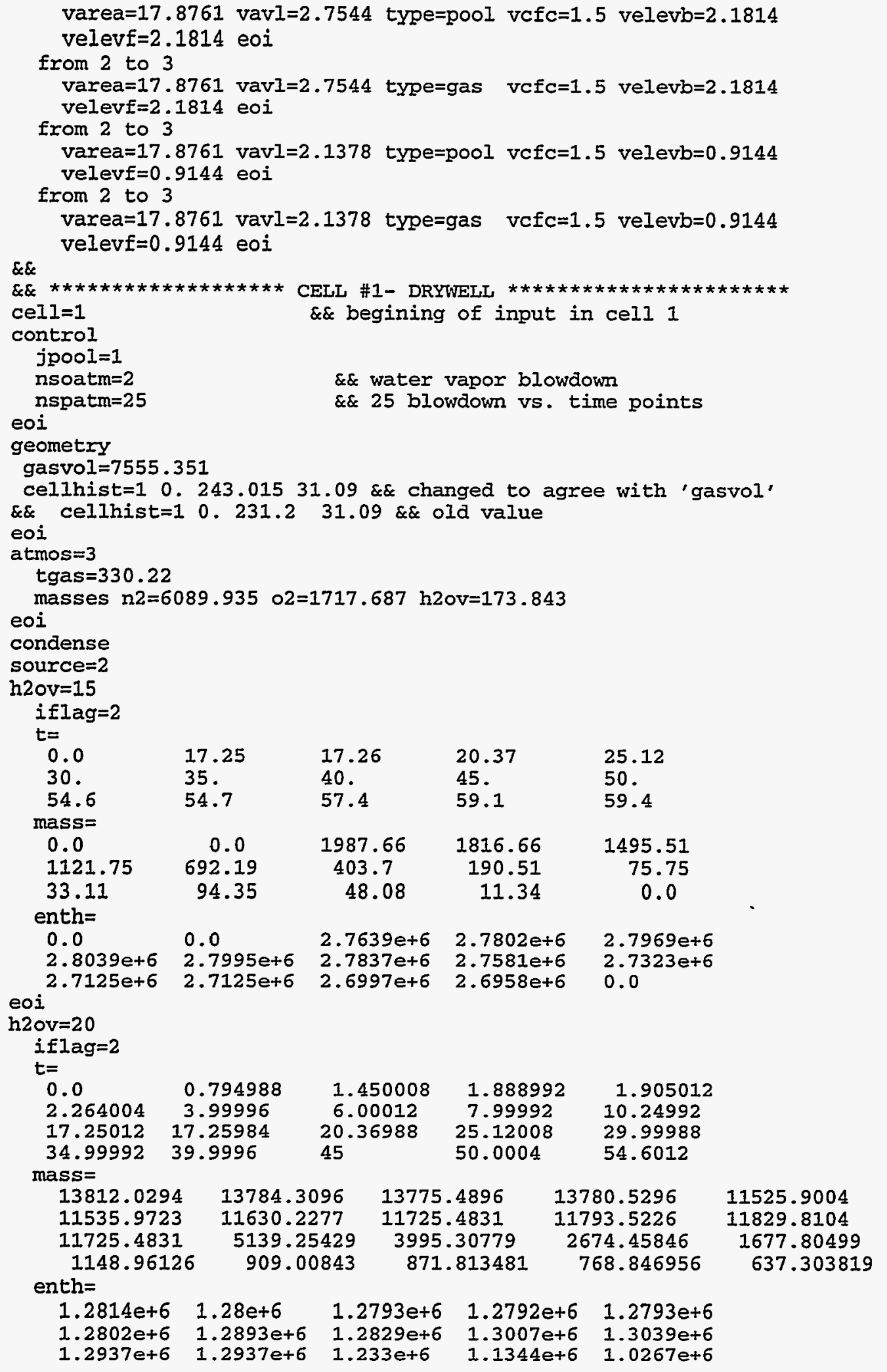

$\begin{array}{lllll}1.2814 e+6 & 1.28 e+6 & 1.2793 e+6 & 1.2792 e+6 & 1.2793 e+6 \\ 1.2802 e+6 & 1.2893 e+6 & 1.2829 e+6 & 1.3007 e+6 & 1.3039 e+6 \\ 1.2937 e+6 & 1.2937 e+6 & 1.233 e+6 & 1.1344 e+6 & 1.0267 e+6\end{array}$


Table 15-1

Grand Gulf Input File (Concluded)

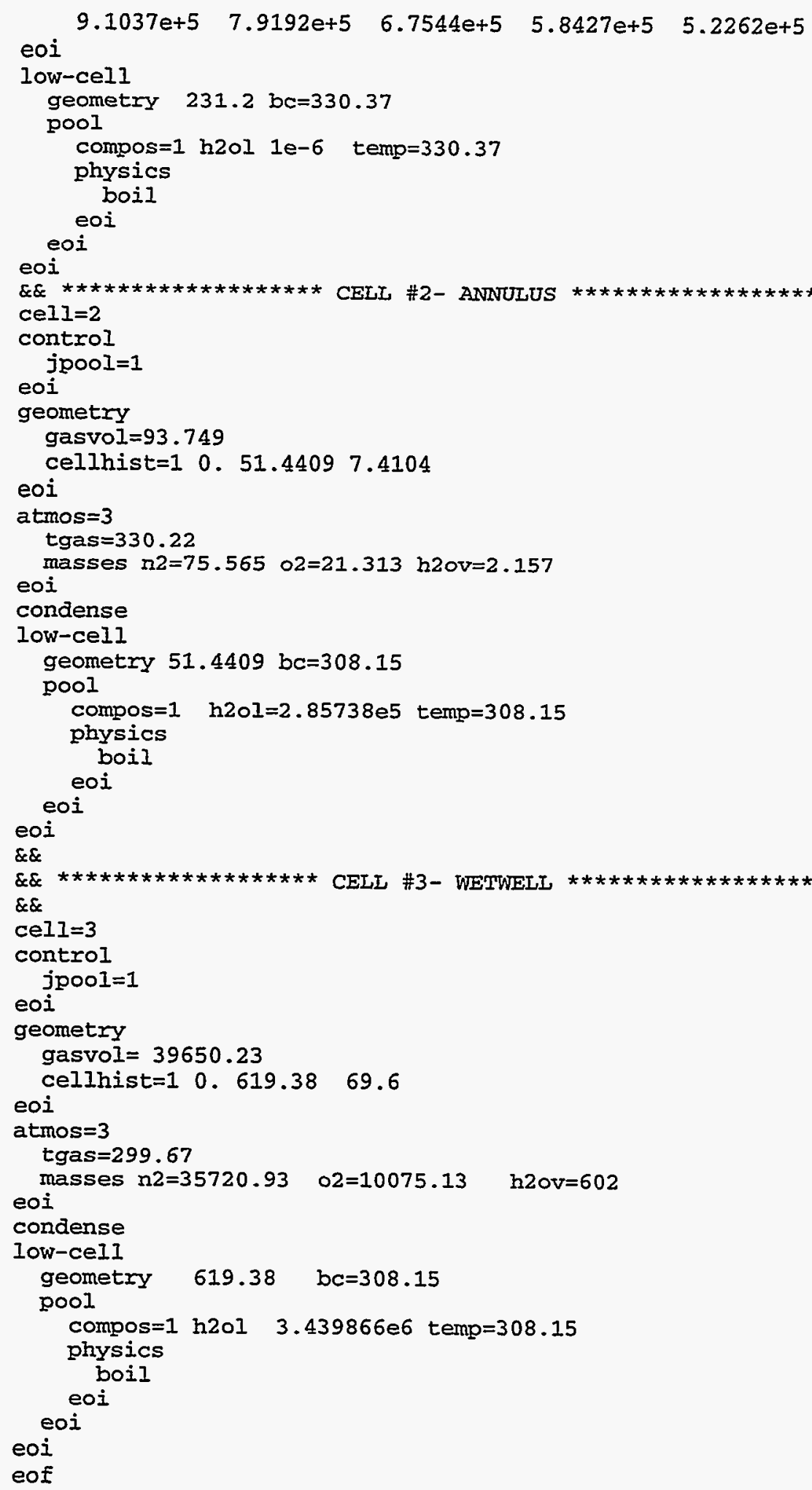




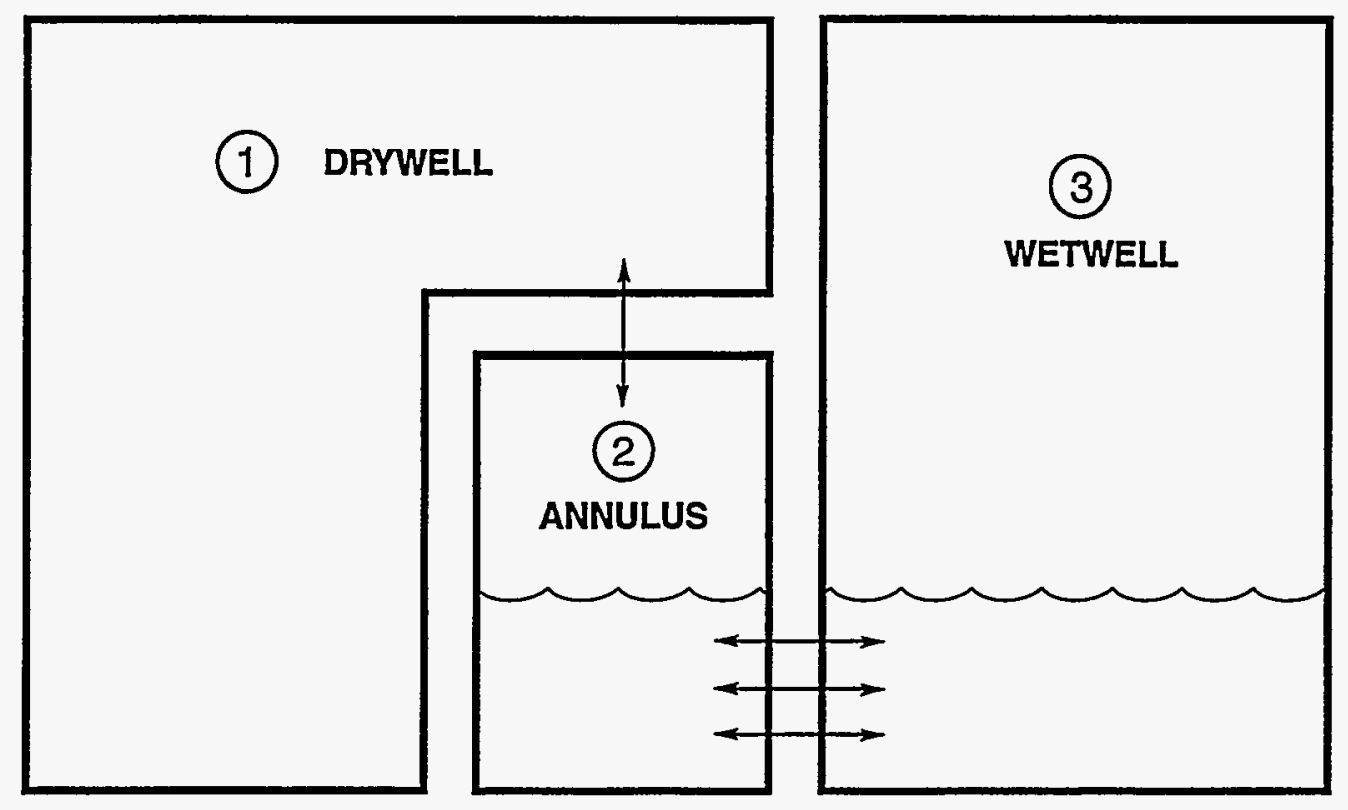

Figure 15-1. Layout of the Grand Gulf Model

Rev. 0 
The recommended implicit flow solver option is used. Since neither DROPOUT nor water aerosol modeling has been specified, water that condenses in the atmosphere remains suspended in this calculation. This suspended liquid is assumed to be in thermal equilibrium with the atmosphere, to flow with the gas, and to contribute thermal and inertial mass to the atmosphere. The pool modeling includes boiling through the BOIL keyword in the POOL input block. The CONDENSE keyword enables condensation heat transfer on surfaces.

All three cells start with a pressure of $102.01 \mathrm{kPa}$. The cell atmospheres all consist of air and a small amount of water vapor. The drywell and annulus atmosphere are at the same temperature of 330.22 $\mathrm{K}$, while the wetwell starts out at a cooler temperature of $299.67 \mathrm{~K}$. The recirculation line break is modeled as a single $\mathrm{H} 2 \mathrm{OV}$ source to the drywell atmosphere with an enthalpy corresponding to the water discharged from the reactor cooling system. This source is specified in the input file in tabular form, after the SOURCE keyword in Cell 1. As this superheated water enters the drywell, it partially flashes to steam. The drywell and annulus are sufficiently pressurized to force liquid through the vents, discharging steam and gas through the suppression pool and into the wetwell.

Figures 15-2 and 15-3, respectively, show the pressure-time and temperature-time histories for the three volumes modeled. The drywell and annulus pressures initially rise very rapidly and then decline rapidly when the suppression pool vents clear. Initially the drywell/wetwell pressure differential remains at a level sufficient to clear all three rows of vents. The pressure differential later declines sufficiently to permit the bottom and the middle vent rows to reflood.

Figure 15-4 shows the vent flow rates as a function of time and Figure 15-5 shows the flow rates on an expanded time scale for the first 5 seconds, during which the vents clear. The time delay in clearing the successive vent rows is clearly evident. Figure $15-4$ also shows that reverse (i.e., negative) flow back through the bottom row of vents begins at about 23 seconds and begins slightly later for the middle row; the top row does not reflood during the time scale of the calculation. There is a damped oscillation in the flows as the vents reflood. Gas flows through the vents are shown in Figure 15-6. The major features of the gas flow history conform with what would be expected based upon the calculated pressure-time history and the vent clearing/reflooding histories.

Pool depths for the annulus and the wetwell are shown in Figure 15-7. The annulus depth initially declines very rapidly as the water is displaced through the vents. There is a corresponding rise in the wetwell depth, but it is much smaller in magnitude because the wetwell cross section is much greater than the annulus cross section. After the lowest row of vents clears, the annulus level holds constant while the wetwell depth increases slowly, owing to the addition of blowdown water. At later times, the annulus level rises as the lower two rows of vents reflood.

It is apparent from these results that a number of details of the containment response could not have been captured without the capability to model multiple suppression pool vents. This capability is not available in code versions prior to CONTAIN 1.2. 


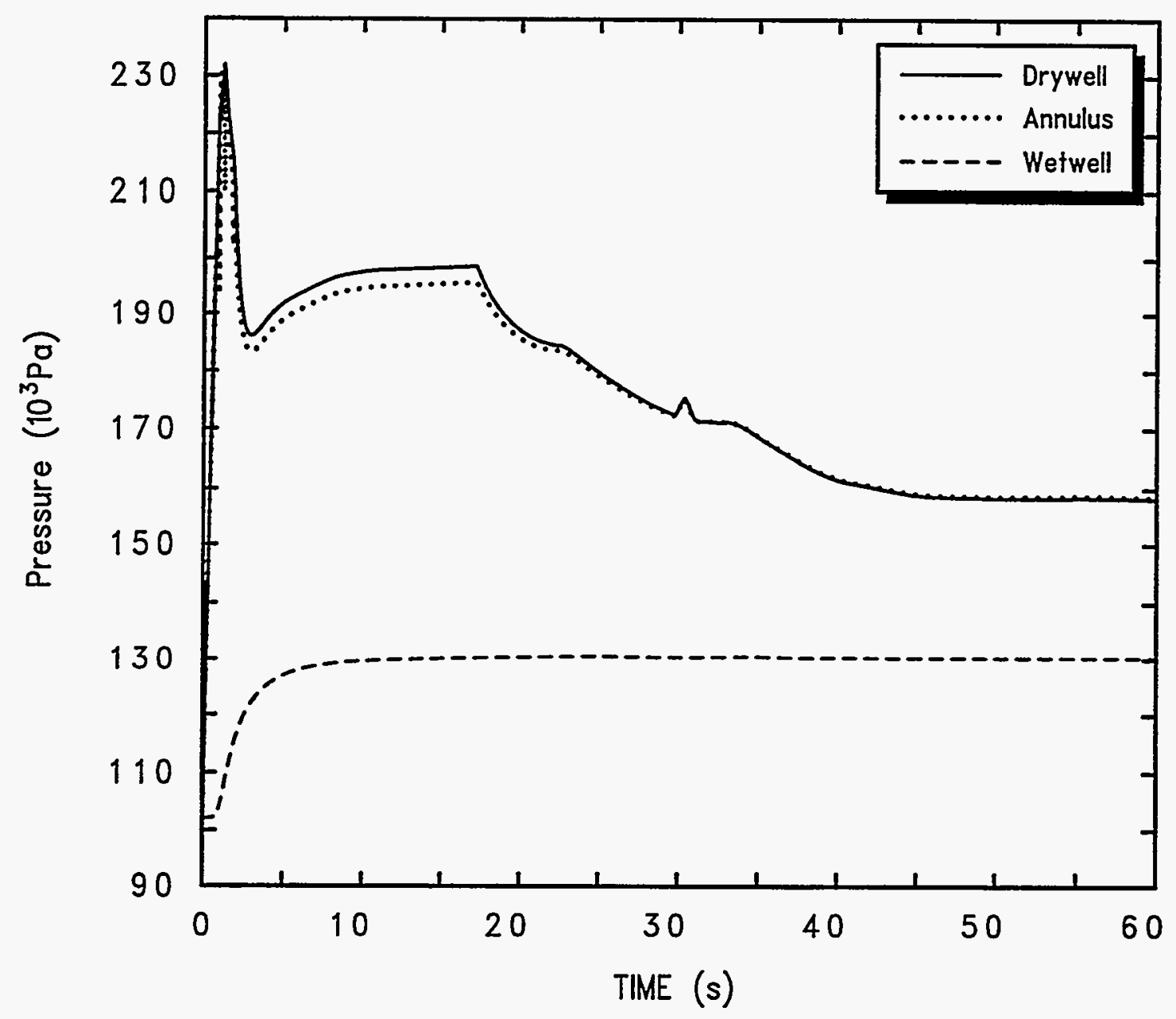

Figure 15-2. Pressure-Time Histories

Rev. 0 


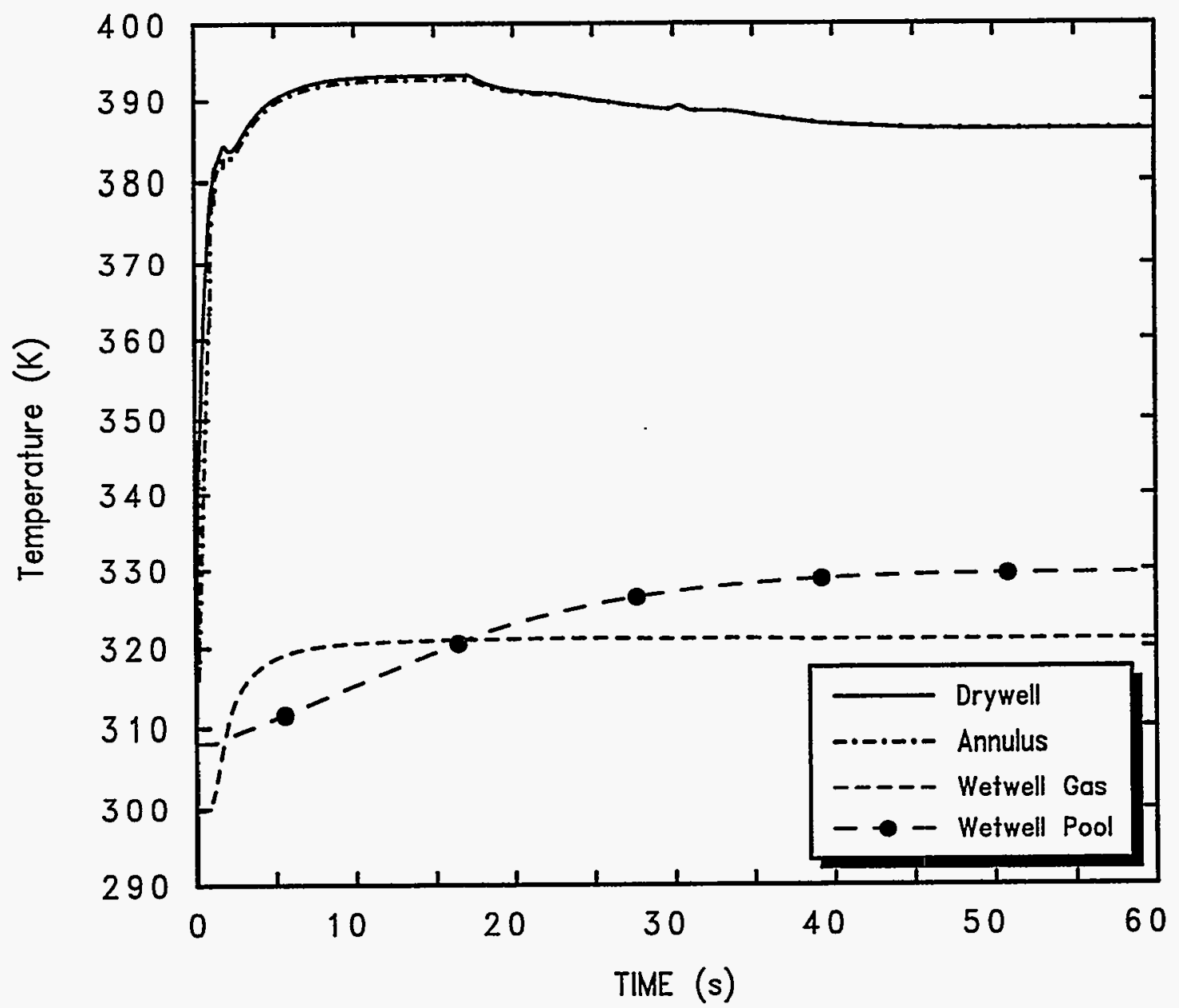

Figure 15-3. Temperature-Time Histories 


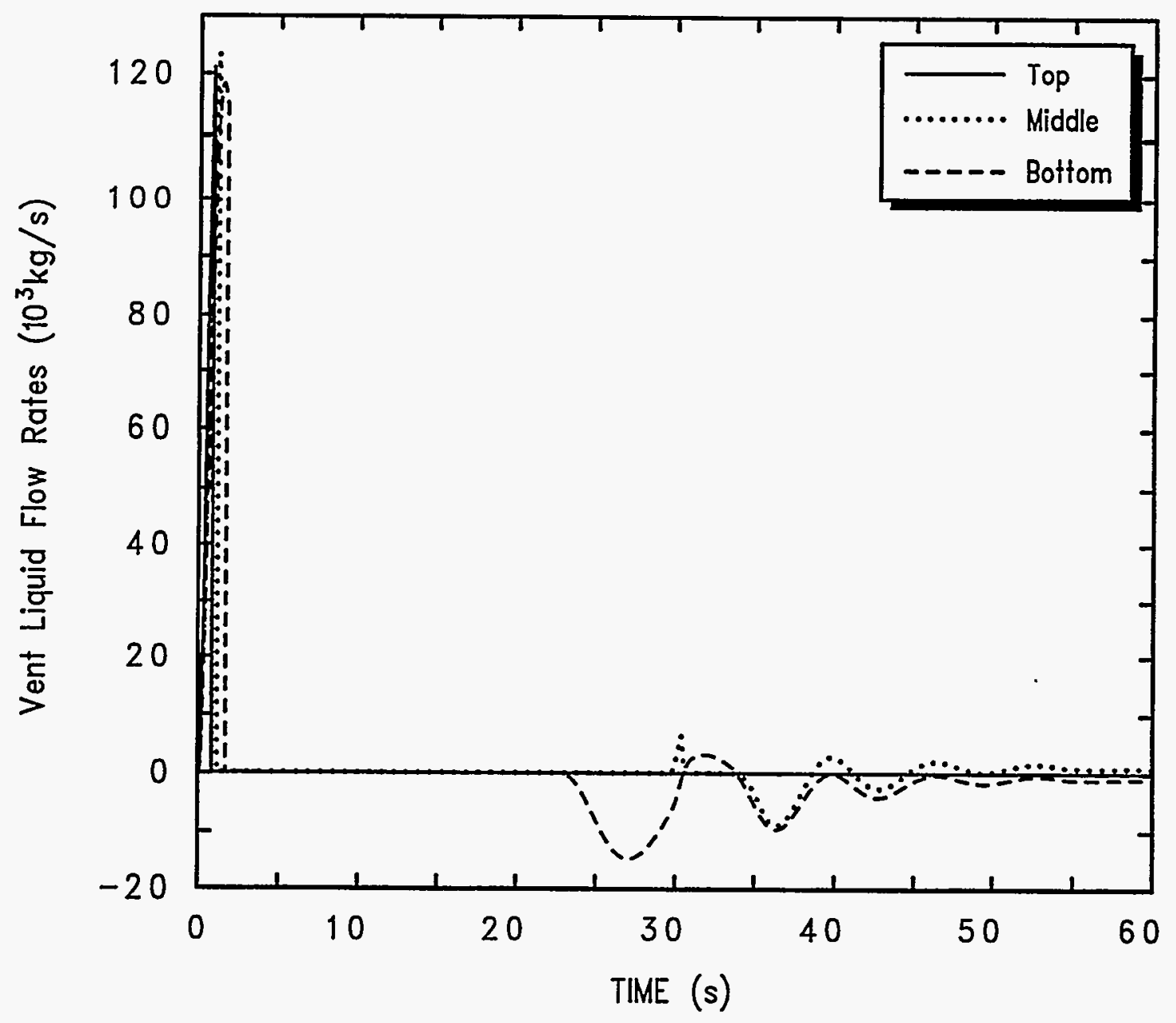

Figure 15-4. Vent Liquid Flow Rates as a Function of Time 


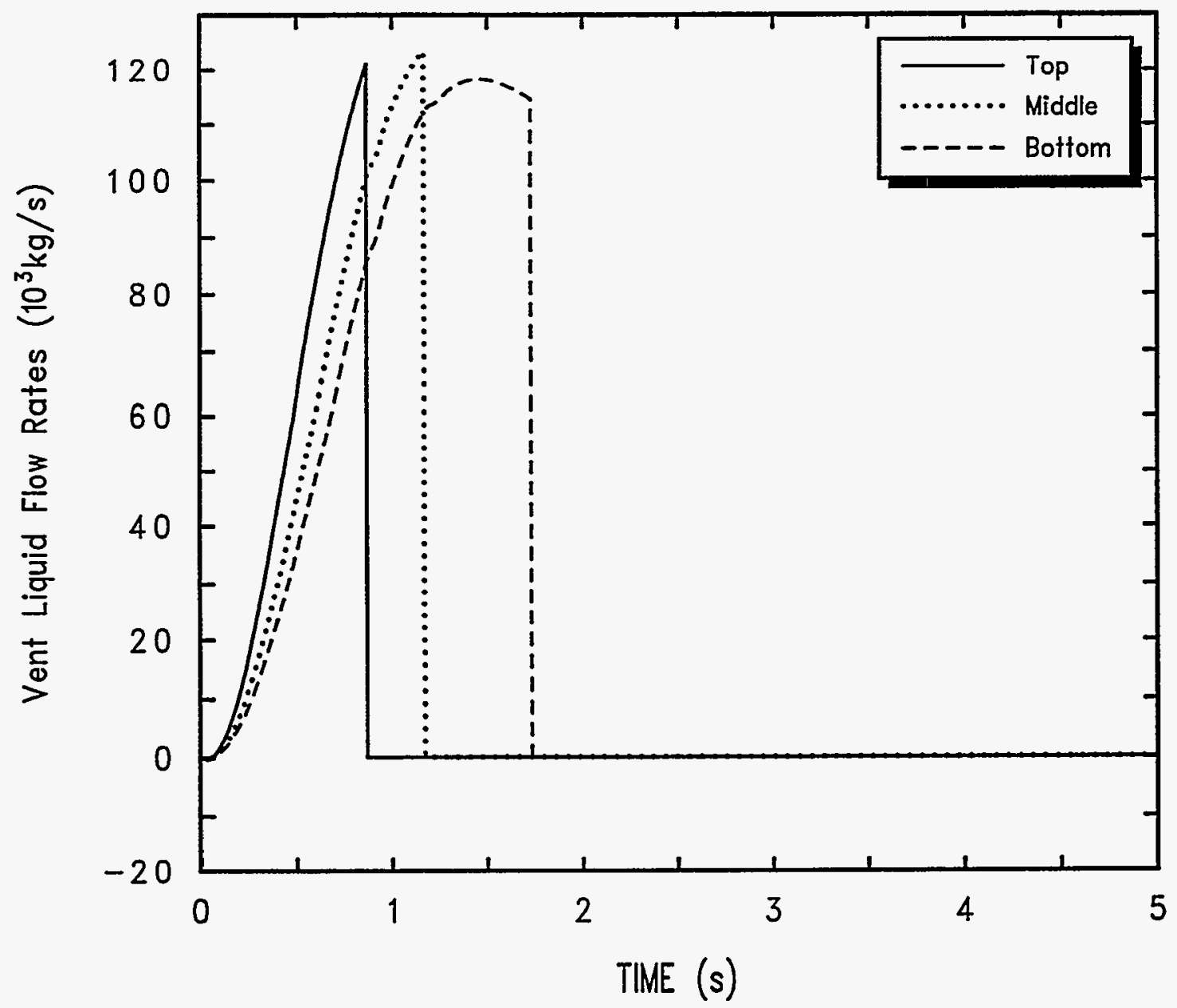

Figure 15-5. Vent Liquid Flow Rates for the First 5 Seconds 


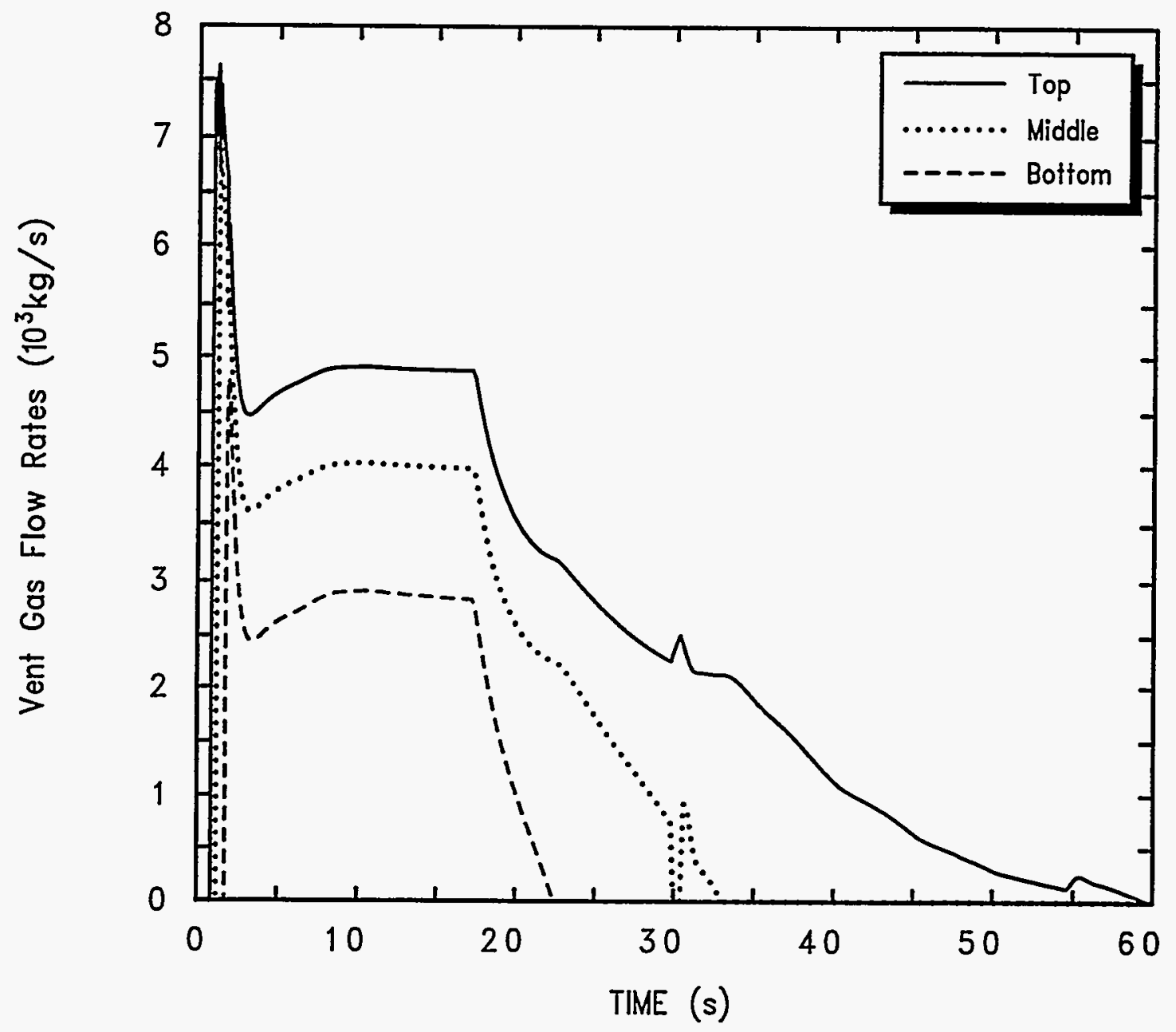

Figure 15-6. Vent Gas Flow Rates as a Function of Time 


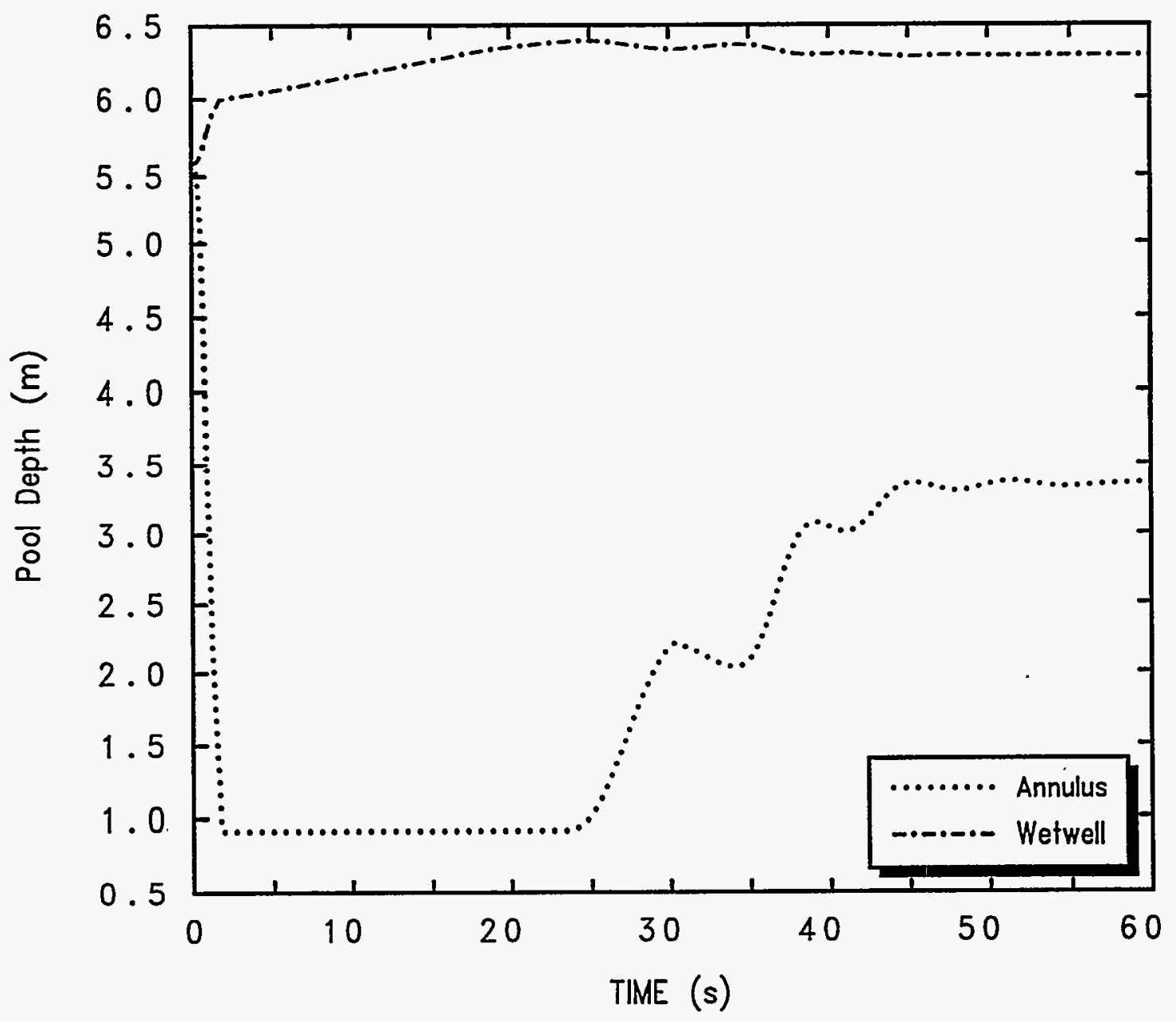

Figure 15-7. Pool Depths for the Annulus and the Wetwell 


\subsection{Surry Plant}

In the second sample problem, a seven-cell model of a dry PWR containment is presented. This model is derived from the subatmospheric containment of the Surry plant. This particular model illustrates the use of the CORCON and VANESA options in CONTAIN and is designed to simulate the containment pressurization that will occur at late times ( $>1$ day) if no containment heat removal systems are available.

The input file is listed in Table 15-2, and the model layout is given in Figure 15-8. In this illustration, the Surry containment is modeled as five computational cells. Two additional cells model the primary system and the environment. Cell 1 is defined as the cavity and the instrument tunnel. Cell 2 encloses the following compartments: basement, lower annulus, residual heat removal area, and upper hoist space. The middle and upper crane wall annulus is represented by Cell 3 . The dome, operating floor, and steam generator cubicles are modeled as Cell 4. Cell 5 includes the pressure relief tank and pressurizer rooms. The primary system cell, which generates blowdown steam and hydrogen sources, is defined as Cell 6 . Cell 7 represents the environment cell.

The implicit flow solver is used for the first six cells (IMPLICIT $=6$ ) and flow is calculated explicitly for the environment cell. Flow paths between cells are specified using engineered vents. Irreversible pressure-dependent flow areas are modeled between cells 1 and 2 and cells 1 and 4. A time-dependent flow area between cell 1 and cell 6 is specified.

The aerosol model is enabled with the AEROSOL keyword. The aerosol option allows the modeling of particle agglomeration and deposition. The AERTIM option allowing input of time-dependent particle sizes is used. The TRAPUNFL and TRAPOVFL keywords are specified to prevent aerosols that become too small or too large for the aerosol mesh from being sent to the WASTE location; with these options, particles that become too small for the mesh are returned to the smallest size class and particles that become too large for the mesh are sent to the pool in the cell designated by the OVERFLOW keyword.

Aerosol condensation and evaporation is disabled in the reactor pressure vessel (RPV) cell by specifying NOCONEVA $=6$ in order to prevent spurious effects that can result when large amounts of water aerosol enter a cell containing much smaller amounts of solid aerosols (see the first paragraph of Section 13.2.4). The fission product data are specified in the FISSION block. Hydrogen burn modeling is enabled with the H-BURN keyword. Radiation heat transfer is modeled with the RAD-HEAT option and condensation/evaporation is activated through use of the CONDENSE keyword. Condensate runoff from structures is diverted to a specific cell also through the OVERFLOW keyword. The specification of OUTGAS in the STRUC block in cell 1 allows the modeling of outgassing from concrete heat structures in that cell only.

The CORCON option in CONTAIN models core-concrete interactions (CCIs) in the cavity cell while the VANESA option models the release of radionuclides and generation of aerosols during $\mathrm{CCI}$. The concrete type specified for the CORCON calculation is generic limestone, which provides for a more active CCI than do other types of concrete; however, the Surry plant actually has a high-silica 
Table 15-2

Surry Input File

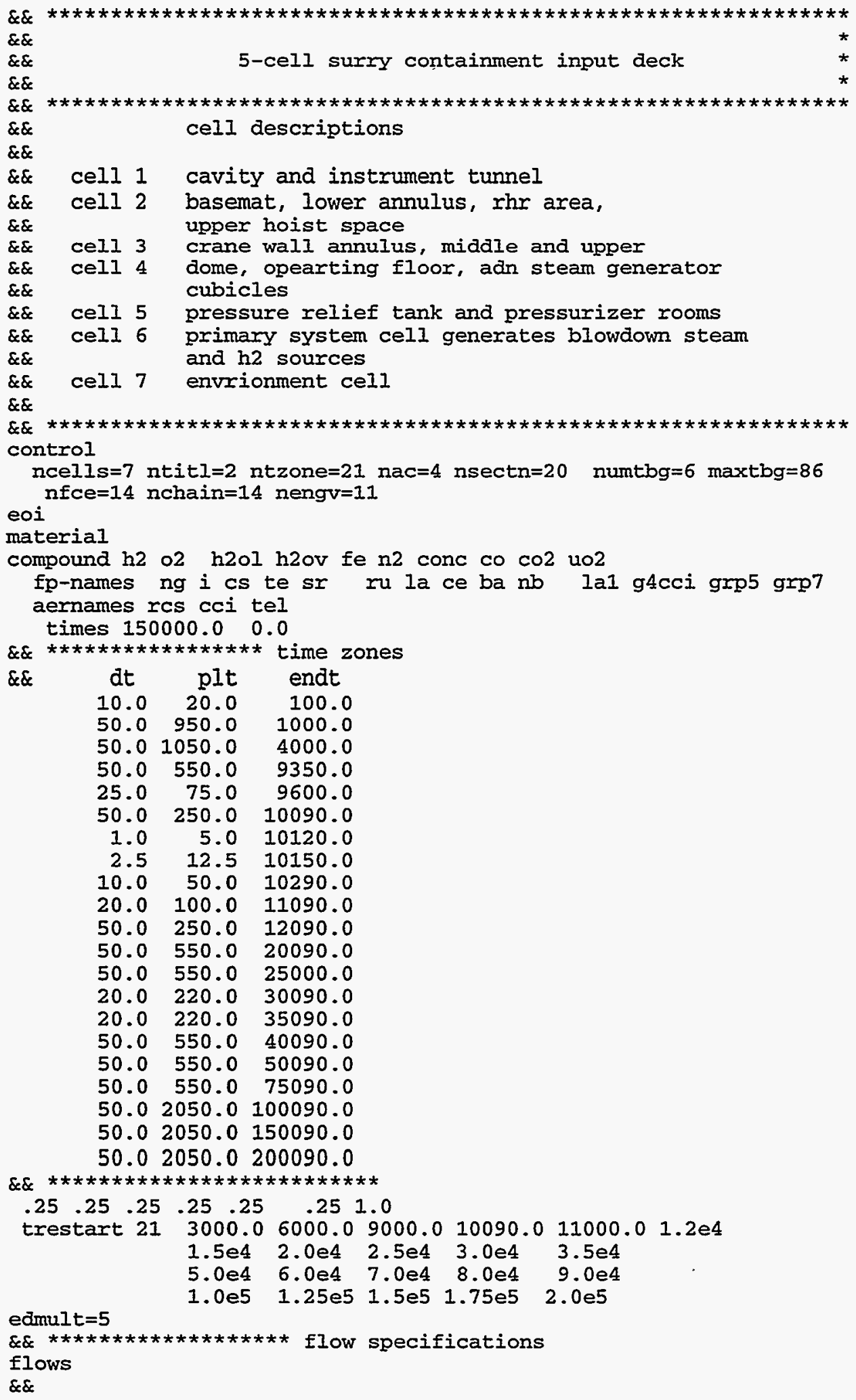


Table 15-2

Surry Input File (Continued)

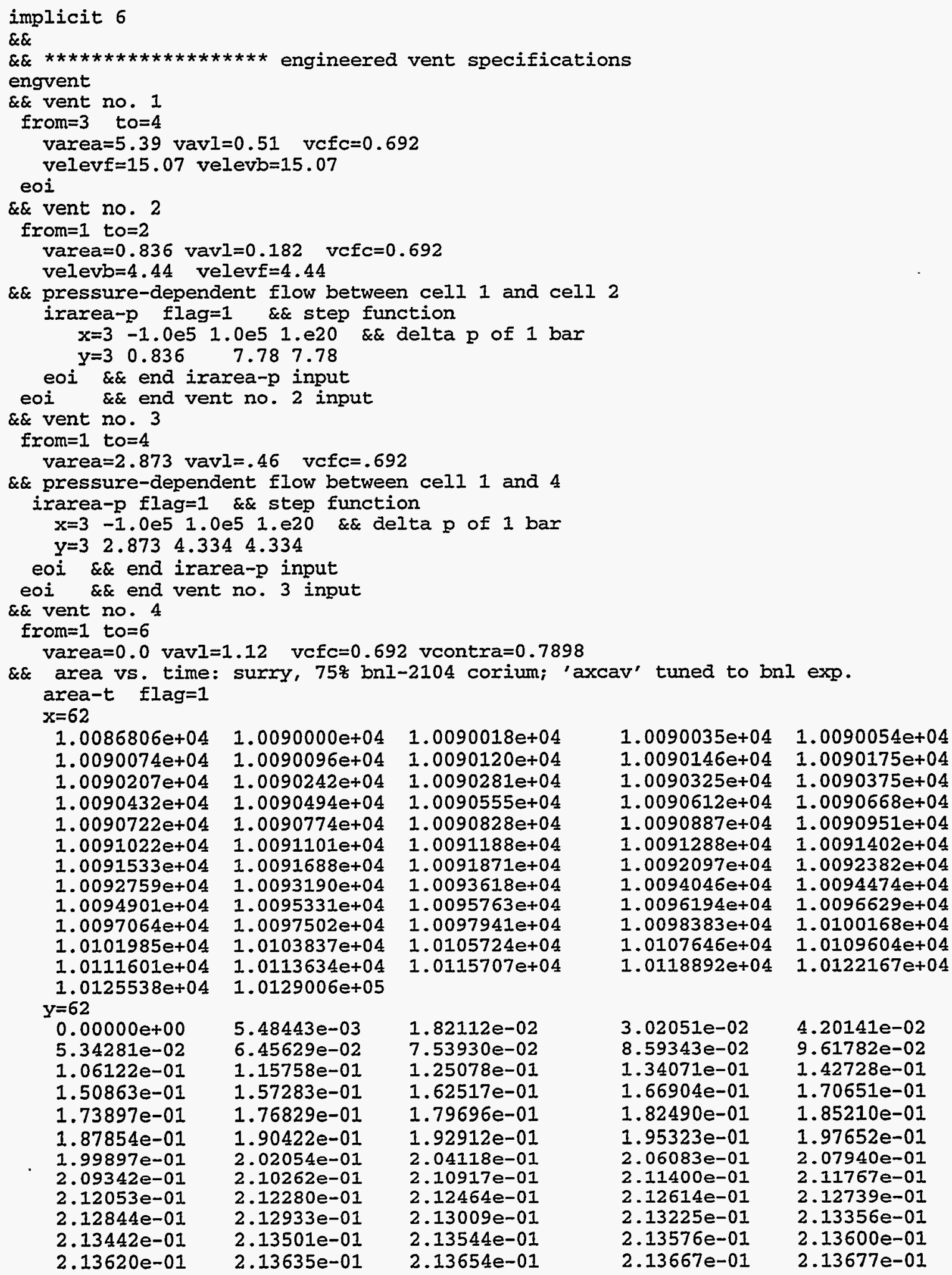

\section{$1.0090000 \mathrm{e}+04$ \\ $1.0090096 \mathrm{e}+04$ \\ $1.0090242 e+04$ \\ $1.0090494 \mathrm{e}+04$ \\ 1. $0090774 \mathrm{e}+04$ \\ $1.0091101 e+04$ \\ $1.0091688 \mathrm{e}+04$ \\ $1.0093190 e+04$ \\ $1.0095331 e+04$ \\ $1.0097502 \mathrm{e}+04$ \\ $1.0103837 e+04$ \\ $1.0113634 \mathrm{e}+04$ \\ $1.0129006 \mathrm{e}+05$}

$5.48443 e-03$

$6.45629 e-02$

$1.15758 \mathrm{e}-01$

$1.57283 \mathrm{e}-01$

$1.76829 \mathrm{e}-01$

1. $90422 \mathrm{e}-01$

$2.02054 \mathrm{e}-01$

$2.10262 \mathrm{e}-01$

$2.12280 e-01$

$2.12933 e-01$

$2.13501 \mathrm{e}-01$

$2.13635 e-01$

$1.0090018 e+04$

$1.0090120 e+04$

$1.0090281 \mathrm{e}+04$

$1.0090555 e+04$

$1.0090828 e+04$

$1.0091188 e+04$

$1.0091871 e+04$

$1.0093618 e+04$

$1.0095763 e+04$

1.0097941e+04

$1.0105724 \mathrm{e}+04$

$1.0115707 \mathrm{e}+04$

1. $82112 \mathrm{e}-02$

$7.53930 \mathrm{e}-02$

$1.25078 \mathrm{e}-01$

$1.62517 \mathrm{e}-01$

$1.79696 \mathrm{e}-01$

$1.92912 \mathrm{e}-01$

2. $04118 \mathrm{e}-01$

2. 10917 e-01

2.12464e-01

2.13009e-01

$2.13544 \mathrm{e}-01$

$2.13654 e-01$

$1.0090035 e+04$

$1.0090146 \mathrm{e}+04$

$1.0090325 e+04$

$1.0090612 \mathrm{e}+04$

$1.0090887 e+04$

$1.0091288 e+04$

$1.0092097 e+04$

$1.0094046 \mathrm{e}+04$

$1.0096194 \mathrm{e}+04$

$1.0098383 e+04$

$1.0107646 e+04$

$1.0118892 \mathrm{e}+04$

3. 02051 e-02

8.59343e-02

$1.34071 \mathrm{e}-01$

$1.66904 \mathrm{e}-01$

$1.82490 \mathrm{e}-01$

$1.95323 \mathrm{e}-01$

$2.06083 e-01$

2.11400 e-0I

$2.12614 e-01$

$2.13225 e-01$

$2.13576 \mathrm{e}-01$

2. $13667 e-01$

$1.0090054 e+04$

$1.0090175 e+04$

$1.0090375 e+04$

$1.0090668 \mathrm{e}+04$

$1.0090951 \mathrm{e}+04$

$1.0091402 \mathrm{e}+04$

$1.0092382 e+04$

$1.0094474 \mathrm{e}+04$

$1.0096629 e+04$

$1.0100168 e+04$

$1.0109604 \mathrm{e}+04$

$1.0122167 \mathrm{e}+04$

4.20141e-02

9.61782e-02

$1.42728 \mathrm{e}-01$

$1.70651 \mathrm{e}-01$

$1.85210 \mathrm{e}-01$

$1.97652 \mathrm{e}-01$

2.07940e-01

2. $11767 \mathrm{e}-01$

2.12739e-01

$2.13356 \mathrm{e}-01$

$2.13600 \mathrm{e}-01$

$2.13677 \mathrm{e}-01$

$2.13683 e-01$

Rev. 0 


\section{Table 15-2}

\section{Surry Input File (Continued)}

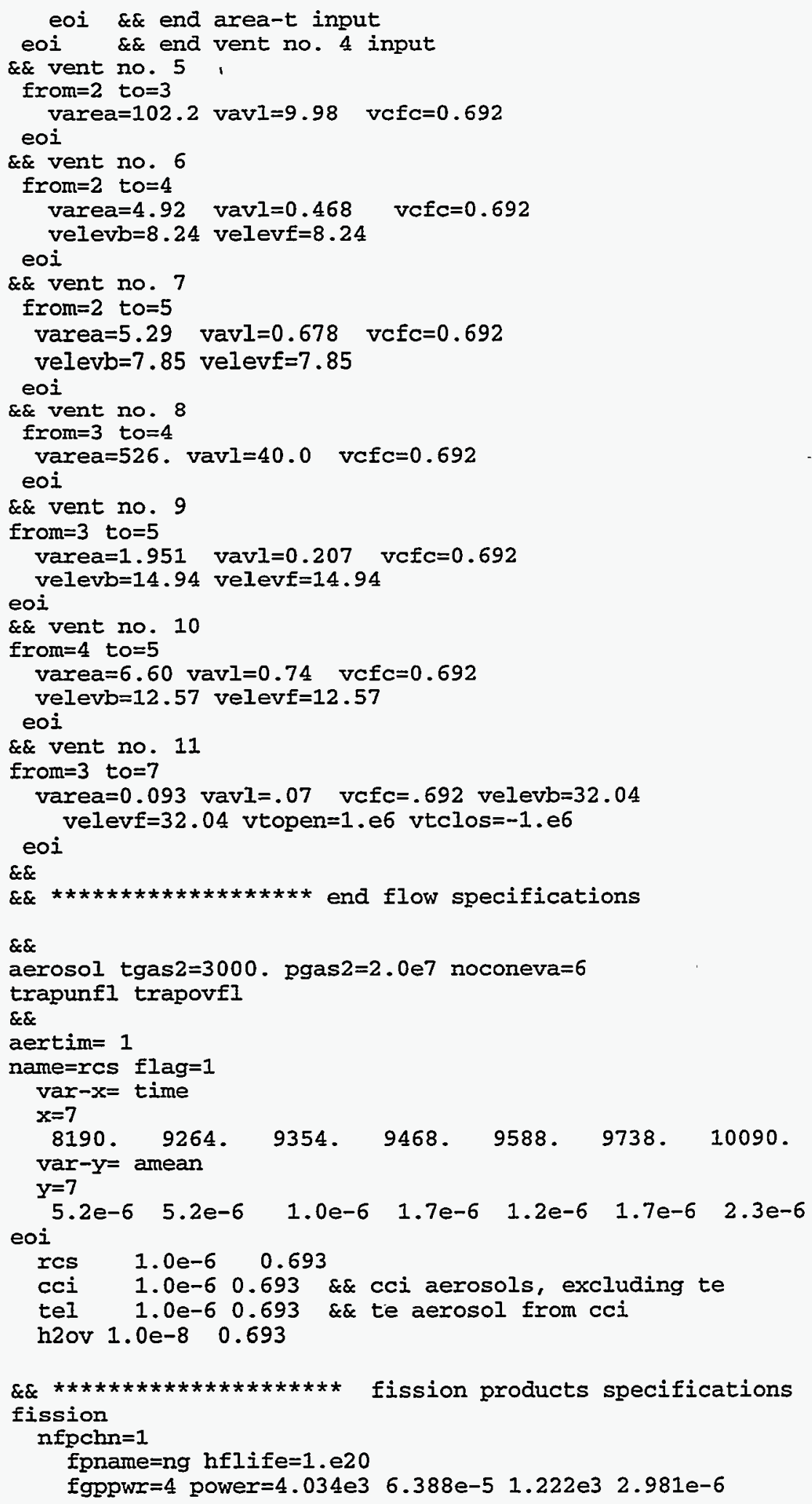




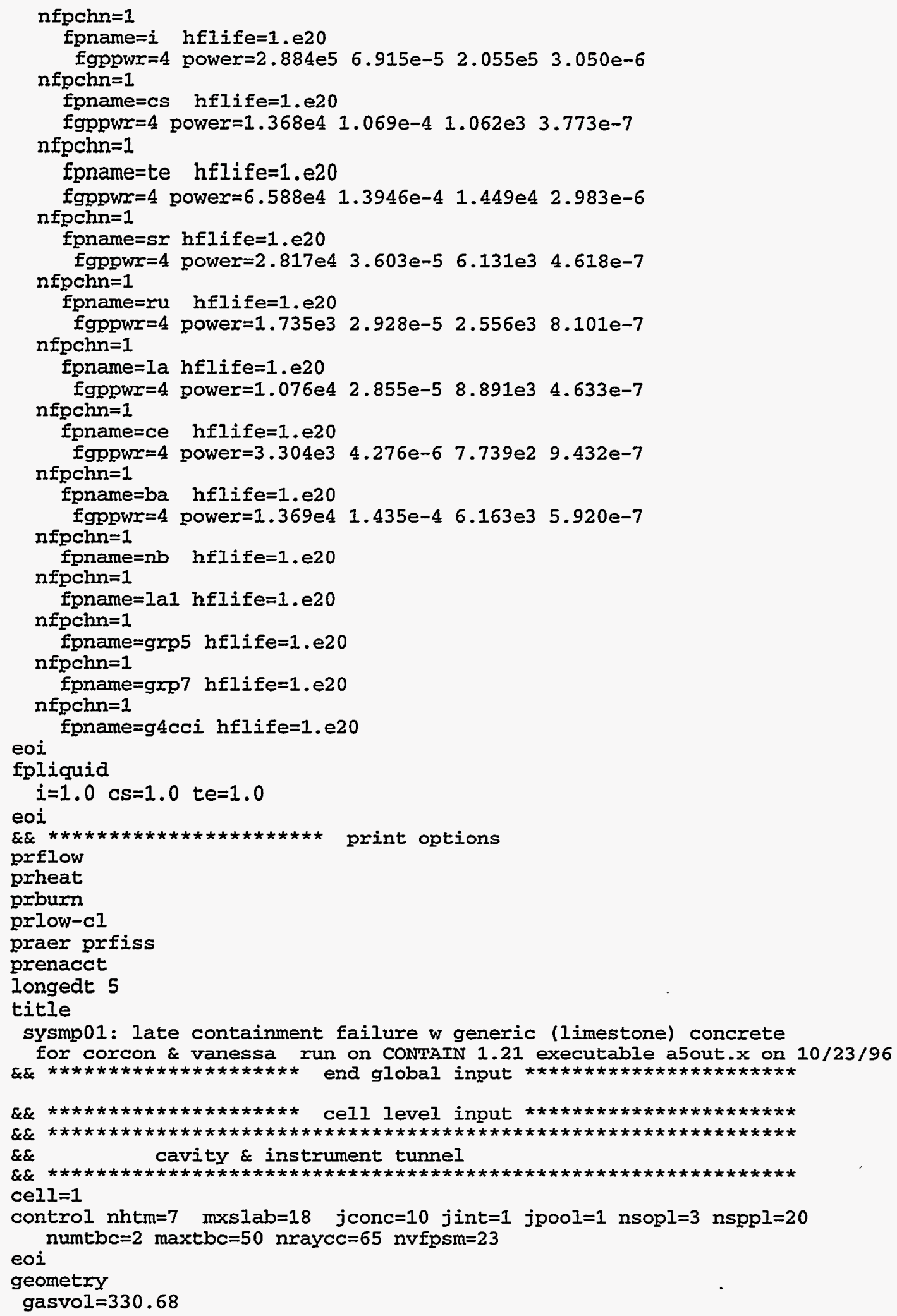


Table 15-2

Surry Input File (Continued)

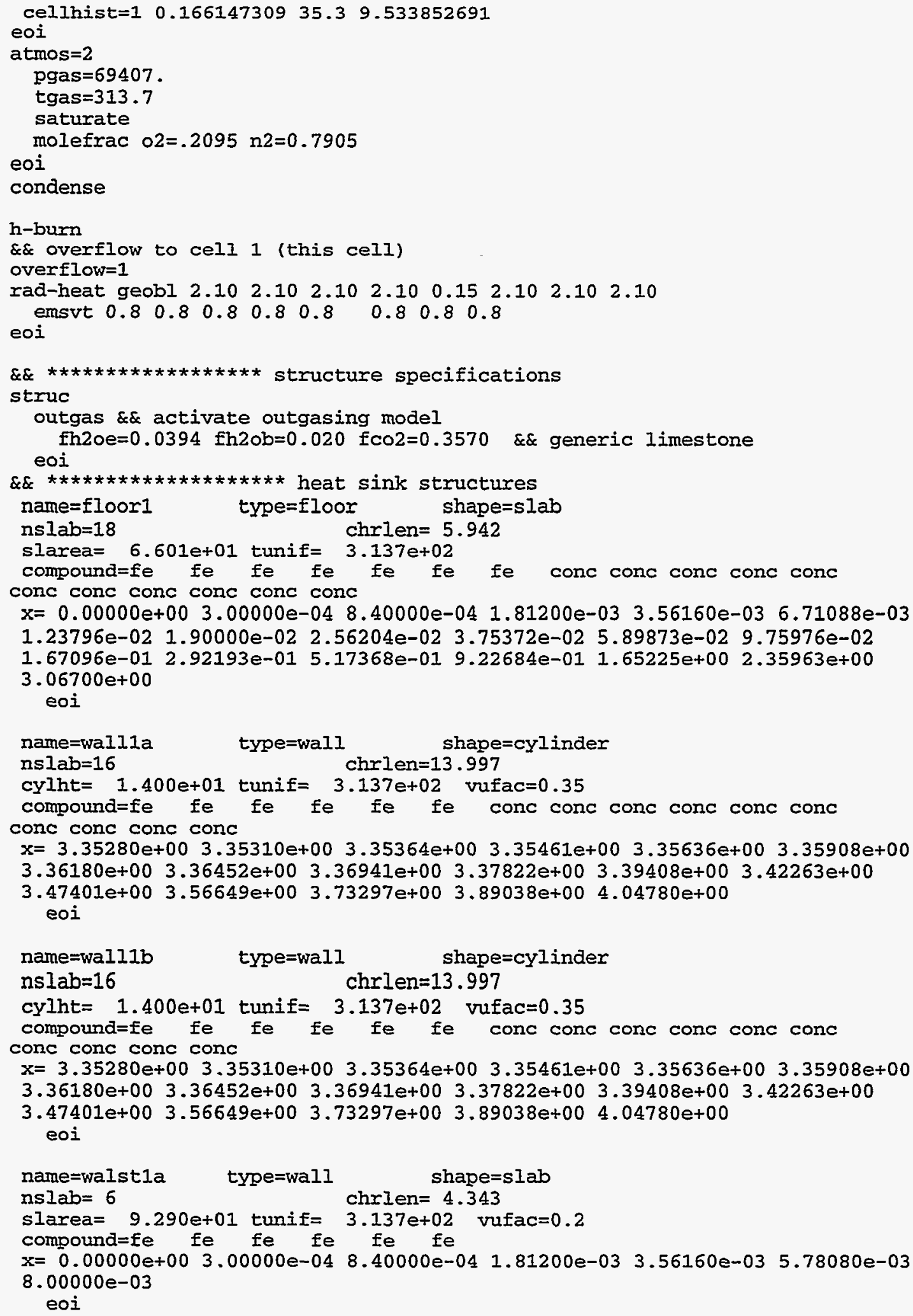


Table 15-2

Surry Input File (Continued)

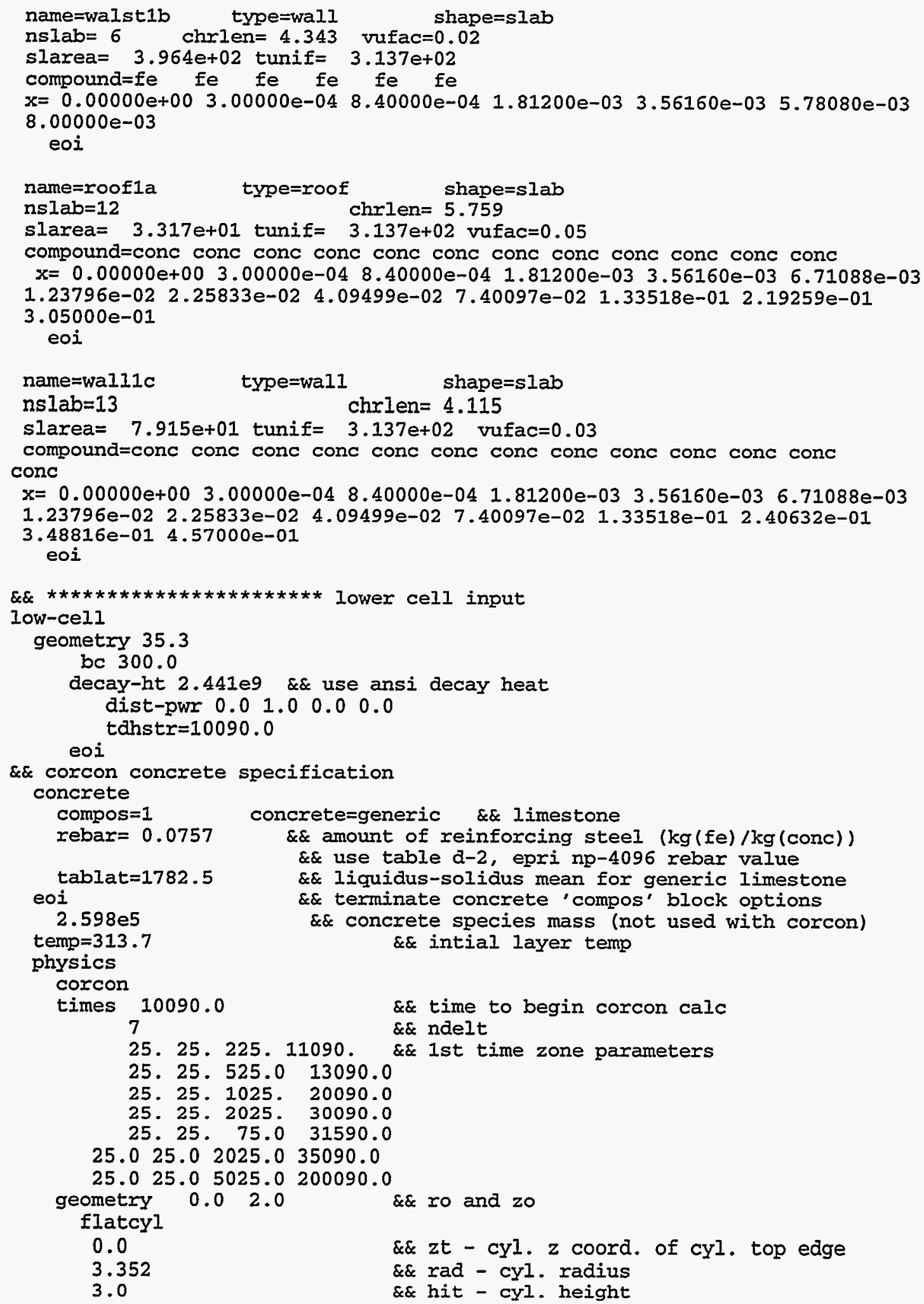




\title{
Table 15-2
}

\section{Surry Input File (Continued)}

\author{
0.2 \\ 10.0 \\ 5.00 \\ \&\& radc - radius of corner \\ \&\& IW - conc. cavity external radius \\ $\& \& \mathrm{hbb}$ - height from external base of \\ \&\& cavity to base of cavity (flat bottom) \\ 25 \\ 10 \\ emisiv \\ \&\& \# ray points along flat bottom of cavity \\ \&\& \# ray points around corner \\ oxide \\ time 2 \\ $\begin{array}{llll}0.0 & 0.8 & 1.0 e 6 & 0.8\end{array}$ \\ metal \\ time 2 \\ $\begin{array}{llll}0.0 & 0.8 & 1.0 e 6 & 0.8\end{array}$ \\ surrnd \\ time 2 \\ eoi \\ $\begin{array}{llll}0.0 & 0.8 & 1.0 e 6 & 0.8\end{array}$ \\ eoi \\ \&\& terminate emisiv option \\ \&\& terminate corcon option \\ $\& \&$ \\ \&\& vanesa specification \\ vanesa \\ scrub \&\& turn on vanesa scrubbing \\ $\varepsilon \&$ \\ eoi \\ \&\& terminate scrub option \\ aerconst \\ 2 \\ \&\& aername ncnams onams \\ tel 2 te $s b$ \\ cci -1 \\ \&\& Eission product track
fptrack=detail 12 \\ \&\& associate contain aerosol w/ vanesa fps \\ $\& \&$ \# aerosol comps. to carry vanesa const. \\ \&\& onam nfp fpnam wfrac

$\begin{array}{ccll}\text { csi } & 2 & i & 0.489\end{array}$ \\ cs 0.511 \\ te 2 te 1.0 \\ g4cci 1.0 \\ sb 2 te 1.0 \\ $\begin{array}{llll} & & \text { g4cci } 1.0 \\ \text { sro } & 2 & \text { sr } & 0.848\end{array}$ \\ $\begin{array}{lll}\text { bao } 2 & \text { grp5 } & 0.848 \\ \text { ba } & 0.896\end{array}$ \\ $\begin{array}{lll}\text { grp5 } & 0.896\end{array}$

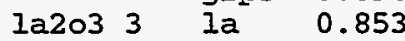 \\ la1 0.853 \\ grp7 0.853 \\ ceo2 2 ce 0.867 \\ $\mathrm{nb205} 3 \mathrm{nb} \quad 0.703$ \\ la $\quad 0.703$ \\ grp7 0.703 \\ ru 1 xu 1.0 \\ mo 1 IU 1.0 \\ zro2 2 grp7 \\ 0.0080 aerosol \\ la \\ 0.0080 aerosol \\ \&\& al1 other species \\ $\& \&$ detail option for 11 vanesa
$\& \&$ constituents \\ ihost comp \# \\ aerosol cci \\ aerosol cci \\ aerosol cci \\ aerosol tel \\ aerosol tel \\ aerosol tel \\ aerosol tel \\ aerosol cci \\ aerosol cci \\ aerosol cci \\ aerosol cci \\ aerosol $\mathrm{cci}$ \\ aerosol cci \\ aerosol cci \\ aerosol cci \\ aerosol cci \\ aerosol cci \\ aerosol $\mathrm{CCi}$ \\ aerosol $\mathrm{cci}$ \\ aerosol cci \\ $\mathrm{cci}$ \\ $\mathrm{cci}$ \\ $\operatorname{cci}$ \\ $\& \&$ \\ \&\& melt composition specification \\ ces $=4.6$ iod $=0.46$ xen=9.4 $\mathrm{kry}=0.49$ te $=18.0$ \\ $\mathrm{ba}=60.6 \mathrm{sn}=249$. $t c=37.1 \quad$ uo2 $=79650$. $\mathrm{zr}=7561.3$ \\ fe $=23200$. mo=155. $s r=47.6$ cr $=6370$. ni=3540.
}




\section{Table 15-2}

\section{Surry Input File (Continued)}

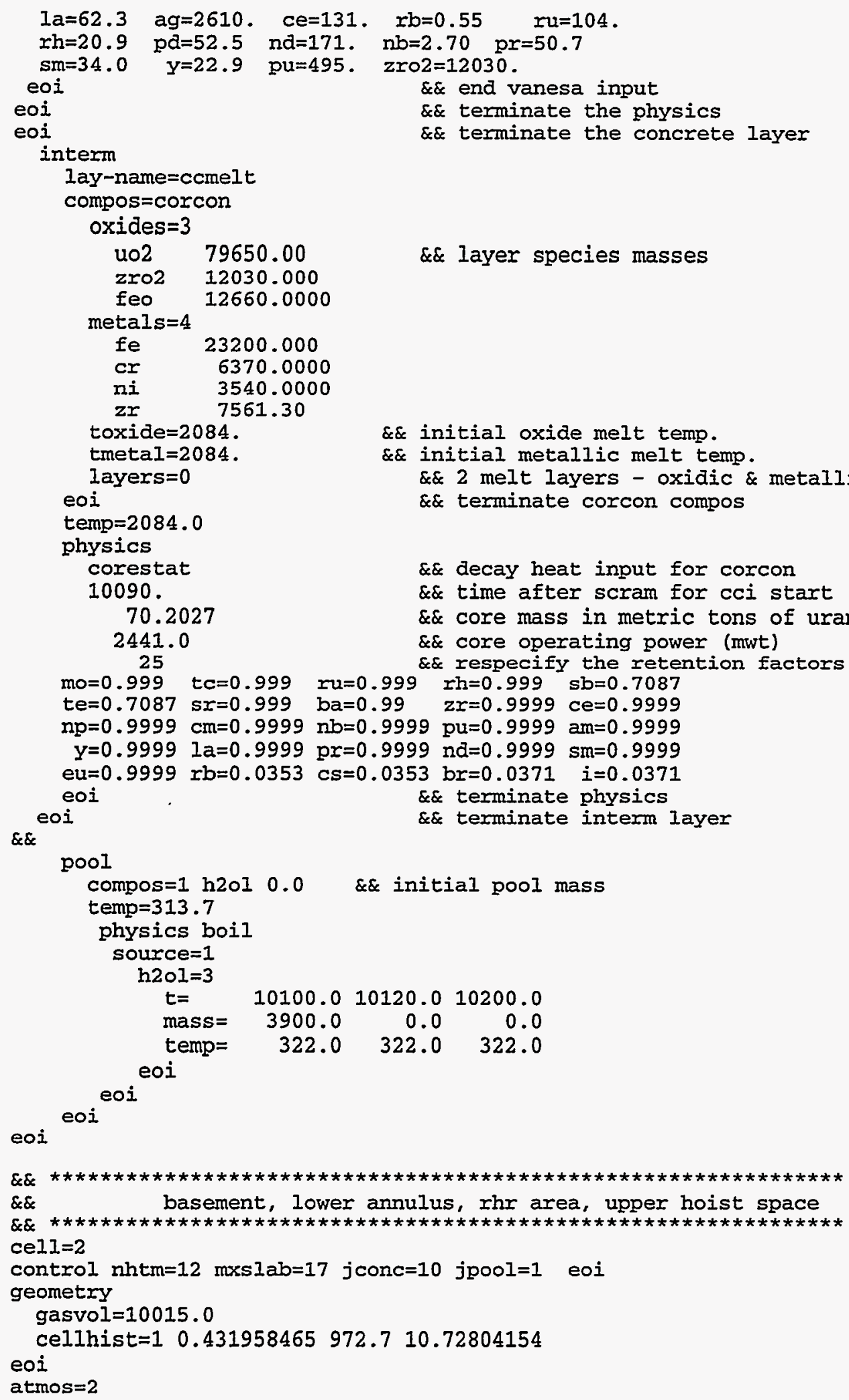


Table 15-2

Surry Input File (Continued)

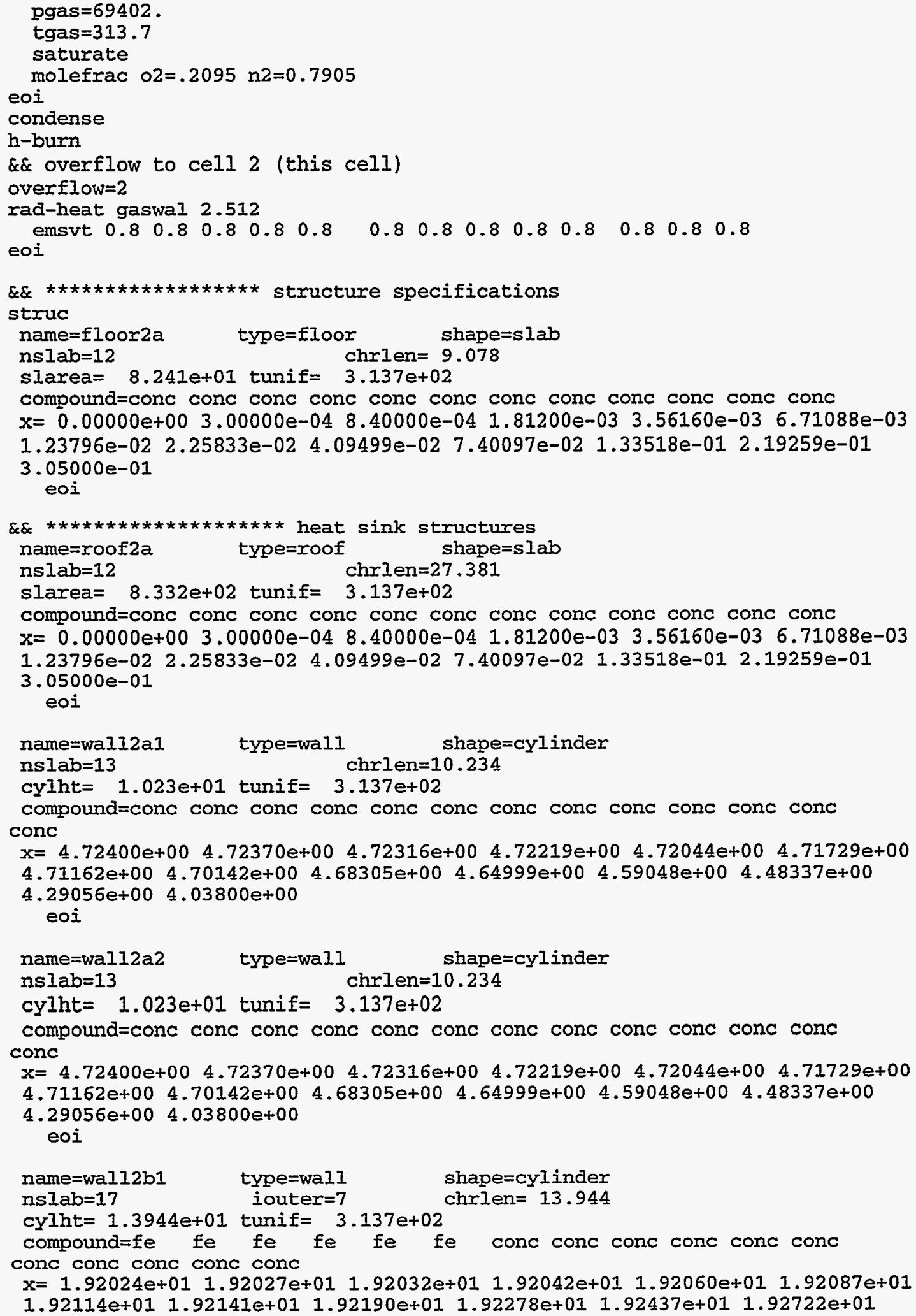


Table 15-2

Surry Input File (Continued)

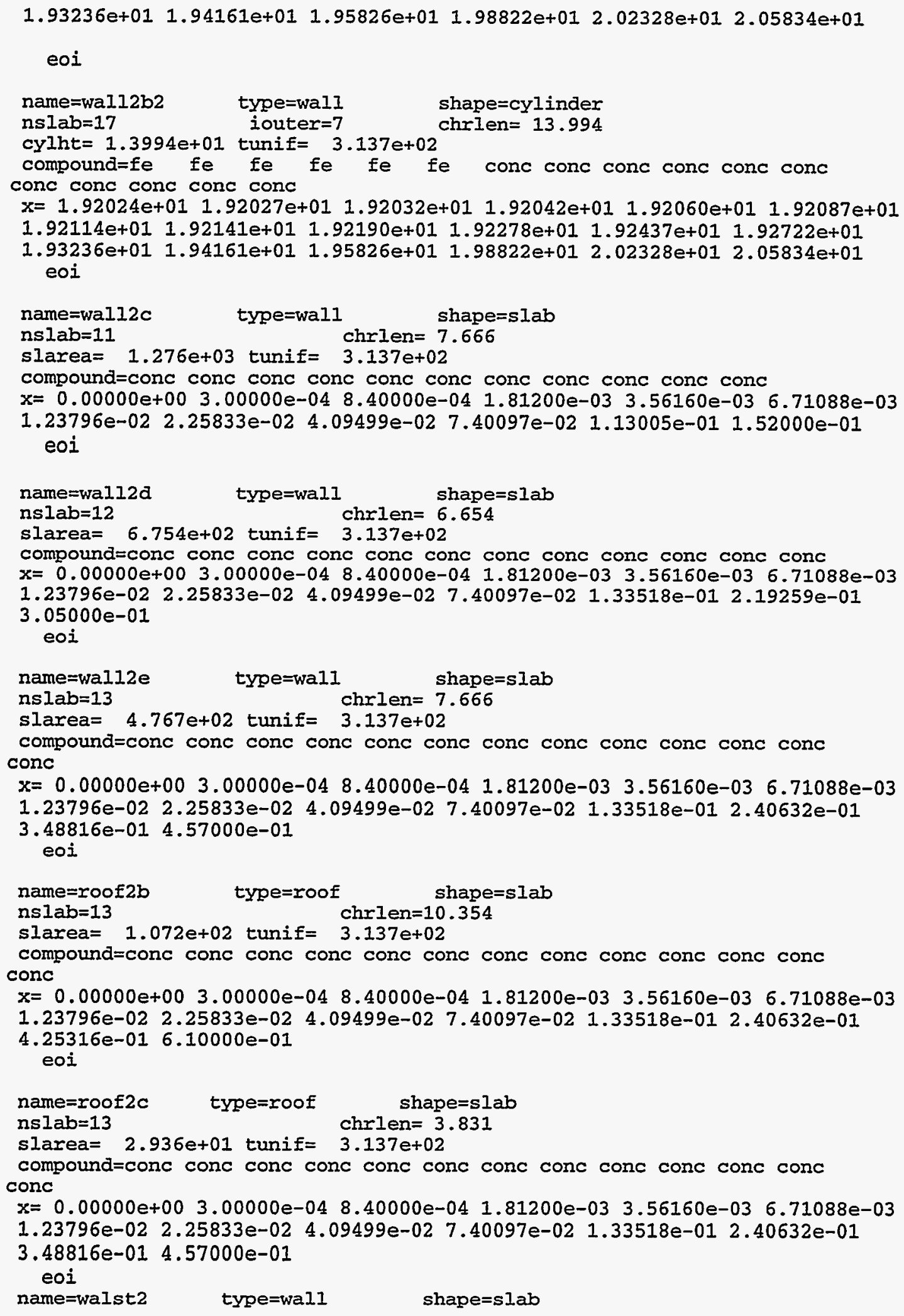


Table 15-2

Surry Input File (Continued)

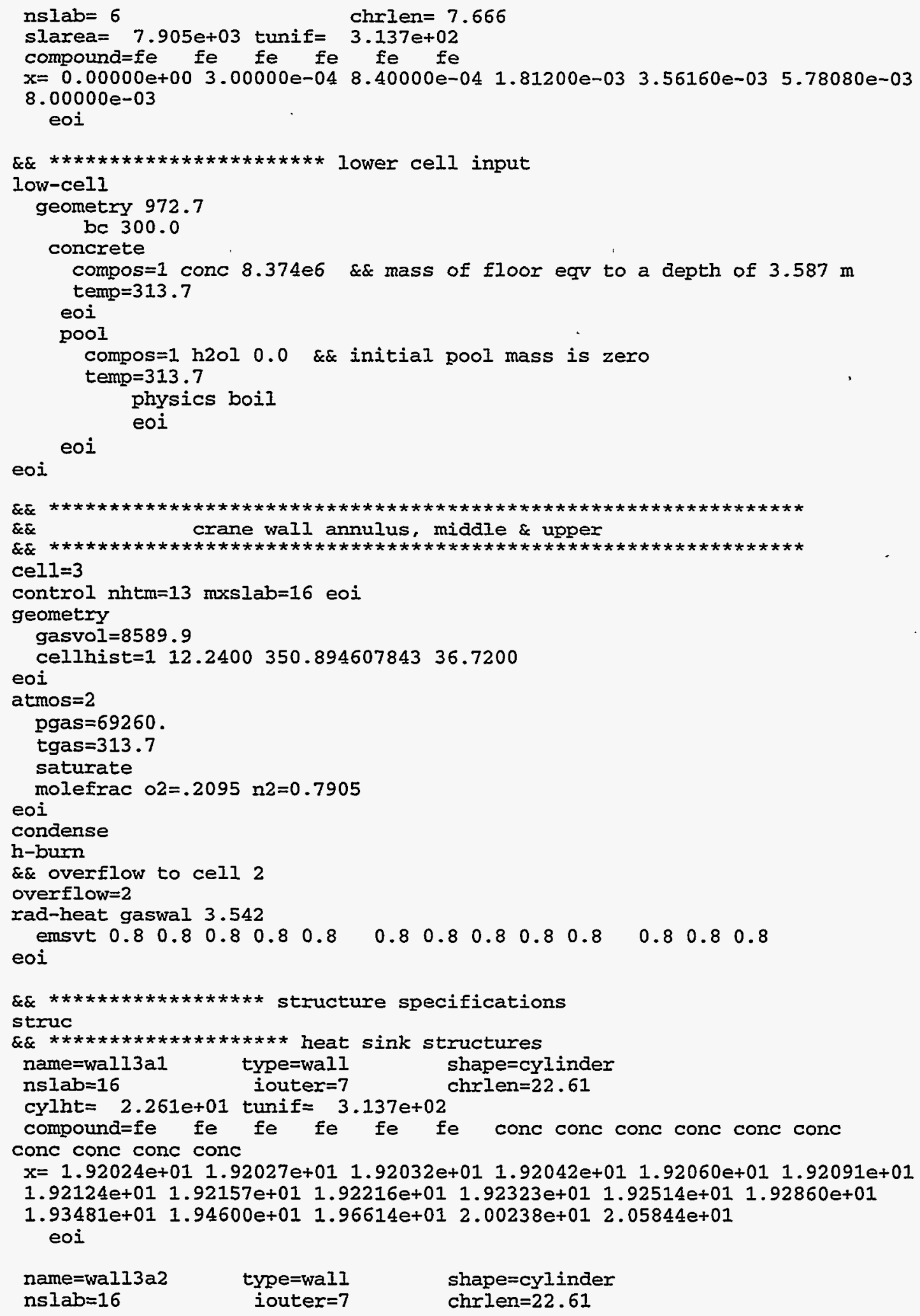


Table 15-2

Surry Input File (Continued)

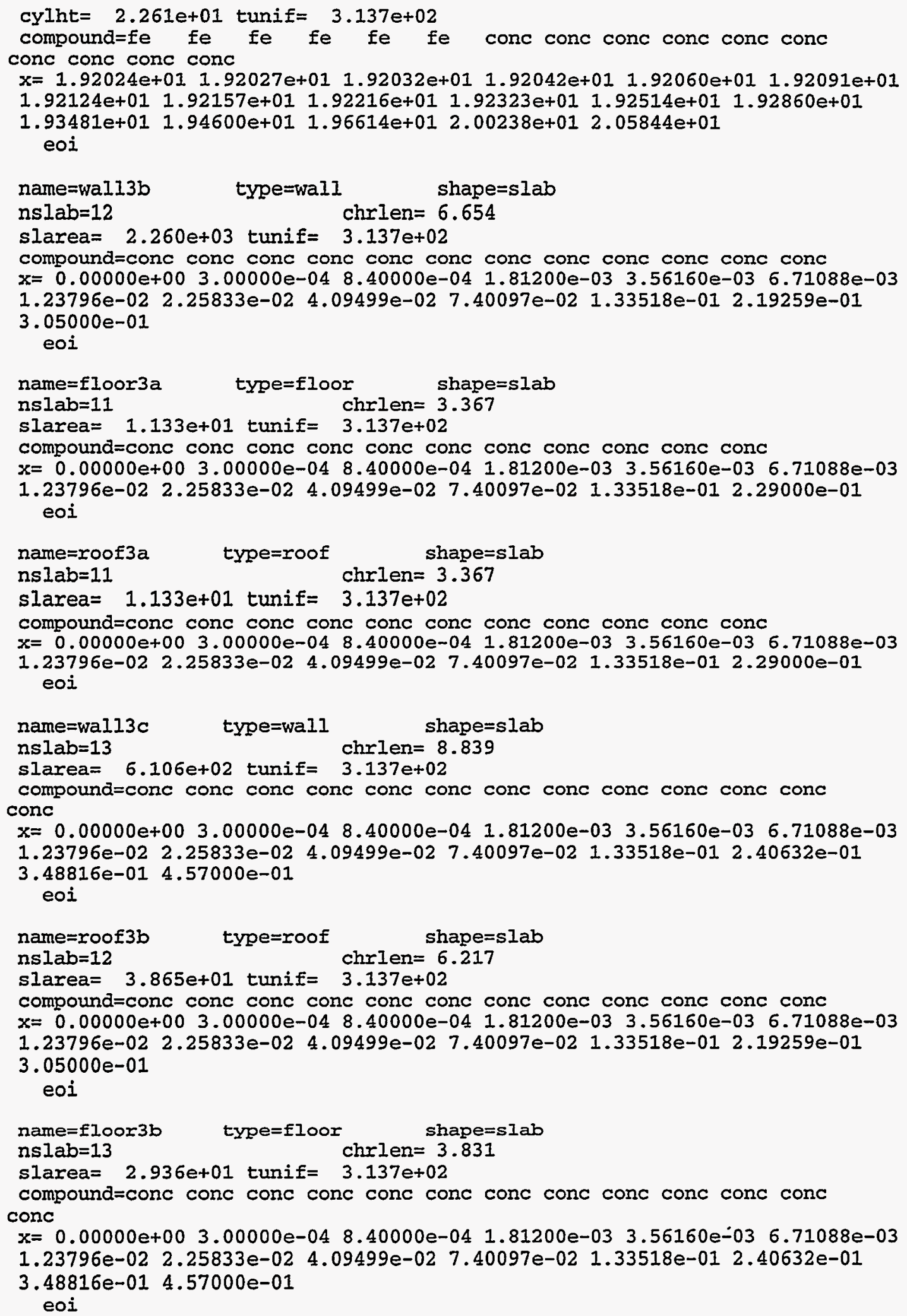


Table 15-2

Surry Input File (Continued)

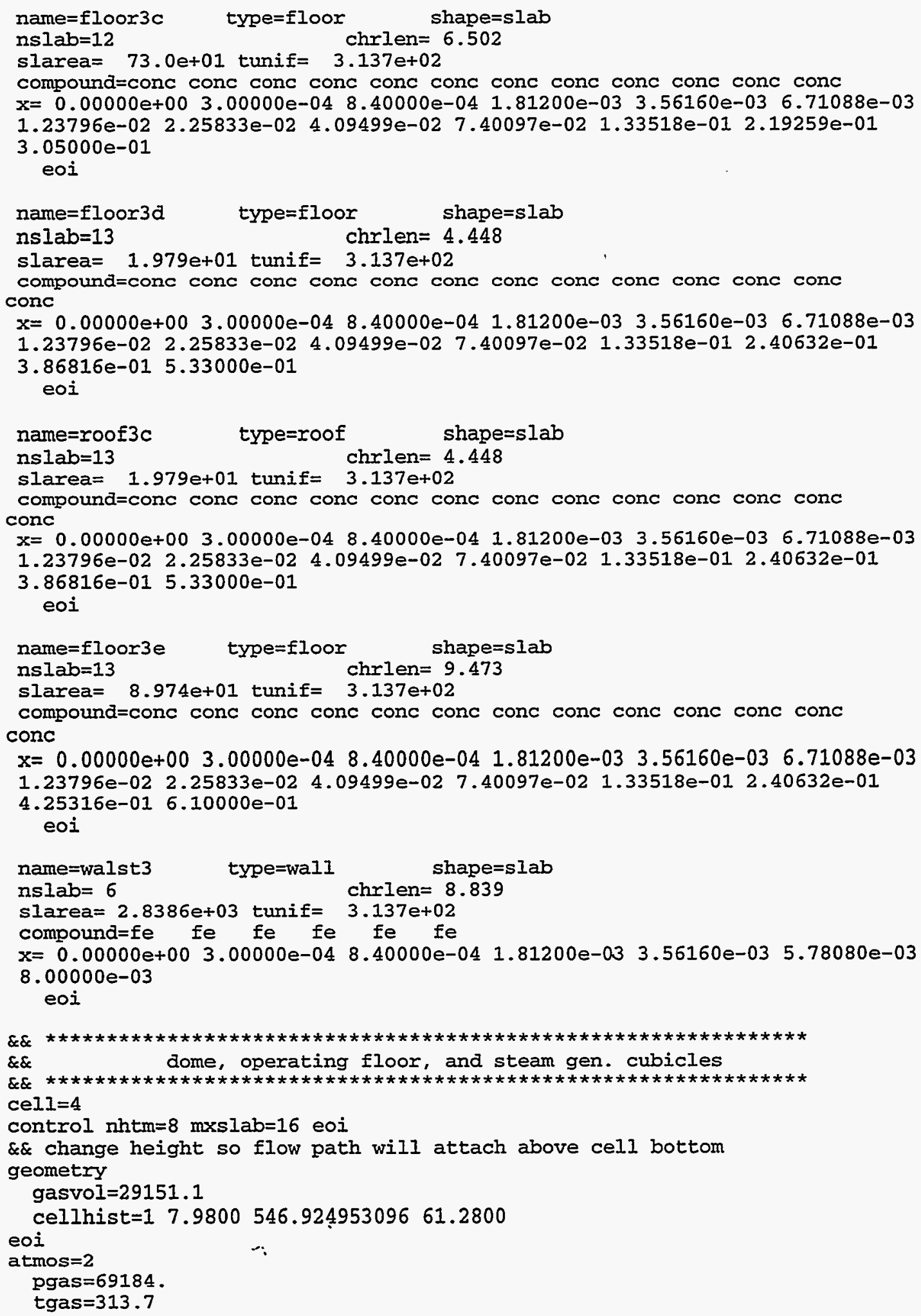


Table 15-2

Surry Input File (Continued)

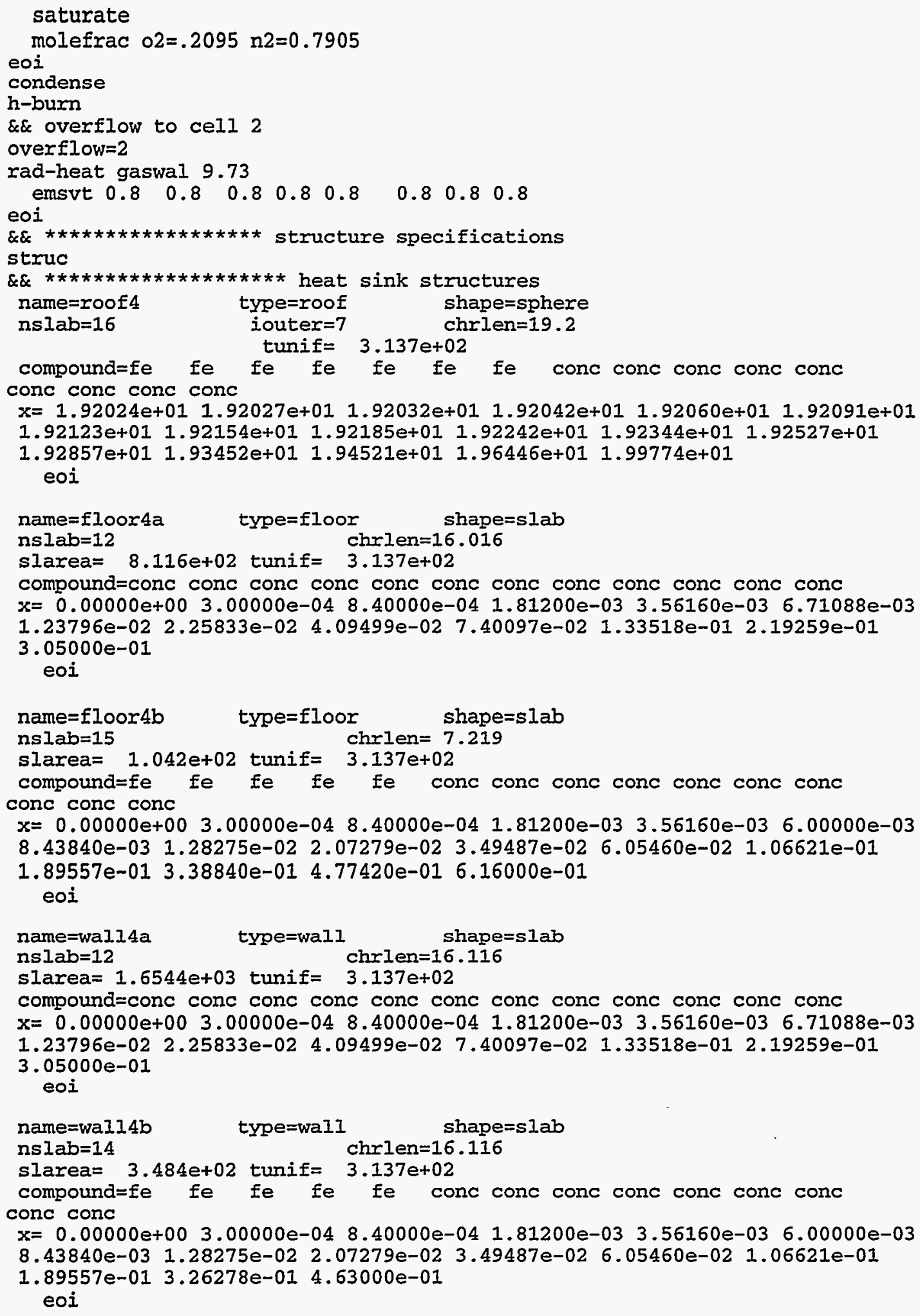


Table 15-2

Surry Input File (Continued)

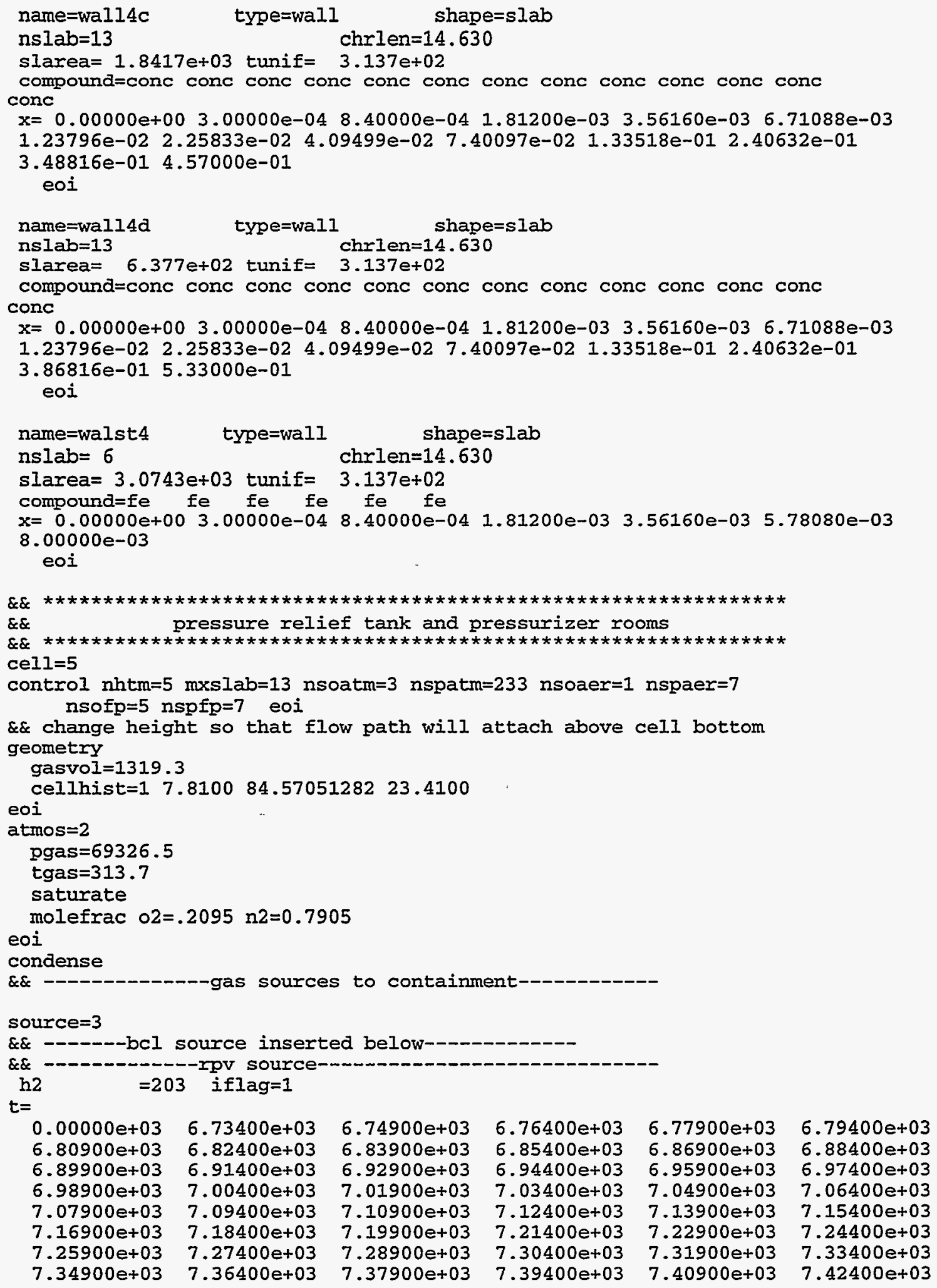


Table 15-2

Surry Input File (Continued)

$7.43900 e+03$

$7.52900 e+03$

$7.61900 e+03$

$7.70900 \mathrm{e}+03$

$7.79900 e+03$

$7.88900 e+03$

$7.97900 e+03$

$8.06900 e+03$

$8.15900 e+03$

$8.24900 e+03$

$8.33900 e+03$

$8.42900 e+03$

$8.51900 e+03$

$8.60900 e+03$

$8.69900 e+03$

$8.78900 e+03$

$8.87900 e+03$

$8.96900 e+03$

$9.05900 \mathrm{e}+03$

$9.14900 \mathrm{e}+03$

$9.23900 e+03$

$9.32900 \mathrm{e}+03$

$9.41900 \mathrm{e}+03$

$9.50900 \mathrm{e}+03$

$9.63631 e+03$

$9.86184 \mathrm{e}+03$ mass $=$

$0.00000 e+00$

$6.94167 e-09$

$2.71090 e-08$

$6.70278 \mathrm{e}-08$

1.77051e-07

$5.81907 \mathrm{e}-07$

$1.99487 \mathrm{e}-06$

$6.84829 e-06$

2.14542 e-05

$5.40412 \mathrm{e}-05$

$1.17482 \mathrm{e}-04$

$2.03393 e-04$

$3.68869 e-04$

$6.56799 e-04$

$1.08152 \mathrm{e}-03$

$2.30692 \mathrm{e}-03$

$3.91777 \mathrm{e}-03$

$5.44028 \mathrm{e}-03$

$6.94832 \mathrm{e}-03$

$8.48433 e-03$

$8.54997 e-03$

$8.12572 \mathrm{e}-03$

$8.67487 e-03$

$9.13365 e-03$

$9.60797 e-03$

$1.01278 \mathrm{e}-02$

$1.10142 \mathrm{e}-02$

2. 81487e-02

$4.99483 e-02$

$7.77180 e-02$

$1.69858 \mathrm{e}+00$

3.95293e-01

$2.39335 e-01$

$1.48462 \mathrm{e}-02$
$7.45400 e+03$

$7.54400 e+03$

$7.63400 \mathrm{e}+03$

$7.72400 e+03$

$7.81400 \mathrm{e}+03$

$7.90400 e+03$

$7.99400 \mathrm{e}+03$

$8.08400 e+03$

$8.17400 e+03$

$8.26400 e+03$

$8.35400 e+03$

$8.44400 \mathrm{e}+03$

$8.53400 e+03$

$8.62400 e+03$

$8.71400 e+03$

$8.80400 e+03$

$8.89400 e+03$

$8.98400 e+03$

$9.07400 e+03$

$9.16400 e+03$

$9.25400 e+03$

$9.34400 e+03$

$9.43400 e+03$

$9.52550 e+03$

$9.66554 \mathrm{e}+03$

$9.91363 e+03$

$0.00000 e+00$

$9.34025 e-09$

$3.16673 \mathrm{e}-08$

$7.92679 \mathrm{e}-08$

$2.14138 \mathrm{e}-07$

$7.14368 \mathrm{e}-07$

$2.44647 e-06$

$8.31157 \mathrm{e}-06$

$2.55974 e-05$

$6.23241 e-05$

$1.30814 \mathrm{e}-04$

$2.25675 e-04$

$4.07083 e-04$

$7.18429 e-04$

$1.18533 e-03$

$2.57861 \mathrm{e}-03$

$4.18557 \mathrm{e}-03$

$5.68173 e-03$

$7.20581 e-03$

8.63621e-03

$8.43477 \mathrm{e}-03$

$8.27745 e-03$

$8.75455 e-03$

$9.21029 e-03$

$9.69056 e-03$

$1.02192 \mathrm{e}-02$

$1.13521 \mathrm{e}-02$

3.30743e-02

$6.72295 e-02$

$8.21113 e-02$

$6.57585 e-01$

3. $62608 \mathrm{e}-01$

$2.25753 \mathrm{e}-01$

1.24285e-02
$7.46900 e+03$

$7.55900 \mathrm{e}+03$

$7.64900 \mathrm{e}+03$

$7.73900 e+03$

$7.82900 e+03$

$7.91900 e+03$

$8.00900 e+03$

$8.09900 e+03$

$8.18900 e+03$

$8.27900 e+03$

$8.36900 e+03$

$8.45900 e+03$

$8.54900 \mathrm{e}+03$

$8.63900 e+03$

$8.72900 e+03$

$8.81900 e+03$

$8.90900 e+03$

$8.99900 e+03$

$9.08900 e+03$

$9.17900 e+03$

$9.26900 e+03$

$9.35900 \mathrm{e}+03$

$9.44900 e+03$

$9.54365 e+03$

$9.69769 e+03$

$9.97059 e+03$

$4.99554 \mathrm{e}-10$

$1.22112 \mathrm{e}-08$

$3.69605 e-08$

$9.22230 \mathrm{e}-08$

$2.60137 e-07$

8.75799e-07

$2.99495 \mathrm{e}-06$

$1.00387 \mathrm{e}-05$

$3.01716 e-05$

$7.15779 \mathrm{e}-05$

$1.44268 \mathrm{e}-04$

2.50071 e- 04

$4.49154 \mathrm{e}-04$

$7.83752 \mathrm{e}-04$

$1.31750 \mathrm{e}-03$

$2.83501 e-03$

$4.44175 e-03$

$5.94638 \mathrm{e}-03$

$7.43687 \mathrm{e}-03$

$8.70633 \mathrm{e}-03$

$8.30132 e-03$

$8.38409 e-03$

$8.83969 e-03$

$9.28847 e-03$

$9.77554 \mathrm{e}-03$

$1.03202 \mathrm{e}-02$

$1.17482 \mathrm{e}-02$

$3.74789 e-02$

8.49543e-02

1. $33978 \mathrm{e}+00$

$5.53473 e-01$

$3.23225 \mathrm{e}-01$

$2.44932 \mathrm{e}-01$

$1.18303 e-02$
$7.48400 e+03$

$7.57400 e+03$

$7.66400 \mathrm{e}+03$

$7.75400 e+03$

$7.84400 e+03$

$7.93400 e+03$

$8.02400 e+03$

$8.11400 e+03$

$8.20400 e+03$

$8.29400 e+03$

$8.38400 e+03$

$8.47400 e+03$

$8.56400 e+03$

$8.65400 e+03$

$8.74400 e+03$

$8.83400 e+03$

$8.92400 \mathrm{e}+03$

$9.01400 e+03$

$9.10400 e+03$

$9.19400 e+03$

$9.28400 \mathrm{e}+03$

$9.37400 \mathrm{e}+03$

$9.46400 \mathrm{e}+03$

$9.56362 \mathrm{e}+03$

$9.73306 \mathrm{e}+03$

$1.00306 \mathrm{e}+04$

$1.50936 \mathrm{e}-09$

$1.55491 e-08$

$4.30839 e-08$

$1.07551 \mathrm{e}-07$

3.17541e-07

$1.07338 \mathrm{e}-06$

$3.67579 \mathrm{e}-06$

$1.21451 \mathrm{e}-05$

$3.55440 \mathrm{e}-05$

$8.17154 \mathrm{e}-05$

$1.57334 \mathrm{e}-04$

$2.75856 e-04$

$4.95180 \mathrm{e}-04$

$8.52476 \mathrm{e}-04$

$1.46515 \mathrm{e}-03$

3. $06524 \mathrm{e}-03$

$4.69484 \mathrm{e}-03$

$6.18722 e-03$

$7.62837 \mathrm{e}-03$

$8.72125 e-03$

$8.12743 \mathrm{e}-03$

$8.46678 \mathrm{e}-03$

$8.90803 e-03$

$9.37006 e-03$

$9.85961 \mathrm{e}-03$

$1.04356 \mathrm{e}-02$

1.22051e-02

4.17424e-02

$8.92390 e-02$

$1.03712 \mathrm{e}+00$

$5.14882 \mathrm{e}-01$

$2.94487 \mathrm{e}-01$

$5.35045 e-02$

$0.00000 e+00$

$7.49900 \mathrm{e}+03$

$7.58900 \mathrm{e}+03$

$7.67900 e+03$

$7.76900 \mathrm{e}+03$

7.85900 e+03

$7.94900 \mathrm{e}+03$

$8.03900 e+03$

$8.12900 e+03$

$8.21900 e+03$

$8.30900 \mathrm{e}+03$

$8.39900 e+03$

$8.48900 e+03$

$8.57900 \mathrm{e}+03$

$8.66900 \mathrm{e}+03$

$8.75900 e+03$

$8.84900 e+03$

$8.93900 e+03$

$9.02900 e+03$

$9.11900 \mathrm{e}+03$

$9.20900 \mathrm{e}+03$

$9.29900 \mathrm{e}+03$

$9.38900 \mathrm{e}+03$

$9.47900 e+03$

$9.58558 \mathrm{e}+03$

$9.77197 e+03$

$1.00900 \mathrm{e}+04$

$3.02442 e-09$

$1.88625 \mathrm{e}-08$

$4.97671 \mathrm{e}-08$

$1.24559 \mathrm{e}-07$

$3.88263 \mathrm{e}-07$

$1.31712 \mathrm{e}-06$

4.52861 e-06

$1.47233 e-05$

$4.08157 e-05$

$9.27499 e-05$

$1.69911 \mathrm{e}-04$

$3.03496 e-04$

$5.45127 e-04$

$9.24445 e-04$

$1.67807 \mathrm{e}-03$

$3.29485 e-03$

$4.93694 \mathrm{e}-03$

$6.45084 e-03$

$7.80603 \mathrm{e}-03$

$8.70076 e-03$

$7.99881 \mathrm{e}-03$ 
Table 15-2

Surry Input File (Continued)

\&\& $* \star 3.65761 \mathrm{~kg} / \mathrm{s}$ of $\mathrm{h} 2$ at $10030.6 \mathrm{~s}$ was taken away and put in cell 4 enth $=$

$0.00000 e+00$

$5.06914 e+06$

$5.07134 e+06$

$5.07373 e+06$

$5.08726 e+06$

$5.10494 \mathrm{e}+06$

$5.12550 e+06$

$5.14552 e+06$

$5.16393 e+06$

$5.18155 e+06$

$5.19776 \mathrm{e}+06$

$5.21149 e+06$

$5.22329 e+06$

$5.23448 \mathrm{e}+06$

$5.24367 e+06$

$5.24955 e+06$

$5.25296 e+06$

$5.25570 \mathrm{e}+06$

$5.25828 e+06$

$5.26024 e+06$

$5.26126 \mathrm{e}+06$

$5.26166 e+06$

$5.26198 \mathrm{e}+06$

$5.26235 e+06$

$5.26277 e+06$

$5.26325 e+06$

$5.26393 e+06$

$5.26831 e+06$

$5.29145 e+06$

$5.34287 e+06$

$7.23934 e+06$

$7.40213 e+06$

$7.34255 e+06$

$7.28732 e+06$ eoi
$0.00000 e \div 00$

$5.06956 \mathrm{e}+06$

$5.07160 \mathrm{e}+06$

$5.07590 e+06$

$5.08976 e+06$

$5.10832 e+06$

$5.12894 \mathrm{e}+06$

$5.14870 e+06$

$5.16689 e+06$

$5.18434 \mathrm{e}+06$

$5.20028 e+06$

$5.21352 \mathrm{e}+06$

$5.22520 e+06$

$5.23623 e+06$

$5.24484 e+06$

$5.25019 e+06$

$5.25343 e+06$

$5.25615 e+06$

$5.25866 e+06$

$5.26046 e+06$

$5.26136 e+06$

$5.26172 e+06$

$5.26204 \mathrm{e}+06$

$5.26243 e+06$

$5.26284 e+06$

$5.26334 e+06$

$5.26411 e+06$

$5.27098 e+06$

$5.29897 e+06$

$5.35166 e+06$

$7.29378 e+06$

$7.40681 e+06$

$7.30781 e+06$

$7.28728 e+06$
$5.06742 e+06$

$5.06994 e+06$

$5.07187 e+06$

$5.07829 e+06$

$5.09251 e+06$

$5.11172 e+06$

$5.13231 e+06$

$5.15181 e+06$

$5.16980 e+06$

$5.18710 e+06$

$5.20277 e+06$

$5.21556 e+06$

$5.22709 e+06$

$5.23792 e+06$

$5.24593 e+06$

$5.25081 e+06$

$5.25388 \mathrm{e}+06$

$5.25659 e+06$

$5.25903 e+06$

$5.26064 e+06$

$5.26145 e+06$

$5.26176 e+06$

5.26211 e+0 6

$5.26249 e+06$

$5.26292 e+06$

$5.26343 e+06$

$5.26428 \mathrm{e}+06$

$5.27425 e+06$

$5.30903 e+06$

$5.66615 e+06$

$7.32917 e+06$

$7.40657 \mathrm{e}+06$

$7.28729 \mathrm{e}+06$

$7.28731 e+06$
$5.06781 e+06$

$5.07033 e+06$

$5.07215 e+06$

$5.08086 e+06$

$5.09541 e+06$

$5.11518 e+06$

$5.13563 e+06$

$5.15488 \mathrm{e}+06$

$5.17288 e+06$

$5.18983 e+06$

$5.20507 e+06$

$5.21751 e+06$

$5.22897 e+06$

$5.23951 e+06$

$5.24700 e+06$

$5.25137 e+06$

$5.25434 \mathrm{e}+06$

$5.25703 e+06$

$5.25938 e+06$

$5.26083 e+06$

$5.26153 e+06$

$5.26182 e+06$

$5.26216 e+06$

$5.26256 e+06$

$5.26300 e+06$

$5.26354 e+06$

$5.26453 e+06$

$5.27806 \mathrm{e}+06$

$5.31918 e+06$

$5.89384 e+06$

$7.35683 e+06$

$7.40053 e+06$

$7.28729 e+06$

$7.28730 \mathrm{e}+06$
$5.06828 e+06$

$5.07067 e+06$

$5.07239 e+06$

$5.08292 \mathrm{e}+06$

$5.09848 e+06$

$5.11860 e+06$

$5.13895 e+06$

$5.15792 e+06$

$5.17585 e+06$

$5.19251 e+06$

$5.20730 \mathrm{e}+06$

$5.21944 \mathrm{e}+06$

$5.23084 \mathrm{e}+06$

$5.24103 e+06$

$5.24800 e+06$

$5.25195 e+06$

$5.25481 e+06$

$5.25746 e+06$

$5.25972 e+06$

$5.26099 e+06$

$5.26157 e+06$

$5.26186 e+06$

$5.26223 e+06$

$5.26263 e+06$

$5.26308 e+06$

$5.26364 e+06$

$5.26496 e+06$

$5.28221 e+06$

$5.32696 e+06$

$6.27285 e+06$

$7.37780 \mathrm{e}+06$

$7.38813 e+06$

$7.28730 e+06$

$0.00000 \mathrm{e}+00$
$5.06872 e+06$

$5.07103 e+06$

$5.07289 e+06$

$5.08501 e+06$

$5.10165 e+06$

$5.12208 e+06$

$5.14225 e+06$

$5.16093 e+06$

$5.17873 e+06$

$5.19518 \mathrm{e}+06$

$5.20940 e+06$

$5.22136 \mathrm{e}+06$

$5.23268 e+06$

$5.24241 e+06$

$5.24886 e+06$

$5.25245 e+06$

$5.25525 \mathrm{e}+06$

$5.25788 \mathrm{e}+06$

5.26000 e+06

$5.26114 \mathrm{e}+06$

$5.26162 e+06$

$5.26192 \mathrm{e}+06$

$5.26229 e+06$

$5.26269 e+06$

$5.26316 e+06$

$5.26379 e+06$

5.26621 e+06

5.28663 e+06

$5.33452 e+06$

$6.72021 e+06$

$7.39271 e+06$

$7.36918 e+06$

$7.28733 e+06$ h2ov $=233$ iflag $=1$ $t=$

$5.91273 e+03$

$6.03169 e+03$

$6.20900 e+03$

$6.38900 e \div 03$

$6.56900 e+03$

$6.71900 e+03$

$6.80900 e+03$

$6.89900 \mathrm{e}+03$

$6.98900 e+03$

$7.07900 e+03$

$7.16900 \mathrm{e}+03$

$7.25900 e+03$

$7.34900 e \div 03$

$7.43900 \mathrm{e}+03$

$7.52900 e+03$

$7.61900 e+03$

$7.70900 e+03$

$7.79900 \mathrm{e}+03$

$7.88900 e+03$

$7.97900 \mathrm{e}+03$

$8.06900 e+03$
$5.92815 e+03$

$6.05900 e+03$

$6.23900 e+03$

$6.41900 e+03$

$6.59900 e+03$

$6.73400 \mathrm{e}+03$

$6.82400 e+03$

$6.91400 e+03$

$7.00400 e+03$

$7.09400 e+03$

$7.18400 e+03$

$7.27400 e+03$

$7.36400 \mathrm{e}+03$

$7.45400 \mathrm{e}+03$

$7.54400 \mathrm{e}+03$

$7.63400 \mathrm{e}+03$

$7.72400 \mathrm{e}+03$

$7.81400 \mathrm{e}+03$

$7.90400 \mathrm{e}+03$

$7.99400 e+03$

$8.08400 e+03$
$5.94511 e+03$ $6.08900 e+03$ $6.26900 e+03$ $6.44900 e+03$ $6.62900 e+03$ $6.74900 \mathrm{e}+03$ $6.83900 e+03$ $6.92900 \mathrm{e}+03$ $7.01900 e+03$ $7.10900 \mathrm{e}+03$ $7.19900 e+03$ $7.28900 \mathrm{e}+03$ $7.37900 e+03$

$7.46900 \mathrm{e}+03$ $7.55900 e+03$ $7.64900 e+03$ $7.73900 e+03$ 7.82900 e+03 $7.91900 e+03$ 8.00900 e +03 $8.09900 e+03$

$5.96376 e+03$ $6.11900 \mathrm{e}+03$ $6.29900 e+03$ $6.47900 e+03$ $6.65900 e+03$ $6.76400 e+03$ $6.85400 e+03$ 
Table 15-2

Surry Input File (Continued)

$8.15900 e+03$

$8.24900 \mathrm{e}+03$

$8.33900 \mathrm{e}+03$

$8.42900 \mathrm{e}+03$

$8.51900 e+03$

$8.60900 e+03$

$8.69900 e+03$

$8.78900 e+03$

$8.87900 e+03$

$8.96900 e+03$

$9.05900 \mathrm{e}+03$

$9.14900 \mathrm{e}+03$

$9.23900 e+03$

$9.32900 e+03$

$9.41900 e+03$

$9.50900 e+03$

$9.63631 e+03$

$9.86184 \mathrm{e}+03$ mass $=$

2.33304e+01

$2.39534 e+01$

$2.60211 \mathrm{e}+01$

$2.58706 e+01$

$2.57534 e+01$

$2.52656 \mathrm{e}+01$

$2.42939 e+01$

$1.93719 e+01$

$1.43671 e+01$

$1.08613 e+01$

$8.42512 \mathrm{e}+00$

$6.64109 e+00$

$5.33163 e+00$

$4.35784 e+00$

$3.57889 e+00$

$3.16321 \mathrm{e}+00$

$2.59161 \mathrm{e}+00$

2. $45099 e+00$

$2.42190 \mathrm{e}+00$

$2.27903 e+00$

$2.74363 e+00$

$2.82433 e+00$

$2.75239 e+00$

$2.69558 e+00$

2. $64659 e+00$

$2.23111 e+00$

$1.85643 e+00$

$1.77217 \mathrm{e}+00$

1. 69191e+00

1. $63098 \mathrm{e}+00$

1. $58841 \mathrm{e}+00$

$1.60566 e+00$

$3.76156 e+00$

$5.76756 \mathrm{e}+00$

$7.47133 e+00$

$1.03452 \mathrm{e}+02$

2. $94257 e+01$

$2.25250 e+01$

$1.51690 e+00$ enth $=$

$2.53000 e+06$

$2.53112 e+06$
$8.17400 \mathrm{e}+03$

$8.26400 \mathrm{e}+03$

$8.35400 \mathrm{e}+03$

$8.44400 \mathrm{e}+03$

$8.53400 \mathrm{e}+03$

$8.62400 \mathrm{e}+03$

$8.71400 \mathrm{e}+03$

$8.80400 e+03$

$8.89400 e+03$

$8.98400 e+03$

$9.07400 e+03$

$9.16400 \mathrm{e}+03$

$9.25400 e+03$

$9.34400 e+03$

$9.43400 e+03$

$9.52550 e+03$

$9.66554 \mathrm{e}+03$

$9.91363 e+03$

$2.33485 e+01$

2.39167e+01

2. $59865 \mathrm{e}+01$

2. $58496 \mathrm{e}+01$

$2.57352 \mathrm{e}+01$

2. 53900 e+01

$2.36136 \mathrm{e}+01$

$1.84571 \mathrm{e}+01$

1. $39237 \mathrm{e}+01$

$1.04011 e+01$

$8.09459 e+00$

$6.37771 e+00$

$5.11495 e+00$

4. $24033 \mathrm{e}+00$

$3.50371 \mathrm{e}+00$

$3.08349 e+00$

$2.56520 e+00$

$2.44356 e+00$

2. $41063 e+00$

$2.27626 e+00$

$2.78064 \mathrm{e}+00$

2. 81887 e+00

$2.73769 e+00$

$2.68845 e+00$

$2.60520 \mathrm{e}+00$

2. $14714 \mathrm{e}+00$

1. $85403 e+00$

$1.75800 \mathrm{e}+00$

$1.68035 \mathrm{e}+00$

$1.62261 \mathrm{e}+00$

$1.58281 e+00$

$1.63524 \mathrm{e}+00$

$4.32261 e+00$

$7.52371 \mathrm{e}+00$

$7.68333 \mathrm{e}+00$

4.11297 e+01

$2.79357 e+01$

$2.23013 e+01$

1. $26987 e+00$

$2.53008 e+06$

2. $53142 e+06$
$8.18900 \mathrm{e}+03$

$8.27900 e+03$

$8.36900 e+03$

$8.45900 \mathrm{e}+03$

$8.54900 e+03$

$8.63900 e+03$

$8.72900 \mathrm{e}+03$

$8.81900 e+03$

$8.90900 \mathrm{e}+03$

$8.99900 e+03$

$9.08900 e+03$

$9.17900 \mathrm{e}+03$

$9.26900 e+03$

$9.35900 e+03$

$9.44900 e+03$

$9.54365 e+03$

$9.69769 e+03$

$9.97059 e+03$

$2.35745 e+01$

$2.62023 e+01$

$2.59589 e+01$

$2.58293 e+01$

$2.57172 e+01$

$2.54639 e+01$

$2.28530 e+01$

$1.75547 e+01$

$1.32767 e+01$

$9.96365 e+00$

$7.76698 \mathrm{e}+00$

$6.11695 e+00$

$4.90136 e+00$

$4.10435 \mathrm{e}+00$

$3.43627 e+00$

$2.98834 \mathrm{e}+00$

$2.54203 e+00$

$2.43955 e+00$

$2.39455 e+00$

$2.30777 e+00$

$2.78958 e+00$

$2.80608 \mathrm{e}+00$

$2.73404 e+00$

$2.67188 e+00$

$2.54363 e+00$

$2.06393 e+00$

1. $84200 \mathrm{e}+00$

$1.74548 \mathrm{e}+00$

$1.66950 \mathrm{e}+00$

$1.61492 \mathrm{e}+00$

$1.57882 \mathrm{e}+00$

$1.67210 e+00$

$4.78378 \mathrm{e}+00$

$9.17335 e+00$

$1.07343 e+02$

$3.59038 e+01$

$2.57997 e+01$

$2.50257 e+01$

$1.20876 \mathrm{e}+00$

$2.53018 e+06$

$2.53172 e+06$
$8.20400 e+03$

$8.29400 e+03$

$8.38400 e+03$

$8.47400 \mathrm{e}+03$

$8.56400 e+03$

$8.65400 e+03$

$8.74400 e+03$

8.83400 e +03

$8.92400 e+03$

$9.01400 \mathrm{e}+03$

$9.10400 \mathrm{e}+03$

$9.19400 \mathrm{e}+03$

$9.28400 e+03$

$9.37400 e+03$

$9.46400 \mathrm{e}+03$

$9.56362 e+03$

$9.73306 e+03$

$1.00306 e+04$

$2.37904 e+01$

2. $61545 e+01$

$2.59355 e+01$

$2.58097 e+01$

$2.56994 \mathrm{e}+01$

$2.54582 e+01$

$2.20368 e+01$

$1.66761 \mathrm{e}+01$

$1.26686 \mathrm{e}+01$

$9.55599 e+00$

$7.45003 e+00$

$5.88618 e+00$

$4.72428 e+00$

$3.99829 e+00$

$3.36906 e+00$

$2.87284 \mathrm{e}+00$

$2.51380 e+00$

$2.43699 e+00$

$2.37268 \mathrm{e}+00$

2.34698e+00

$2.77204 \mathrm{e}+00$

$2.79252 e+00$

$2.72009 e+00$

2. $64296 e+00$

$2.47157 e+00$

$1.97605 e+00$

$1.82542 \mathrm{e}+00$

$1.73026 \mathrm{e}+00$

$1.65964 e+00$

$1.60732 \mathrm{e}+00$

$1.57710 \mathrm{e}+00$

$1.71628 \mathrm{e}+00$

$5.19748 \mathrm{e}+00$

$9.31113 e+00$

$7.54030 \mathrm{e}+01$

$3.45988 e+01$

$2.44033 e+01$

$5.46679 e+00$

$0.00000 \mathrm{e}+00$

$2.53032 e+06$

$2.53199 e+06$

$8.219000+03$

$8.30900 \mathrm{e}+03$

$8.39900 \mathrm{e}+03$

$8.48900 \mathrm{e}+03$

$8.57900 \mathrm{e}+03$

$8.66900 e+03$

$8.75900 e+03$

$8.84900 e+03$

$8.93900 \mathrm{e}+03$

$9.02900 e+03$

$9.11900 e+03$

$9.20900 e+03$

$9.29900 e+03$

$9.38900 \mathrm{e}+03$

$9.47900 \mathrm{e}+03$

$9.58558 e+03$

$9.77197 e+03$

$1.00900 \mathrm{e}+04$

$2.39566 e+01$

. $61059 e+01$

$2.59137 e+01$

$2.57906 e+01$

$5.13636 e+01$

$2.52641 e+01$

$2.11819 e+01$

$1.58255 e+01$

$1.19411 \mathrm{e}+01$

$9.16404 \mathrm{e}+00$

$7.15515 e+00$

$5.69147 e+00$

$4.58518 e+00$

3.82271 e+00

$3.30161 \mathrm{e}+00$

$2.74347 e+00$

$2.48448 \mathrm{e}+00$

$2.43399 e+00$

$2.34483 e+00$

$2.45070 e+00$

$2.75566 e+00$

$2.77443 e+00$

$2.71595 e+00$

$2.61156 e+00$

$2.39538 e+00$

$1.90370 \mathrm{e}+00$

$1.80470 e+00$

$1.71695 \mathrm{e}+00$

$1.64850 e+00$

$1.60085 e+00$

$1.57941 \mathrm{e}+00$

$1.95283 e+00$ 
Table 15-2

Surry Input File (Continued)

\author{
$2.53267 e+06$ \\ $2.53366 \mathrm{e}+06$ \\ $2.53434 e+06$ \\ $2.53469 \mathrm{e}+06$ \\ $2.53489 \mathrm{e}+06$ \\ $2.53519 \mathrm{e}+06$ \\ $2.53547 e+06$ \\ $2.53732 e+06$ \\ $2.53980 e+06$ \\ $2.54270 e+06$ \\ $2.54556 e+06$ \\ $2.54821 e+06$ \\ $2.55076 e+06$ \\ $2.55311 e+06$ \\ $2.55512 e+06$ \\ $2.55685 e+06$ \\ $2.55851 e+06$ \\ $2.55988 \mathrm{e}+06$ \\ $2.56077 e+06$ \\ $2.56132 \mathrm{e}+06$ \\ $2.56177 e+06$ \\ $2.56219 e+06$ \\ 2. 56252 e+06 \\ $2.56272 e+06$ \\ $2.56283 e+06$ \\ $2.56294 \mathrm{e}+06$ \\ $2.56303 e+06$ \\ $2.56316 \mathrm{e}+06$ \\ $2.56328 \mathrm{e}+06$ \\ $2.56342 \mathrm{e}+06$ \\ $2.56396 \mathrm{e}+06$ \\ $2.56732 e+06$ \\ $2.57453 e+06$ \\ $2.84083 e+06$ \\ $2.86602 e+06$ \\ $2.85797 e+06$ \\ $2.88041 e+06$
} eoi

h2ov $=115$ iflag=1

$t=$

$0.00000 e+00 \quad 3.37553 e+03$

$3.43183 e+03$

$3.54640 e+03$

$3.72165 e+03$

$3.90165 e+03$

$4.08165 e+03$

$4.26165 e+03$

$4.44165 e+03$

$4.62165 \mathrm{e}+03$

$4.80165 e+03$

$4.98165 e+03$

$5.16165 e+03$

$5.32620 e+03$

$5.41045 e+03$

$5.49446 e+03$

$5.57822 e+03$

$5.66194 \mathrm{e}+03$

$5.74576 e+03$

$5.82971 \mathrm{e}+03$

$5.91273 e+03$
$2.53287 e+06$ $2.53379 e+06$ $2.53465 e+06$ $2.53496 e+06$ $2.53523 e+06$ $2.53577 e+06$ $2.53767 e+06$ $2.54026 e+06$ $2.54320 \mathrm{e}+06$ $2.54603 e+06$ $2.54865 e+06$ $2.55116 e+06$ $2.55349 e+06$ $2.55542 e+06$ $2.55714 \mathrm{e}+06$ $2.55878 \mathrm{e}+06$ $2.56006 e+06$ $2.56088 e+06$ $2.56139 e+06$ $2.56184 e+06$ $2.56225 e+06$ $2.56256 e+06$ $2.56275 e+06$ $2.56284 e+06$ $2.56295 e+06$ $2.56306 e+06$ $2.56318 \mathrm{e}+06$ $2.56329 e+06$ $2.56345 e+06$ $2.56438 e+06$ $2.56836 e+06$ $2.57567 e+06$ 2.84901 e+ 06 $2.86672 e+06$ $2.85273 e+06$ $2.88263 e+06$ $2.53443 e+06$
$3.44668 \mathrm{e}+03$

$3.57271 e+03$

$3.75165 e+03$

$3.93165 e+03$

$4.11165 e+03$

$4.29165 e+03$

$4.47165 e+03$

$4.65165 e+03$

$4.83165 e+03$

$5.01165 e+03$

$5.19165 e+03$

$5.33953 e+03$

$5.42450 e+03$

$5.50843 e+03$

$5.59217 e+03$

$5.67590 e+03$

$5.75974 e+03$

$5.84364 e+03$
$2.53304 e+06$ $2.53391 \mathrm{e}+06$ $2.53452 e+06$ $2.53463 e+06$ $2.53502 \mathrm{e}+06$ $2.53525 e+06$ $2.53608 e+06$ $2.53805 e+06$ $2.54074 e+06$ $2.54369 e+06$ $2.54648 e+06$ $2.54905 e+06$ $2.55156 \mathrm{e}+06$ $2.55385 e+06$ $2.55572 e+06$ $2.55741 e+06$ $2.55902 e+06$ $2.56022 e+06$ $2.56097 \mathrm{e}+06$ $2.56147 e+06$ $2.56191 \mathrm{e}+06$ $2.56231 e+06$ $2.56259 \mathrm{e}+06$ $2.56277 e+06$ $2.56286 e+06$ $2.56297 e+06$ $2.56308 e+06$ $2.56319 e+06$ $2.56331 \mathrm{e}+06$ $2.56348 e+06$ $2.56488 \mathrm{e}+06$ $2.56957 e+06$ $2.61984 e+06$ $2.85513 e+06$ $2.86781 \mathrm{e}+06$ $2.85022 \mathrm{e}+06$ $2.88502 e+06$
$2.53321 e+06$ $2.53403 \mathrm{e}+06$

$2.53460 e+06$

$2.53465 \mathrm{e}+06$

$2.53507 e+06$

$2.53529 e+06$

$2.53643 e+06$

$2.53845 e+06$

$2.54124 e+06$

$2.54415 e+06$

$2.54691 e+06$

$2.54952 \mathrm{e}+06$

$2.55196 \mathrm{e}+06$

$2.55418 e+06$

$2.55601 e+06$

$2.55770 e \div 06$

$2.55926 e+06$

$2.56038 e+06$

$2.56107 e+06$

$2.56154 e+06$

$2.56198 e+06$

$2.56237 e+06$

$2.56263 e+06$

$2.56278 e+06$

$2.56288 e+06$

$2.56299 e+06$

$2.56309 e+06$

$2.56322 e+06$

$2.56334 \mathrm{e}+06$

$2.56353 e+06$

$2.56542 \mathrm{e}+06$

$2.57104 \mathrm{e}+06$

$2.64950 e+06$

$2.85968 \mathrm{e}+06$

$2.86666 e+06$

$2.87490 \mathrm{e}+06$

$0.00000 e+00$
$2.53337 e+06$

$2.53414 e+06$

$2.53467 e+06$

$2.53472 e+06$

2. $53511 e+06$

2. $53529 e+06$

$2.53673 e+06$

$2.53888 e+06$

$2.54174 \mathrm{e}+06$

$2.54463 e+06$

$2.54736 e+06$

$2.54994 e+06$

$2.55235 e+06$

$2.55452 \mathrm{e}+06$

$2.55629 \mathrm{e}+06$

$2.55796 \mathrm{e}+06$

$2.55949 e+06$

$2.56053 e+06$

$2.56115 e+06$

2.56162e+06

$2.56205 e+06$

$2.56243 e+06$

$2.56267 e+06$

$2.56279 e+06$

$2.56290 \mathrm{e}+06$

$2.56300 \mathrm{e}+06$

$2.56311 e+06$

$2.56323 e+06$

$2.56336 \mathrm{e}+06$

$2.56359 e+06$

$2.56600 e+06$

$2.57230 e+06$

$2.70726 \mathrm{e}+06$

$2.86274 e+06$

$2.86438 e+06$

$2.87662 \mathrm{e}+06$

$0.00000 e+00$
$2.53352 e+06$

$2.53424 e+06$

$2.53471 \mathrm{e}+06$

$2.53482 \mathrm{e}+06$

$2.53516 \mathrm{e}+06$

$2.53536 e+06$

2.53701 e+06

$2.53933 e+06$

$2.54221 e+06$

$2.54509 e+06$

$2.54779 e+06$

$2.55036 e+06$

$2.55273 e+06$

$2.55482 e+06$

$2.55657 e+06$

$2.55824 \mathrm{e}+06$

$2.55970 e+06$

$2.56066 \mathrm{e}+06$

$2.56124 \mathrm{e}+06$

$2.56169 e+06$

$2.56212 \mathrm{e}+06$

$2.56248 \mathrm{e}+06$

$2.56269 e+06$

$2.56281 \mathrm{e}+06$

$2.56292 \mathrm{e}+06$

$2.56302 e+06$

$2.56313 e+06$

$2.56325 e+06$

$2.56340 e+06$

$2.56375 e+06$

$2.56665 e+06$

$2.57353 e+06$

$2.76773 e+06$

$2.86474 e+06$

$2.86161 e+06$

$2.87838 \mathrm{e}+06$

$3.38475 e+03$

$3.46301 e+03$

$3.60165 e+03$

$3.78165 e+03$

$3.96165 e+03$

$4.14165 \mathrm{e}+03$

$4.32165 e+03$

$4.50165 e+03$

$4.68165 e+03$

$4.86165 e+03$

$5.04165 e+03$

$5.22165 e+03$

$5.35327 e+03$

$5.43852 \mathrm{e}+03$

$5.52239 e+03$

$5.60612 e+03$

$5.68986 \mathrm{e}+03$

$5.77373 e+03$ 
Table 15-2

Surry Input File (Continued)

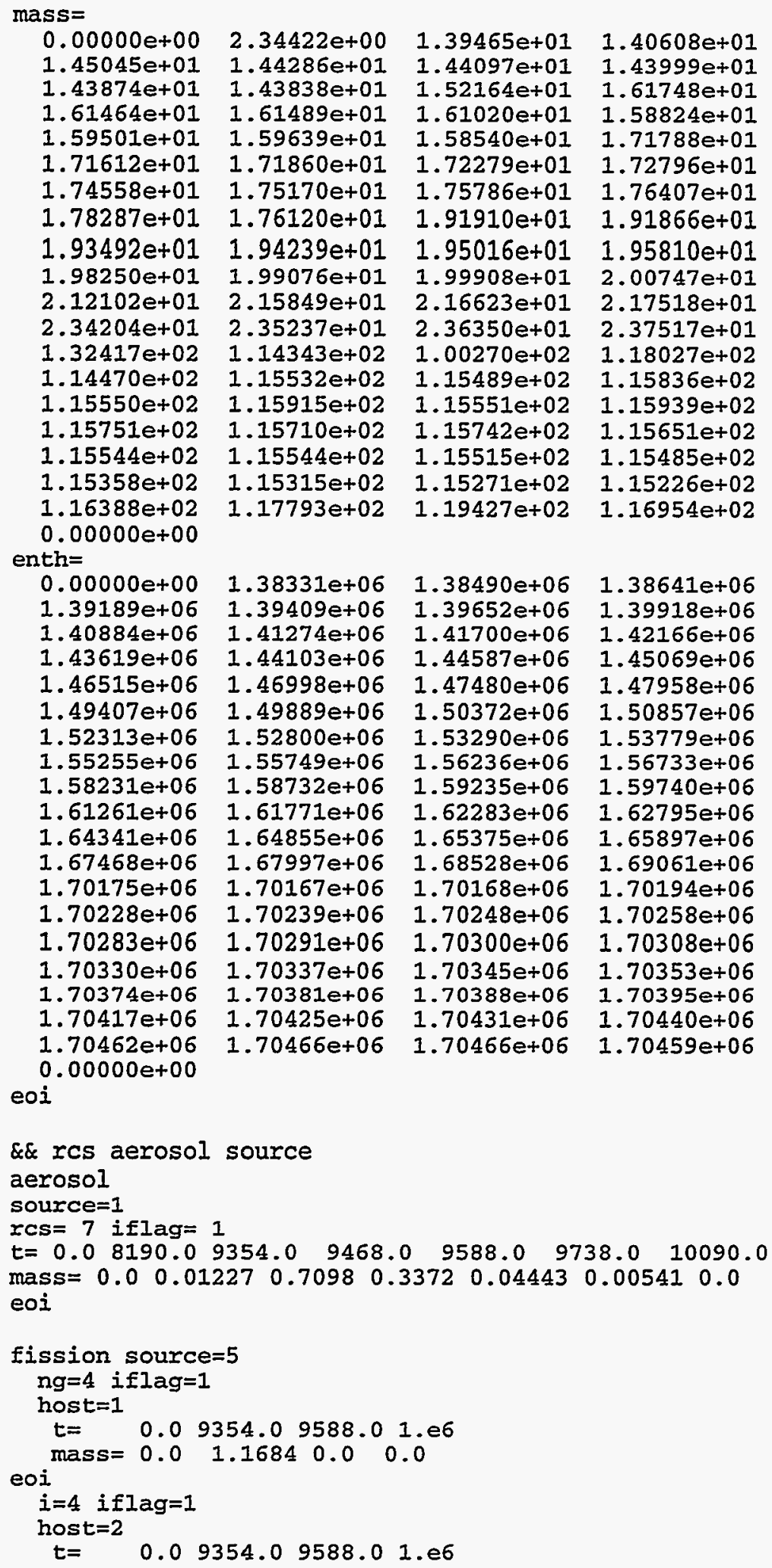

$0.00000 e+00$

$1.45045 e+01$

$1.43874 \mathrm{e}+01$

$1.61464 \mathrm{e}+01$

1.59501 e+01

1. $71612 e+01$

$1.74558 \mathrm{e}+01$

$1.78287 e+01$

1. $93492 \mathrm{e}+01$

$1.98250 e+01$

$2.12102 \mathrm{e}+01$

2. $34204 e+01$

$1.32417 \mathrm{e}+02$

1. $14470 \mathrm{e}+02$

$1.15550 \mathrm{e}+02$

1.15751 e+02

$1.15544 \mathrm{e}+02$

$1.15358 \mathrm{e}+02$

$1.16388 \mathrm{e}+02$

$0.00000 e+00$

enth $=$

$0.00000 e+00$

$1.39189 e+06$

$1.40884 \mathrm{e}+06$

$1.43619 e+06$

$1.46515 e+06$

$1.49407 \mathrm{e}+06$

$1.52313 e+06$

$1.55255 e+06$

$1.58231 \mathrm{e}+06$

$1.61261 e+06$

$1.64341 \mathrm{e}+06$

$1.67468 \mathrm{e}+06$

$1.70175 e+06$

$1.70228 \mathrm{e}+06$

$1.70283 e+06$

$1.70330 e+06$

$1.70374 \mathrm{e}+06$

$1.70417 e+06$

$1.70462 e+06$

$0.00000 e+00$

$2.34422 e+00$

$1.44286 e+01$

$1.43838 \mathrm{e}+01$

1. $61489 e+01$

$1.59639 e+01$

$1.71860 \mathrm{e}+01$

$1.75170 \mathrm{e}+01$

$1.76120 e+01$

$1.94239 e+01$

$1.99076 \mathrm{e}+01$

$2.15849 e+01$

2.35237 et 01

$1.14343 e+02$

$1.15532 \mathrm{e}+02$

$1.15915 e+02$

$1.15710 e+02$

$1.15544 \mathrm{e}+02$

$1.15315 e+02$

$1.17793 e+02$

$1.38331 \mathrm{e}+06$

$1.39409 e+06$

$1.41274 \mathrm{e}+06$

$1.44103 e+06$

$1.46998 \mathrm{e}+06$

$1.49889 \mathrm{e}+06$

$1.52800 \mathrm{e}+06$

$1.55749 \mathrm{e}+06$

$1.58732 \mathrm{e}+06$

$1.61771 \mathrm{e}+06$

1. $64855 e+06$

$1.67997 e+06$

$1.70167 \mathrm{e}+06$

$1.70239 e+06$

$1.70291 \mathrm{e}+06$

$1.70337 \mathrm{e}+06$

$1.70381 \mathrm{e}+06$

$1.70425 e+06$

$1.70466 \mathrm{e}+06$

eoi

$1.39465 e+01$

$1.44097 e+01$

1. $52164 \mathrm{e}+01$

1. $61020 \mathrm{e}+01$

$1.58540 \mathrm{e}+01$

$1.72279 e+01$

1.75786e+01

$1.91910 \mathrm{e}+01$

$1.95016 e+01$

$1.99908 \mathrm{e}+01$

$2.16623 e+01$

$2.36350 e+01$

$1.00270 \mathrm{e}+02$

$1.15489 \mathrm{e}+02$

$1.15551 \mathrm{e}+02$

$1.15742 \mathrm{e}+02$

$1.15515 e+02$

$1.15271 \mathrm{e}+02$

$1.19427 \mathrm{e}+02$

$1.38490 e \div 06$

$1.39652 \mathrm{e}+06$

$1.41700 e+06$

$1.44587 \mathrm{e}+06$

1. $47480 \mathrm{e}+06$

$1.50372 \mathrm{e}+06$

$1.53290 \mathrm{e}+06$

$1.56236 \mathrm{e}+06$

$1.59235 e+06$

$1.62283 e+06$

$1.65375 e+06$

$1.68528 \mathrm{e}+06$

$1.70168 e+06$

$1.70248 \mathrm{e}+06$

$1.70300 e+06$

$1.70345 e+06$

$1.70388 \mathrm{e}+06$

$1.70431 \mathrm{e}+06$

1. $70466 \mathrm{e}+06$

1. $40608 \mathrm{e}+01$

$1.43999 \mathrm{e}+01$

$1.61748 \mathrm{e}+01$

$1.58824 \mathrm{e}+01$

$1.71788 \mathrm{e}+01$

$1.72796 \mathrm{e}+01$

$1.76407 \mathrm{e}+01$

$1.91866 \mathrm{e}+01$

1. $95810 \mathrm{e}+01$

2. $00747 e+01$

$2.17518 e+01$

$2.37517 e+01$

$1.18027 e+02$

$1.15836 \mathrm{e}+02$

$1.15939 e+02$

$1.15651 e+02$

$1.15485 e+02$

$1.15226 \mathrm{e}+02$

$1.16954 \mathrm{e}+02$

$1.38641 e+06$

$1.39918 \mathrm{e}+06$

$1.42166 e+06$

$1.45069 e+06$

$1.47958 \mathrm{e}+06$

$1.50857 e+06$

$1.53779 e+06$

$1.56733 e+06$

$1.59740 \mathrm{e}+06$

1. $62795 \mathrm{e}+06$

$1.65897 \mathrm{e}+06$

$1.69061 e+06$

$1.70194 \mathrm{e}+06$

$1.70258 e+06$

$1.70308 \mathrm{e}+06$

$1.70353 e+06$

$1.70395 e+06$

1.70440 e+06

$1.70459 e+06$

\&\& rcs aerosol source

aerosol

source $=1$

rcs $=7$ iflag $=1$

$t=0.0 \quad 8190.0 \quad 9354.0 \quad 9468.0 \quad 9588.0 \quad 9738.0 \quad 10090.0$

mass $=\begin{array}{llllllll}0.0 & 0.01227 & 0.7098 & 0.3372 & 0.04443 & 0.00541 & 0.0\end{array}$

eoi

fission source $=5$

$\mathrm{ng}=4$ iflag $=1$

host $=1$

$t=0.09354 .09588 .01 . e 6$

eoi

mass $=0.0 \quad 1.1684 \quad 0.0 \quad 0.0$

$i=4$ iflag $=1$

host $=2$

$t=0.09354 .09588 .01 . e 6$

$1.41358 \mathrm{e}+01$

$1.43952 \mathrm{e}+01$

$1.61823 e+01$

$1.59406 e+01$

$1.71471 \mathrm{e}+01$

$1.73363 e+01$

$1.77030 e+01$

$1.92219 \mathrm{e}+01$

$1.96616 \mathrm{e}+01$

$2.01591 e+01$

$2.18477 e+01$

$2.38714 \mathrm{e}+01$

$1.17040 \mathrm{e}+02$

$1.15482 e+02$

$1.15638 \mathrm{e}+02$

$1.15729 e+02$

$1.15440 e+02$

$1.15181 e+02$

$1.14753 e+02$

$1.38807 e+06$

$1.40211 \mathrm{e}+06$

$1.42650 e+06$

$1.45549 e+06$

$1.48441 \mathrm{e}+06$

$1.51341 \mathrm{e}+06$

$1.54270 e+06$

$1.57231 \mathrm{e}+06$

$1.60245 e+06$

$1.63310 \mathrm{e}+06$

1. $66422 \mathrm{e}+06$

$1.69596 \mathrm{e}+06$

$1.70210 \mathrm{e}+06$

$1.70265 e+06$

$1.70316 e+06$

$1.70360 e+06$

$1.70403 e+06$

$1.70447 e+06$

$1.70457 \mathrm{e}+06$
$1.40953 e+01$

$1.43911 \mathrm{e}+01$

$1.61513 \mathrm{e}+01$

$1.59410 \mathrm{e}+01$

$1.71451 e+01$

$1.73954 \mathrm{e}+01$

$1.77657 \mathrm{e}+01$

$1.92802 e+01$

$1.97429 e+01$

2. $01739 e+01$

$2.25624 \mathrm{e}+01$

2.29173 e+02

$1.16141 \mathrm{e}+02$

$1.15837 e+02$

$1.15801 \mathrm{e}+02$

$1.15724 \mathrm{e}+02$

$1.15400 \mathrm{e}+02$

$1.15358 \mathrm{e}+02$

$1.14034 \mathrm{e}+02$

$1.38990 \mathrm{e}+06$

$1.40532 \mathrm{e}+06$

$1.43135 e+06$

$1.46032 \mathrm{e}+06$

$1.48924 \mathrm{e}+06$

$1.51826 e+06$

$1.54762 \mathrm{e}+06$

$1.57730 e+06$

$1.60753 e+06$

$1.63826 \mathrm{e}+06$

$1.66947 e+06$

$1.70133 e+06$

$1.70220 e+06$

$1.70275 e+06$

$1.70323 e+06$

$1.70367 e+06$

$1.70411 e+06$

$1.70455 e+06$

$1.70461 e+06$

Rev. 0

15-33 
Table 15-2

Surry Input File (Continued)

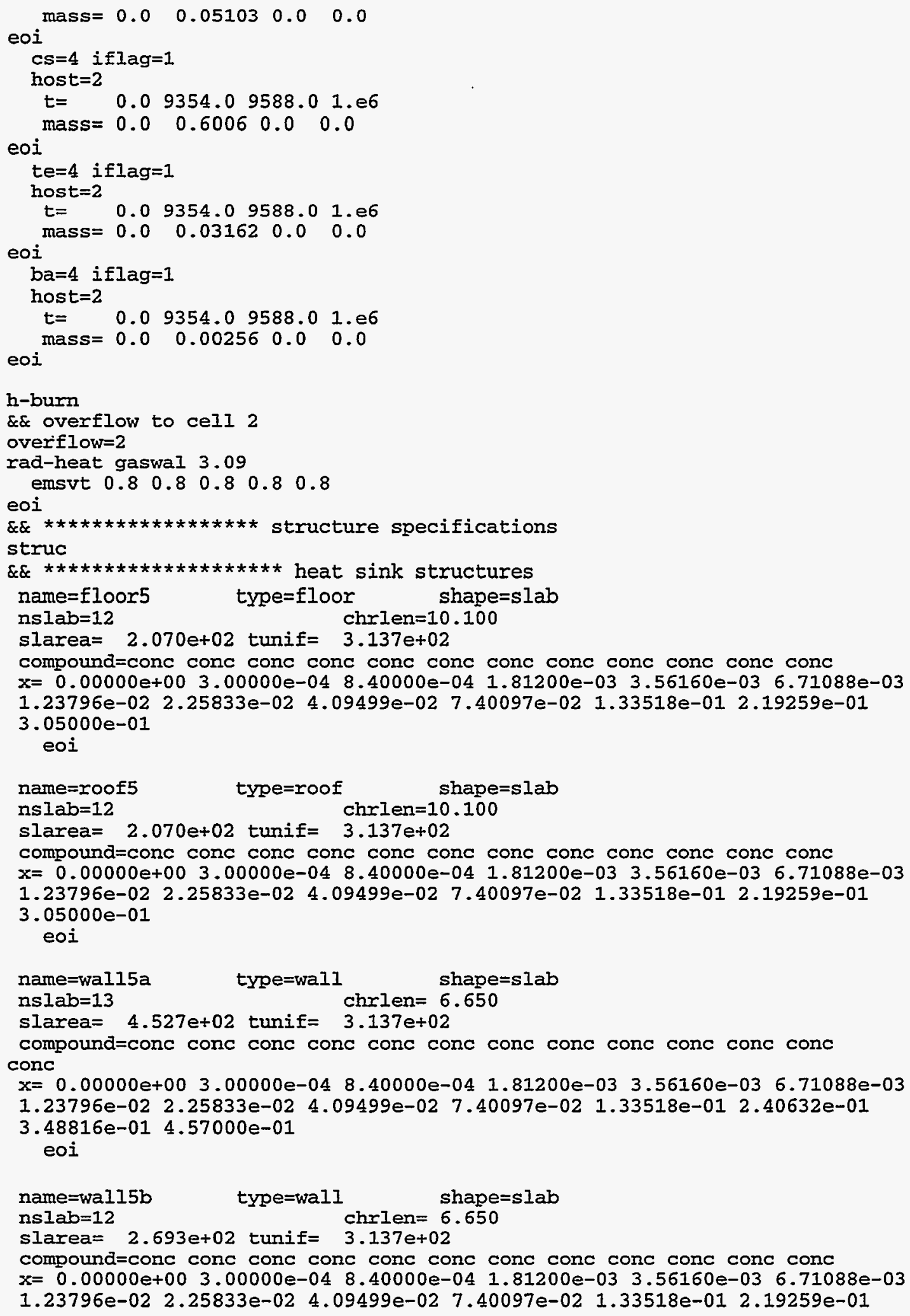




\section{Table 15-2}

Surry Input File (Concluded)

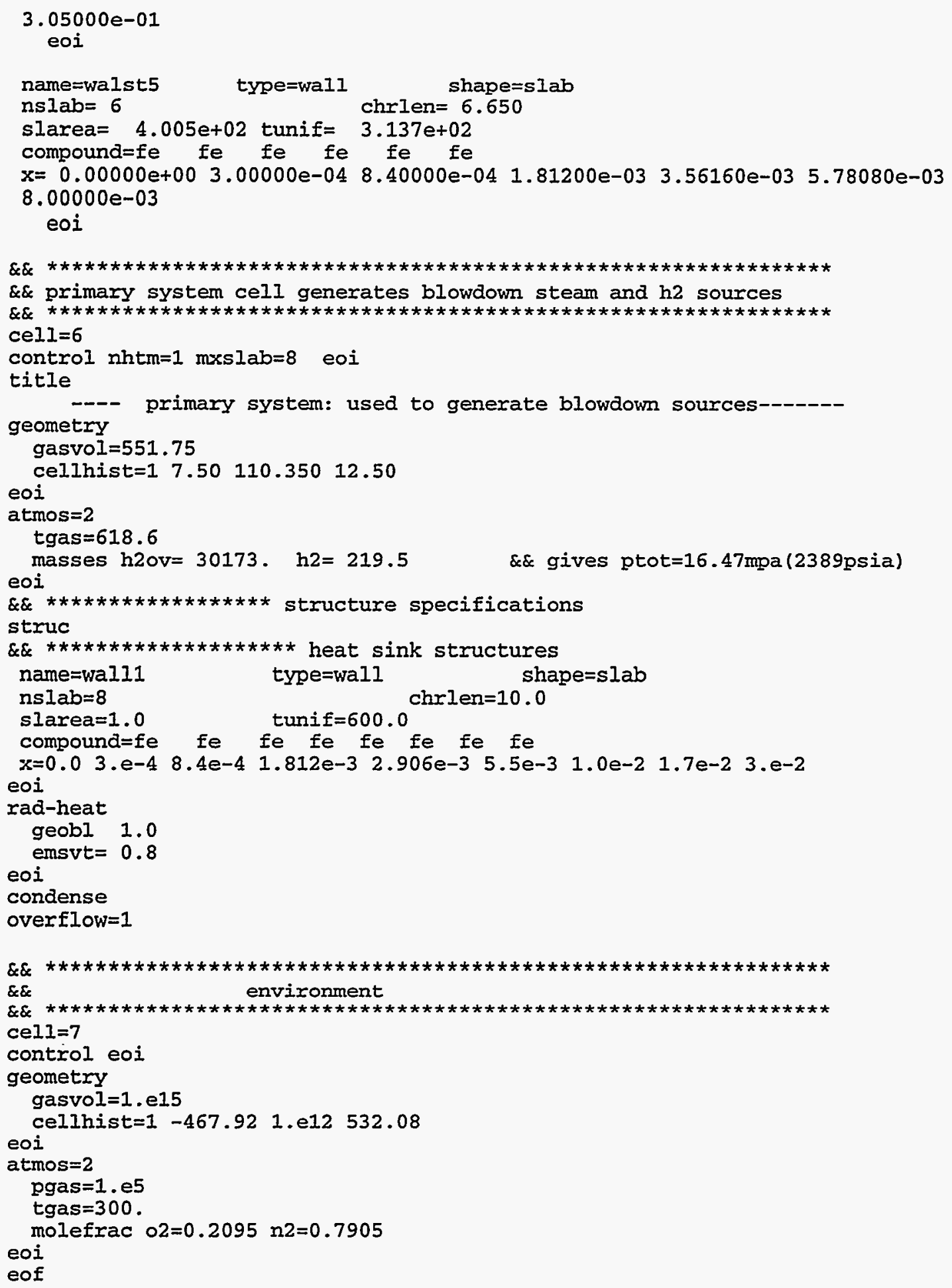




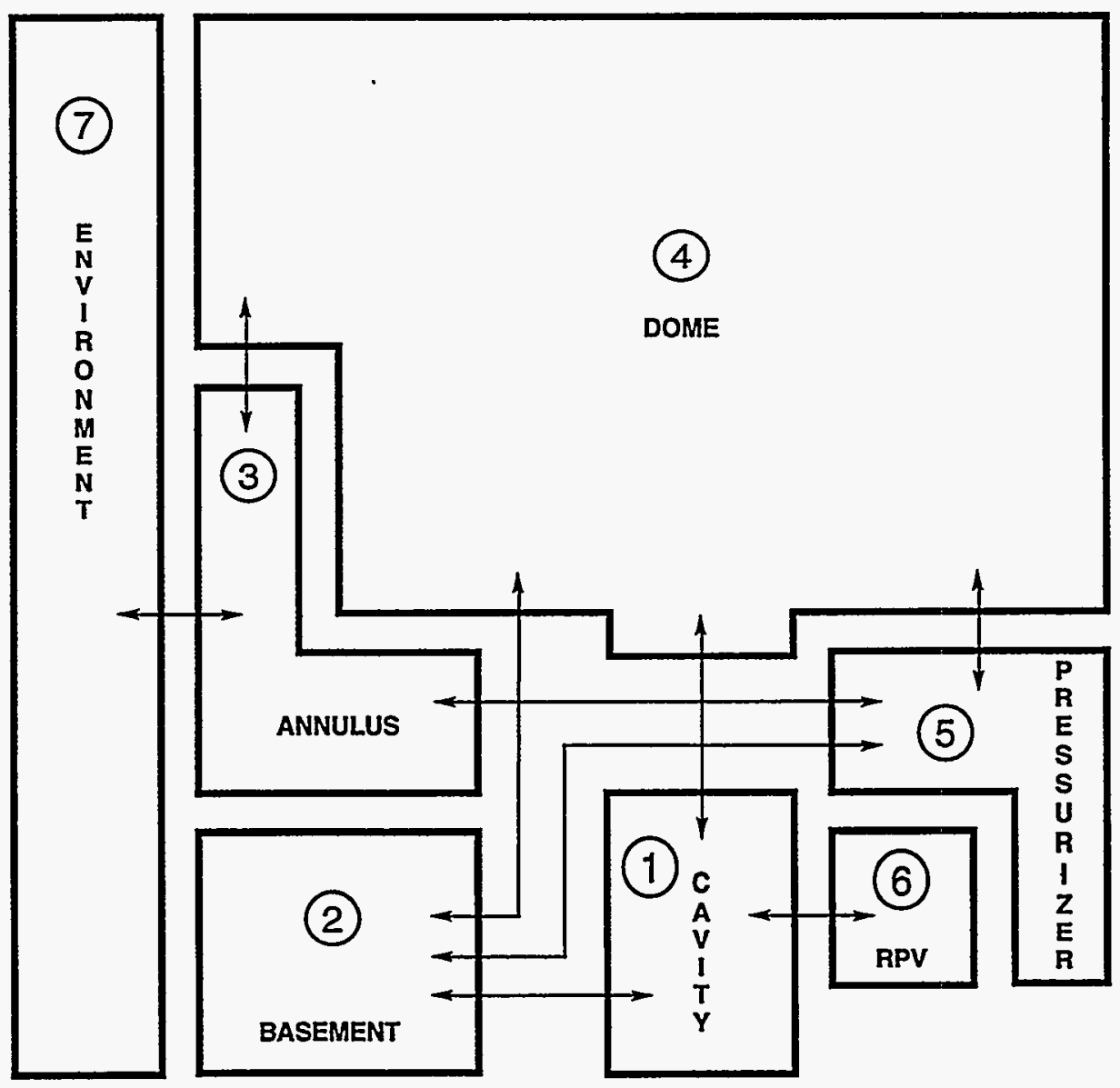

Figure 15-8. Layout of the Surry Model 
concrete in the cavity and thus the results obtained in this problem differ in some ways from what would be obtained for an analysis of the actual Surry plant. CORCON and VANESA are inactive until the time of vessel breach at $10090 \mathrm{~s}$. Detailed mapping of the VANESA constituent materials onto the CONTAIN fission product inventory system is enabled with the FPTRACK=DETAIL specification. The ANSI decay heat data is used. The intermediate layers are defined as stratified with LAYERS $=0$ specified, which means that core debris metals and oxides will be treated as residing in separate layers. The FPLIQUID block in the global input provides that iodine, cesium, and tellurium fission products deposited on structures wash down to the pool when the condensate films drain to the pool. Except for the cavity, condensate films in all cells drain to the basement pool as provided by the OVERFLOW=2 specification. Other fission products are assumed to be insoluble and to remain on the surfaces they were deposited on.

The containment is initially at saturated conditions at $313.7 \mathrm{~K}$. The primary system cell is pressurized at 16.47 MPa. The accident scenario assumes an "early" station blackout in which the primary system remains fully pressurized up until vessel breach. However, in this model, it is assumed that no DCH occurs and the full core melt inventory is assumed to be discharged to the reactor cavity and to participate in the subsequent CCI. It is also assumed that the accumulator discharge follows the melt discharge, that debris beds are noncoolable, and that CCI releases are scrubbed by the overlying water until all the water boils away.

The problem was run out to $2 \times 10^{5}$ seconds (55.6 hours or 2.3 days). Pressure-time histories for the first 40,000 s and for the full time period are shown in Figures 15-9 and 15-10, respectively. The first large pressure spike reflects RPV blowdown following vessel breach and the remaining spikes reflect small burns of hydrogen and carbon monoxide that initially occur in Cell 2 and later occur in Cell 5. These pressure spikes are small because there are no global burns or burns in the large dome volume (Cell 4).

After $40,000 \mathrm{~s}$, the oxygen mole fractions become too low for burns to occur. The containment pressure starts to rise slowly as a result of the continued input of steam, noncondensable gas, and energy into the containment while internal heat sinks become thermally saturated, reducing the ability of the structures to condense steam or cool the atmosphere. This pressure increase would continue until containment-threatening pressures would eventually be reached, but it is evident that at least a few days would be required for this to happen. The present calculation assumes that no action would be taken over this time span in order to restore containment cooling, which is probably unrealistic.

Temperature histories are shown in Figure 15-11. Burns produce some early temperature spikes; most of the burns actually occur in Cell 5 but temperatures are not plotted for this cell as plotting the numerous large temperature excursions would obscure other features of interest in the plot. After hydrogen burns cease, there is a slow rise in temperature that parallels the pressure rise. Except for the cavity, which is heated by the core debris, late-time temperatures in the containment do not reach high values in this scenario. 


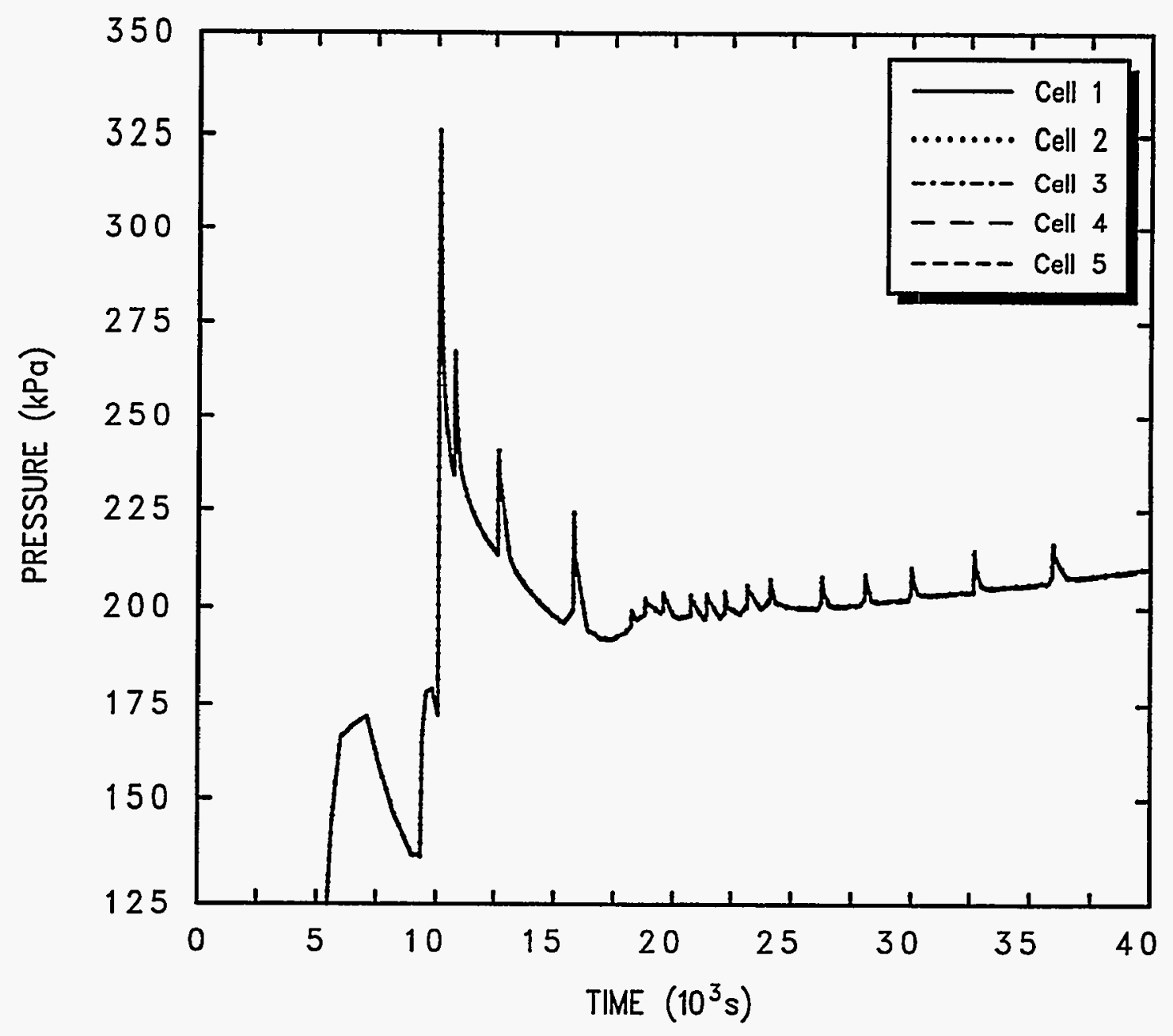

Figure 15-9. Pressure-Time Histories for the First 40,000 Seconds 


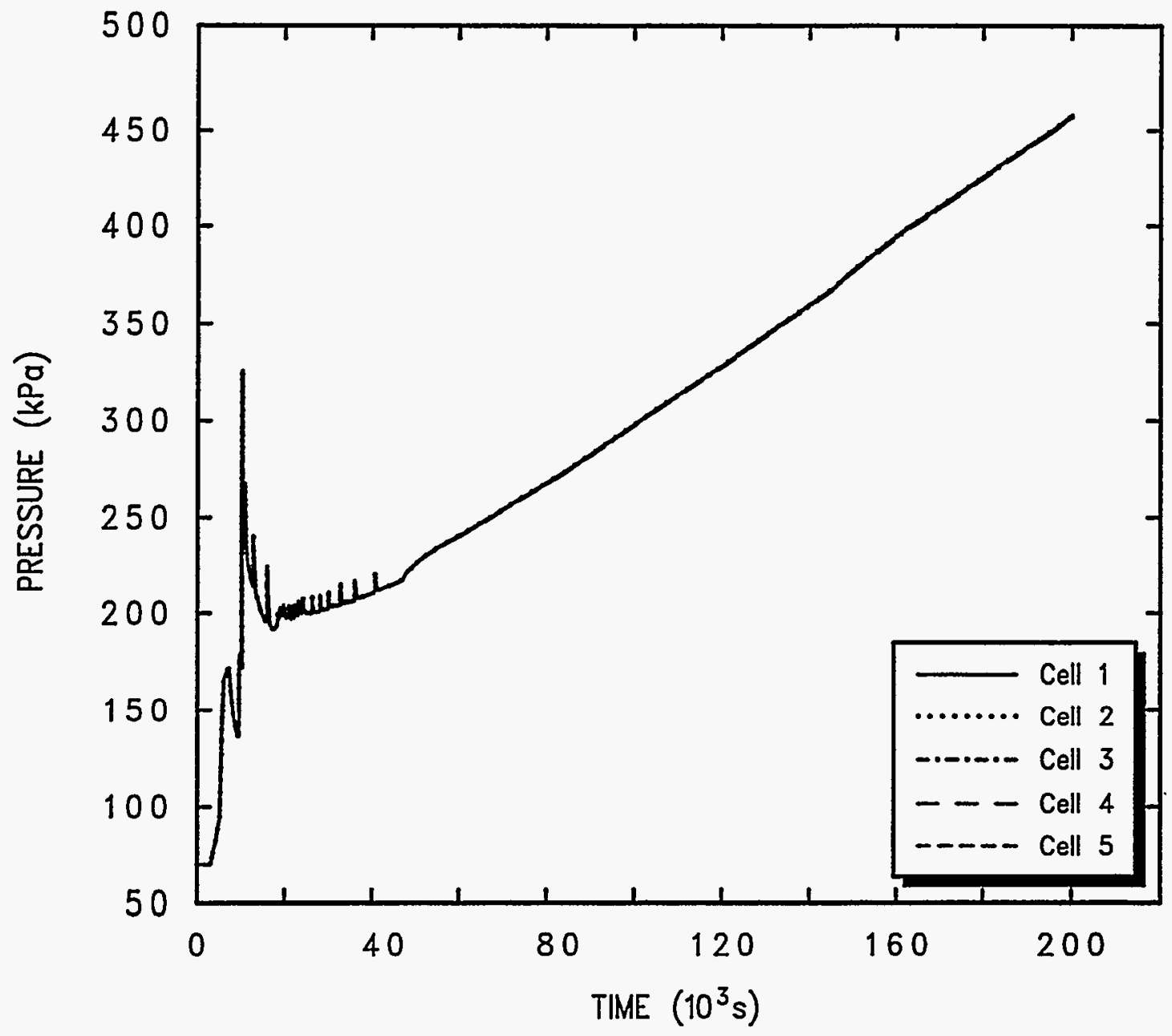

Figure 15-10. Pressure-Time Histories for the Full Time Period 


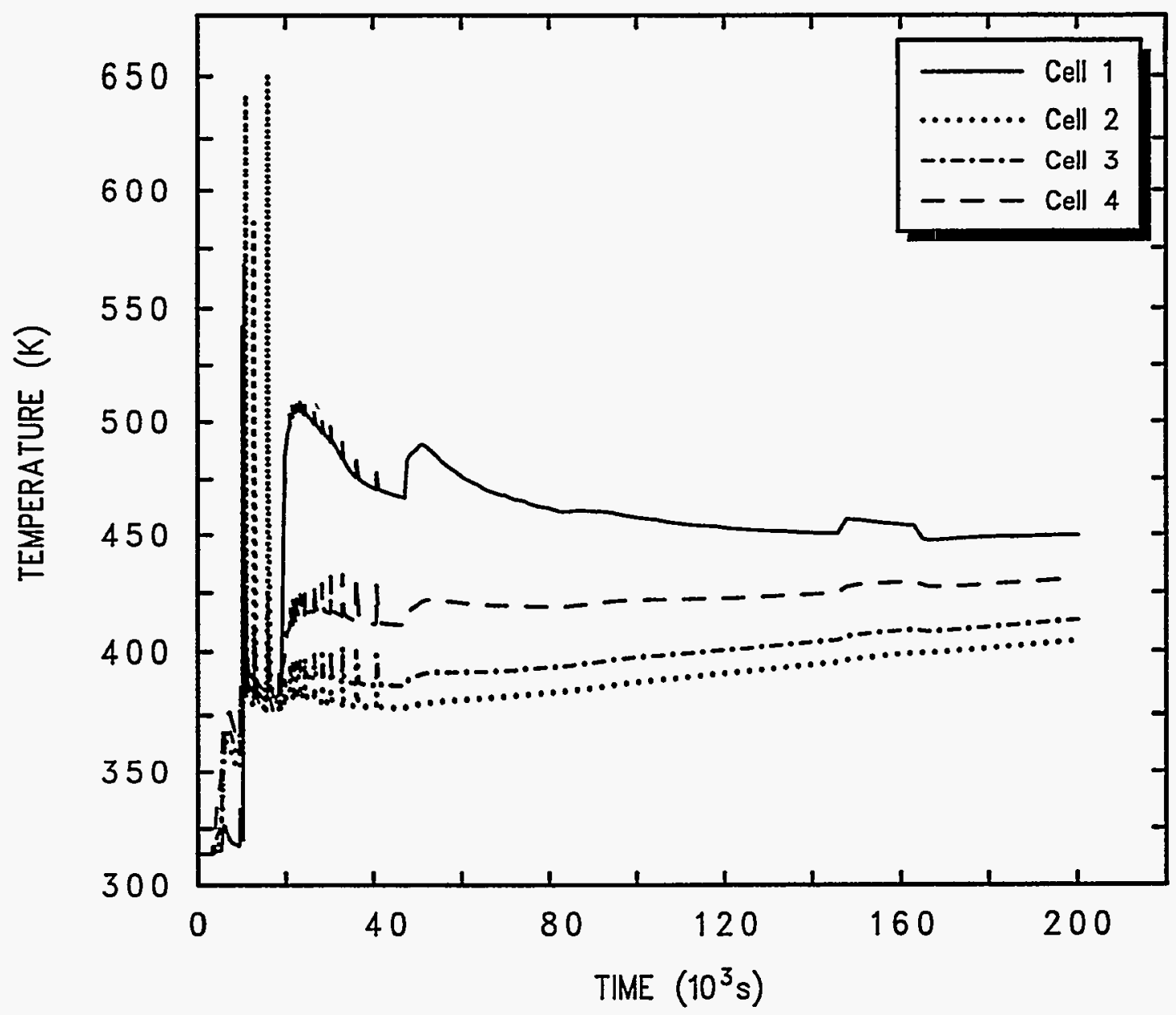

Figure 15-11. Temperature Histories 
The amount of water boiled and evaporated from pools is plotted in Figure 15-12. Water in the cavity (Cell 1) represents the accumulator discharge following vessel breach. It overlies the hot core debris and is boiled off relatively quickly. Boiling from the basement pool (Cell 2) is considerably slower but it continues indefinitely and is an important contributor to the long-term containment pressurization. Decay heating from radionuclides washed from structures into the pool is an important contributor to the rates of water evaporation from the pool in Cell 2. A sensitivity calculation not including the washdown model resulted in considerably slower long-term containment pressurization. [Gid91]

In Figure 15-13, the cumulative quantities of gases evolved, in kg-moles, are plotted along with the amount of zirconium (in $\mathrm{kg}$-moles) remaining in the melt. Zirconium metal is very reactive chemically and its oxidation releases substantial energy. It is relatively quickly depleted by oxidation and, until it is gone, there is virtually no release of the oxidized gas species $\left(\mathrm{CO}_{2}\right.$ and $\left.\mathrm{H}_{2} \mathrm{O}\right)$ because they are all consumed by the zirconium. Another change is apparent at around $90000 \mathrm{~s}$, at which time the release of the reducing gases slows and the release of oxidizing gases increases. The reason is that, at this time, the iron initially present in the melt is depleted and only the iron from rebar in the ablated concrete is available at later times to react with the oxidizing gases released from the concrete. The metal layer after this time consists principally of nickel and silver, which are insufficiently reactive to convert more than a small fraction of the oxidizing gases to the reduced species.

Masses of the melt layers are plotted in Figure 15-14. Initially there is a heavy oxide layer overlain by a metal layer. A light oxide layer composed of ablation products develops on top. At about $46000 \mathrm{~s}$, the density of the heavy oxide layer falls below that of the metal layer. At this point, older CORCON versions would undergo a "layer flip." In the present (MOD3) version of CORCON, the material in the heavy oxide layer is transferred to the light oxide layer. Hence the heavy oxide mass abruptly drops to zero at this time while the light oxide mass abruptly undergoes a corresponding increase. After this time, ablation products and products of metal oxidation continue to be added to the light oxide layer. The metal layer mass steadily decreases as zirconium, chromium, and iron are depleted by oxidation. When the iron is mostly depleted at around $90000 \mathrm{~s}$, the decline in the metal mass slows but some depletion still occurs.

Masses of airborne iodine, tellurium, strontium, cerium, cesium, and (molybdenum plus ruthenium) fission products are shown in Figure 15-15. The shift to more oxidizing conditions that accompanies iron depletion has a dramatic effect upon the rate of molybdenum fission product release from the melt that is calculated by the VANESA model. The reason is that molybdenum metal itself is extremely refractory but it can form volatile oxides under more oxidizing conditions. However, the model may exaggerate this effect because it assumes that molybdenum in the condensed phase will remain in the metallic form even when conditions become sufficiently oxidizing that oxidized forms of molybdenum residing in the oxide layer would be more stable thermodynamically. Under these conditions, assuming the condensed phase molybdenum remains metallic can lead to an overestimated vaporization release rate. No quantitative investigation has been made of the magnitude of this effect, but it can be large. Note also that this limitation applies to all versions of VANESA, including those incorporated into stand-alone versions of CORCON. 


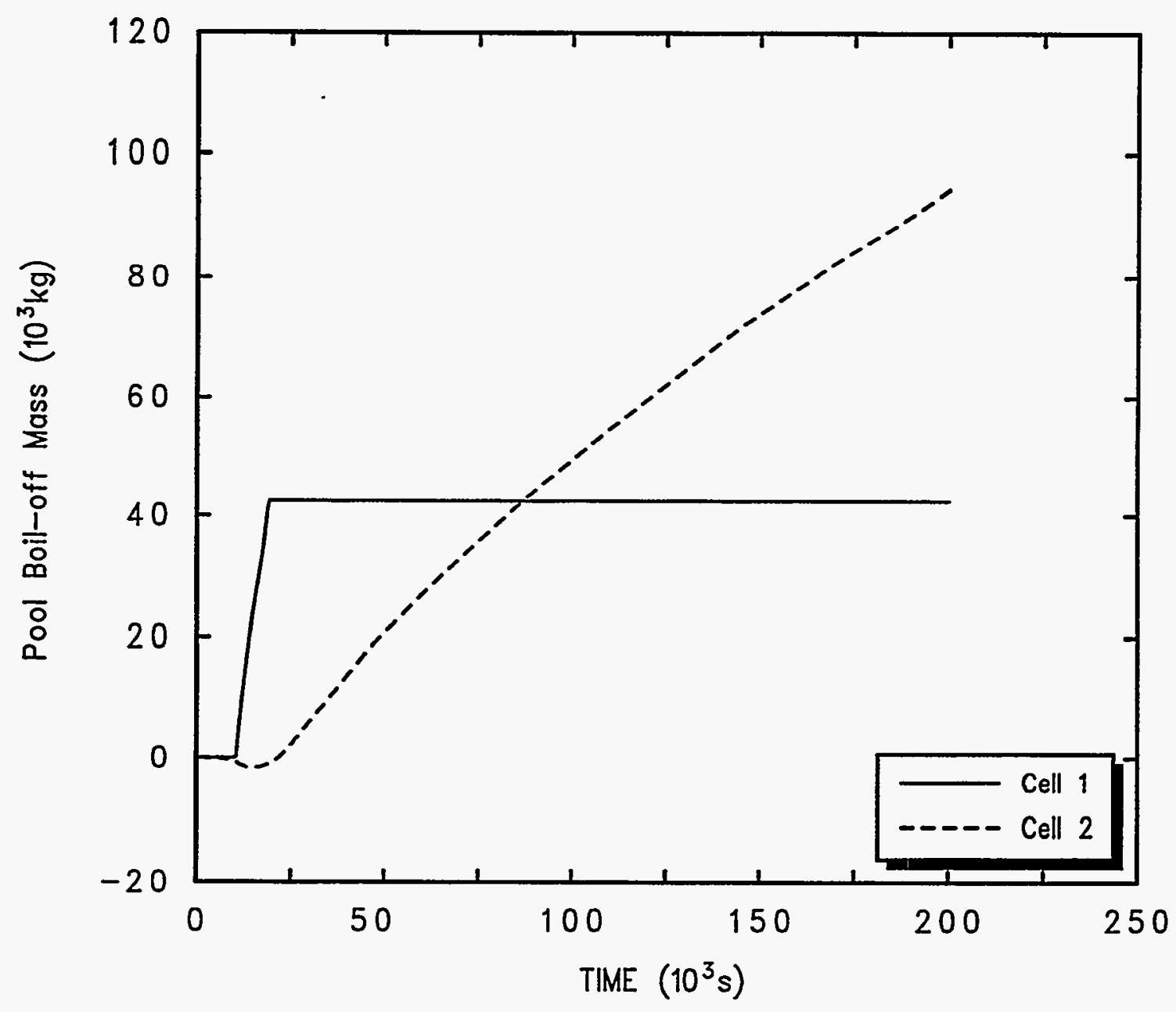

Figure 15-12. Amount of Water Boiled and Evaporated from Pools 


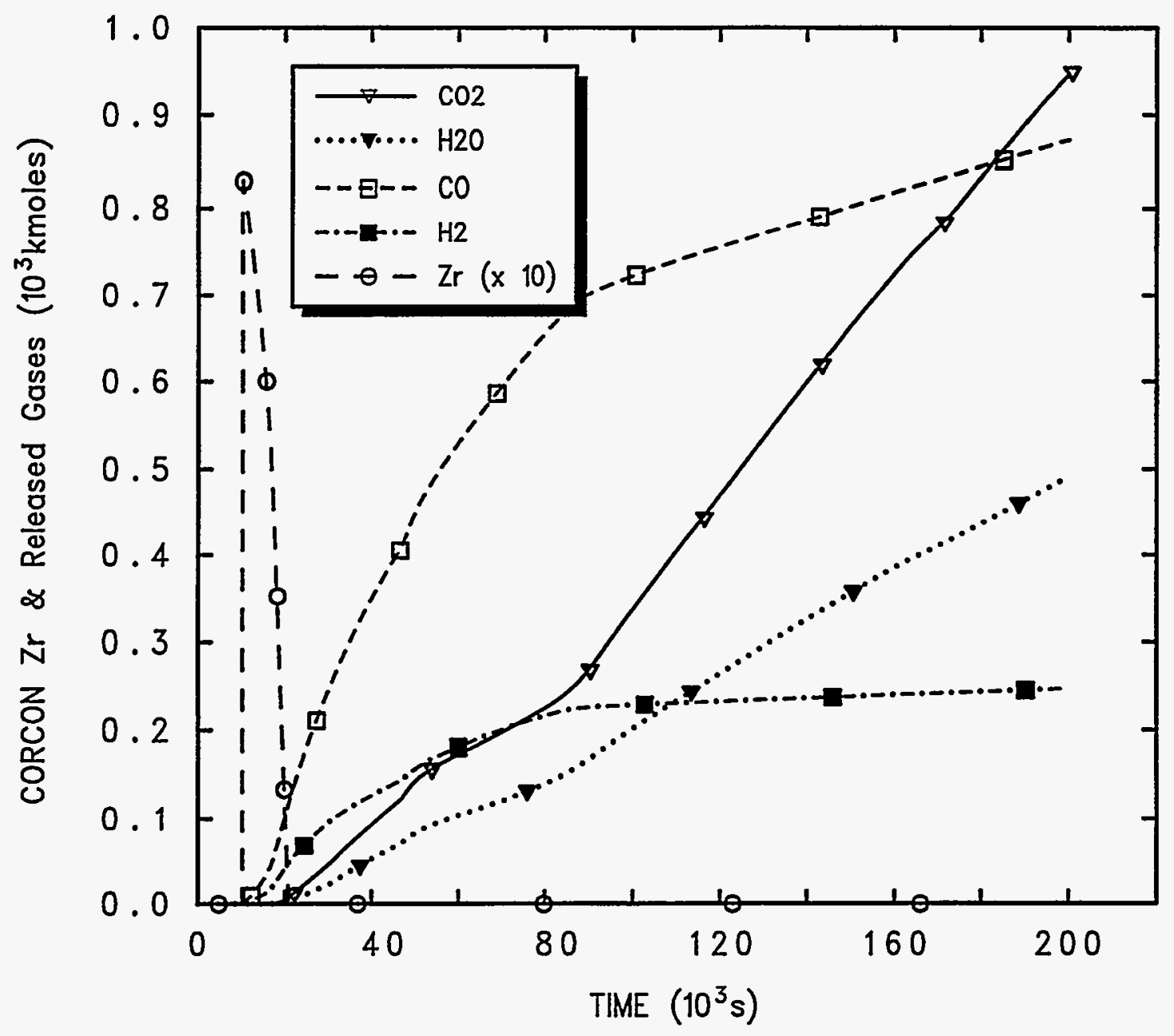

Figure 15-13. Cumulative Quantities of Gases Evolved and Amount of Zirconium Remaining in the Melt 


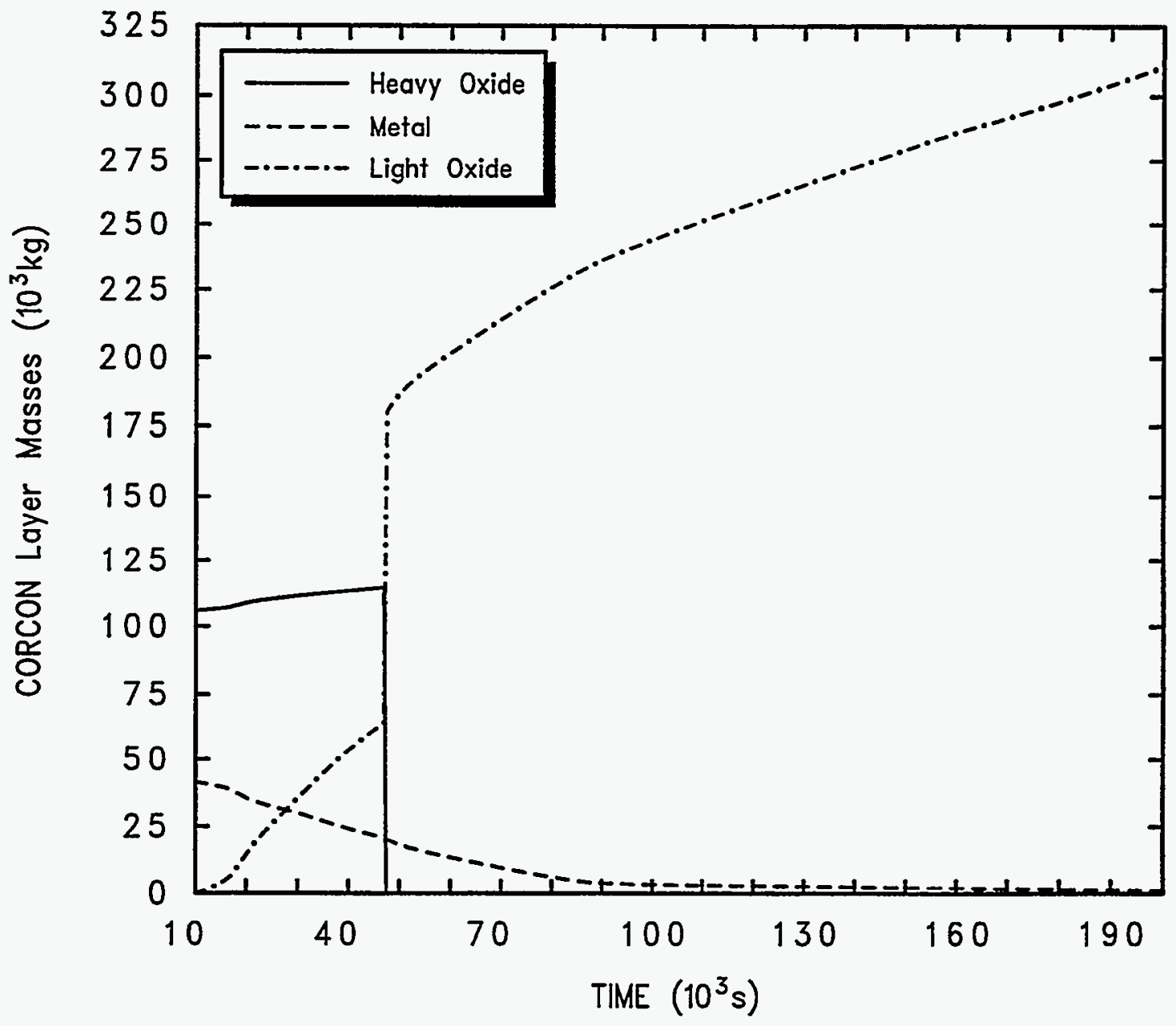

Figure 15-14. Masses of the Melt Layers 


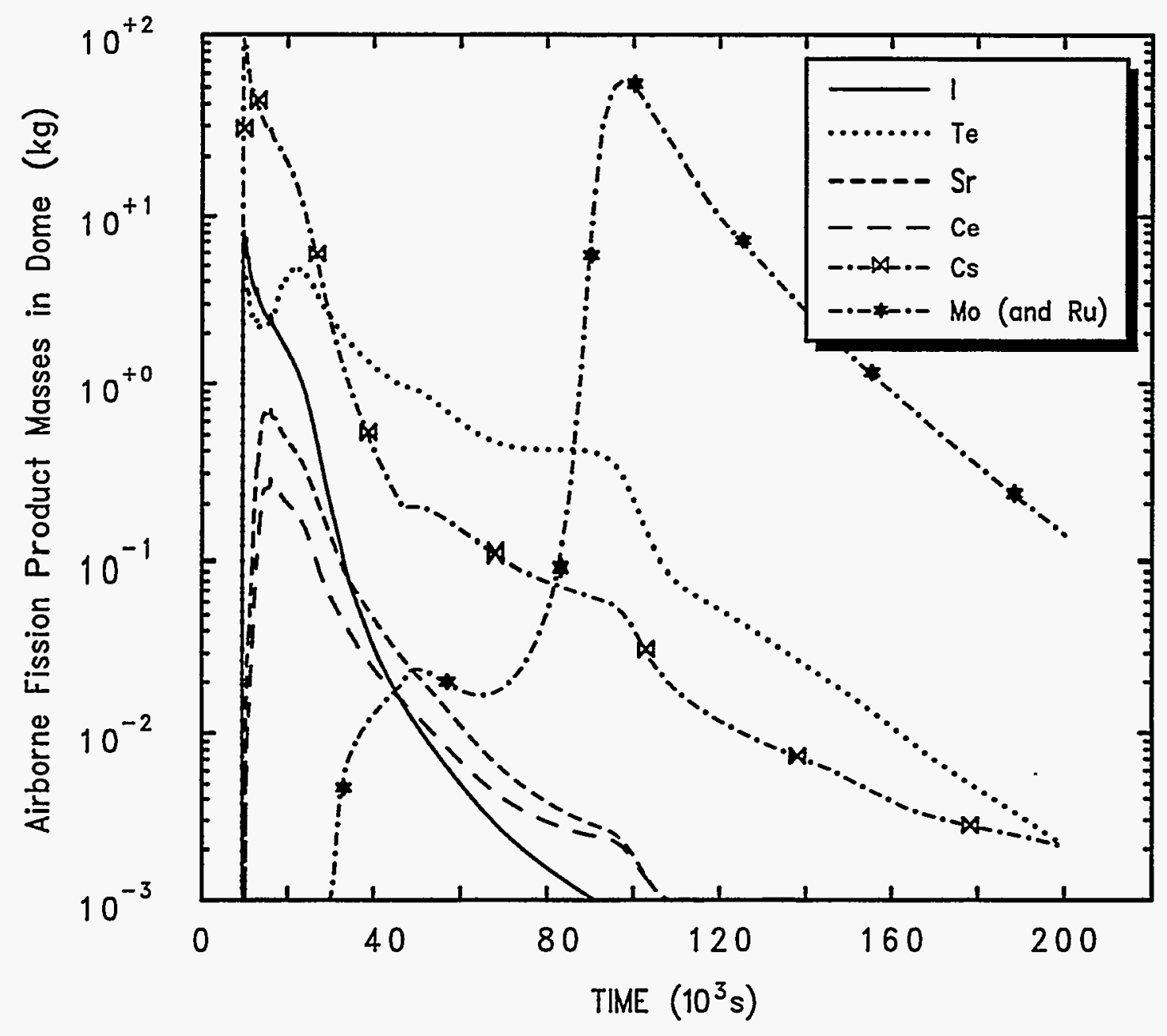

Figure 15-15. Masses of Fission Products 
Except for molybdenum, masses of airborne fission products peak early and then decline, as all fission products are hosted to aerosols which are depleted by various natural processes. Cesium and iodine are released either from the primary system or the very first stages of CCIs and are not subsequently replenished; hence, they decline especially rapidly. Strontium and cerium also decline fairly rapidly because their releases occur principally during the early stages of CCI when temperatures are high and conditions are strongly reducing chemically owing to the presence of zirconium in the melt. Tellurium is released partly from the reactor cooling system (RCS), which accounts for the first peak in the airborne mass curve, and partly from the melt during CCI. Tellurium release during CCI does not decline as rapidly as does release of the other species. There is a second peak in the airborne tellurium at about $20000 \mathrm{~s}$ as a result of the continuing CCI releases and the loss of the scrubbing effect of the cavity pool when boil-off is complete at about 19,000 s.

Cavity dimensions as a function of time are plotted in Figure 15-16. The change in configuration that occurs when the heavy oxide layer is combined with the light oxide layer results in a decrease in the rate of radial ablation and an increase in the downward ablation rates. This change in the configuration also affects the debris temperatures, which are plotted for the lower interfaces of the various layers in Figure 15-17. The high temperatures at early times are at least partly the result of the large energy release that accompanies zirconium oxidation.

\subsection{Sequoyah Plant}

In the third sample problem, a nine-cell model of an ice condenser PWR containment is presented. This model is based on the containment of the Sequoyah plant. In this example, the DCH models and restart capabilities in CONTAIN are exercised.

The initial input file is listed in Table 15-3, and the model layout is shown in Figure 15-18. Nine cells are used. Cell 1 represents the reactor cavity volume. The lower compartment is represented by Cell 2 . Cell 3 is the volume enclosed by the lower plenum. The ice compartment is modeled as Cell 4. The upper plenum volume is represented by Cell 5. Cell 6 models the upper containment volume. The primary system cell which generates the blowdown sources is modeled as Cell 7 . The annular volume between the shield building and steel containment is represented as Cell 8. The ninth cell represents the environment.

As in the Grand Gulf and Surry sample input, the recommended implicit flow solver is used, except for the environment cell. Flow paths are specified using engineered vents. In the engineered vents, examples of reversible and irreversible pressure-dependent flow areas are modeled in some of the vents that simulate the ice condenser doors. An engineered vent with a user-specified volumetric flow rate is used to simulate the air return fans; however, this flow rate is set to zero as the fans are assumed to be inoperative in this scenario. The RESOLVHD keyword allows the code to recover the gravitational head modeling used in previous code versions, and also to accommodate certain limitations of coarse nodalizations typically used with a control volume code. The AEROSOL model is enabled in this sample problem but only water aerosols are included. The steam, water, and hydrogen releases from the primary system prior to vessel breach are represented by user-specified source tables in Cell 2. 


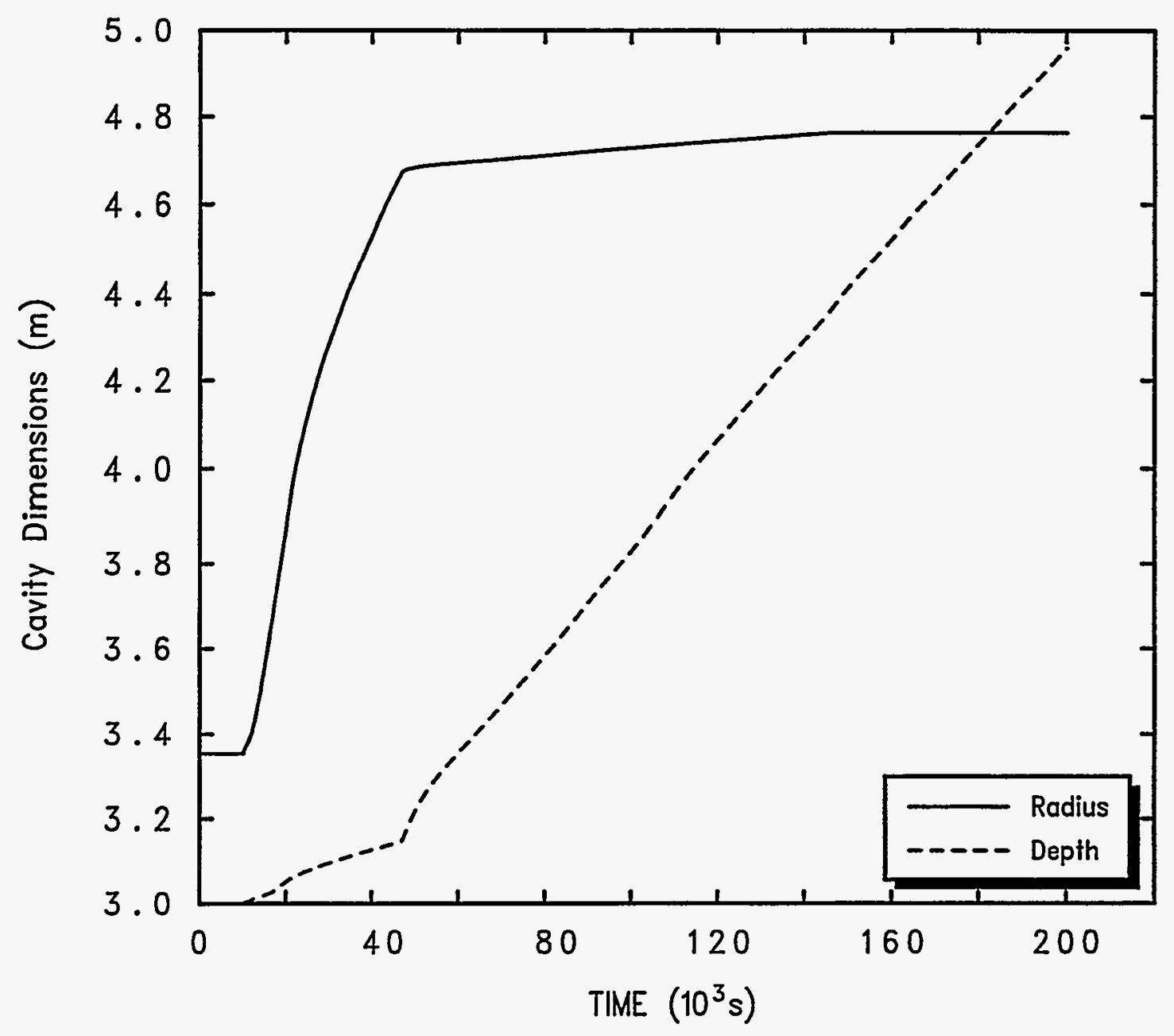

Figure 15-16. Cavity Dimensions as a Function of Time 


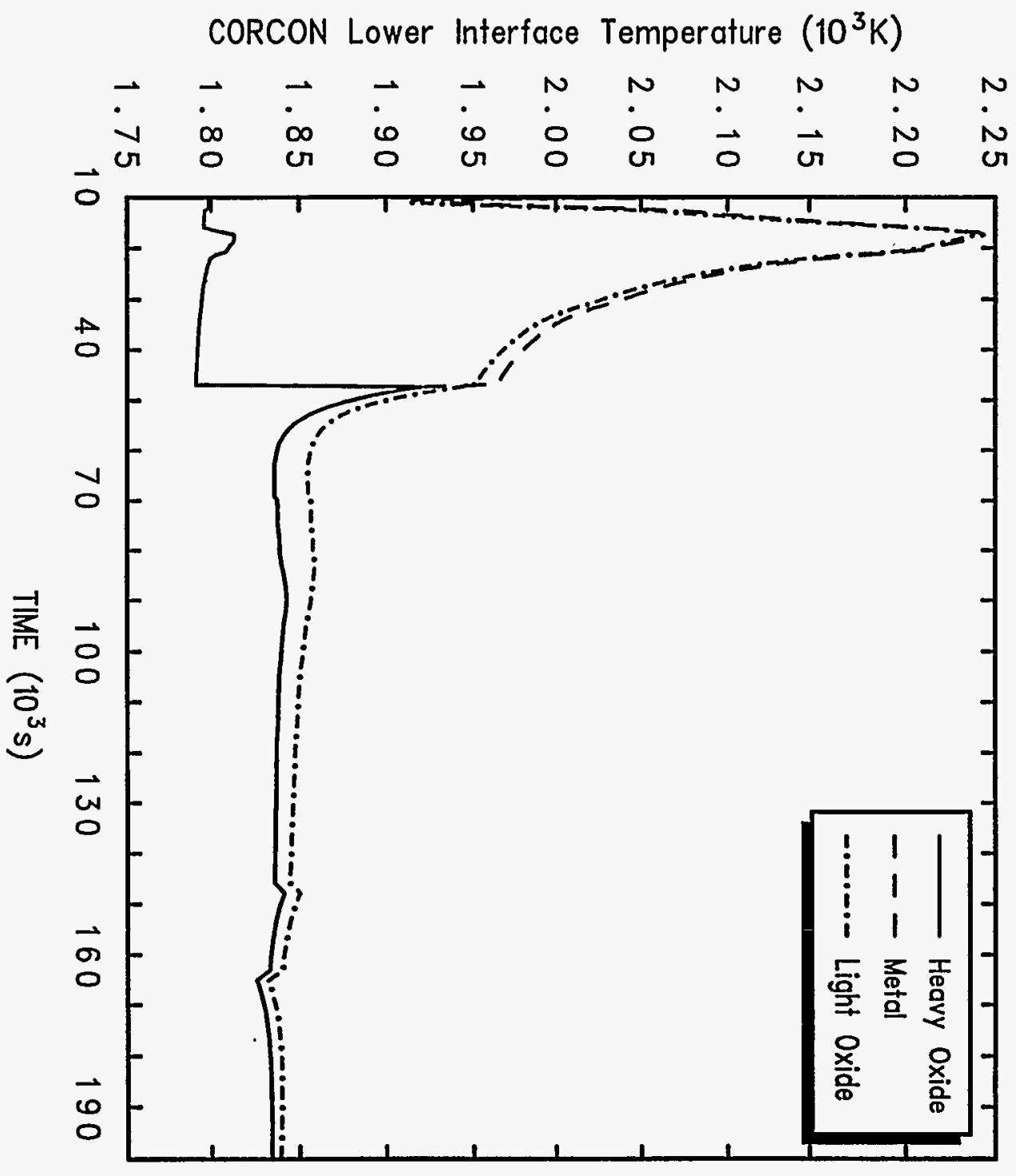


Table 15-3

Sequoyah Input File

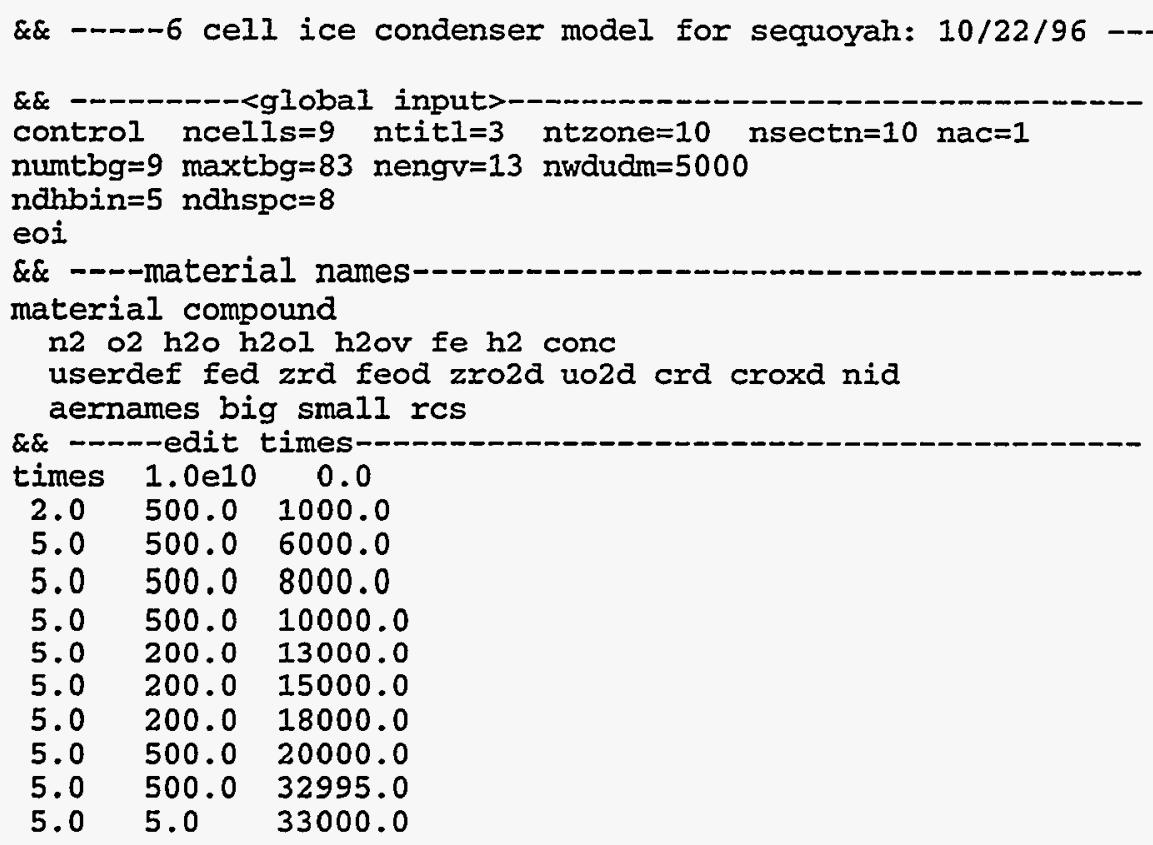

\&\& vent no. 2

from $=2$ to $=6$ varea $=0.29$ vavl $=0.48$ vcf $c=1.25$ velevb $=20.32$ velevf $=20.92$ 
Table 15-3

Sequoyah Input File (Continued)

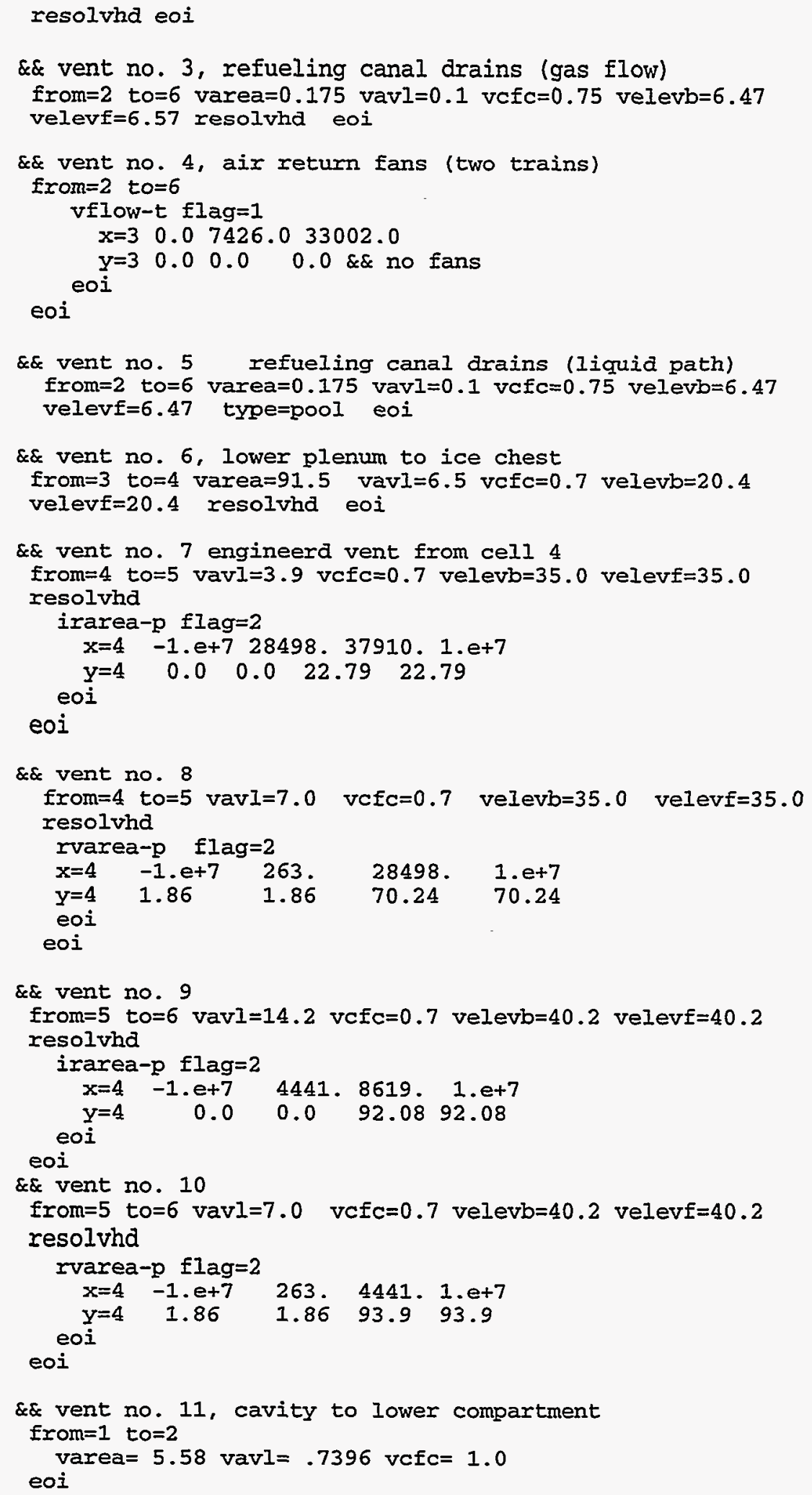


Table 15-3

Sequoyah Input File (Continued)

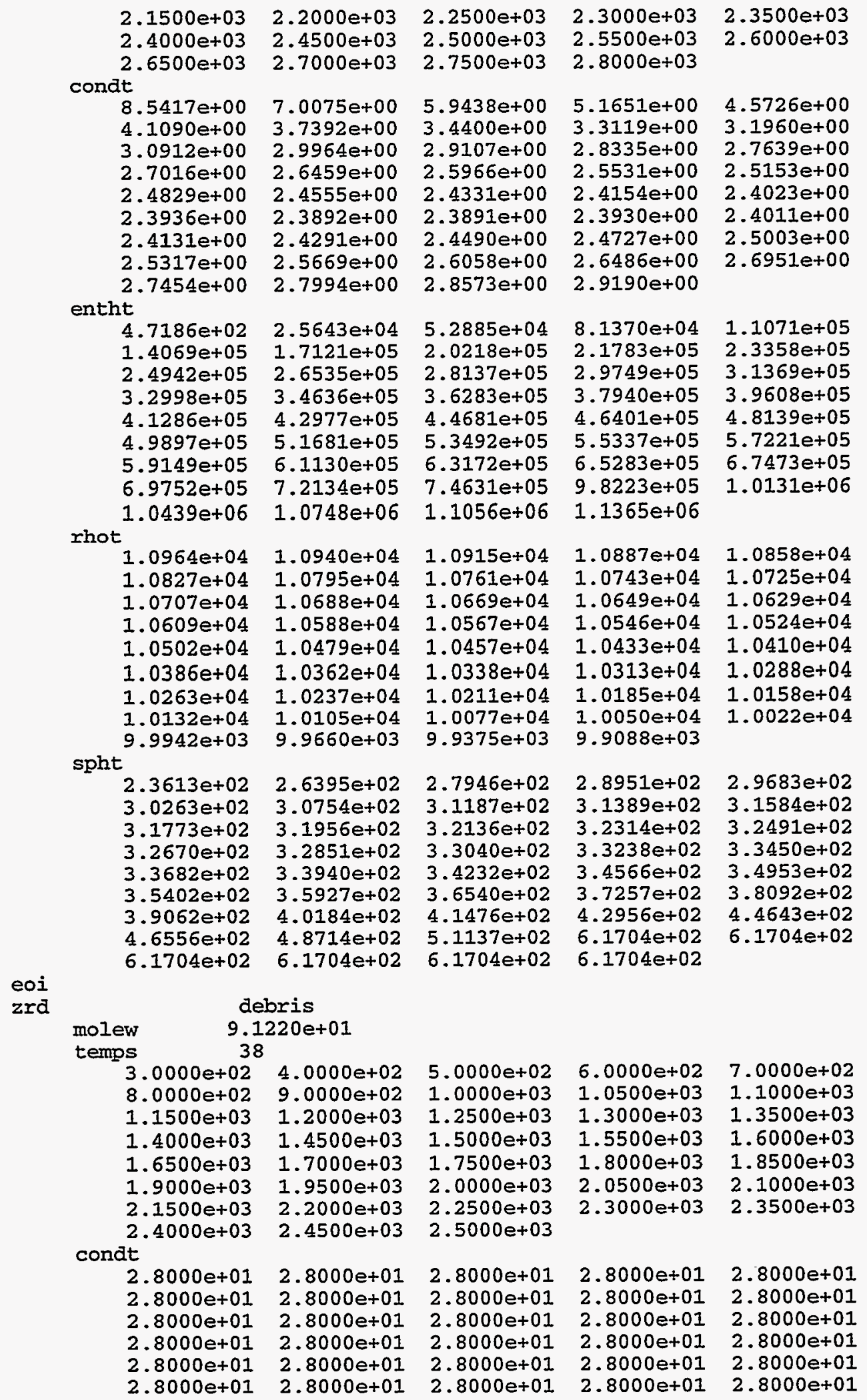


Table 15-3

Sequoyah Input File (Continued)

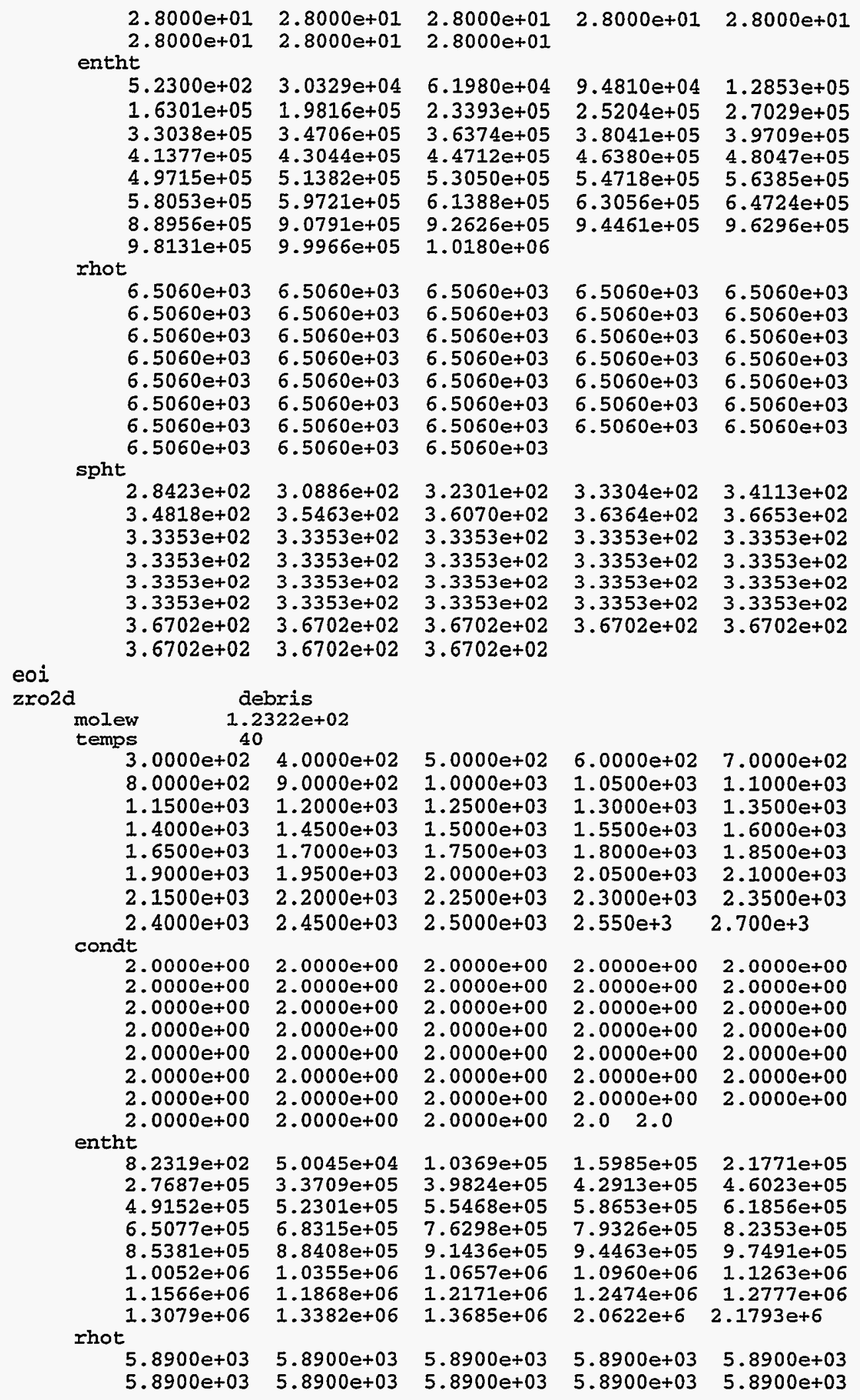


Table 15-3

Sequoyah Input File (Continued)

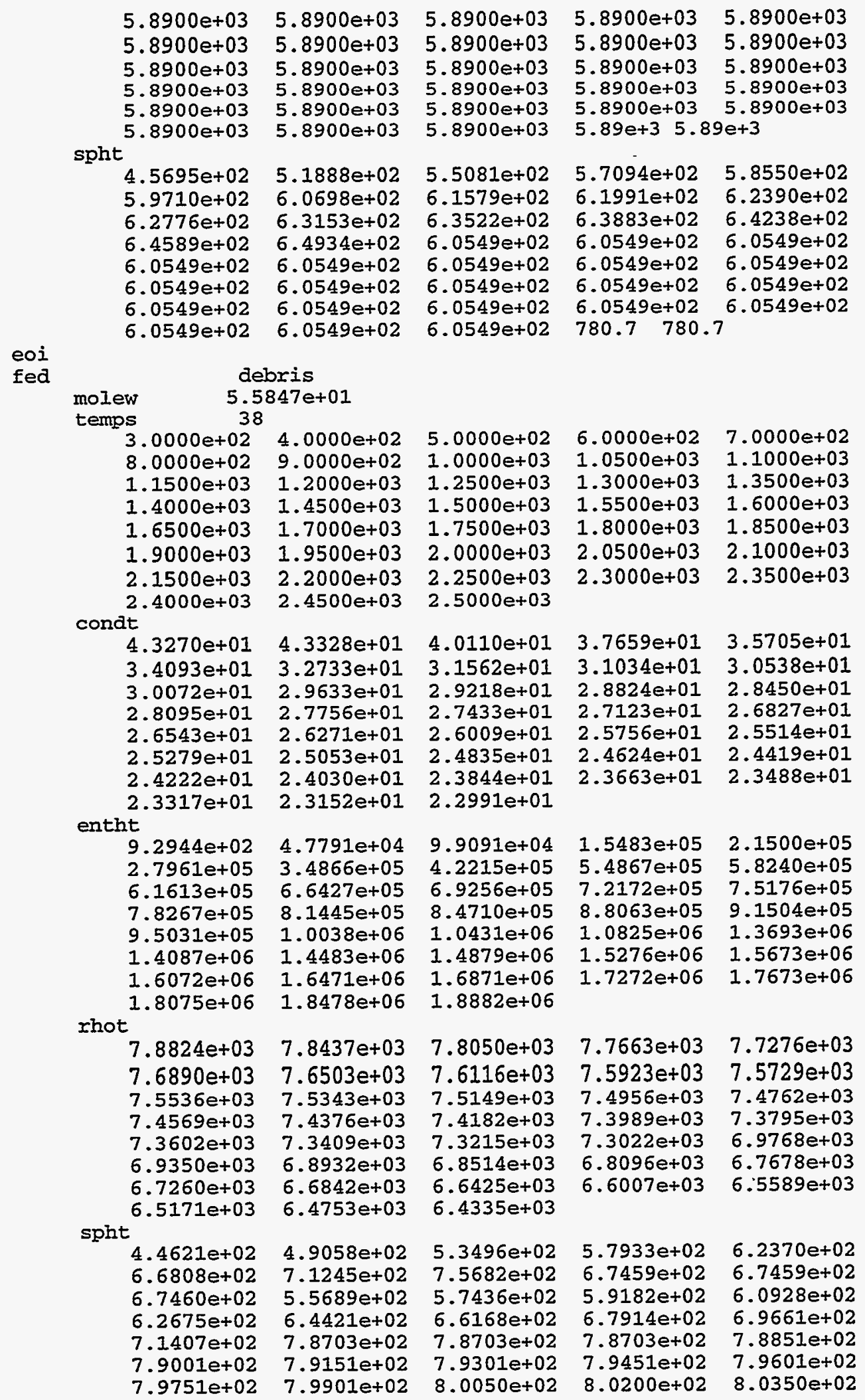


Table 15-3

Sequoyah Input File (Continued)

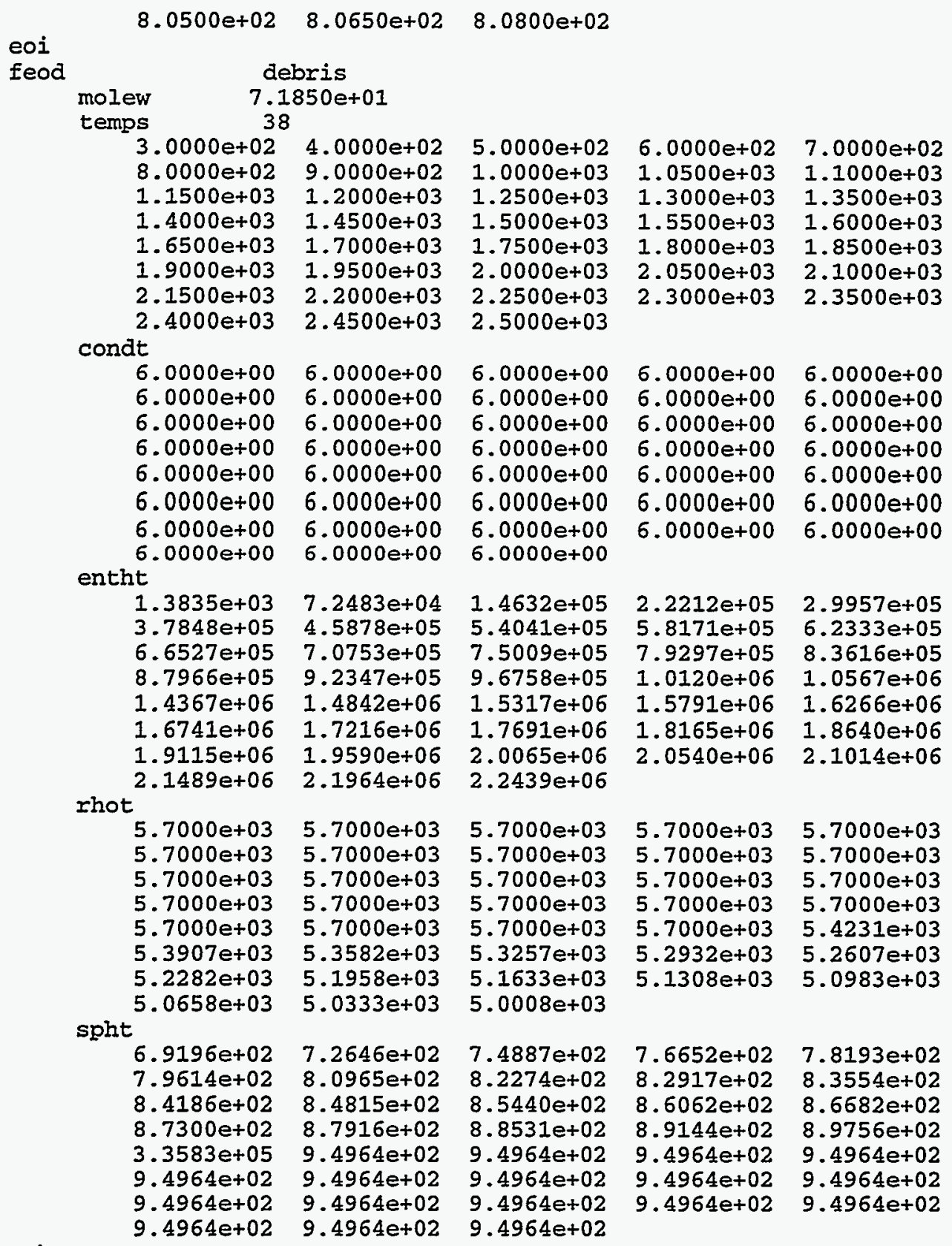

eoi

\&\& add cr and croxd properties from iet309.inp

crd

debris

$\begin{array}{cc}\text { molew } & 5.2010 \mathrm{e}+01 \\ \text { temps } & 45 \\ 3.0000 \mathrm{e}+02 & 4.0000 \mathrm{e}+02 \\ 8.0000 \mathrm{e}+02 & 9.0000 \mathrm{e}+02 \\ 1.1500 \mathrm{e}+03 & 1.2000 \mathrm{e}+03 \\ 1.4000 \mathrm{e}+03 & 1.4500 \mathrm{e}+03 \\ 1.6500 \mathrm{e}+03 & 1.7000 \mathrm{e}+03 \\ 1.9000 \mathrm{e}+03 & 1.9500 \mathrm{e}+03 \\ 2.1500 \mathrm{e}+03 & 2.2000 \mathrm{e}+03\end{array}$

$5.0000 e+02$

$6.0000 e+02$

$7.0000 e+02$

$1.0000 e+03$

$1.0500 e+03$

$1.1000 e+03$

$1.2500 \mathrm{e}+03$

$1.3000 e+03$

$1.3500 e+03$

$1.5000 \mathrm{e}+03$

$1.5500 e+03$

$1.6000 e+03$

$1.7500 e+03$

$1.8000 e+03$

$1.8500 e+03$

$2.0000 e+03$

$2.0500 e+03$

$2.1000 e+03$

$2.2500 e+03$

$2.3000 e+03$

$2.3500 e+03$ 
Table 15-3

Sequoyah Input File (Continued)

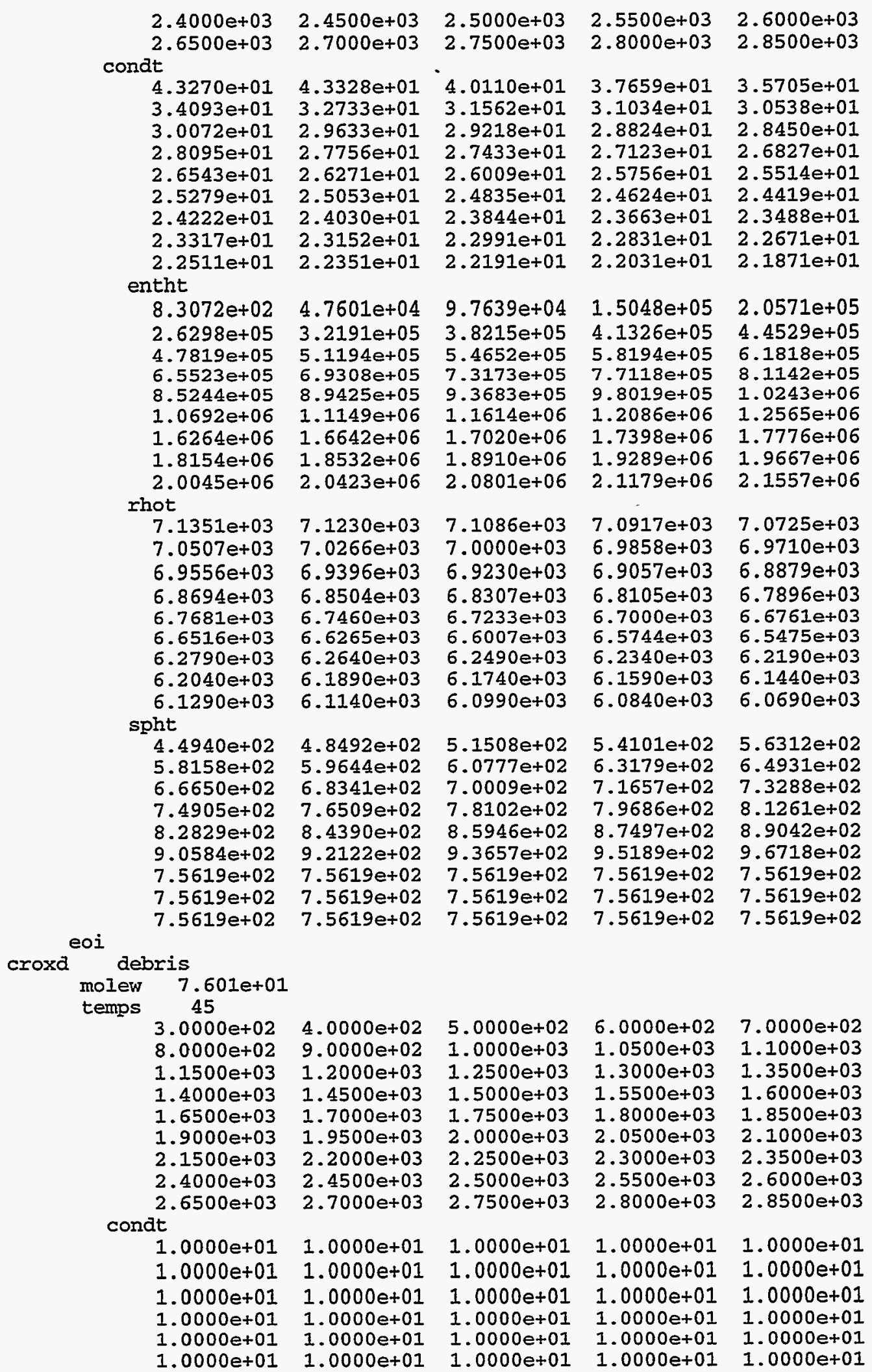


Table 15-3

Sequoyah Input File (Continued)

\begin{tabular}{|c|c|c|c|c|}
\hline $\begin{array}{l}1.0000 e+01 \\
1.0000 e+01 \\
1.0000 e+01\end{array}$ & $\begin{array}{l}1.0000 e+01 \\
1.0000 e+01 \\
1.0000 e+01\end{array}$ & $\begin{array}{l}1.0000 e+01 \\
1.0000 e+01 \\
1.0000 e+01\end{array}$ & $\begin{array}{l}1.0000 e+01 \\
1.0000 e+01 \\
1.0000 e+01\end{array}$ & $\begin{array}{l}1.0000 e+01 \\
1.0000 e+01 \\
1.0000 e+01\end{array}$ \\
\hline $\begin{array}{r}\text { entht } \\
1.2733 e+03 \\
3.8910 e+05 \\
6.8067 e+05 \\
8.9468 e+05 \\
1.1130 e+06 \\
1.3379 e+06 \\
1.5679 e+06 \\
1.7979 e+06 \\
2.8872 e+06\end{array}$ & $\begin{array}{l}7.3338 e+04 \\
4.7134 e+05 \\
7.2312 e+05 \\
9.3801 e+05 \\
1.1571 e+06 \\
1.3839 e+06 \\
1.6139 e+06 \\
1.8439 e+06 \\
2.9388 e+06\end{array}$ & $\begin{array}{l}1.4944 e+05 \\
5.5447 e+05 \\
7.6575 e+05 \\
9.8150 e+05 \\
1.2014 e+06 \\
1.4299 e+06 \\
1.6599 e+06 \\
1.8899 e+06 \\
2.9904 e+06\end{array}$ & $\begin{array}{l}2.2786 e+05 \\
5.9634 e+05 \\
8.0855 e+05 \\
1.0252 e+06 \\
1.2459 e+06 \\
1.4759 e+06 \\
1.7059 e+06 \\
1.9359 e+06 \\
3.0420 e+06\end{array}$ & $\begin{array}{l}3.0787 e+05 \\
6.3841 e+05 \\
8.5153 e+05 \\
1.0690 e+06 \\
1.2919 e+06 \\
1.5219 e+06 \\
1.7519 e+06 \\
1.9819 e+06 \\
3.0936 e+06\end{array}$ \\
\hline \multicolumn{5}{|c|}{ rhot } \\
\hline $\begin{array}{l}5.2091 e+03 \\
5.1409 e+03 \\
5.0931 e+03 \\
5.0590 e+03 \\
5.0249 e+03 \\
4.9908 e+03 \\
4.9567 e+03 \\
4.9226 e+03 \\
4.5736 e+03\end{array}$ & $\begin{array}{l}5.1954 e+03 \\
5.1272 e+03 \\
5.0863 e+03 \\
5.0522 e+03 \\
5.0181 e+03 \\
4.9840 e+03 \\
4.9499 e+03 \\
4.9158 e+03 \\
4.5668 e+03\end{array}$ & $\begin{array}{l}5.1818 e+03 \\
5.1136 e+03 \\
5.0795 e+03 \\
5.0454 e+03 \\
5.0113 e+03 \\
4.9772 e+03 \\
4.9431 e+03 \\
4.9090 e+03 \\
4.5599 e+03\end{array}$ & $\begin{array}{l}5.1682 e+03 \\
5.1068 e+03 \\
5.0727 e+03 \\
5.0386 e+03 \\
5.0045 e+03 \\
4.9704 e+03 \\
4.9363 e+03 \\
4.9022 e+03 \\
4.5531 e+03\end{array}$ & $\begin{array}{l}5.1545 e+03 \\
5.1000 e+03 \\
5.0659 e+03 \\
5.0318 e+03 \\
4.9977 e+03 \\
4.9636 e+03 \\
4.9295 e+03 \\
4.8954 e+03 \\
4.5463 e+03\end{array}$ \\
\hline $\begin{array}{r}\text { spht } \\
6.8902 e+02 \\
8.1759 e+02 \\
8.4708 e+02 \\
8.6475 e+02 \\
8.8136 e+02 \\
9.2001 e+02 \\
9.2001 e+02 \\
9.2001 e+02 \\
1.0321 e+03\end{array}$ & $\begin{array}{l}7.4512 e+02 \\
8.2702 e+02 \\
8.5074 e+02 \\
8.6813 e+02 \\
8.8461 e+02 \\
9.2001 e+02 \\
9.2001 e+02 \\
9.20011 e+02 \\
1.0321 e+03\end{array}$ & $\begin{array}{l}7.7433 e+02 \\
8.3549 e+02 \\
8.5433 e+02 \\
8.7148 e+02 \\
8.8783 e+02 \\
9.2001 e+02 \\
9.2001 e+02 \\
9.2001 e+02 \\
1.0321 e+03\end{array}$ & $\begin{array}{l}7.9297 e+02 \\
8.3947 e+02 \\
8.5786 e+02 \\
8.7480 e+02 \\
8.9105 e+02 \\
9.2001 e+02 \\
9.2001 e+02 \\
9.2001 e+02 \\
1.0321 e+03\end{array}$ & $\begin{array}{l}8.0661 e+02 \\
8.4333 e+02 \\
8.6133 e+02 \\
8.7809 e+02 \\
9.2001 e+02 \\
9.2001 e+02 \\
9.2001 e+02 \\
9.2001 e+02 \\
1.0321 e+03\end{array}$ \\
\hline & & & & \\
\hline
\end{tabular}

\&\& add nickel using physical properties of iron

\&\& for its part of steel

\&\& but also use an artificially large molecular weight to

\&\& get rid of diluent effect on the iron oxide equilibrium nid

debris molew $5.870 e+06$ temps 38

$3.0000 e+02$ $8.0000 e+02$

$1.1500 e+03$

$4.0000 e+02$ $9.0000 e+02$ $1.2000 \mathrm{e}+03$ $1.4500 e+03$

1. 4000 et03

$1.6500 \mathrm{e}+03$

$1.9000 e+03$

$2.1500 \mathrm{e}+03$

$2.4000 e+03$ condt

$4.3270 e+01$

3. $4093 e+01$

3. $0072 e+01$

$2.8095 e+01$

2. $6543 e+01$

2. $5279 e+01$

2. $4222 e+01$ entht $2.3317 \mathrm{e}+01$

$9.2944 \mathrm{e}+02$

$2.7961 e+05$

$1.7000 e+03$

$1.9500 \mathrm{e}+03$

$2.2000 e+03$

$2.4500 \mathrm{e}+03$

$4.7791 e+04$

$3.4866 e+05$
$5.0000 e+02$

$1.0000 e+03$

$1.2500 \mathrm{e}+03$

$1.5000 e+03$

$1.7500 \mathrm{e}+03$

$2.0000 e+03$

$2.2500 e+03$

$2.5000 e+03$

$6.0000 e+02$

$1.0500 \mathrm{e}+03$

$1.3000 e+03$

$1.5500 \mathrm{e}+03$

$1.8000 e+03$

$2.0500 e+03$

$2.3000 e+03$

$7.0000 e+02$

$1.1000 e+03$

$1.3500 e+03$

$1.6000 \mathrm{e}+03$

$1.8500 \mathrm{e}+03$

$2.1000 e+03$

$2.3500 e+03$

$3.5705 e+01$

$3.0538 e+01$

$2.8450 e+01$

$2.6827 e+01$

$2.5514 \mathrm{e}+01$

$2.4419 e+01$

$2.3488 e+01$

$2.4030 e+01 \quad 2.3844 e+01$

$2.3152 e+01 \quad 2.2991 e+01$

$9.9091 e+04$
$2.8824 e+01$

$2.7123 e+01$

$2.5756 e+01$

$2.4624 e+01$

$2.3663 e+01$

$1.5483 e+05$

$5.4867 e+05$
$2.1500 e+05$

$5.8240 e+05$ 
Table 15-3

Sequoyah Input File (Continued)

$\begin{array}{rllll}6.1613 e+05 & 6.6427 e+05 & 6.9256 e+05 & 7.2172 e+05 & 7.5176 e+05 \\ 7.8267 e+05 & 8.1445 e+05 & 8.4710 e+05 & 8.8063 e+05 & 9.1504 e+05 \\ 9.5031 e+05 & 1.0038 e+06 & 1.0431 e+06 & 1.0825 e+06 & 1.3693 e+06 \\ 1.4087 e+06 & 1.4483 e+06 & 1.4879 e+06 & 1.5276 e+06 & 1.5673 e+06 \\ 1.6072 e+06 & 1.6471 e+06 & 1.6871 e+06 & 1.7272 e+06 & 1.7673 e+06 \\ 1.8075 e+06 & 1.8478 e+06 & 1.8882 e+06 & & \\ \text { rhot } & & & & \\ 7.8824 e+03 & 7.8437 e+03 & 7.8050 e+03 & 7.7663 e+03 & 7.7276 e+03 \\ 7.6890 e+03 & 7.6503 e+03 & 7.6116 e+03 & 7.5923 e+03 & 7.5729 e+03 \\ 7.5536 e+03 & 7.5343 e+03 & 7.5149 e+03 & 7.4956 e+03 & 7.4762 e+03 \\ 7.4569 e+03 & 7.4376 e+03 & 7.4182 e+03 & 7.3989 e+03 & 7.3795 e+03 \\ 7.3602 e+03 & 7.3409 e+03 & 7.3215 e+03 & 7.3022 e+03 & 6.9768 e+03 \\ 6.9350 e+03 & 6.8932 e+03 & 6.8514 e+03 & 6.8096 e+03 & 6.7678 e+03 \\ 6.7260 e+03 & 6.6842 e+03 & 6.6425 e+03 & 6.6007 e+03 & 6.5589 e+03 \\ 6.5171 e+03 & 6.4753 e+03 & 6.4335 e+03 & & \\ \text { spht } & & & & \\ 4.4621 e+02 & 4.9058 e+02 & 5.3496 e+02 & 5.7933 e+02 & 6.2370 e+02 \\ 6.6808 e+02 & 7.1245 e+02 & 7.5682 e+02 & 6.7459 e+02 & 6.7459 e+02 \\ 6.7460 e+02 & 5.5689 e+02 & 5.7436 e+02 & 5.9182 e+02 & 6.0928 e+02 \\ 6.2675 e+02 & 6.4421 e+02 & 6.6168 e+02 & 6.7914 e+02 & 6.9661 e+02 \\ 7.1407 e+02 & 7.8703 e+02 & 7.8703 e+02 & 7.8703 e+02 & 7.8851 e+02 \\ 7.9001 e+02 & 7.9151 e+02 & 7.9301 e+02 & 7.9451 e+02 & 7.9601 e+02 \\ 7.9751 e+02 & 7.9901 e+02 & 8.0050 e+02 & 8.0200 e+02 & 8.0350 e+02 \\ 8.0500 e+02 & 8.0650 e+02 & 8.0800 e+02 & & \end{array}$

eoi

eoi

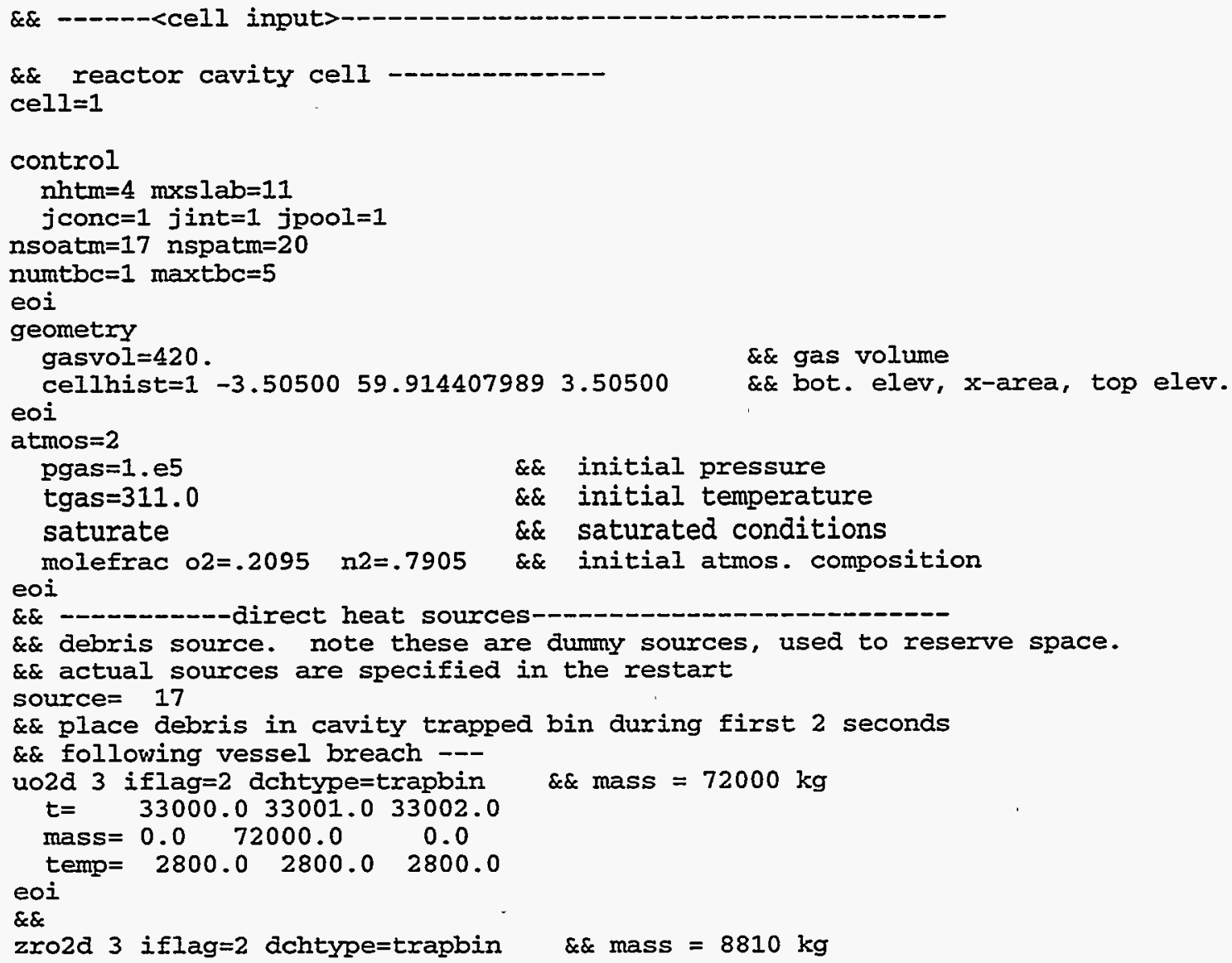


Table 15-3

Sequoyah Input File (Continued)

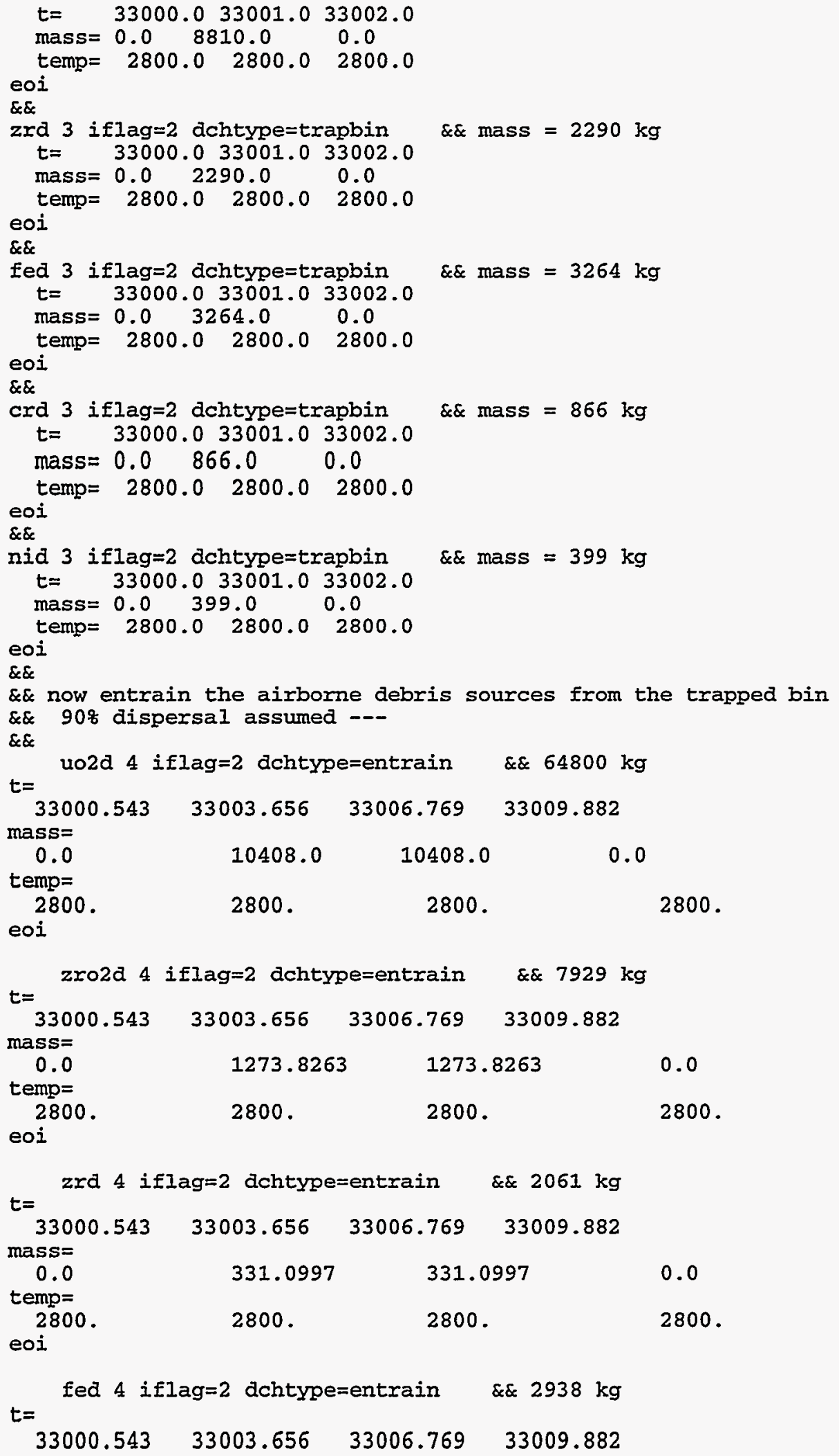

Rev. 0 
Table 15-3

Sequoyah Input File (Continued)

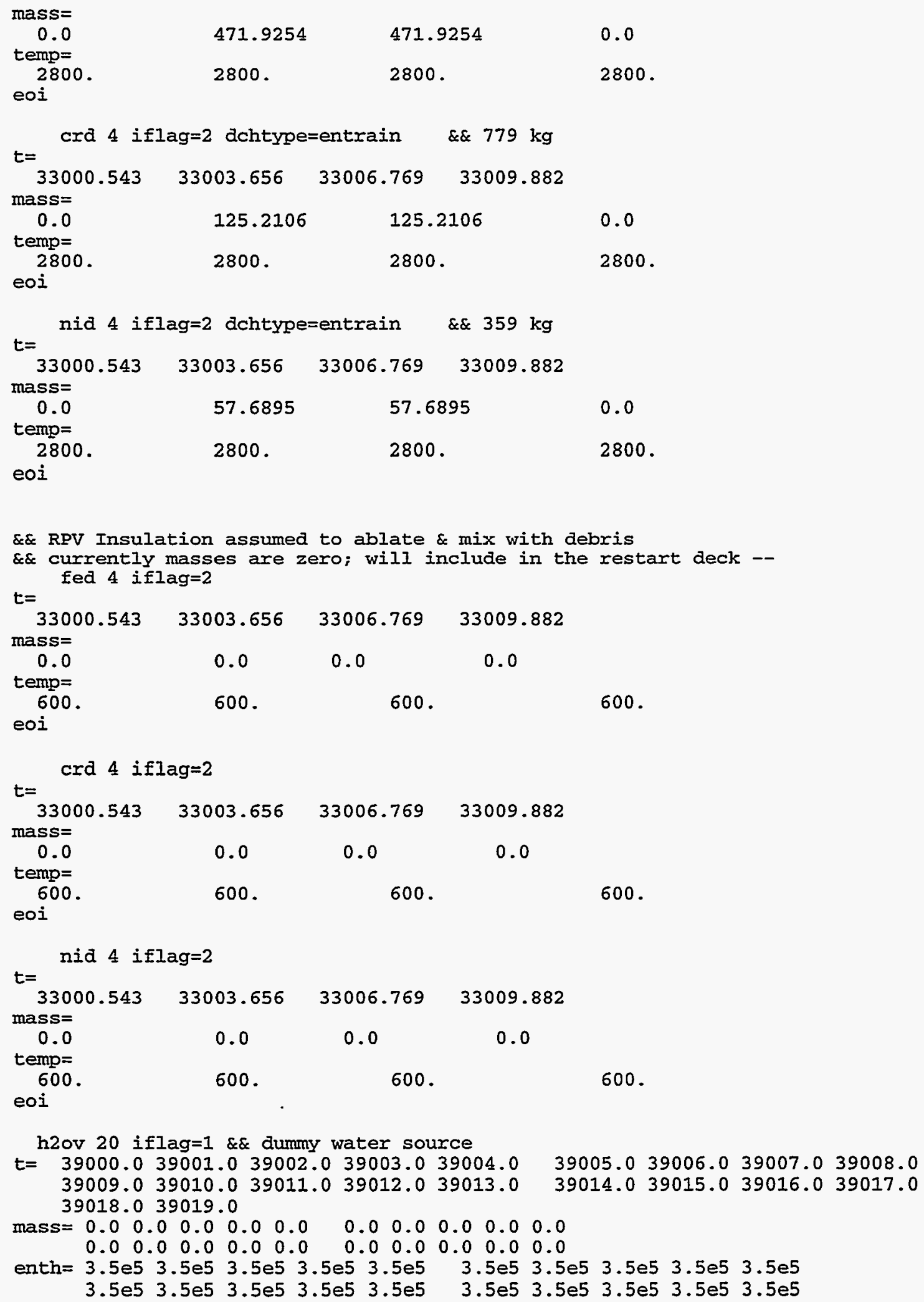


Table 15-3

Sequoyah Input File (Continued)

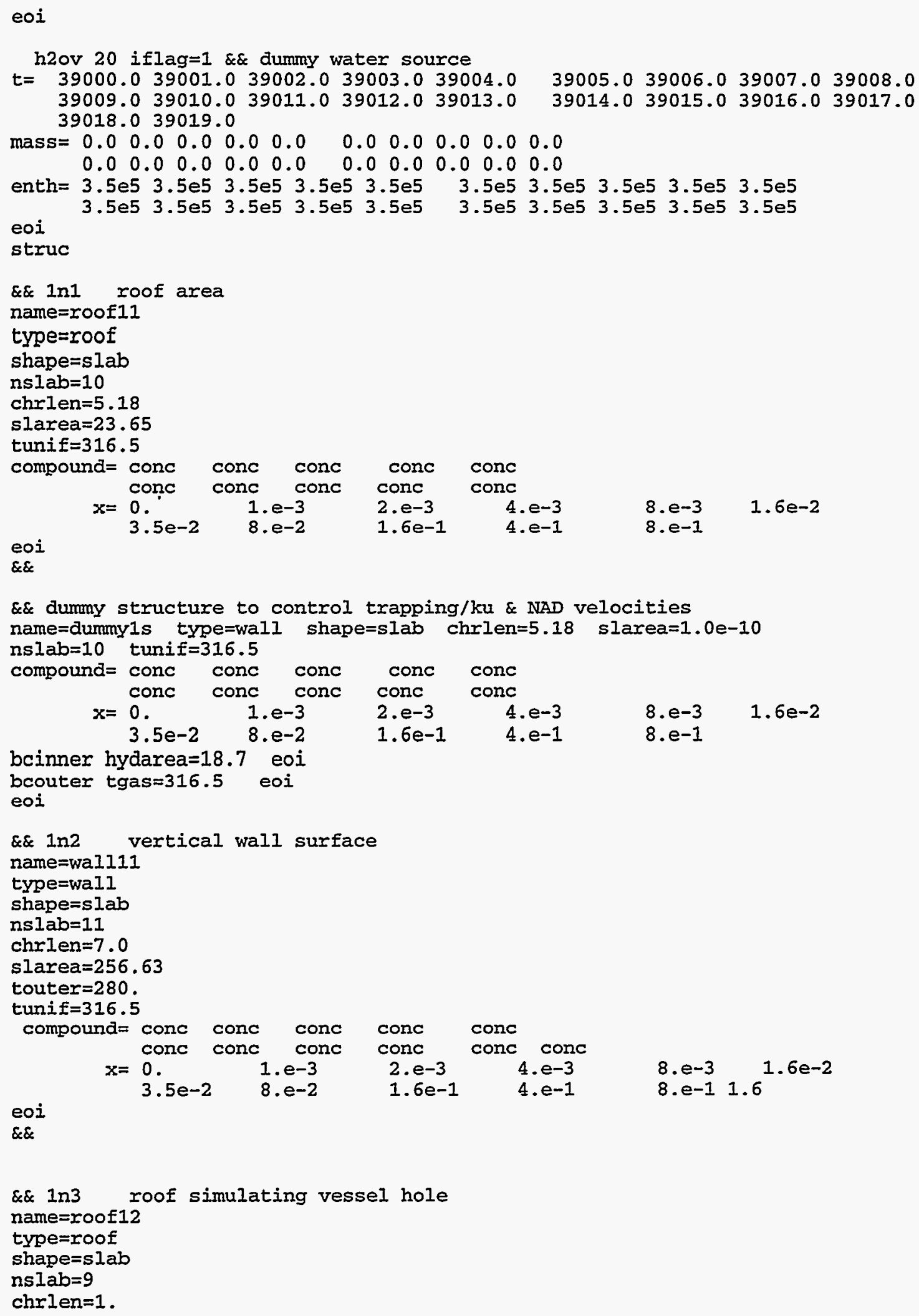

$\& \&$

\&\& in3 roof simulating vessel hole name $=\operatorname{roof} 12$

type $=$ roof shape $=s l a b$ nslab=9

chrlen=1. 


\section{Table 15-3}

\section{Sequoyah Input File (Continued)}

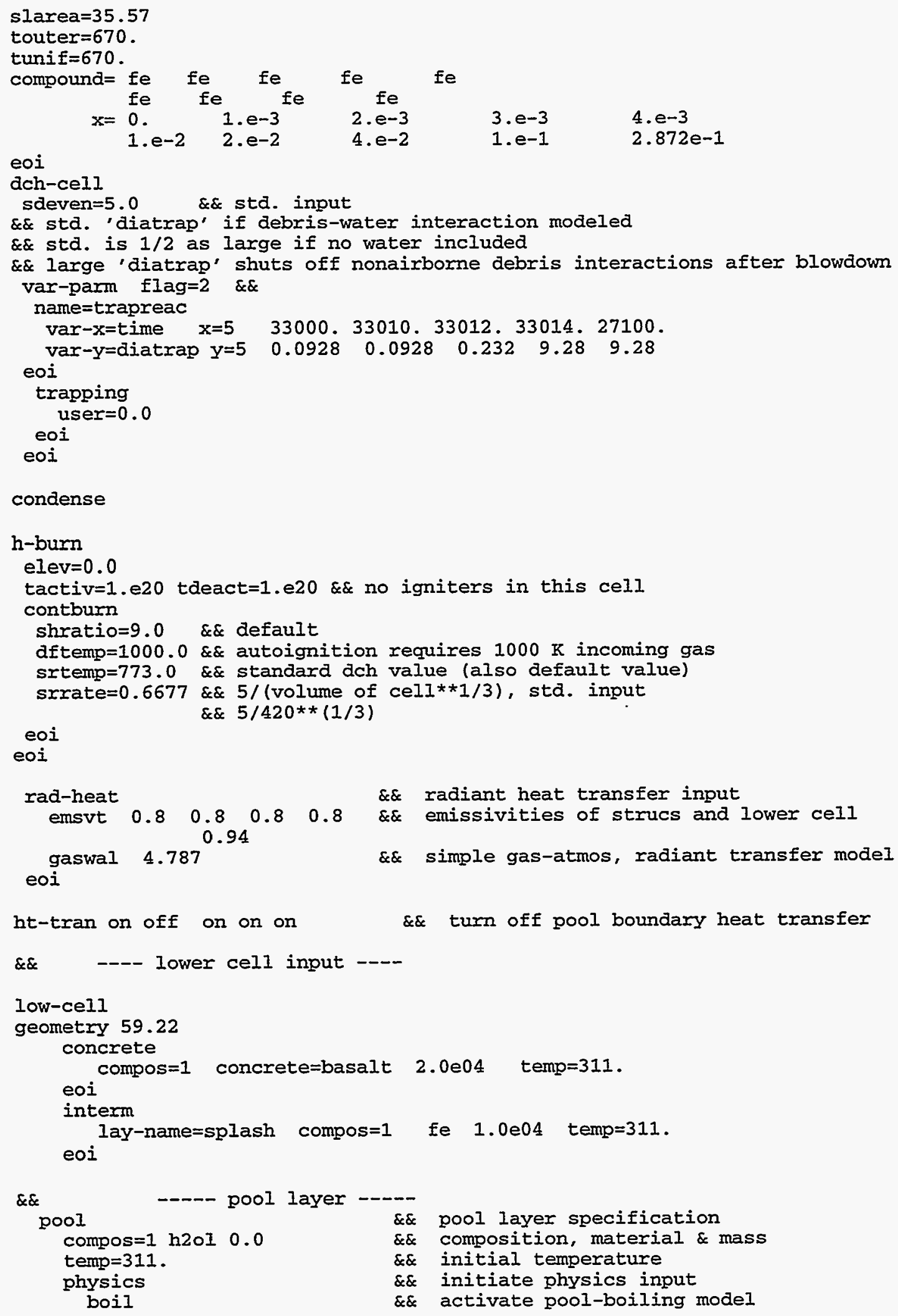


Table 15-3

Sequoyah Input File (Continued)

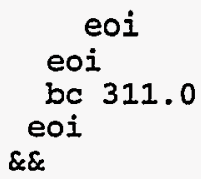

\&\& terminate physics

\&\& terminate pool-layer

$\& \&$ boundary condition temp.

\&\& terminate lower-cell input

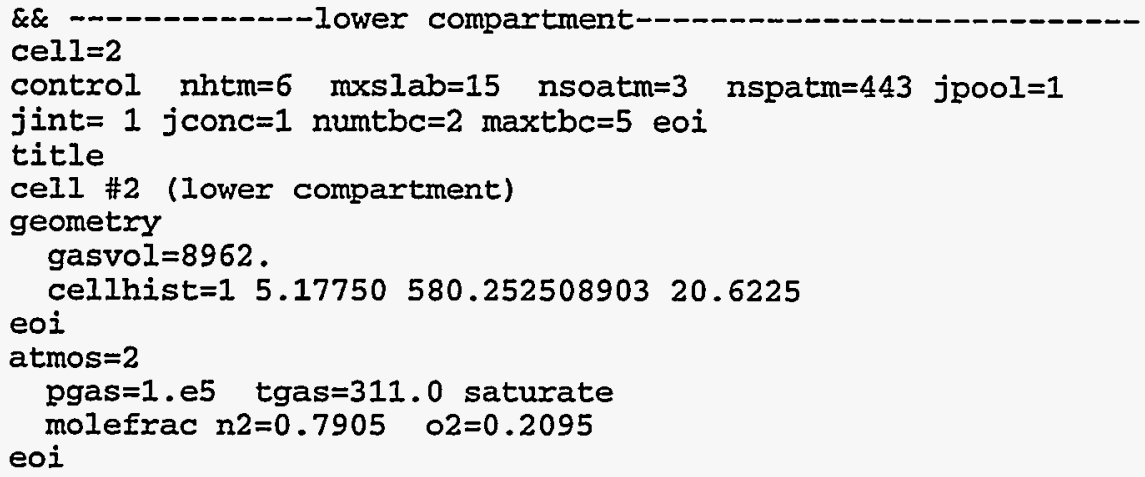

$4.5100000 e+03$

$4.7000000 \mathrm{e}+03$

$4.7250000 \mathrm{e}+03$

$4.7500000 \mathrm{e}+03$

$4.7750000 e+03$

$4.8000000 \mathrm{e}+03$

$4.8250000 \mathrm{e}+03$

$4.8500000 e+03$

$4.8750000 \mathrm{e}+03$

$4.9000000 e+03$

$4.9250000 \mathrm{e}+03$

$4.9500000 e+03$

$4.9750000 e+03$

$5.0000000 e+03$

$5.0250000 e+.03$

$5.0500000 e+03$

$5.0750000 e+03$

$5.1000000 e+03$

$5.1250000 e+03$

$5.1500000 \mathrm{e}+03$

$5.1750000 e+03$

$5.2000000 e+03$

$5.2250000 e+03$

$5.2500000 e+03$

$5.2750000 e+03$

$5.3000000 \mathrm{e}+03$

$5.3250000 e+03$

$5.3500000 \mathrm{e}+03$

$5.3750000 e+03$

$5.4000000 e+03$

$5.4250000 e+03$

$5.4500000 e+03$

$5.4750000 e+03$

$5.5000000 e+03$

$5.5250000 e+03$
$4.6800000 e+03$

$4.7050000 e+03$

$4.7300000 e+03$

$4.7550000 \mathrm{e}+03$

$4.7800000 e+03$

$4.8050000 \mathrm{e}+03$

$4.8300000 \mathrm{e}+03$

$4.8550000 e+03$

$4.8800000 e+03$

$4.9050000 e+03$

$4.9300000 \mathrm{e}+03$

$4.9550000 \mathrm{e}+03$

$4.9800000 e+03$

$5.0050000 \mathrm{e}+03$

$5.0300000 e+03$

$5.0550000 \mathrm{e}+03$

$5.0800000 e+03$

$5.1050000 \mathrm{e}+03$

$5.1300000 \mathrm{e}+03$

$5.1550000 \mathrm{e}+03$

$5.1800000 e+03$

$5.2050000 e+03$

$5.2300000 e+03$

$5.2550000 e+03$

$5.2800000 \mathrm{e}+03$

$5.3050000 \mathrm{e}+03$

$5.3300000 \mathrm{e}+03$

$5.3550000 e+03$

$5.3800000 \mathrm{e}+03$

$5.4050000 e+03$

$5.4300000 \mathrm{e}+03$

$5.4550000 e+03$

$5.4800000 \mathrm{e}+03$

$5.5050000 e+03$

$5.5300000 e+03$
$4.6850000 e+03$

$4.7100000 \mathrm{e}+03$

$4.7350000 e+03$

$4.7600000 e+03$

$4.7850000 \mathrm{e}+03$

$4.8100000 \mathrm{e}+03$

$4.8350000 e+03$

$4.8600000 e+03$

$4.8850000 \mathrm{e}+03$

$4.9100000 \mathrm{e}+03$

$4.9350000 e+03$

4.9600000 e +03

$4.9850000 e+03$

$5.0100000 e+03$

$5.0350000 e+03$

$5.0600000 \mathrm{e}+03$

$5.0850000 e+03$

$5.1100000 e+03$

$5.1350000 e+03$

5.1600000 et 03

$5.1850000 \mathrm{e}+03$

$5.2100000 e+03$

$5.2350000 \mathrm{e}+03$

$5.2600000 \mathrm{e}+03$

$5.2850000 \mathrm{e}+03$

$5.3100000 e+03$

$5.3350000 e+03$

$5.3600000 e+03$

$5.3850000 \mathrm{e}+03$

$5.4100000 e+03$

$5.4350000 \mathrm{e}+03$

$5.4600000 e+03$

$5.4850000 \mathrm{e}+03$

$5.5100000 \mathrm{e}+03$

$5.5350000 e+03$
$4.6900000 e+03$

$4.7150000 e+03$

$4.7400000 e+03$

$4.7650000 e+03$

$4.7900000 e+03$

$4.8150000 \mathrm{e}+03$

$4.8400000 e+03$

$4.8650000 \mathrm{e}+03$

$4.8900000 e+03$

$4.9150000 \mathrm{e}+03$

$4.9400000 e+03$

$4.9650000 \mathrm{e}+03$

$4.9900000 e+03$

$5.0150000 \mathrm{e}+03$

$5.0400000 e+03$

$5.0650000 e+03$

$5.0900000 \mathrm{e}+03$

$5.1150000 e+03$

$5.1400000 e+03$

$5.1650000 \mathrm{e}+03$

$5.1900000 e+03$

$5.2150000 e+03$

$5.2400000 e+03$

$5.2650000 e+03$

$5.2900000 e+03$

$5.3150000 e+03$

$5.3400000 e+03$

$5.3650000 e+03$

$5.3900000 e+03$

$5.4150000 e+03$

$5.4400000 e+03$

$5.4650000 e+03$

$5.4900000 e+03$

$5.5150000 e+03$

$5.5400000 e+03$
$4.6950000 e+03$

$4.7200000 e+03$

$4.7450000 \mathrm{e}+03$

4.7700000 e+03

$4.7950000 \mathrm{e}+03$

$4.8200000 e+03$

$4.8450000 \mathrm{e}+03$

$4.8700000 e+03$

$4.8950000 e+03$

$4.9200000 e+03$

$4.9450000 e+03$

$4.9700000 e+03$

$4.9950000 e+03$

$5.0200000 e+03$

$5.0450000 e+03$

$5.0700000 e+03$

$5.0950000 e+03$

$5.1200000 \mathrm{e}+03$

$5.1450000 \mathrm{e}+03$

$5.1700000 \mathrm{e}+03$

$5.1950000 \mathrm{e}+03$

$5.2200000 e+03$

$5.2450000 e+03$

$5.2700000 e+03$

$5.2950000 \mathrm{e}+03$

$5.3200000 e+03$

$5.3450000 e+03$

$5.3700000 e+03$

$5.3950000 e+03$

$5.4200000 e+03$

$5.4450000 e+03$

$5.4700000 e+03$

$5.4950000 e+03$

$5.5200000 e+03$

$5.5450000 e+03$ 
Table 15-3

Sequoyah Input File (Continued)

$5.5500000 e+03$ $5.5750000 e+03$ $5.6000000 e+03$ $5.6250000 e+03$ $5.6500000 e+03$ $5.6750000 e+03$ $5.7000000 e+03$ $5.7250000 \mathrm{e}+03$ $5.7500000 \mathrm{e}+03$ $5.7750000 \mathrm{e}+03$ $5.8000000 e+03$ $5.8250000 e+03$ $5.8500000 \mathrm{e}+03$ $5.8750000 e+03$ $5.9000000 e+03$ $5.9250000 \mathrm{e}+03$ $5.9500000 \mathrm{e}+03$ $5.9750000 e+03$ $6.0000000 e+03$ $6.0250000 e+03$ $6.0500000 \mathrm{e}+03$ $6.0750000 e+03$ $6.1000000 e+03$ $6.1250000 e+03$ $6.1500000 e+03$ $6.1750000 e+03$ $6.2000000 e+03$ $6.2250000 e+03$ $6.2500000 \mathrm{e}+03$ $6.2750000 \mathrm{e}+03$ $6.3000000 \mathrm{e}+03$ $6.3250000 \mathrm{e}+03$ $6.3500000 \mathrm{e}+03$ $6.3750000 e+03$

$6.4000000 e+03$ $6.4550000 e+03$ mass $=$

$1.64812 \mathrm{e}+00$

$4.18392 \mathrm{e}+01$

$5.10141 e+01$

5.10208e+01

$5.11628 e+01$

5.32720e+01

$5.52245 e+01$

$5.68448 \mathrm{e}+01$

$5.93766 e+01$

$6.23449 e+01$

6. 37747e+01

$6.46145 e+01$

$6.56279 e+01$

$6.70282 e+01$

$6.81875 e+01$

$6.77588 \mathrm{e}+01$

$6.78991 e+01$

$7.15836 e+01$

8.89772e+01

$9.21004 \mathrm{e}+01$

$9.35765 e+01$

$9.53352 \mathrm{e}+01$

$9.69784 \mathrm{e}+01$

$9.83158 \mathrm{e}+01$

$9.92134 \mathrm{e}+01$
$5.5550000 e+03$ $5.5800000 e+03$ $5.6050000 e+03$ $5.6300000 e+03$ $5.6550000 \mathrm{e}+03$ $5.6800000 e+03$ $5.7050000 e+03$ $5.7300000 e+03$ $5.7550000 \mathrm{e}+03$ $5.7800000 e+03$ $5.8050000 e+03$ $5.8300000 e+03$ $5.8550000 \mathrm{e}+03$ $5.8800000 \mathrm{e}+03$ $5.9050000 e+03$ $5.9300000 e+03$ $5.9550000 e+03$ $5.9800000 e+03$ $6.0050000 e+03$ $6.0300000 e+03$ $6.0550000 e+03$ $6.0800000 e+03$ $6.1050000 e+03$ $6.1300000 \mathrm{e}+03$ $6.1550000 \mathrm{e}+03$ $6.1800000 \mathrm{e}+03$ $6.2050000 e+03$ $6.2300000 e+03$ $6.2550000 \mathrm{e}+03$ $6.2800000 e+03$ $6.3050000 e+03$ $6.3300000 \mathrm{e}+03$ $6.3550000 \mathrm{e}+03$ $6.3800000 e+03$ $6.4050000 e+03$ $6.5550000 e+03$
$3.75145 e+01$ $4.39966 e+01$ $5.15472 e+01$ $5.08093 e+01$ $5.15079 e+01$ 5.37697 e+ 01 $5.55252 \mathrm{e}+01$ $5.72944 \mathrm{e}+01$ $5.99014 \mathrm{e}+01$ $6.28975 e+01$ $6.39397 e+01$ $6.47958 e+01$ $6.58415 e+01$ $6.73737 e+01$ $6.81812 e+01$ $6.76373 e+01$ $6.82344 \mathrm{e}+01$ $8.12831 e+01$ $8.97454 e+01$ $9.25631 e+01$ $9.38615 e+01$ $9.55610 \mathrm{e}+01$ $9.71438 e+01$ $9.86424 e+01$ $9.92332 e+01$

$5.5600000 e+03$ $5.5850000 \mathrm{e}+03$ 5. $6100000 e+03$ $5.6350000 \mathrm{e}+03$ $5.6600000 \mathrm{e}+03$ $5.6850000 \mathrm{e}+03$ $5.7100000 \mathrm{e}+03$ $5.7350000 \mathrm{e}+03$ $5.7600000 e+03$ $5.7850000 e+03$ 5.8100000 e +03 $5.8350000 e+03$ $5.8600000 e+03$ $5.8850000 e+03$ $5.9100000 e+03$ $5.9350000 e+03$ $5.9600000 \mathrm{e}+03$ $5.9850000 e+03$ $6.0100000 e+03$ $6.0350000 e+03$ $6.0600000 e+03$ $6.0850000 e+03$ $6.1100000 e+03$ $6.1350000 e+03$ $6.1600000 \mathrm{e}+03$ $6.1850000 \mathrm{e}+03$ $6.2100000 e+03$ $6.2350000 e+03$ $6.2600000 e+03$ $6.2850000 e+03$ $6.3100000 e+03$ $6.3350000 e+03$ $6.3600000 e+03$ $6.3850000 e+03$ $6.4100000 e+03$ $7.5300000 e+03$

$3.81634 \mathrm{e}+01$

$4.62164 \mathrm{e}+01$

$5.17118 \mathrm{e}+01$

$5.07376 e+01$

$5.18744 \mathrm{e}+01$

$5.42254 e+01$

$5.58271 e+01$

$5.78217 e+01$

$6.04436 e+01$

$6.32133 e+01$

$6.41081 \mathrm{e}+01$

$6.49810 e+01$

$6.60974 \mathrm{e}+01$

$6.77421 \mathrm{e}+01$

$6.81575 e+01$

$6.75755 e+01$

$6.86490 e+01$

$8.62033 e+01$

$9.05128 \mathrm{e}+01$

$9.27951 e+01$

$9.41781 e+01$

$9.60178 e+01$

$9.75471 \mathrm{e}+01$

$9.87536 e+01$

$9.92493 e+01$
$5.5650000 e+03$

$5.5900000 \mathrm{e}+03$

$5.6150000 \mathrm{e}+03$

$5.6400000 e+03$

$5.6650000 e+03$

$5.6900000 e+03$

5.7150000 e+03

5.7400000 e +03

$5.7650000 e+03$

$5.7900000 e+03$

$5.8150000 e+03$

$5.8400000 e+03$

$5.8650000 e+03$

$5.8900000 e+03$

$5.9150000 e+03$

$5.9400000 \mathrm{e}+03$

$5.9650000 e+03$

$5.9900000 \mathrm{e}+03$

$6.0150000 e+03$

$6.0400000 e+03$

$6.0650000 e+03$

$6.0900000 \mathrm{e} \div 03$

$6.1150000 \mathrm{e}+03$

$6.1400000 e+03$

$6.1650000 \mathrm{e}+03$

$6.1900000 e+03$

$6.2150000 \mathrm{e}+03$

$6.2400000 e+03$

$6.2650000 e+03$

$6.2900000 e+03$

$6.3150000 e+03$

$6.3400000 e+03$

$6.3650000 \mathrm{e}+03$

$6.3900000 e+03$

$6.4150000 \mathrm{e}+03$

$3.88083 e+01$

$4.82378 e+01$

5. $15905 e+01$

$5.07717 e+01$

$5.23065 e+01$

$5.46054 \mathrm{e}+01$

$5.61269 e+01$

$5.83458 e+01$

$6.10327 e+01$

$6.34431 e+01$

$6.42571 e+01$

$6.52088 \mathrm{e}+01$

$6.63773 e+01$

$6.80532 e+01$

$6.80780 e+01$

$6.76128 e+01$

$6.91989 e+01$

$8.71740 e+01$

$9.10565 e+01$

$9.30981 e+01$

$9.46222 e+01$

$9.63506 \mathrm{e}+01$

$9.79606 \mathrm{e}+01$

$9.87984 \mathrm{e}+01$

9.94855e+01

$5.5700000 e+03$

$5.5950000 e+03$

$5.6200000 e+03$

$5.6450000 e+03$

$5.6700000 e+03$

$5.6950000 e+03$

$5.7200000 \mathrm{e}+03$

$5.7450000 \mathrm{e}+03$

$5.7700000 e+03$

$5.7950000 e+03$

$5.8200000 e+03$

$5.8450000 e+03$

$5.8700000 e+03$

$5.8950000 e+03$

$5.9200000 e+03$

5.9450000 e+03

$5.9700000 e+03$

$5.9950000 e+03$

$6.0200000 e+03$

$6.0450000 e+03$

$6.0700000 e+03$

$6.0950000 \mathrm{e}+03$

$6.1200000 e+03$

$6.1450000 \mathrm{e}+03$

$6.1700000 \mathrm{e}+03$

$6.1950000 \mathrm{e}+03$

$6.2200000 \mathrm{e}+03$

$6.2450000 e+03$

$6.2700000 e+03$

$6.2950000 e+03$

$6.3200000 e+03$

$6.3450000 e+03$

$6.3700000 \mathrm{e}+03$

$6.3950000 \mathrm{e}+03$

$6.4250000 \mathrm{e}+03$

$4.00378 e+01$

4. $99197 e+01$

$5.13187 e+01$

$5.08906 e+01$

$5.27753 e+01$

$5.49258 e+01$

$5.64654 \mathrm{e}+01$

$5.88548 e+01$ 
Table 15-3

Sequoyah Input File (Continued)

\begin{tabular}{|c|c|c|}
\hline $\begin{array}{l}.93685 e+01 \\
.91354 e+01 \\
.85674 e+01 \\
.81128 e+01 \\
.76439 e+01 \\
.74849 e+01 \\
.69474 e+01 \\
.53347 e+01 \\
.56330 e+01 \\
.61959 e+01 \\
.55072 e+01 \\
.30735 e+01 \\
.83708 e+01 \\
.66329 e+01 \\
.46009 e+01 \\
.36380 e+01 \\
.30004 e+01 \\
.26639 e+01 \\
.25733 e+01 \\
.23404 e+01 \\
.25089 e+01 \\
.27759 e+01 \\
.34015 e+01 \\
.39843 e+01 \\
.44521 e+01 \\
.51923 e+01 \\
.55696 e+01 \\
.60173 e+01 \\
.62807 e+01 \\
.68165 e+01 \\
.64060 e+01 \\
.50691 e+01 \\
.36816 e+01 \\
.25698 e+01 \\
.14835 e+01 \\
.06971 e+01 \\
.00737 e+01 \\
.73408 e+01 \\
.43721 e+01 \\
.10444 e+01 \\
.74266 e+01 \\
.37510 e+01 \\
.76946 e+01 \\
.10072 e+00 \\
n t h= \\
.31819 e+06 \\
.31436 e+06 \\
.31469 e+06 \\
.31332 e+06 \\
.31225 e+06 \\
.31203 e+06 \\
.31204 e+06 \\
.31220 e+06 \\
.31300 e+06 \\
.31421 e+06 \\
.31541 e+06 \\
.31667 e+06 \\
.31817 e+06 \\
.32023 e+06 \\
.32219 e+06\end{array}$ & $\begin{array}{l}9.92974 e+01 \\
9.90477 e+01 \\
9.84555 e+01 \\
9.81341 e+01 \\
9.76223 e+01 \\
9.73879 e+01 \\
9.67298 e+01 \\
9.49070 e+01 \\
9.57155 e+01 \\
9.65445 e+01 \\
7.37132 e+01 \\
7.23158 e+01 \\
6.79531 e+01 \\
6.63762 e+01 \\
6.44169 e+01 \\
6.33281 e+01 \\
6.28370 e+01 \\
6.25742 e+01 \\
6.27168 e+01 \\
6.23383 e+01 \\
6.24542 e+01 \\
6.28089 e+01 \\
6.35314 e+01 \\
6.40625 e+01 \\
6.46149 e+01 \\
6.53764 e+01 \\
6.56595 e+01 \\
6.61136 e+01 \\
6.65435 e+01 \\
6.69117 e+01 \\
6.62372 e+01 \\
6.47260 e+01 \\
6.34079 e+01 \\
6.22188 e+01 \\
6.12740 e+01 \\
6.05919 e+01 \\
5.99209 e+01 \\
5.65442 e+01 \\
5.37049 e+01 \\
5.03728 e+01 \\
4.67004 e+01 \\
4.31584 e+01 \\
3.40593 e+01 \\
2.40773 e-01 \\
1.31458 e+06 \\
1.31452 e+06 \\
1.31452 e+06 \\
1.31306 e+06 \\
1.31215 e+06 \\
1.31206 e+06 \\
1.31206 e+06 \\
1.31234 e+06 \\
1.31321 e+06 \\
1.31446 e+06 \\
1.31565 e+06 \\
1.31694 e+06 \\
1.31846 e+06 \\
1.32079 e+06 \\
1.32230 e+06\end{array}$ & $\begin{array}{l}9.92550 e+01 \\
9.88672 e+01 \\
9.81438 e+01 \\
9.80017 e+01 \\
9.75883 e+01 \\
9.72158 e+01 \\
9.64230 e+01 \\
9.48466 e+01 \\
9.58669 e+01 \\
9.68211 e+01 \\
7.56770 e+01 \\
7.10291 e+01 \\
6.74770 e+01 \\
6.58712 e+01 \\
6.42325 e+01 \\
6.31654 e+01 \\
6.26868 e+01 \\
6.25094 e+01 \\
6.27240 e+01 \\
6.24250 e+01 \\
6.25783 e+01 \\
6.28874 e+01 \\
6.36640 e+01 \\
6.41168 e+01 \\
6.47902 e+01 \\
6.54926 e+01 \\
6.57385 e+01 \\
6.60807 e+01 \\
6.67002 e+01 \\
6.69391 e+01 \\
6.60570 e+01 \\
6.43533 e+01 \\
6.32194 e+01 \\
6.19391 e+01 \\
6.11638 e+01 \\
6.04343 e+01 \\
5.95184 e+01 \\
5.58502 e+01 \\
5.29724 e+01 \\
4.98343 e+01 \\
4.59417 e+01 \\
4.25141 e+01 \\
3.33808 e+01 \\
0.00000 e+00 \\
1.31440 e+06 \\
1.31469 e+06 \\
1.31426 e+06 \\
1.31283 e+06 \\
1.31207 e+06 \\
1.31207 e+06 \\
1.31208 e+06 \\
1.31248 e+06 \\
1.31344 e+06 \\
1.31469 e+06 \\
1.31591 e+06 \\
1.31720 e+06 \\
1.31881 e+06 \\
1.32135 e+06 \\
1.32244 e+06\end{array}$ \\
\hline
\end{tabular}

$9.94611 e+01$

95541e+01

$9.85674 \mathrm{e}+01$

$9.81128 \mathrm{e}+01$

$76439 e+01$

$9.69474 e+01$

$9.53347 e+01$

$9.56330 e+01$

$7.30735 e+01$

$6.83708 \mathrm{e}+01$

$6.36380 e+01$

$6.30004 e+01$

$26639 e+01$

$6.25089 e+01$

$6.27759 \mathrm{e}+01$

$6.44521 e+01$

$6.51923 e+01$

$55696 e+01$

$6.68165 e+01$

$6.64060 e+01$

$6.25698 \mathrm{e}+01$

$6.14835 e+01$

$06971 e+01$

$5.43721 e+01$

$5.10444 \mathrm{e}+01$

$4.74266 \mathrm{e}+01$

$4.37510 e+01$

enth=

$1.31819 \mathrm{e}+06$

$1.31436 e+06$

$1.31469 e+06$

1. $31332 \mathrm{e}+06$

$1.312030+06$

1. $31204 e+06$

$1.31220 e+06$

$1.31300 \mathrm{e}+06$

$1.31421 e+06$

$1.31817 \mathrm{e}+06$

$1.32219 e+06$
$6 e+01$

$9.90477 e+01$

$9.84555 e+01$

$.81341 e+01$

$6223 e+01$

$672980+01$

. $49070 \mathrm{e}+01$

$57155 e+01$

$6.63762 \mathrm{e}+01$

$6.44169 e+01$

$281 e+01$

$.27168 e+01$

$6.23383 e+01$

$6.24542 e+01$

$28089 e+01$

$6.46149 \mathrm{e}+01$

$6.53764 e+01$

$6.56595 \mathrm{e}+01$

$6.69117 e+01$

$6.62372 \mathrm{e}+01$

6. $22188 e+01$

$6.12740 e+01$

$6.05919 e+01$

$5.99209 e+01$

$5.65442 \mathrm{e}+01$

$3.40593 e+01$

$1.31458 \mathrm{e}+06$

$1.31452 e+06$

$1.31452 \mathrm{e}+06$

1. $31206 \mathrm{e}+06$

1. $31206 e+06$

1. 31234 e+06

$1.31565 \mathrm{e}+06$

$1.31694 \mathrm{e}+06$

$1.32079 e+06$

$1.32230 e+06$
$9.94966 e+01$

$9.93916 e+01$

$9.92057 e+01$

$9.87893 e+01$

$9.81663 e+01$

$9.79220 e+01$

$9.75076 e+01$

$9.72348 e+01$

$9.61356 e+01$

$9.50619 e+01$

$9.62164 e+01$

$9.68699 e+01$

$7.46816 e+01$

$6.97102 e+01$

$6.70938 e+01$

$6.53343 e+01$

$6.40369 e+01$

$6.30931 e+01$

$6.26920 \mathrm{e}+01$

$6.23904 e+01$

$6.26907 e+01$

$6.24122 e+01$

$6.27207 e+01$

$6.30492 e+01$

$6.37527 e+01$

$6.42435 e+01$

$6.48631 e+01$

$6.55938 e+01$

$6.58146 e+01$

$6.59962 e+01$

$6.67546 e+01$

$6.69285 e+01$

$6.57742 \mathrm{e}+01$

$6.40563 e+01$

$6.30367 e+01$

$6.18139 e+01$

$6.10462 e+01$

$6.02713 e+01$

$5.88818 e+01$

$5.53489 e+01$

$5.25094 e+01$

$4.91111 e+01$

$4.51338 e+01$

$4.18808 \mathrm{e}+01$

$3.05310 e+01$

$1.31424 e+06$

$1.31479 e+06$

$1.31395 e+06$

$1.31260 e+06$

$1.31203 e+06$

$1.31206 e+06$

1. $31211 e+06$

$1.31263 e+06$

$1.31369 e+06$

$1.31496 e+06$

$1.31614 \mathrm{e}+06$

$1.31754 \mathrm{e}+06$

$1.31923 e+06$

$1.32180 e+06$

1.32264e+06
$9.94796 e+01$

$9.94651 e+01$

$9.92293 e+01$

$9.86439 e+01$

$9.81508 e+01$

$9.77797 e+01$

$9.75054 e+01$

$9.71424 \mathrm{e}+01$

$9.57196 e+01$

$9.52946 e+01$

$9.61721 e+01$

$9.82519 e+01$

$7.37618 e+01$

$6.89029 e+01$

$6.68330 \mathrm{e}+01$

$6.49132 \mathrm{e}+01$

$6.38827 e+01$

$6.30737 e+01$

$6.27450 e+01$

$6.23868 e+01$

$6.25487 e+01$

$6.24806 e+01$

$6.27532 e+01$

$6.32424 e+01$

$6.38376 e+01$

$6.43460 e+01$

$6.49607 e+01$

$6.55820 e+01$

$6.59291 e+01$

$6.60912 e+01$

$6.67638 e+01$

$6.66993 e+01$

$6.54133 e+01$

$6.39000 e+01$

$6.28272 e+01$

$6.16852 e+01$

$6.08494 e+01$

$6.01731 e+01$

$5.81382 e+01$

$5.48612 e+01$

$5.19088 e+01$

$4.82596 e+01$

4. $44151 e+01$

$4.14541 e+01$

$9.49465 e+00$

$1.31425 e+06$

$1.31479 e+06$

$1.31362 \mathrm{e}+06$

$1.31240 \mathrm{e}+06$

1. 31201 e+ 06

1. $31204 \mathrm{e}+06$

1. $31214 \mathrm{e}+06$

1. $31281 e+06$

$1.31395 e+06$

$1.31521 \mathrm{e}+06$

$1.31638 \mathrm{e}+06$

$1.31789 e+06$

$1.31971 \mathrm{e}+06$

$1.32205 e+06$

1. 32291 e+06

Rev. 0 
Table 15-3

Sequoyah Input File (Continued)

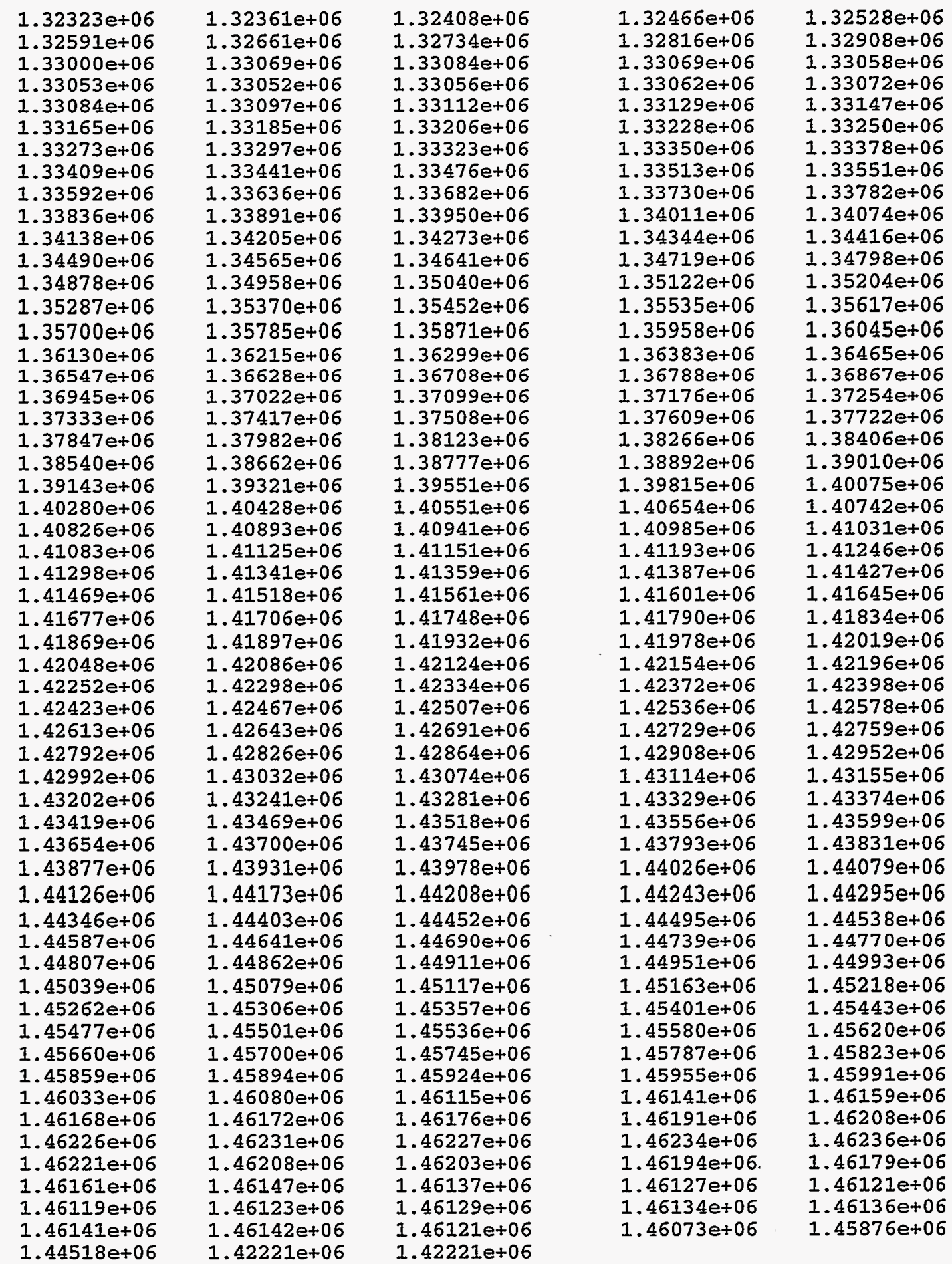

eoi

\&\& INEL Surry steam source, dp on SG dryout, no surge line break, scale to Seq. h2ov 443 iflag $=1$

$t=$

$4.5100000 e+03$

$4.5500000 \mathrm{e}+03$
$4.5200000 e+03$

$4.5600000 \mathrm{e}+03$
$4.5250000 e+03$

$4.5700000 e+03$
$4.5300000 e+03$

$4.5800000 e+03$
$4.5400000 e+03$

$4.5900000 e+03$ 
Table 15-3

Sequoyah Input File (Continued)

$4.6000000 e+03$

$4.6900000 \mathrm{e}+03$

$5.9500000 e+03$

$6.3150000 e+03$

$6.4400000 e+03$

$6.4900000 \mathrm{e}+03$

$6.5400000 e+03$

$6.5900000 e+03$

$6.6400000 e+03$

$6.6900000 e+03$

$6.7400000 e+03$

$6.7900000 e+03$

$6.8400000 e+03$

$6.8900000 e+03$

$6.9400000 e+03$

$6.9900000 e+03$

$7.0600000 e+03$

7.1350000 e+03

$7.2100000 e+03$

$7.2850000 e+03$

$7.3600000 e+03$

$7.4350000 e+03$

$7.5100000 \mathrm{e}+03$

$7.5850000 e+03$

$7.6800000 e+03$

$7.7800000 e+03$

$7.8800000 e+03$

$7.9850000 e+03$

$8.1035000 e+03$

$8.2384902 e+03$

$8.3779902 e+03$

$8.4994902 e+03$

$8.6399902 e+03$

$8.7949902 e+03$

$8.9749902 e+03$

$9.1799902 \mathrm{e}+03$

$9.3899902 e+03$

$9.5899902 e+03$

$9.7649902 e+03$

$9.9399902 e+03$

$1.0140000 e+04$

$1.0350000 \mathrm{e}+04$

$1.0640000 \mathrm{e}+04$

$1.0925000 e+04$

$1.1215000 \mathrm{e}+04$

$1.1490000 \mathrm{e}+04$

$1.1750000 \mathrm{e}+04$

$1.2020000 \mathrm{e}+04$

$1.2245000 e+04$

$1.2475000 \mathrm{e}+04$

$1.2730000 \mathrm{e}+04$

$1.2995000 \mathrm{e}+04$

$1.3200000 \mathrm{e}+04$

$1.3425000 \mathrm{e}+04$

$1.3665000 \mathrm{e}+04$

$1.3930000 \mathrm{e}+04$

$1.4235000 e+04$

$1.4510000 \mathrm{e}+04$

$1.4790000 \mathrm{e}+04$

$1.5100000 \mathrm{e}+04$

$1.5465000 \mathrm{e}+04$

1.5910000 e+04
4. $6150000 e+03$

$4.8000000 e+03$

$6.0450000 \mathrm{e}+03$

$6.3550000 \mathrm{e}+03$

$6.4500000 e+03$

$6.5000000 \mathrm{e}+03$

$6.5500000 \mathrm{e}+03$

$6.6000000 e+03$

$6.6500000 \mathrm{e}+03$

$6.7000000 e+03$

$6.7500000 e+03$

$6.8000000 \mathrm{e}+03$

$6.8500000 e+03$

$6.9000000 e+03$

$6.9500000 \mathrm{e}+03$

$7.0000000 e+03$

$7.0750000 \mathrm{e}+03$

$7.1500000 \mathrm{e}+03$

7.2250000 e+03

$7.3000000 \mathrm{e}+03$

$7.3750000 \mathrm{e}+03$

$7.4500000 \mathrm{e}+03$

$7.5250000 e+03$

$7.6000000 e+03$

$7.7000000 e+03$

$7.8000000 e+03$

$7.9000000 e+03$

$8.0090000 e+03$

$8.1305000 \mathrm{e}+03$

$8.2654902 \mathrm{e}+03$

$8.4004902 e+03$

$8.5264902 \mathrm{e}+03$

$8.6699902 e+03$

$8.8299902 \mathrm{e}+03$

$9.0149902 \mathrm{e}+03$

$9.2199902 \mathrm{e}+03$

$9.4299902 e+03$

$9.6249902 e+03$

$9.7999902 e+03$

$9.9799902 e+03$

$1.0170000 e+04$

$1.0405000 e+04$

$1.0695000 \mathrm{e}+04$

$1.0980000 \mathrm{e}+04$

1.1275000 e+ 04

$1.1540000 \mathrm{e}+04$

1.1805000 e+04

$1.2065000 e+04$

$1.2290000 e+04$

1.2525000 e+04

1.2785000 e+04

$1.3040000 \mathrm{e}+04$

$1.3245000 \mathrm{e}+04$

1.3470000 e+04

$1.3715000 e+04$

$1.3995000 e+04$

$1.4290000 \mathrm{e}+04$

$1.4565000 e+04$

1.4850000 e+04

$1.5170000 e+04$

$1.5550000 \mathrm{e}+04$

1. $6010000 \mathrm{e}+04$
$4.6300000 e+03$

$5.6600000 \mathrm{e}+03$

$6.1350000 e+03$

$6.3900000 \mathrm{e}+03$

$6.4600000 e+03$

$6.5100000 \mathrm{e}+03$

$6.5600000 \mathrm{e}+03$

$6.6100000 \mathrm{e}+03$

$6.6600000 e+03$

$6.7100000 \mathrm{e}+03$

$6.7600000 e+03$

$6.8100000 e+03$

$6.8600000 e+03$

$6.9100000 e+03$

$6.9600000 \mathrm{e}+03$

$7.0150000 \mathrm{e}+03$

$7.0900000 \mathrm{e}+03$

$7.1650000 e+03$

7.2400000 e +03

$7.3150000 \mathrm{e}+03$

$7.3900000 e+03$

$7.4650000 \mathrm{e}+03$

$7.5400000 \mathrm{e}+03$

$7.6200000 e+03$

$7.7200000 e+03$

$7.8200000 e+03$

$7.9200000 e+03$

$8.0315000 e+03$

$8.1575000 e+03$

$\because .2924902 \mathrm{e}+03$

$3.4229902 e+03$

$8.5534902 \mathrm{e}+03$

$8.6999902 e+03$

$8.8649902 e+03$

$9.0549902 e+03$

$9.2599902 \mathrm{e}+03$

$9.4699902 e+03$

$9.6599902 e+03$

$9.8349902 e+03$

$1.0020000 \mathrm{e}+04$

$1.0205000 e+04$

$1.0465000 e+04$

$1.0755000 \mathrm{e}+04$

$1.1035000 e+04$

$1.1330000 \mathrm{e}+04$

$1.1590000 e+04$

1.1865000 e +04

$1.2110000 e+04$

$1.2335000 \mathrm{e}+04$

$1.2575000 \mathrm{e}+04$

$1.2840000 \mathrm{e}+04$

$1.3080000 \mathrm{e}+04$

$1.3290000 \mathrm{e}+04$

$1.3515000 \mathrm{e}+04$

$1.3765000 \mathrm{e}+04$

$1.4065000 \mathrm{e}+04$

$1.4345000 \mathrm{e}+04$

$1.4620000 \mathrm{e}+04$

$1.4910000 e+04$

$1.5240000 \mathrm{e}+04$

$1.5635000 e+04$

1. $6120000 \mathrm{e}+04$
$4.6450000 e+03$

$5.7700000 e+03$

$6.2050000 e+03$

$6.4150000 e+03$

$6.4700000 e+03$

$6.5200000 \mathrm{e}+03$

$6.5700000 e+03$

$6.6200000 e+03$

$6.6700000 \mathrm{e}+03$

$6.7200000 e+03$

$6.7700000 \mathrm{e}+03$

$6.8200000 \mathrm{e}+03$

$6.8700000 e+03$

$6.9200000 e+03$

$6.9700000 \mathrm{e}+03$

$7.0300000 e+03$

$7.1050000 e \div 03$

$7.1800000 \mathrm{e}+03$

$7.2550000 \mathrm{e}+03$

$7.3300000 \mathrm{e}+03$

$7.4050000 \mathrm{e}+03$

$7.4800000 e+03$

7.5550000 e +03

7.6400000 et 03

$7.7400000 e+03$

$7.8400000 \mathrm{e}+03$

7.9400000 e+03

$8.0540000 \mathrm{e}+03$

$8.1845000 e+03$

$8.3239902 \mathrm{e}+03$

$8.4454902 e+03$

$8.5804902 e+03$

$8.7299902 e+03$

$8.8999902 e+03$

$9.0949902 e+03$

$9.2999902 \mathrm{e}+03$

$9.5149902 \mathrm{e}+03$

$9.6949902 e+03$

$9.8699902 \mathrm{e}+03$

$1.0060000 e+04$

$1.0250000 \mathrm{e}+04$

1. $0525000 \mathrm{e}+04$

$1.0815000 \mathrm{e}+04$

$1.1095000 \mathrm{e}+04$

$1.1385000 \mathrm{e}+04$

$1.1640000 \mathrm{e}+04$

1.1925000 e+ 04

$1.2155000 e+04$

1.2380000 et 04

$1.2625000 \mathrm{e}+04$

$1.2900000 \mathrm{e}+04$

$1.3120000 \mathrm{e}+04$

$1.3335000 \mathrm{e}+04$

$1.3565000 \mathrm{e}+04$

1.3820000 e +04

$1.4125000 e+04$

1.4400000 e +04

$1.4675000 e+04$

$1.4970000 \mathrm{e}+04$

$1.5310000 \mathrm{e}+04$

$1.5725000 \mathrm{e}+04$

$1.6175000 \mathrm{e}+04$

$4.6600000 e+03$

$5.8600000 \mathrm{e}+03$

$6.2650000 \mathrm{e}+03$

$6.4300000 e+03$

$6.4800000 e+03$

$6.5300000 \mathrm{e}+03$

$6.5800000 e+03$

$6.6300000 e+03$

$6.6800000 e+03$

$6.7300000 e+03$

$6.7800000 e+03$

$6.8300000 e+03$

$6.8800000 e+03$

$6.9300000 \mathrm{e}+03$

$6.9800000 \mathrm{e}+03$

$7.0450000 e+03$

$7.1200000 e+03$

$7.1950000 \mathrm{e}+03$

$7.2700000 e+03$

$7.3450000 \mathrm{e}+03$

$7.4200000 e+03$

$7.4950000 \mathrm{e}+03$

$7.5700000 \mathrm{e}+03$

$7.6600000 e+03$

$7.7600000 e+03$

$7.8600000 e+03$

$7.9600000 \mathrm{e}+03$

$8.0765000 e+03$

$8.2114902 e+03$

$8.3554902 e+03$

$8.4724902 e+03$

$8.6099902 \mathrm{e}+03$

$8.7599902 e+03$

$8.9349902 \mathrm{e}+03$

$9.1399902 e+03$

$9.3449902 e+03$

$9.5549902 \mathrm{e}+03$

$9.7299902 e+03$

$9.9049902 e+03$

1. $0100000 \mathrm{e}+04$ 
Table 15-3

Sequoyah Input File (Continued)

$1.6270000 e+04$

$1.6554000 e+04$

$1.6890000 e+04$

$1.7310000 e+04$

$1.7838000 \mathrm{e}+04$

$1.8264000 \mathrm{e}+04$

$1.8572000 e+04$

$1.8972000 \mathrm{e} \div 04$

1. $9484000 \mathrm{e}+04$

$2.0148000 e+04$

$2.0596000 e+04$

$2.0764000 e+04$

$2.0996000 e+04$

$2.1324000 e+04$

$2.1772000 e+04$

$2.2332000 e+04$

$2.3036000 e+04$

$2.4036000 e+04$

$2.5100000 e+04$

$2.6507699 e+04$

$2.7355000 e+04$

$2.7760000 e+04$

2.8375000 e +04

$2.9475000 e+04$

$3.1880000 \mathrm{e}+04$ mass $=$

$2.06261 e+01$

$3.98052 e+01$

$2.95703 e+01$

$4.11329 e+00$

$4.83209 e+00$

$1.07355 e+01$

$3.56992 \mathrm{e}+01$

$3.66679 e+01$

$3.47219 e+01$

$3.44213 e+01$

$3.34997 e+01$

$3.18477 \mathrm{e}+01$

$3.27707 e+01$

3. $32608 \mathrm{e}+01$

$3.27007 e+01$

3. $21146 e+01$

$3.15275 e+01$

3. $09423 e+01$

3. $01511 \mathrm{e}+01$

$2.92958 e+01$

$2.83832 \mathrm{e}+01$

$2.73624 \mathrm{e}+01$

$2.63136 e+01$

$2.52959 e+01$

$2.42677 e+01$

$2.32238 e+01$

$2.19682 \mathrm{e}+01$

$2.04966 e+01$

$1.92103 e+01$

$1.80630 \mathrm{e}+01$

$1.66612 \mathrm{e}+01$

$1.51234 \mathrm{e}+01$

$1.93631 \mathrm{e}+01$

$1.66743 e+01$

$1.45599 \mathrm{e}+01$

$1.21565 e+01$
$1.6325000 \mathrm{e}+04$

$1.6614000 \mathrm{e}+04$

$1.6962000 \mathrm{e}+04$

$1.7412000 \mathrm{e}+04$

1.7964000 e +04

$1.8316000 \mathrm{e}+04$

$1.8644000 \mathrm{e}+04$

$1.9060000 \mathrm{e}+04$

$1.9596000 \mathrm{e}+04$

$2.0308000 e+04$

$2.0628000 e+04$

$2.0804000 \mathrm{e}+04$

$2.1052000 \mathrm{e}+04$

$2.1404000 \mathrm{e}+04$

$2.1876000 e+04$

$2.2460000 e+04$

$2.3204000 e+04$

$2.4284000 \mathrm{e}+04$

$2.5364000 e+04$

$2.7100000 e+04$

$2.7425000 e+04$

$2.7860000 e+04$

$2.8545000 e+04$

$2.9805000 e+04$

3.2590000e+04

$6.74371 e+01$

3. $74194 \mathrm{e}+01$

$2.86435 e+01$

$5.32908 \mathrm{e}-01$

$4.96987 \mathrm{e}+00$

1. $25547 \mathrm{e}+01$

3.43191 e+01

$3.57980 \mathrm{e}+01$

$3.45490 e+01$

3. $35812 \mathrm{e}+01$

$3.37327 e+01$

3. $31776 \mathrm{e}+01$

$3.28149 e+01$

3.31564 e+ 01

3. 25752e+01

3.19807e+01

$3.14063 e+01$

3. 08140 e+01

$2.99839 e+01$

2. $91049 e+01$

2. $81993 e+01$

$2.71719 e+01$

2. $60911 e+01$

2.51165e+01

2. $40565 e+01$

2. $29910 e+01$

$2.16456 e+01$

$2.02182 \mathrm{e}+01$

$1.89856 \mathrm{e}+01$

$1.78095 \mathrm{e}+01$

$1.63536 e+01$

$1.48166 \mathrm{e}+01$

$1.82392 \mathrm{e}+01$

$1.62860 e+01$

$1.43151 \mathrm{e}+01$

$1.20021 \mathrm{e}+01$
$1.6380000 e+04$

$1.6680000 \mathrm{e}+04$

$1.7040000 \mathrm{e}+04$

1.7514000 e +04

$1.8102000 \mathrm{e}+04$

$1.8372000 \mathrm{e}+04$

$1.8724000 \mathrm{e}+04$

$1.9156000 \mathrm{e}+04$

$1.9716000 \mathrm{e}+04$

$2.0468000 \mathrm{e}+04$

$2.0660000 e+04$

$2.0844000 e+04$

$2.1116000 \mathrm{e}+04$

$2.1484000 e+04$

$2.1988000 \mathrm{e}+04$

$2.2596000 e+04$

$2.3388000 \mathrm{e}+04$

2.4460000 e+ 04

$2.5652000 e+04$

2. $7180000 e+04$

$2.7500000 e+04$

$2.7970000 e+04$

$2.8735000 e+04$

$3.0200000 \mathrm{e}+04$

$3.3000000 \mathrm{e}+04$

4. $94500 e+01$

3. $52246 e+01$

2.79056e+01

4. 16612 e+00

$6.29786 e+00$

$1.77899 \mathrm{e}+01$

3. $45996 \mathrm{e}+01$

3. $54344 \mathrm{e}+01$

3. 49131 e+ 01

3. $39125 e+01$

3. $36860 e+01$

3. $31159 e+01$

3. $33573 e+01$

3. $30416 e+01$

$3.24533 e+01$

3.18601e+01

$3.12745 e+01$

$3.06596 e+01$

2. $98193 e+01$

$2.89171 e+01$

$2.79628 \mathrm{e}+01$

$2.69835 e+01$

$2.58825 e+01$

$2.49379 e+01$

$2.38531 e+01$

$2.27307 e+01$

$2.13411 e+01$

$1.99505 e+01$

$1.87785 e+01$

$1.75621 \mathrm{e}+01$

$1.60404 \mathrm{e}+01$

$1.45153 \mathrm{e}+01$

$1.75716 \mathrm{e}+01$

$1.58884 \mathrm{e}+01$

$1.40282 \mathrm{e}+01$

$1.18525 e+01$
$1.6435000 e+04$

$1.6746000 e+04$

$1.7124000 \mathrm{e}+04$

$1.7616000 e+04$

$1.8162000 e+04$

$1.8436000 e+04$

$1.8804000 e+04$

$1.9260000 \mathrm{e}+04$

$1.9852000 e+04$

$2.0548000 e+04$

$2.0692000 e+04$

$2.0892000 \mathrm{e}+04$

$2.1180000 e+04$

$2.1572000 \mathrm{e}+04$

$2.2100000 e+04$

$2.2740000 e+04$

$2.3588000 e+04$

$2.4636000 e+04$

$2.5980000 \mathrm{e}+04$

$2.7230000 \mathrm{e}+04$

$2.7580000 \mathrm{e}+04$

$2.8090000 e+04$

$2.8950000 e+04$

$3.0675000 e+04$

$4.48671 e+01$

$3.31923 e+01$

$2.73662 \mathrm{e}+01$

$5.02123 e+00$

$7.27127 e+00$

$2.27872 e+01$

3. $51532 \mathrm{e}+01$

3.39437e+01

3. $42914 \mathrm{e}+01$

3. $41984 \mathrm{e}+01$

$3.31867 e+01$

$3.29846 e+01$

3. $34446 e+01$

3. $29282 \mathrm{e}+01$

$3.23488 \mathrm{e}+01$

$3.17512 e+01$

$3.11508 e+01$

$3.05099 e+01$

$2.96530 e+01$

$2.87357 e+01$

$2.77518 \mathrm{e}+01$

$2.67801 e+01$

$2.56761 e+01$

$2.47319 e+01$

$2.36573 e+01$

$2.24872 e+01$

$2.10482 e+01$

$1.96963 e+01$

$1.85743 e+01$

$1.72825 e+01$

1. $57146 e+01$

$1.39101 e+01$

$1.71273 e+01$

$1.54662 \mathrm{e}+01$

1. $35227 \mathrm{e}+01$

$1.16095 \mathrm{e}+01$

$1.6495000 \mathrm{e}+04$

$1.6818000 \mathrm{e}+04$

$1.7214000 e+04$

$1.7724000 \mathrm{e}+04$

$1.8210000 \mathrm{e}+04$

$1.8500000 e+04$

$1.8884000 \mathrm{e}+04$

$1.9372000 \mathrm{e}+04$

$1.9996000 \mathrm{e}+04$

$2.0572000 e+04$

$2.0724000 e+04$

$2.0940000 \mathrm{e}+04$

$2.1252000 e+04$

2.1668000 e+04

$2.2212000 e+04$

2.2884000 e +04

2.3804000 e+04

$2.4852000 e+04$

$2.6371699 e+04$

$2.7290000 e+04$

$2.7665000 e+04$

$2.8225000 e+04$

$2.9195000 e+04$

3.1245000 e+04

$4.19061 \mathrm{e}+01$

$3.13537 \mathrm{e}+01$

1. $45014 \mathrm{e}+01$

$5.05454 e+00$

$8.88395 e+00$

3. $27381 e+01$

$3.59444 \mathrm{e}+01$

3.30223e+01

$3.46511 \mathrm{e}+01$

$3.37926 e+01$

$3.23296 e+01$

$3.29165 e+01$

$3.33698 e+01$

$3.28131 e+01$

$3.22458 e+01$

$3.16447 e+01$

$3.10384 \mathrm{e}+01$

$3.03295 e+01$

$2.94878 e+01$

$2.85587 e+01$ 
Table 15-3

Sequoyah Input File (Continued)

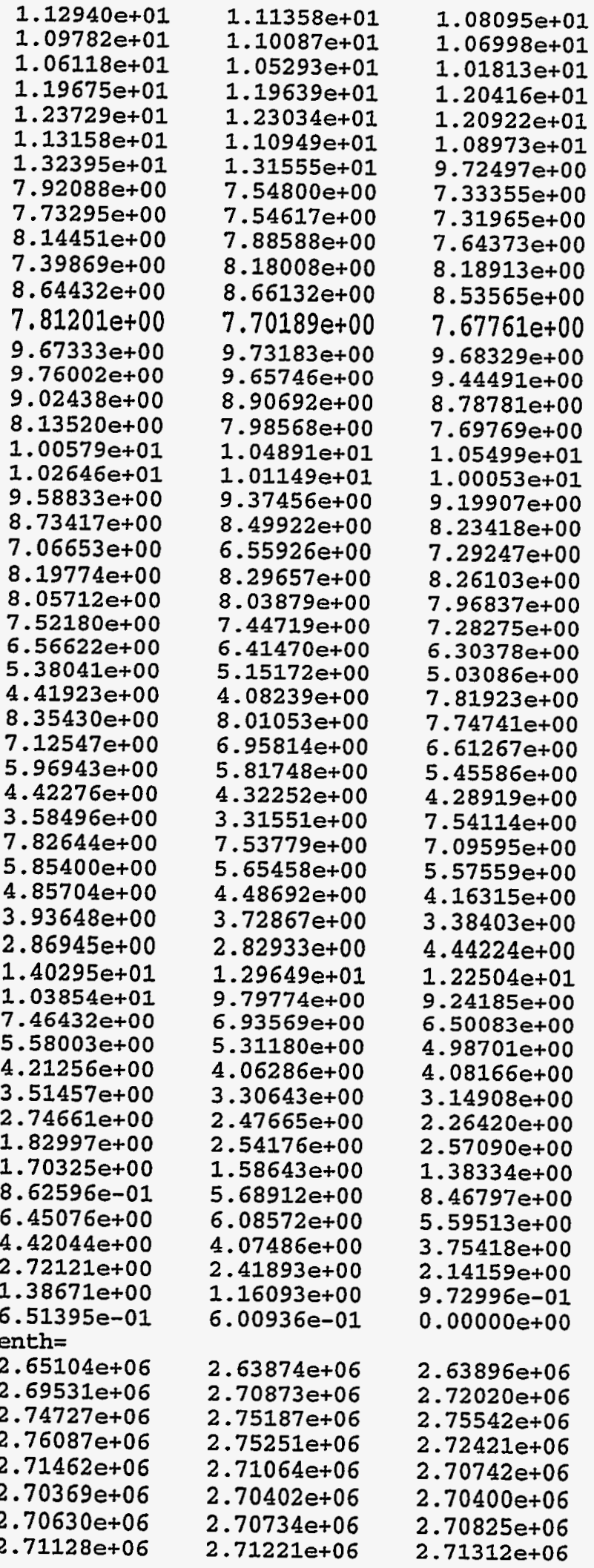

1. $01960 e+01$

$1.02573 e+01$

$1.04410 \mathrm{e}+01$

$1.22490 \mathrm{e}+01$

$1.17129 \mathrm{e}+01$

$1.06179 e+01$

$9.17304 \mathrm{e}+00$

$7.21959 e+00$

7.89687 e +00

$7.51206 e+00$

$8.32295 e+00$

$8.26237 \mathrm{e}+00$

$9.07814 e+00$

$9.71519 \mathrm{e}+00$

$9.27305 e+00$

$8.57050 e+00$

$8.70561 \mathrm{e}+00$

1. $05360 \mathrm{e}+01$

9. $90139 e+00$

$9.04639 e+00$

$8.05106 e+00$

$7.78247 e+00$

$8.15084 e+00$

$7.79855 e+00$

$7.03118 e+00$

$6.04750 \mathrm{e}+00$

$4.89354 \mathrm{e}+00$

$9.83538 \mathrm{e}+00$

$7.55286 \mathrm{e}+00$

$6.32885 e+00$

$5.03522 \mathrm{e}+00$

$4.22091 e+00$

$8.77805 e+00$

$6.61369 e+00$

$5.54465 e+00$

$3.99125 e+00$

$3.14010 \mathrm{e}+00$

$1.54533 \mathrm{e}+01$

$1.17609 \mathrm{e}+01$

$8.60218 e+00$

$6.15315 e+00$

$4.58563 e+00$

$4.06293 \mathrm{e}+00$

$3.07689 e+00$

$2.12184 \mathrm{e}+00$

$2.10612 \mathrm{e}+00$

$1.16365 \mathrm{e}+00$

$7.44956 \mathrm{e}+00$

$5.19787 e+00$

$3.39916 e+00$

$1.88681 \mathrm{e}+00$

8. $05773 e-01$

$2.66126 e+06$

$2.73037 e+06$

2.76058 e+06

$2.72111 e+06$

$2.70512 \mathrm{e} \div 06$

$2.70446 e+06$

$2.70925 e+06$

$2.71389 e+06$
$1.04009 e+01$

$1.01066 \mathrm{e}+01$

$1.16432 \mathrm{e}+01$

$1.23633 e+01$

$1.16114 \mathrm{e}+01$

$1.08656 \mathrm{e}+01$

$8.52424 e+00$

$7.71169 \mathrm{e}+00$

$8.23903 e \div 00$

$7.46037 \mathrm{e}+00$

$8.50866 e+00$

$7.99407 e+00$

$9.40380 e+00$

$9.74930 e+00$

$9.14033 e+00$

$8.32004 e+00$

9.36550 e+00

$1.04976 \mathrm{e}+01$

$9.78534 e+00$

$8.89167 \mathrm{e}+00$

$7.83636 e+00$

$8.01512 e+00$

8.04221 e+00

$7.63726 e+00$

$6.78297 e+00$

$5.70245 e+00$

$4.71093 e+00$

$8.87520 e+00$

$7.37378 e+00$

$6.10375 e+00$

$4.65977 e+00$

$3.90526 e+00$

$8.12034 \mathrm{e}+00$

$6.17418 e+00$

$5.24705 e+00$

$3.96758 e+00$

2. $94201 e+00$

$1.57328 \mathrm{e}+01$

$1.10629 \mathrm{e}+01$

$8.00908 \mathrm{e}+00$

$5.84521 e+00$

$4.34524 e+00$

$3.78932 \mathrm{e}+00$

$2.97675 e+00$

$1.97719 e+00$

$1.83864 \mathrm{e}+00$

$1.09294 \mathrm{e}+00$

$6.75745 \mathrm{e}+00$

$4.80472 e+00$

3. $04703 \mathrm{e}+00$

$1.63921 \mathrm{e}+00$

7. $27215 \mathrm{e}-01$

$2.68043 e+06$

$2.73906 \mathrm{e}+06$

$2.76313 e+06$

$2.71821 e+06$

$2.70374 e+06$

$2.70516 e+06$

$2.71026 e+06$ $2.71465 e+06$ 
Table 15-3

Sequoyah Input File (Continued)

\begin{tabular}{|c|c|c|c|c|}
\hline $\begin{array}{l}2.71543 e+06 \\
2.71907 e+06 \\
2.72216 e+06 \\
2.72525 e+06 \\
2.72820 e+06 \\
2.73096 e+06 \\
2.73376 e+06 \\
2.73668 e+06 \\
2.73936 e+06 \\
2.74195 e+06 \\
2.74552 e+06 \\
2.74945 e+06 \\
2.75340 e+06 \\
2.75796 e+06 \\
2.76191 e+06 \\
2.76606 e+06 \\
2.77013 e+06 \\
2.77426 e+06 \\
2.77856 e+06 \\
2.78399 e+06 \\
2.78797 e+06 \\
2.79213 e+06 \\
2.79488 e+06 \\
2.80057 e+06 \\
2.88244 e+06 \\
2.80875 e+06 \\
2.83809 e+06 \\
2.95601 e+06 \\
2.96917 e+06 \\
2.85790 e+06 \\
2.91579 e+06 \\
2.84536 e+06 \\
2.82127 e+06 \\
2.82446 e+06 \\
3.21542 e+06 \\
5.89916 e+06 \\
6.27988 e+06 \\
6.03473 e+06 \\
5.58502 e+06 \\
5.25680 e+06 \\
4.96603 e+06 \\
4.66574 e+06 \\
4.49122 e+06 \\
4.35803 e+06 \\
4.27967 e+06 \\
4.10695 e+06 \\
3.97704 e+06 \\
3.93173 e+06 \\
3.94295 e+06 \\
3.97707 e+06 \\
3.91333 e+06 \\
4.09083538 e+06+06 \\
4.202834 e+06 \\
4.27638 e+06 \\
4.31448 e+06\end{array}$ & 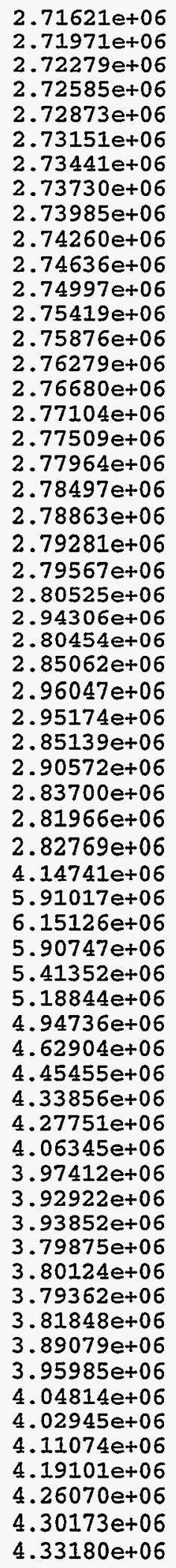 & 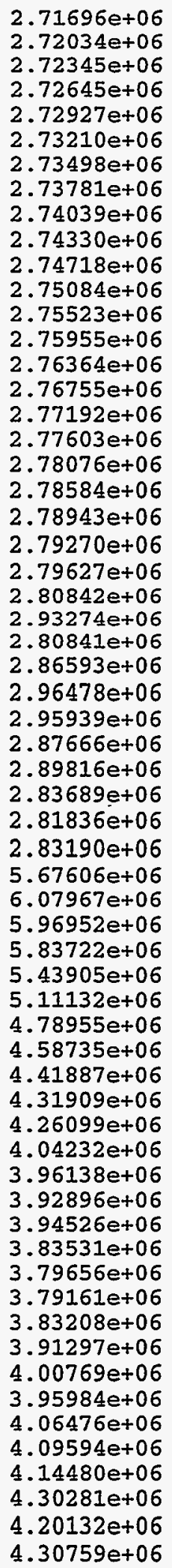 & 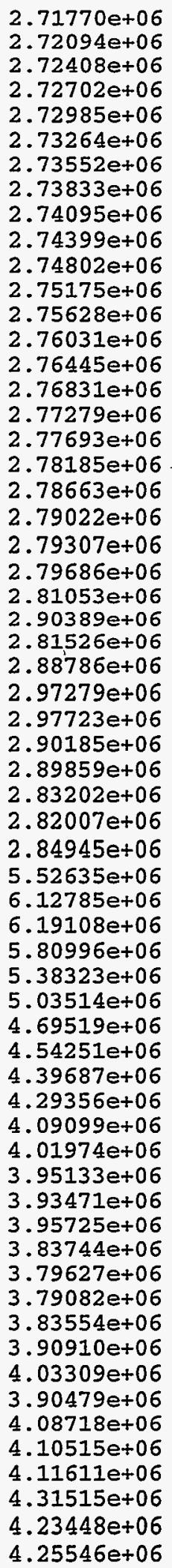 & 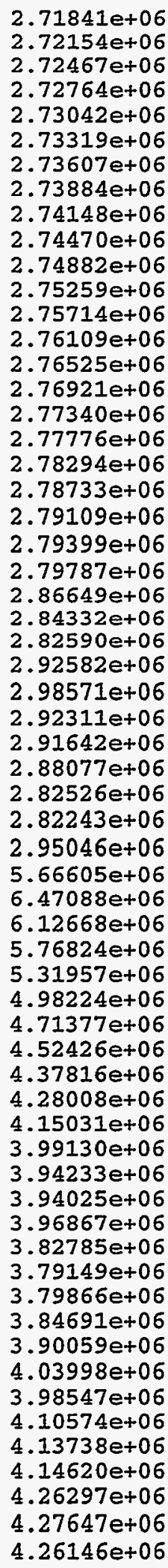 \\
\hline
\end{tabular}


Table 15-3

Sequoyah Input File (Continued)

$\begin{array}{ll}4.30918 e+06 & 4.36513 e+06 \\ 4.34125 e+06 & 4.34307 e+06 \\ 4.52691 e+06 & 4.49108 e+06 \\ 4.59046 e+06 & 4.61207 e+06 \\ 4.02695 e+06 & 4.20500 e+06 \\ 4.25967 e+06 & 4.22246 e+06 \\ 4.21359 e+06 & 4.26638 e+06 \\ 4.39321 e+06 & 4.42248 e+06 \\ 4.32392 e+06 & 4.36543 e+06 \\ 4.35164 e+06 & 4.36378 e+06 \\ 4.37101 e+06 & 4.35214 e+06 \\ 4.63981 e+06 & 4.94178 e+06 \\ 4.76782 e+06 & 4.80023 e+06 \\ 4.09118 e+06 & 3.17054 e+06 \\ 3.47964 e+06 & 3.47543 e+06 \\ 3.49156 e+06 & 3.49650 e+06 \\ 3.47175 e+06 & 3.48030 e+06 \\ 3.50795 e+06 & 3.50759 e+06 \\ 3.49173 e+06 & 3.48643 e+06\end{array}$
$4.41613 e+06$
$4.37680 \mathrm{e}+06$
$4.45452 \mathrm{e}+06$
$4.65594 \mathrm{e}+06$
4. $23572 \mathrm{e}+06$
4. $19920 \mathrm{e}+06$
$4.29273 e+06$
$4.40889 e+06$
$4.39105 e+06$
$4.38456 e+06$
$4.40061 \mathrm{e}+06$
$4.83871 e+06$
$4.73908 \mathrm{e}+06$
$3.07745 e+06$
$3.51766 \mathrm{e}+06$
$3.47149 e+06$
$3.48906 \mathrm{e}+06$
$3.50575 e+06$
$3.48643 e+06$

$4.44813 e+06$
$4.44681 e+06$
$4.48864 e+06$
$3.90104 e+06$
$4.22119 e+06$
$4.21332 e+06$
$4.33010 e+06$
$4.38855 e+06$
$4.38336 e+06$
$4.42193 e+06$
$4.53200 e+06$
$4.72235 e+06$
$4.85600 e+06$
$3.35873 e+06$
$3.51311 e+06$
$3.46327 e+06$
$3.49703 e+06$
$3.48816 e+06$

$4.39053 e+06$

$4.50473 e+06$

$4.51568 \mathrm{e}+06$

3. $70709 e+06$

$4.25469 e+06$

4. 20657 e+06

$4.37323 e+06$

$4.40691 e+06$

$4.33371 \mathrm{e}+06$

$4.40803 e+06$

$4.53309 e+06$

$4.74499 \mathrm{e}+06$

$4.85606 \mathrm{e}+06$

$3.49948 e+06$

3. $50408 \mathrm{e}+06$

$3.46711 e+06$

$3.50516 \mathrm{e}+06$

eoi

\&\& INEL Surry h2 source, dp on SG dryout, no surge line break, scale to seq.

\&\& $722 \mathrm{~kg}$ of $\mathrm{h} 2$ prior to $\mathrm{vb}$ h2 400 iflag=1

$t=$

$7.8200000 \mathrm{e}+03$

$8.4544902 \mathrm{e}+03$

$8.6249902 \mathrm{e}+03$

$8.6699902 \mathrm{e}+03$

$8.6949902 \mathrm{e}+03$

$8.7199902 \mathrm{e}+03$

$8.7449902 e+03$

$8.7699902 e+03$

$8.7949902 e+03$

$8.8199902 \mathrm{e}+03$

$8.8449902 e+03$

$8.8699902 e+03$

$8.8949902 e+03$

$8.9199902 \mathrm{e}+03$

$8.9449902 e+03$

$8.9699902 \mathrm{e}+03$

$8.9949902 e+03$

$9.0199902 \mathrm{e}+03$

$9.0449902 \mathrm{e}+03$

$9.0699902 e+03$

$9.0949902 e+03$

$9.1199902 \mathrm{e}+03$

$9.1449902 \mathrm{e}+03$

$9.1699902 \mathrm{e}+03$

$9.1999902 e+03$

$9.2499902 e+03$

$9.2749902 e+03$

$9.2999902 e+03$

$9.3249902 e+03$

$9.3499902 \mathrm{e}+03$

$9.3749902 e+03$

$9.3999902 \mathrm{e}+03$

$9.4249902 \mathrm{e}+03$

$9.4499902 \mathrm{e}+03$

$9.4749902 e+03$

$9.4999902 \mathrm{e}+03$

$9.5249902 \mathrm{e}+03$

$9.5499902 \mathrm{e}+03$
$8.2069902 e+03$

$8.5174902 e+03$

$8.6349902 e+03$

$8.6749902 e+03$

$8.6999902 \mathrm{e}+03$

$8.7249902 e+03$

$8.7499902 e+03$

$8.7749902 e+03$

$8.7999902 e+03$

$8.8249902 e+03$

$8.8499902 e+03$

$8.8749902 e+03$

$8.8999902 e+03$

$8.9249902 \mathrm{e}+03$

$8.9499902 e+03$

$8.9749902 e+03$

$9.0000000 e+03$

$9.0249902 \mathrm{e}+03$

$9.0499902 \mathrm{e}+03$

$9.0749902 e+03$

$9.0999902 \mathrm{e}+03$

$9.1249902 \mathrm{e}+03$

$9.1499902 \mathrm{e}+03$

$9.1749902 \mathrm{e}+03$

$9.2099902 \mathrm{e}+03$

$9.2549902 e+03$

$9.2799902 \mathrm{e}+03$

$9.3049902 \mathrm{e}+03$

$9.3299902 \mathrm{e}+03$

$9.3549902 \mathrm{e}+03$

$9.3799902 e+03$

$9.4049902 \mathrm{e}+03$

$9.4299902 \mathrm{e}+03$

$9.4549902 e+03$

$9.4799902 e+03$

$9.5049902 e+03$

$9.5299902 \mathrm{e}+03$

$9.5549902 e+03$
$8.3059902 e+03$

$8.5624902 \mathrm{e}+03$

$8.6449902 e+03$

$8.6799902 \mathrm{e}+03$

$8.7049902 e+03$

$8.7299902 e+03$

$8.7549902 e+03$

$8.7799902 e+03$

$8.8049902 e+03$

$8.8299902 e+03$

$8.8549902 e+03$

$8.8799902 \mathrm{e}+03$

$8.9049902 e+03$

$8.9299902 \mathrm{e}+03$

$8.9549902 e+03$

$8.9799902 e+03$

$9.0049902 \mathrm{e}+03$

$9.0299902 e+03$

$9.0549902 e+03$

$9.0799902 e+03$

$9.1049902 \mathrm{e}+03$

$9.1299902 \mathrm{e}+03$

$9.1549902 \mathrm{e}+03$

$9.1799902 \mathrm{e}+03$

$9.2199902 \mathrm{e}+03$

$9.2599902 e+03$

9. $2849902 e+03$

$9.3099902 \mathrm{e}+03$

$9.3349902 \mathrm{e}+03$

$9.3599902 \mathrm{e}+03$

$9.3849902 e+03$

$9.4099902 e+03$

$9.4349902 e+03$

$9.4599902 \mathrm{e}+03$

$9.4849902 \mathrm{e}+03$

$9.5099902 e+03$

$9.5349902 \mathrm{e}+03$

$9.5599902 \mathrm{e}+03$
$8.3554902 e+03$ $8.5894902 e+03$

$8.6549902 \mathrm{e}+03$

8. $6849902 e+03$

$8.7099902 \mathrm{e}+03$

$8.7349902 e+03$

$8.7599902 \mathrm{e}+03$

$8.7849902 e+03$

$8.8099902 e+03$

$8.8349902 e+03$

$8.8599902 e+03$

$8.8849902 e+03$

$8.9099902 \mathrm{e}+03$

$8.9349902 e+03$

$8.9599902 e+03$

$8.9849902 e+03$

$9.0099902 e+03$

$9.0349902 \mathrm{e}+03$

$9.0599902 e+03$

$9.0849902 \mathrm{e}+03$

$9.1099902 e+03$

$9.1349902 \mathrm{e}+03$

$9.1599902 e+03$

$9.1849902 e+03$

$9.2299902 \mathrm{e}+03$

$9.2649902 e+03$

$9.2899902 \mathrm{e}+03$

$9.3149902 \mathrm{e}+03$

$9.3399902 e+03$

$9.3649902 e+03$

$9.3899902 \mathrm{e}+03$

$9.4149902 \mathrm{e}+03$

$9.4399902 \mathrm{e}+03$

$9.4649902 \mathrm{e}+03$

$9.4899902 \mathrm{e}+03$

$9.5149902 \mathrm{e}+03$

$9.5399902 e+03$

$9.5649902 \mathrm{e}+03$
$8.3824902 e+03$ $8.6099902 e+03$ 8. $6649902 e+03$ $8.6899902 \mathrm{e}+03$ $8.7149902 e+03$ $8.7399902 e+03$ $8.7649902 e+03$ $8.7899902 e+03$ $8.8149902 e+03$ $8.8399902 e+03$ $8.8649902 e+03$ $8.8899902 e+03$ $8.9149902 \mathrm{e}+03$ $8.9399902 e+03$ $8.9649902 e+03$ $8.9899902 \mathrm{e}+03$ $9.0149902 e+03$ $9.0399902 \mathrm{e}+03$ $9.0649902 \mathrm{e}+03$ $9.0899902 e+03$ $9.1149902 e+03$ $9.1399902 \mathrm{e}+03$ $9.1649902 \mathrm{e}+03$ $9.1899902 \mathrm{e}+03$ $9.2399902 \mathrm{e}+03$ $9.2699902 e+03$ $9.2949902 \mathrm{e}+03$ $9.3199902 e+03$ $9.3449902 e+03$ $9.3699902 e+03$ $9.3949902 \mathrm{e} \div 03$ $9.4199902 \mathrm{e}+03$ $9.4449902 \mathrm{e}+03$ $9.4699902 e+03$ $9.4949902 e+03$ $9.5199902 \mathrm{e}+03$ $9.5449902 \mathrm{e}+03$ $9.5699902 \mathrm{e}+03$ 
Table 15-3

Sequoyah Input File (Continued)

$9.5799902 \mathrm{e}+03$

$9.6499902 \mathrm{e}+03$

$9.7549902 \mathrm{e}+03$

$9.9399902 \mathrm{e}+03$

$1.0060000 \mathrm{e}+04$

$1.0235000 \mathrm{e}+04$

$1.0380000 \mathrm{e}+04$

$1.0460000 \mathrm{e}+04$

$1.0555000 \mathrm{e}+04$

$1.0705000 \mathrm{e}+04$

$1.0880000 \mathrm{e}+04$

$1.1235000 \mathrm{e}+04$

$1.1900000 \mathrm{e}+04$

$1.2895000 e+04$

$1.5100000 e+04$

$1.7976000 \mathrm{e}+04$

2.0956000e+04

2.2012000e+04

$2.3780000 e+04$

$2.4020000 \mathrm{e}+04$

$2.4068000 \mathrm{e}+04$

$2.4116000 \mathrm{e}+04$

$2.4196000 \mathrm{e}+04$

$2.4268000 e+04$

$2.4340000 e+04$

$2.4380000 e+04$

$2.4476000 \mathrm{e}+04$

$2.4652000 \mathrm{e}+04$

$2.4820000 e+04$

$2.5020000 e+04$

2. $5276000 \mathrm{e}+04$

$2.5708000 e+04$

$2.5812000 e+04$

$2.5908000 e+04$

2.5988000e+04

2. $6068000 \mathrm{e}+04$

2. $6148000 e+04$

$2.6228000 e+04$

2. $6308000 e+04$

$2.6395699 e+04$

$2.6507699 \mathrm{e}+04$

2. $9840000 e+04$ mass $=$

4. 64951e-03

$2.78590 \mathrm{e}-02$

$1.20231 \mathrm{e}-01$

$1.86163 e-01$

$2.32556 \mathrm{e}-01$

$2.90808 \mathrm{e}-01$

$3.79730 \mathrm{e}-01$

$4.88016 e-01$

$5.81677 e-01$

$6.05234 \mathrm{e}-01$

5.79062e-01

$5.63717 \mathrm{e}-01$

$5.72272 \mathrm{e}-01$

$5.96012 e-01$

$6.17089 \mathrm{e}-01$

$6.20887 \mathrm{e}-01$

$5.90387 e-01$

$5.39878 \mathrm{e}-01$

$4.93082 \mathrm{e}-01$
$9.5899902 e+03$

$9.6649902 e+03$

$9.7949902 \mathrm{e}+03$

$9.9649902 \mathrm{e}+03$

$1.0080000 \mathrm{e}+04$

$1.0280000 e+04$

$1.0405000 \mathrm{e}+04$

1.0475000 e +04

$1.0570000 \mathrm{e}+04$

$1.0735000 \mathrm{e}+04$

$1.0950000 e+04$

$1.1300000 \mathrm{e}+04$

$1.2010000 e+04$

$1.3135000 \mathrm{e}+04$

$1.5670000 \mathrm{e}+04$

$1.8908000 \mathrm{e}+04$

$2.1180000 \mathrm{e}+04$

$2.2284000 e+04$

$2.3876000 e+04$

$2.4036000 \mathrm{e}+04$

$2.4076000 e+04$

$2.4132000 e+04$

$2.4212000 e+04$

$2.4276000 e+04$

$2.4348000 e+04$

$2.4388000 e+04$

2. $4516000 \mathrm{e}+04$

2.4684000 e +04

$2.4860000 \mathrm{e}+04$

$2.5060000 \mathrm{e}+04$

$2.5380000 e+04$

$2.5740000 e+04$

$2.5836000 e+04$

$2.5924000 e+04$

$2.6004000 e+04$

$2.6084000 \mathrm{e}+04$

2. $6164000 e+04$

$2.6244000 e+04$

2.6324000 e +04

$2.6419699 e+04$

$2.7100000 e+04$

$3.0840000 e+04$

$1.76428 \mathrm{e}-02$

$3.89000 \mathrm{e}-02$

$1.35498 \mathrm{e}-01$

$1.95371 \mathrm{e}-01$

$2.42918 e-01$

$3.06098 \mathrm{e}-01$

$4.00490 \mathrm{e}-01$

$5.09643 e-01$

$5.93147 e-01$

$6.01154 \mathrm{e}-01$

$5.74154 e-01$

5. $63258 \mathrm{e}-01$

$5.76845 \mathrm{e}-01$

$6.00458 \mathrm{e}-01$

$6.22193 e-01$

$6.17700 \mathrm{e}-01$

$5.80659 e-01$

$5.27608 e-01$

4.94219e-01
$9.6049902 \mathrm{e}+03$

$9.6799902 e+03$

$9.8349902 \mathrm{e}+03$

$9.9899902 e+03$

$1.0100000 \mathrm{e}+04$

$1.0305000 \mathrm{e}+04$

1. $0420000 \mathrm{e}+04$

$1.0495000 \mathrm{e} \div 04$

$1.0600000 e+04$

$1.0765000 e+04$

$1.1025000 e+04$

$1.1400000 \mathrm{e}+04$

$1.2280000 e+04$

$1.3635000 e+04$

1. $6085000 \mathrm{e}+04$

1. $9684000 \mathrm{e}+04$

$2.1404000 \mathrm{e}+04$

2.2732000e+04

$2.3940000 e+04$

$2.4044000 e+04$

2. $4084000 e+04$

$2.4148000 e+04$

$2.4228000 e+04$

$2.4292000 e+04$

$2.4356000 e+04$

$2.4396000 e+04$

$2.4556000 e+04$

$2.4716000 e+04$

$2.4900000 e+04$

$2.5100000 \mathrm{e}+04$

2. 5476000 e +04

$2.5764000 e+04$

2. $5860000 e+04$

$2.5940000 e+04$

$2.6020000 e+04$

2. $6100000 e+04$

2. $6180000 e+04$

$2.6260000 e+04$

2. $6340000 e+04$

$2.6443699 e+04$

2.7380000 e+04

$3.1840000 e+04$

3. $41206 \mathrm{e}-02$

$5.79679 \mathrm{e}-02$

1. $50988 \mathrm{e}-01$

2.05096e-01

$2.53716 \mathrm{e}-01$

3. $22255 e-01$

4. 21809e-01

5.29915e-01

$6.01571 \mathrm{e}-01$

5.96044e-01

$5.70785 e-01$

$5.63781 \mathrm{e}-01$

$5.81455 e-01$

6.04371e-01

$6.23828 e-01$

$6.12774 \mathrm{e}-01$

$5.69159 e-01$

5.14652e-01

$4.93220 \mathrm{e}-01$
$9.6199902 e+03$

$9.6999902 e+03$

$9.8749902 e+03$

$1.0015000 e+04$

$1.0120000 e+04$

$1.0330000 e+04$

$1.0435000 e+04$

$1.0515000 e+04$

$1.0645000 \mathrm{e}+04$

$1.0795000 \mathrm{e}+04$

$1.1085000 e+04$

$1.1670000 e+04$

$1.2555000 e+04$

1. $3885000 \mathrm{e}+04$

$1.6848000 e+04$

$2.0180000 e+04$

$2.1668000 e+04$

$2.3340000 e+04$

$2.3980000 e+04$

$2.4052000 e+04$

$2.4092000 e+04$

2. $4164000 e+04$

$2.4244000 e+04$

$2.4308000 e+04$

2. $4364000 e+04$

$2.4412000 e+04$

$2.4588000 e+04$

$2.4748000 e+04$

2. $4940000 \mathrm{e}+04$

2.5140000 e+04

$2.5572000 e+04$

$2.5780000 e+04$

$2.5876000 e+04$

$2.5956000 e+04$

2. $6036000 e+04$

2. $6116000 e+04$

2. $6196000 e+04$

$2.6276000 e+04$

$2.6355699 e+04$

$2.6467699 e+04$

$2.7900000 e+04$

$3.2840000 e+04$

$5.84220 e-02$ $8.01569 e-02$ $1.66473 e-01$ $2.14028 \mathrm{e}-01$

$2.64690 e-01$

$3.39937 e-01$

$4.43715 e-01$

$5.49203 e-01$

$6.06349 e-01$

$5.90628 e-01$

5. 67420e-01

5.65391e-01

$5.86097 e-01$

$6.08415 e-01$

$6.24485 e-01$.

$6.06885 e-01$

5.59051 e- 01

$5.06647 e-01$

$4.87378 e-01$

$9.6349902 e+03$

$9.7249902 e+03$

$9.9099902 e+03$

$1.0040000 \mathrm{e}+04$

$1.0145000 \mathrm{e}+04$

$1.0355000 e+04$

$1.0450000 \mathrm{e}+04$

$1.0535000 \mathrm{e}+04$

$1.0675000 \mathrm{e}+04$

$1.0830000 \mathrm{e}+04$

$1.1155000 \mathrm{e}+04$

$1.1775000 \mathrm{e}+04$

$1.2730000 \mathrm{e}+04$

$1.4100000 \mathrm{e}+04$

$1.7394000 \mathrm{e}+04$

2. $0668000 e+04$

$2.1900000 \mathrm{e}+04$

2.3604000e+04

$2.4004000 e+04$

$2.4060000 e+04$

2. $4100000 \mathrm{e}+04$

2. $4180000 \mathrm{e}+04$

2. 4260000 e+04

$2.4324000 \mathrm{e}+04$

$2.4372000 \mathrm{e}+04$

$2.4436000 \mathrm{e}+04$

$2.4620000 e+04$

$2.4780000 \mathrm{e}+04$

$2.4980000 \mathrm{e}+04$

$2.5188000 \mathrm{e}+04$

$2.5652000 e+04$

$2.5796000 \mathrm{e}+04$

$2.5892000 e+04$

$2.5972000 e+04$

$2.6052000 e+04$

2. $6132000 \mathrm{e}+04$

$2.6212000 \mathrm{e}+04$

$2.6292000 e+04$

$2.6371699 \mathrm{e}+04$

$2.6491699 e+04$

$2.8840000 e+04$

$3.3000000 e+04$

$2.45114 \mathrm{e}-02$ 
Table 15-3

Sequoyah Input File (Continued)

\begin{tabular}{|c|c|c|c|c|}
\hline 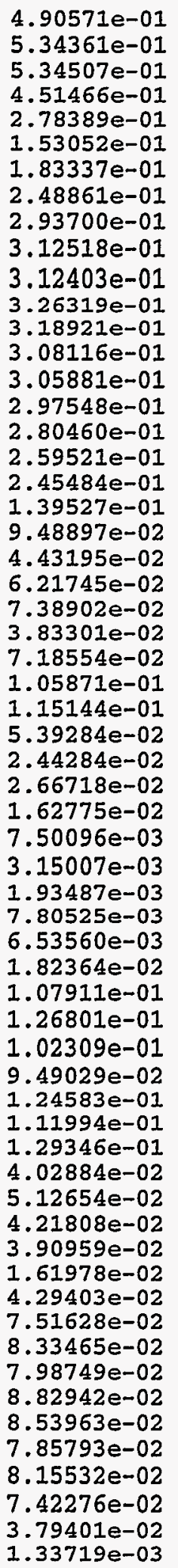 & $\begin{array}{l}4.96375 e-01 \\
5.39884 e-01 \\
5.25869 e-01 \\
4.23424 e-01 \\
2.44949 e-01 \\
1.46078 e-01 \\
1.96147 e-01 \\
2.60075 e-01 \\
2.99052 e-01 \\
3.13733 e-01 \\
3.16215 e-01 \\
3.25237 e-01 \\
3.16510 e-01 \\
3.07821 e-01 \\
3.04917 e-01 \\
2.94450 e-01 \\
2.76794 e-01 \\
2.57979 e-01 \\
2.28574 e-01 \\
1.14942 e-01 \\
9.39427 e-02 \\
4.15089 e-02 \\
6.59300 e-02 \\
7.24626 e-02 \\
6.59102 e-02 \\
1.19616 e-01 \\
8.42447 e-02 \\
5.89816 e-02 \\
5.27213 e-02 \\
2.32740 e-02 \\
1.79644 e-02 \\
6.60373 e-03 \\
3.60538 e-03 \\
4.28132 e-03 \\
2.30148 e-03 \\
7.84414 e-03 \\
3.99611 e-03 \\
2.56421 e-02 \\
1.21129 e-01 \\
1.22432 e-01 \\
9.92830 e-02 \\
9.34262 e-02 \\
1.02515 e-01 \\
1.17317 e-01 \\
1.28016 e-01 \\
4.15323 e-02 \\
5.02552 e-02 \\
4.18960 e-02 \\
3.86123 e-02 \\
1.87244 e-02 \\
6.21213 e-02 \\
7.46572 e-02 \\
8.26705 e-02 \\
8.22202 e-02 \\
8.38400 e-02 \\
9.44588 e-02 \\
7.90357 e-02 \\
7.97722 e-02 \\
7.38605 e-02 \\
6.41553 e-03 \\
1.74878 e-03\end{array}$ & 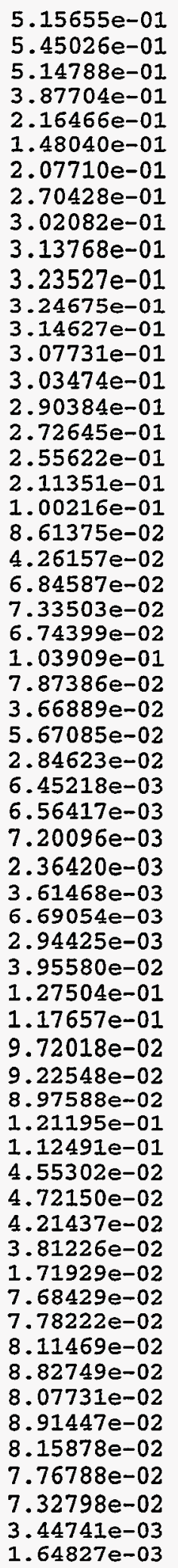 & $\begin{array}{l}5.29776 e-01 \\
5.48542 e-01 \\
4.97637 e-01 \\
3.47911 e-01 \\
1.93988 e-01 \\
1.55778 e-01 \\
2.20039 e-01 \\
2.79399 e-01 \\
3.04495 e-01 \\
3.12645 e-01 \\
3.26712 e-01 \\
3.22030 e-01 \\
3.12832 e-01 \\
3.07149 e-01 \\
3.01390 e-01 \\
2.86754 e-01 \\
2.67191 e-01 \\
2.57163 e-01 \\
1.93539 e-01 \\
9.48927 e-02 \\
7.01974 e-02 \\
5.02003 e-02 \\
7.08609 e-02 \\
6.60372 e-02 \\
6.13895 e-02 \\
9.97600 e-02 \\
8.53535 e-02 \\
5.27714 e-02 \\
4.82486 e-02 \\
2.46000 e-02 \\
1.71722 e-02 \\
1.01689 e-02 \\
8.32855 e-03 \\
3.27991 e-03 \\
3.66037 e-03 \\
7.72528 e-03 \\
6.72831 e-03 \\
6.63618 e-02 \\
1.30936 e-01 \\
1.14963 e-01 \\
9.42550 e-02 \\
1.11256 e-01 \\
9.24247 e-02 \\
1.24465 e-01 \\
6.99341 e-02 \\
4.64099 e-02 \\
4.55213 e-02 \\
4.09100 e-02 \\
3.36948 e-02 \\
2.19336 e-02 \\
7.88030 e-02 \\
8.06412 e-02 \\
7.96722 e-02 \\
9.09773 e-02 \\
8.18892 e-02 \\
8.64764 e-02 \\
8.34943 e-02 \\
7.62498 e-02 \\
7.21088 e-02 \\
1.91866 e-03 \\
1.32282 e-03\end{array}$ & $\begin{array}{l}5.30415 e-01 \\
5.44662 e-01 \\
4.75909 e-01 \\
3.11996 e-01 \\
1.72169 e-01 \\
1.68450 e-01 \\
2.33966 e-01 \\
2.86727 e-01 \\
3.08553 e-01 \\
3.11996 e-01 \\
3.27109 e-01 \\
3.20387 e-01 \\
3.10692 e-01 \\
3.06752 e-01 \\
2.99593 e-01 \\
2.83696 e-01 \\
2.62519 e-01 \\
2.57927 e-01 \\
1.68453 e-01 \\
9.42437 e-02 \\
5.43952 e-02 \\
5.74540 e-02 \\
7.30029 e-02 \\
1.88142 e-02 \\
7.16766 e-02 \\
1.24784 e-01 \\
7.25632 e-02 \\
5.55610 e-02 \\
3.51461 e-02 \\
2.15147 e-02 \\
1.39336 e-02 \\
1.05156 e-02 \\
1.14921 e-03 \\
3.08935 e-03 \\
6.18635 e-03 \\
1.54843 e-02 \\
9.65710 e-03 \\
8.72713 e-02 \\
1.29940 e-01 \\
1.09005 e-01 \\
9.37810 e-02 \\
1.38007 e-01 \\
1.02711 e-01 \\
1.27391 e-01 \\
3.84490 e-02 \\
4.75740 e-02 \\
4.30475 e-02 \\
3.95609 e-02 \\
2.02601 e-02 \\
2.90391 e-02 \\
7.79657 e-02 \\
8.24014 e-02 \\
7.84804 e-02 \\
8.97821 e-02 \\
8.40469 e-02 \\
8.19187 e-02 \\
8.33313 e-02 \\
7.51686 e-02 \\
6.089516 e-02 \\
0.00000 e+00\end{array}$ \\
\hline
\end{tabular}


Table 15-3

Sequoyah Input File (Continued)

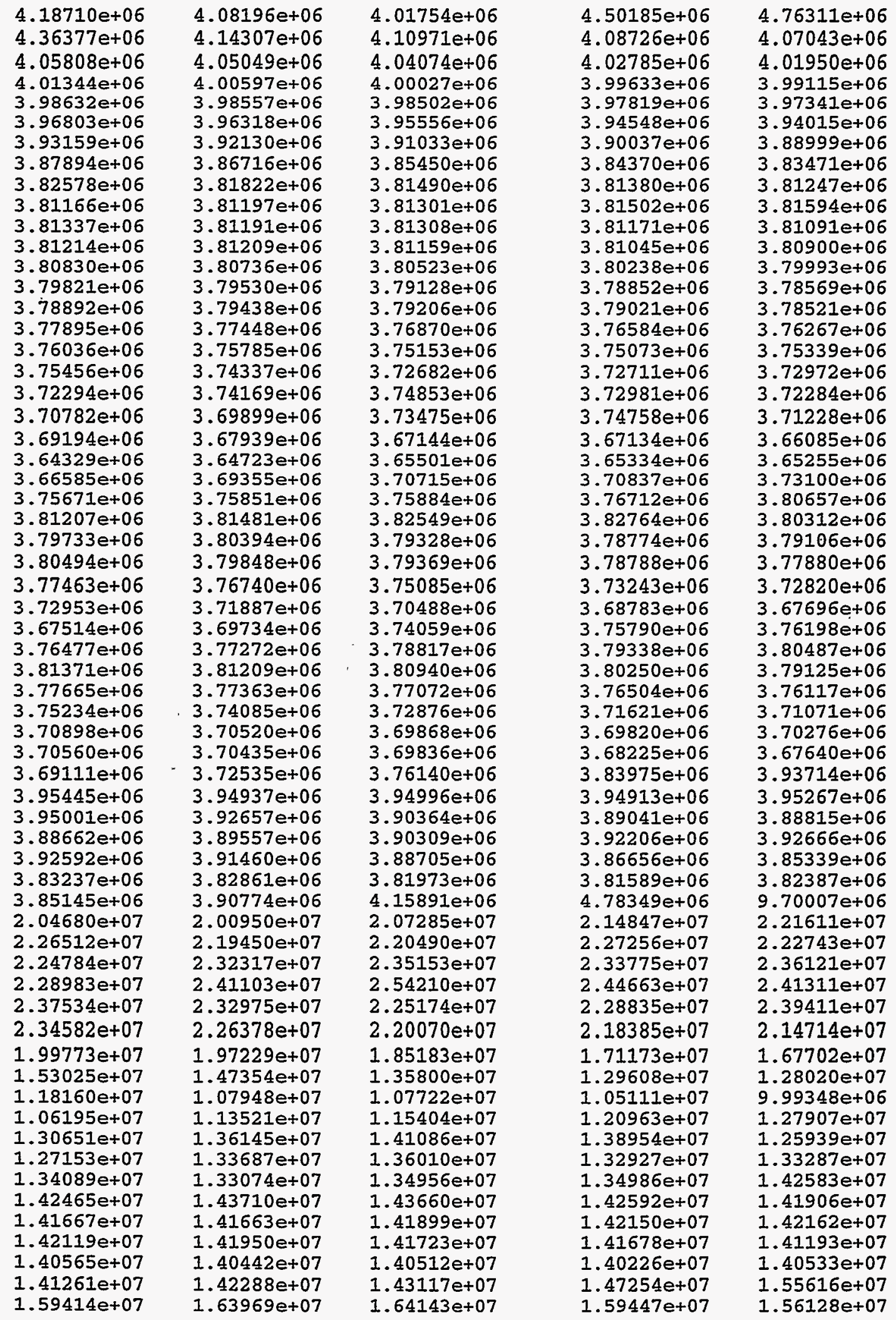


Table 15-3

Sequoyah Input File (Continued)

\author{
$1.55497 e+07$ \\ 1. $58464 \mathrm{e}+07$ \\ $1.65145 e+07$ \\ 1. $47242 \mathrm{e}+07$ \\ $1.53466 \mathrm{e}+07$ \\ $1.54186 e+07$ \\ $1.57783 e+07$ \\ $1.54572 \mathrm{e}+07$ \\ $1.58958 \mathrm{e}+07$ \\ $1.52496 \mathrm{e}+07$ \\ $1.51188 \mathrm{e}+07$ \\ $1.53340 \mathrm{e}+07$ \\ $1.51424 \mathrm{e}+07$ \\ $1.54671 e+07$ \\ $1.51641 e+07$ \\ $1.52421 \mathrm{e}+07$ \\ $1.22693 e+07$ \\ eoi \\ $1.07866 \mathrm{e}+07$ \\ $1.56250 e+07$ \\ $1.60902 \mathrm{e}+07$ \\ $1.59184 \mathrm{e}+07$ \\ $1.53436 \mathrm{e}+07$ \\ $1.53978 \mathrm{e}+07$ \\ $1.52860 \mathrm{e}+07$ \\ 1.59171e+07 \\ $1.57841 e+07$ \\ $1.54723 e+07$ \\ $1.52018 \mathrm{e}+07$ \\ 1. $51016 \mathrm{e}+07$ \\ $1.54261 \mathrm{e}+07$ \\ $1.52446 \mathrm{e}+07$ \\ $1.52611 \mathrm{e}+07$ \\ $1.51809 \mathrm{e}+07$ \\ $1.52303 e+07$ \\ $1.08182 \mathrm{e}+07$ \\ 1. $07005 e+07$ \\ $1.56892 e+07$ \\ $1.64104 \mathrm{e}+07$ \\ $1.53096 \mathrm{e}+07$ \\ $1.55325 \mathrm{e}+07$ \\ $1.55391 e+07$ \\ $1.53232 \mathrm{e}+07$ \\ $1.58051 e+07$ \\ $1.59275 e+07$ \\ $1.53668 e+07$ \\ 1. $51855 e+07$ \\ $1.51261 \mathrm{e}+07$ \\ $1.53425 e+07$ \\ $1.53715 \mathrm{e}+07$ \\ $1.51995 e+07$ \\ $1.51955 e+07$ \\ $1.51971 e+07$ \\ $1.05863 e+07$ \\ $1.06101 \mathrm{e}+07$ \\ $1.57681 e+07$ \\ 1. $65666 \mathrm{e}+07$ \\ $1.48312 \mathrm{e}+07$ \\ 1.54760 e+07 \\ $1.54998 e+07$ \\ $1.57049 e+07$ \\ $1.57087 e+07$ \\ $1.59879 \mathrm{e}+07$ \\ $1.52950 \mathrm{e}+07$ \\ $1.51501 \mathrm{e}+07$ \\ $1.51643 e+07$ \\ $1.52341 e+07$ \\ $1.54860 e+07$ \\ $1.51687 \mathrm{e}+07$ \\ $1.52224 \mathrm{e}+07$ \\ $1.51592 \mathrm{e}+07$ \\ $1.07943 e+07$ \\ 1.06101e+07

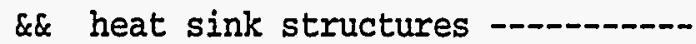 \\ struc \\ st1 wall slab $615311.15 .0 \quad 0.0 \quad 0 \quad 3002.49$ \\ $\begin{array}{llllllllllll}0.0 & 0.003 & 0.0085 & 0.01737 & 0.03444 & 0.05182 & 0.06888\end{array}$ \\ fe fe fe fe fe fe \\ \&\& dummy structure to control trapping/ku \& NAD velocities \\ name=dummy type=wall shape=slab chrlen=15. slarea=1.0e-10 \\ ns $I a b=6 \quad$ tunif $=311$. \\ compound $=\mathrm{fe}$ fe fe fe fe fe

$$
\begin{array}{lllll}
\mathrm{x}=0.0 & 0.003 & 0.0085 & 0.01737 & 0.03444
\end{array}
$$ \\ bcinner hydarea $=103$. eoi \\ bcouter tgas $=311$. eoi \\ eoi

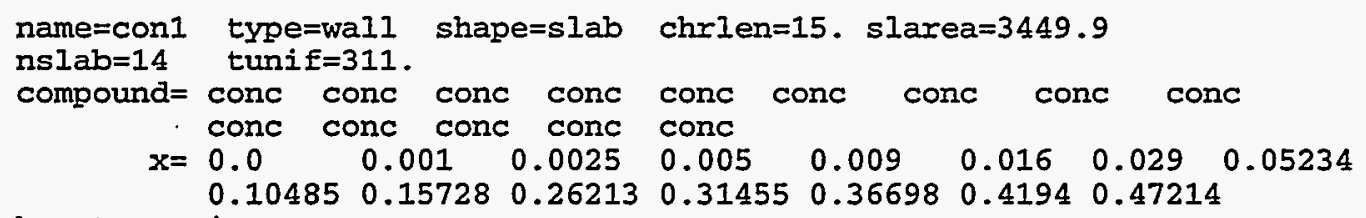 \\ bcouter eoi \\ eoi \\ name=con2 type=wall shape=slab chrlen=15. slarea $=3258.05$ \\ nslab $=15$ tunif $=311$. \\ compound= cone conc conc conc cone conc conc cone cone \\ cone cone cone conc conc conc \\ $\begin{array}{lllllll}\mathrm{x}=0.0 & 0.001 & 0.0025 & 0.005 & 0.009 & 0.016 & 0.029\end{array}$ \\ bcouter eoi \\ $\begin{array}{llllllll}0.04968 & 0.09967 & 0.14935 & 0.19903 & 0.24902 & 0.2987 & 0.34839\end{array}$ \\ eoi \\ name $=$ floor 1 type $=$ floor shape $=$ slab chrlen=15. slarea $=451.16$ \\ nslab $=14$ tunif $=311$. \\ compound= conc conc cone conc cone conc cone cone \\ $\begin{array}{lllllllll}\text { conc } & \text { cone cone conc conc } & \text { conc } & & & & \\ 0.0 & 0.001 & 0.0025 & 0.005 & 0.009 & 0.016 & 0.029 & 0.05234\end{array}$ \\ bcouter eoi

$$
\begin{array}{lllllll}
0.10485 & 0.15728 & 0.26213 & 0.31455 & 0.36698 & 0.4194 & 0.47214
\end{array}
$$

Rev. 0 
Table 15-3

Sequoyah Input File (Continued)

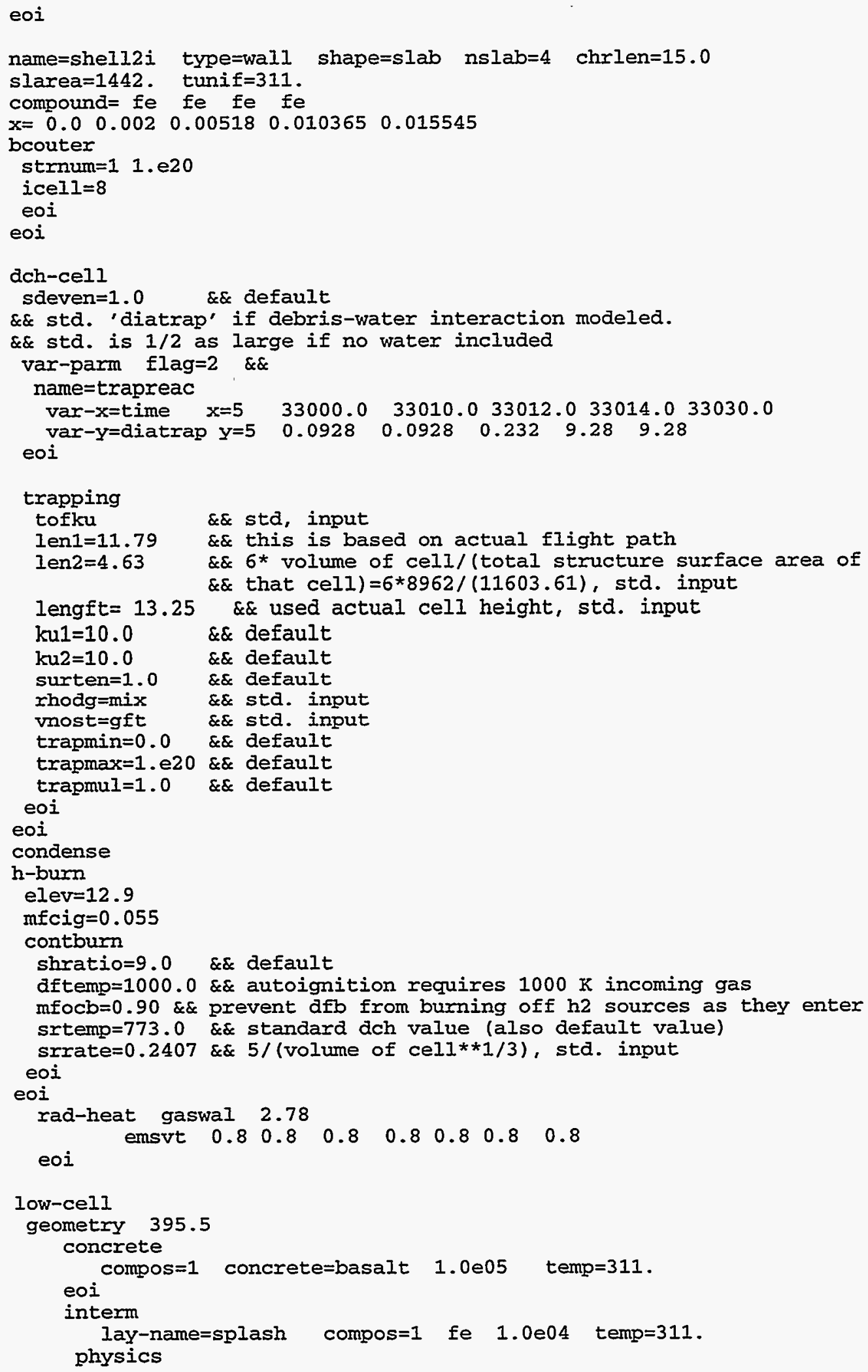


Table 15-3

Sequoyah Input File (Continued)

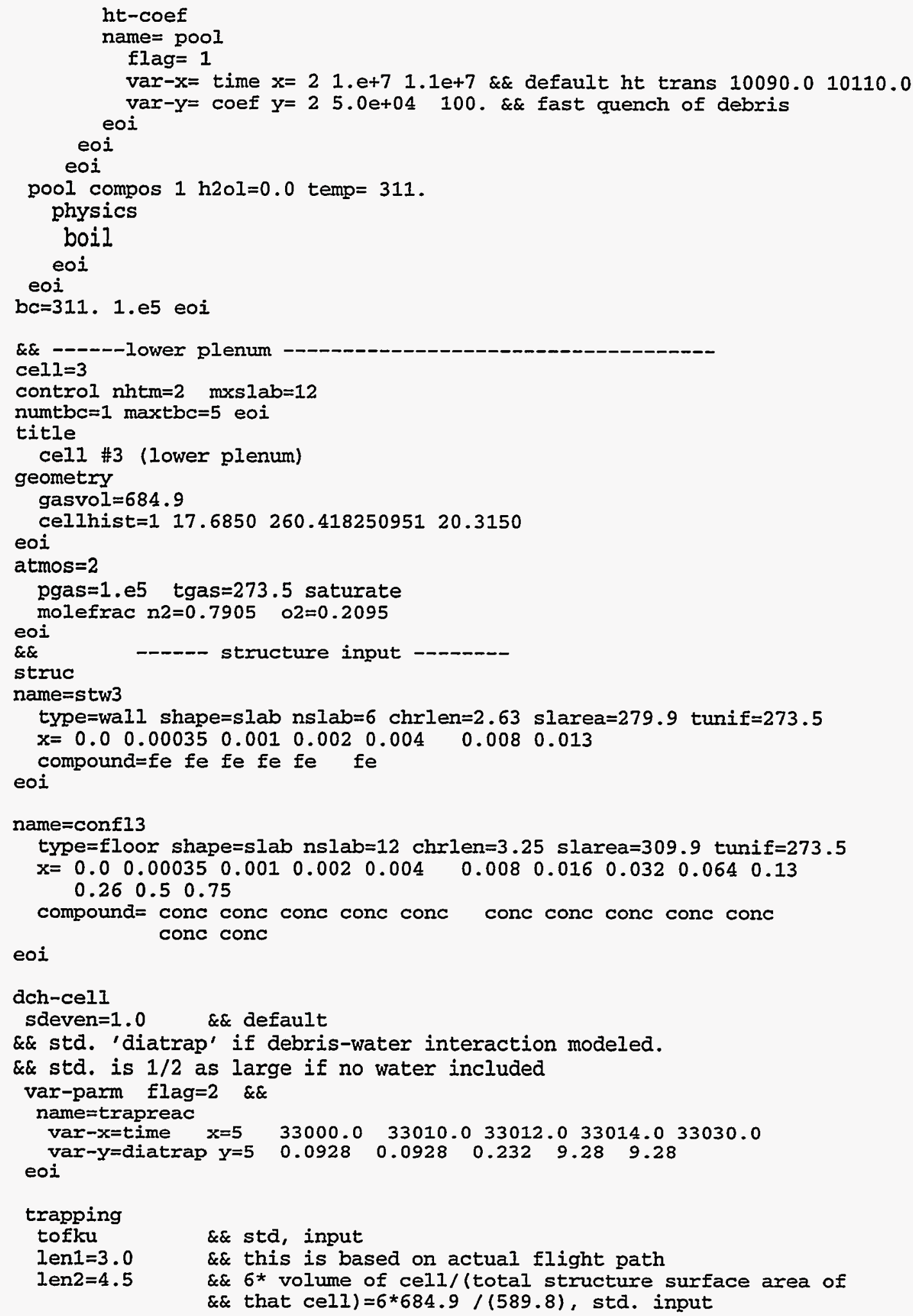


Table 15-3

Sequoyah Input File (Continued)

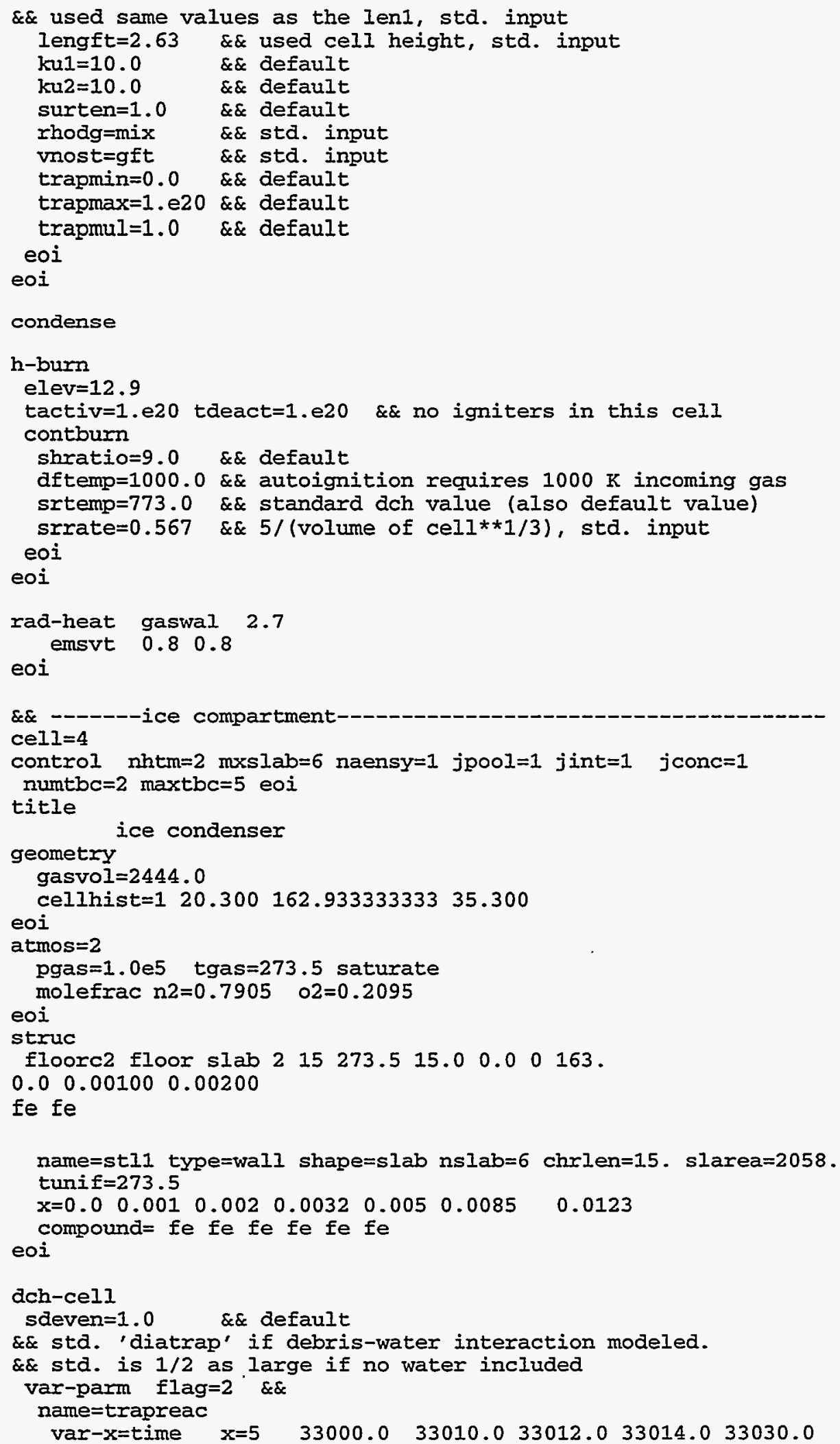


Table 15-3

Sequoyah Input File (Continued)

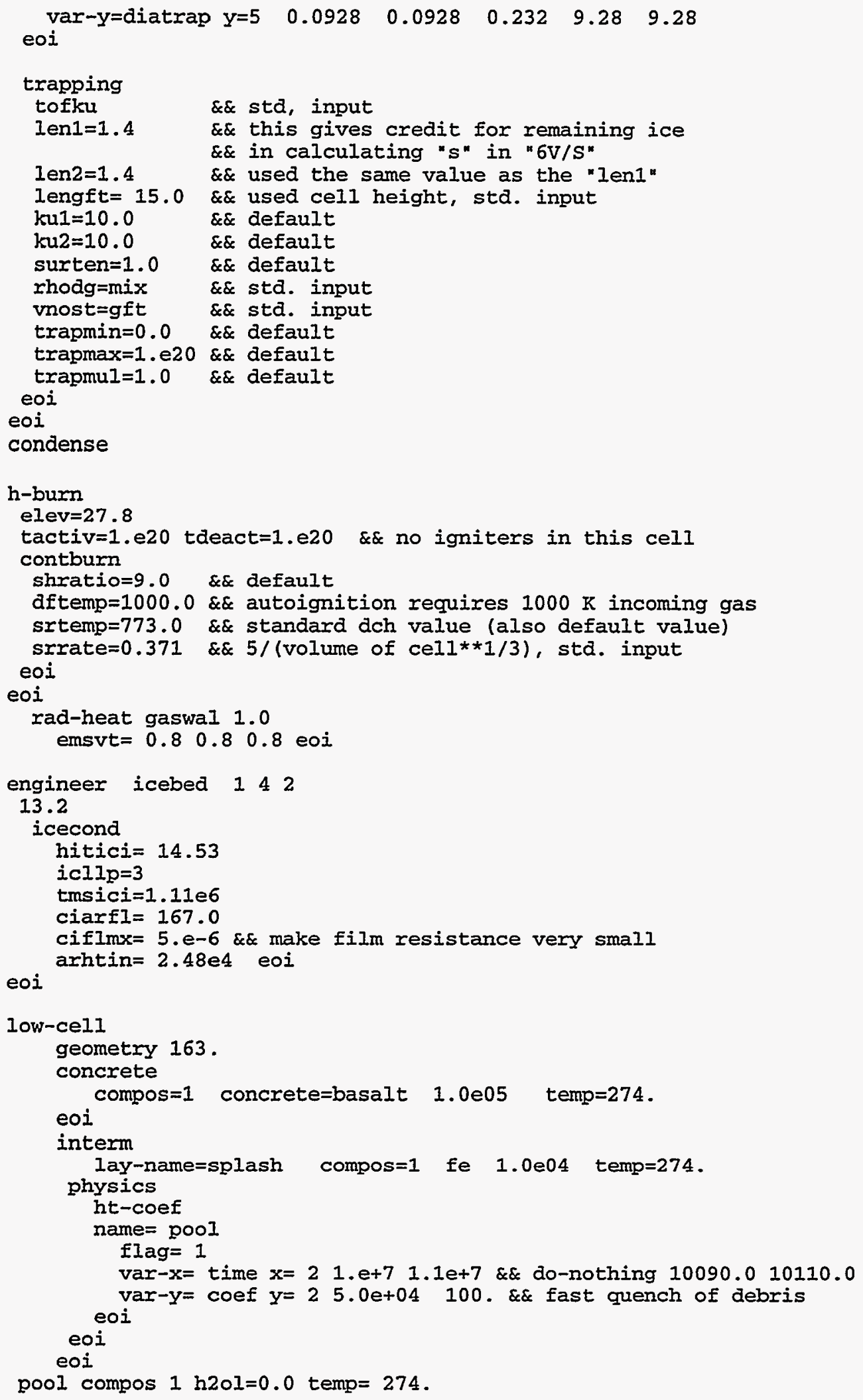


Table 15-3

Sequoyah Input File (Continued)

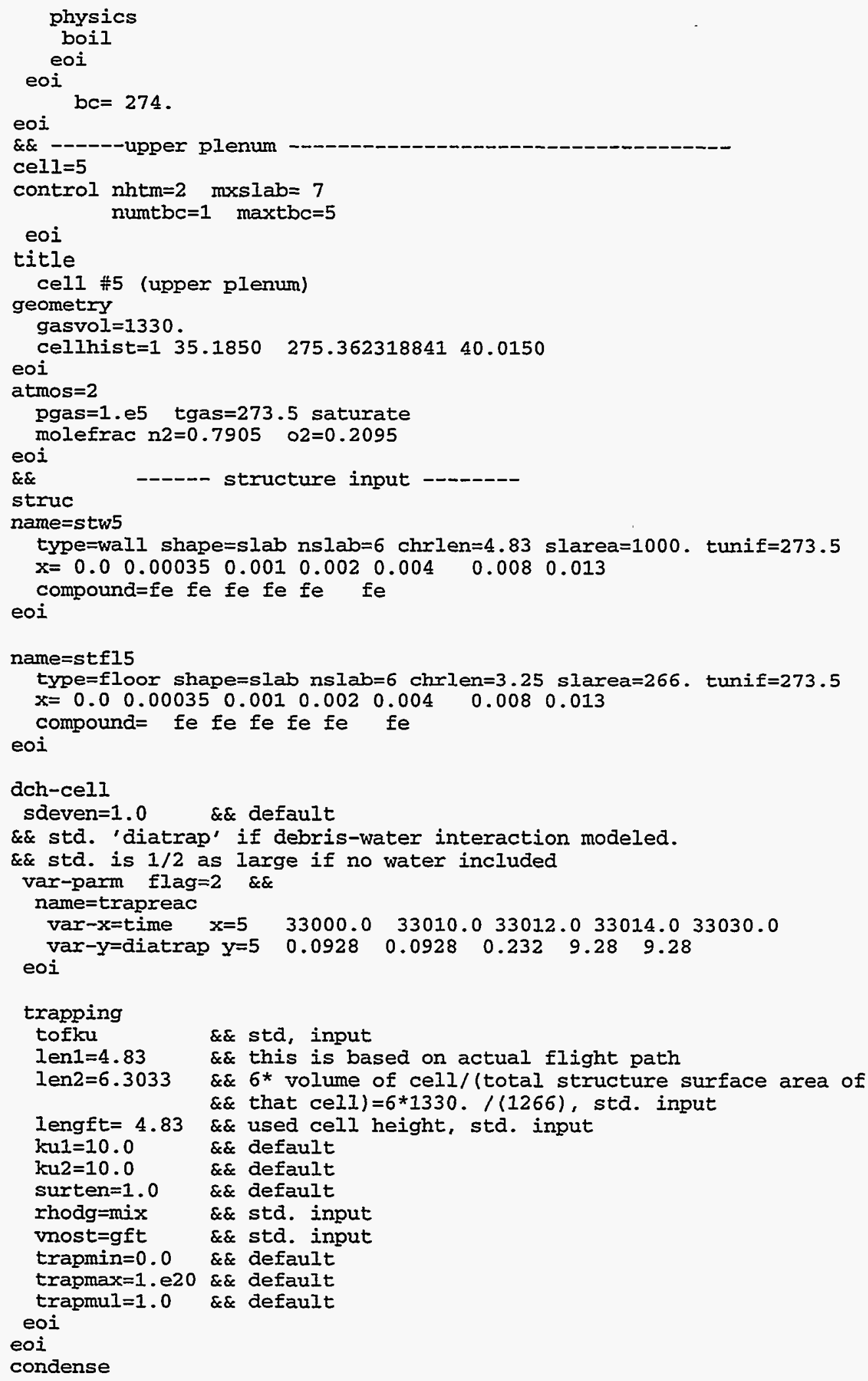




\section{Table 15-3}

\section{Sequoyah Input File (Continued)}

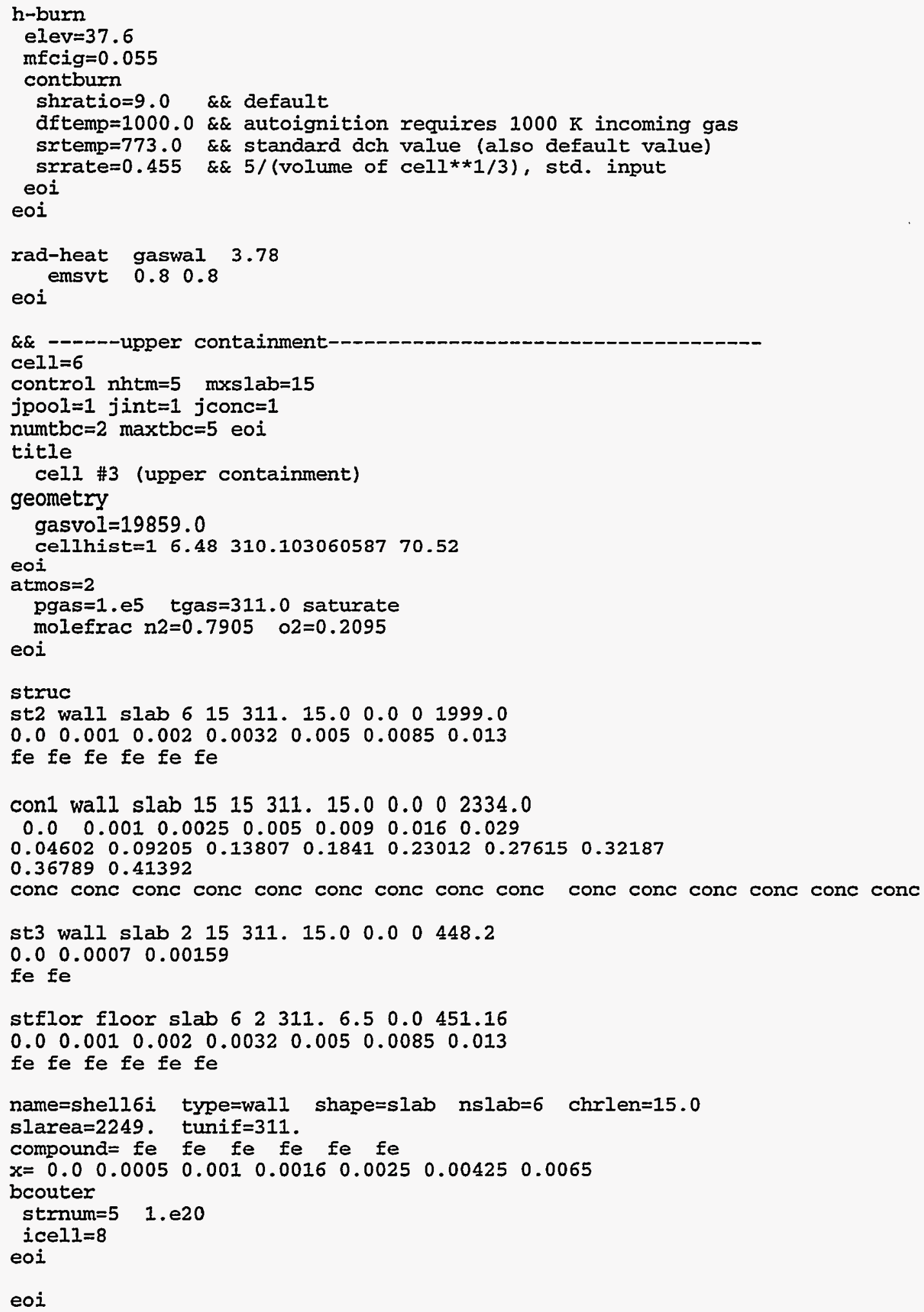


Table 15-3

Sequoyah Input File (Continued)

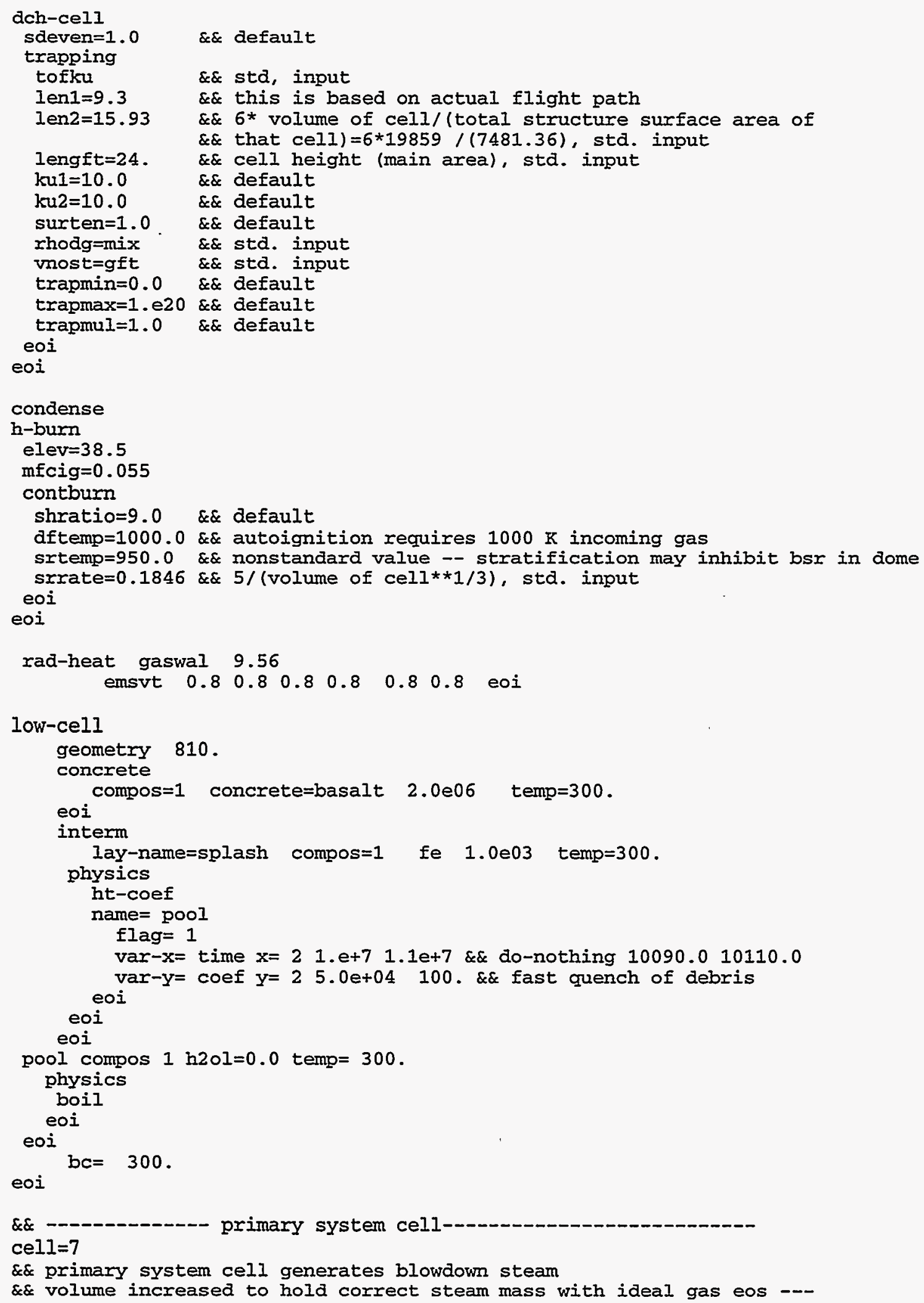




\section{Table 15-3}

\section{Sequoyah Input File (Continued)}

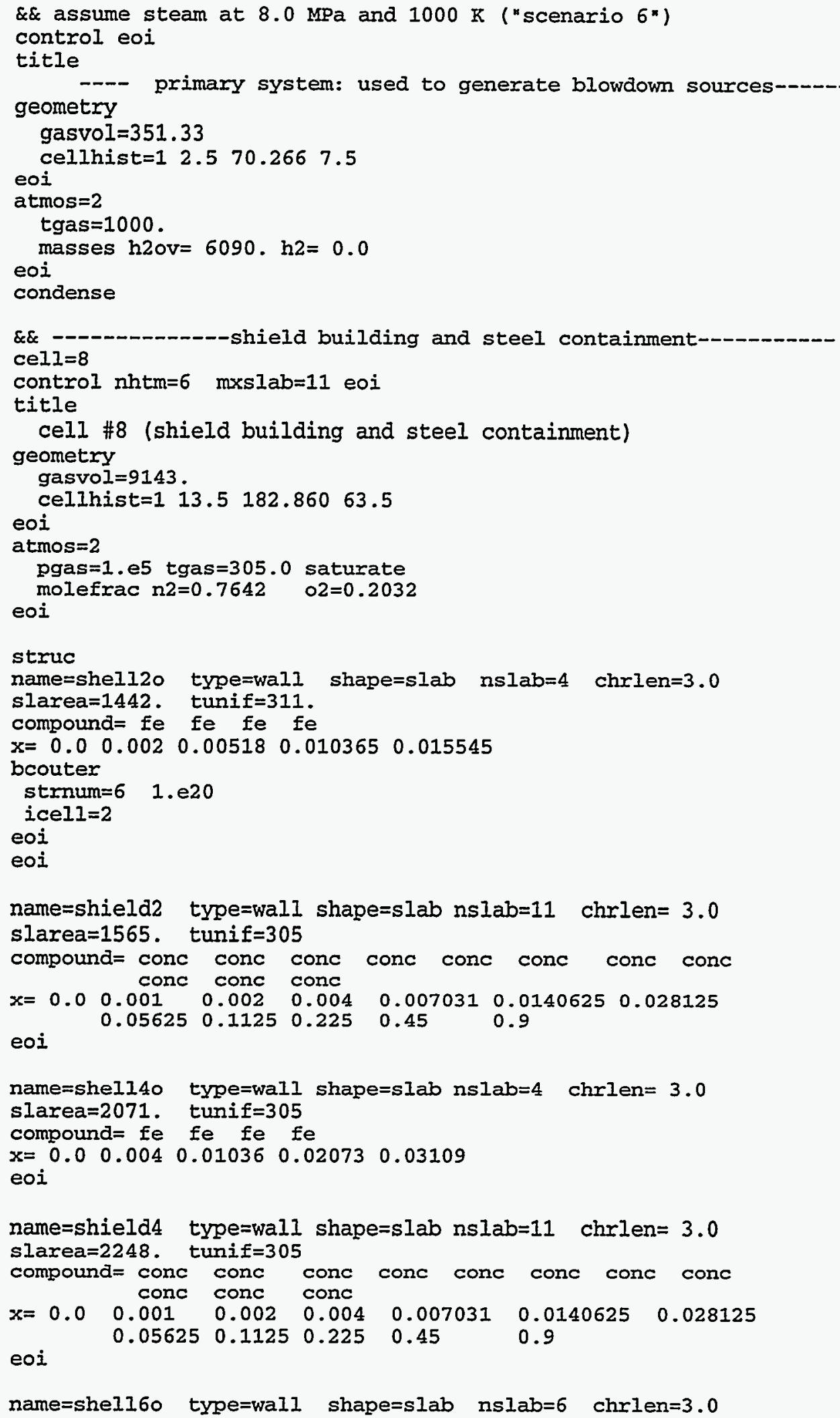


Table 15-3

Sequoyah Input File (Concluded)

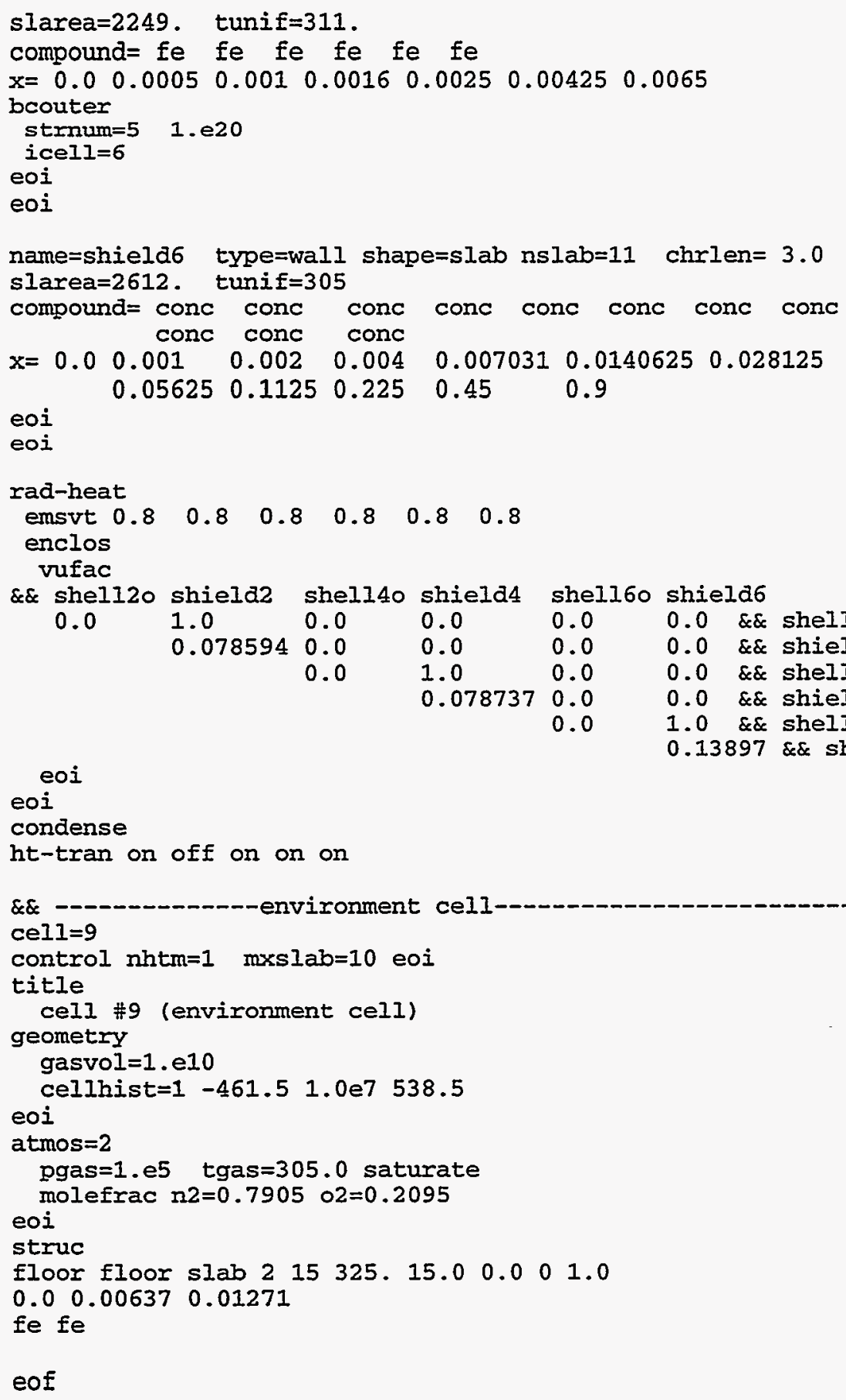




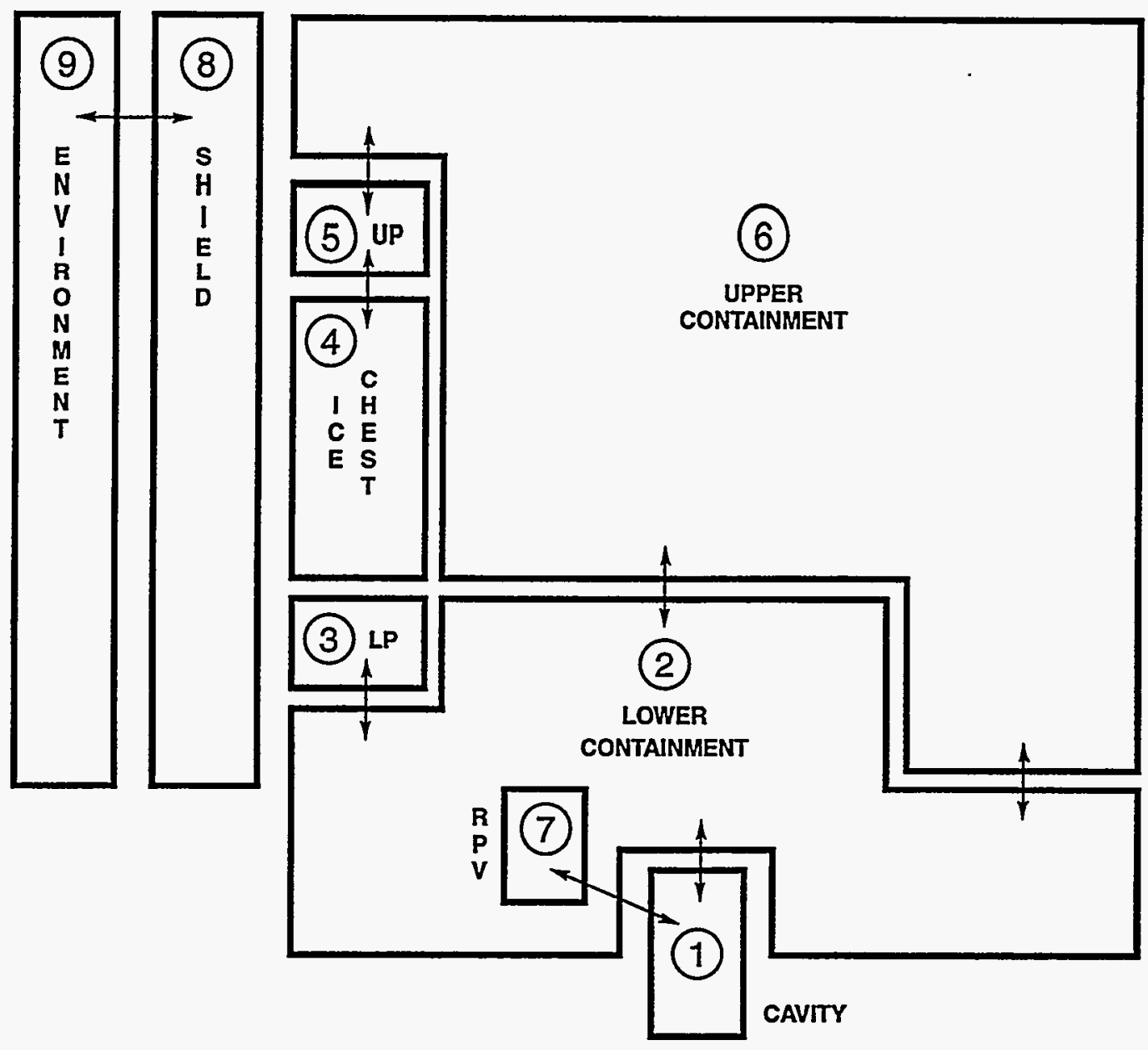

Figure 15-18. Layout of the Sequoyah Model 
Hydrogen combustion models are enabled in the H-BURN block, including the options. Igniters are assumed to be operational but they are present only in Cells 2, 5, and 6; burns initiated in these cells may propagate into other cells if conditions are suitable. Although the igniters are assumed to be operating, it is also assumed that no igniter is sufficiently close to the incoming hydrogen sources to ignite the incoming flows and hence a diffusion flame burn (DFB) is assumed to occur only if the incoming flow is sufficiently hot to autoignite (DFTEMP $=1000$ ). In Cell 2, the DFB model is suppressed prior to vessel breach by specifying an impossible ignition criterion (MFOCB $=0.9$ ) because the DFTEMP temperature threshold is not applied to source tables; i. e., it only applies to incoming flows from another computational cell. After vessel breach, hydrogen burn parameters are reset to values appropriate for $\mathrm{DCH}$, as noted previously.

For DCH calculations, the user must specify the names and properties of core debris materials that can be present. These parameters are given in the USERDEF and USERDAT options block. Global specifications for DCH are given in the DHEAT block. The drop diameter for each debris field is specified individually following the DIABIN keyword. The mass distribution to bins is specified in the FDISTR block. The remaining input keywords in the DHEAT block apply to radiative and convective heat transfer, mass transfer, and iron/steam chemical equilibrium. The values specified in the DHEAT block are used for all cells since they are not overridden in the cell-level DCH-CELL blocks. Other DCH parameters that are specific to each cell are specified under the DCH-CELL blocks.

In this sample problem, vessel breach is assumed to occur at 33,000 s. Because the hydrogen burn parameters appropriate prior to vessel breach are not appropriate during $\mathrm{DCH}$, the calculations were stopped at the time of vessel breach and restarted with the hydrogen burn parameters set to values considered to be suitable for the DCH calculation. Though there are some minor variations, the DCH-related input closely follows the standardized DCH input prescription summarized in Section 13.3.2.2. Although the DCH event begins at $33,000 \mathrm{~s}$, debris sources are also specified in the initial input file to reserve the arrays for the restart run. The debris sources defined in the restart input file are the ones actually used in the calculation. The accompanying restart input file for the initial input file is listed in Table 15-4.

With the exception of the primary system cell, all the cells start with a saturated steam/air mixture in the atmosphere at $100 \mathrm{kPa}$. The primary system is pressurized to $8.0 \mathrm{MPa}$ at a temperature of $1000 \mathrm{~K}$. Pools defined in Cells $1,2,4$, and 6 are initially dry.

In the present accident scenario, it is assumed that $10,000 \mathrm{~kg}$ of water overlie the melt in the RPV. CONTAIN cannot model this configuration explicitly. However, some of the potential effects of the water being co-ejected with the debris are modeled by introducing a user-defined source of $\mathrm{H} 2 \mathrm{OV}$ with the appropriate enthalpy in parallel with the airborne debris sources.

Pressure-time histories for the containment cells are plotted in Figure 15-19 for the time period prior to vessel breach. There are many small pressure spikes and two larger ones corresponding to hydrogen burns. Aside from the hydrogen burns, there is an initial fairly rapid pressure rise at approximately $5000 \mathrm{~s}$ as steam enters the lower compartment and forces the air into the upper 
Table 15-4

Sequoyah Restart Input File

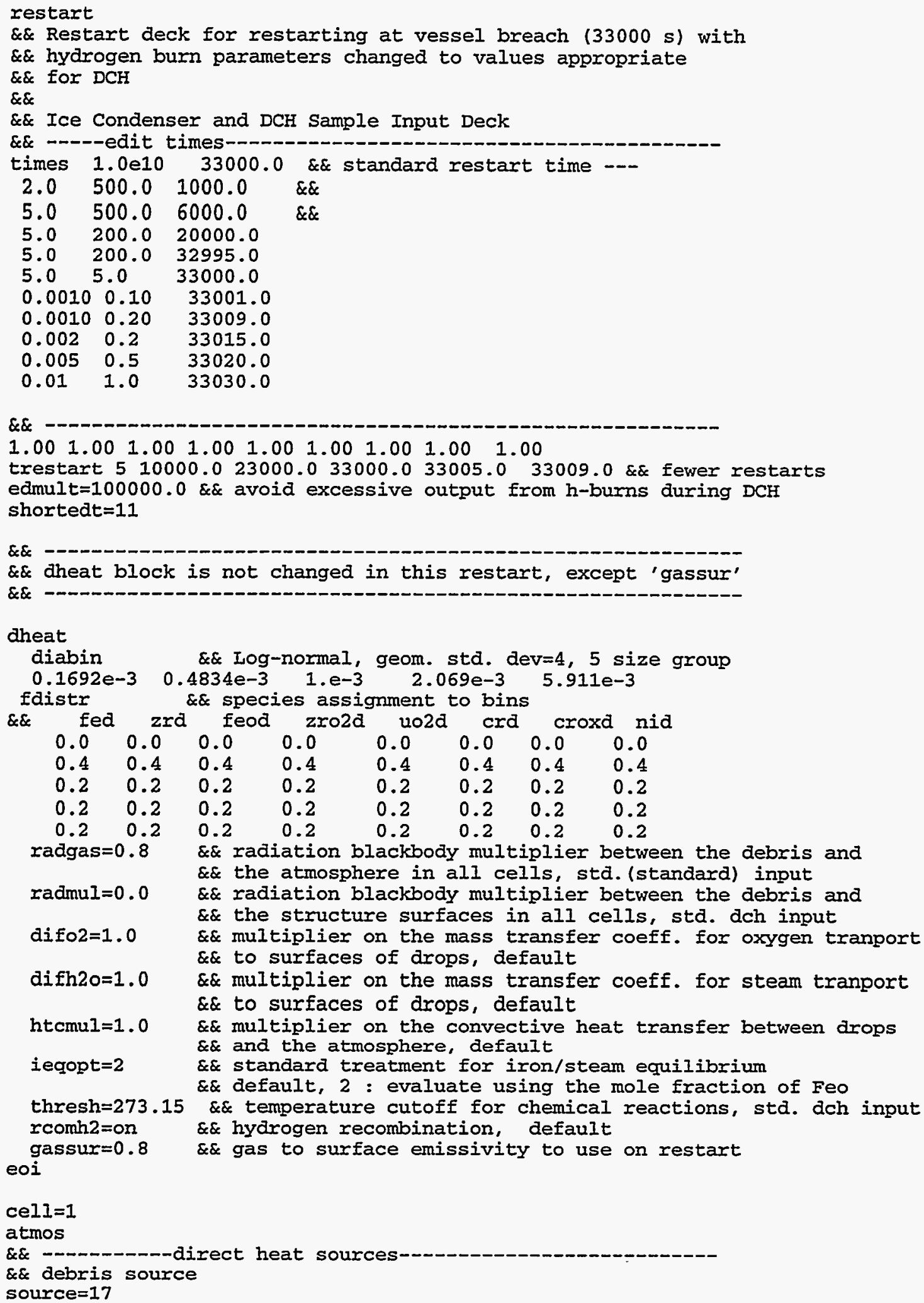


Table 15-4

Sequoyah Restart Input File (Continued)

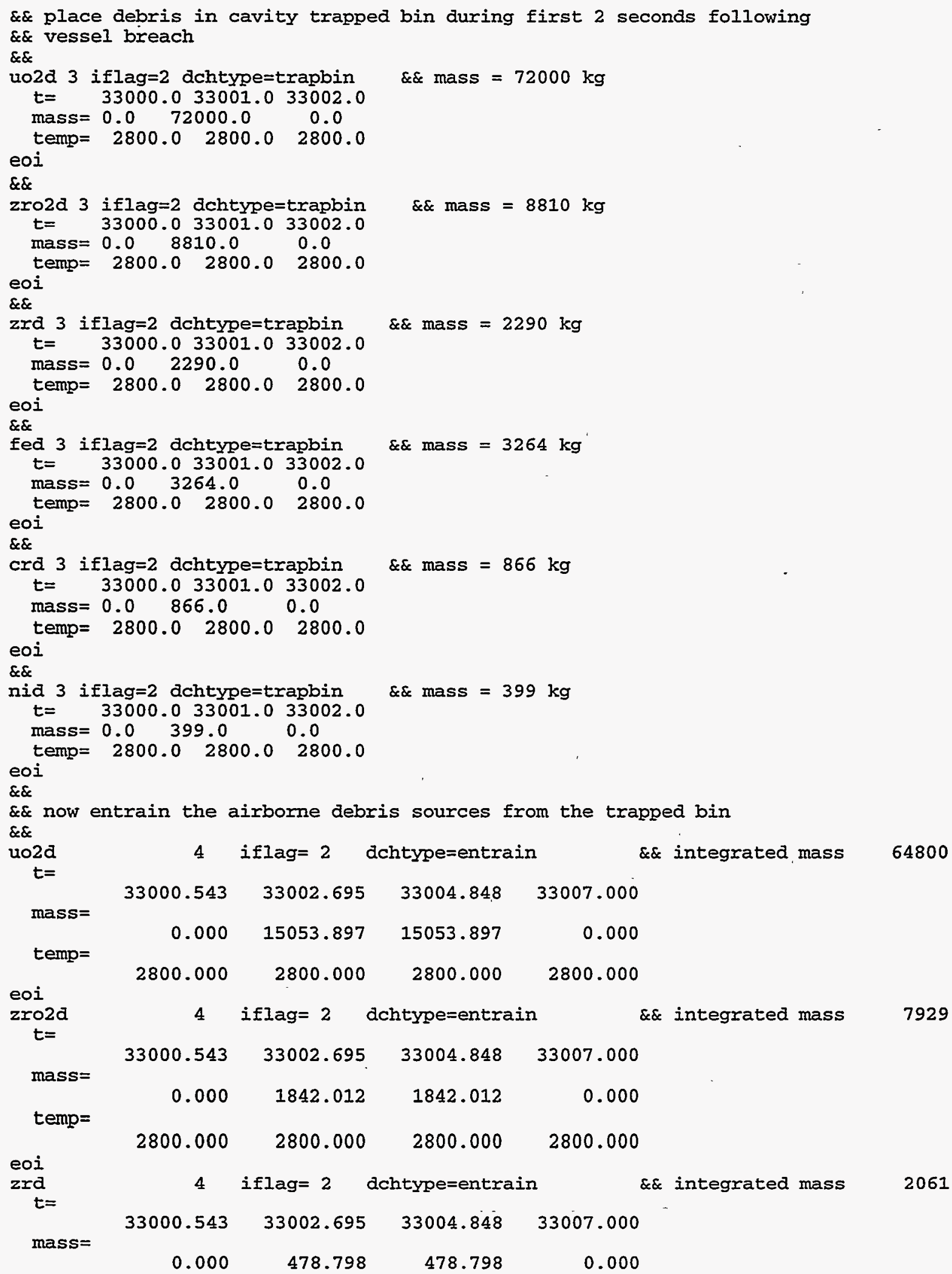


Table 15-4

Sequoyah Restart Input File (Continued)

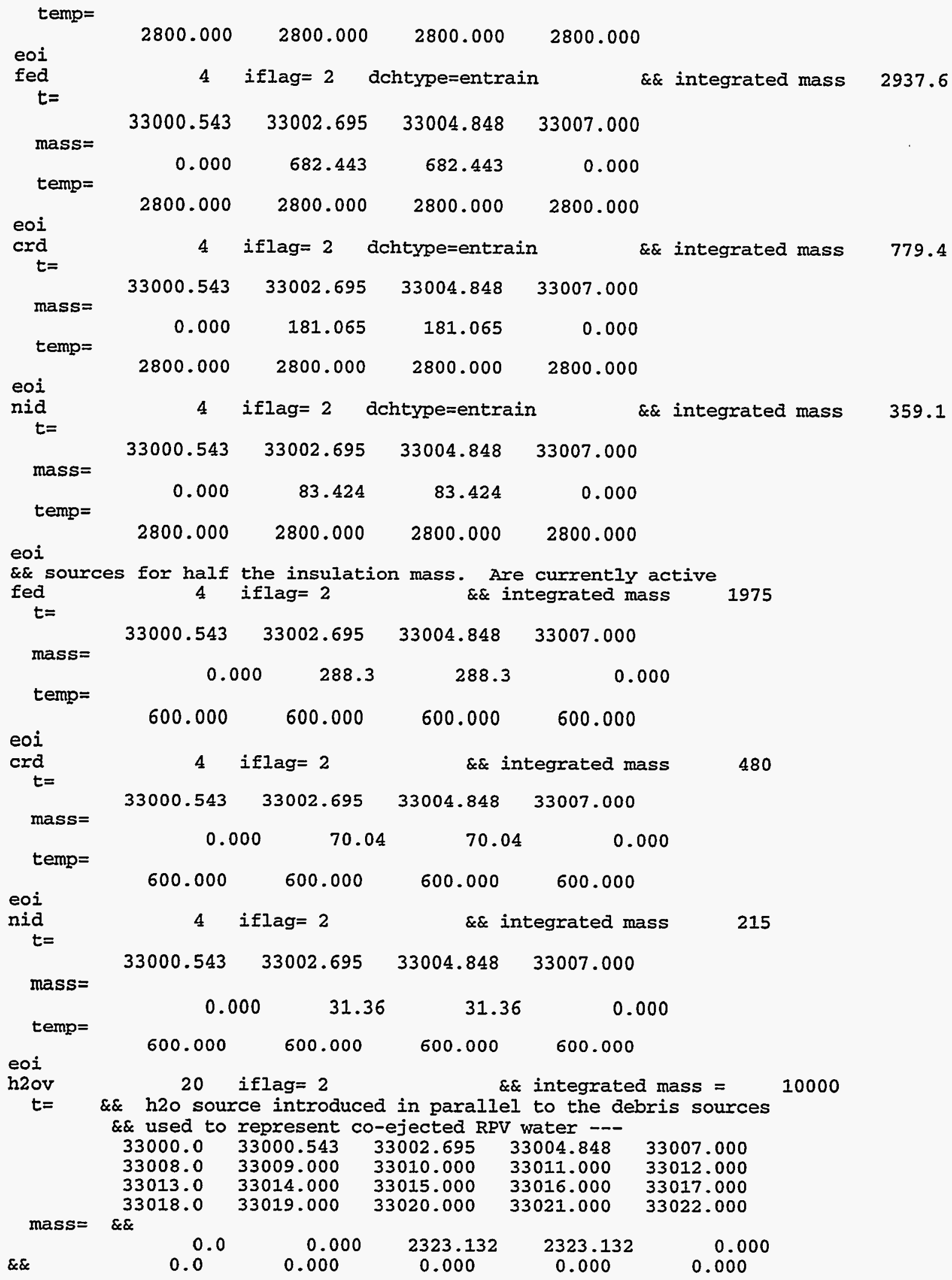

Rev. 0 
Table 15-4

Sequoyah Restart Input File (Continued)

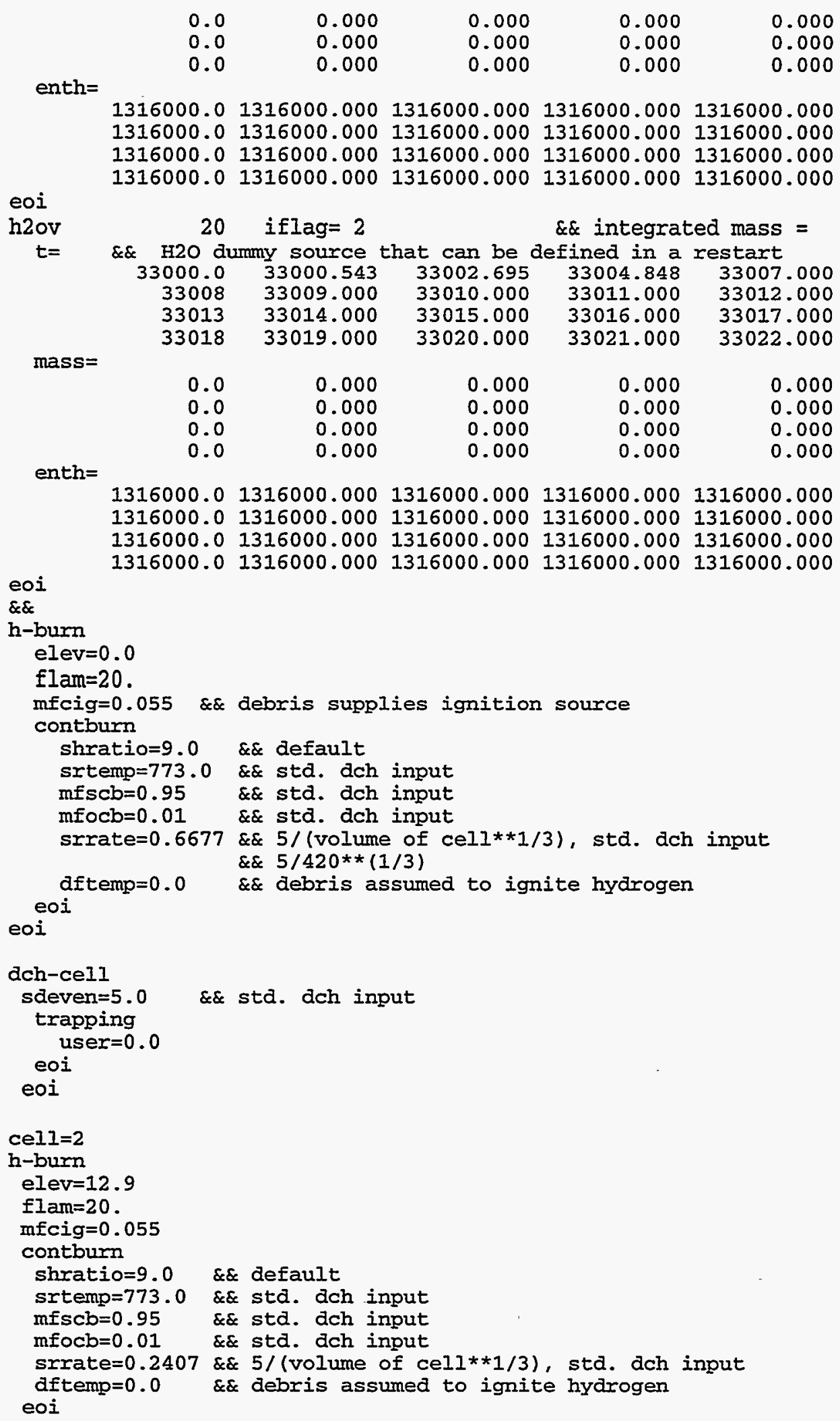

mass=

$\begin{array}{lllll}0.0 & 0.000 & 0.000 & 0.000 & 0.000 \\ 0.0 & 0.000 & 0.000 & 0.000 & 0.000 \\ 0.0 & 0.000 & 0.000 & 0.000 & 0.000 \\ 0.0 & 0.000 & 0.000 & 0.000 & 0.000\end{array}$

enth $=$

$1316000.0 \quad 1316000.000 \quad 1316000.000 \quad 1316000.000 \quad 1316000.000$

$1316000.0 \quad 1316000.0001316000 .000 \quad 1316000.000 \quad 1316000.000$

$1316000.0 \quad 1316000.000 \quad 1316000.000 \quad 1316000.000 \quad 1316000.000$

eoi

1316000.01316000 .0001316000 .0001316000 .0001316000 .000

$\& \&$

h-burn

elev $=0.0$

flam $=20$.

mfcig $=0.055$ \&\& debris supplies ignition source

contburn

shratio=9.0 \&\& default

srtemp $=773.0 \quad \& \&$ std. dch input

mfscb $=0.95 \quad \& \&$ std. dch input

$\mathrm{mfocb}=0.01 \quad \& \&$ std. dch input

srrate $=0.6677 \& \& 5 /$ (volume of $\operatorname{cell} 1 * * 1 / 3)$, sta. dch input

\&\& $5 / 420 * *(1 / 3)$ eoi

dftemp $=0.0 \quad \& \&$ debris assumed to ignite hydrogen

eoi

dch-cell

sdeven=5.0 \&\& std. dch input

trapping

user $=0.0$

eoi

eoi

$\operatorname{cel} 1=2$

h-burn

elev $=12.9$

flam $=20$.

mfcig $=0.055$

contburn

shratio $=9.0 \quad \& \&$ default

srtemp $=773.0$ \&\& std. dch input

mfscb $=0.95$ \&\& sta. dch input

mfocb $=0.01$ \&\& sta. dch input

srrate $=0.2407 \& \& 5 /$ (volume of cell**1/3), std. dch input

dftemp $=0.0 \quad \& \&$ debris assumed to ignite hydrogen

eoi 
Table 15-4

Sequoyah Restart Input File (Continued)

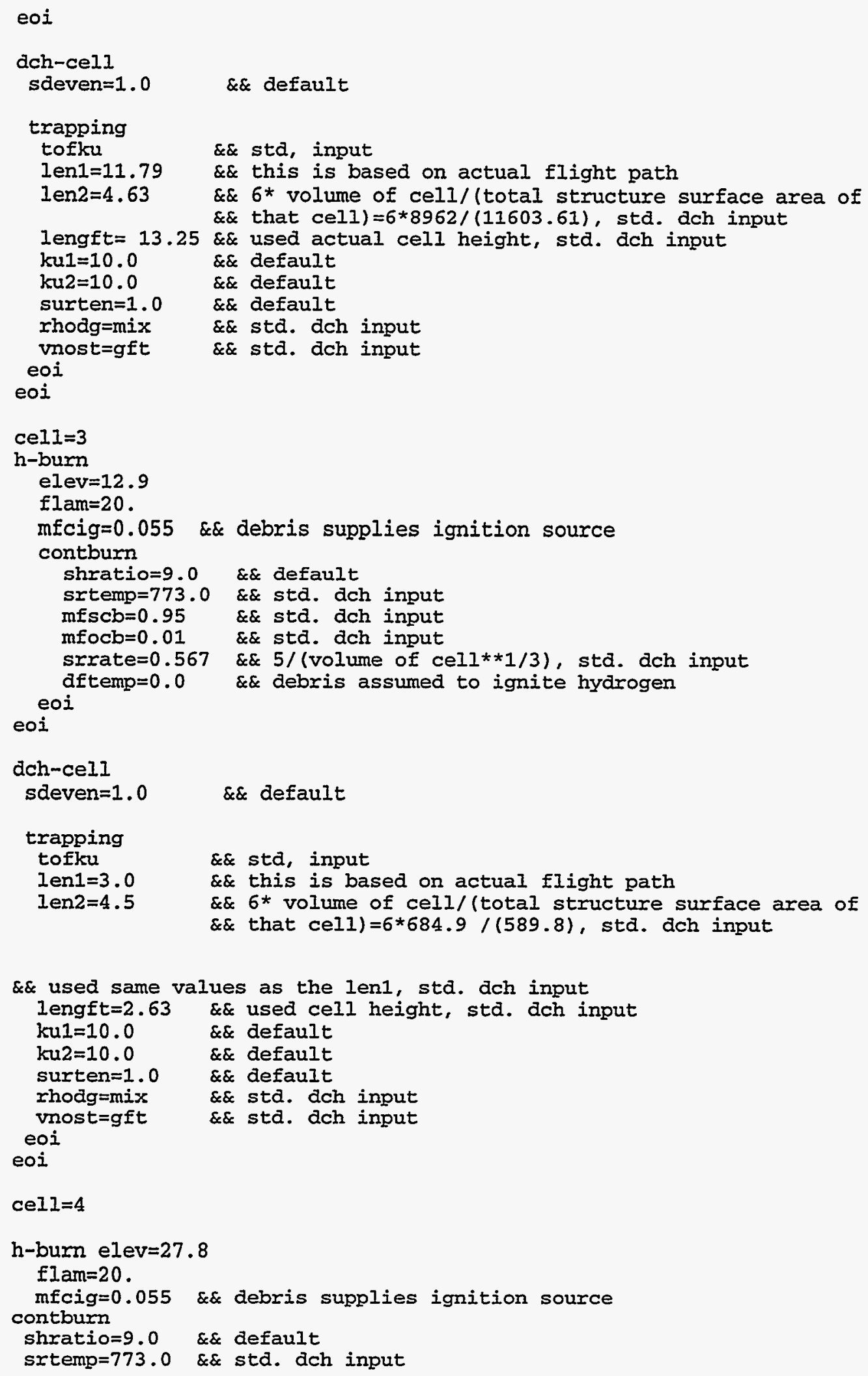


Table 15-4

\section{Sequoyah Restart Input File (Concluded)}

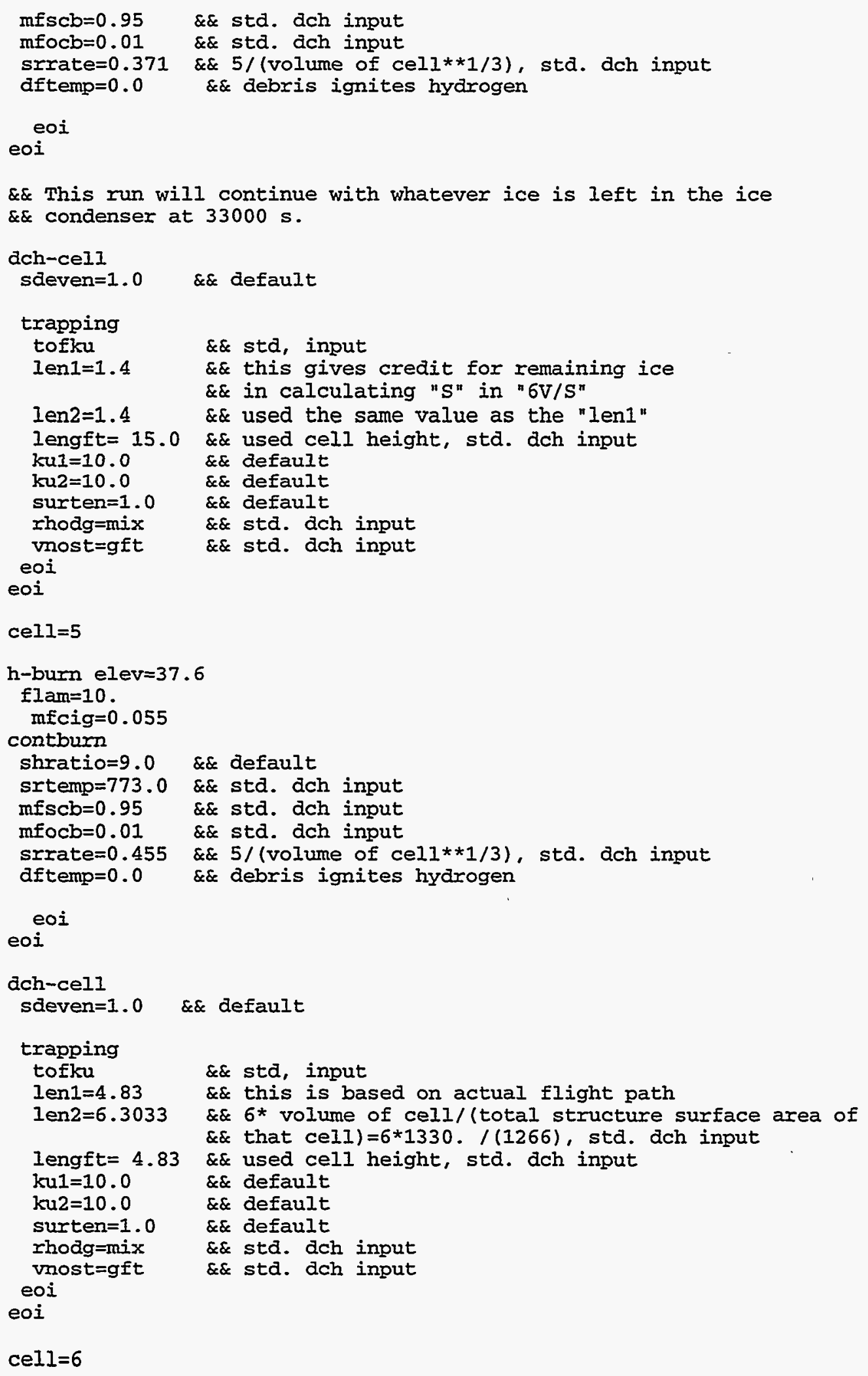


Table 15-4

Sequoyah Restart Input File (Concluded)

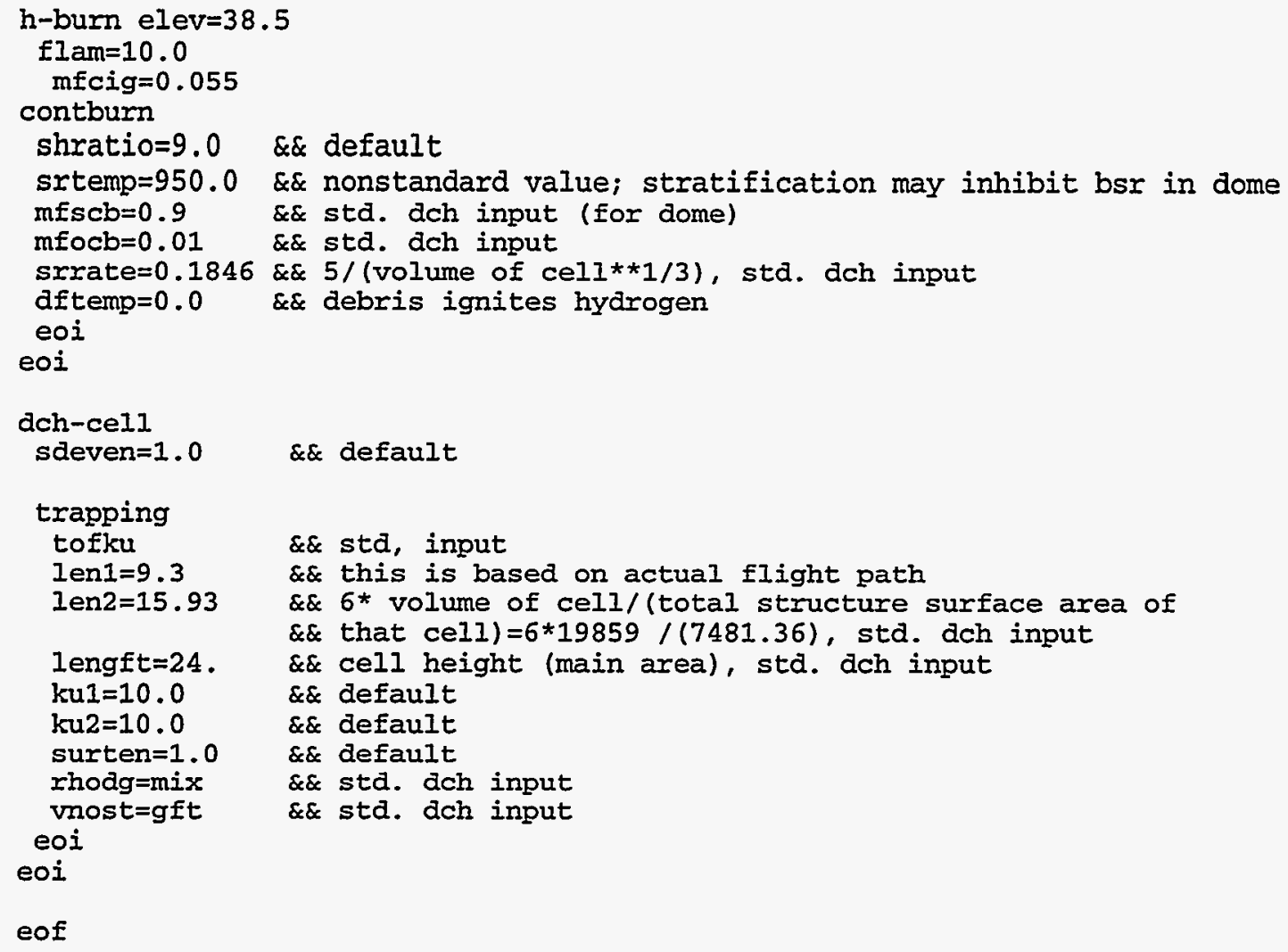




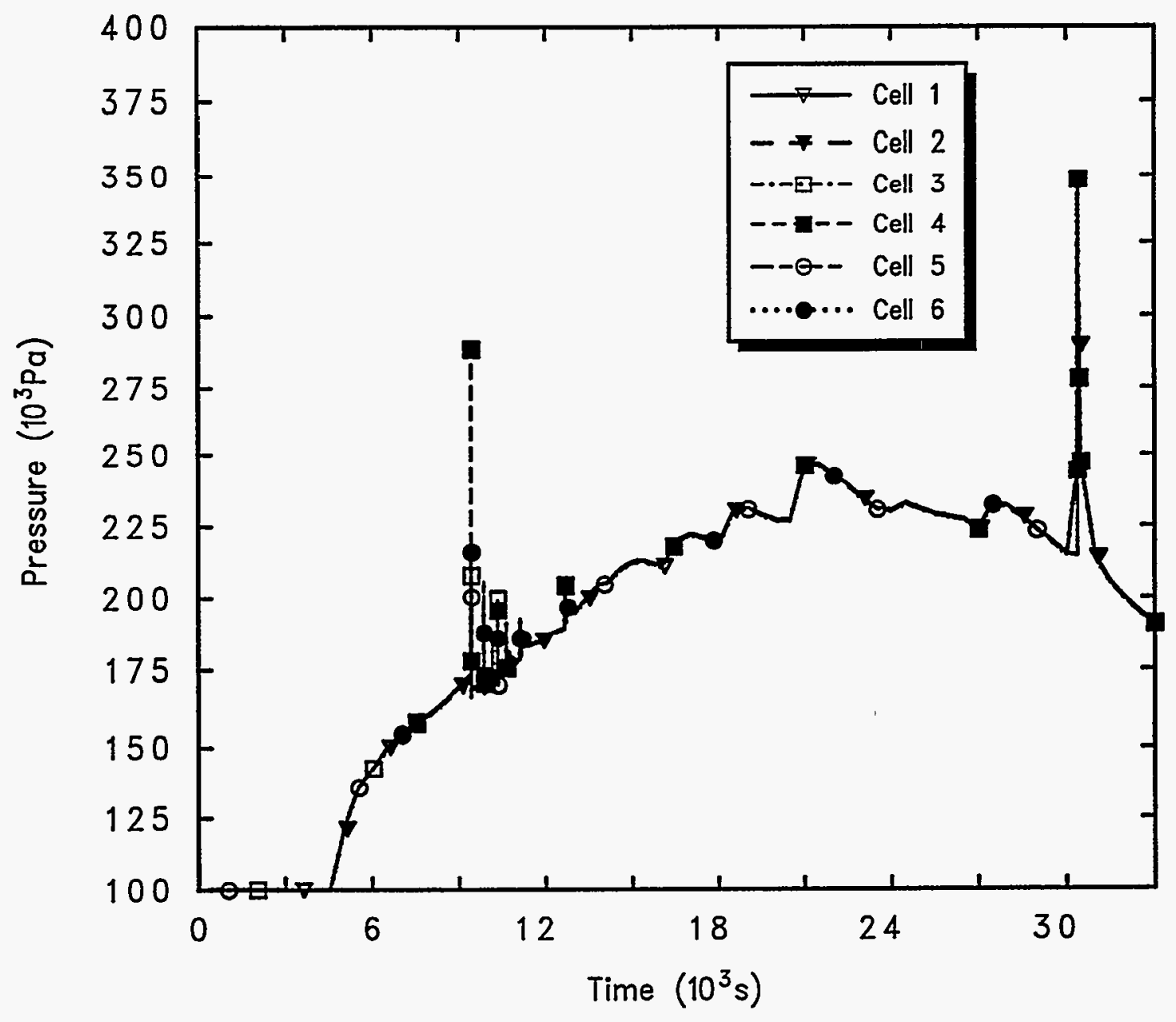

Figure 15-19. Pressure-Time Histories for the Containment Cells 
compartment. The subsequent upward trend that continues until approximately $21,000 \mathrm{~s}$ results from small flow paths that bypass the ice condenser. These paths represent the refueling canal drains (area $0.175 \mathrm{~m}^{2}$, elevation approximately $6.5 \mathrm{~m}$ ) and leakage through the operating file separating upper and lower compartments $\left(0.29 \mathrm{~m}^{2}\right.$ area, elevation approximately $\left.20.5 \mathrm{~m}\right)$. Although small compared with the ice condenser doors ( $78 \mathrm{~m}^{2}$ fully open), large amounts of steam can bypass the ice condenser when time intervals are long and the driving pressure is insufficient to overcome the ice condenser cold head.

Figure 15-20 presents flow rates through the paths leaving the lower compartment (Cell 2). Hydrogen burns cause the abrupt surges in the interval 9000 to $12,000 \mathrm{~s}$ and at about $31,000 \mathrm{~s}$. Initially, flow into the plenum is positive, but at late times it becomes negative, meaning flow is from the plenum back into the lower compartment. This is possible because a small reverse pressure (approximately $14 \mathrm{~Pa}$ ) is required to fully close the doors. Instead of passing through the ice condenser, most of the steam after approximately $14,000 \mathrm{~s}$ is able to flow to the upper containment via the file leakage, and also via the refueling drains until these are flooded at about $23,000 \mathrm{~s}$. These results illustrate how seemingly minor details such as the small bypass flow paths can have an important effect upon the results of the calculation.

Hydrogen burned prior to vessel breach is plotted in Figure 15-21. Large amounts of hydrogen are burned off before vessel breach, as a result of the igniters being operational in this scenario. If there were no ignition sources, this hydrogen would accumulate and result in a very large burn when hot debris provided ignition sources at vessel breach.

Pressure-time histories during the DCH event are plotted in Figure 15-22. Maximum pressures in the containment are slightly under $0.4 \mathrm{MPa}$, while cavity pressures rise to much higher values during the period of debris dispersal. Qualitatively, cavity pressurization during debris dispersal is a wellestablished result of DCH experiments; quantitatively, there are important uncertainties in CONTAIN predictions of cavity pressurization. In the present case, this pressurization is augmented by the assumed co-ejection of RPV water in parallel with the debris sources. There are no experimental results available involving realistic simulation of $\mathrm{DCH}$ events with co-ejected RPV water.

Figure 15-23 gives the mass of trapped debris in the various cells as a function of time. The mass in the cavity peaks as debris is introduced into the cavity using source tables with DCHTYPE = TRAPBIN specified; the mass then decreases as much of it is transferred to the airborne debris fields using source tables with the DCHTYPE = ENTRAIN option. The time-dependence of the latter transfer was defined according to the prescription given in Section 13.3.2.2 (see Equations (13-1) through (13-4) and related discussion). Subsequent transport of debris to other cells and debris trapping in the other cells are controlled by the models in the code. It is seen that most of the debris is de-entrained in the lower compartment (Cell 2), but nontrivial amounts do reach the lower plenum and the ice condenser (Cells 3 and 4). Very little gets beyond the ice condenser, however.

In Figure 15-24, information concerning the mass balance for hydrogen and oxygen is plotted. The curve labeled " $2 \mathrm{x} \mathrm{O}_{2}$ React" is the number of kilogram-moles of oxygen consumed since the start of the calculation at reactor shutdown. The " $\mathrm{H}_{2}$ Burned" curve gives the cumulative hydrogen 


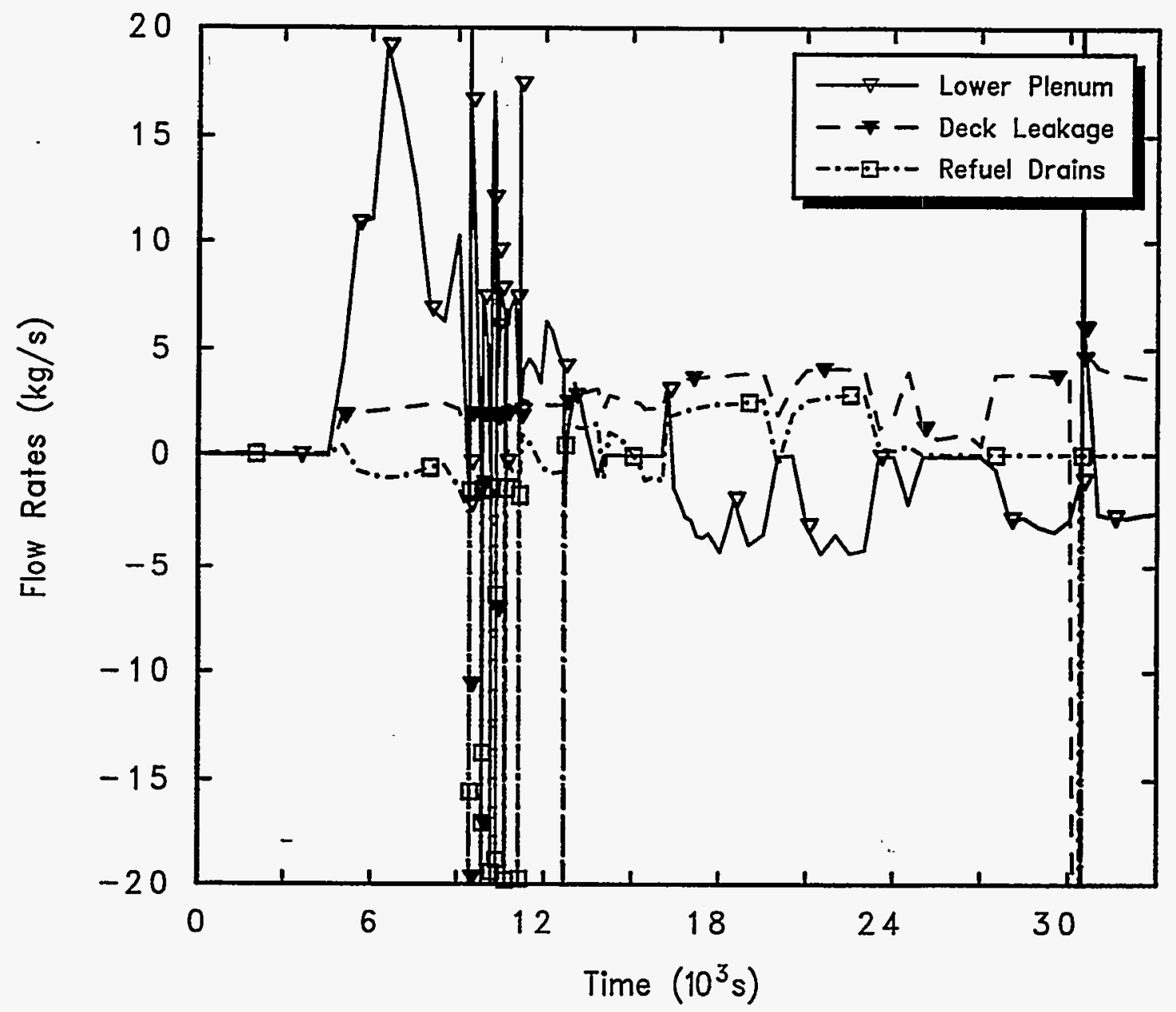

Figure 15-20. Flow Rates Through Paths Leaving the Lower Compartment 


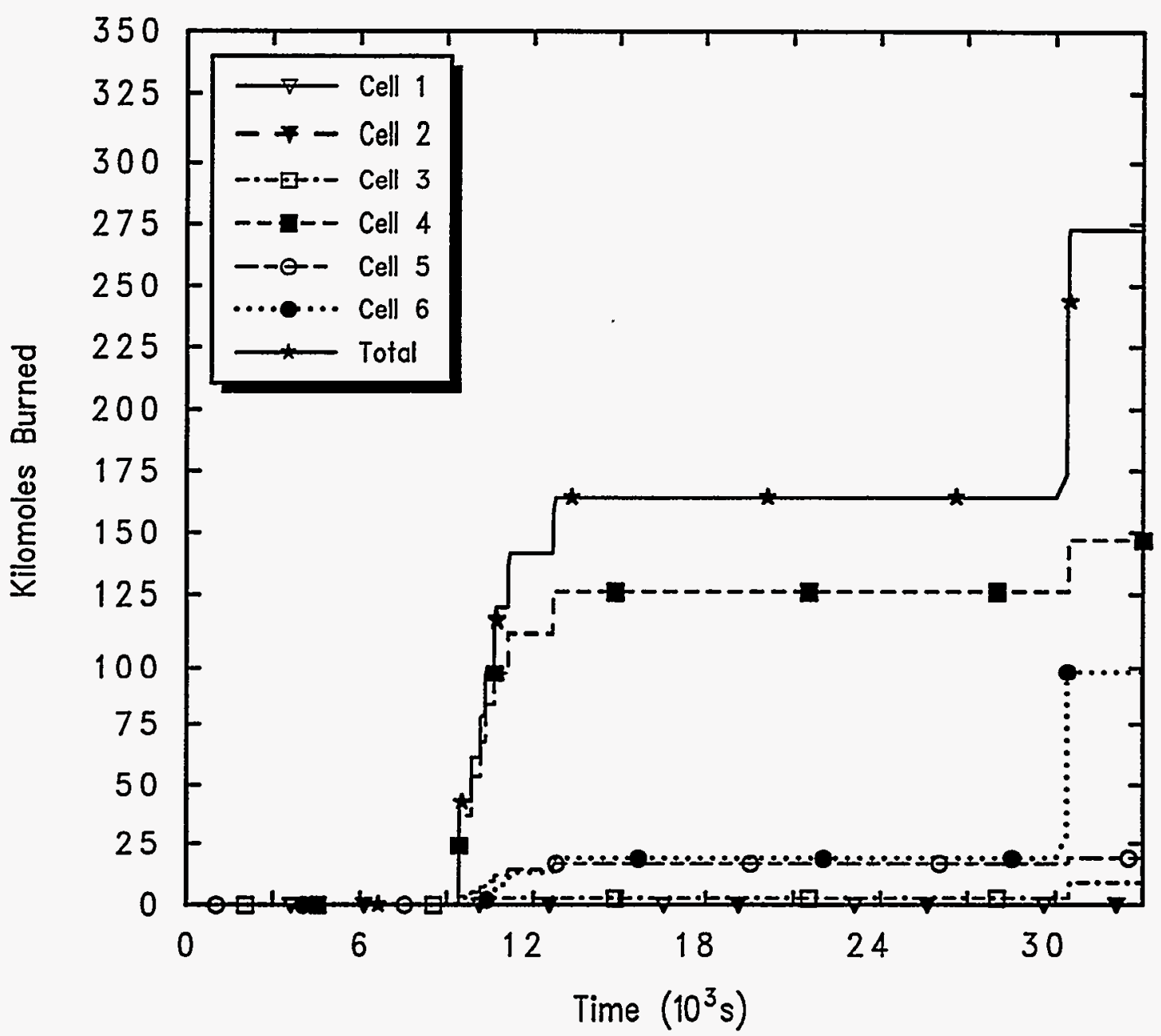

Figure 15-21. Hydrogen Burned Prior to Vessel Breach 


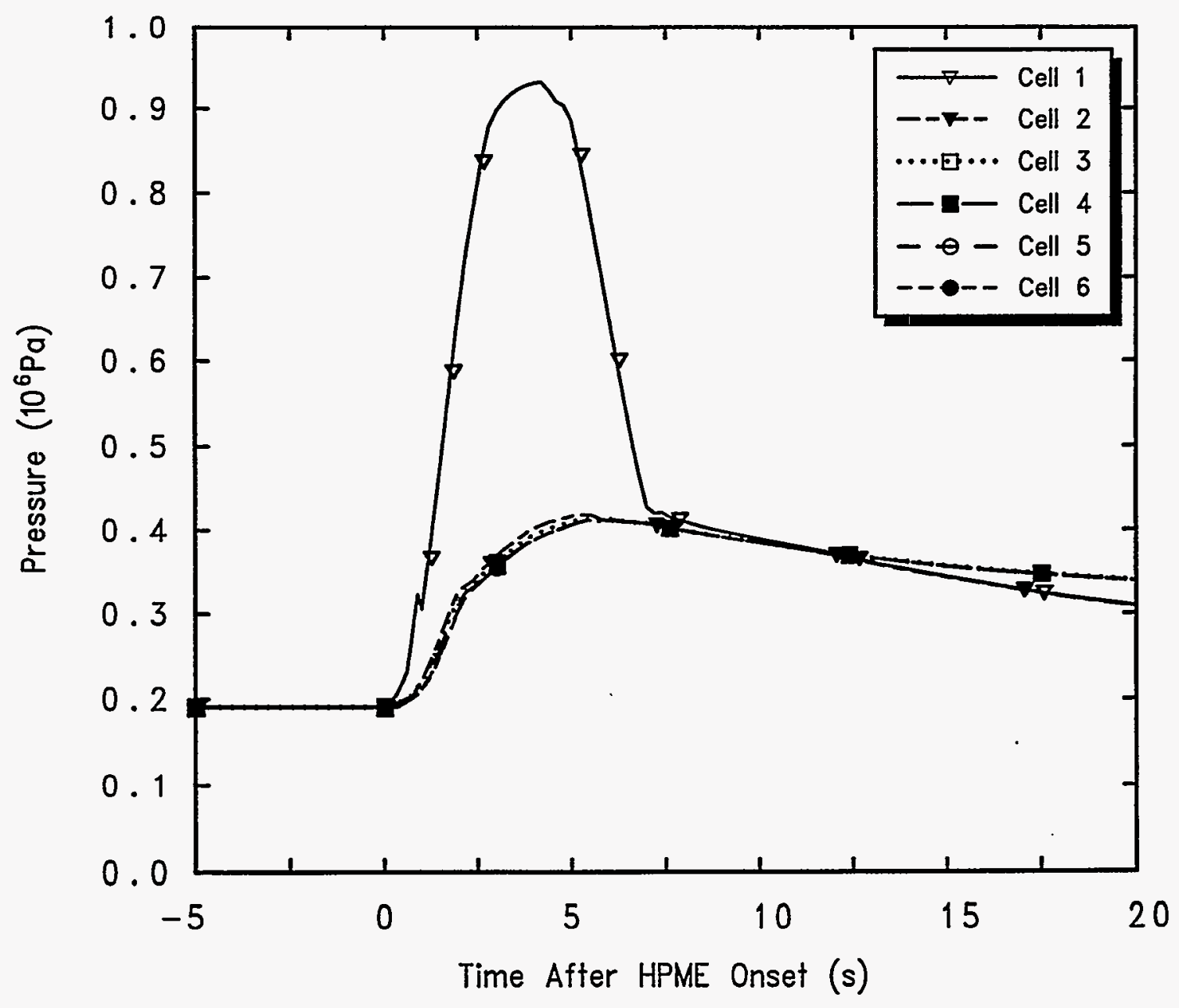

Figure 15-22. Pressure-Time Histories During the DCH Event 


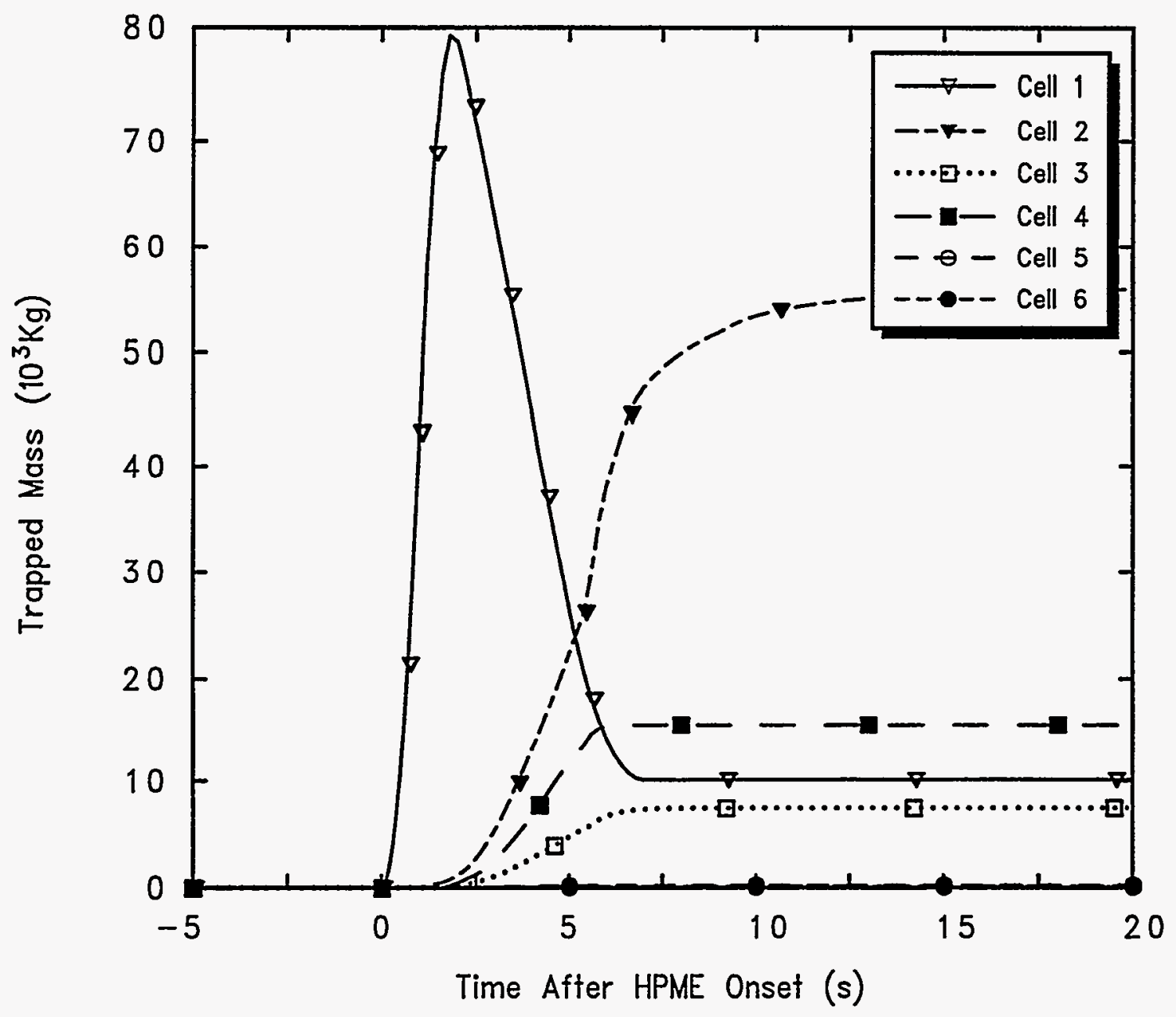

Figure 15-23. Mass of Trapped Debris in Various Cells as a Function of Time 


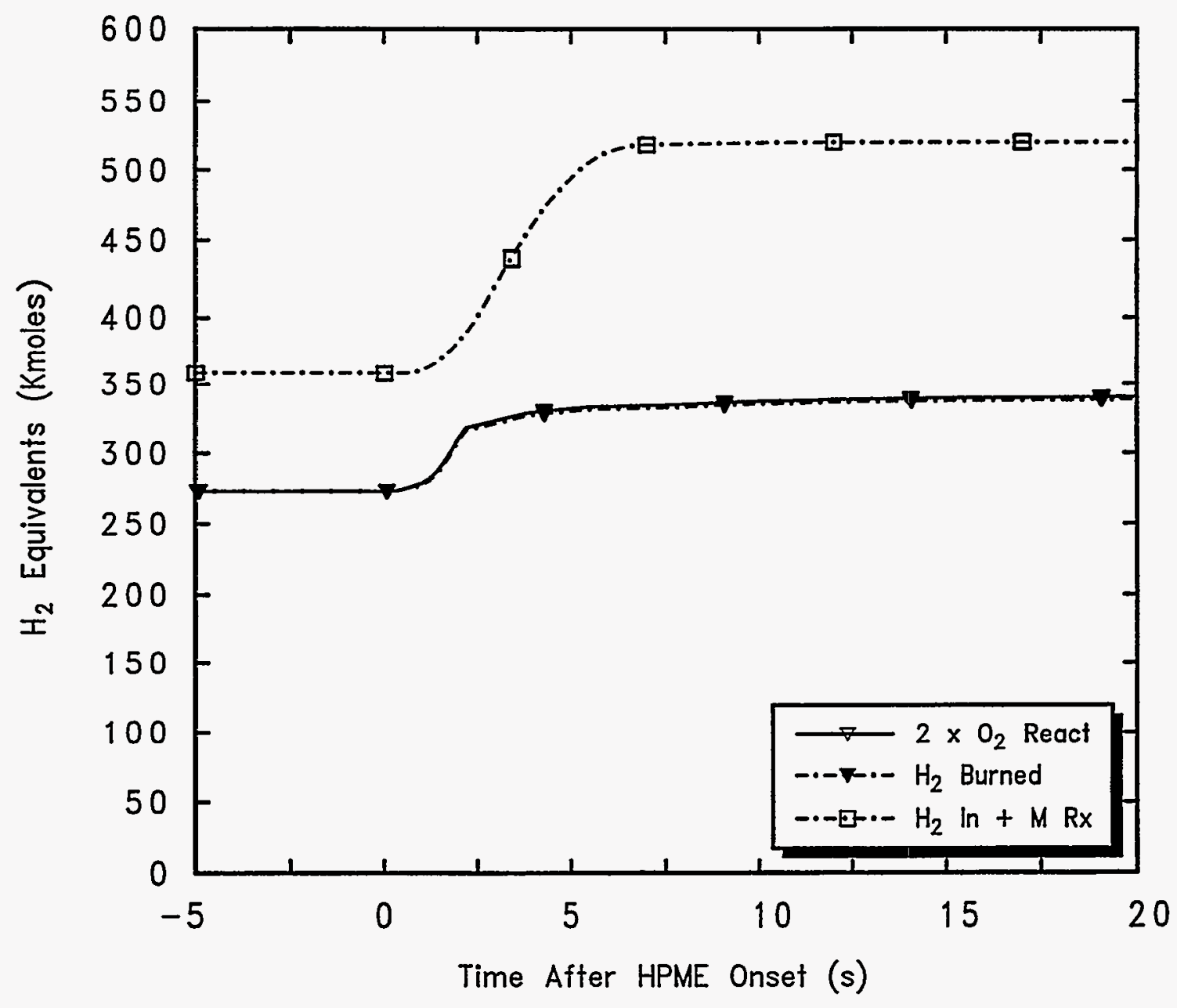

Figure 15-24. Mass Balance Information for Hydrogen and Oxygen 
combustion as calculated by the hydrogen burn models controlled by the H-BURN block. Prior to $\mathrm{DCH}$, the two curves are exactly equal. During $\mathrm{DCH}$, there are processes (direct metal-oxygen reaction and the DCH hydrogen recombination model) that deplete oxygen but that are not reflected in the $\mathrm{H}_{2}$ burned curve; in this instance, the contribution of these additional processes is very minor.

The curve labeled " $\mathrm{H}_{2}$ In $+\mathrm{M} \mathrm{Rx}$ " represents the total hydrogen input to the containment, including both the user-defined sources prior to vessel breach and the hydrogen generated by metal-steam reactions during $\mathrm{DCH}$. The rise in this curve during $\mathrm{DCH}$ is considerably greater than the increase in the amount of hydrogen burned, indicating that a considerable portion of the DCH-produced hydrogen does not burn. The principal reason is that the lower compartment and ice condenser volumes quickly become oxygen-starved, and much of this hydrogen does not get carried as far as the containment dome.

Figure 15-25 plots the height of the ice column, which is proportional to the ice mass. There is substantial decline prior to vessel breach, and there is an abrupt additional decline at vessel breach as the result of energy absorbed by the ice condenser during DCH. The ability of the ice to remove much of the superheated steam generated during the $\mathrm{DCH}$ event is an important mitigating effect.

Referring back to Figure $15-18$, the pressure rise due to $\mathrm{DCH}$ is about $0.205 \mathrm{MPa}$ in this calculation. This is a relatively benign result, considering that the melt mass assumed to participate is relatively large (approximately $88,000 \mathrm{~kg}$ ), the treatment of co-ejected RPV water assumed here may tend to be conservative, and ice condenser containments are relatively small in volume. Three important mitigation effects contribute to obtaining this result: the igniters burn off much of the large quantities of hydrogen assumed to enter the containment prior to vessel breach, some of the DCH-produced hydrogen does not burn, and the ice remaining in the ice condenser is calculated to provide efficient mitigation of DCH, provided there is no large accompanying hydrogen burn. This result should not, of course, be overgeneralized, since there can be other scenarios in which these mitigation effects might not apply. In addition, it should be noted that there is currently no data base for validation of the ice condenser model under DCH conditions. 


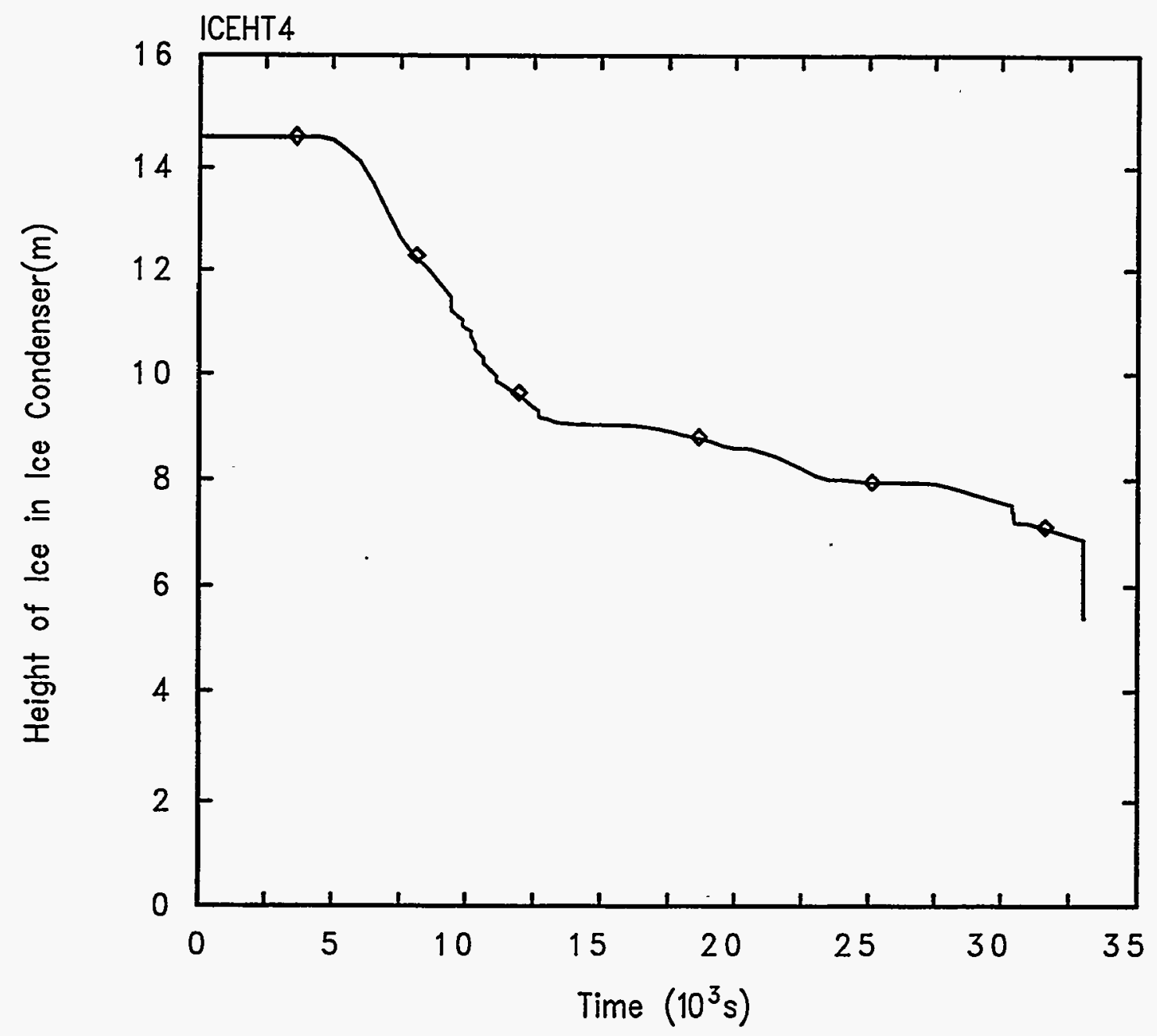

Figure 15-25. Height of the Ice Column 


\subsection{OUTPUT FILES AND POSTPROCESSING}

\subsection{Introduction}

The CONTAIN code generates a vast amount of information in the form of six output files. Three of these files may be read directly, and a fourth requires postprocessing by the POSTCON computer program to convert the information into a usable format. The files generated by CONTAIN and POSTCON, and the relationships between them, are illustrated in Figure 16-1. Given an input file, CONTAIN generates six output files: OUTPUT, PLTFL, TAPE10, TAPE17, TAPE20, and TAPE21. Given the PLTFLL and PINP files, POSTCON then generates five files: PERR, POUT, PVEC, PMIX, and PLTHIS. Brief descriptions of the contents of these files are provided below.

INPUT: The CONTAIN input file, INPUT, contains all of the information required to set up and control a CONTAIN calculation.

OUTPUT: The main CONTAIN output file, OUTPUT, contains the primary output and results of the code calculation and is divided into three major sections. The first part of the output file is an echo of the input file, and provides an accurate record of the input used to generate the output results. The second section of the output file is the input processing section, which presents both the userspecified input data and default values for data that were not specified by the user, and includes both global and cell-level information. The final section of the output consists of the results of the timedependent calculation, written to the file at intervals specified in the input file. Both "long edits" and "short edits" are written to the output file at intervals specified in the input file. Short edits consist of a single summary line of information for pressures, temperatures, and other key values. Long edits contain a much larger amount of information at a greater level of detail.

PLTFIL: The CONTAIN plot file, PLTFIL, contains code output written to the file in binary form for use in producing tabular and graphical displays of code results. PLTFIL contains almost all of the information calculated by CONTAIN, and provides an efficient interface between the calculated results and separate postprocessing and plotting programs such as POSTCON.

TAPE10: The CONTAIN restart file, TAPE10, contains all of the information required to restart a code calculation at a specified time. The time intervals at which restart information is written to TAPE10 is controlled through the input file. Note that TAPE10 is written in binary format, and can only be used in conjunction with CONTAIN's restart capabilities. Further information on restarts is presented in Section 16.11.2.

TAPE17: The main CONTAIN error file, TAPE17, contains most of the error and diagnostic messages generated by the code. In addition, some error messages may also be written to the main output file. In general, the user should check the error file for diagnostic messages after each calculation, even if the calculation did not abort and appeared to run to completion. The error file 


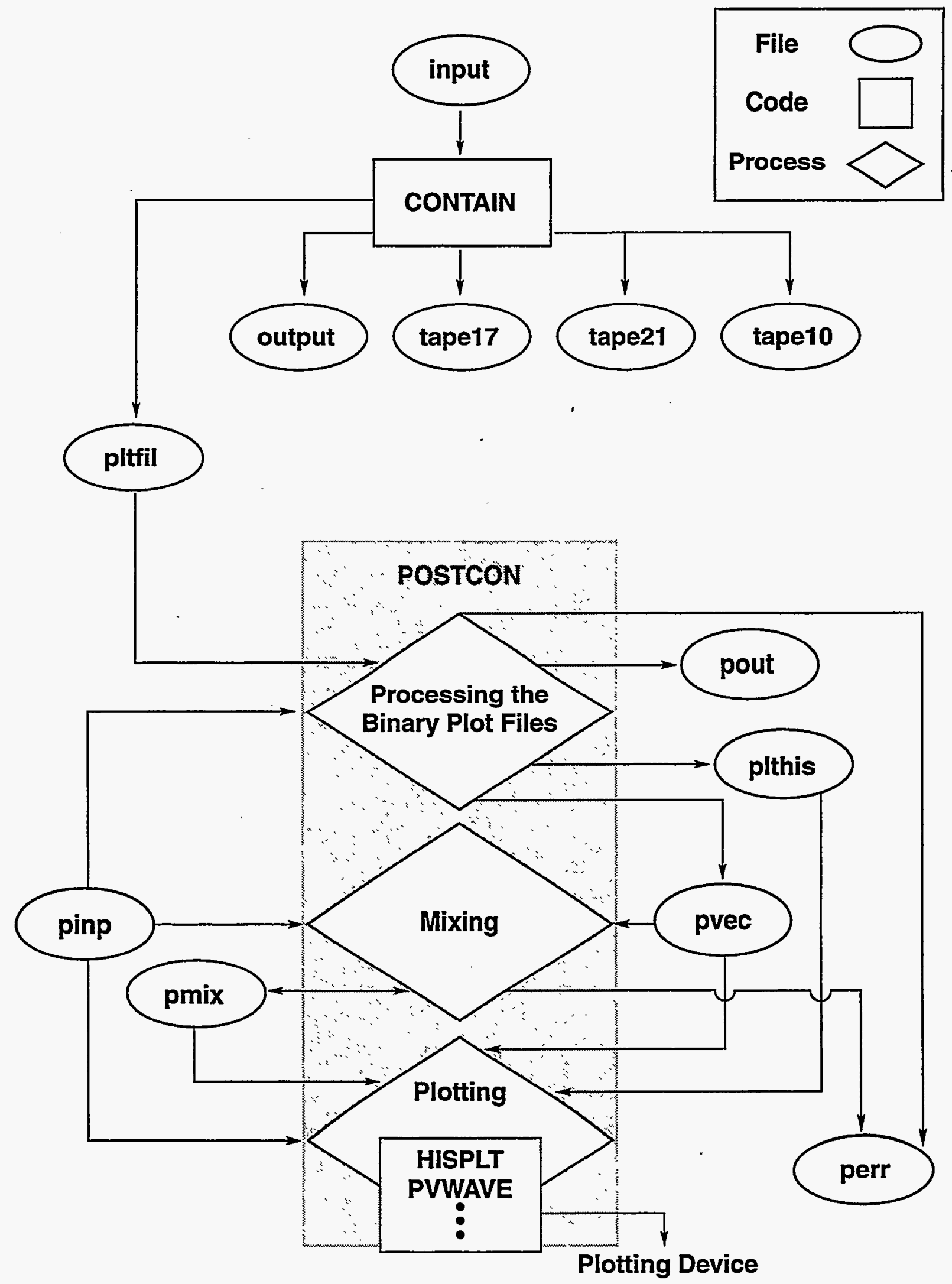

Figure 16-1. CONTAIN and POSTCON Files and Their Interrelationships 
will report conditions that might have adversely affected the results of the calculation, even though the calculation might have continued on to completion.

TAPE20: the aerosol coefficient database file, used to store aerosol kernel and process coefficients for use in future calculations.

TAPE21: The CONTAIN event summary file, TAPE21, contains information about specific events that occur during the course of a calculation. TAPE21 is useful for tracking the initiation and duration of hydrogen deflagrations occurring in a CONTAIN calculation. It is also useful in determining whether burn duration is sufficiently great that the resulting pressures may be sensitive to uncertainties in the burn rate, and to assess burn propagation behavior. The file also lists the number and times of each restart dump put onto the restart and plot files.

PINP: The POSTCON input file, PINP, contains all of the information required to control a POSTCON run. Detailed instructions on the creation of this file are provided later in the chapter.

PERR: The POSTCON error file, PERR, contains an echo of each command read from the PINP file. In addition, any errors reported by POSTCON during the processing of the CONTAIN plot file are written to the PERR file.

POUT: The POSTCON table output file, POUT, contains data generated by POSTCON and presented in tabular form.

PVEC: The POSTCON vector file, PVEC, contains groups of $x-y$ data pairs, or vectors, typically with time as the independent variable. These data pairs can then be plotted by any graphics package.

PMIX: The POSTCON mix file, PMIX, is similar to the PVEC file but contains $x$-y data pairs that have been subjected to algebraic manipulations, referred to below as "mixing."

PLTHIS: The POSTCON HISPLT file, PLTHIS, contains all the data in the PVEC file. However, the information has been reformatted for use in the MELCOR postprocessing package HISPLT [Sum95]

In the past, postprocessing of the CONTAIN data in the binary plot file PLTFIL was performed almost exclusively with the POSTCON program using the commercial software package DISSPLA. As CONTAIN use moved to the UNIX workstation environment, the limited availability of DISSPLA made it difficult to invoke the graphical plotting capabilities of POSTCON, and support for DISSPLA was discontinued. In many cases, POSTCON is now used simply to generate a PVEC file containing $x-y$ data pairs for the variables of interest. The $x-y$ data in the PVEC files can then be plotted using a variety of plotting packages, including XPLOT, PVWAVE, and other commercial software packages.

In an effort to reduce costs and gain more synergy between the MELCOR and CONTAIN projects, a new version of the CONTAIN postprocessing code, POSTCON 2.1, was developed to also generate a HISPLT-compatible plot file, PLTHIS. HISPLT is the MELCOR postprocessing tool 
[Sum95] and is capable of generating graphical output on a wide variety of computer platforms without dependence on DISSPLA. As a result, HISPLT can be used to directly generate CONTAIN plots once POSTCON has been used to postprocess the CONTAIN plot file. Any future development of the HISPLT package for MELCOR will also improve CONTAIN postprocessing capabilities, and CONTAIN and MELCOR users can now learn and apply a single postprocessing tool.

HISPLT is similar to POSTCON in that generating plots from the data requires the construction of a line command input file that generates graphical results in a batch processing mode. To eliminate the need to generate a line command input file for HUSPLT by hand, a graphical user interface (GUT) program called HISPAC has been developed for the postprocessing of both CONTAIN and MELCOR results. The GUI postprocessing tool HISPAC uses the HISPLT-compatible file PLTHIS as input. The GUI serves as a transparent interface to HISPLT, and allows the user to interactively examine and plot CONTAIN results even without specific knowledge of HISPLT commands. A beta test version of HISPAC, and draft documentation, is available to CONTAIN users.

CONTAIN users now have three distinct options in plotting CONTAIN results:

1. Use POSTCON to generate $x-y$ data pairs, and then generate plots with a separate plotting package.

2. Use POSTCON to generate the HISPLT-compatible data file PLTHIS, then use HISPLT directly to generate plots.

3. Use POSTCON to generate the HISPLT-compatible data file PLTHIS, then use the GUIHISPAC to interactively generate plots.

\subsection{Introduction to POSTCON}

The numerical results generated by CONTAIN normally take the form of massive quantities of output data. The POSTCON computer program provides an easily used and efficient method for examining such results. This section describes the capabilities of POSTCON and provides instructions for its use. Its overall capabilities include extraction of all transient data from CONTAIN binary plot files, multiple file handling, a flexible unit conversion system, snapshot and histogram options, generation of output tables, graphics output, and tools for performing arithmetic manipulations on the extracted data. Examples are included to clarify the discussion of input options and output formats.

\subsubsection{Objectives of POSTCON}

CONTAIN calculations typically produce a wealth of numerical output that could potentially overwhelm the investigator and thereby limit the usefulness of the calculations. To solve this problem, POSTCON was created to extract data from CONTAIN binary plot files and to present this data in a form convenient for analysis. 
To achieve this objective, POSTCON provides a wide array of capabilities. A flexible input language allows the user to specify precisely the data to be extracted from one or several binary plot files. A unit conversion capability allows the user to convert the data from the SI units used by CONTAIN to English units or to units defined by the user. Output can be presented in two ways: in tabular form or as a HISPLT-compatible file. [Sum95] Finally, an algebraic data manipulation capability allows data to be prepared for graphing.

While POSTCON's primary objective is to process CONTAIN output, it is not limited to that. By understanding the file structures, the user can find additional uses for the postprocessor. For example, it can be used to graph data generated by means other than the CONTAIN code, provided this data is put in a form readable by POSTCON.

\subsubsection{How to Use This Section}

This chapter is designed to serve as both a user's guide and a reference manual. As a user's guide, it assists in learning how to use POSTCON. As a minimum, the new user should read all of Section 16.3, which provides a general overview of POSTCON. In addition, Sections 16.4.1, 16.4.2, 16.4.3, 16.5.1, and 16.6.1 should be read to gain an understanding of POSTCON input. With this introduction, the examples in Section 16.8 can be studied to get a feel for how POSTCON is typically used. At this point, the user should be ready to start using POSTCON.

For experienced users, the document is organized as a reference manual. Each of Sections 16.4 through 16.7 thoroughly describes a particular POSTCON topic. Each section contains an input details section, which provides a quick reference for the POSTCON input block corresponding to that topic.

\subsubsection{Conventions}

The description of the POSTCON input language follows the same conventions as those used throughout this manual. These conventions are summarized as follows:

- Upper case words are either keywords or character constants (i.e., names), which must be input exactly as shown.

- Lower case words are considered variables, which should be replaced by either a number or a character string. Lower case words representing an integer value follow the FORTRAN convention of starting with a letter between $i$ and $n$, and the value should be specified as an integer. Lower case words representing character constants (i.e., names) should be replaced by the appropriate character constant (i.e., string). (Character constants are also capitalized in the following discussion.) Lower case words representing floating point values should be replaced by values in FORTRAN $F$ or $E$ format. (A decimal point should be present in numbers in the $E$ format. For example, 1.E6 is acceptable but 1E6 is ambiguous.) 
- $\quad$ Parentheses ( ) imply that the enclosed quantity or quantities should be repeated as necessary.

- Square brackets [ ] imply that the enclosed quantity is not always required.

\subsection{General Overview and Capabilities of POSTCON}

POSTCON consists of two major processes: 1) processing the CONTAIN binary plot file, and 2) data manipulation, or mixing. A run can include one or both of these processes. This section gives an overview of these processes and provides general information on how to use POSTCON.

\subsubsection{Processing the CONTAIN Binary Plot File}

To discuss the processing of the CONTAIN binary plot file, it is first necessary to explain what is meant by the terms "plot flag" and "binary plot file."

16.3.1.1 Plot Flags and the CONTAIN Binary Plot File. During every CONTAIN run, a file is created to store general information (also called control data) and computational results. The control data is written to this file at the start of each CONTAIN run, and computational results are written to this file at a frequency designated by the user. All additions to this file are made with unformatted FORTRAN write statements, which imply the storage of data in a compact binary (machinedependent) format. Thus, this file was named the "binary" plot file. This compact storage format is ideal for scanning and extracting large quantities of data for postprocessing.

Since many different types of data are written to the binary plot file, provisions must be made so that POSTCON can distinguish one type from another. Furthermore, the user must have some way to tell POSTCON what particular group of data to process. These functions are performed by plot flags. When CONTAIN writes information to the plot file, it always does so in groups, where each group consists of data that falls into a particular category. For identification purposes, each category has been assigned a unique integer number (normally three digits). For example, the number 101 has been assigned to atmosphere temperatures. These numbers are called "plot flags."

Each record of data written to the plot file begins with an appropriate plot flag and ends with a collection of data items that belong to the identified category. These data records can be as short as one value or can be longer than several hundred values depending upon the category.

16.3.1.2 Extracting Data from the Binary Plot File. Plot flags play a major role in the construction of POSTCON input files in that they identify which groups of data are to be processed. To get a piece of information, the user must specify the appropriate plot flag in the POSTCON input file. Details regarding the role of plot flags as well as other aspects of POSTCON input are discussed in Sections 16.4 through 16.8 .

A complete list of all plot flag categories that pertain to calculational results are given in Table 16-1. Also found in this table are the item keywords, and a list of the values that may be given in response to each item keyword for each plot flag. Since the values associated with some keywords are 
typically very undescriptive, a short description of these is also provided in the table. It should be kept in mind that the keywords listed are only the item keywords, and these can be used with the standard keywords CELL, UNITS, TIME, and VECTOR to select and name data.

If POSTCON is used to read files created with earlier versions of CONTAIN prior to CONTAIN 2.0, many of the flags described in the table will not be available. This is because earlier versions of CONTAIN do not write these flags to the plot file.

An "s" beside a plot flag number indicates that a snapshot (see Section 16.3.1) can be taken of this flag. Values associated with item keywords are often taken from a known list of CONTAIN names such as material names, structure names, aerosol names, and fission-product names. These values will be indicated with a lower case variable that is to be replaced by one of the expected valid names.

In keeping with CONTAIN notational conventions, values that are literal or character constants are given in upper case letters (e.g., COOLMASS). All item keywords are also given in upper case. As discussed in Section 16.6.2, item keywords at times may be followed by another item keyword (e.g., HOST AEROSOL). Both first- and second-level item keywords will be given in boldfaced capital letters in Table 16-1; however, second-level item keywords will be indented to indicate that they must follow the preceding item keyword.

Some of the item keywords require input of an integer (or two integers in a few cases) that must be in a certain range. The inclusive range of integers that will be accepted is described in Table 16-1 with the notation: "min" $\leq n \leq$ "max." Examples of "max" are "nsectn" and "mxslab" for maximum number of aerosol sections and structure nodes respectively. Unless otherwise noted, all range limit names, such as "nsectn," are defined in Chapter 14. The value of "min" is normally unity; however, a few exceptions are noted in Table 16-1. Finally, the underlined word none will be listed in the keyword column for those flags that do not require item keyword input to extract the appropriate data.

16.3.1.3 Unit Conversions. The unit conversion capabilities of POSTCON are extremely flexible. All data written on CONTAIN plot files are either SI units or are dimensionless. Normally, POSTCON does not perform unit conversions. However, if unit conversion is activated for a particular table, the data will, by default, be converted to the most common English unit equivalent. Unit conversion is activated for each table by specifying the UNITS keyword as described in Section 16.6. If other than the default conversions are desired, the user can redefine the conversion factors and unit names by using the UNIT keyword in the control block (see Section 16.5).

Table 16-2 lists the 22 predefined data types, with the appropriate English conversion factors and unit names. Each plot flag is assigned one of these data types. If the UNIT option is used to change the conversion factor for a data type, to produce a new type of unit, then all tables of that data type will use the new unit, whenever conversion is specified. 
Table 16-1

Item Keywords

\begin{tabular}{|c|c|c|c|}
\hline Flag & Description & $\begin{array}{c}\text { Item } \\
\text { Keywords }\end{array}$ & Values \\
\hline 101 & Cell temperature & \multicolumn{2}{|l|}{ none } \\
\hline 102 & Cell pressure & \multicolumn{2}{|l|}{ none } \\
\hline \multirow[t]{2}{*}{103} & $\begin{array}{l}\text { Vapor mass data } \\
\text { mass of coolant vapor } \\
\text { mole fraction of coolant vapor } \\
\text { saturation ratio } \\
\text { total coolant mass in atmosphere }\end{array}$ & TYPE & $\begin{array}{l}\text { COOLMASS } \\
\text { VAPORFRC } \\
\text { SATRAT } \\
\text { TOTMASS }\end{array}$ \\
\hline & \multicolumn{3}{|c|}{$\begin{array}{l}\text { Note: Only pure steam is included in COOLMASS. To perform water mass balance checks, } \\
\text { TOTMASS must be used, which includes the liquid water in the atmosphere. }\end{array}$} \\
\hline 104 & Mole fractions & MATERIAL & matname \\
\hline 105 & Atmospheric masses & MATERIAL & matname \\
\hline 106 & Total condensate mass & $\underline{\text { none }}$ & \\
\hline 107 & Condensate runoff mass & none & \\
\hline 108 & Condensate on structures & $\begin{array}{l}\text { STRUC } \\
\text { SURFACE }\end{array}$ & $\begin{array}{l}\text { strname } \\
\text { INNER } \\
\text { OUTER }\end{array}$ \\
\hline 109 & $\begin{array}{l}\text { DCH time constants } \\
\text { average debris trapping } \\
\text { average debris/gas convection } \\
\text { average debris/gas radiation } \\
\text { average debris/gas total heat transfer } \\
\text { average debris chemical reactions } \\
\text { average debris/wall direct radiation } \\
\text { fastest bin debris trapping } \\
\text { fastest bin debris/gas convection } \\
\text { fastest bin debris/gas radiation } \\
\text { fastest bin debris/gas total heat transfer } \\
\text { fastest bin debris chemical reactions } \\
\text { atmosphere to structure heat transfer } \\
\text { gas convection into/out of cell } \\
\text { debris and gas transport into/out of cell } \\
\text { total gas heat capacity } \\
\text { total debris heat capacity }\end{array}$ & & $\begin{array}{l}\text { AV-TRAP } \\
\text { AV-DGCON } \\
\text { AV-DGRAD } \\
\text { AV-DGHEA } \\
\text { AV-CHEM } \\
\text { AV-DWRAD } \\
\text { FB-TRAP } \\
\text { FB-DGCON } \\
\text { FB-DGRAD } \\
\text { FB-DGHEA } \\
\text { FB-CHEM } \\
\text { AV-GSHEA } \\
\text { GAS-CONV } \\
\text { DG-TRANS } \\
\text { GAS-MCP } \\
\text { DEB-MCP }\end{array}$ \\
\hline 131 & DCH bulk field debris temperature & none & \\
\hline 132 & $\begin{array}{l}\text { DCH total airborne and trapped mass and energy } \\
\text { mass of debris field in cell } \\
\text { energy of debris field in cell } \\
\text { cumulative mass of debris trapped } \\
\text { cumulative energy of debris trapped }\end{array}$ & TYPE & $\begin{array}{l}\text { MASS } \\
\text { ENTH } \\
\text { MASSTRAP } \\
\text { ENTHTRAP }\end{array}$ \\
\hline
\end{tabular}




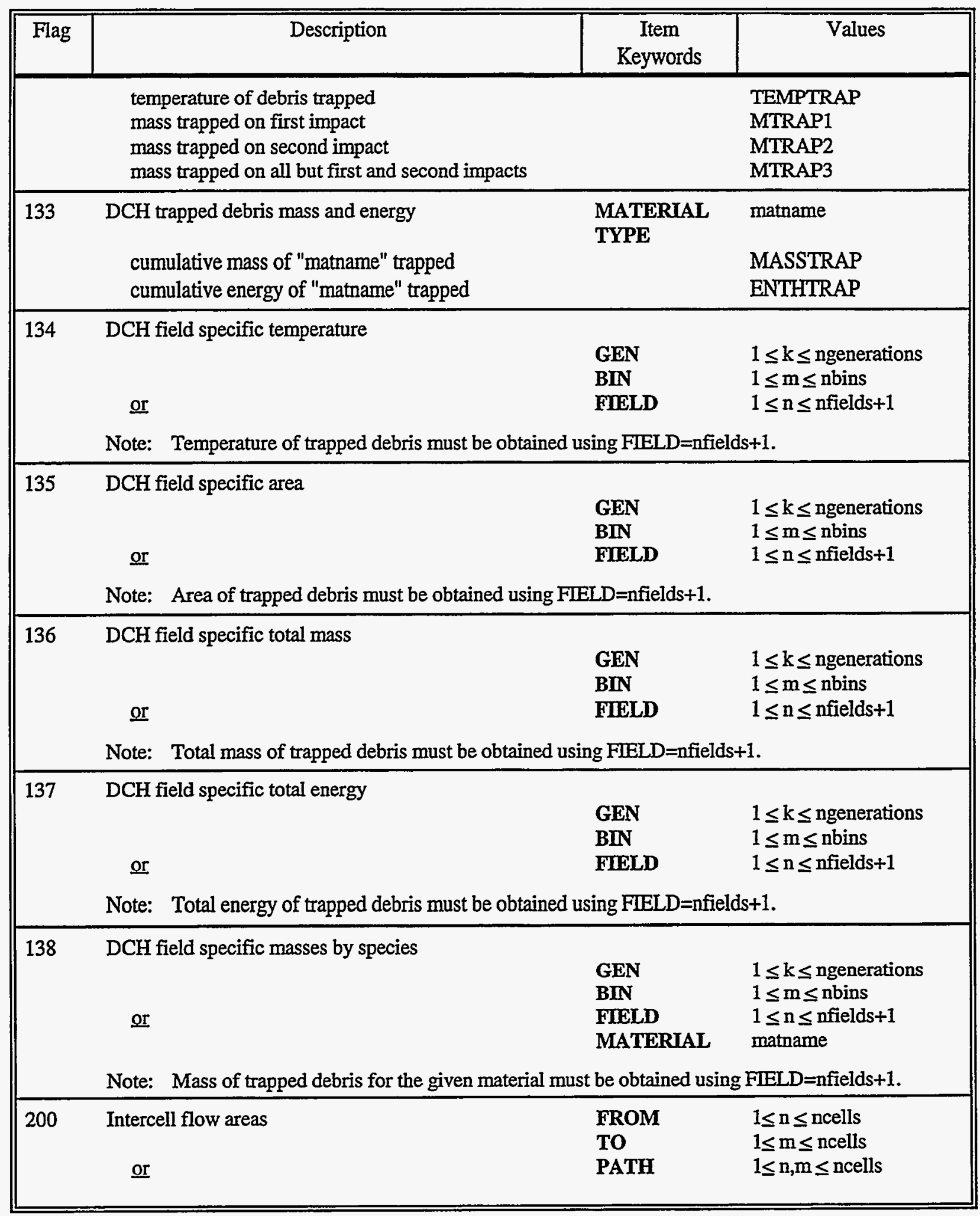




\section{Table 16-1}

Item Keywords (Continued)

\begin{tabular}{|c|c|c|c|}
\hline Flag & Description & $\begin{array}{c}\text { Item } \\
\text { Keywords }\end{array}$ & Values \\
\hline 205 & $\begin{array}{l}\text { Intercell flow rates } \\
\text { or }\end{array}$ & $\begin{array}{l}\text { FROM } \\
\text { TO } \\
\text { PATH }\end{array}$ & $\begin{array}{l}1 \leq \mathrm{n} \leq \mathrm{ncells} \\
1 \leq \mathrm{m} \leq \mathrm{ncells} \\
1 \leq \mathrm{n}, \mathrm{m} \leq \mathrm{ncells}\end{array}$ \\
\hline 206 & $\begin{array}{l}\text { Intercell flow velocities } \\
\text { or }\end{array}$ & $\begin{array}{l}\text { FROM } \\
\text { TO } \\
\text { PATH }\end{array}$ & $\begin{array}{l}1 \leq \mathrm{n} \leq \mathrm{ncells} \\
1 \leq \mathrm{m} \leq \mathrm{ncells} \\
1 \leq \mathrm{n}, \mathrm{m} \leq \mathrm{ncells}\end{array}$ \\
\hline 210 & Engineered vent area & VENT & $1 \leq \mathrm{n} \leq$ nengv \\
\hline \multirow[t]{2}{*}{215} & Engineered vent flow rates & VENT & $1 \leq \mathrm{n} \leq$ nengv \\
\hline & \multicolumn{3}{|c|}{$\begin{array}{l}\text { Note: Engineered vents are defined in the order they appear in the FLOWS block of the CONTAIN } \\
\text { input file. }\end{array}$} \\
\hline 216 & Engineered vent flow velocities & VENT & $1 \leq \mathrm{n} \leq$ nengv \\
\hline 220 & $\begin{array}{l}\text { Suppression pool data } \\
\text { flow rate }\end{array}$ & TYPE & WSUP \\
\hline 225 & Flow timestep criterion for each cell & CELL & $1 \leq \mathrm{n} \leq \mathrm{ncells}$ \\
\hline \multirow[t]{2}{*}{320} & $\begin{array}{l}\text { Pool mass and energy loss } \\
\text { cumulative mass loss } \\
\text { cumulative energy loss }\end{array}$ & TYPE & $\begin{array}{l}\text { BOILMASS } \\
\text { BOILENER }\end{array}$ \\
\hline & \multicolumn{3}{|c|}{$\begin{array}{l}\text { Note: The data in this flag includes all pool mass and energy loss mechanisms such as boiling, } \\
\text { evaporation/condensation, and vaporization. Plot files created with versions of CONTAIN } \\
\text { prior to } 1.10 \text { will only include pool boiling mass and energy loss. }\end{array}$} \\
\hline 340 & Timestep and CPU time & TYPE & $\begin{array}{l}\text { CYCLENUM } \\
\text { STEPSIZE } \\
\text { STEPCPU }\end{array}$ \\
\hline 350 & $\begin{array}{l}\text { CORCON ablation products } \\
\text { cumulative mass of } \mathrm{CO} 2 \text { liberated } \\
\text { cumulative mass of } \mathrm{H} 2 \mathrm{O} \text { liberated } \\
\text { cumulative mass of } \mathrm{CO} \text { liberated } \\
\text { cumulative mass of } \mathrm{H} 2 \text { liberated } \\
\text { mass of } \mathrm{Zr} \text { in metal layer } \\
\text { mass of } \mathrm{C} \text { in metal layer }\end{array}$ & TYPE & $\begin{array}{l}\text { CO2MASS } \\
\text { H2OMASS } \\
\text { COMASS } \\
\text { H2MASS } \\
\text { ZRMASS } \\
\text { CMASS }\end{array}$ \\
\hline 351 & $\begin{array}{l}\text { CORCON heat sources } \\
\text { total decay heat } \\
\text { heat of reaction } \\
\text { heat of ablation } \\
\text { heatup of ablation products } \\
\text { surface heat loss }\end{array}$ & TYPE & $\begin{array}{l}\text { PLDCY } \\
\text { PLRCT } \\
\text { PLABLT } \\
\text { PLHEAT } \\
\text { QLSURF }\end{array}$ \\
\hline
\end{tabular}


Table 16-1

Item Keywords (Continued)

\begin{tabular}{|c|c|c|c|}
\hline Flag & Description & $\begin{array}{c}\text { Item } \\
\text { Keywords }\end{array}$ & Values \\
\hline \multirow[t]{2}{*}{352} & $\begin{array}{l}\text { layer average temperature } \\
\text { total mass of layer } \\
\text { density of layer } \\
\text { void fraction } \\
\text { lower interface temperature } \\
\text { lower interface heat flux }\end{array}$ & $\begin{array}{l}\text { LAYER } \\
\text { TYPE }\end{array}$ & $\begin{array}{l}1 \leq \mathrm{n} \leq 6 \\
\text { TEMPTURE } \\
\text { MASS } \\
\text { DENSITY } \\
\text { VOIDFRAC } \\
\text { TINT } \\
\text { QINT }\end{array}$ \\
\hline & \multicolumn{2}{|l|}{ Note: In CONTAIN, CORCON lay } & $\begin{array}{l}\text { bottom layer and } \\
\text { g record only for the }\end{array}$ \\
\hline 353 & CORCON cavity dimensions & MEASURE & $\begin{array}{l}\text { RADIUS } \\
\text { DEPTH }\end{array}$ \\
\hline 354 & $\begin{array}{l}\text { radial liquid center temperature } \\
\text { axial liquid center temperature } \\
\text { crust thickness on layer bottom } \\
\text { crust thickness in radial direction } \\
\text { crust thickness on layer top }\end{array}$ & $\begin{array}{l}\text { LAYER } \\
\text { TYPE }\end{array}$ & $\begin{array}{l}1 \leq \mathrm{n} \leq 5 \\
\text { TRADIAL } \\
\text { TAXIAL } \\
\text { CRUSTB } \\
\text { CRUSTR } \\
\text { CRUSTT }\end{array}$ \\
\hline 357 & Released masses of VANESA constituents & MATERIAL & matname \\
\hline 358 & Released fractions of VANESA constituents & MATERIAL & matname \\
\hline 360 & $\begin{array}{l}\text { Hydrogen burn data } \\
\text { mass of } h 2 \text { burned } \\
\text { mass of co burned } \\
\text { mass of } 02 \text { burned } \\
\text { mass of } h 20 \text { produced } \\
\text { mass of co2 produced } \\
\text { energy liberated }\end{array}$ & TYPE & $\begin{array}{l}\text { H2BURN } \\
\text { COBURN } \\
\text { O2BURN } \\
\text { H2OPROD } \\
\text { CO2PROD } \\
\text { ENERGY }\end{array}$ \\
\hline \multirow[t]{2}{*}{410} & Lower-cell layer height & $\begin{array}{c}\text { LAYER } \\
\text { NODE }\end{array}$ & $\begin{array}{l}1 \leq k \leq n \text { reg } \\
1 \leq n \leq n n p(k)\end{array}$ \\
\hline & \multicolumn{3}{|c|}{$\begin{array}{l}\text { "nreg" is the total number of lower cell layers defined in the CONTAIN run, and "nnp(k)" is } \\
\text { the maximum number of nodes in layer "k." Also note that lower-cell layers are numbered } \\
\text { from the bottom (one) to the top ("nreg"), even in runs not involving the use of the CORCON } \\
\text { models. }\end{array}$} \\
\hline $415 \mathrm{~s}$ & Lower-cell material masses & $\begin{array}{l}\text { LAYER } \\
\text { NODE } \\
\text { MATERIAL }\end{array}$ & $\begin{array}{l}1 \leq \mathrm{k} \leq \mathrm{nreg} \\
1 \leq \mathrm{n} \leq \mathrm{nnp}(\mathrm{k}) \\
\text { matname }\end{array}$ \\
\hline
\end{tabular}


Table 16-1

Item Keywords (Continued)

\begin{tabular}{|c|c|c|c|}
\hline Flag & Description & $\begin{array}{c}\text { Item } \\
\text { Keywords }\end{array}$ & Values \\
\hline $420 \mathrm{~s}$ & Lower-cell temperatures & $\begin{array}{l}\text { LAYER } \\
\text { NODE } \\
\text { POSITION }\end{array}$ & $\begin{array}{l}1 \leq \mathrm{k} \leq \mathrm{nreg} \\
1 \leq \mathrm{n} \leq \mathrm{nnp}(\mathrm{k}) \\
\text { BOTTOM } \\
\text { MODLE } \\
\text { TOP }\end{array}$ \\
\hline $530 \mathrm{~s}$ & Aerosol airborne mass concentration & $\begin{array}{l}\text { AEROSOL } \\
\text { SECTION } \\
\text { SUM }\end{array}$ & $\begin{array}{l}\text { aername } \\
1 \leq \mathrm{n} \leq \mathrm{nsectn} \\
1 \leq \mathrm{n} 1, \mathrm{n} 2, \leq \mathrm{nsectn}\end{array}$ \\
\hline & \multicolumn{3}{|c|}{ e used in snapshot t } \\
\hline $531 \mathrm{~s}$ & Aerosol airborne mass & $\begin{array}{l}\text { AEROSOL } \\
\text { SECTION } \\
\text { SUM }\end{array}$ & $\begin{array}{l}\text { aername } \\
1 \leq \mathrm{n} \leq \mathrm{nsectn} \\
1 \leq \mathrm{n} 1, \mathrm{n} 2 \leq \mathrm{nsectn}\end{array}$ \\
\hline & \multicolumn{3}{|c|}{ Note: The SECTION and SUM keywords may not be used in snapshot table definition blocks. } \\
\hline 532 & $\begin{array}{l}\text { Aerosol settling information } \\
\text { aerodynamic median diameter } \\
\text { settling mean diameter } \\
\text { deposition rate } \\
\text { standard deviation }\end{array}$ & TYPE & $\begin{array}{l}\text { AERODIAM } \\
\text { SETLDIAM } \\
\text { DEP-RATE } \\
\text { STD-DEV }\end{array}$ \\
\hline \multirow[t]{2}{*}{540} & $\begin{array}{l}\text { Simple aerosol deposition inf } \\
\text { (cumulative) } \\
\text { all deposition locations } \\
\text { all roof surfaces } \\
\text { all wall surfaces } \\
\text { all floor surfaces } \\
\text { bin overflow } \\
\text { bin underflow } \\
\text { summation options }\end{array}$ & $\begin{array}{l}\text { AEROSOL } \\
\text { LOCATION } \\
\text { SUM }\end{array}$ & $\begin{array}{l}\text { aername } \\
\text { TOTAL } \\
\text { ROOF } \\
\text { WALL } \\
\text { FLOOR } \\
\text { OVER-TOP } \\
\text { OVER-BOT } \\
2 \leq \mathrm{n} 1, \mathrm{n} 2 \leq 6\end{array}$ \\
\hline & \multicolumn{3}{|c|}{$\begin{array}{l}\text { Flag } 540 \text { differs from flags } 560 \text { and } 570 \text { described below in that deposited aerosol mass in this } \\
\text { flag is grouped according to generic structure types on the plot tape. This flag is limited in that } \\
\text { pool and individual structure deposition information is not available; however, the TOTAL } \\
\text { value in flag } 540 \text { includes any aerosols in the POOL locations. Finally, it is noted that there is } \\
\text { no "over timestep" version of this flag since it is provided primarily for compatibility with pre- } \\
1.10 \text { versions of CONTAIN. }\end{array}$} \\
\hline 550 & $\begin{array}{l}\text { Aerosol deposition for all components and deposition } \\
\text { locations (cumulative) }\end{array}$ & \multicolumn{2}{|l|}{ none } \\
\hline 560 & Detailed aerosol deposition information (over timestep) & $\frac{\text { see flag_ } 570}{\text { below }}$ & \\
\hline
\end{tabular}


Table 16-1

Item Keywords (Continued)

\begin{tabular}{|c|c|c|c|}
\hline Flag & Description & $\begin{array}{c}\text { Item } \\
\text { Keywords }\end{array}$ & Values \\
\hline \multirow[t]{2}{*}{570} & $\begin{array}{l}\text { inner structure surface } \\
\text { outer structure surface } \\
\text { from ESFs and mesh losses } \\
\text { from BWR and VANESA models } \\
\text { all structure surfaces } \\
\text { structures and pool } \\
\text { summation options }\end{array}$ & $\begin{array}{l}\text { AEROSOL } \\
\text { LOCATION } \\
\text { INNER } \\
\text { OUTER } \\
\text { INNER } \\
\text { OUTER } \\
\text { LOCATION } \\
\text { LOCATION } \\
\text { SUM }\end{array}$ & $\begin{array}{l}\text { aername } \\
\text { strname } \\
\text { strname } \\
\text { POOL } \\
\text { POOL } \\
\text { SETTLED } \\
\text { TOTAL or NONAIR } \\
1 \leq \mathrm{n} 1, \mathrm{n} 2 \leq \text { "nloc"*2 }\end{array}$ \\
\hline & \multicolumn{3}{|c|}{$\begin{array}{l}\text { Note: The INNER second level item keyword following LOCATION may be omitted, in which } \\
\text { case, the inner surface of the structure or the "top" pool location will be assumed. The value of } \\
\text { "nloc" is "nthm"+1. Like flag 540, the TOTAL (or NONAIR) value does not include aerosols } \\
\text { in the WASTE locations. }\end{array}$} \\
\hline 580 & $\begin{array}{l}\text { Aerosol deposition for all components and deposition } \\
\text { locations (over timestep) }\end{array}$ & none & \\
\hline 590 & $\begin{array}{l}\text { Aerosol waste locations } \\
\text { over timestep } \\
\text { cumulative }\end{array}$ & $\begin{array}{l}\text { AEROSOL } \\
\text { TYPE }\end{array}$ & $\begin{array}{l}\text { aername } \\
\text { TOTAL } \\
\text { CUMUL }\end{array}$ \\
\hline \multirow[t]{2}{*}{$610 \mathrm{~s}$} & Structure node temperatures & $\begin{array}{l}\text { STRUC } \\
\text { NODE }\end{array}$ & $\begin{array}{l}\text { strname } \\
1 \leq \mathrm{n} \leq \mathrm{mxslab}\end{array}$ \\
\hline & \multicolumn{3}{|c|}{$\begin{array}{l}\text { Note: The value of n should not exceed the actual number of nodes, "nslab," for the structure } \\
\text { selected. Zeros are written on the plot file for each structure beyond node "nslab" until } \\
\text { "mxslab" values have been written. Also note that the NODE item keyword does not apply to } \\
\text { snapshot table definition blocks. }\end{array}$} \\
\hline 611 & $\begin{array}{l}\text { Cumulative surface energy and interface temperature } \\
\text { energy to inner surface } \\
\text { energy to outer surface } \\
\text { inner surface interface temp } \\
\text { outer surface interface temp } \\
\text { forced convection velocity across inner surface } \\
\text { forced convection velocity across outer surface }\end{array}$ & $\begin{array}{l}\text { STRUC } \\
\text { TYPE }\end{array}$ & $\begin{array}{l}\text { strname } \\
\text { QINNER } \\
\text { QOUTER } \\
\text { TINNER } \\
\text { TOUTER } \\
\text { VINNER } \\
\text { VOUTER }\end{array}$ \\
\hline 612 & $\begin{array}{l}\text { Nusselt and Sherwood numbers } \\
\text { Nusselt number, inner surface } \\
\text { Nusselt number, natural convection, inner surface } \\
\text { Nusselt number, forced convection, inner surface } \\
\text { Sherwood number, inner surface } \\
\text { Sherwood number, natural convection, inner surface } \\
\text { Sherwood number, forced convection, inner surface }\end{array}$ & $\begin{array}{l}\text { STRUC } \\
\text { TYPE }\end{array}$ & $\begin{array}{l}\text { strname } \\
\text { NUINNR } \\
\text { NUNCINNR } \\
\text { NUFCINNR } \\
\text { SHINNN } \\
\text { SHNCINNR } \\
\text { SHFCINNR }\end{array}$ \\
\hline
\end{tabular}


Table 16-1

Item Keywords (Continued)

\begin{tabular}{|c|c|c|c|}
\hline Flag & Description & $\begin{array}{c}\text { Item } \\
\text { Keywords }\end{array}$ & Values \\
\hline & $\begin{array}{l}\text { Nusselt number, outer surface } \\
\text { Nusselt number, natural convection, outer surface } \\
\text { Nusselt number, forced convection, outer surface } \\
\text { Sherwood number, outer surface } \\
\text { Sherwood number, natural convection, outer surface } \\
\text { Sherwood number, forced convection, outer surface }\end{array}$ & & $\begin{array}{l}\text { NUOUTR } \\
\text { NUNCOUTR } \\
\text { NUFCOUTR } \\
\text { SHOUTR } \\
\text { SHNCOUTR } \\
\text { SHFCOUTR }\end{array}$ \\
\hline 613 & $\begin{array}{l}\text { Cumulative heat and mass transfer } \\
\text { cumulative heat transfer, convection, inner surface } \\
\text { cumulative heat transfer, condensed, inner surface } \\
\text { cumulative heat transfer, radiation, inner surface } \\
\text { cumulative heat transfer, liquid deposit, inner surface } \\
\text { cumulative heat transfer, films, inner surface } \\
\text { cumulative heat transfer, condensed, inner surface } \\
\text { cumulative heat transfer, liquid deposit, inner surface } \\
\text { cumulative heat transfer, films, inner surface } \\
\text { cumulative heat transfer, convection, outer surface } \\
\text { cumulative heat transfer, condensed, outer surface } \\
\text { cumulative heat transfer, radiation, outer surface } \\
\text { cumulative heat transfer, liquid deposit, outer surface } \\
\text { cumulative heat transfer, films, outer surface } \\
\text { cumulative mass transfer, condensed, outer surface } \\
\text { cumulative mass transfer, liquid deposit, outer surface } \\
\text { cumulative mass transfer, films, outer surface }\end{array}$ & $\begin{array}{l}\text { STRUC } \\
\text { TYPE }\end{array}$ & $\begin{array}{l}\text { HCNVINNR } \\
\text { HCNDINNR } \\
\text { HRADINNR } \\
\text { HDEPINNR } \\
\text { HFLMINNR } \\
\text { MCNDINNR } \\
\text { MDEPINNR } \\
\text { MFLMINNR } \\
\text { HCNVOUTR } \\
\text { HCNDOUTR } \\
\text { HRADOUTR } \\
\text { HDEPOUTR } \\
\text { HFLMOUTR } \\
\text { MCNDOUTR } \\
\text { MDEPOUTR } \\
\text { MFLMOUTR }\end{array}$ \\
\hline 615 & $\begin{array}{l}\text { Cumulative heat and mass transfer summed over } \\
\text { structures } \\
\text { cumulative heat transfer, convection, total } \\
\text { cumulative heat transfer, condensed, total } \\
\text { cumulative heat transfer, radiation, total } \\
\text { cumulative heat transfer, liquid deposit, total } \\
\text { cumulative heat transfer, films, total } \\
\text { cumulative mass transfer, condensed, total } \\
\text { cumulative mass transfer, liquid deposit, total } \\
\text { cumulative mass transfer, films, total }\end{array}$ & TYPE & $\begin{array}{l}\text { strname } \\
\text { HCNVTOT } \\
\text { HCNDTOT } \\
\text { HRADTOT } \\
\text { HDEPTOT } \\
\text { HFLMTOT } \\
\text { MCNDTOT } \\
\text { MDEPTOT } \\
\text { MFLMTOT }\end{array}$ \\
\hline 620 & $\begin{array}{l}\text { Cumulative } \mathrm{H} 2 \mathrm{O} \& \mathrm{CO} 2 \text { release per structure } \\
\text { evaporable } \mathrm{H} 2 \mathrm{O} \text { release } \\
\text { bound } \mathrm{H} 2 \mathrm{O} \text { release } \\
\text { total } \mathrm{H} 2 \mathrm{O} \text { release } \\
\mathrm{CO} 2 \text { release }\end{array}$ & $\begin{array}{l}\text { STRUC } \\
\text { TYPE }\end{array}$ & $\begin{array}{l}\text { strname } \\
\mathrm{H} 2 \mathrm{OE} \\
\mathrm{H} 2 \mathrm{OB} \\
\mathrm{H} 2 \mathrm{O} \\
\mathrm{CO} 2\end{array}$ \\
\hline 621 & Evaporable $\mathrm{H} 2 \mathrm{O}$ mass per node of structure & $\begin{array}{l}\text { STRUC } \\
\text { NODE }\end{array}$ & $\begin{array}{l}\text { strname } \\
1 \leq n \leq n n p(k)\end{array}$ \\
\hline
\end{tabular}


Table 16-1

Item Keywords (Continued)

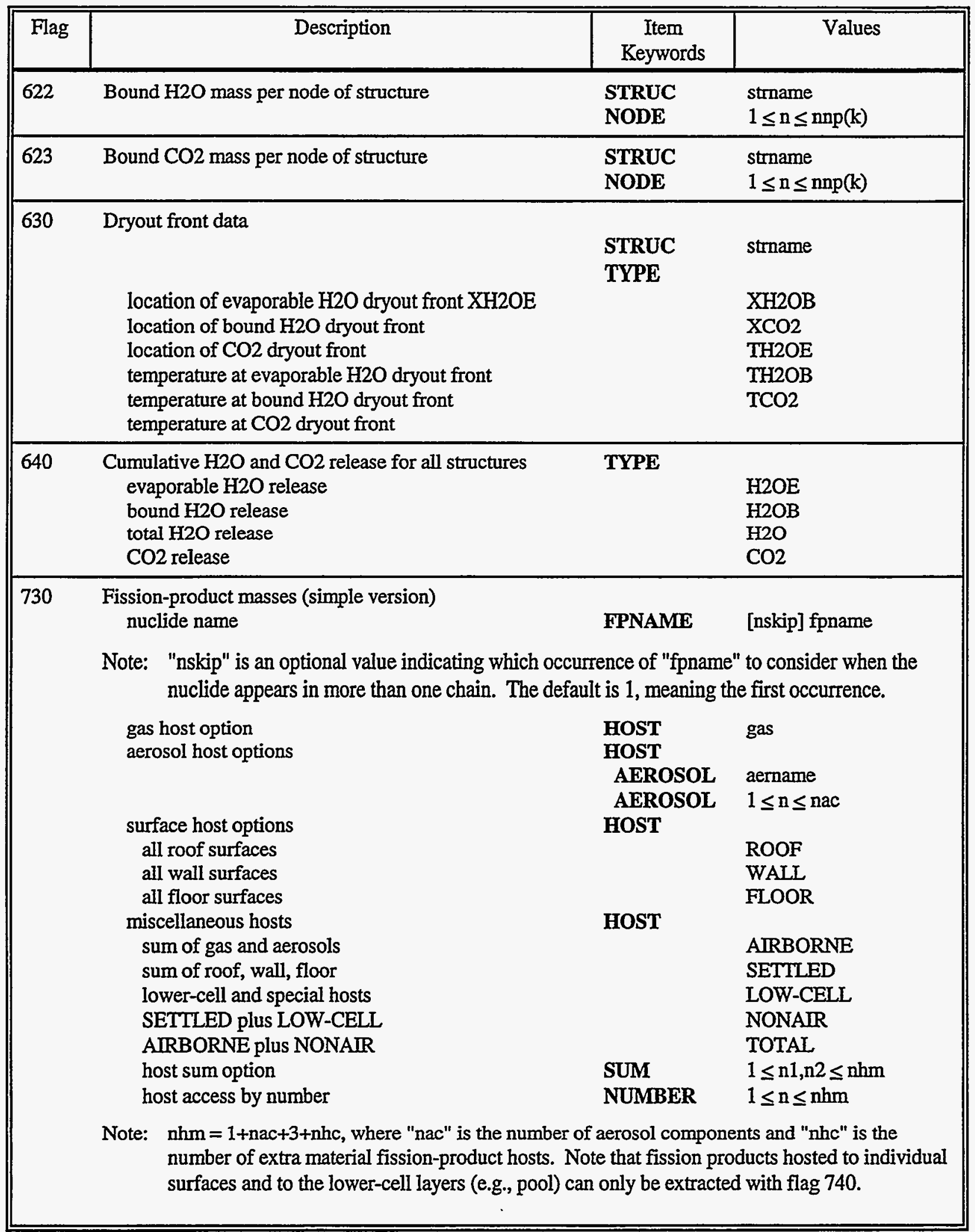


Table 16-1

Item Keywords (Concluded)

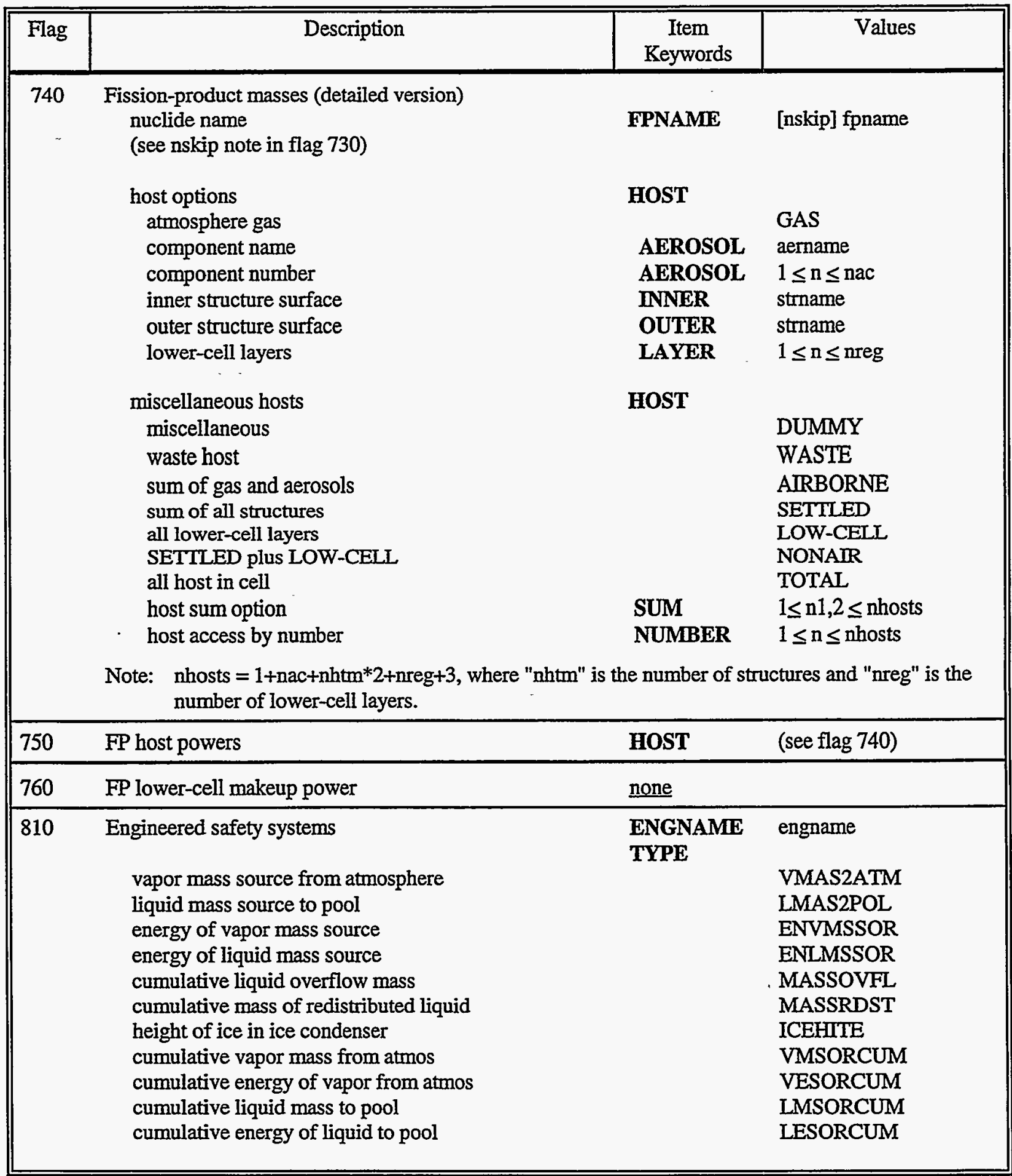


Table 16-2

Default Conversion Factors and Unit Names

\begin{tabular}{|c|c|c|c|c|}
\hline No. & English Name & SIName & $\begin{array}{l}\text { Default } \\
\text { "confac" }\end{array}$ & Description \\
\hline 1 & & & 1.0 & dimensionless \\
\hline 2 & minutes & seconds & 0.016667 & time \\
\hline 3 & hours & seconds & $2.7778 \mathrm{e}-4$ & time \\
\hline 4 & days & seconds & $1.1574 \mathrm{e}-5$ & time \\
\hline 5 & feet & meters & 3.2808 & distance \\
\hline 6 & feet $^{2}$ & meters ${ }^{2}$ & 10.7639 & area \\
\hline 7 & feet $^{3}$ & meters ${ }^{3}$ & 35.3147 & volume \\
\hline 8 & $\mathrm{lb}-\mathrm{m}$ & $\mathrm{kg}$ & 2.205 & mass \\
\hline 9 & psi & $\mathrm{Pa}$ & $1.4504 \mathrm{e}-4$ & pressure \\
\hline 10 & BTU & Joules & $9.4782 e-4$ & energy \\
\hline 11 & BTU/lb-m & Joule $/ \mathrm{kg}$ & $4.2992 e-4$ & specific energy \\
\hline 12 & deg-F & $\mathrm{K}$ & 1.8 & temperature \\
\hline 13 & $\mathrm{lb}-\mathrm{m} / \mathrm{min}$ & $\mathrm{kg} / \mathrm{sec}$ & 132.38 & flow rate \\
\hline 14 & $\mathrm{cfm}$ & $\mathrm{m}^{3} / \mathrm{sec}$ & 2118.9 & vol. flow rate \\
\hline 15 & $\mathrm{BTU} / \mathrm{hr}-\mathrm{ft}^{2}-\mathrm{F}$ & $\mathrm{W} / \mathrm{m}^{2}-\mathrm{K}$ & 0.1761 & thermal conductance \\
\hline 16 & $\mathrm{BTU} / \mathrm{ft}^{3}$ & $\mathrm{~J} / \mathrm{m}^{3}$ & $2.6839 \mathrm{e}-5$ & volumetric heat \\
\hline 17 & $\mathrm{lb}-\mathrm{m} / \mathrm{ft}^{3}$ & $\mathrm{~kg} / \mathrm{m}^{3}$ & $6.2428 \mathrm{e}-2$ & density \\
\hline 18 & BTU/hr & Watts & 3.415 & power \\
\hline 19 & BTU/hr-ft ${ }^{2}$ & $\mathrm{~W} / \mathrm{m}^{2}$ & 0.3170 & energy flux \\
\hline 20 & $\mathrm{ft} / \mathrm{min}$ & $\mathrm{m} / \mathrm{s}$ & 196.85 & velocity; speed \\
\hline 21 & $\mathrm{lb}-\mathrm{m} / \mathrm{ft}^{2}-\min$ & $\mathrm{kg} / \mathrm{m}-\mathrm{sec}$ & 12.289 & mass flux \\
\hline 22 & $\mathrm{lb}-\mathrm{m} / \mathrm{ft}^{2}$ & $\mathrm{~kg} / \mathrm{m}^{2}$ & 0.2048 & integrated mass flux \\
\hline
\end{tabular}

16.3.1.4 Table Output and Vector Output. The processing of the CONTAIN binary plot file produces two types of readable output files; a table output file (hereafter called POUT) and a vector file (hereafter called PVEC). Only POUT is intended for visual inspection; however, there is no reason not to inspect PVEC. The POUT file is a collection of automatically formatted, paginated, and labeled tables, which can be printed for presentation or merely examined on screen. PVEC is 
organized into single columns of $x-y$ data pairs, or vectors, normally with time as the independent variable. The primary function of the PVEC file is to provide an interface between CONTAIN results and a graphics package.

16.3.1.5 Snapshots and Histograms. Snapshots are defined as $x-y$ data pairs with an abscissa different than time. All data in a snapshot curve pertain to the same user-specified time. Snapshots are only available for a subset of the CONTAIN plot flags. These plot flags are labeled with an "s" in Table 16-1. All snapshot data are grouped together and presented in a single table at the front of POUT. Like other tables in POUT, the snapshot table is automatically labeled and, if necessary, may consist of more than one section. User-defined time intervals (described under the TIMEOPT keyword in Section 16.5) do not apply to snapshots; however, all other options, including the unit conversion capabilities, still apply.

Snapshot data may be written in normal format or in histogram format. In histogram format, each abscissa value is assigned two ordinate values to create a square wave effect. This gives plotting programs an easy method for generating histograms.

\subsubsection{The Mixing Process}

During the mixing process, data stored in a POSTCON vector file is manipulated to produce new vectors in a form ready for plotting. By evaluating user-designated expressions, POSTCON generates new vectors as arithmetic combinations of previous vectors. This feature gives the user the ability, for example, to compute the ratio of pressure versus temperature for a set of time points. The vectors generated by the user expressions are written to the PMIX file.

\subsubsection{POSTCON Input}

POSTCON input is free-field and follows the same syntax rules and conventions as CONTAIN. The POSTCON input file (hereafter called PINP) is comprised of four major sections. The first section is an optional file definition block (FDB). This block is used to specify the input plot file names and the output file names. The second section is a control block that is used for memory allocation, time zone definition, and specification of global unit conversion options. The third section consists of a collection of table definition blocks (TDBs). Each TDB defines a separate POSTCON table in the POUT file. Each table consists of one or more columns (also called curves). Each curve of the TDB can also be written to PVEC by using the VECTOR keyword (see Section 16.6.2). Instructions regarding the conversion of units for the curves are also given in the TDB.

The fourth and last section of the PINP file is called the mix block. This block allows the user to specify what arithmetic manipulations will be performed on the vectors stored in PVEC in preparation for plotting.

\subsubsection{Modes of Use}

POSTCON is flexible enough to be used in a variety of modes. Some of the more common modes are described below. 
16.3.4.1 Single Pass. For simple problems, POSTCON processing can be performed in a single pass. All of the necessary information for processing the binary plot file is placed in the POSTCON input file and all processing is done at once. This approach works well if no modification of the output formats is expected.

16.3.4.2 Multiple Passes. A more flexible approach is to perform data extraction separately from the data mixing. If no mixing information is found in the PINP file, POSTCON will terminate after it generates the PVEC and POUT files. POSTCON can then be run with a second PINP file to perform the algebraic manipulations on the data in the PVEC file.

The advantage of doing this is that massaging of the output formats can be done more efficiently since the most time consuming part of a POSTCON run, the reading of the binary plot file, does not need to be repeated. Note, however, that POSTCON must always have a binary plot file as input. If the postprocessing is continued on the same machine used for the original CONTAIN run, the original plot file can be used again; only one pass through this file is made, which is not very time consuming. If the postprocessing is done on a different machine, a dummy plot file must be created on that machine. Figure 16-2 shows a simple FORTRAN program that can be used to create such a dummy file.

16.3.4.3 Processing Results from Sources Other than CONTAIN. Finally, POSTCON can be used independently of CONTAIN manipulate data generated by other sources. The data simply needs to

be put into a file in PVEC format. Once this is accomplished, POSTCON can process it exactly like a vector file.

\subsection{Input and Output Files and the File Definition Block}

\subsubsection{File Descriptions}

A set of input and output files is associated with each POSTCON run. Figure 16-1 illustrates how each of these files is used. Each rectangle represents a code, each diamond represents a process, and each ellipse represents a file labeled with its default name. The arrows indicate the direction of data flow. Notice that some files are associated with all of the major POSTCON processes while others are limited to one or two of the processes. Details of each of the files follows.

16.4.1.1 Input File (PINP). All user instructions to POSTCON are placed in a file called PINP. This file instructs POSTCON on how memory will be initialized, what files will be used, what information will be processed, how output will be formatted, and what mixing operations will be performed. Much of the remaining portion of this chapter deals with how to create this file.

16.4.1.2 Error File (PERR). In order for the user to be able to debug POSTCON runs, an error file is produced to provide information on what went wrong. The name of this file is PERR. Whenever a command is read from the PINP file, it is echoed to the PERR file. Furthermore, whenever an error is detected, an error message is written to the PERR file. 
program makepit

C

c this program creates a small dummy contain plot file

$c$ with as many time points as requested and minimal control info

c

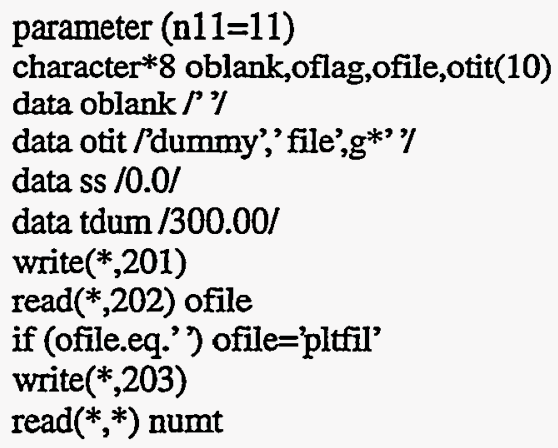

c

oflag='11'

write(n11) oflag,oblank,ss,ss

write(n11) oblank,oblank, h2ol'

c

oflag $=15$,

write(n11) oflag,oblank,ss,ss

write(n11) oblank,oblank,(otit(I),I=1,10)

c

oflag='4444'

c

write(n11) oflag,oblank

$\mathrm{xtpdt}=0.0$

do $10 \mathrm{I}=1$, numt

oflag='100'

write(n11) oflag,oblank,ss, xtpdt

xtpdt=xtpdt+1.0

oflag='101'

write(n11) oflag,oblank, 1 ,tdum

tdum $=$ tdum +1.0

c

10 continue

oflag='5555'

write(n11) oflag,oblank,xtpdt,ss

close(n11,status=keep')

stop

201 format('Enter file name:')

202 format(a8)

203 format ('Enter number of time points: ')

end

Figure 16-2. The MAKEPLT Program for a Dummy Plot File

Rev. 0 
16.4.1.3 Binary Plot File (PLTFIL). POSTCON processes the CONTAIN binary plot file. This file is described in detail in Section 16.3.1.1. The name of this file is PLTFL by default; however, this name can be changed in the FDB of the POSTCON input file (see Sections 16.4.3 and 16.4.4). As can be seen in Figure 16-1, PLTFL is an input to the POSTCON process. Because the plot file is used during POSTCON initialization, it must always be present, even if no processing of the binary plot file is to be performed during a particular POSTCON run.

16.4.1.4 Table Output File (POUT). Postprocessed CONTAIN data, organized in tabular form, are written to a file named POUT by default. As can be seen from Figure 16-1, these tables are generated before the mixing process, so the tables cannot reflect the results of mixing.

The POSTCON tables are comprised of a collection of data curves presented in columns. All curves in any one table correspond to the same plot flag and TBD, with the possible exception of snapshot curves. For example, one column in the table for plot flag 101 may be the temperature in cell 1 , and another may be the temperature in cell 2. Further discussion of table curves is given in Section 16.6.

The tables generated by POSTCON normally consist of a maximum of eight columns per section. If there are more than nine curves in a TBD, then the table will be printed in two or more appropriately labeled sections. An exception to this rule is made when a table can be completed by including a ninth column in the last section. This prevents a single column from being placed in a section by itself (unless of course there is only one column in the table). Each table (or table section) is printed with the correct unit labels and a brief descriptive title. The tables are formatted using FORTRAN carriage control in pages of length 66 with titles appearing on each page and new tables beginning on a fresh page. The minimum, maximum, and average of the extracted values are printed at the bottom of each table column. NOTE: Since POSTCON can extract data at user-defined time intervals, the minimum, maximum, and average values reported may not apply to the entire calculation.

16.4.1.5 Vector File (PVEC). Postprocessed CONTAIN data, organized in vector form, are written to a file named PVEC by default. A vector is an $x-y$ data pair, normally with time as the independent variable. The primary function of the vector file is to provide an interface between CONTAIN results and a graphics package.

The PVEC file also serves as an input file for the mixing process. Vectors are read in from the PVEC file and the manipulated results are output, as vectors, to the PMIX file.

16.4.1.6 Mix File (PMIX). The mix file is created by the POSTCON mixing process. When algebraic manipulations are performed on vectors from the PVEC file, the results are written to a file named PMIX by default. The format of this file is identical to that of the PVEC file.

If a curve, or set of vectors, cannot be found in the PVEC file, the PMIX file is also searched. This allows the user to create a curve with one mix expression and refer to that curve in a later expression. Thus, the PMIX file is both an input to and an output from the mixing process of POSTCON. 


\subsubsection{Renaming Input and Output Files}

As noted above, the names of the PINP and the PERR files are fixed; the names of all other files used by POSTCON can be changed at run time. These file names are restricted to eight characters and must be valid file names for the host system.

\subsubsection{File Definition Block Input Summary}

Reassignment of the PLTFL, POUT, PVEC, and PMXX file names is accomplished by including a file definition block (FDB) at the beginning of the PINP file. The general structure of the FDB is given below in standard CONTAIN User's Manual notation. (See Section 16.2.3 for these notational conventions.)

[PLTFIL=pltfil]

[POUT=pout]

$[\mathrm{PVEC}=\mathrm{pvec}]$

[PMTX=pmix]

Use of this block is completely optional and default names will be used for any keywords omitted.

\subsubsection{File Definition Block Input Details}

PLTFIL keyword to input a user-selected file name for the CONTAIN binary plot file.

pltfil a character string corresponding to the name of the CONTAIN binary plot file.

POUT keyword to input a user-selected file name for the table output file.

pout a character string corresponding to the name for the table output file. Default $=$ POUT.

PVEC keyword to input a user-selected file name for the vector file.

pvec a character string corresponding to the name for the vector file. Default = PVEC.

PMIX

keyword to input a user-selected file name for the mix file.

pmix

a character string corresponding to the name for the mix file. Default $=$ PMIX.

Note: On some systems (e.g., UNIX), any file matching either the POUT, PVEC, or PMIX file names will be overwritten when POSTCON is executed; on other systems (e.g., VAX/VMS), new versions will be created. 


\subsection{Control Block}

The general structure of the control block is similar to that of the CONTAIN global and cell control input blocks. In addition to reserving the required memory, the control block is also used to define alternate unit conversion factors and time zones. The time zones defined here obviously cannot be more finely resolved than those written to the plot file during the CONTAIN run. However, coarser time zones can be specified that will override the time point frequency read from the files.

\subsubsection{Control Block Input Summary}

The CONTROL block input is driven completely by keywords. The keyword names and their usage are summarized below using standard CONTAIN notation.

CONTROL

[DOUBLE] [PADINT]

[MAXTIME=timax] [MAXSIZE=maxsiz] [MAXFLG=maxflg]

[MAXCRV=maxcrv] [MAXTAB=maxtab]

[MAXSNP=maxsnp] [SNPSLZ=snpsiz] [HISTO]

[MAXMIX =maxmix]

[TUNIT=otunit]

[TIMEOPT =tstart (tstep tstop)]

EOI

( [UNIT = idno ouname confac [tcoff] ] )

All keywords in the control block are optional and have reasonable default values. As usual, the keywords may be given in any order. Even though each keyword in the control block is optional, CONTROL must either be the first keyword in the file or must immediately follow the file definition block. An EOI must immediately follow the CONTROL keyword if no other keywords are specified, in which case all default values will be used. Complete descriptions of each keyword and associated parameters and default values are given in the following section.

16.5.2 Control Block Input Details

DOUBLE

keyword to invoke the double precision option when POSTCON is run on a workstation. The double precision option is designed to provide the same accuracy on the workstation as on the Cray. This keyword is required when running on the workstation, otherwise the CONTAIN plot file cannot be read properly.

PADINT

keyword that allows POSTCON to process plot files which have doubled integer fields as well as double precision floating point fields.

MAXTIME keyword that initiates the input of the maximum problem time to be extracted. Use of this keyword is not required if the time zone option is 
exercised as described below. However, if used, the maximum time input will override the maximum time of the last requested time zone.

$\operatorname{timax}$

MAXSIZE

$\operatorname{maxsiz}$

MAXFLG

maxflg

MAXCRV

$\operatorname{maxcrv}$

MAXTAB

maxtab a user-chosen maximum problem time for all tables and vectors (excluding snapshot tables). This value is in the units specified by the TUNIT keyword described below. If TUNIT is not specified, then "timax" is assumed to be in seconds. Default $=10^{20}$.

keyword that initiates the input of the maximum number of data points per curve.

the maximum number of data points that each table column will have (the vertical length). Default $=100$.

keyword that initiates the input of the maximum number of table definition blocks.

the maximum number of table definition blocks, excluding snapshot-related blocks. This is not the same as the number of flags read. For example, if flag 101 is associated with two TDBs, then both must be acknowledged in the value of "maxflg." Default $=20$.

keyword that initiates input for the maximum number of specific vector types, or curves, in all table definition blocks, excluding those related to snapshots. An example of a specific vector type is the gas pressure versus time in a given cell.

the upper limit on the total number of all curves in the table definition blocks (TBD). For example, if two TDBs are defined, one with three columns and another with five columns, then "maxcrv" must be at least eight. If an error condition occurs and the message "real blank common is too small" appears, the first course of action should be to set this value to exactly the number of curves requested. This number will be the number of ENDCURVE keywords in the PINP file. Next, the "maxsiz" value described above should be set as close as possible to the anticipated number of points per curve. Default $=$ 100.

keyword that initiates the input of the maximum number of tables to be read from the plot file.

the maximum number of tables that will be read from the plot file. Default $=0$ 
MAXSNP

maxsnp

SNPSIZ

snpsiz

HISTO

MAXMIX

$\operatorname{maxmix}$

TUNIT

otunit

TIMEOPT

tstart

tstep keyword that initiates input for the maximum number of specific snapshot types, or curves. Snapshots can only be taken of structure node temperatures (flag 610) and aerosol airborne masses (flag 530).

the maximum number of snapshots. This is NOT the number of snapshot blocks, but the number of snapshot curves. A nonzero value of "maxsnp" is mandatory if any snapshots are to be requested. Default $=0$.

keyword that initiates input for the maximum number of points in any given snapshot.

the maximum number of data points in any given snapshot. If "maxsnp" is zero, then this control parameter has no meaning. Default $=20$.

keyword that turns on the histogram vector option for all snapshots. When present, all snapshot vectors will be written in histogram format. Histogram vectors can be of flags 610 and 530 only.

keyword that initiates input for the maximum length of a mix expression.

the maximum number of elements allowed in a mix expression. Default $=$ 30.

keyword that initiates input of the global unit for all time values.

one of the following character strings: SECONDS, MINUTES, HOURS, or DAYS. All time data in POSTCON tables and vector files will be converted to this time unit. Also, any plot flag data having units of time (e.g., CPU time in flag 340) will be converted to this time unit if the conversion option has been activated for that table definition block. Default $=$ SECONDS.

keyword that initiates the input of user-selectable time zones. Up to 20 time zones may be defined in a given run. Normally only one time zone which consists of all time points on the plot file is used. One start time followed by up to 20 pairs of "tstep" and "tstop" can be given. Note: the TIMEOPT input parameters are not terminated with an EOI and there is no control parameter for the number of time zones.

the minimum value of the time that will appear in all tables. This value (as well as "tstep" and "tstop") is in the units specified by "otunit" described above. If there is negative time data, then "tstart" must be negative in order to access it. Default $=0$.

the minimum timestep for data extracted from the plot file in a time zone. If data is found in uneven multiples of the tstep value given, the obtained time 
increments will not be exactly tstep in size. For example, if tstep is 100 and data exists at $0,50,120,170,210,270,320,420,510,530$, and 620 ; then the extracted time points will be: $0,120,270,420,530$, and 620 . Note that the time points are separated by at least 100 seconds with the exception of 620 . The 620 time point was extracted because it is at the end of the time zone. Default $=0$ (i.e., extract all time points).

tstop

UNIT

the end of a time zone. The last point in a time zone will always be extracted regardless of the tstep criterion. An error will result if the stop time of any time zone is earlier than the end of the previous time zone. Default = none.

keyword that initiates the input of user-defined unit conversion factors and names. Actual use of these conversion factors and names are contingent on the specification of the UNITS keyword in the table definition block (see Section 16.6).

idno the data-type identification number. The 18 identification numbers correspond to the appropriate units of 18 various physical quantities. Some of the more common values of "idno" are 8 (mass: $\mathrm{lbm}$ ), 9 (pressure: psia), and 12 (temperature: deg-F). A full list of these numbers and their corresponding default conversion factors and names are given in Table 16-2.

ouname

the user-supplied name for the new unit (e.g., calories). This name will be used in the labeling of converted data in the tables that POSTCON produces.

confac

the conversion factor from SI units to the user-defined unit. Data extracted from the plot file known internally to POSTCON to be of type "idno" will be multiplied by this number if the UNITS keyword is given in the corresponding table definition block. The conversion formula is NEW = OLD $\times$ "confac." A special situation occurs if "idno" is 9 or 12. In these cases, the "tcoff" value is also subtracted in order to support pressure and temperature conversions as explained below.

tcoff

a real value that must be entered if, and only if, "idno" is 9 or 12 . In these cases, the conversion formula used by POSTCON is NEW = OLD $\times$ "confac" - "tcoff." For an "idno" of 9 , this allows conversions between absolute and relative pressure scales. For an "idno" of 12, this allows conversion between the Kelvin absolute temperature scale to Celsius or Fahrenheit. For example, to enable conversions from Kelvin to Celsius, the UNIT entry should be: UNIT=12 deg-C 1.0 273.15. The new unit is activated by the UNITS keyword in the table definition block. NOTE: Once the UNIT keyword is given followed by the "idno" parameter, the default English conversion parameters for that type of unit will be overridden by the required input parameters "ouname," "confac," and "tcoff." 


\subsection{Table Definition Block}

The third section of a POSTCON input file is comprised of a collection of table definition blocks TDBs). The number of TDBs (not flags) cannot exceed the number specified for MAXFLG in the control block (or the default value of 20 if MAXFLG is not in the control block). Table definition blocks beginning with the keyword SNAPSHOT are handled in a special manner and are not included in the normal TDB count. There is no limit on the number of snapshot TDBs since all snapshots will be collected and presented in a single output table. However, the total number of snapshots must not exceed the MAXSNP value specified in the control block.

Each TDB is restricted to a single plot identification flag; however, more than one TDB can reference the same flag. For example, fission-product mass in cell 1 and fission-product mass in cell 2 may be extracted into either one table or two separate ones (since they are stored under the same plot flag). On the other hand, fission-product masses and aerosol masses must be in separate tables, since they are stored under separate plot flags. Each TDB is comprised of a collection of any number of specific data types, or curves from the same flag. The only limitation is that the total number of curves (summed over all TDBs) cannot exceed the value given for MAXCRV in the control block (or the default of 100 if MAXCRV is not in the control block). Curves in snapshot TDBs are not included in this total; however, the total number of snapshot curves must not exceed the value of MAXSNP given in the control block. The last TDB should be followed by the MIX block or an EOF keyword marking the end of the input file.

\subsubsection{Table Definition Block Input Summary}

The input format for each table definition block (TDB) is given below. Again, we use the notation described in Section 16.2.3. Item keywords and their corresponding values are used to specify particular items of data stored in the record of a particular plot flag. Item keywords are described in further detail in the following section. This format summary represents the input structure of a single TDB. A typical PINP file might have many such blocks.

flagnum [UNITS]

( [CELL=cell] [VECTOR=ovnam]

( IKEY1 [IKEY2] = value1 [,value2] )

ENDFLAG ENDCURVE )

or

SNAPSHOT flagnum [UNITS]

( [CELL=cell] [TIME=time] [VECTOR=ovnam]

( IKEY1 = value1)

ENDCURVE )

ENDFLAG 
16.6.2 Table Definition Block Input Details

SNAPSHOT optional keyword that identifies the curves in a table definition block (TDB) as part of the snapshot table. Only flags designated by an " $s$ " in Table 16-1 as snapshot flags can be used with this keyword.

flagnum

an integer plot flag. This integer identifies which category will be used in the extraction of data from the plot file for the TDB being defined. A valid identification flag number, "flagnum," (or the keyword SNAPSHOT followed by "flagnum") is required to start each TDB, and an ENDFLAG is required to terminate each TDB. The identification flag must come from the list of flags described in Table 16-1.

UNITS

optional keyword that activates the unit conversion module in POSTCON for the present table. The unit conversion module acts appropriately on all data retrieved in the table. This unit conversion by default will be from SI to English units; however, the conversion factors and the unit names (printed in the tables) can be readily changed through use of the UNIT keyword described in Section 16.5. The default conversion factors and unit names are given in Table 16-2:

CELL

keyword that initiates the input of the cell from which subsequent curve data will be extracted. In postprocessing multiple cell problems, this keyword should appear before any item keywords in the definition of a curve. This order dependency must be honored if POSTCON is to properly determine the validity of the values given for the item keywords.

cell

the number of the cell from which the data is to be extracted. This value applies to the curve being defined and will be used as the default for subsequent curves in the same TDB. Cell values must be in the range of one to the number of cells in the CONTAIN run, "ncells." Like many other variables, "ncells" is automatically determined from one of the CONTAIN control flags. If an invalid number is given, a value of 1 will be assumed. Default $=1$ or last cell specified.

TIME

keyword that initiates input of the snapshot time for curves in a snapshot TDB only. This keyword can be used only if the snapshot keyword is followed by a snapshot flag.

time the snapshot time for the curve in the snapshot block. The information requested by the snapshot flag will be extracted for this time value as a function of position. This time is normally in seconds, but will be in the units of "otunit" if the TUNIT keyword is given in the control block. Default = first time point extracted. 
VECTOR

ovname

IKEY1 and IKEY2

value1; value2 keyword that initiates input of the name for the specific vector type comprising a given curve. Curves are only written to the vector file if a name is specified through use of this keyword. The VECTOR keyword applies only to individual curves and not the entire TDB. If no vectors are defined, the vector file will not be created or will be created with a zero file size.

the name of the curve that will be written to the vector file. This name can be any string up to eight characters in length and not containing an input delimiter.

item keywords as defined in Table 16-1. It is important that item keywords are understood since they compose the major portion of TDB curve definitions. These keywords are discussed separately because a different set of keywords applies to each individual plot flag. Item keywords are used to identify which element of a data record of a particular flag is to be extracted for the TDB curve being defined. Normally, an item keyword must be followed by either one or two values. These values may be numerical values or character strings. Two item keywords are listed here, IKEY1 and IKEY2, because in some instances an item keyword may be followed by another item keyword, which in turn must be followed by one or two values. Further clarification of item keywords and a few examples are given below.

identifying values for an item keyword. The validity of the value given is determined by POSTCON based on various entries for control information written to the plot file by CONTAIN. Most values are either character strings (eight character maximum) or integers. As mentioned above, some item keywords are followed by an additional item keyword, which in turn must be followed by an appropriate value field. The "value2" field is required in a few cases where the item keyword requires two entries as opposed to one. A short discussion of the contents of a typical CONTAIN plot file record is given below for further clarification of item keywords and values.

When CONTAIN writes information onto a plot file, it labels each record with a flag (and usually a cell number). However, each individual numerical value written to the plot file obviously cannot have its own unique flag. Instead, results are grouped into categories and then written with the same flag. For example, structure temperatures for all structures and all structure nodes are written with a 610 flag. In this case, item keywords followed by the appropriate values would be used to identify which structure and which node temperature to extract. Perhaps some actual examples of IKEY1 and IKEY2 input blocks will clarify the above discussion. In the examples below, the item keywords are boldfaced in order to distinguish them from constant values, since both are capitalized according to standard CONTAIN notational conventions. 
(a) Atmospheric Masses: MATERIAL $=\mathrm{N} 2$

(b) Aerosol Deposition: AEROSOL=NA2O LOCATION=FLOOR

(c)Fission Product Mass: FPNAME=CS137 HOST AEROSOL NA2O

(d) Airborne Aerosol Mass: AEROSOL=NA2O SECTION SUM 1,20

(e) Lower Cell Masses: MATERIAL=K2O LAYER=3 NODE=1

(f) Pool Boil Information: TYPE=BOILMASS

In example (a), the item keyword is MATERIAL, and N2 is the value (an error message would be written to the PERR file followed by a code abort if N2 is not in the material list of the CONTAIN run). In example (b), there are two sets of item keywords and values, each which have the same format as the first example. In example (c), the HOST item keyword (IKEY1) is followed by the AEROSOL item keyword (IKEY2) prior to the specification of the host name. This is necessary since aerosols, structure surfaces, and extra materials may be fission-product hosts, each of which have names. In example (d), the first item keyword set is just as in the first two examples, and the second item keyword set is similar to the third example. However, this example is further complicated by the fact that both "value1" and "value2" parameters follow the SECTION and SUM item keywords. Example (e) is interesting because there are three item keywords and values, each of which is needed to define the desired table column. The item keyword TYPE used in example $(f)$ is reserved for flags that have various types of data that cannot be categorized by a more descriptive term. In some of these cases, the values are the actual names of the variables in the CONTAIN code.

A full list of the item keywords and acceptable values is provided in Table 16-1 for each flag. If an item keyword/value pair is omitted, the first data value in the record is always assumed. If an item keyword appears more than once in a curve definition, all but the last one will be ignored. Note that some item keywords have order dependencies which should be acknowledged. Example (e) above is an example of an order-dependent group of item keywords. In this example, the layer number must be given before the node number because each layer has its own number of nodes. Such dependent item keywords are designated in Table 16-1 through use of indentation levels.

ENDCURVE

keyword that terminates the definition of a curve. Each curve in a TDB normally begins with the CELL keyword, and must end with the ENDCURVE keyword. A multiple column table is created by including several curves in a TDB. The ENDCURVE keyword is mandatory.

ENDFLAG

keyword that terminates the TDB. Following this, another TDB can be specified, or if this is the last TDB, the MIX block or the EOF marker may follow. 
EOF

keyword that terminates the end of the input file. If no MIX block is used, this keyword should follow the last TDB. The physical end of the input file will also serve the same function.

\subsection{Mix Block}

Vectors appearing in a POSTCON PVEC file can be algebraically manipulated by a process called "mixing" to produce new vectors in a form ready for plotting. By evaluating user-designated expressions, POSTCON generates new vectors as arithmetic combinations of previous vectors. This feature gives the user the ability, for example, to compute the ratio of pressure versus temperature for a set of time points.

The vectors generated by the mix function are output to a file named PMIX by default. This file has the same format as the PVEC file.

\subsubsection{Mix Block Input Summary}

Mixing operations can be specified by including a mix block in the input file PINP. This block should follow all TDBs and precede the PLOT block. The format of the MIX block is quite simple:

[MTX

$\mathrm{EOT}$

$$
\text { (VECTOR ovname }=\text { expr) }
$$

Use of this block is completely optional. If it is omitted, no PMIX file will be generated. The MIX block should be followed by a PLOT block or an EOF keyword marking the end of the input file.

16.7.2 Mix Block Input Details

MIX

VECTOR

ovname

expr keyword to initiate the mix block.

keyword to initiate the definition of a new type of vector.

name, with eight or fewer characters, to be given to the newly defined type of vector. NOTE: Each vector name must be unique.

an arithmetic expression involving previously defined vectors. Expressions may use the symbols $+,-, I, *,($, and $)$ as operators, and previously defined vector names or real constants as operands. Several rules apply:

1. Leading minus signs are allowed for constants, but not for vector names. 
2. Vector names used in expressions must have been previously defined in the PVEC file, a TDB, or in a previous expression in the mix block.

3. All vectors used in an expression must have the same length.

4. Every operation must involve at least one vector. For example, $3.0 * 6.5$ is not allowed; however, $3.0 *$ vector $1 * 6.5$ is allowed.

5. Expressions may extend beyond a single line, but must be no longer than the limit set by MAXMIX in the control block (see Section 16.5).

Some examples of legal expressions follow:

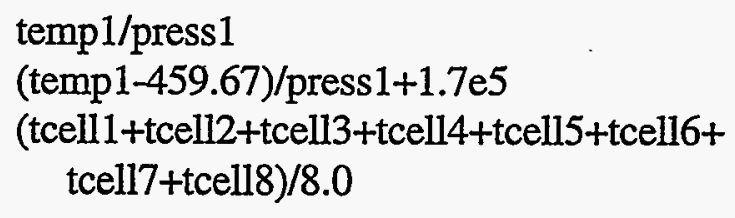

It should be noted that the expressions described above are evaluated element by element. That is, for vectors of length $n$, the equation

$$
\text { VECTOR newvec }=\operatorname{vec} 1 * \operatorname{vec} 2
$$

is equivalent to

$$
\text { newvec(i) }=\operatorname{vec} 1(\mathbf{i}) * \operatorname{vec} 2(\mathbf{i}), \text { for } \mathrm{i}=1 \text { to } \mathrm{n} \text {. }
$$

EOI

keyword that terminates the mix block.

EOF

keyword that terminates the input file. The physical end of the input file will also serve the same function.

\subsubsection{Error Handling}

Division by zero does not cause the program to abort. Instead, an error message is sent to the PERR file, and the quotient is set to zero.

\subsubsection{Adding Functions to the Mix Option}

The mix option was written to allow user-defined functions to be easily incorporated. For example, with very little effort, trigonometric functions can be added as operators to expressions. Adding each function is as simple as choosing a name to represent the function in expressions, modifying a single 
POSTCON routine, and possibly writing a FORTRAN function to perform the necessary calculations.

16.7.4.1 Choosing a Function Name. Mix function names must be no more than eight characters starting with a \$'. For example, \$sin, \$sqrt, \$myfunct, and \$min are all legal function names.

16.7.4.2 Modifying POSTCON for Unary Functions. Provisions are made in POSTCON to process three different classes of mix functions: 1) unary functions, 2) binary functions where both arguments are vectors, and 3 ) binary functions where one argument is a constant.

Unary functions are functions that have only one argument. Examples are sin, log, exp, and sqrt. These functions are handled by a subroutine called OPERU. To implement the function, the user simply needs to add an IF block to this routine. For example, to implement the square root function, the following code should be inserted before the write statement:

10

$$
\begin{aligned}
& \text { if (oper .eq. \$sqrt') then } \\
& \text { do } 10 \mathrm{i}=1 \text {,numpts } \\
& \text { ypts(i) = sqrt(ypts(i)) } \\
& \text { continue } \\
& \text { endif }
\end{aligned}
$$

16.7.4.3 Modifying POSTCON for Binary Functions. Binary functions are functions that have two arguments, such as $\min , \max$, and $y^{x}$. If both arguments are vectors, then the subroutine OPERVV needs to be modified. If only one of the arguments is a vector and one is a scalar, then the subroutine OPERSV needs to be modified. As an example, the following code could be inserted just before the END IF statement of subroutine OPERVV:

$$
\begin{aligned}
& \text { else if (oper .eq. \$ytox') then } \\
& \text { do } 50 \mathrm{i}=1 \text {,numpts } \\
& \text { y1pts(i) = ytox }(\mathrm{y} 1 \mathrm{pts}(\mathrm{i}), \mathrm{y} 2 \mathrm{pts}(\mathrm{i})) \\
& \text { continue }
\end{aligned}
$$

Of course, in this example, the user would also have to supply the FORTRAN function YTOX.

16.7.4.4 Examples of Mix Expressions with Functions. Following are legal mix expressions using the functions that could be easily added to POSTCON.

VECTOR vec1 $=a+\$ s q r t(b)$

VECTOR vec $2=\$ \min (\mathrm{a}, \mathrm{b})$

\subsubsection{Hints for Efficient Use}

Following are some suggestions for using the mix option efficiently. 
1. Defining intermediate vectors. The mix functions uses both the PVEC file and the PMIX file as inputs. This gives the user the capability to define a vector once, and then use that vector in subsequent expressions.

2. Ordering of vectors. Vectors are always extracted from the PVEC and PMIX files from left to right. Time can be saved in computing an expression if the vectors appear in the order they are stored in the files. Furthermore, the PVEC file is always searched first, so vectors stored in the PMIX file should appear last in the expression.

Example: The expression

VECTOR vnew=vthree+vtwo*vone

will take longer to evaluate than the equivalent expression.

VECTOR vnew=vone*vtwo+vthree,

assuming the order of the vectors in PVEC is vone, vtwo, vthree.

Similarly, the sequence of expressions

VECTOR vec1=vtwo-273.15

VECTOR vec2=vone-273.15

would work faster if the lines were reversed.

\subsection{Sample Input and Output}

Examples of POSTCON postprocessing are provided in the following sections.

\subsubsection{Example 1: Extracting Data in a Single Run}

The first sample input file extracts the atmospheric temperatures from each cell of a two cell problem. Figure 16-3 shows the input file. Note that no control keywords are given in this sample problem since the defaults are adequate. The table output is shown in Figure 16-4. A PVEC file is also created by this example, but is not shown here. Sample PVEC files will be shown in later examples.

\subsubsection{Example 2: Unit Conversions, User-Selectable Time Zones, and Snapshots}

The second example extracts information from the binary plot file and performs unit conversions. This example also demonstrates the use of user-selectable time zones and snapshots. The input file is shown in Figure 16-5 and selected portions of the table output file are shown in Figure 16-6. 
Three units are converted in this example. First the time units are changed to minutes by the TUNIT keyword in the control block. Also the temperature and pressure are converted by the UNITS keyword in the TDB. Ordinarily, the conversion would be performed according to Section 16.3.1.3; pressure would be converted from $\mathrm{Pa}$ to $\mathrm{psi}$, and temperature would be converted from $\mathrm{K}$ to deg-F. However, these default conversion factors were changed by the UNIT keywords in the control block. These keywords effectively changed this run so that pressure is converted to ATM if the UNITS keyword is present, and temperature is converted to deg-C.

Note that file names are reassigned in the file definition block (FDB). This provides an easy way to keep results from different problems separate. The vector file from this example is shown in Figure 16-7.

\subsubsection{Example 3: Mixing}

This example demonstrates how to use the MIX option. The temperature from the PVEC1 file in Figure 16-7, from Example 2, is converted to the Rankine temperature scale and then used to compute the pressure divided by the temperature. (The PINP file used to do this is shown in Figure 16-8.) The resulting PMIX file is shown in Figure 16-9.

\subsubsection{Example 4: More Complicated Table Definition Blocks}

The example in Figure 16-10 shows the proper syntax for extracting a number of plot flags from the CONTAIN binary plot file. No output files are shown.

control eoi

101

cell $=1$ vector=temp 1 endcurve

cell $=2$ vector $=$ temp 2 endcurve

endflag

eof

Figure 16-3. PINP File for Example 1 
contain test ht02

heat transfer from hot layer into

Page 0

pool subject to boiling and aerosol fallout

Date of CONTAIN run: 90/20/05 at 19:06:33

CONTAIN version 1.11 last update: $c 110 \mathrm{~m}$

POSTCON version 1.03 last update: p103i made on 09/20/89

POSTCON output file pout

a total of 1 tables definition blocks were written

POSTCON vector file pvec

a total of 2 vectors were written

$\frac{\text { block }}{1} \frac{\text { flag }}{101} \frac{\text { curves }}{2} \frac{\text { convert }}{\text { no }}$

total of 25 time data points in a

total of 1 binary plot files read

1 file name time points

1 pittil 25

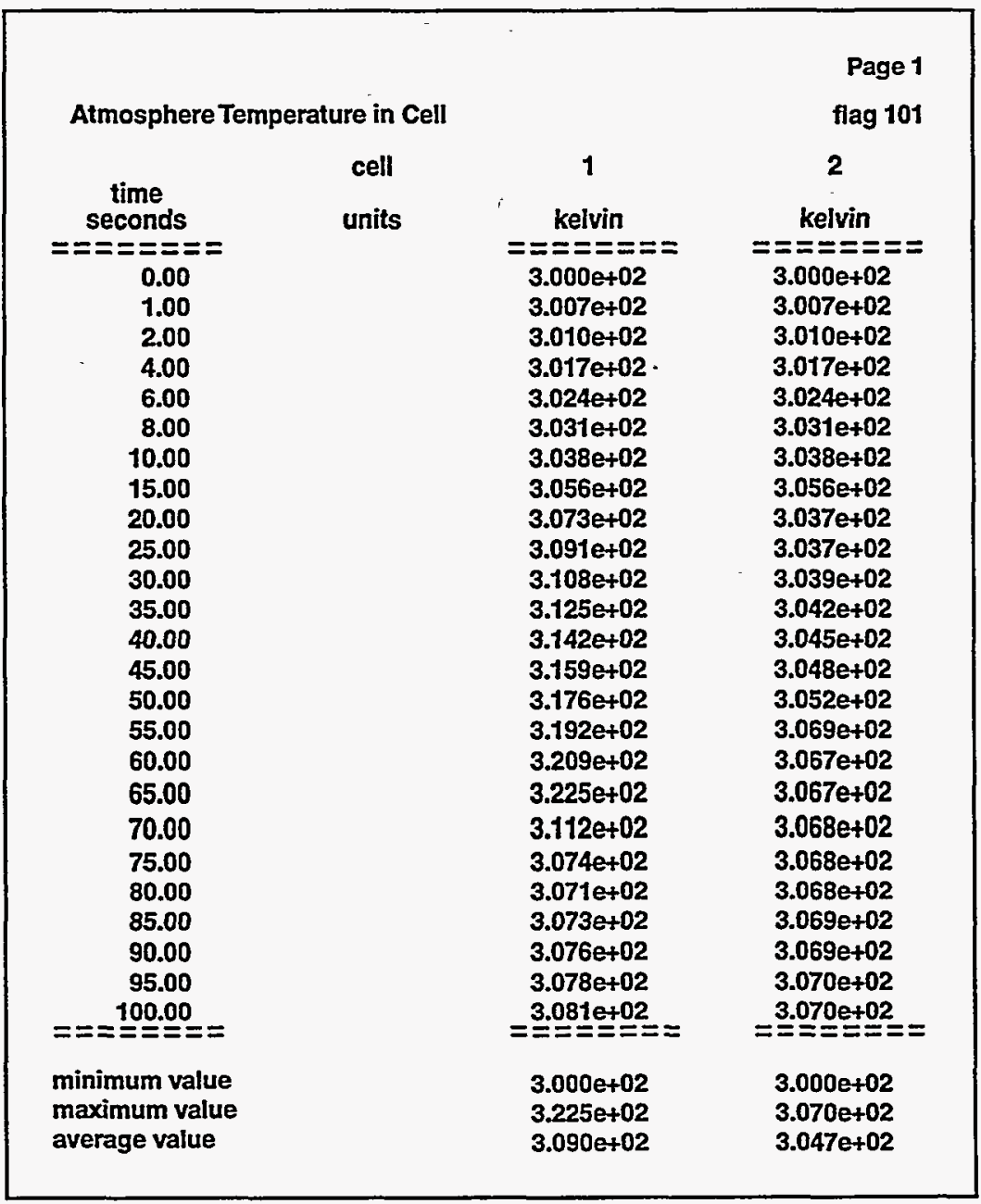

Figure 16-4. POUT File from Example 1 
pltfil $=$ plot 1 pout=pout 1 pvec $=$ pvec 1 control

$\operatorname{maxsize}=100$

maxsnp=10 snpsiz $=20$

tunit=minutes

timeopt 0.0

5.020 .0

10.0100 .0

20.0200 .0

$40.0 \quad 400.0$

100.0800 .0

200.01600 .0

unit=9 ATM 9.86272e-6

unit=12 deg-C 1.0273 .0
$\& \&$ Define file names.

$\& \&$ No more than 100 pts./curve $\& \&$ No more than 10 snapshots, 20 pts/snapshot. \&\& Change time units to minutes. $\& \&$ Define user-selectable time zones.

eoi

\&\& Change pressure units for curves with \&\& UNITS keyword.

\&\& Change temperature units for curves with \&\& UNITS keyword.
101 cell=1 units vector=temp 1 endcurve endflag

102 cell $=1$ units vector=press 1 endcurve endflag

\&\& ----------Snapshot of aerosol airborne densities.

snapshot 530

time $=500$ aerosol $=$ cao vector $=$ csnap endcurve

time $=500$ aerosol $=m n o$ vector $=$ msnap endcurve

endflag

eof

Figure 16-5. PINP File for Example 2
\&\& temperature $\& \&$ pressure 


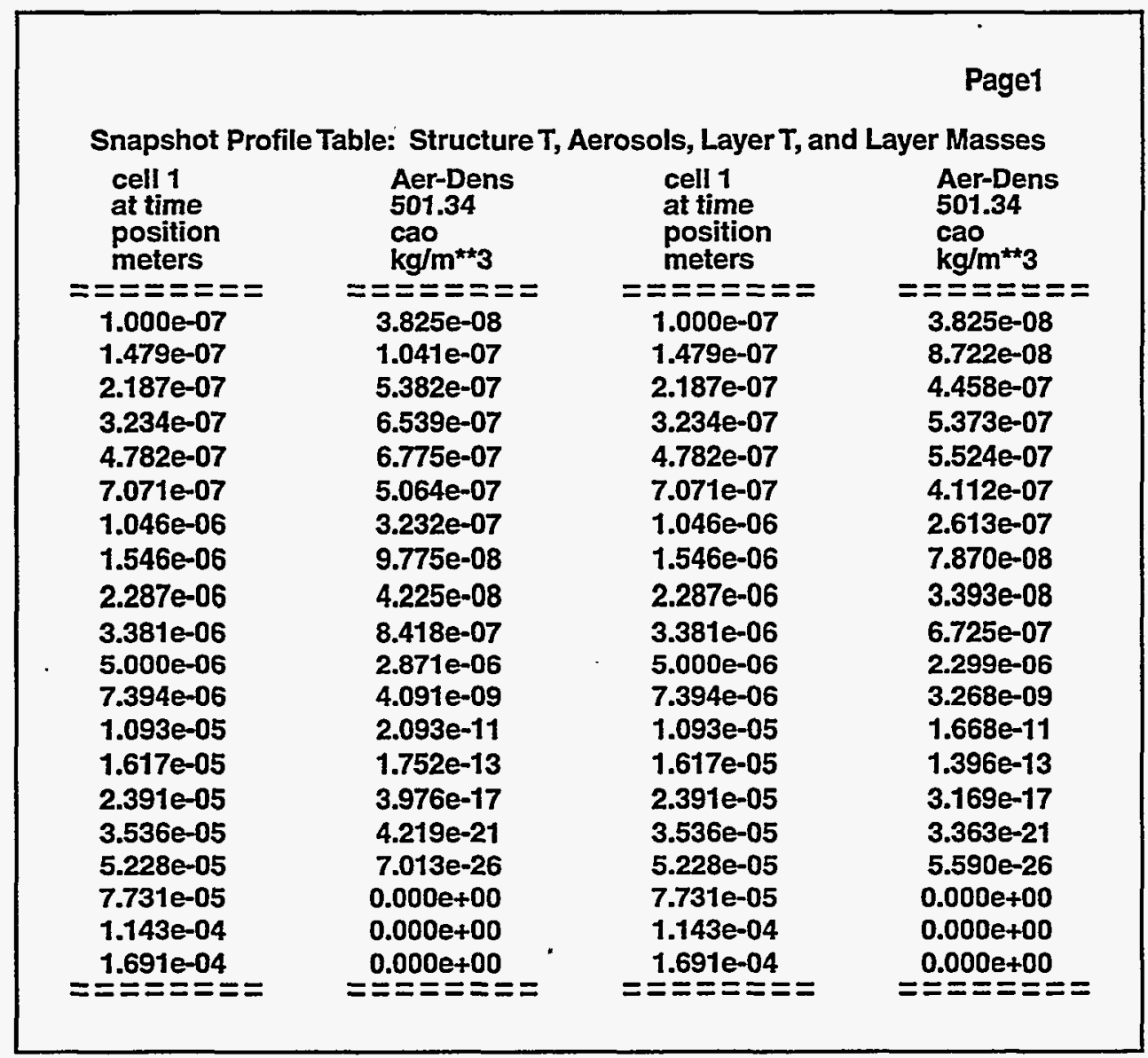

Figure 16-6. Snapshot Table from the POUT1 File for Example 2 
\&\& post snapshot vector for flag: 530

$\begin{array}{lll}\text { c\&nap } & 10001 \quad 20 & \\ 1.00000 e-07 & 3.8254 e-08 \\ 1.47876 e-07 & 1.0412 e-07 \\ 2.18672 e-07 & 5.3820 e-07 \\ 3.23364 e-07 & 6.5390 e-07 \\ 4.78176 e-07 & 6.7749 e-07 \\ 7.07107 e-07 & 5.0641 e-07 \\ 1.04564 e-06 & 3.2322 e-07 \\ 1.54625 e-06 & 9.7752 e-08 \\ 2.28653 e-06 & 4.2247 e-08 \\ 3.38122 e-06 & 8.4184 e-07 \\ 5.00000 e-06 & 2.8714 e-06 \\ 7.39379 e-06 & 4.0912 e-09 \\ 1.09336 e-05 & 2.0926 e-11 \\ 1.61682 e-05 & 1.7518 e-13 \\ 2.39088 e-05 & 3.9755 e-17 \\ 3.53553 e-05 & 4.2193 e-21 \\ 5.22820 e-05 & 7.0134 e-26 \\ 7.73124 e-05 & 0.000 e+00 \\ 1.14326 e-04 & 0.000 e+00 \\ 1.69061 e-04 & 0.000 e+00\end{array}$

\&\& post snapshot vector for flag: 530

\begin{tabular}{|c|c|c|}
\hline \multirow{21}{*}{$\begin{array}{l}\text { \&\& } \\
\text { msnap }\end{array}$} & \\
\hline & & $3.2346 e-08$ \\
\hline & & $8.7220 e-08$ \\
\hline & $2.18672 \mathrm{e}-07$ & 4.4576e-07 \\
\hline & $3.23364 \mathrm{e}-07$ & $5.3734 e-07$ \\
\hline & 4.78176e-07 & $5.5245 \mathrm{e}-07$ \\
\hline & $7.07107 \mathrm{e}-07$ & 4.1122e-07 \\
\hline & $1.04564 \mathrm{e}-06$ & $2.6133 e-07$ \\
\hline & $1.54625 \mathrm{e}-06$ & $7.8702 \mathrm{e}-08$ \\
\hline & $2.28653 \mathrm{e}-06$ & $3.3934 \mathrm{e}-08$ \\
\hline & $3.38122 \mathrm{e}-06$ & $6.7253 e-07$ \\
\hline & $5.00000 e-06$ & $2.2986 e-06$ \\
\hline & $7.39379 \mathrm{e}-06$ & $3.2677 e-09$ \\
\hline & $1.09336 \mathrm{e}-05$ & $1.6683 e-11$ \\
\hline & $1.61682 e-05$ & $1.3962 \mathrm{e}-13$ \\
\hline & $2.39088 \mathrm{e}-05$ & $3.1688 \mathrm{e}-17$ \\
\hline & $3.53553 e-05$ & $3.3630 \mathrm{e}-21$ \\
\hline & $5.22820 \mathrm{e}-05$ & $5.5899 \mathrm{e}-26$ \\
\hline & 7.73124e-05 & $0.000 e+00$ \\
\hline & $1.14326 e-04$ & $0.000 e+00$ \\
\hline & & \\
\hline
\end{tabular}

$\& \&$ post vector for flag: 101 number 1 $\& \&$ templ 025 $0.00000 e+00 \quad 1.0987 e+02$ $5.1668 \quad 1.0575 e+02$ $10.1669 \quad 1.0589 \mathrm{e}+02$ $15.1670 \quad 1.0633 \mathrm{e}+02$ $20.1671 \quad 1.0678 \mathrm{e}+02$ $30.5006 \quad 1.0759 e+02$ $40.5008 \quad 1.0812 e+02$ $51.3344 \quad 1.0874 e+02$ $61.6679 \quad 1.0965 e+02$ $71.6681 \quad 1.1020 \mathrm{e}+02$ $81.6683 \quad 1.0933 e+02$ $91.6685 \quad 1.0635 \mathrm{e}+02$ $101.0020 \quad 1.0553 \mathrm{e}+02$ $121.0024 \quad 1.0513 e+02$ $141.0028 \quad 1.0518 e+02$ $161.0032 \quad 1.0533 e+02$ $182.0036 \quad 1.0550 e+02$ $201.0040 \quad 1.0566 e+02$ $241.0048 \quad 1.0602 e+02$ $282.0056 \quad 1.0399 e+02$ $322.0064 \quad 9.7798 e+01$ $362.0072 \quad 9.3252 e+01$ $400.0080 \quad 8.9864 \mathrm{e}+01$ $501.3434 \quad 9.1635 e+01$ $601.3454 \quad 9.1875 e+01$

\&\& post vector for flag: 102 number 2 $\& \&$ pressl $0 \quad 25$ $0.00000 \mathrm{e}+00$ $10.1669-2.2485 e+00$ $15.1670 \quad 2.2792 e+00$ $20.1671 \quad 2.3135 \mathrm{e}+00$ $30.5006 \quad 2.3904 e+00$ $40.5008 \quad 2.4657 e+00$ $51.3344 \quad 2.5492 e+00$ $61.6679 \quad 2.6323 \mathrm{e}+00$ $71.6681 \quad 2.7127 e+00$ $81.6683 \quad 2.7709 \mathrm{e}+00$ $91.6685 \quad 2.7618 e+00$ $101.0020 \quad 2.7657 e+00$ $121.0024 \quad 2.7793 e+00$ $141.0028 \quad 2.7946 e+00$ $161.0032 \quad 2.8107$ e +00 $182.0036 \quad 2.8278 \mathrm{e}+00$ $201.0040 \quad 2.8436 e+00$ $241.0048 \quad 2.8776 \mathrm{e}+00$ $282.0056 \quad 2.8423 e+00$ $322.0064 \quad 2.0760 \mathrm{e}+00$ $362.0072 \quad 1.5717 e+00$ $400.0080 \quad 1.2486 e+00$ $501.3434 \quad 1.0468 \mathrm{e}+00$ $601.3454 \quad 1.0387 e+00$

Figure 16-7. PVEC1 File for Example 2 


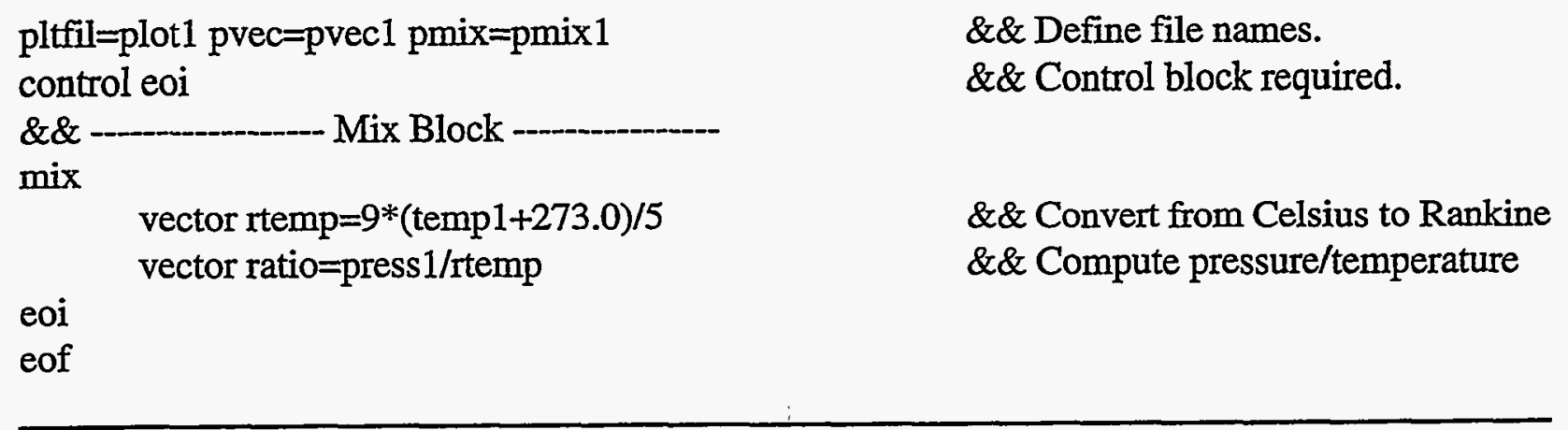

Figure 16-8. PINP File for Example 3 


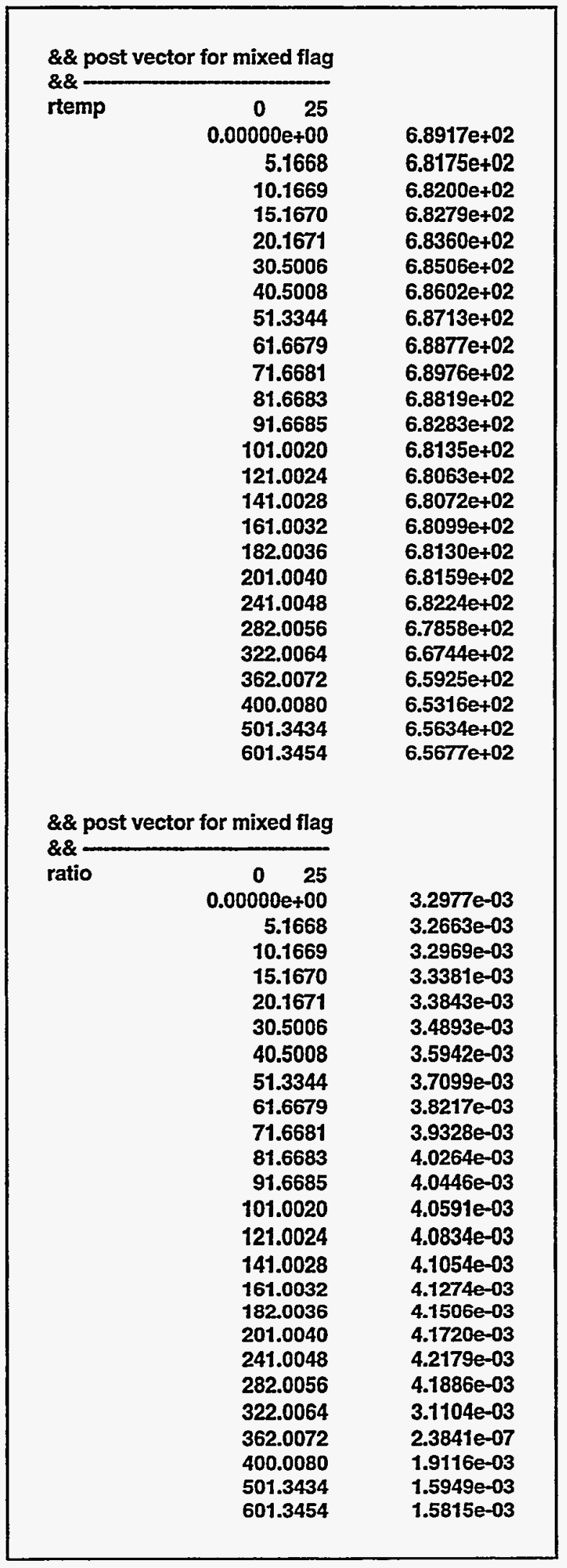

Figure 16-9. PMIX1 File from Example 3 
pltfil=pltfp pout=poutfp pvec=pvecfp

control

eoi

maxsize $=150$ maxflg=11 $\operatorname{maxcrv}=50$

maxsnp $=6$ snpsiz $=20$

\&\& fission product masses from wall and floor

730 cell $=1$ units

fpname $=c s$ host=airborne vector $=$ csair endcurve

fpname $=c s$ host=floor endcurve

fpname $=$ cs host $=$ sum 5,6 vector=csdep endcurve

endflag

fpname=il32 host=airborne endcurve

\&\& aerosol sectional masses snapshots

snapshot 530

cell $=1$ time $=0.0$ aerosol $=$ uo 2 vector $=$ uo2 -0 endcurve

cell $=1$ time $=300.0$ aerosol=uo 2 endcurve

endflag

$\& \&$ aerosol depositions on surfaces

540 cell $=1$

aerosol=uo2 location=wall endcurve

endflag

aerosol=mgo location $=$ wall vector $=$ mgowall endcurve

\&\& time history of structure first node temperature

610

endflag

cell $=1$ struc $=$ wall 1 node $=1$ endcurve

352

cell $=2$ vector $=$ hoxtmp layer $=1$ type $=$ tempture endcurve

cell $=2$ vector $=$ ho $x$ mas layer $=1$ type $=$ mass endcurve

cell $=2$ vector $=$ hoxden layer $=1$ type $=$ density endcurve

cell $=2$ vector $=$ hoxvdf layer $=1$ type $=$ voidfrac endcurve

cell $=2$ vector $=$ hoxint layer $=1$ type $=$ tint endcurve

cell $=2$ vector $=$ hoxqnt layer $=1$ type $=q$ int endcurve

Figure 16-10. PINP File for Example 4 (Continued on Next Page)

Rev. 0 
cell $=2$ vector $=$ mettmp layer $=3$ type=tempture endcurve

cell $=2$ vector $=$ metmas layer $=3$ type $=$ mass endcurve

cell $=2$ vector $=$ metden layer $=3$ type $=$ density endcurve

cell $=2$ vector $=$ metvdf layer $=3$ type $=$ voidfrac endcurve

cell $=2$ vector $=$ metint layer $=3$ type $=$ tint endcurve

endflag

cell $=2$ vector $=$ metqnt layer $=3$ type $=q$ int endcurve

353

endflag

cell $=2$ vector $=$ cavrad measure $=$ radius endcurve

cell $=2$ vector $=$ cavdep measure $=$ depth endcurve

354

cell $=2$ vector $=$ loxtrd layer $=5$ type=tradial endcurve

cell $=2$ vecotr $=$ loxtax layer $=5$ type=taxial endcurve

cell $=2$ vector $=10 \times c r b$ layer $=5$ type $=$ crustb endcurve

cell $=2$ vector $=$ loxcrr layer $=5$ type $=$ crustr endcurve

endflag

cell $=2$ vector $=$ loxcrt layer $=5$ type $=$ crustt endcurve

420

cell $=2$ vector $=$ ptemp layer $=3$ node $=1$ position $=$ top endcurve

cell $=2$ vector $=$ tmphoxb layer $=2$ node $=1$ position $=$ bottom endcurve

cell $=2$ vector $=$ tmploxm layer $=2$ node $=3$ position $=$ middle endcurve

endflag

eof

Figure 16-10. PINP File for Example 4 (Concluded)

\subsection{POSTCON Summary}

The POSTCON postprocessor is an invaluable companion to the CONTAIN code. For quick scoping calculations, the simple user interface of POSTCON combined with the clarity of the output tables should save any user a great deal of time when compared to scanning the CONTAIN output file. For example, using a very simple POSTCON input file (less than ten lines long) the temperatures and pressures (or any other parameter) in any or all cells can be displayed in a collection of neatly formatted and labeled tables. Also, the statistics printed with the POSTCON tables make determining peak pressures or temperatures a completely trivial task. For more demanding situations, POSTCON can extract with great speed nearly any value written onto the plot 
file in a variety of combinations. POSTCON also offers an extremely flexible unit conversion module and an extremely useful snapshot feature for the examination of aerosol density and structure temperature distributions.

Presentation of results is not limited to tables. POSTCON writes a HISPLT-compatible plot file PLTHIS that can be plotted with the HISPLT utility. Finally, an easy to use mix capability is included which allows algebraic manipulations to be performed on postprocessed data in preparation for plotting. This capability can be quite useful for studying relationships between different types of CONTAIN output data, or for evaluating complex functions of CONTAIN data.

\subsection{Useful Suggestions}

\subsubsection{Upward Compatibility Issues}

Every effort has been made to make the CONTAIN 2.0 input compatible with existing input files for prior code versions. However, in some instances the models and/or code architecture have undergone such severe modifications that intelligent translations of old input options are not possible. In other instances, the old input format is accepted by CONTAIN 2.0, but the action taken may differ from the action that the user originally intended. A warning message is always written to the error file when the latter condition is encountered. Therefore, the error file TAPE17 should

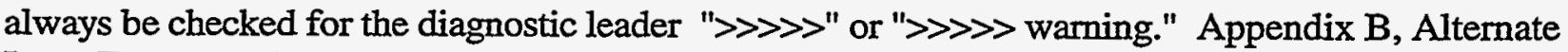
Input Format and Upward Compatibility, discusses obsolete input formats.

\subsubsection{Restarts}

Long-running jobs can be efficiently handled using the restart option. Each restart file should be saved under a file name that is different from the name of any previously generated restart files. Such a backup avoids the loss of previous restart information should an error or system problem occur during the run. The files that need to be saved to invoke a restart are the CONTAIN plot file PLTFL and the restart file TAPE10. Note that PLTFIL and TAPE10 are overwritten on a restart, beginning with the restart time. Thus subsequent restarts at the same restart time will invoke parameter values specified in the previous restart, not those defined in the original run. The output file for each CONTAIN calculation is overwritten during each run; therefore, to save the output file for subsequent restarts, it must be renamed prior to each restart.

Note that if a problem terminates due to the problem time exceeding the maximum CPU time limit "cput," a temporary restart time dump for the last completed edit timestep will be put onto the restart file. However, the restart times listed in either the error or event summary files, TAPE17 and TAPE21, respectively, will not indicate this restart time. To restart from the temporary restart time dump, use a large value for "tstart" that lies beyond any restart time that could be on the file, such

as "tstart" $=10^{10}$. In this manner the user can continue a calculation without having to recalculate from the last listed restart time. 


\subsubsection{Output Handling}

When a user is first getting acquainted with the code or is running a new problem, it is desirable to run a job initially for only a few "long-edit" cycles to test the quantity of output being generated. The quantity of output may require the judicious use of the LONGEDT and SHORTEDT output options, which control the frequency of long and short edits that are written to the output file. The more experienced user is encouraged to use the plot file and post processor for most output purposes.

\subsubsection{Annotated Input}

Users are encouraged to add comments to their CONTAIN or POSTCON input files. This is accomplished using the \&\& sign, followed by a blank and then the appropriate comments. Comments at the beginning of an input file describing the nature of the file, the particular circumstances being modeled, etc., are useful for later referral. One can also use the CONTAIN TITLE option. However, titles cannot be put at the very beginning of a file, while comment lines can be. On the other hand, because titles are printed at the beginning of each long edit and become part of the plot file, they are convenient for labeling the output. 


\section{REFERENCES}

AAF72 "Design and Testing of Fan Cooler Filter Systems for Nuclear Applications," American Air Filter Topical Report AAF-TR-7101, February 20, 1972.

All91 M. D. Allen et al., Experiments to Investigate the Effect of Flight Path on Direct Containment Heating (DCH) in the Surtsey Test Facility," NUREG/CR-5728, SAND91-1105, Sandia National Laboratories, Albuquerque, NM, October 1991.

All92a M. D. Allen et al., "Experiments to Investigate the Effect of Water in the Cavity on Direct Containment Heating (DCH) in the Surtsey Test Facility - The WC-1 and WC-2 Tests," SAND91-1173, Sandia National Laboratories, Albuquerque, NM, March 1992.

All92b M. D. Allen et al., "Experimental Results of Tests to Investigate the Effect of Hole Diameter Resulting from Bottom head Failure on Direct Containment Heating (DCH) in the Surtsey Test Facility - The WC-1 and WC-3 Tests," SAND91-2153, Sandia National Laboratories, Albuquerque, NM, March 1992.

All94 M. D. Allen et al., "Experiments to Investigate Direct Containment Heating Phenomena with Scaled Models of the Zion Nuclear Plant in the Surtsey Test Facility," NUREG/CR-6044, SAND93-1049, Sandia National Laboratories, Albuerque, NM, May 1994.

Ame79 "American National Standard for Decay Heat Power in Light Water Reactors," ANSI/ANS-5.1-1979, available from American National Standards Institute, 1430 Broadway, New York, NY 10018, Copyrighted 1979.

Bak70 L. Baker, Jr., "LOA4 Attenuation of Meltthrough Consequences," ANL-EDP-70, Argonne National Laboratory, 1970.

Bar73 I. Barin and O. Knacke, Thermochemical Properties of Inorganic Substances, Springer-Verlag, Berlin and Heidelberg, Germany; New York, NY, 1973.

Bar77 I. Barin and O. Knacke, Thermochemical Properties of Inorganic Substances, Supplement, Springer-Verlag, Berlin and Heidelberg, Germany; New York, NY, 1977.

Bat70 J. L. Bates, "High Temperature Thermal Conductivity of U.S. Round Robin' Uranium Dioxide," BNWL-1431, July, 1970.

Bau78 T. A. Baumeister, ed., Mark's Standard Handbook for Mechanical Engineers, 8th Ed., McGraw-Hill Book Co., Inc. New York, NY, 1978. 
Ben84 W. B. Benedick, J. C. Cummings, and P. G. Prassinos, "Combustion of Hydrogen: Air Mixtures in the VGES Cylindrical Tank," SAND83-1022, NUREG/CR03273, Sandia National Laboratories, Albuquerque, NM, May 1984.

Ber85a K. D. Bergeron, D. C. Williams, P. E. Rexroth, and J. L. Tills, "Integrated Severe Accident Containment Analysis with the CONTAIN Computer Code," NUREG/CR4343, SAND85-1639, Sandia National Laboratories, Albuquerque, NM, December 1985.

Ber85b K. D. Bergeron et al., "User's Manual for CONTAIN 1.0, A Computer Code for Severe Nuclear Reactor Accident Containment Analysis," NUREG/CR-4085, SAND84-1204, Sandia National Laboratories, Albuquerque, NM, May 1985.

Ber86 K. D. Bergeron, D. E. Carroll, J. L. Tills, K. E. Washington, and D. C. Williams, "Development and Application of the Interim Direct Heating Model for the CONTAIN Computer Code," Fourteenth Water Reactor Safety Information Meeting, Gaithersburg, MD, 1986.

Bil93 S. C. Billups et al., "User's Manual for CONTAIN-HWR/0, Rev. 1: A Computer Code for the Analysis of Heavy Water Nuclear Reactor Containments Under Accident Conditions," SAND91-1482, NPRW-SA91-6, Sandia National Laboratories, Albuquerque, NM, March 1993.

Bin94 J. L. Binder, L. M. McUmber, and B. W. Spencer, "Direct Containment Heating Integral Effects Tests at 1/40 Scale in Zion Nuclear Power Plant Geometry," NUREG/CR-6168, ANL-94/18, Argonne National Laboratory, September 1994.

Bir60 R. B. Bird, W. E. Stewart, and E. N. Lightfoot, Transport Phenomena, John Wiley and Sons, New York, NY, 1960.

Bla94 T. K. Blanchat et al., "Experiments to Investigate Direct Containment Heating Phenomena with Scaled Models of the Surry Nuclear Power Plant," NUREG/ CR-6152, SAND93-2519, Sandia National Laboratories, June 1994.

Boy95 B. E. Boyack, "CONTAIN Independent Peer Review, "LA-12866, Los Alamos National Laboratory, Los Alamos, NM, 1995.

Bra67 Bradfield, W. S., "On the Effect of Subcooling on Wall Superheat in Pool Boiling," Journal of Heat Transfer, pp. 269-270, 1967.

Bra83 E. A. Brandes, ed., Smithells Metals Reference Book, 6th Ed., Butterworths, 1983.

Bra93 D. R. Bradley, D. R. Gardner, J. E. Brockman, and R. O. Griffith, "CORCON-Mod3: An Integrated Computer Model for Analysis of Molten Core-Concrete Interactions. Users Manual," NUREG/CR-5843, SAND92-0167, Sandia National Laboratories, Albuquerque, NM, 1993. 
Brg81 A. E. Bergeles, Two-Phase Flow in the Power and Process Industries, Hemisphere Publishing Corp., NY, 1981.

Bro84 J. E. Broadwell, W. J. A. Dahm, and M. G. Mungal, "Blowout of Turbulent Diffusion Flames," Twentieth Symposium (International) on Combustion, The Combustion Institute, pp. 303-310, 1984.

Bro90 T. D. Brown et al., "Evaluation of Severe Accident Risks: Grand Gulf, Unit 1," NUREG/CR-4551, SAND86-1309, Vol. 6, Rev. 1, Part 2, Sandia National Laboratories, Albuquerque, NM, December 1990.

Ces76 R. D. Cess and M. S. Lian, "A Simple Parameterization for the Water Vapor Emissivity," Transactions, ASME Journal of Heat Transfer, Vol. 98, No. 4, pp. 676678, 1976.

Cha39 S. Chapman and T. G. Cowling, The Mathematical Theory of Nonuniform Gases, Cambridge University Press, New York, NY, 1939.

Cha65 M. W. Chase, Jr., et al., JANAF Thermochemical Tables, Third Edition, Dow Chemical Company, Thermal Research Laboratory, Midland, MI, 1965.

Chu78 T. K. Chu and C. Y. Ho, "Thermal Conductivity and Electrical Resistivity of Eight Selected AISI Stainless Steels," Conductivity, V. V. Mirkovich, ed., Vol. 15, Plenum Press, 1978.

Chu84 T. H. Chung, L. L. Lee, and K. E. Starling, "Applications of Kinetic Theories and Multiparameter Correlation for Prediction of Dilute Gas Viscosity and Thermal Conductivity," Ind. Eng. Chem. Fundam., Vol. 23, pp. 8, 1984.

Col81 J. G. Collier, Convective Boiling and Condensation, 2nd Ed., McGraw-Hill Book Co.(UK) Limited, Great Britain, 1981.

Col84 R. K. Cole, Jr., D. P. Kelly, and M. A. Ellis, "CORCON-MOD2: A Computer Program for Analysis of Molten-Core Concrete Interactions," NUREG/CR-3920, SAND84-1246, Sandia National Laboratories, Albuquerque, NM, 1984.

Con88 R. S. Conti and M. Hertzberg, "Thermal Autoignition Temperatures for HydrogenAir and Methane-Air Mixtures," Journal of Fire Sciences, Vol. 6, 1988.

Cow52 H. F. Coward and G. W. Jones, "Limits of Flammability of Gases and Vapors," Bulletin 503, Bureau of Mines, 1952.

DeS75 G. G. De Soete, "The Flammability of Hydrogen-Oxygen-Nitrogen Mixtures at High Temperatures," La Rivista dei Combustibili, v. 29, pp. 166-172, 1975. 
Dhi78 V. K. Dhir and G. P. Purohit, "Subcooled Film Boiling Heat Transfer from Spheres," Nuclear Engineering and Design, Vol. 47, pp. 49-66, 1978.

Din86 S. E. Dingman, A. L. Camp, C. C. Wong, D. B. King, and R. G. Gasser, "HECTR Version 1.5 User's Manual," NUREG/CR-4507, SAND86-0101, Sandia National Laboratories, Albuquerque, NM, 1986.

Dui89 M. R. Duignan and G. A. Greene, "Enhanced Convective and Film Boiling Heat Transfer by Surface Injection," Dissertation, State University of New York at Stony Brook, NY, 1989.

Dun84 I. H. Dunbar and S. N. Ramsdale, "Improvements in the Modeling of Sedimentation and Gravitational Agglomeration," CSNI Specialists' Meeting on Nuclear Aerosols in Reactor Safety, Karlsruhe, Germany, September 4-6, 1984.

Edw73 D. K. Edwards and A. Balakrishnan, "Thermal Radiation by Combustion Gases," International Journal of Heat and Mass Transfer, Vol. 16, pp. 25-40, 1973.

Elw62 M. M. El-Wakil, Nuclear Power Engineering, McGraw Hill, NY, 1962.

Eng68 T. R. England, "CINDER, A One Point Depletion and Fission Program, U. W. Version," WAPD-TM-334, Bettis Atomic Power Laboratory, Pittsburgh, PA, 1962, revised 1968.

Fel73 J. D. Felske and C. L. Tien, "Calculation of the Emissivity of Luminous Flames," Combustion Science and Technology, Vol. 7, pp. 25-31, 1973.

Fin80 J. K. Fink, M. G. Chasanov, and L. Leibowitz, "Thermodynamic Properties of Uranium Dioxide," ANL-CEN-RSD-80-3, Argonne National Laboratory, 1980.

Fis74 W. A. Fischer, D. Janke, and K. Stahlschmidt, Arch. Eisenhuttwes, Vol. 45, pp. 509, 1974.

Flo79 .Flow of Fluids through Valves, Fittings, and Pipes, "Technical Paper No. 410, Crane Co., New,York, NY, 1979.

Fuc64 N. A. Fuchs, The Mechanics of Aerosols, Pergamon Press, 1964.

Gel80 F. Gelbard and J. H. Seinfeld, "Simulation of Multicomponent Aerosol Dynamics," Journal of Colloid and Interface Science, Vol. 78, December 1980.

Gel82 F. Gelbard, "MAEROS Users Manual," NUREG/CR-1391, SAND80-0822, Sandia National Laboratories, Albuquerque, NM 1982.

Ge190 F. Gelbard, "Modeling Aerosol Growth by Vapor Condensation," Aerosol Science and Technology, Vol. 12, p. 399, 1990. 
Ge191 F. Gelbard, L. A. Moody, and S. E. Ohrt, "A New Method for Determining Hydrodynamic Effects on the Collision of Two Spheres," Journal of Statistical Physics, Vol. 62, p. 945, 1991.

Gid77 R. G. Gido, J. S. Gilbert, and W. J. Jensen, "Containment Ice-Condenser Analysis Using the COMPARE Code," American Nuclear Society, Proceedings of Topical Meeting on Thermal Reactor Safety, Sun Valley, ID, July 31 - August 4, 1977.

Gid84 R. G. Gido and A. Koestal, "Parameters for Containment Hydrogen Burn Analysis," Joint ANS/ASME Conference on Design, Construction, and Operation of Nuclear Power Plants, Portland, OR, August 5-8, 1984.

Gid91 R. G. Gido, D. C. Williams, and J. J. Gregory, "PWR Dry Containment Parametric Studies," NUREG/CR-5630, SAND90-2339, Sandia National Laboratories, Albuquerque, NM, April 1991.

Gol73 L. A. Goldsmith and J. A. M. Douglas, "Measurements of the Thermal Conductivity of Uranium Dioxide at $670^{\circ}-1270^{\circ} \mathrm{K}$," Journal of Nuclear Materials, Vol. 47, pp. 31, 1973.

Gre90 J. J. Gregory et al., "Evaluation of Severe Accident Risks: Grand Gulf, Unit 1," NUREG/CR-4551, SAND86-1309, Vol. 6, Rev. 1, Part 2, Sandia National Laboratories, Albuquerque, NM, December 1990.

Gri82 D. R. Grist, "Spray Removal of Fission Products in PWR Containments," SRDR267, UKAEA Safety and Reliability Directorate, 1982.

Hag81 D. L. Hagerman, A. R. Gregory, and R. E. Mason, eds., "MAPRO - Version 11 (revision 2), A Handbook of Materials Properties for Use in the Analysis of Light Water Reactor Fuel Rod Behaviors," EG\&G Idaho, Inc., Idaho Falls, ID, 1981.

Hen87 J. O. Henrie and A. K. Postma, "Lessons Learned from Hydrogen Generation and Burning During the TMI-2 Event," RHO-RE-EV-95 P, Rockwell Hanford Operations, Hanford, WA, March 1987.

Her83 M. Hertzberg and K. L. Cashdollar, "Flammability Behavior and Pressure Development of Hydrogen Mixtures in Containment Volumes," presented at The Second International Topical Meeting on Nuclear Reactor Thermal-Hydraulics, Santa Barbara, CA, January 11-14, 1983.

Hih95 E. Hihara and P. F. Peterson, "Mixing in Thermally Stratified Fluid Volumes by Buoyant Jets," Proceedings of the ASME/JSME Thermal Engineering Conference, Vol. 1, ASME, 1995.

Hil71 R. K. Hilliard et al., "Removal of Iodine and Particles by Sprays in the Containment Systems Experiment," Nuclear Technology, Vol. 10, 1971. 
Hin82 W. C. Hinds, Aerosol Technology, John Wiley \& Sons, New York, NY, 1982.

Ho68 C. Y. Ho, R. W. Powell, and P. E. Liley, "Thermal Conductivity of Selected Materials Part 2," NSRDS-NBS 16, National Bureau of Standards, USDC, 1968.

Hol68 J. P. Holman, Heat Transfer, McGraw-Hill Book Co., New York, NY, 1968.

Hot67 H. C. Hottell and A. F. Sarofim, Radiative Transfer, McGraw-Hill Book Co., Inc., New York, NY, 1967.

Hus88 J.E. Hustad and O. K. Sonju, "Experimental Studies of Lower Flammability Limits of Gases and Mixtures of Gases at Elevated Temperatures," Combustion and Flame, Vol. 71, pp. 283-294, 1988.

Ide60 I. E. Idel'chik, Handbook of Hydraulic Resistance, Coefficients of Local Resistance and Friction, Moscow, GEI, 1960. (English translation from Russian--AEC-TR6630, available from NTIS.)

Ive62 H. J. Ivey, "Acceleration and the Critical Heat Flux in Pool Boiling," Chartered Mechanical Engineering, Vol. 9, pp. 413-427, 1962.

Kal81 G. T. Kalghatgi, "Blow-Out Stability of Gaseous Diffusion Flames. Part 1: In Still Air," Combustion Science and Technology, Vol. 26, pp. 233-239, 1981.

Kan92 T. F. Kanzleiter, K. Fischer, and D. Wennerberg, "Experimental Results and Modeling Strategies for Multi-Compartment Hydrogen Deflagration," Proceedings of the Workshop on Hydrogen Behavior and Mitigation in Water-Cooled Nuclear Power Plants, March 4-8, 1991, Brussels, Commission of the European Communities document EUR 14039, 1992.

Kay64 W. M. Kays and A. L. London, Compact Heat Exchangers, 2nd Edition, McGrawHill Book Co., Inc., New York, NY, 1964.

Kee78 J. H. Keenan, F. G. Keys, and J. G. Moore, Steam Tables, Thermodynamic Properties of Water Including Vapor, Liquid, and Solid Phases (International System of Units S.I.), John Wiley \& Sons, New York, NY, 1978.

Kee92a R. J. Kee, F. M. Rupley, and J. A. Miller, "Chemkin-II: A Fortran Chemical Kinetics Package for the Analysis of Gas Phase Chemical Kinetics," SAND89-8009B, Sandia National Laboratories, Albuquerque, NM, Reprinted April 1992.

Kee92b R. J. Kee, F. M. Rupley, and J. A. Miller, "The Chemkin Thermodynamic Data Base," SAND87-8215B, Sandia National Laboratories, Albuquerque, NM, Reprinted February 1992. 
Ker72 J. F. Kerrisk and D. G. Clifton, "Smoothed Values of the Enthalpy and Heat Capacity of UO2," Nuclear Technology, Vol. 16, pp. 531, 1972.

Koc81 D. C. Kocher, "Radioactive Decay Data Tables," DOE/TIC-11026, Technical Information Center, U.S. Department of Energy, Washington, DC, 1981.

Kre58 F. Kreith, Principles of Heat Transfer, Wiley, New York, NY, 1958.

Kre73 F. Kreith, Principles of Heat Transfer, 3rd edition, Harper \& Row, Publishers, New York, NY, 1973.

Kum84 R. K. Kumar, H. Tamm, and W. C. Harrison, "Intermediate-Scale Combustion Studies of Hydrogen-Air-Steam Mixtures," EPRI NP-2955, Electric Power Research Institute, June 1984.

Kum85 R. K. Kumar, "Flammability Limits of Hydrogen-Oxygen-Diluent Mixtures," Journal of Fire Sciences, Vol. 3, July-August 1985.

Lam45 H. Lamb, Hydrodynamics, Dover Pub., New York, NY, 1945.

Lan48 I. Langmuir, "The Production of Rain by a Chain Reaction in Cumulus Clouds at Temperatures Above Freezing," Journal of Meteorology, Vol. 5, p. 175, 1948.

Lec72 B. Leckner, "Spectral and Total Emissivity of Water Vapor and Carbon Dioxide," Combustion and Flame, Vol. 19, pp. 33-48, 1972.

Lee80 C. E. Lee, "ASH: An Isotope Transmutation Program Using Matrix Operators," Proceedings, International Conference on Nuclear Waste Transmutation, Austin, TX, 1980.

Lei71 L. Leibowitz, M. G. Chasanov, L. W. Mishler, and D. F. Fisher, "Enthalpy of Liquid Uranium Dioxide to $3500^{\circ} \mathrm{K}$," Journal of Nuclear Materials, Vol. 39, pp. 115, 1971.

Lei84 C. D. Leigh and J. C. Helton, "A Sensitivity Study of Aerosol Agglomeration and Deposition using the MAEROS Model," Proceedings of the ANS Topical Meeting on Fission Product Behavior and Source Term Research, Snowbird, UT, July 15-18, 1984.

Lev91 S. Levy, "Debris Dispersal from Reactor Cavity During Low Temperature Simulant Tests of Direct Containment Heating (DCH), Proceedings of the Eighteenth Water Reactor Safety Information Meeting, NUREG/CP-0114, Vol. 2, April 1992.

Lip85 R. J. Lipinski et al., "Uncertainty in Radionuclide Release Under Specific LWR Accident Conditions," SAND84-0410, Vols. 2 and 3, Sandia National Laboratories, Albuquerque, NM, 1985.

Rev. 0

R-7

$6 / 30 / 97$ 
Low82 W. E. Lowry, B. R. Bowman, and B. W. Davis, "Final Results of the Hydrogen Igniter Experimental Program," NUREG/CR-2486, UCRL-53036, Lawrence Livermore National Laboratory, Livermore, CA, February 1982.

Lui83 D. D. S. Lui and R. MacFarlane, "Laminar Burning Velocities of Hydrogen-Air and Hydrogen-Air-Steam Flames," Combustion and Flame, pp. 59-71, 1983.

Lut91 A. E. Lutz, R. J. Kee, and J. A. Miller, "SENKIN: A Fortran Program for Predicting Homogeneous Gas Phase Chemical Kinetics with Sensitivity Analysis," SAND878248, Sandia National Laboratories, Albuquerque, NM, Reprinted August 1991.

Mak70 R. C. Makhija and R. A. Stairs, Can. J. Chem., Vol. 48, p. 12, 1970.

Mar86 B. W. Marshall, "Hydrogen:Air: Steam Flammability Limits and Combustion Characteristics in the FITS Vessel," NUREG/CR-3468, SAND84-0383, Sandia National Laboratories, Albuquerque, NM, December 1986.

Mas58 E. A. Mason and S. C. Saxena, The Physics of Fluids, Vol. 1, pp. 361-369, 1958.

Mod79 A. T. Modak, "Radiation from Products of Combustion," Fire Research, Vol. 1, pp. 339-361, 1979.

Mur89a K. K. Murata et al., "User's Manual for CONTAIN 1.1, A Computer Code for Severe Nuclear Reactor Accident Containment Analysis," NUREG/CR-5026, SAND872309, Sandia National Laboratories, Albuquerque, NM 1989.

Mur89b K. K. Murata et al., "User's Manual for CONTAIN 1.1, A Computer Code for Severe Nuclear Reactor Accident Containment Analysis (Revised for Revision 1.11)," NUREG/CR-5026, SAND87-2309, Sandia National Laboratories, Albuquerque, NM, 1990.

Mur91 K. K. Murata et al., "New Containment Modeling Features of the CONTAIN Code," Nineteenth Water Reactor Safety Information Meeting, Bethesda, MD, October 28 30, 1991.

Mur93 K. K. Murata, D. E. Carroll, K. D. Bergeron, and G. D. Valez, "CONTAIN LMR-IBMod1, A Computer Code for Containment Analysis of Accidents in Liquid-MetalCooled Nuclear Reactors," SAND91-1490, Sandia National Laboratories, Albuquerque, NM, 1993.

Mur96 K. K. Murata and D. W. Stamps, "Development and Assessment of the CONTAIN Hybrid Flow Solver," SAND96-2792, Sandia National Laboratories, Albuquerque, NM, 1996. 
NRC90 U. S. Nuclear Regulatory Commission (USNRC), "Severe Accident Risks: An Assessment for Five U.S. Nuclear Power Plants," Final Summary Report, NUREG1150, USNRC, Washington, DC, December 1990.

NRC92 United States Nuclear Regulatory Commission (USNRC), Office of Nuclear Regulatory Research, "Severe Accident Research Program Update," NUREG-1365, Rev. 1, USNRC, Washington, DC, December 1992.

Owc85a P. C. Owczarski and R. I. Schrenk, "Technical Bases and User's Manual for the Prototype of a Suppression Pool Aerosol Removal Code," NUREG/CR-3317, Pacific Northwest Laboratory, Richland, WA, 1985.

Owc85b P. C. Owczarski et al., "ICEDF: A Code For Aerosol Particle Capture in Ice Compartments," NUREG/CR-4130, PNL-5379, Pacific Northwest Laboratory, Richland, WA, 1985.

Per73 R. H. Perry and C. H. Chilton, Chemical Engineer's Handbook, 5th Ed., pp. 23-39, McGraw-Hill, New York, NY, 1973.

Pet94 P. F. Peterson, "Scaling and Analysis of Mixing in Large Stratified Volumes," International Journal of Heat and Mass Transfer, Vol. 37, Suppl. 1, pp. 97-106, 1994.

Pet96 P. F. Peterson, "Theoretical Basis for the Uchida Correlation for Condensation in Reactor Containments," Nuclear Engineering and Design, Vol. 162, pp. 301-306, 1996.

Pi170 M. J. Pilat and D. S. Ensor, "Plume Opacity and Particulate Mass Concentration," Atmospheric Environment, Vol. 4, pp. 163-173, 1970.

Pil92a M. Pilch and R. O. Griffith, "Liquid-Phase and Gas-Phase Discharge Coefficients for Use in the Analysis of High Pressure Melt Ejection (HPME) Events," SAND920444, Sandia National Laboratories, Albuquerque, NM, January 1992.

Pi192b M. Pilch and R. O. Griffith, "Gas Blowthrough and Flow Quality Correlations for Use in the Analysis of High Pressure Melt Ejection (HPME) Events," SAND912322, Sandia National Laboratories, Albuquerque, NM, June 1992.

Pi194a M. Pilch, H. Yan, and T. G. Theofanous, "The Probability of Containment Failure by Direct Containment Heating in Zion," NUREG/CR-6075, SAND93-1535, Sandia National Laboratories, Albuquerque, NM, December 1994.

Pil94b M. M. Pilch et al., "The Probability of Containment Failure by Direct Containment Heating in Zion," NUREG/CR-6075, SAND93-1535, Supplement 1, Sandia National Laboratories, Albuquerque, NM, December 1994. 
Pi195

Pi196

Pit89

Pon90

Pow86

Pow93

Pru78

$\operatorname{Ran} 52$

Rat85

$\operatorname{Raz} 76$

Rei77

Rei87

M. M. Pilch et al., "The Probability of Containment Failure by Direct Containment Heating in Surry," NUREG/CR-6109, SAND93-2078, Sandia National Laboratories, Albuquerque, NM, May 1995.

M. M. Pilch et al., "Resolution of the Direct Containment Heating Issue for All Westinghouse Plants with Large Dry Containments or Subatmospheric Containments," NUREG/CR-6338, SAND95-2381, Sandia National Laboratories, Albuquerque, NM, February 1996.

W. M. Pitts, "Importance of Isothermal Mixing Processes to the Understanding of Lift-Off and Blowout of Turbulent Jet Diffusion Flames," Combustion and Flame, Vol. 76, pp. 197-212, 1989.

L. T. Pong, "Assessment of the Combustion Model in the HECTR Code," NUREG/CR-5590, SAND90-7080, Sandia National Laboratories, Albuquerque, NM, November 1990.

D. A. Powers, J. E. Brockmann, and A. W. Shiver, "VANESA: A Mechanistic Model of Radionuclide Release and Aerosol Generation During Core Debris Interactions with Concrete," NUREG/CR-4308, SAND85-1370, Sandia National Laboratories, Albuquerque, NM, 1986.

D. A. Powers and S. B. Burson, "A Simplified Model of Aerosol Removal by Containment Sprays," NUREG/CR-5966, SAND92-2689, Sandia National Laboratories, Albuquerque, January 1993.

H. R. Pruppacher and J. D. Klett, Microphysics of Clouds and Precipitation, D. Reidel, Boston, MA, 1978.

W. E. Ranz and W. R. Marshall, "Evaporation from Drops," Chemical Engineering Progress, Vol. 48, No. 3, April 1952.

A. C. Ratzel, "Data Analyses for Nevada Test Site (NTS), Premixed Combustion Tests," NUREG/CR-4138, SAND85-0135, Sandia National Laboratories, Albuquerque, NM, May 1985.

K. Raznjevic, Handbook of Thermodynamic Tables and Charts, McGraw-Hill, New York, NY, 1976.

R. C. Reid, J. M Prausnitz, and T. K. Sherwood, The Properties of Gases and Liquids, 3rd Ed., McGraw-Hill, New York, NY, 1977.

R. C. Reid, J. M. Prausnitz, and T. K. Sherwood, The Properties of Gases and Liquids, 4th Ed., McGraw-Hill, New York, NY, 1987. 
Ric61 F. P. Ricou and D. B. Spalding, "Measurements of Entrainment by Axisymmetrical Turbulent Jets," Journal of Fluid Mechanics, Vol. 11, pp. 21-32, 1981.

Roh52 W. M. Rohsenow, "A Method of Correlating Heat Transfer for Surface Boiling of Liquids," Trans. ASME, Vol. 74, pp. 979-976, 1952.

Roh73 W. M. Rohsenow, "Boiling," in Handbook of Heat Transfer, McGraw-Hill, New York, NY, pp. 13-28, 1973.

Roh85 W. M. Rosenhow, J. P. Harnett, and E. N. Ganic, Handbook of Heat Transfer Fundamentals, McGraw-Hill, 1985.

Rus90a N. A. Russell et al., "Quality Assurance Procedures for the CONTAIN Severe Reactor Accident Computer Code," NUREG/CR-5518, SAND90-0011, Sandia National Laboratories, Albuquerque, NM, August 1990.

Rus90b N. A. Russell and D. C. Williams, "Comparison of CONTAIN Code to Experimental Ice Condenser Data," Proceedings of the Second International Conference on Containment Design and Operation, Canadian Nuclear Society, Toronto, Canada, October 14-17, 1990.

Saf56 P. G. Saffman and J. S. Turner, "On the Collision of Drops in Turbulent Clouds," Journal of Fluid Mechanics, Vol. 1, p. 16, 1956.

Sha57 Z. M. Shapiro and R. T. Moffette, "Hydrogen Flammability Data and Application to PWR Loss-of-Coolant Accident," WAPD-SC-545, Westinghouse Electric Corporation, September 1957.

She85 J.E. Shepherd, "Hydrogen-Steam Jet-Flame Facility and Experiments," NUREG/CR3638, SAND84-0060, Sandia National Laboratories, Albuquerque, NM, February 1985.

She87 J. E. Shepherd, "Analysis of Diffusion Flame Tests," NUREG/CR-4534, SAND860419, Sandia National Laboratories, Albuquerque, NM, August 1987.

Sie81 R. Siegel and J. R. Howell, Thermal Radiation Heat Transfer, 2nd Edition, McGrawHill Co., Inc., New York, NY, 1981.

Siv70 J. B. Siviour and A. J. Ede, "Heat Transfer in Subcooled Film Boiling," paper B3.12, Proceedings of the Fourth International Heat Transfer Conference, Paris-Versailles, France, 1970.

Sta95 D. W. Stamps, "CONTAIN Assessment of the NUPEC Mixing Experiments," SAND94-2880, Sandia National Laboratories, Albuquerque, NM, August 1995. 
Sum95 R. M. Summers et al., "MELCOR Computer Code Manuals, Vol. 2," NUREG/CR6119, SAND93-2185, Sandia National Laboratory, Albuquerque, NM, March 1995.

Tal80 L. Talbot et al., "Thermophoresis of Particles in a Heated Boundary Layer," Journal of Fluid Mechanics, Vol. 101, pp. 737-758, 1980.

Tam85 H. Tamm, R. McFarlane, and D. D. S. Liu, "Effectiveness of Thermal Ignition Devices in Lean Hydrogen-Air-Steam Mixtures," EPRI NP-2956, Electric Power Research Institute, March 1985.

Tam87 H. Tamm, M. Ungurian, and R. K. Kumar, "Effectiveness of Thermal Ignition Devices in Rich Hydrogen-Air-Steam Mixtures," EPRI NP-5254, Electric Power Research Institute, July 1987.

Tam88 F. Tamanini, E. A. Ural, J. L. Chaffee, and J. F. Hosler, "Hydrogen Combustion Experiments in a 1/4-Scale Model of Nuclear Power Plant Containment," TwentySecond Symposium (International) on Combustion, The Combustion Institute, pp. 1715-1722, 1988.

Tar88 W. W. Tarbell, J. E. Brockman, K. E. Washington, M. Pilch, and K. D. Marx, "Direct Containment Heating and Aerosol Generation During High Pressure Melt Ejection Experiments," Sixteenth Water Reactor Safety Information Meeting, Gaithersburg, MD, October 24-27, 1989.

Tem63 L. J. Templin, ed., "Reactor Physics Constants," ANL-5800, Argonne National Laboratory, 1963.

Tho88a R. T. Thompson et al., "Large-Scale Hydrogen Combustion Experiments, Volume 1: Methodology and Results," EPRI NP-3878, Vol. 1, Electric Power Research Institute, October 1988.

Tho88b R. T. Thompson et al., "Large-Scale Hydrogen Combustion Experiments, Volume 2: Data Plots," EPRI NP-3878, Vol. 2, Electric Power Research Institute, October 1988.

Tie93 S. R. Tieszen et al., "Hydrogen Distribution and Combustion," Chapter 6 in ExVessel Severe Accident Review for the Heavy Water New Production Reactor, SAND90-0234, K. D. Bergeron (ed.), Sandia National Laboratories, Albuquerque, NM, January 1993.

Tod82 S. Toda, and M. Mori, "Subcooled Film Boiling and the Behavior of Vapor Film on a Horizontal Wire and Sphere," Seventh International Heat Transfer Conference, Munich, Germany, pp. 173-178, 1982.

Tor83 R. Torok et al., "Hydrogen Combustion and Control Studies in Intermediate Scale, EPRI NP-2953, Electric Power Research Institute, June 1983. 
Tou79 Y. S. Touloukian and C. Y. Ho, Thermophysical Properties of Matter: The TPRC Data Series, Plenum Press, 1970 - 1979.

Tut90 N. K. Tutu et al., "Melt Dispersal Characteristics of the Watts Bar Cavity," Technical Report A-3024, 4/90, Brookhaven National Laboratory, Upton, NY, April 1990.

Tut91 N. K. Tutu, "An Idealized Transient Model for Melt Dispersal from Reactor Cavities During Pressurized Melt Ejection Accident Scenarios," BNL-46305 Informal Report, Brookhaven National Laboratory, Upton, NY, June 1992.

Uch65 H. Uchida, A. Oyama, and Y. Togo, "Evaluation of Post-Incident Cooling Systems of Light-Water Power Reactors," Proceedings of the Third International Conference on the Peaceful Uses of Atomic Energy, Geneva, August 31-September 9, 1964, Vol. 13, pp. 93-104, United Nations, New York, NY, 1965.

Val88 G. D. Valdez, "Review and Assessment of Thermodynamic and Transport Properties for the CONTAIN Code," NUREG/ CR-5173, SAND88-7101, Sandia National Laboratories, Albuquerque, NM, December 1988.

Van78 G. J. Van Wylen and R. E. Sonntag, Fundamentals of Classical Thermodynamics, 2nd Ed., SI Version, John Wiley \& Sons, New York, NY, 1978.

Wal66 L. Waldmann and K. H. Schmitt, "Thermophoresis and Diffusiophoresis of Aerosols," in C. N. Davies, ed., Aerosol Science, Academic Press, New York, NY, 1966.

Wal69 G. B. Wallis, One-dimensional Two-phase Flow, McGraw-Hill, New York, NY, 1969.

Was83 K. E. Washington, "High Order Numerical Solutions to Time Dependent AdvectionDiffusion Problem," Thesis, Texas A\&M University, 1983.

Was87 K. E. Washington, "POSTCON: A Postprocessor and Unit Conversion Program for the CONTAIN Computer Code," NUREG/CR-4887, SAND87-0562, Sandia National Laboratories, Albuquerque, NM, 1987.

Was88 K. E. Washington and D. E. Carroll, "Assessment of Models for Steam Release from Concrete and Implications for Modeling Corium Behavior in Reactor Cavities," SAND88-2329C, Sandia National Laboratories, Albuquerque, NM, 1988.

Was91 K. E. Washington et al., "Reference Manual for the CONTAIN 1.1 Code for Containment Severe Accident Analysis," NUREG/CR-5715, SAND91-0835, Sandia National Laboratories, Albuquerque, NM, 1991. 
Was95 K. E. Washington and D. C. Williams, "Direct Containment Heating Models in the CONTAIN Code," SAND94-1073, Sandia National Laboratories, Albuquerque, NM, August 1995.

Wea85 R. C. Weast, ed., CRC Handbook of Chemistry and Physics, 65th Ed., CRC Press, Inc., Boca Raton, FL, 1984 - 1985.

Web92 C. F. Weber, E. C. Beahm, and T. S. Kress, "Models of Todine Behavior in Reactor Containments," ORNL/TM-12202, Oak Ridge National Laboratory, Oak Ridge, TN, 1992.

Wei72 J. C. Weilbacher, "Diffusivité Thermique de l'Oxyde d'Uranium et de l'Oxyde de Thorium a Haute Temperateur," High Temperatures -High Pressure, Vol. 4, p. 431, 1972.

Wha78 P. B. Whalley and G. F. Hewitt, "The Correlation of Liquid Entrainment Fraction and Entrainment Rate in Annular Two-Phase Flow," AERE-R9187, HTFS RS237, AERE Harwell, England, July 1978.

Wil87a D. C. Williams, K. D. Bergeron, D. E. Carroll, G. D. Gasser, J. L. Tills, and K. E. Washington, "Containment Loads Due to Direct Containment Heating and Associated Hydrogen Behavior: Analysis and Calculations With the CONTAIN Code," NUREG/CR-4896, SAND87-0633, Sandia National Laboratories, Albuquerque, NM, 1987.

Wil87b D. C. Williams K. D. Bergeron, P. E. Rexroth, and J. L. Tills, "Integrated Phenomenological Analysis of Containment Response to Severe Core Damage Accidents," Progress in Nuclear Energy, Vol. 19, 1987.

Wil88a D. C. Williams and D. L. Y. Louie, "CONTAIN Analyses of Direct Containment Heating Events in the Surry Plant," ANS/ENS International Meeting, Washington, DC, 1988.

Wil88b D. C. Williams, D. A. Powers, and A. W. Shiver, "Some Uncertainties in Radionuclide Release During Core-Concrete Interactions," SAND88-1550C, Second Nuclear Reactor Severe Accident Chemistry Symposium, Toronto, Canada, June 5, 1988.

Wi190 D. C. Williams and J. J. Gregory, "Mitigation of Direct Containment Heating and Hydrogen Combustion Events in Ice Condenser Plants," NUREG/CR-5586 SAND90-1102, Sandia National Laboratories, Albuquerque, NM, October 1990.

Wi195 D. C. Williams et al., "CONTAIN Code Analyses of Direct Containment Heating (DCH) Experiments: Model Assessment and Phenomenological Interpretation," Proceedings of the ANS Winter Meeting, Thermal Hydraulics Division, San Francisco, October 29-November 2, 1995. 
Wil96 D. C. Williams and R. O. Griffith, "Assessment of Cavity Dispersal Correlations for Possible Implementation Into the CONTAIN Code," SAND94-0015, Sandia National Laboratories, Albuquerque, NM, February 1996.

Win83 W. K. Winegardner, A. K. Postma, and M. W. Jankowski, "Studies of Fission Product Scrubbing Within Ice Compartments," NUREG/CR-3248, PNL-4691, Pacific Northwest Laboratory, Richland, WA, 1983.

Won88 C. C. Wong, "HECTR Analyses of the Nevada Premixed Combustion Experiments," NUREG/CR-4916, SAND87-0956, Sandia National Laboratories, Albuquerque, NM, November 1988.

Woo80 R. O. Wooten and H. I. Avci, "MARCH (Meltdown Accident Response Characteristics), Code Description and User's Manual," NUREG/CR-1711, BMI2064, Battelle Columbus Laboratories, Columbus, OH, 1980.

Woo83 R. O. Wooten and P. Cybulskis, "MARCH 2 (Meltdown Accident Response Characteristics), Code Description and User's Manual," NUREG/CR-3988, BMI2115, Battelle Columbus Laboratories, Columbus, OH, 1983.

Yea38 J. S. Yeaw and L. Shnidman, "The Extinction of Gas Flames by Steam," Proceedings, American Gas Association, Technical Section, pp. 717-745, 1938.

Yos79 T. Yoshida, Y. Kousaka, and K. Obseyama, Aerosol Science for Engineers, University of Osaka Prefecture, Power Company Ltd., Tokyo, Japan, 1979.

Zab56 M. G. Zabetakis, "Research on the Combustion and Explosion Hazards of HydrogenWater Vapor-Air Mixtures," AECU-3327, U.S. Atomic Energy Commission, September 4, 1956.

Zio00 "Zion Station, Final Safety Analysis Report, Vol VI," Commonwealth Edison Company, U.S. Atomic Energy Commission Docket 50295-18.

Zub58 N. Zuber, "On Stability of Boiling Heat Transfer," Trans. ASME, Vol. 80, pp. 711$720,1958$.

Zub61 N. Zuber et al., "The Hydrodynamic Crisis in Pool Boiling of Saturated and Subcooled Liquids," International Developments in Heat Transfer, Part II, ASME, New York, NY, pp. 230-235, 1961. 


\section{APPENDIX A \\ DETAILS OF THE MASS AND ENERGY ACCOUNTING SCHEME}

\section{A.1 Introduction}

This appendix discusses the details of the independent mass and energy accounting scheme available in CONTAIN. An introduction to the mass and energy accounting scheme and example output are given in Section 3.4 of the CONTAIN User's Manual.

\section{A.2 Mass and Energy Tracking Strategy}

The objective of the mass and energy accounting scheme is to allow the user to assess the discrepancies in the code bookkeeping entities where mass or energy can be gained or lost. In CONTAIN, these entities are the repositories of mass and energy, such as a cell atmosphere, or, in some cases, because of intentionally nonconservative treatment of the energy flux associated with mass fluxes, the interfaces between repositories. (Examples of such nonconservative treatment are given below.) There is broad latitude in how such a scheme might be set up. The present method is repository-oriented. This means, for example, that the mass and energy accounting errors can be directly identified with individual repositories, without the need for extensive side calculations or sums. To allow this identification, the energy gain or loss in the nonconservative interfaces must be dealt with, since an interface gain or loss cannot be assigned to a specific repository. To eliminate the need to track the interfaces separately, best-estimate conservative expressions are constructed for the energy transfer associated with mass transfer across such interfaces, and the energy in each repository is evaluated, in effect, with respect to the best estimate for the energy transfer. The bestestimate energies specifically constructed for use in the mass and energy accounting scheme will be referred to as audit energies in the discussion below. One disadvantage of this method is that considerable overhead is required to calculate the audit energies. Another is that the audit energy transfers cannot be conveyed by the standard interface array architecture but require a new parallel and independent architecture. This independent architecture, which is necessitated by the bestestimate nature of the audit energies, can conveniently be defined to accommodate the fact that the audit energies are total energies, which are conserved, whereas the energies loaded in the standard interface arrays correspond to internal energy contributions.

Aerosol specific heats and contributions to gravitational potential energies are examples of what is included in an audit energy but not in an interface array energy. Although CONTAIN normally neglects the specific heat of aerosols in evaluating the thermodynamic state of a repository, the internal energy of aerosol components that are thermodynamic materials is considered in the audit energy, calculated according to the assumption that the aerosols are in thermal equilibrium with the repository. Also, although aerosols are not currently considered in the gravitational potential energy terms used in repository state calculations, the aerosol components that are thermodynamic materials are considered in the audit potential energy terms. In addition, the enthalpy flux carried by such aerosol components is tracked in the flow between two cells in the energy accounting scheme, even though this energy transport is ignored in the CONTAIN atmosphere state calculations themselves. It should be noted, in contrast, that the enthalpy flux associated with water aerosol deposition is taken into account in the temperatures of structures and pools on which the deposition occurs, as well as in the audit energies. 
For purposes of the present discussion, two types of interfaces and repositories are defined: internal and external. Internal interfaces are those that connect internal repositories, which are defined as ones that consistently utilize the CONTAIN set of materials and thermodynamic functions. External interfaces are those that connect an internal repository with either an external source, external repository, or external boundary condition. A repository is defined as external when it is incompatible with the internal repositories with respect to the set of materials or thermodynamic functions used. Such repositories are typically the result of importing codes with similar but incompatible representations into CONTAIN. In the present scheme, mass and energy accounting is done only for the internal repositories. The heat and mass fluxes from the external repositories are treated as if they were external sources applied to the internal repositories.

By design, certain CONTAIN internal interfaces are treated as nonconservative with respect to the energy loaded into the standard interface arrays (even when the potential energy change is considered). In general, an internal interface should be conservative unless a good reason exists for a nonconservative one. One reason for a "nonconservative" interface is to compensate for the effect of considering the coolant to have a finite volume in some repositories, such as the pool, but not in others, such as structures. An example is the interface for coolant film overflow from structures into pools. Since displacement effects on the atmosphere by a coolant film are ignored, but those of a pool are considered, the new pressure term in the enthalpy (see Equation (3-3) or (3-8)), which should in general be present in the liquid water enthalpy transferred from the film to pool on overflow, is included in the energy added to the pool but omitted from the energy subtracted from the structure film. This nonconservative treatment gives a more accurate representation of the thermodynamic state of the film when the work done against the system constraints is considered, and as a minor side benefit, it also reduces the coding involved in implementing the pressure term.

Interfaces associated with a source table or an external user-specified temperature boundary condition are examples of external interfaces. An important example of an external interface connecting two repositories is the interface between CONTAIN and the lower cell layers representing CORCON. The latter layers are considered to be external repositories because of representational incompatibilities between the materials and thermodynamic functions used by CONTAIN and by CORCON. To accommodate this representational incompatibility, the flux of aerosol materials from VANESA, the aerosol generation module of CORCON, into the CONTAIN domain is determined by mapping the larger set of VANESA materials onto the smaller set of CONTAIN materials and the enthalpy flux of released gases is determined by matching temperatures, not enthalpies, of the released gases across the interface. (These gases are presently assumed to be ideal, and therefore knowledge of the pressure is not required.)

The decision to exclude external repositories as separate accounting entities in the mass and energy accounting scheme is based on the observation that energy is in general not formally conserved across the interface to an external repository but the significance of the lack of conservation cannot be determined in a simple manner. (It also should be noted that CORCON, the principal external repository, has its own internal energy accounting scheme.) It is possible that the lack of formal conservation has little or no impact on the calculated thermodynamic states. For example, if the same set of materials with the same thermodynamic derivative functions (such as the specific heat) is used on both sides of such an interface, but the arbitrary zeroes of enthalpies are substantially different on the two sides, then temperature and pressure matching would lead to the correct 
thermodynamic states. However, the energy associated with mass transport would not be formally "conserved" across such an interface. While in this simple first example, one could adjust the energy accounting to account for the difference in enthalpy zeroes, in more realistic situations, it is not clear how to make the adjustment. An example is a situation in which the same material sets are used but somewhat different yet still acceptable approximations to the thermodynamic properties (within the accuracy of the experimental data) are used on the two sides of the interface. In such a case, one cannot properly evaluate the effect of the formal energy conservation error on the repository states, because in general the precise degree to which the respective thermodynamic properties are correct is not known (i.e., one does not know how to construct the best-estimate expression).

Another problem with the CONTAIN-CORCON interface is the fact that the CORCON lower cell layers are switched discontinuously between the CONTAIN representation and CORCON representation when CORCON becomes active and when it deactivates. This domain switching produces discontinuities that are difficult to interpret with respect to mass and energy conservation. Therefore, to avoid such complications when CORCON is invoked, the CORCON intermediate and concrete lower cell layers are considered to be external repositories even when CORCON is not active.

\section{A.3 Repository Accounting}

In this section, the internal repositories discussed in the preceding section are defined explicitly. Also, expressions are given for the audit energies reported in mass and energy accounting output.

The internal repositories are defined in the following list. Each item in the list defines one bookkeeping entity for the purposes of mass and energy accounting, even though some of the items actually consist of a collection of repositories. For example, the first item defines the internal repository referred to as the "atmosphere" repository in the present discussion.

1. Each cell atmosphere, including gases and condensables; aerosols that are composed of materials with internally-defined or user-defined thermodynamic properties; and other such materials that are not considered part of a debris field.

2. The suspended and trapped debris fields considered collectively in each cell but excluding any trapped debris transferred to the lower cell.

3. Each heat transfer structure, including the surface condensate films and deposited aerosols on both faces. The heat and mass transfer to and from a structure, including aerosol deposition and concrete outgassing fluxes, are tracked for each structure. The aerosol enthalpy for deposited aerosols is tracked for thermodynamic aerosol materials.

4. Each lower cell layer, with the exception of the CORCON layer and concrete layer when CORCON is invoked. As discussed above, the latter layers are treated as external repositories in the mass and energy accounting. To avoid having to define the basemat as a repository, interactions with the lower cell basemat are treated as an external source to the repository adjacent to the basemat. This treatment is compatible with direct basemat-tostructure radiation, since this radiation is treated as occurring in two steps: basemat-to- 
atmosphere and atmosphere-to-structure, regardless of whether the atmosphere actually participates in the radiative transfer.

5. A waste repository, which keeps track of audit energies and masses. This repository is used as a destination for processes that have no obvious user-specified or mechanistically determinable path or destination. The waste repository includes the contributions of the aerosols that are comprised of thermodynamic materials in the aerosol waste locations. However, the principal purpose of the waste repository is to track coolant that would normally be directed to a pool but is lost from the problem because a pool is not defined. The energy accounting for waste locations is similar to that of any other repository, except that there is no energy outflow or thermodynamic state information, and the repository energy is set equal to the sum of the influx and source enthalpies.

Entities that are not considered to be internal repositories in the present accounting scheme include the following:

1. External repositories, which are discussed above in Section A.2. An example of an external repository is the lower cell configuration representing CORCON.

2. Engineered systems, which in CONTAIN play the role of interfaces between repositories but are not considered repositories in themselves. For example, a containment spray is considered a model for determining the mass and enthalpy to be removed from a cell atmosphere and directed to a recipient pool. In the mass and energy accounting, injected spray water is treated as a two-step process involving injection into a cell atmosphere and transfer of effluent to a recipient pool. Since the suspended spray drops are not considered to be a repository, it is necessary to treat the effects of the spray drops, integrated over the drop fall, as if they occurred during the timestep in which a set of spray drops is injected. The spray effluent which forms is also transferred to the recipient pool in the same timestep. The liquid mass and energy contributed by an engineered systems reservoir, such as a containment spray tank or the ice in an ice condenser, is treated as an external source in the repository to which the liquid mass and energy are introduced.

3. The pool scrubbing models for injected gas/aerosol mixtures are treated in a manner similar to that for engineered systems: they determine the partitioning of heat and mass between the atmosphere and pool but the gas and associated aerosol inventory in transit within a pool at any one time is not considered to be a repository.

Prior to defining the audit energies, it is convenient to define the specific internal energy and enthalpy functions used for the CONTAIN thermodynamic materials with respect to the energy accounting. The specific energy is defined as

$$
\begin{aligned}
& u_{k}\left(T, P_{k}\right)=\text { specific internal energy of material } \mathrm{k} \\
& =h_{k}(T)-P_{k} / \rho_{k}\left(T, P_{k}\right)=u_{k}(T) \quad \text { (for gases and water vapor with the ideal } \\
& \text { equation of state) } \\
& =h_{k}\left(T, P_{k}\right)-P_{k} / \rho_{k}\left(T, P_{k}\right) \\
& \text { (for water vapor with the non-ideal equation }
\end{aligned}
$$




$$
\begin{aligned}
& =\mathrm{h}_{\mathrm{k}}(\mathrm{T}) \\
& =\mathrm{u}_{\mathrm{l}}(\mathrm{T})
\end{aligned}
$$

(for non-coolant liquids, solids, or liquid water with the ideal equation of state)

(for liquid water with the non-ideal equation of state)

where $h_{k}(T)$ is the specific enthalpy function stored in CONTAIN or defined by the user and $u_{f}(T)$ for liquid water with the non-ideal equation of state is the saturated specific energy. Also $\rho_{k}\left(T, P_{k}\right)$ is the density of material $k, T$ is the temperature, and $P_{k}$ is either the partial pressure for a gas, or the total pressure for a liquid or solid. It should be noted that all liquids and solids in CONTAIN are presently treated as incompressible; that is, there is no explicit dependence of the density on pressure. The specific enthalpy is similarly defined as

$$
\begin{aligned}
& h_{k}\left(T, P_{k}\right)=\text { specific enthalpy of material } \mathrm{k} \\
& =\mathrm{h}_{\mathrm{k}}(\mathrm{T}) \\
& =h_{k}\left(T, P_{k}\right) \\
& =h_{k}(T) \\
& =h_{\ell}(T)+P_{\ell} / \rho_{\ell}(T) \\
& =\mathrm{u}_{\ell}(\mathrm{T})+\mathrm{P}_{\ell} / \rho_{\ell}(\mathrm{T})
\end{aligned}
$$

It should be noted that the pressure term in the enthalpy for liquid water is required to properly account for work done on and by the liquid water. Strictly speaking, for liquid water, it is incorrect to use the enthalpy function $h_{\ell}(T)$ as the energy in the ideal equation of state, and it should be replaced by the specific energy function for the non-ideal equation of state. Under containment conditions, the difference between the two is small. The interchangeability between enthalpy and energy assumed in the above expressions for non-coolant liquids and solids is in general a good approximation and consistent with the fact that work done on or by these materials is neglected in the code.

The audit mass and energy contributions reported in the code output are defined in the following discussion. All of the audit energies are total energies including gravitational potential energies. Although only the audit energy is explicitly defined below, the reported mass contributions are organized in the same manner as the energy.

Prior to discussing the audit energies, the reference elevations used for the potential energy terms will be discussed. The reference elevation for computing gravitational potential energy for an upper cell repository, such as the atmosphere, is always the gas center of elevation, which depends on the coolant pool depth. The upper cell quantities controlled by this elevation include (1) liquid films on structures, (2) trapped debris residing on structures, (3) suspended debris in the atmosphere, (4) gases outgassed from concrete structures, and (5) gases, aerosols, and homogeneously dispersed liquids and solids. The nodes of a heat transfer structure are treated somewhat differently from the above because they can in principal be submerged below the coolant pool surface. The reference elevation for structures is therefore taken to be the cell center of elevation. 
A number of reference elevations are also used for the lower cell repositories in a given cell, depending on the material and whether a transfer is involved. With the exception of liquid coolant and gases and aerosols directed to and from the coolant pool, the reference elevation for computing the gravitational potential energy for a lower cell repository is the cell-bottom elevation. For transfers of liquid coolant to and from the pool, the reference elevation is taken to be the pool surface elevation, computed on the basis of the cell-bottom elevation, the pool cross-sectional area, and the pool coolant density. For purposes of calculating the repository potential energy of the pool coolant, the reference elevation is the pool-center elevation. With respect to gases and aerosols introduced into the pool through flow path or the boiling water reactor safety relief valve or suppression vent models, the reference elevation for the point of introduction is given by the injection height above cell bottom. With respect to gases and aerosols introduced into the pool through core-concrete interactions modeled by CORCON, the reference elevation for the point of introduction is the cellbottom elevation. The reference elevation for the point of departure from the pool of gases and aerosols is the pool surface elevation defined above. For all other materials in the coolant pool and for all materials in all other lower cell layers, the reference elevation is the cell-bottom elevation. It should be noted that the latter elevation is fixed and not allowed to change in conjunction with concrete or intermediate layer inventory changes in the course of a problem.

Repository pressures are also needed to define the repository energy and inter-repository energy transfers and are defined in the following way. For repositories adjacent to the gas space, or free volume, in a cell, the gas pressure is taken to be the gas center-of-volume pressure, without adjustment for the variation in gas pressure in a cell due to gas gravitational heads. Such an assumption affects processes such as the intercell flow of liquid between pools. Note, however, that in this case, the pressure is adjusted for the liquid heads present. The neglect of gas pressure variation within a cell should affect only slightly the partitioning of liquid between pools in different cells, or the partitioning of energy between the atmosphere and pool.

The initial energy $E_{0, i}$ is the total energy for repository $i$ at the start of the calculation

$$
E_{0, i}=\sum_{k=1}^{N_{\text {mat }}} m_{i, k}^{0}\left(u_{k}\left(T_{i}^{0}, P_{i, k}^{0}\right)+g H_{i, k}^{0}\right)
$$

where $N_{m a t}$ is the number of thermodynamic materials in the problem; $m_{i, k}^{0}$ is the initial mass of thermodynamic material $k$ in repository $i ; T_{i}^{0}$ is the initial temperature of repository $i ; P_{i, k}^{0}$ is the initial pressure of material $k$ in repository $i ; g$ is the acceleration due to gravity; and $\mathrm{H}_{\mathrm{i}, \mathrm{k}}^{0}$ is the initial repository-average reference elevation of material $\mathrm{k}$ in repository $\mathrm{i}$. As discussed above, for liquid water in a lower cell pool, this elevation is the pool-center elevation. All other average reference elevations are either the gas-center-of-volume (gas-center) elevation, cell-center elevation, or cellbottom elevation, depending on the repository.

The external source energy $E_{e, j}$ is defined as the sum of the energies due to external source tables, the coupling to external repositories, and heat conduction boundary conditions at external boundaries: 


$$
E_{e, i}=\int_{t_{0}}^{t}\left(q_{e, i}+\sum_{n=1}^{N_{e x t i}} W_{e, i, n}\left(h_{e, i, n}+g H_{i, n}^{\prime}\right)\right) d t^{\prime}
$$

where $\mathrm{N}_{\text {extij }}$ is the number of external material sources and individual material flows through external interfaces; $t$ is the problem time; $q_{e, i}$ is the net heat flow rate from external sources, repositories, and boundary conditions into repository $\mathrm{i} ; \mathrm{W}_{\mathrm{e}, \mathrm{n}, \mathrm{n}}$ is the mass flow rate for external flow $\mathrm{n}$ into repository $\mathrm{i} ; \mathrm{h}_{\mathrm{e}, \mathrm{i}, \mathrm{n}}$ is the specific enthalpy associated with the material in external flow $\mathrm{n}$, as discussed below; and $\mathrm{H}_{\mathrm{i}, \mathrm{n}}^{\prime}$ is the reference elevation for external transfers of the material in external flow $n$ to repository i. For liquid coolant directed to or taken from the pool, this elevation is the pool surface elevation. For gases and aerosols vented into the pool, it corresponds to either the "elevnt" height above cell bottom for the boiling water reactor vent models or the cell-bottom elevation for contributions from CORCON. (It should be noted that the reference elevation for departure of gases and aerosols from the pool is at the pool surface. However, since negative gas and aerosol sources to the pool are not supported, there should be no ambiguity about the point of introduction and departure of such materials.) All other reference elevations for external transfers are either the gascenter elevation or cell-bottom elevation depending on the repository and do not depend on the material involved.

The manner in which $h_{\text {e, in }}$ is defined depends on the thermodynamic information (i.e., the enthalpies or temperatures and pressures) available about the external process in question. In some cases, a specific enthalpy is given, as in a source table, or the temperature and pressure required to define the enthalpy are known, such as at the interface with an external repository. However, it should be noted that in other cases, such as for aerosol source tables and for specified-temperature source tables for liquid water or steam, the information required to define the audit energies is not readily available. The approach taken here in the last two cases is to use nominal values for the missing information. It should be noted that the contributions in question to the energy are minor for the aerosol loadings and pressures for which the present CONTAIN thermodynamic state calculations are valid. Nominal values, which would at present be used only to define audit energies, should suffice until improvements in the source tables and in the state calculations allow a consistent treatment of aerosol enthalpies and pressure dependencies in the water equation of state.

For all source tables for thermodynamic materials, except for ones involving aerosols or liquid water, the specific enthalpy is specified by the table:

$$
\begin{aligned}
h_{\mathrm{e}, \mathrm{i}, \mathrm{n}} & =\mathrm{h}_{\mathrm{k}(\mathrm{n})}\left(\mathrm{T}_{\mathrm{i}, \mathrm{n}}\right) \text { (for temperature tables, } \mathrm{k}(\mathrm{n}) \neq \text { liquid water or steam) } \\
& =\mathrm{h}_{\mathrm{i}, \mathrm{n}}(\text { for enthalpy tables, } \mathrm{k}(\mathrm{n}) \neq \text { liquid water or steam) }
\end{aligned}
$$

where $\mathrm{k}(\mathrm{n})$ is the material index associated with external flow $\mathbf{n}$ (specified here through a source table), $T_{i, n}$ is the source table temperature, and $h_{i, n}$ is the source table specific enthalpy.

Similarly, for nonaerosol source tables of liquid water or steam, $\mathrm{h}_{\mathrm{e}, \mathrm{i}, \mathrm{n}}$ is defined as

$$
\begin{aligned}
\mathrm{h}_{\mathrm{e}, \mathrm{i}, \mathrm{n}}=\mathrm{h}_{\mathrm{k}(\mathrm{n})}\left(\mathrm{T}_{\mathrm{i}, \mathrm{p}} \mathrm{P}_{\mathrm{i}}\right) \quad & \text { (for temperature tables, } \mathrm{k}(\mathrm{n}) \\
& =\text { liquid water or steam) }
\end{aligned}
$$




$$
\begin{array}{r}
=h_{i, \mathrm{n}} \quad \text { (for enthalpy tables, } \mathrm{k}(\mathrm{n}) \\
=\text { liquid water or steam) }
\end{array}
$$

It should be noted that the repository pressure $\mathrm{P}_{\mathrm{i}}$ used in this equation is a nominal pressure because the actual source pressure is not known. The repository pressure used for the purposes of energy accounting is taken for simplicity to be the gas pressure at the gas-center elevation, for all repositories in a cell. Contrary to what one might expect, this nominal pressure is not adjusted for the variation in pressure from gas and liquid heads within a cell, since this adjustment would have a small effect. This choice for the nominal pressure, coupled with the effect of the work done by the atmosphere on the pool in the pool energy, leads to substantial cancellations of the pressure terms in the pool state calculations. (The cancellations are related to the fact that the pool state should be affected by the pressure terms only with respect to viscous losses and the volume by which the pool is expanded or compressed. Because the pool is treated as incompressible, the effects of the pressure terms on the pool state should reflect only viscous losses, which vanish in the limit that the source pressure is equal to the cell pressure.)

Enthalpies for nonaerosol materials from external repositories, such as the gas influxes from the CORCON model, are defined on the basis of temperature and pressure matching across the interface:

$$
\mathrm{h}_{\mathrm{e}, \mathrm{i}, \mathrm{n}}=\mathrm{h}_{\mathrm{k}(\mathrm{n})}\left(\mathrm{T}_{\mathrm{i}, \mathrm{n}} \mathrm{P}_{\mathrm{i}, \mathrm{n}, \mathrm{k}(\mathrm{n})}\right) \quad \begin{aligned}
& \text { (for nonaerosol materials from } \\
& \text { external repositories) }
\end{aligned}
$$

where $T_{i, n}$ here is the matching temperature and $P_{i, n, k(n)}$ is the matching pressure.

For all aerosol external fluxes, $h_{\mathrm{e}, \mathrm{in}}$ is nominally defined at the temperature and pressure of the repository into which the aerosols are introduced. That is,

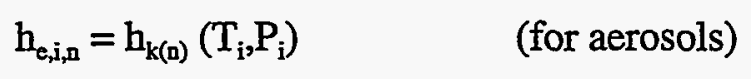

where $T_{i}$ is the repository temperature and $P_{i}$ is the repository pressure as defined above.

The chemical reaction and fission product decay energy $E_{\mathrm{r}, \mathrm{i}}$ is the sum of the integrated rate at which heats of reaction are generated from chemical reactions and integrated decay heating rates.

$$
E_{r, i}=\int_{t_{0}}^{t}\left(q_{c h e m, i}+q_{F P, i}\right) d t^{\prime}
$$

where $q_{\text {chem, }}$ is the rate of generation of heats of reaction, referenced to $273.15 \mathrm{~K}$, in repository $\mathrm{i}$; and $\mathrm{q}_{\mathrm{F}, \mathrm{i}}$ is the fission product decay heating rate in repository $i$.

Chemical reaction heats include those from hydrogen burns (including deflagrations, diffusion flames, and bulk recombination), debris droplet chemistry, and concrete outgassing. Note that in the last case only the heats of reaction for release of bound water and $\mathrm{CO}_{2}$ are included. Evaporable water, even with a user-specified heat of evaporation, is treated as usual through an appropriate offset 
in the liquid and vapor enthalpies. The fission product decay heating includes radionuclides hosted to the ith repository and the ANSI-standard decay heat model, if applicable.

The net energy influx $E_{\mathrm{f}, \mathrm{i}}$ includes heat fluxes, potential energies, and the enthalpy of thermodynamic materials (including aerosols) flowing into a given repository from other internal repositories.

$$
E_{f, i}=\int_{t_{0}}^{t}\left(q_{f, i}+\sum_{m=1}^{N_{\text {inti }}} W_{f, i, m}\left(h_{f, i, m}+g H_{u, m}^{\prime \prime}\right)\right) d t^{\prime}
$$

where $\mathrm{N}_{\mathrm{inti}}$ is the number of individual material flows through internal interfaces into or out of repository $\mathrm{i} ; \mathrm{q}_{\mathrm{f}, \mathrm{i}}$ is the net heat flow rate from internal repositories into repository $\mathrm{i} ; \mathrm{W}_{\mathrm{f}, \mathrm{i}, \mathrm{m}}$ is the mass flow rate of internal flow $m$ into repository $i$ (can be negative to represent outflow); and $h_{\mathrm{f}, \mathrm{m}}$ equals $h_{k(m)}\left(T_{v}, P_{u, k(m)}\right)$, the specific enthalpy associated with the material $k(m)$ in internal flow $m$. This is defined in terms of the donor or upstream repository temperature $T_{u}$ and pressure $P_{u, k(m)}$. As discussed above, the latter is defined on the basis of cell-center gas pressures without adjustment for gas head variations within a cell. $\mathrm{H}_{\mathrm{u}, \mathrm{m}}^{\prime \prime}$ is the reference elevation for internal transfers of the material in internal flow $\mathrm{m}$ to repository $\mathrm{i}$. This elevation is based on the upstream repository. As discussed above, for liquid coolant taken from a pool and for gases and aerosols exiting a pool, this elevation is the pool surface elevation. All other reference elevations for internal transfers are either a cellcenter or cell-bottom elevation and do not depend on the material involved.

Note that the bookkeeping for $\mathrm{E}_{\mathrm{f}}$ in some cases is done for convenience through a two-step transfer. For example, direct thermal radiation between structures or from the lower cell to structures is treated as occurring in a two-step fashion, from surface-to-atmosphere and atmosphere-to-surface, even when the atmosphere is transparent to radiation. Also, the transport of gases and aerosols from a lower cell is treated as if they are first injected into the coolant pool repository (if defined) and then transferred to the atmosphere, even if the gases and aerosols are not actually scrubbed.

The committed energy $E_{c, i}$ can consist of any of the $E_{e}, E_{i}$, or $E_{f}$ types of energy. It is the audit energy that corresponds to energy committed to the $i$ th repository in an interface array but not yet reflected in the calculated thermodynamic state. (The audit committed energy is not necessarily the same as the interface array energy, for reasons discussed above.) The committed energy term is required because the sequential processing method used to advance CONTAIN models and repositories over a system timestep in some cases allows mass and energy to remain in interface arrays at the end of a system timestep. For example, since cells are processed in numerical order, committed mass and energy could arise from processes in higher numbered cells that direct coolant mass and energy into the pool of a lower numbered cell. Committed mass and energy could also arise from aerosol deposition, since the deposited water aerosols are not incorporated into the recipient structure film or pool until the next timestep. The accounting scheme assumes that any committed mass and energy are processed in the next system timestep.

The present energy $E_{p, i}$ is the current total energy for repository $i$. It is defined in a manner similar to the initial energy in Equation (A-3), except that current timestep values are used instead of initial values for masses, temperatures, pressures, and reference elevations. 
The energy balance condition that would hold if energy were perfectly conserved is

$$
E_{p, i}=E_{0, i}+E_{c, i}+E_{r, i}+E_{f, i}-E_{c, i}-E_{w, i}
$$

where $\mathrm{E}_{\mathrm{w}}$ is the work done by the system against simplifying geometric constraints, as discussed below.

$\mathrm{E}_{\mathrm{w}}$ is related to the assumptions made about the system geometry to simplify repository state calculations. For example, the volume of water films on structures is assumed to be negligible compared to the gas volume. In general, this is a good approximation, and therefore film-gas volume displacement work is not explicitly considered in the code. However, in a general formulation of energy conservation accounting, it is desirable to have a well-defined prescription for characterizing the effects of gas-film displacement, as well as a number of other similar energy effects, without explicitly evaluating the energy terms involved. One way to do this is to impose geometric constraints on the system so that the neglected energy terms vanish. In the case of film-gas displacement, the geometric constraint is to define the system volume so that the gas volume is not altered by the film volume. It may seem that this approach simply shifts the problem from one part of the energy accounting to another part, since the presence of such constraints means that in general work is done by the system against the constraints, and this work in principle should be evaluated as part of the energy accounting. In addition, the constrained system may not correspond precisely to the physical one. The point is that the work against the geometric constraints given below is typically negligible, but both the magnitude of the constraint work and the effect of removing such constraints are easily estimated, if necessary, by the user. This approach is preferable to burdening the code with evaluating a large number of generally negligible terms in the energy.

The geometric constraints are as follows:

1. The sum of the gas and pool coolant volume in a cell is taken to be constant for a given problem. The gas volume consists of the free volume in a cell not taken up by suspended materials such as aerosols, homogeneously dispersed non-coolant liquids or solids, core debris particles, liquid films on structures, trapped debris considered to reside on structures, or the structures themselves. The gas volume does include homogeneously dispersed liquid coolant. The pool coolant volume is that displaced by only the liquid water in the lower cell pool and does not include materials suspended in the pool, such as solid materials, noncoolant aerosols, or fission products. It should be noted that coolant aerosols deposited in the pool are automatically transferred to the pool coolant inventory and thus do contribute to the pool volume. Also, other lower cell layers such as intermediate or concrete layers are àssumed not to displace the pool coolant or the atmosphere.

2. The gas and pool coolant volume, as defined above, is assumed to fill the cell. The gas, furthermore, is assumed to lie above the pool free surface. This assumption and the filling assumption define a center of volume for the gas. Other repositories, such as the debris and aerosol fields, are constrained to be on the average at their reference elevations. For suspended debris, aerosols, and structure films, this elevation is that of the gas center of volume. For structures, it is the cell center of volume. For non-coolant materials in the pool and lower cell layers other than the pool, it is the cell-bottom elevation. 


\section{APPENDIX B \\ ALTERNATE INPUT FORMATS AND UPWARD COMPATIBILITY}

As the CONTAIN code has evolved through several versions, an attempt has been made to keep the structure of the input the same. In some cases, however, input revisions were made. The old input options have been maintained in most cases, so that old input files are still upward compatible. However, new features are not in general added to the obsolete input. Therefore, any change in an input block to take advantage of a new feature will probably require changing over to the new format. In some cases, however, upward compatibility could not be maintained. In such cases the input is still read but a diagnostic is given indicating that a non-upward compatible change has been made. This appendix describes all such alternative formats for input and as needed refers the reader to appropriate sections, tables, figures, and equations in the CONTAIN 2.0 Code Manual.

\section{B.1 Alternative Global Input Formats}

\section{B.1.1 Alternative Global CONTROL Input Block Format}

CONTROL number (ncells ntitl ntzone nfce nchain nsectn nac nhm numtbg maxtbg)
number
the number of global control values to follow.
cells
number of cells.
ntitl
number of title lines (maximum of 80 characters/line).
ntzone
number of time zones.
nfce
number of fission product chain elements.
nchain
number of fission product chains.
nsectn
number of aerosol particle size classes ( 20 recommended).
nac
number of aerosol components.
nhm
number of additional fission product host materials (besides the atmosphere gas, all aerosols, floors, walls, and roofs).
numtbg
number of times a global table is used.
maxtbg
maximum number of entries used in any global table option.

If any value is to be specified, all values preceding it must also be specified. For example, if "nac" is the last value to be specified, then "number" $=7$ and seven values are specified, corresponding to 
the first seven variables in the control block; the default value of 0 will be automatically used for "nhm," "numtbg," and "maxtbg." The default value is 0 for all of the above control parameters.

\section{B.1.2 Alternative Fission Product Names Keyword}

The keyword FISSION can be used in place of the keyword FP-NAMES in the global MATERIAL block. All else remains as described in Section 14.2.1. Thus, the input format for fission product names would appear as follows:

FISSION (fname)

fname

the names of user-defined fission products. See Section 14.2.1.

\section{B.1.3 Alternative FLOWS Input Block Options}

The following input options may be specified in the FLOWS input block.

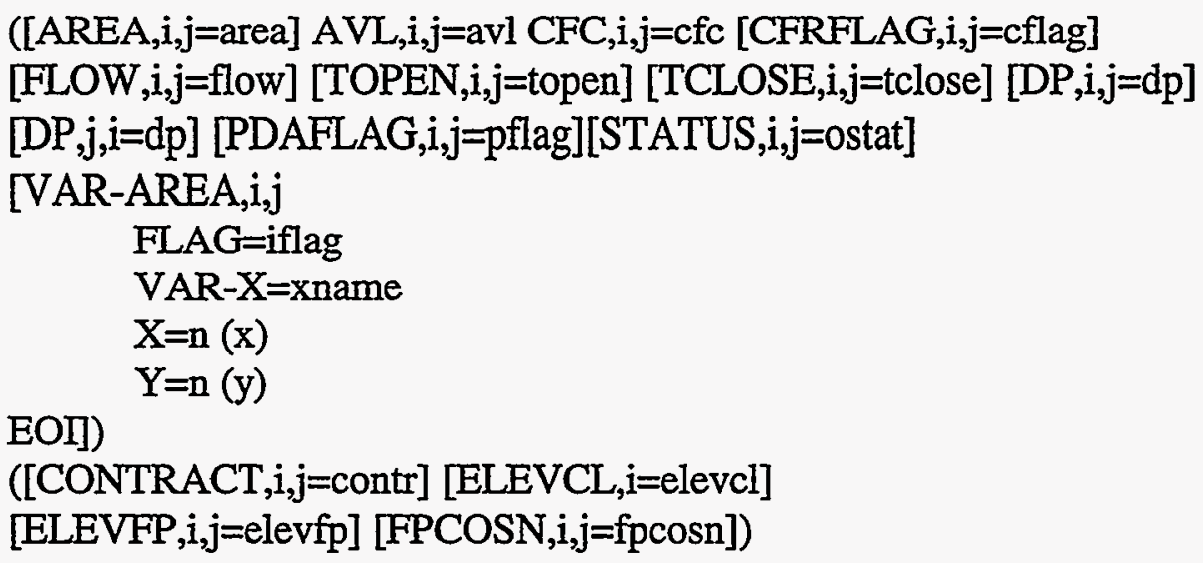

These keywords determine the characteristics of the regular flow paths, which can be only of type GAS. For these flow paths, the gas-pool equilibration lengths, discussed in Section 4.4.7, are set to $0.01 \mathrm{~m}$ and cannot be changed. Also, aerosols and fission products in flow scrubbed by a downstream pool are completely removed and placed in that pool. The keywords immediately below should all be followed by cell indices $i$ and $j$ representing the cells connected by the flow path and then by a numerical value for the variable represented by the keyword. Unless otherwise noted, only one permutation of the indices $i$ and $j$ need be specified. (Note that in the input, a comma is simply a field separator just like a blank, parenthesis, or equal sign.) Only the AVL keyword and the CFC keyword are always required for each regular flow path. If not explicitly stated, the quantities discussed in the descriptor blocks below refer to the numerical value to be given after the cell indices. 
AREA,i,j

$=$ area

AVL,i,j

$=\mathrm{avl}$

$\mathrm{CFC}, \mathbf{i}, \mathbf{j}$

$=\mathrm{cfc}$

CFRFLAG, $i, j$

$=$ cflag

FLOW,i,j

= flow

TOPEN,i,j

$=$ topen

TCLOSE,i,j

$=$ tclose

$\mathrm{DP}, \mathbf{i}, \mathbf{j}$

$=\mathrm{dp}$

PDAFLAG, $\mathbf{i}, \mathbf{j}$

$=$ pflag the (constant) cross-sectional area of flow path when open. $\left(\mathrm{m}^{2}\right)$

the ratio of effective flow path area to inertial length. The value corresponds to $A_{i j} / L_{i j}$ in the conservation of momentum equation in Table 4-2. (m)

the flow loss coefficient. This coefficient includes entrance, exit, and other discontinuity losses as well as frictional losses. The value corresponds to $C_{F C}$ in the conservation of momentum equation given in Table 4-2. (dimensionless)

the flag that specifies that the "flow" value discussed below is a constant rate, as opposed to the initial flow rate. Specify 1 if the units of the specified "flow" value are $\mathrm{kg} / \mathrm{s}$, or -1 if the units are $\mathrm{m}^{3} / \mathrm{s}$. If CFRFLAG is not specified, the value specified for "flow" is interpreted as an initial flow rate rather than a constant flow rate.

a constant or initial flow rate. (CFRFLAG, discussed above, is a flag that indicates that the value specified for "flow" is a constant flow rate. If CFRFLAG is not specified, then the value for "flow" is taken to be an initial flow rate.) The value for "flow" should be positive if the flow is in the direction from cell $i$ to cell $j$. Note $\mathrm{kg} / \mathrm{s}$ or $\mathrm{m}^{3} / \mathrm{s}$ units are allowed if a constant flow rate is specified (see CFRFLAG above); only $\mathrm{kg} / \mathrm{s}$ is allowed if an initial flow rate is specified. Default $=0$. $\left(\mathrm{kg} / \mathrm{s}\right.$ or $\left.\mathrm{m}^{3} / \mathrm{s}\right)$

the time to open the flow path. Default $=-10^{30}$. (s)

the time to close the flow path. Default $=10^{30}$. (s)

the positive definite pressure difference to open the flow path. The pressure difference across the flow path is compared to the value of the appropriate "dp." If the pressure difference exceeds this value and the flow path is closed, the flow path is opened. The pressure difference is checked only until "dp" is exceeded the first time, even if the flow path is open at that point. Once the flow path is open, it remains open even if the actual pressure difference drops below the opening value. DP, $i, j$ determines the opening pressure difference when the pressure in cell $i$ is greater than that in cell $j$. The value for $\mathrm{DP}, \mathrm{i}, \mathrm{j}$ does not have to be same as that for $\mathrm{DP}, \mathrm{j}, \mathrm{i}$. It is assumed that the values are equal unless both DP,i,j and DP,j,i are specified. $(\mathrm{Pa})$

a flag used with the area-versus-pressure option within the VAR-AREA table option. A value of 1 implies a reversible pressure-dependent area. A value 
STATUS, $, \mathbf{i}, \mathbf{j}$ = ostat of -1 implies an irreversible pressure-dependent area that can only stay the same or increase in size. Default $=1$.

the initial state of the flow path. If "ostat" = OPEN, the flow path will be initially open; if "ostat" = CLOSED, it will be initially closed. By default, it will be initially closed if TOPEN is specified without DP or TCLOSE or if DP is specified without TOPEN or TCLOSE. Otherwise, the flow path by default will be initially open. Note that if more than one of the key words $D P, T O P E N$, or TCLOSE is specified, the initial state of the flow path may not be the same in versions prior to CONTAIN 1.1 as it is in CONTAIN 1.1 and higher versions. (A diagnostic will be written to the error file in such cases.) Note also that the STATUS keyword may not be used on a restart. A change of state may, however, be initiated through the TOPEN, TCLOSE, or DP keywords.

A flow path to be used in the calculation must be specified with a positive nonzero value of "avl" for all flow options; otherwise, that flow path will be ignored. A flow path may be either open or closed. If a flow path is open, the flow is calculated according to the cell pressure differences or set to a constant user-specified rate, set by the "flow" value and "cflag." "Avl" must be the area/length ratio for the flow path in the default and IMPLICIT flow options. Note that an area specification is necessary for aerosol settling in the case of a user-specified constant flow rate even though it is not necessary for calculating the gas flow rate. If the flow path is closed, no gas flow or aerosol settling through the flow path occurs.

The logical state (open or closed) of a flow path is controlled by DP, TOPEN, or TCLOSE. By default, a flow path is open. The state of the flow path is initially closed if DP is specified, or if TOPEN is specified without TCLOSE, or if both TOPEN and TCLOSE are specified with the value for "topen" less than that for "tclose." Once a flow path is open, the flow area can be controlled through the VAR-AREA option discussed below.

The VAR-AREA keyword allows the user to specify a global table for the flow area. The table keywords FLAG, VAR-Y, X, and $Y$ are discussed in Section 14.4.2, as are the values associated with FLAG. Discussion of other keywords and values follows. These tables are stored at the global level in CONTAIN. Consequently the number of global tables "numtbg" and maximum global table size "maxtbg" specified in the global CONTROL block should take any tables specified in the following option into account.

VAR-AREA,i,j VAR-AREA initiates the input of a global table for specifying the flow area as a function of time or pressure difference. VAR-AREA should be followed by the indices I and $j$, which refer to the cells connected by the regular flow path which is to be governed by the table, and then by other table keywords.

VAR-X

$=\mathrm{xname}$ the name of the independent variable in the table. The value "xname" can either be specified as TIME, which indicates that the " $x$ " independent variable corresponds to time, or as DELTA-P, which indicates that the " $x$ " variable corresponds to a pressure difference. Note that for the DELTA-P option, the 
PDAFLAG keyword discussed above determines whether the area corresponding to the dependent variable " $y$ " is reversible or irreversible.

n

$\mathrm{X}$

y

the number of points in the table.

the independent variable in the table. It corresponds to time if $V A R-X=T I M E$ is specified or to pressure difference if VAR-X=DELTA-P is specified. The " $x$ " value for the DELTA-P table corresponds to $\triangle P_{i j}$ in the conservation of momentum equation, Table 4-2. The values must be monotonically increasing. Specify " $n$ " values. Note that outside of the range of the independent variable of the table, the table is extrapolated. A constant value equal to the closest endpoint value is used in the extrapolation. ( $\mathrm{s}$ or $\mathrm{Pa})$

the dependent variable in the table, which corresponds to area. Specify " $n$ " values. $\left(\mathrm{m}^{2}\right)$

An example of table input follows:

$\begin{array}{cl}\text { VAR-AREA,1,2 } & \text { \&\& table for flow between cells } 1 \text { and } 2 \\ \text { FLAG=2 } & \text { \&\& linear interpolation } \\ \text { VAR-X=DELTA-P } & \text { \&\& pressure difference is independent } \\ & \text { \&\& variable } \\ \mathrm{X}=3-1 . \mathrm{E} 40.1 . \mathrm{E} 4 & \text { \&\& three values of pressure difference } \\ \mathrm{Y}=30.0 .10 . & \text { \&\& three values of area } \\ \text { EOI } & \text { \&\& table terminator }\end{array}$

CONTRACT, $\mathrm{i}, \mathrm{j}$ the area ratio for the vena contracta that may develop downstream of the flow $=$ contr path. The value between zero and one specified for "contr" is the ratio of the cross-sectional area of the vena contracta to the geometric cross-sectional area of the flow path. "Contr" is used only for choked flow. Default $=1$.

\section{ELEVCL, $;$ \\ $=$ elevcl}

the initial absolute elevation of the center of volume of the gas in cell I. This input is used only if CELLHIST input is not given in the cell GEOMETRY block. It is used to calculate the fixed elevations of the entire cell from the gas and pool volumes and gas "height" input. The CELLHIST input is recommended for all cells with pools or pool flow paths. The gas center-ofvolume elevation is adjusted as the pool depth changes. See Figure 4-3. Default $=0$. $(\mathrm{m})$

the absolute elevation of one end of the regular flow path connecting cells $i$ and $j$. The value "elevfp" given after ELEVFP,, $\mathrm{j} j$ defines the end of the path between $\mathrm{i}$ and $\mathrm{j}$ that is attached to cell $\mathrm{j}$. In general both ELEVFP, $i, j$ and ELEVFP,j, i need to be defined. The default value of "elevfp," as for other gas paths, is the top of the cell to which the end is attached $\left(\mathrm{H}_{\mathrm{t}, \mathrm{i}}\right.$ as shown in Figure 4-4). The minimum value, as with other gas paths, is the elevation of the bottom of the cell. The user should consider the pool-gas hierarchy 
discussed in Section 4.2 prior to specifying the flow path elevation and in particular should note that a flow path connected to the top of a cell is treated differently from side-connected or bottom-connected paths. In general, the robustness of the code will be improved if the gas head space associated with the onset of gas or pool flow in a side-connected path is fairly substantial (on the order of $20 \%$ of the cell volume). Side-connected paths attached very close to the top of the cell are not recommended. To avoid accidental specification of such a side connection when a top connection is intended, it is recommended that the user rely on the CELLHIST input in the cell GEOMETRY block to specify cell elevations directly, rather than rely on values that would otherwise be calculated by the code from the initial cell gas center-of-volume elevation, the cell gas "height," and the initial gas and pool volumes. (m)

FPCOSN, $, \mathrm{i}, \mathrm{j}$ the cosine of the angle of the flow path axis with respect to the vertical $=\mathrm{fpcosn}$ direction. The angle is measured between the upward direction and the flow path axis in the direction from $i$ to $j$. The value is used to calculate aerosol settling through the flow path. The value for "fpcosn" should be 1 if the end of the flow path at cell $j$ is directly above that at cell $i$, and -1 if the reverse is true. Only the component of aerosol settling velocities parallel to the flow path axis is considered. Default $=0$. (dimensionless)

\section{B.1.4 Alternative Global AEROSOL Keyword Format}

The global AEROSOL keyword may be followed by an optional parameter string.

AEROSOL [newcof diam1 diam2 tgas1 tgas2 pgas1 pgas2]

The parameters have the following definitions:

$\begin{array}{ll}\text { newcof } & \text { flag for calculating aerosol coefficient sets. Default }=1 . \\ \text { diam1 } & \text { aerosol minimum diameter. Default }=1.0 \times 10^{-7} .(\mathrm{m}) \\ \text { diam2 } & \text { aerosol maximum diameter. Default }=1.0 \times 10^{-4} .(\mathrm{m}) \\ \text { tgas1 } & \text { lower interpolation temperature. Default }=273 .(\mathrm{K}) \\ \text { tgas2 } & \text { upper interpolation temperature. Default }=673 .(\mathrm{K}) \\ \text { pgas1 } & \text { lower interpolation pressure. Default }=5.0 \times 10^{4} .(\mathrm{Pa}) \\ \text { pgas2 } & \text { upper interpolation pressure. Default }=8.0 \times 10^{5} .(\mathrm{Pa})\end{array}$

The above parameters are followed by the rest of the global aerosol input block, discussed in Section 14.2.5. If a keyword entry following the above parameters redefines any of the parameters in the 
parameter string, the redefined value will be used. A zero value for a parameter in the string implies the default value for that parameter.

\section{B.1.5 Alternative Global FISSION Input Block Format}

Alternate input options and formats are available for specifying fission product input parameters in the global FISSION input block. First, the decay chain structure and decay power coefficients may be specified with the alternate format shown below at the beginning of the FISSION block. Second, the FPM-CELL blocks may be specified at the global level as shown below. This is true regardless of the format used to specify other fission product input parameters in the FISSION block. If these blocks are placed at the global level then the FPM-CELL keyword must be followed by the cell number for which the following parameters are to apply. Also, global FPM-CELL keyword blocks do not end with an EOI; rather, they end with the next FPM-CELL keyword or the EOI for the entire FISSION block. Third, in place of (or in addition to) the TARGET keyword, RELEASE and ACCEPT may be used as shown below to activate the nontargeted release and acceptance model. It should be noted that this model is entirely different from and less flexible than the targeted release and acceptance model. Also note that when ACCEPT is given, $1+"$ nac" $+3+" n h$ " $^{\text {" values for each }}$ host are expected to follow even though this number in general will not be the proper number of hosts for the cell in CONTAIN 1.1 and later versions (in older versions of CONTAIN, this is the number of hosts in a given cell). The code will automatically reassign the acceptance fractions to the actual CONTAIN hosts in the cell. Fourth, in place of actual structure names, the "hname" following the HOST keyword may be ROOF, WALL, or FLOOR. In this instance, mass will be distributed among structure surfaces of the appropriate type according to surface area. If no surfaces of the specified type exist in the cell, then the mass will be diverted to a waste holding location. Fifth, extra material hosts ("ehnames") are accepted as input; however, any reference to such hosts will be assumed to apply to the DUMMY host, unless the extra material host name matches that of the coolant (H2OL). Any reference to coolant extra hosts are assumed to apply to the pool as opposed to the DUMMY host, if a pool is defined. If extra material hosts are specified, then the keyword NHM must also be specified in the global CONTROL block as follows:

NHM = total number of extra material hosts in all cells. Even though extra materials nhm are no longer hosts in CONTAIN, this parameter must be entered accurately if the old FISSION format is used and extra material hosts are defined in the FISSION block

The alternate FISSION block input format is given below. The format shown below assumes that the FPM-CELL blocks are at the global level.

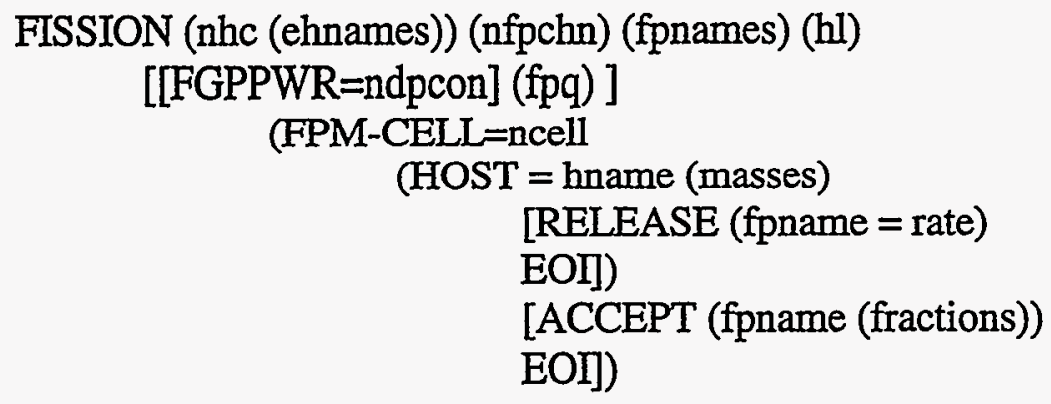


nhe

ehnames

nfpchn

fpnames

hl

FGPPWR

ndpcon

fpq

FPM-CELL

ncell

HOST

hname

masses number of obsolete extra material fission product hosts in each cell. Specify "cells" values, one for each cell. The sum of "nhc" over all cells must equal "nhm" in the global CONTROL block even though the code no longer uses extra material hosts.

names of the extra material hosts. Specify "nhc" names for each value of "nhc" that is nonzero. If "nhc" is zero for a cell, then no names should be entered for that cell. These extra host names must come from the names of the materials listed in the COMPOUND block in order for CONTAIN to properly reassign the extra host references to the DUMMY host.

number of fission products in each chain ("nchain" values must be specified). The sum of "nfpchn" over all chains must be equal to "nfce" in the global CONTROL block. Unlike hosts; fission product chains are common to all cells.

names of fission products in the order of appearance in the decay chains. Specify "nfce" values for all chains.

half-lives corresponding to each of the fission products named above. (s)

initializes input of "ndpcon." If this keyword is not given, then "ndpcon" will have the value of 1 .

number $(\leq 4)$ of decay power coefficients per nuclide. All nuclides must have the same value of "ndpcon" when using this old input format.

the power coefficients $a_{1}, a_{2}, \ldots$ Specify "ndpcon" values for each fission chain element. The definition of the coefficients is given in Equation (8-5). $\left(\mathrm{W} / \mathrm{kg} \mathrm{or} \mathrm{s}^{-1}\right)$

keyword to specify initial fission products masses and constant release rates.

number of the cell to which the masses and release rates apply.

initializes input for specification of initial fission product mass. Note that this keyword must be given if the nontargeted release model is to be used, even if no initial mass specification is desired (zeros should be given in such a case for the masses).

is either a single word or several words that identify a CONTAIN host in the specified cell. The valid host names are described in Section 14.3.1.10.

exactly "nfce" initial fission product masses for the host specified. $(\mathrm{kg})$ 
RELEASE

fpname

rate

ACCEPT

fpname

fractions specifies nontargeted release rates for fission products hosted to the previously specified "hname" host.

one of the names given in the FP-NAMES block. All fission chain elements having this name will have the specified release rate from the "hname" host in the cell.

a constant release rate for the "fpname" fission product chain elements. Note that in the nontargeted release model, the sum of the acceptance rates of the various hosts does not equal this release rate if the host acceptance fractions do not sum to unity. (See the ACCEPT input below.) $\left(\mathrm{s}^{-1}\right)$

initializes input for the host acceptance fractions in the cell. If this keyword is omitted, the acceptance fraction for all hosts will be zero except for that for the cell atmosphere which will be unity.

one of the names given in the FP-NAMES block. All fission chain elements having this name will be accepted by the various hosts in the cell according the fractions described below.

exactly $1+$ "nac" $+3+$ "nhc" values representing the fraction of the released fission product accepted by each host. Note that in the nontargeted release model, mass will not be conserved if these fractions do not sum to unity.

NOTE: If the first quantity following the FISSION keyword is a numerical value (i.e., the first "nhc" value), then the old input format described above will be assumed. Otherwise, the keyword formats are described in Sections 14.2.6 and 14.3.1.10. Mixing of the two formats is not allowed with the exception that the FPM-CELL blocks may be placed at either the global or cell level regardless of the format used for the rest of the FISSION block.

\section{B.2 Alternative Cell-Level Input Formats}

\section{B.2.1 Alternative Cell CONTROL Input Block Format}

CONTROL number (nz nreg nhtm mxslab nsopl nsppl nsoatm nspatm nsospr nspspr nsoaer nspaer nsofp nspfp nstrlw naensy nsensy nchmre nsoeng nspeng jconc jint jpool numtbc maxtbc nraycc nvfpsm)

number number of cell control values to follow.

nz indexing variable; use value of 1 .

nreg indexing variable; use value of 1.

nhtm number of heat transfer structures in the cell. 
mxslab

nsopl

nsppl

nsoatm

nspatm

nsospr

nspspr

nsoaer

nspaer

nsofp

nspfp

nstrlw

naensy

nsensy

nchmre

nsoeng

nspeng

jconc

jint

jpool

numtbc

$\operatorname{maxtbc}$ maximum number of nodes in any heat transfer structure.

number of lower cell source tables.

maximum number of entries in lower cell source tables.

number of external atmosphere sources.

maximum number of entries in atmosphere source tables.

number of external sodium spray fire sources.

maximum number of entries in sodium spray fire source tables.

number of external aerosol sources.

maximum number of entries in aerosol source tables.

number of external fission product sources.

maximum number of entries in fission product source tables.

indexing variable; use value of 0 .

number of engineering systems to be defined in atmosphere.

indexing variable; use value of 0 .

indexing variable, use value of 0 .

number of engineered system sources.

maximum number of entries in engineered system source tables.

designator indicating presence of concrete layer in lower cell. The possible values and their meanings are described in Section 5.3.

number of intermediate layers in lower cell.

designator indicating presence of pool layer in lower cell; 1 if pool layer present, 0 if not present.

number of cell-level tables used for this cell.

maximum number of entries used in any cell-level table within this cell. 
nraycc number of rays used to model the CORCON cavity; allows use of the CORCON option in this cell.

nvfpsm

number of individually tracked VANESA fission products.

If any value is to be specified, all variables preceding it in the above list must also be given. For example, if "nspspr" is the last value to be specified, then "number" $=10$ and ten values are specified, corresponding to the first 10 variables in the control block. The default value of 0 will be automatically set for the remaining variables.

\section{B.2.2 Alternative ATMOS Input Block Format}

This alternative input format specifies the initial atmosphere conditions and atmosphere sources in the upper cell.

ATMOS nma pgas tgas (gas frac)

[SOURCE $=$ nso

(oname $=\mathrm{n}$

[IFLAG=ival] $\mathrm{T}=$ (times) MASS=(masses)

EOI)]

$\{$ TEMP $=$ (temps) or ENTH=(enths)\}

ATMOS keyword to initiate input of atmosphere initial conditions and sources.

nma number of materials initially in the atmosphere. Specify at least one gas or the coolant vapor.

pgas gas pressure or an input option flag. See the discussion below of the options available for the initial conditions. $(\mathrm{Pa})$

tgas $\quad$ gas temperature. $(\mathrm{K})$

The following group of two variables is repeated "nma" times, once for each material initially present in the atmosphere:

gas

frac material name; must be among those specified after either the COMPOUND keyword or the USERDEF keyword in the MATERIAL block.

molar fraction (dimensionless) or material mass. (See the discussion below of the options available for the initial conditions.) ( $\mathrm{kg})$

The keyword SOURCE may be used here to introduce any nonaerosol material declared after the COMPOUND keyword or USERDEF keyword in the MATERIAL block to the upper cell atmosphere. Sources of condensed phases of materials that are not aerosol components will be carried along in the atmosphere gas as homogeneously dispersed material. For a discussion of the keywords following SOURCE, see Section 14.4.1. 
SOURCE keyword to initiate input of source information.

nso

number of source tables to follow.

Three different types of input options for the initial conditions are available to give the user considerable flexibility. The option type depends on the sign of the "pgas" parameter. Only completely dry and/or saturated initial conditions are allowed in the first two options; arbitrary conditions are allowed in the third.

If "pgas" is specified to be positive, then "pgas" is taken to be the initial atmosphere pressure, "gas" should be the name of a gas or the coolant vapor, and "frac" should be the gas or vapor molar fraction. Furthermore, if the coolant vapor, $\mathrm{H} 2 \mathrm{OV}$, is specified as one of the gases, the atmosphere will be assumed to be at the dew point (saturation), and the input value of the coolant vapor molar fraction will be ignored. The coolant vapor fraction will be redefined internally as the value that will bring the atmosphere to the dew point. The other values of "frac" will be rescaled so as to bring the total to one. If the coolant vapor is not specified as one of the gases, the sum of all values of "frac" will be renormalized to one if necessary, and the atmosphere will have the composition given by the renormalized molar fractions. The atmosphere will be completely dry.

If "pgas" is specified to be negative, "gas" should be the name of a gas or the coolant vapor, and "frac" should be the corresponding molar fraction. One of the gases specified must be the coolant vapor, and the corresponding value of "frac" must be positive and less than 1 . If the sum of all the molar fractions is not 1 , the fractions of the gases other than the coolant vapor are rescaled so that the sum of all molar fractions becomes unity. The pressure is calculated as the value that places the atmosphere at the dew point and gives a composition corresponding to the adjusted molar fractions. If no other gases are present, the coolant vapor fraction must be unity. Note that the CONDENSE option models the diffusion of the condensable through the noncondensable gas boundary layer at a structure surface. This process is not defined if noncondensables are not present.

In the two options above, acceptable names for "gas" are $\mathrm{N} 2, \mathrm{O} 2, \mathrm{H} 2, \mathrm{CO} 2, \mathrm{HE}, \mathrm{CO}, \mathrm{AR}, \mathrm{H} 2 \mathrm{OV}$ or the name of a gas defined through the user-defined material option.

If "pgas" is specified to be zero, "gas" may be the name of any nonaerosol or non-fission-product material, and "frac" is taken to be a material mass. The pressure is calculated from equations of state at the temperature "tgas."

\section{B.2.3 Alternative STRUC Input Block Format}

An alternative format for the STRUC input block for heat transfer structures is as follows:

STRUC (name istr ishape nslab ibc tint chrl vufac [bctr] [heit] (x) (names))

The following group of variables is repeated for each structure:

name arbitrary name with eight or fewer characters for the structure.

istr structure type (ROOF, WALL, or FLOOR). 

ishape
structure shape (SLAB, CYLINDER, or SPHERE).
nslab
number of nodes in structure.
$\mathrm{ibc}$
number of the cell adjacent to outer face of structure.
tint
initial temperature of structure. (K)
chrl
characteristic length of structure for condensation model. (m)
vufac
a value which depends on the emissivities of the structure surfaces and the uppermost lower cell layer, and on their geometric relationships. (Must be $\leq 1$; see Equation (10-63))
bctr
outer face boundary temperature. (K)
heit
if the structure is a SLAB, "heit" is the surface area (m); if the structure is a CYLINDER, "heit" is the height; if the structure is a SPHERE, "heit" is ignored and must be omitted. (m)
$\mathrm{x}$
node interface positions relative to the inner face of a SLAB or to the center of curvature of a CYLINDER or a SPHERE. (Specify "nslab" + 1 values, starting at inner face.) (m)
names
material name for each node (specify "nslab" names).

This block provides the characteristics of the structures modeled in the cell. Three structural shapes are allowed: slabs, cylinders, and spheres. Cylinders and spheres are actually half-cylinders and hemispheres whose inner surfaces act as roofs, walls, or floors for aerosol deposition. Thus, to model a whole cylinder or a complete sphere, two structures are required. When "ibc" is a valid cell number ( $1 \leq$ "ibc" $\leq$ "ncells"), "bctr" must not be present. When "ibc" is not a valid cell number, "bctr" is used as the temperature seen by the outer boundary; if "bctr" is zero, an adiabatic (insulated) outer boundary is assumed. An invalid cell number is one that is greater than the maximum number of cells specified for the problem, i.e., it corresponds to a "fictitious" cell. Radiative heat transfer from the lower cell to the outer face is not currently modeled, regardless of the location of the face. Neither condensation nor aerosol deposition is included on the outer face if the face is in another cell.

The above alternate input format may be used in conjunction with the format presented below and in Section 14.3.1.3. However, the format in Section 14.3.1.3 must always follow the alternative format above. 


\section{B.2.4 Alternative STRUC Input Block Keywords}

The following keywords may be used within the STRUC input block for heat transfer structures to define the outer surface boundary condition. These keywords must be given in the input stream corresponding to each relevant structure, but outside of the BCOUTER block.

\section{[IOUTER=iouter $]$ [TOUTER=touter $]$}

IOUTER $=$ iouter

TOUTER $=$ touter number of the cell adjacent to the outer surface of the structure. The number must be that of a cell in the problem.

temperature of the gas adjacent to the outer face. Specify only if the outer surface is not exposed in a cell and if nonadiabatic, fixed temperature boundary conditions on the outer face are desired. Note that a heat transfer coefficient will control the heat transfer between the outermost node and the gas, which will be assumed to be at the temperature specified. (K)

\section{B.2.5 Alternative CONDENSE Input Block Keywords}

The CONDENSE condensation heat transfer input block has a number of obsolete modeling options, which are specified following the CONDENSE keyword (see Section 14.3.1.4). The principal reason for obsolescence is the unfortunate logic created by this input that condensate films and forced convection heat transfer must be associated with condensation heat transfer. There are a number of ways that films can form on structures in the absence of condensation heat transfer, and forced convection is associated with convective heat transfer as well as condensation heat transfer. Equivalent input in the BCINNER and BCOUTER blocks of the heat transfer structure input (see Section 14.3.1.3) supercedes the following input.

\section{[FLMAX=flmax] [FORCED nmtb}

$$
\begin{array}{ll} 
& ([\mathrm{IFLAG}=\mathrm{iflag}] \\
\mathrm{X}=\mathrm{n}(\mathrm{x}) \\
\text { VAR-Y=option } \\
\mathrm{Y}=\mathrm{n}(\mathrm{y}) \\
\text { EOI) } \\
\text { STR-COND nprs (istruc itabl) }
\end{array}
$$

FLMAX

$=$ fimax the maximum condensate film depth. This option is obsolete and will cause an abort; see the keyword MINDEPTH, the minimum depth prior to flow, in the BCINNER and BCOUTER blocks of the heat transfer structure input (see Section 14.3.1.3).

The FORCED keyword activates the forced convection option for selected structures in a cell. This option utilizes a number of cell level tables to specify either the velocity, the Reynolds number, or the forced convection Nusselt number as a function of time. A given table is associated with a given structure through the STR-COND input discussed below. The definitions of the standard table 
keywords FLAG, $X$, and $Y$ are given in Section 14.4.2. The other keywords and values associated with FORCED are defined below.

FORCED keyword to specify that a number of forced convection tables follow.

nmtb

number of tables to be used with the FORCED option. The tables are numbered in the order that they are entered, the first being 1 , the second 2 , and so forth.

$\mathrm{n} \quad$ the number of points in the table.

$\mathrm{x}$

the independent variable of the table, corresponding to time. Specify " $n$ " values in ascending order. Note that outside of the range of times in the table, the degree of forced convection will be assumed to be zero.

VAR-Y

option

y

EOI

STR-COND

nprs

istruc

itabl keyword to specify the type of dependent variable represented by the table.

character variable determining the dependent variable in the table. Specify either VELOCITY, REY-NUM, or NUS-NUM depending on whether velocity, Reynolds number, or forced convection Nusselt number is specified by the table.

the dependent variable of the table. Specify " $n$ " values appropriate for the choice made for "option."

keyword used to terminate each table.

keyword to specify the correspondence between the forced convection tables and the structures. The default forced convection treatment, based on gas flow path velocities, is used for any structure not specified.

the number of pairs of values of "istruc" and "itabl" to follow.

the number of the structure associated with the table "itabl." The structure number is determined by the order of input of the structures in the heat transfer structure input block. Both the inner and outer surfaces will be assigned the forced convection conditions specified in the table, if both lie within the cell; otherwise, only the inner surface will be assigned.

the number of the table containing the forced convection information for structure "istruc."

The following is an example of the STR-COND input:

STR-COND $3(1,1)(2,1)(3,2)$ 
This example implies at least two tables and at least three structures in the cell. In this example,the first table defines the forced convection associated with the first and second structures, while the second table defines the forced convection associated with the third structure.

\section{B.2.6 Alternative Radiation Input Block Format}

The RADIAT keyword and the associated alternative radiation input may be used in place of the keyword RAD-HEAT to activate the radiation model. This alternate input format is capable of activating only the direct gas-structure radiation model. In order to use the net enclosure model, the RAD-HEAT input block must be used.

\section{RADIAT}

BEAML (beaml)

EMSVT (emsvt)

[PBEAML pbml PEMSVT pevt]

EOI [MODAK]

The BEAML and EMSVT keywords are always followed by only "nhtm" values (one for each structure in the cell). The beam length and emissivity of the topmost lower cell layer (usually the pool) are input via the keywords PBEAML and PEMSVT. The beam lengths in this input block are the same as the geometric beam lengths given under the GEOBL keyword in the RAD-HEAT block and should not be confused with the net enclosure inter-surface beam lengths. Also note that when using the RADIAT input, the Cess-Lian correlation is used by default for the gas mixture emissivity calculations. The MODAK keyword is used to override this default. Use of the RADIAT input format is discouraged as new options are not accessible within this format.

\section{B.2.7 Alternative KMX Input Option}

The use of negative value for $\mathrm{KMX}$ in the RAD-HEAT radiation input block overrides the gas mixture emissivity calculation.

$\mathrm{KMX}=-\mathrm{kmx}$

$\mathrm{KMX}$

$=-\mathrm{kmx}$ when "kmx" is entered with an overall negative sign it overrides the calculated gas emissivity. The value specified is the negative of the desired gas mixture emissivity. The negative sign is required since the $\mathrm{KMX}$ keyword followed by a positive number is used as an aerosol density multiplier as described in Section 14.3.1.5. 


\section{APPENDIX C \\ VALIDATION SUMMARY}

\section{C.1 Introduction}

The CONTAIN code has been used extensively in the United States and abroad to perform analyses of nuclear reactor containments and of experiments. CONTAIN is internationally recognized as a standard for performing containment analyses. This recognition stems from the maturity and robustness of the code and its demonstrated ability to be applied to a broad class of problems and containment configurations. CONTAIN has also been extensively validated against experimental data and through analytic and code-to-code comparisons. This appendix summarizes the CONTAIN validation data base.

The major modeling categories in CONTAIN include: intercell flow, hydrogen phenomena, heat and mass transfer processes (radiation, convection, conduction, etc.), aerosol behavior, fission product behavior (decay, heating, and transport), engineered safety features (sprays, fan coolers, and ice condensers), boiling water reactor (BWR) specific models (suppression pool and safety relief valve venting), core-concrete interactions, and direct containment heating (DCH). These major modeling categories can be further divided into numerous separate phenomenological models; a few of which are noted parenthetically above. Taken collectively, these models provide CONTAIN with the capability to analyze a wide variety of light water reactor (LWR) plants and accident scenarios. Through proper user input large-dry, sub-atmospheric, and ice condenser pressurized water reactor (PWR) containments, and BWR suppression pool containments can be modeled with CONTAIN. Recently added models for liquid film and pool tracking have extended the modeling capability of CONTAIN to advanced light water reactors (ALWRs). Experimental facilities, such as LACE, HDR, Surtsey, and other configurations can also be represented; this has proven to be important for performing code validation calculations.

The remainder of this introductory section is devoted to a history of the CONTAIN code, and an introduction to the types of comparisons used to validate the code. Section C.2 discusses the objectives of the code validation program and describes the nature and format of the code validation summaries presented in this appendix. Section C.3 summarizes the code validation and assessment (CV\&A) for individual models. A summary of the code performance in the major modeling areas is given in Section C.4, and conclusions are presented in Section C.5.

\section{C.1.1 History of the CONTAIN Code}

The first official version of CONTAIN, CONTAIN 1.0, was completed, documented, and distributed in 1984. Since that time, numerous modeling improvements and code enhancements have been made to stay abreast of changes in the technical data base for severe accident phenomena. These improvements have resulted in several minor code revisions, CONTAIN 1.01 through CONTAIN 1.06, and one major new version, CONTAIN 1.1. Three code revisions, CONTAIN 1.11 and 1.12 (released together) and CONTAIN 1.2 have occurred since the completion of CONTAIN 1.1. The most recent major release of the code is CONTAIN 2.0. The previous code versions are listed in Table C-1, with a summary of the major improvements and new models contained in each version. 
(Because of their unwieldy nature, the tables and figures referenced in this appendix are all placed at the end.)

\section{C.1.2 Experimental Programs}

Experimental programs are of interest primarily because they have provided the principal means for code validation. Specific experiments that are clearly relevant are those on DCH (Surtsey), molten core-concrete interactions (SURC), aerosol behavior (ABCOVE, LACE), and gas/hydrogen distribution (HDR, NUPEC). Recently emphasis in CONTAIN validation has been placed on comparisons to the LACE aerosol experiments, the HDR gas transport experiments, the Surtsey DCH tests, and some recently performed ALWR experiments conducted by Westinghouse Electric Company and General Electric. Some of the main experimental facilities that have been modeled with CONTAIN are listed in Table C-2.

\section{C.2 Validation and Assessment of CONTAIN}

The purpose of this section is to discuss the objectives of code validation and the format of the code validation summaries presented in this appendix. Validation is used in the present context to mean the capability of the CONTAIN code to accurately represent the physical processes that could occur within a reactor containment. Section C.2.1 describes the objectives of code validation and assessment (CV\&A). Section C.2.2 describes the format used to summarize the CONTAIN validation and assessment efforts.

\section{C.2.1 Objectives of Code Validation and Assessment (CV\&A)}

Validation is a necessary part of the development of the CONTAIN code. Code models are often developed based on an incomplete or approximate understanding of separate, isolated physical processes. When these models are added to CONTAIN, where many physical processes are interacting, code predictions are rightfully questioned. The main objective of the CONTAIN CV\&A is to provide an information base broad enough answer such questions and establish confidence in the code predictions. Other objectives include providing direction for model development, support for experiment planning, and education for the code user.

Often the issue of CV\&A encompasses that of verification. In the present terminology, "verification" means efforts to ensure that there are no coding errors, and that the calculations represent correct solutions to the model equations. The term "validation" is used to refer to efforts to determine whether the models are accurate representations of the physical reality of containment phenomena by comparing code predictions with experimental results. The verification activities for CONTAIN are performed as part of the formal testing specified in the Quality Assurance (QA) program described in Appendix D. In particular, a systematic set of procedures is used for testing CONTAIN against hand calculations or other codes to identify any model implementation errors or logical errors in the code. For example, Reference Sci84 is a joint verification and validation investigation primarily involving the verification of CONTAIN during the code's early development. However, parts of the investigation are considered to be validation and are included in the individual CV\&As below. 
Validation is considered outside the formal QA program since it involves a great deal of technical judgment about what experiments are suitable and what degree of accuracy is needed, and also requires that opportunities for validation be available. One form of validation and assessment has been the so-called "blind post-test" prediction exercises. In such programs, code predictions are made after the experiments have been conducted but without prior knowledge of the results by the persons making the predictions, except for the data needed to define the initial and boundary conditions for the calculations.

For example, the ABCOVE series provided important blind validation of CONTAIN for dry aerosol behavior; the German HDR experiments were used to provide blind validation of gas mixing and atmosphere thermal-hydraulic behavior with respect steam blowdowns; the LACE experiment series provided the opportunity to validate both thermal hydraulic and aerosol behavior aspects of CONTAIN. In general, the CONTAIN results from these three major validation exercises showed that the code could successfully predict the time evolution of the experimental data. (The reader should consult the appropriate table for the references for the above comparisons.)

In addition to direct validation of CONTAIN, there is another way that the code's predictive ability has been validated. Numerous stand-alone codes and models have been imported into CONTAIN with little or no modification. These include the hydrogen combustion models from HECTR, [Din86, Pon90] the CORCON models for core-concrete interactions, [Bra93] the SPARC pool scrubbing model, [Owc85] and others. Numerous comparisons with experiments have been performed with these codes or models individually to determine the validity of the modeling. Therefore, the validation of the stand-alone codes and models, along with verification that the models are working as expected in CONTAIN, gives a considerable degree of "inherited validation" to these models within CONTAIN.

\section{C.2.2 Nature and Format of the CV\&A}

The validation efforts summarized in this report include studies in which CONTAIN results were compared to one or more of the following:

- Experimental results

- Calculated results from other codes

- Analytical results

The emphasis is placed on experimental comparisons. However, because the experimental data base for code comparisons remains somewhat limited, other codes and analytic solutions have proven to be a critical source of data to complement the existing experimental results in the validation of CONTAIN. In addition, a selected number of integral plant calculations that demonstrate the general capabilities of CONTAIN are also summarized.

The individual CV\&A summaries are discussed by modeling categories:

- Atmosphere thermal hydraulics and intercell flow

- Heat and mass transfer 
- $\quad$ Direct containment heating $(\mathrm{DCH})$

- Aerosol behavior

- Hydrogen burns

- $\quad$ Pressure suppression models

- Miscellaneous models

Each modeling category includes a validation summary in tabular form, along with selected figures that highlight the validation effort.

The summary tables include information on the type of validation (experiment, analytical, or codeto-code), facility, accident simulation or procedure, references, modeling particulars, code version, model ranking for key parameters(where appropriate), and comments. The comments can include brief statements concerning the validation and assessment results, recommendations, and actions taken.

The specific phenomenological model or group of models addressed by the CV\&A are identified in the title of each summary table (Tables C-3 through C-9). Note that an investigation may involve more than one model so that a reference may be used in more than one summary table. More than one reference may be noted in a particular entry if the basic work was presented in more than one publication or when several investigations cover the same basic modeling area. Note that an evaluation of a particular model may require the use of supporting models. For example, an evaluation of the heat and mass transfer modeling typically requires the use of models for the atmosphere thermodynamics. The supporting models are not identified in the individual CV\&A summaries. Also, the summaries identify the use of the material properties models only when the investigation is specifically directed at material properties modeling.

In the case of heat and mass transfer modeling, an additional summary table, Table C-5, is included. This gives the validation experience relevant to the modeling approach that is used in CONTAIN for predicting condensation/evaporation processes, but acquired without use of CONTAIN itself. Inclusion of this table is for supporting purposes only.

The code version is an important element of each CV\&A because CONTAIN has been under continuous development and many of the problems identified with earlier versions of the code have been resolved. In a few cases, the code version "Early" is denoted, and in other cases variant CONTAIN versions were used. The "Early" version of CONTAIN predates CONTAIN 1.0. Validation performed with this code version consisted mainly of testing. The CONTAIN DCH variant was originally used to perform CV\&A on the $\mathrm{DCH}$ models. These models were eventually implemented into the mainstream code in CONTAIN 1.12.

An evaluation of model performance has been included for some models; in these instances an attempt was made to evaluate models according to criteria applied to the principal quantity involved. The ranking of models can be either low, medium, and high, according to the following criteria:

1) High = prediction of prime quantity to within $10 \%$ and trends predicted; 
2) Medium = prediction of prime quantity to within $20-40 \%$ and trends predicted; and,

3) Low $=$ prediction of prime quantity less than $50 \%$ and only a few trends predicted.

\section{C.3 Individual CV\&A Summaries}

\section{C.3.1 Atmosphere Thermal Hydraulics and Intercell Flow}

Table C-3 presents a summary of the validation and assessment studies for the atmosphere thermal hydraulics and intercell flow models in CONTAIN. One area of specific concern for model adequacy was whether CONTAIN could predict the observed stable stratifications that typically occur for elevated source injection locations within a containment. A review of the performance of the code in the ISP-29 test seemed to suggest that CONTAIN 1.11 could not handle this class of problems. As a result of experiences like these a code improvement effort was undertaken to address the problem of overmixing in lumped parameter codes. A hybrid flow solver was developed for CONTAIN to solve this problem of overmixing for stably stratified containments. [Mur96] A comparison of data and code results for the light gas distribution in the upper dome of the HDR facility for the ISP-29 test is shown in Figure C-1. The improvement in the results for the hybrid flow solver in CONTAIN 1.2 is clearly evident. This case shows how the CV\&A procedure can identify shortcomings in code performance, provide guidance for additional code development, and finally establish confidence in a revised model.

\section{C.3.2 Heat and Mass Transfer}

Table C -4 presents a summary of the validation and assessment studies for the heat and mass transfer models in the CONTAIN code. Energy transport by condensation/evaporation processes are very important to the predictions of atmospheric pressure, temperature, and gas composition. The modeling approach for such processes is based on diffusion boundary layer theory in conjunction with a heat and mass transfer analogy (HMTA). Table C-5 presents additional validation studies that have been completed for this approach, in addition to the CONTAIN-specific efforts described in Table C-4. An example of the type of experimental comparison used to show the adequacy of the modeling is the comparison between the experimentally derived heat transfer coefficient for condensation and the CONTAIN blind post-test results for the LACE LA-4 experiment, as shown in Figure C-2. A code-to-code comparison exercise between CONTAIN and the Purdue finite difference boundary layer code was recently completed. This comparison showed that the CONTAIN treatment gives good results for high evaporative mass transfer rates when simulating the evaporation occurring in an asymmetrically heated vertical channel cooled by a falling water film. The CONTAIN comparisons for high condensation rates in condenser tubes also show good agreement with data, as illustrated in Figure C-3. Agreement such as this shows not only the adequacy of the HMTA modeling methods, but also the appropriateness of the correction factors used to modify the HMTA at high mass fluxes. Recent ALWR test comparisons involving condensation/evaporative processes have also confirmed that the HMTA, as implemented in CONTAIN, provides very good accuracy for mass transfer in both free and forced convective regimes. 


\section{C.3.3 Direct Containment Heating}

The direct containment heating (DCH) model validation and assessment studies include the modeling of airborne debris interactions and reactor pressure vessel (RPV) and cavity phenomena. Some of the experimental DCH validation comparisons are summarized in Table C-6.

The DCH modeling has been tested by performing numerous calculations comparing predicted peak pressures, hydrogen production, and debris carryover fractions to experimental data for the Sandia $\mathrm{DCH}, \mathrm{LFP}$, and WC tests. Calculations for all tests in these three series were performed, including DCH-1, DCH-3, LFP-A, 1B, 2A, 2B, 2C, WC-1, WC-2, and WC-3. The comparisons for pressure in an early DCH-1 test are shown in Figure C-4. For the LFP and WC series, two cases were evaluated, the first case of which excluded interaction of non-airborne debris, and the second case included interaction with an effective 10-mm particle diameter. Integral results for the LFP and WC tests compared well with the experimental results.

In addition to the normal functional testing that accompanied the development and implementation of the RPV and cavity models, a number of the modeling options were tested in a simulation of the IET-9 high pressure melt ejection (HPME) experiment conducted at SNL. All of the RPV models were exercised, as well as the entrained fraction option, two of the entrainment rate options, the Weber debris droplet size model, the time-dependent gas flow area option, and both the userspecified and internally calculated entrainment time option. A more exhaustive testing of the various available modeling options may be performed in the future. Four test cases were performed to simulate the IET-9 test.

Based on the results of the four IET-9 test cases, the RPV models appear to be functioning correctly, with one exception - the model for predicting gas blowthrough seems to underpredict the time by a significant amount. In the four test cases, gas blowthrough was forced to occur at $t=0.3 \mathrm{~s}$ to match the experimentally observed result. The gas blowthrough model is based on low-temperature tests with nonprototypic materials and has never been assessed against the high-temperature HPME experimental data base. An assessment activity might be necessary to determine if the gas blowthrough model needs to be modified.

The cavity models also seem to be functioning well. The entrained fraction option works well and was exercised with two of the five available entrainment rate models. The Weber model also appears to function well, although it did not have a large effect on the results. However, the choice of entrainment rate model appears to have a significant effect on both the shape and magnitude of the pressure and temperature results. The TDISP option functioned correctly to create a specific entrainment interval, but did cause an abrupt drop in the cavity pressure and temperature when entrainment was terminated at the designated time in the calculation. The time-dependent gas flow area option also worked well. Although more assessment is necessary, the RPV and cavity models appear to have been successfully integrated into CONTAIN and are functioning as designed. 


\section{C.3.4 Aerosol Behavior}

Table C-7 presents a summary of validation and assessment studies for aerosol modeling. In its early development, the CONTAIN aerosol modeling was tested to determine its adequacy for applications as a source term code. Most of that testing is reflected in the summary table. A significant advance in aerosol modeling occurred with the introduction of a model for soluble aerosol behavior. The LACE LA-4 experiment was used as a blind-post-test exercise to evaluate the new model. The improvement in the aerosol airborne concentrations predicted with the soluble aerosol model is shown in Figure C-5. In aerosol behavior modeling, agreement to within a factor of two for airborne concentrations is generally considered to be good agreement. The LACE test indicated that the aerosol modeling in the code was adequate for applying the code to source term analyses.

\section{C.3.5 Hydrogen Burns}

The CONTAIN validation summary for hydrogen burning is presented in Table C-8. The validation summary indicates that difficulty was encountered in the modeling of burn completeness and burn times for hydrogen deflagrations. Partially as a result of such observations, the hydrogen burn models in CONTAIN were revised in CONTAIN 1.12. They are now based on the models in the HECTR 1.8 code, [Din86, Pon90] which incorporated the available data base for deflagrations in open volumes under well-mixed conditions. However, as discussed in Chapter 9, the models have been improved as new experimental or analytical results became available.

\section{C.3.6 Pressure Suppression Models}

There is limited validation of the pressure suppression models, which include models for BWR suppression pool behavior, ice condensers, and water sprays. Table C-9 presents the validation summary for such models. Experiments in this modeling area are limited. For ice condenser modeling, unpublished thermal hydraulic results from the Battelle Pacific Northwest Laboratories (PNL) ice condenser experiments were used for model assessment. These experiments used a prototypic full height ice condenser section. Some of the results from this validation exercise are shown in Figure C-6. This exercise showed that CONTAIN qualitatively simulated major features of the flow in the PNL experiments. Results from this exercise support the use of CONTAIN to qualitatively predict the response of ice condenser containments to conditions that include natural circulation. Recently, the water spray modeling has been evaluated against data obtained from the NUPEC series of 1/4-scale PWR tests. Such comparisons show that qualitative agreement can be expected, yet accurate quantitative agreement is lacking.

\section{C.3.7 Miscellaneous Models}

The validation of miscellaneous models is summarized in Table C-10. These assessments cover fission product behavior, heat and mass transfer models not covered in Section C.3.2 (concrete outgassing and conduction), and material properties. 


\section{C.4 Overall CV\&A Summary}

This section summarizes the overall results of the CV\&A presented in this appendix.

- Atmospheric thermal hydraulics and intercell flow - The atmosphere thermodynamics modeling was found to be generally acceptable. However, it was noted that the aerosol options should be used to avoid unrealistically high degrees of supersaturation. The intercell flow model was observed to have the following characteristics: (a) The predictions for the mass flow rate through an orifice at low ratios of the upstream to downstream pressure were good. (b) The mass flow rate through an orifice under choked conditions was approximately $16 \%$ high. (c) The proper characterization of flow in a long duct required five or more nodes. (d) With adequate nodalization in the vertical direction, the ability to model stratified conditions with CONTAIN 1.2 was remarkably improved.

- Heat and mass transfer - The condensation heat and mass transfer model was found to be adequate for most conditions expected in containments.

- $\quad \mathrm{DCH}$ - The DCH modeling was found to adequately represent debris chemical interactions. Improvements in the model for the radiative emissivity when debris is present as a cloud and a more mechanistic model for debris interacting with structures are needed.

- Aerosol behavior - For aerosol modeling it was observed that (a) condensation, agglomeration and deposition were well predicted; (b) diffusiophoresis was acceptably predicted for small temperature differences but may not be acceptable if it plays a major role in aerosol depletion.

- Hydrogen burns - The original model for deflagrations was found to be inadequate, especially with respect to the fraction of hydrogen burned and the burn time. The present, revised model reflects the available data base for deflagrations in open volumes under well-mixed conditions. However, the model should continue to be assessed, especially under less than ideal conditions, to more fully characterize its limitations.

- $\quad$ Pressure suppression models - The spray model was found to be acceptable based on qualitative agreement with data.

- Miscellaneous models - The miscellaneous models (see Table C-10) performed satisfactorily.

\section{C.5 Conclusions}

This appendix has summarized the overall capabilities of the CONTAIN code and efforts taken to validate and assess the code against experimental data and other sources. The capabilities of the code include the ability to predict containment pressures, temperatures, and material distributions within the containment for assessing containment loads and associated threats to containment integrity under accident conditions. Because the code also has models for aerosol and fission 
product behavior, it can be used to predict radiological releases to the environment in the event of containment failure.

In addition to the code validation discussed above, it should be noted that numerous integrated analyses or code applications have been performed which are not directly associated with the validation and assessment effort. However, such applications are important contributors to the overall objective of code validation in the sense that they often identify modeling weaknesses and/or code errors.

The following two conclusions are made regarding the overall capabilities of CONTAIN and efforts to validate and assess these capabilities:

1. The CONTAIN code is a fully operational containment analysis tool that can be used with a reasonable degree of confidence to address a broad class of containment issues.

2. A critical component of the development of CONTAIN and the establishment of the current level of confidence has been the existence of programs to validate and assess the code against experimental data and other appropriate sources. 


\section{Table C-1 CONTAIN Code Release History}

\begin{tabular}{|c|c|}
\hline $\begin{array}{c}\text { Code Version } \\
\text { (Date) }\end{array}$ & Major Improvements, New Models and Items \\
\hline $\begin{array}{c}1.0 \\
\text { (Aug. 1984) }\end{array}$ & First official release of code \\
\hline $\begin{array}{c}1.01 \\
\text { (December 1984) }\end{array}$ & Modifications to correct nonstandard use of character variables \\
\hline $\begin{array}{c}1.02 \\
1.03 \\
\text { (May 1985) }\end{array}$ & $\begin{array}{l}\text { Modifications to make code conform to FORTRAN-77 standard } \\
\text { Improved lower-cell (pool and basemat) nodalization process, and } \\
\text { engineering safety systems models }\end{array}$ \\
\hline $\begin{array}{c}1.04 \\
\text { (November 1985) }\end{array}$ & $\begin{array}{l}\text { Add water aerosol deposition into structure surface film layer, time-dependent } \\
\text { aerosol size distribution input parameters, and implicit flow solver for multi-cell } \\
\text { gas transport } \\
\text { Improved atmosphere-to-structure radiation model and hydrogen burn timestep } \\
\text { adjustment, and method to estimate liquid film boundary layer interface } \\
\text { temperature }\end{array}$ \\
\hline $\begin{array}{c}1.05 \\
\text { (July 1986) }\end{array}$ & $\begin{array}{l}\text { Add carbon monoxide combustion, fission product targeted release and acceptance, } \\
\text { choked flow limit for intercell gas flow, and activation and deactivation keywords } \\
\text { for combustion model } \\
\text { Improved thermal properties, burn completeness correlation, and flexibility in } \\
\text { specifying combustion burn parameters }\end{array}$ \\
\hline $\begin{array}{c}1.06 \\
\text { (February 1987) }\end{array}$ & $\begin{array}{l}\text { Add integrated implementation of the CORCON-Mod2 model for molten core } \\
\text { concrete interaction (MCCD, VANESA model for aerosol generation and } \\
\text { radioisotope release from MCCIs, water dropout model to remove water from } \\
\text { atmosphere without using aerosol dynamics model, and aerosol settling through } \\
\text { flow paths } \\
\text { Improved models for radiant heat transfer (net enclosure model) }\end{array}$ \\
\hline $\begin{array}{c}1.10 \\
\text { (October 1987) }\end{array}$ & $\begin{array}{l}\text { Add specialized models for boiling water reactor features (safety relief valves and } \\
\text { pressure suppression pools), new flow path type (engineering vent) to provide } \\
\text { added flexibility to plant nodalization, and user-defined material properties } \\
\text { Improved method for tracking radioisotopes, the heat conduction algorithm for } \\
\text { lower-cell concrete floor model, various solution techniques including semi- } \\
\text { implicit coupling of the thermal radiation model with flow equations, and } \\
\text { automatic recovery from nonconvergence in the flow solver }\end{array}$ \\
\hline
\end{tabular}


Table C-1 CONTAIN Code Release History (Concluded)

\begin{tabular}{|c|c|}
\hline $\begin{array}{l}\text { Code Version } \\
\text { (Date) }\end{array}$ & Major Improvements, New Models and Items \\
\hline $\begin{array}{c}1.11 \\
\text { (March 1991) }\end{array}$ & $\begin{array}{l}\text { Add moving-grid calculation technique for solving the equations for aerosol } \\
\text { growth by water vapor condensation, concrete outgassing of both bound and } \\
\text { evaporable water and carbon monoxide, generalized treatment of heat structure } \\
\text { boundary conditions, flexibility for variable setting on restart, aerosol settling into } \\
\text { pools, volumetric displacement of atmosphere by pools, and an averaging scheme } \\
\text { to approximate gas flow velocities within a cell, used for calculating forced } \\
\text { convection for heat structures } \\
\text { Improved material property library; heat and mass transfer models for } \\
\text { condensation/evaporation at structure surface, and in ice condenser and fan } \\
\text { coolers; and lower cell modeling of transient pool layers } \\
\text { Improved flexibility of user control over plot files }\end{array}$ \\
\hline $\begin{array}{c}1.12 \\
(\operatorname{March} 1991)\end{array}$ & $\begin{array}{l}\text { Add direct containment heating }(\mathrm{DCH}) \text { modeling, reactor cavity models for high } \\
\text { pressure debris dispersal and vessel blowdown, and new model for the vapor } \\
\text { saturation of noncondensable gas vented into pools } \\
\text { Improve concrete outgassing modeling to include ability to outgas from behind } \\
\text { liners }\end{array}$ \\
\hline $\begin{array}{c}1.12 \mathrm{~V} \\
\text { (August 1993) }\end{array}$ & Workstation version of release 1.12 \\
\hline $\begin{array}{c}1.2 \\
\text { (October 1995) }\end{array}$ & $\begin{array}{l}\text { Add film flow on wall structures, energy and mass conservation tracking, hybrid } \\
\text { flow solver, pool tracking, non-ideal equation of state for water, specific reactor } \\
\text { pressure vessel and cavity models for DCH, CORCON Mod3, and fission product } \\
\text { library } \\
\text { Improved DCH model setup and heat and mass transfer for convection and } \\
\text { condensation on structures }\end{array}$ \\
\hline $\begin{array}{c}2.0 \\
\text { (June 1997) }\end{array}$ & Improvements in the DCH and hydrogen burn models and miscellaneous bug fixes. \\
\hline
\end{tabular}


Table C-2 Containment Test Facilities Used for CONTAIN Integral Validation

\begin{tabular}{|c|c|c|c|c|c|}
\hline Facility & Location & $\begin{array}{l}\text { Free Volume } \\
\qquad\left(\mathrm{m}^{3}\right)\end{array}$ & $\begin{array}{l}\text { Sub- } \\
\text { compartments }\end{array}$ & Heat Sinks & Comment \\
\hline HDR & Germany & 11,300 & 62 & $\begin{array}{l}\text { Upper steel shell } \\
\text { internal concrete walls } \\
\text { misc. steel }\end{array}$ & $\begin{array}{l}\text { Large-scale containment with } \\
\text { vertical/horizontal aspect ratio significantly } \\
\text { larger than commercial plants. Well } \\
\text { instrumented for stratification studies }\end{array}$ \\
\hline NUPEC & Japan & 1310 & 28 & $\begin{array}{l}\text { Steel shell } \\
\text { steel interior walls }\end{array}$ & $\begin{array}{l}1 / 4 \text { scale model of PWR plant, with } \\
\text { non-prototypical internal heat sinks }\end{array}$ \\
\hline $\mathrm{BMC}$ & Germany & 600 & 9 & $\begin{array}{l}\text { Concrete exterior and } \\
\text { interior walls }\end{array}$ & $\begin{array}{l}\text { Scale model of German PWR plant, with } \\
\text { some leakage rate uncertainty }\end{array}$ \\
\hline CSTF & USA & 850 & 1 & Insulated steel shell & $\begin{array}{l}\text { Domed cylindrical tank } \\
\text { used mainly for iodine and aerosol } \\
\text { deposition testing }\end{array}$ \\
\hline Surtsey & USA & 59 & 1 & Insulated steel shell & Domed cylindrical tank \\
\hline Falcon & UK & 0.3 & 1 & Insulated steel shell & $\begin{array}{l}\text { Open tank (inlet and outlet) with heaters } \\
\text { used for aerosol deposition tests }\end{array}$ \\
\hline $\begin{array}{l}\text { WEC Large Scale Test } \\
\text { facility (LST) }\end{array}$ & USA & 84 & 1 and 4 & $\begin{array}{l}\text { Passive cooled steel shell } \\
\text { steel partition walls } \\
\text { misc. Al heat sinks }\end{array}$ & $1 / 8$ scale model of the AP 600 containment \\
\hline $\begin{array}{l}\text { WEC Small Scale Test } \\
\text { facility (SST) }\end{array}$ & USA & 4.7 & 1 & Passive cooled steel shell & $\begin{array}{l}\text { Facility consists of a steam distribution } \\
\text { pipe fitted to the axis of a tall cylindrical } \\
\text { tank or shell }\end{array}$ \\
\hline
\end{tabular}


Table C-3 Validation Matrix for Atmosphere Thermal Hydraulics and Intercell Flow Modeling

\begin{tabular}{|c|c|c|c|c|c|c|c|c|}
\hline \multirow{2}{*}{$\begin{array}{c}\text { Integral } \\
\text { Experiment }\end{array}$} & \multirow{2}{*}{$\begin{array}{c}\text { Test } \\
\text { Facility }\end{array}$} & \multirow{2}{*}{$\begin{array}{l}\text { Accident Type } \\
\text { or Process } \\
\text { Simulated }\end{array}$} & \multirow[t]{2}{*}{ Reference } & \multirow[t]{2}{*}{ Nodes } & \multirow{2}{*}{$\begin{array}{l}\text { Code } \\
\text { Version }\end{array}$} & \multicolumn{2}{|c|}{ Model Ranking ${ }^{\mathrm{a}}$} & \multirow[t]{2}{*}{ Comment } \\
\hline & & & & & & Pressure & $\begin{array}{c}\text { Gas } \\
\text { Mixing }\end{array}$ & \\
\hline \multicolumn{9}{|l|}{ Analytic: } \\
\hline$-\cdots$ & $\ldots$ & Intercell flow & Lan88a & 2 & 1.10 & --- & $\mathrm{H}$ & $\begin{array}{l}\text { Mass flow rates from the explicit and } \\
\text { implicit flow solvers were compared to } \\
\text { results from the Bernoulli equation. } \\
\text { Observed discrepancies in mass flow } \\
\text { rates were attributed to the CONTAIN } \\
\text { use of the upstream density for the flow } \\
\text { path inertial density. A different flow } \\
\text { path density may be required for high } \\
\text { speed flows. }\end{array}$ \\
\hline \multicolumn{9}{|c|}{ Experiment (International Standard Problems): } \\
\hline $\begin{array}{l}\text { ISP-16 } \\
\text { (V44) }\end{array}$ & HDR & $\begin{array}{l}\text { DBA } \\
\text { blowdown, } \\
\text { mid-elevation } \\
\text { injection of } \\
\text { steam }\end{array}$ & $\begin{array}{l}\text { Val83 } \\
\text { Wol83 }\end{array}$ & 5 & Early & M-H & M-H & $\begin{array}{l}\text { The explicit flow solver was used, with } \\
\text { no forced convection modeling. Slight } \\
\text { overprediction of the peak blowdown } \\
\text { pressure resulted. Very good prediction } \\
\text { of early-time break-room to adjoining } \\
\text { room differential pressure was found. }\end{array}$ \\
\hline $\begin{array}{l}\text { ISP-23 } \\
\text { (T31.5) }\end{array}$ & HDR & $\begin{array}{l}\text { DBA } \\
\text { blowdown, } \\
\text { mid-elevation } \\
\text { injection of } \\
\text { steam }\end{array}$ & Kar89 & 33,60 & 1.10 & $\mathrm{H}$ & M & $\begin{array}{l}\text { The implicit flow solver was used, with } \\
\text { forced convection modeling. }\end{array}$ \\
\hline
\end{tabular}


Table C-3 Validation Matrix for Atmosphere Thermal Hydraulics and Intercell Flow Modeling (Continued)

\begin{tabular}{|c|c|c|c|c|c|c|c|c|}
\hline \multirow{2}{*}{$\begin{array}{c}\text { Integral } \\
\text { Experiment }\end{array}$} & \multirow{2}{*}{$\begin{array}{l}\text { Test } \\
\text { Facility }\end{array}$} & \multirow{2}{*}{$\begin{array}{l}\text { Accident Type } \\
\text { or Process } \\
\text { Simulated }\end{array}$} & \multirow[t]{2}{*}{ Reference } & \multirow[t]{2}{*}{ Nodes } & \multirow{2}{*}{$\begin{array}{l}\text { Code } \\
\text { Version }\end{array}$} & \multicolumn{2}{|c|}{ Model Ranking ${ }^{a}$} & \multirow[t]{2}{*}{ Comment } \\
\hline & & & & & & Pressure & $\begin{array}{c}\text { Gas } \\
\text { Mixing }\end{array}$ & \\
\hline \multirow[t]{2}{*}{$\begin{array}{l}\text { ISP-29 } \\
\text { (E11.2) }\end{array}$} & \multirow[t]{2}{*}{ HDR } & \multirow{2}{*}{$\begin{array}{l}\text { SBLOCA/SA } \\
\text { with steam } \\
\text { and light gas } \\
\text { injection at } \\
\text { mid-elevation, } \\
\text { followed by } \\
\text { steam } \\
\text { injection at } \\
\text { low elevation }\end{array}$} & $\begin{array}{l}\text { Kar92; } \\
\text { see Note } \\
\text { b }\end{array}$ & 14 & 1.11 & $\mathrm{H}$ & L-M & $\begin{array}{l}\text { A tendency to overmix the containment } \\
\text { atmosphere was observed. The amount of } \\
\text { light gas in the dome region was } \\
\text { severely underpredicted. }\end{array}$ \\
\hline & & & Mur96 & 14,15 & 1.2 & M-H & M-H & $\begin{array}{l}\text { The hybrid flow solver improved } \\
\text { substantially the ability to predict the } \\
\text { stable stratifications. Uncertainties in test } \\
\text { specifications were believed to be } \\
\text { responsible for the medium ranking } \\
\text { given to the pressure prediction. }\end{array}$ \\
\hline $\begin{array}{l}\text { ISP-35 } \\
(\mathrm{M}-7-1)\end{array}$ & NUPEC & $\begin{array}{l}\text { SA, steam and } \\
\text { helium } \\
\text { injection at } \\
\text { low elevation, } \\
\text { with sprays }\end{array}$ & Sta95 & 28,35 & $1.12 \mathrm{~V}$ & $\mathrm{M}-\mathrm{H}$ & $\mathrm{M}-\mathrm{H}$ & $\begin{array}{l}\text { Steam preheating ( } 210 \text { minutes) was } \\
\text { followed by } 30 \text { minutes of steam and } \\
\text { helium injection and water sprays. The } \\
\text { sprays tended to create currents that } \\
\text { mixed gases; the extent of the mixing } \\
\text { was slightly underpredicted since the } \\
\text { sprays induced gas currents that were not } \\
\text { modeled. }\end{array}$ \\
\hline $\begin{array}{l}\text { ISP-37 } \\
\text { (VANAM M-3) }\end{array}$ & BMC & $\begin{array}{l}\mathrm{SA}, \text { core melt } \\
\text { transient }\end{array}$ & Fir96 & 12 & $1.12 \mathrm{~V}$ & M & $\mathrm{M}-\mathrm{H}$ & $\begin{array}{l}\text { Steam injection at mid- and low- } \\
\text { elevations resulted in convection loops } \\
\text { and stratification. Five participants used } \\
\text { the CONTAIN code with significant } \\
\text { scatter in the pressure predictions. }\end{array}$ \\
\hline
\end{tabular}


Table C-3 Validation Matrix for Atmosphere Thermal Hydraulics and Intercell Flow Modeling (Continued)

\begin{tabular}{|c|c|c|c|c|c|c|c|c|}
\hline \multirow{2}{*}{$\begin{array}{l}\text { Integral } \\
\text { Experiment }\end{array}$} & \multirow{2}{*}{$\begin{array}{c}\text { Test } \\
\text { Facility }\end{array}$} & \multirow{2}{*}{$\begin{array}{l}\text { Accident Type } \\
\text { or Process } \\
\text { Simulated }\end{array}$} & \multirow[t]{2}{*}{ Reference } & \multirow[t]{2}{*}{ Nodes } & \multirow{2}{*}{$\begin{array}{c}\text { Code } \\
\text { Version }\end{array}$} & \multicolumn{2}{|c|}{ Model Ranking ${ }^{\mathbf{a}}$} & \multirow[t]{2}{*}{ Comment } \\
\hline & & & & & & Pressure & $\begin{array}{c}\text { Gas } \\
\text { Mixing }\end{array}$ & \\
\hline \multicolumn{9}{|c|}{ Experiment (Other): } \\
\hline \multirow[t]{2}{*}{ M-8-1 } & \multirow[t]{4}{*}{ NUPEC } & \multirow{2}{*}{$\begin{array}{l}\text { SA, no } \\
\text { preheat, steam } \\
\text { and helium } \\
\text { injection at } \\
\text { mid-elevation } \\
\text { at top of } \\
\text { pressurizer, no } \\
\text { water sprays }\end{array}$} & \multirow[t]{2}{*}{ Mur96 } & \multirow[t]{2}{*}{35} & $\begin{array}{l}1.2 \\
\text { (using old } \\
\text { flow } \\
\text { solver) }\end{array}$ & $\mathrm{M}-\mathrm{H}$ & L-M & $\begin{array}{l}\text { The old flow solver tended to overmix } \\
\text { gases such that stratification boundary at } \\
\text { the top of the pressurizer was smeared. }\end{array}$ \\
\hline & & & & & $\begin{array}{l}1.2 \text { (using } \\
\text { hybrid } \\
\text { flow } \\
\text { solver) }\end{array}$ & M-H & M-H & $\begin{array}{l}\text { The hybrid flow solver maintained a } \\
\text { stratification boundary layer near the top } \\
\text { of the pressurizer in good agreement with } \\
\text { the observed behavior. }\end{array}$ \\
\hline M-4-3 & & $\begin{array}{l}\text { SA, no pre- } \\
\text { heat, steam } \\
\text { and helium } \\
\text { injection at } \\
\text { low elevation, } \\
\text { no water } \\
\text { sprays }\end{array}$ & Sta95 & 28,35 & $\begin{array}{l}1.12 \mathrm{~W} \\
1.12 \mathrm{XBG}\end{array}$ & $\mathrm{H}$ & $\mathrm{H}$ & $\begin{array}{l}\text { The old ( } 1.12 \mathrm{~W} \text { ) and hybrid (1.12XBG) } \\
\text { flow solvers gave nearly identical results. } \\
\text { The low injection elevation resulted in a } \\
\text { nearly well-mixed containment. }\end{array}$ \\
\hline M-5-5 & & $\begin{array}{l}\text { SA, no pre- } \\
\text { heat, helium } \\
\text { injection at } \\
\text { low elevation, } \\
\text { water sprays }\end{array}$ & & & & $\mathbf{H}$ & M-H & $\begin{array}{l}\text { The old ( } 1.12 \mathrm{~W} \text { ) and hybrid ( } 1.12 \mathrm{XBG}) \\
\text { flow solvers gave nearly identical results. } \\
\text { The low injection elevation and sprays } \\
\text { resulted in a nearly well-mixed } \\
\text { containment. }\end{array}$ \\
\hline
\end{tabular}


Table C-3 Validation Matrix for Atmosphere Thermal Hydraulics and Intercell Flow Modeling (Continued)

\begin{tabular}{|c|c|c|c|c|c|c|c|c|}
\hline \multirow{2}{*}{$\begin{array}{c}\text { Integral } \\
\text { Experiment }\end{array}$} & \multirow{2}{*}{$\begin{array}{c}\text { Test } \\
\text { Facility }\end{array}$} & \multirow{2}{*}{$\begin{array}{l}\text { Accident Type } \\
\text { or Process } \\
\text { Simulated }\end{array}$} & \multirow[t]{2}{*}{ Reference } & \multirow[t]{2}{*}{ Nodes } & \multirow{2}{*}{$\begin{array}{l}\text { Code } \\
\text { Version }\end{array}$} & \multicolumn{2}{|c|}{ Model Ranking ${ }^{a}$} & \multirow[t]{2}{*}{ Comment } \\
\hline & & & & & & Pressure & $\begin{array}{c}\text { Gas } \\
\text { Mixing }\end{array}$ & \\
\hline$M-8-2$ & NUPEC & $\begin{array}{l}\text { SA, preheat, } \\
\text { steam and } \\
\text { helium } \\
\text { injection at } \\
\text { mid-elevation, } \\
\text { water sprays }\end{array}$ & Sta95 & 28,35 & $\begin{array}{l}1.12 \mathrm{~W} \\
1.12 \mathrm{XBG}\end{array}$ & L-M & M-H & $\begin{array}{l}\text { The old (1.12W) and hybrid (1.12XBG) } \\
\text { flow solvers gave similar results. The } \\
\text { sprays tended to produce a nearly well- } \\
\text { mixed containment. Good prediction of } \\
\text { local temperatures but significant } \\
\text { overprediction of total pressure was } \\
\text { found. This overprediction was believed } \\
\text { to be the result of the sensitivity of steam } \\
\text { pressure to the predicted gas } \\
\text { temperatures. Discrepancies in local } \\
\text { temperatures were the result of an } \\
\text { inability to model the hydrodynamic } \\
\text { effects of water sprays on local mixing. }\end{array}$ \\
\hline LACE LA-4 & CSTF & $\begin{array}{l}\text { SA, preheat } \\
\text { phase fol- } \\
\text { lowed by } \\
\text { steady state } \\
\text { steam injec- } \\
\text { tion and then } \\
\text { depressuriza- } \\
\text { tion, steam } \\
\text { injected in } \\
\text { bottom third } \\
\text { of vessel } \\
\end{array}$ & $\begin{array}{l}\text { Gel90 } \\
\text { Sla87 }\end{array}$ & 1 & 1.11 & $\mathrm{H}$ & $M$ & $\begin{array}{l}\text { The low injection elevation resulted in } \\
\text { well-mixed conditions in the upper two } \\
\text { thirds of the vessel. Agreement in } \\
\text { containment gas temperatures was good } \\
\text { except below the injection point where } \\
\text { the code overmixed the gases. The vessel } \\
\text { steel walls were insulated. The small late } \\
\text { time difference between the measured } \\
\text { and predicted pressures was believed to } \\
\text { be the result of uncertainties in insulation } \\
\text { properties. }\end{array}$ \\
\hline
\end{tabular}

Rev. 0 
Table C-3 Validation Matrix for Atmosphere Thermal Hydraulics and Intercell Flow Modeling (Continued)

\begin{tabular}{|c|c|c|c|c|c|c|c|c|}
\hline \multirow{2}{*}{$\begin{array}{c}\text { Integral } \\
\text { Experiment }\end{array}$} & \multirow{2}{*}{$\begin{array}{c}\text { Test } \\
\text { Facility }\end{array}$} & \multirow{2}{*}{$\begin{array}{l}\text { Accident Type } \\
\text { or Process } \\
\text { Simulated }\end{array}$} & \multirow[t]{2}{*}{ Reference } & \multirow[t]{2}{*}{ Nodes } & \multirow{2}{*}{$\begin{array}{c}\text { Code } \\
\text { Version }\end{array}$} & \multicolumn{2}{|c|}{ Model Rankinga } & \multirow[t]{2}{*}{ Comment } \\
\hline & & & & & & Pressure & $\begin{array}{c}\text { Gas } \\
\text { Mixing }\end{array}$ & \\
\hline ST-3 & Surtsey & $\begin{array}{l}\text { SA, mid- } \\
\text { elevation } \\
\text { injection of } \\
\text { hydrogen into } \\
\text { ambient air }\end{array}$ & Mur96 & $\begin{array}{l}2,8,9 \\
\text { and } 15\end{array}$ & $1.12 \mathrm{~V}$ & $N / A^{c}$ & $\mathrm{H}$ & $\begin{array}{l}\text { The high Froude number injection of } \\
\text { hydrogen caused a marginally unstable } \\
\text { condition to exist such that the mixing } \\
\text { observed below the injection point was } \\
\text { well represented by the code - a } \\
\text { fortuitous result caused by numerical } \\
\text { effects. The ability to predict the } \\
\text { observed mixing was dependent on the } \\
\text { nodalization scheme. }\end{array}$ \\
\hline ST-3 & Surtsey & $\begin{array}{l}\text { SA, mid- } \\
\text { elevation } \\
\text { injection of } \\
\text { hydrogen into } \\
\text { ambient air }\end{array}$ & Mur96 & $\begin{array}{l}2,8,9 \\
\text { and } 15\end{array}$ & 1.2 & N/A & $\mathrm{M}-\mathrm{H}$ & $\begin{array}{l}\text { The tendency of hybrid flow solver to } \\
\text { maintain a stable stratification and lack } \\
\text { of momentum-driven mixing resulted in } \\
\text { an underprediction of mixing below the } \\
\text { injection point. }\end{array}$ \\
\hline $\begin{array}{l}202.3,203.2 \\
212.1,213.1, \\
214.1,222.2 \\
224.1,224.2\end{array}$ & $\begin{array}{l}\text { WEC } \\
\text { LST } \\
\text { facility }\end{array}$ & $\begin{array}{l}\text { Long-term } \\
\text { passive } \\
\text { containment } \\
\text { cooling for } \\
\text { AP600, steam } \\
\text { injection into } \\
\text { the steam } \\
\text { generator } \\
\text { room open to } \\
\text { above deck } \\
\text { region, } \\
\text { varying initial } \\
\text { air masses }\end{array}$ & Ti196 & $\begin{array}{l}2,4 \text {, and } \\
17\end{array}$ & 1.12 & $\mathrm{H}$ & $\mathrm{M}-\mathrm{H}$ & $\begin{array}{l}\text { The external passive containment cooling } \\
\text { system (PCS) was modeled with } \\
\text { additional nodes. The facility } \\
\text { arrangement did not permit convection } \\
\text { loops between above deck and below } \\
\text { deck regions. Steam injected into the } \\
\text { steam generator room was released to the } \\
\text { above deck region. Stratification between } \\
\text { open regions above deck and dead ended } \\
\text { regions below deck were well } \\
\text { approximated with } 4 \text { nodes. Stratification } \\
\text { in the open region above the operation } \\
\text { deck could not be modeled. Adding more } \\
\text { cells resulted in an overmixing of gases. }\end{array}$ \\
\hline
\end{tabular}


Table C-3 Validation Matrix for Atmosphere Thermal Hydraulics and Intercell Flow Modeling (Continued)

\begin{tabular}{|c|c|c|c|c|c|c|c|c|}
\hline \multirow{2}{*}{$\begin{array}{c}\text { Integral } \\
\text { Experiment }\end{array}$} & \multirow{2}{*}{$\begin{array}{c}\text { Test } \\
\text { Facility }\end{array}$} & \multirow{2}{*}{$\begin{array}{l}\text { Accident Type } \\
\text { or Process } \\
\text { Simulated }\end{array}$} & \multirow[t]{2}{*}{ Reference } & \multirow[t]{2}{*}{ Nodes } & \multirow{2}{*}{$\begin{array}{c}\text { Code } \\
\text { Version }\end{array}$} & \multicolumn{2}{|c|}{ Model Ranking } & \multirow[t]{2}{*}{ Comment } \\
\hline & & & & & & Pressure & $\begin{array}{c}\text { Gas } \\
\text { Mixing } \\
\end{array}$ & \\
\hline \multirow[t]{2}{*}{$221.1,222.1$} & \multirow[t]{3}{*}{$\begin{array}{l}\text { WEC } \\
\text { LST } \\
\text { facility }\end{array}$} & \multirow[t]{2}{*}{$\begin{array}{l}\text { Long-term } \\
\text { passive with } \\
\text { an elevated } \\
\text { injection point }\end{array}$} & \multirow[t]{3}{*}{ Til96 } & 4,17 & 1.12 & $\mathbf{H}$ & M & $\begin{array}{l}\text { The 17-cell nodalization effectively } \\
\text { allowed convection loops resulting in the } \\
\text { overmixing of gas. A more restrictive } \\
\text { nodalization scheme prevented some of } \\
\text { the mixing and gave better results for } \\
\text { local gas temperatures. }\end{array}$ \\
\hline & & & & 17. & 1.2 & $\mathrm{H}$ & M-H & $\begin{array}{l}\text { The overmixing of gas below the } \\
\text { injection point was reduced, giving better } \\
\text { agreement with measurements. }\end{array}$ \\
\hline 219.1 & & $\begin{array}{l}\text { Long-term } \\
\text { passive with } \\
\text { helium } \\
\text { injected into } \\
\text { the steam } \\
\text { generator } \\
\text { room }\end{array}$ & & 4 & 1.12 & $\mathrm{H}$ & L-M & $\begin{array}{l}\text { The long-term mixing of helium was } \\
\text { underpredicted. Gas mixing was believed } \\
\text { to be dominated by gas diffusion which } \\
\text { was not modeled. }\end{array}$ \\
\hline
\end{tabular}




\begin{tabular}{|c|c|c|c|c|}
\hline \multicolumn{2}{|c|}{ 莒 } & 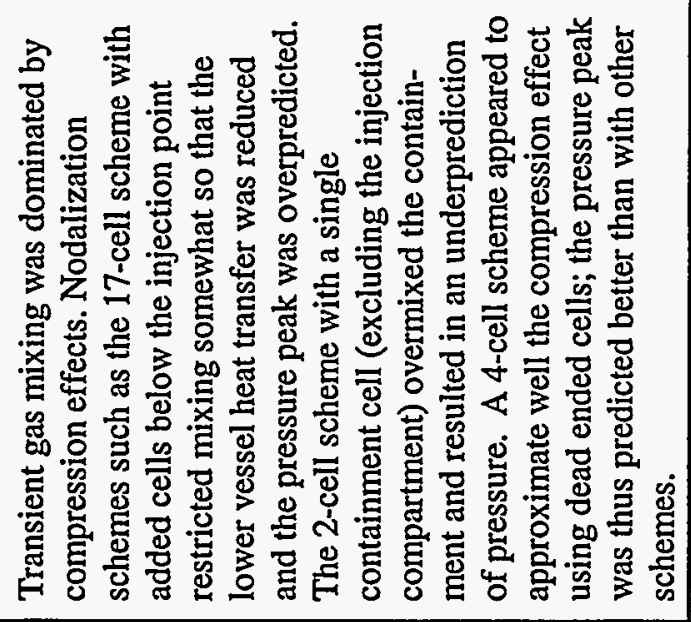 & 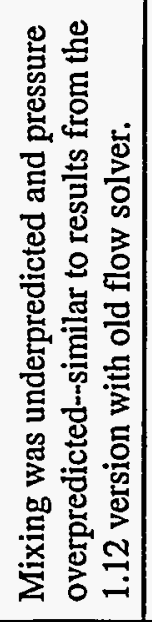 & 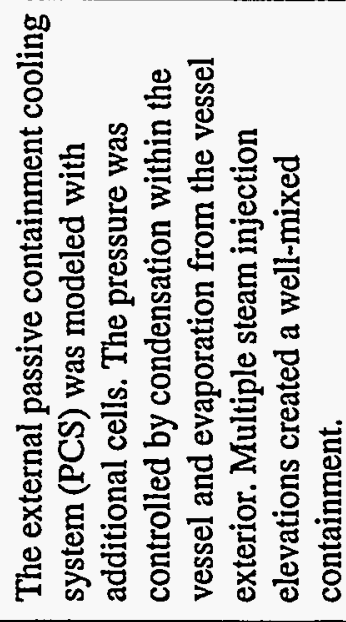 \\
\hline \multirow{2}{*}{ 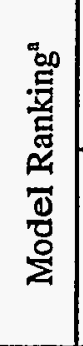 } & Ðึ & $\sum_{\Sigma}^{5}$ & $\stackrel{5}{\Sigma}$ & $\widehat{z}$ \\
\hline & 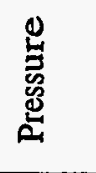 & 帘 & $\Sigma$ & : \\
\hline \multicolumn{2}{|c|}{ 吾 } & $\cong$ & $\Im$ & $\exists$ \\
\hline \multicolumn{2}{|l|}{$\begin{array}{l}\frac{3}{2} \\
\frac{8}{2}\end{array}$} & 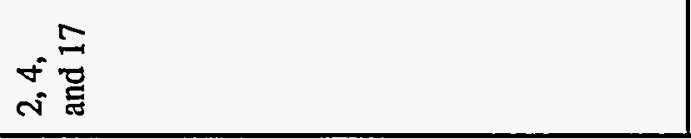 & $\approx$ & - \\
\hline \multicolumn{2}{|l|}{ 递 } & ฏ & & $\vec{a}$ \\
\hline \multicolumn{2}{|c|}{ 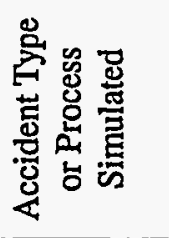 } & 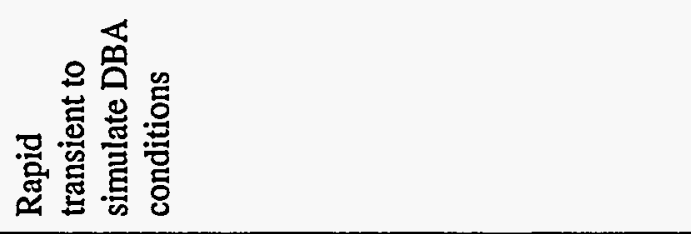 & & 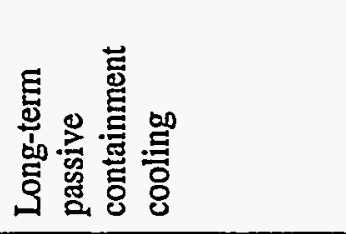 \\
\hline \multicolumn{2}{|c|}{ 岁言 } & 兽焉 & & 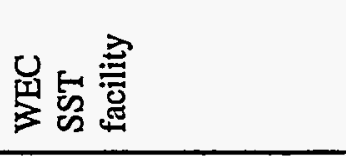 \\
\hline \multicolumn{2}{|c|}{ 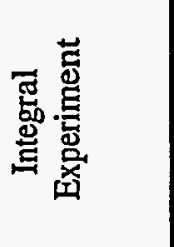 } & 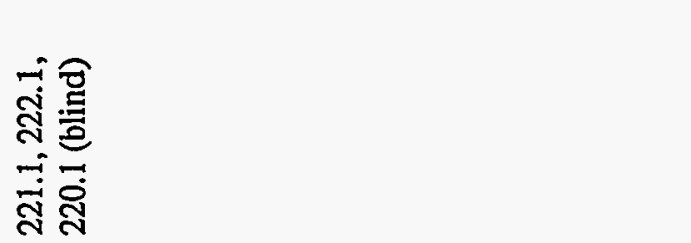 & & 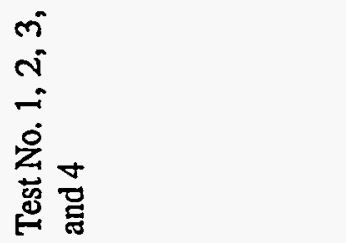 \\
\hline
\end{tabular}

ঠ̊̊̆ 
Table C-3 Validation Matrix for Atmosphere Thermal Hydraulics and Intercell Flow Modeling (Continued)

\begin{tabular}{|c|c|c|c|c|c|c|c|c|}
\hline \multirow{2}{*}{$\begin{array}{c}\text { Integral } \\
\text { Experiment }\end{array}$} & \multirow{2}{*}{$\begin{array}{c}\text { Test } \\
\text { Facility }\end{array}$} & \multirow{2}{*}{$\begin{array}{l}\text { Accident Type } \\
\text { or Process } \\
\text { Simulated }\end{array}$} & \multirow[t]{2}{*}{ Reference } & \multirow[t]{2}{*}{ Nodes } & \multirow{2}{*}{$\begin{array}{c}\text { Code } \\
\text { Version }\end{array}$} & \multicolumn{2}{|c|}{ Model Ranking ${ }^{\mathfrak{a}}$} & \multirow[t]{2}{*}{ Comment } \\
\hline & & & & & & Pressure & $\begin{array}{c}\text { Gas } \\
\text { Mixing }\end{array}$ & \\
\hline FAL-3A & $\begin{array}{l}\text { Falcon } \\
\text { facility, } \\
\text { open } \\
\text { system } \\
\text { with inlet } \\
\text { and } \\
\text { outlet. }\end{array}$ & $\begin{array}{l}\text { SA, injection } \\
\text { of steam, } \\
\text { helium, and } \\
\text { solid aerosols }\end{array}$ & Smi91 & 1 & 1.12 & $\begin{array}{l}\text { M-H for } \\
\text { tempera- } \\
\text { ture }^{\text {d }}\end{array}$ & $\mathrm{N} / \mathrm{A}$ & $\begin{array}{l}\text { Slight underprediction of the degree of } \\
\text { superheat was observed. This was } \\
\text { believed to be the result of the high } \\
\text { concentration of helium in the } \\
\text { atmosphere and the assumed use of an air } \\
\text { diffusivity to approximate the helium } \\
\text { diffusivity. }\end{array}$ \\
\hline $\mathrm{ACE}$ & CSTF & $\begin{array}{l}\text { SA, injection } \\
\text { of steam, } \\
\text { solid aerosols, } \\
\text { and iodine }\end{array}$ & Smi92a & 1 & 1.11 & $\mathrm{H}$ & N/A & $\begin{array}{l}\text { The steam/gas mixture in the vessel was } \\
\text { well mixed. The temperature and } \\
\text { saturation ratio were predicted well. }\end{array}$ \\
\hline $\begin{array}{l}\text { FIPLOC } \\
\text { verification } \\
\text { experiment, F2 }\end{array}$ & $\mathrm{BMC}$ & $\begin{array}{l}\text { T/H } \\
\text { conditioning } \\
\text { for aerosol } \\
\text { deposition, } \\
\text { steam } \\
\text { injection }\end{array}$ & Smi92b & $\begin{array}{l}1,5, \\
\text { and } 11\end{array}$ & 1.10 & $\mathrm{M}-\mathrm{H}$ & L-M & $\begin{array}{l}\text { Uncertainties in the containment leak rate } \\
\text { made accurate pressure and local } \\
\text { temperature comparisons difficult. } \\
\text { Significant stratification was observed-- } \\
\text { trends were generally predicted. The } \\
\text { transition to well-mixed conditions was } \\
\text { not predicted due to an unknown lower } \\
\text { compartment leakage rate. A single cell } \\
\text { calculation significantly underpredicted } \\
\text { the measured pressure rise. }\end{array}$ \\
\hline $\begin{array}{l}\text { DEMONA } \\
\text { A9 }\end{array}$ & $\mathrm{BMC}$ & $\begin{array}{l}\mathrm{T} / \mathrm{H} \\
\text { conditioning } \\
\text { for aerosol } \\
\text { deposition, } \\
\text { steam } \\
\text { injection }\end{array}$ & Smi92c & 5 & 1.11 & $\mathbf{M}-\mathbf{H}$ & M-H & $\begin{array}{l}\text { Periods of saturated and superheated } \\
\text { conditions were observed--the pressure } \\
\text { and local temperature were predicted } \\
\text { well. A small degree of stratification was } \\
\text { observed. }\end{array}$ \\
\hline
\end{tabular}

Rev. 0 
Table C-3 Validation Matrix for Atmosphere Thermal Hydraulics and Intercell Flow Modeling (Continued)

\begin{tabular}{|c|c|c|c|c|c|c|c|c|}
\hline \multirow{2}{*}{$\begin{array}{l}\text { Integral } \\
\text { Experiment }\end{array}$} & \multirow{2}{*}{$\begin{array}{c}\text { Test } \\
\text { Facility }\end{array}$} & \multirow{2}{*}{$\begin{array}{l}\text { Accident Type } \\
\text { or Process } \\
\text { Simulated }\end{array}$} & \multirow[t]{2}{*}{ Reference } & \multirow[t]{2}{*}{ Nodes } & \multirow{2}{*}{$\begin{array}{l}\text { Code } \\
\text { Version }\end{array}$} & \multicolumn{2}{|c|}{ Model Ranking ${ }^{a}$} & \multirow[t]{2}{*}{ Comment } \\
\hline & & & & & & Pressure & $\begin{array}{c}\text { Gas } \\
\text { Mixing }\end{array}$ & \\
\hline $\begin{array}{l}\text { DEMONA } \\
\text { B2 }\end{array}$ & BMC & $\begin{array}{l}\mathrm{T} / \mathrm{H} \\
\text { conditions for } \\
\text { dry aerosols }\end{array}$ & Smi92d & 1 & 1.11 & M & N/A & $\begin{array}{l}\text { This experiment was well-mixed and } \\
\text { used only noncondensable gas. } \\
\text { Depressurization was dependent on } \\
\text { containment leakage. The relevant } \\
\text { laminar leakage flow models were not } \\
\text { available. The temperature relaxation was } \\
\text { predicted well, confirming the dry heat } \\
\text { transfer modeling. }\end{array}$ \\
\hline $\begin{array}{l}\text { DEMONA } \\
\text { B3 }\end{array}$ & BMC & $\begin{array}{l}\mathrm{T} / \mathrm{H} \\
\text { conditions for } \\
\text { wet aerosols, } \\
\text { steam } \\
\text { injection }\end{array}$ & Smi92e & 1 & 1.11 & $\mathbf{H}$ & N/A & $\begin{array}{l}\text { This experiment was well-mixed and } \\
\text { used a steam/gas mixture. The steam } \\
\text { partial pressure was found to be in good } \\
\text { agreement with the data. }\end{array}$ \\
\hline E11.4 & HDR & $\begin{array}{l}\text { SBLOCA/SA, } \\
\text { low-elevation } \\
\text { steam } \\
\text { injection, } \\
\text { depressuriza- } \\
\text { tion by } \\
\text { venting }\end{array}$ & $\begin{array}{l}\text { See } \\
\text { Note e }\end{array}$ & 13 & 1.12 & $\mathrm{H}$ & M-H & $\begin{array}{l}\text { The low-elevation injection of steam } \\
\text { tended to mix the containment } \\
\text { atmosphere thereby producing a } \\
\text { relatively small degree of stratification } \\
\text { (compared to E11.2). Slight } \\
\text { overprediction of light gas concentrations } \\
\text { in the dome region was observed-- } \\
\text { general trends in temperature and } \\
\text { concentration throughout the } \\
\text { containment were predicted. }\end{array}$ \\
\hline $\mathrm{T} 31.6^{\mathrm{r}}$ & HDR & $\begin{array}{l}\text { LOCA/mid- } \\
\text { elevation } \\
\text { steam } \\
\text { injection } \\
\end{array}$ & Lan88b & 39 & 1.10 & $\mathbf{H}$ & M-H & Test simulated a blowdown \\
\hline
\end{tabular}


Table C-3 Validation Matrix for Atmosphere Thermal Hydraulics and Intercell Flow Modeling (Concluded)

\begin{tabular}{|c|c|c|c|c|c|c|c|c|}
\hline \multirow{2}{*}{$\begin{array}{l}\text { Integral } \\
\text { Experiment }\end{array}$} & \multirow{2}{*}{$\begin{array}{l}\text { Test } \\
\text { Facility }\end{array}$} & \multirow{2}{*}{$\begin{array}{l}\text { Accident Type } \\
\text { or Process }\end{array}$} & \multirow[t]{2}{*}{ Reference } & \multirow[t]{2}{*}{ Nodes } & \multirow{2}{*}{$\begin{array}{l}\text { Code } \\
\text { Version }\end{array}$} & \multicolumn{2}{|c|}{ Model Ranking ${ }^{a}$} & \multirow[t]{2}{*}{ Comment } \\
\hline & & & & & & Pressure & $\begin{array}{c}\text { Gas } \\
\text { Mixing }\end{array}$ & \\
\hline \multicolumn{9}{|l|}{ Code/Code: } \\
\hline $\begin{array}{l}\text { RELAP/ } \\
\text { CONTAIN }\end{array}$ & N/A & \multirow{2}{*}{$\begin{array}{l}\text { Intercell flow, } \\
\text { buoyancy } \\
\text { driven }\end{array}$} & Jac89 & & 1.10 & N/A & $\mathrm{H}$ & $\begin{array}{l}\text { Good agreement found with adequate } \\
\text { CONTAIN noding }\end{array}$ \\
\hline $\begin{array}{l}\text { PHOENIX/ } \\
\text { CONTAIN }\end{array}$ & N/A & & Hei86 & 16,64 & 1.04 & N/A & M-H & $\begin{array}{l}\text { Comparisons showed that CONTAIN } \\
\text { could predict the effects of buoyancy in a } \\
\text { simple open geometry. }\end{array}$ \\
\hline \multirow[t]{2}{*}{$\begin{array}{l}\text { CONTEMPT/ } \\
\text { CONTAIN }\end{array}$} & PWR & $\begin{array}{l}\text { LOCA and } \\
\text { MSLB with } \\
\text { sprays }\end{array}$ & \multirow[t]{2}{*}{ Alm93 } & 1 & \multirow[t]{2}{*}{1.12} & $\mathrm{H}$ & N/A & $\begin{array}{l}\text { The LOCA pressures and temperatures } \\
\text { compared very well. Modeling differ- } \\
\text { ences for MSLB conditions resulted in } \\
\text { CONTAIN temperatures that were } \\
\text { significantly lower than CONTEMPT's. }\end{array}$ \\
\hline & BWR & $\begin{array}{l}\text { Recirculation } \\
\text { line break; } \\
\text { MSLB }\end{array}$ & & 2 & & $\begin{array}{l}\mathrm{H} \\
\text { (drywell) } \\
\mathrm{M} \\
\text { (wetwell) }\end{array}$ & $\mathrm{H}$ & $\begin{array}{l}\text { Modeling differences in mass/energy } \\
\text { transport from the wetwell pool to gas } \\
\text { bubbles were mainly responsible for the } \\
\text { medium ranking in the wetwell. }\end{array}$ \\
\hline
\end{tabular}

${ }^{2} \mathrm{H}$ - prediction of prime quantity (pressure, temperature, or concentration) to within $10 \%$ and trends predicted; $\mathrm{M}$ - prediction of prime quantity to within 20 to $40 \%$ and trends predicted; $\mathrm{L}$ - prediction of prime quantity less than $50 \%$ and only a few trends predicted

bJ. Tills, Modeling and Analysis Department, Sandia National Laboratories, Albuquerque, NM, letter report “Analysis of the HDR Test E11.2," 1989.

${ }^{`}$ Not applicable (not measured or reported)

${ }^{\mathrm{G}} \mathrm{Gas}$ temperature comparison only

cJ. Green and K. Almenas, Nuclear Engineering Program, University of Maryland, College Park, MD," letter report to the USNRC with title "Modeling of the HDR E11.4 Experiment Using CONTAIN 1.12," March 1992.

'Test similar to ISP-23 (T31.5), except the break room was located at a slightly higher elevation, in same location as in ISP-29 
Table C-4 Validation Matrix for Heat and Mass Transfer Modeling

\begin{tabular}{|c|c|c|c|c|c|c|c|c|}
\hline $\begin{array}{l}\text { Validation } \\
\text { Type/Basis }\end{array}$ & Reference & $\begin{array}{l}\text { Code } \\
\text { Version }\end{array}$ & $\begin{array}{c}\text { Transfer } \\
\text { Type/ } \\
\text { Regime }\end{array}$ & $\begin{array}{c}\text { Gas } \\
\text { Mixture }\end{array}$ & Geometry & $\begin{array}{l}\text { Heat Flux, q } \\
\left(\mathrm{W} / \mathrm{m}^{2}\right) \text {, or } \mathrm{h} \\
\left(\mathrm{W} / \mathrm{m}^{2}-\mathrm{K}\right)\end{array}$ & $\begin{array}{c}\text { Model } \\
\text { Ranking }\end{array}$ & Comments \\
\hline \multirow{2}{*}{$\begin{array}{l}\text { Integral } \\
\text { effects/ LACE } \\
\text { LA-4 }\end{array}$} & \multirow[t]{2}{*}{ Gel90 } & \multirow[t]{2}{*}{1.11} & \multirow{8}{*}{$\begin{array}{l}\text { Condensa- } \\
\text { tion/natural }\end{array}$} & \multirow[t]{8}{*}{ Air/steam } & \multirow{2}{*}{$\begin{array}{l}\text { Vertical steel } \\
\text { wall, CSTF } \\
\text { facility }\end{array}$} & $q(0-1500)$ & $\mathrm{H}$ & \multirow{2}{*}{$\begin{array}{l}\text { Steam injected into a steel vessel at a low } \\
\text { elevation resulted in nearly steady state } \\
\text { conditions. Flux and coefficient data were } \\
\text { obtained from instrumented locations } \\
\text { along the vessel vertical steel wall. }\end{array}$} \\
\hline & & & & & & $h(0-300)$ & M-H & \\
\hline \multirow{2}{*}{$\begin{array}{l}\text { Integral } \\
\text { effects/ HDR } \\
\text { E11.4 }\end{array}$} & \multirow{2}{*}{$\begin{array}{l}\text { See } \\
\text { Note a }\end{array}$} & \multirow{2}{*}{1.12} & & & \multirow{2}{*}{$\begin{array}{l}\text { Concrete block; } \\
\text { lead block }\end{array}$} & & & \multirow{2}{*}{$\begin{array}{l}\text { Steam was injected into a } \\
\text { compartmentalized large scale } \\
\text { containment -- transient and steady state } \\
\text { responses were investigated for structures } \\
\text { at various locations within the contain- } \\
\text { ment. }\end{array}$} \\
\hline & & & & & & h $(0-200)$ & $\mathrm{M}-\mathrm{H}$ & \\
\hline $\begin{array}{l}\text { Separate } \\
\text { effects/SAFE } \\
\text { (Uchida data) }\end{array}$ & $\begin{array}{l}\text { (See } \\
\text { Chapter } \\
13)\end{array}$ & 1.2 & & & $\begin{array}{l}\text { Vertical cooled } \\
\text { plate }\end{array}$ & h $(100-1000)$ & $\mathrm{H}$ & $\begin{array}{l}\text { CONTAIN results were compared to the } \\
\text { Uchida data [Uch65] for steady state } \\
\text { condensation in the presence of air. The } \\
\text { results of the analyses confirmed } \\
\text { conclusions by Peterson [Pet } 96] \text { that the } \\
\text { Uchida data can be reproduced by an } \\
\text { HTMA treatment. }\end{array}$ \\
\hline \multirow{3}{*}{$\begin{array}{l}\text { Integral } \\
\text { effects/ WEC } \\
\text { LST Phase } 2 \text { \& } \\
3\end{array}$} & \multirow[t]{3}{*}{ Ti196 } & \multirow[t]{3}{*}{1.12} & & & \multirow{3}{*}{$\begin{array}{l}\text { Vertical cooled } \\
\text { vessel wall }\end{array}$} & q (15000 - & M-H & \multirow{3}{*}{$\begin{array}{l}\text { Predicted condensation rates were } \\
\text { compared to analyses for a vertical wall } \\
\text { cooled by an evaporating water film that } \\
\text { wetted the exterior of the vessel wall and } \\
\text { were found to be consistent with } \\
\text { experiment. }\end{array}$} \\
\hline & & & & & & & & \\
\hline & & & & & & h $(300-700)$ & M-H & \\
\hline \multirow{2}{*}{$\begin{array}{l}\text { Integral } \\
\text { effects/ WEC } \\
\text { LST Phase 2 \& } \\
3\end{array}$} & \multirow[t]{2}{*}{ Til96 } & \multirow[t]{2}{*}{1.12} & \multirow[t]{2}{*}{$\begin{array}{l}\text { Evaporation/ } \\
\text { forced }\end{array}$} & \multirow[t]{2}{*}{$\begin{array}{l}\text { Air/water } \\
\text { vapor }\end{array}$} & \multirow{2}{*}{$\begin{array}{l}\text { Vertical heated } \\
\text { vessel wall } \\
\text { within a duct }\end{array}$} & $\begin{array}{l}q(15000- \\
20000)\end{array}$ & M-H & \multirow[t]{2}{*}{$\begin{array}{l}\text { Duct heat transfer was simulated using a } \\
\text { Colburn equation for forced convection. }\end{array}$} \\
\hline & & & & & & h $(200-400)$ & M-H & \\
\hline
\end{tabular}


Table C-4 Validation Matrix for Heat and Mass Transfer Modeling (Concluded)

\begin{tabular}{|c|c|c|c|c|c|c|c|c|}
\hline $\begin{array}{l}\text { Validation } \\
\text { Type/Basis }\end{array}$ & Reference & $\begin{array}{l}\text { Code } \\
\text { Version }\end{array}$ & $\begin{array}{l}\text { Transfer } \\
\text { Type/ } \\
\text { Regime }\end{array}$ & $\begin{array}{c}\text { Gas } \\
\text { Mixture }\end{array}$ & Geometry & $\begin{array}{c}\text { Heat Flux, } q \\
\left(\mathrm{~W} / \mathrm{m}^{2}\right) \text {, or } \mathrm{h} \\
\left(\mathrm{W} / \mathrm{m}^{2}-\mathrm{K}\right) \text {, or } \\
\text { Other }\end{array}$ & $\begin{array}{c}\text { Model } \\
\text { Ranking }\end{array}$ & Comments \\
\hline $\begin{array}{l}\text { Separate } \\
\text { effects/ } \\
\text { GE } \\
\text { PANTHERS }\end{array}$ & $\begin{array}{l}\text { See } \\
\text { Note b }\end{array}$ & 1.2 & $\begin{array}{l}\text { Condensa- } \\
\text { tion/forced }\end{array}$ & Air/steam & $\begin{array}{l}\text { Vertical } \\
\text { condenser tube } \\
\text { cooled by water } \\
\text { bath }\end{array}$ & $\begin{array}{l}q(80000- \\
100000)\end{array}$ & $\mathbf{H}$ & $\begin{array}{l}\text { CONTAIN results were compared to total } \\
\text { heat removal for a range of air/steam } \\
\text { concentrations and flow rates. Pressures } \\
\text { ranged from } 1.7 \text { to } 3.9 \text { bars; Reynolds } \\
\text { number from } 12,000 \text { to } 14,000 \text {; inlet air } \\
\text { concentrations from } 0.01 \text { to } 0.1 \text {. }\end{array}$ \\
\hline \multirow{3}{*}{$\begin{array}{l}\text { Separate } \\
\text { effects/ } \\
\text { UCB } \\
\text { condenser tests }\end{array}$} & \multirow[t]{3}{*}{$\begin{array}{l}\text { See } \\
\text { Note c }\end{array}$} & \multirow[t]{3}{*}{1.12} & \multirow[t]{3}{*}{$\begin{array}{l}\text { Condensa- } \\
\text { tion/forced }\end{array}$} & \multirow[t]{2}{*}{$\begin{array}{l}\text { Pure } \\
\text { steam }\end{array}$} & \multirow{3}{*}{$\begin{array}{l}\text { Vertical } \\
\text { condenser tube } \\
\text { cooled by } \\
\text { exterior water } \\
\text { jacket }\end{array}$} & $\begin{array}{l}q(80000- \\
100000)\end{array}$ & $\mathrm{H}$ & \multirow{3}{*}{$\begin{array}{l}\text { The prediction of Nusselt condensate film } \\
\text { formation was compared to experiment } \\
\text { with the intent of simulating the Passive } \\
\text { Containment Cooling System (PCCS) } \\
\text { proposed for the GE Simplified Boiling } \\
\text { Water Reactor (SBWR). }\end{array}$} \\
\hline & & & & & & $\begin{array}{l}\text { h }(5000- \\
10000)\end{array}$ & $\mathrm{H}$ & \\
\hline & & & & $\begin{array}{l}\text { Air/ } \\
\text { steam }\end{array}$ & & $h(200-3000)$ & M-H & \\
\hline \multirow{2}{*}{$\begin{array}{l}\text { Code-to-Code/ } \\
\text { Purdue } \\
\text { Boundary } \\
\text { Layer Calc. }\end{array}$} & \multirow[t]{2}{*}{ Vij97 } & \multirow[t]{2}{*}{1.2} & \multirow[t]{2}{*}{$\begin{array}{l}\text { Evaporation/ } \\
\text { forced and } \\
\text { natural }\end{array}$} & \multirow[t]{2}{*}{$\begin{array}{l}\text { Air/water } \\
\text { vapor }\end{array}$} & \multirow{2}{*}{$\begin{array}{l}\text { Asymmetrically } \\
\text { heated vertical } \\
\text { channel cooled } \\
\text { by falling water } \\
\text { film }\end{array}$} & $\begin{array}{l}q(14000 \text { - } \\
18000)\end{array}$ & M-H & \multirow{2}{*}{$\begin{array}{l}\text { The CONTAIN simulation used the } \\
\text { default mixed convection model with the } \\
\text { default natural convection model and a } \\
\text { Colburn equation for the forced } \\
\text { convection model. Comparisons were } \\
\text { made to a finite difference boundary layer } \\
\text { code developed by Purdue University. }\end{array}$} \\
\hline & & & & & & $\begin{array}{l}\mathrm{N}_{\mathrm{Nu}} \text { and } \mathrm{N}_{\mathrm{Sh}} \\
(25-30)\end{array}$ & M-H & \\
\hline
\end{tabular}

aJ. Green and K. Almenas, Nuclear Engineering Program, University of Maryland, College Park, MD, letter report to the USNRC with title "Modeling of the HDR E11.4 Experiment Using CONTAIN 1.12," March 1992.

'J. Tills, Modeling and Analysis Department, Sandia National Laboratories, Albuquerque, NM, letter report to the USNRC with title "Analysis of the GE PANTHERS Tests Using the CONTAIN Code," June 1996.

'J. Tills, Modeling and Analysis Department, Sandia National Laboratories, Albuquerque, NM, letter report to the USNRC with title "Letter Report on PCCS Modeling for SBWR," March 1994. 
Table C-5 Validation Matrix for Heat and Mass Transfer Modeling (Non-CONTAIN Comparisons)

\begin{tabular}{|c|c|c|c|c|c|c|c|c|}
\hline $\begin{array}{l}\text { Validation } \\
\text { Type/Basis }\end{array}$ & $\begin{array}{l}\text { Refer- } \\
\text { ence }\end{array}$ & $\begin{array}{l}\text { Transferl } \\
\text { Regime }\end{array}$ & Mixture & Model & Geometry & $\begin{array}{c}\text { Parameter } \\
\mathrm{h}\left(\mathrm{W} / \mathrm{m}^{2}-\mathrm{K}\right), \text { or } \\
\text { Other }\end{array}$ & $\begin{array}{c}\text { Model } \\
\text { Ranking }\end{array}$ & Comments \\
\hline $\begin{array}{l}\text { Separate } \\
\text { effects/Uchida } \\
\text { tests }\end{array}$ & Pet96 & $\begin{array}{l}\text { Condensation/ } \\
\text { turbulent natural }\end{array}$ & $\begin{array}{l}\text { Steam/air, } \\
\text { steam } / \mathrm{N}_{2} \\
\text { steam } / \mathrm{Ar}\end{array}$ & HMTA $^{\mathrm{a}}$ & $\begin{array}{l}\text { Vertical plate } \\
\text { in small } \\
\text { vessel (thin } \\
\text { film) }\end{array}$ & $h(100-1500)$ & $\mathrm{H}$ & $\begin{array}{l}\text { Steady state analysis } \\
\text { confirmed the one-to-one } \\
\text { correspondence between } \\
\text { HTMA and the Uchida } \\
\text { correlation. }\end{array}$ \\
\hline \multirow{2}{*}{$\begin{array}{l}\text { Separate } \\
\text { effects/ } \\
\text { ECOTRA }\end{array}$} & \multirow{2}{*}{ Ver87 } & $\begin{array}{l}\text { Condensation/ } \\
\text { laminar forced }\end{array}$ & \multirow{2}{*}{ Steam/air } & \multirow{2}{*}{ HMTA } & \multirow{2}{*}{$\begin{array}{l}\text { Vertical plate } \\
\text { in small } \\
\text { vessel (thin } \\
\text { film) }\end{array}$} & $h(100-7000)$ & $\mathbf{L}$ & $\begin{array}{l}\text { Predictions were conserva- } \\
\text { tive, with small scatter in } \\
\text { data }\end{array}$ \\
\hline & & $\begin{array}{l}\text { Condensation/ } \\
\text { turbulent natural }\end{array}$ & & & & $h(100-1500)$ & L-H & $\begin{array}{l}\text { Predictions were conserva- } \\
\text { tive, with large scatter in } \\
\text { data }\end{array}$ \\
\hline \multirow{3}{*}{$\begin{array}{l}\text { Separate } \\
\text { effects/ } \\
\text { Goodykoontz } \\
\text { experiment }\end{array}$} & \multirow{3}{*}{$\operatorname{Kim} 90$} & \multirow{3}{*}{$\begin{array}{l}\text { Condensation/ } \\
\text { turbulent forced at } \\
\text { high velocity, } v= \\
53.3 \mathrm{~m} / \mathrm{s}\end{array}$} & \multirow{3}{*}{ Steam } & \multirow{3}{*}{$\begin{array}{l}\text { Condensate film } \\
\text { model } \\
\text { comparison }\end{array}$} & \multirow{3}{*}{$\begin{array}{l}\text { Condensa- } \\
\text { tion in a } \\
\text { vertical tube }\end{array}$} & \multirow{3}{*}{$\begin{array}{l}\mathrm{h}(5000-30000) \\
\text { (local) }\end{array}$} & M-H & $\begin{array}{l}\text { Turbulent film model with } \\
\text { smooth interface }\end{array}$ \\
\hline & & & & & & & L-H & $\begin{array}{l}\text { Laminar film with wavy } \\
\text { interface }\end{array}$ \\
\hline & & & & & & & $\mathbf{H}$ & $\begin{array}{l}\text { All models in the low } h \\
\text { range. }\end{array}$ \\
\hline
\end{tabular}


Table C-5 Validation Matrix for Heat and Mass Transfer Modeling (Non-CONTAIN Comparisons) (Continued)

\begin{tabular}{|c|c|c|c|c|c|c|c|c|}
\hline $\begin{array}{l}\text { Validation } \\
\text { Type/Basis }\end{array}$ & $\begin{array}{c}\text { Refer- } \\
\text { ence }\end{array}$ & $\begin{array}{c}\text { Transfer Type/ } \\
\text { Regime }\end{array}$ & $\begin{array}{c}\text { Gas } \\
\text { Mixture }\end{array}$ & Model & Geometry & $\begin{array}{c}\text { Parameter } \\
\mathrm{h}\left(\mathrm{W} / \mathrm{m}^{2}-\mathrm{K}\right), \text { or } \\
\text { Other }\end{array}$ & \begin{tabular}{c|} 
Model \\
Ranking
\end{tabular} & Comments \\
\hline $\begin{array}{l}\text { Separate } \\
\text { effects/Asano } \\
\text { tests }\end{array}$ & Kim90 & $\begin{array}{l}\text { Condensation/ } \\
\text { laminar forced }\end{array}$ & $\begin{array}{l}\text { Steam/air } \\
\text { (air mass } \\
\text { fractions } \\
\text { from } 0.02 \text { - } \\
0.6 \text { ) }\end{array}$ & $\begin{array}{l}\text { HMTA with } \\
\text { laminar } \\
\text { condensate film }\end{array}$ & $\begin{array}{l}\text { Vertical flat } \\
\text { plate, } 20.8 \\
\text { mm long }\end{array}$ & Total heat flux & M-H & $\begin{array}{l}\text { The flux was underpredicted } \\
\text { at low air mass fractions and } \\
\text { overpredicted at high air } \\
\text { mass fractions }\end{array}$ \\
\hline \multirow{2}{*}{$\begin{array}{l}\text { Integral } \\
\text { blowdown test/ } \\
\text { CVTR }\end{array}$} & & \multirow{2}{*}{$\begin{array}{l}\text { Condensation/ } \\
\text { turbulent forced } \\
(6-10 \mathrm{~m} / \mathrm{s})\end{array}$} & \multirow[t]{2}{*}{ Steam/air } & \multirow{2}{*}{$\begin{array}{l}\text { HMTA with } \\
\text { laminar film with } \\
\text { and without } \\
\text { waves }\end{array}$} & \multirow{2}{*}{$\begin{array}{l}\text { Vertical heat } \\
\text { plug on large } \\
\text { vessel wall }\end{array}$} & \multirow[t]{2}{*}{$h(100-1500)$} & $\mathrm{H}$ & $\begin{array}{l}\text { Applicable to low h, } 100- \\
700 \text {, with and without waves }\end{array}$ \\
\hline & & & & & & & M-H & $\begin{array}{l}\text { Applicable to high h. } \\
\text { Laminar with waves gave } \\
\text { better results; without waves } \\
\text { conservative results. }\end{array}$ \\
\hline $\begin{array}{l}\text { Integral } \\
\text { blowdown test/ } \\
\text { CVTR }\end{array}$ & Kro78 & $\begin{array}{l}\text { Condensation/ } \\
\text { turbulent forced } \\
(3-5 \mathrm{~m} / \mathrm{s})\end{array}$ & Steam/air & $\begin{array}{l}\text { HMTA with } \\
\text { laminar film }\end{array}$ & $\begin{array}{l}\text { Large vertical } \\
\text { wall of vessel }\end{array}$ & $h(100-1500)$ & $\mathrm{L}$ & $\begin{array}{l}\text { A single cell representation } \\
\text { of a blowdown vessel that } \\
\text { was not well mixed } \\
\text { produced transient heat } \\
\text { transfer coefficients that } \\
\text { were significantly over- } \\
\text { predicted even though the } \\
\text { maximum predicted } \\
\text { coefficients agreed within a } \\
\text { medium ranking with the } \\
\text { measured peak values. }\end{array}$ \\
\hline $\begin{array}{l}\text { Separate } \\
\text { effects/MIT } \\
\text { steam con- } \\
\text { densation } \\
\text { experiment }\end{array}$ & Has96a & $\begin{array}{l}\text { Condensation/ } \\
\text { turbulent natural }\end{array}$ & Steam/air & $\begin{array}{l}\text { HMTA with film } \\
\text { equations as in } \\
\text { RELAP5-MOD3 }\end{array}$ & $\begin{array}{l}\text { Vertical } \\
\text { cylinder, } \\
\text { outside } \\
\text { condensation }\end{array}$ & $h(200-1000)$ & M-H & $\begin{array}{l}\text { The heat transfer } \\
\text { coefficients were over- } \\
\text { predicted (local analysis). }\end{array}$ \\
\hline
\end{tabular}


Table C-5 Validation Matrix for Heat and Mass Transfer Modeling (Non-CONTAIN Comparisons) (Continued)

\begin{tabular}{|c|c|c|c|c|c|c|c|c|}
\hline $\begin{array}{l}\text { Validation } \\
\text { Type/Basis }\end{array}$ & $\begin{array}{l}\text { Refer- } \\
\text { ence }\end{array}$ & $\begin{array}{l}\text { Transfer Type/ } \\
\text { Regime }\end{array}$ & $\begin{array}{c}\text { Gas } \\
\text { Mixture }\end{array}$ & Model & Geometry & $\begin{array}{c}\text { Parameter } \\
\mathrm{h}\left(\mathrm{W} / \mathrm{m}^{2}-\mathrm{K}\right), \text { or } \\
\text { Other }\end{array}$ & $\begin{array}{c}\text { Model } \\
\text { Ranking }\end{array}$ & Comments \\
\hline $\begin{array}{l}\text { Separate } \\
\text { effects/MIT } \\
\text { single tube } \\
\text { experiments }\end{array}$ & Has96a & $\begin{array}{l}\text { Condensation/ } \\
\text { turbulent forced }\end{array}$ & Steam/air & $\begin{array}{l}\text { HMTA with film } \\
\text { equations }\end{array}$ & $\begin{array}{l}\text { Vertical tube, } \\
\text { inside } \\
\text { condensation }\end{array}$ & $\begin{array}{l}\mathrm{h}(2000-8000) \\
\text { (local) }\end{array}$ & L-M & $\begin{array}{l}\text { The heat transfer } \\
\text { coefficients were over- } \\
\text { predicted (local analysis). }\end{array}$ \\
\hline $\begin{array}{l}\text { Separate } \\
\text { effects/UCB } \\
\text { single tube } \\
\text { experiments }\end{array}$ & Has96a & $\begin{array}{l}\text { Condensation/ } \\
\text { turbulent forced }\end{array}$ & Steam $/ \mathbf{N}_{2}$ & & $\begin{array}{l}\text { Vertical tube, } \\
\text { inside } \\
\text { condensation }\end{array}$ & $\begin{array}{l}\mathrm{h}(0-7000) \\
\text { (local) }\end{array}$ & L & $\begin{array}{l}\text { The heat transfer } \\
\text { coefficients were over- } \\
\text { predicted (local analysis). }\end{array}$ \\
\hline $\begin{array}{l}\text { Separate } \\
\text { effects/MIT } \\
\text { single tube } \\
\text { experiments }\end{array}$ & Has $96 \mathrm{~b}$ & $\begin{array}{l}\text { Condensation/ } \\
\text { turbulent forced }\end{array}$ & $\begin{array}{l}\text { Steam/air/ } \\
\text { He }\end{array}$ & $\begin{array}{l}\text { HMTA with film } \\
\text { equations }\end{array}$ & $\begin{array}{l}\text { Vertical tube, } \\
\text { inside } \\
\text { condensation }\end{array}$ & $h(500-4500)$ & $\mathrm{H}$ & $\begin{array}{l}\text { Results of local analysis } \\
\text { were compared. }\end{array}$ \\
\hline $\begin{array}{l}\text { Separate } \\
\text { effects/Kim } \\
\text { experiment }\end{array}$ & Kan94 & $\begin{array}{l}\text { Condensation/ } \\
\text { turbulent forced }\end{array}$ & $\begin{array}{l}\text { Steam/air } \\
\text { (air mass } \\
\text { fraction = } \\
0.4 \text { ) } \\
\end{array}$ & $\begin{array}{l}\text { HMTA with film } \\
\text { equations }\end{array}$ & $\begin{array}{l}\text { Horizontal } \\
\text { plate }\end{array}$ & Total heat flux & $\mathrm{H}$ & $\begin{array}{l}\text { Laminar condensate flow } \\
\text { without waves was present. }\end{array}$ \\
\hline $\begin{array}{l}\text { Separate } \\
\text { effects/Pisa } \\
\text { experiments }\end{array}$ & Amb95 & $\begin{array}{l}\text { Evaporation/ } \\
\text { turbulent forced ( } 0 \\
\text { to } 10 \mathrm{~m} / \mathrm{s})\end{array}$ & Water/air & HMTA & Vertical duct & $\mathrm{N}_{\mathrm{Sh}}(90-400)$ & M-H & $\begin{array}{l}\text { AP600 PCS wall evapora- } \\
\text { tion was studied within a } \\
\text { thin film approximation. }\end{array}$ \\
\hline $\begin{array}{l}\text { Separate } \\
\text { effects/ } \\
\text { U. Wisc. } \\
\text { experiments }\end{array}$ & Huh93 & $\begin{array}{l}\text { Condensation/ } \\
\text { turbulent forced (1 } \\
\text { to } 3 \mathrm{~m} / \mathrm{s} \text { ) }\end{array}$ & Steam/air & HMTA & $\begin{array}{l}\text { Horizontal to } \\
\text { vertical duct }\end{array}$ & $h(100-1000)$ & $\mathrm{M}-\mathrm{H}$ & $\begin{array}{l}\text { Condensation on inclined } \\
\text { ceilings was studied. }\end{array}$ \\
\hline
\end{tabular}

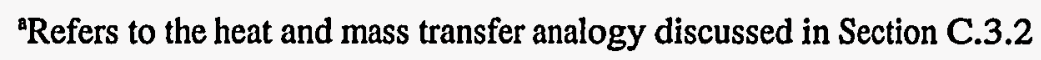


Table C-5 Validation Matrix for Heat and Mass Transfer Modeling (Non-CONTAIN Comparisons) (Concluded)

\begin{tabular}{|c|c|c|c|c|c|c|c|c|}
\hline $\begin{array}{l}\text { Validation } \\
\text { Type/Basis }\end{array}$ & $\begin{array}{l}\text { Refer- } \\
\text { ence }\end{array}$ & $\begin{array}{l}\text { Transfer Type/ } \\
\text { Regime }\end{array}$ & $\begin{array}{c}\text { Gas } \\
\text { Mixture }\end{array}$ & Model & Geometry & $\begin{array}{c}\text { Parameter } \\
h\left(W / m^{2}-K\right), \text { or } \\
\text { Other }\end{array}$ & $\begin{array}{c}\text { Model } \\
\text { Ranking }\end{array}$ & Comments \\
\hline $\begin{array}{l}\text { Separate } \\
\text { effects/Fujii } \\
\text { experiments }\end{array}$ & Fuj93 & $\begin{array}{l}\text { Condensation/ } \\
\text { laminar forced and } \\
\text { laminar natural }\end{array}$ & $\begin{array}{l}\text { Ethanol/ } \\
\text { water and } \\
\text { air/water }\end{array}$ & $\begin{array}{l}\text { Similarity } \\
\text { solutions of } \\
\text { boundary layers }\end{array}$ & $\begin{array}{l}\text { Vertical flat } \\
\text { plate }\end{array}$ & Similarity ratio & $\mathrm{H}$ & $\begin{array}{l}\text { Provided a fundamental } \\
\text { theoretical confirmation of } \\
\text { the analogy between } \\
\text { convection and mass transfer } \\
\text { for forced and natural } \\
\text { convection condensation }\end{array}$ \\
\hline $\begin{array}{l}\text { Separate } \\
\text { effects/Kroger } \\
\text { experiments }\end{array}$ & Kro68 & $\begin{array}{l}\text { Condensation/ } \\
\text { stagnant }\end{array}$ & $\begin{array}{l}\text { Potassium/ } \\
\text { helium }\end{array}$ & Diffusion analysis & $\begin{array}{l}\text { Under-side of } \\
\text { a horizontal } \\
\text { plate }\end{array}$ & $h(40-4000)$ & $\mathrm{M}-\mathrm{H}$ & $\begin{array}{l}\text { Provided basic confirmation } \\
\text { of condensation heat transfer } \\
\text { in the presence of noncon- } \\
\text { densable gas through the } \\
\text { mechanism of molecular } \\
\text { diffusion }\end{array}$ \\
\hline $\begin{array}{l}\text { Separate } \\
\text { effects/ } \\
\text { Borishansky } \\
\text { experiments }\end{array}$ & Wan88 & $\begin{array}{l}\text { Condensation/ } \\
\text { turbulent forced }\end{array}$ & Steam/air & $\begin{array}{l}\text { HMTA with } \\
\text { laminar film flow, } \\
\text { interfacial shear }\end{array}$ & $\begin{array}{l}\text { Vertical tube, } \\
\text { inside con- } \\
\text { densation }\end{array}$ & Total heat flux & $\mathrm{H}$ & $\begin{array}{l}\text { Condensation was studied in } \\
\text { a confined space. }\end{array}$ \\
\hline \multirow{2}{*}{$\begin{array}{l}\text { Separate } \\
\text { effects/UCB - } \\
\text { MIT experi- } \\
\text { ments }\end{array}$} & \multirow[t]{2}{*}{ Kag93 } & \multirow[t]{2}{*}{$\begin{array}{l}\text { Condensation/ } \\
\text { turbulent forced }\end{array}$} & \multirow{2}{*}{$\begin{array}{l}\text { Steam/air; } \\
\text { steam/ } \\
\text { helium }\end{array}$} & \multirow[t]{2}{*}{$\begin{array}{l}\text { HMTA with film } \\
\text { equations }\end{array}$} & \multirow{2}{*}{$\begin{array}{l}\text { Vertical tube, } \\
\text { inside con- } \\
\text { densation }\end{array}$} & \multirow[t]{2}{*}{ Total heat flux } & M-H & $\begin{array}{l}\text { Applicable to steam/air, with } \\
\text { predictions conservative }\end{array}$ \\
\hline & & & & & & & L-M & $\begin{array}{l}\text { Applicable to steam/helium, } \\
\text { with predictions conserva- } \\
\text { tive }\end{array}$ \\
\hline
\end{tabular}


Table C-6 Validation Matrix for DCH Modeling

\begin{tabular}{|c|c|c|c|c|c|c|c|}
\hline \multirow{2}{*}{$\begin{array}{l}\text { Experiment } \\
\text { (Test } \\
\text { Facility) }\end{array}$} & \multirow{2}{*}{$\begin{array}{l}\text { Experiment } \\
\text { Description }\end{array}$} & \multirow[t]{2}{*}{ Reference } & \multirow[t]{2}{*}{ Nodes } & \multirow{2}{*}{$\begin{array}{l}\text { Code } \\
\text { Version }\end{array}$} & \multicolumn{2}{|c|}{ Model Ranking } & \multirow[t]{2}{*}{ Comments } \\
\hline & & & & & $\Delta \mathrm{P}$ & $\begin{array}{c}\mathrm{H}_{2} \\
\text { Prod. }\end{array}$ & \\
\hline $\begin{array}{l}\text { SNL/DCH-1 } \\
\text { (Surtsey) }\end{array}$ & $\begin{array}{l}\sim 20 \mathrm{~kg} \text { melt, } 1 / 10- \\
\text { scale Surry cavity, } \\
\mathrm{N}_{2} \text { driving gas, air } \\
\text { atmosphere }\end{array}$ & Wil87 & 1 & $\begin{array}{l}1.04 / \\
\text { DCH }\end{array}$ & $\mathrm{H}$ & N/A & $\begin{array}{l}\text { Blind post-test analysis, using the experimentally } \\
\text { determined mass dispersed into containment, gave } \\
\text { excellent agreement with the experimental pressure- } \\
\text { time history. The user-defined trapping rate was based } \\
\text { on gravitational fall time. }\end{array}$ \\
\hline $\begin{array}{l}\text { SNL/DCH-3 } \\
\text { (Surtsey) }\end{array}$ & $\begin{array}{l}\sim 80 \mathrm{~kg} \text { melt, } 1 / 10 \text { - } \\
\text { scale Surry cavity, } \\
\mathrm{N}_{2} \text { driving gas, air } \\
\text { atmosphere }\end{array}$ & $\begin{array}{l}\text { Wil88, } \\
\text { Tar88 }\end{array}$ & 1,3 & $\begin{array}{l}1.1 / \\
\text { DCH }\end{array}$ & $\mathrm{L}$ & N/A & $\begin{array}{l}\text { Large overprediction of } \Delta \mathrm{P} \text { occurred when the } \\
\text { gravitational fall rate was used to estimate the trapping } \\
\text { rate. Agreement was improved by using estimated time } \\
\text { to reach dome [Wil88] or subdividing containment to } \\
\text { represent debris self-shielding from the full } \\
\text { containment atmosphere ("cloud effect") [Tar88]. }\end{array}$ \\
\hline $\begin{array}{l}\text { SNL/WC-1, 3; } \\
\text { SNL/LFP-8A } \\
\text { (Surtsey) }\end{array}$ & $\begin{array}{l}50 \mathrm{~kg} \text { melt, } 1 / 10 \text { - } \\
\text { scale Zion cavity, } \\
\text { steam-driven, open } \\
\text { containment } \\
\text { geometry, } \mathrm{Ar} \\
\text { atmosphere }\end{array}$ & Wil $^{\mathrm{a}}$ & $\begin{array}{l}5(1 \\
\text { node for } \\
\text { main } \\
\text { volume) }\end{array}$ & $1.12 \mathrm{Z}$ & M-H & M-H & $\begin{array}{l}\text { Used DCH standard prescription (DCHSP) (see Section } \\
\text { 13.3.2.2, for details). } \triangle \mathrm{P} \text { was overpredicted by } 8-17 \% \text {; } \\
\text { this was somewhat sensitive to trapping and some other } \\
\text { trapping options would give a greater overprediction. } \\
\text { Some sensitivity to the particle size was presumably } \\
\text { present in the calculation. }\end{array}$ \\
\hline $\begin{array}{l}\text { SNL/WC-2 } \\
\text { (Surtsey) }\end{array}$ & $\begin{array}{l}\text { As in WC-1 } \\
\text { except } 11.76 \mathrm{~kg} \\
\text { water in cavity }\end{array}$ & Wil95 & $\begin{array}{l}5(1 \\
\text { node for } \\
\text { main } \\
\text { volume) }\end{array}$ & $1.12 \mathrm{Z}$ & $\mathrm{M}-\mathrm{H}$ & M & $\begin{array}{l}\text { Used DCHSP, which neglected the water. The } \mathrm{H}_{2} \\
\text { production was underpredicted by } \sim 20 \% \text {. Comparison } \\
\text { with WC- } 1 \text { suggested water increased } \mathrm{H}_{2} \text { by } \sim 24 \% \text {. } \\
\text { Assuming } 15 \% \text { water available to interact with debris } \\
\text { overpredicted } \mathrm{H}_{2} \text { production by } \sim 16 \% ; 100 \% \text { water } \\
\text { overpredicted } \mathrm{H}_{2} \text { by factor of } 2 \text {. Water had little effect } \\
\text { on calculated } \Delta \mathrm{P} \text {, in agreement with experiment; note } \\
\text { contrast with compartmentalized geometries in Zion } \\
\text { IET below. }\end{array}$ \\
\hline
\end{tabular}


Table C-6 Validation Matrix for DCH Modeling (Continued)

\begin{tabular}{|c|c|c|c|c|c|c|c|c|}
\hline \multirow{2}{*}{$\begin{array}{c}\text { Experiment } \\
\text { (Test } \\
\text { Facility) }\end{array}$} & \multirow{2}{*}{$\begin{array}{l}\text { Experiment } \\
\text { Description }\end{array}$} & \multirow[t]{2}{*}{ Reference } & \multirow{2}{*}{$\begin{array}{c}\text { Code } \\
\text { Version }\end{array}$} & \multicolumn{4}{|c|}{ Model Ranking } & \multirow[t]{2}{*}{ Comments } \\
\hline & & & & $\Delta \mathrm{P}$ & $\begin{array}{c}\mathrm{H}_{2} \\
\text { Prod. }\end{array}$ & $\underset{\text { Burn }}{\mathrm{H}_{2}}$ & $\begin{array}{c}\text { Debris } \\
\text { Trans- } \\
\text { port }\end{array}$ & \\
\hline $\begin{array}{l}\text { SNL/LFP: } \\
\# 1 \mathrm{~A}, 1 \mathrm{~B}, 2 \mathrm{~A}, \\
2 \mathrm{~B}, 2 \mathrm{C}\end{array}$ & $\begin{array}{l}1 / 10 \text {-scale } \\
\text { Surry cavity, } \\
\text { Steam-driven } \\
\text { melt dispersal, } \\
\text { concrete slab } \\
\text { limits } \\
\text { unobstructed } \\
\text { flight path } \\
\end{array}$ & Wil95 & $1.12 Z$ & L-M & M-H & N/A & $\mathrm{L}$ & $\begin{array}{l}\text { DCHSP overpredicted debris transport to the dome } \\
\left(f_{\text {dome }}\right) \text { by up to an order of magnitude, which } \\
\text { resulted in an overprediction of } \triangle P \text {. Decoupling of } \\
\text { debris trajectories from gas flow was a suggested } \\
\text { cause of the discrepancy. Model options improving } \\
\text { agreement in } f_{\text {dome }} \text { (and } \triangle P \text { ) for LFP underpredicted } \\
\mathrm{f}_{\text {dome }} \text { for IET experiments and were not } \\
\text { recommended for the DCHSP. }\end{array}$ \\
\hline $\begin{array}{l}\text { SNL/IET: } \\
\# 1,1 \mathrm{R} \\
\text { (Surtsey) }\end{array}$ & $\begin{array}{l}1 / 10 \text {-scale Zion } \\
\text { cavity and } \\
\text { containment } \\
\text { structures; } \mathrm{N}_{2} \\
\text { atmosphere; } \\
3.48 \mathrm{~kg} \text { water } \\
\text { in cavity } \\
\end{array}$ & Wil $^{\mathrm{a}}$ & $1.12 Z$ & $\mathrm{M}-\mathrm{H}$ & M-H & N/A & M-H & $\begin{array}{l}\text { Good agreement for } \mathrm{f}_{\text {dome }}{ }^{b} \text { Obtaining good } \\
\text { agreement for } \Delta \mathrm{P} \text { and especially } \mathrm{H}_{2} \text { production } \\
\text { required modeling nonairborne debris (NAD) } \\
\text { interactions and/or interactions of debris with cavity } \\
\text { water. }{ }^{\circ} \text { (SNL/IET-1 was not entirely satisfactory for } \\
\text { code validation because the accumulator pressure } \\
\text { history was anomalous.) }\end{array}$ \\
\hline $\begin{array}{l}\text { SNL/IET: } \\
\# 3,4 \\
\text { (Surtsey) }\end{array}$ & $\begin{array}{l}\text { As in IET-1,1R } \\
\text { except } \sim 50: 50 \\
\text { air- } \mathrm{N}_{2} \text { contain- } \\
\text { ment atm. } \\
\text { Water on } \\
\text { basement floor } \\
\text { in IET-4 }\end{array}$ & Wil95 ${ }^{a}$ & $1.12 Z$ & $\mathrm{M}-\mathrm{H}$ & $\mathrm{M}-\mathrm{H}$ & $\mathrm{M}-\mathrm{H}$ & $M$ & $\begin{array}{l}\text { SNL/IET-1, } 1 \mathrm{R} \text { comments apply. In the calculation, } \\
\text { much of } \mathrm{DCH} \text {-produced } \mathrm{H}_{2} \text { burns in } \mathrm{DFB} \text { mode and } \\
\text { contributes }>50 \% \text { of the total } \triangle \mathrm{P} \text {, in agreement with } \\
\text { experiment. }{ }^{\mathrm{d}} \text { Mitigation by atmosphere-strucure } \\
\text { heat transfer and incomplete } \mathrm{H}_{2} \text { combustion was } \\
\text { very important. }{ }^{\mathrm{C}} \text { Water on the basement floor in } \\
\text { IET-4 was not modeled and made little difference in } \\
\text { the experiment. } \triangle \mathrm{P} \text { and } \mathrm{H}_{2} \text { production was not } \\
\text { sensitive to nodalization but } \mathrm{f}_{\text {dome }} \text { was more } \\
\text { sensitive; } \triangle \mathrm{P} \text { and } \mathrm{H}_{2} \text { production were insensitive to } \\
\mathrm{f}_{\text {dome }} \text { within limits. NAD and/or debris-water } \\
\text { interactions were very important.c }\end{array}$ \\
\hline
\end{tabular}

Rev. 0 
Table C-6 Validation Matrix for DCH Modeling (Continued)

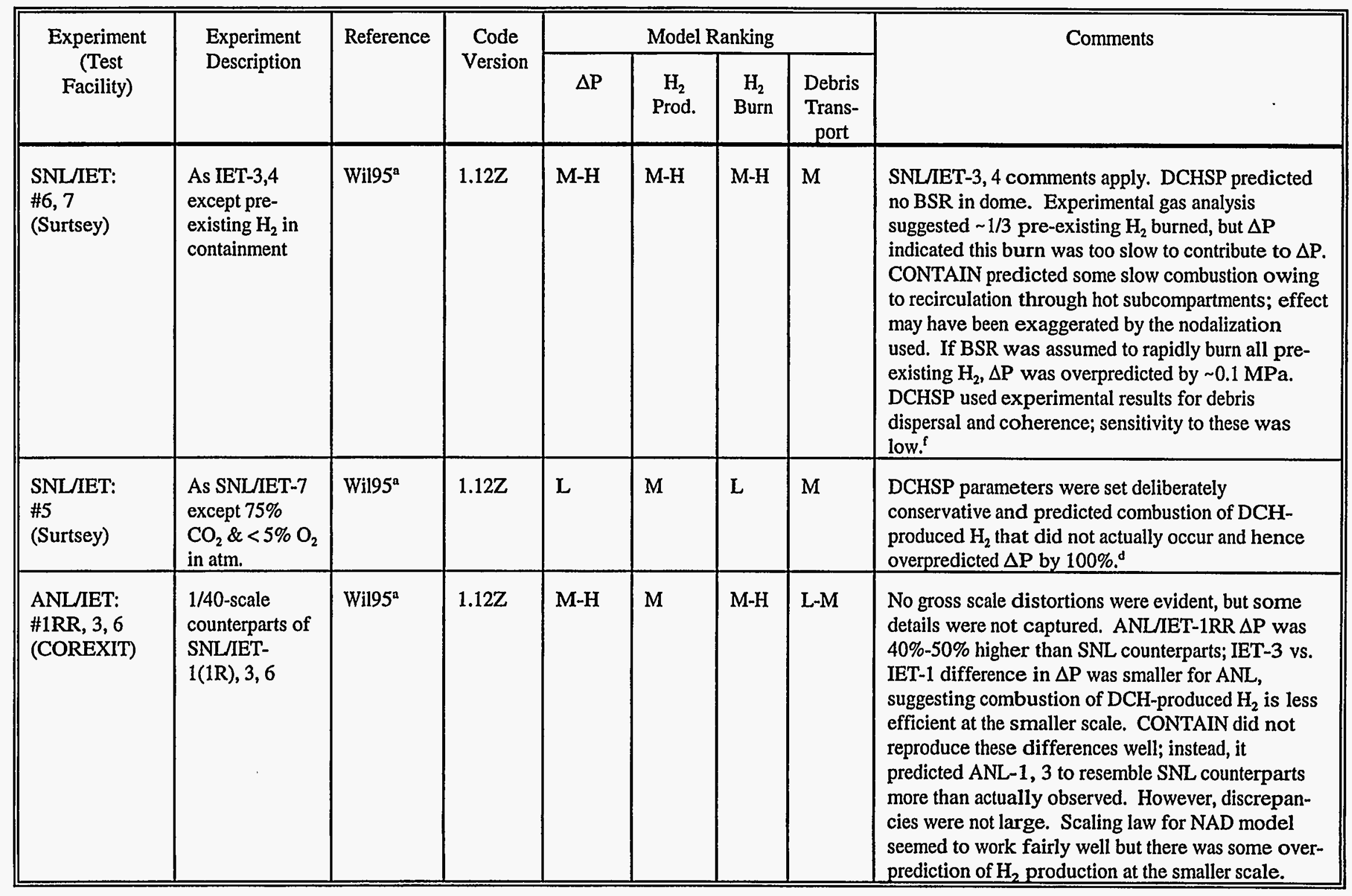


Table C-6 Validation Matrix for DCH Modeling (Continued)

\begin{tabular}{|c|c|c|c|c|c|c|c|c|}
\hline \multirow{2}{*}{$\begin{array}{c}\text { Experiment } \\
\text { (Test } \\
\text { Facility) }\end{array}$} & \multirow{2}{*}{$\begin{array}{l}\text { Experiment } \\
\text { Description }\end{array}$} & \multirow[t]{2}{*}{ Reference } & \multirow{2}{*}{$\begin{array}{c}\text { Code } \\
\text { Version }\end{array}$} & \multicolumn{4}{|c|}{ Model Ranking } & \multirow[t]{2}{*}{ Comments } \\
\hline & & & & $\Delta \mathrm{P}$ & $\begin{array}{c}\mathrm{H}_{2} \\
\text { Prod. }\end{array}$ & $\begin{array}{c}\mathrm{H}_{2} \\
\text { Burn }\end{array}$ & $\begin{array}{c}\text { Debris } \\
\text { Trans- } \\
\text { port }\end{array}$ & \\
\hline $\begin{array}{l}\text { SNL/IET: } \\
9,10,11 \\
\text { (Surtsey) }\end{array}$ & $\begin{array}{l}1 / 6-s c a l e \text { Surry } \\
\text { cavity and } \\
\text { containment } \\
\text { structures; air- } \\
\text { steam } \\
\text { atmosphere; } \\
\text { small amount } \\
\text { of pre-existing } \\
\mathrm{H}_{2}\end{array}$ & Wil95 & $1.12 \mathrm{Z}$ & $\mathrm{H}$ & M-H & $\mathrm{M}-\mathrm{H}$ & M & $\begin{array}{l}\text { DCHSP reproduced well the net effects of the many } \\
\text { differences between the Surry and Zion geometry } \\
\text { IET experiments. The code predicted BSR would } \\
\text { burn pre-existing } \mathrm{H}_{2} ; \text { however pre-existing } \mathrm{H}_{2} \\
\text { amounts were small (equivalent to } 14-24 \% \text { in-vessel } \\
\mathrm{Zr} \text { oxidation) and contributed only } 5-15 \% \text { to } \Delta \mathrm{P} \text {, } \\
\text { and other analysis uncertainties precluded } \\
\text { conclusions about whether pre-existing } \mathrm{H}_{2} \text { actually } \\
\text { contributed to } \triangle \mathrm{P} \text {. Some sensitivity to nodalization } \\
\text { was found, with the } 12 \text {-cell deck yielding } 0.025 \text { - } \\
0.045 \mathrm{MPa} \text { higher } \triangle \mathrm{P} \text { than the 8-cell deck. }\end{array}$ \\
\hline $\begin{array}{l}\text { SNL/IET-9 } \\
\text { (Surtsey) }\end{array}$ & $\begin{array}{l}\text { As described } \\
\text { above }\end{array}$ & $\begin{array}{l}\text { See } \\
\text { Note g }\end{array}$ & $1.12 Z$ & \multicolumn{5}{|c|}{$\begin{array}{l}\text { The principal purpose was verification that the models appeared to be functioning properly. } \\
\text { All RPV models were exercised. Cavity options tested included the user-specified debris } \\
\text { dispersal fraction with Whalley-Hewitt and Levy entrainment rate options, Weber number } \\
\text { debris particle size option, the time-dependent gas flow area option, and both the user- } \\
\text { specified and internally-calculated entrainment time option. Other options were tested in the } \\
\text { course of development and implementation of the models. All models appeared to be } \\
\text { functioning reasonably except that the gas blowthrough model predicted blowthrough } \\
\text { considerably earlier than it actually occurred. The code tended to overpredict } \Delta \mathrm{P} \text { by } 10-20 \% \\
\text { in these analyses, in part because the degree of coherence between blowdown steam and } \\
\text { debris dispersal in the calculation was higher than in the experiment. }\end{array}$} \\
\hline
\end{tabular}


Table C-6 Validation Matrix for DCH Modeling (Continued)

\begin{tabular}{|c|c|c|c|c|}
\hline $\begin{array}{c}\text { Experiment } \\
\text { (Test } \\
\text { Facility) }\end{array}$ & $\begin{array}{l}\text { Experiment } \\
\text { Description }\end{array}$ & Reference & $\begin{array}{c}\text { Code } \\
\text { Version }\end{array}$ & Comments \\
\hline $\begin{array}{l}\text { SNL/IET-3 } \\
\text { (Surtsey) }\end{array}$ & $\begin{array}{l}\text { As described } \\
\text { above }\end{array}$ & Wil96 & $1.12 \mathrm{AB}$ & 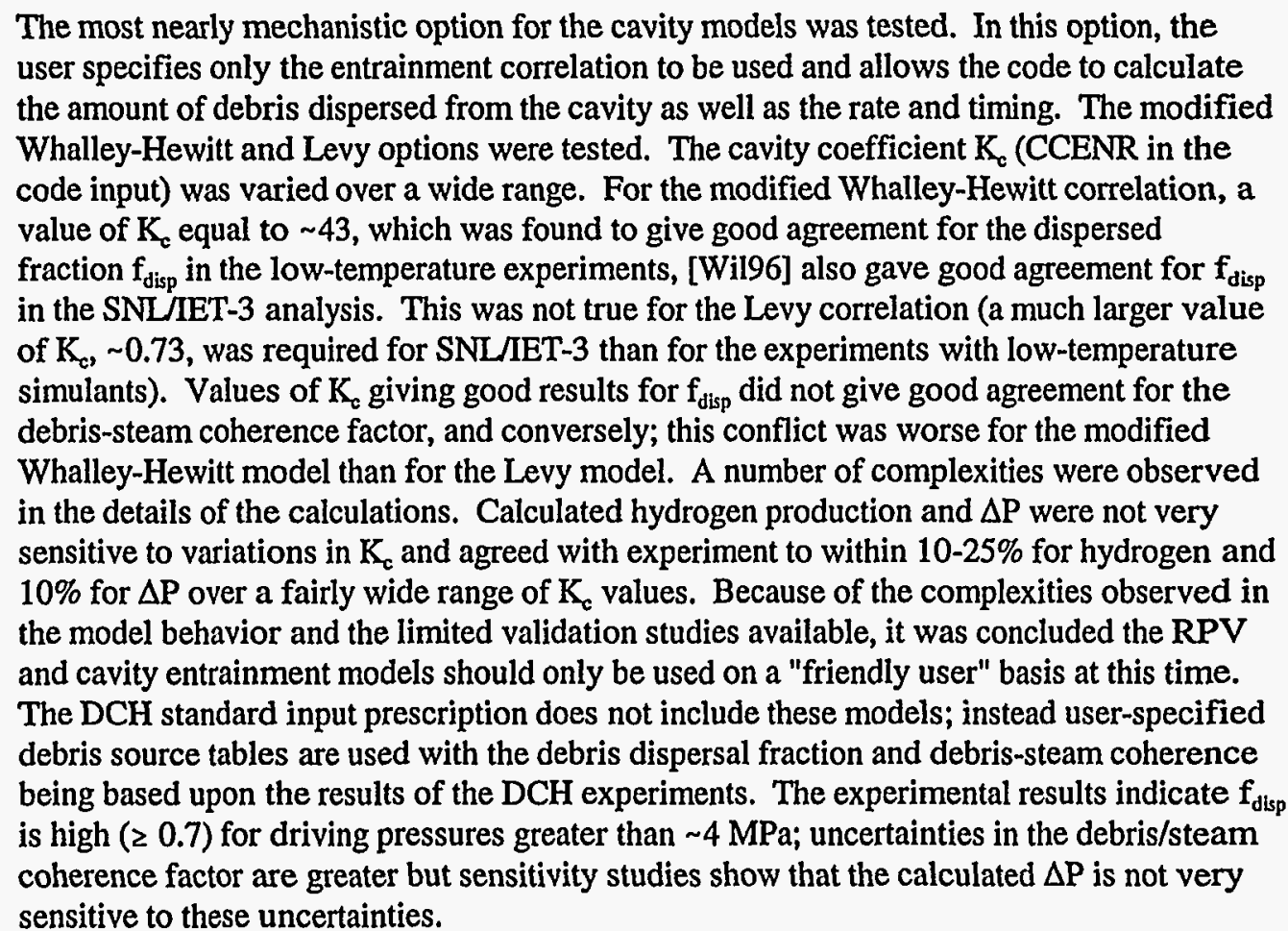 \\
\hline
\end{tabular}


Table C-6 Validation Matrix for DCH Modeling (Concluded)

\begin{tabular}{|c|c|c|c|c|}
\hline $\begin{array}{c}\text { Experiment } \\
\text { (Test } \\
\text { Facility) } \\
\end{array}$ & $\begin{array}{l}\text { Experiment } \\
\text { Description }\end{array}$ & Reference & $\begin{array}{l}\text { Code } \\
\text { Version }\end{array}$ & Comments \\
\hline $\begin{array}{l}\text { LFP and WC } \\
\text { Experiments } \\
\text { (Surtsey) }\end{array}$ & $\begin{array}{l}\text { As described } \\
\text { above }\end{array}$ & Wil92 & N/A & $\begin{array}{l}\text { CONTAIN calculates metal oxidation rates using the gas-phase heat/mass transfer analogy, } \\
\text { and the standard input prescription assumes debris is well mixed and that drop-side limits to } \\
\text { mass transport are negligible. These concepts were tested against the results of the LFP and } \\
\text { WC experiments using an analytical calculation based upon the same heat/mass transfer } \\
\text { analogy as that used elsewhere in CONTAIN. In the LFP and WC experiments, hydrogen } \\
\text { production appeared to be largely limited to debris-steam interactions occurring in the } \\
\text { cavity. The experimental values of hydrogen production and the heat/mass transfer analogy } \\
\text { were used to estimate the amount of debris-gas energy transfer occurring in the cavity. A } \\
\text { simple model incorporating this treatment successfully correlated the experimental values of } \\
\Delta \mathrm{P} \text { to a high degree }\left(\mathrm{R}^{2}>0.98\right) \text {. The model required assuming well-mixed debris and } \\
\text { assuming that drop-side mass transport limits were unimportant, and the model's success } \\
\text { was therefore interpreted as providing support for use of these assumptions in the } \\
\text { CONTAIN DCH standard input prescription. }\end{array}$ \\
\hline $\begin{array}{l}\text { SNL/IET and } \\
\text { ANL/IET } \\
\text { (Surtsey, } \\
\text { COREXIT, } \\
\text { and CTTF) }\end{array}$ & $\begin{array}{l}\text { As described } \\
\text { above }\end{array}$ & Wil95 ${ }^{a}$ & $1.12 Z$ & $\begin{array}{l}\text { The CONTAIN models for non-airborne debris and debris-water interactions include some } \\
\text { parametric features, and calculated results for the IET experiments (especially the Zion IET } \\
\text { experiments) exhibited some sensitivity to these features. However, the ratio of energy } \\
\text { transfer to hydrogen produced in the calculation is governed by the heat/mass transfer } \\
\text { analogy and is considerably less sensitive to the parametric features of the model. In } \\
\text { sensitivity studies, it was found that the code either predicted both } \triangle \mathrm{P} \text { and } \mathrm{H}_{2} \text { production } \\
\text { reasonably well for the Zion IET experiments or else failed to predict either } \Delta \mathrm{P} \text { or } \mathrm{H}_{2} \text { well, } \\
\text { which was interpreted as providing additional support for use of the CONTAIN heat/mass } \\
\text { transfer analogy in calculating hydrogen production. }\end{array}$ \\
\hline
\end{tabular}

${ }^{\mathrm{A}}$ Reference Wi195 provides a relatively high-level summary of some of the results that are reported in much greater detail in unpublished work.

'The DCH standard input prescription substantially overpredicted debris transport to the dome $\left(f_{\text {dome }}\right)$ for the LFP experiments, reproduced the average behavior well for the Zion-geometry IET experiments, and underpredicted $f_{\text {dome }} 15-40 \%$ for the Surry geometry experiments. $\triangle P$ was sensitive to $f_{\text {dome }}$ in the LFP experiments and was insensitive to $f_{\text {dome }}$ for both the Zion and Surry IET experiments.

(Footnotes Continued Overleaf) 


\section{(Footnotes for Table C-6 Continued)}

'Hydrogen production was underpredicted in almost all cases if NAD interactions were not modeled, and $\triangle \mathrm{P}$ and hydrogen were both underpredicted by about a factor of two for the Zion IET experiments without NAD interactions. However, the original standard input prescription did not include the cavity water and it is now believed that both the water and the NAD made significant contributions to $\triangle \mathrm{P}$ and $\mathrm{H}_{2}$ production in the Zion IET experiments. A clean separation of the effects of NAD and cavity water cannot be defined with the existing data base, but calculated pressure-time histories agree best with experiment when it is assumed that both contribute.

II the standard prescription, the concentration parameters for the receiving cell are set to values that essentially eliminate the possibility that inerting will suppress DFB unless there is virtually no oxygen. This prescription was found to work well for those experiments in which the DCH-produced hydrogen did burn, but it is deliberately conservative with respect to the threshold concentrations for combustion of DCH-produced hydrogen.

'Mitigation by atmosphere-structure heat transfer was found to be very important in both the Zion and Surry geometry IET analyses. Oxygen starvation in the subcompartment volumes played an important role by delaying or preventing complete combustion of DCH-produced hydrogen, thereby giving the heat transfer more time to be effective.

In the standard prescription, RPV and cavity entrainment models are not used. Instead user-defined source tables are used, based on experimental results for debris dispersal. For plant calculations, debris dispersal fractions $\left(f_{\text {disp }}\right)$ are based upon the experimental observation that $f_{\text {disp }} \geq 0.7$ except when the driving pressure is quite low, and the coherence factor between debris dispersal and blowdown steam is based upon an empirical correlation. Sensitivity studies indicate impact upon the calculated $\Delta \mathrm{P}$ is not large when dispersal fractions and coherence factors are varied over an uncertainty range consistent with the experimental results, except that there could be high sensitivity to dispersal fraction uncertainties for open containment geometries when the RPV pressure is low.

8R. O. Griffith, Modeling and Analysis Department, Sandia National Laboratories, Albuquerque, NM, letter report to the USNRC with title "Verification of the DCH Models for RPV and Cavity Phenomena Incorporated into CONTAIN in Update C110Z," July 29, 1993. 
Table C-7 Validation Matrix for Aerosol Modeling

\begin{tabular}{|c|c|c|c|c|c|}
\hline $\begin{array}{l}\text { Validation } \\
\text { Type/Basis }\end{array}$ & Facility & Reference & $\begin{array}{c}\text { Code } \\
\text { Version }\end{array}$ & Models & Comments \\
\hline $\begin{array}{l}\text { ABCOVE (dry) } \\
\text { aerosol tests/ AB5, } \\
\mathrm{AB} 6, \mathrm{AB} 7\end{array}$ & -..- & $\begin{array}{l}\text { Ber85 } \\
\text { Mur83a } \\
\text { Mur83b }\end{array}$ & 1.0 & $\begin{array}{l}\text { Aerosol } \\
\text { agglomeration and } \\
\text { deposition }\end{array}$ & $\begin{array}{l}\text { CONTAIN results compared well with the test results for the } A B 5 \text { and } \\
A B 7 \text { tests. The } A B 7 \text { comparison was not as good later in the test, but it } \\
\text { was believed that the test conditions deviated from the conditions } \\
\text { originally planned. }\end{array}$ \\
\hline $\begin{array}{l}\text { Sodium fire } \\
\text { (closed vessel) test } \\
\text { data and code-to- } \\
\text { code comparison, } \\
\text { PARDISEKO } \\
\text { code }\end{array}$ & ---- & $\begin{array}{l}\text { Jon88a } \\
\text { Jon88b }\end{array}$ & 1.04 & $\begin{array}{l}\text { Aerosol } \\
\text { deposition and } \\
\text { coagulation }\end{array}$ & $\begin{array}{l}\text { CONTAIN results were compared with a) measurements for tests } \\
\text { involving sodium-fire aerosols and metallic sodium drops in closed } \\
\text { vessels and b) calculated results from other codes, particularly the } \\
\text { PARDISEKO code. CONTAIN was found to provide a realistic } \\
\text { simulation of aerosol behavior with the modification, implemented by the } \\
\text { authors, in the procedure for accounting for turbulent deposition. Note } \\
\text { that these references supersede Jon87 and, in particular, correct a } \\
\text { conclusion made in this earlier reference that the CONTAIN aerosol } \\
\text { calculations were grossly in error. }\end{array}$ \\
\hline $\begin{array}{l}\text { Sodium fire tests } \\
\text { and code-to-code/ } \\
\text { PARDISEKO, } \\
\text { AEROSIM, and } \\
\text { AEROSOL/B2 } \\
\text { codes }\end{array}$ & $\ldots$ & Lhi90 & 1.10 & $\begin{array}{l}\text { General aerosol } \\
\text { behavior, } \\
\text { including } \\
\text { agglomeration and } \\
\text { deposition }\end{array}$ & $\begin{array}{l}\text { The CONTAIN aerosol modeling was validated by the test measurements } \\
\text { and the comparisons with other codes with the exception that the wall } \\
\text { deposition calculated by CONTAIN was lower than measured. }\end{array}$ \\
\hline $\begin{array}{l}\mathrm{ABCOVE} \text { (dry) } \\
\text { aerosol tests/ } \\
\mathrm{AB} 5, \mathrm{AB} 6, \text { and } \\
\mathrm{AB} 7\end{array}$ & --- & Lei87 & 1.10 & $\begin{array}{l}\text { Aerosol } \\
\text { deposition }\end{array}$ & $\begin{array}{l}\text { CONTAIN results were compared with test data and MELCOR-code } \\
\text { results. They compared a) within approximately } 10 \% \text { of the test results for } \\
\text { the gravitational and diffusional deposition, b) poorly with the test results } \\
\text { for deposition caused by turbulence, and c) very well with the MELCOR } \\
\text { results. }\end{array}$ \\
\hline $\begin{array}{l}\text { ABCOVE } \\
\text { experimental } \\
\text { program }\end{array}$ & $-\cdots$ & $\begin{array}{l}\text { Lei87 } \\
\text { Ber85 } \\
\text { Mur83a }\end{array}$ & 1.0 & $\begin{array}{l}\text { Aerosol } \\
\text { agglomeration, } \\
\text { co-agglomeration } \\
\text { and deposition }\end{array}$ & $\begin{array}{l}\text { Participation in the ABCOVE program included comparisons of } \\
\text { CONTAIN results with a) calculated results from other codes and b) test } \\
\text { results. In addition to the models mentioned, the CONTAIN discrete } \\
\text { modeling approach was compared to the lognormal approach. The } \\
\text { comparisons provided strong confirmation of CONTAIN's ability to } \\
\text { model one- and two-component aerosol behavior in dry conditions. }\end{array}$ \\
\hline
\end{tabular}

Rev. 0 
Table C-7 Validation Matrix for Aerosol Modeling (Continued)

\begin{tabular}{|c|c|c|c|c|c|}
\hline $\begin{array}{l}\text { Validation } \\
\text { Type/Basis }\end{array}$ & Facility & Reference & $\begin{array}{c}\text { Code } \\
\text { Version }\end{array}$ & Models & Comments \\
\hline $\begin{array}{l}\text { ORNL test } 504 \\
\text { (with steam } \\
\text { environment) }\end{array}$ & -.-- & Mur83a & Early & $\begin{array}{l}\text { Aerosol } \\
\text { diffusiophoresis }\end{array}$ & $\begin{array}{l}\text { The comparison confirmed CONTAIN's ability to model diffusiophoresis } \\
\text { for small atmosphere to wall temperature differences, and the model } \\
\text { should suffice except in those cases in which diffusiophoresis plays a } \\
\text { major role in aerosol depletion. }\end{array}$ \\
\hline $\begin{array}{l}\text { Experimental and } \\
\text { code-to-code } \\
\text { comparison with } \\
\text { the HAARM-3 } \\
\text { and QUICK codes }\end{array}$ & $-\cdots$ & Sci84 & Early & $\begin{array}{l}\text { Aerosol } \\
\text { agglomeration and } \\
\text { deposition }\end{array}$ & $\begin{array}{l}\text { The comparisons were applicable to agglomeration and deposition in cases } \\
\text { in which a) turbulent agglomeration is not important and b) there is no } \\
\text { water vapor condensation on aerosols. The numerical solution scheme in } \\
\text { the code was found to be highly satisfactory. It was recommended } \\
\text { however that aerosol condensation modeling with an improved numerical } \\
\text { scheme be added to the code. }\end{array}$ \\
\hline $\begin{array}{l}\text { Sodium fire } \\
\text { aerosol tests and } \\
\text { code-to-code } \\
\text { comparisons/ } \\
\text { ABC-INTG code }\end{array}$ & --- & Sei90 & 1.10 & $\begin{array}{l}\text { General aerosol } \\
\text { behavior, } \\
\text { including } \\
\text { agglomeration and } \\
\text { deposition }\end{array}$ & $\begin{array}{l}\text { The CONTAIN aerosol modeling was validated by the test measurements } \\
\text { with the exception that the wall deposition calculated by CONTAIN was } \\
\text { lower than measured. The CONTAIN calculated wall deposition was } \\
\text { improved by using a smaller fixed thermal boundary layer thickness. }\end{array}$ \\
\hline $\begin{array}{l}\text { Code-to-code } \\
\text { comparison/ } \\
\text { PARDISEKO } \\
\text { code }\end{array}$ & --- & Wie88 & 1.05 & $\begin{array}{l}\text { Aerosol } \\
\text { deposition and } \\
\text { coagulation }\end{array}$ & $\begin{array}{l}\text { This study compared results from the SNR } 300 \text { reactor study. The results } \\
\text { of this CV\&A were not (readily) obtainable because this report is in } \\
\text { German. Adequate results of similar nature are available elsewhere in this } \\
\text { table. }\end{array}$ \\
\hline LACE/LA-4 & CSTF & $\begin{array}{l}\text { Gel90 } \\
\text { Smi92f }\end{array}$ & 1.11 & $\begin{array}{l}\text { Moving-grid } \\
\text { numerical model } \\
\text { for aerosol } \\
\text { condensation and } \\
\text { growth }\end{array}$ & $\begin{array}{l}\text { This experiment investigated soluble and insoluble aerosol behavior in a } \\
\text { steam environment. Previous CONTAIN aerosol models could only treat } \\
\text { insoluble aerosols. When hygroscopic effects were modeled, through a } \\
\text { new moving-grid model for aerosol growth by condensation, the predicted } \\
\text { deposition rates for this experiment increased significantly and brought the } \\
\text { rate into good agreement with the experimental data. }\end{array}$ \\
\hline
\end{tabular}


Table C-7 Validation Matrix for Aerosol Modeling (Concluded)

\begin{tabular}{||l|l|l|l|l|l|}
\hline $\begin{array}{c}\text { Validation } \\
\text { Type/Basis }\end{array}$ & Facility & Reference & $\begin{array}{c}\text { Code } \\
\text { Version }\end{array}$ & Models & Comments \\
\hline DEMONA/ A9 & BMC & Smi92c & $1.11 \mathrm{UK}^{\mathrm{a}}$ & $\begin{array}{l}\text { Insoluble aerosol } \\
\text { agglomeration and } \\
\text { deposition }\end{array}$ & $\begin{array}{l}\text { This experiment measured aerosol deposition in a multicompartment } \\
\text { facility. The CONTAIN input model consisted of 5 interconnected cells. } \\
\text { CONTAIN calculations correctly predicted the washout of airborne } \\
\text { aerosols by steam condensation, although the predicted airborne } \\
\text { condensation rates were initially too low and persisted too long. It was } \\
\text { recommended that the number of aerosol size classes be increased to } \\
\text { improve convergence of the condensation calculation. }\end{array}$ \\
\hline
\end{tabular}

a CONTAIN 1.11 modified to allow the user to input an initial temperature profile in the basemat. 
Table C-8 Validation Matrix for Hydrogen Burn Modeling

\begin{tabular}{|l|l|l|l|l|l||}
\hline $\begin{array}{l}\text { Validation } \\
\text { Type/Basis }\end{array}$ & Facility & Reference & $\begin{array}{l}\text { Code } \\
\text { Version }\end{array}$ & Models & \multicolumn{1}{|c|}{ Comments } \\
\hline $\begin{array}{l}\text { Analysis of } \\
\text { the TMI } \mathrm{H}_{2} \\
\text { burn }\end{array}$ & $\begin{array}{l}\text { TMI } \\
\text { contain- } \\
\text { ment }\end{array}$ & $\begin{array}{l}\text { Mur88 } \\
\text { Mur89 }\end{array}$ & 1.10 & $\begin{array}{l}\mathrm{H}_{2} \text { deflagration } \\
\text { model for } \\
\text { fraction } \mathrm{H}_{2} \\
\text { burned }\end{array}$ & $\begin{array}{l}\text { The CONTAIN predicted fraction of } \mathrm{H}_{2} \text { burned was too high. Otherwise, the } \\
\text { code gave a reasonable accounting for the effects of heat transfer (radiation and } \\
\text { convective) and burning on pressure. Recommendations were made that the } \\
\text { discrepancy in the fraction of } \mathrm{H}_{2} \text { burned be investigated further. }\end{array}$ \\
\hline $\begin{array}{l}\text { Analysis of } \\
\text { NTS burn } \\
\text { data }\end{array}$ & $\begin{array}{l}\text { NTS } \\
\text { Large- } \\
\text { Scale } \\
\text { Hydrogen } \\
\text { Combus- } \\
\text { tion } \\
\text { Facility }\end{array}$ & Rij86 & 1.04 & $\begin{array}{l}\text { Single } \\
\text { deflagrations }\end{array}$ & $\begin{array}{l}\text { Comparison of CONTAIN predictions with NTS data for burns with premixed } \\
\text { gases. In comparison to the measurements, the code predictions agreed a) well } \\
\text { for peak pressure and peak temperature, b) poorly for the burn duration, and c) } \\
\text { reasonably well for the wall heat fluxes considering that the experimental data } \\
\text { may have had considerable uncertainty. Recommendations were made that the } \\
\text { discrepancy regarding burn duration be investigated further. }\end{array}$ \\
\hline $\begin{array}{l}\text { Comparison } \\
\text { with hand } \\
\text { calculations }\end{array}$ & --- & Sci84 & Early & $\begin{array}{l}\text { Burn criterion for } \\
\text { initiation, } \\
\text { timestep control }\end{array}$ & $\begin{array}{l}\text { Checks were made on the implementation of } \mathrm{H}_{2} \text { burn threshold concentrations, } \\
\mathrm{O}_{2} \text { threshold concentrations, and } \mathrm{H}_{2} \mathrm{O} \text { concentrations for inerting. In addition, } \\
\text { the calculated pressure and temperature were evaluated, and the effect of } \\
\text { varying the timestep was investigated. Comparisons with hand calculations } \\
\text { were made. }\end{array}$ \\
\hline
\end{tabular}


Table C-9 Validation Matrix for Pressure Suppression Modeling

\begin{tabular}{||l|l|l|l|l|l||}
\hline $\begin{array}{l}\text { Validation } \\
\text { Type/Basis }\end{array}$ & Facility & Reference & $\begin{array}{l}\text { Code } \\
\text { Version }\end{array}$ & \multicolumn{1}{|c|}{ Models } & Comments \\
\hline $\begin{array}{l}\text { HEDL test } \\
\text { CSE A-9 }\end{array}$ & CSTF & Mur83a & Early & $\begin{array}{l}\text { Model for } \\
\text { removing gas } \\
\text { and aerosol } \\
\text { fission products } \\
\text { by sprays }\end{array}$ & $\begin{array}{l}\text { CONTAIN predictions were compared with results from the HEDL test CSE } \\
\text { A-9, which introduced a spray into a steam environment. The general ability } \\
\text { of CONTAIN to model the effect of sprays on radionuclides was } \\
\text { demonstrated. }\end{array}$ \\
\hline $\begin{array}{l}\text { NUPEC test } \\
\text { M-7-1 and } \\
\text { M-8-2 }\end{array}$ & $\begin{array}{l}\text { NUPEC } \\
\text { model } \\
\text { contain- } \\
\text { ment }\end{array}$ & Sta95 & 1.12 & $\begin{array}{l}\text { Spray heat and } \\
\text { mass transfer } \\
\text { model }\end{array}$ & $\begin{array}{l}\text { Spray effects in a multi-compartment containment were investigated using 28- } \\
\text { and 35-cell CONTAIN models of the highly compartmentalized NUPEC } \\
\text { containment, simulating a PWR containment. Trends in the pressure and } \\
\text { compartment temperature after the introduction of sprays were predicted for } \\
\text { both experiments although there was some obvious disagreement in the details. } \\
\text { The disagreement was believed to be caused in part by the code's inability to } \\
\text { model spray-induced air currents. }\end{array}$ \\
\hline $\begin{array}{l}\text { Ice } \\
\text { condenser } \\
\text { tests }\end{array}$ & $\begin{array}{l}\text { Battelle- } \\
\text { Pacific } \\
\text { Northwest } \\
\text { Laboratory } \\
\text { (PNL) Ice } \\
\text { Condenser } \\
\text { Test } \\
\text { Facility }\end{array}$ & Rus90 & 1.11 & $\begin{array}{l}\text { General ice } \\
\text { condenser } \\
\text { modeling, } \\
\text { including effects } \\
\text { of buoyancy and } \\
\text { heat transfer to } \\
\text { ice heat sinks }\end{array}$ & $\begin{array}{l}\text { CONTAIN predictions were compared to test results from the PNL facility. } \\
\text { The capability of CONTAIN to predict backflow and temperature stratification } \\
\text { was demonstrated qualitatively. }\end{array}$ \\
\hline
\end{tabular}


Table C-10 Validation Matrix for Miscellaneous Modeling

\begin{tabular}{|c|c|c|c|c|c|}
\hline Topic & $\begin{array}{l}\text { Validation } \\
\text { Type/Basis }\end{array}$ & Reference & $\begin{array}{l}\text { Code } \\
\text { Version }\end{array}$ & Models & Comments \\
\hline \multirow[t]{3}{*}{$\begin{array}{l}\text { Fission } \\
\text { product } \\
\text { behavior }\end{array}$} & \multirow[t]{3}{*}{ Analytical check } & \multirow[t]{3}{*}{ Sci84 } & \multirow[t]{3}{*}{ Early } & $\begin{array}{l}\text { Fission product } \\
\text { decay, release and } \\
\text { acceptance }\end{array}$ & $\begin{array}{l}\text { Code performed as intended. However, one must be careful to choose an } \\
\text { adequately small timestep. }\end{array}$ \\
\hline & & & & $\begin{array}{l}\text { Fission product } \\
\text { intercell transport }\end{array}$ & Agreement between code predictions and hand calculations was excellent. \\
\hline & & & & $\begin{array}{l}\text { Fission product } \\
\text { heating of } \\
\text { structures }\end{array}$ & $\begin{array}{l}\text { Agreement between code predictions and hand calculations was satisfactory. A } \\
\text { minor bug with the heating when both left and right sides of a structure are in a } \\
\text { cell was identified; in addition, errors in the conduction solution for structures } \\
\text { were noted. (Such problems have since been corrected.) }\end{array}$ \\
\hline \multirow[t]{3}{*}{$\begin{array}{l}\text { Mass and } \\
\text { energy } \\
\text { transfer }\end{array}$} & $\begin{array}{l}\text { Code-to-code } \\
\text { comparison and } \\
\text { data }\end{array}$ & Lan90 & 1.10 & $\begin{array}{l}\text { Outgassing and } \\
\text { heat conduction }\end{array}$ & $\begin{array}{l}\text { The CONTAIN predictions were compared with a) measured results and b) } \\
\text { calculated results from the DATRAN code, which provides a more mechanistic } \\
\text { accounting of the mass and energy transfer. The CONTAIN predictions were } \\
\text { found to compare relatively satisfactorily with a) measured results and b) the } \\
\text { DATRAN results. }\end{array}$ \\
\hline & $\begin{array}{l}\text { Code-to-code } \\
\text { comparison }\end{array}$ & Was88 & 1.10 & $\begin{array}{l}\text { Outgassing and } \\
\text { core-concrete } \\
\text { interactions }\end{array}$ & $\begin{array}{l}\text { The CONTAIN predictions were compared with calculations with the USINT } \\
\text { and SLAM codes. Also effects of outgassing on the CONTAIN/ } \\
\text { CORCON core-concrete interaction modeling were investigated by variation of } \\
\text { parameters. The CONTAIN outgassing model predictions were found to be in } \\
\text { reasonable agreement with the USINT and SLAM codes. It was concluded that } \\
\text { the treatment of the thermophysical concrete properties as a function of } \\
\text { temperature and gas release may be important for long-term problems. With } \\
\text { regard to core-concrete interactions, it was demonstrated that the modeling } \\
\text { should be extended beyond the present steady state treatment. }\end{array}$ \\
\hline & $\begin{array}{l}\text { Code-to-code } \\
\text { comparison }\end{array}$ & Sci84 & Early & Heat conduction & $\begin{array}{l}\text { The CONTAIN predictions were compared with calculations with the SINDA } \\
\text { and CONDUC codes. The CONTAIN conduction solution was confirmed to be } \\
\text { approximately correct. }\end{array}$ \\
\hline $\begin{array}{l}\text { Material } \\
\text { properties }\end{array}$ & $\begin{array}{l}\text { Comparison to } \\
\text { property data and } \\
\text { equations }\end{array}$ & Val88 & 1.10 & $\begin{array}{l}\text { Thermodynamic } \\
\text { and transport } \\
\text { properties }\end{array}$ & $\begin{array}{l}\text { This reference gives a thorough evaluation of the properties used by the } \\
\text { CONTAIN code, including the deviations from the preferred behavior. (This } \\
\text { resulted in a subsequent material properties upgrade.) }\end{array}$ \\
\hline
\end{tabular}




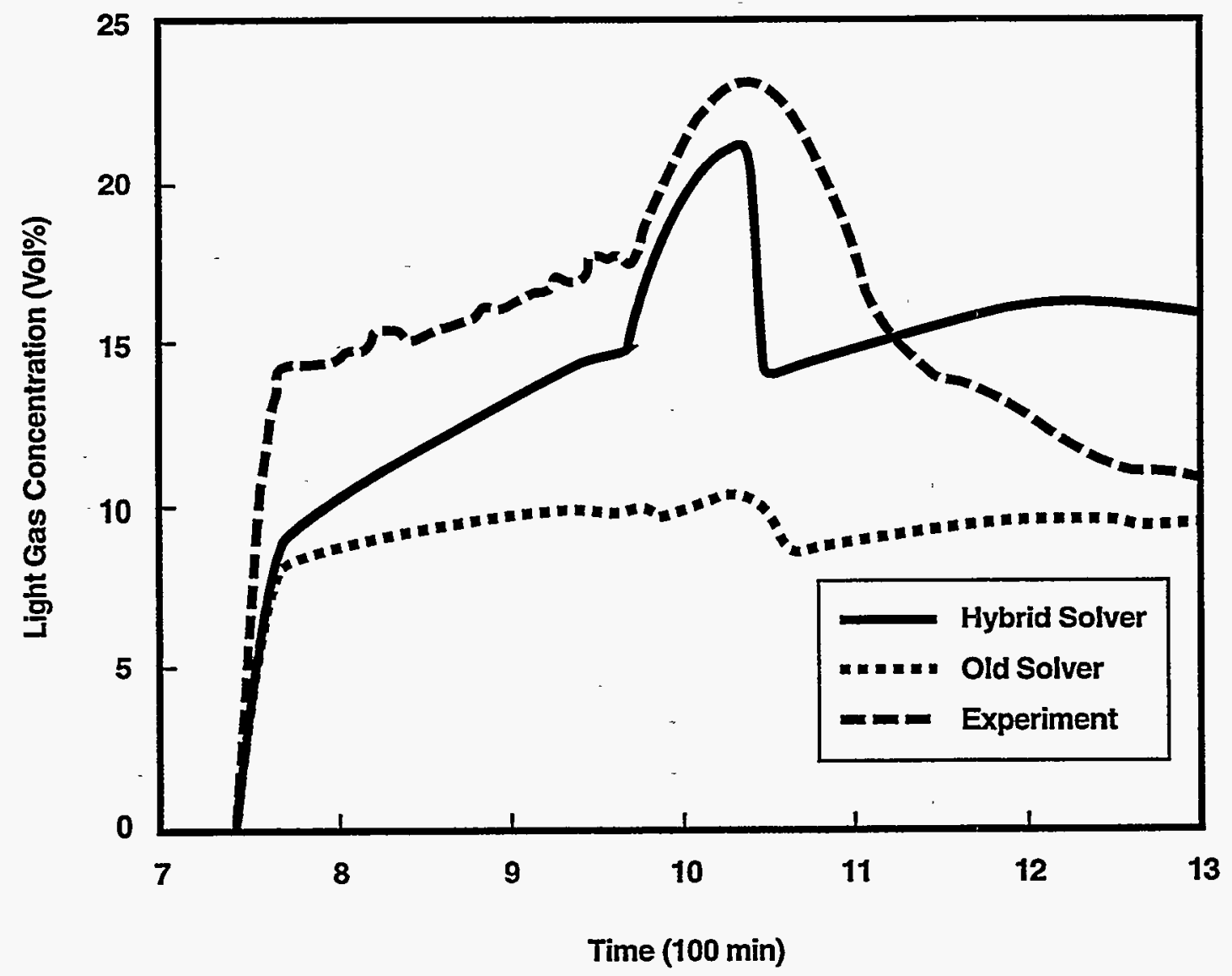

Figure C-1. Comparison of the Predicted Light Gas Concentrations in the Dome (Cell 9) in the 15Cell Base Case with the Experimental Measurements [Mur96] 


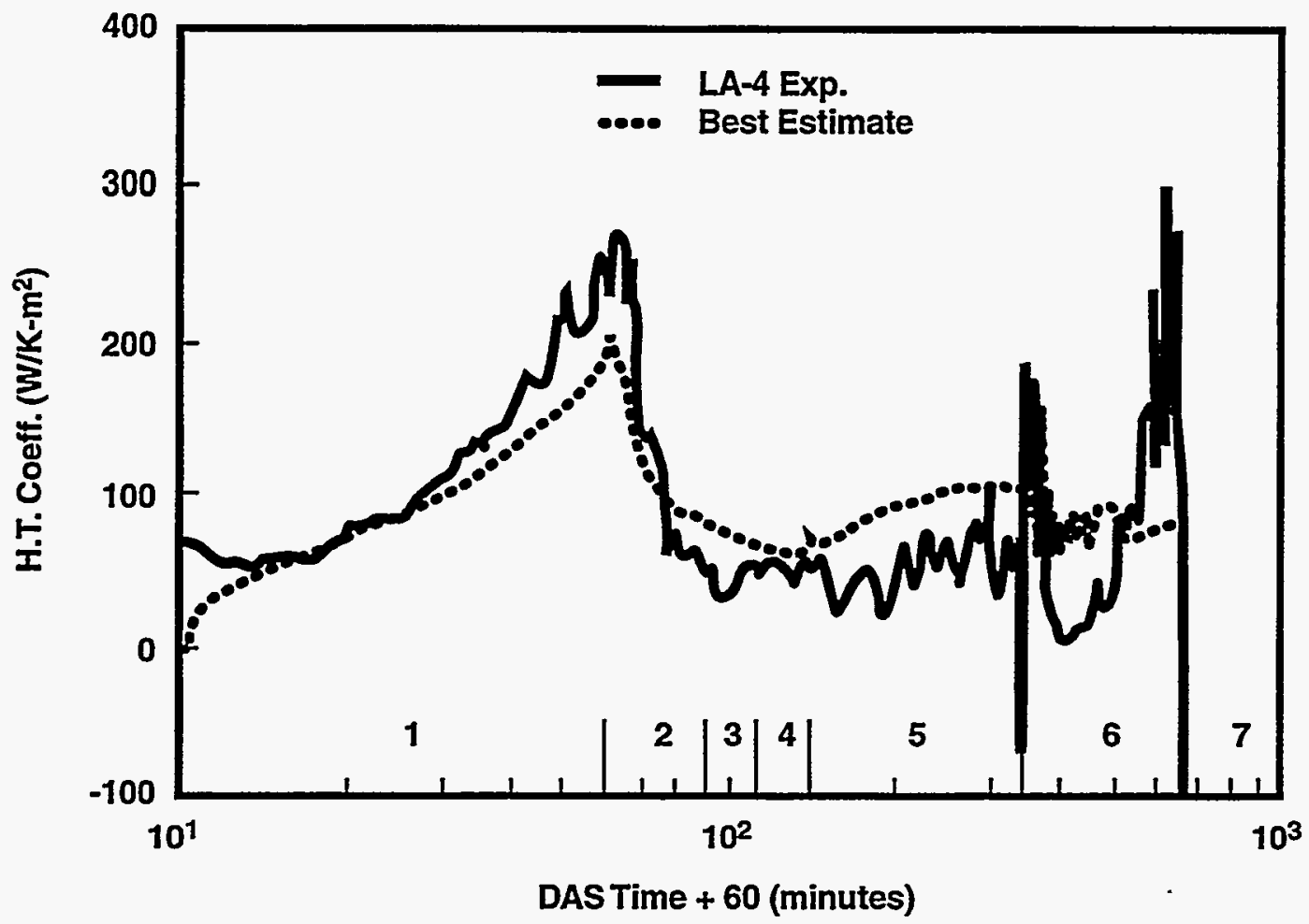

Figure C-2. Estimated Total Heat Transfer Coefficients for the LA-4 Vessel at Location WCC2 [Ge190] 


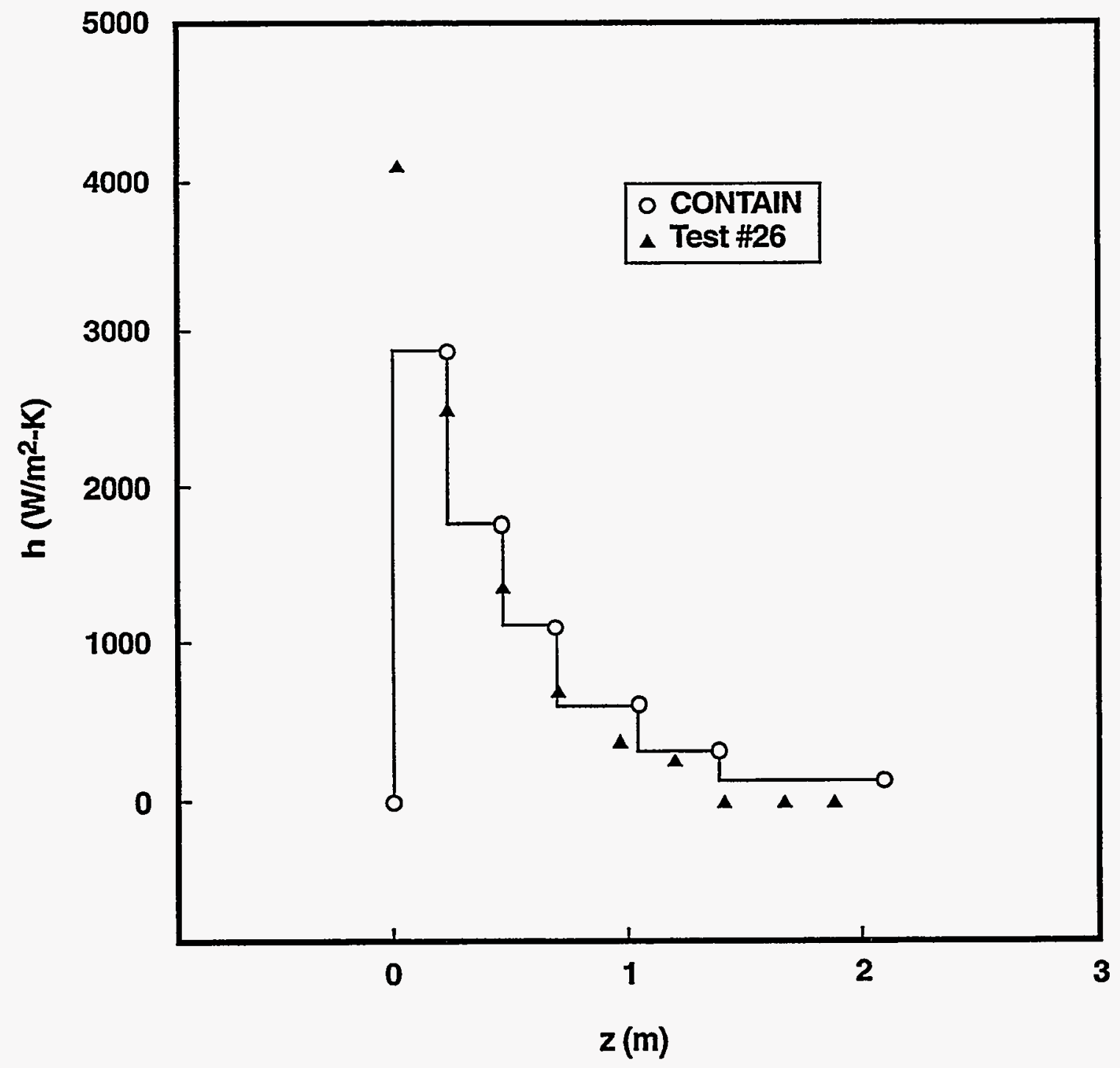

Figure C-3. CONTAIN Simulation of the University of California at Berkeley Condenser Tube Heat Transfer Experiment for Air/Stream Mixtures. The inlet is at $\mathrm{z}=0 \mathrm{~m}$. The air concentration increases along the condenser length as steam is condensed on the tube wall. 


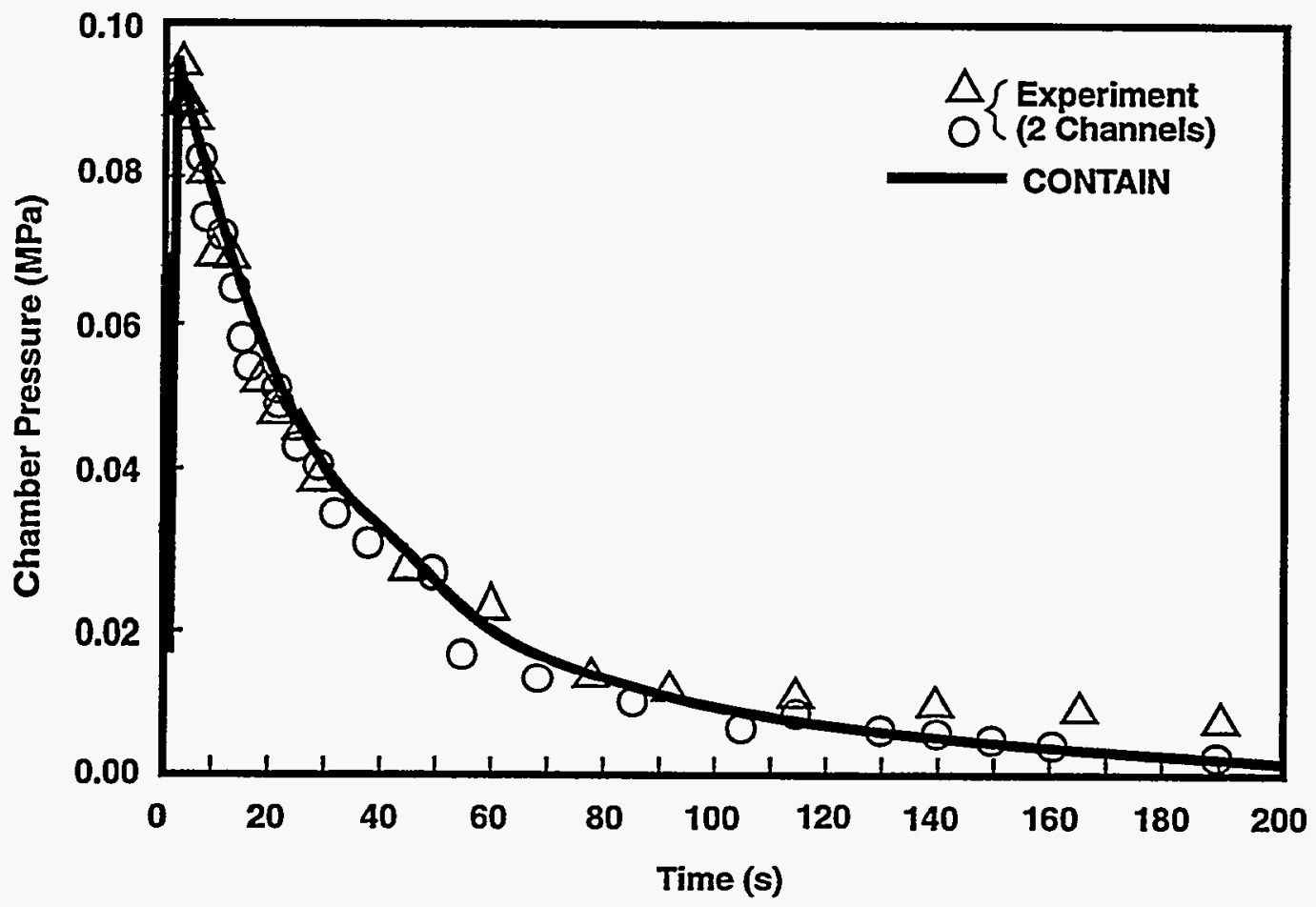

Figure C-4. CONTAIN DCH-1 Pressure Prediction and Comparison to Experiment [Tar88] 


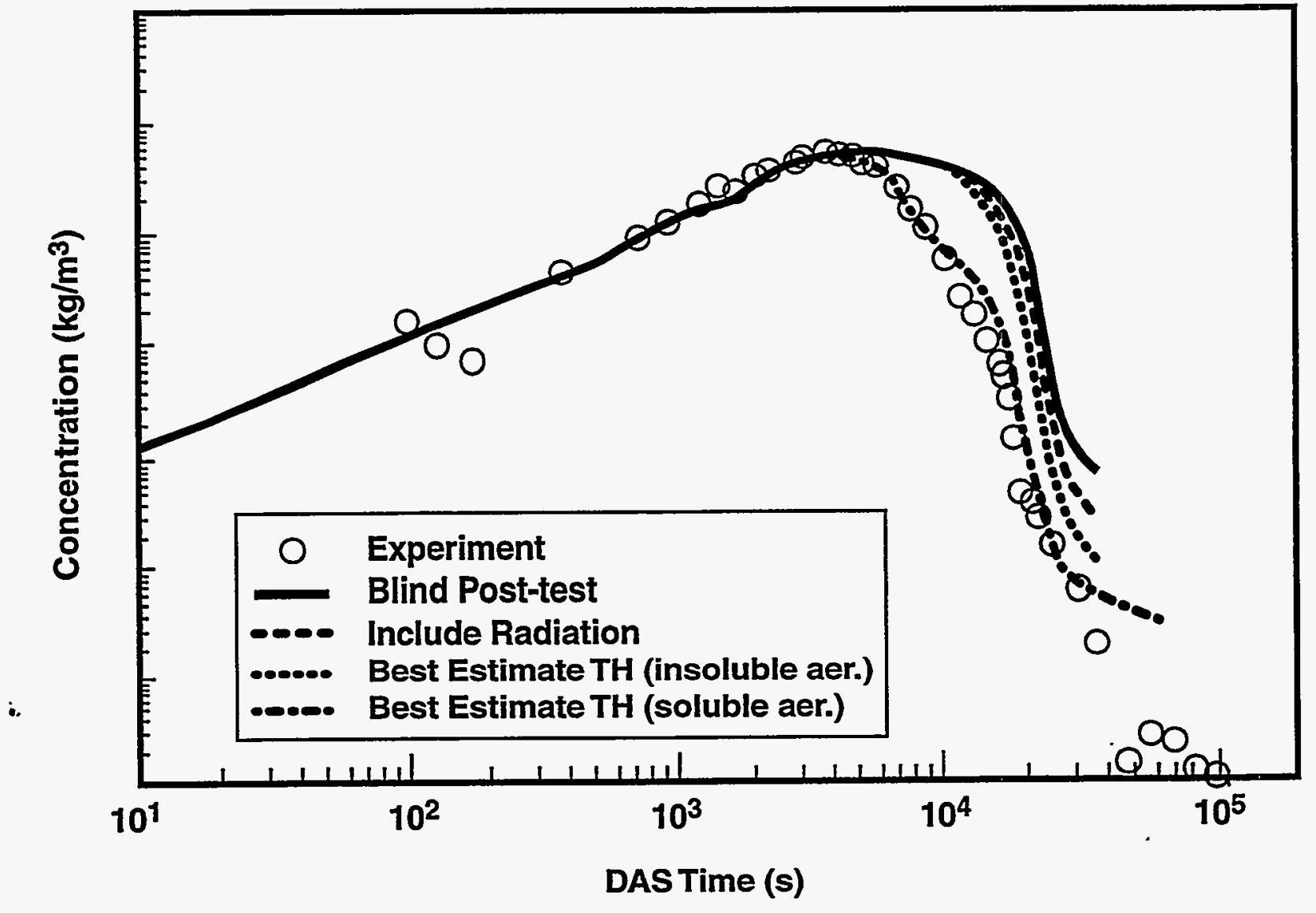

Figure C-5. Suspended Solid Aerosol Concentrations Predicted in the LA-4 Experiment, Compared to Measurements [Ge190] 


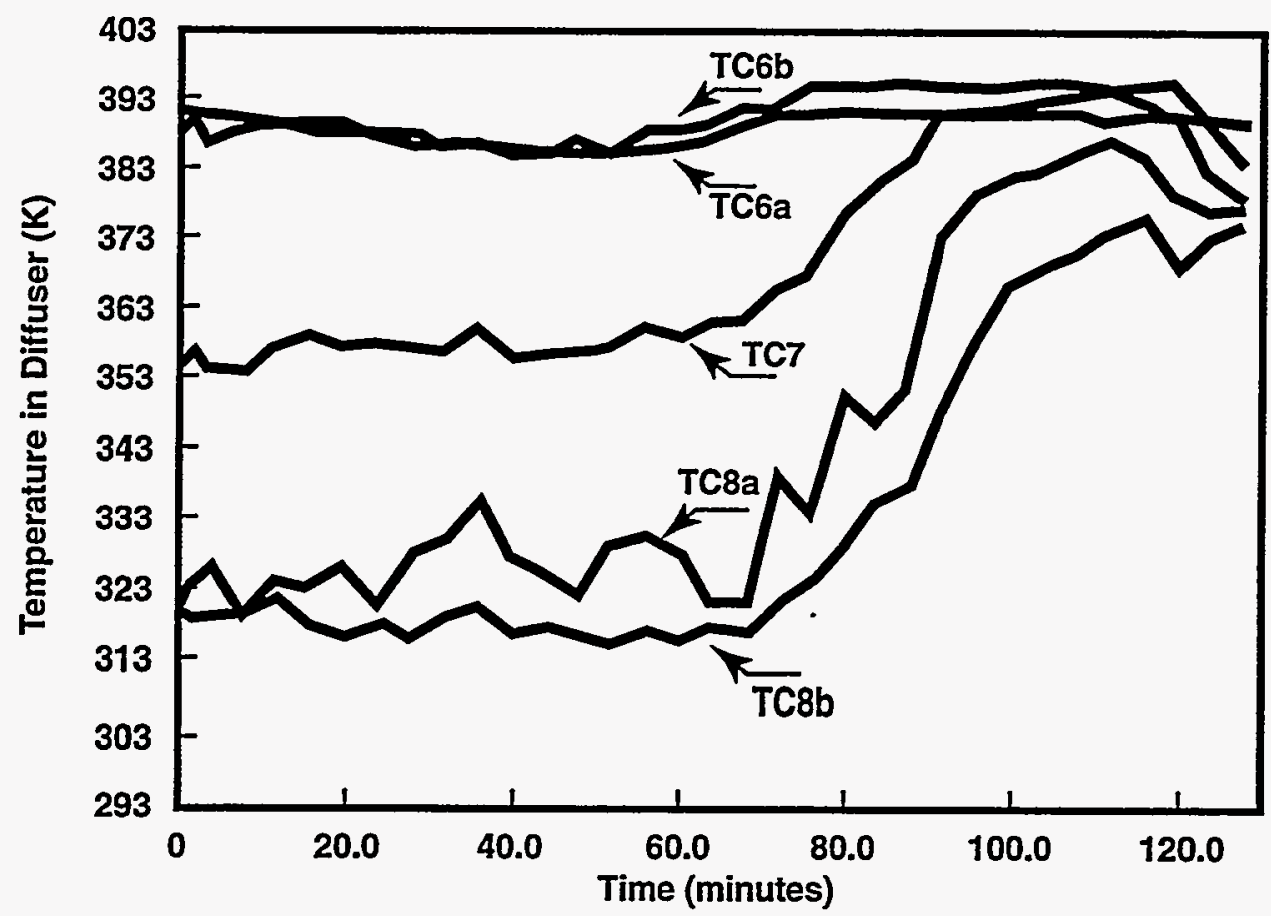

b) PNL Temperature Versus Time in the Diffuser (SAA Flow Rate $=0.15 \mathrm{~m}^{3} / \mathrm{s}, \mathrm{SMF}=0.6$ ).

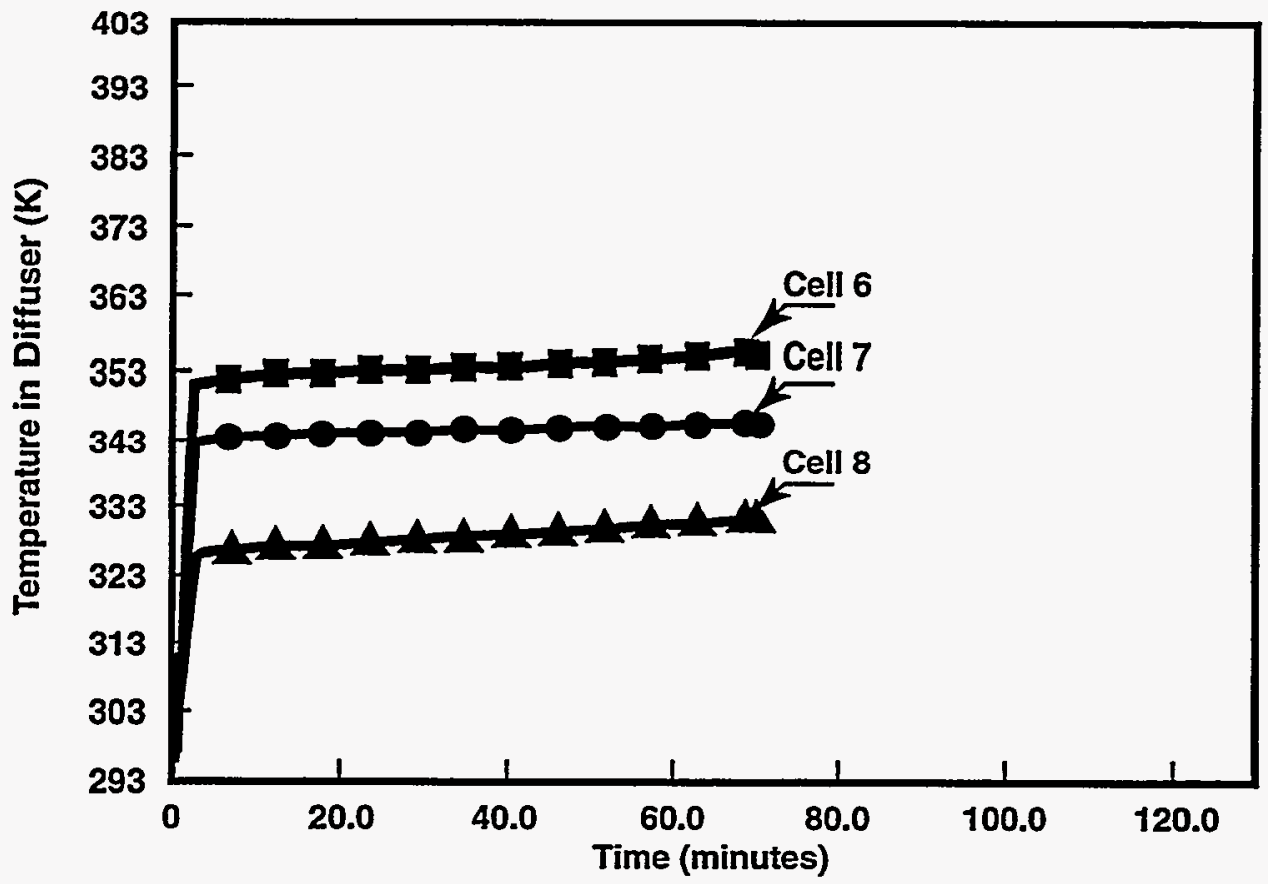

a) CONTAIN Temperature Versus Time in the Diffuser (Same Inlet Conditions).

Figure C-6. Comparison of the PNL Experiment 11-6 Thermocouple Data and the CONTAIN Calculated Temperatures, Showing the Effects of Backflow and Stratification on Temperatures in the Diffuser Inlet [Rus90] 


\section{REFERENCES}

Alm93 K. Almenas, F. J. Munno, N. Ennaciri, and H. Esmaili, "Recomputation of Selected CONTEMPT Code Design Basis Containment Transients Using the CONTAIN Code," ERI/NRC 93-209, Energy Research, Inc., Rockville, MD, August, 1993.

Amb95 W. Ambrosini, A. Manfredini, F. Mariotti, and F. Oriolo, "Heat Transfer From a Plate Cooled by a Water Film with Countercurrent Air Flow," Nuclear Technology, Vol. 112, pp. 227-237, 1995.

Ber85 K. D. Bergeron, M. J. Clauser, B. D. Harrison, K. K. Murata, P. E. Rexroth, F. J. Schelling, F. W. Sciacca, M. E. Senglaub, P. R. Shire, W. R. Trebilock, and D. C. Williams, "User's Manual for CONTAIN 1.0, A Computer Code for Severe Nuclear Reactor Accident Containment Analysis," NUREG/CR-4085, SAND841204, Sandia National Laboratories, Albuquerque, NM, May 1985.

Boy95 B. E. Boyack et al., "CONTAIN Independent Peer Review," LA-12866, Los Alamos National Laboratory, Los Alamos, NM, 1995.

Bra93 D. R. Bradley, D. R. Gardner, J. E. Brockman, and R. O. Griffith, "CORCON-Mod3: An Integrated Computer Model for Analysis of Molten Core-Concrete Interactions. Users Manual," NUREG/CR-5843, SAND92-0167, Sandia National Laboratories, Albuquerque, NM, 1993.

Din86 S. E. Dingman, A. L. Camp, C. C. Wong, D. B. King, and R. G. Gasser, "HECTR Version 1.5 User's Manual," NUREG/CR-4507, SAND86-0101, Sandia National Laboratories, Albuquerque, NM, 1986.

Fir96 M. Firnhaber, T. Kanzleiter, S. Schwarz, and G. Weber, "VANAM M3 - A MultiCompartment Aerosol Depletion Test with Hygroscopic Aerosol Material - Draft Comparison Report," OECD-CSNI-ISP-37, Organisation for Economic Cooperation and Development - Committee on the Safety of Nuclear Installations, February, 1996.

Fuj93 T. Fujii, K. Shinzato, and J. B. Lee, "Various Formulas and Their Accuracy Concerning Convective Heat and Mass Transfer in the Vapor Boundary Layer in the Case of Laminar Film Condensation of Binary Vapor Mixtures," Int. Journal of Heat and Mass Transfer, Vol. 36, No. 1, pp. 27-33, 1993.

Ge190 F. Gelbard, J. L. Tills, and K. K. Murata, "CONTAIN Code Calculations for the LA-4 Experiment," Proceedings of the Second International Conference on Containment Design and Operation, Vol. 2, Canadian Nuclear Society, Toronto, Ontario, Canada, October 14-17, 1990.

Has96a Y. A. Hassin and S. Banerjee, "Implementation of a Non-Condensable Model in RELAP5/MOD3," Nuclear Engineering and Design, Vol. 162, pp. 281-300, 1996. 
Has96b H. A. Hasanein, M. S. Kazimi, and M. W. Golay, "Forced Convection In-Tube Steam Condensation in the Presence of Noncondensable Gases," Int. Journal of Heat and Mass Transfer, Vol. 39, No. 13, pp. 2625-2639, 1996.

Hei86 J. A. Heil, H. van Rij, J. E. Speelman, and L. Winters, "Aspects of In- and Ex-Vessel Thermal Hydraulics in Severe Accident Sequences," Paper for ENC-4 Contributed Session Enclosed in Letter to Dr. Peter Bucher, ENC-4 Conference Secretary, European Nuclear Society, from J. E. Speelman, Netherlands Energy Research Foundation (ECN), February 14, 1986.

Huh93 I. K. Huhtiniemi and M. L. Corradini, "Condensation in the Presence of Noncondensable Gases," Nuclear Engineering and Design, Vol. 141, pp. 429-446, 1993.

Jac89 G. Jacobs and W. Scholtyssek, "Study of a Convection Loop Using the Codes CONTAIN and RELAP5," INR-1630, Forschungszentrum Karlsruhe, Karlsruhe, Germany, February 1989.

Jon87 R. Jonas and H. Bunz, "Aerosol Behavior Calculations Performed with the CONTAIN Code in Comparison to PARDISEKO Calculations and to Experiments," Journal of Aerosol Science, Vol. 18, No. 6, 1987.

Jon88a R. Jonas, "Consideration of Turbulent Deposition in Aerosol Behavior Modeling With the CONTAIN Code and Comparison of the Computations to Sodium Release Experiments," KfK 4431, Laboratorium for Aerosolphysik und Filtertechnik, Projekt Schneller Bruter, Forschungszentrum Karlsruhe, Karlsruhe, Germany, September 1988.

Jon88b R. Jonas, G. Schwientek, and W. Schutz, "Consideration of Turbulent Deposition in Aerosol Behavior Modeling With the CONTAIN Code and Comparison of the Computations With the FAUNA 2 Sodium Fire Experiment," 16th Annual Conference of the Gesellschaft fur Aerosolforschung, Lund, Sweden, September 8-9, 1988.

Kag93 T. Kageyama, P. F. Peterson, and V. E. Schrock, "Diffusion Layer Modeling for Condensation in Vertical Tubes with Noncondensable Gases," Nuclear Engineering And Design, Vol. 141, pp. 289-302, 1993.

Kan94 H. C. Kang and M. H. Kim, "Effect of Non-Condensable Gas and Wavy Interface on the Condensation Heat Transfer in a Nearly Horizontal Plate," Nuclear Engineering and Design, Vol. 149, pp. 313-321, 1994.

Kar89 H. Karwat, "ISP-23, Rupture of a Large Diameter Pipe in the HDR Containment," Vols. 1 and 2, CSNI Report No. 160, Committee on the Safety of Nuclear Installations - OECD Nuclear Energy Agency, Paris, France, 1989. 
Kar92 H. Karwat, "Distribution of Hydrogen Within the HDR-Containment Under Severe Accident Conditions - Final Comparison Report," OECD Standard Problem OECDCSNI-ISP-29, Organisation for Economic Cooperation and Development Committee on the Safety of Nuclear Installations, August 1992.

Kim90 M. H. Kim and M. L. Corradini, "Modeling of Condensation Heat Transfer in a Reactor Contäinment," Nuclear Engineering and Design, Vol. 118, pp. 193-212, 1990.

Kro68 D. G. Kroger and W. M. Rohsenow, "Condensation Heat Transfer in the Presence of a Noncondensable Gas," Int. Journal of Heat and Mass Transfer, Vol. 11, pp. 15-26, 1968.

Kro78 W. J. Krotiuk and M. B. Rubin, "Condensing Heat Transfer Following a Loss-ofCoolant Accident," Nuclear Technology, Vol. 37, pp. 118-128, 1978.

Lan88a J. Langhans, "Verifikation der Berechnung von Druckausgleichsstromungen in CONTAIN," GRS-A-1406, Gesellschaft fur Reaktorsicherheit (GRS) mbH, Koln, Germany, 1988.

Lan88b J. Langhans, "CONTAIN-Vergleichsrechnung zum HDR-Containment Versuch T31.4," Report No. 73 880/GRS-A-1495, Gesellschaft fur Reaktorsicherheit (GRS) $\mathrm{mbH}$, Koln, Germany, October 1988.

Lan90 J. Langhans, "Recent Progress in Verification and Development of the CONTAIN Code," Proceedings of the 1990 International Fast Reactor Safety Meeting, Snowbird, Utah, Vol. 1, p. 279, August 12-16, 1990.

Lei87 C. D. Leigh, ed., "MELCOR Validation and Verification - 1986 Papers," NUREG/CR-4830, SAND86-2689, Sandia National Laboratories, Albuquerque, NM, March 1987.

Lhi90 G. Lhiaubet, G. Bunz, M. P. Kissane, H. Seino, O. Miyake, Y. Himeno, C. Casselman, J. M. Such, and R. Rzekiechi, "Comparison of Aerosol Behavior Codes with Experimental Results from a Sodium Fire in a Containment," Proceedings of the 1990 International Fast Reactor Safety Meeting, Snowbird, UT, Vol. 2, p. 457, August 12-16, 1990.

Mur83a K. K. Murata, K. D. Bergeron, P. E. Rexroth, M., J. Clauser, J. L. Tills, F. W. Sciacca, M. E. Senglaub, W. Trebilcock, and D. C. Williams, "CONTAIN: Recent Highlights in Code Testing and Validation," Int. Meeting on Light Water Reactor Severe Accident Evaluation, Cambridge, MA, August 28-September 1, 1983. 
Mur83b K. K. Murata, K. D. Bergeron, and J. L. Tills,, "Experimental Validation of the CONTAIN Code," Eleventh Water Reactor Safety Research Information Meeting, Gaithersburg, MD, October 24-28, 1983.

Mur88 K. Muramatsu, and K. Soda, "Analysis of Hydrogen Burn in the TMI-2 Accident with the CONTAIN 1.1 Computer Code," TMI-2 Topical Meeting at the ANS/ENS International Meeting, Washington DC, October-November, 1988.

Mur89 K. Muramatsu and K. Soda, "Analysis of Hydrogen Burn in the Three Mile Island Unit 2 Accident with the CONTAIN 1.1 Computer Code," Nuclear Technology, Vol. 87, pp. 907-911, December 1989.

Mur96 K. K. Murata and D. W. Stamps, "Development and Assessment of the CONTAIN Hybrid Flow Solver," SAND96-2792, Sandia National Laboratories, Albuquerque, NM, November 1996.

Owc85 P. C. Owczarski and R. I. Schrenk, "Technical Bases and User's Manual for the Prototype of a Suppression Pool Aerosol Removal Code," NUREG/CR-3317, Pacific Northwest Laboratory, Richland, WA, 1985.

Pet96 P. F. Peterson, "Theoretical Basis for the Uchida Correlation for Condensation in Reactor Containments," Nuclear Engineering and Design, Vol. 162, pp. 301-306, 1996.

Pon90 L. T. Pong, "Assessment of the Combustion Model in the HECTR Code," NUREG/CR-5590, SAND90-7080, Sandia National Laboratories, Albuquerque, NM, November 1990.

Rij86 H. van Rij, "Hydrogen Burn Assessment with the CONTAIN Code," SAND852771C, Third Workshop on Containment Integrity, Washington, DC, May 21-23, 1986.

Rus90 N. A. Russell and D. C. Williams, "Comparison of CONTAIN Code to Experimental Ice Condenser Data," Proceedings of the Second International Conference on Containment Design and Operation, Canadian Nuclear Society, Toronto, Canada, October 14-17, 1990.

Vij97 R. Vijaykumar and M. Khatib-Rahbar, "Evaluation of the Applicability of the CONTAIN Heat and Mass Transfer Models to Asymmetrically Heated Vertical Channels," ERI/NRC 97-202 (Proprietary), Energy Research, Inc., Rockville, MD, July 1997.

Sci84 F. W. Sciacca, K. D. Bergeron, K. K. Murata, and P. E. Rexroth, "Testing of the CONTAIN Code," NUREG/CR-3310, SAND83-1149, Sandia National Laboratories, Albuquerque, NM, 1984. 
Sei90 H. Seino, O. Miyake, S. Morikawa, and Y. Himeno, "Validation of CONTAIN Code for Sodium Aerosol Behavior," Proceedings of the 1990 International Fast Reactor Safety Meeting, Snowbird, UT, Vol. 2, p. 489, August 12-16, 1990.

Sla87 D. Slaughterbeck, "Pre- and Post-Test Thermal-Hydraulic Comparisons of LACE LA-4," LACE TR-027, EPRI RP2802-4, Electric Power Research Institute, Palo Alto, CA, December 1987.

Smi91 P. N. Smith and P. L. Mason, "Analysis of Falcon Test FAL-3A Using the CONTAIN Code," AEA-TRS-5095, AEA Technology, Winfrith Technology Centre, Dorchester, Dorset DT2 8DH, United Kingdom, March 1991.

Smi92 P. N. Smith and P. Ellicot, "Analysis of the ACE Iodine-Aerosol Test using the CONTAIN Code," AEA-RS-5287, AEA Technology, Winfrith Technology Centre, Dorchester, Dorset DT2 8DH, United Kingdom, March 1992.

Smi92a P. N. Smith and P. Ellicot, "UK Calculations of the Heatup Phase of the F2 Experiment in the Battelle Model Containment Using the CONTAIN Code," AEARS-5203, AEA Technology, Winfrith Technology Centre, Dorchester, Dorset DT2 8DH, United Kingdom, March 1992.

Smi92b P.N. Smith and P. Ellicot, "On the Analysis of the DEMONA Experiment A9 Using CONTAIN 1.11(UK)," AEA-RS-5121, AEA Technology, Winfrith Technology Centre, Dorchester, Dorset DT2 8DH, United Kingdom, March 1992.

Smi92c P. N. Smith and P. Ellicot, "On the Analysis of the DEMONA Experiment B2 Using CONTAIN 1.11(UK)," AEA Technology, Winfrith Technology Centre, Dorchester, Dorset DT2 8DH, United Kingdom, March 1992.

Smi92d P.N. Smith and P. Ellicot, "Analysis of DEMONA B3 Using CONTAIN 1.11(UK)," AEA-RS-5290, AEA Technology, Winfrith Technology Centre, Dorchester, Dorset DT2 8DH, United Kingdom, March 1992.

Smi92e P.N. Smith, and P. Ellicott, "On the Analysis of the LACE LA-4 Experiment Using CONTAIN 1.11(UK)," AEA-RS-5122, AEA Technology, Winfrith Technology Centre, Dorchester, Dorset DT2 8DH, United Kingdom, March 1992.

Sta95 D. W. Stamps, "CONTAIN Assessment of the NUPEC Mixing Experiments," SAND94-2880, Sandia National Laboratories, Albuquerque, NM, August 1995.

Tar88 W. W. Tarbell et al., "Direct Containment Heating and Aerosol Generation During High Pressure Melt Ejection," in Proceedings of the ANS/ENS Winter Meeting, Thermal Hydraulics Division, American Nuclear Society, Washington, DC, November 1988. 
Til91 J. Tills, V. Nicolette, and K. Washington, "Initial Performance Assessment of the Westinghouse AP600 Containment Design and Related Safety Issues," NUREG/CP0118, Proceedings of the U. S. Nuclear Regulatory Commission Nineteenth Water Reactor Safety Information Meeting, Bethesda, MD, October 28-30, 1991.

Ti196

Uch65

Val83

Val88

Ver87

Wan88

Was88

Wie88

Wil87
J. Tills, "Calculations and Analysis for the Large-Scale Passive Containment Cooling System (PCS) Tests," SAND96-1089 (Proprietary), Sandia National Laboratories, Albuquerque, NM, May 1996.

H. Uchida, A. Oyama, and Y. Togo, "Evaluation of Post-Incident Cooling Systems of Light-Water Power Reactors," Proceedings of the Third International Conference on the Peaceful Uses of Atomic Energy, Geneva, August 31-September 9, 1964, Vol. 13, pp. 93-104, United Nations, New York, 1965.

L. Valencia and T. F. Kanzleiter, "Blowdown-Investigations in a Reactor Containment - Rupture of a Steam Pipe -- Quick Look Report for Tests V42, V43, and V44," Technical Report PHDR 38-83, Forschungszentrum, Karlsruhe, Germany, May 1983.

G. D. Valdez, "Review and Implementation of Thermodynamic and Transport Properties for the CONTAIN Code," NUREG/CR-5173, SAND88-7101, Sandia National Laboratories, Albuquerque, NM, November 1988.

P. J. Vernier and P. Solignac, "A Test of Some Condensation Models in the Presence of a Noncondensable Gas Against the ECOTRA Experiment," Nuclear Technology, Vol. 77, pp. 82-91, 1987.

C. Wang and C. Tu, "Effects of Non-Condensable Gas on Laminar Film Condensation in a Vertical Tube," Int. Journal of Heat and Mass Transfer, Vol. 31, No. 11, pp. 2339-2345, 1988.

K. E. Washington and D. E. Carroll, "Assessment of Models for Steam Release from Concrete and Implications for Modeling Corium Behavior in Reactor Cavities," SAND88-2329C, Sixteenth Water Reactor Safety Information Meeting, Gaithersburg, MD, October 24-27, 1988.

J. Wiesemes, "CONTAIN-Rechnungen zum Aerosol Verhalten fur Fall 7 Der SNRRisikostudie," (in German), GRS-A-1488, Gesellschaft fur Reaktorsicherheit (GRS) mbH, Koln, Germany, October 1988.

D. C. Williams et al., "Containment Loads Due to Direct Containment Heating and Associated Hydrogen Behavior: Analysis and Calculations with the CONTAIN Code," NUREG/CR-4896, SAND87-0633, Sandia National Laboratories, Albuquerque, NM, May 1987. 
Wil88 D. C. Williams, and D. L.Y. Louie, "CONTAIN Analyses of Direct Containment Heating Events in the Surry Plant," in Proceedings of the ANS/ENS Winter Meeting, Thermal Hydraulics Division, American Nuclear Society, Washington, DC, 1988.

Wi192 D. C. Williams, "An Interpretation of the Results of Some Recent Direct Containment Heating (DCH) Experiments in the Surtsey Facility," SAND92-0442, Proceedings of the NURETH-5 Conference, Salt Lake City, UT, September 1992.

Wil95 D. C. Williams et al., "CONTAIN Code Analyses of Direct Containment Heating (DCH) Experiments: Model Assessment and Phenomenological Interpretation," Proceedings of the ANS Winter Meeting, Thermal Hydraulics Division, San Francisco, October 29-November 2, 1995.

Wi196 D. C. Williams and R. O. Griffith, "Assessment of Cavity Dispersal Correlations for Possible Implementation Into the CONTAIN Code," SAND94-0015, Sandia National Laboratories, Albuquerque, NM, February 1996.

Wol83 L. Wolf, L. Valencia, and T. Kanzleiter, "Overview of the HDR-Containment Tests," NUREG/CP-0048, Proceedings of the U. S. Nuclear Regulatory Commission Eleventh Water Reactor Safety Reasearch Information Meeting, Gaithersburg, MD, Vol. 3, October 24-28, 1983. 


\section{APPENDIX D \\ QUALITY ASSURANCE PROCEDURES}

\section{D.1 Introduction}

The primary purpose of CONTAIN and of the program under which it is developed is to provide the U. S. Nuclear Regulatory Commission (USNRC) with a state-of-the-art containment analysis tool to address critical safety issues. An important aspect of achieving this goal has been and continues to be a well-thought-out and executed quality assurance program. The quality assurance procedures are a set of guidelines that ensure the quality of the code by monitoring key aspects of the development process. These include the code development, code configuration control, code development documentation, change tracking, testing, problem reporting and tracking, and user support procedures. The objective of this appendix is to describe the CONTAIN quality assurance program.

\section{D.1.1 Quality Assurance Program Scope}

The CONTAIN quality assurance program is concerned solely with the protection and sound development of the CONTAIN code and associated documentation. The quality assurance procedures neither monitor nor ensure the purity of code developed outside the CONTAIN project. A separate program, POSTCON, [Was87] has been developed to postprocess CONTAIN plot files. This program is available to users, and information about it appears in the Code Manual (since the integration of all user documentation into one code manual for CONTAIN 2.0, the Code Manual and User's Manual are one and the same). However, POSTCON's development and maintenance are not covered by the CONTAIN quality assurance program. Nor does the program cover code modifications developed outside the CONTAIN project. The CONTAIN project recognizes that users may need to modify the released code to accommodate site-specific needs, but it does not support these changes. While acknowledging that individual sites have unique needs, the CONTAIN project also recognizes that its commitment is to protect and support its own baselines.

As an established, complex computer code, CONTAIN attracts the interest of outside agencies who want to modify CONTAIN to handle slightly different applications. To accommodate these needs, multiple development projects might be performed by the same SNL staff working in the USNRC CONTAIN project. Because the sponsors of the CONTAIN variants may each require different levels of quality assurance, modifications developed for one variant may not be suitable for others. In general, though, the variant codes benefit from the strict quality assurance procedures enforced for the baseline CONTAIN. The relation of variants of CONTAIN to baseline CONTAIN is discussed in Section D.2.5.3.

\section{D.1.2 CONTAIN Quality Assurance Organization}

The organizational structure (Figure D-1) of the CONTAIN quality assurance system is designed to control and verify the quality of the change process. In general, as the development and testing process continues, more constraints are imposed on the change process and correspondingly higher levels of authority are needed for approving a change and authorizing its movement to another stage. 
The primary functions within the organizational structure are those of project supervision, revision management, code development, internal "beta testing," and integration testing.

The project leader provides overall direction to the CONTAIN project. The line manager and project leader negotiate to select the project staff. The line manager, project leader, and revision manager negotiate with the USNRC to determine whether the current change process is just a revision or actually a new version. This decision is based on such matters as commitments to users for improvements or corrections, the inefficiencies involved in running the current baseline, and the significance of the phenomenological changes being implemented. The line manager also resolves major conflicts within the project and authorizes the release of a revision or version of CONTAIN.

The revision manager plans and implements the overall strategy of the new development, taking responsibility for the software configuration and the documentation. The revision manager is responsible for protecting the developmental baseline version of CONTAIN. This manager is responsible for grouping the requests for change into updates, determining the priority of each update, informing the staff of revision status, and performing a continuing informal quality assurance function. Other responsibilities include authorizing the movement of an update from one stage to the next, and giving approval for the initial implementation of the change, the final acceptance of the update, and the associated code documentation changes prior to release. The revision manager supervises the testing of a revision or version to ensure that as many tests as feasible are run. The revision manager is also responsible for maintaining the operational baseline code.

The code developers take sets of problems that are grouped into update sets and define, design, test, and implement new coding and code manual revisions. The developers do this in consultation with the rest of the staff and, in particular, with the revision manager and any affected code developers. The developers are responsible for resolving all conflicts with other updates before the start of integration testing. Their work is reviewed by the revision manager and verified by the internal beta testers. For each update, the developer, internal beta tester, and revision manager jointly decide when to move the update from one stage of development and testing to another. Should there be a conflict, the revision manager makes the final decision.

The internal testing is performed by members of the CONTAIN project, as well as experienced users outside of the CONTAIN project. The internal testing is intended to detect problems or errors not uncovered in the developmental stages. Because the internal testers are typically not the developers of the code modification in question, this internal testing can be thought of as an informal form of beta testing. No formal documentation is generated as part of the internal beta stage, other than problem reports when major problems are uncovered that are not immediately addressed.

The integration tester runs the integration and acceptance test program, submitting the collected updates to a verification process that establishes the integrity of the physics, coding, and documentation revisions. The integration tester also demonstrates that the updates are compatible with the operational baseline code.

For any change to CONTAIN, informal peer review occurs when the CONTAIN staff members discuss the options for modeling and implementing the code changes. For many of the code changes 


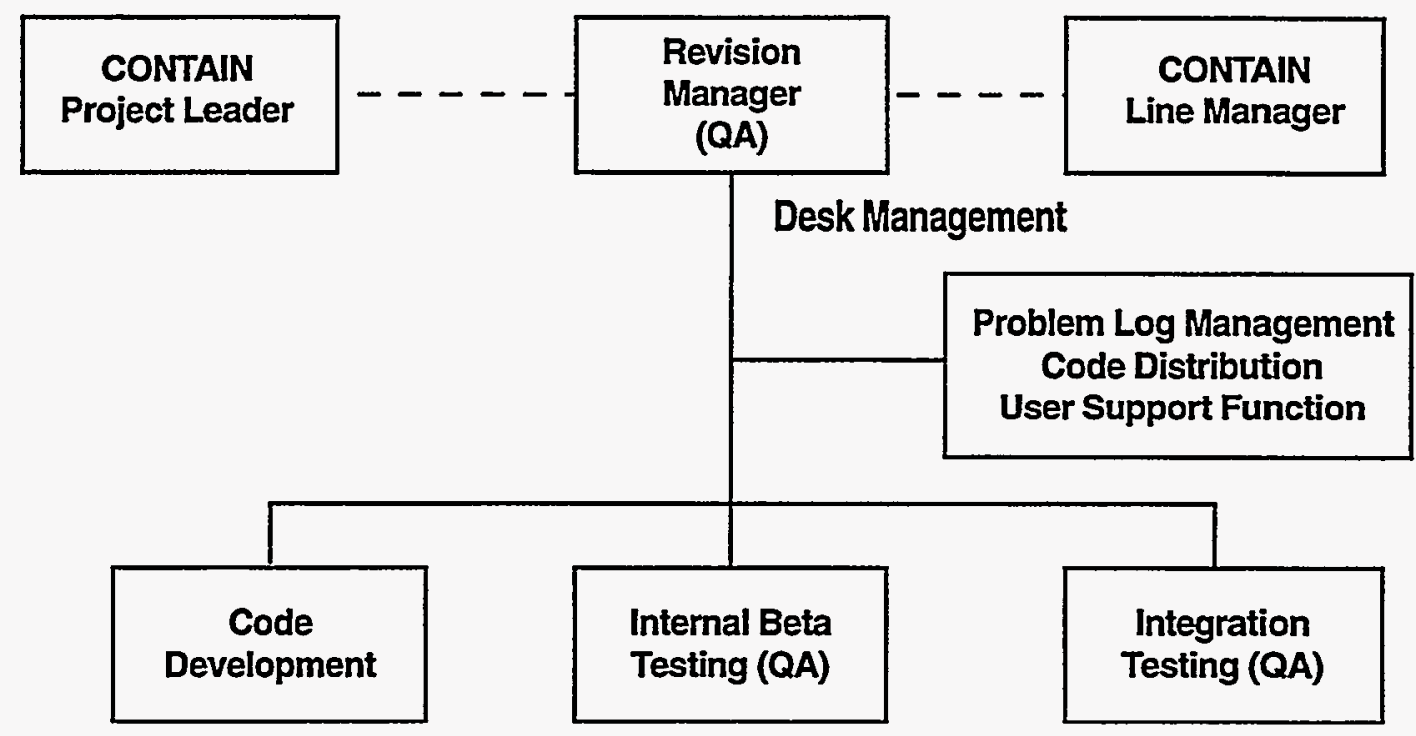

Figure D-1. CONTAIN Quality Assurance (QA) Organization 
up to CONTAIN 1.11, the code developers circulated memos describing the changes to the CONTAIN staff for review. Code changes for revisions and versions following CONTAIN 1.11 are required to go through a formal peer review process. This review process requires that the code developer prepare a requirements specification document for the code change and offers the code developer the option of holding a review meeting with the CONTAIN staff. The review meeting provides formal peer review and helps ensure that the resources of the entire CONTAIN staff are best utilized for each code change. The formal review process is described in more detail in Section D.2.4.

\section{D.2 The CONTAIN Code Development/Maintenance System}

The CONTAIN code development/maintenance system is implemented as six major functions, as shown in Figure D-2. In this data flow diagram, data sources and sinks are represented as rectangles, functions as circles (bubbles), and data stores as single horizontal lines. Arrows show data flow and direction, and words on the arrows indicate what data items are involved in the flow. Each of these six major functions is described briefly in this section with respect to the class of error it detects or the control it exercises.

\section{D.2.1 User Support}

The user support function mediates all communication with the user community, as illustrated in Figure D-3. CONTAIN users can obtain user support by contacting the USNRC for authorization or through an independent contract with Sandia National Laboratories. The user support function disseminates information to users by official releases of new revisions and versions of the CONTAIN code (including both software and user documentation). The user support function also maintains a data base on the user community.

The CONTAIN user support function accepts and evaluates user requests for revision and additions to the code, and it disseminates information about innovative or particularly useful ways of using CONTAIN discovered by individual users. The phone numbers and electronic mail addresses of any member of the project are made available to any code user, who can then contact the person involved in the area closest to the area of interest. After the first contact is made further support is channeled through the project leader so that USNRC concurrence can be obtained.

When requested by the USNRC, the CONTAIN code will be distributed to organizations that request the code for use in specific USNRC-sponsored programs or members of the Cooperative Severe Accident Research Partners (CSARP) at USNRC expense. Distribution of the code to all others (non-USNRC sponsored programs/organizations) is at the discretion of the USNRC. Requests from non-USNRC programs that are not approved by the NRC may be considered by the manager of the CONTAIN program. The option exists for the manager to establish an independent contract with the requesting organization.

The user support function also helps control requirement specification errors. For example, if a modification is not warranted, the CONTAIN staff may offer the user suggestions on minimizing 


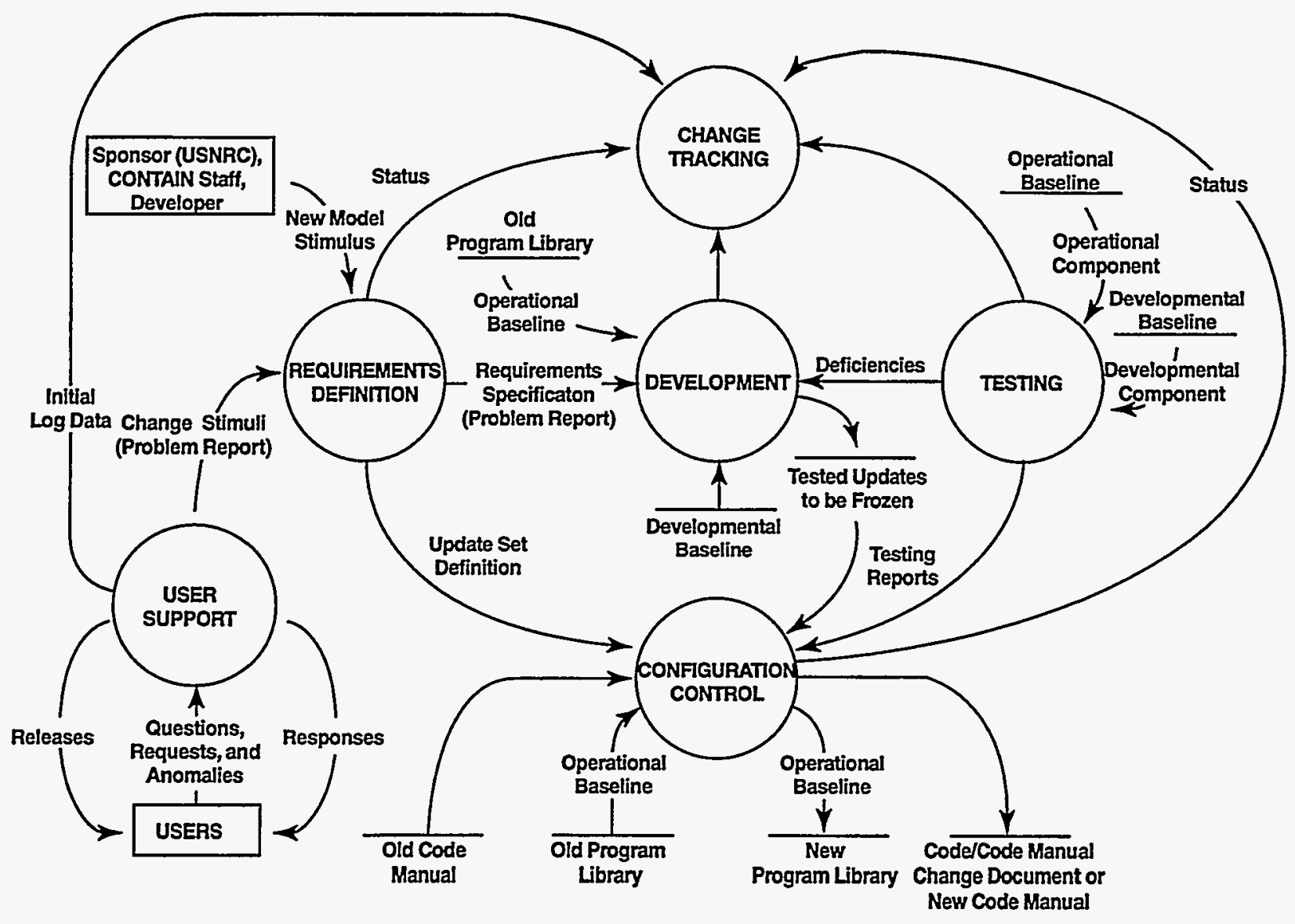

Figure D-2. CONTAIN Code Development/Maintenance System 


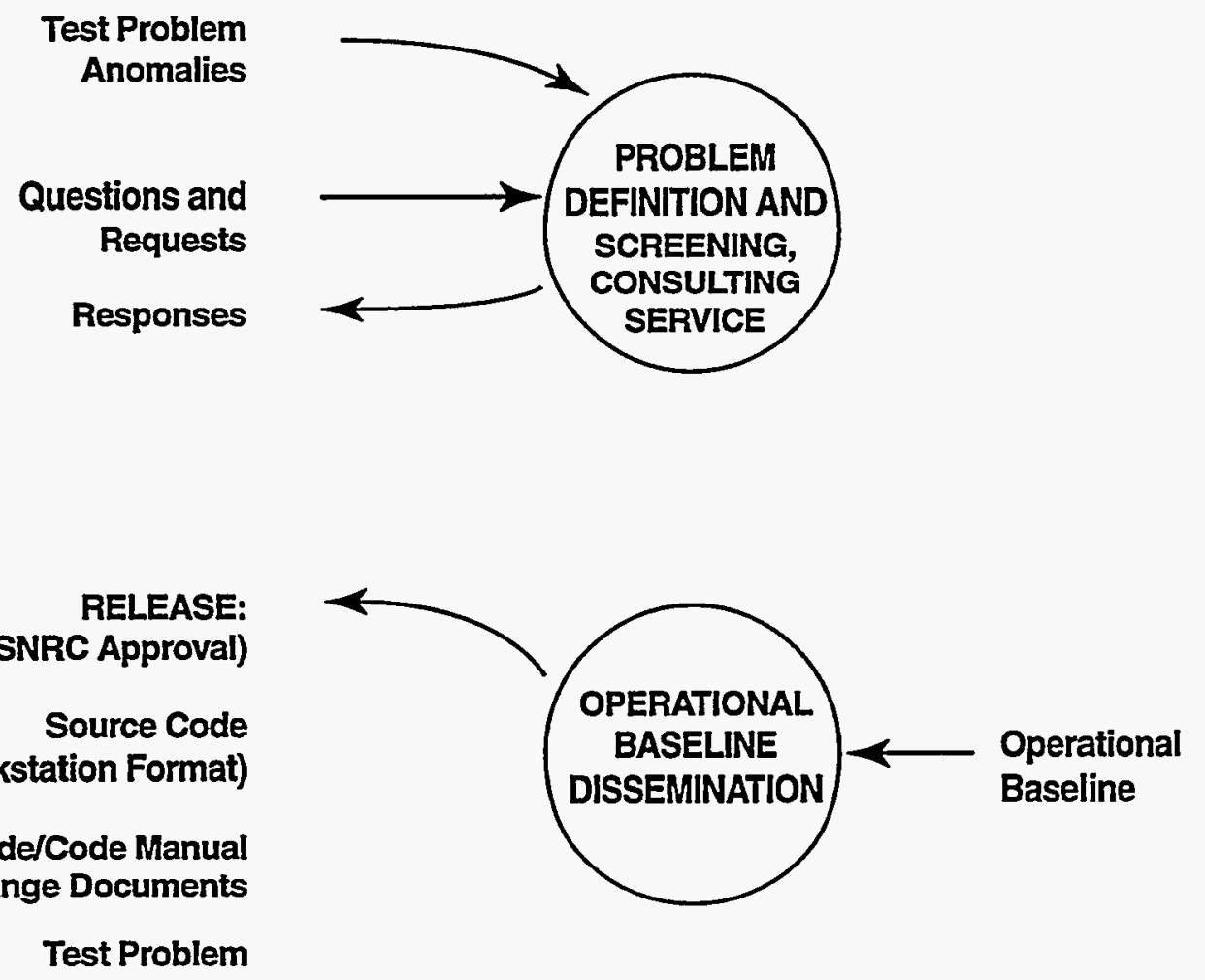

Figure D-3. The User Support Function 
the difficulty, resolving the issue without resorting to code modification. For every apparent need that can be resolved without changing the code, there is concomitant elimination of the opportunity for all the types of error that may be generated by a code change.

D.2.1.1 Change Stimuli. The CONTAIN user community, the CONTAIN staff, and the USNRC are the principal sources of change stimuli. A planned update comes from the USNRC through the statement of work. As with any complex software system, the operational baseline of CONTAIN inevitably contains errors. When discovered by internal and/or external use of the code or in the testing process, errors become stimuli for change. Users also discover innovative ways to use CONTAIN. When these innovations are of broad interest, they too become change stimuli.

D.2.1.2 CONTAIN Code Manual. Quality assurance procedures concerning the release of a new Code Manual require that the manual pass through a review process that checks for consistency, accuracy, and clarity. The Code Manual is subjected to a thorough internal review in which the document goes through the SNL standard publication review process, which requires that the first two levels of SNL management approve the document. Prior to management review, the document is reviewed by other staff members. Any necessary changes are incorporated before publication of the new Code Manual.

Any subsequent changes are addressed by a Code/Code Manual Change Document. The changes may be due to code changes, peer review, or other stimuli. The Change Document completely describes the models and provides the appropriate user guidance. The Change Document also includes the update to CONTAIN and a discussion on the assessment of the models. The developer of the new model or revision is responsible for generating the Change Document. The draft Change Document is first reviewed by the author. Next, the document is sent to the CONTAIN staff and interested individuals for internal review. Prior to submission to the revision manager, the author is responsible for resolving the comments received from the internal review. The revision manager then reviews the document and signs off on the cover sheet when the Change Document is judged to be satisfactory. After the completion of the internal review, the document is reviewed by the USNRC, and their recommendations are subsequently resolved by the author and the CONTAIN staff.

D.2.1.3 Code Releases. The CONTAIN code is distributed only in source code format. This ensures that the updates have been assembled correctly, since the work is done by the CONTAIN staff. Also, it ensures that any machine-specific modifications are correctly included for a given workstation. Workstation issues are presently available for SUN, HP, DEC, and IBM machines. The Cray version is still available in source form, but it is expected that few, if any, users will request this platform. As a final measure against the introduction of errors, the user is sent sample input and output for a test problem. The user can then verify that the code installation is correct and current.

\section{D.2.2 Requirements Definition}

The requirements definition function provides a number of mechanisms for controlling requirements specification errors. The code developer assigned to correct a known problem or add a new model 
documents the proposed correction or model in a requirements specification document. This document is reviewed by the revision manager and other members of the CONTAIN staff for completeness and design flaws. The purpose of the review is to ensure that all aspects of the known problem or desired modeling need are covered by the proposed approach. Review comments are then addressed by the developer in a revised specification. Either the developer or the revision manager may at their discretion request a review meeting. The need for such a meeting is typically driven by the extent of comments or when various review comments from the staff are conflicting. Often this meeting is between the revision manager and the developer, but it can include other members of the CONTAIN staff as needed.

\section{D.2.3 Development}

The development process begins once an update has been defined and the project manager has assigned a developer. The developer is responsible for designing, implementing, and documenting each code modification.

Much of the responsibility for ensuring quality in the software lies with the developer. The developer has the primary responsibility for controlling coding errors. In addition, the developer is responsible for code completeness (i.e., for ensuring that the code performs all the required functions); for the consistency of the new code relative to other parts of CONTAIN (e.g., the code must use the same data formats and structures as those used by other CONTANN code elements with which it communicates); and for avoiding regression (i.e., the new code must not introduce unintended changes to the operation of other portions of the CONTAIN code).

The developer defines, designs, debugs, and tests the update in accordance with CONTAIN conventions. As part of the test responsibilities, the developer must ensure the compatibility of the update with all previous updates. Communication with other developers is encouraged, in part to facilitate conflict resolution between or among updates. While free to consult with staff members, the developer may not involve the internal beta tester for the update in the design of the developmental tests. The quality assurance procedures that govern testing activities are detailed in Section D.2.6.

\section{D.2.4 Change Tracking}

Upon completion of the update, an update review is held to ensure that the update adheres to the requirements specification document and that the software guidelines were followed. Deficiencies in the code structure are reviewed by the revision manager to determine whether they are severe enough to warrant rework of the software. Deficiencies in the documentation are generally corrected immediately.

The format of the code review is decided by the revision manager. In the case of very simple updates, the revision manager may review the update personally. For more complicated updates, the revision manager may assign a review team of one or more individuals. The revision manager receives comments from the review team and resolves these with the code developer. It is the ultimate responsibility of the revision manager to approve the update. 
When the review is complete, the update is delivered to the revision manager. The revision manager inspects the package to check for completeness, accuracy, and adherence to project standards. The manager creates the source code from the update and compiles this source code to check for compile errors and warning messages. The manager or an internal beta tester runs the test problems used by the developer and checks the results qualitatively for error messages, code bombs, etc. The manager inspects the documentation to ensure that it contains all mandatory sections, such as descriptions of testing and new input, and that upward compatibility has been maintained.

When the revision manager is satisfied with the update and documentation, the manager signifies acceptance by signing the Change Document cover sheet. A sample copy of the Change Document cover sheet is shown in Figure D-4. This signoff constitutes a process called "freezing." Once the update is frozen, it can no longer be modified. Any further modifications or error corrections must be addressed in a subsequent update.

\section{D.2.5 Configuration Control}

The CONTAIN quality assurance program includes a strictly enforced configuration control component. The objective of this component is to ensure that whenever users or developers access the CONTAIN code, they are presented with a complete, consistent, and tested code.

D.2.5.1 Baseline Control. CONTAIN configuration control employs a baseline system to ensure consistency and to control context. A baseline consists of a set of updates that are used without modification and that can be changed only under strictly controlled procedures. CONTAIN configuration control enforces two baselines: operational and developmental. The operational baseline is the software released to the user community as a "version" or "revision." A version embodies major changes and entails the publication of a new edition of the Code Manual. A revision consists of less significant, but often still substantial, changes and includes the distribution of change documents to the most recent version of the Code Manual. The developmental baseline, derived from the operational baseline, provides a controlled environment for development and testing. The same mechanism is used to control both baselines, but control is exercised over each baseline independently.

D.2.5.2 The Control Mechanism. Prior to CONTAIN 1.2, the UPDATE utility was used to enforce baseline control. UPDATE is a CRAY utility designed to facilitate the maintenance of a large code project by managing and tracking software changes. [UPD84] It is a line-oriented text editor that creates and maintains program libraries (data sets) and other data on the basis of line and deck identifiers. A library can be modified by adding or purging decks, or by adding, modifying, or deleting cards in existing decks. UPDATE can be used to generate new program libraries, source data sets (with UPDATE directives embedded), compile data sets (ready for compilation), or selected listings. Duplicated code can be defined once and referenced for inclusion in other decks. Options in UPDATE ensure correct line numbers by assigning new sequence numbers using the UPDATE 4.0 scheme. UPDATE produces output that can be used as input to other programs, particularly compilers and assemblers. Although there are many alternative ways of enforcing baseline control, UPDATE was chosen for CONTAIN because it 


\section{CONTAIN}

\section{Code and/or Code Manual Change Document}

Code/Code Manual Change

Date

CD Number

Reference Problem Number (s)

Update Identifier (s)

Date

Responsible Individual

Date

Cognizant Individuals

Date

Date

Revision Manager Approvals:

Requirements Specification Document

Date

Revision Meeting

Date

Code Change

Date

Code Manual

Date

Comments:

Figure D-4. Sample Change Document Cover Sheet

Rev. 0

D-10 
- ensures that modifications will promulgate in the correct order,

- maintains the completeness of a set of modifications,

- maintains the historical integrity of the code, and

- provides a controlled means for reversing modifications, allowing a clean return to a previous well-defined baseline.

To simplify the use of UPDATE emulators, CONTAIN developers are restricted to the use of a limited subset of Cray's UPDATE features. These features include

$\begin{array}{ll}* \text { deck } & \text { to introduce a deck } \\ * \text { comdeck } & \text { to introduce a COMMON DECK } \\ *_{\mathrm{i}} & \text { to insert } \\ \text { * }_{\mathrm{d}} & \text { to delete or replace } \\ * \text { compile } & \text { to specify compile or source data set } \\ * \text { \% } & \text { to comment } \\ * \text { purgedk } & \text { to remove a deck. }\end{array}$

In addition to the above restriction, blank lines are not allowed in the update files.

With the ongoing evolution of computing platforms, the CONTAIN code must be adaptable if it is to keep pace with the changing environment. Following CONTAIN 1.2, CMP [Tho91] will be used for creating updates. CMP is similar to UPDATE and has demonstrated compatibility in the workstation environment. Under the new environment, it will be possible to make changes to CONTAIN using a more direct approach than before, while still retaining the level of protection offered by UPDATE. In this approach an old and new copy of the code will be kept in separate directories by each of the various developers and a "differences" program provided as part of the CMP tools will be used to generate code updates.

CMP consists of four major functions to maintain computer programs and other data sets. The first function is an UPDATE processor with system independent libraries that allow corrections and retain a correction history. Program libraries can be transported between systems without loss of information. The second function is a sorting processor that alphabetically sorts all subroutines, functions, and other program units allowed under FORTRAN 77. Another option allows for sorting of decks and comdecks in a program library. The third function is a differences and correction set generator that operates by finding differences between two files. An update correction set is produced to modify an update library. The fourth function is a collection of text file copying utilities. A complete description of the CMP utility is given in Reference Tho91.

An important part of CONTAIN configuration control is the concept of update freezing, introduced in Section D.2.3. Should a need for modifying a frozen update be discovered during internal beta testing, the modification would be implemented with a follow-on correction update. The follow-on 
could consist either of significant changes required to complete the original update, or could incorporate fixes to miscellaneous small errors. Such errors could pertain to more than one update.

As update sets are developed and frozen (see Section D.2.3), the revision manager adds them to the developmental baseline. The developmental baseline thus consists of the most recently released operational baseline in the form of a CMP program library plus all frozen update sets for the next operational baseline. When all update sets have been frozen and tested, they are added to the old operational baseline to create the new operational baseline. Only then is the code ready for release. At the time of release of the new operational baseline, the developmental baseline and the new operational baseline are identical. The process of using CMP to maintain the code baseline is depicted in Figures D-5 and D-6. A detailed data flow diagram of the configuration control function is presented in Figure D-7.

When all the update sets in a revision have been incorporated into the developmental baseline and internal beta testing of all update sets has been completed to the satisfaction of the revision manager, the revision manager notifies the integration tester to initiate integrated testing of the (now complete) developmental baseline as a whole. When integration testing has been completed, the operational baseline is updated by application of the complete set of revision updates. Testing is discussed at greater length in Section D.2.6.

D.2.5.3 Control of Variant Codes. Once a large simulation code has been developed, it can be used as a base to create codes for related applications. An example is CONTAIN-LMR, [Mur93] which has been developed with foreign funding coordinated through the USNRC. Its base is the light water version of CONTAIN with added features unique to sodium-cooled reactors. While there are substantial advantages to using this approach, care must be taken in managing the quality control and development of both the base code and the alternate codes. Each code sponsor's needs must be considered to ensure that work performed for the new customer does not create unnecessary complications for the original sponsor.

If two codes have different procedures or standards of quality assurance, improvements in one may not satisfy the needs of the other. Ideally, new features in a variant would be useful, or at least have no negative effect, for the users of the base code. Incorporating variant features into the base code, however, may impose a burden on the mainstream development process, since the more a code is enlarged, the harder it is to maintain. If the complexity of the code also increases, then its maintenance is even more demanding. For example, future improvements of the base code must be tested against a variety of new problems, and the existence of these variant features complicates this process.

When developing variants of the base code and performing updates of the base and variant codes, the CONTAIN team follows a process designed to ensure that the new features do not interfere with old ones. The CMP utility and CONTAIN's code configuration control mechanism (described in Section D.2.5.2) govern the relationship between the base and variant codes. A variant is independently developed as an update of a particular base code version or revision. The update 

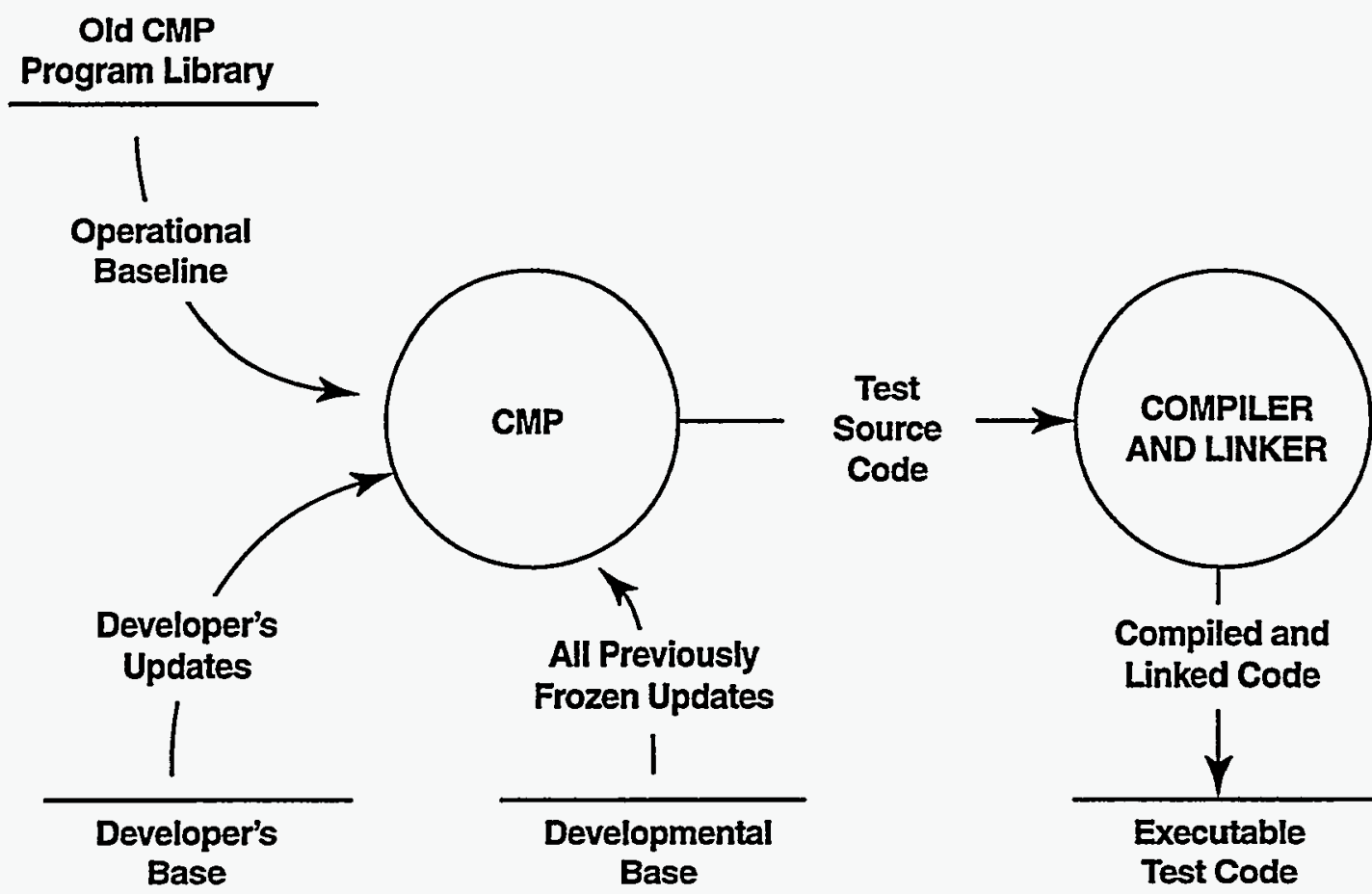

Compiled and

Linked Code

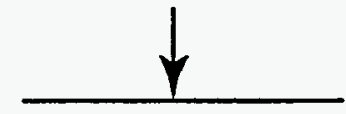

Executable Test Code

Figure D-5. Use of CMP for Testing

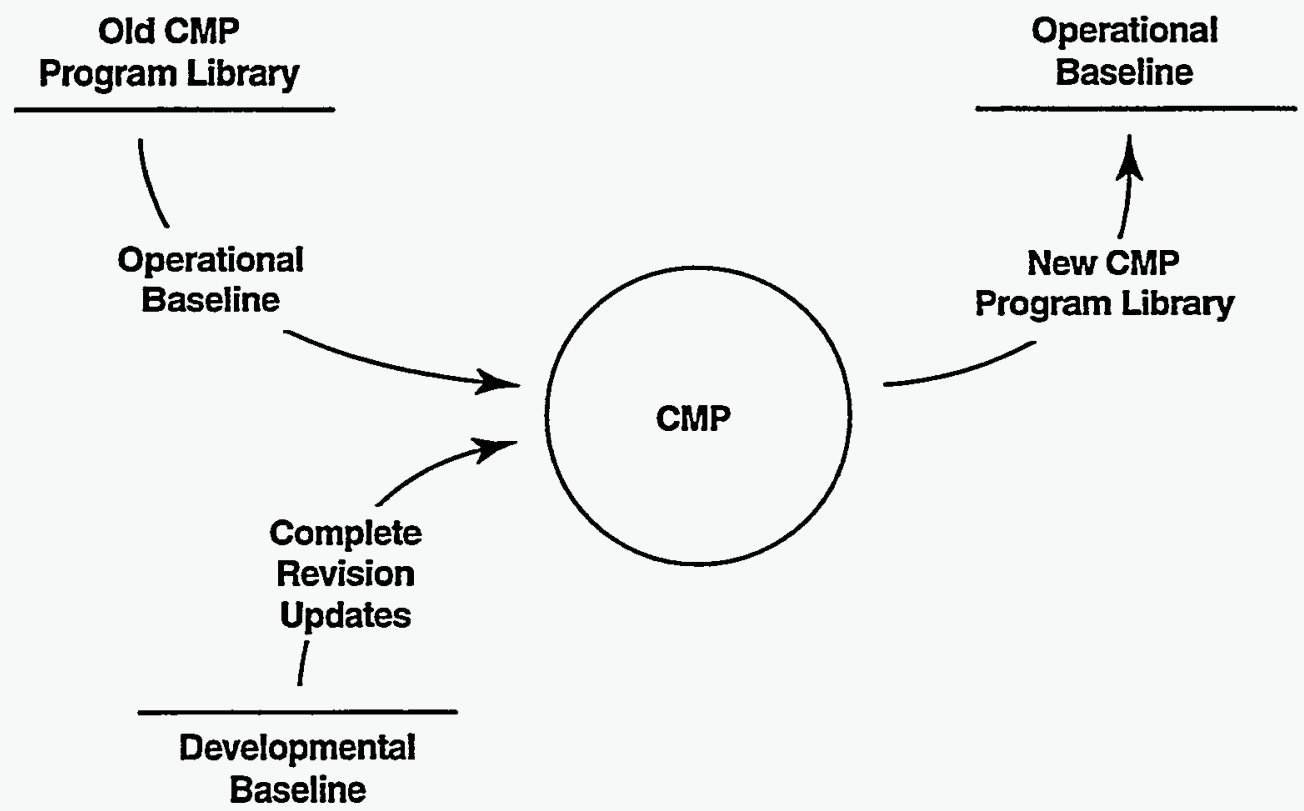

Figure D-6. Use of CMP for Operational Baseline Control 


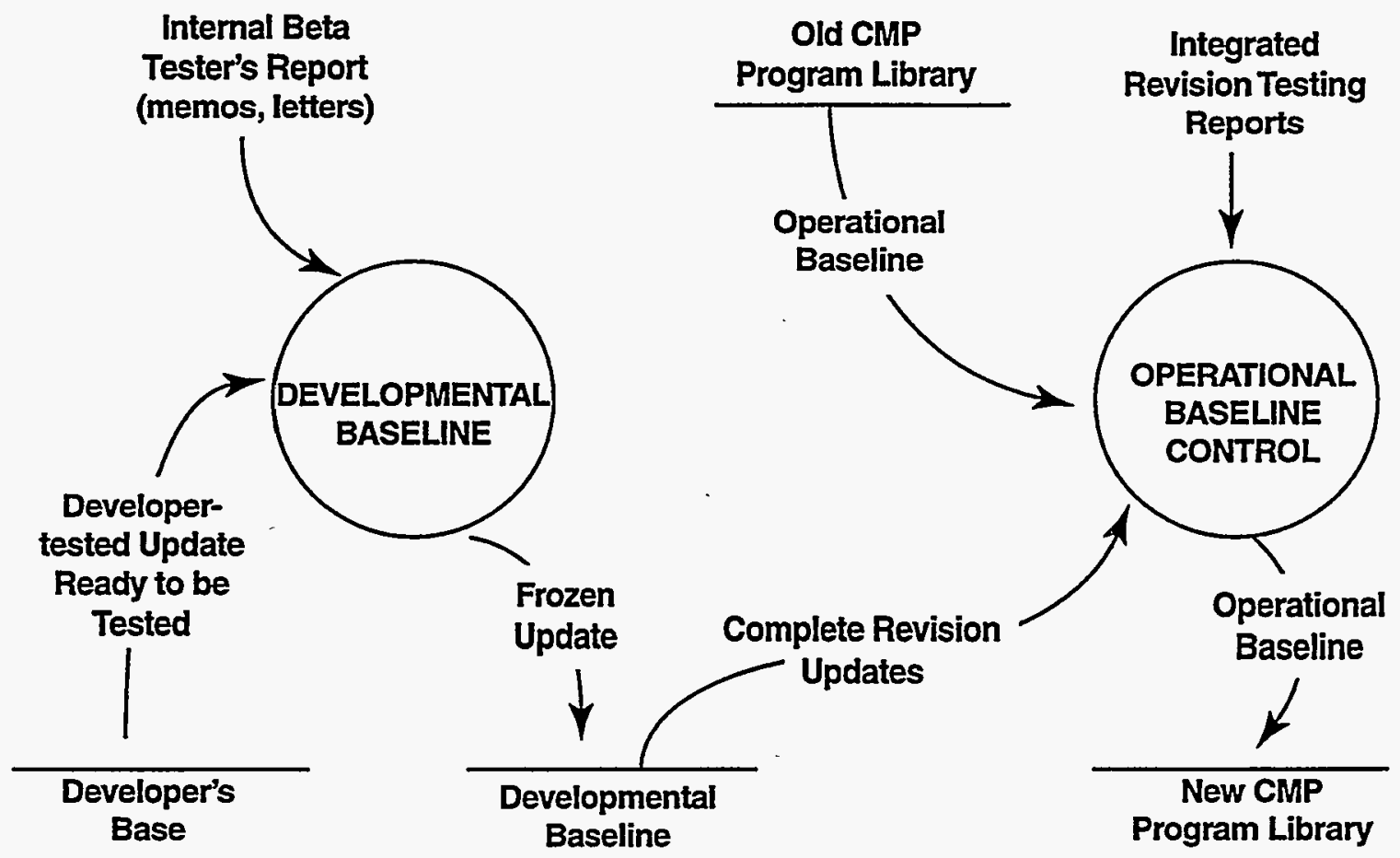

Figure D-7. The Configuration Control Function 
instruction set that creates the variant will work only on that particular base code, because other versions of the base code will have different line sequencing.

As time passes, the base code evolves away from the variant code, and cross-fertilization between the two variations becomes desirable. Code features are transferred in two ways:

1) from the variant version to the base code, and

2) from the base to the variant version.

Staff in the base code project determine whether or not to carry out a transfer of the first type based on a strict cost/benefit assessment done from the viewpoint of the base code project sponsor. They then implement the desired changes in essentially the same way that a code revision is done, by assigning the update a location in the sequencing of code changes, modifying the code documentation, and completing formal testing.

A similar assessment from the viewpoint of the variant code project is done for the second type of transfer. The desired changes are accomplished periodically and systematically by a process called an "accommodation." Using this method, an update set that creates an existing, working variant version from a particular base code is modified to reflect the line numbering of a subsequent base code release. This part of the process is straightforward. In addition, it is usually necessary to examine the interactions between features in the variant code and new features in the base code that have developed after the original base version was frozen. Some features may have to be disabled and/or significantly reworked. When complete, the accommodation will provide a new variant code that has the original variant features, and those new features in the base code that were deemed appropriate. The cost of performing this accommodation is borne by the variant code project.

Note that at times the variant code based on one release of the base code coexists in time with a later release of the base code. This situation occurs because it takes time to perform an accommodation, and also because there may be reasons to postpone an accommodation until a natural point in the variant development schedule occurs. The relationship between the mainstream CONTAIN code and variant versions of CONTAIN is shown in Figure D-8.

Documentation of variant versions using this approach can be simplified by providing supplementary reports that discuss the differences between a variant release and the documented base version from which it stems. This was done for CONTAIN-HWR. [Bil94]

\section{D.2.6 Testing}

Testing verifies the integrity of both individual updates and code revisions and of the combination of revisions that becomes a new version. Testing has four objectives: verification, validation, integration, and regression prevention. The objective of verification is to determine that the update set performs as specified by the code developer in the code specification requirements. Validation testing determines that the update set performs calculations accurately with respect to the physical model represented. Validation testing may include comparison of calculated results with available 


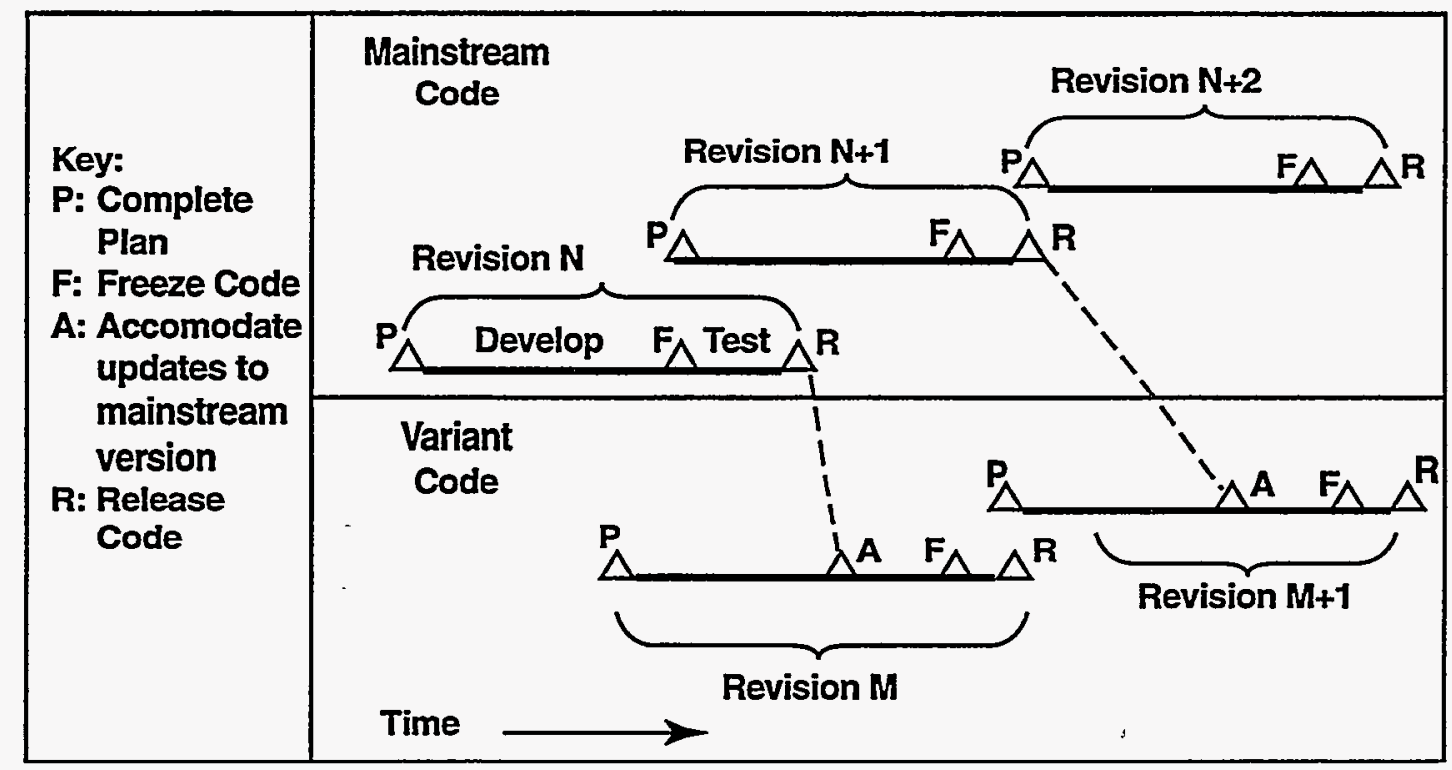

Figure D-8. The Relationship Between the Mainstream CONTAIN Code and its Variant Versions 
experimental results. The objective of integration is to exercise aspects of an update that will interact with other parts of the developmental baseline to ensure that in-context transformations are correctly performed. The objective of regression prevention is to ensure that the addition of an update set does not make any unintended changes in CONTAIN results.

Testing is carried out in three distinct stages. The developer is responsible for the first testing stage, which focuses on determining that the update functions correctly and executes as intended. During the second phase, one or more internal beta testers use the modified code and focus on determining that there are no undetected errors in the update. The integration tester is responsible for the final testing stage, which determines that the new release, with all updates in place, functions correctly. Table D-1 summarizes the relationship between the development/maintenance functions and indicates the type of errors they protect against.

D.2.6.1 Developmental Testing. In developmental testing, the developer formulates test problems and executes them to verify the update design. The developmental testing program is used to uncover and correct problems with the update. The developmental tests are initially used to determine that the update code is indeed executable. Once the executable code is created, the developmental tests are used to verify that the update executes as the developer intended. Finally, the developmental tests are used to verify that the code produces results that are accurate.

Table D-1

Quality Assurance Functions

\begin{tabular}{|c|c|c|c|c|c|c|c|c|}
\hline \multirow[b]{3}{*}{ Type of Error } & \multicolumn{8}{|c|}{ Location of Quality Assurance Functions } \\
\hline & \multirow[b]{2}{*}{ 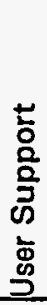 } & \multirow[b]{2}{*}{ 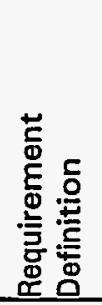 } & \multirow[b]{2}{*}{ 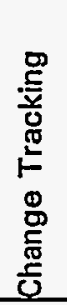 } & \multirow[b]{2}{*}{ 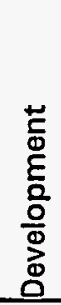 } & \multirow[b]{2}{*}{ 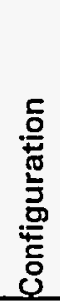 } & \multicolumn{3}{|c|}{ Testing } \\
\hline & & & & & & 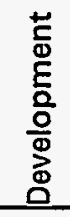 & 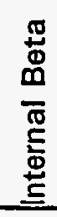 & 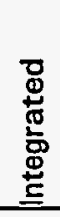 \\
\hline Requirements Specification & - & $\bullet$ & & & & & & \\
\hline Model & & & & & & - & - & \\
\hline Design & & & & & & & - & - \\
\hline Coding & & & & $\cdot$ & & - & - & - \\
\hline Regression & & & & - & - & - & - & - \\
\hline Operational Consistency & & & & - & $\cdot$ & & $\cdot$ & \\
\hline Communication & - & & & & & - & - & \\
\hline Completeness & & & - & $\cdot$ & $\cdot$ & & - & \\
\hline
\end{tabular}


In addition to simpler calculations traditionally done at this testing stage, developmental testing also includes actual plant calculations. Including more complex calculations at this phase of testing exposes errors not previously discovered. Also, the calculations help in finding any unexpected results related to model interdependencies. It should be noted, however, that developmental testing cannot be very comprehensive, especially for code updates that make changes in many areas of the code. Therefore, the Change Document must indicate what developmental testing has been performed, to identify the aspects of the updates have been checked or verified.

When the Change Document for the update is complete and approved, the update is frozen and a transition is made from developmental testing to internal beta testing. In the developmental testing stage, the developer is allowed to change the update to correct any problems discovered. In contrast, in the internal beta testing program the update is frozen and direct modifications to the update are not allowed. Any changes to the update that are subsequently found to be desirable must be implemented through subsequent updates.

D.2.6.2 Internal Beta Testing. After approval of the Change Document for an update, testing can proceed to the internal beta testing stage. In the internal beta testing phase, the purpose is to exercise the update to uncover problems or errors not detected in the developmental testing stages. No formal documentation is generated as part of the internal beta testing. Internal beta testing is typically performed by a member of the CONTAIN staff other than the developer. Experienced users outside of the CONTAIN staff may also perform internal beta testing.

Internal beta testing replaces independent testing, which was the prescribed practice prior to CONTAIN 1.12. Independent tests were performed for the earlier versions of CONTAIN and as a result, CONTAIN had reached a demonstrated level of robustness and maturity. Consequently, independent testing has become outmoded and internal beta testing is considered more appropriate and cost-effective means of verifying revisions and new models implemented in CONTAIN.

D.2.6.3 Integrated Testing. When the internal beta testing is successfully completed and the Change Document has been approved, the revision manager includes the update in the revision. At this stage, the update has been tested against the established code and against all updates accepted ahead of it in the revision. Before the final revision is released, all updates are tested together using the complete set of standard tests, plus selected plant calculations deemed appropriate by the revision manager. This process is defined as integrated testing. This integration analysis verifies the ability of the collected updates to perform accurately and consistently as part of an integrated code. This pattern of testing causes several tests to be applied more than once-a redundancy that is necessary for a good quality assurance program.

The present set of standard tests (see Table D-2) provides a measure of quality assurance for the compatibility of all the updates. In addition, an automated process has been developed to perform a quantitative check of differences between CONTAIN results performed on different computer systems (SUN, HP, DEC, and IBM machines) to help identify and guard against machine-dependent coding. In this automated process, standard tests are run on the different machines and the results are then compared. 
D.2.6.4 Test Documentation. In every aspect of testing, documentation is necessary and important for accountability, reproducibility, and manageability. Throughout the testing phase of any new revision or version of CONTAIN, the process is documented either formally or informally. During the developmental testing stage, the developer generates the Change Document to describe the changes or new models implemented in CONTAIN.

The internal beta testing does not require a formal record. It is documented through informal memos from the internal beta testers, as well as letters from experienced external testers. These memos and letters are stored in the department filing system.

In integration testing, all the standard tests are performed and documented. The documentation is then provided to the revision manager for review and approval. The documentation for the integration testing phase also includes a brief description of the results of machine dependency investigations, if required. 
Table D-2

CONTAIN 2.0 Standard Tests

\begin{tabular}{||l|l||}
\hline Test ID & \\
\hline ST01 & Forced and Natural Condensation on Structures \\
\hline ST02 & Aerosol Fall Through Test \\
\hline ST03 & Aerosol Flow (Intercell Transport of Aerosols) \\
\hline ST04 & Intercell Gas Flow - Adiabatic Flow \\
\hline ST05 & CORCON/VANESA Standard Test Problem \\
\hline ST06 & Engineering Vent - Parameter - Table Input Test \\
\hline ST07 & Fission Product Intercell Flow Test \\
\hline ST08 & Hydrogen Burn Test \\
\hline ST09 & Ice Condenser Test \\
\hline ST10 & Improved Host Package - Fission Product Decay Heating and Pool Boiling Test \\
\hline ST11 & Improved Host Package - Engineering Safety Features \\
\hline ST12 & Improved Host Package - Fission Product Transport Through Structural Host \\
\hline ST13 & Integrated Workshop Problem \\
\hline ST14 & Radiation Enclosure with Intervening Gas Test \\
\hline ST15 & Boiling Water Reactor Test - SRV and SPV with Pool Boiling \\
\hline ST16 & Connected Structure Option Test \\
\hline ST17 & Film Flow Test with Fission Products \\
\hline ST18 & Heat Transfer Correlation Update Test \\
\hline ST19 & Pool Tracking with Drain Down \\
\hline ST20 & Grand Gulf Plant Model \\
\hline ST21 & Diffusion Frame Burn Test with Blow Down \\
\hline ST22 & Non-Ideal Eguation of State Water Test with a Source \\
\hline ST23 & Fission Product Library and the S-Host Option \\
\hline ST24 & Lower Cell Heat Conduction Test with Upward and Downward Heat Flow \\
\hline & \\
\hline
\end{tabular}




\section{REFERENCES}

Bil94 S. C. Billups et al., "User's Manual for CONTAIN-HWR/0, Rev. 1: A Computer Code for the Analysis of the Heavy Water Nuclear Reactor Containments Under Accident Conditions," SAND91-1482/NPRW-SA91-6, SandiaNational Laboratories, Albuquerque, NM, January 1994.

Mur93 K. K. Murata et al., "CONTAIN LMR/1B-Mod 1, A Computer Code for Containment Analysis of Accidents in Liquid-Metal-Cooled Nuclear Reactors," SAND91-1490, Sandia National Laboratories, Albuquerque, NM, January 1993.

Rus91 N. A. Russell et al., "Quality Assurance Procedures for the CONTAIN Severe Reactor Accident Computer Code," NUREG/CR-5518, SAND90-0011, Sandia National Laboratories, Albuquerque, NM, January 1991.

Tho91 S.L. Thompson, "CMP Code Maintenance Package, Reference Manual, Version 2.0" SAND85-0825, Sandia National Laboratories, Albuquerque, NM, 1991.

UPD84 "UPDATE Reference Manual," SR-0013, CRAY X-MP and CRAY-1 Computer Systems, CRAY Research, Inc., Mendota Heights, MN, 1984.

Was87 K. E. Washington, "POSTCON: A Postprocessor and Unit Conversion Program for the CONTAIN Computer Code," NUREG/CR-4887, SAND87-0562, Sandia National Laboratories, Albuquerque, NM, May 1987. 


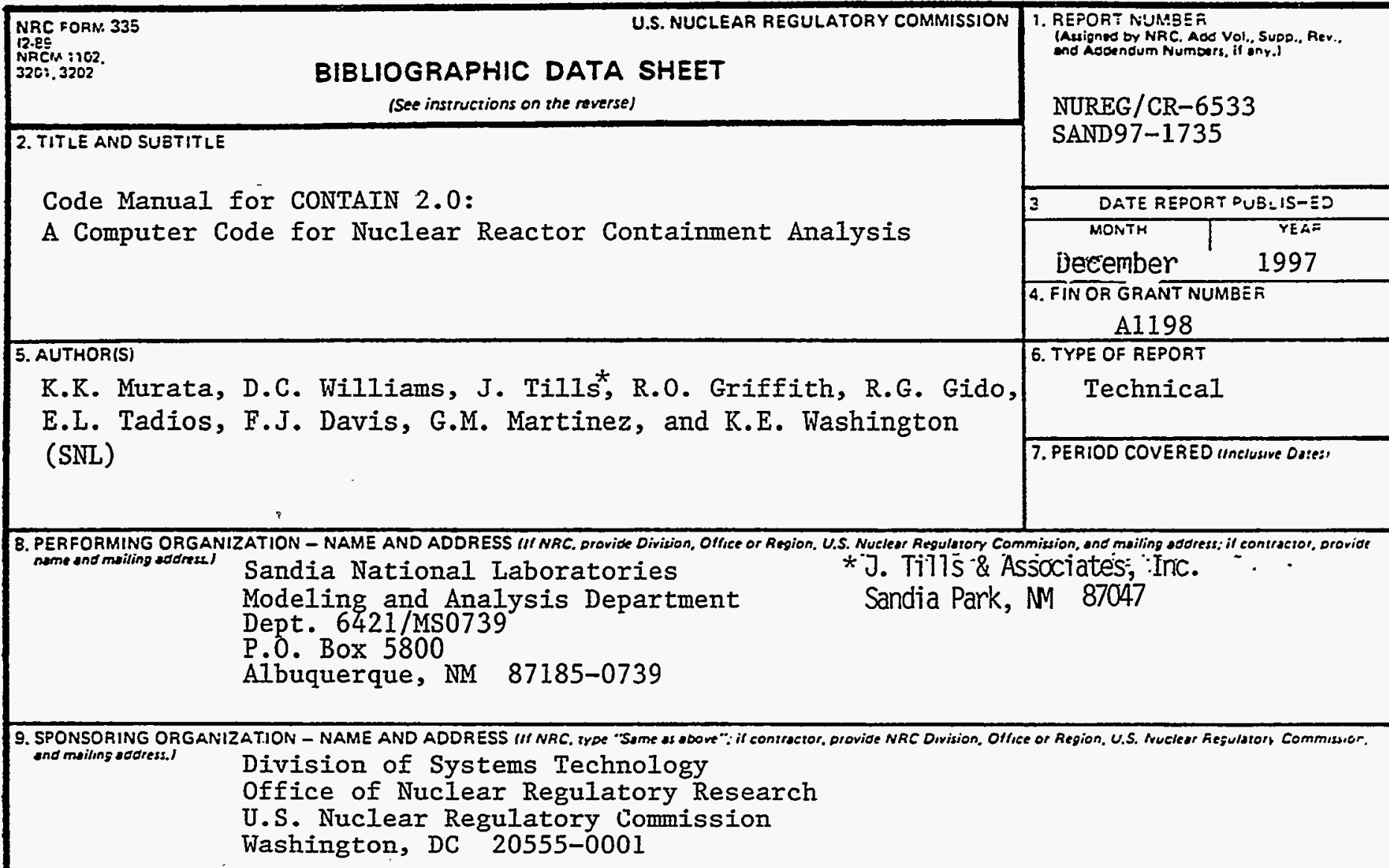

10. SUPPLEMENTARY NOTES

A. Notafrancesco, NRC Project Manager

11. ABSTRACT 200 wores or less)

The CONTAIN 2.0 computer code is an integrated analysis tool used for predicting the physical, chemical, and radiological conditions inside a containment building following the release of material from the primary system in a light-water reactor (LWR) accident. It can also predict the source term to the environment. The purpose of this Code Manual is to provide full documentation of the features and models in CONTAIN 2.0. Besides complete descriptions of the models, this Code Manual provides a complete description of the input and output from the code. The code includes atmospheric models for steam/air thermodynamics, intercell flows, condensation/evaporation on structures and aerosols, aerosol behavior, and gas combustion. It also includes models for reactor cavity phenomena such as core-concrete interactions and coolant pool boiling. Heat conduction in structures, fission product decay and transport, radioactive heating, and the thermalhydraulic and fission product decontamination effects of engineered safety features are also modeled. These models allow selected design basis and severe accidents to be analyzed, for both current and advanced LWR designs.

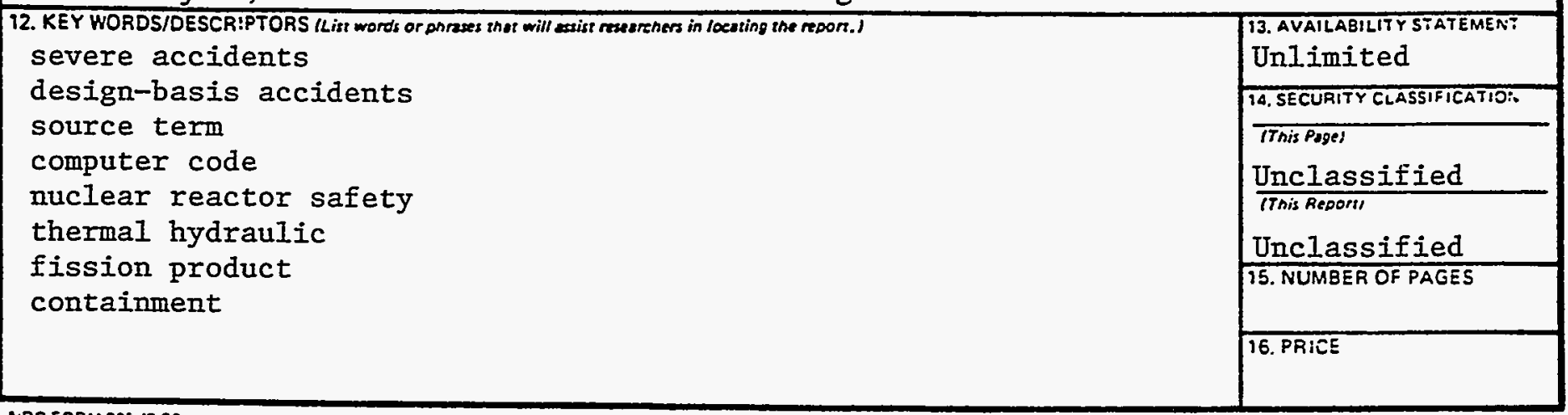

